\title{
Tools for investigating electronic excitation: experiment and multi-scale modelling
}

Edited by:

T. Apostolova J. Kohanoff N. Medvedev E. Oliva A. Rivera 



\section{Tools for investigating electronic excitation: experiment and multi-scale modelling}

Edited by:

T. Apostolova, J. Kohanoff, N. Medvedev, E. Oliva, and A. Rivera

Cover design, typsetting and layout: Ana Benítez Mayobre Published by: Instituto de Fusión Nuclear "Guillermo Velarde"

Universidad Politécnica de Madrid

First published in October 2021
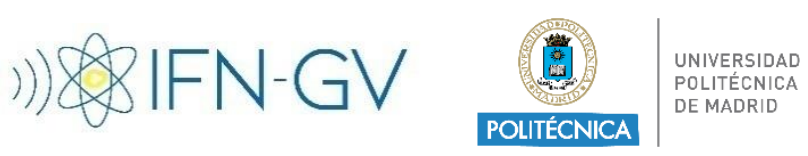
This book is based upon work from COST Action TUMIEE (CA17126), supported by COST (European Cooperation in Science and Technology).

COST (European Cooperation in Science and Technology) is a funding agency for research and innovation networks. Our Actions help connect research initiatives across Europe and enable scientists to grow their ideas by sharing them with their peers. This boosts their research, career and innovation. www.cost.eu
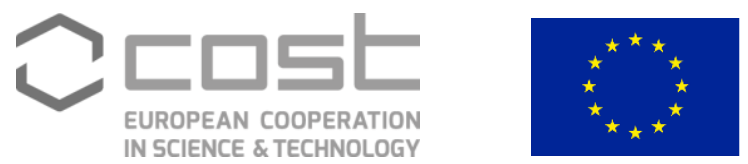

Funded by the Horizon 2020 Framework Programme

of the European Union

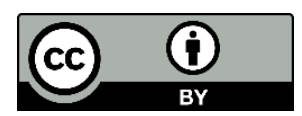

(C) The Editor(s) (if applicable) and The Author(s) 2020. This book is an open access publication.

Open Access This book is licensed under the terms of the Creative Commons Attribution 4.0 International License (http://creativecommons.org/licenses/by/4.0/), which permits use, sharing, adaptation, distribution and reproduction in any medium or format, as long as you give appropriate credit to the original author(s) and the source, provide a link to the Creative Commons licence and indicate if changes were made.

The images or other third party material in this book are included in the book's Creative Commons licence, unless indicated otherwise in a credit line to the material. If material is not included in the book's Creative Commons licence and your intended use is not permitted by statutory regulation or exceeds the permitted use, you will need to obtain permission directly from the copyright holder.

The use of general descriptive names, registered names, trademarks, service marks, etc. in this publication does not imply, even in the absence of a specific statement, that such names are exempt from the relevant protective laws and regulations and therefore free for general use.

The publisher, the authors, and the editors are safe to assume that the advice and information in this book are believed to be true and accurate at the date of publication. Neither the publisher nor the authors or the editors give a warranty, expressed or implied, with respect to the material contained herein or for any errors or omissions that may have been made. The publisher remains neutral with regard to jurisdictional claims in published maps and institutional affiliations.

The content of this publication and any liabilities arising from it remain the responsibility of the authors.

ISBN: 978-84-09-36032-1

DOI: 10.20868/UPM.book.69109 


\section{LIST OF CONTRIBUTORS}

\section{Tzveta Apostolova}

Institute for Advanced Physical Studies, New Bulgarian University, Bulgaria

Institute for Nuclear Research and Nuclear Energy, Bulgarian Academy of Sciences

\section{Emilio Artacho}

CIC nanoGUNE research center, Spain

\section{Fabrizio Cleri}

Institute of Electronics, Microelectronics and Nanotechnology, CNRS - University of Lille I, France

\section{Manuel Cotelo}

Instituto de Fusión Nuclear “Guillermo Velarde”, Universidad Politécnica de Madrid, Spain

\section{Miguel L. Crespillo}

Centro de Micro-Análisis de Materiales, Universidad Autónoma de Madrid, Spain

\section{Fabiana Da Pieve}

Royal Belgian Institute for Space Aeronomy (BIRA-IASB), Belgium

\section{Vasilis Dimitriou}

Institute of Plasma Physics \& Lasers (IPPL), Hellenic Mediterranean University, Greece

\section{Flyura Djurabekova}

Department of Physics, University of Helsinki, Finland

\section{Dorothy M. Duffy}

Department of Physics and Astronomy, University College London, United Kingdom

\section{Gastón García}

Centro de Micro-Análisis de Materiales, Universidad Autónoma de Madrid, Spain

\section{Mario Garcia-Lechuga}

Departamento de Física Aplicada, Universidad Autónoma de Madrid, Spain

Centro de Micro-Análisis de Materiales, Universidad Autónoma de Madrid, Spain

\section{Bin Gu}

Atomistic Simulation Centre, Queen's University Belfast, United Kingdom

\section{Thomas Jarrin}

CEA-DAM-DIF, France

LAAS-CNRS, Université de Toulouse, France 


\section{Evaggelos Kaselouris}

Institute of Plasma Physics \& Lasers (IPPL), Hellenic Mediterranean University, Greece

\section{Jorge Kohanoff}

Instituto de Fusión Nuclear "Guillermo Velarde", Universidad Politécnica de Madrid, Spain

Atomistic Simulation Centre, Queen's University Belfast, United Kingdom

\section{George Koundourakis}

Institute of Plasma Physics \& Lasers (IPPL), Hellenic Mediterranean University, Greece

\section{Natalia Koval}

CIC nanoGUNE research center, Spain

\section{Vladimir Lipp}

Center for Free-Electron Laser Science CFEL, Deutsches Elektronen-Synchrotron DESY, Germany

\section{Layla Martin-Samos}

CNR-INFM Democritos National Simulation Center, Italy

\section{Nikita Medvedev}

Institute of Physics, Czech Academy of Sciences, Czech Republic

Institute of Plasma Physics, Czech Academy of Sciences, Czech Republic

\section{Alejandro Molina-Sánchez}

Instituto de Ciencia de Materiales (ICMUV), Universidad de Valencia, Spain

\section{Daniel Muñoz-Santiburcio}

CIC nanoGUNE research center, Spain

\section{Samuel T. Murphy}

Department of Engineering, Lancaster University, United Kingdom

\section{Kai Nordlund}

Department of Physics, University of Helsinki, Finland

\section{Eduardo Oliva}

Instituto de Fusión Nuclear "Guillermo Velarde”, Universidad Politécnica de Madrid, Spain

\section{José Olivares}

Centro de Micro-Análisis de Materiales, Universidad Autónoma de Madrid, Spain

Instituto de Óptica, CSIC, Spain

\section{Nektarios A. Papadogiannis}

Institute of Plasma Physics \& Lasers (IPPL), Hellenic Mediterranean University, Greece 


\section{Andrés Redondo-Cubero}

Centro de Micro-Análisis de Materiales, Universidad Autónoma de Madrid, Spain

Departamento de Física Aplicada, Universidad Autónoma de Madrid, Spain

\section{Antonio Rivera}

Instituto de Fusión Nuclear “Guillermo Velarde”, Universidad Politécnica de Madrid, Spai

\section{Andrea E. Sand}

Aalto University, Finland

\section{Davide Sangalli}

Istituto di Struttura della Materia, Italian National Research Council (CNR), Italy

\section{Jan Siegel}

Laser Processing Group, Instituto de Óptica, CSIC, Spain

\section{Andrey V. Solov'yov}

MBN Research Center, Germany

\section{Ilia A. Solov'yov}

Department of Physics, Carl von Ossietzky Universität Oldenburg, Germany

\section{Johannes Teunissen}

Royal Belgian Institute for Space Aeronomy (BIRA-IASB), Belgium

\section{Elisa Vázquez}

Instituto de Fusión Nuclear "Guillermo Velarde”, Universidad Politécnica de Madrid, Spain

\section{Alexey V. Verkhovtsev}

MBN Research Center, Germany

\section{Sílvia Viñals}

Centro de Micro-Análisis de Materiales, Universidad Autónoma de Madrid, Spain

\section{María Dolores Ynsa}

Centro de Micro-Análisis de Materiales, Universidad Autónoma de Madrid, Spain Departamento de Física Aplicada, Universidad Autónoma de Madrid, Spain 



\section{Table of contents}

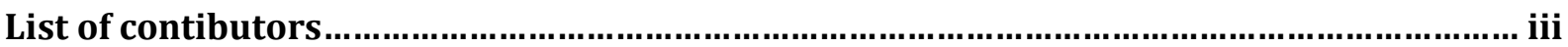

\section{SECTION I: INTRODUCTION}

1. COST Action TUMIEE (CA17126): this book

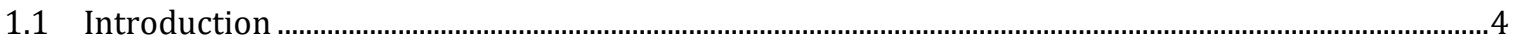

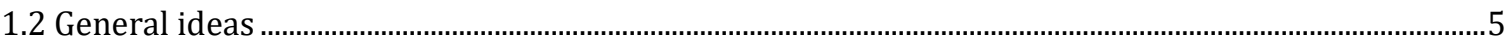

1.3 Modelling of irradiation phenomena: state-of-the-art....................................................................................

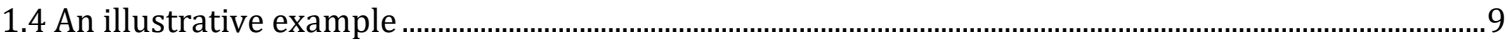

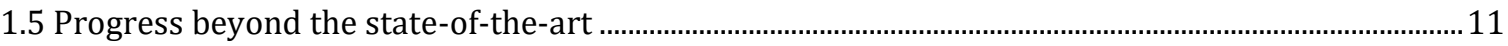

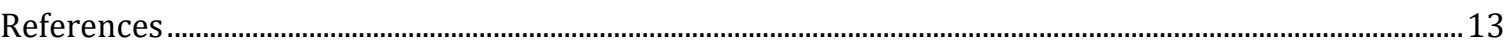

\section{SECTION II: EXPERIMENTAL METHODS}

2. Laser-matter interaction dynamics probed by time-resolved techniques..................... 19

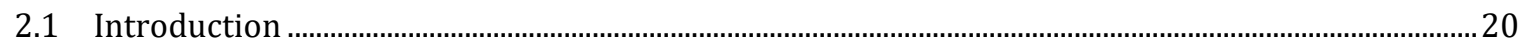

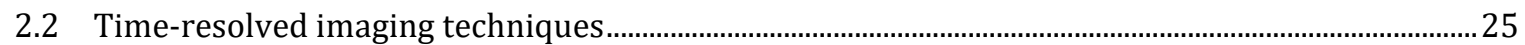

2.3 An experimental case study: visualizing bound and free electrons with time-resolved

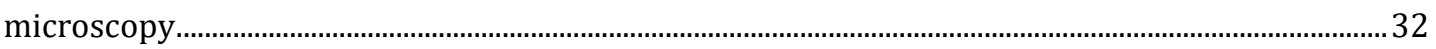

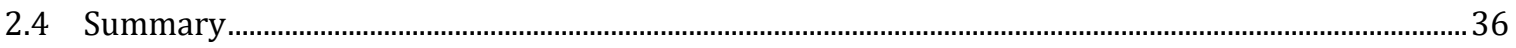

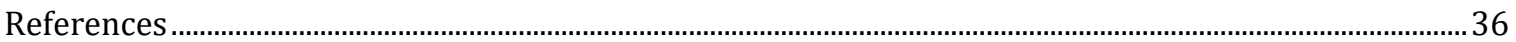

3. Ultrafast-laser high electronic excitation for nano-acoustic wave generation: new horizons in the materials probing

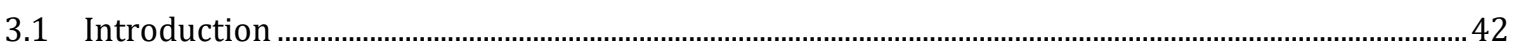

3.2 The role of the metals as optoacoustic transducers ………...................................................................... 43

3.3 Ultrafast longitudinal ultrasonic waves generated by ultrafast lasers ................................................. 48

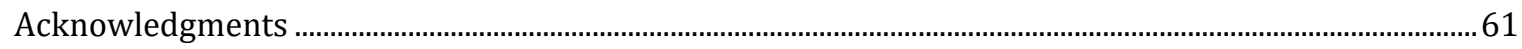

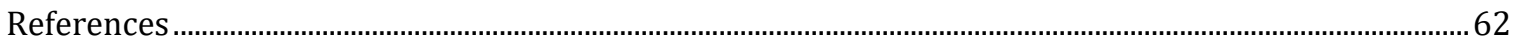

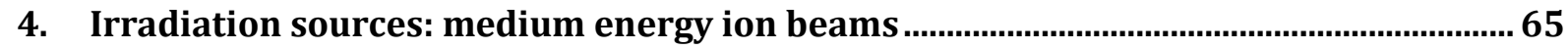

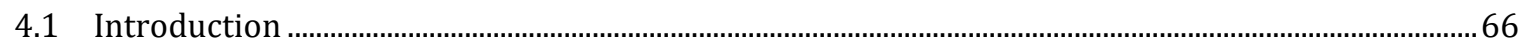

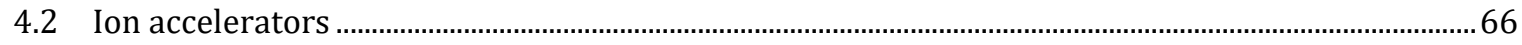

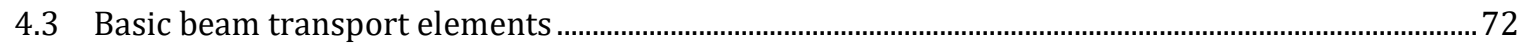

4.4 Some examples of ion-matter interaction...............................................................................................

4.5 Ion beam analytical techniques and experimental setup examples..........................................................90

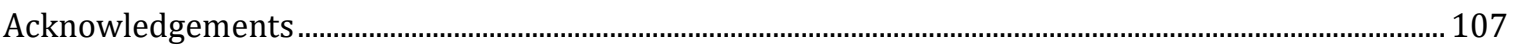

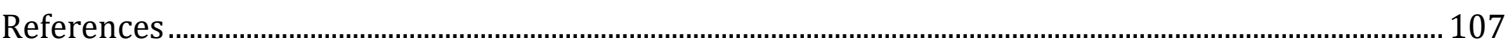




\section{SECTION III: SIMULATION METHODS}

5. Electron dynamics from first-principles ....................................................................117

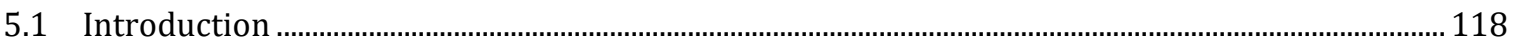

5.2 Ground state and excited states .............................................................................................................. 118

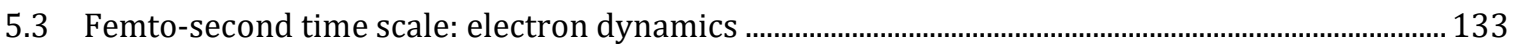

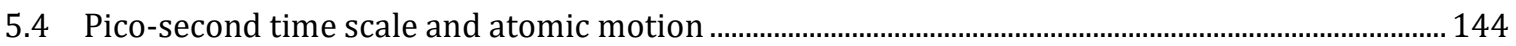

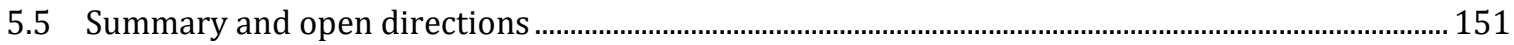

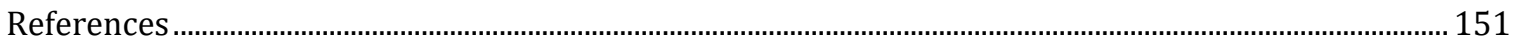

6. Quantum and semiclassical dynamics of semiconductors and dielectrics....................159

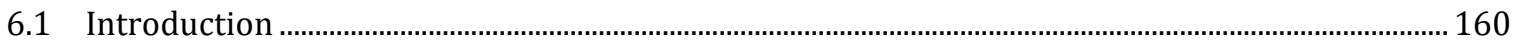

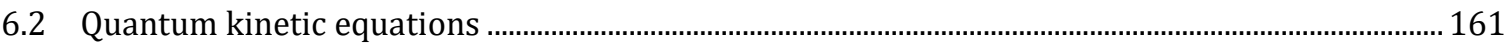

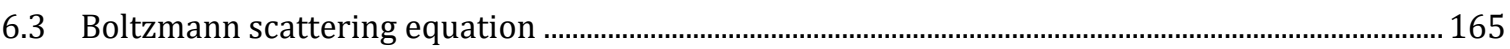

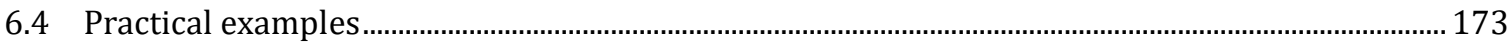

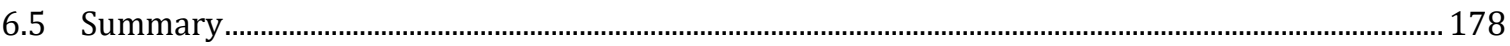

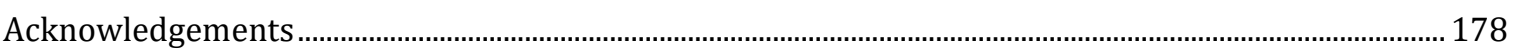

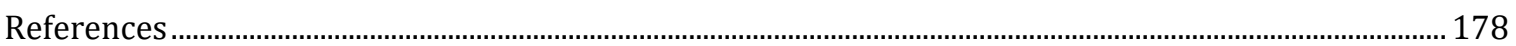

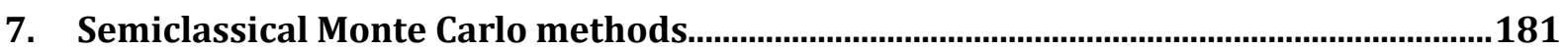

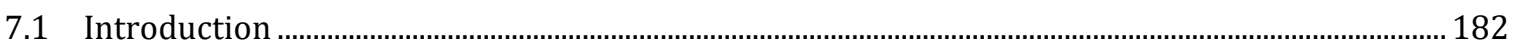

7.2 Core principles of Monte Carlo …………………………........................................................................... 183

7.3 Practical implementation details ............................................................................................................. 192

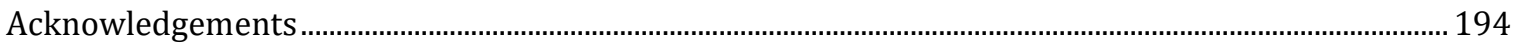

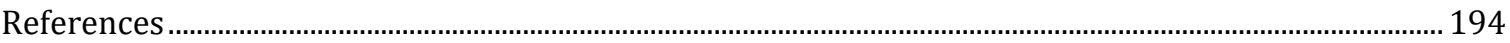

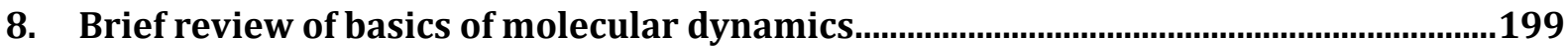

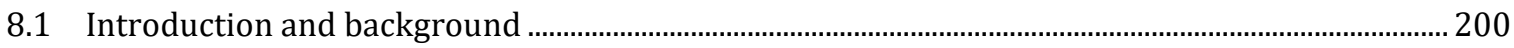

8.2 Basics of molecular dynamics simulations ............................................................................................ 201

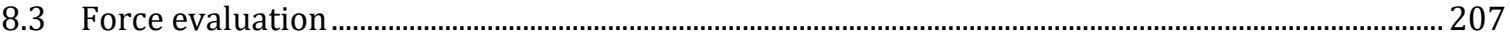

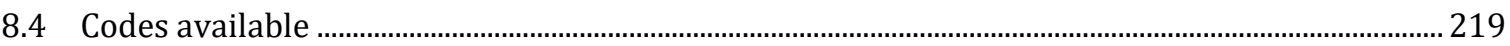

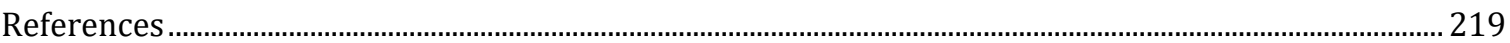

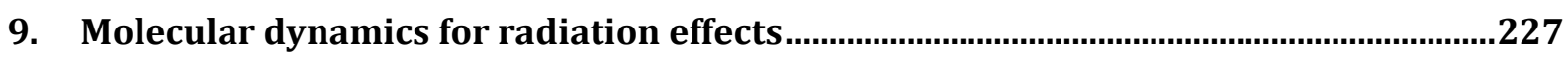

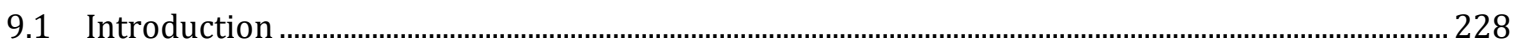

9.2 Molecular dynamics for collision cascades ...................................................................................... 228

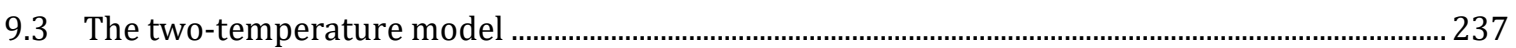

9.4 Molecular dynamics for swift heavy ion effects ................................................................................ 244

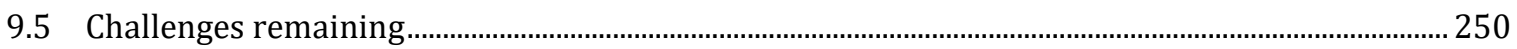

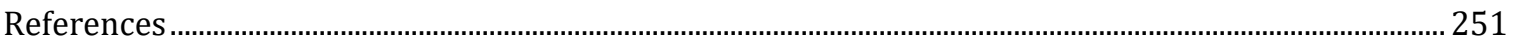




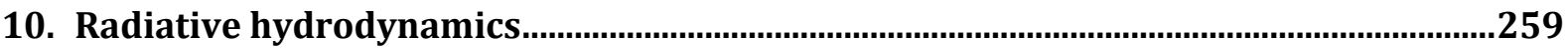

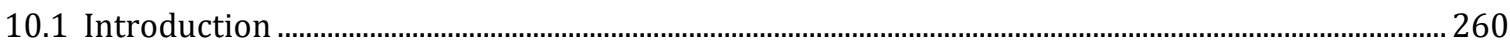

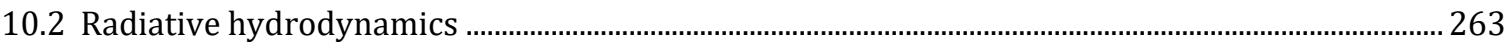

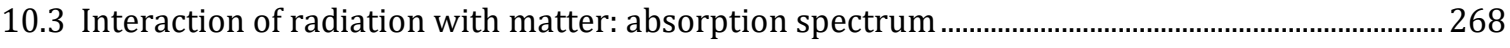

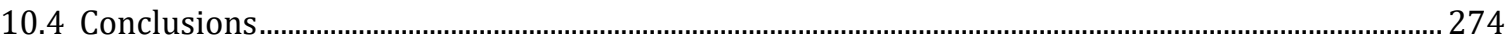

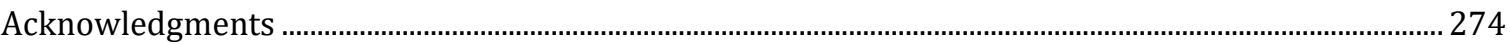

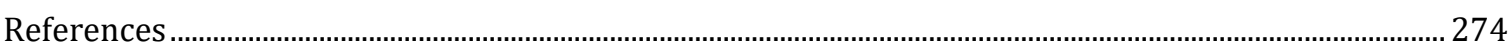

11. FEM Modeling and Simulations in Laser Matter Interactions and Plasma MHD

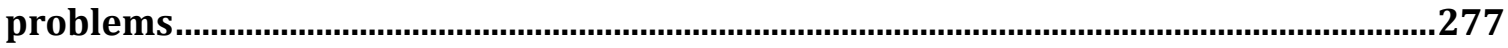

11.1 The finite element method............................................................................................................................ 278

11.2 Multiphysics FEM simulations for MHD analysis .................................................................................... 287

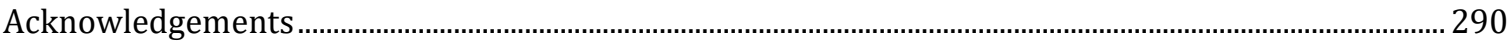

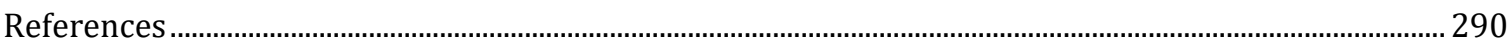

\section{SECTION IV: CASE STUDIES}

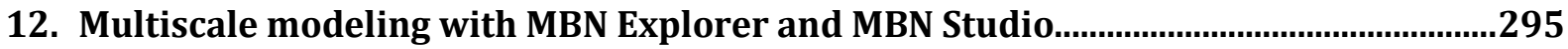

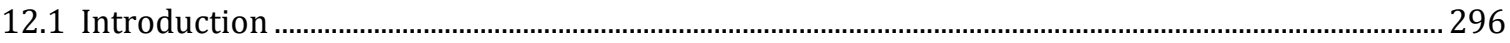

12.2 Computational approaches in Meso-Bio-Nano Science ……………………………………………....... 296

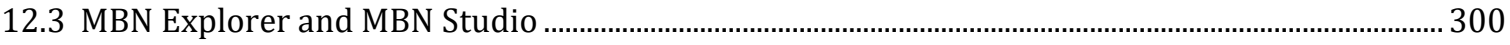

12.4 Computational modeling of novel crystal-based gamma-ray light sources ....................................... 302

12.5 Computational modeling of the focused electron beam induced deposition process ..................... 308

12.6 Computational modeling of ion-induced DNA damage in relation to ion-beam cancer

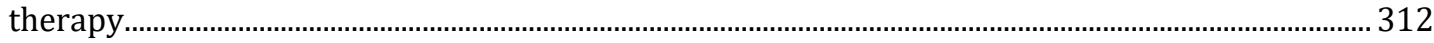

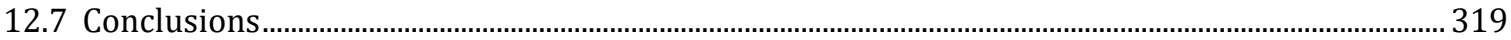

Acknowledgements .................................................................................................................................. 319

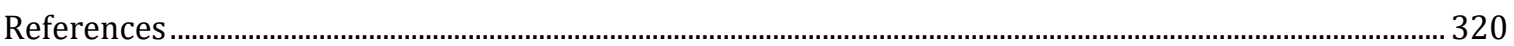

13. Irradiation driven molecular dynamics with reactive CHARMM force field.................325

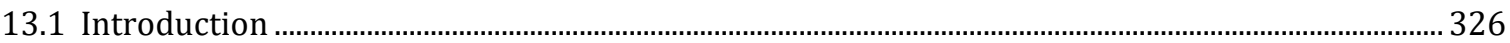

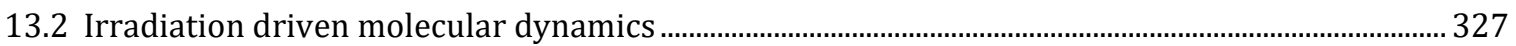

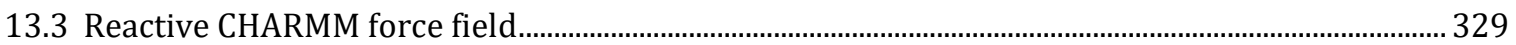

13.4 Multi-fragmentation of carbon fullerenes upon collision …………………………................................ 332

13.5 Transport of chemically reactive species around energetic ion tracks .................................................. 334

13.6 Atomistic simulation of the FEBID process ................................................................................................. 337

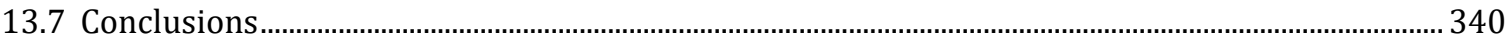

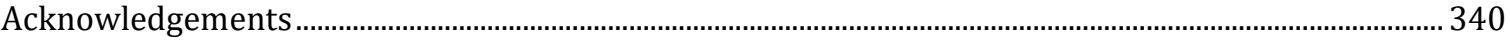

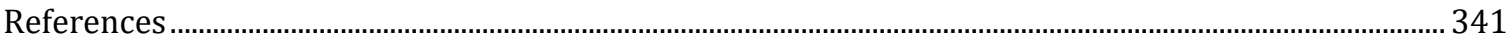


14. Fundamentals of Monte Carlo particle transport and synergies with quantum dynamics for applications in ion-irradiated materials in Space and radiobiology ....345

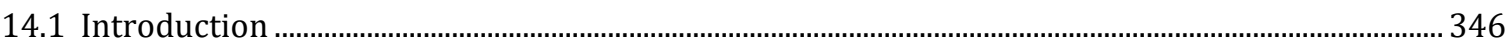

14.2 The Boltzmann equation .................................................................................................................... 349

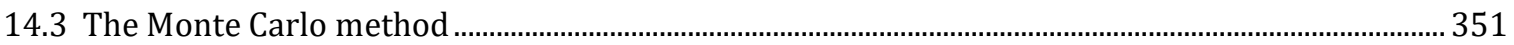

14.4 Examples of applications of Monte Carlo particle transport and synergies with quantum dynamics in the electromagnetic sector ............................................................................................ 358

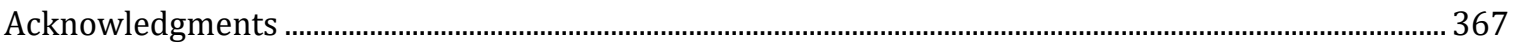

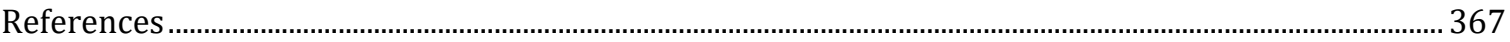

15. Combining Monte Carlo methods with other techniques …............................................375

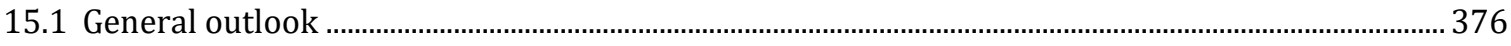

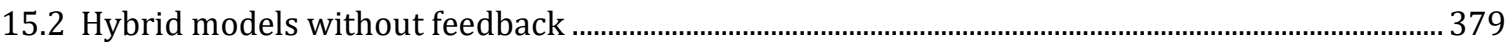

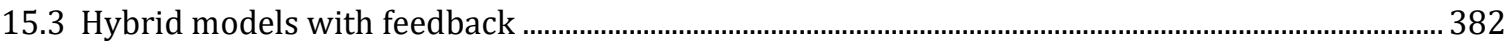

Acknowledgements ......................................................................................................................................... 387

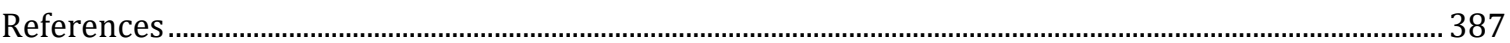

16. Molecular dynamics simulations of collision cascades in semiconducting

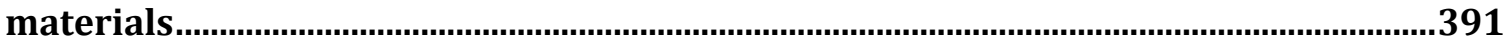

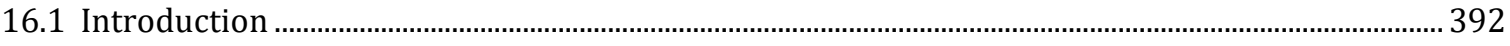

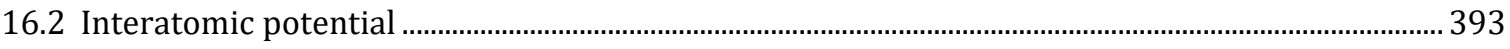

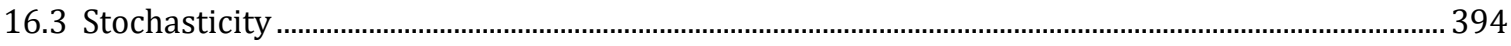

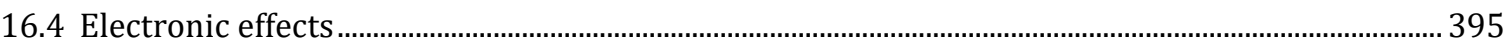

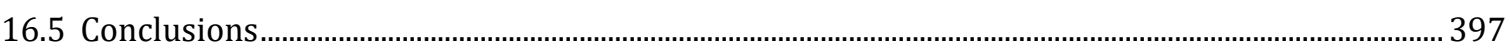

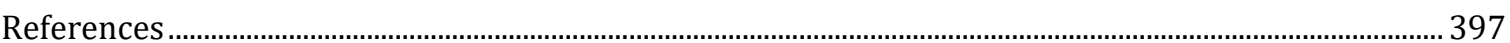

17. Modelling ion implantation ranges with $a b$ initio electronic stopping power ....401

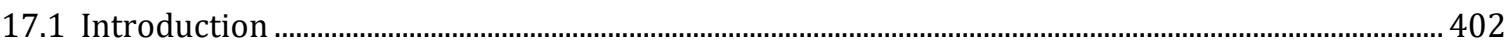

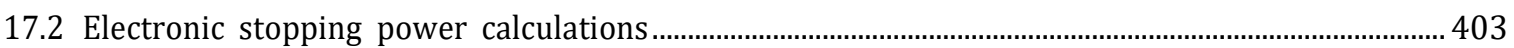

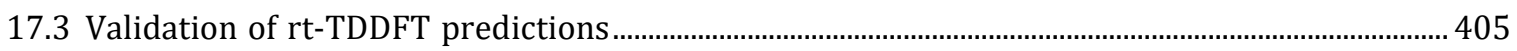

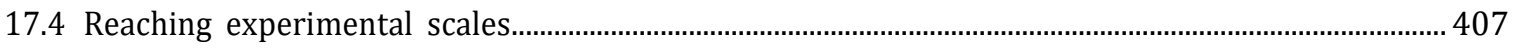

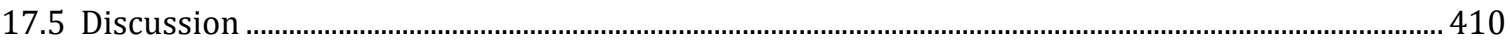

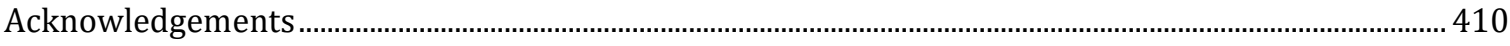

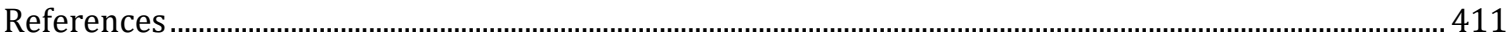

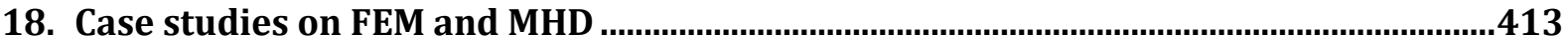

18.1 Laser matter interaction FEM simulations- Introduction using LS-DYNA/LS-PREPOST ............... 414

18.2 Laser matter interaction FEM simulations - Tensile stress \& thermal stress problem.................. 429

18.3 Laser matter interaction FEM simulations- Laser heating of a metal ................................................... 442

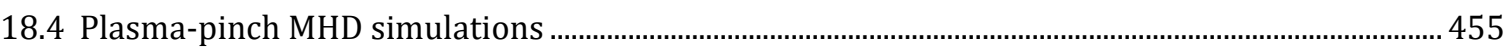

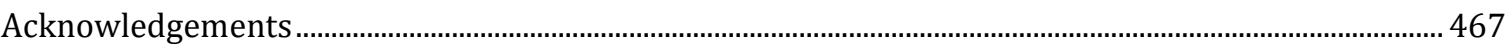


SECTION I

INTRODUCTION 



\title{
1. COST Action TUMIEE (CA17126): this book
}

\author{
Tzveta Apostolova, Jorge Kohanoff, Nikita Medvedev, \\ Eduardo Oliva, and Antonio Rivera
}

This adventure started with a CECAM Workshop organized by some of us in 2015 in Dublin. One of the recommendations that emerged from the Workshop was to propose a COST Action focusing on the theme of multi-scale modelling of intense electronic excitation, which we did in 2017. The proposal was approved, and the Action started in 2018 with a kick-off meeting in Brussels (Belgium), followed by meetings in Madrid (Spain), Porto (Portugal), Warsaw (Poland) and Marseille (France), several online workshops, and a Training School in Rethymno (Greece). Interspersed between these activities were a substantial number of short-term scientific missions in which scientists spend time at an institution in another country, carrying out collaborative research. This book collects the lectures presented in the school, supplemented with specific applications that illustrate how the multi-scale approach is implemented in specific cases of interest. The book is intended both as a reference in the field and as a textbook for people becoming interested or entering the field. The first part focuses on experimental methods, the second on theoretical approaches, and the third on cases of interest. In the rest of this introductory chapter, we set the scene by discussing some applications, the associated phenomenology, and the multi-scale paradigm. 


\subsection{Introduction}

Electronic excitation reaching high energy density is central in many different applications, from materials processing to medical treatments. It emerges when intense radiation arising from sources such as lasers, swift ions, or high-flux X-ray or electron pulses, interact with matter. Processes occurring in a target after irradiation span many orders of magnitude in space and time. It is currently impossible to treat them within a single rigorous approach. In general, only partial aspects related to the excitation produced by this type of sources are treated. The lack of a systematic methodology to face the simulation of the underlying phenomena makes it essential to involve scientists from different fields, theoreticians, simulators, and experimentalists. A successful methodology will require smart strategies to make existing solutions, which are appropriate within restricted scopes, work together within a multiscale formalism. COST Action TUMIEE (CA17126) was designed to tackle this challenge through the following approach:

1. Identify and propose experiments to validate simulations as an optimal way to generate progress in the field of intense electronic excitation. One may think this is obvious and simple. However, effects that depend on whether various processes are consecutive or coexistent (e.g., electron-electron and electron-phonon collisions), require dedicated time-resolved experiments to assess their role and the degree of coupling among them. Moreover, laser-matter interaction strongly depends on the structure of the target at the nanoscale.

2. Identify the specific role of different radiation sources on electronic excitation-induced effects. This requires connecting different communities that explore similar effects (e.g., intense laser and swift ion irradiation) to contribute to a better understanding of the general picture. It includes participation of experimentalists and theoreticians from different fields.

3. Identify strategies to connect computational methods on different timescales (see e.g. Figure 1.1 below). This is particularly important when the different methods do not run consecutively but simultaneously, overlapping over a certain period of time. This will be a central point of the project, since most methods operate reasonably well within their scope of applicability but their coupling to other approaches is not straightforward (e.g., Molecular Dynamics and Boltzmann transport equation).

4. Transfer the newly acquired knowledge to industry and societal applications.

A better understanding of phenomena induced by an intense initial electronic excitation is of interest for a broad range of disciplines: Solid-State Physics, Plasma Physics, Chemistry, Materials Engineering, Computational Science, Electronics, Photonics, Medicine, Astrophysics. The following are examples of straightforward applications that will benefit from progress in understanding intense electronic excitation: plume formation by intense laser irradiation, lasergenerated particle beams, planet and star core studies, medical applications such as hadron and $\mathrm{X}$-ray therapies, generation of high order harmonics, radiation detectors, transducers, materials 
processing with swift ions, intense lasers and plasma discharges, materials and devices for nuclear and space environments.

The field of intense electronic excitation turns out to have some peculiarities with respect to others. It is necessary to understand them to anticipate how the field will progress in the coming years.

(i) Multi-scale simulations of highly excited matter require the coupling of different methods (see Figure 1.1 below), which typically cover different time and space scales. The methods are relatively mature and have shown success when utilized within their natural scope of application. The major difficulty arises when trying to couple them.

(ii) A general solution is not expected. The goal is to apply multi-scale modelling to selected cases of interest.

Taking into account these points, one can realize that progress will be fast in some of the selected areas, but not in all of them. When the solution to a problem depends on different coupled codes that only slightly overlap in time and space (weak coupling), then the probability of success greatly increases. That is, when the fine details of the physical evolution at a particular time scale do not have a strong influence on the time scale of interest, then the development of a multi-scale approach is easier. In general, the fewer the number of involved codes the easier the implementation and, vice versa, the larger the number of involved codes and the stronger the coupling between methods, the harder the implementation.

\subsection{General ideas}

The central theme of this book is to describe methods to model irradiation processes across many space and time scales, where the initial stage of the phenomenon consists of intense electronic excitation and ionization. Depending on the material and conditions, excitations and ejected electrons may diffuse away from the region where they were created. In the case of ionization, electrons will leave positively charged ions that repel each other and can lead to a Coulomb explosion. Electronic excitations in semiconductors and insulators will form excitons that can also diffuse away. Alternatively, excitations and ejected electrons may remain in the vicinity, affecting the cohesion of the material and weakening chemical bonds. When this energy that has been transferred to electrons is deposited into vibrations via electron-phonon processes, it will induce a temperature increase that can lead, in a longer temporal and spatial scale, to mechanical and thermodynamic effects like swelling, fracture and phase transitions.

Electromagnetic radiation interacts with matter in different ways depending on its energy (or wavelength). The photon energy should match typical energy differences between levels in the target. For example, infrared radiation, with energy in the order of tens of meV, will typically interact with atomic vibrations. Lower energies like microwaves, will interact with rotational degrees of freedom. Visible light, with energies starting at $1.6 \mathrm{eV}$ in the red, are too energetic to interact with vibrations. At these energies and above, covering the whole range of UV, X-rays, and up to the $\gamma$ regime, radiation interacts with electrons. It can excite electrons or, if sufficiently energetic, it can remove them altogether ionizing the sample. 
A second important aspect is the intensity of the radiation, arhow many photons arrive per unit time. Low-intensity visible or infrared radiation will take the electronic system slightly out of equilibrium, so that the ensuing electron dynamics is characterised by small oscillations and photon emission. This is the basis of optical pump-probe experiments, which are reviewed in Chapters $\mathbf{2}$ and $\mathbf{3}$, within the context of imaging. Intense electronic excitation like the one produced in free electron lasers (FEL) can lead to non-linear effects photoionizing the sample and producing highly-charged ions like $\mathrm{Xe}^{+21}$ [1]. In a similar vein, multi-photon absorption, a non-linear electronic excitation effect, is at the root of high-harmonic generation $[2,3]$ which has been instrumental in producing attosecond, soft X-ray pulses to study electron dynamics with unprecedented resolution [4]. Similarly, soft X-ray intense radiation can make a metal look like an insulator and induce melting without heating, due to the weakening of interatomic interactions upon excitation. This has been observed, e.g., in $\mathrm{Al}$ [5].

On the other hand, when an energetic ion travels through matter it generally slows down by continually transferring energy to both the electronic and nuclear degrees of freedom of the target [6,7]. Ion beams as irradiation sources are discussed in Chapter 4. We consider ions as classical particles, which is justified as long as their velocity and/or mass is not too low (e.g., electrons with kinetic energies below $20 \mathrm{eV}$ will not fall within this category). Therefore, at low projectile velocities we have to consider the collision between the projectile and atomic nuclei. This is a very well-studied classical collision problem [8]. The energy deposition spatial rate, i.e., the nuclear stopping power, increases with the projectile's velocity, peaks, and then decays at high velocities because the nuclei in the target cannot respond as rapidly. Still, there is a non-zero probability that energetic ions interact directly with the atoms in the system, knocking them out of place and generating defects. As in a billiard, the displaced atoms go on to hit other atoms, and so on generating what is known as a radiation cascade. If the velocity of the secondary or tertiary atoms in this cascade is large enough, they can also produce electronic excitation. However, when the projectile reaches low enough velocities, the probability of exciting electrons becomes vanishingly small.

In the case of metals, the energy deposition rate on electronic degrees of freedom increases linearly with velocity. For semiconductors and insulators, the finite band gap may introduce a threshold for electronic excitation, although this is currently under debate. Similarly, to the case of nuclear stopping, the energy spatial deposition rate onto electronic degrees of freedom, i.e., the electronic stopping power, increases with the velocity of the ion, peaks at a velocity comparable to that of the valence electrons, and then decays like $v^{-2}$ until relativistic effects kick in $[9,10]$. Hence, the location of the peak in velocity is determined by the electronic properties of the target. The location in energy, however, is determined by the mass of the projectile via a kinetic conversion. If the peak for protons is located at $100 \mathrm{keV}$, for electrons it will be located at around $50 \mathrm{eV}$. As for photons, depending on intensity, ions can induce temporary opacity in insulators returning then to their original state [11], or can produce unrecoverable damage in the material if the intensity is too high as, e.g., in nuclear reactor pressure vessels [12] or in biological matter [13].

\subsection{Modelling of irradiation phenomena: state-of-the-art}

An overwhelmingly large fraction of the theoretical work aimed at describing the properties of materials makes use of the Born-Oppenheimer (adiabatic) approximation, which assumes that 
electrons follow a "slave" dynamics dictated by the motion of the nuclei, where transitions between electronic states are not allowed. The treatment of non-adiabatic effects, i.e., electronic excitation and decay, requires a different approach. In recent times, various methodologies have been developed and applied to study interesting phenomena. Perhaps the simplest one is TimeDependent Density Functional Theory (TDDFT) [14,15] combined with the Ehrenfest approximation for the nuclei [16]. While electronic excitation processes are captured correctly by this approach [17], the mean-field character of Ehrenfest distorts the characteristics of energy transfer from electrons to phonons, e.g., Ehrenfest cannot describe properly ubiquitous phenomena like Joule heating [18]. A second limitation is related to the exchange-correlation approximations used in TDDFT. In most cases, these are semi-local in space (GGA) and local in time. Therefore, memory effects in the electronic evolution are ignored, thus leading to another host of pathologies, mainly related to the lack of electronic decoherence (no electron-electron collisions term) $[19,20]$. In addition, incoherent electron-phonon scattering by ionic vibrations, which are not explicitly represented in classical molecular dynamics, are not accounted for in TDDFT calculations [21]. While Ehrenfest dynamics is straightforward to implement and computationally efficient, it is not trivial to go beyond it.

To improve on the electron-nuclear correlation, a possibility that has attracted interest amongst chemists is "surface hopping" [22]. In this method the forces on the nuclei are determined from single electronic potential energy surfaces (PES) but hops between surfaces are allowed to include non-adiabatic effects. Surface hopping works reasonably well when non-adiabatic transitions occur between a small number of PES [23], but not for a dense manifold of excited states. Perhaps the most sophisticated way to go beyond Ehrenfest in a controlled manner is the Correlated electron-ion dynamics approach (CEID) [24]. CEID relies on expansions of the quantum Liouville equation for the electron-nuclear system, with different formulations proposed in the limits of weak [25] and strong [26] electron-nuclear coupling. It has also been implemented in a similar spirit to quantum chemistry methods in electronic structure. Although CEID is still rather young and costly, it has emerged as a powerful tool for problems in which the transfer of energy among electrons and nuclei is crucial. Simulation methodologies for correlated electron and electronnuclear dynamics will be discussed at length in Chapter 5.

On the electronic front, a possible way forward is to start from the very general equations of nonequilibrium quantum statistical mechanics, e.g., the Balescu-Resibois formalism [27] or the timedependent non-equilibrium Green's functions approach (NEGF) [28-30]. When applied to intense laser irradiation of matter, this ambitious program may in principle describe both the coherent and incoherent interaction of electrons with sub-picosecond optical pulses as well as spatially inhomogeneous particle (e.g., ion) irradiation. Calculating the sub-picosecond kinetics of observable quantities entails the solution of the Green's function (Kadanoff-Baym) equations of motion and leads to extremely arduous numerical computations when correlations and screening are included [31]. None of these approaches is capable, with present day computers and algorithms, of reaching time scales beyond a few femtoseconds (maybe up to picoseconds if using simplified electronic structure methods like tight-binding), since the time step (typically around an attosecond) is dictated by the time-dependent electronic Schrödinger equation. The electron dynamics following electronic excitation, however, develops in a longer time scale where processes like electron-hole recombination, electron-phonon interactions and diffusion become significant. These time scales require a different theoretical approach. 
This is the realm of kinetic models, where one starts from Boltzmann-like kinetic equations and develops complex, yet tractable numerical schemes. Kinetic models like the Master and Boltzmann's equations can be formally derived from the theory of non-equilibrium Green's functions above, within the framework of the so-called quasi-particle approximation. These, however, are limited to the semi-classical regime, i.e., when the electron-hole plasma dynamics is controlled by collisions (Markovian) rather than by coherent quantum effects [32]. This condition is often satisfied in the sub-picosecond regime, with the mean time-independent collision rates calculated via Fermi's golden rule. Instead of tracing the motion of the individual particles, one follows the time evolution of the particle distribution function in phase space, starting from the Liouville equation. This approach may be useful in describing photo-induced phase transitions, where atomic transformations are driven on a ps timescale [33]. Simplifications of the latter lead to the BBGKY hierarchy [34], Boltzmann's kinetic equation [35], and the Fokker-Planck equation [36]. When Markovianity is not fulfilled, the semi-classical approach no longer applies and a full quantum approach based on the evolution of the density matrix (quantum Liouville equation) should be used [37]. This is the scope of Chapter 6.

An alternative approach to Boltzmann equations is to describe the semi-classical carrier dynamics according to Langevin equations and then solve them by Monte Carlo methods [38]. Transport Monte Carlo method trace propagation of individual particles, sampling occurring events with help of random numbers. It results into a probabilistic approach, delivering statistical results such as particle distributions. These methods are in principle equivalent to directly solving the Boltzmann equations, with certain advantages of being an integral instead of a differential method, having a reduced cost and an easy parallelization. They are reviewed in Chapter 7.

Simulations of ion-solid interactions have traditionally been driven by state-of-the-art molecular dynamics (MD) simulations based on the Born-Oppenheimer approximation and using empirical potentials that have limited realism and accuracy. Electronic energy losses are generally not included, and the effects of electron-hole pair production and charge-density redistributions are ignored. To go forward in this direction and access the ps-ns regime one needs to incorporate electronic energy losses in MD simulations. This has been done based on the Langevin equation, where the electronic losses are taken care of by a friction term and then this energy is redistributed to the atomic system via a random force [39]. The most recent works in this area consider friction forces whose direction and magnitude depend on the local, time-dependent environment [40], or simplified descriptions of the environment in which a structured bath coupled to the nuclear motion via a Generalized Langevin equation [41]. Molecular dynamics simulations, in equilibrium, and applied to the irradiation scenario are discussed in detail in

\section{Chapters 8 and 9.}

If the intensity of the radiation is high enough, it will generate a large density of highly excited electrons. Some of these electrons will be energetic enough to become free and ionize further electrons. This cascade will lead to the development of a plasma, a quasi-neutral medium composed of electrons and ions, among other species. Following all the processes that take part in plasma dynamics is a complex multiscale, multiphysics problem. Free electrons collide with ions in picosecond time scales, exciting and ionizing them. Atomic physics codes can model these phenomena by using different approaches like rate equations or Fokker-Planck. Further propagation of the intense laser through the plasma and its interaction with free electrons, spanning timescales from femtoseconds to tens of picoseconds or even nanoseconds can be 
modelled using Particle in Cell (PIC) codes. In longer timescales (nano- to microseconds), the plasma will expand (evolve) following hydrodynamic equations. All these processes must take into account the mutual interaction with the radiation field, that can be critical when tackling detailed atomic processes or plasmas created inside höhlraums, for example. These aspects are discussed in Chapter 10. Alternatively, laser-matter and plasma MHD problems can be treated using finite element simulations, as discussed in Chapter 11.

\subsection{An illustrative example}

We consider an example related to the development of the particle detectors for future hadronic machines, such as the High-Luminosity Large Hadron Collider (HL-LHC) at CERN. The major challenge of designing the detectors is the reconstruction of the trajectories of an unprecedented density of charged particles. State-of-the-art spatial resolution in the measurement of the energy deposition is necessary to distinguish the tracks, together with a good time resolution to distinguish the many hadronic collisions. The design and the fabrication of timing 3D diamond sensors, with electrodes obtained through a pulsed laser graphitisation technique and improved by the application of adaptive optics to reduce the electrode resistance has been achieved recently [42]. A Ti:Sapphire femtosecond laser source was used to induce a transformation of the carbon phase from diamond to a mixture of $\mathrm{sp}^{2}$ and $\mathrm{sp}^{3}$ phases which is sufficiently conductive to behave as a resistive electrode buried into the diamond. To engrave electrodes, extremely short laser pulses are preferred to make the non-thermal multi-photon interaction mechanism dominant and to obtain thinner and more conductive electrodes. In this way the influence of the crystal defects as well as the charge collection times are substantially reduced resulting in some of the fastest and radiation hardest radiation sensors ever fabricated.

The effect of laser processing parameters on the properties of graphite wires formed inside synthetic mono- and polycrystalline diamonds is of great importance [43]. Wires were fabricated in CVD diamond samples using different light polarizations, processing speeds and laser pulse energies. A comparison of their quality was made by measuring their electrical resistance and radius. For many of the columns a barrier potential was observed. The output current was negligible until the applied voltage reached a threshold value. For values of the applied voltage exceeding the threshold voltage the wires exhibited ohmic behavior [43]. The properties of the wires were almost independent of the polarization of the laser beam. The correction of the optical aberrations by adaptive optics, aimed to control the laser spot size during fabrication made a great impact on the quality of the formed wires. Applying aberration correction resulted in a negligible barrier potential and a minimum value of the wire resistance.

Below we describe, in this specific example, how the various physical phenomena relate to the diagram in Figure 1.1. 


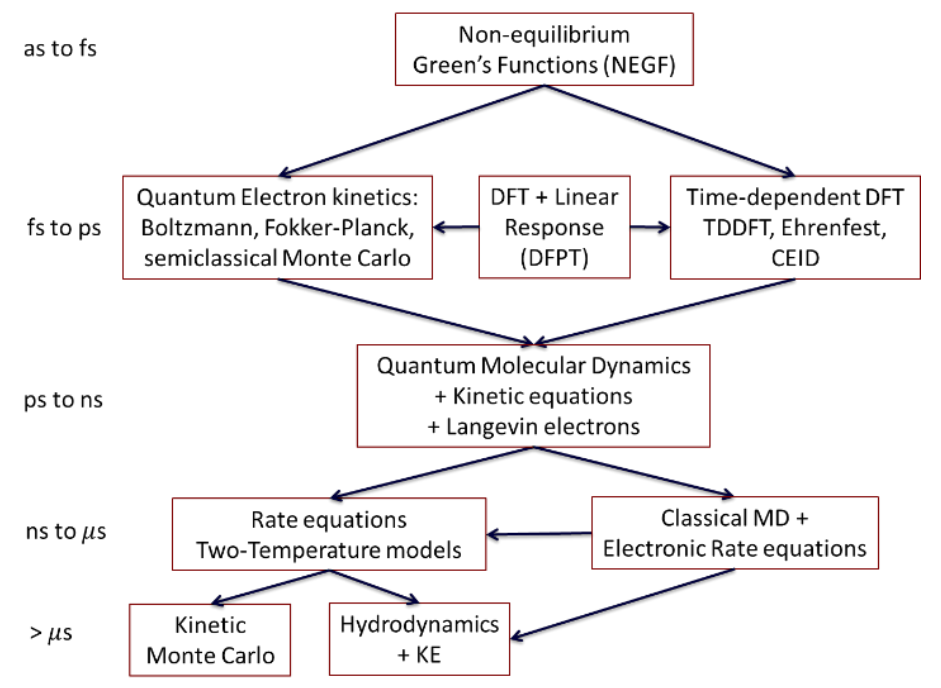

Figure 1.1: A possible hierarchy of theoretical and computational methods to implement a multi-scale modelling approach to materials irradiation.

The mechanisms involved in the laser engraving of electrodes in diamond are essentially governed by femtosecond pulsed laser-electronic system interaction that can trigger ultrafast optical and material response. More elaborate first principles approaches based on many-body perturbation theories provide an ab initio description of both the photo-excitation and of the time dependence of the carrier scattering rates. They allow for the inclusion, on the same footing, of the evolution of carrier and phonon populations due to both excitation and scattering which, for the case of ultrashort laser pulse excitation, may overlap in time. Recently a first-principles version of the Kadanoff-Baym equations [44] employing the collision approximation [45] to reduce the non-equilibrium Green's function dynamics to a single time variable was used to reproduce the carrier dynamics in bulk silicon. The equation for the evolution of the nonequilibrium electron (hole) occupation contains a coherent generation term, which describes the creation of carriers via the interaction of the polarization and the laser electric field, and a term describing how carriers dissipate energy and scatter with phonons and electrons. This equation is coupled to the polarization equation self-consistently and the generation term is derived from Green's functions approach based on DFT Kohn-Sham Hamiltonian and includes Hartree and exchange correlation effects. The expressions for carrier-carrier and carrier-phonon lifetimes that govern the carrier relaxation include the statically screened Coulomb interaction and the screened ionic potential derivatives, calculated within density-functional perturbation theory [46] and formally correspond to the carrier-carrier and carrier-phonon scattering in the semi classical Boltzmann equation obtained using Fermi Golden Rule. Graphitization requires changes in the bonding characteristics of the material $\left(\mathrm{sp}^{3}\right.$ to $\left.\mathrm{sp}^{2}\right)$ driven by the motion of the $\mathrm{C}$ atoms following irradiation. If a substantial fraction of electron-hole pairs is generated in the material, there is a strong modification of the electron density distribution. Consequently, the ions experience strong repulsive inter-atomic forces due to the dramatic changes of the potential energy surface (PES). The functional form of the potential energy surface (PES) strongly depends on the occupation of the electronic levels. The modified interatomic forces can cause coherent atomic motions. If they are sufficiently strong, they can induce permanent structural modification of crystalline diamond on a very short time scale [47] without invoking significant energy transfer between electrons and ions and without significant increase of the ionic temperature. Hence, the ultrafast disordering can occur as a nonthermal phase transition [48]. Ab initio molecular 
dynamics based on finite-temperature density functional theory uses the calculated rearrangement of a material's electron density during laser irradiation to determine the modified nonthermal forces on the atoms of the crystal lattice. This leads to coherent atomic motions resulting in structural phase transformation on very short time scales [49]. Alternatively, first principles simulations can be performed using the quantum mechanically-calculated stress tensor [50] to determine the free energy difference between the two phases of the material [51]. Nonthermal graphitization due to interatomic bond breaking induced by electronic excitation may occur at sub-picosecond timescale, as illustrated in Chapter 15. In case of thermally induced graphitization, this phenomenon occurs in the pico- to nanosecond timescale, which is just about accessible to the methods in the previous timescale. Ehrenfest molecular dynamics (EMD), which involves electronic excitations, can hardly reach the ps. Ground state ab initio MD can access the sub-nanosecond scale, but does not include electronic excitations. Therefore, a methodology is required that includes electronic excitations within a framework of classical force fields. This can be achieved through Langevin dynamics for the atoms coupled to a diffusion equation for the electronic temperature. Such Two-Temperature Models coupled to MD (TTMMD), assume that electrons are always in thermal equilibrium. Moreover, this can be supplemented with electronic temperature-dependent force fields that take into account that electronic excitations weaken interatomic bonds. It is, however, unclear whether electrons are effectively in thermal equilibrium, and this will again depend on the characteristics of the excitation, e.g., whether a fs-, ps- or a ns-laser is used.

Nowadays, permanent modification of materials is described to a large extent through such phenomenological TTM, which depend on a number of empirical parameters, calibrated from experimental data, like the size of the damaged zone. Can we obtain such parameters using the multi-scale approach? Moving forward, if the laser field is intense enough (over $50 \mathrm{TW} / \mathrm{cm}^{2}$ ), it will generate a large density of highly excited electrons that will develop into a plasma. Further interaction of intense laser with the plasma can be modelled using Particle in Cell (PIC) codes. This plasma will expand (evolve) in the nano- to microsecond timescale following hydrodynamic equations. This evolution is driven by the kinetic energy stored in the electron fluid, but part of this energy is also used to heat the lattice as described in the previous paragraph. Therefore, there is again a superposition of time scales, requiring a description where plasma and lattice evolution may not be separable. This also involves spatial scales far larger than before, in the order of the micrometres. Classical MD simulations can reach up to a billion atoms, i.e., about $100 \mathrm{~nm}$ as a linear dimension. Therefore, again simpler models are required. One possibility is twotemperature models, which describe the coupled evolution of the two continuous temperature fields, electronic and nuclear, in time. Any of these models, PIC, hydrodynamics, TTM, or kinetic Monte Carlo, will require input in the form of equations of state, transport coefficients, etc., as well as validation against explicit MD methodologies.

\subsection{Progress beyond the state-of-the-art}

Many of the points raised in Section 1.3 have, in fact, a widely open end. This concerns all kinetic models and stochastic descriptions, particularly when dealt with at a quantum-mechanical level. The present methodologies (electronic friction, Langevin equation, random forces, etc.) are limited to relatively weak intensities. An aspect that is often ignored is that, under intense electronic excitation, the interatomic forces are weakened and thus the empirical potentials 
should be modified [52]. Therefore, there is a need for improvement in this classical description, which manifests itself more clearly in the extreme excitation regime. This point is discussed in Chapter 8. Damage models based on SRIM tables proved inaccurate when swift heavy ions (e.g. $\mathrm{Br}$ ) are used to irradiate semiconductors (e.g. SiC) [53]. Connecting the electronic dynamics at the NEGF/TDDFT and/or kinetic equations' level with the lattice evolution scale is one of the key outstanding problems in the field.

Distribution functions obtained either via kinetic equations or MD simulations can then be used to compute macroscopic observables by means of thermodynamic relations. While equilibrium thermodynamics is a well-established discipline, its time-dependent non-equilibrium counterpart is still under development. Therefore, extracting thermodynamic relations where neither particles nor their distributions enter the equations is very relevant. In fact, these methods may allow us to trace changes in the experimentally observable values (temperature, pressure, volume) without considering the detailed description of processes occurring with individual particles [54]. The main advantage is the computational cost that can be decisive for many applications of interest.

Finally, methods usually employed in plasma physics, namely, hydrodynamic equations, may turn out advantageous for a number of situations in which long term evolution of highly ionized matter upon certain initial conditions play an important role, e.g. plume production and evolution. This will require putting on firm grounds the connection between kinetic and hydrodynamic equations [55].

The state-of-the-art in modelling intense electronic excitation consists of a variety of computational methods, many of them mature enough to provide accurate results within their scope of application. However, in general the effects produced by this type of electronic excitation extend to different timescales and evolve in large space regions. In order to provide quantitative results, one must develop a multi-scale methodology. Similar multi-scale paradigms have been developed and applied to a number of studies in the irradiation of solids, e.g., within the nuclear materials or the biological or soft matter contexts, using different methods for different scales: DFT, MD, transport Monte Carlo, Coarse graining, Kinetic Monte Carlo, Dislocation theory, Continuum theory.

The multi-scale approach has not been fully realized yet in the field of highly excited matter. The difficulty stems from the need to follow the electronic evolution of the highly perturbed, nonequilibrium system, and to propagate this information to other scales by coupling various methodologies designed for different goals. Therefore, any such coupling strategy is unique and beyond state-of-the-art. This is precisely the merit of this COST Action, where a large effort is devoted to couple different computational methods involving highly excited electrons, with the aim of solving relevant problems, of scientific or industrial impact.

As an example to facilitate the understanding on the important role of coupling, we discuss some strategies to tackle the laser-irradiated diamond case described in Section 1.4, going beyond the state-of-the-art by developing interfaces between codes. Quantum mechanical effects are essential for the correct description of early stages of photoionization and photoexcitation on a femtosecond time scale beyond the conventional Keldysh approach. They can be incorporated via ab initio approaches (TDSE, TDDFT). The coherent connection between the generated charged carriers and the irradiating laser field is given by the laser-induced polarization. The dephasing of 
the polarization is caused by inter-band electron-electron interaction (leading to impact ionization and Auger recombination) and by electron-phonon scattering. These processes can be described by interfacing the TDSE with Boltzmann quantum kinetic equation by solving them selfconsistently. The photoionization term from the first principles calculation can be substituted into the kinetic equation containing the collision integrals. Depending on the pulse duration a nonthermal melting of the material can take place in which the energy absorbed into the generated electron-hole pairs will not cause thermal effects but rather change the bonding strength of the lattice ions due to high density of the generated carriers [46]. For longer pulse durations or after the end of the pulse the energy deposited into the electron system will be transferred to the phonon modes of the lattice via electron-phonon interactions, thus effecting the lattice temperature. The time evolution of the thermal energy deposition in the lattice and the space distribution of the lattice temperature can be modeled by interfacing Boltzmann quantum kinetic equation with Molecular Dynamics simulation. This requires considering all the energy transfer channels to the lattice (not only electron-phonon coupling) and a way to include the transferred energy as lattice energy in MD. A continuous feedback from MD (temperature, atomic density, defect structure) must be passed back in each iteration step to the Boltzmann code [56]. This way the structural changes in the material due to dissipation of the absorbed energy that causes changes into the lattice bonding through a thermal mechanism can be modeled.

Chapters 12 to 18 illustrate the multiscale approach by applying it to specific cases of interest. Some of these applications required the development of new simulations techniques.

\section{References}

[1] A. Sorokin, S. V. Bobashev, T. Feigl, K. Tiedtke, H. Wabnitz, and M. Richter, Photoelectric effect at ultrahigh intensities. Phys. Rev. Lett. 99, 213002 (2007)

[2] P. A. Franken, A. E. Hill, C. W. Peters, and G. Weinreich, Generation of opticalharmonics. Phys. Rev. Lett. 7, 118-119 (1961).

[3] A. Wardlow and D. Dundas. High-order-harmonic generation in benzene with linearly and circularly polarized laser pulses. Phys. Rev. A 93, 023428 (2016).

[4] F. Krausz and M. Ivanov, Attosecond physics, Rev. Mod. Phys. 81, 163-234, (2009).

[5] B. Nagler et al., Turning solid aluminium transparent by intense soft $x$-ray photoionization, Nature Physics 5, 693-696 (2009).

[6] N. Bohr, On the constitution of atoms and molecules. The London, Edinburgh, and Dublin Philosophical Magazine and Journal of Science 26, 1-25 (1913).

[7] W. H. Bragg and R. Kleeman, On the $\alpha$ particles of radium, and their loss of range in passing through various atoms and molecules. The London, Edinburgh, and Dublin Philosophical Magazine and Journal of Science 10, 318-340 (1905).

[8] Roger Smith, ed. Atomic and Ion Collisions in Solids and at Surfaces: Theory, Simulation and Applications. (Cambridge University Press, 1997). doi: 10.1017/CB09780511524325

[9] H. Bethe, Bremsformel fue elektronen relativistischer geschwindkeit, Zeitschrift fuer Physik 76, 293299, (1932).

[10] J. F. Ziegler, Stopping of energetic light ions in elemental matter, J. Appl. Phys. 85, 1249-1272 (1999)

[11] M Coughlan et al., Ultra- fast dynamics and evolution of ion-induced opacity in transparent dielectrics, New J. Phys. 22, 103023 (2020) 
[12] C. English and J. Hyde, Radiation damage of reactor pressure vessel steels, in Rudy J. M. Konings, ed. Comprehensive Nuclear Materials, (Elsevier, Oxford, 2012), pages 151-180.

[13] Julie A. Reisz, Nidhi Bansal, Jiang Qian, Weiling Zhao, and Cristina M. Furdui, Effects of ionizing radiation on biological molecules-mechanisms of damage and emerging methods of detection, Antioxidants \& Redox Signalling 21, 260-292 (2014)

[14] A. V. Krasheninnikov, Y. Miyamoto, and D. Tománek, Phys. Rev. Lett. 99, 016104 (2007)

[15] C. A. Ullrich, Time-dependent density functional theory: Concepts and Applications (Oxford University Press, 2011). doi: 10.1093/acprof:oso/9780199563029.001.0001

[16] G. D. Billing, Chem. Phys. Lett. 100, 535 (1983)

[17] A. A. Correa, J. Kohanoff, E. Artacho, D. Sánchez-Portal, and A. Caro, Phys. Rev. Lett. 108, 213201 (2012)

[18] A.P. Horsfield, D.R. Bowler, A.J. Fisher, T.N. Todorov and C.G. Sanchez, J. Phys.: Condens. Matter 17, 4793 (2005)

[19] E. J. McEniry et al., Eur. Phys. J. B, 77, 305 (2010)

[20] B. Arnaud and Y. Giret, Phys. Rev. Lett. 110, 016405 (2013)

[21] A. K. Rajam, I. Raczkowska, N. T. Maitra, Phys. Rev. Lett. 105, 113002 (2010)

[22] J. C. Tully, J. Chem. Phys. 93, 1061 (1990)

[23] R. Long, N.J. English, O.V. Prezhdo, J. Am. Chem. Soc. 135, 18892 (2013)

[24] A.P. Horsfield, D.R. Bowler, A.J. Fisher, T.N. Todorov and C.G. Sanchez, J. Phys.: Condens. Matter 17, $4793(2005)$

[25] L. Stella, M. Meister, A.J. Fisher, and A.P. Horsfield, J. Chem. Phys. 127, 214104 (2007)

[26] R. P. Miranda, A. J. Fisher, L. Stella, and A. P. Horsfield, J. Chem. Phys. 134, 244102 (2011)

[27] R. Balescu, Statistical Physics of charged particles (London Press, 1963)

[28] L.P. Kadanoff and G. Baym, Quantum Statistical Mechanics (Benjamin, New York 1963); J. Math. Phys. 3, 605, (1968)

[29] L. V. Keldysh, Soviet Phys. JETP 20, 1018 (1965)

[30] K. Henneberger and H. Haug, Phys. Rev. B 38, 9759 (1988)

[31] C. Attaccalite, M. Grüning, and A. Marini, Phys. Rev. B 84, 245110 (2011)

[32] A. Kaiser, B. Rethfeld, M. Vicanek, and G. Simon, Phys. Rev. B 61, 11437 (2000)

[33] A. Cavalleri et al. Phys. Rev. B 70, 161102 (2004); D. Fritz et al., Science 315, 633-636 (2007)

[34] N. N. Bogoliubov and K. P. Gurov, Journal of Physics USSR 10, 265 (1946)

[35] L. Boltzmann, Lectures on Gas Theory. (U. of California Press, Berkeley, 1964)

[36] H. Risken; The Fokker-Planck Equation: Methods of Solutions and Applications. Springer Series in Synergetics, Heidelberg, 1996

[37] F. Rossi and T. Kuhn, Rev. Mod. Phys. 74, 895, 2002

[38] N.A. Medvedev, R.A. Rymzhanov, A.E. Volkov, J. Phys. D. Appl. Phys. 48, 355303 (2015)

[39] A. Caro and M. Victoria, Phys. Rev. A 40, 2287 (1989), D. M. Duffy and A. M. Rutherford, J. Phys.: Condens. Matter 19, 016207 (2007)

[40] C. Race, D. Mason, and A. Sutton, J. Nucl. Materials 425, 33 (2012) 
[41] L. Stella, C. D. Lorenz, and L. Kantorovich. Phys. Rev. B 89, 134303, (2014)

[42] L. Anderlini et al., Front. Phys. 8, 474 (2020)

[43] I. Haughton et al., Diamond Relat. Mater. 111, 108164 (2020)

[44] D. Sangalli and A. Marini, Europhys. Lett. 110, 47004 (2015)

[45] D. Sangalli and A. Marini, J. Phys.: Conf. Ser. 609, 12006 (2015)

[46] M. Bernardi, Eur. Phys. J. B 89, 239 (2016)

[47] H. O. Jeschke, M. E. García, and K. H.Bennemann, Phys. Rev. B 60, R3701-4 (1999); K. H. Bennemann, J. Phys.: Condens. Matter 16, R995-R1056 (2004); K. H. Bennemann, J. Phys.: Condens. Matter 23, $073202(2011)$

[48] H. W. K. Tom, G. D. Aumiller, and C. H. Brito-Cruz, Phys. Rev. Lett. 60, 1438-41 (1988)

[49] P. L. Silvestrelli, A. Alavi, M. Parrinello, D. and Frenkel, Phys. Rev. Lett. 77, 3149-52 (1996); Phys. Rev. B 94, 184310 (2016)

[50] O. H. Nielsen and R. M. Martin, Phys. Rev. Lett. 50, 697 (1983)

[51] F. Dorner, Z. Sukurma, C. Dellago, and G. Kresse, Phys. Rev. Lett. 121, 195701 (2018)

[52] S. Khakshouri, D. Alfè, and D. M. Duffy, Phys. Rev. B 78, 224304 (2008)

[53] K. Jin, Y. Zhang, Z. Zhu, D. A. Grove, H. Xue, J. Xue, and W. J. Weber, J. Appl. Phys. 115, 044903 (2014)

[54] J. Gibbs. Elementary Principles in Statistical Mechanics (C. Scribner's Sons, NY, 1902)

[55] Yu. L Klimontovich, Physics Letters A 170, 434, (1992)

[56] N. Medvedev, V. Tkachenko, V. Lipp, Z. Li, B. Ziaja, 4open 1, 3 (2018) 

SECTION II

EXPERIMENTAL METHODS 



\title{
2. Laser-matter interaction dynamics probed by time-resolved techniques
}

\author{
Mario Garcia-Lechuga* and Jan Siegel
}

This chapter provides a brief review of the physical phenomena behind the modification of materials with lasers and the experimental tools to characterize them. Special emphasis is placed on the phenomena involved when using ultrashort pulses, since the extreme processes to which the material is subjected (strong ionization, high temperatures and pressures, etc.) are of great fundamental and applied interest. To analyse these inherently transient processes, time-resolved techniques are required. In particular, this chapter presents techniques based on ultrafast imaging, which provide access to transient optical changes and whose interpretation is relatively intuitive (changes in reflectivity, absorption, etc.). At the end on the chapter is presented an experimental case of study using time-resolved microscopy. The reflectivity images obtained allows to characterize the effects of bound electrons (Kerr effect) and free electrons (photoionization) when subjected to an intense ultrashort pulse.

\footnotetext{
*Contact: mario.garcialechuga@uam.es
} 


\subsection{Introduction}

Since their invention, lasers have become very interesting scientific and industrial tools because they allow matter to reach extreme thermodynamic conditions. As a direct consequence, highprecision materials machining is possible, which has become one of the topics that benefits most of this unique property of lasers [1]. The versatility of laser technology (broad range of pulse durations and wavelengths) and the adaptable conditions of irradiation (energy, number of pulses, strong focusing, etc.) enables the modification on any material, irrespective of its chemical composition, optical properties, hardness, etc. Additionally, the nature of the induced modification is manifold, ranging from ablation (ejection of material) to more subtle structural changes (melting, solidification, density changes, changes in the crystal structure, etc). This enables the processing of materials for diverse applications, such as surface texturing, generation of nanoparticles or waveguide writing.

For laser processing of materials, the use of ultra-short (fs and ps) laser pulses has several advantages over the use of longer-lasting (typically ns) pulses or continuous wave (cw) lasers [2]. The first one is the high electric field intensity achieved, which even allows modification of transparent materials by means of non-linear absorption processes. The second one is the ultrafast material excitation process. In this way, the energy deposition in the material ends before any significant heat transfer can occur, unlike what happens when processing with nanosecond lasers.

Additionally, ultra-short pulses are also widely employed as 'time-resolved measurement tools', since their short duration can be used to probe the ultrafast dynamics during laser-induced material transformation at any given moment. In this book chapter, a series of time-resolved experimental techniques, known as pump-probe techniques, will be presented, in which an ultrashort laser pulse is split into two pulses. One of them induces the modification in the material and the other one "interrogates" the material state with a certain delay with respect to the arrival of the excitation beam. In particular, among the vast number of related techniques, the focus will be placed on imaging techniques.

\subsubsection{How laser light interacts with matter}

As every electromagnetic field, laser light propagation is described by Maxwell equations. While these equations are appropriate, their solution can be rather difficult. In this chapter, considering the context of laser processing of materials, some simplifications are introduced. First, only nonmagnetic media are considered. Using two of the Maxwell equations, namely the Ampère's law and the Maxwell-Faraday equation [3], allows to express the wave equation for the electric field, $\vec{E}$, as,

$$
\nabla \times(\nabla \times \vec{E})+\frac{1}{c^{2}} \frac{\partial^{2} \vec{E}}{\partial t^{2}}=-\frac{1}{c^{2} \epsilon_{0}} \frac{\partial^{2} \vec{P}}{\partial t^{2}}-\frac{1}{c^{2} \epsilon_{0}} \frac{\partial \vec{J}}{\partial t}
$$

Being $c$ the speed of light in free space and $\epsilon_{0}$ the permittivity of free space. The two terms on the right-hand side are the called source terms and describe the interaction of light with matter, and more in particular, of light with electrons. 
Vector $\vec{P}$ is the polarization of the material. This term, mostly relevant for non-conductive media, accounts for the oscillation of bound electrons when facing the oscillating external E-field, expressed mathematically as,

$$
\vec{P}(t)=\chi \epsilon_{0} \vec{E}(t)
$$

with $\chi$ being the electric susceptibility of the medium, a tensor for anisotropic media and a scalar for isotropic ones. When only accounting the first source term of eq. (2.1), related with the bound electrons, one simple option to solve the wave equation is to apply the semiclassical Lorentz model. This method provides a reasonably good approximation of the optical behaviour of dielectrics, which lack free electrons.

On the other hand, vector $\vec{J}$ in eq. (2.1) corresponds to the electric current density, representing the behavior of free electrons when subjected to an external electric field. This term is expressed following Ohm's law,

$$
\vec{J}(t)=\sigma \vec{E}(t)
$$

where $\sigma$ is the electric conductivity of the medium. If we only consider the corresponding source term related to free electrons, the wave equation can be solved by the semi-classical Drude model. This model represents in a simplified way the optical behaviour of metals.

Independent of considering one or both source terms, a simplified wave equation can be expressed for an isotropic medium interacting with a monochromatic and periodic E-field,

$$
\nabla^{2} \vec{E}=-\frac{\omega^{2}}{c^{2}} \cdot \epsilon(\omega) \cdot \vec{E}
$$

with $\omega$ being the frequency of the E-field and $\epsilon$ the dielectric function. The dielectric function is of major importance since it describes the optical behaviour of the material, whatever its nature: conductive or dielectric. This function is directly linked to the complex refractive index, $\tilde{n}$, by the expression,

$$
\epsilon(\omega)=\tilde{n}^{2}=(n+i \kappa)^{2}
$$

where $n$ is the real part of the refractive index and $\kappa$ the absorption coefficient of the material. In the frame of laser processing of materials, the absorption coefficient is the key factor, since it is one of the responsible properties that determine the energy deposition. As examples, Figure 2.1 shows the optical behavior of three different material classes. In the case of a dielectric material, e.g. quartz, the absorption is zero $(\kappa=0)$, except when the incident light reaches the ultraviolet spectral range. This range corresponds to photon energies that are higher than the material bandgap $\left(E_{\text {gap }}\right)$. Therefore, linear absorption occurs a dielectric material only for UV light.

In the case of a metal, for instance silver, absorption occurs at any wavelength of the visible spectral range. Light absorption is produced by the free electrons that are present in metals at high densities $\left(\sim 10^{21} \mathrm{~cm}^{-3}\right)$. Finally, semiconductors are known to have lower bandgap energies than dielectrics, showing relative high absorption in the visible and near infrared, but less or none in the mid- and far-infrared (as shown for silicon in Figure 2.1). However, mathematically describing the optical behavior of semiconductors is more complex and both sources terms need to be taken into account, since the free electron contribution is non-negligible at room temperature. 

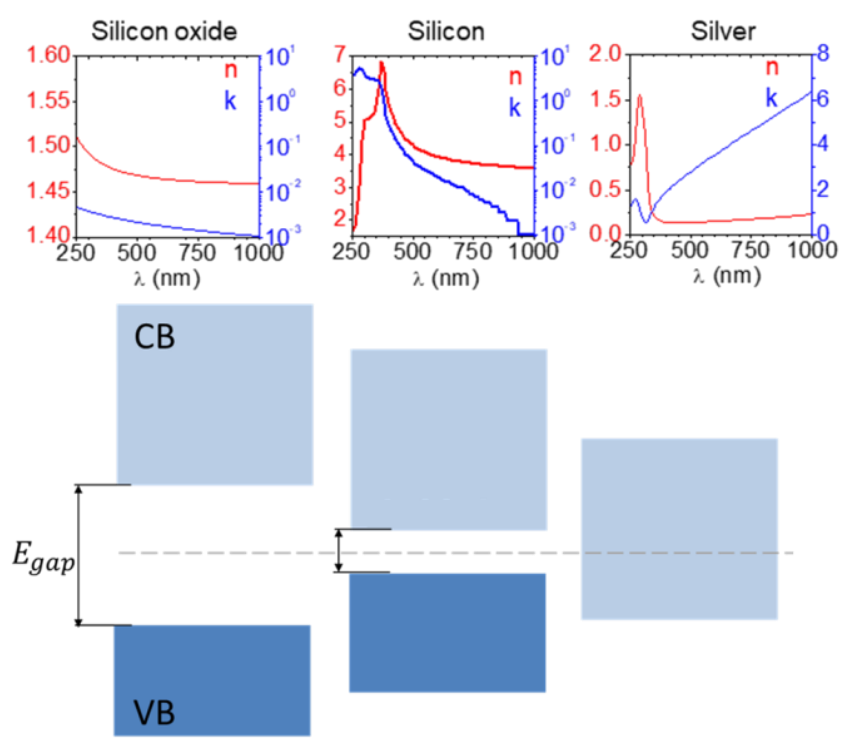

Figure 2.1. (Top) Representation of the refractive index, $n$, and the absorption coefficient, $k$, as a function of the wavelength for a dielectric material (silicon oxide), a semiconductor (silicon) and a metal (silver). Data are extracted from [4-6]. (Bottom) Schematic representation of the valence bands (VB) and conduction bands (CB) of the three different classes of materials.

\subsubsection{Basics of material modification by laser}

In the field of laser processing of materials, two basic conditions should be fulfilled to induce a permanent modification. First, the material should absorb part of the incident light. This condition, as expressed on the previous subsection, is dependent of the linear optical properties of the material at the corresponding wavelength of irradiation. But also, as will be explained in the following sections, absorption may occur even in transparent materials by means of non-linear absorption, triggered by high-intensity laser pulses.

Apart from that, a second necessary condition to be fulfilled is the need for achieving a sufficient amount of deposited energy in the free electron subsystem. This means, that free electrons with a sufficiently high temperature (or equivalently, a high kinetic energy) has to be generated in order to initiate processes that alter the material. This is necessary, since the role of hot free electrons is to transfer their energy to the lattice through electron-phonon coupling processes, thus heating the material. If the transferred energy causes the lattice to reach temperatures and pressures above the melting or boiling points of the material, a first order phase transition occurs, often accompanied by a permanent modification after the subsequent cooling and solidification process. The irradiation conditions at which these modifications occur are referred to as fluence thresholds for a certain material modification, normally expressed as $F_{t h}$. The term fluence refers to the incident pulse energy divided by the area of the laser-exposed region.

\subsubsection{Benefits of using ultrashort laser pulses}

\subsubsection{Any material can be modified}

Ultrashort pulses refer to laser pulses with duration of less than a few tens of picoseconds $(1 \mathrm{ps}=$ $10^{-12} \mathrm{~s}$ ). Nowadays, their generation is a very consolidated and accessible technology. Pulse 
durations ranging from few tens of femtoseconds $\left(1 \mathrm{fs}=10^{-15} \mathrm{~s}\right)$ to few picoseconds are obtained with commercial laser systems. However, the spectral range of operation is mostly limited to the near-infrared: $\lambda \approx 800 \mathrm{~nm}$ for lasers based on Ti:Sapphire technology and $\lambda \approx 1050 \mathrm{~nm}$ for lasers based on rare earth-doped crystals. Higher harmonics, typically second and third and fourth harmonics, can be obtained for these fundamental wavelengths with commercial frequencyconversion systems (for instance for $\lambda \approx 800 \mathrm{~nm}, \lambda_{\mathrm{SHG}}=400 \mathrm{~nm}, \lambda_{\mathrm{THG}}=267 \mathrm{~nm}$ ), although at the great expense of pulse energy.

According to the optical properties above mentioned, dielectrics could in principle not be modified upon irradiation with lasers pulses at the fundamental frequency in the near infrared, since the bandgap is larger than the photon energy $\left(\hbar \omega<E_{\text {gap }}\right)$. However, materials are not perfect and present defects (e.g. $\sim 10^{12} \mathrm{~cm}^{-3}$ for a high purity glass). Assisted by the presence of defects, light can be absorbed, thus promoting electrons to the conduction band, as sketched in the left part of Figure 2.2. This is the main mechanism that triggers absorption when irradiating with nanosecond laser pulses.

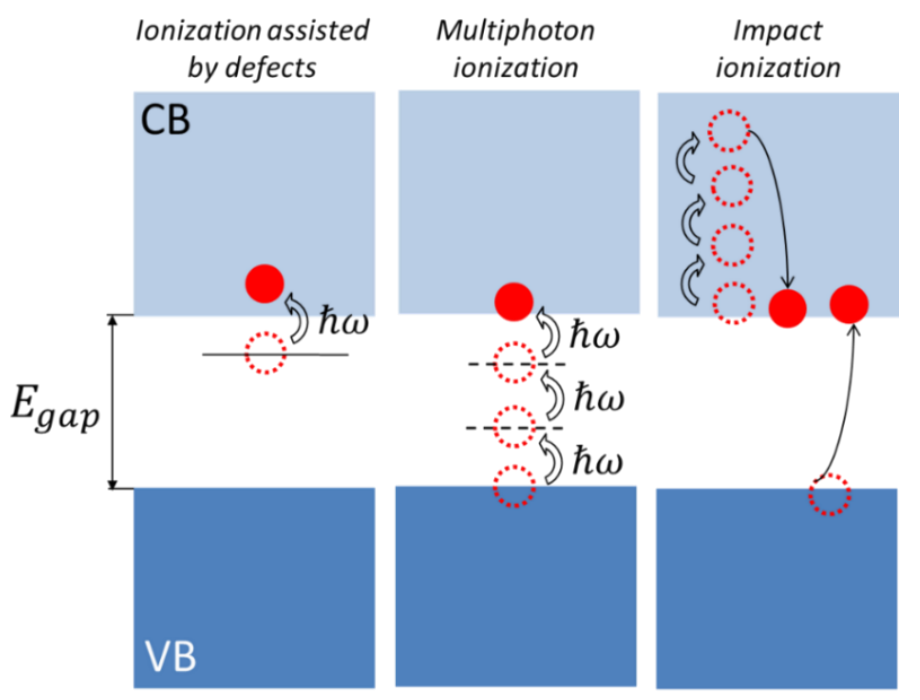

Figure 2.2. (Left) Sketch of ionization assisted by defects. The defect is represented with an energy level within the bandgap. (Center) Sketch of multiphoton absorption. An electron is promoted from the valence band (VB) to the conduction band (CB) by the simultaneous absorption of multiple photons through virtual levels, represented with dashes lines. (Right) Sketch of impact ionization. A free electron in the conduction band gains sufficient kinetic energy for promoting via collision another electron from the VB into CB.

Moreover, when irradiating non-absorbing materials with ultrashort pulses there is an additional absorption process that dominates over defect-induced absorption. Due to the elevated peak power of those pulses (high energy concentrated on a very short time) non-linear absorption processes become statistically possible [7]. Among them, multiphoton ionization (MPI) is an absorption process produced by the simultaneous absorption of various photons, occurring typically for intensities above $10^{14} \mathrm{~W} / \mathrm{cm}^{2}$ [8]. As sketched in the central image of Figure 2.2, the simultaneous absorption of several photons promotes an electron from the valence band to the conduction band through intermediate virtual states. As an example, the band gap of the dielectric material lithium niobate is $E_{g a p}=3.5 \mathrm{eV}$ and the energy of a photon at $800 \mathrm{~nm}$ is $E(800 \mathrm{~nm})=$ $1.55 \mathrm{eV}$, requiring the absorption of three photons to generate free electrons. 
This generation of free electrons causes the optical properties of the material to turn transiently into semi-metallic. To model this change, one can use the semi-classical Drude-Lorentz model, where the complex refractive index is expressed as,

$$
\tilde{n}^{2}=\tilde{n}_{0}^{2}-\left(\Delta \tilde{n}_{\text {Drude }}\right)^{2}
$$

Being $\tilde{n}_{0}$ the complex refractive index of the material before being excitation, and $\Delta \tilde{n}_{D r u d e}$ the contribution of the generated free electrons, being mathematically described as,

$$
\left(\Delta \tilde{n}_{\text {Drude }}\right)^{2}=\frac{n_{e}}{n_{c}} \frac{1}{1+i /(\omega \cdot \tau)}
$$

where $n_{e}$ is the free electron density, $\omega$ the light frequency of interest (irradiation or illumination), $\tau$ the scattering time of free electrons and $n_{c}$ the so-called critical electron density. This parameter is obtained throughout the expression $n_{c}=m^{*} \epsilon_{0} \omega^{2} / e^{2}$, being $m^{*}$ the reduced mass of the electron and $e$ the charge of the electron.

Obviously, this transient change of optical properties due to the presence of free electrons has consequences for the absorption process. Now, photons can be also absorbed by free electrons via a process called inverse bremsstrahlung, which increases their kinetic energy. Once this kinetic energy becomes larger than the bandgap, the free electrons can promote other electrons to the conduction band via a collision impact, a process called impact ionization and sketched on the right part of Figure 2.2. This process is a dynamic process that occurs on the timescale of the laser excitation. The modelling of this dynamic process is complex and out of the scope of this chapter. For that purpose we refer to standard models based on single- or multi-rate equations $[9,10]$

\subsubsection{Deterministic response}

The energy threshold for material transformation by laser pulses moves from stochastic for long and short pulses to deterministic for ultrashort pulses [11,12]. This makes the interaction extremely controllable and repeatable, an aspect with evident interest for fundamental science but also for industrial applications [13].

This deterministic behavior is a consequence of the characteristic timescales of different mechanisms involved in the modification process [14]. As mentioned, the first process is the electron excitation, which lasts while the laser pulse is present, i.e. the time scale is given by the pulse duration. The second process is the energy transfer from the electronic subsystem to the lattice, until both reach the same temperature. This transfer is produced by electron-phonon coupling and takes place in the picosecond timescale. Therefore, the excitation process and the thermalization of electrons and lattice occurs separately upon ultrashort pulse excitation, one in the femtosecond timescale and the other one in the picosecond timescale.

Consequently, this leads to a reduced heat flux compared to excitation with longer pulses, making the modification edges sharper. Figure 2.3 gives a visual example of this behavior, showing a modified region on a thin film of $G e_{2} S b_{2} T e_{5}$ (GST) generated after single-pulse irradiation (120 fs duration). Three different regions with different optical contrast can be observed: a central round dark grey area corresponding an ablated region, a surrounding grey annular region corresponding to amorphized material and an exterior brighter region corresponding to the unchanged crystalline material. The separation between regions is sharp and symmetrical. These two aspects are consequences of a strict fluence threshold response. To further illustrate this 
aspect, the Gaussian spatial profile of the irradiating pulse with a peak fluence of $F_{0}$ is superimposed to the image. Ablation is only produced for local fluences above a the fluence threshold for ablation, $F_{t h, a b}$, and amorphization is limited to fluences above the fluence threshold of amorphization, $F_{t h, a m}$. Therefore, by controlling the irradiation fluence the spatial extent and nature of the induced modification will be determined.

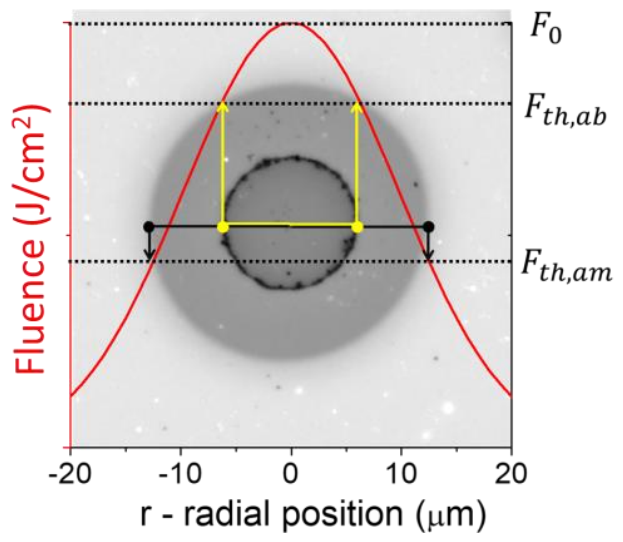

Figure 2.3. Optical microscope image of a GST sample after single-pulse irradiation $(\lambda=800 \mathrm{~nm}, \Delta t=120 \mathrm{fs})$. The Gaussian-shaped intensity profile with a peak fluence of $F_{0}$ is plotted to illustrate the sharp threshold response (ablation: $F_{t h, a b}$, amorphization: $F_{t h, a m}$ ).

\subsection{Time-resolved imaging techniques}

\subsubsection{Introduction}

Our perception of moving objects is limited by our physiology, since our brain can perceive changes at a maximum rate of around 45 frames per second ( $\mathrm{fps}$ ). I other words, our response time to very fast changes is approximately 20 milliseconds. For that reason, technologies were developed to overcome this limit. As a first historical example of such a technique breaking the limit imposed, Figure 2.4 (a) shows the famous set of photographs of a running horse taken by E. Muybridge at the end on the $19^{\text {th }}$ century. His technique was based on the use of multiple cameras (one per frame, lined up along the race track) with short exposure times and an ingenious mechanical system, allowing to synchronize each individual camera shutter with the horse passing in front of each camera. Using this strategy, it was possible to capture events with a high temporal resolution (= exposure time) although with a low sampling rate (= number of frames within a given time window).

Further developments allowed further increasing the temporal resolution to the microsecond time scale. This was possible by changing the strategy, using a short illumination pulse to further narrow down the limited exposure time offered by a mechanical shutter. Figure 2.4 (b) shows an image recorded in the 1960's, in which is possible to observe a bullet in flight. The recording of frozen movement at velocities higher than the speed of sound was possible by a combination of a short illumination time (microsecond flash) and a perfect synchronization between the event (bullet travelling), the flash and the camera shutter. 
(a)

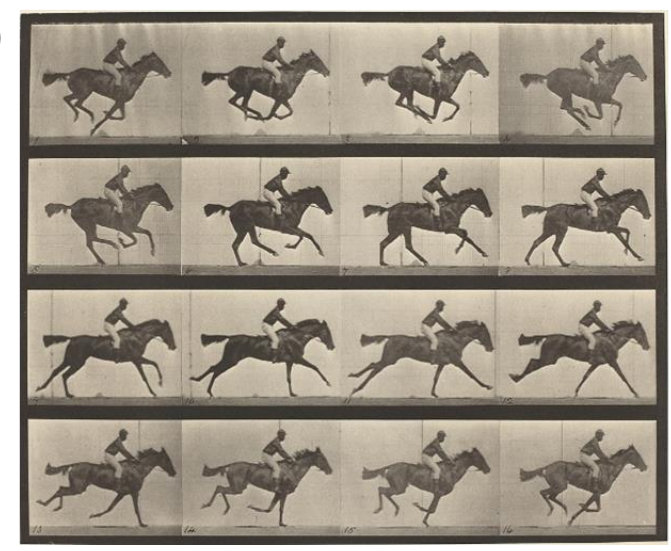

(b)

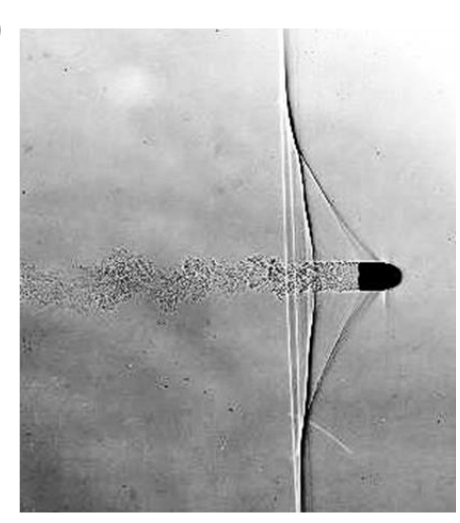

Figure 2.4. (a). Sequence of photographs of a horse running recorded by E. Muybridge, using an imaging system with millisecond time-resolution

(https://commons.wikimedia.org/wiki/File:Eadweard Muybridge, Animal Locomotion, Plate 626, 1887, NGA 1365

36.jpg). (b) Shadowgraph of a bullet in flight recorded by D. P. B. Smith with a microsecond time-resolved imaging equipment (Image Creative Commons: https://commons.wikimedia.org/wiki/File:Shockwave.jpg)

These two examples demonstrate that time-resolved images can be obtained if there is a sufficiently fast probing and a perfect synchronization between the event and the recording process. It also shows that time resolution can be improved by reducing the illumination time, i.e. by using shorter and shorter light pulses. Therefore, the development of short and ultrashort pulsed lasers represented the perfect opportunity for accessing dynamics that occur on the submicrosecond to femtosecond time scale.

In particular, femtosecond laser sources paved the way for studying ultrafast processes, by using the so-called pump-probe techniques. The main characteristic of those techniques is the separation of a single femtosecond pulse into two pulses. The pump pulse induces a modification in the target under study (gas, liquid or solid) and the probe beam monitors this change at a selected time delay after the pump. The selection of the time delay of observation is made by controlling the optical path difference between pump and probe pulse. That way, the synchronization between the event and the recording of it is extremely precise, because it is based on a physical distance (and thus jitter-free) rather than on electronics, inherently limited by jitter. As a relevant example of the importance of these techniques, they triggered the emergence of an entirely new field, so-called femtochemistry [15], for which Ahmed Zewail was awarded with the Nobel Prize in Chemistry in 1999.

In the following sections, we present the imaging techniques developed for the observation and characterization of the excitation and transformation dynamics of materials upon pulsed laser irradiation. Special emphasis is devoted to time-resolved microscopy, a direct imaging technique with allows to monitor changes of the sample reflectivity with femtosecond temporal and micrometer spatial resolution.

\subsubsection{Femtosecond-resolved microscopy}

In 1985, M.C. Downer, R.L. Fork and C.V. Shank introduced a novel technique, combining a pumpprobe approach with an optical microscope [16]. This new experimental system was presented as the natural evolution of the previous work of C.V. Shank et al. [17], in which the temporal evolution 
of the reflectance in the center of the excited area, probed by a delayed pulse, was monitored with a photodiode. Using the novel pump-probe microscopy technique, the temporal reflectivity evolution at every point of the laser-excited area could be obtained. That way, the material response at different local fluences is obtained, since the fluence distribution of the excitation pulse normally shows a known Gaussian shape (as represented in Figure 2.3).

Figure 2.5 (a) shows the basic concept of femtosecond-resolved microscopy. The probe pulse illuminates an area much larger than the pump pulse and arrives with a user-defined time delay with respect to the pump pulse, interrogating the surface reflectivity at that moment. The image of the surface is then formed by means imaging system on photographic film and recorded. Normally, pump and probe beams are not collinear, which allows to efficiently prevent the entrance of the pump pulse (normally more energetic) into the imaging system.

The interest of this technique lies in two main aspects. First, it provides direct images of the surface, which makes the qualitative analysis of the transformation dynamics simple that other analytical techniques. Second, it gives simultaneously access to the reflectivity evolution of the material both in time, $t$, and space, $r$. This radial resolution is of high relevance since it allows to characterize the material evolution at different local fluences (see Figure 2.3) upon single pulse irradiation.

(a)

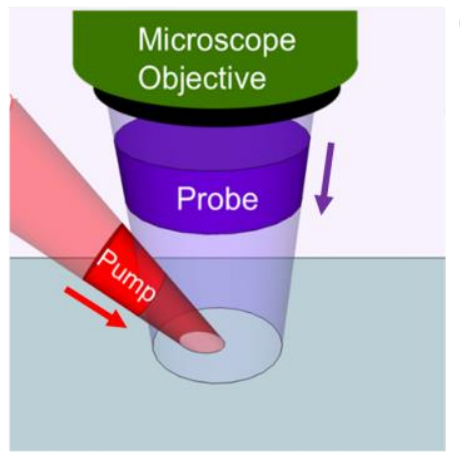

(b)
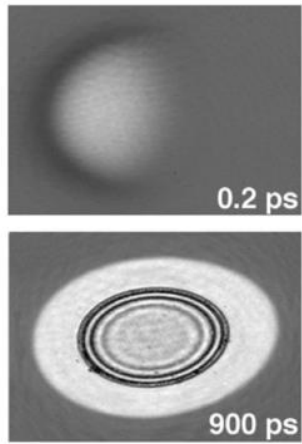
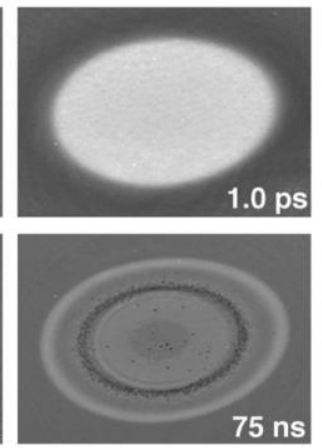

Figure 2.5. (a) Sketch of femtosecond-resolved microscopy. In this particular case, the pump pulse is incident at an oblique angle and the probe pulse normal to the surface. (b) Surface of a silicon wafer after irradiation with a $120 \mathrm{fs}$ laser pulse of $0.47 \mathrm{~J} / \mathrm{cm}^{2}$. Frame size $220 \mu \mathrm{m}$ x $300 \mu \mathrm{m}$. (Reprinted with permissions from [19]).

A step forward in the development and importance of this technique was made in 1997 by D. von der Linde, K. Sokolowski-Tinten and coworkers [18], by capturing the evolution of the reflectivity with a CCD camera instead of photographic paper. This resulted in high quality digital images, as shown in the Figure 2.5 (b) for a silicon sample, which allowed better quantitative analysis by digital processing. As an example of the high spatial and temporal resolution, the image recorded at a delay $0.2 \mathrm{ps}$ resolves the landing of the pump pulse (incident from the left). Since the incidence angle of the pump beam is $45^{\circ}$ there is a region where a change of reflectivity is triggered (due to the generation of free electrons) and another region without change, since the pulse has not yet reached the surface. This first visual qualitative analysis can be turned into quantitative by analyzing the reflectivity evolution, $R(r, t)$, extracted from these images. From the Fresnel equation relating the reflectivity (at normal incidence) and the complex refractive index,

$$
R(r, t)=\left|\frac{\tilde{n}(r, t)-1}{\tilde{n}(r, t)+1}\right|^{2}
$$


and by using equation (2.6) that relates the change of refractive index to the free electron density, it is possible to estimate the temporal evolution of free electron density, $n_{e}(r, t)$. A practical example will be shown in subsection 2.3.2

A second demonstration of the huge potential of this technique to unravel ultrafast laser-matter interaction mechanisms are the interference rings formed at a delay on the scale of hundreds of picoseconds ( 900 ps on the image of Figure 2.5 (b)). Their appearance and temporal evolution denote the formation of an expanding surface layer due to partial ablation of material. The temporal evolution of those rings allowed to measure the speed of material ejection $(\sim 1000 \mathrm{~km} / \mathrm{h})$ and to understand the mechanisms responsible for the ablation process [19-21].

This technique has been also applied to the study of transformation dynamics of transparent materials. The group of Solis and Siegel took advantage of this technique to investigate surface excitation and ablation processes in transparent materials with technological interest [22-24]. In addition, and what denotes even more the versatility of this technique, they applied this technique to the study of non-linear propagation, excitation and modification inside transparent materials [25]. Figure 2.6 (a) displays shadowgraphs recorded at different time delays, visualizing regions where free electrons are generated (dark regions) during pump pulse propagation. This study contributed to the understanding of the role of free electrons during laser fabrication of optical waveguides in glasses.

(a)

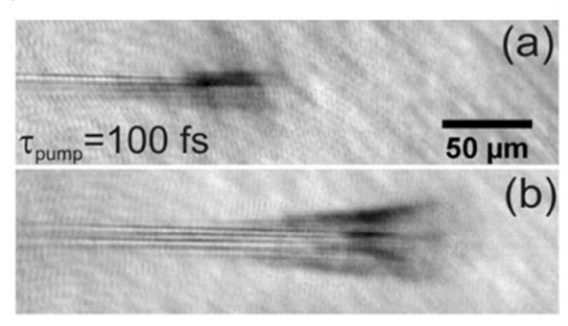

(b)

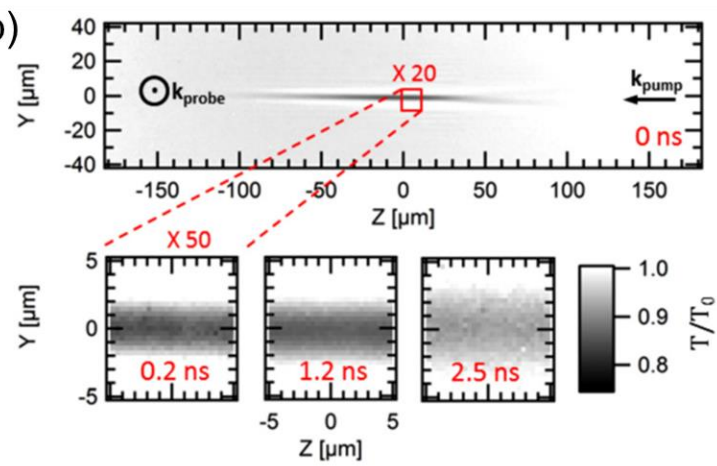

Figure 2.6. (a) Shadowgraphs recorded in bulk glass upon irradiation with a single pulse $(\lambda=800 \mathrm{~nm}, \Delta t=100 \mathrm{fs}$ ). The upper image correspond to a delay of $0.65 \mathrm{ps}$ the lower image to $1.2 \mathrm{ps}$ after the pump irradiation. The laser pulse propagates from left to right. (Reprinted with permissions from [25]). (b) Shadowgraphs recorded in bulk silicon after the irradiation with a single pulse $(\lambda=1300 \mathrm{~nm}, \Delta t=90 \mathrm{fs})$. For longer time delays (bottom part), diffusion of free electrons is observed. The laser pulse propagates from right to left. (Reprinted with permissions from [27]).

The group of Grojo has also applied femtosecond-resolved microscopy to the study of volumetric excitation in silicon $[26,27]$. The originally and difficulty of these works lies in the need of using wavelengths in the spectral range where silicon becomes transparent $(\lambda>1100 \mathrm{~nm})$, requiring the use of pump and probe lasers in this range as well as the use of cameras with infrared sensitivity. Figure 2.6 (b) displays time-resolved shadowgraphs recorded with an infrared camera, revealing that the laser-excited dark filament (where electrons are generated) becomes laterally broader with time. This observation allows the characterization of electron diffusion in silicon. 


\subsubsection{Time-resolved ellipsometry}

Ellipsometry encompasses techniques that analyse the intensity and state of polarisation of a light beam after it has been reflected off a surface, allowing access to the dielectric function of the material under study. Wang and Downer [28] carried out the first work on ellipsometry with temporal resolution upon irradiation of an ultra-short pulse. More recently, the group of Huber has developed a technique called ultrafast pump-probe ellipsometry [29,30], in which femtosecond time-resolved microscopy is combined with ellipsometric measurements, recording a set of images for each time delay, each recorded at different angles of a polarizer (analyser). By means of image analysis and application of Fresnel equations, the two components of the refractive index can be obtained for a full image. This is technique is especially relevant for the scale of ten picoseconds after irradiation, where the images recorded with the standard technique are usually dark, and it is not clear if due to an interferential effect (destructive interference) or an absorptive process. The determination of the complex refractive index allows to extract optical penetration depth, which clarifies whether an absorption, interference or a combination of both is responsible.

\subsubsection{Time resolved-interferometry in the space domain}

For a complete characterization of a monochromatic electromagnetic field it is necessary to know its amplitude and phase. All previously mentioned techniques provide information about the change of the amplitude the probe beam after being reflected or transmitted. Here, two techniques characterizing also the change in phase are described, which allows to directly measure transient changes of refractive index and/or transient surface deformations.

In 2004, von der Linde, Sokolowski-Tinten and coworkers designed a complementary variant of their time-resolved microscopy experiment [31,32], represented in Figure 2.7. On the left side, the experimental scheme is shown, which differs from the conventional time-resolved microscopy in the fact that the illumination beam is split in two, using a Michelson interferometer configuration. One beam is reflected from the sample and the other from a reference mirror forming an interference pattern in the plane of the CCD camera, provided that spatial and temporal overlap between the beams is obtained. On the right side of the figure, an image of the recorded interference pattern is shown, at a delay of $1.8 \mathrm{~ns}$ after the irradiation of GaAs with a femtosecond pulse. By analysing the phase shift (curvature of the interference bands) and the evolution of the reflectivity, both the phase and amplitude variation of the reflected beam can be extracted, which is sufficient for a complete characterization. Despite the fact that this technique requires laborious image processing and complex analysis, it offers a great potential for the measurement of transient surface deformations of a few tens of nanometers [32]. 


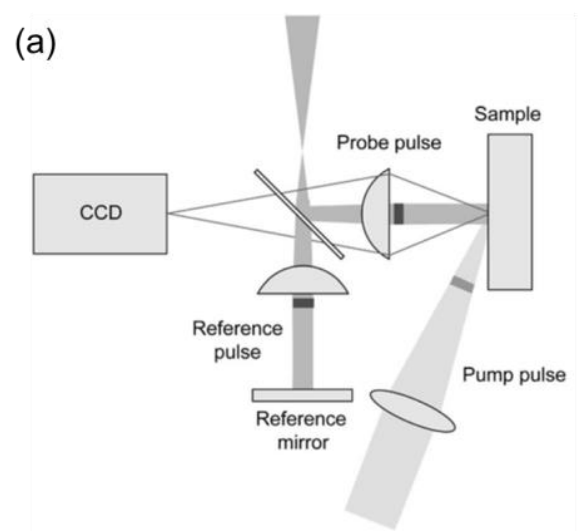

(b)

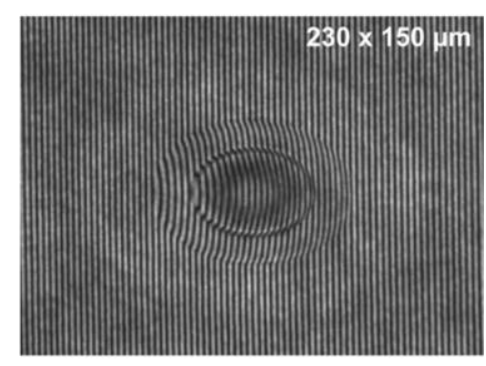

Figure 2.7. (a) Setup for ultrafast interferometry with imaging configuration. (b) Transient interferogram image of an ablating GaAs surface recorded at a temporal delay of $1.8 \mathrm{~ns}$. (Reprinted with permissions from [32]).

More recently, L. Gallais and S. Monneret presented a novel time-resolved microscopy technique [33], allowing to retrieve amplitude and phase in a single step and without the need of moving elements. The key is the placement of a type of mask (modified Hartmann mask) in front of a CCD camera, which causes an interference phenomenon of the probe beam recorded on the camera. After mathematical treatment [34], two different images are extracted from a single image: one containing amplitude information (as in conventional time-resolved microscopy) and another one containing phase information.

\subsubsection{Time resolved-interferometry in the time domain}

In 1994, Audebert et al. presented a novel pump-probe technique based on an Michelson interferometer and an imaging spectrograph [35,36]. S. Guizard of CEA-Saclay (Paris) has continued developing and exploiting this strategy to the present day [37,38]. As shown in Figure 2.8 (a), the Michelson interferometer is located behind the sample, which divides the probe beam that illuminates a region much larger than the excited region into two beams. The interferometer is slightly misaligning, projecting onto the spectrograph slit the two beams laterally displaced. This causes that one beam acts as a probe beam (carrying information of the excited region) and the other as a reference beam (carrying information of the non-excited area close to the excited one). Both beams enter the spectrograph and the spectral interference pattern is imaged onto a CCD. It should be mentioned that the introduction of a slit and grating, required for dispersion of the different spectral components of the probe beam to generate the interference pattern, reduces the spatial information to 1D. However, due to the circular symmetry of the excitation beam, this information is sufficient for the study of the influence of local incident fluence.

Figure 2.8 (a) also includes two examples of interference images recorded with the CCD situated at the spectrograph output. The upper image shows a featureless interference pattern. This represents a situation without excitation (pump beam blocked) or when the probe beam arrives earlier than the pump beam at the sample surface (delay time $<0$ ). In contrast, the lower image corresponds to a situation of laser excitation, showing a strong distortion and increase in contrast of the fringes, caused by the phase shift and absorption experienced by the probe beam while traversing the laser excited region. By recording a series of images at different time delays the temporal evolution of the excitation dynamics can be obtained. 
(a)

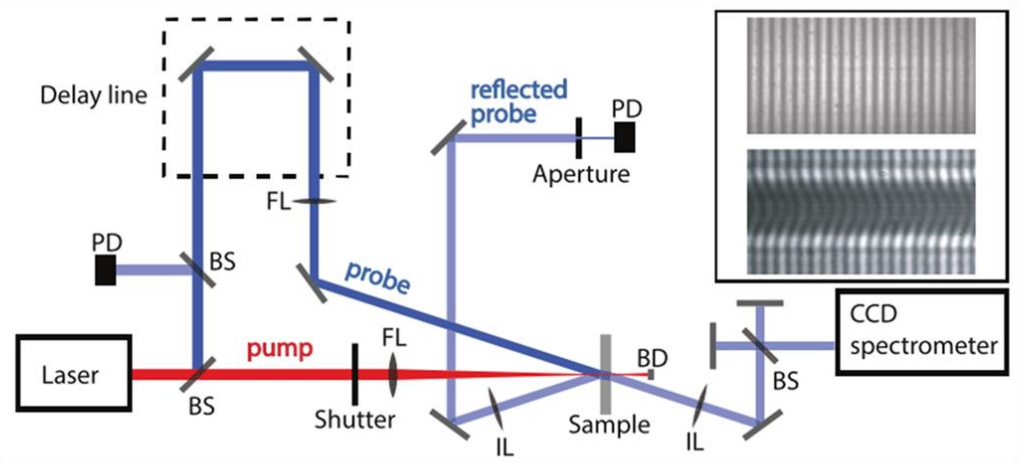

(b)
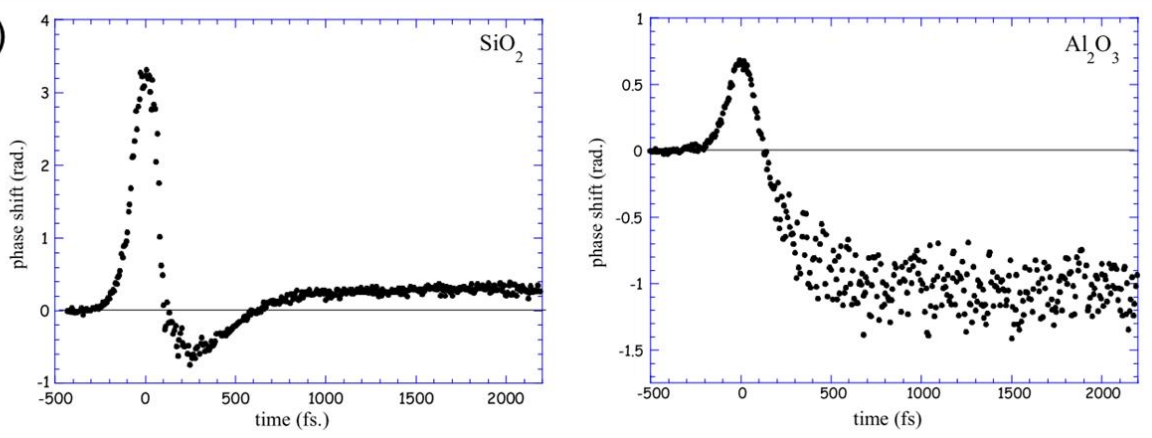

Figure 2.8. (a) Schematic of a time resolved-interferometry setup. BS: beam splitter. FL: focusing lens. IL: imaging lens. PD: photodiode. BD: beam dump. The inset shows two examples of CCD images recorded with (bottom) and without (top) laser excitation. (Reprinted with permission from [37]). (b) Temporal evolution of the measured phase shift in fused silica $\left(\mathrm{SiO}_{2}\right)$ and sapphire $\left(\mathrm{Al}_{2} \mathrm{O}_{3}\right)$. The pump irradiation intensities are well below the ablation threshold fluence. (Reprinted with permission from [38])

Depending on the time delay and the material, different evolutions on the measured phase shifts can be observed, as shown on Figure 2.8 (b) for fused silica $\left(\mathrm{SiO}_{2}\right)$ and sapphire $\left(\mathrm{Al}_{2} \mathrm{O}_{3}\right)$. The phase shift is directly related to the change of the real part of the refractive index, which depends on the following terms [38]:

$$
\Delta n(t)=n_{N L} I(t)+\frac{e}{2 n_{0} \epsilon_{0}}\left(-\frac{n_{e}(t)}{m^{*} \omega^{2}}+\frac{n_{S T E}(t)}{m\left(\omega_{S T E}^{2}-\omega^{2}\right)}\right)
$$

The first term corresponds to the non-linear optical behavior of bound electrons ( $n_{N L}$ is the nonlinear refractive index) caused by the high intensity $(I)$ of the excitation beam. This phenomenon, called non-linear Kerr effect, appears at very short time delays (during the pulse duration) and contributes with a positive phase shift, as observed for both materials in Figure 2.8 (b). The observation of the Kerr effect is vital for a precise determination of pump pulse arrival time at the sample surface and therefore a necessary temporal reference.

The second term in equation (2.9) is a negative contribution to the refractive index and consequently produces a negative phase shift. The term is dependent on the temporal evolution of the photo-generated free electrons density $\left(n_{e}\right)$, which corresponds to the already mentioned Drude model. For both materials, a decrease of the phase shift due to the generation of free electrons it is observed after the initial increase caused by the Kerr effect. Finally, a recovery of the phase shift to positive values is observed for fused silica, which does not occur for sapphire. This recovery is due to the formation of self-trapped excitons (STE) in fused silica, which 
contribute optically with an increase of the refractive index (third term of equation (2.9), being $n_{S T E}$ the density of STE and $\omega_{S T E}$ the resonance frequency of STE).

Operating in transmission, this technique also provides information about the relative absorption experienced by the sampling beam, obtaining information about the imaginary part of the refractive index. However, it has two main disadvantages. First, only transparent materials or very thin films on transparent substrates can be studied. Second, the sampling beam does not only probe the surface (where the energy deposition is highest) but also collects laser-induced changes occurring in deeper regions, effectively providing and average measurement in depth. As a solution for clarifying emerging ambiguities, it has been proposed to combine this technique with femtosecond-resolved microscopy [39].

\subsection{An experimental case study: visualizing bound and free electrons with time-resolved microscopy}

In this section, we present an experimental example that allows us to temporally observe and characterize the transient and permanent optical changes produced by bound and free electrons. In particular, the chosen material is lithium niobate $\left(\mathrm{LiNbO}_{3}\right.$, optical bandgap of $\sim 3.5 \mathrm{eV}$ ), an interesting dielectric material with exceptional electrical and optical properties that give rise to its use in a large number of applications in photonics [40]. The results presented here are a summary of the research by Garcia-Lechuga et al. [24,41,42]. They also include investigation of the material ablation dynamics (experimental and modelling) and characterization of the longlasting photorefractive effect.

(a)

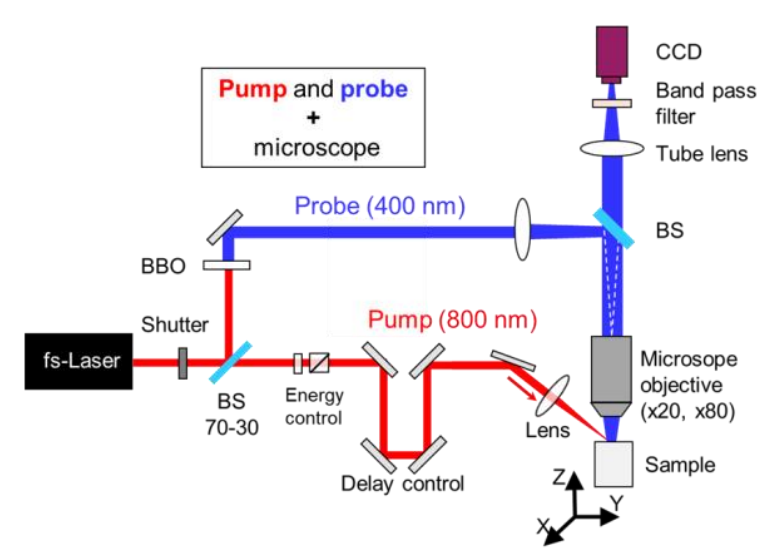

(b)

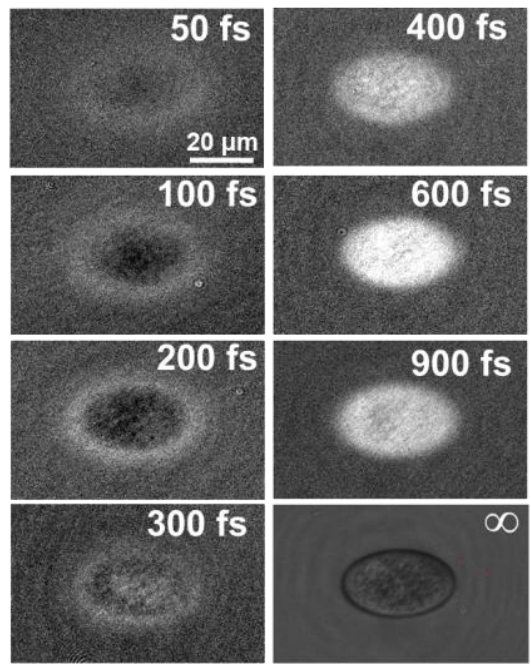

Figure 2.9. (a) Sketch of the femtosecond-resolved microscopy set-up. BBO: Beta barium borate crystal for second harmonic generation. BS: Beam Splitter; CCD: charge-coupled device (Camera). (b) Time-resolved surface reflectivity images $\left(\lambda_{\text {probe }}=400 \mathrm{~nm}\right)$ of lithium niobate at short delay times (see labels) after exposure to a pump pulse $\left(\lambda_{\text {probe }}=\right.$ $800 \mathrm{~nm}, \Delta t=130 \mathrm{fs}, F=2.1 \mathrm{~J} / \mathrm{cm}^{2}$ ) incident at $53^{\circ}$. The image labeled as infinite corresponds to the state of permanent modification (recorded 1 second after pump exposure).

The experimental pump-and-probe set-up used for recording time-resolved images of an excited surface is sketched in Figure 2.9 (a). The irradiation/probing process starts by selecting a single 
pulse from a femtosecond laser amplifier $(\lambda=800 \mathrm{~nm}$, pulse duration $130 \mathrm{fs})$. This pulse is divided into a pump and a probe pulse by means of a beam splitter. The pump pulse, which is more energetic, is focused onto the sample surface at an angle of $53^{\circ}$. Because of this oblique angle of incidence, the excitation spot intensity distribution has an elliptical Gaussian shape (59 $\mu \mathrm{m} \times 97$ $\mu \mathrm{m} 1 / \mathrm{e}^{2}$-diameters). The probe beam is frequency-doubled $\left(\lambda_{\text {probe }}=400 \mathrm{~nm}\right)$ by a non-linear BBO crystal. This step is important to allow discrimination of the probe light from the scattered pump light by means of a narrow spectral bandpass filter when imaging the sample surface. The probe light is directed to a microscope objective to illuminate the sample surface, and the reflection is recollected by the microscope and finally directed to a CCD camera. The time delay between pump-and-probe pulse is controlled with a motorized delay line introduced in the pump beam path. This allows recording images of the sample surface at different time delays after the arrival of the pump pulse.

Figure 2.9 (b) shows several images of the sample surface, for delays ranging from 50 fs to $900 \mathrm{fs}$ after being exposed to a pump pulse with a peak fluence of $2.1 \mathrm{~J} / \mathrm{cm}^{2}$. The recorded images have been normalized, using as a reference an image recorded before irradiation. At the center of the irradiated region, where the local fluence is maximum, the reflectivity initially decreases before undergoing a subsequent increase. Interestingly, the reflectivity behaviour observed in the outer region of the irradiated zone is the opposite, with an initial fast increase followed by a subsequent decrease. The origin of each of these two observations, central region and outer ring, are explained in more detail in the next sections.

As a last general comment, the consequence of image normalization is that the observed parameter is not the reflectivity, but the relative reflectivity, $R_{r e l}$. This $R_{r e l}$ relates to the reflectivity as,

$$
R_{\text {rel }}=\frac{R}{R_{0}}
$$

Being $R_{0}$ the reflectivity of the unexcited surface, that can be calculated with the tabulated data of refractive indexes and applying the Fresnel relationship shown in equation (2.8).

\subsubsection{Visualizing the influence of bound electrons: Kerr effect}

In this subsection, the origin of the annular reflectivity change shown in Figure 2.9 (b) is analysed. It is worth emphasizing that this ultrafast response, since it is off-center, appears at a considerably lower fluence than the peak fluence and corresponds to a local fluence of aprox. $1.5 \mathrm{~J} / \mathrm{cm}^{2}$. As can be seen in Figure 2.10 (a), this local fluence corresponds to the subablative regime, since most of the high reflectivity ring lies outside the ablated crater (image labelled with $\infty$ ).

Much more information can be extracted from this data by analysing the temporal evolution of the reflectivity at the central part of the ring, represented with a cyan circle in Figure 2.10 (a). Figure 2.10 (b) shows the obtained reflectivity evolution, where each point represents the measured transient reflectivity at that local fluence for each of the recorded images. This ultrafast increase and decrease of reflectivity suggest that this is ultrafast transient phenomenon, in the absence of ablation. Therefore, this reflectivity change can be associated to an interaction of the pump laser light with the bound electrons in the valence band, instead of free electrons in the conduction band. Bound electrons that are excited with the intense electric field (pump pulse) 
produce a change in the optical properties of the material, a non-linear process known as the Kerr effect. This effect relates the dependence of the refractive index, $n(t)$, with the irradiance, $I(t)$, as a function of time, being mathematically expressed as,

$$
n(t)=n_{0}+n_{N L} I(t)
$$

where $n_{0}$ is the refractive index of the unexcited material and $n_{N L}$ is the non-linear refractive index.

(a)

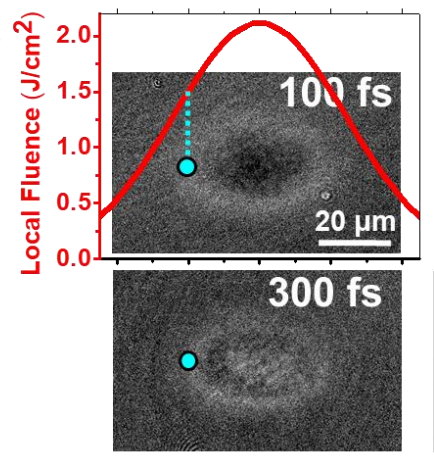

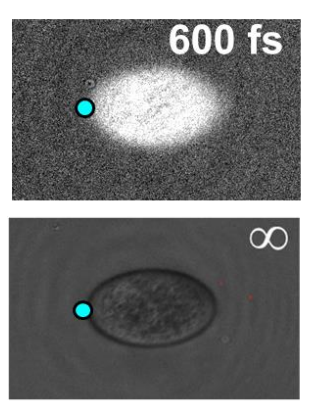

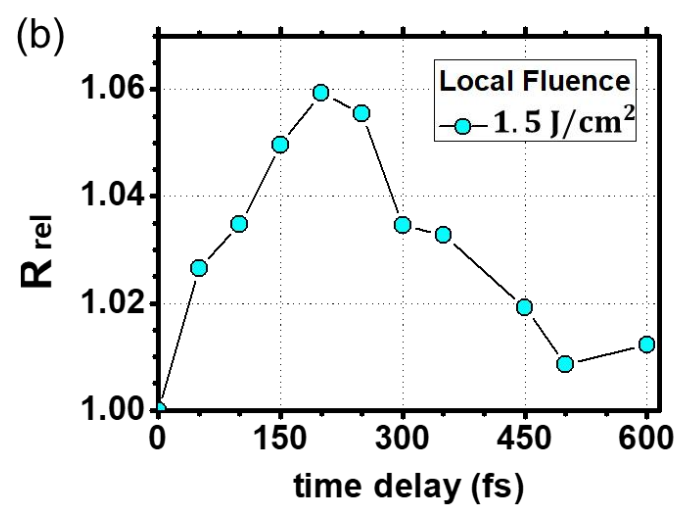

Figure 2.10. (a) Time-resolved surface reflectivity images (delay times are labelled). The cyan circle represents the lateral position corresponding to a local fluence equal to $1.5 \mathrm{~J} / \mathrm{cm}^{2}$. The dark ring that appears in the image labeled as infinite marks the border of the ablation crater. (b) Transient reflectivity curve extracted from time-resolved images (a) at a local fluence slightly below the ablation threshold.

Since the change in $n$ is proportional to $I$, and the reflectivity is observed to increase when $n$ increases, the peak reflectivity observed in Figure 2.10 (b) corresponds to the moment when the sample is subjected to the intensity peak of the pump pulse. This peak irradiance is calculated to be $I=10.5 \mathrm{TW} / \mathrm{cm}^{2}$, considering the local fluence of $1.5 \mathrm{~J} / \mathrm{cm}^{2}$ and knowing the pulse has a Gaussian temporal shape of $130 \mathrm{fs}$ at full width at half maximum (FWHM).

This observation has a practical consequence, the precise observation of the pump pulse arrival at the sample surface. This is an important observation for pump-and-probe experiments, since it allows to determine the so-called zero delay, marking the origin in time of the laser-matter interaction processes that follow.

Additionally, the non-linear refractive index, $n_{N L}$, can be retrieved with this experimental data. Experimentally, a maximum increase in reflectivity of $\Delta R=5.9 \%$ is observed, for a local irradiance of $I=10.5 \mathrm{TW} / \mathrm{cm}^{2}$. By using the relationship shown in equation (2.8), the increase of refractive index corresponding to this increase of reflectivity is $\Delta n=0.074$ can be obtained. Knowing that the initial reflectivity of lithium niobate at the pump laser wavelength $(800 \mathrm{~nm})$ is $R_{0}=0.175$ (with $n_{0}=2.44$ ), and taking into account the Kerr effect dependence of the refractive index with the irradiance (equation (2.10), the non-linear refractive index of lithium niobate can be estimated as: $n_{N L} \approx 7.3 \cdot 10^{-3} \mathrm{~cm}^{2} / T W$. This value is consistent with the ones found by other groups [43,44]. 


\subsubsection{Free electron generation and physical mechanisms}

In this subsection, the reflectivity evolution at the spot centre is analysed, where the local fluence $\left(F=2.1 \mathrm{~J} / \mathrm{cm}^{2}\right)$ corresponds to excitation levels above the ablation threshold. Figure 2.11 (b) shows the temporal evolution of the sample reflectivity, where each point represents the measured transient reflectivity at that local fluence for each of the recorded images, as represented in Figure 2.11(a) with a yellow triangle. In this graph, the reflectivity is observed to initially decrease before undergoing a subsequent increase, reaching a maximum value for a delay of $\sim 750 \mathrm{fs}$, which is a completely different behaviour from the one observed in Figure 2.10 (b).

This behaviour corresponds to a regime in which laser-induced generation of free electrons determines the optical response. This is demonstrated by simulating, employing the Drude model, the reflectivity change when increasing artificially the free electron population in lithium niobate. The result of this calculation is represented in Figure 2.11 (c). It can be seen that the shape of both curves (experimental and modelled) is essentially similar, featuring an initial drop in reflectivity, followed by a steep rise caused by the further increase in free carrier density. Therefore, the observed maximum reflectivity in Figure 2.11(b) corresponds to the moment of maximum free electron generation. From Figure 2.11(c) the maximum free electron density can be estimated by identifying the point that corresponds to the experimental maximum reflectivity, obtaining $n_{e, \max } \approx 6 \cdot 10^{22} \mathrm{~cm}^{-3}$.

(a)

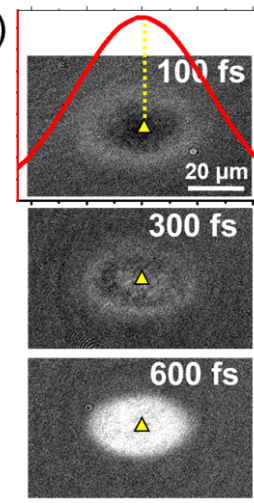

(b)

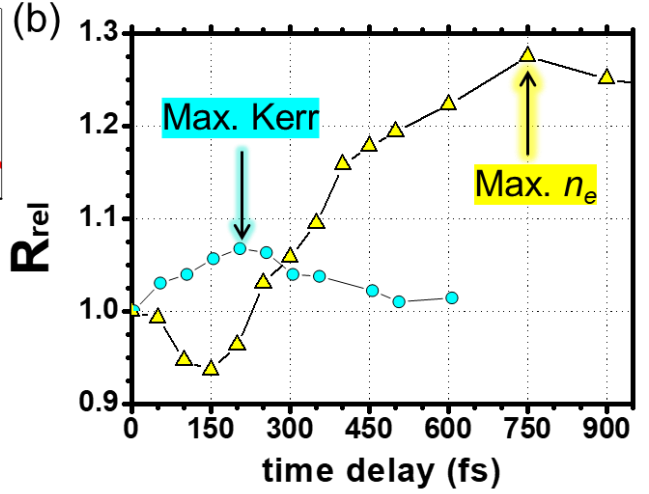

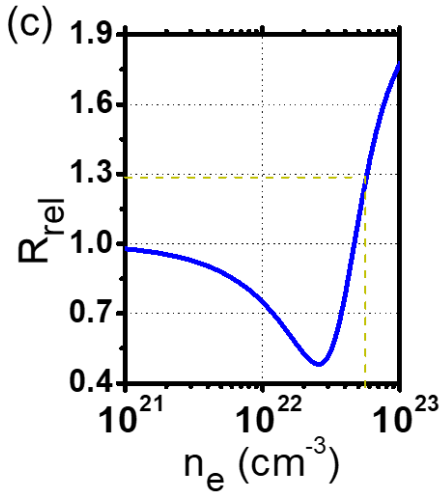

Figure 2.11. (a) Time-resolved surface reflectivity images (delay times are labelled). The yellow triangles represent the spatial position corresponding to a local fluence equal to the peak fluence, $2.1 \mathrm{~J} / \mathrm{cm}^{2}$ (b) (yellow triangles) Transient reflectivity curve extracted from the time-resolved images shown in (a) above the ablation threshold, corresponding to the center of the irradiation spot. (cyan circles) Data taken from Figure 2.10 (b). (c) Simulation of the surface reflectivity as a function of the free electron density, using the Drude model. The dashed horizontal line indicates the maximum reflectivity observed experimentally, and the vertical line indicates the free electron density corresponding to that reflectivity.

An important observation derived from these results is that the reflectivity peak is reached $550 \mathrm{fs}$ after the pump pulse intensity peak (maximum of the Kerr effect). This observation indicates the existence of a delayed electron excitation mechanism, such as impact ionization. This issue has led in the past to a vivid debate and some controversial results $[22,37,45,46]$. This study, with a precise and reliable determination of the zero-delay based on the observation of Kerr-effect, and direct space-and-fluence resolved measurements, confirms the presence of delayed carrier generation, at least for this material $\left(\mathrm{LiNbO}_{3}\right)$. 


\subsection{Summary}

In this chapter it was reviewed the relevance of electronic excitation in the field of laser processing of materials. The first part of the chapter described the optical properties of materials, according to their nature (conductive or dielectric) and to the spectral range of interest. These optical properties make possible to explain the mechanisms of light absorption, both under low and under intense irradiation conditions, such as those produced by ultrashort laser pulses. In particular, the mechanisms of non-linear absorption and impact ionization that occur in dielectric materials are described in detail. The second part of the chapter reviews different experimental ultrafast imaging techniques, whose interest lies in its capacity to characterize the excitation and transformation dynamics of materials after irradiation. These techniques, usually referred as pump-probe techniques, provide access to the optical transient changes by measuring reflectivity changes (including ellipsometry), transmission evolution or phase shifts. Depending on the time delay of observation, the optical changes can be associated to different processes, as the free electron generation occurring at ultrashort time delays or subsequent transformation effects, as material melting or material ablation. Finally, in the third part of the chapter, an experimental case of study was shown: the excitation dynamics of a dielectric material by using time-resolved microscopy. By the analysis of images recorded at different time delays, it was characterized the ultrafast non-linear Kerr effect, associated to the interaction with bound electrons in the valence band, and the free electrons generation dynamics, which allows to retrieve the relative role of the different ionization mechanisms.

\section{References}

[1] W.M. Steen, J. Mazumder, Laser Material Processing, Springer London, London, 2010. https://doi.org/10.1007/978-1-84996-062-5.

[2] E.G. Gamaly, a. V. Rode, Physics of ultra-short laser interaction with matter: From phonon excitation to ultimate transformations, Prog. Quantum Electron. 37 (2013) 215-323. https://doi.org/10.1016/j.pquantelec.2013.05.001.

[3] G.R. Fowles, Introduction to Modern Optics, Dover Publications, 1975. https://books.google.es/books?id=SL1n9TuJ5YMC.

[4] L. V. Rodríguez-de Marcos, J.I. Larruquert, J.A. Méndez, J.A. Aznárez, Self-consistent optical constants of SiO_2 and Ta_20_5 films, Opt. Mater. Express. 6 (2016) 3622. https://doi.org/10.1364/0ME.6.003622.

[5] M.A. Green, M.J. Keevers, Optical properties of intrinsic silicon at 300 K, Prog. Photovoltaics Res. Appl. 3 (1995) 189-192. https://doi.org/10.1002/pip.4670030303.

[6] A.D. Rakic, A.B. Djurisic, J.M. Elazar, M.L. Majewski, Optical properties of metallic films for verticalcavity optoelectronic devices, Appl. Opt. 37 (1998) 5271. https://doi.org/10.1364/A0.37.005271.

[7] R.W. Boyd, Nonlinear optics, Academic Press, 2008.

[8] F. Krausz, T. Brabec, Intense few-cycle laser fields: Frontiers of nonlinear optics, Rev. Mod. Phys. 72 (2000) 545-591.

[9] B.C. Stuart, M.D. Feit, S. Herman, A.M. Rubenchik, B.W. Shore, M.D. Perry, Nanosecond-tofemtosecond laser-induced breakdown in dielectrics, Phys. Rev. B. 53 (1996) 1749-1761. https://doi.org/10.1103/PhysRevB.53.1749.

[10] B. Rethfeld, Free-electron generation in laser-irradiated dielectrics, Phys. Rev. B. 73 (2006) 035101. https://doi.org/10.1103/PhysRevB.73.035101. 
[11] B.C. Stuart, M.D. Feit, S. Herman, a. M. Rubenchik, B.W. Shore, M.D. Perry, Optical ablation by highpower short-pulse lasers, J. Opt. Soc. Am. B. 13 (1996) 459. https://doi.org/10.1364/JOSAB.13.000459.

[12] M. Garcia-Lechuga, G. Gebrayel El Reaidy, H. Ning, P. Delaporte, D. Grojo, Assessing the limits of determinism and precision in ultrafast laser ablation, Appl. Phys. Lett. 117 (2020) 171604. https://doi.org/10.1063/5.0023294.

[13] M. Malinauskas, A. Žukauskas, S. Hasegawa, Y. Hayasaki, V. Mizeikis, R. Buividas, S. Juodkazis, Ultrafast laser processing of materials: from science to industry, Light Sci. Appl. 5 (2016) e16133. https://doi.org/10.1038/lsa.2016.133.

[14] B. Rethfeld, K. Sokolowski-Tinten, D. von der Linde, S.I. Anisimov, Timescales in the response of materials to femtosecond laser excitation, Appl. Phys. A. 79 (2004). https://doi.org/10.1007/s00339-004-2805-9.

[15] A.H. Zewail, Laser Femtochemistry, Science (80-. ). 242 (1988) 1645-1653. https://doi.org/10.1126/science.242.4886.1645.

[16] M.C. Downer, R.L. Fork, C. V. Shank, Femtosecond imaging of melting and evaporation at a photoexcited silicon surface, J. Opt. Soc. Am. B. 2 (1985) 595-599. https://doi.org/10.1364/JOSAB.2.000595.

[17] C. Shank, R. Yen, C. Hirlimann, Time-Resolved Reflectivity Measurements of Femtosecond-OpticalPulse-Induced Phase Transitions in Silicon, Phys. Rev. Lett. 50 (1983) 454-457. https://doi.org/10.1103/PhysRevLett.50.454.

[18] D. von der Linde, K. Sokolowski-Tinten, J. Bialkowski, Laser-solid interaction in the femtosecond time regime, Appl. Surf. Sci. 109-110 (1997) 1-10. https://doi.org/10.1016/S01694332(96)00611-3.

[19] K. Sokolowski-Tinten, J. Bialkowski, A. Cavalleri, D. von der Linde, A. Oparin, J. Meyer-ter-Vehn, S.I. Anisimov, Transient States of Matter during Short Pulse Laser Ablation, Phys. Rev. Lett. 81 (1998) 224-227. https://doi.org/10.1103/PhysRevLett.81.224.

[20] N.A. Inogamov, Y. V. Petrov, S.I. Anisimov, a. M. Oparin, N. V. Shaposhnikov, D. von der Linde, J. Meyer-ter-Vehn, Expansion of matter heated by an ultrashort laser pulse, J. Exp. Theor. Phys. Lett. 69 (1999) 310-316. https://doi.org/10.1134/1.568029.

[21] D. von der Linde, K. Sokolowski-Tinten, The physical mechanisms of short-pulse laser ablation, Appl. Surf. Sci. 154-155 (2000) 1-10. https://doi.org/10.1016/S0169-4332(99)00440-7.

[22] D. Puerto, J. Siegel, W. Gawelda, M. Galvan-Sosa, L. Ehrentraut, J. Bonse, J. Solis, Dynamics of plasma formation, relaxation, and topography modification induced by femtosecond laser pulses in crystalline and amorphous dielectrics, J. Opt. Soc. Am. B. 27 (2010) 1065. https://doi.org/10.1364/JOSAB.27.001065.

[23] J. Hernandez-Rueda, D. Puerto, J. Siegel, M. Galvan-Sosa, J. Solis, Plasma dynamics and structural modifications induced by femtosecond laser pulses in quartz, Appl. Surf. Sci. 258 (2012) 9389-9393. https://doi.org/10.1016/j.apsusc.2011.12.020.

[24] M. Garcia-Lechuga, J. Siegel, J. Hernandez-Rueda, J. Solis, Femtosecond laser ablation of dielectric materials in the optical breakdown regime: Expansion of a transparent shell, Appl. Phys. Lett. 105 (2014) 112902. https://doi.org/10.1063/1.4895926.

[25] W. Gawelda, D. Puerto, J. Siegel, A. Ferrer, A. Ruiz de la Cruz, H. Fernández, J. Solis, Ultrafast imaging of transient electronic plasmas produced in conditions of femtosecond waveguide writing in dielectrics, Appl. Phys. Lett. 93 (2008) 121109. https://doi.org/10.1063/1.2988275.

[26] A. Mouskeftaras, A. V. Rode, R. Clady, M. Sentis, O. Utéza, D. Grojo, Self-limited underdense microplasmas in bulk silicon induced by ultrashort laser pulses, Appl. Phys. Lett. 105 (2014) 1-5. https://doi.org/10.1063/1.4901528.

[27] A. Mouskeftaras, M. Chanal, M. Chambonneau, R. Clady, O. Utéza, D. Grojo, Direct measurement of ambipolar diffusion in bulk silicon by ultrafast infrared imaging of laser-induced microplasmas, Appl. Phys. Lett. 108 (2016) 041107. https://doi.org/10.1063/1.4941031. 
[28] X.Y. Wang, M.C. Downer, Femtosecond time-resolved reflectivity of hydrodynamically expanding metal surfaces, Opt. Lett. 17 (1992) 1450. https://doi.org/10.1364/OL.17.001450.

[29] S. Rapp, M. Kaiser, M. Schmidt, H.P. Huber, Ultrafast pump-probe ellipsometry setup for the measurement of transient optical properties during laser ablation, Opt. Express. 24 (2016) 17572. https://doi.org/10.1364/OE.24.017572.

[30] J. Winter, S. Rapp, M. Spellauge, C. Eulenkamp, M. Schmidt, H.P. Huber, Ultrafast pump-probe ellipsometry and microscopy reveal the surface dynamics of femtosecond laser ablation of aluminium and stainless steel, Appl. Surf. Sci. $511 \quad$ (2020) 145514. https://doi.org/10.1016/j.apsusc.2020.145514.

[31] V.V. Temnov, K. Sokolowski-Tinten, P. Zhou, D. von der Linde, Femtosecond time-resolved interferometric microscopy, Appl. Phys. A Mater. Sci. Process. 78 (2004) 483-489. https://doi.org/10.1007/s00339-003-2408-x.

[32] V. V Temnov, K. Sokolowski-Tinten, P. Zhou, D. von der Linde, Ultrafast imaging interferometry at femtosecond-laser-excited surfaces, J. $\quad$ Opt. Soc. Am. $\quad$ B. $23 \quad$ (2006) 1954. https://doi.org/10.1364/JOSAB.23.001954.

[33] L. Gallais, S. Monneret, Time-resolved quantitative-phase microscopy of laser-material interactions using a wavefront sensor, Opt. Lett. 41 (2016) 3245. https://doi.org/10.1364/OL.41.003245.

[34] P. Bon, G. Maucort, B. Wattellier, S. Monneret, Quadriwave lateral shearing interferometry for quantitative phase microscopy of living cells, Opt. Express. 17 (2009) 13080. https://doi.org/10.1364/OE.17.013080.

[35] P. Audebert, P. Daguzan, A. Dos Santos, J.C. Gauthier, J.P. Geindre, S. Guizard, G. Hamoniaux, K. Krastev, P. Martin, G. Petite, A. Antonetti, Space-time observation of an electron gas in si02, Phys. Rev. Lett. 73 (1994) 1990-1993. https://doi.org/10.1103/PhysRevLett.73.1990.

[36] J.P. Geindre, P. Audebert, A. Rousse, F. Falliès, J.C. Gauthier, A. Mysyrowicz, A. Dos Santos, G. Hamoniaux, A. Antonetti, P. Audebert, A. Rousse, G. Hamoniaux, A. Antonetti, F. Falliès, J.C. Gauthier, A. Mysyrowicz, A. Dos Santos, G. Hamoniaux, A. Antonetti, P. Audebert, A. Rousse, G. Hamoniaux, A. Antonetti, F. Falliès, J.C. Gauthier, Frequency-domain interferometer for measuring the phase and amplitude of a femtosecond pulse probing a laser-produced plasma, Opt. Lett. 19 (1994) 1997. https://doi.org/10.1364/OL.19.001997.

[37] K.J. Waedegaard, D.B. Sandkamm, A. Mouskeftaras, S. Guizard, P. Balling, Probing ultrashort-pulse laser excitation of sapphire: From the initial carrier creation to material ablation, Europhys. Lett. 105 (2014) 47001. https://doi.org/10.1209/0295-5075/105/47001.

[38] A. Mouskeftaras, S. Guizard, N. Fedorov, S. Klimentov, Mechanisms of femtosecond laser ablation of dielectrics revealed by double pump-probe experiment, Appl. Phys. A. 110 (2013) 709-715. https://doi.org/10.1007/s00339-012-7217-7.

[39] M. Garcia-Lechuga, L. Haahr-Lillevang, J. Siegel, P. Balling, S. Guizard, J. Solis, Simultaneous timespace resolved reflectivity and interferometric measurements of dielectrics excited with femtosecond laser pulses, Phys. Rev. B. $95 \quad$ (2017) 214114. https://doi.org/10.1103/PhysRevB.95.214114.

[40] L. Arizmendi, Photonic applications of lithium niobate crystals, Phys. Status Solidi. 201 (2004) 253283. https://doi.org/10.1002/pssa.200303911.

[41] M. Garcia-Lechuga, J. Siegel, J. Hernandez-Rueda, J. Solis, Imaging the ultrafast Kerr effect, free carrier generation, relaxation and ablation dynamics of Lithium Niobate irradiated with femtosecond laser pulses, J. Appl. Phys. 116 (2014) 113502. https://doi.org/10.1063/1.4895833.

[42] M. Garcia-Lechuga, J. Solis, J. Siegel, Key stages of material expansion in dielectrics upon femtosecond laser ablation revealed by double-color illumination time-resolved microscopy, Appl. Phys. A Mater. Sci. Process. 124 (2018) 1-8. https://doi.org/10.1007/s00339-018-1650-1.

[43] H. Li, F. Zhou, X. Zhang, W. Ji, Picosecond Z-scan study of bound electronic Kerr effect in LiNbO 3 crystal associated with two-photon absorption, Appl. Phys. B Lasers Opt. 64 (1997) 659-662. https://doi.org/10.1007/s003400050229. 
[44] H.-T. Hsieh, D. Psaltis, O. Beyer, D. Maxein, C. von Korff Schmising, K. Buse, B. Sturman, Femtosecond holography in lithium niobate crystals., Opt. Lett. 30 (2005) 2233-5. http://www.ncbi.nlm.nih.gov/pubmed/16190428.

[45] A. Wu, I. Chowdhury, X. Xu, Femtosecond laser absorption in fused silica: Numerical and $\begin{array}{lllllll}\text { experimental investigation, Phys. Rev. B. } 72 & \text { (2005) } & 085128 .\end{array}$ https://doi.org/10.1103/PhysRevB.72.085128.

[46] B. Rethfeld, Free-electron generation in laser-irradiated dielectrics, Phys. Rev. B. 73 (2006) 035101. https://doi.org/10.1103/PhysRevB.73.035101. 



\title{
3. Ultrafast-laser high electronic excitation for nano-acoustic wave generation: new horizons in the materials probing
}

\author{
Nektarios A. Papadogiannis*
}

To probe the ultrafast high electronic excitation phenomena that occur on the surface or inside the bulk of materials, at proper time scales, it is required to develop laser secondary sources that offer the ability of monitoring phenomena deep in the matter with extremely small spatiotemporal windows. Although laser technology provides electromagnetic sources in the visible and near-infrared light (ultra-short lasers technology) their penetration inside the materials is often very small and the observation capacity is limited in the very few surface layers of materials, i.e. for metals is of the order of few nanometers. In this chapter we will present laser secondary sources of nano-mechanical waves that will be, in our opinion, the future of nanoimaging of materials in small spatial dimensions much less than a micrometer and in very short time scales in the order of picoseconds or less. All presented here ultrasonic sources could penetrate matter in micrometer or even in millimeter scales thus are able to probe the bulk. These sources are the picosecond longitudinal ultrasonic mechanical pulses generated by the localized interaction of ultrafast laser pulses with the metallic film lattices.

\footnotetext{
*Contact: npapadogiannis@hmu.gr
} 


\subsection{Introduction}

In the last decades, there exist a fast development of ultrafast laser pulse technology providing laser pulses with duration well below 1 ps with the state of the art in our days to be in the order of few as ( 1 as=10-18 $\mathrm{s}$ ). Initially, by mode-locking various chromatic components of a broadband fluorescence of an appropriate active laser material light pulses less than 1 ps down to few fs are routinely developed. Since the duration of such pulses is very short, even if the energy per pulse is in the $\mathrm{nJ}$ region, their power easily reaches the $1 \mathrm{MW}$ per pulse.

In early nineties, the Nobelists Gérard Mourou and Donna Strickland [1] discover a method to increase considerably the energy of ultrafast pulses without destroying the optical parts of the laser cavity. Their method is the Chirp Pulse Amplification (CPA) [2]. By spreading the energy of various chromatic components in different times (chirp) in an optical stretcher, they were able to safely amplify the light in an optical amplifier and then compress back the laser pulse to its Fourier-Limited duration in an optical compressor. This technique allows to increase the energy per pulse from the $\mathrm{nJ}$ to the $\mathrm{mJ}$ region and by using many stages of optical amplifiers and vacuum compressor to a maximum of about $100 \mathrm{~J}$. Thus, the ultrafast laser pulse power reaches, today, in values of the order of few PW.

At the same period there were parallel tries to decrease the pulse duration from about 10-20 fs that allows the natural fluorescence spectrum of some laser materials (eg. Titanium Sapphire crystals) down to 5 fs region, i.e., only few optical cycles of the laser EM field [3]. The timebandwidth product of a laser pulse shows whether the spectral width is the necessary for the given pulse duration. For a few-cycle laser pulses, a bandwidth of more than $200 \mathrm{~nm}$ is necessary in the optical and NIR region. This cannot be fulfilled by the natural fluorescence bandwidths of the common laser materials. Therefore, non-linear technique of the self-phase modulation (SPM) is used. The SPM of the Electric femtosecond laser field inside a neutral gas hollow capillary gave the best results for spectral broadening. The chirp of these broad bandwidth pulses spans, sometimes, in more than one color octave is taken back through specially design chirp multilayer mirror compressor [4]. This way, pulses with duration of few fs are generated. The nowadays technology allows for optoelectronic techniques in order the carrier envelope phase of such pulses to be monitored and controlled in pulse-to-pulse frame. Thus, the maximum E-field of the laser pulse can be kept constant, fact important for the non-linear interaction of these high-power pulses with the matter. Off course, the laser pulse quality includes additional aspects such as details of the temporal and spectral pulse shape, such as the presence of temporal or spectral pedestals or side lobes.

Furthermore, the need for even shorter than fs laser pulses should be based on a different region of spectrum, i.e., VUV or XUV. The non-linear phenomenon of high harmonic generation (HHG) is typically used to extend the laser coherent light to VUV and XUV spectral region [5]. In this phenomenon, a fs laser pulse excited the external electrons in the continuum by tunneling effect. After half E-field laser period the sign of the E-field changes and the electrons are driven back to the atomic nuclei. This happens twice the laser E-field period and the electrons oscillating around the atomic core. Every time that an electron approaches the atomic core a non-zero probability to be captured exist. The electron re-capturing from the atomic core accompanied by the emission of high energy photon. The maximum energy of the photon depends on the atomic target ionization potential the ponderomotive energy that the electron gains by its oscillation from the 
laser E-Field around the atomic nuclei. Since the phenomenon occurs twice per laser period which is, for optical laser $\sim 2-3 \mathrm{fs}$, the secondary emitted photon has a spectrum of individual odd harmonics covering the spectrum from the UV up to soft-Xray EM-field region [6-8]. The first idea for as laser pulse generation is based on the mode-locking of these harmonic coherent spectral lines and thus XUV light can be localized in a "train" of attosecond wagons [9]. The experimental demonstration of such ideas appears few years after the theoretical proposal $[10,11]$. Additionally, the same idea is used for a single as laser pulse fabrication [12]. Now, the XUV generating IR laser pulse last no more than 1-2 laser period and thus the harmonic spectrum in the cut-off region is continuous. By Fourier adding this part of spectrum single as pulses are generated and measured.

All these evolution of ultrafast laser pulses enable a numerous direct application starting for the monitoring of phenomena happening in microcosmos and even in the atomic level. Among them is worthy to refer to a) the femto-chemistry ideas by Nobelist Ahmed Zewail [13] that monitoring chemical reaction in their natural time scales and control them, b) the nanoscale fs Laser-material processing which is now a major component of the manufacturing process [1], c) the novel kinds of laser surgery are now available that utilize ultrafast high-intensity laser processing of tissue, i.e., Laser-Assisted In-Situ Keratomileusis (LASIK) [14], where the laser pulse scalpels to make incisions in the eyeball in order to improve eyesight, d) the tries for ultra-intense and ultrafast laser-driven inertial confinement fusion for energy production [15].

Furthermore, more important seems to be the applications of the secondary sources in the interaction of ultrafast and ultra-intense laser pulses with matter. Among them are: i) the generation of localized nano-acoustic (mechanical) pulses with picosecond duration and down to few nm carrier wavelengths, ii) the nanoscale coherent sub-fs XUV EM pulses (HHG) and iii) the microscale coherent X-Ray betatron-type sources generated by the accelerated electrons inside the plasma waves (Laser weak Field Acceleration - LWFA) induced by the interaction of an ultraintense relativistic laser with a gas target [16].

The physics and technology of the generation of such secondary laser sources, the role of the high electronic excitation on their final characteristics and their applicability on the probing materials in unexplored spatiotemporal limits is crucial.

In this chapter, it will be presented a tutorial review of the research that take place in Institute of Plasma Physics and Laser of the Hellenic Mediterranean University on the physics and technology of ultrafast laser generated nano-acoustic waves and their specific application in material probing.

\subsection{The role of the metals as optoacoustic transducers}

Since a reasonable elementary model for a metal is that of an isotropic free electron gas, which is a highly linear system when excited by electromagnetic waves, the non-linear mechanisms are very weak. Thus, experimental investigations require relatively high optical power laser sources and are performed very close to the damage threshold of the metal surfaces. The damage takes place when the lattice temperature exceeds the melting point of the metal.

The only way to apply very intense electromagnetic fields on a metallic surface, without destroying it, is to use ultrashort laser pulses. When the laser pulse is shorter than the electron- 
phonon energy relaxation time, the electrons are rapidly thermalized in a high temperature Fermi-Dirac distribution, but the lattice remains at relatively low temperature due to its high specific heat $[17,18]$. After a few picoseconds (or faster), electron energy relaxation occurs in the lattice [19-22].

The penetration depth of a laser beam in a metal, is of the order of few $10 \mathrm{~nm}$ for the wavelength range of the lasers used in these studies. In fact, the thickness of a metallic surface that participates in the above-mentioned phenomena is of the order of a few $10 \mathrm{~nm}$.

In the Sommerfeld theory, a metal is described, quantum mechanically, by a step potential model as shown in Figure 3.1. The electrons are free to move in the left part of the step potential (bulk). The electronic energy states can be calculated quantum mechanically [23]. Pauli exclusion principle requires a Fermi-Dirac distribution for the electrons. The electron occupation probability of a state with energy $E$ is given by the expression:

$$
f(E)=\frac{1}{1+e^{\frac{E-E_{F}}{k_{B} T}}}
$$

Where $E_{F}$ is the Fermi energy of the metal, $k_{\mathrm{B}}$ is the Boltzmann constant and $T$ is the electrons temperature. The range of Fermi energies, for the densities of metallic elements, is between 1.5 and $15 \mathrm{eV}$ [23].

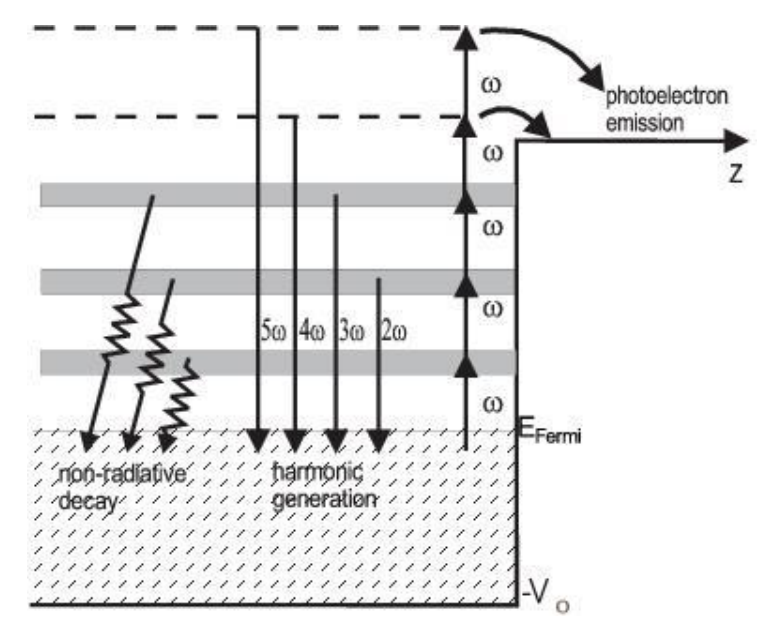

Figure 3.1. The electrons on a metallic surface are attracted by the bulk positive ions and the potential of this attraction can be described by a step potential. When an external electromagnetic field excites the electrons of the metal three phenomena may occur: Multiphoton absorption, harmonic generation and non-radiative energy decay by electronelectron and electron-phonon scattering [24].

The electrons on a metallic surface are attracted by the bulk positive ions and the potential of this attraction can be described by a step potential. When an external electromagnetic field excites the electrons of the metal three phenomena may occur: Single and multiphoton absorption and re-emission, harmonic generation and non-radiative energy decay by electron-electron and electron-phonon scattering. In the above feature the external fields are assumed to be unable to change the profile of the surface potential -perturbation region- but at the same time are intense enough to induce multiphoton phenomena. The thick metal can be described by the one- 
dimensional step potential. This dimension is the one perpendicular to the surface, because in the plain of the metal surface the electrons are free particles and cannot absorb any photons. If $z$ is the axis perpendicular to the metal surface which lies at $z=0$, the one-dimensional potential step is given by:

$$
V(z)=\left\{\begin{array}{c}
-V_{o} \text { for } z \leq 0 \\
0 \text { for } z>0
\end{array}\right.
$$

The band theory is the basis of the distinction between metallic conductors, semiconductors and insulators. In the case of insulators, all the lower bands are full, the first empty band being separated by a relatively large band gap from the upper empty one. In the case of semiconductors, thermal excitation of electrons across the band gap occurs, leaving a few empty states in the lower band and a few filled states at the bottom of the almost empty band. In the case of metals having a partially filled upper band (conduction band), all the states are partially filled within a region of $k_{\mathrm{B}} T$ around the Fermi level. For some cases, a crystal has metallic behavior due to overlapping of the two upper bands, one with few empty states and another one with few filled states.

Even the conduction electrons of a metal are not completely free particles. The electrons interact predominantly with the phonons (lattice vibrations) and in special situations with other electrons. The number of phonons in any normal mode is given by the Bose statistics and it is expressed by the equation [24]:

$$
n_{q}=\frac{1}{e^{\frac{E_{p h o n}}{k_{B} T_{L}}}-1}
$$

where $E_{\text {phon }}$ is the energy of the phonons and $T_{L}$ is the lattice temperature.

In the electron-phonon interaction creation or destruction of one phonon may occur. Assuming a constant density of states, the electron phonon scattering time depends on the total probability for a phonon scattering event. The phonon creation probability is proportional to $n_{q}+1$ and the destruction one is proportional to $n_{q}$ [25]. Since $n_{q}$ is temperature dependent, the electron-photon relaxation rate depends on the lattice temperature $[23,26]$.

In the limit, where the lattice temperature is higher than the Debye temperature, $T_{D}, n_{q}$ in (3.3) is equal to $k_{B} T / E_{\text {phon. }}$. This happens because $T_{D}$ is the respective temperature of the most energetic phonons. In this situation, the electron-phonon relaxation collision time, $\tau_{\mathrm{e}-\mathrm{ph}}$, i.e., the time between two scattering of an electron by phonons, is inversely proportional to the lattice temperature. Note that, in each electron-phonon scattering, only a few percent of the electron energy is transferred for the phonon creation, which, typically, have energy a few meV. Thus, an electron with an energy of a few $\mathrm{eV}$, above the Fermi level, requires more than one thousand scatterings to transfer its energy to the lattice. The statistical average time, which an excited part of the electron gas above the $E_{F}$ requires to relax giving its energy to the lattice is the electronphonon energy relaxation time. The energy relaxation is one or two (or more) orders of magnitude longer than the electron-phonon scattering time. According to the Fermi liquid theory, i.e., consequences of the exclusion principle on electron-electron scattering near the Fermi energy, an electron cannot be scattered by another electron, if the first one is less or equal to the Fermi- 
energy [23]. Suppose, for example, that the $\mathrm{N}$-electron state consists of a filled Fermi-sphere $\left(T_{e}=0\right)$, plus an excited electron of energy $E_{1}$ slightly higher than $E_{F}$.

For this electron to be scattered, it must interact with another electron of energy $E_{2}$. In our case, $E_{2}$ is less than $E_{F}$, since only the states with energy lower than $E_{F}$ are occupied. The Pauli exclusion principle requires that the two electrons must scatter into unoccupied levels, effectively greater than $E_{F}$. Thus, $E_{1}>E_{F}, E_{2}<E_{F}, E_{3,4}>E_{\mathrm{F}}$. Additionally, energy conservation requires: $E_{1}+E_{2}=E_{3}+E_{4}$.

The above conditions are not satisfied together, except in the limited case where $E_{1}=E_{2}=E_{3}=E_{4}=E_{\mathrm{F}}$. Thus, the allowed wave vectors for electrons 2,3,4 occupy a region of $\mathrm{k}$-space of zero volume, (i.e., the Fermi surface), and therefore give a vanishingly small contribution to the cross-section of the process. Consequently, the lifetime of an electron at the Fermi-surface at $T=0$ is infinite.

In the case where $E_{1}$ is larger than $\mathrm{EF}$, the available phase-space for the scattering process is a shell of thickness of order $E_{1}-E_{\mathrm{F}}$. This leads to a scattering rate of order $\sim\left(E_{1}-E_{\mathrm{F}}\right)^{2}$. The quantity appears squared rather than cubed, because once $E_{2}$ and $E_{3}$ have been chosen within the shell of allowed energies, energy conservation allows no further choice for $E_{4}$. In fact, very low excitation in elastic region, the electron Fermi-Dirac temperature has a minimum value equal to the room one, i.e., $\sim 0.025 \mathrm{eV}$. This provides an additional range of choice of the order of $k_{B} T$ because there will be partially occupied levels in a shell of width $k_{B} T$ around the $E_{F}$. Combining the above considerations, we can express the electron-electron scattering rate as [23,24]:

$$
\frac{1}{\tau_{e-e}}=s_{1}\left(E_{1}-E_{F}\right)^{2}+s_{2}\left(k_{B} T\right)^{2}
$$

where the coefficients $s_{1}, s_{2}$ are independent of $E_{1}$ and $T$.

For a metallic material, the energy of the femtosecond laser pulse is initially absorbed by the electrons, thus altering their thermal energy and temperature. Note, here that at the very initial times of the interaction the energy distribution of the electrons doesn't obey a Fermi-Dirac distribution (non-thermal electrons) and thus the use of the term "temperature" is abusive. Fastly, in few tens of femtoseconds, the electron gas is thermalized mainly through electron-electron collisions and an initial Fermi-Dirac electron distribution is formed. The electron thermal energy is then transferred to the phonons via electron-phonon interactions; therefore, a part of the electron thermal energy diffuses in the sample. The electron heat capacity is much smaller than that of the lattice, thus the electrons acquire a higher temperature than the lattice. During this non-equilibrium process a localized acoustic strain pulse wave is generated by the sudden transport of excess electron energy to the lattice.

Metals with strong electron-phonon coupling constant seems to be the best optoacoustic transducers. In this scheme, the electron and lattice temperatures are modeled separately in the metal surface using the Anisimov heat equations $[17,24]$. The dimension of the area illuminated by the laser pulse is assumed to be large compared to the absorption length and the total lattice length of the sample. Therefore, the problem can be solve only in one-dimensional (z-direction is normal to the metal surface). The basic equations of electron temperature $T_{\mathrm{e}}$ and lattice temperature $T_{\mathrm{L}}$ spatiotemporal evolution are: 


$$
\begin{gathered}
C_{e} \frac{\partial T_{e}(z, t)}{\partial t}=k \frac{\partial^{2} T_{e}}{\partial z^{2}}-U\left(T_{e}, T_{L}\right)+G(z, t) \\
C_{L} \frac{\partial T_{L}(z, t)}{\partial t}=U\left(T_{e}, T_{L}\right)
\end{gathered}
$$

where $C_{\mathrm{e}}, C_{\mathrm{L}}$, are the heat capacities of the electrons and lattice, respectively, and $k$ is the electron thermal conductivity. The term $U\left(T_{\mathrm{e}}, T_{\mathrm{L}}\right)$ corresponds to the amount of energy per unit volume and per unit time transferred from the electrons to the lattice, and is given by $U\left(T_{\mathrm{e}}, T_{\mathrm{L}}\right)=G \times\left(T_{\mathrm{e}}\right.$ $T_{\mathrm{L}}$ ) where $g$ accounts for the electron-phonon coupling constant. The laser pulse power density is $G(\mathrm{z}, \mathrm{t})=a I_{0} \mathrm{e}^{-a z} I(t)$ where $a$ is the surface absorptivity (equivalently, $\zeta=1 / a$ is the absorption length), $I_{0}$ is the incident laser peak absorbed intensity and $I(t)$ is the temporal profile of the laser pulse which here is assumed to be Gaussian with a characteristic duration, $\tau$, the full temporal width at half maximum (FWHM). The equation for the electron temperature (3.5) is a nonhomogenous heat equation, while the equation for the lattice temperature is coupled to the electron one through the interaction term $U\left(T_{\mathrm{e}}, T_{\mathrm{L}}\right)$.

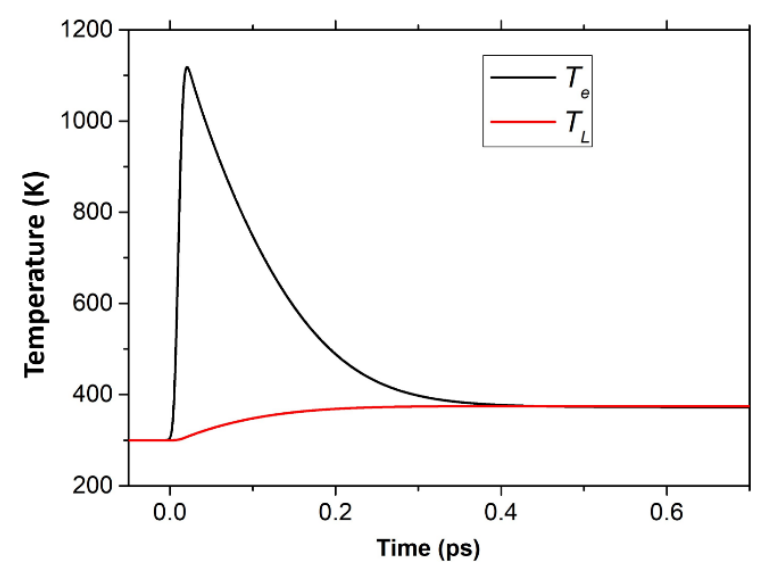

Figure 3.2. Typical solution of Anisimov coupled equations (3.5) at a surface of a noble metal (Ag) after an excitation of a 35 fs laser pulses with a peak intensity $\sim 10^{12} \mathrm{~W} / \mathrm{cm}^{2}$. The electron temperature, $T_{\mathrm{e}}$, is shown with the black line while the lattice temperature, $T_{\mathrm{L}}$, is shown with red line.

Typical solution for electron and lattice temperature of Anisimov coupled equations (3.5) at a surface of a noble metal after an excitation of a 25 fs laser pulses with a peak intensity $\sim 10^{12}$ $\mathrm{W} / \mathrm{cm}^{2}$ is illustrated in Figure 3.2. The maximum of the laser intensity is taken at time $t=0$. It is clear that the initial fast (less than a ps) electron temperature rise is followed by a relatively slow decrease while, at the same scale, the lattice temperature is also slowly increase. After about 10 ps from the laser excitation a thermal equilibrium between electron gas and lattice is reached. Note that, the lattice temperature increases in few picosecond timescales. Take also into account that laser energy absorption is localized in a range of a few tens of nanometres. These effects are the triggering for the generation of a very high and localized strain pulse that moves outwards the interaction region in all directions. 


\subsection{Ultrafast longitudinal ultrasonic waves generated by ultrafast lasers}

The generation and characterization of very-high frequency nano-acoustic waves in metal/substrate layered systems by ultrafast laser pulses has been a subject of primary interest in the field of ultrafast laser-matter interaction [27-32]. After ultrafast laser excitation, very-high frequency nano-acoustic waves are generated that propagate at all directions outward the interaction region. Various laser-based optical experimental techniques have been implemented for the study the dynamics [30-32] of said waves. Femtosecond laser pulses are used to generate longitudinal nano-acoustic pulses of sub-THz frequencies in metal/semiconductor layered systems. A variety of experimental laser-based techniques is employed among with appropriate theoretical simulation models to study and understand the spatiotemporal dynamics and the physical mechanisms following ultrafast laser electron excitation. For example, femtosecond laser pulses were used to excite different metal thin-film transducers deposited on $\mathrm{Si}(100)$ monocrystal thick substrates to examine the role of the optoacoustic transducer [32]. Utilizing a degenerate femtosecond pump-probe transient reflectivity technique giant longitudinal nano-mechanical strains transferred in semiconductor substrates was observed, manifested experimentally as strong Brillouin oscillations. To explain the experimental findings, theoretical thermo-mechanical approaches based on a revised two-temperature model and elasticity theory are developed.

Furthermore, the influence of femtosecond laser pulse chirp on the laser-generated longitudinal acoustic strains in metal/semiconductor substrates is studied. Experimental results show that acoustic strains, manifested as strong Brillouin oscillations, show that the dependence of the Brillouin amplitude and the lattice strain is a non-monotonous function of the laser chirp parameter are more effectively induced when negatively chirped femtosecond laser pulses pump the metallic transducer [33]. A detailed thermomechanical model satisfactorily supports the experimental findings. The model is based in a modified thermo-mechanical model based on the combination of a revised two-temperature model and elasticity theory which considers the instantaneous frequency of the chirped femtosecond laser pump pulses for the first time. That way shows that the suppression or enhancement of the induced nanoacoustic strain amplitude is possible to be controlled with an all-optically scheme that affects the initial high electronic excitation in the optoacoustic transducer [33].

\subsubsection{Degenerate femtosecond transient reflectivity method for the generation and detection of longitudinal nano-acoustic waves in nano-structures materials}

The femtosecond degenerate transient reflectivity experimental arrangement is schematically depicted in Figure 3.3. Typically few $\mu J$ femtosecond Laser pulses are derived from a Ti:Sapphirebased amplifier, at a high repetition rate typically of $1 \mathrm{kHz}$, having a FWHM time duration in the order of a few tens of fs, and center wavelength of $\sim 800 \mathrm{~nm}$. The amplifier's output is split into pump and probe beams. Typically, the pump and probe are orthogonally polarized by means of a low dispersion $\lambda / 2$ waveplate. The pump beam is incident perpendicularly to the metal film surface, loosely focused by means of a spherical broad bandwidth metallic mirror to achieve a fluence of $\sim 10 \mathrm{~mJ} / \mathrm{cm}^{2}$, which is within the thermoelastic regime for most metals. The probe beam 
was incident at an angle of $\sim 45^{\circ}$ relative to the pump beam, focused by means of a parabolic metal mirror, while its fluence is kept at significantly lower levels compared to the pump to avoid contribution to the excitation dynamics [32,33].
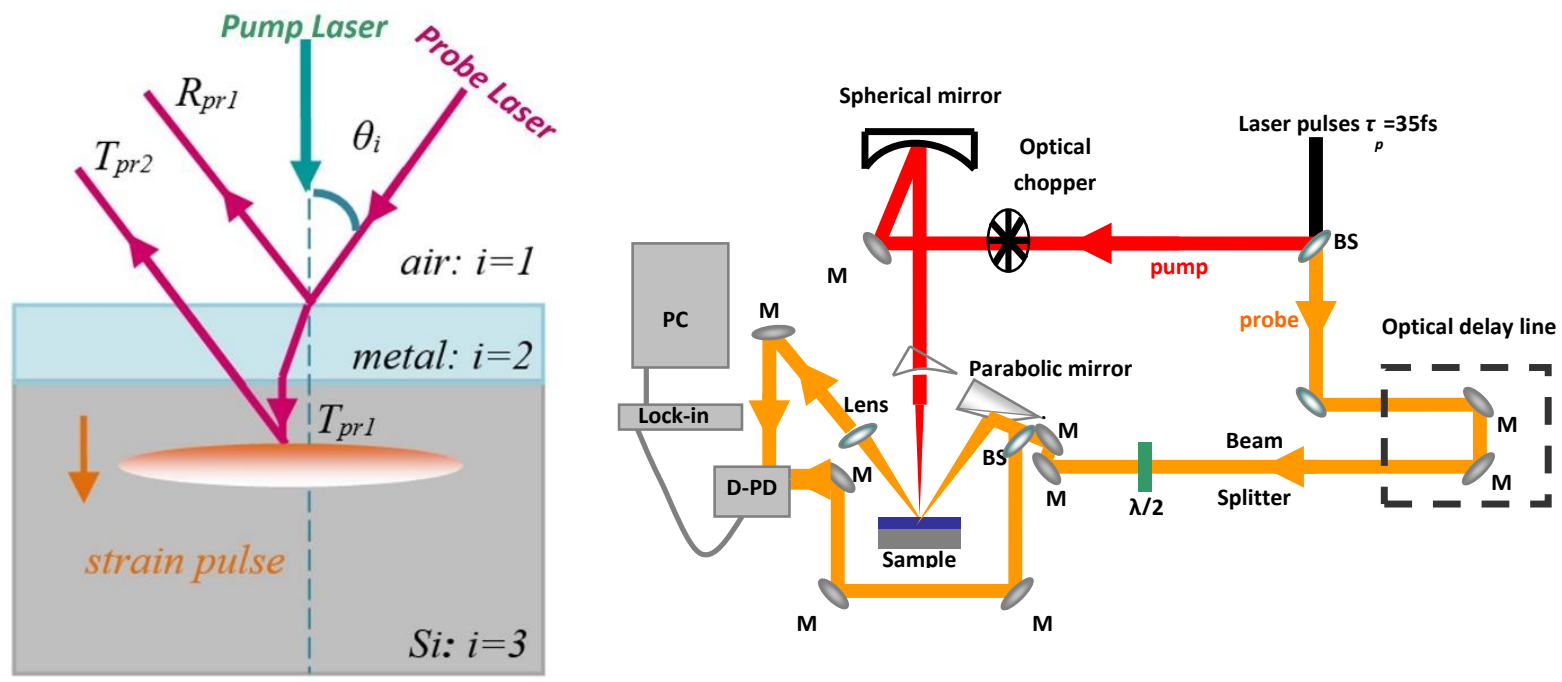

Figure 3.3. Typical femtosecond degenerate transient reflectivity experimental arrangement for nano-acoustic generation strain and echo detection. Left is the detail of the beams in the vicinity of the sample probed [32].

An imaging system is used to monitor the relative position and size of the pump and probe beams on the interaction area. A variable high accuracy ( $<1 \mathrm{fs}$ ) optical delay is introduced between the pump and probe pulses, by means of motorized stage. Since the reflectivity changes from acoustical echoes is very tiny the detection of reflectivity changes take place with a lock-in detection technique. For the use of the lock-in a mechanical chopper modulates the pump beam at a certain frequency. A small portion of the probe beam is split before the target and is directed onto a balanced photodiode along with the probe beam reflected from the sample surface. The output of the photodiode is the difference of these two signals. The balanced photodiode output signal is directed to a dual channel lock-in amplifier which detects signal differences at the pump beam modulating frequency. This detection scheme allows for reflectivity changes of the order of $\sim 10^{-6}$ to be resolved. Specially developed software is used to simultaneously control the temporal delay and detection instruments, and for data recording.

The incident pump laser energy is partially absorbed from the metallic film within few nanometers and is finally converted into metal lattice movement, thus generating a localized acoustic longitudinal strain pulse in the metal film. A small portion of the laser pump beam is also transmitted into the substrate, for example for $25 \mathrm{~nm}$ thick Ti metal and Si substrate is typically $\sim 15 \%$. This transmitted energy for all cases is typically not sufficient for any measurable excitation in the substrate. Strain reflections from the metal/substrate interface can appear in the transient reflectivity data at time delays multiples of $\tau=2 d_{\mathrm{m}} / u_{\mathrm{m}}$, where $d_{\mathrm{m}}$ is the thin metal film thickness and $u_{\mathrm{m}}$ is the longitudinal sound velocity in the metal.

The generated in the metal longitudinal acoustic strain wave enters and propagates inside the substrate. The coupling efficiency of the strain into substrate is determined by the acoustical impedance matching between the metallic material and the substrate. The metal films should be 
sufficiently thin for a fraction of the probe pulse to enter the substrate area and to probe the strain wave as it propagates inside it. The probing process is as follows (Figure 3.3): a fraction of the probe beam is initially reflected upon incidence on the metal/substrate sample $\left(R_{p r 1}\right)$. The small fraction of the probe beam that is not absorbed from the thin metal film is transmitted into the substrate $\left(T_{p r 1}\right)$. Part of the probe beam that enters the substrate will be reflected from the longitudinal strain wave and travels back towards the metal/substrate sample surface experiencing absorption and reflection losses at the interfaces. Eventually, a small fraction of the probe beam will be transmitted out of the metal surface $\left(T_{p r 2}\right)$. The observed signal is the interference of $R_{p r 1}$ and $T_{p r 2}$ beams, manifested as oscillations. The period of the observed oscillations effectively corresponds to a $2 \pi$ optical phase difference between the $R_{p r 1}$ and $T_{p r 2}$ beams. This prerequisite that the longitudinal acoustic strain has to travel a distance that corresponds to an optical delay of the probe beam $\sim 2.7 \mathrm{fs}$, which is the probe beam $E$-field period (for $800 \mathrm{~nm}$ laser), $E_{T}$. Maxima in the oscillations are observed when the optical time delay between the $R_{p r 1}$ and $T_{p r 2}$ pulses is an integer multiple of the $E$-field period (i.e. $k E_{T}$, where $k=1$, $2,3 \ldots)$. Since the $E$-field of the interfering pulses $\left(R_{p r 1}\right.$ and $\left.T_{p r 2}\right)$ has a Gaussian-like temporal envelope (with $\tau_{\text {FWнM }} \sim 20-40 \mathrm{fs}$ ), as the relative temporal delay between them increases, their interference oscillations amplitude decreases. These oscillations are the so-called Brillouin oscillations, and they originate from the Brillouin scattering mechanism. Another way to understand the same phenomenon is to apply Bragg condition, so that the optical path difference, $d_{\text {opt }}$ between two successive maxima must be equal to the probe wavelength, $\lambda$ (constructive interference between $R_{p r 1}$ and $T_{p r 2}$ ). Therefore [32]:

$$
d_{o p t}=2 \tau_{o s c} v=\frac{E_{T} c}{n}=\frac{\lambda}{n} \Rightarrow f_{B r}=\frac{2 n v}{\lambda}
$$

which is the well-known Brillouin scattering frequency (for normal incidence) observed when light experiences refractive index variations inside an optically transparent material due to lattice deformations. $\lambda$ is the central wavelength of the EM wave, $v$ is the sound speed inside the material, $n$ is the index of refraction of the material and $f_{B r}$ is the Brillouin oscillation frequency. The period of these oscillations is given by:

$$
\tau_{o s c}=\frac{\lambda}{2 n v \cos \theta}
$$

where $\theta$ is the scattering angle. Brillouin scattering is an interaction between an electromagnetic wave and the crystalline lattice matter waves. In most of the cases the photons of the electromagnetic wave lose energy (Stokes process) and the energy is transformed in one of the three quasiparticle types (phonon, polariton, magnon). Of course, there exist the opposite transition where the photon absorbed one quasiparticle and increase its energy (anti-Stokes process).

\subsubsection{Profilometry of nanostructured materials using the longitudinal laser generated nano-acoustic waves}

Using the set up for pump-probe degenerate transient reflectivity described previously the reflectivity changes for thin free-standing films can be measured. In Figure 3.4 the reflectivity 
changes $\Delta R$ from two Au thin films as a function of delay time between pump and probe laser pulses is shown. The polycrystalline Au films have different width as measured by a classical profilometer to be $25 \mathrm{~nm}$ and $100 \mathrm{~nm}$. The Ti:Sa laser pulses $(\sim 790 \mathrm{~nm})$ have a duration $\sim 35 \mathrm{fs}$ and their energy per pulse was $\sim 20 \mu \mathrm{J}$.

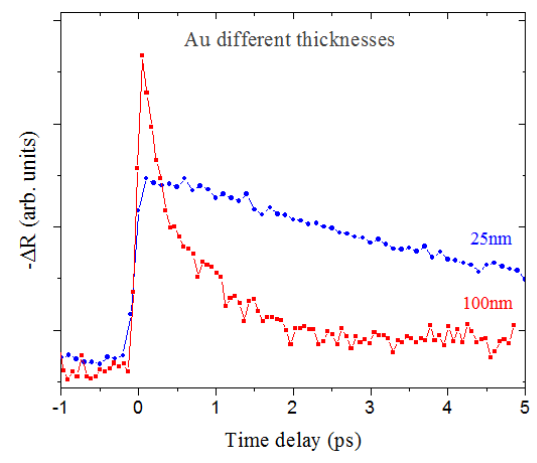

Figure 3.4. Typical femtosecond degenerate transient reflectivity results for Au thin films using a 35fs laser pulses [33].

In both films there is a rapid increase of the reflectivity that attributed to fast increase $(\sim 100 \mathrm{fs})$ of the electron gas mean energy by the laser pulse absorption (single or multiphoton). Then, the rapid increase of $R$ is followed by a slow recovery ( $>2 \mathrm{ps}$ ) which is obviously slower in the case of the thick film. This slow recovery is attributed to the transfer of electron excess energy to the lattice via electron-phonon scattering and the diffusion of the electron gas heat outside the interaction region via, mainly, ballistic electron-electron scattering. This behavior is explained very well by Anisimov's equations (3.5). Note here that the slower recovery of the thinner film is attributed to lower energy diffusion in z-direction (perpendicularly to the surface) since the laser excites the whole z-depth of the film $(\sim 13 \mathrm{~nm}$ penetration depth). This data shown clearly that the excitation is localized in space within few nanometers.

The same experiment was repeated for a semiconductor wafer, Si (100). The reflectivity change is also initially rapidly change but a very slow ( $>100 \mathrm{ps}$ ) recovery (that can be fitted by a two different slopes) is observed (Figure 3.5). This result proves that the localization of the heat transferred by laser pulses to the semiconductor material is in the order of $\sim 10 \mu \mathrm{m}$ which is also more or less the penetration depth of the $790 \mathrm{~nm}$ light inside the Si [33]. This clearly proves that the energy transferred by the laser to semiconductor lattice is spread over a few $\mu \mathrm{m}$ depth and thus cannot be considered as localized. This is the reason why no high acoustical strains is expected to induced is these materials where the laser penetration depth is almost macroscopic.

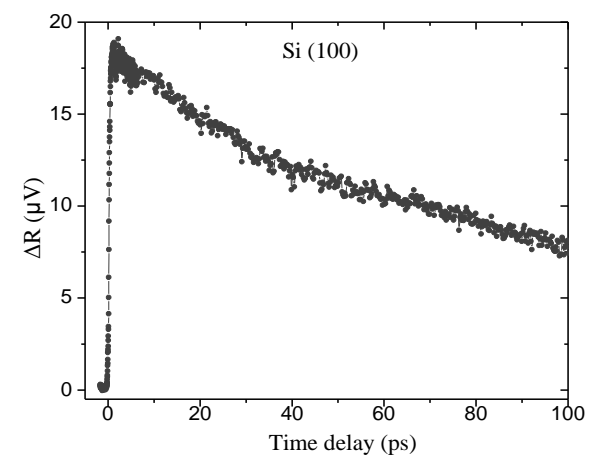

Figure 3.5. Typical femtosecond degenerate transient reflectivity results for $\mathrm{Si}(100)$ thick substrate without the metallic transducer using a $35 \mathrm{fs}$ laser pulses [33]. 
Then, the experiment is repeated using a nanostructured material with a relatively thick metallic film (Ti) of $180 \mathrm{~nm}$ placed on on a thick $(1 \mathrm{~mm}) \mathrm{Si}(100)$ substrate. The results are depicted in Figure 3.6.

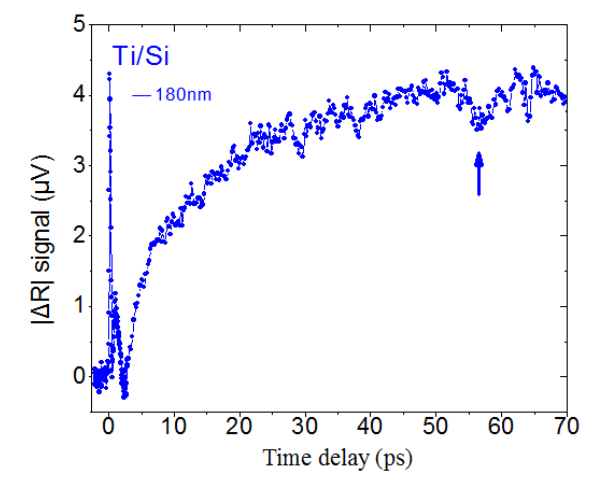

Figure 3.6. Typical femtosecond degenerate transient reflectivity experimental results in Ti/Si multilayer sample for nano-acoustic generation strain and echo detection. The thickness of the Ti film is $180 \mathrm{~nm}$. The echo from the Ti/Si interface is noted with the arrow [33].

In Figure 3.6 the electric initial excitation is obvious with the fast increase of the reflectivity signal which is followed by an also fast decrease attributed to high electron-phonon coupling constant of the Ti metal. Then, in picosecond time scales, there is a slow increase of the reflectivity that attributed to lattice thermalization and electron temperature diffusion that affects also topical the electron gas energy. With the blue arrow is indicated a tiny but evident decrease of the reflectivity at about $\tau_{e c h o}=57 \mathrm{ps}$ delay. This feature is originated by the reflection of the acoustical strain at the boundary between the interface $\mathrm{Ti} / \mathrm{Si}$. The acoustical echo of reflections at the boundary of Ti/Si, because of the different acoustical impedance, arrives at the surface of the Ti and is probed by the probe beam at the appropriate delay. Considering that the speed of sound in Ti is $u_{s} \sim 6100 \mathrm{~m} / \mathrm{s}$ the Ti film depth can be estimated to be $d_{T i}=2 u_{s} \times \tau_{\text {echo }}=186 \mathrm{~nm}$ which agrees perfectly with the Ti film depth $\sim 180 \mathrm{~nm}$ as measured by a typical profilometer.

The above analysis proves the applicability of the fs-laser generated ultrasonic waves to measure the stratification profiles of a nano-structured materials in the nanoscale.

To understand clearly the physical processes and mechanisms participating in the picosecond ultrasonic profilometry. Following the analysis by Thomsen et. al. [27] the variation of the index of diffraction $N(N=n$-i $k)$ of a material is given by:

$$
\begin{aligned}
& \Delta n(x, t)=\frac{\partial n}{\partial \varepsilon_{x x}} \varepsilon_{x x}(x, t) \\
& \Delta \kappa(x, t)=(3.8) \frac{\partial \kappa}{\partial \varepsilon_{x x}} \varepsilon_{x x}(x, t)
\end{aligned}
$$

where $\varepsilon_{x x}$ is the deformation of the material in the axis of the strain propagation. Then the variation of the reflectivity at the surface of the material is given by [27]:

$$
\Delta R(x=0, t)=\int_{0}^{\infty} f(x) \varepsilon_{x x}(x, t) d x
$$


Where $f(x)$ is the sensitivity function given by:

$$
\begin{aligned}
& f(x)=f_{o} e^{-\frac{x}{\beta}}\left\{\frac{\partial n}{\partial \varepsilon_{x x}} \operatorname{Sin}\left[\frac{4 \pi n x}{\lambda_{o}}-\varphi\right]+\frac{\partial \kappa}{\partial \varepsilon_{x x}} \operatorname{Cos}\left[\frac{4 \pi n x}{\lambda_{o}}-\varphi\right]\right\} \\
& f_{o}=8 \frac{\omega\left[n^{2}\left(n^{2}+\kappa^{2}-1\right)^{2}+\kappa^{2}\left(n^{2}+\kappa^{2}+1\right)^{2}\right]^{1 / 2}}{c\left[(n+1)^{2}+\kappa^{2}\right]^{2}}, \tan \varphi=\frac{\kappa\left(n^{2}+\kappa^{2}+1\right)}{n\left(n^{2}+\kappa^{2}-1\right)}
\end{aligned}
$$

Where $\beta$ is the optical penetration depth.

The deformation variations in the axis perpendicularly to the material surface can be estimated by the equation [27]:

$$
\varepsilon_{x x \text { var }}(x, t)=(1-R) \frac{F a}{\beta c \rho} \frac{1+v}{1-v} r_{a c}\left[\frac{1}{2} e^{\frac{-\left|z+v_{L} t\right|}{\beta}} \operatorname{Sign}\left(z+v_{L} t\right)+\frac{1}{2} e^{\frac{-\left|z-v_{L} t\right|}{\beta}} \operatorname{Sign}\left(z-v_{L} t\right)\right]
$$

Where $r_{a c}=\frac{Z_{2}-Z_{1}}{Z_{2}+Z_{1}}$ is the acoustical reflection coefficient in the boundary of the two materials with $Z_{1}$ and $Z_{2}$ are the corresponding acoustical impedances, $v$ is the Poison ratio of the transducer material, $v_{L}$ is the sound speed, $c$ is the speed of light, $R$ is the optical reflectivity (air or vacuum and the upper material), $a$ is the th ermal expansion coefficient.

Finally, the strain is calculation from the deformation by the equation $[27,33]$ :

$$
\sigma_{x x}(x)=\rho v_{L}^{2} \varepsilon_{x x}-3 B a \Delta \mathrm{T}_{L}(x)
$$

where $B$ is the modulus of volume elasticity and $\Delta T_{L}$ is the lattice temperature variations calculated by the coupled Anisimov equations.

The above modelling is applied in the Ti $(180 \mathrm{~nm}) / \mathrm{Si}$ substrate experiments as they described before with all the parameters need taking from the bibliography. Typical results are presented in the Figure 3.7.
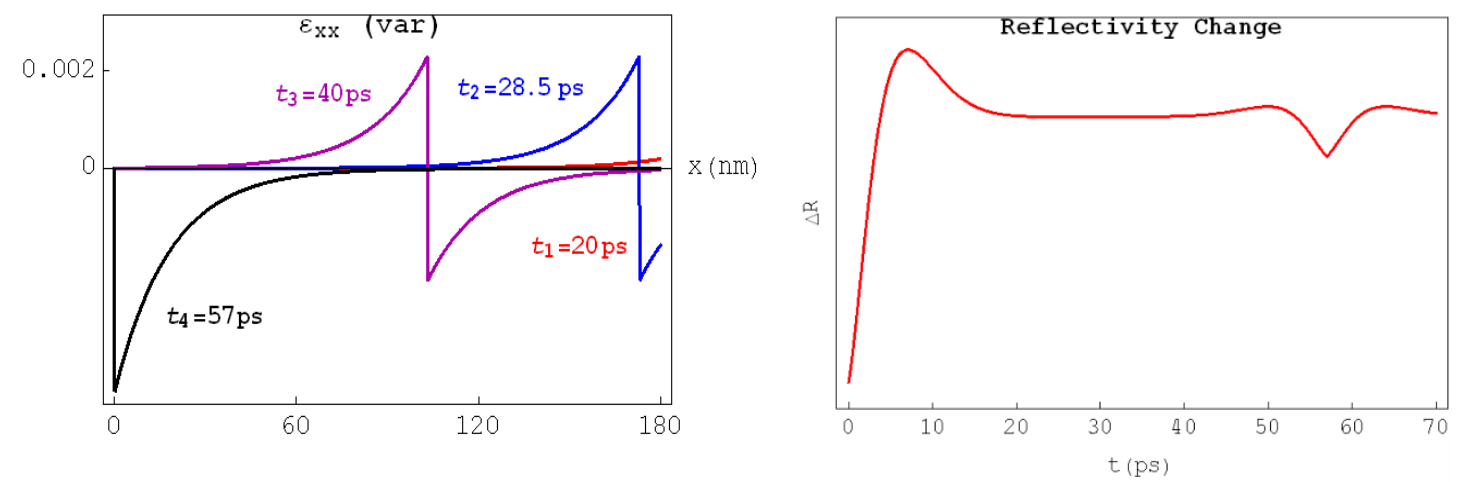

Figure 3.7. Calculated stains (left, $\mathrm{x}$-axes is the depth in $\mathrm{nm}$ ) and transient reflectivity (right) in Ti/Si multilayer sample for nano-acoustic generation strain and echo detection. The thickness of the Ti film is $180 \mathrm{~nm}$. Note echo from the Ti/Si interface at 57 ps (right) $[27,33]$. 
Note here that the reflectivity variations based on the electronic component is not included in the model. Comparing the experimental data for the transient reflectivity presented in Figure 3.6 and Figure 3.7 (right part) the agreement is obvious. Note the echo of the acoustical pulse that appears 57 ps after the pump laser excitation.

\subsubsection{Comparing different metals as optoacoustic transducer}

The optoacoustic transducing is of fundamental significance for the ultrasonic strain characteristics like the localization, the amplitude, and its propagation in the substate under examination. For this purpose, metals with completely different electron-phonon coupling factors are examined. For a proof of principle study two metals are chosen. Namely these metals are the $\mathrm{Ti}$ and Ag. Theoretical studies by Z. Lin et. [34] calculated the electron-phonon coupling factor $G$ as a function with electron temperature considering the density of states for each material. In Figure 3.8 their results are depicted.
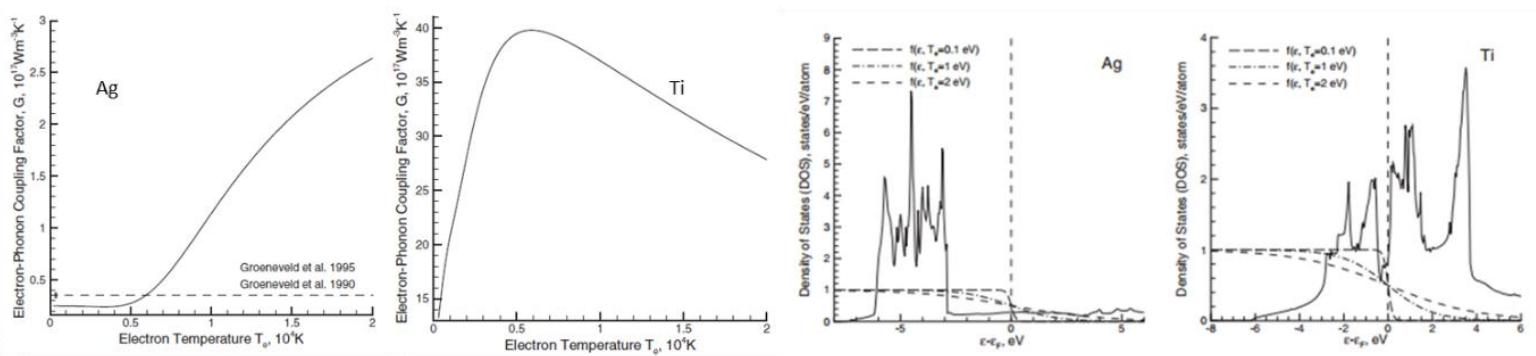

Figure 3.8. Electron-phonon coupling constant for $\mathrm{Ag}$ and $\mathrm{Ti}$ as a function of electron temperature (first and second left) and Density of states curves for Ag and Ti (third and fourth right). The data is taken from Ref.[34]

As seen in Figure 3.8 the $G$ for Ti metal has more than 40-times higher value than Ag metal at $10^{4}$ $\mathrm{K}$ electron temperature. This means that the electron excess energy taking for the fs laser very fast is transferred to the Ti lattice. Additionally, this causes an abrupt change in the lattice temperature of $\mathrm{Ti}$ and consequently a high and localized generation of an acoustical strain. In order to examine in more detail, the above effects a novel optoacoustical model is introduced that taking into account the initial non-thermal electron distribution and all the layers of the nanoacoustic material. The following set of equations is employed to investigate the spatiotemporal distribution of the produced thermalized electron $\left(T_{e}\right)$ and lattice $\left(\mathrm{T}_{L}\right)$ temperatures of the assembly $[32,35,36]$ :

$$
\begin{gathered}
C_{e}\left(T_{e}\right)^{(2)}{\frac{\partial T_{e}}{\partial t}}^{(2)}=\vec{\nabla} \cdot\left(K_{e}{ }^{(2)} \vec{\nabla} T_{e}^{(2)}\right)-G^{(2)}\left(T_{e}^{(2)}-T_{l}^{(2)}\right)+\frac{\partial U_{e e}}{\partial t} \\
C_{l}\left(T_{l}\right)^{(2)}{\frac{\partial T_{l}}{\partial t}}^{(2)}=G^{(2)}\left(T_{e}^{(2)}-T_{l}^{(2)}\right)+\frac{\partial U_{e l}}{\partial t}-\left(3 \lambda^{(2)}+2 \mu^{(2)}\right) \alpha^{(2)} T_{l}^{(2)} \sum_{j=1}^{3} \varepsilon_{j j}{ }^{(2)} \\
\left(C_{l}\left(T_{l}\right)^{(3)}{\frac{\partial T_{l}{ }^{(3)}}{\partial t}}^{(3)}=\vec{\nabla} \cdot\left(K_{l}^{(3)} \vec{\nabla} T_{l}^{(3)}\right)-\left(3 \lambda^{(3)}+2 \mu^{(3)}\right) \alpha^{(3)} T_{l}^{(3)} \sum_{j=1}^{3} \varepsilon_{j j}{ }^{(3)}\right.
\end{gathered}
$$

$k_{e}$ is the thermal conductivity of the electrons, $C_{e}$ and $C_{L}$ are the heat capacity of electrons and lattice, respectively, $G$ is the electron-phonon coupling factor while superscripts $i$ correspond to the $\mathrm{Ag} / \mathrm{Ti}(i=2)$ and $\mathrm{Si}$ materials $(i=3)$, respectively, and $T_{0}=300 \mathrm{~K}$. On the other hand, $\varepsilon_{j j}$ 
correspond to components of thermally induced strains while $\lambda, \mu$, and $\alpha^{\prime}$ are the Lamé constant, shear modulus and thermal expansion coefficients of the materials. Due to the large radius of the laser beam compared to the thickness of the irradiated metal films, the solution of the equations can be simplified by assuming a one-dimensional approach. Therefore, the following equation can be used for two materials with density $\rho^{(i)}(i=2,3)$ to determine the spatio-temporal distribution of thermally induced lattice displacement $u$ [32]:

$$
\rho^{(i)} \frac{\partial^{2} u^{(i)}}{\partial t^{2}}=\left(\lambda^{(i)}+2 \mu^{(i)}\right) \frac{\partial^{2} u^{(i)}}{\partial z^{2}}-\left(3 \lambda^{(i)}+2 \mu^{(i)}\right) \alpha^{\prime(2)} \frac{\partial T_{L}^{(i)}}{\partial t}
$$

where the strain and stress along the z-axis are given by the expressions [32]:

$$
\varepsilon^{(i)}=\frac{\partial u^{(i)}}{\partial z}, \quad \sigma_{z}^{(i)}=\left(\lambda^{(i)}+2 \mu^{(i)}\right) \varepsilon^{(i)}-\left(3 \lambda^{(i)}+2 \mu^{(i)}\right) \alpha^{\prime(2)}\left(T_{L}^{(i)}-T_{0}\right)
$$

respectively. The energy densities per unit time transferred from the non-thermal electrons to thermal electrons $\left(\partial U_{e e} / \partial t\right)$ and lattice $\left(\partial U_{e l} / \partial t\right)$ require modification with respect to the initial model to account for the dynamic character of the absorption coefficient during irradiation that alters the absorption $[32,36]$ :

$$
\begin{aligned}
& \frac{\partial}{\partial t}\left\{\begin{array}{l}
U_{e e} \\
U_{e L}
\end{array}\right\}=\frac{2 A \sqrt{\ln 2} J}{\sqrt{\pi}(h v)^{2} t_{p}} \int_{0}^{t}\left[\frac{1}{1-\exp \left(-\frac{d m}{\alpha^{-1}+\Lambda}\right)} \frac{1}{\alpha^{-1}+\Lambda}\right. \\
&\left.\times \exp \left(-4 \ln 2\left(\frac{t^{\prime}-t_{0}}{t_{p}}\right)^{2}\right) \exp \left(-\int_{0}^{z} \frac{1}{\alpha^{-1}+\Lambda} d z^{\prime}\right)\left\{\begin{array}{l}
H_{e e}\left(t-t^{\prime}\right) \\
H_{e L}\left(t-t^{\prime}\right)
\end{array}\right\}\right] d t^{\prime}
\end{aligned}
$$

where $J$ is the fluence of the laser beam, $h v$ is the one-photon energy, $t_{p}$ is the pulse duration, $A$ is the absorbance of the laser energy, $d_{m}$ is the metal film thickness, $\Lambda$ is the ballistic depth that is characteristic for each metal, $\alpha$ is the absorption coefficient and $t_{0}=-3 t_{p}$ while $H_{e e}$ and $H_{e p}$ are functions that contain details and parameters related to the transient creation of non-thermal electron distribution. Deferent models for the computation of the heat electron conductivity for Ag and Ti was performed [32].

The calculated spatio-temporal evolution of lattice temperature of (a) $25 \mathrm{~nm}$ Ti thin film, and (b) $25 \mathrm{~nm}$ Ag thin film, are illustrated in Figure 3.9.
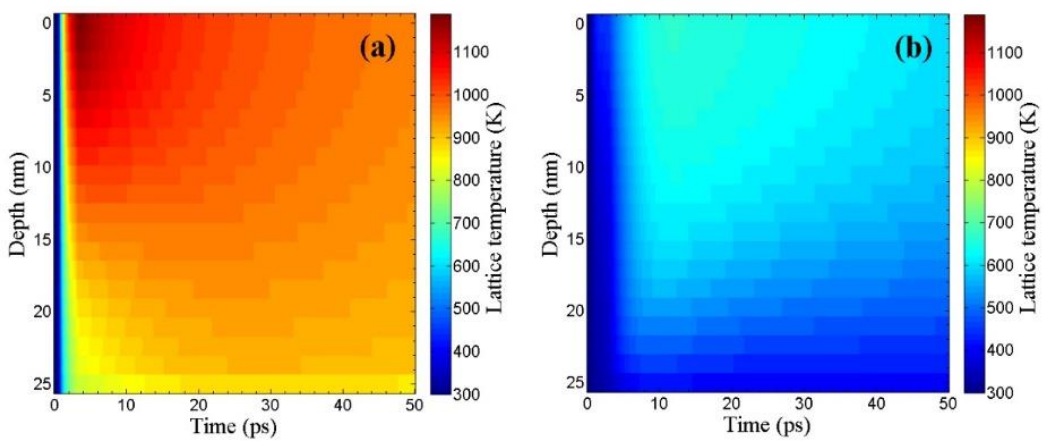

Figure 3.9. Calculated spatiotemporal evolution of lattice temperature for Ti (left) and Ag (right) thin films after the excitation of 35 fs laser Ti:Sa laser pulses (data is taken from Ref.[32]) 
From Figure 3.9 is clear that the lattice temperature after the femtosecond laser excitation is higher and more abrupt than in $\mathrm{Ag}$ as expected on the basis of the previous analysis. In Figure 3.10 the calculated strains in different time after the femtosecond laser excitation is plotted. Its clear that the strain formation and transmission to Si substrate is much higher in amplitude and more localized in the case of Ti transducer compare to that of Ag.

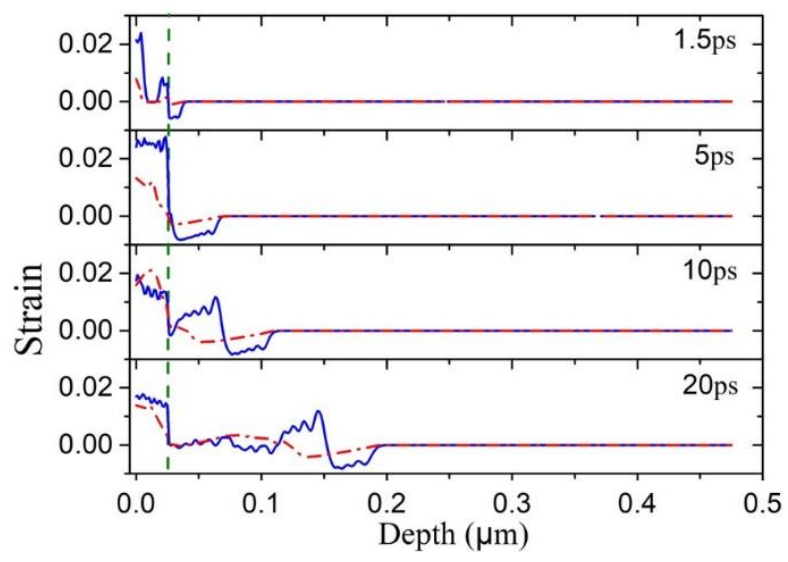

Figure 3.10. Calculated acoustical strains induced by $35 \mathrm{fs}$ Ti:Sa laser pulses on Ti/Si (blue curve) and Ag/Si (red curve) for various delays after the laser excitation. Dashed vertical line indicate the position of metal:Si interface (metal thickness $25 \mathrm{~nm}$ ) [32].

Experiments have been performed to test the theroretical findings using the degenerated femtosecond transient reflectivity set-up. Very thin films of Ti and Ag metals have been used as optoacoustic transducers placed on $\mathrm{Si}(100)$ substrates. The thickness of optoacoustic transducers is very important since the probe beam should be able to penetrate the metal and thus probe the acoustical strain pulse as running with the speed of sound inside the substrate.

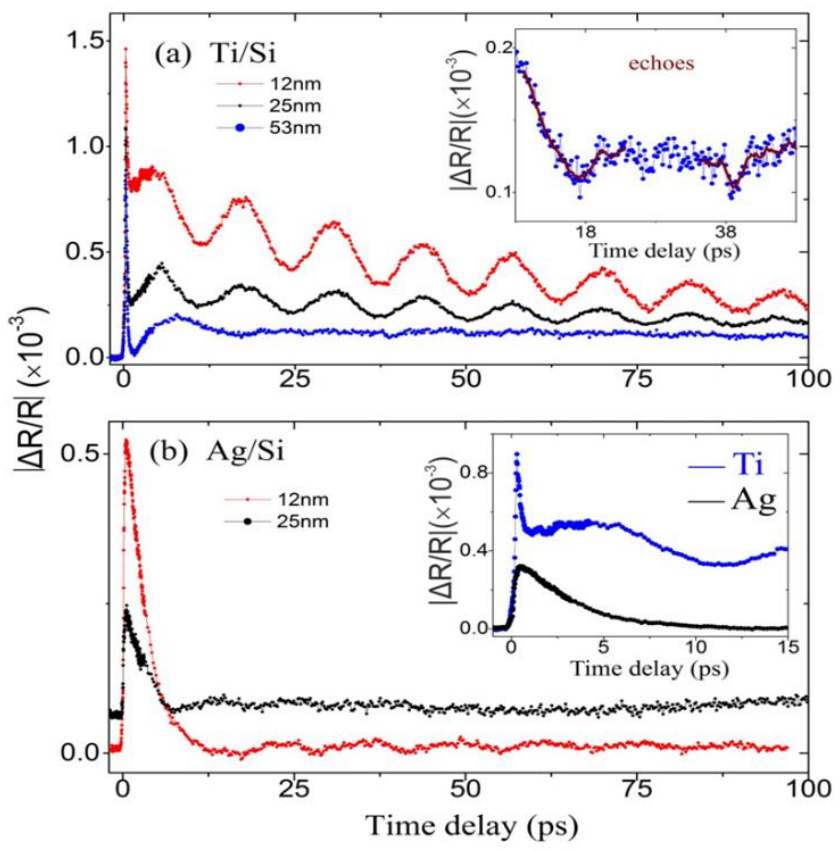

Figure 3.11. Experimental transient reflectivity curves for $\mathrm{Ti} / \mathrm{Si}$ (a) and $\mathrm{Ag} / \mathrm{Si}$ (b) nanostructure materials. The metallic optoacoustic transducer has various thicknesses. Details of the curves are shown in the two insets. Note the Brillouin oscillations (data is taken from Ref[32]). 
Typical experimental results are presented in Figure 3.11. The Brillouin oscillation amplitude is decreased as the thickness of each material increases because less energy of probe beam penetrates the metallic film and enter the Si substrate. Initially there observed a rapid increase of the reflectivity attributed to the fast energy transfer from laser to electron gas via absorption. Then there exist a relatively slower decay which is much more for Ag metal compared to that of Ti which behavior reflects the faster transfer of energy to lattice in the case of Ti. This is because the electron-phonon coupling constant of $\mathrm{Ti}$ is much higher than that of $\mathrm{Ag}$ as is explained previously. The Brillouin oscillation occurs in delays that the acoustical strain pulse is inside the $t$ Si substrate. The amplitude of Brillouin oscillations is higher in Ti/Si case compare to that of $\mathrm{Ag} / \mathrm{Si}$ case where is barely observed. This is because the acoustical strain has higher amplitude in $\mathrm{Ti} / \mathrm{Si}$ and it is much more spatially localized. The experimental data agrees very well with the funding by the theoretical calculation and validate very well the model and its assumptions.

Furthermore, considering that the refractive index of $S i$ at the probe wavelength, $n_{S i}=3.7$, the optical path of the probe beam in $S i$ is $d_{o p t}=E_{T} c / n_{S i} \approx 219 \mathrm{~nm}$, where $c$ is the speed of light in vacuum and $E_{T}$ is the period of the oscillation of the laser electric field ( $\sim 2.7 \mathrm{fs}$ in our case). The time required for the longitudinal acoustic strain to travel this distance is $\tau_{o s c}=\frac{d_{o p t}}{2 u_{S i}} \approx 13 \mathrm{ps}$, where $u_{S i}$ is the longitudinal sound velocity in Si. This is extactly the period of Brillouin oscillations measured experimentally in Figure 3.11. It is obvious for the above analysis that the measurement of the period of Brillouin oscillations in an unknown material used as a substrate means also measurement of its sound speed.

\subsubsection{Coherent control of the acoustical stains via femtosecond laser chirp variations}

Given the important application of laser generated ultrasonic laser pulses appear to be very important the control of their characteristics for a given transducer. Up to now only Fourierlimited femtosecond laser pulses (unchirp) are used for the generation of the nanoacoustical strains. This means that in any time instant the optical pulse all the different optical frequencies of the laser spectrum participate with their weight according to the pulse spectrum. There are optical techniques that the different frequencies can be spread in time and the light peak intensity of the pulse drops while its pulse duration increases (chirp). If the blue components of the laser spectrum precede the red part the pulse is negative chirped while if happens the opposite the pulse is positive chirped. In Figure 3.12 the phenomenon of pulse chirping is illustrated.

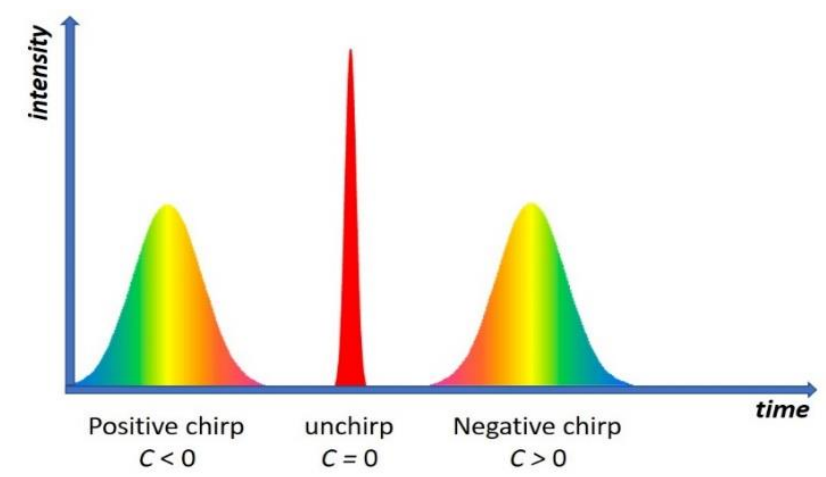

Figure 3.12. Schematic illustration of fs laser chirp. $C$ is the laser chirp parameter as described in the text. 
The main idea of altering the chirp of the laser pulse is to control in time the energy transferred in the electronic gas of the metallic transducer and thus the characteristics of the energy transferring to lattice via electron-phonon scattering. In Figure 3.13 this idea is illustrated schematically.

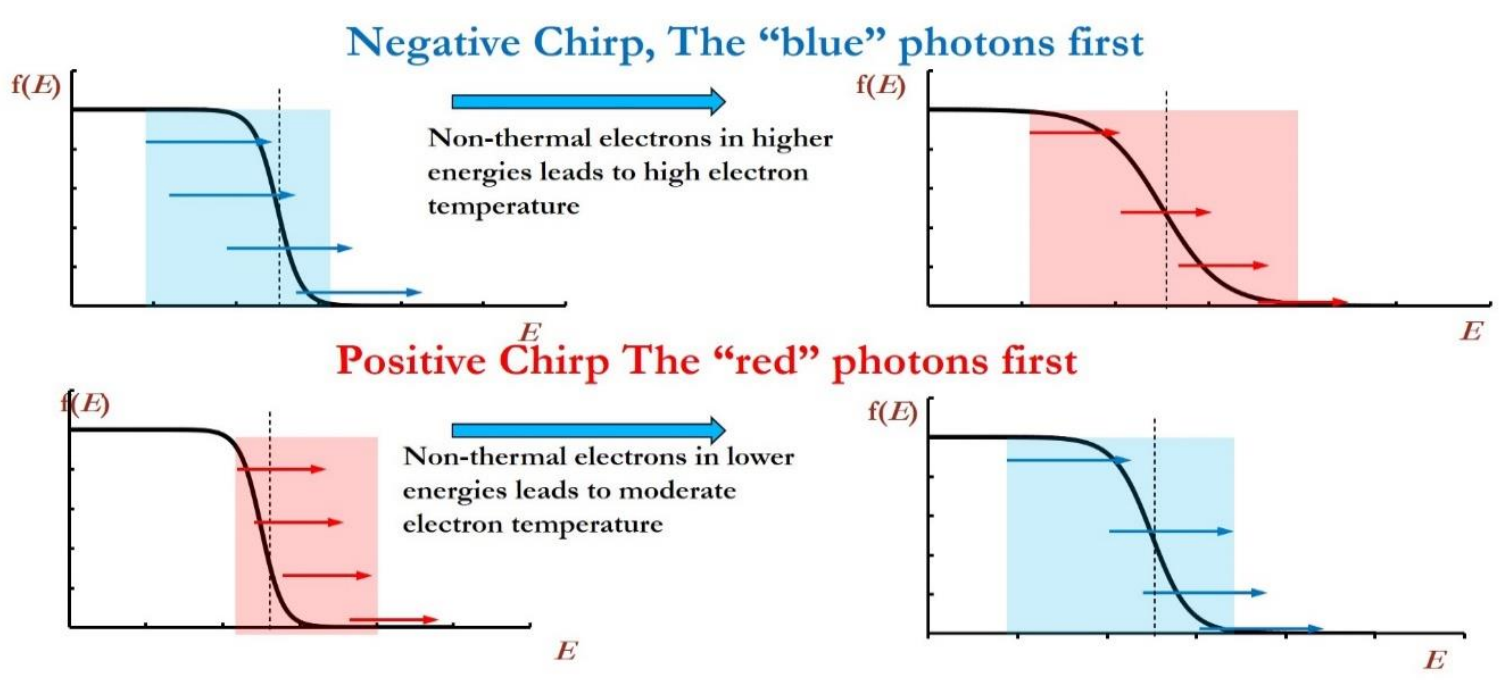

Figure 3.13. Schematic illustration of the excitation of Fermi-Dirac electron distribution function of a metal with negative and positive chirped femtosecond laser pulses. With the colored zone indicated schematically the electrons participating in the excitation with the "blue" (blue arrows) and "red" (red arrows) parts of the laser spectrum.

Following the schematic analysis of Figure 3.13 the negatively chirped pulses have not the same behavior with the positively one in a metallic transducer even if the pulse duration is same. The metal nature of the transducer gives rise to the absorption of the laser energy when the "blue" part of the laser pulse spectrum arrives first (negative chirp). Since this is the more energetic part of the laser spectrum it excites, in high energy states, a high number of nonthermal electrons. That opens a lot off empty positions deep below the Fermi energy and allows for the trailing part of the laser pulse that contains the "red", lower energy photons to excite electrons located very deep in the energy diagram of electron states and thus to be more efficiently absorbed. This is shown in Figure 3.13 with the colored zones of the electrons participate in excitation which is much wider in the case of negatively chirped pulses.

To throw more light to the above ideas a theoretical model is applied that for first time includes the laser chirp in the extended Anisimov equation model together with elasticity theory. Following the analysis presented in Ref. [37] the electric field of the linearly chirped Gaussian laser pulse is given by:

$$
E(t)=E_{0} \exp \left[-(1+i C)\left(\frac{t}{\tau_{G}}\right)^{2}\right]
$$

where $C$ is the chirp parameter, and the laser pulse $E$-Field FWHM duration, $\tau_{p}$, is given by $\tau_{p}=$ $\sqrt{2 \ln 2} \tau_{G}$. Note that with the above definition the chirp parameter is negative for positively chirped pulses and vice versa (i.e. $\frac{d \varphi}{d t}=\frac{-2 C t}{\tau_{G}^{2}}$ ). In order to take into account the changes that are 
laser chirp-dependent, we make the following replacements: (a) the carrier frequency of the laser pulse, $\omega$ is replaced with the instantaneous frequency, $\omega_{\text {inst }}$ :

$$
\omega_{\text {inst }}=\omega-\frac{2 C\left(t-t_{0}\right)}{\tau_{G J}^{2}}
$$

where $t_{0}$ is the time that the laser intensity is maximized (in the simulations $t_{0}=3 \tau_{p}$ ), (b) the peak laser fluence $J$ (which is proportional to the number of photons per unit area that the target is excited) is replaced by the following function that accounts for the different energy given in the system at each time instant because of the time variation of the photon frequency [38-39]:

$$
J=J_{0} \times\left(\omega-\frac{2 C\left(t-t_{0}\right)}{\tau_{G J}^{2}}\right) \times \frac{1}{\frac{\omega \tau_{p} \sqrt{\pi}}{2 \sqrt{\ln 2}}+2 C\left[-\left(\frac{\tau_{p}}{\tau_{G J}}\right)^{2} \times \frac{1}{2^{4\left(\frac{\tau_{p}}{\tau_{G J}}\right)^{2}+3} \ln 2}\right]}
$$

where $J_{0}$ is the fluence of the FL pulse and the last product term is for calibration reasons so that the time integral of the fluence to be $J_{0}$ for all chirp cases, (c) the absorbance of thћhe laser energy by the metal, $A$, is replaced by a time dependent function that accounts for time dependent changes of the Fermi-Dirac electron distribution function inside the metal that alters the single photon transition probability [38-42]:

$$
A(t)=A_{0}\left(\omega_{\text {inst }}, T_{e 0}\right) \times \frac{\int_{\varepsilon} f\left(\varepsilon, T_{e}\right) \times\left[1-f\left(\varepsilon+\hbar \omega_{\text {inst }}, T_{e}\right)\right] \times D O S \times d \varepsilon}{\int_{\varepsilon} f\left(\varepsilon, T_{e 0}\right) \times\left[1-f\left(\varepsilon+\hbar \omega_{\text {inst }}, T_{e 0}\right)\right] \times D O S \times d \varepsilon}
$$

where, $\varepsilon$ is the electron energy, $T_{e 0}$ is the initial electron temperature (here $300 \mathrm{~K}$ ), $\mathrm{T}_{e}$ is the instantaneous electron temperature, $f\left(\varepsilon, T_{e}\right)$ is the Fermi-Dirac distribution function at electron temperature $T_{e}, A_{0}\left(\omega_{\text {inst }}, T_{e 0}\right)$ is the absorbance of the Ti:Si system at $T_{e 0}$ which changes as a function of $\omega_{\text {inst }}$ and is derived through the computation of the dielectric constant ${ }^{25}, f(\varepsilon)$ is the probability that the state $\varepsilon$ is occupied (thus $1-f(\varepsilon)$ is the probability that the energy state $\varepsilon$ is unoccupied), and DOS is the density of states of the metal.

In Figure 3.14 the results of the calculation of the electron temperature for the Ti metal for the various laser chirps of the $35 \mathrm{fs}$ laser pulses are presented.
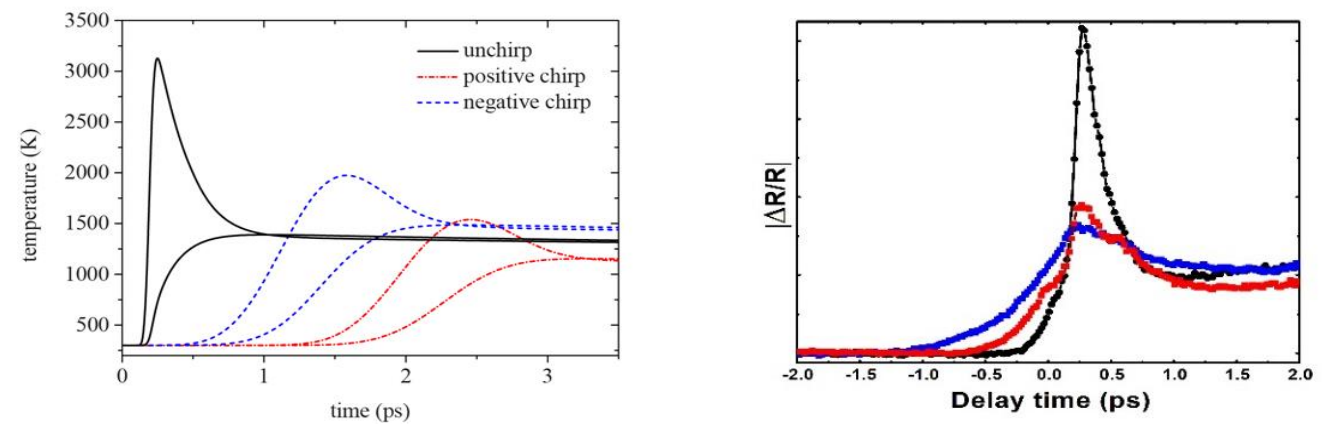

Figure 3.14. Left: Calculated Ti-surface electron temperature evolution after the excitation from upchirp 35 fs laser pulses (black line), positive chirped (red line) and negative chirped (blue line). Right: Experimental data with initial stage of electronic transient reflectivity changes via a pump-probe measurements in Ti/Si after the pump from upchirp 35 fs laser pulses (black line), positive chirped (red line) and negative chirped (blue line). 
Very interesting features appears in Figure 3.14. Note here that the number of laser photons (laser energy) is the same for all the three cases presented. The highest temperature appear for the case of unchirp pulses which is attributed to the higher laser intensity there since the pulse duration is the shorter one. Additionally, is clear that the negative and positive pulse temperatures are different even the laser intensity is exactly the same. The maximum electron temperature induced by the negatively chirped pulse is higher than the one induced by the positively chirped pulse. More importantly, the lattice temperature (equal with the value of the flattening of the temperature curves) is higher in the case of negatively laser pulses compared to both positively and unchirped pulses.

Solving the all the model equations the acoustical strain can be extracted for the three laser pulse cases. Typical strain results for Ti/Si multilayer material are presented in Figure 3.15.

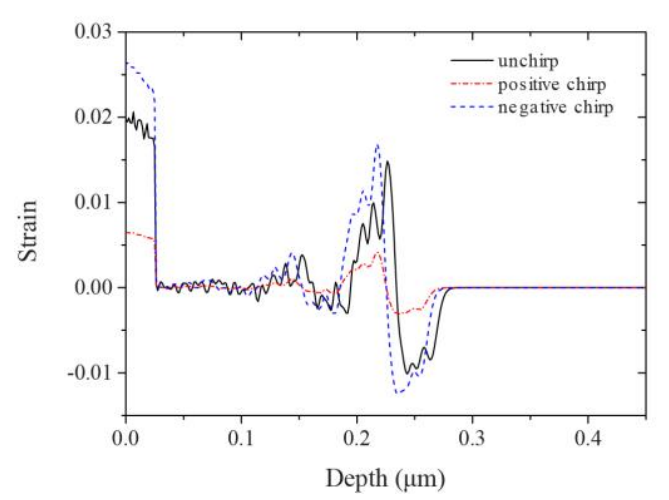

Figure 3.15. Acoustic strains induced on Ti/Si nanostructured material with unchirp 35 fs laser pulses (black), positive chirped (red) and negative chirped (blue).

For the Figure 3.15 is clear that the strain induced inside Si using Ti thin film as optoacoustic transducer could be controlled. Using negatively chirp pulses one should expected to have higher acoustical strain generation efficiency.

The next step is to design the appropriate experiments for prove that the idea is correct and valid the model findings. The chirp of the femtosecond laser pulses could be altered by detuning the compressor gratings of the laser amplifier relative to the position that yields unchirped laser pulses. The schematic of this experimental method is illustrated to Figure 3.16.

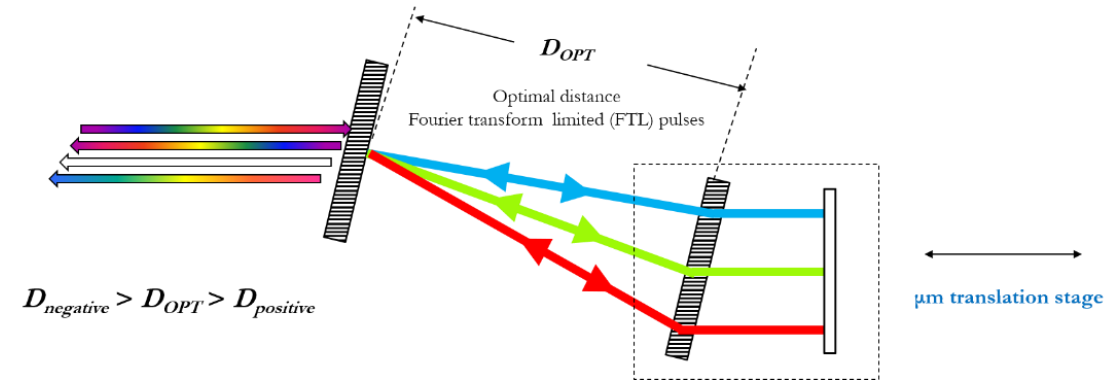

Figure 3.16. Schematic illustration of a femtosecond laser compressor based on two transmition Typical femtosecond degenerate transient reflectivity experimental arrangement for nano-acoustic generation strain and echo detection.

A second-order interferometric autocorrelator could be used to measure by fitting both the laser pulse duration (assuming Gaussian envelope) and its linear chirp parameter (assuming only linear chirp). The parameters $\tau_{G}$ and $C$ that characterize under the previous assumptions the 
chirped laser pulses are determined by fitting the second-order interferometric autocorrelation data with the equation:

$$
\begin{aligned}
& G_{2}(\tau)=1+2 \exp \left(-\frac{\tau^{2}}{\tau_{G}^{2}}\right)+4 \exp \left(-\frac{C^{2}+3}{4} \frac{\tau^{2}}{\tau_{G}^{2}}\right) \cos \left(\frac{C}{2} \frac{\tau^{2}}{\tau_{G}^{2}}\right) \cos (\omega \tau) \\
& +\exp \left[-\left(1+C^{2}\right) \frac{\tau^{2}}{\tau_{G}^{2}}\right] \cos (2 \omega \tau) .
\end{aligned}
$$

where $\omega$ is the laser pulse carrier frequency and $\tau$ the time delay between the two pulses. The $1 / \mathrm{e}$ duration of the intensity $\left(\tau_{G J}\right)$ of a Gaussian laser pulse is $\sqrt{2}$ shorter than its real $E$-field amplitude $\left(\tau_{G J}=\tau_{G} / \sqrt{2}\right)$.

The experimental results of the acoustical strains for the various chirps are illustrated in Figure 3.17 [38-39].
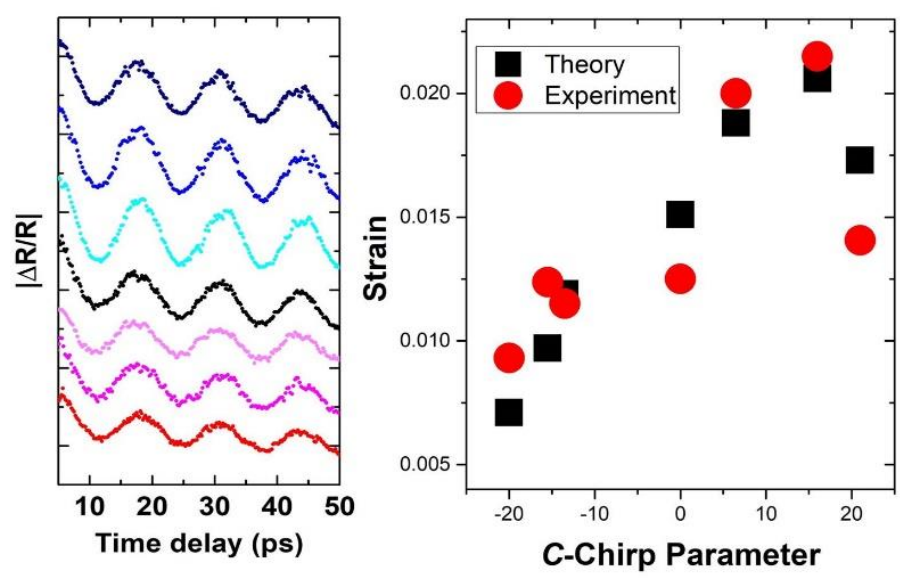

Figure 3.17. Brillouin oscillation in Ti/Si nanostructured materials induced by ultrafast Ti:Sa laser pulses at various linear chirps. On the left figure is presented the transient reflectivity variation signals for different chirp parameter, $C$ (negative values for positive chirp and positive value for negative chirp), dispersed vertically for clarity reasons (down with red-like colors are the positive chirps, middle with black color is the unchirp and upper with blue-type colors are the negative chirps). On the right picture theoretical calculations of the induced acoustical strains in Si are presented for the various chirp parameters along with the experimental findings (exported by the left figure data) [38-39].

From the Figure 3.17 is clear that the acoustic strain pulses could be fully controlled by adjusting the generator femtosecond laser pulse chirp. A little negatively chirped laser pulses proved to be favorable in the efficient generation of the nano-acoustic strains.

\section{Acknowledgments}

This educational material is based mostly on research performed and/or supported by several people under the supervision of the author. I would like to thank the Dr. Irene Tzanaki (former $\mathrm{PhD}$ student of mine) for her effort in experimental work on picosecond ultrasonics, my colleague Prof. Makis Bakarezos (HMU) for his valuable collaboration and support on all results presented here, my colleague Prof. Michael Tatarakis (HMU) for his valuable collaboration being the leader of the Institute of Plasma Physics and Lasers at Hellenic Mediterranean University (HMU) that all the experiments presented here took place. I also like to thank Dr. George Tsibidis (FORTH-IESL) for his valuable theoretical support, most of the theoretical findings presented here was 
developed by him. Finally, I would like also to thank my colleagues Prof. Vasilios Dimitriou (HMU), Dr. Yannis Orphanos and Mr Stylianos Petrakis as well as Dr. Panayiotis Loukakos (FORTH-IESL), Prof. Kostantine Kosmidis (UoI) and Prof. Panos Patsalas (AUTH) for their support and collaboration.

\section{References}

[1] https://www.nobelprize.org/prizes/physics/2018/summary/

[2] Donna Strickland, Gerard Mourou, Compression of amplified chirped optical pulses, Opt. Com. 56, 1985, 219.

[3] Few-cycle Laser Pulse Generation and its Applications, Franz X. Kärtner, Springer; 2004th edition (September 14, 2004).

[4] T. Brabec and F. Krausz, "Intense few-cycle laser fields: frontiers of nonlinear optics," Rev. Mod. Phys. 72, 2000, 545-591.

[5] Li, X. F.; L'Huillier, A.; Ferray, M.; Lompre, L. A.; Mainfray, G. Multiple-harmonic generation in rare gases at high laser intensity. Phys. Rev. A. 39 , (1989) 5751-5761.

[6] Corkum, P.B., Plasma perspective on strong field multiphoton ionization. Phys. Rev. Lett. 71, 1993, 1994-1997.

[7] A. Willner, F. Tavella, M. Yeung, T. Dzelzainis, C. Kamperidis, M. Bakarezos, D. Adams, R. Riedel, M. Schulz, M.C. Hoffmann, W. Hu, J. Rossbach, M. Drescher, V.S. Yakovlev, N.A. Papadogiannis, M. Tatarakis, B. Dromey, M. Zepf, Efficient control of quantum paths via dual-gas high harmonic generation" New Journal of Physics 13, 2011, 113001.

[8] A. Willner, F. Tavella, M. Yeung, T. Dzelzainis, C. Kamperidis, M. Bakarezos, D. Adams, M. Schulz, R. Riedel, M.C. Hoffmann, W. Hu, J. Rossbach, M. Drescher, V.S. Yakovlev, N.A. Papadogiannis, M. Tatarakis, B. Dromey, M. Zepf, Coherent control of high harmonic generation via dual-gas multijet arrays" Physical Review Letters 107, 2011, 175002.

[9] Gy.Farkas, Cs.Tóth, Proposal for attosecond light pulse generation using laser induced multipleharmonic conversion processes in rare gases, Phys. Lett. A 168, 1992, 447-450.

[10] N.A. Papadogiannis, B. Witzel, C. Kalpouzos and D. Charalambidis, Observation of attosecond light localization in higher order harmonic generation, Physical Review Letters 83, 1999, 4289.

[11] P. Tzallas, D. Charalambidis, N.A. Papadogiannis, K. Witte and G.D. Tsakiris, Direct observation of attosecond light bunching, Nature 426, 2003, 267.

[12] M. Schultze, E. Goulielmakis, M. Uiberacker, M. Hofstetter, J. Kim, D. Kim, F. Krausz and U. Kleineberg, Powerful 170-attosecond XUV pulses generated with few-cycle laser pulses and broadband multilayer optics. New Jour. of Phys. 9 (2007) 243

[13] https://www.nobelprize.org/prizes/chemistry/1999/zewail/facts/

[14] Kerry D Solomon, Luis E Fernández de Castro, Helga P Sandoval, Joseph M Biber, Brian Groat, Kristiana D Neff, Michelle S Ying, John W French, Eric D Donnenfeld, Richard L Lindstrom, LASIK world literature review: quality of life and patient satisfaction, Ophthalmology 116, 2009, 691-701.

[15] http://www.hiper-laser.org/

[16] Mangles, S., Murphy, C., Najmudin, Z. et al. Monoenergetic beams of relativistic electrons from intense laser-plasma interactions. Nature 431, 2004, 535-538.

[17] Anisimov S. I., Kapeliovich B. L., and Perelman T. L., Electron emission from metal surfaces exposed to ultrashort laser pulses, Sov. Phys. JEPT 39, (1974) 375.

[18] Kaganov M. I., Lifshitz I. M., and Tanatarov L. V., Relaxation of electrons with crystalline lattice, Sov. Phys. JEPT 4, (1957), 173.

[19] Fujimoto J. G., Liu J. M., Ippen E. P., and Bloembergen N., Femtosecond Laser Interaction with Metallic 
Tungsten and Nonequilibrium Electron and Lattice Temperatures, Phys. Rev. Lett. 53, (1984) 1837.

[20] Elsayed-Ali H. E., Norris T. B., Pessot M. A., and Mourou G. A., Time-resolved observation of electronphonon relaxation in copper, Phys. Rev. Lett. 58, (1987),1212

[21] Schoenlein R. W., Lin W. Z., Fujimoto J. G., and Eesley G. L., Femtosecond studies of nonequilibrium electronic processes in metals, Phys. Rev. Lett. 58, (1987), 1680

[22] N.A. Papadogiannis, S.D. Moustaizis and J.P. Girardeau-Montaut, Electron relaxation phenomena on a copper surface via nonlinear ultrashort single-photon photoelectric emission, Journal of Physics D: Applied Physics 30, (1997) 2389.

[23] Ashcroft N. and Mermin C, Solid State Physics, Saunders Philadelphia (1976).

[24] N. A. Papadogiannis, PhD Thesis, Interaction of ultrashort intense laser pulses with metallic surfaces, Elocus library University of Crete (1996).

[25] Spicer W. E., Optical Properties of Solids, edited by Abeles F., North-Holland Publishing CompanyAmsterdam, London (1972) pp. 796-801

[26] Harisson W. A., Electronic Structure and Properties of Solids, Freeman W. H. and Company, SanFrancisco (1980).

[27] C. Thomsen, H.T. Grahn, H.J. Maris, and J. Tauc, Surface generation and detection of phonons by picosecond light pulses, Phys. Rev. B 34, 4129 (1986).

[28] H. Hirori, T. Tachizaki, O. Matsuda, and O.B. Wright, Electron dynamics in chromium probed with 20fs optical pulses, Phys. Rev. B 68, 113102 (2003).

[29] Y. Sugawara, O.B. Wright, O. Matsuda, M. Takigahira, Y. Tanaka, S. Tamura, and V.E. Gusev, Watching Ripples on Crystals, Phys. Rev. Lett. 88, 185504 (2002).

[30] V. Dimitriou, E. Kaselouris, Y. Orphanos, E. Bakarezos, N. Vainos, M. Tatarakis, and N.A. Papadogiannis, Appl. Phys. Lett. 103, 114104 (2013).

[31] V. Dimitriou, E. Kaselouris, Y. Orphanos, M. Bakarezos, N. Vainos, I.K. Nikolos, M. Tatarakis, and N.A. Papadogiannis, Appl. Phys. A: Mater. Sci. Process. 118, 739 (2015).

[32] E. Tzianaki, M. Bakarezos, G.D. Tsibidis, Y. Orphanos, P.A. Loukakos, C. Kosmidis, P. Patsalas, M. Tatarakis, and N.A. Papadogiannis, High acoustic strains in Si through ultrafast laser excitation of Ti thin-film transducers, Optics Express 23, 17191 (2015).

[33] Eirini Tzianaki, PhD Thesis University of Ioannina, Study of high frequency elastic waves in layered Metal/Silicon samples by femtosecond laser pulses, (2016).

[34] Z. Lin, L. V. Zhigilei, and V. Celli, Electron-phonon coupling and electron heat capacity of metals under conditions of strong electron-phonon nonequilibrium, Phys. Rev. B 77, 075133 (2008)

[35] Apostolova T., Perlado J.M., Rivera A (2015) Femtosecond laser irradiation induced-high electronic excitation in band gap materials: a quantum-kinetic model based on Boltzmann equation. Nucl Instrum Methods Phys Res, Sect B 352:167-170

[36] E. Carpene, Ultrafast laser irradiation of metals: Beyond the two-temperature model, Phys. Rev. B 74, (2006) 024301

[37] J.-C. Diels and W. Rudolph, Ultrashort Laser Pulse Phenomena (Academic Press, San Diego, 1996).

[38] Tzianaki, E., Bakarezos, M., Tsibidis, G.D., Petrakis, S., Loukakos, P.A., Kosmidis, C., Tatarakis, M., Papadogiannis, N.A., Controlling nanoscale acoustic strains in silicon using chirped femtosecond laser pulses, Applied Physics Letters 108, 254102 (2016)

[39] M. Bakarezos, E. Tzianaki, S. Petrakis, G. D. Tsibidis, P. A. Loukakos, V. Dimitriou, C. Kosmidis, M. Tatarakis, N. A. Papadogiannis, Ultrafast laser pulse chirp effects on laser-generated nanoacoustic strains in Silicon, Ultrasonics 86C, 14 (2018)

[40] N. A. Papadogiannis and S. D. Moustaizis, Opt. Commun. 137, 174 (1997).

[41] N. A. Papadogiannis, P. A. Loukakos, and S. D. Moustaizis, Opt. Commun. 166, 133 (1999).

[42] C. Guo, G. Rodriguez, and A. J. Taylor, Phys. Rev. Lett. 86, 1638 (2001). 



\title{
4. Irradiation sources: medium energy ion beams
}

\author{
Gastón García*, Miguel L. Crespillo, José Olivares, Andrés Redondo-Cubero, \\ Sílvia Viñals, and María Dolores Ynsa
}

This chapter deals with basic aspects on the usage of medium energy ion beams (meaning from hundreds of $\mathrm{keV}$ to tens of $\mathrm{MeV}$ ), as sources of electronic excitation of suitable target materials. Ion-matter interaction involves both electronic excitation and nuclear processes. Even if the focus of this work is electronic excitation, it is also very relevant to keep in mind nuclear processes. On the other hand, the usage of ion beams involves two complementary approaches: modification and analysis of materials. Both will be addressed in the following pages. The purpose of the present chapter is to provide the reader with basic introductory information, complemented by selected references, on the basic experimental context elements relevant to the experimental usage of medium energy ion beams.

\footnotetext{
*Contact: gaston.garcia@uam.es
} 


\subsection{Introduction}

Ion beams in the range from hundreds of $\mathrm{keV}$ to tens of $\mathrm{MeV}$ are an extremely useful tool for both modification and analysis of materials. Ions interact with matter both via electronic and nuclear excitation, with variable contributions depending on the ion beam species and energy range. Both excitation channels are strongly related for two main reasons: the effects of electronic excitation by a given ion beam may frequently be characterized with another ion beam by using analysis techniques based on nuclear interactions; on the other hand electronic and nuclear excitation are simultaneously present in a modification experiment and thus a minimum reference to any of them is needed even if one focuses on the other.

The contents of the chapter are necessarily touching upon many different aspects, with a miscellaneous approach and making an effort to avoid too much detail, which will always be available via the references. After this brief introduction, section 4.2 provides some basics on ion accelerators, their most usual configuration and basic parts. Section 4.3 introduces some basic beam transport elements for ion beams. Section 4.4 changes perspective and illustrates with examples how different ion beams interact with matter, in order to give the reader a flavour on the involved penetration ranges and energy densities, along with several illustrative applications examples. Analytical techniques based on ion beams are briefly explained in section 4.4, including only the most basic aspects and relying heavily on references for a deeper insight, and along with some examples of experimental setups in which several key elements for ion-matter experiments are present. At the end of the chapter references are given.

\subsection{Ion accelerators}

\subsubsection{Introduction: types of accelerators}

Particle accelerators exist in many different configurations and are used for a wide variety of purposes. In this paragraph we will briefly comment on ion accelerators, describing some of the most frequent types. As with any other particle, an ion accelerator may use only electrostatic fields for the particle acceleration, or else use time-varying fields. Electrostatic accelerators are frequently used for limited energy ranges, as the energy to be reached is limited by the maximum voltage available. Very high voltages are difficult to handle or eventually impossible. Therefore, higher energies are usually obtained with time-varying fields (cyclotron, synchrotron, radiofrequency linac). In all cases the working principle is based on the proper synchronization of an electromagnetic field with the time of passage of a pulsed beam. Further information may be obtained in [1] or in the references therein.

Electrostatic accelerators are conceptually simpler, as they inject energy to the ion beams by just letting ions drift between two points with different electrostatic potential, thereby obtaining an energy

$$
\Delta E=-q \Delta V
$$

Where $\mathrm{q}$ stands for the ion charge and $\Delta \mathrm{V}$ is the potential difference between the two points. If the voltage is given in volts and the charge is given in electron charge units, the energy is directly 
obtained in $\mathrm{eV}$ units, whose multiples $(\mathrm{keV}, \mathrm{MeV}$, etc...) are regularly used in the framework of particle accelerators. Notice that the ion gains energy E, starting at rest, only if the potential difference and the ion charge have the proper signs. Otherwise the ion would decelerate. It is interesting to observe at this point that, as electrostatic accelerators rely on a time-independent field configuration, they are compatible with both pulsed and continuous beams.

The most intuitive way in which equation (4.1) may be translated into an actual accelerating scheme is that of the so-called single-ended machine. In this case ions are produced with positive sign at a point which has positive potential. Then ions are accelerated down to ground voltage and extracted for their subsequent usage in whatever beam transport elements, which are all at ground voltage as well. This scheme has the disadvantage that the ion production elements (the so-called ion sources) must be at a large electrostatic voltage and are therefore not easily accessible for servicing.

There is a second, less intuitive way, in which the principle underlying (4.1) may be used: this is the so-called tandem accelerator. In this case ions are produced at ground voltage (eventually implying low electrostatic voltages for extraction, which may be switched on and off easily and require only simple insulation configurations) with negative sign. Negative ions are not widely known, but they may be produced efficiently for most atomic species with charge $q=-1$. For those species where this may not be possible there are some alternative tricks, such as using molecular ions containing the atom of interest or picking up extra electrons on the fly by making a lowenergy positive ion beam cross a properly prepared electron-rich atmosphere. A comprehensive review on negative ion production for usage in a tandem accelerator is available in [2]. In any case a negative ion is accelerated from ground voltage to a positive voltage $V_{t}$, present at a point of the accelerator which is denominated terminal, as suggested by the subscript. Then at the terminal the ion beam crosses a section of the beam pipe where it is forced to interact with a target called stripper, which may be solid (typically a thin carbon foil) or gas (frequently high purity nitrogen). Upon interaction with the stripper the negative ions present in the beam keep their energy almost intact, whereas they have a high probability of losing one or more electrons. Therefore a superposition of different charge states is generated, all with the same energy, as given by (4.2). After the stripper the beam runs across a second accelerating section in which voltage decreases from $V_{t}$ again to ground. As the ions have switched (with a high probability) to a positive charge $\mathrm{q}$, which may range from +1 to higher values, the decreasing voltage along the beam path is able to inject energy to the beam again. The final beam energy once the (positively charged) beam reaches ground voltage again is therefore

$$
\Delta E=(q+1) V_{t}
$$

where $\mathrm{q}$ takes different possible values and therefore beams with different energies are superimposed to one another. A simplified scheme of a tandem accelerator is given in Figure 4.1. 


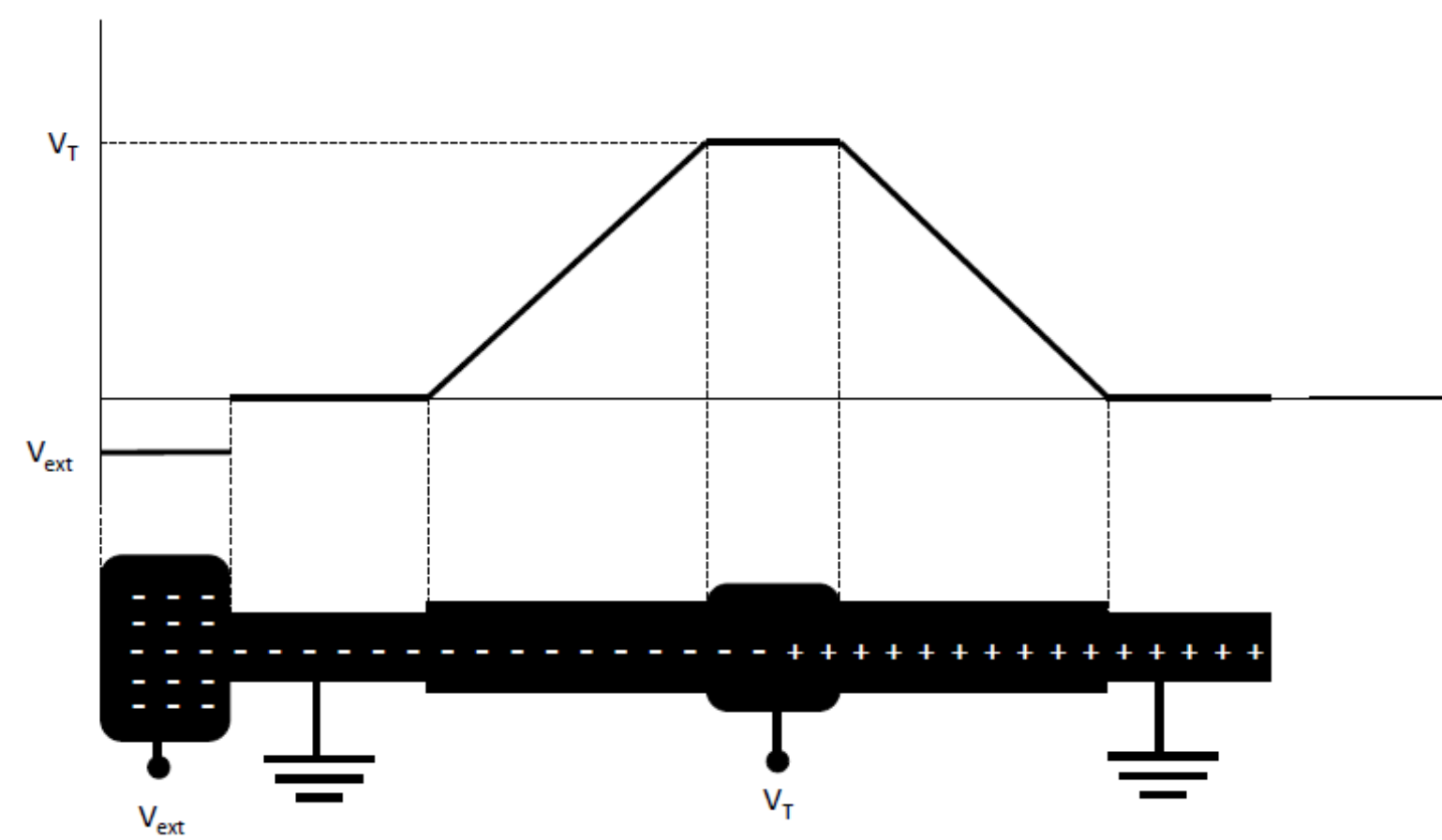

Figure 4.1. Schematics of a tandem accelerator (lower), with the conceptual graph of the voltage at the different points along the device (upper).

The main parts of a tandem accelerator, which is taken here as a representative example, are briefly described in the following section.

\subsubsection{Main elements of an electrostatic tandem accelerator}

\subsubsection{Injector}

The first element is called the injector. It includes first the ion source or sources, where negative ions are prepared and extracted, typically with voltages in the range of tens of $\mathrm{kV}$. A detailed description of ion sources is outside the scope of this work. As an example, two of the usual types of ion sources are very briefly described below.

Plasma ion sources are using a pressurized vessel of a gas which contains the type of ion wanted. This gas is let into a suitable chamber by a leak valve and then proper electrostatic and magnetic fields are provided in order to obtain, sustain and confine the plasma, which contains positive and negative ions, as well as electrons. The field configuration must be properly designed in such a way that the negative ions may be extracted efficiently through a narrow diaphragm via application of a suitable extraction voltage with the proper sign. Several gas vessels may be available with a manifold, in such a way that the same source may be used to produce different ion species depending on the experiment. A notable case is that of He beams, which are frequently used in tandem accelerators. Since He- is metastable in this case the opposite polarity is used for the extraction voltage, so that $\mathrm{He}^{+}$is extracted from the source. Then within the injector the beam is made to pass across a device in which an alkali metal (frequently Li) is evaporated locally with a small oven. Upon crossing such an electron rich atmosphere, already with the velocity corresponding to the extraction voltage, $\mathrm{He}^{+}$ions are made to pick up two electrons and become, with a relevant probability, $\mathrm{He}^{-}$with the same velocity. Then the negative ions may be propagated 
into the subsequent accelerator stages before they decay, as it is done with any other ion beam directly produced as negative at the ion source.

Sputtering ion sources are based on bombarding a solid target cathode with $\mathrm{Cs}^{+}$ions, which are produced at a small oven and accelerated by a cathode voltage at the level of a few $\mathrm{kV}$. The target atoms are sputtered away and upon traversing the target surface, which gets coated with Cs atoms, provide a significant yield of negative ions, as this surface layer is efficiently providing electrons to the sputtered fragments. These negative ions are then repelled from the cathode by the same cathode voltage used to attract the $\mathrm{Cs}^{+}$ions. Then negative ions are extracted out of the source by a subsequent extraction stage, with a suitable diaphragm and extraction voltage with the proper sign. As compared to the previous example, in this case the extracted ions may be any component of a solid target chemically stable with a negative ion configuration. Therefore this type of source is extremely flexible and gives access to most atomic species of the periodic table, with higher or lower yields.

The low energy negative ion beams produced at the ion source are then guided with suitable beam transport elements, such as steerers (electrostatic and/or magnetic) and lenses towards the main accelerator stage. Before reaching this main acceleration stage it is usual to have a mass analysis system, normally a magnet followed by a horizontal slit with a suitable beam diagnostic. Upon bending the beam extracted from the ion source with the magnetic field of such magnet, the output angle depends on the magnetic rigidity of the beam, as defined below in this chapter. Therefore the magnet provides a dispersive map of all masses present in the beam. As frequently ion sources may give rise to different ions, in addition to the one to be selected, this mass analysis stage is essential to identify and select the proper beam. As an example, Figure 4.2 presents the mass spectrum at the injector of a tandem accelerator where $\mathrm{Cl}$ ions are being prepared from a solid target sputtering ion source. It is evident that the beam directly extracted from the target contains many species other than the one wanted.

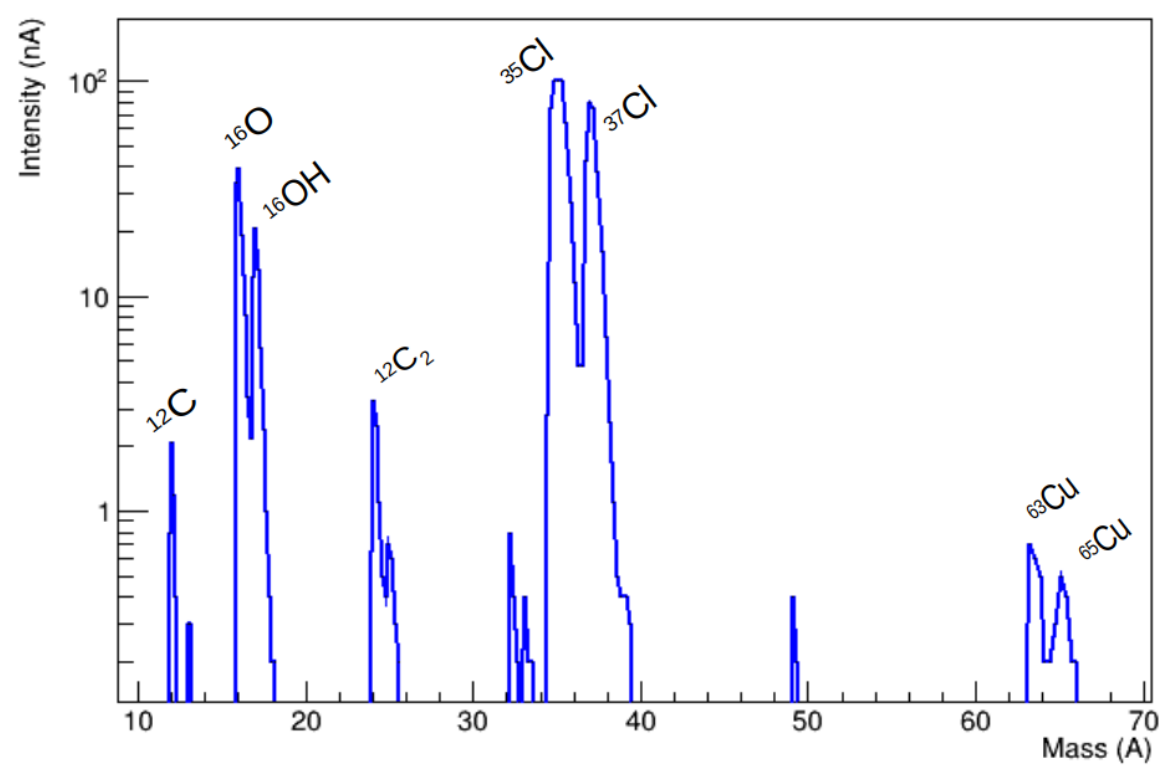

Figure 4.2. Beam current as a function of the ion mass obtained by a mass-analysis magnet at the exit of a sputtering ion source in a tandem accelerator. The two isotopes of $\mathrm{Cl}$ (masses 35 and 37), being the target beam in this experiment, may be readily observed, along with other alien species such as oxygen, carbon and copper. This graph makes evident that mass-analyzing the beam is an essential step after extraction from the ion source. 


\subsubsection{Main accelerating stage}

As briefly explained above, once the negative beam leaves the injector it enters an accelerating tube, in which it propagates across a region where voltage increases gradually and thereby the beam energy increases. The maximum voltage is attained at the so-called terminal. One of the crucial elements for this type of accelerator is the system allowing to set a high electrostatic voltage at the terminal in a robust and stable way. The maximum voltage attainable for a given machine may vary from a few million volts (MV) to several tens of MV is some singular machines. Then this voltage is transmitted along the accelerating tubes with proper resistors in such a way that at each point of the accelerating tubes the proper voltage value is present and the beam sees a gradual voltage change along its path. This applies to the two accelerating tube sections: the one that takes the negative beam from the injector to the terminal and the one that takes the positive beam to the output of the accelerator.

These voltage levels require extreme insulation measures: therefore the terminal and accelerating tubes are housed inside large pressurized vessels, filled with a suitable dielectric. A frequent choice is $\mathrm{SF}_{6}$. On the other hand one needs to generate and regulate the voltage at the terminal. The two most frequently used voltage generation systems are the so-called Van de Graaf and Cockcroft-Walton ones. The former is based on an insulating movable mechanical assembly which looks like a belt, extracting charge from the ground voltage side and depositing it on the high voltage side. Simple as it may look, this concept works and is widely used. The Cockcroft-Walton system is based on an electronic circuit based on alternating capacitors and diodes which produces DC output with a voltage level that multiplies the amplitude of an input AC signal by a large number. In both cases online monitoring of the voltage at the terminal and sophisticated feedback systems for proper stabilization are essential to have a stable enough voltage value at the terminal and thereby a narrow beam energy distribution. A description on electrostatic accelerator voltage supply systems may be found in [1].

The stripper has been conceptually defined above and no further details will be given on its technical details here. Notice that at the output of the stripper a superposition of beams with different charges is always present. The abundance of each charge state depends on the beam species and energy and on the characteristics of the stripper. An estimate may be obtained from [3]. Figure 4.3 shows the expected abundance of the different charge states for an illustrative example. It may be observed that only moderate charge states are present and that the probability of the most abundant ones is of the order of unity. Less abundant, higher charge states may be selected and used to reach higher beam energies (as indicated by formula (4.2)), for a given value of $V_{t}$, at the cost of having smaller ion flux. 


\section{Si@ @ MV}

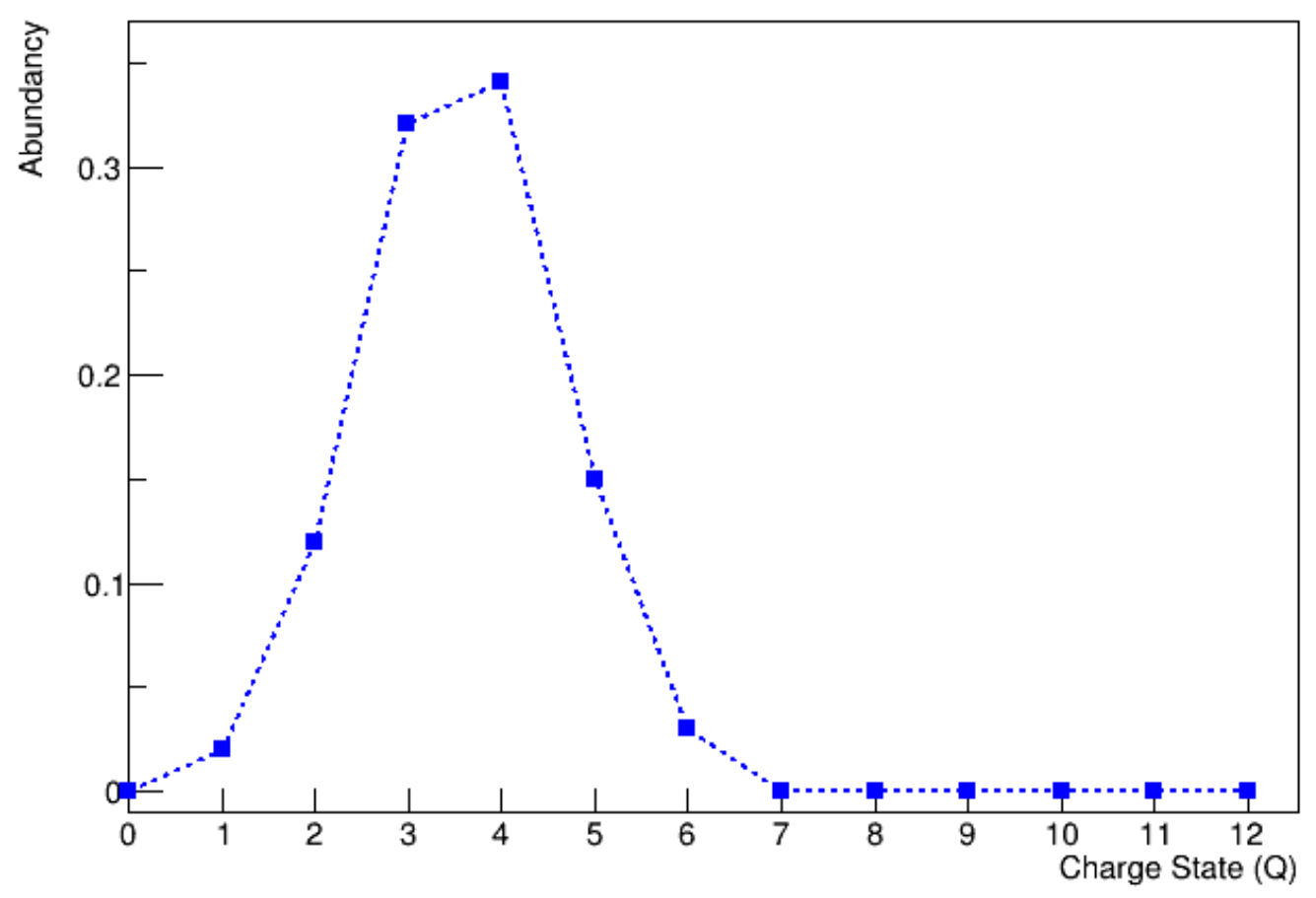

Figure 4.3. Abundancy calculation of the different charge states for a Tandem accelerator with a gas stripper for a $\mathrm{Si}$ beam which reaches the terminal at an energy of $5 \mathrm{MeV}$ (i.e. $\mathrm{V}_{\mathrm{t}}=5 \mathrm{MV}$ ).

\subsubsection{Switching magnet}

At the output of the main accelerating stage, described in the previous section, it is usual to have several beamline extensions, so that the ion beams may be guided alternatively to each of them for specific experimental needs. The key element allowing to select the beamline to which the beam is guided is the switching magnet. This magnet has the proper geometry so that, adjusting the magnetic field properly, a given beam (defined by its energy and mass) is deviated by an angle corresponding precisely to one of the beamlines. Figure 4.4 provides a panoramic view of the different beamlines emerging from the switching magnet at the CMAM ion beam facility [4].

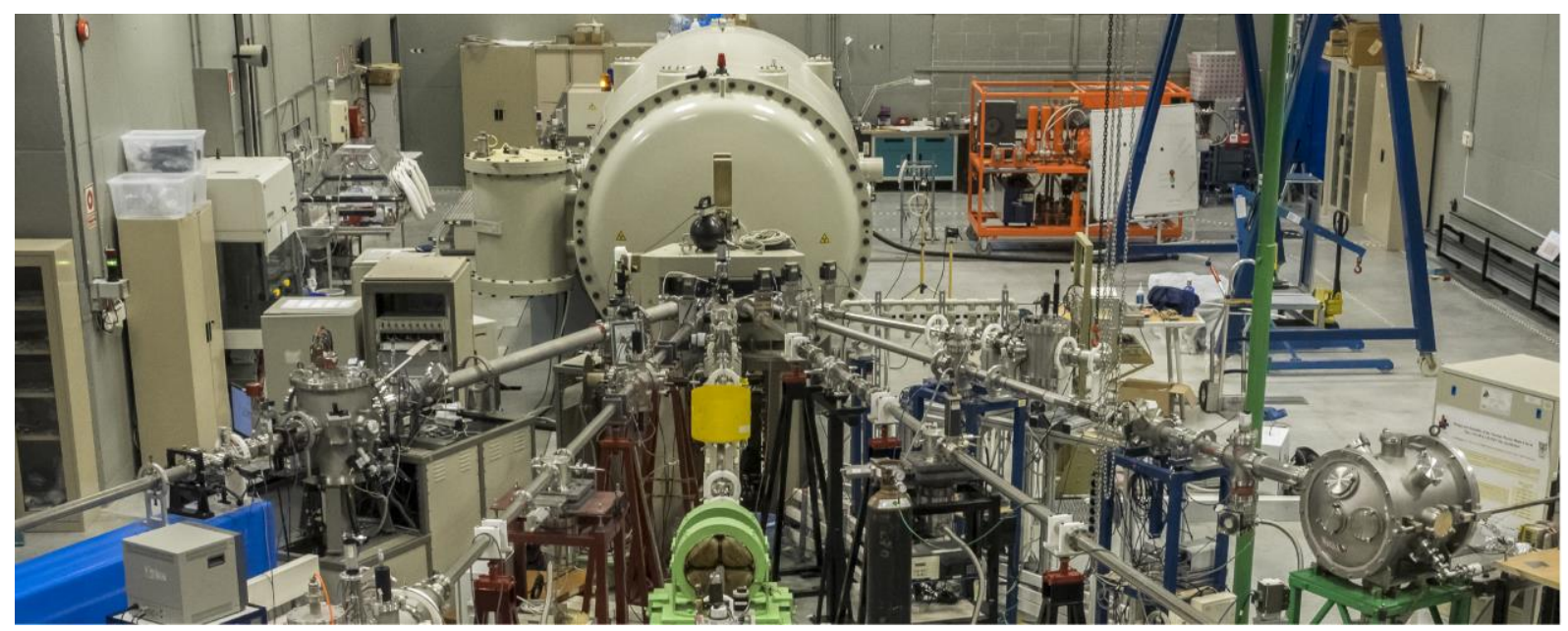

Figure 4.4. Panoramic view of the beamlines at the Center for Micro-Analysis of Materials (CMAM), operated by University Autónoma Madrid (UAM). The switching magnet is visible at the point where all beamlines converge. 


\subsubsection{Ion accelerator landscape in Europe}

Numerous ion accelerator facilities exist in Europe and other regions of the world. These facilities are frequently of small or moderate size and support user access open to a multidisciplinary scientific and industrial community, combined with internal usage. The weight of the different science areas varies from case to case, and includes materials science, nuclear physics, biomedical sciences, archaeometry and environmental science, among others. The European landscape may be explored at [5], where many of the ion beam facilities are identified, whereas a more global view is available at [6]. Recently an interesting focus point issue has been published revising the status of some small size European ion beam facilities [7].

\subsection{Basic beam transport elements}

\subsubsection{Basic ideas on ion beam transport}

In this section a brief introduction is given on how an ion beam is transported from the output of the accelerator stage downstream across a given beamline, with the aim of illustrating, in a nonexhaustive way, the most relevant aspects and providing the reader with some insight which may be improved by consulting the references. For the sake of simplicity only continuous beams (i.e. not pulsed) will be considered.

A beam of charged particles (e.g. ions) may be considered and modelled in the same way as classical light optics. The main elements one may find across the beam path have their analogues in light optics and a simple matrix formalism may be applied in the same way. The optical axis is taken as the ideal trajectory of an ion going through the symmetry axis of the different elements along the beam path. At any point of the optical axis a plane perpendicular to the axis may be defined. Each possible ion trajectory cuts the successive planes at given points and with given angles with respect to the axis. The ideal trajectory cuts all these planes at point $(0,0)$ and with angle $(0,0)$ by definition. The coordinates $(x, y)$ and angles $\left(\theta_{x}, \theta_{y}\right)$ in one of these planes allow to propagate the ion trajectory to an arbitrary plane downstream, if the different beam elements are properly defined. The beam can then be considered a set of points in the phase space defined by these four coordinates, and the propagation of the beams may be seen as the evolution of this cloud of points, which changes its shape according to given rules. In a typical case of interest one takes an input plane where the beam is frequently defined as a homogeneous density in a given area of phase space (such as a rectangle). Then this area of phase is space is propagated downstream up to a given target plane (which may be the position where an aperture is placed or the sample plane). The phase space at this final plane yields the beam size and divergence. We will also assume that the beam particles have a given energy distribution, which may be considered as a gaussian with a given width. In many practical (simple) examples the beam energy may be considered as a delta distribution and therefore energy may be taken as a fixed parameter. Notice that such an approximation may only hold for continuous beams, as for pulsed beams the energy and time of passage of each particle across a given plane are two conjugate variables to be dealt with in the same way as the pair position-angle. 
The propagation of a beam thus defined along free space is then very easy to model. Taking one of the two spatial directions as an example (x), it is very easy to see that for small angles if a beam crosses plane 1 at position $x$ and angle $\theta_{x}$, then it crosses plane 2 at a distance $L$ along the beam axis at position $x^{\prime}=x+L \theta_{x}$ and with the same angle $\theta_{x}{ }^{\prime}=\theta_{x}$. In matrix form one has

$$
\left(\begin{array}{l}
\mathrm{x}^{\prime} \\
\theta_{x}^{\prime}
\end{array}\right)=\left(\begin{array}{ll}
1 & L \\
0 & 1
\end{array}\right)\left(\begin{array}{c}
\mathrm{x} \\
\theta_{x}
\end{array}\right)
$$

The matrix given in (4.3) is the one corresponding to a drift space of length L. Each of the different elements described below in this section may be assigned matrices analogous to this one, so that the problem of propagating the beam is just the succession of matrix multiplications. Such matrices will not be given in detail, as they may be consulted in proper textbooks, such as [8]. We will concentrate in the following on describing beam elements which do not change the beam energy. Therefore, in practice, we are considering the ion beam once it has left the accelerator, concentrating on its propagation along a given beamline.

\subsubsection{Electrostatic and magnetic elements}

The beam elements which do not change the beam energy may use electrostatic or magnetic fields. By looking at the fundamental equations of motion of a charged particle in an electrostatic or a magnetic field two important magnitudes may be defined: electrostatic and magnetic rigidity. For the detailed definition of these magnitudes the reader is referred to [8]. We will here only indicate that the electrostatic (magnetic) rigidity of a beam is a way to quantify its resistance to modify its trajectory when crossing a region with a given electrostatic (magnetic) field. Electrostatic rigidity scales as $\mathrm{E} / \mathrm{q}$ and magnetic rigidity as $\sqrt{\frac{M E}{q^{2}}}$, where $\mathrm{E}$ stands for the beam energy, $\mathrm{q}$ is the ion charge state and $\mathrm{M}$ its mass. Therefore, electrostatic elements may modify an ion beam depending only on its energy and charge state, the beam being more rigid to bending for larger energies and lower charge states. With magnetic elements the rigidity increases both with the beam energy and its mass, and it decreases with the charge state.

In general one may do simple calculations based on the concept of electrostatic and magnetic rigidity based on scaling rules and without the need of using the detailed formulas, just by making the following observation: if we have an electrostatic (magnetic) beam element which is tuned to providing the right trajectory for an ion beam with a given electrostatic (magnetic) rigidity and we change the properties of the beam in such a way that its electrostatic (magnetic) rigidity is multiplied by a factor $\alpha$, then by multiplying the electrostatic (magnetic) fields present in the element by $\alpha$, the original beam tuning is recovered. For example, if the charge state is changed from 1 to 2 , then the electrostatic rigidity becomes half its original value and then the electrostatic field has to be reduced by the same factor to recover the right beam trajectory. In the same way, if we replace a He beam (i.e. with mass 4 ) by an 0 beam (with mass 16), both with the same energy and charge state, the magnetic field needs to be scaled up by a factor of 2 . 


\subsubsection{Dipoles}

Dipole magnets are used to deviate the beam by generating a uniform magnetic field in a given volume. It may be seen by analyzing the equations of motion that the trajectory of a charged particle in a uniform magnetic field is a circle arc, with a bending radius which scales with the value of the magnetic field and with the inverse of the magnetic rigidity. The magnetic field is normally set by regulating the DC current across coils which embrace a soft iron nucleus. In practice the scaling rules sketched in the previous section may be applied directly to the coil current setting, as it is proportional to the generated magnetic field. Depending on the characteristics of the boundary between the region with and without field, that is, the boundary of the magnet coils, the magnet has in addition focusing properties. This means that the particles deviating from the reference trajectory get bent towards the optical axis. The geometry of a magnet may be fine-tuned in such a way that the focusing properties are similar in the horizontal and vertical directions. This is the so-called double focusing magnet, which is usually doing the function of switching magnet at the output of electrostatic accelerators.

\subsubsection{Quadrupoles}

Focusing of ion beams in the MeV range is usually obtained with quadrupoles, which may be magnetic or electrostatic. A magnetic quadrupole is a device which generates a field varying linearly as a function of the distance to the optical axis on the plane normal to it, according to the formulae

$$
\left(\begin{array}{l}
B_{x} \\
B_{y}
\end{array}\right)=K\left(\begin{array}{ll}
0 & 1 \\
1 & 0
\end{array}\right)\left(\begin{array}{l}
\mathrm{x} \\
\mathrm{y}
\end{array}\right)
$$

that is, the field has an increasing y component as one moves along the $\mathrm{x}$ component, and reciprocally. This type of field configuration generates focusing properties. A quadrupole behaves as a focusing lens along one axis and as a defocusing lens in the axis perpendicular to it. Therefore, in order to have focusing in both directions one needs at least two quadrupoles in series. This is the usual way of disposing quadrupoles in a beam transport system, and it is called a quadrupole doublet. When modelling beam transport quadrupoles may be approximated as thin lenses, characterized by a single parameter, the focal length, which depends on the field parameter $\mathrm{K}$ given in (4.4) as

$$
f=\frac{\chi_{m}}{K L}
$$

$L$ being the length of the region with quadrupole field along the beam axis, i.e. the quadrupole thickness, and $\chi_{m}$ being the magnetic rigidity of the beam. It becomes evident that for a given field configuration (i.e. K and L), the beam is more strongly affected (i.e. has shorter focal length) when $\chi_{\mathrm{m}}$ is smaller (i.e. less rigid). Quadrupole sets may have larger multiplicities, such as quadrupole triplets, in order to further fine tune the focusing properties.

The usual way of transporting ion beams is to have the beam focused at a given point along the optical axis, where an aperture is placed, frequently together with a proper diagnostic and where the beam is tuned to reach a beam waist (i.e. a local minimum of beam dimensions in both 
directions perpendicular to the axis and to one another). Then the beam diverges again, as given by (4.3), until it reaches a suitable quadrupole multiplet, where it is focused again to reach a new beam waist downstream. Usually the last beam waist along the beam transport line is on a plane where one positions a sample, to be illuminated by the beam for analysis of modification purposes. The on-sample beam waist allows for having optimal beam size so that irradiation is selective in the sample plane and may be used to analyze or modify the material with spatial lateral resolution.

The quadrupole configuration described in this section may also be obtained with electrostatic fields, in which case it is called an electrostatic quadrupole. Its properties are similar to those given above for magnetic quadrupoles, but its action on the beam depends on the electrostatic rigidity instead of the magnetic one, as defined in 4.3.2. Electrostatic quadrupole sets have thus the feature that their focusing properties are independent of the ion mass, which turns out to be very useful, for example at the output of the acceleration stage in a machine which is frequently used to accelerate many different ion species. Further details on quadrupole configurations and their effects on a charged particle beam may be found in [8].

\subsubsection{Beam apertures}

As mentioned in the previous section beam apertures may be used at selected points of the beam path in order to properly define the beam or support the optimization of the operational parameters of other beam transport devices such as dipoles or quadrupoles. From the point of view of beam transport calculations apertures work as filters in phase space. Once the beam is transferred to the plane where the aperture sits, its extent in phase space is cut according to the aperture size, in such a way that only the part of it fulfilling the aperture condition is transmitted downstream.

A common way to use an aperture is to have protected from the beam an element placed directly downstream (e.g. the vacuum chamber placed within the gap of a dipole magnet). The size of the aperture is defined in such a way that the beam coming from the most limiting point upstream may not touch the element protected downstream (e.g. the walls of the vacuum chamber along all its extension). This is usually done with simple geometrical calculations. For this purpose, usually static apertures are used, more frequently called diaphragms, since there is no need to vary the aperture size and shape.

A second typical way of using an aperture is the optimization of beam transport along a given set of optical elements. The aperture is then frequently placed downstream a focusing element, such as a quadrupole doublet or triplet. The aperture has normally adjustable size and shape, in such a way that the beam conditions downstream may be regulated according to the experiment needs. Once the aperture has been adjusted to the size and shape required the focusing element settings may be optimized in such a way that the beam flux passing through the aperture is maximized. This procedure may be done in terms of beam transport calculations with a proper software tool, or experimentally, provided one has proper diagnostics, ideally immediately downstream the aperture. Different geometries may be used for such a tunable aperture, the most frequent and simple being a set of four blades which move independently from one another, allowing for the definition of a rectangular aperture with different horizontal and vertical sizes and even with a horizontal and vertical offset with respect to the nominal beam axis. 
Beam apertures must be carefully designed taking into account the amount of power which the beam may deposit on them, including when necessary water cooling. Four-blade apertures are also frequently built in such a way that they may be used also as a diagnostic in its own, as it will be explained in the next section.

\subsubsection{Beam diagnostics}

Beam diagnostics are devices which allow to monitor the beam properties and assist accelerator operation in such a way that the desired beam properties may be obtained in a simple and robust way. In this section a few examples of frequent diagnostics for continuous beam ion accelerators are given. The dynamical aspects inherent to pulsed beams imply very different types of additional diagnostics, which are outside the scope of this work.

Faraday cups (FC) are devices which measure the beam flux of charged particles at a given point of the beam path. Normally they are insertable metal blocks, which are introduced or removed from the beam path with a pneumatic device or a motorized axis. Once inserted, the FC blocks the beam. The metal block is properly insulated in such a way that the charge of the ions reaching the device per unit time may be measured as an electrical current with suitable readout electronics. When ions impact on a solid target, such as the FC, secondary electrons are ejected from the solid. Therefore, the net charge flow measured by the readout device will be the sum of the inward current corresponding to the (usually positive) incoming ions, plus the outward (negative) current of the ejected electrons, which implies an overestimation of the real ion flux, if not corrected properly. The geometry of the FC must be such that the outgoing path of the electrons is efficiently blocked. In addition, it is frequent to add a bias ring immediately upstream the FC, polarized to a moderate voltage (frequently in the range of 50-200 V). This is enough to suppress most of the escaping electron current and guarantee a reliable correspondence of the readout current with the incoming ion flux. A FC is frequently placed immediately downstream an aperture, in such a way that its reading is used to optimize the beam flux across the aperture by tuning the setting of the upstream optical elements, such as dipoles or sets of quadrupoles.

Slits, as described in the previous paragraph, may be designed in such a way that each blade is electrically insulated and connected to a current readout device. With this configuration two blades cutting the beam horizontally provide two independent current measurements which scale with the beam flux cut by each of the two blades. Even if the current reading is usually not a good quantitative estimate of the beam flux actually cut by the corresponding blade (due to secondary electrons, as explained above), the relation between the reading of the two blades is a very good way to adjust the position of the beam with respect to the center of the aperture. This configuration may be used connected to an automatic feedback mechanism which steers the beam upstream the aperture in such a way that the beam goes centered through it. As it is evident fourblade slits with current readout may be used to determine and control the beam position in both directions.

Finally, there is a wide variety of systems which allow to monitor the beam properties by dynamically cutting a small fraction of the beam at a given point along the transport line, whereas most of the beam goes through unaffected. This may be implemented by a vibrating string which oscillates crossing the beam or any other suitable mechanical setup with similar properties. The element which dynamically interrupts part of the beam may be connected to a current readout 
device, thus operating as a transmission Faraday cup, or may be used to generate a secondary signal picked up by a suitable detector placed nearby, outside the beam path (such as visible light, characteristic X-rays or backscattered particles). In all cases the time-averaged signal is used as a way to sample and thus estimate the average beam flux across the plane where the diagnostic sits. In the case that the passage of the oscillating element across the beam is used to measure the resulting signal synchronously, the time-dependent readout signal may be used to provide a graphical view of the beam shape. This is the so-called beam profile monitor, which is normally designed in such a way that the output signal allows to visualize both the vertical and the horizontal shape of the beam, by a proper mechanical configuration of the vibrating element. Beam profile monitors are sometimes used as qualitative assistance to visualize easily the presence of a beam and its shape when optical element settings are being scanned or optimized.

\subsection{Some examples of ion-matter interaction}

\subsubsection{Basic concepts: energy loss, range and straggling}

When ions of medium energy (higher than some hundreds keV) impinge matter they initially interact mainly with the electrons of the sample via excitation and ionization. The penetrating ion loses energy along the ion trajectory through these inelastic collisions with the electrons. The radii of atomic nuclei are so small compared with atomic dimensions that nuclear scattering is rare compared to interaction with electrons. Therefore, in a first approximation, nuclear interactions maybe neglected in the slowing down process. Microscopically, energy loss due to excitation and ionization is a discrete process. Macroscopically, it is a good assumption that the ion loses energy continuously. The average energy loss (Se, $\mathrm{dE} / \mathrm{dx}$ ) is the relevant magnitude for most analysis and experiments regarding Ion Beam Analysis (described in the next Section) and Ion Beam Modification of Materials [9] [10]. Some frequently used units are $d E / d x: e V / \AA ;(1 / \rho) d E / d x$ : $\mathrm{eV} /\left(\mu \mathrm{g} / \mathrm{cm}^{2}\right)$. At high energies the electronic stopping of energetic particles in amorphous solids is well described by the Bethe-Bloch formula [11] [12] [13]. The elastic nuclear interaction will be progressively significant at lower ion velocity, reaching its maximum values near the buried layer where the ions are stopped and "implanted".

Charge exchange (loss or gain of electrons) takes place as an ion moves amongst the atoms of a sample and it adopts an equilibrium charge which depends on the velocity. For heavy ions at velocities less than the velocity of orbital electrons, quasi-molecular electron orbits may be temporarily established between the ion and a target atom. At MeV energies, light ions such as alpha particles are fully stripped after they enter into a solid sample and only the positive nuclei penetrate the sample. After they have been slowed down to low energies they pick up electrons again from the sample atoms. Heavier ions, with velocities greater than those of orbiting electrons in sample atoms reach an equilibrium charge state after travelling through sufficient thickness of sample (typically 5 to $50 \mu \mathrm{g} \mathrm{cm}^{-2}$ ). The mean charge is the mean value of a broad, approximately Gaussian charge state distribution which, being velocity dependent, changes as the ion slows down [14].

The energy loss rate, $\mathrm{dE} / \mathrm{dx}$, can be calculated using the computer program "stopping and ion ranges in matter" (SRIM) (http://www.srim.org/) [15] (see Figure 4.5 and Figure 4.6 as examples 
for several cases of ion species and energies). SRIM is a group of programs that calculate the stopping and range of ions (up to $2 \mathrm{GeV} / \mathrm{amu}$ ) into matter using a quantum mechanical treatment of ion-atom collisions (assuming a moving atom as an ion and all target atoms as atoms). This calculation is made very efficient using statistical algorithms, which allow the ion to make jumps between calculated collisions and then average the collision results over the intervening gap. During the collisions, the ion and atom have a screened Coulomb collision, including exchange and correlation interactions between the overlapping electron shells. The ion has long-range interactions creating electron excitations and plasmons within the target. The charge state of the ion within the target is described using the concept of effective charge, which includes a velocity dependent charge state and long-range screening due to the collective electron sea of the target [16]. A full description of the calculation is found in the tutorial book, SRIM-The Stopping and Range of Ions in Solids, by J. F. Ziegler and J. P. Biersack in 1985 (a new edition was published in 2008). The SRIM book offers an interesting and broad historical review of the stopping power theories and model developed along the past century.
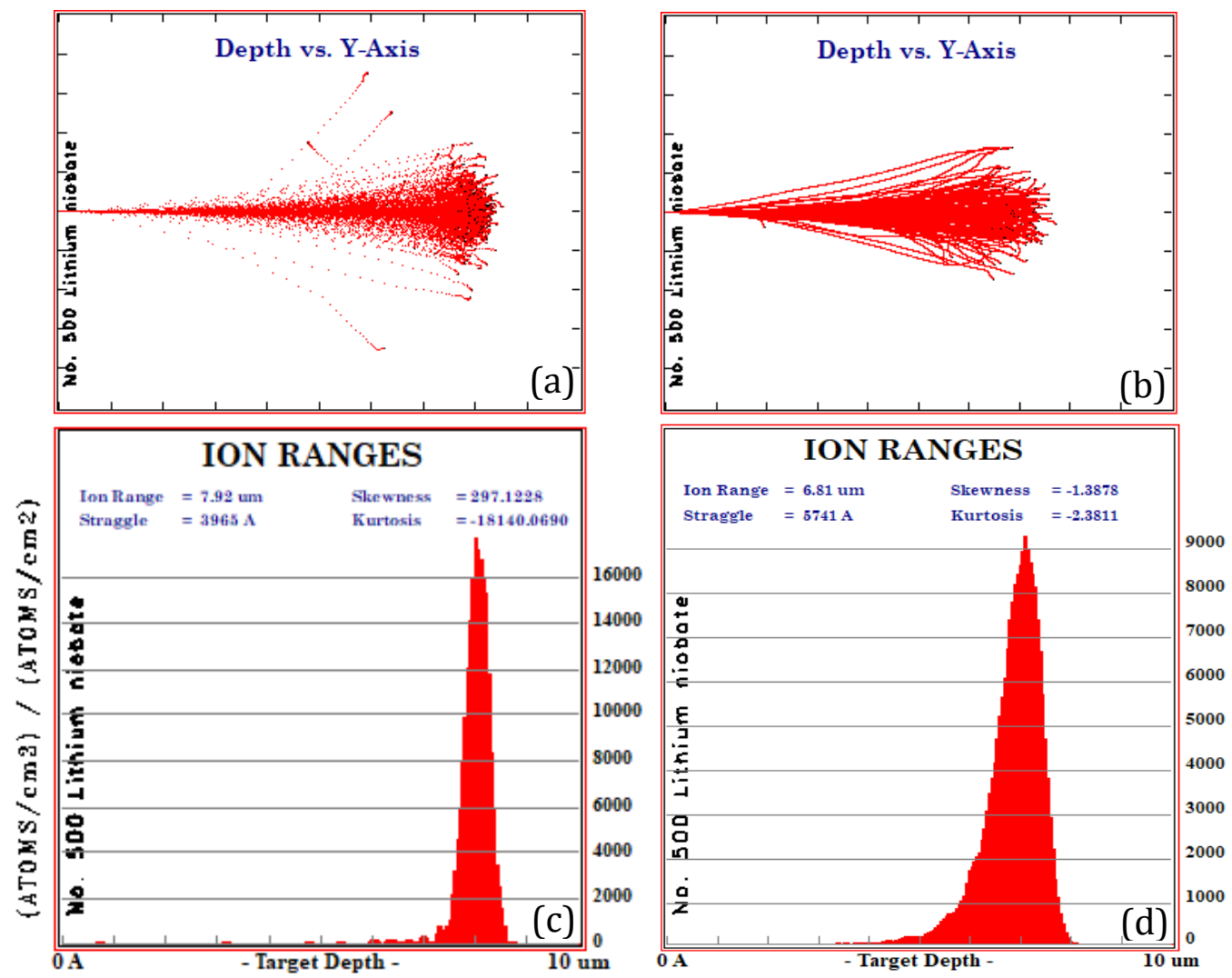

Figure 4.5. SRIM simulations showing the straggling and ion range for $850 \mathrm{keV} \mathrm{H}$ ions (a,c) and $50 \mathrm{MeV}$ I ion (b,d) beams of similar range in $\mathrm{LiNbO}_{3}$ 

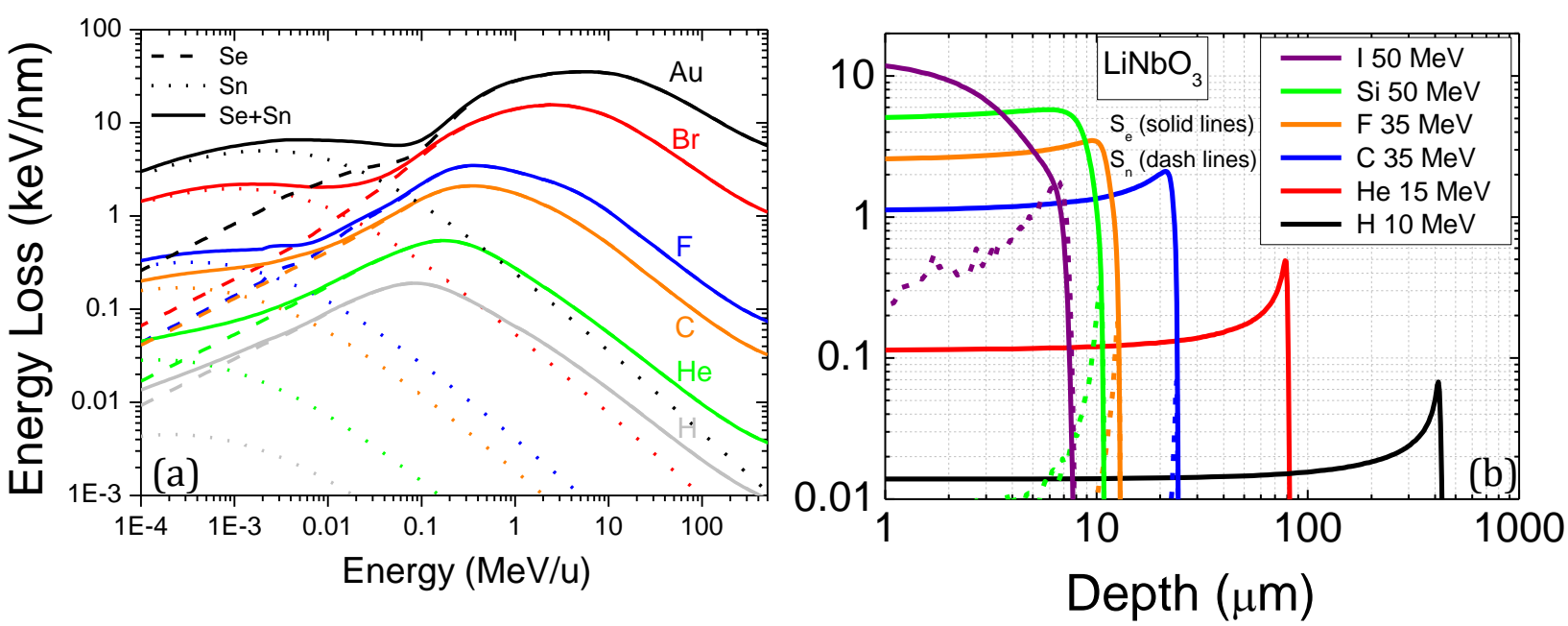

Figure 4.6. (a) Distributions of electronic (Se), nuclear ( $\mathrm{Sn}$ ) and total (Se+Sn) energy loss of ions listed in the legend as a function of ion energy per atomic mass unit (specific energy). (b) Energy loss profiles (Se, Sn) vs depth. (a, b) show the broad range of energy loss values and ranges calculated by SRIM 2008 [15] from the lightest to the heaviest possible ions, for the particular relevant material $\mathrm{LiNbO}_{3}$.

\subsubsection{Ion implantation with light $(\mathrm{H}, \mathrm{He})$ and medium mass ion beams (C to Si)}

Ions at energies above $100 \mathrm{keV}$ are likely to penetrate into the substrate rather than sputter the target [17] [18]. Implanters and tandem accelerators are used to generate energetic beams of both light and heavy ions. The incident ions lose their energy mainly through electronic and nuclear energy-transferring mechanisms [17] [19]. The ions, implanted at different stopping powers determined by the ion mass, energy, and target materials, will cause damage through these two mechanisms. High-dose (typically above $\sim 10^{16}$ ions $/ \mathrm{cm}^{2}$ ) light ions, typically referring to $\mathrm{H}$ or $\mathrm{He}$ particles, at energies of several $\mathrm{keV}$ to $\mathrm{MeV}$ induce an obvious nuclear damage layer buried inside $\mathrm{LiNbO}_{3}$ crystals [19] [20] [21] [22]. The electronic damage of light ion implantation mainly consists of point defects or colour centres, which can be removed to some extent by thermal annealing at moderate temperatures of $200-400^{\circ} \mathrm{C}$ [19] [21]. However, heating treatments of $\mathrm{LiNbO}_{3}$ in this temperature range do not destroy the nuclear damaged layers, and at most cause slight recovery of the disordered lattices in the region.

Some middle-light-mass ion such as $\mathrm{C}, \mathrm{N}, \mathrm{O}, \mathrm{F}, \mathrm{Si}, \mathrm{Cl}, \mathrm{Ni}$, or $\mathrm{Cu}$ at energies of several $\mathrm{MeV}$, can cause relatively strong modifications in the implanted regions in $\mathrm{LiNbO}_{3}$ even at lower doses of $\sim 10^{14}$ atoms $/ \mathrm{cm}^{2}$. In these cases, the electronic stopping powers are much higher than those of light ions [23] [24] [25] [26] [27]. Nevertheless, low-dose heavy-ion-induced lattice disorders can also be removed by suitable thermal annealing $\left(\sim 200-300^{\circ} \mathrm{C}\right)$. Figure 4.7 shows the distributions of the electronic and nuclear energy deposition of $1.75 \mathrm{MeV}$ He and $5 \mathrm{MeV} O$ ions implanted into $\mathrm{LiNbO}_{3}$ crystals from numerical simulations of SRIM 2008 [15].

Completing the framework, the use of heavier ions has been explored, for which in some of these irradiations electronic damage was beginning to come in to play as an important variable. In particular, results have been published with Si $3 \mathrm{MeV}$ ions [27], $\mathrm{Cu}$ ions at 0.7, 1.4, 2.6 $\mathrm{MeV}$ (a multi-energetic irradiation), $\mathrm{O}$ ions at $5 \mathrm{MeV}$ [23], $\mathrm{B}$ ions at $3 \mathrm{MeV}$ [28], $\mathrm{Ni}$ ions at $3 \mathrm{MeV}$ [29], and 
$\mathrm{C}$ ions at $6 \mathrm{MeV}$ [30]. For all the mentioned irradiations $S_{e}$ has much higher values than in the case of He implantations because they have higher $Z$ value. The increase in the extraordinary refractive index of $\mathrm{LiNbO}_{3}$ can be reached with much lower fluences to produce waveguides by this procedure $\left(\sim 10^{14} \mathrm{at} / \mathrm{cm}^{2}\right)$, being several orders in magnitude lower than those used in the implantation method.

For the purpose of photonic applications, ion implantation has proven to be an efficient method for fabricating optical waveguide structures in many materials due to its accurate control of the refractive indices of the substrates [19] [21]; in fact, in some crystals with low phase transition temperatures or very stable chemical properties, ion implantation is one of the most effective techniques to guide structures within them. $\mathrm{LiNbO}_{3}$ waveguides produced by the implantation of light and heavy (medium-mass) ion beams have been realized for many years and exhibit attractive properties for various photonic applications.
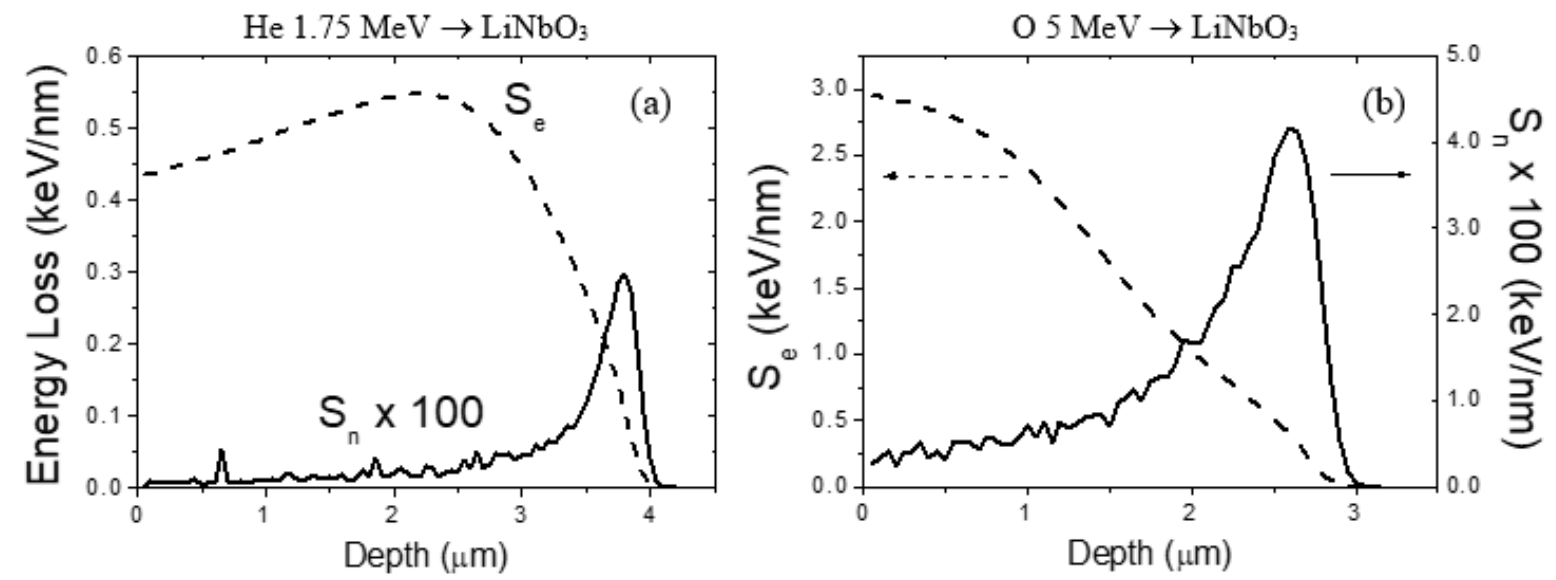

Figure 4.7. Distributions of electronic (dashed lines) and nuclear (solid lines) energy deposition of (a) $1.75 \mathrm{MeV} \mathrm{He}$ and (b) $5 \mathrm{MeV} O$ ions implanted into $\mathrm{LiNbO}_{3}$ crystals calculated by SRIM 2008 [15].

\section{$\underline{\text { Refractive index tailoring }}$}

$\mathrm{LiNbO}_{3}$ is a negative birefringent crystal, with a difference between the extraordinary and ordinary index $\Delta n=n_{e}-n_{o} \approx-0.08$ (at a wavelength of $632.8 \mathrm{~nm}$ ). Several types of refractive index profiles of lithium niobate waveguides can be generated using different ion beam techniques.

\section{"Barrier"- type index profiles}

The optical barrier refers to low refractive index layers buried inside the substrate generated by nuclear damage through energy transfer from implanted ions (particularly light ions, e.g., He or H) to the target matrix [19] [21]. These elastic collisions between the incident ions and target nuclei mainly occur at the end of the ion range, forming a disordered layer accompanied by a reduction in the physical density of the substrate and resulting in a decrease in the refractive index. Meanwhile, within most regions along the incident ions trajectory inside the target, the nuclear damage is quite small; therefore, there is almost no change (or only slight changes) in the refractive index. In this way, the regions between the surface of the substrate (i.e., air) and the optical barrier are surrounded by low-index regions, which act as waveguides [19]. These barrier- 
confined refractive index profiles are quite typical for ion-implanted waveguides in various materials, and are particularly common for those related to light ion implantation [19] [21].

Figure 4.8(a) shows a typical barrier-type profile of the ordinary index $\left(n_{0}\right)$ of $\mathrm{LiNbO}_{3}$ waveguides produced by the implantation of protons or He. The $n_{o}$ value in the waveguide regions only slightly decreases after implantation (typically $\Delta n_{o} \sim-0.1 \%$ to - $0.5 \%$ ), while in the barrier regions a large reduction in $n_{o}$ is found, ranging from $-1 \%$ to $-5 \%$ depending on the ion fluence [19] [20] [21] [22]. In this case, the low index barrier is the only confinement for the waveguide, which normally results in relatively strong light leakage through the barrier into the substrate regions due to optical tunneling effects, particularly when the barrier layers are very thin [19] Nevertheless, by broadening the barrier (through multiple-energy ion implantations, see Figure 4.8(a), the tunneling effect can be considerably reduced, resulting in a better confinement of the light modes. It should be stressed that for normal barrier distributions, the index value does not decrease significantly in the waveguide regions, just as in the case of $n_{o}$ for light ion-implanted $\mathrm{LiNbO}_{3}$ waveguides.

However, for heavier ions such as $\mathrm{O}, \mathrm{Si}$, or $\mathrm{P}$, implantation at energies of several $\mathrm{MeV}$ will induce large reductions (typically from $\sim-2 \%$ to $-3 \%$ ) in the waveguide regions [31], while a larger $n_{o}$ decrease (typically $\sim-5 \%$ ) occurs at the end of the ion track (Figure 4.8(b)). This non-typical barrier-type index profile theoretically makes the confined structures act as waveguides; however, more leakage of light into the substrate will occur due to the smaller index difference between the waveguide and the barrier, as well as the poor transmission properties. Therefore, in this a configuration, waveguides are not adequate for practical applications due to very high propagation losses.
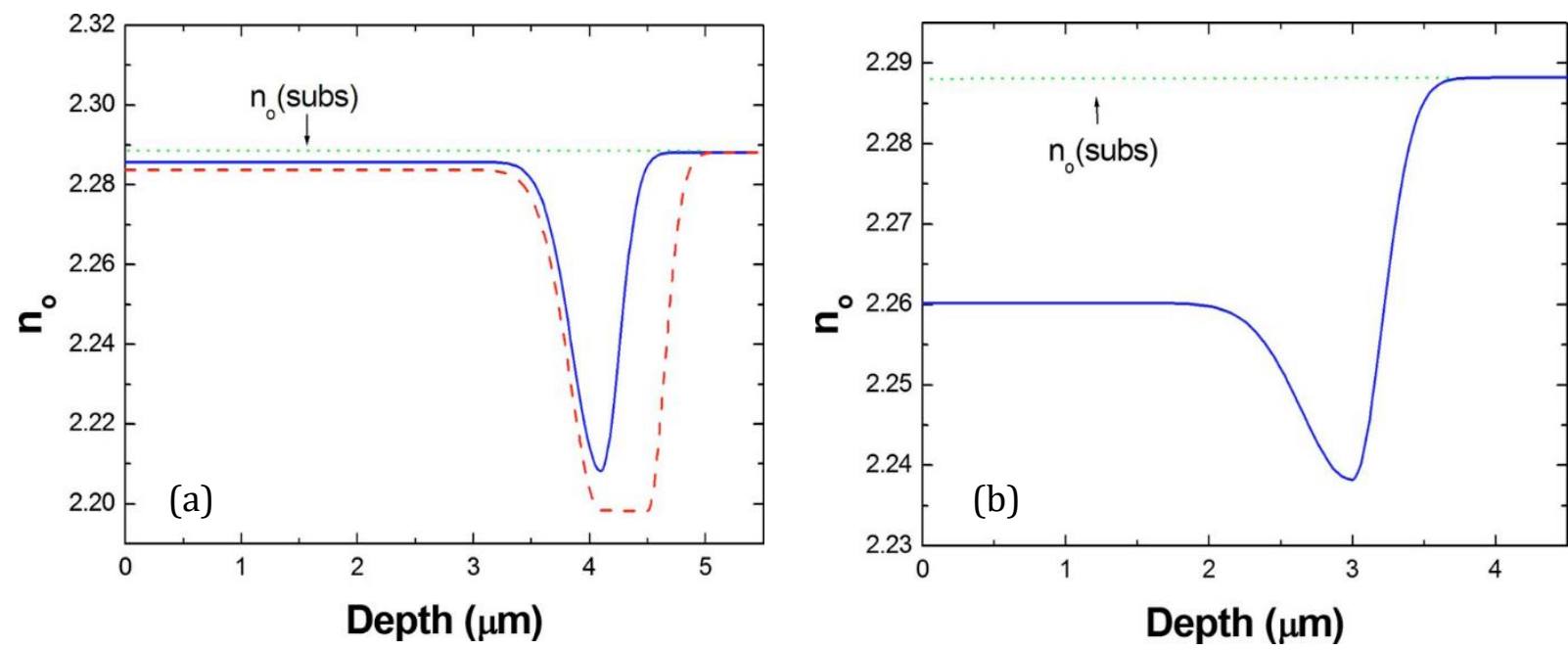

Figure 4.8. Typical barrier-confined no profile of (a) light ion-implanted $\mathrm{LiNbO}_{3}$ waveguide. The solid and dashed lines represent the single and multiple-energy implant configurations, respectively. (b) after heavy ion implantation in $\mathrm{LiNbO}_{3}$ (from Ref. [32]).

\section{“Enhanced-well + barrier-type" index profiles}

The mechanism for modification of the extraordinary refractive index $\left(n_{e}\right)$ is much more complicated. The implantation of light ions (He or $\mathrm{H}$ ) will cause positive changes in $n_{e}$ (for example, $\Delta n_{e} \sim+0.5 \%$ ) in the near-surface regions, resulting in an enhanced index well. At the end of the ion track, a negative index barrier, just as in the case of $n_{o}$ profiles, is built up due to nuclear 
energy deposition (nuclear damage) [19]. This profile is called a typical "enhanced well + barrier" distribution (Figure 4.9(a)). On the other hand, for the implantation of heavy (medium-mass) ions (with energies of several $\mathrm{MeV}$ ) such as $\mathrm{C}, \mathrm{O}$, or $\mathrm{Si}$, a larger increase in $n_{e}$ (up to $\sim 2 \%$ ) will occur in the near-surface region, while a negative index barrier might be constructed at the end of the ion range inside the crystal (Figure 4.9(a)).

As a representative example of the manufactured waveguides described by increasing the extraordinary refractive index, $n_{e}$, it is shown in Figure 4.9(b) (from Ref. [23]) the $n_{e}$ profile of 5 $\mathrm{MeV}$ at $1 \times 10^{14} \mathrm{O} / \mathrm{cm}^{2}$ implanted $\mathrm{LiNbO}_{3}$ optical waveguide. An increasing value of the index appears at the surface, with $\Delta \mathrm{n}_{\mathrm{e}} \approx 0.03$. Moreover, it also created a low index value to the depth where the ions are slowed down completely $(\approx 2.6 \mu \mathrm{m})$, i.e., where the collisions of incident ions with the lattice atoms happen, responsible for the nuclear stopping.

For $\mathrm{H}$ - or $\mathrm{He}$-implanted $\mathrm{LiNbO}_{3}$ waveguides, the light confinement is achieved by the combination of an enhanced well and a negative barrier, although theoretically, the enhanced well is sufficient for a fairly good restriction of light propagation [19] [21]. As for heavy-ion-implanted $\mathrm{LiNbO}_{3}$ waveguides, the large positive index well is mainly responsible for the light confinement, through which the light inside the waveguide can propagate in a non-leaky manner [21]. With respect to the mechanism by which such a $n_{e}$ profile is produced, positive $n_{e}$ well formation, it is still under study. Nevertheless, one may assume that the $n_{e}$ increase is related to electronic-energydeposition-induced effects on the $\mathrm{LiNbO}_{3}$ cell matrix. Specifically, in the network of oxygen octahedrons $\left(\mathrm{BO}_{6}\right)$, because most of the optical properties of oxygen-octahedron ferroelectrics depend on the presence of $\mathrm{BO}_{6}$ octahedron building blocks [33].
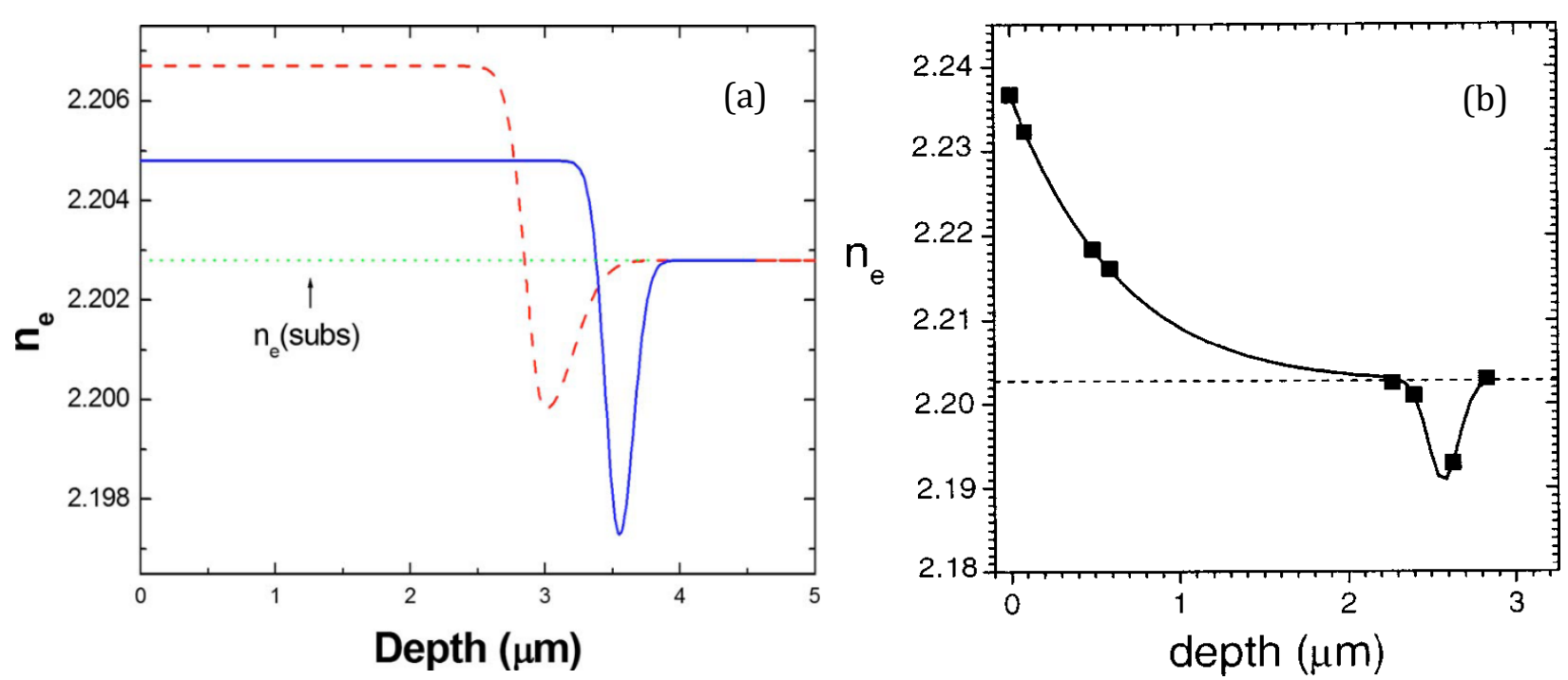

Figure 4.9. (a) "Enhanced well+barrier" confined ne profile of $\mathrm{LiNbO}_{3}$ waveguide formed by light (solid line) and heavy (dashed line) ion implantation (from Ref. [32]). (b) Extraordinary index (ne) profile (solid line) of $5 \mathrm{MeV}, 1 \times 10^{14} 0 / \mathrm{cm}^{2}$ implanted $\mathrm{LiNbO}_{3}$. The dashed line represents the bulk value (from Ref. [23])

\subsubsection{Ion irradiation with heavy and swift heavy ion beams}

Swift heavy ions (SHIs) are available at large accelerator facilities that can produce beams of high mass ions with kinetic energies in the $\mathrm{MeV}-\mathrm{GeV}$ range and above. In many solids SHIs release sufficient energy to generate long, nanometer-sized damage trails often denoted as 'latent tracks' 
because they are not discernable by the naked eye or optical microscopy. The initial interest in ion tracks goes back to the late 1950s, when Young reported the etching of tracks from fission fragments in LiF [34], and shortly after, when Silk and Barnes published the first transmission electron microscopy images of fission tracks in mica [35]. At that time, these discoveries generated a boom in track research and motivated numerous applications in nuclear detector physics, geochronology, archaeology, and many other fields [36] [37]. This interest was further stimulated with the advent of large heavy-ion accelerators in the 1980s and inspired intensive and systematic basic research on track formation as well as applied projects in material science, nanophysics, biophysics, and simulations of cosmic ray effects [38] [39] [40] [41].

SHIs are usually characterized by their specific energy in units of $\mathrm{MeV}$ per nucleon $(\mathrm{MeV} / \mathrm{u})$. In this velocity regime, the projectiles mainly interact with the target electrons, resulting in dense electronic excitations and ionizations of the target atoms (electronic stopping). Energy loss by elastic collisions with target atoms (nuclear stopping) is up to 2-3 orders of magnitude smaller and thus plays only a minor role for track formation. Since the majority of interactions occur with target electrons, no large-angle scattering of the ion projectiles occurs; this results in straight, highly parallel ion tracks. Transmission electron microscopy (TEM) studies and small angle X-ray scattering (SAXS) experiments revealed that the track shape is nearly cylindrical with a constant diameter for most of the ion range, while over the last micrometer or so, before the ions come to rest, the track diameter narrows and the shape changes into a cigar-shaped and irregular form [41] [42] [43]. This behavior is related to elastic collisions with the target atoms which become increasingly important when the ion has slowed down and is no longer able to efficiently ionize the target material.

A characteristic feature of SHI irradiation is that formation of tracks requires a critical minimum electronic $\mathrm{dE} / \mathrm{dx}$. This threshold strongly depends on the material and slightly increases with ion velocity. It can be below $1 \mathrm{keV} / \mathrm{nm}$ for polymers and up to a few tens of $\mathrm{keV} / \mathrm{nm}$ for metals. In metals, the large heat conductivity of the electrons dissipates the deposited energy before the track has time to form [44]. In contrast, tracks readily form in complex systems, in materials with polymorphism and electronic defects, and if radiation can induce radiolysis in combination with volatile radiation products. The most sensitive materials are polymers where SHI irradiation leads to chain scissions and the formation of small volatile fragments that leave the sample through outgassing. In many oxides, the tracks consist of amorphous cylinders embedded in the crystalline matrix ( $\mathrm{SiO}_{2}$, apatite, mica, etc.) [45]. In other materials, the track structure can be more complex, e.g., consisting of an amorphous core surrounded by a disordered crystalline shell (e.g., $\mathrm{Gd}_{2}(\mathrm{Ti}, \mathrm{Zr})_{2} \mathrm{O}_{7}$ and $\mathrm{Gd}_{2} \mathrm{TiO}_{5}$ ) [46]. Tracks even exist in amorphous materials such as in vitreous $\mathrm{SiO}_{2}$ and metallic glasses, where the amorphous state within the track slightly differs from the disorder of the surrounding matrix. In semiconductors the effects of SHIs are not completely understood, but it seems that the band gap has some influence. Some insulators $\left(\mathrm{UO}_{2}, \mathrm{ThO}_{2}, \mathrm{CeO}_{2}, \mathrm{MgO}\right.$, etc. $)$ are extremely resistant under SHI irradiation and their response includes mainly the creation of isolated defects and strain [47]. Figure 4.10 shows ion tracks in different materials.

The diameters of ion tracks are typically a few nanometers, being material specific. Depending on the material and its radiation sensitivity, the track size becomes larger with increasing energy loss of the projectile. Above a critical threshold, each ion creates an individual, continuous, homogeneous cylindrical track, whereas close to the threshold, as well as in rather insensitive materials (metals and semiconductors), the damage morphology can be discontinuous and tracks 
may consist of a sequence of dotted damage fragments. Additionally, the track diameter depends on the ion velocity and is, for similar $\mathrm{dE} / \mathrm{dx}$ values, larger for low-velocity ions [48].

\subsubsection{Applications of Swift Heavy Ions}

Over the past several decades, ion-beam facilities that were developed for nuclear physics research have impacted many other fields, including atomic physics, plasma physics, material science, condensed matter physics, geosciences, environmental physics, and bio-medical sciences. The number of SHI-beam applications in basic research as well as for industrial applications is still growing. A unique feature of $\mathrm{MeV}-\mathrm{GeV}$ heavy ions is that each individual projectile produces a cylindrical nanometer-sized track. Heavier projectiles are preferred as a structuring tool, because they produce tracks of continuous damage with the largest track diameters as a result of their high energy loss. The penetration depth of ions in a material can be adjusted by the beam energy. The track density is adjusted by the fluence which typically ranges from a single ion impact per sample up to the regime of multiple track overlap (typically above $\sim 10^{13} \mathrm{ions} / \mathrm{cm}^{2}$ ). Tracks are usually stochastically distributed over the exposed target area, but can also be precisely placed on predefined positions using a heavy-ion microprobe. The application of targeted irradiations includes writing specific patterns [49], testing microelectronic circuits [50], and delivering a preset number of ions to the nuclei of individual living cells [51]. A few examples of exciting research topics based on SHIs are presented in the following paragraphs.

\subsection{Micro- and nanostructures formed by ion track technology. Applications of latent- and etched tracks}

One has to distinguish between the application of latent, i.e. as-implanted ion tracks, and etched tracks. Latent tracks emerge from the energy deposition of swift heavy ions inside a tiny target

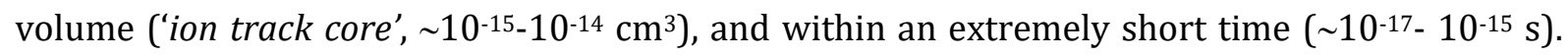
These extraordinary transient conditions lead to dramatic modifications of the materials via chemical and structural changes, with accompanying heat and pressure pulses.

Amorphous tracks formed by electronic excitation are well aligned with beam direction and are themselves nanostructures, offering very interesting capabilities in nanotechnology [39] [52] [53] [54] [55] [56]. Four major strategies for latent track applications show up: (1) exploitation of the modified transport properties along ion tracks, (2) letting metallic atoms or clusters precipitate along the tracks, (3) exploitation of the material's chemical changes, and (4) making use of ioninduced phase transitions [57] [58].

Dissolution of the latent track material by suitable chemical agents ('etching') leads to etched track formation; the so-called "etched tracks", that is, generating nanopores. By careful selection of projectile, target, etchant, and etching conditions, etched tracks can be tailored towards any required shape, such as cylindrical, conical, or hyperbolic, transmittent (in thin foils) or nontransmittent, that can act as traps for foreign molecular species (sensors). On the other hand, etched tracks can be filled with other substances to form nanocomposite materials with novel optical, electrical and magnetic properties; moreover, the embedded matter can be arranged as either massive wires (also called: 'fibers, fibrilles') or tubules, or it just can be dispersed discontinuously as small nanoparticles along the track length. There is a wide range of applications linked either to direct patterning of materials or to depositing material into the pores of etched ion track membranes [55] [59] [60] [81][142]. 


\section{a) Ion track membranes}

Selective attack by a suitable chemical etchant of ion tracks, convert the damage of each track into an individual nanopore. The etching time determines the pore size and specific etching conditions control the pore shape and geometry (cylindrical, conical, double conical, etc) [61] (see as example Figure 4.11 a). The discovery of track-etching has triggered applications in a wide range of scientific and industrial areas. During the last decade, the rapidly increasing activities and recent developments in nanoscience have boosted the interest in track-etched channels in polymers in particular [39] [40] [62] [63] [64]. Several small companies process commercially large quantities of track-etched membranes with pores of extremely uniform diameter

Transport properties of membranes with a single track-etched nanopore became of great interest to mimic conditions of ion channels in biological cell membranes [61]. The asymmetrical geometry of nanopores allows preferred transportation of chemical and biological species in one direction like an ionic diode [65] [66]. Using different chemical and physical modification or decoration strategies, it is possible to tailor systems with sensor properties [67] [68] [69] [70]. Ion track membranes are also of interest as nanofluidic devices for biosensing, separation of drug molecules, desalination, electro osmosis, electrochemical energy storage in batteries, and fuel cells and supercapacitors, just naming a few topics.

\section{b) From etched tracks to nanowires}

Another very active research field in nanoscience uses the ion-track technology for the fabrication of nanowires by filling the pores of ion-track membranes via electrodeposition (see as example Figure $4.11 \mathrm{~b}$ ). The combination of chemical etching, electrochemical deposition, and surface modification techniques led to the development of an enormous flexibility to synthesize tailored nanostructures of various metals and semiconducting compounds and exploit size dependent physical and chemical properties of materials at the nanoscale. As an advantage, ion track membranes allow the control of the size, geometry, aspect ratio, and surface morphology of the nanowires. Recent investigations provide results on size effects on optical, electrical, and thermal properties, surface plasmon resonances, and thermal instabilities [71] [72] [73] [74]. 

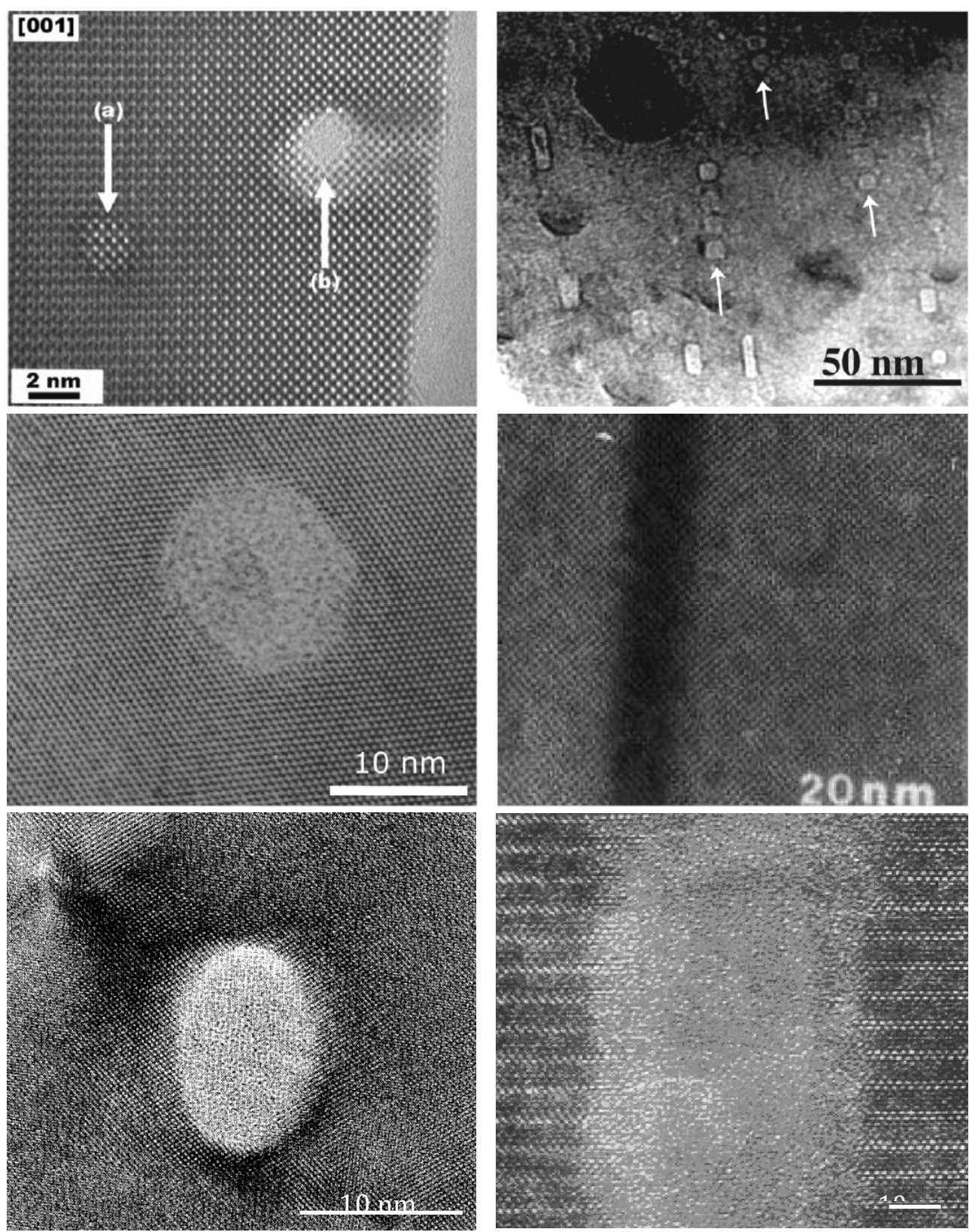

Figure 4.10. High-resolution transmission electron microscopy (TEM) images of ion tracks in non-amorphisable (top) and amorphisable (medium/bottom) insulators/semiconductors. Top left: Cross section of two tracks (a and b) in nonamorphisable $\mathrm{SnO}_{2}$ irradiated with $9 \mathrm{MeV} / \mathrm{u}$ Cd ions [75]. Top right: $\mathrm{CaF}_{2}$ irradiated with $10 \mathrm{MeV} / \mathrm{u} \mathrm{Bi}$ ions. The arrows indicate the trajectories of non-continuous facetted defect clusters [76]. Medium left: Cross section of a single track of a $\mathrm{Pb}$ ion in mica. The amorphous track zone is surrounded by the intact crystal matrix [77]. Medium right: Continuous amorphous track region created along the trajectory of a Xe ion $(\sim 24 \mathrm{MeV} / \mathrm{u})$ in $\mathrm{Y}_{3} \mathrm{Fe}_{5} \mathrm{O}_{12}$ [78]. Bottom left: Latent tracks of Dy ions $(13 \mathrm{MeV} / \mathrm{u})$ in GeS irradiated normal to the crystal surface [79]. Bottom right: Amorphous track in $\mathrm{Bi}_{2} \mathrm{Sr}_{2} \mathrm{CaCu}_{2} \mathrm{O}_{8}$ irradiated with swift Au ions [80]. 


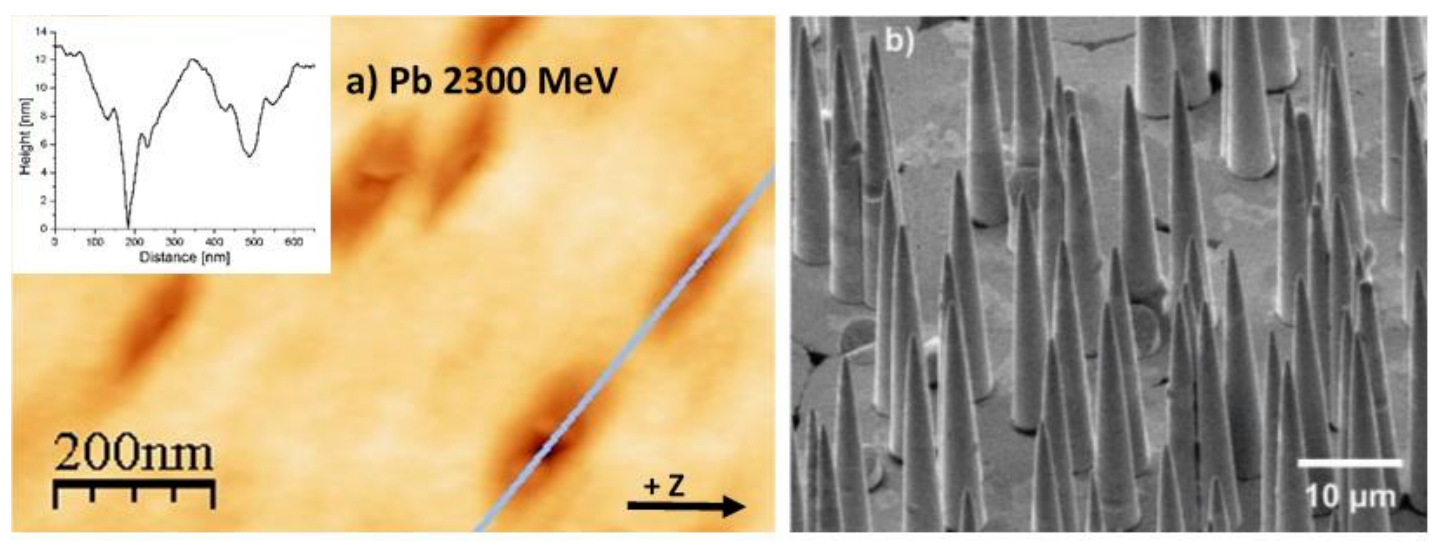

Figure 4.11. (a) AFM image of etch pit morphologies in $\mathrm{LiNbO}_{3}$ substrate obtained after etching tracks (HF $40 \%$ at $\mathrm{RT}$ for $10 \mathrm{~min}$ ) produced with $\mathrm{Pb} 2300 \mathrm{MeV}$ ions [55]. The inset shows the depth profile recorded along the line across the long axis of the two elongated pores. (b) Scanning electron microscopy image of Au nanocones electrodeposited in conical nanopores [82].

\subsection{Functional Bulk Material Testing}

Studies on the radiation hardening of bulk materials under extreme conditions including radiation dose, stress, temperature, and pressure, have received significant attention in the context of nuclear materials and of, more recently, next-generation accelerators. Functional materials under a constant flux of radiation that may modify their properties need to be tested and appropriate mitigation methods must be developed. Irradiation experiments with SHIs are an efficient and controlled way to test bulk materials and identify specific physical and structural property changes. For reliability tests and lifetime estimates, it is important to understand how a given material responds to extreme radiation fields, what is the nature of the specific damage, and how thermal treatment can mitigate the radiation damage. An important research area addresses the radiation hardening of materials for new high-power accelerator facilities (e.g., FAIR, FRIB, ESS, etc.), operational limits of materials in high dose and high energy density environments, lifetime predictions, and the development of new material solutions for extreme cases. Unique material requirements also arise with the development of facilities with the highest pulse intensities. The dynamic response (pressure wave propagation and damping) under high-power beam impacts of beam intercepting devices (collimators, targets, and beam dump materials) are currently tested with SHIs and provide helpful experimental data for benchmarking respective finite element simulations [83] [84] [85] [86].

\subsection{Simulation of cosmic radiation effects with $\mathrm{SHI}$}

Electronic components in space missions are sensitive to radiation effects and require reliability tests before being installed. High-energy particles produce local charges and electron cascades that generate multiple types of artifacts, such as the increase of leakage currents, local memory errors (e.g., single event upsets), and single event transient errors at the system level. In some cases, more dramatic and destructive events like dielectric rupture, burn-out, or latch-up render the component completely unusable. For safe operation of satellites or devices for exploration of the solar system, the importance of accelerator-based studies and the need to develop countermeasures are acknowledged by all space agencies and give radiation tests at accelerator facilities high priority [87] [88]. The advantage of SHIs are their large penetration depths that allows tests without disassembling the electronic components. 


\subsection{Swift heavy ion related research in nuclear materials}

Irradiation experiments with SHIs have relevance for nuclear materials research, particularly to simulate the effects of fission fragments in nuclear fuels. The primary sources of radiation damage in nuclear fuels are fissile actinides, such as uranium, which fission into two fragments of $\sim 100$ $\mathrm{MeV}$ kinetic energy. It is interesting to note that end-of-range ballistic collisions by fission fragments are almost entirely responsible for the displacement damage near the end of the $\mathrm{UO}_{2}$ fuel life ( 4 to 6 years) in current nuclear power plants [89]. Only about $\sim 20$ displacements per atom (dpa) are from collisions with fission neutrons, while fission fragments produce 1200 to $\sim 1800$ dpa between the center and outer periphery of the fuel pellet. This damage process, which is based on elastic collisions, has been studied in detail and is well understood. However, many open questions remain on the effects of the electronic energy loss and the extremely high energy densities that are induced along the major part of the trajectory of fission fragments. The intense ionization processes during the slowing down of fission fragments can also influence the formation and stability of fission-gas bubbles and alter the chemical composition of the fuel via radiation enhanced diffusion. Some studies suggest that fission fragments can result in the stabilization of low-swelling metastable phases [90] [91] and fine precipitate structures that act as noble gas nucleation sites (thereby suppressing fuel swelling), as well as the formation of favorable gas bubble architectures [92] [93] [94] [95]. Experimentally, large accelerator facilities for SHIs provide the most suitable conditions for simulating fission-fragment type damage in nuclear fuel because the irradiation conditions are well-controlled and the ion species as well as their energies can be adjusted. This allows irradiation experiments at various electronic stopping powers, including tests below and above the threshold for track formation.

\subsection{Generation of waveguides structures using SHI irradiation}

Recently, swift ion irradiation in $\mathrm{LiNbO}_{3}$ has received considerable attention due to its novel modification of structures and refractive indices of the crystal [55] [96] [97] [98] [99] [100] [101] [102] [103]. In these cases, the refractive index changes are mainly due to the electronic-damagerelated structural modifications. The electronic stopping power $\left(S_{e}\right)$ of the incident ions, is a key parameter that affects the structures and refractive indices of the $\mathrm{LiNbO}_{3}$ substrate [100]. In addition, there is an intrinsic amorphization threshold value $S_{t h} \approx 5 \mathrm{keV} / \mathrm{nm}$, which strongly influences the formation of nanometer-sized amorphous tracks occurring during the irradiation process [97] [100].

a) "Buried amorphous layer" confined waveguides. Heavy ion irradiation at low fluence: high index jump waveguides

When the incident ions have $S_{e}<S_{t h}$, tracks with partial damage will be created along the ion trajectory, and at sufficiently high fluences (for $\mathrm{LiNbO}_{3}, \sim 10^{14}-10^{15}$ ions/cm²), the tracks overlap and a homogenously amorphous layer of damage accumulation will be generated within the crystal [96] [97] [99], whose thickness increases with the ion fluence. This amorphous layer is related to electronic energy deposition, and its thickness can be tuned by choosing suitable ion fluences (see Figure 4.12.) 

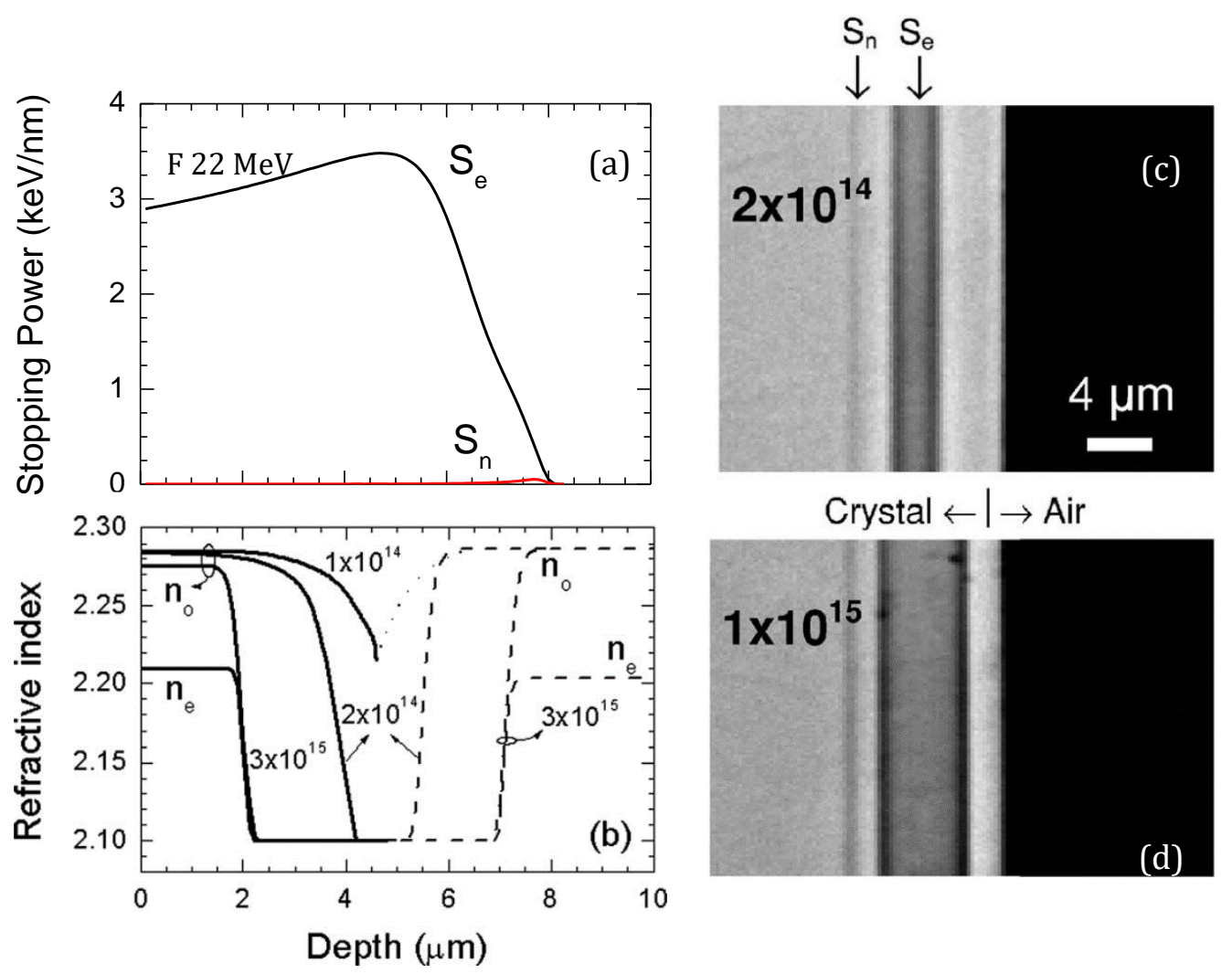

Figure 4.12. (a) The electronic stopping power versus depth for $22 \mathrm{MeV} \mathrm{F}^{4+}$ ions in $\mathrm{LiNbO}_{3}$ [96], (b) The refractive index profiles $\left(\mathrm{n}_{\mathrm{o}}\right.$ and $\mathrm{n}_{\mathrm{e}}$ ) of z-cut samples with this irradiation at different ion fluences (at/cm2) [96], (c) and (d) Optical microphotographs of a polished y-cut cross section for samples irradiated with $\mathrm{F}^{4+}$ at fluences of $2 \times 10^{14} \mathrm{at} / \mathrm{cm}^{2}(\mathrm{c})$, and $1 \times 10^{15} \mathrm{at} / \mathrm{cm}^{2}(\mathrm{~d})$. The depths of the maximum electronic stopping power, $\left(\mathrm{S}_{\mathrm{e}}\right)$ and nuclear stopping power $\left(\mathrm{S}_{\mathrm{n}}\right)$ are indicated with arrows. Ion beam is penetrating the material coming from left side in (c, d).

Figure 4.12 (b) shows the $n_{e}$ and $n_{o}$ profiles of $22 \mathrm{MeV} \mathrm{F-ion-irradiated} \mathrm{LiNbO}_{3}$ waveguides. As it is indicated, the profile has a "steplike" distribution with sharp boundaries. Both $n_{e}$ and $n_{o}$ have the same value, i.e., $\sim 2.10$, in the amorphous region, while the surface indices for both $n_{e}$ and $n_{o}$ are only slightly modified. In this way, the waveguide layer is therefore confined in the region surrounded by the surface (air) and the buried amorphous layer. Moreover, the profile index shapes obtained with this new technique are sharper, the index change is higher, and the barrier can be thicker.

b) Swift heavy ion irradiation at ultra-low fluences: nanostructured gradient index waveguides

On the other hand, when the ion energy increases further, $S_{e}>S_{t h}$, amorphous tracks with dimensions of a few nanometers will be created, and at ultralow fluences $\left(10^{12}\right.$ ions $\left./ \mathrm{cm}^{2}\right)$, [55] [102] [103] effective waveguides can be formed with buried damage layer confined refractive index profiles. In this case, each single ion impact creates a nanometer-sized latent amorphous track with an isotropic refractive index $\left(n_{a}=2.1\right)$. The radius of each track increases monotonically with $S_{e}$, forming a depth-shaped distribution [103]. The refractive index of the irradiated regions will follow the electronic damage $\left(S_{e}\right)$ behaviour. By this way, the fluence required for waveguide formation is remarkably reduced to $\sim 10^{12}$ ions $/ \mathrm{cm}^{2}$. The advantage of this method is the avoidance of overlapping nanotracks, which requires ultralow doses.

c) Novel thick optical waveguides in lithium niobate induced by swift heavy ions ( $10 \mathrm{MeV} / \mathrm{amu})$ at ultralow fluences 
In addition, it is possible to use a much higher energy for ion irradiation, e.g., several GeVs, to fabricated in seconds very deep optical waveguides (tens of microns) for applications in the infrared wavelength bands [104]. In this way, since $S_{e}>>S_{\text {th }}$, a much lower dose is required for waveguide formation in $\mathrm{LiNbO}_{3}$. Specifically, heavy mass ions, $\mathrm{Kr}$ and $\mathrm{Xe}$, having energies in the $\sim 10 \mathrm{MeV} / \mathrm{amu}$ range have been used to produce thick planar optical waveguides at the surface of lithium niobate. The waveguides have a thickness of 40-50 micrometers, depending on ion energy and fluence, smooth profiles and refractive index jumps up to $0.04(\lambda=633 \mathrm{~nm})$. It implies the generation of a buried low-index layer (acting as optical barrier), made up of amorphous nanotracks embedded into the crystalline lithium niobate crystal matrix. This would allow the fabrication of much thicker waveguides (up to tens of microns) with ultralow fluences $\left(\ll<10^{12}\right.$ $\mathrm{cm}^{-2}$ ). They propagate ordinary and extraordinary modes with low losses keeping a high nonlinear optical response (SHG) turning them into great candidates for many applications. This first test demonstration could be extended to other crystalline materials useful as photonic devices for the mid-Infrared applications, such as astrophysics studies.

As a summary we can state that, swift heavy ion irradiation presents a novel way to fabricate waveguides in $\mathrm{LiNbO}_{3}$. In this case of heavy ions, the electronic damage is the main cause of the index change. This technique overcomes some of the disadvantages of light ion implantation, such as the large fluence of ions required $\left(10^{16}-10^{17} \mathrm{~cm}^{2}\right)$ and the contamination of the material with the irradiated ions in the optical barrier. Irradiation parameters such as ion species, energy, and fluence must be chosen by carefully considering the $S_{e}$ values of the ions in $\mathrm{LiNbO}_{3}$ crystals.

Table 4.1. Typical parameters for $1 \mathrm{D}$ waveguides production in $\mathrm{LiNbO}_{3}$ by ion beam techniques.

\begin{tabular}{|c|c|c|c|}
\hline Ion beam techniques & Ion species & Ion energies & Ion fluences $\left(\mathrm{at} / \mathrm{cm}^{2}\right)$ \\
\hline Light ion implantation & $\mathrm{H}$ and $\mathrm{He}$ & $\begin{array}{l}100 \mathrm{keV}-3 \\
\mathrm{MeV}\end{array}$ & $\sim 10^{16}-10^{17}$ \\
\hline $\begin{array}{l}\text { Heavy (medium- mass) } \\
\text { ion implantation }\end{array}$ & $\begin{array}{l}\mathrm{C}, \mathrm{N}, \mathrm{O}, \mathrm{F}, \mathrm{Si} \\
\mathrm{P}, \mathrm{Cu}, \mathrm{Ni}, \text { etc. }\end{array}$ & $2-7 \mathrm{MeV}$ & $\sim 1 \times 10^{14}-2 \times 10^{15}$ \\
\hline $\begin{array}{l}\text { Heavy and medium-mass } \\
\text { ion irradiation }\end{array}$ & $\begin{array}{l}\mathrm{O}, \mathrm{Si}, \mathrm{Cl}, \mathrm{Cu} \text {, } \\
\text { etc. }\end{array}$ & $12-50 \mathrm{MeV}$ & $\sim 10^{12}-10^{14}$ \\
\hline
\end{tabular}

\subsection{Ion beam analytical techniques and experimental setup examples}

\subsubsection{Techniques based on elastic nuclear processes: RBS and ERD}

Ions can interact with matter in different ways but the simplest scenario is the interaction via elastic nuclear processes. Because in elastic processes we can assume a two-body collision controlled by a central Coulombic force the physics can be described in a straightforward way. Indeed, since the involved velocities are not high, the elastic collision between the ion and the target can be treated within the classical mechanics framework. The idea that elastically scattered ions can be used to get information about matter was primarily developed in the famous Rutherford experiment (actually performed by Geiger and Marsden) in 1909 [105] [106]. It is important to note that, although in Rutherford's experiment a light projectile (alpha particle) impinges into a heavy target (gold), the physical problem is identical for the opposite conditions 
(a heavy ion and a light target). These two cases correspond to the most basic IBA techniques: the first one is known as Rutherford backscattering spectrometry (RBS) and the latter is known as elastic recoil detection (ERD).

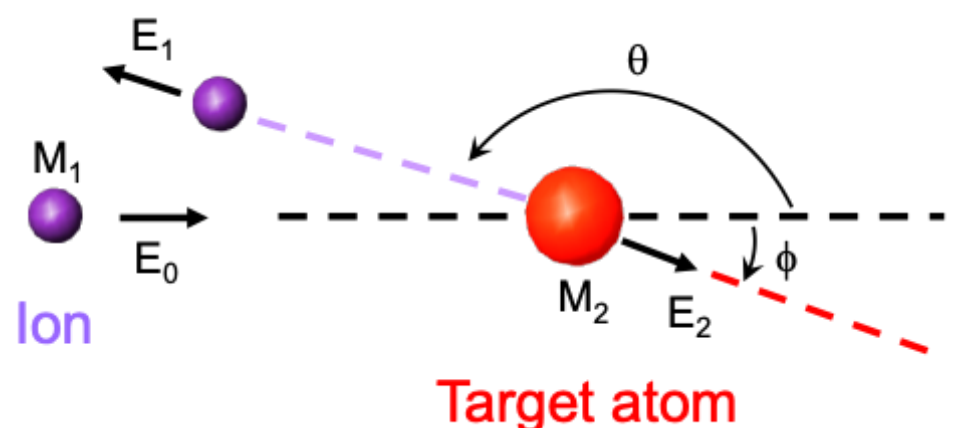

Figure 4.13. Kinematics of a two-body elastic collision. Depending on the mass of the projectile and the target atom, scattered ions (RBS) or recoils (ERD) can be detected.

Figure 4.13 shows the basic scheme of the ion-target collision that takes place both in RBS and ERD experiments. The main difference between the techniques is that for RBS the projectile satisfies the condition $M_{1}<M_{2}$ and the scattering angle $(\theta)$ is high, while in ERD the working conditions satisfy $M_{1}>M_{2}$ and we use a forward scattering angle ( $\left.\Phi\right)$. Imposing conservation laws for the energy and the momentum, the velocity and scattering angle of the projectiles can be obtained as a function of the initial parameters of the incoming ion. Moreover, the ratio between the final and the initial energies of the ion, known as the kinematic factor $(K)$ can be analytically determined [107]:

$$
\begin{gathered}
K_{R B S}=\frac{E_{1}}{E_{0}}=\left(\frac{\left(M_{1} / M_{2}\right) \cos \theta+\sqrt{1-\left(M_{1} / M_{2}\right)^{2} \sin ^{2} \theta}}{1+\left(M_{1} / M_{2}\right)}\right)^{2} \\
K_{E R D}=\frac{E_{2}}{E_{0}}=\frac{4 M_{1} / M_{2}}{\left(1+M_{1} / M_{2}\right)^{2}} \cos ^{2} \phi
\end{gathered}
$$

The kinematic factor is always lower than 1 because the energy of the projectile always exceed the energy of the scattered ions or recoils. In addition, the different values of $K$ for the different ion-atom combinations allow us separating the elements in the spectrum. In particular, if we assume for simplicity that the collision takes place only at the surface, the mass of the element can be measured only by the reduction of the energy of the projectile: $\Delta E=E-K E$. This is one of the characteristic features of RBS and ERD: the mass resolution is determined by the kinematic factor. If the difference in the kinematic factor of two elements is large, then the resolution is high; whereas similar values of $K$ lead to a difficult separation of the elements.

Figure 4.14 shows the kinematic factors for representative RBS (a) and ERD (b) conditions, including different target elements. Ideally, the particle detector should be placed at a scattering angle where $K$ is almost constant. Due to this fact and the additional technical limitations, the typical angles are around $\theta=170^{\circ}$ for RBS and $\Phi=30^{\circ}$ for ERD. 

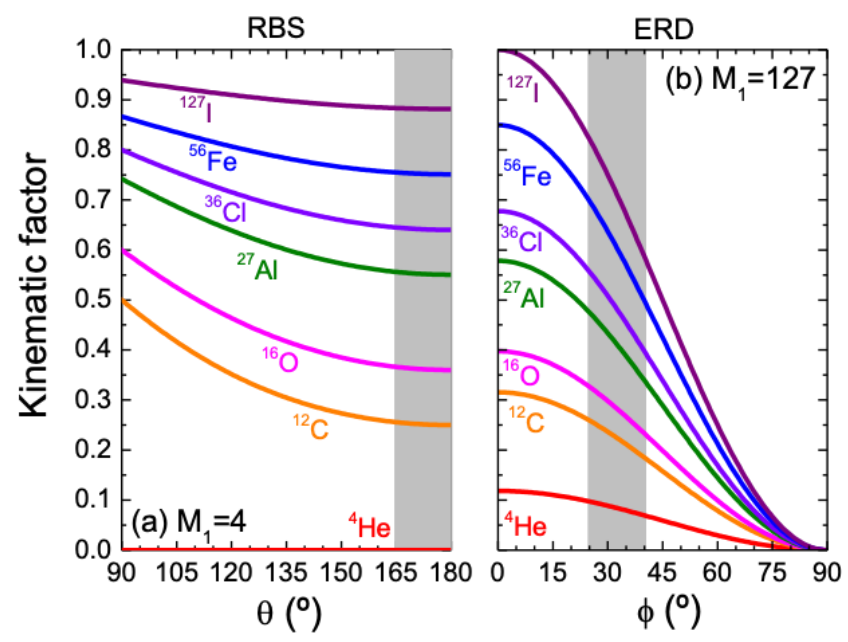

Figure 4.14. Values of the kinematic factor for (a) RBS with ${ }^{4} \mathrm{He}$ and (b) ERD with ${ }^{127}$. The shaded region corresponds to the typical range for the detection of the scattered particles.

Although the kinematic factor is very useful to read the spectrum in terms of the mass of the elements, it does not provide information about the concentration of these elements. Logically, the number of scattered particles (yield) must be proportional to the number of target atoms per unit area (concentration), but also to other factors such as the number of incident particles or dose $(Q)$ and the solid angle of the detector $(\Omega)$. Since both the dose and the solid angle can be determined experimentally, the only remaining unknown to calculate the concentration of the sample is the scattering probability.

Atoms with different masses have different scattering probabilities because the Coulombic potential depends on the atomic number and, therefore, the repulsing force will be higher for heavy elements than for light ones. The physical quantity representing the scattering probability is the cross section $(d \sigma / d \Omega)$, which describes the effective area of each nucleus presented to the beam of incident particles. The calculation of the cross section was first derived by Rutherford for the Coulombic potential and, in the laboratory frame of reference. It can be expressed as [108]:

$$
\begin{gathered}
\frac{d \sigma_{R B S}}{d \Omega}=\left(\frac{Z_{1} Z_{2} e^{2}}{16 \pi \varepsilon_{0} E}\right)^{2} \frac{4}{\sin ^{4} \theta}\left[\frac{\cos \theta+\sqrt{1-\left(M_{1} / M_{2}\right)^{2} \sin ^{2} \theta}}{\sqrt{1-\left(M_{1} / M_{2}\right)^{2} \sin ^{2} \theta}}\right]^{2} \\
\frac{d \sigma_{E R D}}{d \Omega}=\left(\frac{Z_{1} Z_{2} e^{2}}{4 \pi \varepsilon_{0} E}\right)^{2} \frac{1}{\cos ^{3} \phi}\left(1+\frac{M_{1}}{M_{2}}\right)^{2}
\end{gathered}
$$

where $E$ is the energy of the ion and $Z_{1}$ and $Z_{2}$ are the atomic numbers of the ion and the target atom, respectively.

The cross section depends on many parameters, but due to the quadratic dependence the most important factor is the first one. This factor, which is shared for both RBS and ERD, indicates that the cross section decreases fast with the beam energy and also increases fast with the atomic number of the detected element. Figure 4.15 shows the cross sections for representative situations in RBS (a) and ERD (b) experiments. The fact that cross sections are tabulated and well- 
known means that RBS and ERD do not require standards to quantify the composition of the element, which is an advantage over other IBA techniques such as PIXE.
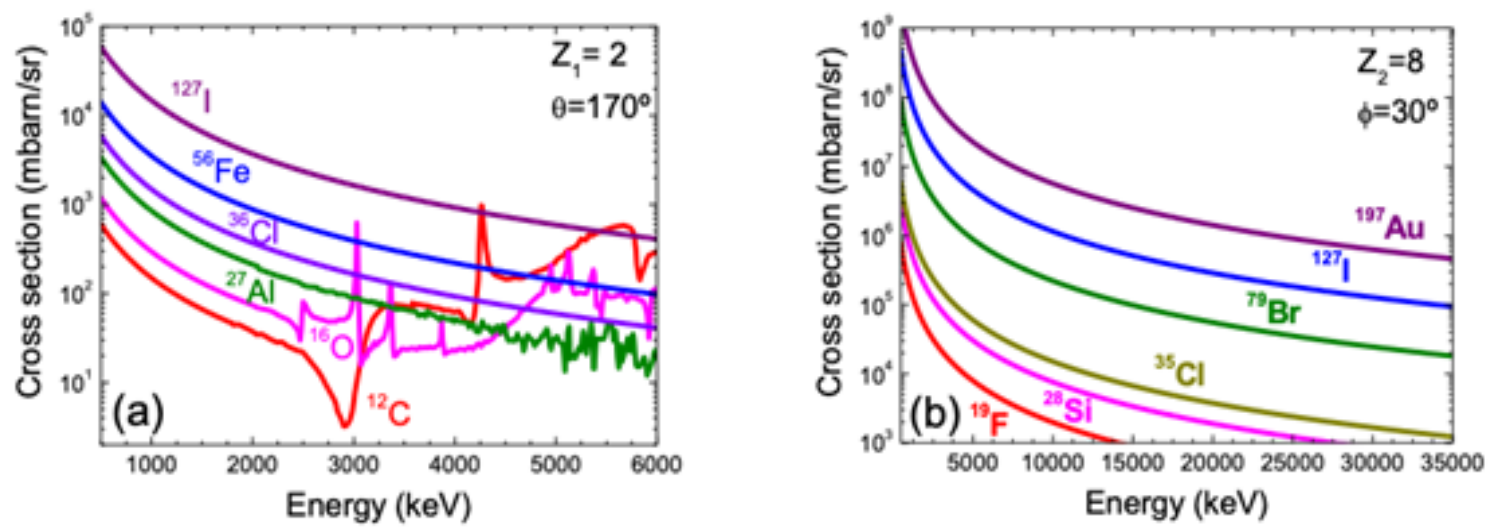

Figure 4.15. (a) Cross section for different elements using ${ }^{4} \mathrm{He}$ at different energies $\left(170^{\circ}\right.$ scattering angle). (b) Cross sections for 160 using different ions and energies $\left(30^{\circ}\right.$ scattering angle).

Figure 4.15a shows an RBS example with a ${ }^{4} \mathrm{He}$ beam. The cross section for the different elements increases with the target mass and decreases with energy as mentioned before (note the semi-log scale). It is important to note that Rutherford's formula is satisfied below $2 \mathrm{MeV}$ for almost all the elements, but there are important deviations for higher energies (see, for instance, $\mathrm{C}$ and $\mathrm{O}$ ). These deviations are due to nuclear interactions leading to resonances (discussed in the next section). Since these resonances can increase the cross section by one or even two orders of magnitude they are extremely useful to detected light elements that typically have a low yield. In the case of ERD, the Figure $4.15 \mathrm{~b}$ shows the cross section for ${ }^{16} 0$ recoils when using different ion beams. As it can be seen, the heavier the ion, the higher the cross section. Thus, when measuring light elements, heavy ions are especially convenient to increase the statistics in the spectra.

Once the cross section is known, the quantification of the composition can be developed as a function of the solid angel $(\Omega)$, the dose $(\mathrm{Q})$ and the experimental backscattering yield (Y). In the simplest case of RBS on a thin film under normal incidence, the areal density (number of atoms per unit area) can be calculated as $N_{A}=Y / Q \sigma \Omega$, where $\sigma$ is the average cross section (coinciding with the $\mathrm{d} \sigma / \mathrm{d} \Omega$ for small $\Omega$ ). However, this formula is only a surface approximation. When the beam enters into the target, the ions slow down and lose energy and to take this effect into account a new variable must be considered: the stopping force.

The stopping force is the physical link between energy and depth and, therefore, it is the mechanism providing depth resolution to RBS and ERD. The total energy loss by an ion can be divided in three steps: the path of the ion from the surface towards the target atom, the ion-solid collision, and the ion/recoil path towards the surface again. The effect of the stopping force is that the same ion-atom collision occurring at different depths will be detected at different energies, allowing depth concentration profiling.

Due to the large number of parameters that must be considered for a complete analysis of an RBS or ERD spectrum, there are many commercial programs to develop the simulation and fit of the experimental data, such as SimNRA [109], NDF [110] or RBX [111]. Typically, these programs take into account the kinematic factor, the scattering cross section, the stopping cross section and the 
straggling, but also adds several other theoretical and experimental corrections. A systematic intercomparison of most used software packages can be found in reference [112].

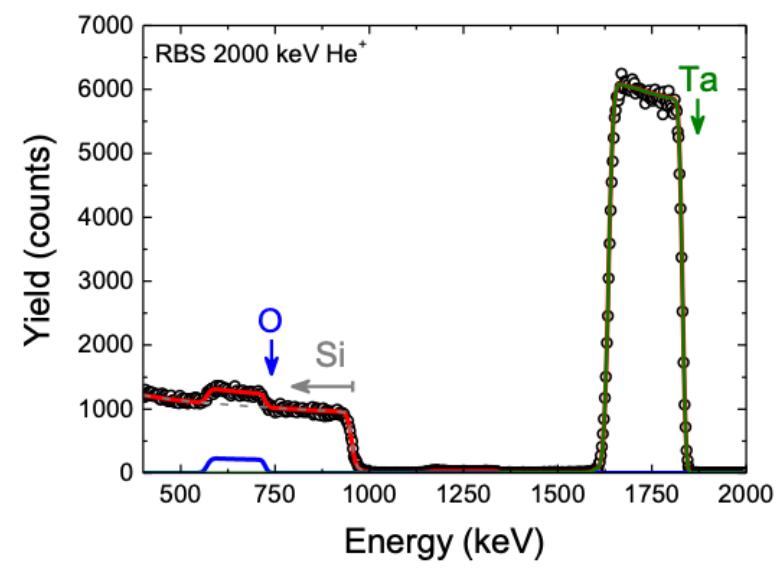

Figure 4.16. Experimental (dots) and simulated (lines) RBS spectrum of a sample composed of a thin layer of $\mathrm{Ta}_{2} \mathrm{O}_{5}$ on a silicon substrate.

Figure 4.16 shows an example of a RBS spectrum for a $\mathrm{Ta}_{2} \mathrm{O}_{5}$ thin layer grown on a silicon substrate. The line represents the simulation, which fits perfectly with the experimental data. The Ta and $\mathrm{O}$ energies marked with an arrow correspond to the surface and, therefore, coincide with the ones obtained through the kinematic factor. Note that the very different yield for Ta versus 0 is not due to the higher concentration but to the ( $\sim 100$ times $)$ higher cross section of Ta. The uniform yield of the elements evidence the homogeneity of the concentration with depth. The thickness of the layer can be estimated due to the energy loss of the ions.

\subsubsection{Techniques based on inelastic nuclear processes: NRA and PIGE}

RBS and ERD are elastic techniques that involve two-body collisions in which the energy and the momentum are conserved. However, $\mathrm{MeV}$ ions can produce nuclear processes that need to be treated in a more complex way. In particular, a nuclear reaction is not a two-body but a 4-particle problem. For instance, the reaction ${ }^{16} \mathrm{O}(\mathrm{d}, \mathrm{p}){ }^{17} \mathrm{O}$ is produced when a deuterium ion $\left({ }^{2} \mathrm{H}\right)$ interacts with a ${ }^{16} \mathrm{O}$ nucleus, producing a proton $\left({ }^{1} \mathrm{H}\right)$ and the isotope ${ }^{17} \mathrm{O}$. In this process both the target and the scattered ion change, so it does not fit in our previous definition of RBS and ERD techniques. On the contrary, this is a new method and we must now consider the conservation of nucleons (A) and charge (Z) too. This method is known as nuclear reaction analysis (NRA), because we analyse the products of the nuclear interaction such as protons, alpha particles, nuclei or gamma-rays (this latter case known as particle induced gamma-ray emission or PIGE). Since nuclear reactions are very specific, this technique is particularly useful for the detection of certain isotopes (typically light elements) with high sensitivity. 

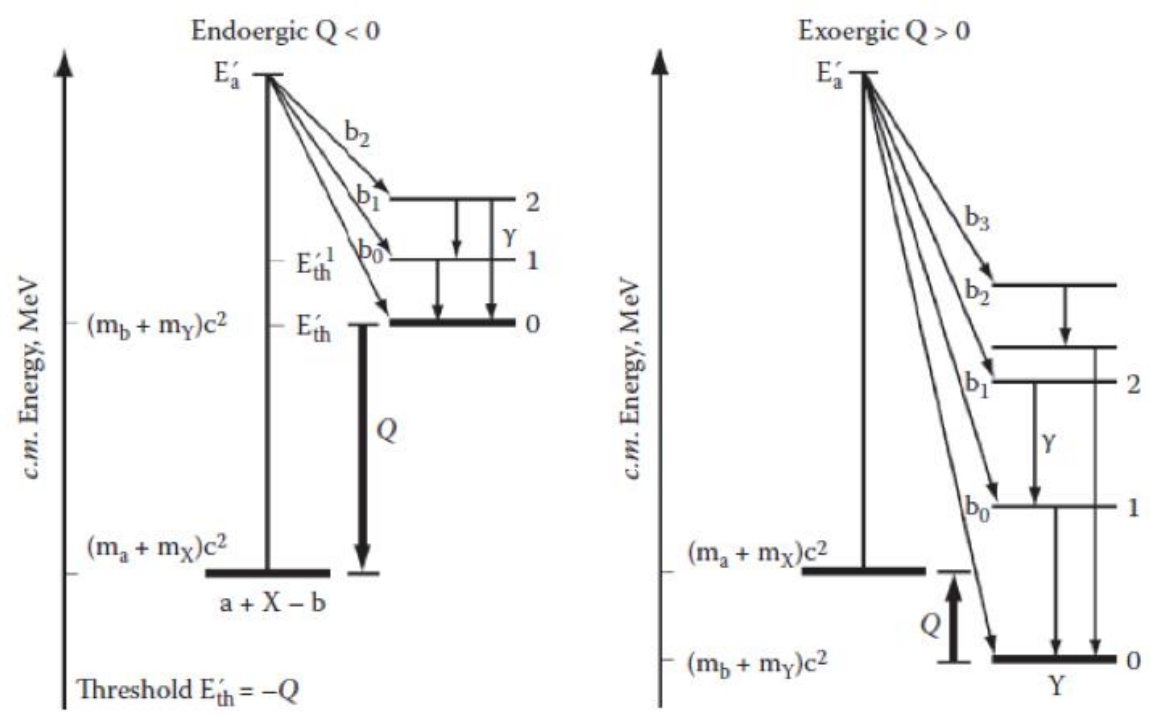

Figure 4.17. Energy diagram of an endoergic and an exoergic nuclear reaction [16]

Nuclear reactions can be generally divided into different classes depending on the energy balance and the value of $\mathrm{Q}=\mathrm{E}$ (products)-E(particles). Figure 4.17 shows two examples of $\mathrm{X}(\mathrm{a}, \mathrm{b}) \mathrm{Y}$ reactions where the final products have lower and higher energy than the initial particles [16]. When $Q \neq 0$ the reaction is inelastic (either endoergic or exoergic), while $\mathrm{Q}=0$ represents a particular case that correspond to an elastic collision. However, there are many possible decay channels and several of them can occur simultaneously, what makes NRA complex.

In IBA, we can highlight three relevant cases [113]:

- $\quad$ Ion-ion $(p, q)$ reactions:

- Elastic $(\mathrm{Q}=0)$. In some cases both the projectile and the target are not modified by the nuclear interaction. This can be due to a Rutherford (only Coulomb force dominates) or non-Rutherford scattering. In the latter, there is formation of a transient quasibound state but the target remains in the ground state. As we mentioned in the previous section, this kind of reaction can interfere with RBS experiments because alters the cross section. An example of an elastic reaction is ${ }^{16} \mathrm{O}(\alpha, \alpha)^{16} \mathrm{O}$.

- Inelastic $(Q \neq 0)$. These reactions can be due to excitation or rearrangement. In the first one there is no change in the particle but the target is left in an excited state. For example, the reaction ${ }^{19} \mathrm{~F}\left(\mathrm{p}, \mathrm{p}^{\prime}\right){ }^{19} \mathrm{~F}^{*}$ with $\mathrm{Q}=-0.011 \mathrm{MeV}$. In the second one, there is a compound reaction where all particles are combined. For example, the reaction ${ }^{14} \mathrm{~N}(\mathrm{~d}, \alpha){ }^{12} \mathrm{C}$ with $\mathrm{Q}=13.575 \mathrm{MeV}$.

- Ion-gamma $(p, \gamma)$ reactions:

- Due to radiative capture, sometimes a small part of the nucleons are combined to form a nucleus in a highly excited state that emits $\gamma$-rays promptly. For example, the reaction ${ }^{27} \mathrm{Al}(\mathrm{p}, \gamma){ }^{28} \mathrm{Si}$ with different released energies.

- Gamma-ray emission can also accompany other ion-ion reactions where an excited state is formed. This is the case of the ${ }^{1} \mathrm{H}\left({ }^{15} \mathrm{~N}, \alpha \gamma\right)^{12} \mathrm{C}$, where gammas at 4.43 
$\mathrm{MeV}$ are emitted for sufficiently high energies of the projectile.

- Ion-neutron $(\mathrm{p}, \mathrm{n})$ reactions:

For example, ${ }^{19} \mathrm{~F}(\mathrm{p}, \mathrm{n}){ }^{19} \mathrm{Ne}$ with $\mathrm{Q}=-4.020 \mathrm{MeV}$. Some of these reactions are used to produce radioactive isotopes and be used in particle induced activation analysis.

The underlying principle of NRA is not very different to that of RBS or ERD. Essentially, the yield of a certain particle (ions, gammas, or neutrons) indicates the concentration of a target element. The main complexity arises from the variety of decay channels and the special set-ups required (involving additional detectors, foils, shielding, etc.). Another important fact to consider is that the cross sections for nuclear reactions need to be measured. Typically, the probability follows a Lorentzian shape determined by three main factors: the resonant energy, the width and the cross section. Fortunately, most of the nuclear cross-sections of interest are relatively well known and accessible through data bases (simulation codes incorporate them), but often reference samples are needed (especially when using PIGE).

One of the remarkable cases where NRA demonstrates its power over RBS or ERD is for $\mathrm{H}$ detection. As mentioned before the ${ }^{1} \mathrm{H}\left({ }^{15} \mathrm{~N}, \alpha \gamma\right){ }^{12} \mathrm{C}$ can be used for that via gamma detection. Note that this is an inverse reaction, i.e., the projectile used is ${ }^{15} \mathrm{~N}$ and not ${ }^{1} \mathrm{H}$ as usually. The resonance for this nuclear reaction is ideal because it is narrow (1.8 keV), it has a high cross section (1650 $\mathrm{mb}$ ) and it is isolated from others (4.43 MeV gammas can be detected without overlapping signals). Thus, depth profiling of $\mathrm{H}$ can be obtained with high resolution (few $\mathrm{nm}$ ) and high sensitivity (some ppm). In order to get the concentration profile, the yield of the gamma rays is determined as a function of the beam energy. Because there is a threshold energy of $E\left({ }^{15} \mathrm{~N}\right)=6385$ $\mathrm{keV}$ for the reaction to happen, different probing energies correspond to different probing depths. Figure 4.18 shows an example of this kind of analysis for two AlGaN/GaN samples grown with different plasma conditions.

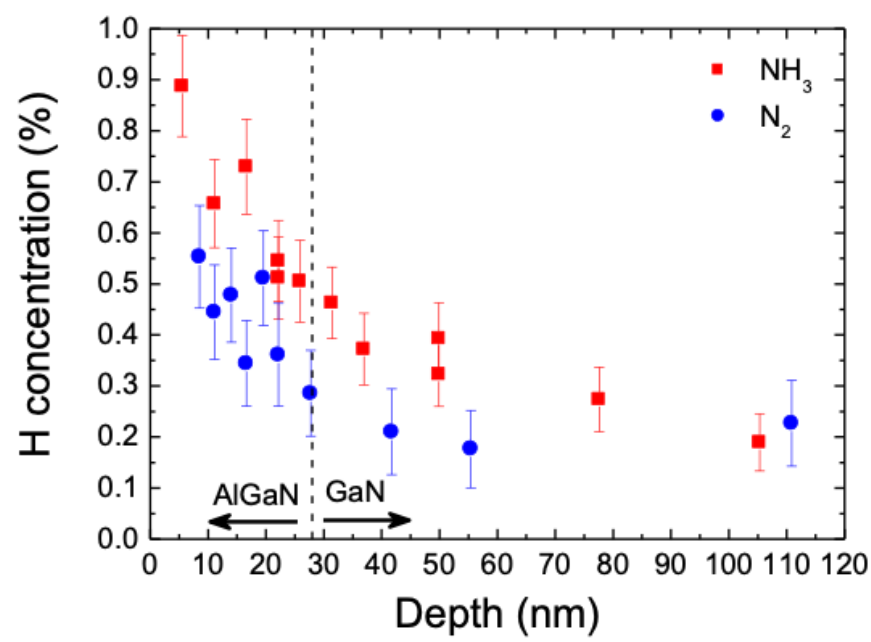

Figure 4.18. Hydrogen depth profile for two AlGaN/GaN samples grown with $\mathrm{NH}_{3}$ and with $\mathrm{N}_{2}$ plasma conditions. The profile was obtained through ${ }^{1} \mathrm{H}\left({ }^{15} \mathrm{~N}, \alpha \gamma\right){ }^{12} \mathrm{C}$ nuclear reaction analysis. The data are consistent with an in-diffusion process from surface [114]. 


\subsubsection{Techniques based on electronic excitation: PIXE}

Since its introduction in the early 1970s [115], Particle Induced X-ray Emission (PIXE) has been one of the most widely applied IBA techniques. When a MeV ion beam impacts on a target, the atomic electrons of the inner shells are ejected resulting vacancies. The atoms are not stable with this configuration and the vacancies are filled by the electrons falling from outer shells. The released energy during this process can be emitted as X-rays. Figure 4.19 shows the schematic of the physical process.

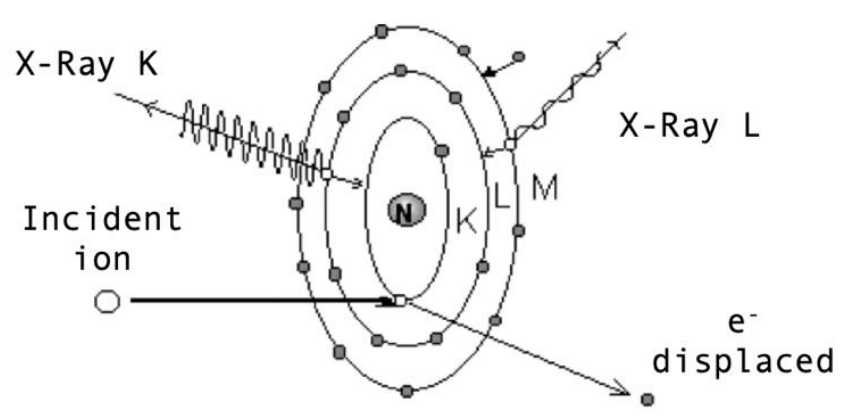

Figure 4.19. Schematic of the PIXE physical process.

In order to determine the yield equation for X-ray peaks, the different physical phenomena which take place must be considered:

- The number of vancancies produced in the layer. This number depends on the probability of ionization of the layer for the particle energy E called the ionization cross-section, $\sigma_{Z}(E)$.

- The transmission factor of the ion beam in the sample matrix and the stopping power. The ion beam energy decreases on its way through the sample according with the density and sample thickness.

- The X-ray detector efficiency and its geometry.

Consequently, the mathematical PIXE equation is:

$$
Y(Z)=\frac{N_{A v} \omega_{Z} b_{Z} \varepsilon_{Z}^{i}(\Omega / 4 \pi)}{A_{Z}} N_{p} C_{Z} \int_{E_{0}}^{E_{r}} \frac{\sigma_{Z}(E) T_{Z}(E)}{S_{M}(E)} d E
$$

where $Y(Z)$ is the yield of the $\mathrm{X}$-ray peak corresponding the element with atomic number $\mathrm{Z}, N_{A v}$ Avogadro's number, $\omega_{Z}$ the fluorescence yield of the peak, $b_{Z}$ the fraccion of the X-rays that appear in the line, $\varepsilon_{Z}^{i}$ the absolute detection efficiency, $\Omega$ the solid angle of X-ray detector, $N_{p}$ the number of incident particles, $C_{z}$ the element concentration, $E_{0}$ the incident energy of the ion beam, $E_{r}$ the final energy of the ion beam, $\sigma_{Z}(E)$ the ionization cross-section for the particle energy $\mathrm{E}$, $T_{Z}(E)$ the transmission of particles from succesive depths in the sample and $S_{M}(E)$ the sample matrix stopping power.

PIXE is a multielemental technique. The energies of the detected X-rays are characteristic of the sample elements. The PIXE spectra consist of the different X-ray peaks on a continuous bremsstrahlung background (Figure 4.20). Measuring the intensities of the characteristic X-ray lines, the elemental concentrations may be determined. 


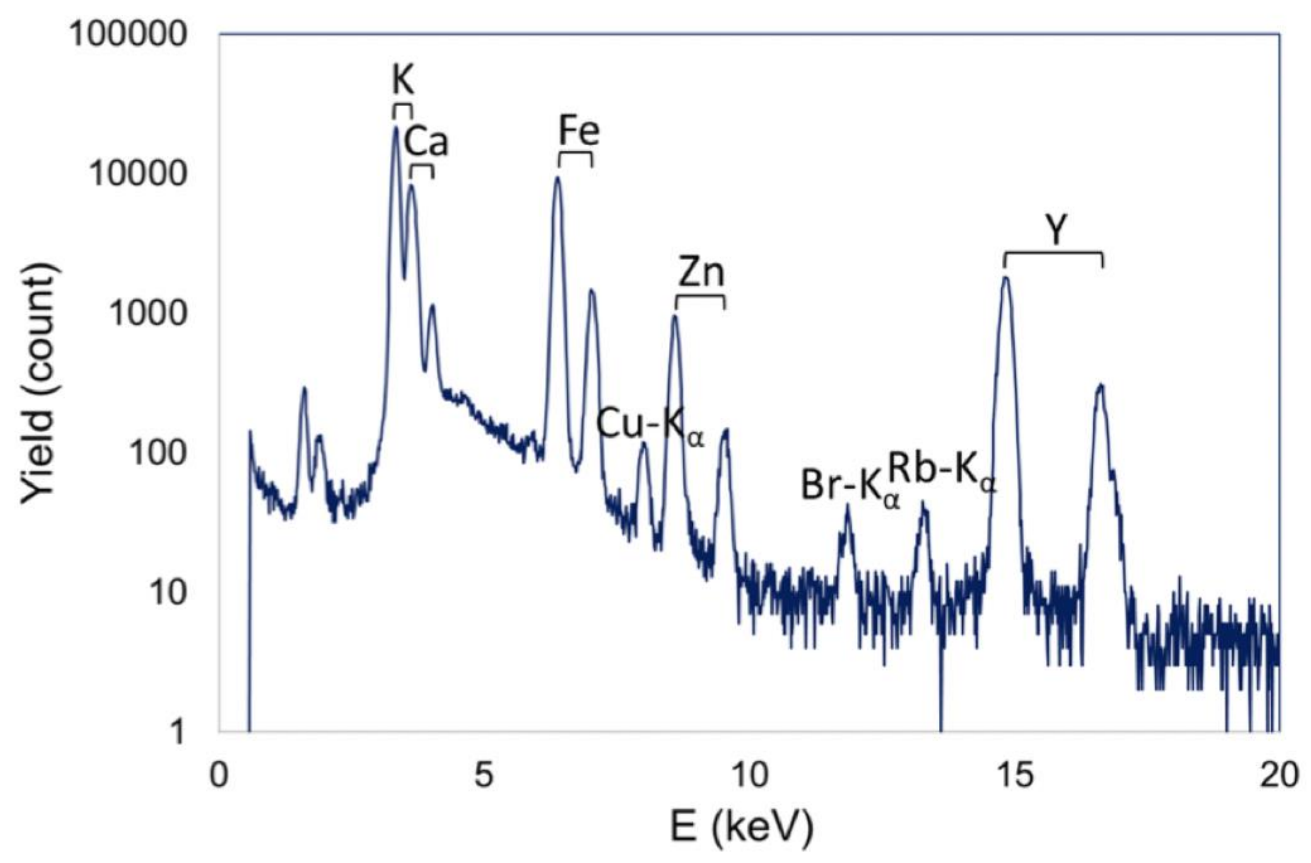

Figure 4.20. PIXE spectrum of an uterus sample treated with yttrium.

PIXE is an excellent analytical technique for the detection and quantification of low elemental concentration starting from sodium onwards. It allows to map trace element concentrations at the ppm level. The process is similar to EDX (Energy-dispersive X-ray spectroscopy) where an electron beam excites the sample atoms, but PIXE uses particles to eject inner shell electrons. This leads to orders of magnitude reduction in the bremsstrahlung background under the characteristic X-ray lines compared to EDX and consequently to a much better elemental sensitivity. In addition, the penetration in the sample of $\mathrm{MeV}$ ions is higher than that of keV electrons, therefore PIXE allows the study of thicker samples than EDX.

PIXE analysis uses semiconductor X-ray radiation detectors. During years Si(Li) and LEGe (Low Energy Germanium) detectors [116] cooled by liquid nitrogen and with conventional acquisition electronics have been most employed although, in the last years, the use of detectors cooled by the Peltier effect is increasing.

The elemental concentrations from PIXE spectra are generally determined with computer fitting programs [117]. GUPIX code [118] [119] is the most widely used software for PIXE analysis although there are others such as GEOPIXE [120], DATTPIXE [121] and PIXAN [122].

\subsubsection{Ion channeling}

So far, we introduced IBA techniques without taking into account the nature of the sample under analysis. We implicitly assumed that the methods will lead to the same results independently of the structure of the material, i.e., that the ion-solid interaction is the same in all the cases. However, there is one specific case in which this is not true: crystalline targets.

Crystals are solids that exhibit a uniform geometric periodicity due to a highly ordered microscopic structure. As a consequence, their properties are substantially different when compared to those of amorphous or polycrystalline materials. In particular, because crystals are 
formed by lattices, their atomic density will strongly depend on the orientation of the sample, which alters the stopping force and the scattering cross section. More importantly, for certain orientations the atoms of the crystals are perfectly aligned in arrays, providing empty "channels" to travel through for particles or radiation. This effect was already proposed in 1912 by Johannes Stark [123], but it was not confirmed until 1963, both experimentally and computationally [124] [125].

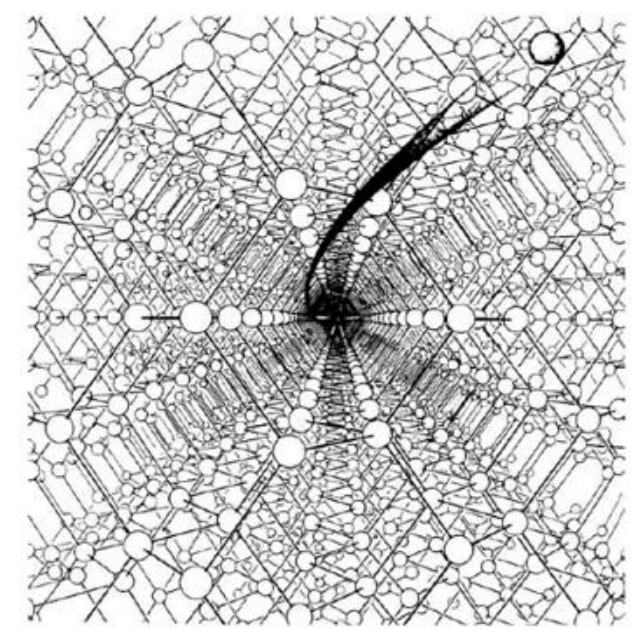

Figure 4.21. Artistic view of ion channelling. From [126]

We can define ion channeling as the directional effect that takes place when a charged particle beam is aligned with a major symmetry direction of a crystal [127]. Figure 4.21 illustrates this effect, which can be divided into two classes: axial channeling (when the direction of the beam is parallel to an axis) and planar channeling (the direction is parallel to a plane). The main manifestation of channeling in the experimental spectra is an intense reduction of the scattering events. This reduction in the yield is a consequence of the correlated series of small-angle screened Coulomb collisions between the ions and the lattice atoms. Therefore, the close encounter probability decreases and ions can travel large distances inside the material.

For most depth profiling applications, channeling is an inconvenience because the composition of the sample cannot be properly determined (we cannot assume ordinary cross sections and stopping forces). However, channeling is a very powerful method to determine any lattice perturbation in crystals such as extended defects (interstitials, dislocations, stacking faults, etc.), strain, impurities, phase separation or implantation damage. Hence, channeling is a widely used technique for crystallography, most often in combination with RBS (RBS/C), although it can be also used for PIXE or NRA. RBS in channeling mode is indeed an excellent complement to other analytical methods such as X-ray diffraction or transmission electron microscopy, because it provides direct information on defects with depth-resolution and mass sensitivity. Comprehensive reviews of ion channeling available in references [128] [129] [130] [131].

A simple example of the channeling effect in the RBS spectra is shown in Figure 4.22 for a GaN crystal. When the crystal is aligned with a major axis (a) the backscattering yield is strongly reduced, evidencing a surface peak at the surface (the surface is essentially a type of defect, and ions cannot be channeled in it). When the crystal is aligned with a plane (b) the yield is reduced but less than in axial channeling. In addition, the spectrum shows characteristic oscillations due to the harmonic movement of the ions in the channel. Finally, when the crystal is in a random 
orientation we recover the conventional RBS spectrum, i.e., the spectrum equivalent to the one obtained for an amorphous target. Nevertheless, it is important to keep in mind that getting a proper random spectrum can be a challenge and, most of the times, requires rotating the sample during the measurement.
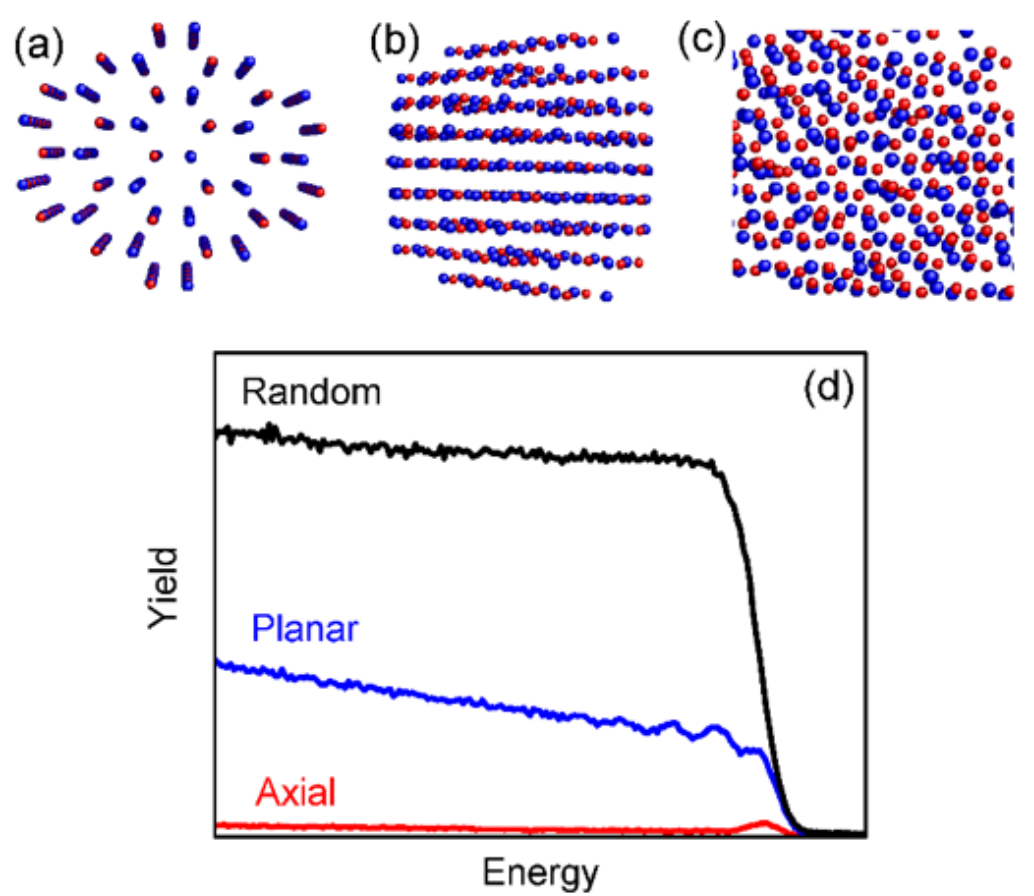

Figure 4.22. GaN crystal aligned in three different geometries for: (a) axial channeling, (b) planar channeling, and (c) random orientation. (d) RBS spectra for the three geometries. It can be seen that the minimum yield is obtained for axial channeling.

Channeling can be explained in terms of a correlated series of elastic two-body collisions, but the classical theory developed by Jens Lindhard assumes, instead, a continuum collision model [127]. This means that the ion trajectory can be described using the force of a continuous string, and not calculated from individual atomic interactions. Thus, the effect of the lattice can be incorporated in an average transverse string potential. The hypotheses for Lindhard's model are: (a) the scattering angles are small, (b) the successive collisions are strongly correlated, (c) the collisions are elastic encounters between two bodies and (d) the crystal can be approximated by perfect strings of atoms with a uniform spacing. The choice of this potential requires a compromise between accuracy and simplicity, and nowadays the universal Ziegler-Biersack-Littmark (ZBL) repulsive potential is the most extended one [132]. Lindhard's theory predicts the existence of two essential parameters controlling the oscillatory movement of the ions inside the channels: the critical angle and the minimum yield.

Channeling is not only a geometrical phenomenon but mainly a steering phenomenon, because the potential of the atomic strings forces the ions to remain channeled. Hence, there is a certain tolerance for the incidence angle that allows the channeling of the particles. The critical angle for channeling $\left(\psi_{1}\right)$ is defined as the maximum value to get steering of the ions inside the channels of the crystal. This angle can be calculated as the maximum angle that allows the application of the Lindhard's continuum model, and its value is 


$$
\psi_{1}=\sqrt{\frac{2 Z_{1} Z_{2} e^{2}}{4 \pi \varepsilon_{0} E d}}
$$

where $Z_{1}$ is the atomic number of the ion, $Z_{2}$ is the atomic number of the target, $e$ is the charge of the electron, $\varepsilon_{0}$ is the vacuum permittivity, $E$ is the kinetic energy of the ion, and $d$ is the spacing between atoms along the string direction. For energies of few $\mathrm{MeV}$, the critical angle is typically in the range of $1^{\circ}$.

In practical terms, the effect of the critical angle can be measured performing an angular scan along a certain crystallographic direction. For angles below $\psi_{1}$ the effect is very important and the scattering events decrease significantly, while for higher angles the yield recovers the expected level for random conditions. However, it has been demonstrated that the experimental half-angle $\psi_{1 / 2}$ does not correspond exactly to the value $\psi_{1}$, but it is proportional to it. This is due to the fact that atoms in the crystal are not fixed at the lattice positions, but affected by thermal vibrations. The vibrational amplitude of these atoms modifies the effective potential and, therefore, the final value of the critical angle. This effect can be well-described with Monte Carlo simulations as shown in Figure 4.23 for the particular case of a GaN crystal [133].

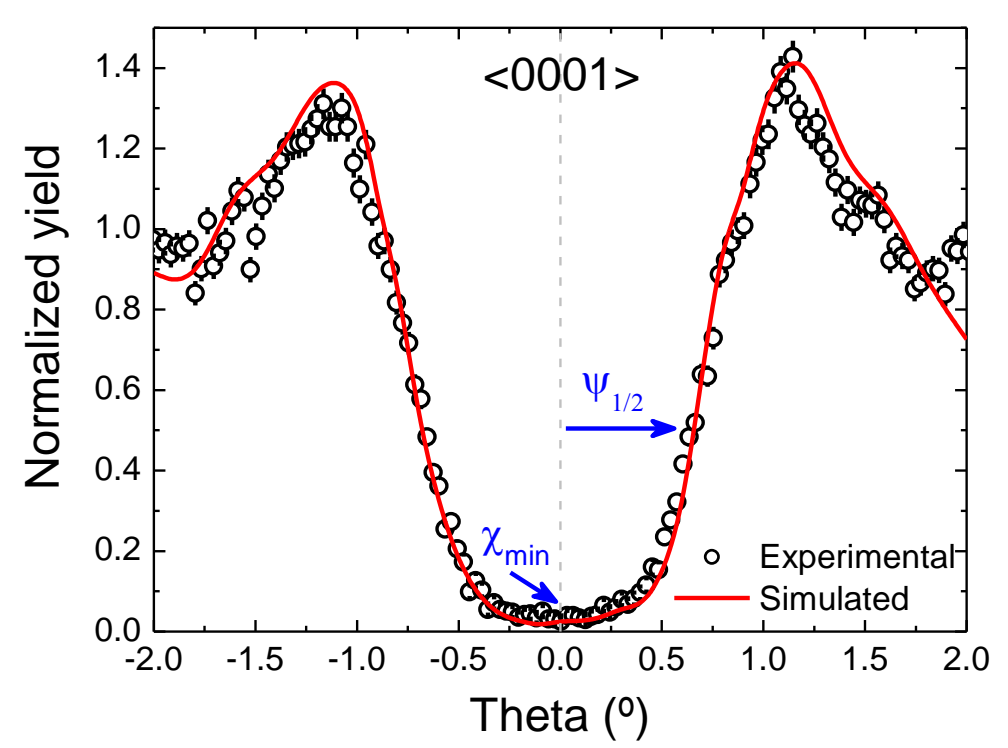

Figure 4.23. Angular scan (dip) of the $<0001>$ axis for a GaN crystal obtained with a $2 \mathrm{MeV} \mathrm{He}^{+}$beam. Half-angle is $\psi_{1 / 2}=0.66^{\circ}$ and minimum yield is $\chi_{\min }=2.5 \%$. Solid line is the Monte Carlo simulation, which shows an excellent agreement with the experiment. Adapted from [133]

The other important parameter obtained from ion channeling is the minimum yield $\left(\chi_{\min }\right)$. This value is the fraction of the channel area which is forbidden by the vibrating strings of atoms and can be theoretically calculated as:

$$
\chi_{\min }=\pi N d u_{2}^{2}
$$

where $N$ is the atomic density, $d$ is the interatomic spacing, and $u_{2}$ is the thermal vibrational amplitude of the atoms. Interestingly, $\chi_{\min }$ is independent of beam parameters and it is determined solely by the properties of the crystal. Indeed, it is a good indicator of the crystal quality. 
Experimentally, the minimum yield is obtained from the ratio of the aligned and random yields. For perfect crystals, this value is typically below 4\%. An example for GaN is shown in Figure 4.22.

Evidently, the ion flux inside the channel varies with depth and several scattering processes such as defects, electrons and thermally displaced atoms affect it. Those processes are known as dechanneling. Despite the modelling of dechanneling is complex, it also provides useful information about the defect density in crystals with proper simulation programs.

From the experimental point of view, ion channeling experiments require some specific elements in the set-up. Perhaps the most critical points are the need of a well-collimated ion beam (in the order of $0.05^{\circ}$ divergence) and a precise motor-controlled goniometer (typically a 3-axis system with $0.01^{\circ}$ resolution). In addition, channeling demands an accurate measurement of the dose to compare random and aligned yields. Detectors play also an important role. The solid angle should be large enough to avoid possible double-alignments (beam entering and exiting channeled). Interestingly, in the last years position-sensitive detectors have become widely available, which have improved the accuracy and speed of the alignment. Finally, an important limitation of channeling is the requirement of monocrystalline samples with a well-defined surface plane.

To illustrate a practical example of ion channeling in combination with RBS,

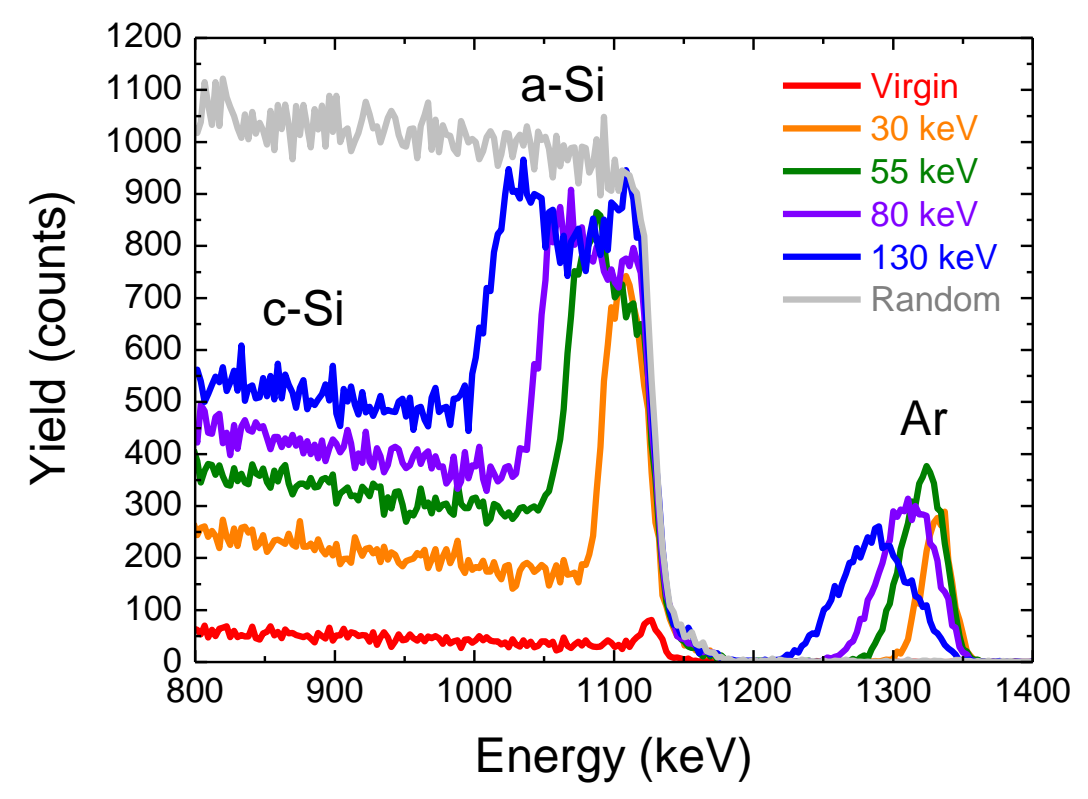

Figure 4.24 shows the spectra of Ar-implanted silicon samples at different energies: from 30 to 130 $\mathrm{keV}$. For comparison, a virgin sample is also shown in the figure. Since ion implantation damages the original crystal structure of $\mathrm{Si}$, the backscattering yield of those damaged or amorphous layers (a-Si) is highlighted by the channeling effect. Two features can be clearly distinguished. First, the thickness of the damaged layers can be precisely determined due to the channeling effect in the crystalline substrate (c-Si). This thickness corresponds well with the implantation energy. Second, the damage of the layer can be determined due to the direct comparison with the random spectrum. The increasing yield of the damage with the energy evidences the higher damage of the layers. 


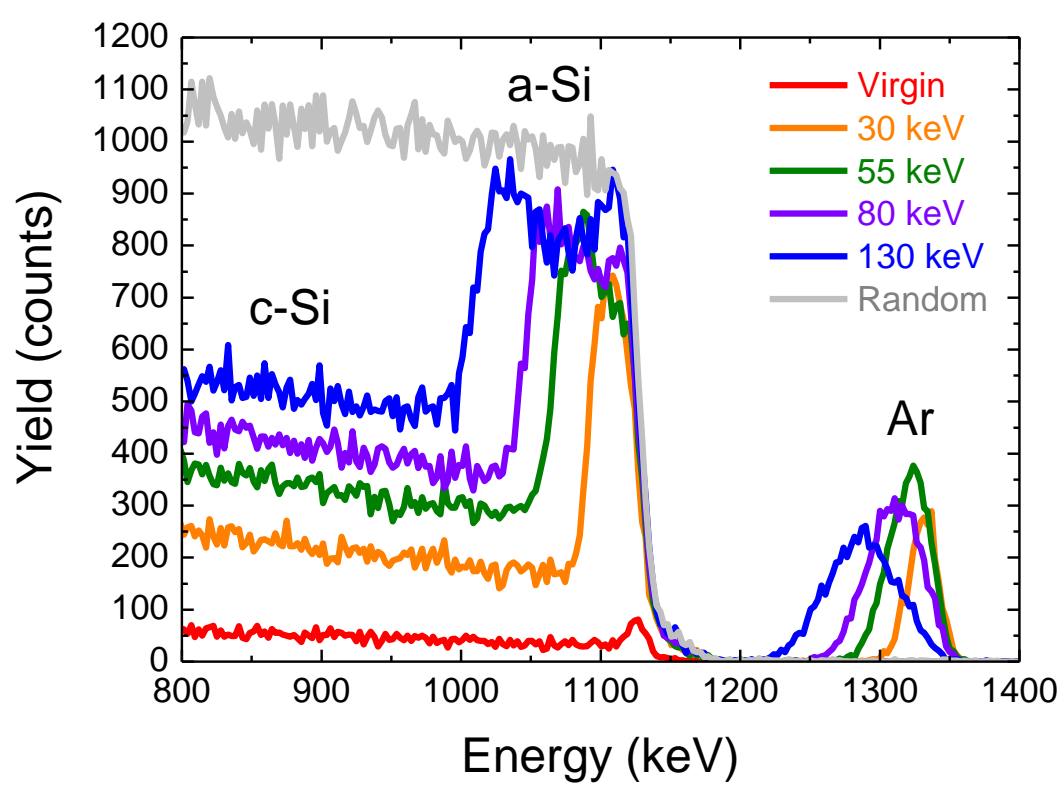

Figure 4.24. RBS/C spectra of Ar-implanted silicon samples with different energies. Thanks to the channeling effect in the substrate, the damage of the amorphous layers is highlighted in the spectra. The experiment was performed with 2 $\mathrm{MeV} \mathrm{He}+$ ions.

\subsubsection{Microbeams}

The ion beam versatility significantly increases for both material modification and analysis when micrometric or sub-micrometric beam sizes are achieved. In order to successfully reach these small sizes, beam collimation and focusing are required. During these processes an ion current loss arises which is conditioned by the quality of the beam produced by the ion source and the accelerator and the efficiency of the focusing systems.

This section provides a short and simple summary of the instrumentation required to produce and to work with micrometric or submicrometric $\mathrm{MeV}$ ion beams. A more detailed overview may be found in [134] [135].

\subsubsection{Collimation}

The beam collimation is a two-stage process and it is the previous step to the precise beam focusing.

The collimation systems may be made up of a set of diaphragms with different hole sizes or a set of micrometric slits. The slit systems may be comprised by four independent slits or a vertical slit and a horizontal slit and the movement of the slits can be manual or motorized. Both the slits and the diaphragms must meet a set of requirements:

- They must be manufactured with a resistant material to the impact of the high ion beam currents.

- They must be good heat dissipators to avoid the expansion during the experiments.

- The construction material must be chosen in order to minimize radiation levels.

- The edge roughness must be extremely small in order to minimize the scattering and the straggling of beam. 
The first collimator is the object collimator which determines the beam size. The aperture dimensions of this collimator are conditioned by the demagnification of the quadrupole lenses to focus the beam and the final beam size required. The collimated beam consists of a central core and a halo of scattered particles with variable energies mainly generated by this first collimation process. The aperture collimator is typically positioned a few meters downstream the object collimator. This collimator reduces the beam dispersion and consequently the beam aberrations. To achieve this, the aperture collimator must be more open than the object collimator avoiding new scattered particles and removing those produced by the object collimator. Figure 4.25 shows a general view of the CMAM internal microbeam line where object and collimator slits can be seen.

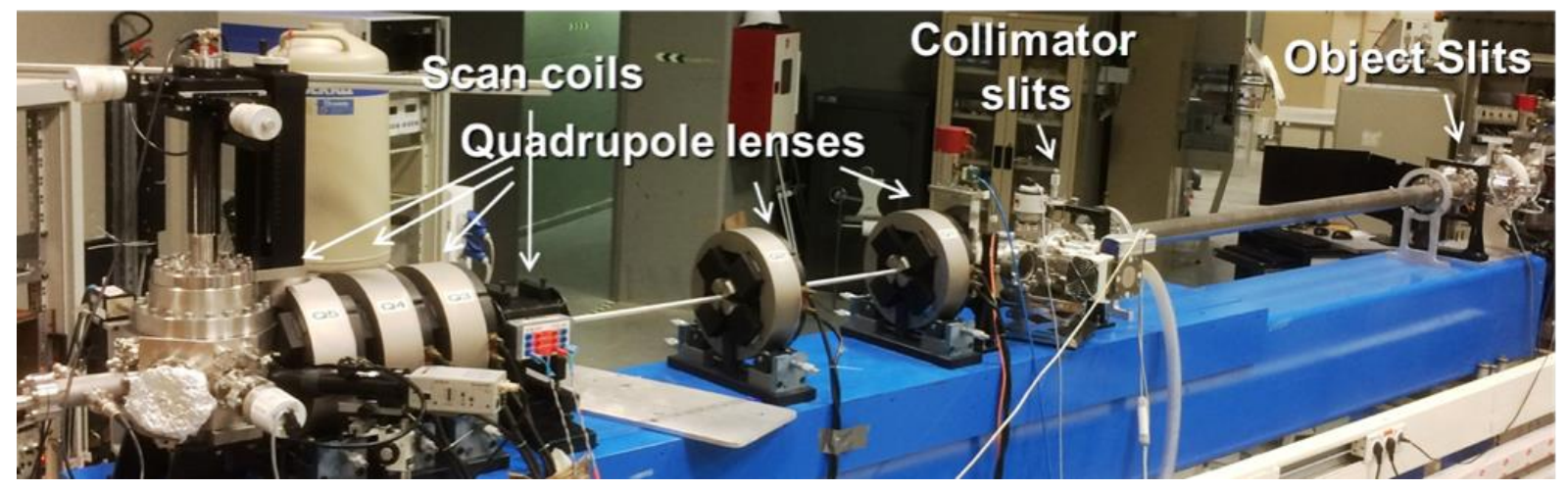

Figure 4.25. General view of the internal microbeam line at CMAM. The different focusing, steering and collimating elements of the line are labelled.

\subsubsection{Quadrupole lenses}

The experiments with micrometric ion beams have often followed the example of the works with electrons, however the lenses used to focus electron beams cannot be used for ions because ion mass and energy are higher and consequently the magnetic field necessary to focus ions is much larger than to focus electrons. The best systems to focus ions are the quadrupole lenses used for the ion beam transport in the ion accelerators, previously described.

The microbeam focus systems are diverse. The lenses can be electrostatic or magnetic (Figure 4.25 shows magnetic quadrupole lenses at CMAM internal microbeam line). The number of lenses of these systems is usually between two and six, because the punctual focusing with one lens is not possible and with a high number it is significantly difficult to work due to the increased optical aberrations. The different quadrupole configurations are characterized by a demagnification and a maximum magnetic rigidity. A high demagnification leads to small beam sizes while the maximum magnetic rigidity restricts the ion beam that can be focused.

In order to reach an optimum focalization, the optical aberrations, chromatic and spherical aberrations, have to be minimized. The chromatic aberrations are typically exhibited when the beam ions have different energies, therefore these aberrations are minimized when the beam halo is removed with a correct collimation. The spherical aberrations appear as the result of the ions not travelling parallel to the quadrupole lens axis, so in order to avoid this aberration the alignment between the ion beam and the lenses has to be as perfect as possible. The tables supporting quadrupole lenses are designed with micrometric screws to allow the precise vertical, horizontal and rotational movements of each lens making it easier to align.

The precision of the power supplies of the lenses has to be extremely high to achieve small beam size and variations of the focal point must be avoided. Therefore, the power supplies have to be 
exceptionally stable. Frequently, the beam size variations are due to thermal changes consequence to the high currents used. This problem is resolved switching on the lenses in advance until the heat stability is reached, or with a good cooling system for the lenses.

\subsubsection{Scanning systems}

In order to get the most out of all focused ion beam capabilities, its impact point on the sample must vary allowing the distribution maps for the analysis experiments or the patterning implantation for modification studies. There are two alternative options: the sweeping of the ion beam with magnetic or electrostatic deflector plates, or the sample movement with motorized systems. While the former requires simpler sample stages and the reaction time is significantly shorter, its deflection amplitude is limited. Motorized systems allow the scanning of large regions preserving the beam focus. However sometimes the degree of accuracy determined by the beam size is difficult to reach. In some set-ups, both systems are combined to carry out millimetric scans in conjunction with precise submicrometric small patterns.

\subsubsection{Analysis chamber}

The analysis chambers of microbeam set-ups, together with the sample stage, basic detectors and charge acquisition systems, have an optical microscope with the necessary magnification to visualize in detail the samples.

The chamber pressure must be as low as possible to avoid the beam degradation. A pressure of about $10^{-7}$ Torr is considered good. Another major consideration is the chamber vibrations. Any vibration has to be avoided in order to minimize the beam focus variation.

\subsubsection{Acquisition system}

The information recorded and processed by microbeam acquisition systems is very ample, therefore these systems are relatively complex. The microbeam acquisition systems collect the detector voltage pulses in conjunction with the scanning voltages from the deflector systems. The perfect synchronization between the different signals is mandatory to be able to unequivocally assign each detector signal to the corresponding beam coordinates.

Over these years, several hardware and systems have been developed for this purpose. Oxford Microbeams OMDAQ system [136] is widely used by microbeam scientific community and some laboratories with microprobe lines built in the Micro-Analytical Research Centre (MARC, Melbourne) use the software package MPSYS [137]. However, the acquisition systems need to adapt to increasingly complex experimental configurations and some groups have developed their own systems to help the users to process their mapping data acquired on the microbeam lines [138] [139] [140] [141]. Although all have their own particularities, all microprobe acquisition systems provide the possibility to store list-mode data to obtain a full power of the collected data and their operator interfaces allow the display screen of different energy spectra and distribution maps during their acquisitions. 


\subsubsection{External beam}

The size, fragility or delicacy of certain samples, for example, biological or archaeological materials, prevent their analysis and/or irradiation in vacuum chambers. The alternative is the use of an external beam set-up where the beam is extracted to air through an exit window. Figure 4.26 shows the exit window of CMAM external beam line.

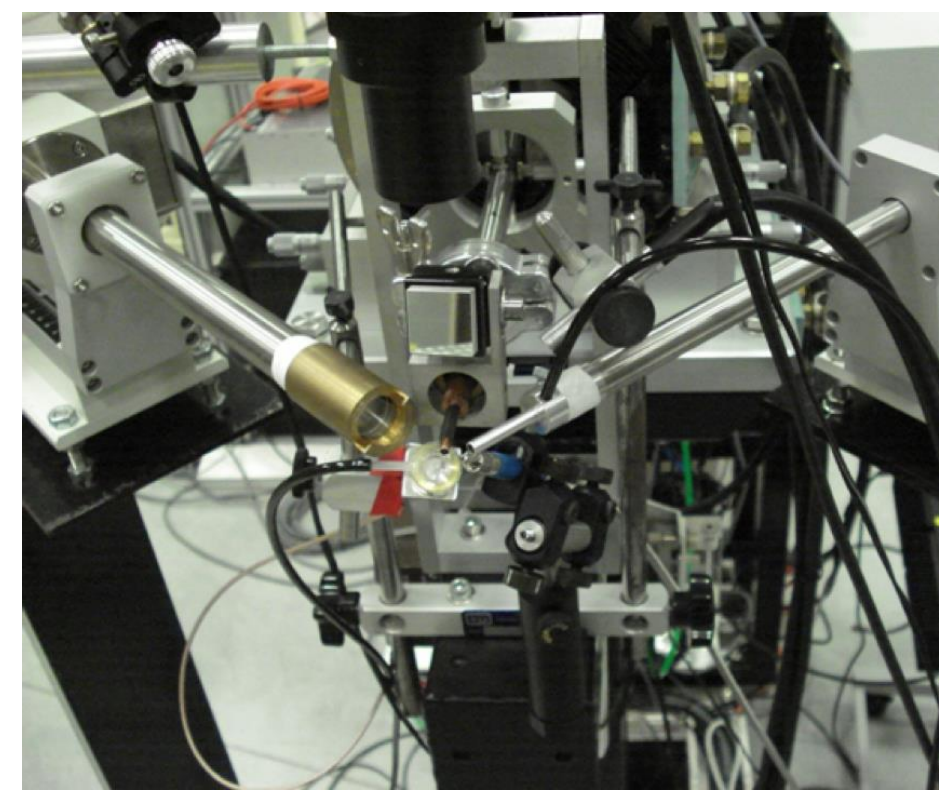

Figure 4.26. Exit window of the external microbeam line at CMAM. At the sides, the PIXE and PIGE detectors pointing at the beam-sample interaction point. The exit window may be seen, attached to the nozzle through which the beam is extracted from the vacuum line to the outside.

The composition and the thickness of the exit window determine the energy loss and dispersion of the beam. Typically, working with microbeams, $100 \mathrm{~nm}$ or $200 \mathrm{~nm}$ silicon nitride windows are used to minimize the ion scattering. For defocused ion beams with ion currents of few nanoamperes more resistant windows are needed and generally Kapton, Mylar or Al foil with a thickness of few micrometres are used.

The sample is usually placed few millimetres from the exit window. To reduce the absorption and dispersion effect of air, helium can be blown between the sample and the exit window. The region under irradiation is visualized with a CCD video microscope. When the distance between the exit window and the sample is fixed, the impact right points are typically determined on the sample with the help of a low-power laser.

The measurement of the ion charge delivered by an external beam on the target is not easy. If the beam passes through the sample, this charge can be measured with a faraday cup positioned just behind the sample. Whereas for thick samples, the charge can be determined from the signal given by the exit window due to the beam impact. Despite this handicap, the work with an external beam offers several advantages, for example, the easy and quick positioning of the sample, the fact that heat dissipation in air avoids the damage of the sample, no electrical charging of insulating material and for valuable objects sampling is not required. 


\section{Acknowledgements}

We are grateful to the support provided by the CMAM technical staff. C. García Iturriaga is acknowledged for Figure 4.1.

J. Olivares acknowledges the projects TechnofusionIII (S2018/EMT-4437) from Comunidad de Madrid and PID2019-105156GB-I00 from Ministerio de Ciencia e Innovación.

\section{References}

[1] K. S. Krane, Introductory Nuclear Physics, John Wiley \& Sons, 1988.

[2] R. Middleton, "Negative ion cookbook," University of Pennsylvania, unpublished, 1989. [Online] Available: pelletron.com/cookbook.pdf.

[3] R. O. Sayer, Rev. Phys. Appl., vol. 12, p. 1543, 1977.

[4] A. Redondo-Cubero, M. J. G. Borge, N. Gordillo, P. C. Gutiérrez, J. Olivares, R. Pérez-Casero and M. D. Ynsa, The European Physical Journal Plus, pp. 136-175, 2021.

[5] [Online]. Available: www.ionbeamcenters.eu.

[6] [Online]. Available: http://www-naweb.iaea.org.

[7] M. A. Ramos and J. Gómez-Camacho, "Focus point on Small and Medium Particle Accelerator Facilities in Europe," European Physical Journal Plus.

[8] H. Wollnik, Optics of charged particles, Elesevier, 2021.

[9] T. Aldford, L. Feldman and J. Mayer, Fundamentals of nanoscale films analysis, New York: Springer Science, 2007.

[10] J. Bird and J. Williams, Ion Beams for Materials Analysis, Australia: Academic Press Australia, 1989.

[11] H. Bethe, "Zur theorie des durchgangs schneller korpuskularstrahlen durch materie," Annalen der Physik, no. 397.3, pp. 325-400, 1930.

[12] F. Bloch, "Zur bremsung rasch bewegter teilchen beim durchgang durch materie.," Annalen der Physik, no. 408.3, pp. 285-320, 1933.

[13] F. Bloch, "Bremsvermögen von Atomen mit mehreren Elektronen.," Zeitschrift für Physik, vol. 81, no. 5, pp. 363-376, 1933.

[14] H. Betz, "in Condensed Matter," Academic Press, Orlando, 1983.

[15] F. Ziegler, J. Biersack and M. Ziegler, "SRIM - The Stopping and Range of Ions in matter," Nuclear instruments and Methods B, vol. 268, pp. 1818-1123, 2010.

[16] M. Nastasi, J. W. Mayer and Y. Wang, Ion beam analysis: fundamentals and applications, London: CRC Press, 2015.

[17] J. Ziegler, Handbook of ion implantation and beam processing, Orlando: Academic, 1984.

[18] J. Williams and J. Poate, Ion implantation and beam processing, Orlando: Academic, 1984.

[19] P. Townsend, P. Chandler and L. Zhang, Optical effects of ion Implantation, Cambridge: Cambridge University Press, 1994. 
[20] G. Destefanis, P. Townsend and J. Gailliard, "Optical waveguides in LiNb03 formed by ion implantation of helium,", Appl. Phys. Lett., vol. 32, no. 293, 1978.

[21] F. Chen, X. -L. Wang and K. -M. Wang, "Development of ion-implanted optical waveguides in optical materials: A review," Opt. Mater., vol. 29, pp. 1523-1542, 2007.

[22] H. Hu, F. Lu, F. Chen, F. -X. Wang, J. -H. Zhang, X. -D. Liu, K. -M. Wang and B. -R. Shi, "Optical waveguide formation by MeV H+ implanted into LiNbO3 crystal," Optics Commun., vol. 177, pp. 189-193, 2000.

[23] G. Bentini, M. Bianconi, M. Chiarini, L. Correra, C. Sada, P. Mazzoldi, N. Argiolas, M. Bazzan and R. Guzzi, "Effect of low dose high energy 03+ implantation on refractive index and linear electro-optic properties in X-cut LiNb03:Planar optical waveguide formation and characterization," J. Appl. Phys, vol. 92 , no. $6477,2002$.

[24] G. Bentini, M. Bianconi, L. Correra, M. Chiarini, P. Mazzoldi, C. Sada, N. Argiolas, M. Bazzan and R. Guzzi, "Damage effects produced in the near-surface region of $\mathrm{x}$-cut LiNbO3 by low dose, high energy implantation of nitrogen, oxygen, and fluorine ions," J. Appl. Phys, vol. 96, no. 242, 2004.

[25] M. Bianconi, N. Argiolas, M. Bazzan, G. G. Bentini, M. Chiarini, A. Cerutti, P. Mazzoldi, G. Pennestri and C. Sada, "Nuclear and electronic energy loss synergy in the process of damage growth in ion implanted LiNbO3," Nucl. Instr. Meth. B, vol. 249, pp. 122-125, 2006.

[26] G. Bentini, M. Bianconi, A. Cerutti, M. Chiarini, G. Pennestri, C. Sada, N. Argiolas, M. Bazzan, P. Mazzoldi and R. Guzzi, "Structural and compositional characterization of X-cut LiNbO3 crystals implanted with high energy oxygen and carbon ions," Nucl. Instr. Meth. B, vol. 240, pp. 174-177, 2005.

[27] H. Hu, F. Lu, F. Chen, B. -R. Shi, K. -M. Wang and D. -Y. Shen, "Monomode optical waveguide in lithium niobate formed by MeV Si+ ion implantation," J. Appl. Phys., vol. 89, no. 5224, 2001.

[28] G. Fu, K. M. Wang, F. Chen, X. L. Wang, S. L. Li, D. Y. Shen, H. J. Ma and R. Nie, "Extraordinary refractiveindex increase in MeV B3+ ion-implanted LiNbO3 waveguide," Nucl. Instr. and Meth. B, vol. 211, pp. 346-350, 2003.

[29] F. Lu, T. Zhang, X. Wang, S. Li, K. Wang, D. Shen and H. Ma, "Formation of waveguides by implantation of 3.0 MeV Ni2+," J. Appl. Phys., vol. 96, no. 3463, 2004.

[30] S. Li, K. M. Wang, F. Chen, X. L. Wang, G. Fu, D. Y. Shen, H. J. Nie and R. Ma, "Monomode optical waveguide excited at $1540 \mathrm{~nm}$ in LiNbO3 formed by MeV carbon ion implantation at low doses," Opt. Express, vol. 12, pp. 747-752, 2004.

[31] F. Chen, H. Hu, J. H. Zhang, F. Lu, B. R. Shi, K. M. Wang, D. Y. Wang and C. Q. Shen, "Optical waveguide in X-cut LiNbO3 crystals by MeV P+ ion implantation with low dose," Phys. Stat. Sol. A, vol. 187, pp. 543-548, 2001.

[32] F. Chen, "Photonic guiding structures in lithium niobate crystals produced by energetic ion beams," J. Appl. Phys. 106, p. 081101, 2009.

[33] S. Wemple, M. DiDomenico and I. Camlibel, "Relationship between linear and quadratic electro-optic coefficients in LiNb03, LiTa03, and other oxygen-octahedra ferroelectrics based on direct measurement of spontaneous polarization," Appl. Phys. Lett., vol. 12, no. 209, 1968.

[34] W. Elcock and E. W. Young, Proc. Phys. Soc., vol. 89, no. 735, 1966.

[35] E. Barnes and R. S. Silk, Philos. Mag. A J. Theor. Exp. Appl. Phys., vol. 4, no. 970, 1959.

[36] P. Henderson, Mineral. Mag, vol. 42, no. 306, 1978.

[37] G. Haute and W. P. den, Fission-Track Dating, Dordrecht: Springer Netherlands, 1992.

[38] A. Audouard, E. Balanzat, S. Bouffard, J. C. Jousset, A. Chamberod, A. Dunlop, D. Lesueur, G. Fuchs, R. Spohr, J. Vetter and L. Thomé, Phys. Rev. Lett., vol. 65, no. 875, 1990.

[39] M. Toulemonde, C. Trautmann, E. Balanzat, K. Horjt and A. Weidinger, "Track formation and 
fabrication of nanostructures with MeV-ion beams," Nucl. Instr. Meth. B, vol. 216, pp. 1-8, 2004.

[40] C. Trautmann, Ion Beams Nanosci. Technol, Berlin, heidelberg: Springer Berlin Heidelberg, 2010.

[41] P. Apel, Nucl. Instruments Methods Phys. Res. Sect. B Beam Interact. with Mater. Atoms, vol. 208, no. $11,2003$.

[42] W. Li, M. Lang, A. J. W. Gleadow, M. V. Ewing and R. C. Zdorovets, Earth Planet. Sci. Lett, vol. 121, 2012.

[43] D. Schauries, B. Afra, T. Bierschenk, M. Lang, M. D. Rodriguez, C. Trautmann, W. Li, R. C. Kluth and P. Ewing, Nucl. Instruments Methods Phys. Res. Sect. B Beam Interact. with Mater. Atoms, vol. 326, no. $117,2014$.

[44] Z. Wang, C. Dufour, E. Paumier and M. Toulemonde, "The Se sensitivity of metals under swift-heavyion irradiation: a transient thermal process," J. Phys.: Condens. Matter, vol. 6, pp. 6733-6750, 1994.

[45] P. Kluth, C. Schnohr, O. Pakarinen, F. Djurabekova, D. Sprouster, R. Giulian, M. C. Ridgway, A. Byrne, C. Trautmann, D. Cookson, K. Toulemonde and M. Nordlund, Phys. Rev. Lett, vol. 101, no. 175503, 2008.

[46] J. Zhang, M. Toulemonde, M. Lang, J. Costantini, S. della-negra and R. Ewing., J. Mater. Res., vol. 30, no. 2456, 2015.

[47] W. Cureton, R. I. Palomares, J. Walters, C. L. Tracy, C.-H. Chen, R. C. Ewing, G. Baldinozzi, J. Lian, C. Trautmann and M. Lang, Acta Mater., vol. 160, no. 47, 2018.

[48] M. Lang, F. Djurabekova, M. N., M. Toulemonde and C. Trautmann, Fundamental Phenomena and Applications of Swift Heavy Ion Irradiations, Oxford, UK: Elsevier, 2020.

[49] P. Rajasekaran, C. Zhou, M. Dasari, K. O. Voss, C. Trautmann and P. Kohli, Sci. Adv., vol. 3, no. e1602071, 2017.

[50] A. Evans, D. Alexandrescu, V. Ferlet-Cavrois and K. Voss, IEEE Int. Reliab. Phys. Symp., no. p.SE.6.1.SE.6.6, 2015.

[51] B. Fischer, K.-O. Voss and G. Du, Nucl. Instruments Methods Phys. Res. Sect. B Beam Interact. with Mater. Atoms, vol. 267, no. 2122, 2009.

[52] D. Fink, P. Alegaonkar, A. Petrov, M. Wilhelm, P. Szimkowiak, M. Behar, D. Sinha, W. Fahrner, K. Hoppe and L. Chadderton, "High energy ion beam irradiation of polymers for electronic applications," Nucl. Instr. Meth. B, vol. 236, pp. 11-20, 2005.

[53] Z. Siwy, P. Apel, D. Baur, D. Dobrev, Y. Korchev, R. Neumann, R. Spohr, C. Trautmann and K.-O. Voss, "Preparation of synthetic nanopores with transport properties analogous to biological channels," Surf. Sci., Vols. 532-535, pp. 1061-1066, 2003.

[54] R. Sanz, J. Jensen, A. Johansson, M. Skupinski, G. Possnert, M. Boman, M. Hernández-Vélez, M. Vázquez and K. Hjort, "Well-ordered nanopore arrays in rutile TiO2 single crystals by swift heavy ion-beam lithography," Nanotechnology, vol. 12, no. 305303, 2007.

[55] M. Crespillo, M. Otto, A. Muñoz-Martín, J. Olivares, F. Agulló-López, M. Seibt, M. Toulemonde and C. Trautmann, "Optimization of nanopores obtained by chemical etching on swift-ion irradiated lithium niobate," Nucl. Instr. Meth. B, vol. 267, pp. 1035-1038, 2009.

[56] D. Kanjilal, "Swift heavy ion-induced modification and track formation in materials," Current Science, vol. 80, pp. 1560-1566, 2001.

[57] D. Fink, P. Alegaonkar, A. Petrov, A. Berdinsky, V. Rao, M.Müller, K. Dwivedi and L. Chadderton, "The emergence of new ion track applications," Radiation Meas., vol. 36, pp. 605-609, 2003.

[58] C. Trautmann, "Modifications induced by swift heavy ions," Bull. Mater. Sci., vol. 22, pp. 679-686, 1999. 
[59] E. Ferain and R. Legras., "Pore shape control in nanoporous particle track etched membrane," Nucl. Instr. Meth. B, vol. 174, pp. 116-122, 2001.

[60] R. Spohr, Ion Tracks and Microtechnology: Basic Principles and Applications, Vieweg, Braunchsweig,: Bethge K, (Ed.)., 1990.

[61] P. Apel, I. V. Blonskaya, O. L. Orelovitch, P. Ramirez and B. A. Sartowska, Nanotechnology, vol. 22, no. 175302, 2011.

[62] P. Apel, Radiat. Phys. Chem, vol. 159, no. 25, 2019.

[63] P. Apel, I. V. Blonskaya, N. E. Lizunov, K. Olejniczak, O. L. Orelovitch, M. E. Toimil-Molares and C. Trautmann, Small, vol. 14, no. e1703327, 2018.

[64] P. Wang, M. Wang, F. Liu, S. Ding, X. Wang, G. Du, J. Liu, P. Apel, P. Kluth, C. Trautmann and Y. Wang, Nat. Commun. 9, vol. 569, 2018

[65] Z. Siwy, P. Apel, D. Dobrev, R. Neumann, R. Spohr, C. Trautmann and K. Voss, Nucl. Instruments Methods Phys. Res. Sect. B Beam Interact. with Mater. Atoms, vol. 208, no. 143, 2003.

[66] I. Vlassiouk, T. R. Kozel and Z. S. Siwy, J. Am. Chem. Soc, vol. 131, no. 8211, 2009.

[67] T. Ma, E. Balanzat, J. M. Janot and S. Balme, Biosens Bioelectron, vol. 137, no. 207, 2019.

[68] G. Perez-Mitta, J. S. Tuninetti, W. Knoll, C. Trautmann, M. E. Toimil-Molares and O. Azzaroni, J Am Chem Soc, vol. 137, no. 6011, 2015.

[69] G. Pérez-Mitta, A. G. Albesa, W. Knoll, C. Trautmann, M. E. Toimil-Molares and O. Azzaroni, Nanoscale, vol. 7, no. 15594, 2015.

[70] A. Spende, N. Sobel, M. Lukas, R. Zierold, J. C. Riedl, L. Gura, I. Schubert, J. M. Moreno, K. Nielsch, B. Stuhn, C. Hess, C. Trautmann and M. E. Toimil-Molares, Nanotechnology, vol. 26, no. 335301, 2015.

[71] M. Toimil-Molares and J. Beilstein, Nanotechnology, vol. 3, no. 860, 2012.

[72] R. Delalande, L. Burr, E. Charron, M. Jouini, M. E. Toimil-Molares and L. Belliard, Appl. Phys. Lett., vol. 115, no. 83103, 2019.

[73] L. Movsesyan, A. W. Maijenburg, N. Goethals, W. Sigle, A. Spende, F. Yang, B. Kaiser, W. Jaegermann, S. Y. Park, G. Mul, C. Trautmann and M. E. Toimil-Molares, "ZnO Nanowire Networks as Photoanode Model Systems for Photoelectrochemical Applications," Nanomater, vol. 8, no. 693, 2018.

[74] I. Schubert, C. Huck, P. Kröber, F. Neubrech, A. Pucci, M. E. Toimil-Molares, C. Trautmann and J. Vogt, "Porous Gold Nanowires: Plasmonic Response and Surface-Enhanced Infrared Absorption," Adv. Opt. Mater., vol. 4, pp. 1838-1845, 2016.

[75] A. Berthelot, S. Hémon, G. Gourbilleau, C. Dufour, B. Domengès and E. Paumier, "Behaviour of a nanometric SnO2 powder under swift heavy-ion irradiation: from sputtering to splitting," Phil. Mag. $A$, vol. 80, pp. 2257-2281, 2000.

[76] N. Khalfaoui, C. Rotaru, S. Bouffard, M. Toulemonde, J. Stoquert, F. Haas, C. Trautman, J. Jensen and A. Dunlop, "Characterization of swift heavy ion tracks in CaF2 by scanning force and transmission electron microscopy," Nucl. Instr. and Meth. B, vol. 240, pp. 819-829, 2005.

[77] J. Vetter, R. Scholz, D. Dobrev and L. Nistor, "HREM investigation of latent tracks in GeS and mica induced by high energy ions.," Nucl. Ins. and Meth. B, vol. 141, pp. 747-752, 1998.

[78] M. Toulemonde and F. Studer, "Comparison of the radii of latent tracks induced by high-energy heavy ions in Y3Fe5012 by HREM, channeling Rutherford backscattering and Mössbauer spectrometry," Phil. Mag. A, vol. 58, pp. 799-808, 1988.

[79] J. Vetter, R. Scholz and N. Angert, "Investigation of latent tracks from heavy ions in GeS crystals by high resolution TEM," Nucl. Ins. Meth. B, vol. 91, pp. 129-133, 1994. 
[80] J. Wiesner, C. Traeholt, J. G. Wen, H. W. Zandbergen, G. Wirth and H. Fuess, "High resolution electron microscopy of heavy-ion defects in superconducting Bi-2212 thin films in relation to their effect on Jc," Physica C, vol. 268, pp. 161-172, 1996.

[81] M.C.G. Toro, M.L. Crespillo, J. Olivares and J.T. Graham, "Anisotropic nanostructure formation by vapor etching of ion tracks in $\alpha$-quartz", Nuclear Instruments and Methods in Physics Research Section B: Beam Interactions with Materials and Atoms, Volume 498, 52-60 (2021).

[82] L. Burr, Ion-Track Technology Based Synthesis and Characterization of Gold and Gold Alloys Nanowires and Nanocones (Ph. D Thesis), Darmstadt: Technische Universitaet Darmstadt, 2016.

[83] F. Pellemoine, M. Avilov, M. Bender, R. C. Ewing, S. Fernandes, M. Lang, W. X. Li, W. Mittig, M. Schein, D. Severin, M. Tomut, C. Trautmann and F. X. Zhang, "Study on structural recovery of graphite irradiated with swift heavy ions at high temperature," Nucl. Instruments Methods Phys. Res. Sect. B Beam Interact. with Mater. Atoms, vol. 365, pp. 522-524, 2015.

[84] K. Kupka, A. A. Leino, W. Ren, H. Vázquez, E. H. Ahlgren, K. Nordlund, M. Tomut, C. Trautmann, P. Kluth, M. Toulemonde and F. Djurabekova, Diam. Relat. Mater, vol. 83, no. 134, 2018.

[85] J. Habainy, Y. Lee, K. B. Surreddi, A. Prosvetov, P. Simon, S. Iyengar, Y. Dai and M. Tomut, Nucl. Instruments Methods Phys. Res. Sect. B Beam Interact. with Mater. Atoms, vol. 439, no. 7, 2019.

[86] C. Hubert, K. O. Voss, M. Bender, K. Kupka, A. Romanenko, D. Severin, C. Trautmann and M. Tomut, Nucl. Instruments Methods Phys. Res. Sect. B Beam Interact. with Mater. Atoms, vol. 365, no. 509, 2015.

[87] M. Shaneyfelt, J. R. Schwank, P. E. Dodd and J. A. Felix, IEEE Trans. Nucl. Sci., vol. 55, no. 1926, 2008.

[88] S. Hoeffgen, M. Durante, V. Ferlet-Cavrois, R. Harboe-Sorensen, W. Lennartz, T. Kuendgen, J. Kuhnhenn, C. LaTessa, M. Mathes, A. Menicucci, S. Metzger, P. Nieminen, R. Pleskac, C. Poivey, D. Schardt and U. Weinand., IEEE Trans. Nucl. Sci., vol. 59, no. 1161, 2012.

[89] H. Matzke, Radiat. Eff. Solids, Dordrecht: Springer Netherlands, 2007.

[90] M. Bleiberg, J. Nucl. Mater, vol. 1, no. 182, 1959.

[91] A. Shoudy, W. E. Mchugh and M. A. Silliman, "The Effect of Irradiation Temperature and Fission Rate on the Radiation Stability of the Uranium-10 Wt \% Molybdenum Alloy,," International Atomic Energy Agency (IAEA), 1963.

[92] B. Ye, L. Jamison, Y. Miao, S. Bhattacharya, G. L. Hofman and A. M. Yacout, J. Nucl. Mater., vol. 488, no. 134, 2017.

[93] J. Gan, D. D. Keiser, B. D. Miller, A. B. Robinson, D. M. Wachs and M. K. Meyer, J. Nucl. Mater., vol. 464, no. $1,2015$.

[94] Y. Miao, K. Mo, B. Ye, L. Jamison, Z.-G. Mei, J. Gan, B. Miller, J. Madden, J.-S. Park, J. Almer, S. Bhattacharya, Y. S. Kim, G. L. Hofman and A. M. Yacout, Scr. Mater., vol. 114, no. 146, 2016.

[95] B. Miller, J. Gan, D. D. Keiser, A. B. Robinson, J. F. Jue, J. W. Madden and P. G. Medvedev, J. Nucl. Mater., vol. 458, no. 115, 2015.

[96] J. Olivares, G. García, A. García-Navarro, F. Agulló-López, O. Caballero and A. García-Cabañes, "Generation of high-confinement step-like optical waveguides in LiNbO3 by swift heavy ion-beam irradiation," Appl. Phys. Lett., vol. 86, no. 183501, 2005.

[97] F. Agulló-López, G. García and J. Olivares, "Lattice preamorphization by ion irradiation: Fluence dependence of the electronic stopping power threshold for amorphization," J. Appl. Phys, vol. 97, no. $093514,2005$.

[98] J. Olivares, G. García, F. Agulló-López, F. Agulló-Rueda, A. Kling and J. Soares, "Generation of amorphous surface layers in LiNbO3 by ion-beam irradiation: thresholding and boundary propagation," Appl. Phys. A, vol. 81, pp. 1465-1469, 2005. 
[99] F. Agulló-López, A. Méndez, G. García, J. Olivares and J. M. Cabrera, "Synergy between thermal spike and exciton decay mechanisms for ion damage and amorphization by electronic excitation," Phys. Rev. B, vol. 74, no. 174109, 2006

[100] J. Olivares, A. García-Navarro, A. Méndez, F. Agulló-López, G. García, A. García-Cabañes and M. Carrascosa, "Novel optical waveguides by in-depth controlled electronic damage with swift ions," Nucl. Instr. Meth. B, vol. 257, pp. 765-770, 2007.

[101] T. Ruiz, A. Méndez, M. Carrascosa, J. Carnicero, A. García-Cabañez, J. Olivares, F. Agulló-López, A. García-Navarro and G. García, "Tailoring of refractive index profiles in LiNb03 optical waveguides by low-fluence swift-ion irradiation," J. Phys. D: Appl. Phys., vol. 40, pp. 4454-4459, 2007.

[102] A. García-Navarro, A. Méndez, J. Olivares, G. García, F. Agulló-López, M. Zayat, D. Levy and L. Vazquez, "Morphology of ion tracks and nanopores in LiNb03 produced by swift-ion-beam irradiation," Nucl. Instr. Meth. B, vol. 249, pp. 172-176, 2006.

[103] J. Olivares, A. García-Navarro, G. García, A. Méndez, F. Agulló-López, A. García-Cabañes, M. Carrascosa and 0. Caballero, "Nonlinear optical waveguides generated in lithium niobate by swift-ion irradiation at ultralow fluences," Optics Letters, vol. 32, pp. 2587-2589, 2007.

[104] J. Olivares, M. Crespillo, O. Caballero-Calero, M. Ynsa, A. García-Cabañes, M. Toulemonde, C. Trautmann and F. Agulló-López., "Thick optical waveguides in lithium niobate induced by swift heavy ions ( 10 MeV/amu) at ultralow fluences," Optics Express, vol. 17, pp. 24175-24182, 2009.

[105] H. Geiger and E. Marsden, "On a diffuse reflection of the alpha-particles," Proceedings of the Royal Society $A$, vol. 82, no. 557, 1909.

[106] E. Rutherford, "The scattering of alpha and beta particles by matter and structure of the atom," Philosophical Magazine and Journal Science, vol. 21, no. 125, pp. 669-688, 1911.

[107] J. R. Tesmer and M. Nastasi, Handbook of modern ion beam material analysis, Pittsburg: MRS, 1995.

[108] W. K. Chu, J. W. Mayer and M. A. Nicolet, "Backscattering spectrometry," 1978.

[109] M. Mayer, AIP Conference Proceeding, vol. 475, p. 541, 1999.

[110] N. P. Barradas, C. Jeynes and R. P. Webb, Applied Physics Letter, vol. 71, p. 291, 1997.

[111] E. Kótai, Nuclear Instruments and Methods B, vol. 85, no. 588, 1994.

[112] N. Barradas, K. Arstila, G. Battistig, M. Bianconi, N. Dytlewski, C. Jeynes, E. Kótai, G. Lulli, M. Mayer, E. Rauhala, E. Szilágyi and M. Thompson, Nuclear Instruments and methods B, vol. 262, no. 281, 2007.

[113] R. C. Birds and J. S. Williams, "Ion beams for materials analysis," 1989.

[114] F. G.-P. Flores, A. Redondo-Cubero, R. Gago, A. Bengoechea, A. Jiménez, D. Grambole, A. F. Braña and E. Muñoz, Journal of physics D: Applied Physics, vol. 42, no. 055406, 2009.

[115] T. B. Johansson, R. Akselsson and S. A. E. Johansson, "X-ray analysis: Elemental trace analysis at the 10-12g level," Nuclear Instruments and Methods, vol. 84, pp. 141-143, 1970.

[116] G. F. Knoll, Radiation detection and measurement, John Wiley \& Sons, 2010.

[117] M. Blaauw, J. L. Campbell, S. Fazini, M. Jaksi, I. Orlic and P. Van Espen, "The 2000 IAEA interocmparison of PIXE spectrum analysis software," Nuclear Instruments and Methods B, vol. 189, pp. 113-122, 2002.

[118] J. L. Campbell, "GUPIXWIN," 2017. [Online]. Available: http://pixe.physics.uoguelph.ca/GUPIX/main/.

[119] J. L. Campbell, N. I. J. L. Boyd, N. Grassi, P. Bonnick and J. A. Maxwell, "The Guelph PIXE software package IV," Nuclear Insturments and Methods B, vol. 268, pp. 3356-3363, 2010.

[120] C. G. Ryan and D. Jamieson, "Dynamic analysis: on-line quantitative PIXE microanalysis and its use 
in overlap-resolved elemental mapping," Nuclear Instruments and Methods B, vol. 77, pp. 203-214, 1993.

[121] M. A. Reis and L. C. Alves, "DATTPIXE, a computer package for TTPIXE data analysis," Nuclear Instruments and Methods B, vol. 68, pp. 300-304, 1992.

[122] E. Clayton, P. Duerden and D. D. Cohen, "A discussion of PIXAN and PIXANPC: The AAEC PIXE analysis computer packages," Nuclear Instruments and Methods B, vol. 22, pp. 64-67, 1987.

[123] J. Stark, "Bemerkung über Zerstreuung und Absorption von $\beta$-strahlen und Röntgenstrahlen in Kristallen," Phys. Z, vol. 13, pp. 973-977, 1912.

[124] G. R. Piercy, F. Brown, J. A. Davies and M. McCargo, "Experimental evidence for the increase of heavy ion ranges by channeling in crystalline structure," Physical Revier Letters, vol. 10.9, p. 399, 1963.

[125] M. T. Robinson and O. S. Oen, "Computer studies of the slowing down of energetic atoms in crystals," Physical Review, vol. 132, p. 2385, 1963.

[126] W. Brandt, "Channeling in crystals," Scientific American, vol. 218, no. 3, pp. 90-101, 1968.

[127] J. Lindhard, "Influence of crystal lattice on motion of energetic charges particles," Kongel. Dan. Vidensk. Selsk., Mat.-Fys. Medd., vol. 34, no. 1, 1965.

[128] L. C. Feldman, J. W. Mayer and S. T. Picraux, "Materials analysis by ion channeling," Academic Press, 1982.

[129] M. L. Swanson, "The study of lattice defects by channelling," Reports on Progress in Physics, vol. 45, no. $47,1982$.

[130] D. S. Gemmell, "Channeling and related effects in the motion of charged particles through crystals," Reviews of Modern Physics, vol. 46, no. 129, 1974.

[131] A. Vantomme, "50 years of ion channeling in materials science," Nuclear Instruments and Methods in Physics Research section B, vol. 371, pp. 12-26, 2016.

[132] J. F. Ziegler, J. P. Biersack and U. Littmark, "The stopping and range of ions in solids," Pergamon Press, 1985.

[133] A. Redondo-Cubero, Ion beam analysis of wide band gap semiconductor heterostructures, 2010.

[134] F. Watt and G. W. Grime, Principles and Applications of High-Energy Ion Microbeams, Bristol: IOP Publishing Ltd, 1987.

[135] M. Breese, D. Jamieson and P. King, Material Analysis Using a Nuclear Microprobe, New York: Wiley, 1996.

[136] G. W. Grime and M. Dawson, Nuclear Instruments and Methods B, vol. 104, pp. 107-113, 1995.

[137] P. M. O'Brien, G. Moloney, A. O'Connor and G. J. F. Legge, "A versatile system for the rapid collection, handling and graphics analysis of multidimensional data," Nuclear Instruments and Methods B, vol. 77, pp. 52-55, 1993.

[138] M. Jakšić, I. B. Radović, M. Bogovac, V. Desnica, S. Fazinić, M. K.', Z. Medunić, H. Muto, Z. Siketić, N. Skukan and T. Tadić, "New capabilities of the Zagreb ion microbeam system," Nuclear Instruments and Methods, vol. 260, pp. 114-118, 2007.

[139] C. Udalagama, X. Chen, A. A. Bettiol and F. Watt, "An ion beam analysis software based on ImageJ," Nuclear Instruments and Methods B, vol. 306, pp. 59-63, 2013.

[140] G. Deves, L. Daudin, A. Bessy, F. Buga, J. Ghanty, A. Naar, V. Sommar, C. Michelet, H. Seznec and P. Barberet, "An ImageJ plugin for ion beam imaging and data processing at AIFIRA facility," Nuclear Instruments and Methods B, vol. 348, pp. 62-67, 2015. 
[141] J. S. Laird, C. G. Ryan, R. Kirkham, T. Satoh and A. Pagès, "Next generation data acquisition systems for the CSIRO Nuclear Microprobe: Highly scaled versus customizable," Nuclear Instruments and Methods B, vol. 404, pp. 15-20, 2017.

[142] M.C.G. Toro, M.L. Crespillo, J. Olivares and J.T. Graham, "Raman characterization of phonon confinement and strain effects from latent ion tracks in $\alpha$-quartz", J. Raman Spectrosc. 52:1185-1192 (2021). https://doi.org/10.1002/jrs.6108 
SECTION III

SIMULATION METHODS 



\title{
5. Electron dynamics from first-principles
}

\author{
Davide Sangalli*, Layla Martín-Samos, Alejandro Molina-Sánchez, \\ and Jorge Kohanoff
}

\begin{abstract}
Here we present a microscopic approach to describe electron dynamics in a many-body system fully ab initio. First, we introduce the state-of-the-art methods to model extended systems in equilibrium: Density Functional Theory (DFT) and $a b$ initio Many-Body perturbation theory (MBPT), with the so-called GW+BSE scheme. We then move onto their non-equilibrium extensions, Time-Dependent (TD) DFT and Non-Equilibrium Green Functions (NEGF). Both approaches can be successfully employed to model coherent electron dynamics in the femtosecond regime. A few examples with practical applications are shown. We then turn our attention onto how these approaches can be extended to account for dissipative processes, such as electronphonon scattering, and to include the (coherent) atomic motion coupled to the electron dynamics. The connection between the full Quantum Kinetics equation of NEGF, and the semi-classical Boltzmann equation (which will be explored in more detail in next Chapter) is also discussed.
\end{abstract}

\footnotetext{
*Contact: davide.sangalli@ism.cnr.it
} 


\subsection{Introduction}

The central theme of this book is to describe methods to model irradiation processes across many space and time scales, where the initial stage of the phenomenon consists of intense electronic excitation and ionization. Depending on the material and conditions, excitations and ejected electrons will either diffuse away from the region where they were created leaving positively charged ions that can repel each other and lead to a Coulomb explosion or will remain in the neighbourhood effecting the cohesion of the material and weakening chemical bonds. Excitation will also induce temperature increases when the energy transferred to electrons is deposited back into vibrations. This leads to mechanical and thermodynamic effects like swelling, fracture or phase transitions, that occur in longer times and larger distances.

In this Chapter we will focus on the firststage, i.e., electronic excitation and, more generally, electron dynamics. We will discuss the conceptual, theoretical and computational frameworks necessary to describe electronic excitation, in particular intense electronic excitation. We start in Section 5.2 by reviewing ground state electronic structure approaches, including Hartree-Fock and density functional theory (DFT), and paying particular attention to the concept of mean-field approximation. We then focus on band structures and introduce electronic ex-citations in terms of quasiparticles, via Green's functions and the GW approximation, within the framework of Many-Body Perturbation Theory (MBPT). In Section 5.2.3 we introduce the main techniques to describe optical excitations, including time-dependent DFT and the Bethe-Salpeter equation (BSE). A final subsection introduces nuclear motion and electron-phonon coupling at the level of ground state DFT, which becomes useful in Section 5.4. In Section 5.3 we focus on the description of electron dynamics in real time. Coherent dynamics is described via rt-TDDFT, and illustrated in the case of ion irradiationand electronic stopping power. The MBPT counterpart, embodied into the Kadanoff-Baym equations (KBE), is described next and illustrated by comparing the optical spectrum of BN with the one obtained using the BSE. Connections between the two approaches are explored here. The KBE equations supplemented with a collisions term is then used in Section 5.3.3 to describe incoherent dynamics within the MBPT framework. This is illustrated by following the evolution of the electron and hole occupations in bulk $\mathrm{Si}$, showing that both equilibrate to the Fermi-Dirac distribution, something that is not possiblein coherent dynamics. Connections to the semi-classical Boltzmann transport equation, which will be described in detail in Chapter 6, are explored here. Section 5.4 focuses on the pico-second time scale, inwhich electronphonon coupling or electron-nuclear interactions allow for energy transfer between electronic and nuclear degrees of freedom. We first describe methods based on classical molecular dynamics for the nuclei, and then introduce the quantization of the nuclear motion by means of the $N$-body density matrix and simplified, efficient schemes based on the evolution of the one-body reduced density matrix. Finally, in Section 5.5 we summarize and indicate somepossible directions for future research.

\subsection{Ground state and excited states}

Before entering in the hard part of the problem of many-interacting electrons let us first define some physical quantities and terminology, that might appear sometimes confusing because of "language abuse". When nothing is acting on a system, the system is said to be in its Ground-State. 
Contrary to classical systems, in quantum mechanics ground-state does not mean frozen. Indeed, a many-electron system is a dynamical system in which electrons/holes are exchanging energy and momentum with each other (and with the nuclei) constantly. The ground-state properties and ground-state total energy of an $N$-electron system, $E(N)$, contain implicitly the average of all such "self-" excitations. Hence, averaged, ground-state quantities might be seen as time-independent.

To bring the whole system into an excited state it is necessary to apply a perturbation. Trivially, as it is well known from basic quantum mechanics, any measurement is a perturbation. It is, therefore, crucial to know what is the effect of a given measurement on the system and which observable it selects. If a measurement/experiment removes and/or add electrons, like photoemission and inverse-photo emission, the system will explore (partly or fully) the ensemble of its "charged" excitations, with total energies $E^{S}(N-1)$ or $E^{S}(N+1)$, respectively. The energies required to afford such excitations are addressed as Ionization potentials (IP) or Electron Affinities (EA), with $I P^{s}=E^{s}(N-1)-E(N)$ and $A E^{s}=E(N)-E^{s}(N+1)$. The band structure, which is an observable measured through photo-emission- and inverse-photo emission-like experiments is the ensemble of Brillouin-zone-resolved IPs and EAs (with a minus sign) of a material. It is, therefore, not a ground-state property. What is usually addressed as "electronic

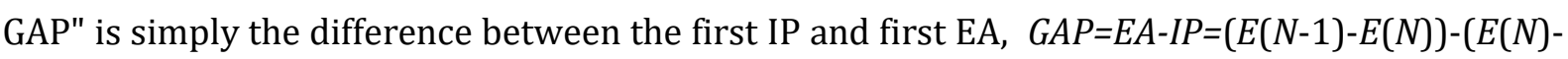
$E(N+1))=E(N+1)+E(N-1)-2 E(N)$. The electronic GAP is, therefore, not a ground-state property. If the measurement involves excitations in which the number of electrons does not change (an electron is taken from the occupied orbitals and promoted to an empty orbital), as it is the case for optical absorption, then the system will explore its neutral excitations $E^{S}(N)$. The energies required to afford such excitations are $E^{S}(N)-E(N)$, and the optical GAP is the smallest energy difference. In general, the electronic GAP and the optical GAP are not the same, as in the later, electron-hole interactions take place.

\subsubsection{Ground state electronic properties}

For an interacting many-electron system, the Hamiltonian operator can be written as a sum of a Kinetic term $(\boldsymbol{T})$ a many-body electron-electron Coulomb Interaction $(\boldsymbol{W})$ and some external potential $(\boldsymbol{V})$ that accounts for the electrostatic ion-electron interaction:

$$
\widehat{H}=\widehat{T}+\widehat{W}+\widehat{V} \equiv \sum \frac{\hat{p}_{i}^{2}}{2 m}+\frac{1}{2} \sum \frac{e^{2}}{\hat{r}_{i}-\hat{r}_{j}}-\sum \int \frac{e Z}{\left|R-\hat{r}_{i}\right|} d \Omega
$$

One can also include other external agents that couple to the electronic degrees of freedom like a laser field or an ionic projectile. The ground state total energy, $E_{0}$, is the expectation value: $E_{0}=$ $\left\langle\Psi_{0}|H| \Psi_{0}\right\rangle$.

The problem of many interacting electrons subject to ion-electron interaction is not exactly solvable for more than two electrons. However, as the electron-electron interaction, $\widehat{W}$, depends on the bare Coulomb potential, it is, therefore, natural, to address the problem by a perturbative series expansion on the bare Coulomb potential. Such series expansion is at the root of most of Quantum Chemical approaches, such as Hartree-Fock, Møller-Plesset and Coupled Clusters perturbation theory, and multi-reference Configuration Interaction [1]. In the following, we briefly describe the two simplest approximations, namely Hartree and Hartree-Fock, in order to 
gain some insight into the meaning and limitations of Mean-field theories. We will assume that the nuclei act on electrons only as a fixed external potential. This is usually referred as the BornOppenheimer approximation (atomic motion will be treated in some detail in section 5.4).

\subsubsection{Second quantization}

Before going into Mean field theories, we introduce an alternative mathematical formulation of the problem, usually known as second quantization, which is useful for indistinguishable particles like electrons. Here the single-particle states are filled with a certain number of identical particles. The number of such particles can be modified by means of creation and annihilation operators, which replace the differential operators that appear in first quantization. In this formulation the Hamiltonian takes the following form:

$$
\widehat{T}+\widehat{W}=\sum_{r s} c^{\dagger}\langle r|T| s\rangle c_{s}+\frac{1}{2} \sum_{r s t u} c_{r}^{\dagger} c_{s}^{\dagger}\langle r s|W| t u\rangle c_{u} c_{t}
$$

where

$$
\begin{gathered}
\langle r|T| s\rangle=\int \phi_{r}^{\dagger}(x) T_{r s} \phi_{s}(x) d x, \\
\langle r s|W| t u\rangle=\int \phi_{r}^{\dagger}(x) \phi_{s}^{\dagger}\left(x^{\prime}\right) \frac{1}{r-r^{\prime}} \phi_{t}(x) \phi_{u}\left(x^{\prime}\right) d x d x^{\prime} .
\end{gathered}
$$

and $\phi_{r}$ are single-particle wave functions.

\subsubsection{Mean Field Approximations}

Mean Field (MF) is the simplest way to approximate the problem of many interacting electrons by considering the effect of interactions only in average. At a first glance such a simplified picture might be considered naïve. However, it provides useful information that is often qualitatively correct. The MF approach consists of replacing the two-body interaction $(\widehat{W})$, which is a product of four fermion operators, by a one-body term that considers the mean value of the product of two of the four fermion operators, thus linearising the Hamiltonian. In other words, the integrals that involves the product of two operators, symbolically, $\mathrm{AB}$ is replaced by $\langle A\rangle B+\langle B\rangle A-\langle A\rangle\langle B\rangle$, where the last term is just a scalar that only modifies the energy reference. By linearizing, $\mathrm{MF}$ approaches neglect higher-order terms, usually referred to as correlation, and fluctuations with respect to the mean. It is important to note that the eigenstates of the linearised Hamiltonian have to be consistent with the states that have been used to compute the mean interactions in the aforementioned Hamiltonian. As such, the solution can only be found through a self-consistent calculation. In fact, often these approaches are called self-consistent field (SCF).

By linearising the Hamiltonian, two different types of terms can be identified: direct terms (usually referred to as Coulomb or Hartree term)

$$
\left\langle c_{r}^{\dagger} c_{t}\right\rangle c_{s}^{\dagger} c_{u}+c_{r}^{\dagger} c_{t}\left\langle c_{s}^{\dagger} c_{u}\right\rangle
$$


where the mean quantities $\left\langle c^{\dagger} c\right\rangle$ correspond to single-particle state densities; and indirect terms (usually referred to as Fock or EXchange term)

$$
-\left\langle c_{r}^{\dagger} c_{u}\right\rangle c_{s}^{\dagger} c_{t}-c_{r}^{\dagger} c_{u}\left\langle c_{s}^{\dagger} c_{t}\right\rangle
$$

where $\left\langle c_{r}^{\dagger} c_{u}\right\rangle$ (and $\left\langle c_{s}^{\dagger} c_{t}\right\rangle$ ) are overlap integrals. The minus sign arises from commutation and anticommutation rules.

In the case of the homogeneous electron gas, the Hamiltonian can be simplified by using the eigenfunctions of the kinetic energy operator for $c^{\dagger}$ and $c$, and by exploiting the translational invariance by resorting to Fourier space, to obtain:

$$
H=\sum_{k \sigma} \epsilon_{k} c_{k \sigma}^{\dagger} c_{k \sigma}+\sum_{\substack{q \neq 0 \\ k k^{\prime} \\ \sigma \sigma^{\prime}}} \frac{2 \pi}{q^{2}} c_{k+q \sigma}^{\dagger} c_{k \prime-q \sigma^{\prime}}^{\dagger} c_{k \prime} \sigma^{\prime} c_{k \sigma},
$$

where $\epsilon_{k}$ is the kinetic energy corresponding to a state of momentum $k$, and the spin $\sigma$ has been explicitly written. The $q=0$ term has been excluded assuming a neutralizing background charge and, as the nuclear-electron interaction is not included, the basis for $\widehat{T}$ are simply plane waves. A similar form can be derived for Bloch states in crystalline periodic systems featuring nuclearelectron interactions.

\section{The Hartree approximation}

It consists of taking into account only the direct terms (which is equivalent to neglecting the antisymmetrization of the wave functions) at $q=0$ (we assume that the electronic density does not have components for $q \neq 0$ ). This is equivalent to postulate that the many-body wave function is a simple product of one-electron terms. The linearized Hamiltonian reduces, therefore, to:

$$
H_{\text {Hartree }}=\sum \epsilon_{k} c_{k \sigma}^{\dagger} c_{k \sigma}
$$

In essence, the solutions are those of a free electron gas, i.e., plane waves, with the total energy equal to:

$$
E_{\text {Hartree }}=\sum_{\epsilon<E_{F}} \epsilon_{m}
$$

where $\epsilon_{m}$ are one-electron energies and $E_{F}$ is the Fermi energy, i.e., the energy of the highest occupied state.

If the nuclear-electron interaction, $\widehat{V}$, is taken into account, the solutions would be Bloch states that respect the periodicity of the crystal, and the Hartree Hamiltonian would include, in Fourier space, a term proportional to the product of Bloch components ( $q$-vector components) of the density (excluding $q=0$ ). 
The Density of States (DOS) of the Hartree electron gas, as for the non-interacting electron gas goes as $\sim \sqrt{E-E_{0}}$, which does not exhibit an energy gap. In the Hartree world all systems are, therefore, seen as metallic.

In this case, the excitations, band structure, IP and EA, which are, by definition, total energy differences, do correspond to the single-particle energies $\epsilon_{m}$.

\section{The Hartree-Fock approximation}

At variance with Hartree, the Hartree-Fock approximation (HF) includes both direct and indirect terms. If we assume that the symmetry of the HF ground state is the same as the one of the noninteracting electron gas, the mean values are non-zero only if $\sigma=\sigma^{\prime}$ and $k+q=k^{\prime}$. The HartreeFock Hamiltonian is:

$$
H_{H F}=\sum \epsilon_{k} c_{k \sigma}^{\dagger} c_{k \sigma}-\sum \frac{4 \pi}{q^{2}}\left\langle n_{k+q \sigma}\right\rangle c_{k}^{\dagger} \sigma c_{k} \sigma
$$

where $\left\langle n_{k+q \sigma}\right\rangle$ is the electronic density. The one-electron HF energies, $\epsilon_{k \sigma}^{H F}$, are given by:

$$
\epsilon_{k \sigma}^{H F}=\epsilon_{k}-\frac{1}{\Omega} \sum_{k^{\prime}<k_{F}} \frac{4 \pi e^{2}}{\left|k-k^{\prime}\right|^{2}}
$$

Contrary to the case of Hartree, the HF DOS drops to zero at the Fermi energy. HF penalizes, therefore, partial occupations. Thus, in the HF world all systems are "insulators".

In the more general HF case the minimization of the total energy $E=\langle\Psi|\widehat{T}+\widehat{W}| \Psi\rangle$, under the subsidiary condition $\int \phi_{i}(x) \phi_{j}(x)=\delta_{i j}$ leads to the Hartree-Fock equations for $\phi$ :

$$
\left(T+V_{H}+V_{e x}\right) \phi_{k}=\epsilon_{k}^{H F} \phi_{k},
$$

with

$$
V_{H}(x)=\int \frac{1}{\left|r-r^{\prime}\right|}\left[\sum_{k=1}^{N}\left|\phi_{k}(x)\right|^{2}\right] d x^{\prime}
$$

and

$$
V_{e x}\left(x, x^{\prime}\right)=-\frac{1}{\left|r-r^{\prime}\right|}\left[\sum_{k=1}^{N} \phi_{k}(x) \phi_{k}^{\dagger}\left(x^{\prime}\right)\right] .
$$

The HF total energy can be obtained as:

$$
E^{H F}=\frac{1}{2} \sum_{o c c}\left(T_{k k}+\epsilon_{k}^{H F}\right)
$$

where the sum runs over occupied states. 
The significance of $\epsilon_{k}^{H F}$ as one-electron energies has been discussed by Koopmans. Considering the energy difference between the ground-state and a state with an electron in the one-electron state $k$ missing, it can be shown that:

$$
E(N)-E(N-1, k \text { empty }) \approx \epsilon_{k}^{H F} .
$$

$\epsilon_{k}^{H F}$ represents the energy required to ionize the system leaving a hole in state $k$, if we assume that the wave functions $\phi_{k}$ in the $N$ and $(N-1)$-particle states are the same. In the so-called Koopmans approximation, Ionization Potentials and Electronic Affinities are directly related to HF one-electron energies.

HF theory serves as a model example of self-consistent theories, but in which polarization effects, also called correlation effects, are not included. In other words, all interactions/effects that are not included within HF, are defined as correlation.

\section{Higher order approximations}

The expansion in the Coulomb interaction quickly becomes computationally prohibitive, except for small molecules. It is important to note that, within the "expansion" framework, effective polarization effects are very difficult to capture, as they arise as a consequence of including higherorder excitations, starting from double excitations (or "doubles"), in which two electrons are promoted from occupied to empty states, and building up to include triples, quadruples, etc. A particularly appealing solution to this is coupled cluster theory, which includes infinite resummations of excitations by reducing them to doubles, triples and quadruples. Nevertheless, the scaling with system size makes this theory of limited use for large and extended systems. In view of this situation the formulation of Density Functional Theory was a revolution! It is somehow as reverting the problem like a sock. For a very clear review see Ref. [2].

\subsubsection{Density Functional Theory}

\section{The Hohenberg-Kohn theorem (1964)}

He Hohenber-Kohn theorem states that an exact representation of the ground state properties of a stationary, non-relativistic many-particle system is possible in terms of the ground state density alone. The ground state total energy can, therefore, be written as a functional of the density:

$$
E_{v}[n]=\langle\Psi[n]|\hat{T}+\widehat{W}+\widehat{V}| \Psi[n]\rangle
$$

This first statement of HK theorem is often addressed as the invertibility of the map statement. Despite its surprising simplicity compared, in particular, to MBPT, the Hohenberg-Kohn theorem has a rigorous mathematical foundation. It represents one of the major advances in theoretical and computational material science. Walter Kohn was, indeed, awarded by Nobel prize in chemistry in 1998 for this. The HK theorem also establishes the variational character of the energy functional. Thus, the exact ground state density can be determined by minimizing a functional of the density $(n)$ at a given external potential $\left(v_{0}\right)$; in short: 


$$
E_{0}=\min _{n \in \mathcal{N}} E_{v_{0}}[n],
$$

where $\mathcal{N}$ is the ensemble of ground state densities and $E_{0}$ is the ground state energy. In addition, as the first statement is universal, i.e., it does not depend on the external potential of the particular system under consideration, the last statement of HK theorem establishes the universality of the functional:

$$
F[n] \equiv\langle\Psi[n]|\widehat{T}+\widehat{W}| \Psi[n]\rangle
$$

The functional form is, however, unknown.

Along the years, they have been several derivations of lower and upper bounds for the functional, and, also, critical extensions of the formalism (as the extension to degenerate ground states, fractional occupation and relativistic systems).

One year after HK original formulation, Kohn and Sham (1965), proposed a scheme that has been the key for the success of DFT implementations and its broad adoption.

\section{The Kohn-Sham scheme}

In the Kohn-Sham scheme, the variation with respect to the density is formulated through the introduction of an auxiliary set of one-electron orbitals. The central assumption is the following: for any interacting system, there exist a local single-particle potential such that the exact groundstate density of the interacting system equals the ground state density of the auxiliary problem. In other words, if we consider a particular interacting system with external potential $v_{0}(r)$ and ground state density $n_{0}(r)$, the auxiliary potential $v_{0, s}$ that generates the ground state density via a sum over KS orbitals, $\phi_{i, 0}(r)$, satisfies the Schrödinger-like equation:

$$
\left(-\frac{\hbar^{2}}{2 m} \nabla^{2}+v_{s, 0}(r)\right) \phi_{i, 0}(r)=\varepsilon_{i} \phi_{i, 0}(r)
$$

In this scheme the total ground state energy can be re-written as:

$$
E_{v_{0}}[n]=T_{s}[n]+\int v_{0}(r) n(r) d^{3} r+\frac{1}{2} \iint n(r) \frac{1}{r-r^{\prime}} n\left(r^{\prime}\right) d^{3} r d^{3} r^{\prime}+E_{x c}[n],
$$

which is a sum of the KS non-interacting kinetic energy $\left(T_{S}\right)$, the ion-electron Coulomb interaction (external potential $V_{0}$ ), a Hartree term, and an Exchange-Correlation functional of the density, $E_{x c}[n]$, that accounts for all remaining interactions. Since the HK variational principle ensures that the total energy is stationary for small variations of the ground state density, it can be proved that

$$
v_{s, 0}=v_{0}(r)+\int \frac{n\left(r^{\prime}\right)}{r-r^{\prime}} d^{3} r^{\prime}+v_{x c}\left(\left[n_{0}\right] ; r\right)
$$

with $v_{x c}\left(\left[n_{0}\right] ; r\right)=\left.\frac{\delta E_{x c}[n]}{\delta n(r)}\right|_{n_{0}}$. As in HK, the exchange-correlation functional is universal, but in this case it is unknown. 


\section{Kohn-Sham auxiliary orbitals}

Strictly speaking, the Kohn-Sham auxiliary orbitals do not carry a precise physical meaning. The theory is in principle exact for the ground state density, the ground state energy and any quantity that is derived from the density alone. Moreover, within DFT and KS, empty orbitals have no grounds. Indeed, they are often called virtual orbitals. KS bands, or sometimes called "ground state electronic structure" is used as indication (sometimes in very good agreement, because nature is mainly Mean Field), or as an initial guess for theories that address other aspects than the ground state, like meaningful band structures, optical spectra as it will be shown in next sections.

A word of caution: DFT is not a Mean Field Theory, and it is not a Perturbation Theory (it is not an expansion), as it is not an approximation to the Many-Body Hamiltonian of Equation (5.7). DFT is, in principle, exact. Its limitations arise from the approximate character of the exchangecorrelation functional.
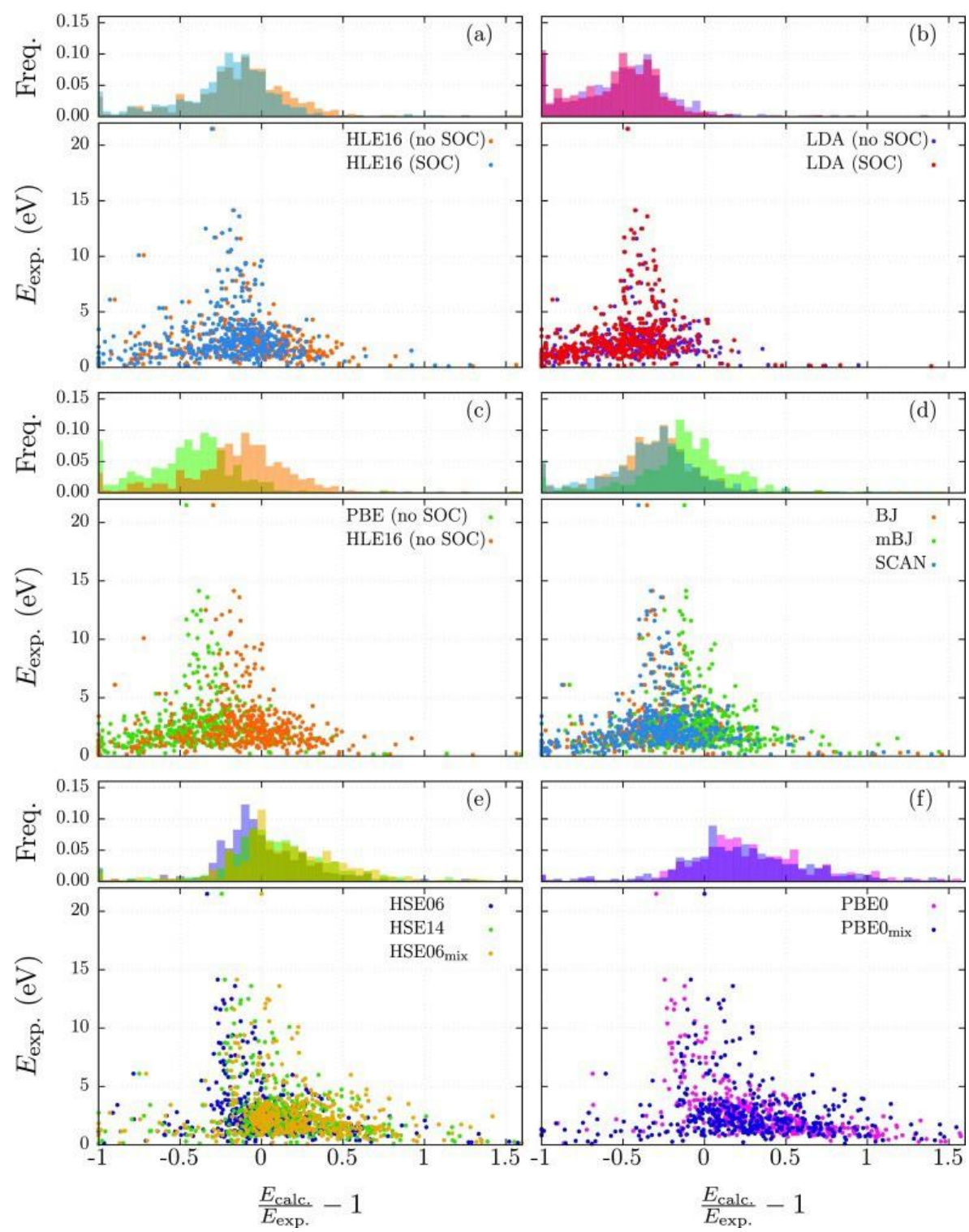

Figure 5.1. Large-scale benchmark of band gaps computed within several DFT exchange-correlation flavors, including hybrids. Picture from Ref. [3]. 


\section{Practical aspects}

Since the universal functional is unknown, several approximations to the exchange-correlation functional have been proposed. Some, as the Local Density Approximation (LDA) and the several flavours of Gradient Corrections (GGA's) are local or semi-local functionals of the density. Others, such as hybrids (short ranged, long ranged, range separated) are orbital dependent. A word of caution: since DFT is not a Mean Field Theory and it is not a perturbative expansion, there is no warranty of improvement. Not because a functional includes a gradient (or higher-order derivatives), it is better than the LDA for all materials and applications, and for all derived quantities It is not because a functional gives an "accurate" gap for many systems that it will exhibit the same accuracy for a specific system, or that it will predict "accurate" fusion temperatures. For a large benchmark of functionals, see Figure 5.1 and Ref. [3]. Within a modelling scenario it is very important to try several functional flavours, to understand, to benchmark, to compare directly or indirectly with experiments, and to try and extract trends and relative quantities that are less sensitive to the specific functional.

Some functional rely heavily on parametersthat can be modified. Therefore, the results of such calculations can be easily tuned. For instance, the hybrids PBE0 [4] and B3LYP rely on the Fock EXchange fraction parameter, that is by default 0.25 and 0.2 , respectively. The range separated HSE hybrid functional [5] relies not only on the Fock EXchange fraction but also on the screening length parameter. Even simple GGAs like PBE have be re-parameterized to better describe molecular properties (revPBE) and solid-state systems (RPBE).

\section{The band "GAP" problem}

This is often addressed as a major DFT drawback, but in reality, it is an issue related to the use of approximate exchange-correlation functionals. DFT could, in principle, provide the correct gap (which is related to Janak's Theorem). Indeed, the only two Kohn-Sham energies that have a meaning in the DFT framework are the highest occupied KS state and the lowest empty KS state. In practice, the exchange part of LDA and GGAs are shorter in range than the real Fock exchange, and do not exactly cancel the self-interaction present in the Hartree term. As a consequence, the exchange-correlation potential decays, incorrectly, exponentially fast into low-density regions, while it should decay following a power law. This has a critical influence in the value of the GAP, and of course, on the value of the first IP and AE. For an extended discussion, we strongly encourage the reading of the book in Ref. [6].

\section{DFT open source freely available community codes:}

Quantum-Espresso [7], ABINIT [8], SIESTA [9] are just a few of the myriad of electronic structure codes, many of them open source and freely available. An exhaustive list can be found in the Webpage referred in [10]. 


\subsubsection{Band structure: QP energies and the GW approximation}

Similar to people interaction, we are not alone but we are we plus our interactions with our family, friends, co-workers etc, the problem of many-interacting electrons is addressed by averaging ( $m e$ and my average interaction with the others), as in the case of mean-field approximations like Hartree-Fock, or expanding with respect to excitations (me plus my two-people interaction, plus my three-people interaction etc) as in the case of Multi-Reference Configuration interaction. An alternative, way of tackling with many-interacting particles is the concept of quasi-particles and the Green Functions formalism.

\section{The one-electron Green's function}

The one-electron Green's function in coordinate space is defined as the time ordered product of two field operators:

$$
G\left(x t, x^{\prime} t^{\prime}\right)=-i\left\langle N\left|T\left[\psi(x, t) \psi^{\dagger}\left(x^{\prime}, t^{\prime}\right)\right]\right| N\right\rangle
$$

For $t>t^{\prime}$ the Green function describes the propagation of an additional electron injected at time $\mathrm{t}^{\prime}$, whereas for $t<t^{\prime}$ it describes the propagation of a hole. With some manipulation, it can be shown that in Fourier space for time, i.e., Fourier transforming into energies $(\omega)$, the Green function takes the following form:

$$
G\left(x, x^{\prime}, \omega\right)=\sum_{s}\left[\frac{f_{s}(x) f_{s}^{\dagger}\left(x^{\prime}\right)}{\omega-\epsilon_{s} \pm i \delta}\right] .
$$

Each pole of the Green's function corresponds to a N-1 or N+1 excitation energy of the manyelectron system. Such energies are called quasi-particle energies. The term Quasi-particle, instead of particle, arises because the peaks corresponding to each excitation energy are not Dirac deltas but they do exhibit a finite broadening. This broadening is related to the quasi-particle lifetime, which is finite because of the interactions, i.e. because the many-particle system is entangled.

Now, from the equation of motion of the field operators in the Heisenberg picture, and introducing a small perturbing potential $\phi(x, t)$, we can obtain an equation of motion for the Green's function:

$$
G\left(x t, x^{\prime} t^{\prime}\right)+i \int v(x, y) G^{(2)}\left(x t y t, x^{\prime} t y t^{\prime}\right) d y=\delta\left(x, x^{\prime}\right) \delta\left(t, t^{\prime}\right)
$$

which depends on the two-particle Green's function,

$$
G^{(2)}\left(x t x^{\prime} t^{\prime}, y z y^{\prime} z^{\prime}\right)=\left\langle N\left|T\left\{\psi^{\dagger}(x, t) \psi\left(x^{\prime}, t^{\prime}\right) \psi(y, z) \psi^{\dagger}\left(y^{\prime}, z^{\prime}\right)\right\}\right| N\right\rangle
$$

This equation could be taken as the starting point for generating an infinite chain of equations (expansions) introducing successively more complicated interactions. But, instead of proceeding in that way, one could introduce a set of non-linear equations in which the interaction between a particle and the rest of the system is "hidden" inside a non-local time- (or energy-) dependent quantity called the Self-Energy operator.

\section{The Self-Energy}

This quantity is, therefore, a "box" in which all the complexity of the many-body problem is encapsulated: 


$$
[i(\partial / \partial t)-T(x)-V(x, t)] G\left(x t, x^{\prime} t^{\prime}\right)-\int \Sigma(x t, y z) G\left(y z, x^{\prime} t^{\prime}\right) d y d z=\delta\left(x, x^{\prime}\right) \delta\left(t, t^{\prime}\right)
$$

The quantity $V(x, t)$ is the total average potential in the system.

In Fourier space for the energy:

$$
G\left(x, x^{\prime}, \omega\right)-\int \Sigma(x, y, \omega) G\left(y, x^{\prime}, \omega\right)=\delta\left(x, x^{\prime}\right) .
$$

The complicated many-body character of $G$ is due to the energy dependence in the Self-Energy $\Sigma$. Within the quasi-particle and Self-Energy picture, Hartree and Hartree-Fock are two simple approximations in which the Self-Energy is energy independent. In particular, the corresponding Hartree-Fock Self-energy is:

$$
\Sigma^{H F}=i G\left(x, x^{\prime}\right) v\left(x, x^{\prime}\right)
$$

The HF approximation involves a self-consistency requirement regarding the density matrix of the system. When effects of the dynamical interaction are included, the self-consistency requirement must be augmented to include the full Green Function rather than the equal-time limit. In theories beyond HF the Self-Energy could be regarded as a functional of a fully dynamical Green's function.

\section{The GW approximation}

The GW approximation is nowadays considered one of the standards to obtain accurate Ionization Potentials and Electron Affinities (accurate band structures). It has its roots in the seminal work of Hedin and Lundqvist [11], and it is an approximation to the more complete self-consistent $G W \Gamma$ theory.

The extremely smart idea beyond $G W \Gamma$ (and GW) was to express formally the Self-Energy functional as a series expansion in a dynamical screened interaction $W$ (see also Ref. [12]):

$$
W(12)=\int v(13) \epsilon^{-1}(32) d(3)
$$

where 123 are abbreviations for $(1)=\left(x_{1}, t_{1}\right)$ and $v(12)=v\left(r_{1}-r 2\right) \delta\left(t_{1}-t 2\right)$, and $\epsilon^{-1}$ is the inverse dielectric function.

Within the $G W \Gamma$ theory, the poles of the Green Function are N-1 and N+1 excitations of the electron gas (IP and EA, the band structure) that take into account the response of the electron gas to such excitations and includes electron-hole interactions via $\Gamma$ (usually addressed as Vertex). If the electron-hole interactions are neglected $(\Gamma=\delta)$, one gets a set of three equations, one for the irreducible polarizability $P$, one for the screened Coulomb potential $W$ and one for the SelfEnergy:

$$
\begin{aligned}
P(12) & =-i G(12) G(21) \\
W(12) & =v(12)+\int W(13) P(34) v(42) d(34) \\
\Sigma(12) & =i W\left(1^{+} 2\right) G(12)
\end{aligned}
$$


The complexity of the Hedin's equations and the calculation of the screened potential and selfenergy is usually reduced to the calculation of a one-shot correction, G0W0, to some approximate single-particle wave functions and energies, usually Density Functional Theory or hybrids (including HF). Still, the effect of some level of self-consistency has been investigated, see for instance $[13,14]$. The self-consistency, however, tend to provide with less accurate (with respect to experiments) excitations energies [15]. The reason is that, with respect to the whole GW $\Gamma$ theory, GW, alone, neglects the effect of the vertex (neglects $\Gamma$ ). If the starting transition energies are underestimated, as in the case of DFT, $W_{0}^{D F T}$ over-screens with respect to self-consistent $W$, and this compensates for the missing $\Gamma$.
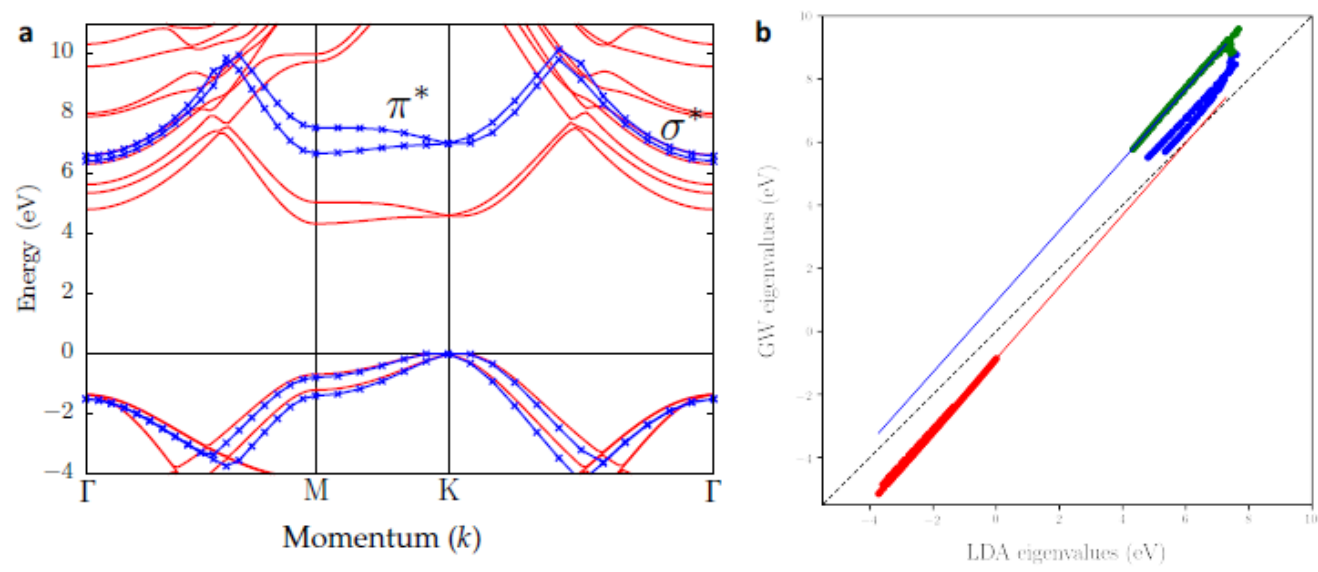

Figure 5.2. (a) LDA (red solid lines) and GW (blue dots) band structure of bilayer BN. (b) GW eigenvalues as a function of LDA eigenvalues. Figure adapted from Ref. [16].

\section{Practical aspects}

There are plenty of further approximations (plasmon-poles for the energy dependence) and numerical tricks (long-wave length limit and the divergence in Coulomb-like integrals [17-20] in plane-wave implementations) that are implemented and sometimes applied, and, that are, in many cases code-dependent. Therefore, unless knowing exactly how a code implements its own GW, it is difficult to compare "numbers" and convergence parameters. In Ref. [21], for instance, the GW gap of silicon is computed with several codes, with differences of the order of $0.3 \mathrm{eV}(20 \%$ of the silicon gap). Ref. [22], investigated the effect of pseudo-potentials versus all-electron in molecules, finding differences of up to $1 \mathrm{eV}$ in Ionization Potentials and up to $0.4 \mathrm{eV}$ in HOMOLUMO gaps.

Finally, in standard implementations, GW calculations involve a sum over a large number of empty states for the Green's function G in the Self-Energy part. The convergence is, however, very slow and requires very careful calculations. Some mitigation strategies have been proposed, but they all rely in further numerical tricks and/or further approximations [23-25].

In general, a reasonable use of GOW0 requires the best starting point and very careful understanding of the approximation and tricks. Nevertheless, materials with bandgap inversion like TiSe2 are especially problematic and require further care for obtaining meaningful results [26]. Figure 5.2 shows the GW calculation of the band structures of bilayer BN. In some cases, the 
GW correction on the whole band structure might be reasonably approximated by a rigid shift of the bands plus some stretching parameter, often referred to as scissor operator, as evidenced in Figure 5.2(b).

\section{GW total energies}

The total energies calculated in GW have only been marginally investigated because of their prohibitive computational cost, see for instance Ref. [27,28].

Non-exhaustive list of freely available open-source GW and Bethe-Salpeter codes: YAMBO [29], ABINIT [8], SaX [30], WEST [31], BerkeleyGW [32] MolGW [XX], GPAW [XX]

\subsubsection{Optical properties}

The calculation of the optical properties requires going beyond ground state calculations performed by DFT. The light-matter interaction is usually approached with a semi-classic description, combining quantum mechanics and classical electromagnetism, assuming the dipole approximation in which the light momentum is negligible. The basics optical properties are described starting from the transition probability induced by the light field, calculated with perturbation theory and Fermi's Golden rule [33].

\subsubsection{Time-dependent DFT (TDDFT): Linear Response}

Time-dependent DFT (TDDFT), either in the frequency domain, or in real time, can describe (neutral) electronic excitations, via the linear response function which relates the TD density to a small external perturbation $\delta \rho=\chi \delta V^{e x t}$. Within TDDFT, electron-electron correlation is included in the time-dependent exchange-correlation potential, $v_{X C}$, which is included in $v_{K S}$. There are two important aspects to address in $v_{X C}$ : non-locality in space, and non-locality in time. The first one is the same as in ground state DFT, and it is taken care of by gradient expansions (GGA, MGGA), HF-KS hybrids for exchange, and RPA-VDW for dynamical correlations. Non-locality in time is absent in ground state DFT, and it should take into account memory effects, e.g., what is the influence of an electron being at point $r$ in space at time $t$, on another electron being at point $r$ ' in space at time $t^{\prime}$ ? While this is precisely what the Green's function addresses, it is by no means obvious how to include it via a functional of the time-evolving density. A good account of these aspects of DFT and TDDFT can be found in [34]. An excellent reference for TDDFT is [35].

In the linear response regime TDDFT can be used to describe the optical properties of a material. Formally a Dyson equation for the linear response function $\chi(\omega, \boldsymbol{q})$ is defined as

$$
\chi(\omega, \boldsymbol{q})=\chi^{K S}(\omega, \boldsymbol{q})+\chi^{K S}(\omega, \boldsymbol{q})\left(v+f^{x c}(\omega, \boldsymbol{q})\right) \chi(\omega, \boldsymbol{q})
$$

where $f^{x c}=\delta v^{x c}[\rho] / \delta \rho$ is the exchange-correlation kernel. Non-locality in time enters its expression. The simplest approach to the TDDFT XC functional is to ignore non-locality in time by proposing a time-local functional, which basically ignores memory effects with $f^{x c}(\omega, \boldsymbol{q}) \approx$ $f^{x c}(\boldsymbol{q})$. For finite systems such as molecules, approximations such as the adiabatic GGA (AGGA), 
which is local in time and semi-local in space, corrects the DFT excitation energies quite well, unless they are of the charge-transfer type. For extended systems the approximation is more questionable, as the corrections introduced are diluted and tend to be ineffective. For example, there is practically no correction to the bandgaps of solids. The use of relatively inexpensive SICTDDFT proved very good at describing ionization and optical spectra of gas-phase targets [36,37]. More sophisticated functionals derived from Green's function approaches like the Bethe-Salpeter equation (BSE) have been also proposed [38]. However, in practice this has the same computational cost of a direct solution of the BSE which we introduce in the following sub-section.
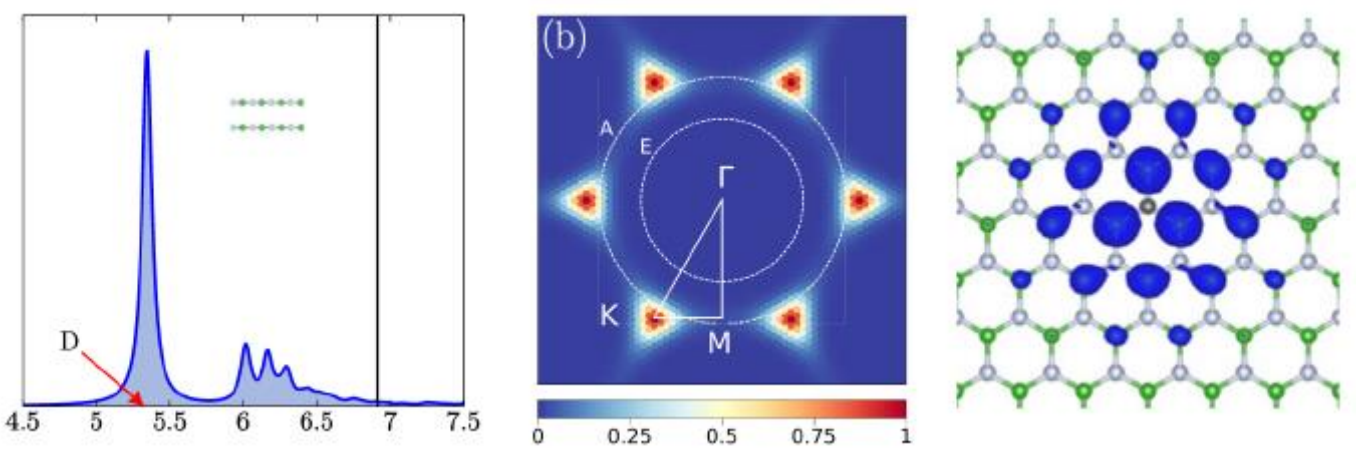

Figure 5.3. (a) Optical Spectra as obtained with GW-BSE of bilayer boron nitride. (b) Excitonic weights in the Brillouin zone. (c) Wave function of the excitonic ground state (hole fixed on top of boron atom). Figure adapted from Ref.[39].

\subsubsection{Electron-hole interactions: The Bethe-Salpeter equation}

The Bethe-Salpeter equation (BSE) in the context of solid-state physics solves the problem of neutral excitations resulting of the Coulomb electron-hole interaction [40]. It is the MBPT version of eq. (5.31), a Dyson equation for the four-point response function $L$ whose space and time contraction gives $\chi$. In practice, BSE is written as an eigenproblem of an effective Hamiltonian $H^{X}$ of two-particle in the basis of electron-hole (e-h) pairs. The Hamiltonian is:

$$
H_{e h, e h^{\prime}}^{X}=\Delta \epsilon_{e h} \delta_{e e^{\prime}} \delta_{h, h^{\prime}}-W_{e h, e h^{\prime}}+V_{e h, e h^{\prime}} .
$$

The diagonal term is the e-h energy differences. The electron-hole energy differences are usually calculated from DFT eigenvalues corrected with the GW approximation. The direct Coulomb interaction is the term $W$ and the exchange interaction is $V$. In general, the direct interaction $W$ is calculated using static screening, i.e., by assuming that dynamical effects in the electron-hole screening and in the one particle Green's function tend to cancel each [41]. In other words, BSE in its standard derivation solves the Hamiltonian for neutral excitons composed by one electron and one hole [42]. Nevertheless, the theoretical framework can be extended to charged excitons like trion states.

The calculation of the BSE Kernel is usually the most demanding part computationally speaking. The solutions of the eigenvalue problem are the excitonic states with energy $\Omega^{S}$ and exciton eigenfunctions with coefficients $A_{e, h, \boldsymbol{k}}^{S}$ in the basis of electron-hole transitions, where $e, h$ denotes the electron-hole bands and $\mathrm{k}$ runs in the full Brillouin zone. The macroscopic dielectric tensor is then directly obtained $[40,43]$, and optical properties like absorption, reflectance, etc... are easily derived [44]. 
Figure 5.3 shows an example of the optical absorption spectra including excitonic effects for a paradigmatic 2D material like hexagonal boron nitride. The optical spectrum of multilayer BN is characterized by a very strong renormalization of the spectra when the excitonic effects are included (black vertical line marks the electronic bandgap without excitonic effects). The excitonic wave functions can be represented in the k-space and in real space, as shown in Figure 5.3(b) and (c), respectively. In the case of the representation in real space, we have to fix either the hole or the electron position in order to plot.

\subsubsection{Phonons and density functional perturbation theory}

So far, we have considered the electronic problem at fixed nuclei, within the BO approximation. Once the electronic problem is solved and the total energy of the system is found in its ground state, the classical forces acting on the atoms can be defined and, minimizing such forces, the equilibrium structure can be obtained. The nuclei then oscillate around such equilibrium positions with characteristic frequencies called phonon frequencies. In general, phonon frequencies are obtained from the solution of the secular equation

$$
\left|\frac{1}{\sqrt{M_{i} M_{j}}} C_{i \alpha, j \beta}(\boldsymbol{q})-\omega^{2}(\boldsymbol{q})\right|=0,
$$

where $M_{i}$ and $M_{j}$ are the atomic masses of atoms $i, j$, and $\alpha, \beta$ indicate the direction of the displacement along the cartesian axis. The dynamical matrix is the second derivative of the total energy with respect the atomic displacements:

$$
C_{i \alpha, j \beta}(\boldsymbol{q})=\frac{\partial^{2} E}{\partial u_{i, \alpha}^{*}(\boldsymbol{q}) \partial u_{i, \alpha}(\boldsymbol{q})^{\prime}}
$$

where $u(\boldsymbol{q})$ denotes displacement of atoms $i, j$ in the cartesian directions $\alpha, \beta$. In density functional perturbation theory (DFPT), the atomic displacements are taken as a perturbation potential and the changes in the electronic density and total energy are calculated self-consistently, analogously to the Kohn-Sham equations $[45,46]$. The main advantage with respect to approaches such as the frozen-phonon scheme is that the calculations of the frequencies for any wavevector $\boldsymbol{q}$ are performed using the primitive unit cell, avoiding the use of supercells [47]. Moreover, DFPT automatically includes mid and long-range interactions.

In addition, the coupling of electrons with the lattice vibrations is fundamental for simulations of carrier dynamics including electron-phonon interaction, phonon-assisted absorption, renormalization of electronic excitation energies and lifetimes [48]. The first-order electronphonon matrix elements account for a large extent of the electron-lattice coupling and are defined as:

$$
g_{m n}^{v, \boldsymbol{q}}(\boldsymbol{k})=\frac{1}{\sqrt{2 \omega_{\boldsymbol{q} v}}}\left\langle\psi_{m \boldsymbol{k}+\boldsymbol{q}}\left|\partial_{\boldsymbol{q} v} V\right| \psi_{n \boldsymbol{k}}\right\rangle
$$

that quantifies the amplitude probability of the scattering process between states $\psi_{m \boldsymbol{k}+\boldsymbol{q}}$ and $\psi_{n \boldsymbol{k}}$, where $\partial_{\boldsymbol{q} v} V$ is the derivative of the self-consistent potential associated to the phonon mode $v$ of 
frequency $\omega$ and wavevector $\boldsymbol{q}$. Efficient ways of calculating electron-phonon matrix elements in dense $\boldsymbol{k}$-grids make use of maximally localized Wannier functions [49]. From the electron-phonon matrix elements it is possible to calculate the electron and phonon self-energies related to the electron-lattice coupling, as described elsewhere [50].

\subsection{Femto-second time scale: electron dynamics}

When the many-body $N$-particle (electrons) ground state $\left|\Psi_{0}^{N}\right\rangle$, or simply $\left|\Psi_{0}\right\rangle$, is perturbed, it ends up in a coherent superposition of many-body states, $\left|\Psi_{I}\right\rangle$, of energy $E_{I}^{N}$. Such superposition determines the ensuing dynamics of the system. After the end of the perturbation, the solution of the time-dependent Schrödinger equation can be written as

$$
|\Psi(t)\rangle=\sum_{I} c_{I} e^{-i E_{I}^{N} t}\left|\Psi_{I}\right\rangle
$$

and is characterized by coherent oscillations at specific frequencies. For small perturbations, the coherent oscillations frequency will be dominated by the energies $E_{I}^{N}$ which enter the poles of the linear response function $\omega_{I 0}=\left(E_{I}^{N}-E_{0}^{N}\right)$. When stronger perturbations are considered the description in terms of linear superposition of states holds, although in general one needs to consider frequencies $\omega_{I J}=\left(E_{I}^{N}-E_{J}^{N}\right)$ which are not limited to the poles of the linear response function. Electron-electron interactions and electron-atoms interactions are responsible for the renormalization of such energies ${ }^{1}$. At this point it is important to distinguish the case of isolated atoms and molecules from extended systems.

In atoms and molecules, since few states are involved, the dynamics can be understood in terms of such linear superposition. It can be modelled either directly computing the wave-function, or using approaches based on the density $n(\mathbf{r}, t)$, such as time-dependent density functional theory (TDDFT), the reduced density matrix $\rho\left(\mathbf{r} \mathbf{r}^{\prime}, t\right)$, or the non-equilibrium Green's function $G\left(\mathbf{r} t, \mathbf{r}^{\prime} t^{\prime}\right)$, like the Kadanoff-Baym Equation (KBE). These quantities will oscillate at the frequencies $\omega_{I 0}$. Methods like TDDFT and KBE become convenient when the size of the electronic system grows.

In extended systems, the number of possible states is so big that, in practice, eq. (5.35) is not useful. To get physical insights in the dynamics, it can be convenient to label the possible states in terms of "effective particles" such as quasi-particles, excitons, or magnons ${ }^{2}$. For example, a state with only a specific level $|\lambda \mathbf{q}\rangle$ populated can be written as $\left|\Psi_{I}^{N}\right\rangle \approx\left|\Psi\left(n_{\lambda}(\boldsymbol{q})\right)\right\rangle \approx\left(\hat{b}_{\lambda \boldsymbol{q}}^{\dagger}\right)^{n}\left|\Psi_{I}^{N}\right\rangle$ with energy $E_{I}^{N} \approx E_{0}^{N}+E\left[n_{\lambda}(\mathbf{q})\right]$. The concept of "effective particles" partially accounts for the electron-electron interaction. Assuming to deal with non-interacting "effective particles" at low densities $E\left[n_{\lambda}(\mathbf{q})\right] \approx n_{\lambda}(\boldsymbol{q}) \omega_{\lambda}(\boldsymbol{q})$ is a reasonable approximation. The early times coherent

\footnotetext{
${ }^{1}$ Also in case the laser frequency is high enough to photo-emit electrons the early time dynamics still involves $\left|\Psi_{I}^{N}\right\rangle$ states, which are however in the continuum of the spectrum

${ }^{2}$ Here we use the jargon "quasi-particle" specifically for the states $\psi_{n \mathbf{k}}$ with energies $\epsilon_{n \mathbf{k}}$ which well describe photoemission experiments, as commonly done in the literature of Many-Body Perturbation Theory. Than we refer, more generally, to quasi-particles, an excitons, magnons, etc ..., as "effective particles"
} 
dynamics are then described in terms of coherent excitons, magnons or phonons. Such coherent states are a linear superposition of different states with different populations.

$$
|\Psi(t)\rangle \approx e^{-i E_{0} t} \sum_{n_{\lambda}(\mathbf{q})} c_{\lambda}(\mathbf{q}) e^{-i n_{\lambda}(\mathbf{q}) \omega_{\lambda}(\mathbf{q}) t}\left|\Psi\left(n_{\lambda}(\mathbf{q})\right)\right\rangle
$$

with $n_{\lambda}(\mathbf{q})=\left|c_{\lambda}(\mathbf{q})\right|^{2}$. If the populations of one or few states $|\lambda \mathbf{q}\rangle$ dominates the sum, then one or few specific frequencies are singled out. This can be measured in pump and probe experiment and corresponds to characteristic oscillations at such frequencies of a macroscopic and classical physical quantity: i.e., polarization for excitons, magnetization for magnons. If a continuum of frequencies enters eq. (5.36) instead, the coherent dynamics quickly "disappears" since oscillations rapidly go out of phase. This leads to the so called "free induction decay" or "free polarization decay". Such decay typically takes place on the time scale of few tens/hundreds of femtoseconds in electronic system. After that, despite coherences are still there, they are not measurable via macroscopic quantities.

This simplified picture, provided by Eq. (5.36), gives physical insight, but it is not useful in practice. Formal approaches like TDDFT and KBE are needed. With such approaches the electronelectron interactions are accounted for via effective potentials. Such potentials, at equilibrium, account for "effective particles", while their adiabatic changes out of equilibrium describes a fully coherent, dynamics which, only at low densities, can be understood in terms of the picture outlined above.

Moreover, even at low densities, non-adiabatic corrections are responsible for changes in the "effective particles" properties, such as energies renormalization $\omega_{\lambda}(\mathbf{q}, t)$, quasi-particle lifetimes $\gamma_{\lambda}(\mathbf{q}, t)$, and the introduction of electron-electron scattering mechanisms. Despite the dynamics of the many-body electronic system remains coherent i.e., can be captured by a pure state, the dynamics in the "effective particles" introduces extra decoherence terms. For the one-body reduced density matrix, the relation $\rho^{2} \neq \rho$ does not hold anymore and the system must be described as a statistical mixture? ${ }^{3}$.

Finally, if the environment is considered, for example due to the interaction with phonons, even the many-body electronic dynamics becomes non coherent the dynamics can only be described in terms of a many-body density-matrix $\mathrm{Q}^{N}$. $\mathrm{Q}^{N}$ evolves according to the Liouville-von Neumann equation ${ }^{4}$ and the system moves towards a non-pure state, i.e. $\left(\mathrm{Q}^{N}\right)^{2} \neq \mathrm{Q}$. Similarly, to the wavefunction case, the use of the many-body density matrix $Q^{N}$ becomes quickly impractical when the size of the system grows and a description in terms of a reduced density matrix, like KBE, is more convenient on short times. Within KBE, electron-phonon scattering is an additional source of decoherence.

Due to all these decoherence mechanisms, the dynamics beyond the first few hundreds of femtoseconds can be often reasonably well described in terms of the semi-classical Boltzmann equation (SCBE) for the populations $n_{\lambda}(\mathbf{q})$ only. Such approach fully neglects electronic coherences. SCBE will be introduced at the end of the present chapter as a special limit of the KBE and developed further in chapters 6 and 7.

\footnotetext{
${ }^{3}$ While exact TDDFT is still valid in this regime, KBE is more prone to describe it, since it is not based on wave-functions.

4 The latter is equivalent to the Schrödinger equation when no environment is present
} 


\subsubsection{Coherent dynamics: TDDFT}

TDDFT allows for the real-time propagation of the electronic density without constraining it to the ground state density. This is particularly useful to describe the initial stage of the irradiation process, in which, e.g., the potential introduced by the moving ionic projectile excites the electronic degrees of freedom. In real-time TDDFT within the Kohn-Sham scheme (TDKS), the electronic orbitals evolve in time but there is no change in the occupations of these orbitals. If they were initially occupied, they remain so, and if they were empty, they will not become occupied during the evolution. The TDKS equations of motion are:

$$
i \partial_{t} \varphi_{n}(\boldsymbol{r}, t)=\left(-\frac{\hbar^{2} \nabla^{2}}{2 m}+v_{K S}[n](\boldsymbol{r}, t)\right) \varphi_{n}(\boldsymbol{r}, t)
$$

where $n(\boldsymbol{r}, t)$ is the time-evolving electronic density and $v_{K S}[n](\boldsymbol{r}, t)$ is the Kohn-Sham potential defined in Section 5.2.1. This includes an external potential that takes into account the interaction of electrons with the nuclei and with other external time-dependent fields like those produced by a laser or by an ionic projectile. Notice that, at variance with the traditional ground state DFT approach, here the electronic density follows its own time evolution. The main advantage of the real time domain, compared to the frequency domain where TDDFT is used to define response functions, is that, at least in principle, any order in perturbation theory can be achieved.

\subsubsection{Laser irradiation, ionization and photoemission}

In the definition of $v^{K S}$, the part of the external potential which describes a laser pulse can be expressed, within the dipole approximation, as

$$
v^{e x t}(t)=\mathbf{E}(t) \mathbf{P}(t)
$$

This expression constitutes the so-called length gauge, where $\mathbf{E}(\mathrm{t})$ is the time dependent electric field at zero momentum $(\mathbf{q}=0)$, while $\mathbf{P}(\mathrm{t})$ is the time dependent polarization constructed from from the KS orbitals. Within TDDFT the exact polarization can be obtained for isolated systems via $\boldsymbol{P}(t)=\int d^{3} \mathbf{r} n(\mathbf{r}, t)$. However, such expression is ill defined for extended systems for which DFT and TDDFT are exact only for finite momentum perturbations. In such cases, D-PolarizationFT (DPFT) and TDDPFT are formally needed [51]. However, the polarization is a many-body operator and can be defined only via a Berry-Phase approach [52]. This is strictly needed in practice whenever physical phenomena beyond the linear regime are needed. Even within TDDPFT however the Drude response of metals cannot be described. An alternative solution is the velocity gauge, where the external potential enters via the replacement $\nabla^{2} \rightarrow(\nabla-A(t))^{2}$ and hence couples to the time-dependent current, which can be formally described within TDCurrent-DFT (TDCDFT). TDCDFT is formally exact for extended systems, metals included, at $\mathbf{q}=0$. However, in practical numerical implementation a number of sum rules need to be carefully fulfilled, which makes the approach problematic [53,54].

For all this reason the first applications of TDDFT where focused on isolated systems or limited to the linear regime. However, also due to the growing interest in ultra-fast electron dynamics, triggered by experimental advances in thegeneration of ultra-short laser pulses, TDDFT have been used and developed for many applications, such as pump and probe experiments, high harmonic 
generation, photoemission experiments in both molecule and complex materials [55-59]. More recently also the response to time-dependent magnetic fields was explored [60]. TDDFT has been also used to model ion irradiation and electronic stopping power. In the next subsection we focus on the latter.

\subsubsection{Ion irradiation and electronic stopping power}

Let us consider an $\alpha$-decay event in which an $\alpha$-particle is emitted with energies of the order of several $\mathrm{MeV}$ (e.g., $5 \mathrm{MeV}$ in the case of ${ }^{238} \mathrm{U}$ ). Due to energy and momentum conservation, the energy of the recoil atom is of the order of $100 \mathrm{keV}$. Since recoil atoms are generally heavy atoms, their velocity tends to be sufficiently low to fall within the adiabatic regime, in which there is no electronic excitation of the target. Strictly speaking, in metallic systems there is electronic excitation at any velocity, but the density of electron-hole pairs is very small at low velocity. Instead, the energy scale is set by the plasmon, which is in the order of several eV. In the case of insulators, the finite energy gap suggests the existence of a velocity threshold below which there is no electronic excitation. In the case of LiF, this has been experimentally [61] and theoretically [62] estimated to be in the region 0.1-0.2 a.u., i.e., 4-8 Bohr/fs. Therefore, the recoil event does not require electronic excitations and can be simulated using classical or first-principles molecular dynamics (see below). Recent works showed a richer threshold structure in insulators [63-66]. The velocity of the $\alpha$-particle, on the other hand, is about $4 \%$ the speed of light, hence about 5 a.u. ( $c=$ 137 a.u.). These velocities fall well into the electronic excitation and ionization regime, independently of the material. Collisions with the target nuclei at these velocities are practically irrelevant due to the exceedingly small cross sections for He-atom interactions. In fact, the maximum of nuclear stopping lies in the region of a few $\mathrm{keV}$, corresponding to about 0.1 a.u. [67], rapidly decreasing for increasing energy due tothe reduction of the interaction time between projectile and target.

Therefore, the description of the initial stages of swift ion irradiation requires the consideration of electronic excitation and ionization in the absence of motion of the target atoms, as these processes occur in a time scale significantly faster than that of nuclear motion. However, in a longer time scale or for low energies and sufficiently close ion-target collisions, the motion of the nuclei becomes important. These two situations can be modelled via real-time TDDFT (rt-TDDFT) simulations, either with fixed nuclei, or using Ehrenfest dynamics (ED, see Section 5.4.2.3).

A central concept in ion irradiation is that of stopping power, defined as the energy loss of the projectile per unit length travelled $(L)$,

$$
S=-\frac{d E_{p}}{d L}
$$

where $E_{p}$ is the kinetic energy of the projectile. The stopping power has two components. The first one, called nuclear stopping $\left(S_{n}\right)$, is due to the collisions of the projectile with the nuclei. It is important at low projectile velocities. The second one, called electronic stopping $\left(S_{e}\right)$, is due to electronic excitation and ionization, and it operates at higher velocities. For historical reasons this quantity is called stopping power, but in fact it is has the units of force. It represents the retarding force due to the energy transfer to the target. 
The rt-TDDFT calculation of electronic stopping power is based on a simple idea first introduced in [63]. Instead of integrating the equations of motion for the nuclei as in ED, the projectile is driven at constant velocity. In the original work of Pruneda et al., the projectile was represented by a moving external potential. In successive works, the projectile was treated explicitly, as any other atom in the system, but forcing it to move with constant velocity while the target atoms were kept fixed at their positions. In ED, the projectile would transfer energy to the electronic excitations while reducing its velocity. However, at typical projectile velocities, this correction represents an extremely small fraction of the projectile's kinetic energy, and no information is lost by keeping the velocity constant. Moreover, this simplifies the simulations because forces on the nuclei do not need to be computed as these will not be allowed to move. One may think that allowing nuclei to move is important, and it certainly is the case within the regime of low projectile velocities when electronic stopping begins to overlap with nuclear stopping. This is also important when the path of the projectile passes close to the host atoms, being moderately relevant for light projectiles like protons or $\alpha$-particles but becoming increasingly important for heavier atoms that cannot move easily through the target without colliding with host atoms. Under the conditions just described, the projectile will travel through the host material at constant velocity, while the electronic energy increases at a spatial rate given by

$$
S_{e}=\frac{d E_{K S}}{d L}
$$

This is precisely the electronic stopping power, i.e. the amount of energy deposited per unit distance travelled by the projectile. Therefore, the electronic stopping power is given by the slope of the KS energy vs. projectile displacement curves, that are shown in Figure 5.4 for the case of a proton travelling along a $\langle 100\rangle$ channel in $\mathrm{Al}[81]$.

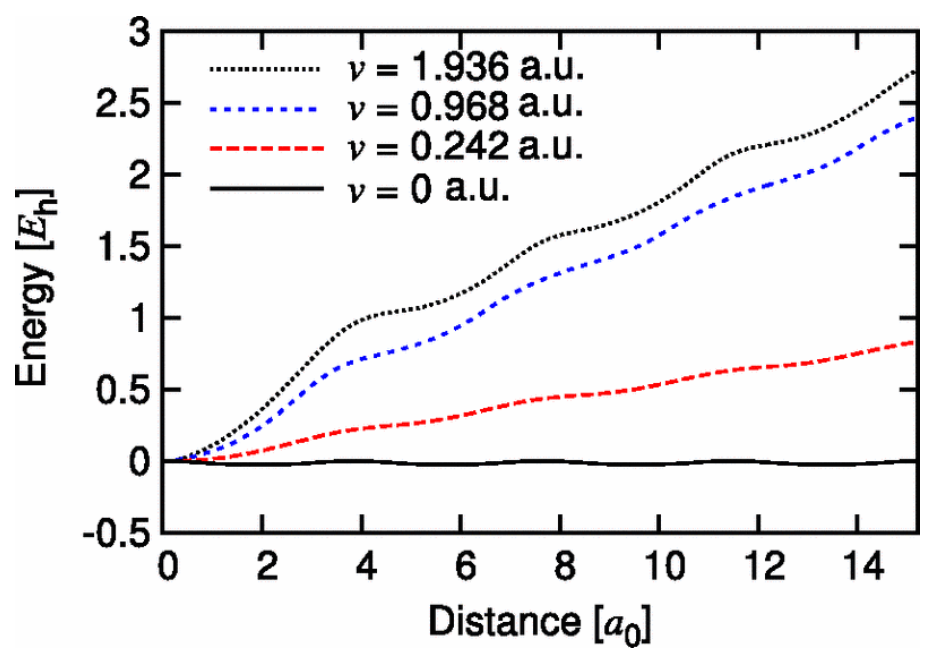

Figure 5.4. Energy deposited by a moving proton into the electronic subsystem of bulk Al as a function of travelled distance, for different velocities. Notice the oscillations due to the periodicity of the lattice. The slopes correspond to the electronic stopping power. Figure reproduced from [68].

There are several aspects to discuss about this procedure. Firstly, as can be seen in Figure 5.4, the evolution of the KS energy with displacement is not a straight line. It will generally exhibit oscillations that reflect the passage close to the host atoms. In the case of a regular solid, this will 
correspond to the periodicity of the crystal lattice. Here, the relative direction between the path of the projectile and the lattice vectors of the host is important. When they are perfectly aligned, this is called hyperchannelling. More generally, if the projectile moves at a small angle with respect to the lattice vector, it will be periodically smoothly reflected by the host atoms, thus exhibiting an oscillatory trajectory without abandoning the channel. These are called channelling trajectories. To simulate these, one would need very large systems. Instead, it is more convenient to simulate hyperchannelling trajectories and average over the impact parameter, i.e. moving away from the center of the channel (off-center trajectories). Under these conditions the projectile samples regions with larger electronic density of the host, and hence the stopping power increases, as it is shown in Figure 5.5.

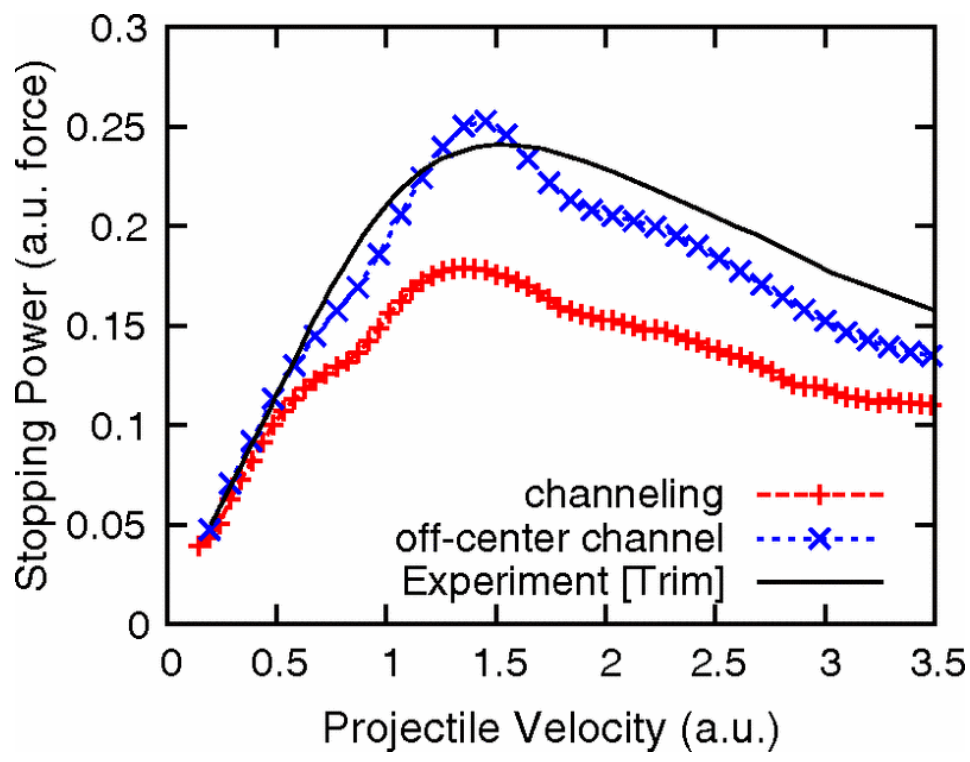

Figure 5.5. Electronic stopping power for protons in bulk $\mathrm{Al}$ as a function of velocity. SRIM values (black line) are compared to a hyperchanneling trajectory (red) and a channelling off-centre trajectory (blue). Figure reproduced from [68].

An important aspect to consider for off-center trajectories is that core electrons can start to play a role. A simple way to treat this is by includingthe shallower core states, usually called semi-core states, explicitly into the valence. Schleife et al. showed that this is important even for protons [69]. Averaging over impact parameter leads to stopping powers that are about a factor of 2 larger than the hyperchannelling result through the center ofthe channel [70]. For amorphous or liquid systems, since the irradiation process is so fast, the projectile will see a static picture of the host. Therefore, to compute the stopping power it will be necessary to averageover several trajectories. This issue has been recently addressed in [71].

Since the initial proof of concept, this approach has been used for an increasing number of applications. The initial focus was on simple crystalline solid like pure elements, salts, and oxides. These calculations were always compared to SRIM tables (SRIM) with the initial aim of assessing the reliability ofthe methodology, and more recently the other way around, to verify the accuracy of the methods used in SRIM for compounds, such as the Bragg additivityrule and the bond and core approximations [72].

Another important aspect that has been recently investigated is the role of inner electronic shells in stopping, i.e., core and semi-core electrons. It has been shown that for heavy projectiles as it the 
case of self-irradiation of $\mathrm{Ni}$, thestopping curve at high energies converges slowly with the number of explicit electrons in the description, particularly those in the projectile [73]. This raises the issue of the use of atomic pseudo-potentials in stoppingcalculations. For heavy projectiles at high energies, these must allow for an increasing number of explicit electrons from inner shells.

Along the same lines, but computationally more efficient is the time-dependentdensity-functional tight-binding (TD-DFTB) approach, which proved useful fordescribing electron dynamics in large systems [74,75]. However, such approaches are limited in the description of the excitation spectrum at high energies, and they are hence questionable when it comes to applying them to the calculation of electronic stopping. They are more useful to describe energy redistribution following optical excitation, e.g., as in visible and near UV (3-4 eV) irradiation.

\subsubsection{Quantum kinetics: the Kadanoff-Baym equations}

The Kadanoff-Baym Equation (KBE) derives from the Dyson equation for the one body Green's function $G$ written on the Keldish contour. Projecting back the Dyson equation to the real axis, an EOM for the lesser green function $G^{<}\left(t, t^{\prime}\right)$ is obtained which describes the exact quantum kinetics of the system. The electron-electron and electron-phonon interactions are captured via the many-body self-energy $\Sigma$. Within the so called generalized Kadanoff-Baym ansatz (GKBA) a closed EOM for the density matrix $\rho(t)=-i G^{<}(t, t)$ can be obtained.

$$
i \partial_{t} \rho(t)=-i\left[h^{e q}+\Delta \Sigma^{s}[\rho(t)]+v^{e x t}(t), \rho(t)\right]-I[\rho](t)
$$

If only the static part of the self-energy, $\Sigma^{s}$, is retained then $I[\rho](t)=0$. Such equation can be conveniently represented in the equilibrium KS basis set, i.e. $\rho_{n m \mathbf{k}}=\left\langle\psi_{n \mathbf{k}}^{0}|\rho(t)| \psi_{m \mathbf{k}}^{0}\right\rangle$. Its solution has been shown to describe the formation of coherent excitons [76] if $\Sigma^{s}=\Sigma^{h s e x}$, the Hartree plus Screened Exchange (HSEX) self-energy with the screening frozen to its equilibrium value, is used. TD-HSEX is equivalent of the Bethe-Salpeter Equation in the low pumping regime.
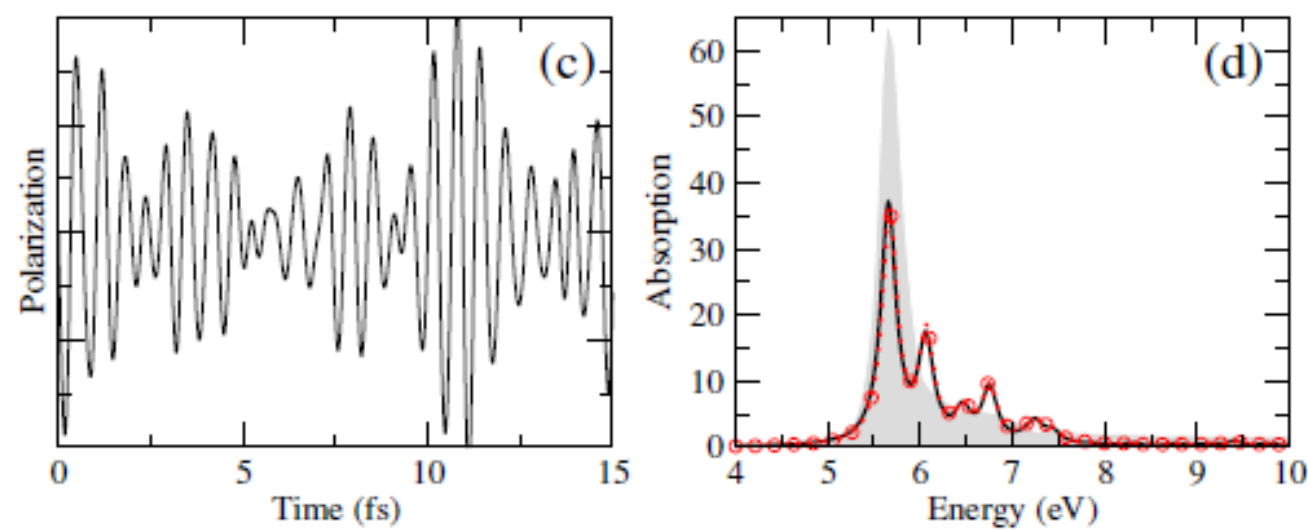

Figure 5.6. Figure adapted from Ref.[76]. It shows the time dependent polarization (panel a) obtained propagating the density matrix and the absorption (panel b) spectrum derived from its Fouriertransform (red circles). The latter is also compared with a BSE calculation andexperimental data from Ref. [76].

In Figure 5.6, adapted from Ref. [76], we show numerical results on hexagonal Boron Nitrite (hBN) comparing absorption computed from the standard GW+BSE scheme with TD-HSEX approach. 
The $\Delta \Sigma^{h s e x}(t)$ term shifts the spectrum from the IP poles to the excitonic ones. Broadening of the peaks is obtained by introducing a smearing parameter $\eta$ when performing the Fourier transform of the polarization constructed from the density matrix.

Although conceptually different, TDDFT in the adiabatic approximation can be seen as a possible approximation to the static self-energy. Using $\Sigma^{S}[\rho(t)]=v_{x c}^{\text {adia }}[n(t)]$ eq. (5.41) one would obtain the TDDFT density matrix. Selecting as a starting point the equilibrium KS density matrix (i.e. $\rho_{n m \mathbf{k}}=\delta_{n, k}$ if $n$ occupied, 0 otherwise) the resulting $\rho_{n m \mathbf{k}}(t)$ is equivalent to propagate the TDKS equations described in the previous section and later constructing

$$
\rho_{n m \mathbf{k}}(t)=\sum_{j \in o c c}\left\langle\psi_{n \mathbf{k}}^{0} \mid \psi_{j \mathbf{k}}(t)\right\rangle\left\langle\psi_{j \mathbf{k}}(t) \mid \psi_{n \mathbf{k}}^{0}\right\rangle
$$

In the IP case, i.e. $\Sigma^{S}=0$, the diagonal elements of the density matrix can be interpreted as the occupations of the equilibrium KS orbitals, while the off-diagonal elements represent the coherences between two orbitals. This is the starting point for the connection with the semiclassical Boltzmann equation we will discuss in the next section. Together with eq. (5.42), this also shows how the time propagation of occupied-only orbitals can describe the generation of nonequilibrium occupations, via the external field, on the equilibrium KS band structure. The interpretation in terms of occupations however is not formally possible beyond IP.

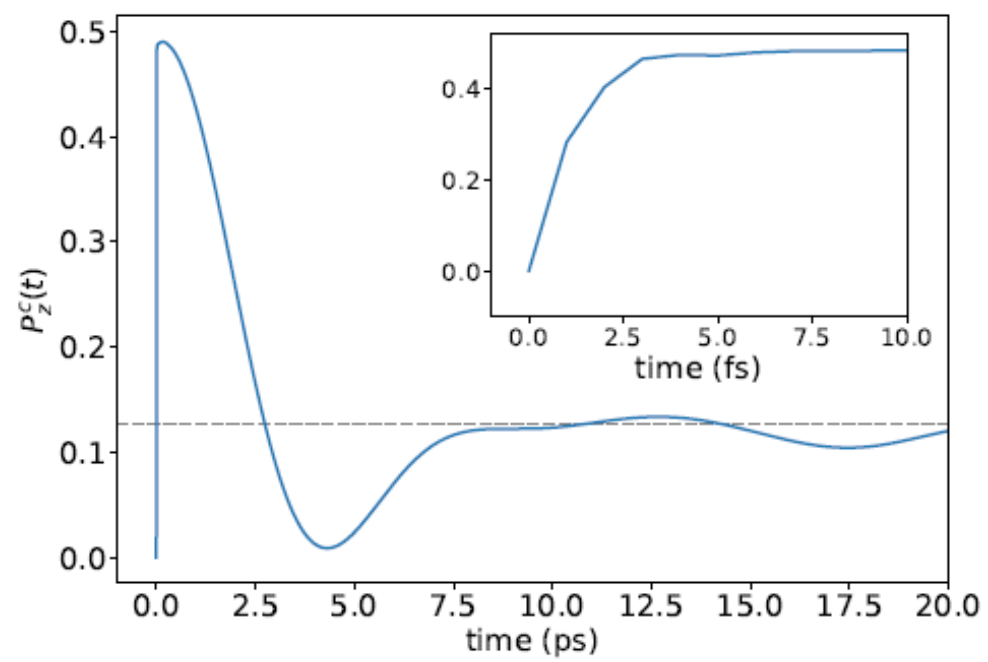

Figure 5.7. An example of free induction decay. Degree of spin polarization of electrons Pc, injected in the conduction band of GaAs via a circularly polarized pulse, quickly decays in absence of any scattering mechanism. The horizontal dashed line represents the non-coherent contribution due to the populations only. In the inset a focus on the generation during the optical pulse. Reprinted with permission from Ref. [77].

The GKBA-KBE dynamics contains both coherent and non-coherent terms. In the next section we discuss how the SCBE, which describes instead the non-coherent dynamics only, can be derived from such equation. Within the SCBE the coherent terms are neglected. This is reasonable on long time scales, because the contribution of these terms tends to vanish due to dephasing. In theIP picture dephasing can be understood in terms of the so called "Free Induction Decay" or "Free Polarization Decay". It happens whenever coherent terms involve a continuum of frequencies which quickly get out of phase [78]. The mechanism has been realized also with ab-initio simulations [77] (see Figure 5.7) The process is even faster if the interaction between particles is accounted for. 
Electron-electron interaction leads to the generation of morepossible excitation frequencies which, in the language of KBE (and TDDFT) are captured by the frequency dependence/memory of the Self-energy (and $v^{x c}$ potential). Only in case of isolated poles coherences can be measured for longer times.

\subsubsection{Incoherent dynamics and connection to the Boltzmann equation}

The connection to the semiclassical Boltzmann equation (SCBE) can be made starting from the KBE-GKBA equation and choosing $\Sigma^{\mathrm{s}}=0$, but including dynamical correlations $\Sigma^{\mathrm{d}}(\mathrm{t}, \mathrm{t})\left[\mathrm{G}^{<}\right]$in the collision integral. The approximations needed to obtain the SCBE are discussed in the literature in general [79], and in its ab-initio implementation [80,81]. Expanding the KBE-GKBA with respect to the perturbation, a set of two equations is obtained:

$$
\begin{gathered}
\partial_{\mathrm{t}} \rho_{n m k}^{(1)}(\mathrm{t})=-\mathrm{i}\left[\mathrm{h}^{\mathrm{eq}}+\mathrm{U}^{\text {ext }}(\mathrm{t}), \rho^{(0)}(\mathrm{t})\right]_{\mathrm{nmk}} \\
\partial_{\mathrm{t}} \rho_{n n k}^{(2)}(\mathrm{t})=-\mathrm{i}\left[\mathrm{U}^{\mathrm{ext}}(\mathrm{t}), \rho^{(1)}(\mathrm{t})\right]_{n n k}-\mathrm{I}\left[\rho_{n n k}^{(2)}(\mathrm{t})\right]
\end{gathered}
$$

where the first order equation involves only $n \neq m$ (this results from the approximation that the equilibrium density matrix is diagonal), and describes the generation of coherences, while the second equation, $\rho_{n n k}^{(2)}(t)=f_{n k}(t)$ describes the dynamical evolution of the occupations with a source term $\left[U^{\text {ext }}(t), \rho^{(1)}(t)\right]$, and a collision integral $I\left[\rho_{n n k}^{(2)}(t)\right]$ which is almost identical to the one heuristically derived in the formulation of the SCBE and which satisfies the detailed balance.

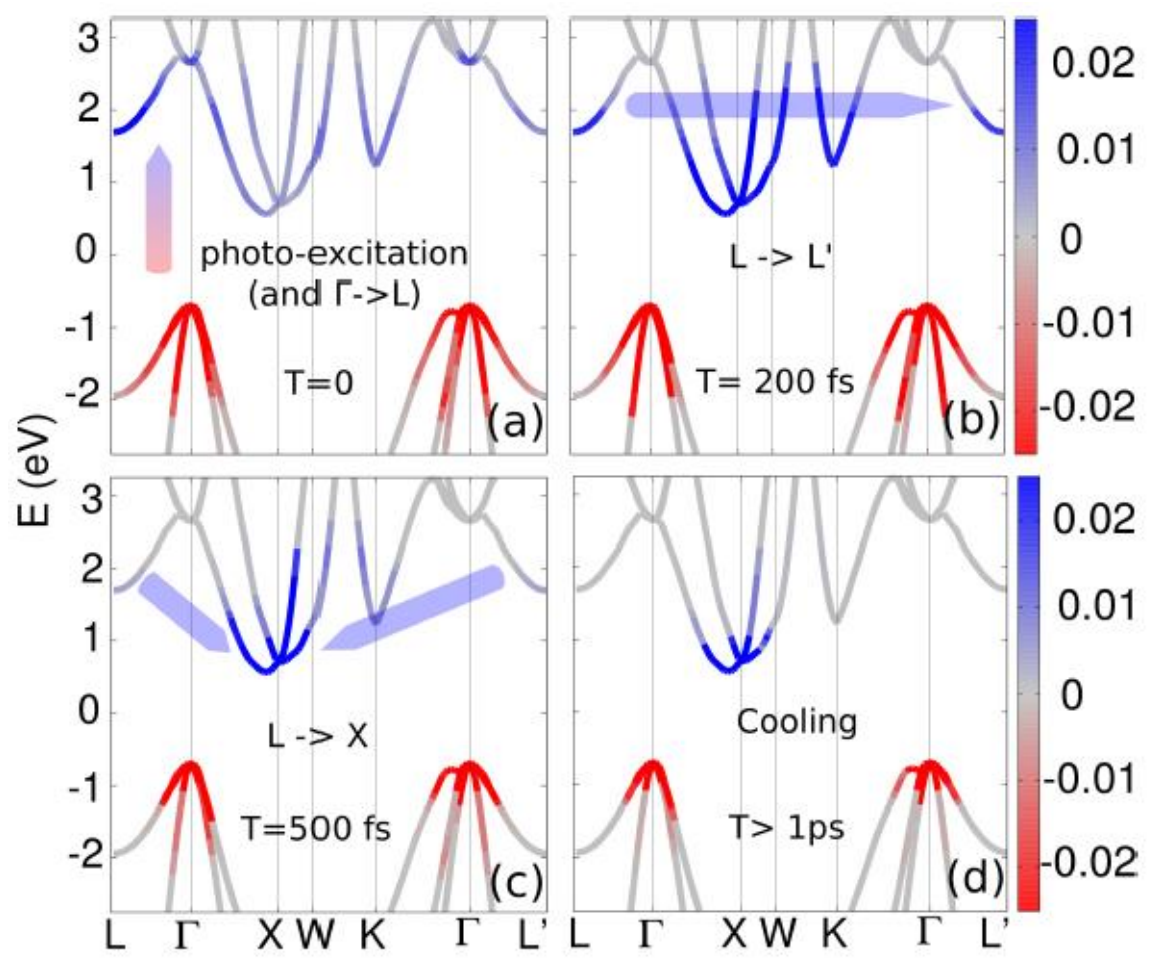

Figure 5.8. Adapted from Ref. [82]. The time dependent occupations on the band structure are shown for the case of bulk silicon under the action of an external laser pulse centred at the optical gap of the material. 
An example of the application of this scheme from first principles can be found in Ref. [82]. The authors study (see Figure 5.8) the time evolution of the occupations in bulk silicon during the action of an external laser pulse, and with a collision integral derived using the electron-phonon self-energy for $\Sigma_{d}$. The derivation within MBPT naturally leads to compare the concept of quasiparticles lifetime, from equilibrium MBPT, $\gamma^{e q}=\operatorname{Im}[\Sigma]$, with the dynamics resulting from the SCBE. Indeed, the equilibrium lifetime naturally brings to a ("multi-band") relaxation time approximation (RTA) equation of the form $\partial_{t} f_{n k}(t)=-\gamma_{n k} f_{n k}(t)$. The collision integral, instead, can be expressed as

$$
I_{n n \mathbf{k}}[f](t)=-\gamma_{n \mathbf{k}}^{(e)}(t) f_{n \mathbf{k}}^{(e)}(t)+\gamma_{n \mathbf{k}}^{(h)}(t) f_{n \mathbf{k}}^{(h)}(t)
$$

where the detailed balance is ensured by the fact that the resulting lifetimes depends on the occupations and by the existence of two lifetimes for "electrons in" and "electrons out" processes. For the electron-phonon case we obtain

$$
\begin{gathered}
\gamma_{n \mathbf{k}}^{(e)}=\frac{2 \pi}{\hbar} \sum_{v \boldsymbol{q} m} \sum_{I= \pm 1} \frac{\left|g_{n m \boldsymbol{k}}^{v \boldsymbol{q}}\right|^{2}}{N_{\boldsymbol{q}}} \delta\left(\Delta \epsilon_{n m \boldsymbol{k}}^{\boldsymbol{q}}+I \omega_{\lambda \boldsymbol{q}}\right)\left(n_{\lambda \boldsymbol{q}}+\frac{1-I}{2}\right)\left(1-f_{m \boldsymbol{k}+\boldsymbol{q}}\right), \\
\gamma_{n \mathbf{k}}^{(h)}=\frac{2 \pi}{\hbar} \sum_{v \boldsymbol{q} m} \sum_{I= \pm 1} \frac{\left|g_{n m \boldsymbol{k}}^{v \boldsymbol{q}}\right|^{2}}{N_{\boldsymbol{q}}} \delta\left(\Delta \epsilon_{n m \boldsymbol{k}}^{\boldsymbol{q}}+I \omega_{\lambda \boldsymbol{q}}\right)\left(n_{\lambda \boldsymbol{q}}+\frac{1+I}{2}\right) f_{m \boldsymbol{k}+\boldsymbol{q}}
\end{gathered}
$$

The full SCBE can be compared with the RTA via the definition of effective NEQ lifetimes:

$$
\bar{\gamma}_{n \mathbf{k}}^{(e / h)}(\mathrm{t})= \pm \frac{\gamma_{n \mathbf{k}}^{(e)}(t) f_{n \mathbf{k}}^{(e)}(t)-\gamma_{n \mathbf{k}}^{(h)}(t) f_{n \mathbf{k}}^{(h)}(t)}{f_{n \mathbf{k}}^{(e / h)}(t)}
$$

which allows to rewrite the equation for the occupations in the form

$$
\partial_{t} f_{n \mathbf{k}}^{(e / h)}(t)=-\bar{\gamma}_{n \mathbf{k}}^{(e / h)}(\mathrm{t}) f_{n \mathbf{k}}^{(e / h)}(t)
$$

The complex SCBE dynamics is now absorbed by the NEQ lifetimes definition.
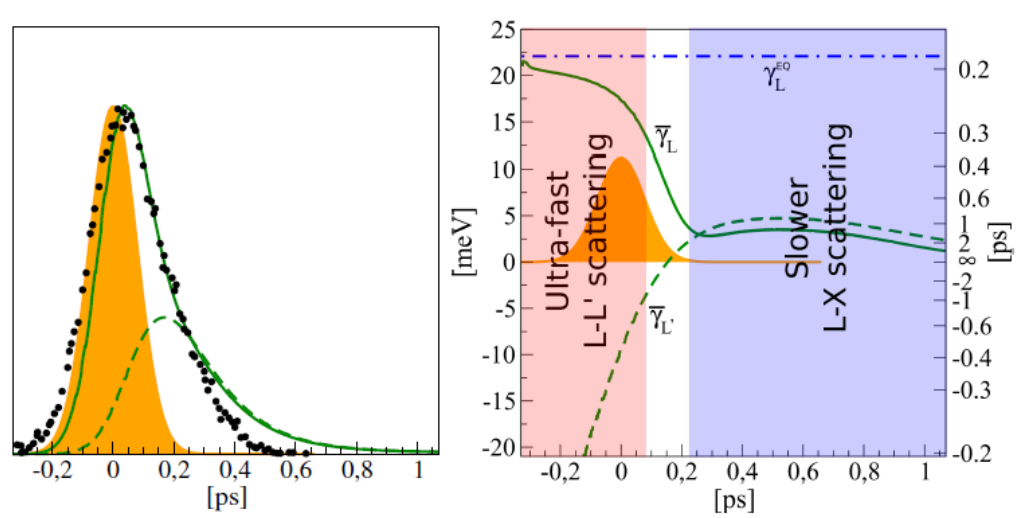

Figure 5.9. The equilibrium lifetime, $\gamma \mathrm{EQ}$ (blue dot-dashed line), is compared with the time dependent out-ofequilibrium lifetimes defined in Eq. for the L1 and L1 states. Due to the symmetry breaking induced by the laser pulse (the orange shadow represents its Gaussian envelope) we have two in-equivalent lifetimes at L1 (green line) and L1 (green dashed line). Their relative intensity defines the ultra-fast $(\gamma \mathrm{L} » \gamma \mathrm{L})$ and the slow $(\gamma \mathrm{L} \approx \gamma \mathrm{L})$ time regimes. 
In Figure 5.9 the NEQ lifetime for bulk silicon at the $L$ point in conduction compared with the equilibrium one. As a general result the NEQ lifetime is always smaller that the equilibrium one. In particular the equilibrium lifetime is a good representation of the dynamics only when the system is in a strongly NEQ situation with electrons mainly localized nearby the few $L$ point(s) in the BZ. It is however remarkable that the NEQ lifetimes at the $L^{\prime}$ points, i.e. the equivalent points of the BZ where no carriers are injected, becomes negative in such situation. As soon as the electrons spread in a quasi-equilibrium distribution the lifetimes at $L$ and $L^{\prime}$ becomes nearly equivalent and the dynamics becomes much slower.

Another advantage of the formulation within NEQ-MBPT is that the obtained occupations can be used to construct the NEQ response function and thus to describe pump and probe experiments via an approximation of $\chi^{n e q}\left[G^{<}\right] \approx \chi^{n e q}\left[f_{n \boldsymbol{k}}(\tau)\right](\omega)$. This approach has been used to compute the transient reflectivity in bulk silicon from first principles [83] and the transient transmission of molybdenum disulphide [84]. Results are shown in Figure 5.10 for the case of bulk silicon. Computing the response function using the occupations at delay $\tau$ makes possible to isolate different contribution to the transient signal and in particular the changes in the screening induced by the neq occupations. The NEQ response function can then be computed for selected delays $\tau$ between the pump and the probe within the GW+BSE scheme. In Figure 5.10 it is shown that the main contribution to the transient reflectivity signal in bulk silicon is due to the update of the screening. The main feature inthe spectrum is due to the optical gap renormalization defined in Ref. [83] as the sum of two separate and computing effect: the renormalization of the band gap and the renormalization of the electron-hole binding energy.
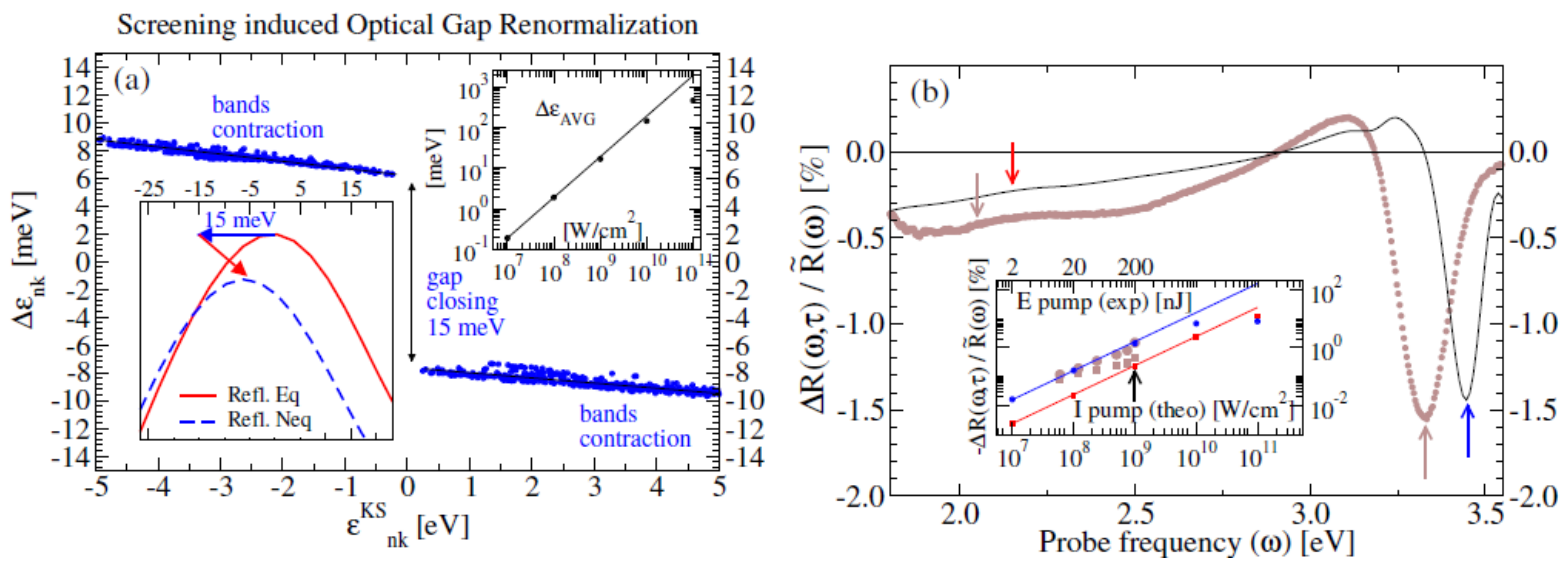

Figure 5.10. Renormalization of the quasi-particles energies (panel a) from the COHSEX self-energy due to the screening of the non-equilibrium carrier's density. The change induced in the reflectivity near the main peak is shown in the inset (blue arrow) together with the effect due to the renormalization of the electron-hole interaction (red arrow). Transient reflectivity (panel b) for a pump intensity of $109 \mathrm{~W} / \mathrm{cm} 2$ and $\tau=200 \mathrm{fs}$; the variation of the BSE poles due to the screening of the non-equilibrium carrier's density is considered. In the insets the change in the gap (panel a) and the signal at $3.45 \mathrm{eV}$ (panel b) are shown as a function of the pump peak intensity. Theoretical results are compared with experimental data (brown circles).

\subsubsection{Beyond quasi-particles}

Both coherent and non-coherent dynamics can be extended beyond the approximation of noninteracting electrons and holes, to describe the dynamics of complex particles like excitons and magnons. In the non-coherent regime this can be done heuristically, for example defining a semiclassical Boltzmann equation for the exciton populations coupling with the phonons. NEQ- 
MEBT offers a formal way to define exciton dynamics equations, exciton populations, and exciton lifetimes [85]. This topic is at the forefront of the present research, expecially within the ab-initio community, with some first promising results $[86,87]$ and many possible applications. An example of the excitonic lifetimes is shown in Figure 5.11.

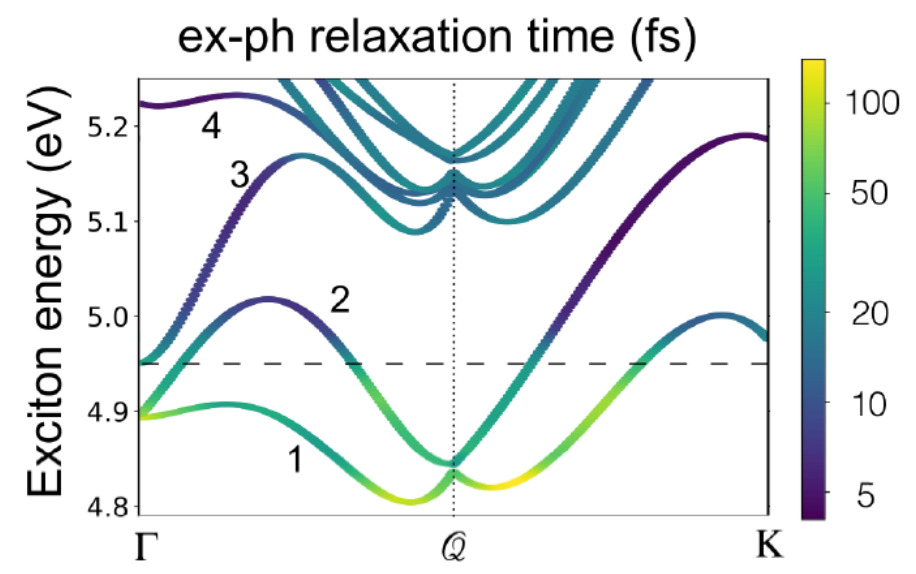

Figure 5.11. Excitonic lifetimes of monolayer hBN computed fully ab-initio, represented on the excitonic band-structure. Figure adapted from Ref. [87].

\subsection{Pico-second time scale and atomic motion}

\subsubsection{Atomic motion and ion irradiation}

The next step in the description of irradiation processes involves the inelastic transport of excited electrons through the target. While electromagnetic radiation is generally quite spatially homogeneous, ion irradiation is very directional. Ions produce a cylindrical track in which the electrons are excited, moving radially outwards from the track. In the stopping simulations described in Section 5.3.1.2, it has been observed an outward propagating electronic density wave. In the longer run, such excited electrons will deposit their energy back into the nuclear subsystem in a time scale that depends on the material and on the energy of the excitations. Traditionally, the energy loss process has been described in terms of a random walk of classical electrons, which is a good approximation at high energies. The random walk is equivalent to a hydrodynamic description in terms of a diffusion equation [88]. This approach, however, becomes questionable at low kinetic energies when the quantum character of the electrons becomes important, typically under $50 \mathrm{eV}$. Notice that the average energy of secondary electrons produced by electron impact ionization is of the order of $20 \mathrm{eV}$, precisely in the quantum regime. Therefore, the use of random walks rests on dangerous waters.

The resulting initial electronic distribution can then be converted into an initial condition for a classical molecular dynamics simulation. To this end, the simplest idea is to deposit the electronic energy back locally as kinetic energy for the nuclei. This is the essence of the thermal spike model [89], that has been successfully applied to model the damage ensuing ion irradiation [90]. In metallic systems, however, the excited electrons can diffuse away due to their high electronic conductivity. They will come back sooner or later in order to neutralize the system. However, if this takes long enough, the (charged) nuclei will experience a strong repulsive electrostatic 
interaction leading to a vigorous radial motion, or Coulomb explosion. The occurrence of one or the other scenario depends on the characteristics of the material, e.g., $\mathrm{Cu}\left(\sigma=5.9 \times 10^{7} \mathrm{~S} / \mathrm{m}\right)$ behaves quite differently from $\mathrm{Ni}\left(\sigma=1.4 \times 10^{7} \mathrm{~S} / \mathrm{m}\right)$. In classical simulations of radiation cascades (see Chapter 9), the combined effect of electronic excitation and decay is modelled using a Langevin approach that includes a friction term that reduces the velocities of the atoms and a random force that mimics the electron-phonon interaction in a stochastic way. Such decay rates can be interpreted in terms of probabilities given by Fermi Golden Rule, i.e., within timedependent quantum perturbation theory. In Langevin dynamics these two terms compensate each other leading to thermal equilibration at a desired temperature. In the methodology developed by Dorothy Duffy and coworkers, the energy transferred to the electronic degrees of freedom is collected in an electronic temperature field that is governed by a diffusion equation [91]. This approach combines the two-temperature model with molecular dynamics (TTM-MD). The latest developments in this field connect the friction coefficients to TDDFT calculations like those in Section 5.3.1.2, enforcing the reproduction of the electron-phonon lifetimes computed using ground-state density-functional perturbation theory [92]. This is the state-of-the-art in the field of classical simulations.

One of the original limitations of this approach is that interatomic forces are generally described through a classical force field that is independent of electronic excitation. Typically, this will be an embedded atom model (EAM) for metals, a Tersoff potential for semiconductors, or a CoulombBuckingham potential for ionic systems (e.g., ceramics). Recently, in [93] the authors used an electronic-temperature dependent force-field based on an embedded atom model, which was described in [94]. The force field was parameterized following a methodology proposed in [95]. The EAM functional form was retained. The pair potential and the Finnis-Sinclair form of the embedding function were kept, but the embedding function was allowed to depend on the electronic temperature. This method, which is described in more detail in Chapter 9 in this book, assumes the existence of a well-defined, largely homogeneous, quasi-equilibrium electronic temperature, whose time evolution can be described through a diffusion equation. This, however, is a poor description when the electronic excitations are local, and remain localized in space for a certain period of time. This is what is likely to happen in the case of ion irradiation of insulators, in which electrons can remain in localized excited states for sufficiently long times for the nuclei to feel the weakening of the interatomic potential and start moving. If the excitation is intense enough, then nuclear motion can lead to cold melting or amorphization. In this case the thermal spike model becomes questionable.

While the methodologies described above approach the question of the energy transfer between electronic and nuclear degrees of freedom after irradiation, they only incorporate the electronic component via an energy reservoir (Langevin) or an electronic temperature classical field (TTMMD). Not only is the electron dynamics ignored in these, but also the correlated motion of electrons and nuclei.

Further progress in this direction requires incorporating explicitly the electronic component, while introducing the nuclear dynamics requires also addressing the issues of electron-nuclear correlation and the quantum character of the nuclei. In the following Sections we introduce a few approaches that accomplish this task in various ways and are useful in different situations. 


\subsubsection{Classical nuclear motion}

\subsubsection{Adiabatic molecular dynamics}

The simplest way to introduce the electronic component along with the atomic motion is to consider that the electrons follow instantaneously the motion of the nuclei, adjusting their wave function (Hartree-Fock) or density (DFT) so that it represents the electronic ground state along a molecular dynamics trajectory. If this ground state electronic structure optimization is carried out at every step [96], the scheme is called Born-Oppenheimer molecular dynamics (BOMD), because the electronic component moves strictly on the Born-Oppenheimer surface, excluding any kind of transitions between electronic states. This scheme requires an excellent convergence of the electronic component at each MD step. The equation of motion for the nuclei is the Newtonian equations

$$
M_{I} \ddot{\boldsymbol{R}}_{I}(t)=-\left\langle\nabla_{I} \widehat{H}(\boldsymbol{R})\right\rangle
$$

Within BOMD $\left\langle\nabla_{I} \widehat{H}(\boldsymbol{R})\right\rangle$ is replaced with $\nabla_{I} E_{K S}[n](\boldsymbol{R})$, the Kohn-Sham energy calculated for the time-evolving nuclear configuration $R$. Since the electronic density is assumed to be instantaneously in the ground state, then the gradient $\nabla_{I}$ also involves the gradient of the electronic density $n(R)$.

\subsubsection{Car-Parrinello dynamics}

A related scheme was proposed in 1985 by Car and Parrinello, within the context of DFT electronic structure. Here, the Kohn-Sham orbitals are allowed to evolve according to a second order fictitious dynamics. The orbitals are assigned a mass that makes them oscillate at frequencies higher than those of the atomic motion. Therefore, the system is never on the Born-Oppenheimer surface, but it is always very close and oscillating around it [97]. This is accomplished by introducing the Kohn-Sham orbitals as dynamical variables in an extended Lagrangian formalism, whose properties have been thoroughly studied in [98]. Typical integration time steps are of the order of fs. This scheme is called Car-Parrinello molecular dynamics (CPMD). A huge number of systems have been studied using either BOMD or CPMD (which are equivalent in practice). In both approaches there is a strong correlation between electronic and nuclear motion, as electrons are slaves of nuclear motion.

BOMD or CPMD are useful and reliable when the time evolution of the system is adiabatic, i.e. there are no transitions between electronic states or, in other words, in the absence of electronic excitation. Therefore, within the irradiation context, these are only useful once the excitation has decayed completely into atomic motion or when there is a constant and homogeneous finite electronic temperature leading to a thermally averaged electronic density. In this case, the KohnSham orbitals are weighted with the Fermi-Dirac distribution.

The equation of motion for the nuclei is the same as for BOMD, however the gradient $\nabla_{I}$ is now to be applied only to the terms in the Hamiltonian that depend explicitly on the nuclear coordinates, as the electronic density has its own time evolution and is treated as an independent variable. 


\subsubsection{Ehrenfest dynamics}

While the CPMD scheme involves the dynamical evolution of the KS orbitals, this is not the actual electron dynamics because the orbitals do not follow the time-dependent Kohn-Sham (TDKS) equations. Moreover, the CPMD approach requires the strict orthonormalization of the KS orbitals at every MD step for stability. To simulate the proper electron dynamics, Theilhaber developed a scheme to integrate the TDKS equations without the need of orthonormalization, using the Suzuki-Trotter split operator method. This early implementation of rt-TDDFT was devised to simulate liquid metals and degenerate plasmas [99]. In this work, the nuclei moved according to a Newtonian dynamics in which the forces on the atoms were calculated in correspondence with the instantaneous, time-evolving electronic density, which is precisely Ehrenfest Dynamics (ED), i.e., the simplest way to combine electronic and nuclear dynamics.

This work was followed by a series of articles on Ehrenfest dynamics by Alonso et al. [100]. The practical implementation is quite similar to CPMD, but since now the electronic dynamics is real, the integration time step should correspond to the real electronic mass, i.e., in the order of attoseconds instead of fs. Therefore, ED simulations reach total times about a thousand times shorten than BOMD of CPMD simulations. The picosecond time scale in ED is only achievable for fairly small molecular systems, i.e., the photoisomerization of small molecules [101].

In extended systems ED was successfully applied to describe the generation of coherent phonons [102] in bulk silicon. Recently the approach was extended to describe also light propagation with a multi-scale set of equations (Maxwell + TDDFT + ED) able to deal in real space with long wavelength perturbations. The approach is very demanding, and the application was done on diamond focusing on a single dimension of propagation [103].

As in BOMD and CPMD, in ED there is a strong correlation between electronic and nuclear motion. On the one hand, TDDFT remembers too much of the previous evolution due to the lack of electron-electron collisions. Hence, the electron dynamics is fully coherent, and electrons never equilibrate to the Fermi- Dirac distribution. On the other hand, in ED the nuclear dynamics correlates directly to the instantaneous electronic density, ignoring fluctuations on both sides. In other words, the electron-nuclear dynamics is coherent, not allowing for inelastic processes. Part of the blame for this should be put in the classical description of the nuclear degrees of freedom. In any case, progress beyond ED requires approximations for the electron-nuclear correlation.

\subsubsection{Beyond TDDFT}

The coupled dynamics of electrons and ions described within ED is fully coherent. It is coherent because the atoms are described classically on the one hand, and, on the other hand, the electrons evolve via adiabatic interactions. Indeed, the exchange-correlation potential $v^{x c}$ of DFT is evaluated within the adiabatic approximation. One possible way forward could be to go beyond TDDFT and couple the nuclear dynamics, i.e., eq. (5.50), with the KBE-GKBA for the electronic density matrix. The extension of Ehrenfest to KBE-GKBA is formally straightforward (at least as long as static self-energies are considered) since the classical force entering eq. (5.50) only involves the density which can be constructed from the density matrix $n(r, t)=\rho(r, r, t)$. Such an approach has never been implemented also because the first ab-initio implementations of KBEGKBA for extended systems are quite recent. It constitutes then an interesting path for future developments. 
On the other hand, an MBPT version of MD is more demanding because it requires replacing the KS total energy, and its gradient, with a MBPT version. Within MBPT the total energy $E$ is not a functional of the density matrix alone but requires the two-times Green's function. Attempts to define MBPT total energies and forces within an ab-initio scheme can be found in the literature $[104,105]$ but so far have never been extended to the time domain.

\subsubsection{Quantum nuclear motion}

While electronic excitation processes are captured correctly by ED, its mean-field character distorts the characteristics of energy transfer from electrons to phonons, in particular failing at properly describing some very ubiquitous phenomena such as Joule heating [106] or the thermalization between electronic and nuclear degrees of freedom [107]. A second limitation is related to the exchange-correlation approximations used in TDDFT, which have been discussed in Section 5.2.3.1. In addition, incoherent electron-phonon scattering by nuclear vibrations (phonons), which are not explicitly represented in classical molecular dynamics, are not accounted for in ED simulations.

\subsubsection{Surface Hopping: a tool for photoinduced dynamics}

ED is straightforward to implement and computationally efficient but going beyond it is far from trivial. To improve on the electron-nuclear correlation, a possibility that has attracted interest in the past decades is the Surface Hopping approximation (SHA) [108]. In this method the forces on the nuclei are determined from single electronic potential energy surfaces (PES) but hops between surfaces are probabilistically allowed in order to include non-adiabatic effects. SHA introduces the quantum character of the nuclei in a minimalistic way, by modifying their kinetic energy to ensure energy conservation every time there is a discrete electronic transition. SHA works reasonably well when non- adiabatic transitions occur between a small number of PES, but not for a dense manifold of excited states [109]. SHA has been combined with LR-TDDFT to calculate the forces on excited electronic states $[110,111]$ and has evolved into widely available codes, e.g. [112,113]. This approach has enabled the calculation of photoinduced dynamical phenomena, as for example the degradation of materials under light irradiation [114].

\subsubsection{Beyond Mean-field: the $\boldsymbol{N}$-body density matrix}

To make progress beyond the classical nuclei and surface hopping approximations it is convenient to move away from the traditional TDKS formulation in terms of the evolution of Kohn-Sham orbitals. The TDKS formulation does not render itself naturally to the inclusion of decoherence effects as discussed in the introduction. Instead, a formulation in terms of a master equation for the density matrix is needed. Here we write the Lindblad master equation

$$
\partial_{t} \hat{\varrho}^{N}=-i\left[\widehat{H}, \hat{\varrho}^{N}\right]+\sum_{i} \gamma_{i}\left(L_{i} \varrho^{N} L_{i}^{\dagger}-\frac{1}{2}\left\{L_{i} L_{i}^{\dagger}, \varrho^{N}\right\}\right)
$$

eq. (5.51) describes the evolution of the N-body density matrix, $\hat{\rho}^{N}$, which can account for both mixed and pure quantum states. The term between round brackets incorporates the interaction between electrons and the environment, e.g., electrons and phonons, within a Markovian 
approximation, and hence it is able to address decoherence. If such term is zero one recovers the Liouville-Von Neumann ( $\mathrm{LvN}$ ) equation, which is equivalent to the Schrödinger equation. The Lindblad master equation is a general framework for introducing the interaction of a quantum many-body open system with the environment, which is central for the success of quantum technologies like quantum computing [115].

A possible, but by no means unique, way to go beyond ED in a controlled manner is the Correlated Electron-Ion Dynamics approach (CEID) [106,116]. CEID starts from the bare electron-nuclear Hamiltonian and solves the LvN equation by separating the electronic and nuclear density matrices and treating the nuclear part approximately by a perturbative expansion in powers of the fluctuations about the mean trajectory. The termination of the expansion at second order leads to a closed system of equations for the center and width of the position and momentum nuclear operators. CEID has emerged as a powerful tool for problems in which the transfer of energy between electrons and nuclei is crucial. A cost-effective alternative that limits the nu-clear motion to harmonic vibrations, named Effective CEID (ECEID), has been proposed recently [117], and applied to inelastic electron transport in water chains [118] and thermoelectric phenomena [119]. In ECEID the evolution of the phonon system is described through the dynamics of phonon occupation numbers, thus simplifying enormously the original CEID formulation in terms of momenta. In Figure 5.12 we show the evolution of the population of electronic states starting from an inverted population, in a tight-binding atomic chain interacting with a phonon bath. It can be seen that, while ED evolves, incorrectly, towards a flat distribution corresponding to infinite electronic temperature, ECEID evolves towards a Fermi-Dirac distribution at a finite temperature that coincides with that of the phonon bath.

(a) Ehrenfest

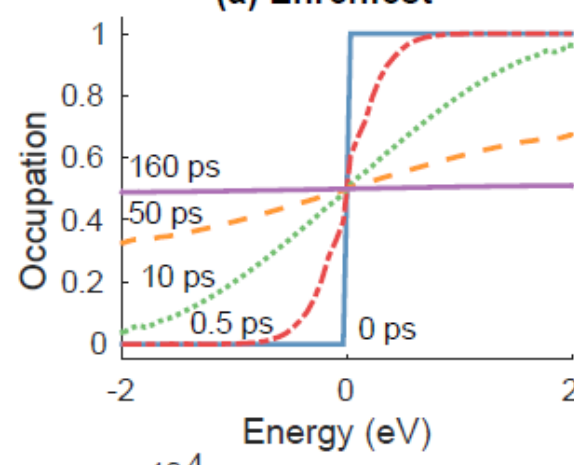

(b) ECEID

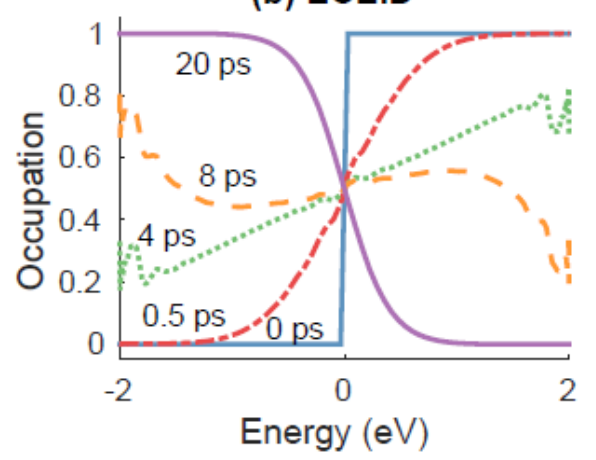

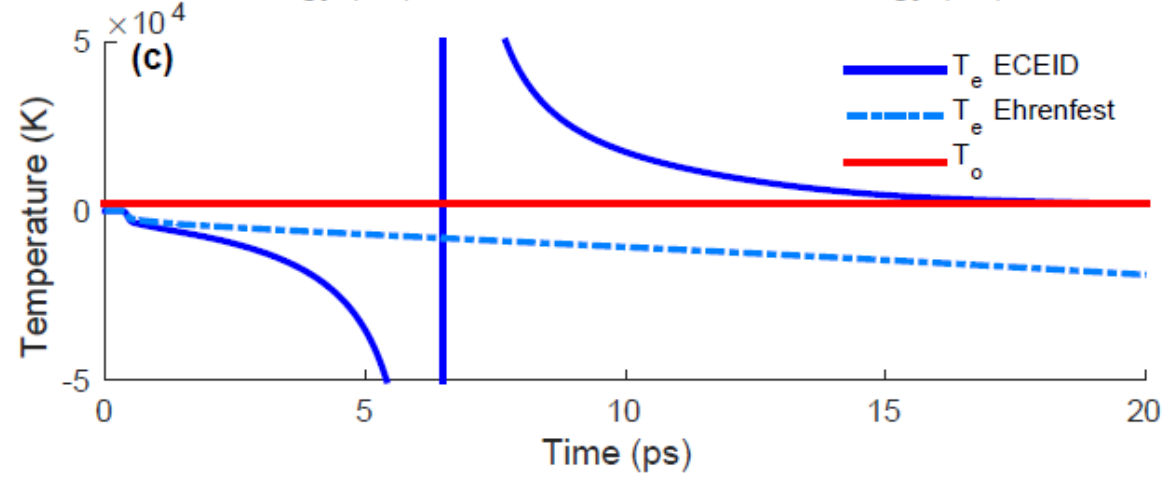

Figure 5.12. Population inversion simulation with the oscillators held at constant temperature. Snapshots of the population of the electronic states and shown for (a) ED and (b) ECIED, at successive times. Panel (c) show the temperature evolution during the simulation for both approaches compared with the fixed oscillator temperature (T0). Figure reproduced from Ref. [107]. 
In [107] it was also shown that the evolution of the electronic occupations could be reproduced to an excellent extent using a kinetic model. This was very recently followed by a formulation of the problem purely in terms of rate equations for the coupled evolution of the electronic and phonon occupations [119], which in addition includes coherences that were absent in [107]. This further simplification, which is correct to first order in perturbation theory, is extremely efficient. There are two main limitations of this approach. Firstly, it applies to weak electron-phonon coupling, and secondly, it is spatially homogeneous and hence applicable to laser, but not to ion irradiation. An important point is that this formulation is closely related to the Boltzmann transport equation discussed in Chapters 6 and 14 and to the simplified formulation discussed below, in sec. 5.4.3.3.

\subsubsection{Quantum Kinetics and the one-body reduced density matrix}

NEQ-MBPT offers an alternative to the Lindblad equation, with a set of coupled equations for the electronic propagator $G^{<}\left(t, t^{\prime}\right)$ and the ions propagator $D^{<}\left(t, t^{\prime}\right)$ which would capture both the coherent and the non-coherent dynamics. The main advantage of such an approach is the use of reduced quantities in place of the many-body density-matrix. A possible example of such approach for small atomic displacements has been discussed in [120], where the atoms propagator is replaced by the bosonic phonon propagator. In this work, the bosonic GKBA is discussed, leading to a generalized version of the equations for the density matrix which we write here in the notation of the reference

$$
\begin{array}{rrr}
\partial_{t} \rho(t) & =-i[h(t), \rho(t)]-\left(I(t)+I^{\dagger}(t)\right), \\
\partial_{t} \boldsymbol{\rho}_{b}(t) & =-i\left[\boldsymbol{\alpha} \boldsymbol{\Omega} \boldsymbol{\rho}_{b}(t)-\boldsymbol{\rho}_{b}(t) \boldsymbol{\alpha} \boldsymbol{\Omega}\right]-\left(\mathbf{I}_{b}(t)\right) .
\end{array}
$$

Such a scheme is very demanding and, in practice, not feasible, in particular for the $a b$ initio simulation of extended systems, or beyond small atomic displacements. Following the discussion in sec. 5.3.3, the approach could be in principle simplified into the form of a SCBE for the electrons and phonon populations, i.e., for the non-coherent part of the dynamics, although a direct derivation is still to be proven. The couplings, i.e., the scattering mechanism, originate from the nonadiabaticity of the many-body self-energy. An ab-initio implementation of the coupled SCBE has been shown possible recently in [121-123]. At variance with the phonon-mediated electron dynamics discussed in sec. 5.3.3, the phonon populations are also updated

$$
\begin{gathered}
\partial_{t} n_{\lambda \boldsymbol{q}}=\gamma_{\lambda \boldsymbol{q}}^{\text {in }}\left(n_{\lambda \boldsymbol{q}}+1\right)-\gamma_{\lambda \boldsymbol{q}}^{\text {out }} n_{\lambda \boldsymbol{q}}, \\
\gamma_{\lambda \boldsymbol{q}}^{\text {in }}=\frac{4 \pi}{\hbar} \sum_{m n \boldsymbol{k}} \frac{\left|g_{n m \boldsymbol{k}}^{v \boldsymbol{q}}\right|^{2}}{N_{\boldsymbol{q}}} \delta\left(\Delta \epsilon_{n m \boldsymbol{k}}^{\boldsymbol{q}}-\omega_{\lambda \boldsymbol{q}}\right)\left(1-f_{n \boldsymbol{k}}\right) f_{m \boldsymbol{k}+\boldsymbol{q}}, \\
\gamma_{\lambda \boldsymbol{q}}^{\text {out }}=\frac{4 \pi}{\hbar} \sum_{m n \boldsymbol{k}} \frac{\left|g_{n m \boldsymbol{k}}^{\text {vq }}\right|^{2}}{N_{\boldsymbol{q}}} \delta\left(\Delta \epsilon_{n m \boldsymbol{k}}^{\boldsymbol{q}}-\omega_{\lambda \boldsymbol{q}}\right) f_{n \boldsymbol{k}}\left(1-f_{m \boldsymbol{k}+\boldsymbol{q}}\right)
\end{gathered}
$$

The full description of the entangled phonon and electron relaxation dynamics would also require the phonon-phonon matrix elements. The evolution of phonons population is complementary to ED. Indeed, while ED describes a displacement of the atoms away from the equilibrium position, 
SCBE describes a situation where the expectation value of the displacement operator of the ions is always identically zero $\langle\Delta \widehat{R}\rangle=0$.

\subsection{Summary and open directions}

Nowadays, $a b$ initio methods for calculating electron dynamics are efficient in covering a rich phenomenology in time scales of femto- and pico-seconds. At the present stage, dynamical approaches focus on elementary particles like electrons and phonons. Scattering mechanisms like electron-electron and electron-phonon scattering are well described, together with light-matter interaction, including laser fields of high intensity. Light, however, is almost invariably treated as a classical external (dipolar) field. Only very recently the quantum character of light began to be considered, thus opening the doors of DFT to quantum electrodynamics [124]. One of the main limitations of time-dependent methods is the system size. In many approaches, a full ab initio study requires the realization of precise ground and excited state calculations (DFT, GW, electronphonon matrix elements) as starting point for more sophisticated calculations of electron and phonon dynamics. Open directions are the description of more complex excitations like the dynamics of excitons, polaritons, the coupling to magnetic fields and spin polarization. The formulation for these is still in the early days and so far, only applicable to small systems or using model Hamiltonians.

\section{References}

[1] Jorge Kohanoff. Electronic Structure Calculations for Solids and Molecules: Theory and Computational Methods. Cambridge University Press, 2006. doi: 10.1017/CB09780511755613

[2] R. M. Dreizler and E. K. U. Gross. Density Functional Theory: an approach to the quantum many-body problem. Springer-Verlag, 1990.

[3] Pedro Borlido, Thorsten Aull, Ahmad W. Huran, Fabien Tran, Miguel A. L. Marques, and Silvana Botti. Large-Scale Benchmark of Exchange-Correlation Functionals for the Determination of Electronic Band Gaps of Solids. Journal of Chemical Theory and Computation 15(9):5069-5079, SEP 2019. ISSN 1549-9618. doi: \{10.1021/acs.jctc.9b00322\}.

[4] JP Perdew, M Ernzerhof, and K Burke. Rationale for mixing exact ex- change with density functional approximations. JOURNAL OF CHEMICAL PHYSICS, 105(22):9982-9985, DEC 1996. doi: $\{10.1063 / 1.472933\}$.

[5] Jochen Heyd, Gustavo E. Scuseria, and Matthias Ernzerhof. Hybrid functionals based on a screened Coulomb potential (vol 118, pg 8207, 2003). JOURNALOF CHEMICAL PHYSICS, 124(21), JUN 72006. ISSN 0021-9606. doi: 10.1063/1.2204597

[6] R. M. Dreizler and E. K. U. Gross. Density Functional Theory: an approach tothe quantum many-body problem. Springer-Verlag, 1990.

[7] www.quantum-espresso.org

[8] www.abinit.org

[9] https://departments.icmab.es/leem/siesta/

[10] https://en.wikipedia.org/wiki/List of quantum chemistry and solid-state physics software

[11] Lars Hedin. New method for calculating the one-particle green's function withapplication to the electron-gas problem. Phys. Rev., 139:A796-A823, Aug 1965. doi: 10.1103/PhysRev.139.A796. URL https://link.aps.org/doi/ 10.1103/PhysRev.139.A796

[12] F Aryasetiawan and O Gunnarsson. The GW method. Reports on Progress in Physics, 61(3):237-312, mar 1998. URL https://doi.org/10.1088/0034-4885/61/3/002 
[13] Fabien Bruneval, Samia M. Hamed, and Jeffrey B. Neaton. A systematic benchmark of the ab initio Bethe-Salpeter equation approach for low-lying opticalexcitations of small organic molecules. The Journal of Chemical Physics, 142(24):244101, 2015. URL https://doi.org/10.1063/1.4922489

[14] Michiel J. van Setten, Fabio Caruso, Sahar Sharifzadeh, Xinguo Ren, Matthias Scheffler, Fang Liu, Johannes Lischner, Lin Lin, Jack R. Deslippe, Steven G. Louie, Chao Yang, Florian Weigend, Jeffrey B. Neaton, Ferdinand Evers, and Patrick Rinke. GW100: Benchmarking G0W0 for molecular systems. Journal of Chemical Theory and Computation, 11(12):5665-5687, 2015. doi: 10.1021/acs.jctc.5b00453. URL https://doi.org/10.1021/acs.jctc. 5b00453. PMID: 26642984

[15] Wei Chen and Alfredo Pasquarello. Band-edge positions in GW: Effects of starting point and selfconsistency. Phys. Rev. B, 90:165133, Oct 2014. doi: 10.1103/PhysRevB.90.165133. URL https://link.aps.org/doi/10.1103/ PhysRevB.90.165133

[16] Fulvio Paleari. First-principles approaches to the description of indirect absorption and luminescence spectroscopy: exciton-phonon coupling in hexagonal boron nitride. PhD thesis, University of Luxembourg, Luxembourg, 2019. URL https://aip.scitation.org/doi/10.1063/1.5115323

[17] Andrea Ferretti, Giuseppe Mallia, Layla Martin-Samos, Giovanni Bussi, Alice Ruini, Barbara Montanari, and Nicholas M. Harrison. Ab initio complex band structure of conjugated polymers: Effects of hydrid density functional theory and GW schemes. Phys. Rev. B, 85:235105, Jun 2012. doi: 10.1103/PhysRevB.85.235105. URL https://link.aps.org/doi/10.1103/ PhysRevB.85.235105

[18] Falco Hüser, Thomas Olsen, and Kristian S. Thygesen. How dielectric screening in two-dimensional crystals affects the convergence of excited-state calculations: Monolayer $\mathrm{MoS}_{2}$. Phys. Rev. B, 88:245309, Dec 2013. URL https://link.aps.org/doi/10.1103/ PhysRevB.88.245309

[19] S. Massidda, M. Posternak, and A. Baldereschi. Hartree-Fock LAPW approach to the electronic properties of periodic systems. Phys. Rev. B, 48:5058-5068, Aug 1993 . doi: 10.1103/PhysRevB.48.5058. URL https://link.aps.org/ doi/10.1103/PhysRevB.48.5058

[20] James Spencer and Ali Alavi. Efficient calculation of the exact exchange energyin periodic systems using a truncated coulomb potential. Phys. Rev. B, 77: 193110, May 2008. doi: 10.1103/PhysRevB.77.193110. URL https://link. aps.org/doi/10.1103/PhysRevB.77.193110

[21] Murilo L. Tiago, Sohrab Ismail-Beigi, and Steven G. Louie. Effect of semicore orbitals on the electronic band gaps of Si, Ge, and GaAs within the GW approximation. Phys. Rev. B, 69:125212, Mar 2004. URL https://link.aps.org/doi/10.1103/PhysRevB.69.125212

[22] C. Rostgaard, K. W. Jacobsen, and K. S. Thygesen. Fully self-consistent GW calculations for molecules. Phys. Rev. B, 81:085103, Feb 2010. doi: 10.1103/PhysRevB.81.085103. URL https://link.aps.org/doi/10.1103/ PhysRevB.81.085103

[23] Fabien Bruneval and Xavier Gonze. Accurate GW self-energies in a plane-wavebasis using only a few empty states: Towards large systems. Phys. Rev. B, 78:085125, Aug 2008 . doi: 10.1103/PhysRevB.78.085125. URL https://link.aps.org/doi/10.1103/PhysRevB.78.085125

[24] Jack Deslippe, Georgy Samsonidze, David A. Strubbe, Manish Jain, Mar-vin L. Cohen, and Steven G. Louie. BerkeleyGW: A massively parallel computer package for the calculation of the quasiparticle and optical properties of materials and nanostructures. Computer Physics Communications, 183(6):1269-1289, June 2012. ISSN 0010-4655. doi: 10.1016/j.cpc.2011.12.006. URL

https://www.sciencedirect.com/science/article/pii/S0010465511003912

[25] P. Umari, Geoffrey Stenuit, and Stefano Baroni. G W quasiparticle spectra from occupied states only. Phys. Rev. B, 81:115104, Mar 2010. URL https://link.aps.org/doi/10.1103/ PhysRevB.81.115104

[26] Maria Hellgren, Jacopo Baima, Raffaello Bianco, Matteo Calandra, Francesco Mauri, and Ludger Wirtz. Critical role of the exchange interaction for the electronic structure and charge-density-wave formation in tise. Phys. Rev. Lett., 119:176401, Oct 2017. doi: 10.1103/PhysRevLett.119.176401. URL https://link.aps.org/doi/10.1103/PhysRevLett.119.176401

[27] Fabio Caruso, Patrick Rinke, Xinguo Ren, Angel Rubio, and Matthias Scheffler.Self-consistent gw: Allelectron implementation with localized basis functions. Phys. Rev. B, 88:075105, Aug 2013. doi: 10.1103/PhysRevB.88.075105. URL https://link.aps.org/doi/10.1103/PhysRevB.88.075105

[28] P. Garáa-González and R. W. Godby. Self-consistent calculation of total energies of the electron gas using many-body perturbation theory. Phys. Rev. B, 63:075112, Jan 2001. doi: 10.1103/PhysRevB.63.075112. URL https://link.aps.org/doi/10.1103/PhysRevB.63.075112

[29] http://www.yambo-code.org/ 
[30] Layla Martin-Samos and Giovanni Bussi. SAX: An open-source package for electronic-structure and optical-properties calculations in the GW approximation. Computer Physics Communications, 180(8):1416-1425, 2009. ISSN 0010-4655. doi: https://doi.org/10.1016/j.cpc.2009.02.005. URL https://www.sciencedirect.com/science/article/pii/S0010465509000496

[31] http://www.west-code.org/

[32] https://berkeleygw.org/

[33] Physics of Photonic Devices, 2nd Edition Wiley. URL https://www.wiley.com/enus/Physics+of+Photonic+Devices\%2C+2nd+Edition-p-9780470293195

[34] Carsten A. Ullrich and Zeng-Hui Yang. A brief compendium of time-dependentdensity functional theory. Brazilian Journal of Physics, 44(1):154-188, 2014. ISSN 0103-9733. doi: 10.1007/s13538-013-0141-2

[35] C. Ullrich. Time-Dependent Density-Functional Theory: Concepts and Applications. Oxford Graduate $\begin{array}{llllll}\text { Texts. OUP } & \text { Oxford, } & \text { 2012. ISBN } & \text { 9780199563029. URL }\end{array}$ https://books.google.co.uk/books?id=hCNNsC4sEtkC

[36] D. Hofmann and S. Kümmel. Self-interaction correction in a real-time Kohn-Sham scheme: Access to difficult excitations in time-dependent density functional theory. The Journal of Chemical Physics, 137(6):064117, 2012. doi: 10.1063/1.4742763. URL https://doi.org/10.1063/1.4742763

[37] J. Messud, P.M. Dinh, P.-G. Reinhard, and E. Suraud. On the exact treatment of time-dependent selfinteraction correction. Annals of Physics, 324 (4):955-976, 2009. ISSN 0003-4916. doi: https://doi.org/10.1016/j.aop. 2008.12.001. URL https://www.sciencedirect.com/science/article/ pii/S0003491608001735

[38] Lucia Reining, Valerio Olevano, Angel Rubio, and Giovanni Onida. Excitonic effects in solids described by time-dependent density-functional theory. Phys. Rev. Lett., 88:066404, Jan 2002. doi: 10.1103/PhysRevLett.88.066404. URL https://link.aps.org/doi/10.1103/PhysRevLett.88.066404

[39] Fulvio Paleari, Thomas Galvani, Hakim Amara, François Ducastelle, Alejandro Molina-Sánchez, and Ludger Wirtz. Excitons in few-layer hexagonal boron nitride: Davydov splitting and surface localization. 2D Materials, 5(4):045017, aug 2018. doi: 10.1088/2053-1583/aad586. URL https://doi.org/10.1088/2053-1583/aad586

[40] Michael Rohlfing and Steven G. Louie. Electron-hole excitations and optical spectra from first principles. Phys. Rev. B, 62:4927-4944, Aug 2000. doi: 10.1103/PhysRevB.62.4927. URL https://link.aps.org/doi/10.1103/ PhysRevB.62.4927

[41] Stefan Albrecht, Lucia Reining, Rodolfo Del Sole, and Giovanni Onida. Ab initio calculation of excitonic effects in the optical spectra of semiconductors. Phys. Rev. Lett., 80:4510-4513, May 1998. doi: 10.1103/PhysRevLett.80.4510. URL https://link.aps.org/doi/10.1103/PhysRevLett.80.4510

[42] D Sangalli et al., Many-body perturbation theory calculations using the Yambocode. J. Phys. Condens. Matter, 31(32):325902, August 2019

[43] R. Del Sole and E. Fiorino. Macroscopic dielectric tensor at crystal surfaces. Phys. Rev. B, 29:46314645, Apr 1984. URL https://link.aps.org/doi/10.1103/PhysRevB.29.4631

[44] Andrea Marini, Conor Hogan, Myrta Grüning, and Daniele Varsano. Yambo: An ab initio tool for excited state calculations. Computer Physics Communications, 180(8):1392 - 1403, 2009. URL http://www.sciencedirect.com/science/ article/pii/S0010465509000472

[45] Stefano Baroni, Stefano de Gironcoli, Andrea Dal Corso, and Paolo Giannozzi.Phonons and related crystal properties from density-functional perturbation theory. Rev. Mod. Phys., 73:515-562, Jul 2001. URL https://link.aps.org/doi/10.1103/RevModPhys.73.515

[46] Xavier Gonze. First-principles responses of solids to atomic displacements and homogeneous electric fields: Implementation of a conjugate-gradient algorithm. Phys. Rev. B, 55:10337-10354, Apr 1997. URL https://link.aps.org/doi/10.1103/PhysRevB.55.10337

[47] K. Parlinski, Z. Q. Li, and Y. Kawazoe. First-principles determination of the soft mode in cubic $\mathrm{ZrO}_{2}$. Phys. Rev. Lett., 78:4063-6 (1997). URL https://link.aps.org/doi/10.1103/ PhysRevLett.78.4063

[48] Feliciano Giustino. Electron-phonon interactions from first principles. Rev. Mod. Phys., 89:015003, Feb 2017. URL https://link.aps.org/doi/10.1103/RevModPhys.89.015003

[49] S. Poncé, E. R. Margine, C. Verdi, and F. Giustino. EPW: Electron-phonon coupling, transport and superconducting properties using maximally localized Wannier functions. Computer Physics 
Communications, 209:116-133, De- cember 2016. ISSN 0010-4655. doi: 10.1016/j.cpc.2016.07.028. URL https://www.sciencedirect.com/science/article/pii/S0010465516302260

[50] X. Gonze et al., Recent developments in the abinit software package. Comput. Phys. Commun.,205:106131, August 2016. URL https://doi.org/10.1016/j.cpc.2016.04.003.

[51] X. Gonze, Ph. Ghosez, and R. W. Godby. Density-polarization functional theoryof the response of a periodic insulating solid to an electric field. Phys. Rev. Lett., 74:4035-4038, May 1995. doi: 10.1103/PhysRevLett.74.4035. URL https://link.aps.org/doi/10.1103/PhysRevLett.74.4035

[52] Raffaele Resta. Macroscopic polarization in crystalline dielectrics: the geometric phase approach. Rev. Mod. Phys., 66:899-915, Jul 1994. URL https://link.aps.org/doi/10.1103/RevModPhys.66.899

[53] Davide Sangalli, J. A. Berger, Claudio Attaccalite, Myrta Grüning, and Pina Romaniello. Optical properties of periodic systems within the current-current response framework: Pitfalls and remedies. Phys. Rev. B, 95:155203, Apr 2017. doi: 10.1103/PhysRevB.95.155203. URL https://link.aps.org/doi/ 10.1103/PhysRevB.95.155203

[54] Kuljit S. Virk and J. E. Sipe. Semiconductor optics in length gauge: A general numerical approach. Phys. Rev. B, 76:035213, Jul 2007. doi: 10.1103/PhysRevB.76.035213. URL http://link.aps.org/doi/10.1103/ PhysRevB.76.035213

[55] G. F. Bertsch, J.-I. Iwata, Angel Rubio, and K. Yabana. Real-space, real-time method for the dielectric function. Phys. Rev. B, 62:7998-8002, Sep 2000. doi: 10.1103/PhysRevB.62.7998. URL https://link.aps.org/doi/ 10.1103/PhysRevB.62.7998

[56] Umberto De Giovannini, Hannes Hübener, and Angel Rubio. A first-principles time-dependent density functional theory framework for spin and time- resolved angular-resolved photoelectron spectroscopy in periodic systems. Journal of Chemical Theory and Computation, 13(1):265-273, 2017. doi: 10.1021/acs.jctc.6b00897. URL http://dx.doi.org/10.1021/acs.jctc. 6b00897. PMID: 27981832

[57] Guillaume Le Breton, Ángel Rubio, and Nicolas Tancogne-Dejean. High- harmonic generation from fewlayer hexagonal boron nitride: Evolution from monolayer to bulk response. Phys. Rev. B, 98:165308, Oct 2018. doi: 10.1103/PhysRevB.98.165308. URL https://link.aps.org/doi/10.1103/ PhysRevB.98.165308

[58] K. Yabana and G. F. Bertsch. Time-dependent local-density approximation inreal time. Phys. Rev. B, 54:4484-4487, Aug 1996. URL https://link.aps.org/doi/10.1103/PhysRevB.54.4484

[59] K. Yabana, T. Sugiyama, Y. Shinohara, T. Otobe, and G. F. Bertsch. Time-dependent density functional theory for strong electromagnetic fields in crystalline solids. Phys. Rev. B, 85:045134, Jan 2012. URL http://link.aps.org/doi/10.1103/PhysRevB.85.045134

[60] N. Tancogne-Dejean, F. G. Eich, and A. Rubio. Time-dependent magnons from first principles. Journal of Chemical Theory and Computation, 16(2): 1007-1017, Feb 2020. ISSN 1549-9618. doi: 10.1021/acs.jctc.9b01064. URL https://doi.org/10.1021/acs.jctc.9b01064

[61] M. Draxler, S. P. Chenakin, S. N. Markin, and P. Bauer. Apparent veloc-ity threshold in the electronic stopping of slow hydrogen ions in LiF. Phys. Rev. Lett., 95:113201, Sep 2005. doi: 10.1103/PhysRevLett.95.113201. URL https://link.aps.org/doi/10.1103/PhysRevLett.95.113201

[62] J. M. Pruneda, D. Sánchez-Portal, A. Arnau, J. I. Juaristi, and Emilio Artacho. Electronic stopping power in LiF from first principles. Phys. Rev. Lett., 99: 235501, Dec 2007. doi: 10.1103/PhysRevLett.99.235501. URL https:// link.aps.org/doi/10.1103/PhysRevLett.99.235501

[63] Emilio Artacho. Electronic stopping in insulators: a simple model. Journal of Physics: Condensed Matter, 19(27):275211, jun 2007. URL https://doi.org/10.1088/0953-8984/19/27/275211

[64] Nicolò Forcellini and Emilio Artacho. Floquet theory for the electronic stopping of projectiles in solids. Phys. Rev. Research, 2:033151, Jul 2020. URL https://link.aps.org/doi/10. 1103/PhysRevResearch.2.033151

[65] Andrew P. Horsfield, Anthony Lim, W. M. C. Foulkes, and Alfredo A. Correa. Adiabatic perturbation theory of electronic stopping in insulators. Phys. Rev. B, 93:245106, Jun 2016. doi: 10.1103/PhysRevB.93.245106. URL https://link.aps.org/doi/10.1103/PhysRevB.93.245106

[66] A. Lim, W. M. C. Foulkes, A. P. Horsfield, D. R. Mason, A. Schleife, E. W. Draeger, and A. A. Correa. Electron elevator: Excitations across the band gap via a dynamical gap state. Phys. Rev. Lett., 116:043201, Jan 2016. URL https://link.aps.org/doi/10.1103/ PhysRevLett.116.043201 
[67] Jorge Kohanoff and Emilio Artacho. Water radiolysis by low-energy carbon projectiles from firstprinciples molecular dynamics. PLOS ONE, 12(3):1-11, 03 2017. URL https://doi.org/10. 1371/journal.pone.0171820

[68] Alfredo A. Correa, Jorge Kohanoff, Emilio Artacho, Daniel Sánchez-Portal, and Alfredo Caro. Nonadiabatic forces in ion-solid interactions: The initial stages of radiation damage. Phys. Rev. Lett., 108:213201, May 2012. doi: 10.1103/PhysRevLett.108.213201. URL https://link.aps.org/doi/ 10.1103/PhysRevLett.108.213201

[69] André Schleife, Yosuke Kanai, and Alfredo A. Correa. Accurate atomistic first- principles calculations of electronic stopping. Phys. Rev. B, 91:014306, Jan 2015. doi: 10.1103/PhysRevB.91.014306. URL https://link.aps.org/doi/ 10.1103/PhysRevB.91.014306

[70] José J. Dorado and F. Flores. Molecular-orbital theory for the stopping power of atoms in the low-velocity regime: The case of helium in alkali metals. Phys. Rev. A, 47:3062-3072, Apr 1993. doi: 10.1103/PhysRevA.47.3062. URL https://link.aps.org/doi/10.1103/PhysRevA.47.3062

[71] Bin Gu, Brian Cunningham, Daniel Muñoz Santiburcio, Fabiana Da Pieve, Emilio Artacho, and Jorge Kohanoff. Efficient ab initio calculation of electronic stopping in disordered systems via geometry presampling: Application to liquid water. The Journal of Chemical Physics, 153(3):034113, 2020. doi: 10.1063/5.0014276. URL https://doi.org/10.1063/5.0014276

[72] Wolfgang Neuwirth and Georg Both. Aggregates of atoms and their stop- ping cross sections. Nuclear Instruments and Methods in Physics Research Section B: Beam Interactions with Materials and Atoms, 12(1):67- 72, 1985. ISSN 0168-583X. doi: https://doi.org/10.1016/0168-583X(85)90702-5. URL https://www.sciencedirect.com/science/article/pii/ $0168583 X 85907025$

[73] Rafi Ullah, Emilio Artacho, and Alfredo A. Correa. Core electrons in the electronic stopping of heavy ions. Phys. Rev. Lett., 121:116401, Sep 2018. doi: 10.1103/PhysRevLett.121.116401. URL https://link.aps.org/doi/10.1103/PhysRevLett.121.116401

[74] Christian F. A. Negre, Valeria C. Fuertes, M. Belén Oviedo, Fabiana Y. Oliva, and Cristián G. Sánchez. Quantum dynamics of light-induced charge injection in a model dye-nanoparticle complex. The Journal of Physical Chemistry C, 116(28):14748-14753, 2012. doi: 10.1021/jp210248k. URL https://doi.org/10.1021/ip210248k

[75] Fabio Trani, Giovanni Scalmani, Guishan Zheng, Ivan Carnimeo, Michael J. Frisch, and Vincenzo Barone. Time-dependent density functional tight binding: New formulation and benchmark of excited states. Journal of ChemicalTheory and Computation, 7(10):3304-3313, 2011. doi: 10.1021/ct200461y. URL https://doi.org/10.1021/ct200461y. PMID: 26598164

[76] C. Attaccalite, M. Grüning, and A. Marini. Real-time approach to the optical properties of solids and nanostructures: Time-dependent Bethe-Salpeter equation. Phys. Rev. B, 84:245110, Dec 2011. doi: 10.1103/PhysRevB.84.245110.URL http://link.aps.org/doi/10.1103/PhysRevB.84.245110

[77] M. D'Alessandro and D. Sangalli. Real-time modelling of optical orientation in GaAs: Generation and decay of the degree of spin polarization. Phys. Rev. B, 102:104437, Sep 2020. doi: 10.1103/PhysRevB.102.104437. URL https://link.aps.org/doi/10.1103/PhysRevB.102.104437

[78] M.W. Wu, J.H. Jiang, and M.Q. Weng. Spin dynamics in semiconductors. Physics Reports, 493:61-236, 2010. URL http://www.sciencedirect.com/science/ article/pii/S0370157310000955

[79] Hartmut Haug and Antti-Pekka Jauho. Quantum Kinetics in Transport and Optics of Semiconductors. Springer-Verlag, Berlin, 2008

[80] Andrea Marini. Competition between the electronic and phonon-mediated scat- tering channels in the outof-equilibrium carrier dynamics of semiconductors: an ab-initio approach. Journal of Physics: Conference Series, 427(1):012003, 2013. URL http://stacks.iop.org/1742-6596/427/i=1/a=012003

[81] Davide Sangalli and Andrea Marini. Complete collisions approximation tothe Kadanoff-Baym equation: a first-principles implementation. Journal ofPhysics: Conference Series, 609(1):012006, 2015a. URL http://stacks. iop.org/1742-6596/609/i=1/a=012006

[82] Davide Sangalli and Andrea Marini. Ultra-fast carriers' relaxation in bulk silicon following photoexcitation with a short and polarized laser pulse. Europhys. Lett., 110(4):47004, M ay 2015b. ISSN 0295-5075. doi: 10.1209/0295-5075/110/47004. URL http://arxiv.org/abs/1409.1706

[83] Davide Sangalli, Stefano Dal Conte, Cristian Manzoni, Giulio Cerullo, and Andrea Marini. Nonequilibrium optical properties in semiconductors from first principles: A combined theoretical and 
experimental study of bulk silicon. Phys. Rev. B, 93:195205, May 2016. doi: 10.1103/PhysRevB.93.195205. URL http://link.aps.org/doi/10.1103/PhysRevB.93.195205

[84] E. Pogna, M. Marsili, D. De Fazio, S. Dal Conte, C. Manzoni, D. Sangalli, D. Yoon, A. Lombardo, A. Ferrari, A. Marini, G. Cerullo, and D. Prezzi. Photo-induced bandgap renormalization governs the ultrafast response of single-layer mos2. ACS Nano, 10:1188, 2016. doi: http://dx.doi.org/ 10.1021/acsnano.5b06488. URL http://pubs.acs.org/doi/full/10.1021/ acsnano.5b06488

[85] Davide Sangalli, Enrico Perfetto, Gianluca Stefanucci, and Andrea Marini. An ab-initio approach to describe coherent and non-coherent exciton dynamics. The European Physical Journal B, 91(8):171, Aug 2018. URL https://doi.org/10.1140/epjb/ e2018-90126-5

[86] Hsiao-Yi Chen, Vatsal A. Jhalani, Maurizia Palummo, and Marco Bernardi. Ab initio calculations of exciton radiative lifetimes in bulk crystals, nanostructures, and molecules. Phys. Rev. B, 100:075135, Aug 2019. doi: 10. 1103/PhysRevB.100.075135. URL https://link.aps.org/doi/10.1103/ PhysRevB.100.075135.

[87] Hsiao-Yi Chen, Davide Sangalli, and Marco Bernardi. Exciton-phonon interaction and relaxation times from first principles. Phys. Rev. Lett., 125: 107401, Aug 2020. URL https://link.aps.org/doi/10.1103/PhysRevLett.125.107401

[88] E. Surdutovich and A. Solov'yov. Random walk approximation for the radial dose dependence. Eur. Phys. J. D, 66:245, 2012

[89] G. H. Vineyard. Thermal spikes and activated processes. Radiation Effects, 29(4):245-248, 1976. doi: 10.1080/00337577608233050. URL https://doi. org/10.1080/00337577608233050

[90] Ch Dufour, V Khomenkov, G Rizza, and M Toulemonde. Ion-matter interaction: the three-dimensional version of the thermal spike model. application to nanoparticle irradiation with swift heavy ions. Journal of Physics D: Applied Physics, 45(6):065302, jan 2012. doi: 10.1088/0022-3727/45/6/065302. URL https://doi.org/10.1088/0022-3727/45/6/065302

[91] A M Rutherford and D M Duffy. The effect of electron-ion interactions on radiation damage simulations. Journal of Physics: Condensed Matter, 19 (49):496201, nov 2007. doi: 10.1088/09538984/19/49/496201. URL https://doi.org/10.1088/0953-8984/19/49/496201

[92] A. Tamm, G. Samolyuk, A. A. Correa, M. Klintenberg, A. Aabloo, and A. Caro. Electron-phonon interaction within classical molecular dynamics. Phys. Rev. B, 94:024305, Jul 2016. doi: 10.1103/PhysRevB.94.024305. URL https://link.aps.org/doi/10.1103/PhysRevB.94.024305

[93] Samuel T. Murphy, Yvelin Giret, Szymon L. Daraszewicz, Anthony C. Lim, Alexander L. Shluger, Katsumi Tanimura, and Dorothy M. Duffy. Contribution of electronic excitation to the structural evolution of ultrafast laser-irradiated tungsten nanofilms. Phys. Rev. B, 93:104105, Mar 2016. doi: 10.1103/PhysRevB.93.104105. URL https://link.aps.org/doi/10.1103/PhysRevB.93.104105

[94] Samuel T. Murphy, Szymon L. Daraszewicz, Yvelin Giret, Matthew Watkins, Alexander L. Shluger, Katsumi Tanimura, and Dorothy M. Duffy. Dynamicalsimulations of an electronically induced solid-solid phase transformation in tungsten. Phys. Rev. B, 92:134110, Oct 2015. doi: 10.1103/PhysRevB.92. 134110. URL https://link.aps.org/doi/10.1103/PhysRevB.92.134110

[95] S. Khakshouri, D. Alfè, and D. M. Duffy. Development of an electron-temperature-dependent interatomic potential for molecular dynamics simulation of tungsten under electronic excitation. Phys. Rev. B, 78:224304, Dec2008. URL https://link.aps.org/doi/ 10.1103/PhysRevB.78.224304

[96] Paul Bendt and Alex Zunger. Simultaneous relaxation of nuclear geometries and electric charge densities in electronic structure theories. Phys. Rev. Lett., 50:1684-1688, May 1983. doi: 10.1103/PhysRevLett.50.1684. URL https://link.aps.org/doi/10.1103/PhysRevLett.50.1684

[97] R. Car and M. Parrinello. Unified approach for molecular dynamics anddensity-functional theory. Phys. Rev. Lett., 55:2471-4 (1985). URL https://link.aps.org/doi/10.1103/ PhysRevLett.55.2471

[98] G. Pastore, E. Smargiassi, and F. Buda. Theory of ab initio molecular-dynamicscalculations. Phys. Rev. A, 44:6334-6347, Nov 1991. URL https://link.aps.org/doi/10.1103/PhysRevA.44.6334

[99] Joachim Theilhaber. Ab initio simulations of sodium using time-dependent density-functional theory. Phys. Rev. B, 46:12990-13003, Nov 1992. doi: 10.1103/PhysRevB.46.12990. URL https://link.aps.org/doi/10.1103/ PhysRevB.46.12990 
[100] José L. Alonso, Alberto Castro, Pablo Echenique, and Angel Rubio. On the Combination of TDDFT with Molecular Dynamics: New Developments, pages 301-315. Springer Berlin Heidelberg, Berlin, Heidelberg, 2012. URL "https://doi.org/10.1007/978-3-642-23518-4_15"

[101] Yoshiyuki Miyamoto, Yoshitaka Tateyama, Norihisa Oyama, and Takahisa Ohno. Conservation of the pure adiabatic state in Ehrenfest dynamics of thephotoisomerization of molecules. Scientific Reports, 5(1):18220, 2016. URL https://dx.doi.org/10.1038/ srep18220

[102] Y. Shinohara, K. Yabana, Y. Kawashita, J.-I. Iwata, T. Otobe, and G. F. Bertsch. Coherent phonon generation in time-dependent density functional theory. Phys. Rev. B, 82:155110, Oct 2010. doi: 10.1103/PhysRevB.82. 155110. URL https://link.aps.org/doi/10.1103/PhysRevB.82.155110

[103] Atsushi Yamada and Kazuhiro Yabana. Multiscale time-dependent density functional theory for a unified description of ultrafast dynamics: Pulsed light, electron, and lattice motions in crystalline solids. Phys. Rev. B, 99:245103, Jun 2019. doi: 10.1103/PhysRevB.99.245103. URL https://link.aps.org/ doi/10.1103/PhysRevB.99.245103

[104] Sohrab Ismail-Beigi and Steven G. Louie. Excited-state forces within a first- principles green's function formalism. Phys. Rev. Lett., 90:076401, Feb 2003. doi: 10.1103/PhysRevLett.90.076401. URL https://link.aps.org/doi/ 10.1103/PhysRevLett.90.076401

[105] Pierre-François Loos, Anthony Scemama, Ivan Duchemin, Denis Jacquemin, and Xavier Blase. Pros and cons of the Bethe-Salpeter formalism for ground-state energies. J. Phys. Chem. Lett., 11(9):3536-3545 (2020). URL https://doi.org/10.1021/ acs.jpclett.0c00460. PMID: 32298578

[106] Andrew P Horsfield, D R Bowler, A J Fisher, Tchavdar N Todorov, and Cristián G Sánchez. Correlated electron-ion dynamics: the excitation of atomic motion by energetic electrons. J. Phys.: Condens. Matter, 17(30):4793-4812 (2005). URL https://doi.org/10.1088/0953-8984/17/30/006

[107] Valerio Rizzi, Tchavdar N. Todorov, Jorge J. Kohanoff, and Alfredo A. Correa. Electron-phonon thermalization in a scalable method for real-time quantum dynamics. Phys. Rev. B, 93:024306, Jan 2016. URL https://link.aps.org/doi/10.1103/PhysRevB.93.024306

[108] John C. Tully. Molecular dynamics with electronic transitions. The Journal of Chemical Physics, 93(2):1061-1071, 1990. doi: 10.1063/1.459170. URL https://doi.org/10.1063/1.459170

[109] Joseph E. Subotnik, Wenjun Ouyang, and Brian R. Landry. Can we derive Tully's surface-hopping algorithm from the semiclassical quantum Liouville equation? almost, but only with decoherence. The Journal of Chemical Physics, 139(21):214107, 2013. URL https://doi.org/10.1063/1.4829856

[110] Colleen F. Craig, Walter R. Duncan, and Oleg V. Prezhdo. Trajectory surface hopping in the timedependent Kohn-Sham approach for electron-nuclear dynamics. Phys. Rev. Lett., 95:163001, Oct 2005. URL https://link.aps.org/doi/10.1103/PhysRevLett.95.163001

[111] Enrico Tapavicza, Ivano Tavernelli, and Ursula Rothlisberger. Trajectory surface hopping within linear response time-dependent density-functional theory. Phys. Rev. Lett., 98:023001, Jan 2007. URL https://link.aps.org/doi/10.1103/PhysRevLett.98.023001

[112] Alexey V. Akimov and Oleg V. Prezhdo. The pyxaid program for non-adiabaticmolecular dynamics in condensed matter systems. Journal of Chemical The- ory and Computation, 9(11):4959-4972, 2013. doi: 10.1021/ct400641n. URL https://doi.org/10.1021/ct400641n. PMID: 26583414.

[113] Alexey V. Akimov and Oleg V. Prezhdo. Advanced capabilities of the pyxaid program: Integration schemes, decoherence effects, multiexcitonic states, and field-matter interaction. Journal of Chemical Theory and Computation, 10(2): 789-804, 2014. doi: 10.1021/ct400934c. URL https://doi.org/10.1021/ct400934c. PMID: 26580053

[114] Jinlu He, Wei-Hai Fang, Run Long, and Oleg V. Prezhdo. Why oxygen increases carrier lifetimes but accelerates degradation of $\mathrm{CH}_{3} \mathrm{NH}_{3} \mathrm{PbI}_{3}$ under light irradiation: Time-domain ab initio analysis. Journal of the American Chemical Society, 142(34):14664-14673, 2020. doi: 10.1021/jacs.0c06769. URL https://doi.org/10.1021/jacs.0c06769. PMID: 32786790

[115] Daniel Manzano. A short introduction to the Lindblad master equation. AIP Advances, 10(2):025106, 2020. doi: 10.1063/1.5115323. URL https://doi. org/10.1063/1.5115323

[116] Andrew P Horsfield, D R Bowler, A J Fisher, Tchavdar N Todorov, and Cristián G Sánchez. Beyond Ehrenfest: correlated non-adiabatic molecular dynamics. Journal of Physics: Condensed Matter, 16(46):8251-8266, nov 2004. doi: 10.1088/0953-8984/16/46/012. URL https://doi.org/10.1088/0953-8984/16/46/012 
[117] Valerio Rizzi, Tchavdar N. Todorov, and Jorge J. Kohanoff. Inelastic electron injection in a water chain. Scientific Reports, 7:45410, 2017

[118] P. Chudzinski. Anharmonic coupling between electrons and to phonons in the vicinity of a ferroelectric quantum critical point. Phys. Rev. Research, 2: 012048, Feb 2020. URL https: //link.aps.org/doi/10.1103/PhysRevResearch.2.012048

[119] Carlos M. Bustamante, Tchavdar N. Todorov, Cristián G. Sánchez, Andrew Horsfield, and Damian A. Scherlis. A simple approximation to the elec- tron-phonon interaction in population dynamics. The Journal of Chemi- cal Physics, 153(23):234108, 2020. doi: 10.1063/5.0031766. URL https://doi.org/10.1063/5.0031766

[120] Daniel Karlsson, Robert van Leeuwen, Yaroslav Pavlyukh, Enrico Perfetto, andGianluca Stefanucci. Efficient non-equilibrium Green's function simulations of correlated electron-boson systems, 2020

[121] Fabio Caruso. Nonequilibrium lattice dynamics in monolayer MoS2. The Journal of Physical Chemistry Letters, 12(0):1734-1740, 2021. doi: 10.1021/acs.jpclett.0c03616. URL https://doi.org/10.1021/acs.jpclett.0c03616.

[122] F. Murphy-Armando, É. D. Murray, I. Savic, M. Trigo, D. Reis, and S. Fahy. Evolution of non-thermal phonon and electron populations in photo-excited germanium on picosecond timescales, 2020

[123] Xiao Tong and Marco Bernardi. Toward precise simulations of the coupled ultrafast dynamics of electrons and atomic vibrations in materials, 2020

[124] M. Ruggenthaler, J. Flick, C. Pellegrini, H. Appel, I. V. Tokatly, and A. Rubio, Quantum-electrodynamical density-functional theory: Bridging quantum optics and electronic-structure theory, Phys. Rev. A 90, 012508 (2014) 


\title{
6. Quantum and semiclassical dynamics of semiconductors and dielectrics
}

\author{
Tzveta Apostolova*
}

\begin{abstract}
A set of quantum kinetic equations that can be applied to study the ultrafast coherent and relaxation carrier dynamics in femtosecond laser excitation of semiconductors and dielectrtics is introduced. These semiconductor Bloch equations contain important many-body effects, electronhole interactions and contributions of phase-space filling. Under certain approximations they lead to semiclassical Boltzmann kinetic equation describing the evolution of the electron energy distribution induced by different processes. The initial interband photo-ionization is followed by impurity- and phonon-assisted photon absorption resulting in impact ionization. Coulomb scattering between two electrons leads to thermalization of the electron system. The process of Auger recombination reducing the number of conduction electrons is included into the formalism. Formation of free excitons is also described. The energy exchange between the electrons and phonons is given by a separate equation for the lattice temperature where the rates of energy transfer from the electrons to the lattice per unit volume are defined quantum mechanically. The electron energy distribution function, conduction electron number density and average kinetic energy of the conduction electrons are obtained as a function of laser parameters such as peak laser electric field, laser wavelength and laser pulse duration.
\end{abstract}

\footnotetext{
*Contact: tzveta.apostolova@nbu.bg
} 


\subsection{Introduction}

Ultrashort pulsed laser irradiation has the ability to bring transparent materials into a state of strong electronic, optical, thermal and structural nonequilibrium by initially creating electronhole pairs across the band gap.

Time-resolved nonlinear optical spectroscopy has been applied to study microscopic carrier dynamics on the ultrashort time scale. Various experimental techniques are designed to study incoherent dynamics - the nonequilibrium dynamics of distribution functions, as well as coherent phenomena - the dynamics of optically created interband and intraband polarizations. The thermalization and relaxation processes have been studied by luminescence, in which the photons created by the radiative recombination of electrons and holes are detected, and also by by pump probe measurements, in which the change in the absorption (or reflection) of a probe beam caused by the prior excitation of electron-hole pairs by the pump beam is observed. In an intrinsic semiconductor the luminescence is due to the recombination of an electron in the conduction band with a hole in the valence band. In a fully incoherent picture, according to Fermi's golden rule, the signal is essentially proportional to the product of the distribution functions of electrons and holes which can be monitored [1-3]. An alternative is the use of doped semiconductors, e.g., $p$-doped samples, in which the band-to-acceptor luminescence directly monitors the distribution function of electrons [4,5], and pump-probe experiments [6] providing data on the sum of the distribution functions. The most commonly used technique is transmission or reflection spectroscopy, in which the change in the transmission or reflection of the probe pulse induced by the pump pulse is measured as a function of the time delay between the two pulses. A detailed analysis of luminescence and pump-probe spectra in the ultrafast regime may also provide information on coherent phenomena in the semiconductor.

Besides pump-probe and luminescence measurements, there are other techniques that provide direct information on coherent phenomena in band gap materials such as the dephasing of electron-hole plasma or the dynamical Stark effect. The most prominent of them is the four-wavemixing (FWM) spectroscopy [7-11].

In addition to the experimental data the ultrafast dynamics of photocarriers in an irradiated band gap material was described by theoretical methods varying from model equations using parametrization of the distribution functions [12-15], direct integration of Boltzmann equation $[16,17]$ or Monte Carlo simulations [18-21]. Complementarity of experimental results and theoretical calculations provide relevant data on scattering rates and coupling constants. On the other hand, the buildup of polarization due to the coherence of the external optical field interacting with the material and the Coulomb interaction between electrons and holes requires a full quantum mechanical treatment. Some of the quantum kinetic theoretical methods used to describe the coherent phenomena are non-equilibrium Green's-functions techniques [22-24] discussed in chapter 5 and density-matrix theory $[25,26]$. On short time scales, the coherent and incoherent processes cannot be separated. For time dependent processes such as ultrashortpulsed laser irradiation of semiconductor (dielectric) materials the density matrix method provides quantities directly comparable with physical observables. 


\subsection{Quantum kinetic equations}

The carrier dynamics induced by an ultrashort (sub-picosecond to picosecond) laser pulse in the bulk of a direct-gap semiconductor (dielectric) at initial lattice temperature $T$ with two isotropic, parabolic bands is studied starting with the the noninteracting part of the Hamiltonian describing the free carriers interacting with a classical light field together with the free phonons in the second quantization is [27-32]:

$$
\widehat{H}_{0}(t)=\sum_{\vec{k}} \varepsilon_{\vec{k}}^{e} \hat{a}_{\vec{k}}^{\dagger} \hat{a}_{\vec{k}}+\sum_{\vec{k}} \varepsilon_{\vec{k}}^{h} \hat{d}_{\vec{k}}^{\dagger} \hat{d}_{\vec{k}}+\sum_{\vec{q}} \hbar \omega_{\vec{q}} \hat{b}_{\vec{q}}^{\dagger} \hat{b}_{\vec{q}}+\sum_{\vec{k}} F_{\vec{k}} \hat{a}_{\vec{k}}^{\dagger} \hat{d}_{-\vec{k}}^{\dagger}+F_{\vec{k}}^{*} \hat{d}_{-\vec{k}} \hat{a}_{\vec{k}}
$$

with well-defined dispersion relations of the quasiparticles

$$
\varepsilon_{\vec{k}}^{e}=E_{g}+\frac{\hbar^{2} k^{2}}{2 m_{e}}, \varepsilon_{\vec{k}}^{h}=\frac{\hbar^{2} k^{2}}{2 m_{h}}
$$

In Eq. (6.2) $E_{g}$ is the material bandgap, $\varepsilon_{\vec{k}}^{e}\left(\varepsilon_{\vec{k}}^{h}\right)$ is the electron (hole) kinetic energy in the conduction (valence) band with electron (hole) effective mass $m_{e}\left(m_{h}\right)$, and $\hbar \omega_{\vec{q}}$ is the phonon energy. $\hat{a}_{\vec{k}}^{\dagger},\left[\hat{d}_{\vec{k}}^{\dagger}\right]$ and $\hat{a}_{\vec{k}},\left[\hat{d}_{\vec{k}}\right]$ are the creation and annihilation operators of an electron [hole] with wave vector $\vec{k}$ which satisfy Fermi-Dirac statistics, while $\hat{b}_{\vec{q}}^{\dagger},\left[\hat{b}_{\vec{q}}\right]$ stand for the phonon creation and annihilation operators of a phonon with wave vector $\vec{q}$ obeying Bose- Einstein statistics. The noninteracting part of the Hamiltonian includes contributions that can be treated exactly within a single-particle picture.

In addition, the carriers interact with the classical field of the laser, with phonons and with each other via the Coulomb potential. The interacting part of the Hamiltonian includes contributions that have to be treated within some approximation scheme [27-34]:

$$
\widehat{H}_{I}(t)=\widehat{H}_{I}^{c f}(t)+\widehat{H}_{I}^{c p}(t)+\widehat{H}_{I}^{c c}(t)+\widehat{H}_{I}^{c c(1)}(t)
$$

with the single-particle Hamiltonian describing the free carriers interacting with a classical electric field of the laser

$$
\widehat{H}_{I}^{c f}(t)=\sum_{\vec{k}}\left[F_{\vec{k}} \hat{a}_{\vec{k}}^{\dagger} \hat{d}_{-\vec{k}}^{\dagger}+F_{\vec{k}}^{*} \hat{d}_{-\vec{k}} \hat{a}_{\vec{k}}\right]
$$

the carrier-phonon Hamiltonian

$$
\widehat{H}_{I}^{c p}(t)=\sum_{\vec{k}, \vec{q}}\left[C_{\vec{q}}^{e} \hat{a}_{\vec{k}+\vec{q}}^{\dagger} \hat{b}_{\vec{q}} \hat{a}_{\vec{k}}+C_{\vec{q}}^{e *} \hat{a}_{\vec{k}}^{\dagger} \hat{b}_{\vec{q}}^{\dagger} \hat{a}_{\vec{k}+\vec{q}}+C_{\vec{q}}^{h} \hat{d}_{\vec{k}+\vec{q}}^{\dagger} \hat{b}_{\vec{q}} \hat{d}_{\vec{k}}+C_{\vec{q}}^{h *} \hat{d}_{\vec{k}}^{\dagger} \hat{b}_{\vec{q}}^{\dagger} \hat{d}_{\vec{k}+\vec{q}}\right]
$$

the carrier-carrier Hamiltonian

$$
\widehat{H}_{I}^{c c}(t)=\sum_{\vec{k}, \vec{k} \prime \vec{q}} V_{\vec{q}}^{c}\left[\frac{1}{2} \hat{a}_{\vec{k}}^{\dagger} \hat{a}_{\vec{k}}^{\dagger} \hat{a}_{\vec{k} \prime+\vec{q}} \hat{a}_{\vec{k}-\vec{q}}+\frac{1}{2} \hat{d}_{\vec{k}}^{\dagger} \hat{d}_{\vec{k}}^{\dagger} \hat{d}_{\vec{k}^{\prime}+\vec{q}} \hat{d}_{\vec{k}-\vec{q}}-\hat{a}_{\vec{k}}^{\dagger} \hat{d}_{-\vec{k}}^{\dagger} \hat{d}_{-\vec{k} \prime+\vec{q}} \hat{a}_{\vec{k}-\vec{q}}\right]
$$


and the carrier-carrier Hamiltonian describing processes which do not conserve the number of carriers per band

$$
\begin{aligned}
& \hat{H}_{I}^{c c(1)}(t)=\sum_{\vec{k}, \vec{k}^{\prime} \vec{q}}\left[M_{e}(q) \hat{a}_{\vec{k}^{\prime}+\vec{q}}^{\dagger} \hat{a}_{\vec{k}^{\prime}-\vec{q}}^{\dagger} \hat{d}_{-\vec{k}}^{\dagger}, \hat{a}_{\vec{k}}+M_{e}^{*}(q) \hat{a}_{\vec{k}}^{\dagger} \hat{d}_{-\vec{k}}, \hat{a}_{\vec{k}^{\prime}-\vec{q}} \hat{a}_{\vec{k}+\vec{q}}\right] \\
& +\sum_{\vec{k}, \vec{k}^{\prime} \vec{q}}\left[M_{h}(q) \hat{d}_{\vec{k}+\vec{q}}^{\dagger} \hat{d}_{\vec{k}^{\prime}-\vec{q}}^{\dagger} \hat{a}_{-\vec{k}}^{\dagger} \hat{d}_{\vec{k}}+M_{h}^{*}(q) \hat{d}_{\vec{k}}^{\dagger} \hat{a}_{-\vec{k}} \hat{d}_{\vec{k}^{\prime}-\vec{q}} \hat{d}_{\vec{k}+\vec{q}}\right]
\end{aligned}
$$

In Eq. (6.3a) $F_{\vec{k}}=d_{\vec{k}} E_{0}(t) \exp \left(-i \omega_{L} t\right)$ is the interband dipole-coupling coefficient between the laser field and electrons, $E_{0}(t)$ denotes the pulse shape of the external light field centered at frequency $\omega_{L}, d_{\vec{k}}$ is the dipole matrix element between valance band and conduction band and the interaction is treated in dipole and rotating wave approximations [30,31]. In Eq. (6.3b) $C_{\vec{q}}^{e, h}$ are the coupling matrix elements for polar or deformation-potential interaction for electrons (holes). In the case of a polar interaction, due to the opposite charge of electrons and holes, the coupling constants are related by $C_{\vec{q}}^{e}=-C_{\vec{q}}^{h}=C_{\vec{q}}$.

In Eq. (6.3c) $V_{\vec{q}}^{c}=\frac{e^{2}}{V \varepsilon_{r} \varepsilon_{0} q^{2}}$ is the the Fourier transform of the Coulomb potential where $e$ is the electron charge, $\varepsilon_{r}$ and $\varepsilon_{0}$ are the relative and the absolute permittivity, respectively and $\mathrm{V}$ is the crystal volume.

Within the electron-hole picture, carrier-carrier interaction splits into several conceptually different contributions - electron electron interaction (first term in Eq. (6.3c)), hole-hole interaction (second term in Eq. (6.3c)) and electron-hole interaction (third term in Eq. (6.3c)) which conserve the number of particles per band.

In Eq. (6.3d) the first term represents impact ionization and the second term is its inverse process - Auger recombination, which do not conserve the number of electron-hole pairs [31,34].

$M_{e}(q)=\frac{e^{2}}{\varepsilon_{r} \varepsilon_{0} \mathrm{~V}} \frac{\mathcal{F}_{c c} \mathcal{F}_{v c}}{q^{2}+\lambda^{2}}$
$M_{h}(q)=\frac{e^{2}}{\varepsilon_{r} \varepsilon_{0} \mathrm{~V}} \frac{\mathcal{F}_{v v} \mathcal{F}_{c v}}{q^{2}+\lambda^{2}}$

are the Debye-like screened Coulomb matrix elements with the inverse screening length $\lambda$. $F_{i j}(\vec{k}, \vec{q})$ are the overlap (or Bloch) integrals. The matrix elements for the electron and hole processes are different due to the different overlap integrals involved. In the definition of the Hamiltonian the additional dependence on the spin variables has been ignored. The last two terms in eq (6.3) represent impact ionization and its inverse process - Auger recombination, which do not conserve the number of electron-hole pairs [31,34]. These processes are usually considered to become important at very high carrier densities or at high carrier energies.

The time evolution of the physical system described by the total Hamiltonian $\widehat{H}(t)=\widehat{H}_{0}(t)+\widehat{H}_{I}(t)$ is refiected in the time evolution of the associated density matrix. The basic variables describing the kinetics of the irradiated material are the distribution functions intraband single-particle density matrices of electrons, holes, and phonons [35] 
$n_{\vec{k}}^{e}=\left\langle\hat{a}_{\vec{k}}^{\dagger} \hat{a}_{\vec{k}}\right\rangle, n_{\vec{k}}^{h}=\left\langle\hat{d}_{\vec{k}}^{\dagger} \hat{d}_{\vec{k}}\right\rangle, N_{\vec{q}}=\left\langle\hat{b}_{\vec{q}}^{\dagger} \hat{b}_{\vec{q}}\right\rangle$ where \langle\rangle denotes the ensemble average. The expectation value is taken with respect to the initial state of the system.

To take into account the coherence induced by the external laser field the interband polarization (interband density matrix) has to be considered:

$p_{\vec{k}}=\left\langle\hat{d}_{-\vec{k}} \hat{a}_{\vec{k}}\right\rangle, p_{\vec{k}}^{*}=\left\langle\hat{a}_{\vec{k}}^{\dagger} \hat{d}_{-\vec{k}}^{\dagger}\right\rangle$.

Using the Heisenberg equations of motion for electron and hole operators a closed set of equations of motion for the electron and hole distribution functions and the polarization is obtained:

$$
\begin{gathered}
\frac{d}{d t} n_{\vec{k}}^{(e)}=\frac{d}{d t} n_{-\vec{k}}^{(h)}=g_{k}^{(0)}(t) \\
\frac{d}{d t} p_{\vec{k}}=\frac{1}{i \hbar}\left(\varepsilon_{\vec{k}}^{(e)}-\varepsilon_{-\vec{k}}^{(h)}\right) p_{\vec{k}}+\frac{1}{i \hbar} d_{\vec{k}} E_{0}(t) \exp \left(-i \omega_{L} t\right)\left(1-n_{\vec{k}}^{(e)}-n_{-\vec{k}}^{(h)}\right)
\end{gathered}
$$

and the complex-conjugate equation for $p_{\vec{k}}^{*}$. The equation for the generation rate is:

$g_{\vec{k}}^{(0)}=\frac{1}{i \hbar}\left[d_{\vec{k}} E_{0}(t) \exp \left(-i \omega_{L} t\right) p_{k}^{*}-d_{\vec{k}}^{*} E_{0}^{*}(t) \exp \left(i \omega_{L} t\right) p_{k}\right]$.

Without interactions, equations (6.4) describe the model two level system driven by an external field with detuning $\Delta \omega_{\vec{k}}=\omega_{L}-\frac{1}{\hbar}\left(\varepsilon_{\vec{k}}^{(e)}+\varepsilon_{-\vec{k}}^{(h)}\right)$ of a given transition with wave vector $\vec{k}$ from resonance. These semiconductor Bloch equations describe fully the coherent dynamics.

The equation for the polarization can be formally integrated:

$p_{\vec{k}}=\frac{1}{i \hbar} \exp \left(-i \omega_{L} t\right) \int_{0}^{\infty} d \tau \exp \left(-i\left(\omega_{\vec{k}}-\omega_{L}\right) \tau\right) d_{\vec{k}} E_{0}(t-\tau)\left(1-n_{\vec{k}}^{(e)}(t-\tau)-n_{\vec{k}}^{(h)}(t-\tau)\right)$

where $\omega_{\vec{k}}=\frac{1}{\hbar}\left(\varepsilon_{\vec{k}}^{(e)}+\varepsilon_{-\vec{k}}^{(h)}\right)$.

When polarization is eliminated from the equation for the generation rate [27-29] the generation rate at time $t$ depends not only on the distribution functions and the optical field at time $t$ but also on earlier times. Within the Markov approximation it can be assumed that that the dominant time dependence is given by the exponential in the expression for the polarization and the carrier distribution and field amplitude (rotating wave approximation) are sufficiently slowly varying so that their values at time $t$ can be taken out of the integral. In order to have a well-defined initial condition the laser field is adiabatically switched on according to $E_{0}(t)=\lim _{\eta \rightarrow 0} \widetilde{E}_{0}(t) \exp (\eta t)$. Then, the polarization is an instantaneous function of the carrier distribution and the external field:

$p_{\vec{k}}=-\frac{i \pi}{\hbar} d_{\vec{k}} E_{0}(t) \exp \left(-i \omega_{L} t\right)\left(1-n_{\vec{k}}^{(e)}(t)-n_{\vec{k}}^{(h)}(t)\right) D\left(\omega_{\vec{k}}-\omega_{L}\right)$,

where $D(x)=-\frac{i}{\pi} \lim _{\eta \rightarrow 0} \frac{1}{x-i \eta}=\delta(x)-\frac{i}{\pi} \frac{P}{x}$ with $P$ denoting the principal value. Then the semiclassical generation rate containing the energy conserving $\delta$ function is recovered, (Fermi's golden rule):

$g_{\vec{k}}=\frac{2 \pi}{\hbar^{2}}\left[d_{\vec{k}} E_{0}(t) \exp \left(-i \omega_{L} t\right)\right]^{2}\left(1-n_{\vec{k}}^{(e)}(t)-n_{\vec{k}}^{(h)}(t)\right) \delta\left(\omega_{\vec{k}}-\omega_{L}\right)$

For ultrashort laser pulses, this approximation is not valid and the energy time uncertainty leads 
to a broadening of the generation rate. This broadening can be introduced by multiplying the generation rate by the spectral intensity of the pulse and integrating over the light frequency. In the case of a Gaussian pulse $E_{0}(t)=E_{0} \exp \left(-t^{2} / \tau_{L}^{2}\right)$ :

$g_{\vec{k}}=\frac{\sqrt{2 \pi}}{\hbar^{2}}\left[d_{\vec{k}} E_{0}\right]^{2} \tau_{L} \exp \left(-2 t^{2} / \tau_{L}^{2}\right) \exp \left[-\frac{1}{2} \tau_{L}^{2}\left(\omega_{\vec{k}}-\omega_{L}\right)^{2}\right]\left(1-n_{\vec{k}}^{(e)}(t)-n_{-\vec{k}}^{(h)}(t)\right)$

It is assumed that during the laser pulse the polarization is not influenced by any other interaction, in particular, there are no phase-breaking processes during carrier generation. Thus, the only density dependence of the generation rate is due to Pauli blocking.

Equations (6.4) can be modified by the different interaction mechanisms such as carrier-phonon interaction and carrier-carrier interaction, etc.

$$
\begin{aligned}
& \frac{d}{d t} n_{\vec{k}}^{(e)}=g_{\vec{k}}^{(0)}(t)+\left.\sum_{\alpha} \frac{d}{d t} n_{\vec{k}}^{(e)}\right|^{\alpha} \\
& \frac{d}{d t} n_{-\vec{k}}^{(h)}=g_{\vec{k}}^{(0)}(t)+\left.\sum_{\alpha} \frac{d}{d t} n_{\vec{k}}^{(\mathrm{h})}\right|^{\alpha} \\
& \frac{d}{d t} p_{\vec{k}}=\frac{1}{i \hbar}\left(\varepsilon_{\vec{k}}^{(e)}-\varepsilon_{-\vec{k}}^{(h)}\right) p_{\vec{k}}+\frac{1}{i \hbar} d_{\vec{k}} E_{0}(t) \exp \left(-i \omega_{L} t\right)\left(1-n_{\vec{k}}^{(e)}-n_{-\vec{k}}^{(h)}\right)+\left.\sum_{\alpha} \frac{d}{d t} p_{\vec{k}}\right|^{\alpha}
\end{aligned}
$$

where summation is over the various types of interactions.

The case of carrier polar-optical-phonon scattering will be considered. In the absence of an external optical field the electron states are eigenstates of an ideal periodic lattice. Deviations from this idealized periodicity due to lattice vibrations lead to a coupling of the different electron states. This interaction is described by the carrier-phonon part of the Hamiltonian $\widehat{H}_{I}(t)$. Contributions up to second order in the carrier-phonon coupling matrix element to the equations of motion of distribution functions and polarization are given by:

$$
\begin{aligned}
& \frac{d}{d t} n_{\vec{k}}^{(e)}=g_{\vec{k}}^{(0)}(t)+\left.\sum_{\alpha} \frac{d}{d t} n_{\vec{k}}^{(e)}\right|^{\alpha} \\
& \frac{d}{d t} n_{-\vec{k}}^{(h)}=g_{\vec{k}}^{(0)}(t)+\left.\sum_{\alpha} \frac{d}{d t} n_{\vec{k}}^{(\mathrm{h})}\right|^{\alpha} \\
& \frac{d}{d t} p_{\vec{k}}=\frac{1}{i \hbar}\left(\varepsilon_{\vec{k}}^{(e)}-\varepsilon_{-\vec{k}}^{(h)}\right) p_{\vec{k}}+\frac{1}{i \hbar} d_{\vec{k}} E_{0}(t) \exp \left(-i \omega_{L} t\right)\left(1-n_{\vec{k}}^{(e)}-n_{-\vec{k}}^{(h)}\right)+\left.\sum_{\alpha} \frac{d}{d t} p_{\vec{k}}\right|^{\alpha}
\end{aligned}
$$

where summation is over the various types of interactions.

The case of carrier polar-optical-phonon scattering will be considered. In the absence of an external optical field the electron states are eigenstates of an ideal periodic lattice. Deviations from this idealized periodicity due to lattice vibrations lead to a coupling of the different electron states. This interaction is described by the carrier-phonon part of the Hamiltonian $\widehat{H}_{I}(t)$. Contributions up to second order in the carrier-phonon coupling matrix element to the equations of motion of distribution functions and polarization are given by: 


$$
\begin{aligned}
& \left.\frac{d}{d t} n_{\vec{k}}^{(e)}\right|^{(c p, 2)}=-\sum_{\vec{q}}\left[W_{\vec{k}-\bar{q}, \vec{k}}^{(e)(\mathrm{cp})} n_{\vec{k}}^{(e)}-W_{\vec{k}, \vec{k}-\bar{q}}^{(e)(c p)} n_{\vec{k}-\vec{q}}^{(e)}\right]+\frac{1}{i \hbar}\left[\Delta_{\vec{k}}^{e(c p)} p_{\vec{k}}^{*}-\Delta_{\vec{k}}^{* \mathrm{e}(c p)} p_{\vec{k}}\right] \\
& \frac{d}{d t} n_{\vec{k}}^{\left.(\mathrm{h})\right|^{(c p, 2)}}=-\sum_{\vec{q}}\left[W_{\vec{k}-(\vec{q}, \vec{k})}^{(h)(c p)} n_{\vec{k}}^{(h)}-W_{\vec{k}, \vec{k}-\vec{q}}^{(h)(c p)} n_{\vec{k}-\vec{q}}^{(\mathrm{h})}\right]+\frac{1}{i \hbar}\left[\Delta_{-\vec{k}}^{\mathrm{h}(c p)} p_{-\vec{k}}^{*}-\Delta_{-\vec{k}}^{* \mathrm{~h}(c p)} p_{-\vec{k}}\right] \\
& \left.\frac{d}{d t} p_{\vec{k}}\right|^{(c p, 2)}=-\sum_{\vec{q}}\left[W_{\vec{k}-\vec{q}, \vec{k}}^{(p)(\mathrm{cp})} p_{\vec{k}}-W_{\vec{k}, \vec{k}-\vec{q})}^{(p)(\mathrm{cp})} p_{\vec{k}-\vec{q}}\right]
\end{aligned}
$$

with scattering rates:

$$
\begin{aligned}
& W_{\vec{k}-\vec{q}, \vec{k}}^{e, h(c p)}=\frac{2 \pi}{\hbar} \sum_{ \pm}\left|C_{\vec{q}}\right|^{2} \delta\left(\varepsilon_{\vec{k}-\vec{q}}^{(e, h)}-\varepsilon_{\vec{k}}^{(e, h)} \pm \hbar \omega_{\vec{q}}\right)\left(N_{\vec{q}}+\frac{1}{2} \pm \frac{1}{2}\right)\left(1-n_{\vec{k}-\vec{q}}^{(e, h)}\right) \\
& W_{\vec{k}-\vec{q}, \vec{k}}^{p(c p)}=\frac{\pi}{\hbar} \sum_{\nu=e, h} \sum_{ \pm}\left|C_{\vec{q}}\right|^{2} D\left[\varepsilon_{\vec{k}-\vec{q}}^{(\nu)}-\varepsilon_{\vec{k}}^{(\nu)} \pm \hbar \omega_{\vec{q}}\right]\left[\left(N_{\vec{q}}+\frac{1}{2} \mp \frac{1}{2}\right) n_{\vec{k}-\vec{q}}^{(\nu)}+\left(N_{\vec{q}}+\frac{1}{2} \pm \frac{1}{2}\right)\left(1-n_{\vec{k}-\vec{q}}^{(\nu)}\right)\right] \\
& \Delta_{\vec{k}}^{\mathrm{e}, \mathrm{h}(c p)}=i \pi \sum_{\vec{q}}\left|C_{\vec{q}}\right|^{2} \sum_{ \pm}\left( \pm p_{\vec{k}-\vec{q}}\right) D\left[\varepsilon_{\vec{k}}^{(h, e)}-\varepsilon_{\vec{k}-\vec{q}}^{(h, e)} \pm \hbar \omega_{\vec{q}}\right]
\end{aligned}
$$

where $N_{\vec{q}}$ is the distribution of phonons and in the case of a polar interaction. It can be seen that $\Delta_{\vec{k}}^{e(c p)}$ appear in the same way as the electric field of the laser in equations (6.5) and (6.6) and the explicit expression for $\left|C_{\vec{q}}\right|^{2}$ is given in 6.3. Therefore, they could be interpreted as internal field that are different for electrons and holes. Also the structure equations (6.5) and (6.6) shows symmetry between distribution functions and polarization. There are terms identifying "outscattering" processes $\left(\propto n_{\vec{k}}, p_{\vec{k}}\right)$ and terms identifying "out-scattering" processes $\left(\propto n_{\vec{k}-\vec{q}}, p_{\vec{k}-\vec{q}}\right)$. The main difference is that, in contrast to the distribution functions, the polarization and the respective matrices are complex quantities.

\subsection{Boltzmann scattering equation}

Apart from details of the generation process itself, where the coherence of the excitation is essential [36] the relaxation is satisfactorily described by a semi classical theory which can be obtained from the semiconductor Bloch equations by adiabatic elimination of the coherent interband polarization and a Markov approximation. Under the assumption that the initial coherence and correlation of the generated electron-hole pairs is rapidly damped out and that the approximations are reasonable the resulting Boltzmann equation for the electron distribution is used for description of femtosecond to picosecond pulsed laser interaction with semiconductors and dielectrics [37]. The use of a Boltzmann transport equation with a drift term [38] under a time-dependent electric field can be justified only within the limit of $v_{L} \tau_{p} \ll 1$, where $v_{L}$ is the frequency of the external field and $\tau_{p}$ is the momentum-relaxation time of carriers. This approach can no longer be physically justified for an incident electromagnetic field with $v_{L} \geq 1 \mathrm{THz}$, and $\tau_{p}=1 p s$ [39]. The electrons are expected to be spatially localized when the time period $T=1 / v_{L} \leq \tau_{p}$. As a result, no drift of electrons can occur under such an electromagnetic field. 
The non-equilibrium electron distribution is governed by the Boltzmann equation for a homogeneous system and a Markovian process assumed for the dynamics of electron scattering where $k$ is a wave number for electrons, $n_{k}^{e}$ is the nonequilibrium distribution of electrons [40]

$$
\begin{gathered}
\frac{\partial n_{k}^{e}}{\partial t}=W_{k}^{(i n)(\alpha)}\left(1-n_{k}^{e}\right)-W_{k}^{(o u t)(\alpha)} n_{k}^{e}+G_{k}^{P I}-W_{k}^{(e x t)} n_{k}^{e} \\
W_{k}^{(\alpha)(\text { in }, \text { out })}=W_{k}^{(p h)}+W_{k}^{(i m p)}+W_{k}^{(e e)}
\end{gathered}
$$

represents the total rate of scattering-in the final electron state $|\vec{k}\rangle$ and scattering-out of the final electron state $|\vec{k}\rangle$ for electrons and $\alpha=(e-p h n-p h t),(e-p h),(e-e)$, (imp), (ar) denotes photon-assisted phonon, phonon, impurity and Coulomb scattering of electrons in the conduction band and scattering in and out of the conduction band. As in section 6.1 a bulk substrate at finite lattice temperature $T$ with two isotropic, parabolic bands, the conduction band and valence band, is considered. The laser electric field is $\vec{E}(t)=E_{0} \cos \left(\omega_{L} t\right) \vec{e}_{x}$ where $E_{0}$ is its amplitude of the field and $\vec{e}_{x}$ is its polarization. The amplitude $E_{0}$ of the pulsed laser field should be time dependent as in section 6.2. A standard Gaussian profile can be assumed for the laser intensity $\left(I_{L} \propto E_{0}^{2}\right)$ and the time dependence of the laser intensity is characterized by both the peak intensity and the pulse duration $\tau_{L}$.

\subsubsection{Photoionization}

The photoionization term in equation (6.7) is derived using Keldysh theory. It provides the transition rate $\dot{W}_{p i}$ from an initial state (valence band) to a final state (conduction band) due to a monochromatic field with amplitude $E_{0}$ and frequency $\omega_{L}$ [41-44]:

$$
G_{k}^{P I}=\frac{2 \pi}{\hbar}\left|\mathrm{M}_{p i}\right|^{2} \delta\left(\varepsilon_{k}^{e}-\varepsilon_{k^{\prime}}^{h}-l_{p i} \hbar \omega_{L}\right) n_{k^{\prime}}^{h}\left(1-n_{k}^{e}\right)
$$

where $n_{k^{\prime}}^{h}$ is the distribution function in the valence band and the matrix element for parabolic band structure is given by:

$\left|\mathrm{M}_{p i}\right|^{2}=\frac{\hbar}{2 \pi} \frac{2 \pi^{2} \hbar^{3} \dot{W}_{p i}}{\sqrt{2 m_{e} \mu^{2} \varepsilon}} \delta_{\varepsilon, \varepsilon_{p i}}$ with

$\varepsilon_{p i}=\frac{\mu}{m_{e}}\left(l_{p i} \hbar \omega_{L}-E_{G}\right)$,

where $\mu^{-1}=m_{e}^{-1}+m_{h}^{-1}$ is the reduced mass. A complete Keldysh photoionization rate for parabolic band is given by [43]:

$\dot{W}_{p i} \approx \frac{2 \omega_{L}}{9 \pi}\left(\frac{\mu \omega_{L}}{\hbar \gamma_{2}}\right)^{\frac{3}{2}} Q(\gamma, x) \times \exp \left[-\pi l_{p i} \frac{K\left(\gamma_{2}\right)-E\left(\gamma_{2}\right)}{E\left(\gamma_{1}\right)}\right]$

with

$Q(\gamma, x)=\sqrt{\pi / K\left(\gamma_{1}\right)} \sum_{m=0}^{\infty} \exp \left[-\pi m \frac{K\left(\gamma_{2}\right)-E\left(\gamma_{2}\right)}{E\left(\gamma_{1}\right)}\right] \times \phi\left[\sqrt{\pi^{2} \frac{2 l_{p i}-x+m}{2 K\left(\gamma_{1}\right) E\left(\gamma_{1}\right)}}\right]$ 
where

$\gamma=\frac{\omega_{L} \sqrt{\mu E_{G}}}{e E_{0}}$

is the Keldysh parameter.

$\gamma_{1}=\frac{1}{\sqrt{1+\gamma^{2}}}, \gamma_{2}=\frac{\gamma}{\sqrt{1+\gamma^{2}}}, \phi$ is a Dawson integral and $K$ and $E$ are complete elliptic integrals of the first and second kinds, respectively.

$\tilde{E}_{G}=\frac{2 E_{G}}{\pi \gamma_{1}} E\left(\gamma_{1}\right) \approx E_{G}\left(1+\frac{1}{4 \gamma^{2}}\right)$ is the effective effective band gap,

$x=\frac{\tilde{E}_{G}}{\hbar \Omega}$

and $l_{p i}=[x+1]$, where [] denotes the integer part.

The transition of a valence band electron to the conduction band requires absorbing minimum number of photons $l_{p i}$ necessary to cross the bandgap. However, the electron energy in the final state is limited, because the process occurs in the first Brillouin zone.

$\varepsilon_{p i, l_{\max }} \leq \varepsilon_{B Z}$

where $l_{\max }=\left[\frac{2 \varepsilon_{B Z}+E_{G}}{\hbar \omega_{L}}-l_{p i}\right]$

if $\hbar \omega_{L} \ll \varepsilon_{B Z}$ then $l_{\text {max }} \rightarrow \infty$.

In the spectral range used commonly the photon energy is several $\mathrm{eV}$, which is comparable to the Brillouin edge energy and the limitations in $l_{\max }$ have to be taken into account.

\subsubsection{Electron scattering}

Using the Hamiltonian from equation 6.2, the transition rate from the initial state to the final state is calculated by Fermi's golden rule

$W_{i \rightarrow f}=\frac{2 \pi}{\hbar}|\langle f|\widehat{H}| i\rangle|^{2} \delta\left(\varepsilon_{f}-\varepsilon_{i}\right)$

The effect of an incident oscillating electric field is reflected in the impurity- and phonon-assisted photon absorption through modifying the scattering of electrons with impurities and phonons. The matrix elements in the transition rate are calculated using the dressed final and initial states or the so called Volkov phases obtained from the solution of the time dependent Schrodinger equation for the conduction electron wave function [45].

$i \hbar \frac{\partial \psi(\vec{r}, t)}{\partial t}=\frac{1}{2 m^{*}}\left[-i \hbar \vec{\nabla}_{\vec{r}}+\frac{e}{c} \vec{A}(\vec{r}, t)\right]^{2} \psi(\vec{r}, t)$

with the vector potential of the laser field

$\vec{A}(t)=\left(E_{0} c / \omega_{L}\right) \sin \left(\omega_{L} t\right) \vec{e}_{x}$

In the Volkov state, the eigenstate of a free electron in electromagnetic field, absorbs the oscillatory time-dependence in a phase factor. In this case the electron (hole) operators introduced in section 6.2 are: 
$\hat{c}_{\vec{k}}(t)=\hat{a}_{\vec{k}}(t) \exp \left[i \zeta_{0} k_{x}\left(1-\cos \omega_{L} t\right)\right] \exp \left[i \zeta_{1} t\left(\sin \left(2 \omega_{L} t\right) / \omega_{L} t-2\right) / \hbar\right]$

where

$\zeta_{0}=e E_{0} / m^{*} \omega_{L}^{2}$

is the mean distance travelled by an electron for one period of the laser pulse and

$\zeta_{1}=\left(e E_{0}\right)^{2} / 4 m^{*} \omega_{L}^{2}$

is the ponderomotive energy shift.

\subsubsection{Photon assisted electron phonon scattering}

When the incident electromagnetic field is spatially uniform, electrons in the conduction band of a bulk semiconducting (dielectric) material in the two-band model considered can not directly absorb photons through an intraband transition since $\vec{A}(t) \cdot\left\langle u_{B}^{c}(\vec{r})|\hat{p}| u_{B}^{c}(\vec{r})\right\rangle=0$ where $u_{B}^{c}(\vec{r})$ is the is the Bloch function for conduction electrons and $\hat{p}$ is the momentum operator [31,37]. This is a direct result of the impossibility of conserving energy and momentum simultaneously during the absorption of a photon by an electron in a single conduction band. The quantum correction to the electron-phonon coupling which is referred to as phonon-assisted free-carrier absorption from the laser field is included. This correction term becomes important when the laser field is strong and either the lattice temperature is very high or the electron initial kinetic energy is very large.

The total scattering-in/scattering-out rates for the final electron state $|\vec{k}\rangle$ due to phonons, including phonon assisted photon absorption, are given by

$$
\begin{aligned}
& W_{k}^{(\mathrm{in})(e-p h n-p h t)} \\
& =\frac{2 \pi}{\hbar} \sum_{\vec{q} \lambda}\left|C_{q \lambda}\right|^{2} \sum_{M=-\infty}^{+\infty} \mathrm{J}_{|M|}^{2}\left(\mathrm{~F}_{q}\right) \\
& \times\left[n_{|\vec{k}-\vec{q}|}^{e} N_{q \lambda}^{p h} \delta\left(\varepsilon_{k}^{e}-\varepsilon_{|\vec{k}-\vec{q}|}^{e}-\hbar \omega_{q \lambda}-M \hbar \omega_{L}\right)\right. \\
& \left.+n_{|\vec{k}+\vec{q}|}^{e}\left(N_{q \lambda}^{p h}+1\right) \delta\left(\varepsilon_{k}^{e}-\varepsilon_{|\vec{k}+\vec{q}|}^{e}+\hbar \omega_{q \lambda}+M \hbar \omega_{L}\right)\right] \\
& =\frac{2 \pi}{\hbar} \sum_{\vec{q} \lambda}\left|C_{q \lambda}\right|^{2} \sum_{M=-\infty}^{+\infty} \mathrm{J}_{|M|}^{2}\left(\mathrm{~F}_{q}\right) \\
& \times\left[\left(1-n_{|\vec{k}+\vec{q}|}^{e}\right) N_{q \lambda}^{p h} \delta\left(\varepsilon_{|\vec{k}+\vec{q}|}^{e}-\varepsilon_{k}^{e}-\hbar \omega_{q \lambda}-M \hbar \omega_{L}\right)\right. \\
& \left.+\left(1-n_{|\vec{k}-\vec{q}|}^{e}\right)\left(N_{q \lambda}^{p h}+1\right) \delta\left(\varepsilon_{|\vec{k}-\vec{q}|}^{e}-\varepsilon_{k}^{e}+\hbar \omega_{q \lambda}+M \hbar \omega_{L}\right)\right]
\end{aligned}
$$

where $\mathrm{J}_{|M|}^{2}(x)$ is the $M$ th order first-kind Bessel function, $m^{*}=\frac{m_{e} m_{h}}{m_{e}+m_{h}}$ is the effective mass of electrons and $\omega_{L}=2 \pi v_{L}$ is the incident photon energy. 
$\mathrm{F}_{q}=\frac{e q \mathrm{E}_{0}}{\sqrt{2} m^{*} \omega_{L}^{2}}$, where $\hbar \omega_{q \lambda}$ represents the phonon energy in different phonon branches.

In Equations (6.9), (6.10) the effect of Pauli exclusion is included in the factors $\left(1-n_{|\vec{k} \pm \vec{q}|}^{e}\right)$ for the final electron states during the transitions. An additional equation may be included for the time evolution of the phonon distribution. It can be solved self consistently with the Boltzmann kinetic equation for the conduction electron distribution. For this treatment, phonons are assumed to be in thermal equilibrium with an external heat bath at a fixed temperature $T$.

The distribution of phonons is a Bose-Einstein function given by

$N_{q \lambda}^{p h}=\frac{1}{\exp \left(\frac{\hbar \omega_{q \lambda}}{k_{B} T}\right)-1}$

If the lattice temperature is not too high, only the scattering with longitudinal phonons can be considered by neglecting the umklapp process. For longitudinal optical (LO) phonons in polar semiconductors or dielectrics, Frohlich electron-phonon coupling [46] is used

$\left|C_{q \lambda=L O}\right|^{2}=\left(\frac{\hbar \omega_{L O}}{2 \mathrm{~V}}\right)\left(\frac{1}{\varepsilon_{r}(\infty)}-\frac{1}{\varepsilon_{r}(0)}\right) \frac{e^{2}}{\varepsilon_{0}\left(q^{2}+Q_{S}^{2}\right)}$, where $\mathrm{v}$ is the volume of the simple, $\omega_{L O}$ is the frequency of the dominant longitudinal-optical (LO) phonon modes at high temperatures, $\varepsilon_{r}(\infty)$ and $\varepsilon_{r}(0)$ are the relative optical and static dielectric constants and

$Q_{s}^{2}=\frac{e^{2}}{\varepsilon_{0} \varepsilon_{r}} \frac{m_{e}^{*}}{\pi^{2} \hbar^{2}}\left(3 \pi^{2} n_{3 D}\right)^{1 / 3}$

is the static Thomas-Fermi screening factor which depends on the conduction-electron density $n_{3 D}$ and effective electron mass.

For acoustic phonon scattering, the deformation potential approximation is used [31,47].

$\left|C_{q \lambda}\right|^{2}=\frac{\hbar \omega_{q l}}{2 \mathrm{~V} \rho_{\text {ion }} v_{l}^{2}}\left[D^{2}+\frac{9}{32 q^{2}}\left(e h_{12}\right)^{2}\right]\left(\frac{q^{2}}{q^{2}+Q_{S}^{2}}\right)^{2}$

$\left|C_{q \lambda}\right|^{2}=\frac{\hbar \omega_{q t}}{2 \mathrm{~V} \rho_{\text {ion }} v_{t}^{2}} \frac{13}{64 q^{2}}\left(e h_{12}\right)^{2}\left(\frac{q^{2}}{q^{2}+Q_{s}^{2}}\right)^{2}$

where $\lambda=l, t$ corresponds to one longitudinal and two transverse acousticphonon modes, $v_{l}$ and $v_{t}$ are the sound velocities for these modes, $\rho_{\text {ion }}$ is the ion mass density, $D$ is the deformationpotential coefficient and $h_{12}$ is the piezoelectric constant. Applying the Debye model to low-energy acoustic phonons, we get $\omega_{\vec{q} \lambda}=c_{\lambda} q$ with $\lambda=l, t$.

For longitudinal acoustic (LA) phonons in both polar and nonpolar semiconductors and dielectrics [48]:

$\left|C_{q L A}\right|^{2}=\frac{q n_{i o n}}{2 M_{i o n} v_{L A} \mathrm{~V}}\left[\frac{e^{2}}{\varepsilon_{0} \varepsilon_{r}(0)\left(q^{2}+Q_{S}^{2}\right)}\right]^{2}$

where $M_{i o n}$ is the ion mass, $n_{\text {ion }}$ is the ion density, and $v_{L A}$ is the sound velocity of longitudinal acoustic phonons. 


\subsubsection{Photon assisted electron-impurity scattering}

The total scattering-in/scattering-out rates for the final electron state $|\vec{k}\rangle$ due to impurities are given by [37]

$$
\begin{aligned}
& W_{k}^{(\mathrm{in})(i m)}= N_{I} \frac{2 \pi}{\hbar} \sum_{\vec{q}}\left|U^{i m}(\mathrm{q})\right|^{2} \sum_{M=-\infty}^{+\infty} \mathrm{J}_{|M|}^{2}\left(\mathrm{~F}_{q}\right)\left[n_{|\vec{k}-\vec{q}|} \delta\left(\varepsilon_{k}^{e}-\varepsilon_{|\vec{k}-\vec{q}|}^{e}-M \hbar \omega_{L}\right)\right. \\
&\left.+n_{|\vec{k}+\vec{q}|} \delta\left(\varepsilon_{k}^{e}-\varepsilon_{|\vec{k}+\vec{q}|}^{e}+M \hbar \omega_{L}\right)\right] \\
& W_{k}^{(\mathrm{in})(i m)}=N_{I} \frac{2 \pi}{\hbar} \sum_{\vec{q}}\left|U^{i m}(\mathrm{q})\right|^{2} \sum_{M=-\infty}^{+\infty} \mathrm{J}_{|M|}^{2}\left(\mathrm{~F}_{q}\right)\left[\left(1-n_{|\vec{k}+\vec{q}|}\right) \delta\left(\varepsilon_{|\vec{k}+\vec{q}|}^{e}-\varepsilon_{k}^{e}-M \hbar \omega_{L}\right)\right. \\
&\left.+\left(1-n_{|\vec{k}-\vec{q}|}^{e}\right) \delta\left(\varepsilon_{|\vec{k}-\vec{q}|}^{e}-\varepsilon_{k}^{e}+M \hbar \omega_{L}\right)\right]
\end{aligned}
$$

with $\left|U^{(i m)}(q)\right|=\frac{Z e^{2}}{\varepsilon_{0} \varepsilon_{r}\left(q^{2}+Q_{S}^{2}\right) \mathrm{v}}$ where $Z$ is the charge number of an ionized impurity atom.

\subsubsection{Electron- electron binary scattering. Impact ionization. Auger recombination}

Coulomb scattering of electrons is a relative motion between electrons. As a result, the external optical field does not directly couple to it. This scattering contributes to the thermalization of the electrons while the optical field is still on .

The total scattering-in/scattering-out rates for the final electron state $|\vec{k}\rangle$ due to the Coulomb interaction between electrons are given by $[47,49,50]$

$$
\begin{gathered}
W_{k}^{(\mathrm{in})(c)}=\frac{2 \pi}{\hbar} \sum_{\vec{k}^{\prime}, \vec{q}}\left|V^{(c)}(\mathrm{q})\right|^{2}\left(1-n_{k^{\prime}}^{e}\right) n_{|\vec{k}-\vec{q}|}^{e} n_{\left|\vec{k}^{\prime}+\vec{q}\right|}^{e} \delta\left(\varepsilon_{k}^{e}+\varepsilon_{k^{\prime}}^{e}-\varepsilon_{|\vec{k}-\vec{q}|}^{e}-\varepsilon_{\left|\vec{k}^{\prime}+\vec{q}\right|}^{e}\right) \\
W_{k}^{(\text {out })(c)}=\frac{2 \pi}{\hbar} \sum_{\vec{k}^{\prime}, \vec{q}}\left|V^{(c)}(\mathrm{q})\right|^{2} n_{k^{\prime}}^{e}\left(1-n_{|\vec{k}-\vec{q}|}^{e}\right)\left(1-n_{\left|\vec{k}^{\prime}+\vec{q}\right|}^{e}\right) \delta\left(\varepsilon_{|\vec{k}-\vec{q}|}^{e}+\varepsilon_{\left|\vec{k}^{\prime}+\vec{q}\right|}^{e}-\varepsilon_{k}^{e}-\varepsilon_{k^{\prime}}^{e}\right)
\end{gathered}
$$

where

$$
\left|V^{(c)}(q)\right|=\frac{e^{2}}{\varepsilon_{0} \varepsilon_{r}\left(q^{2}+Q_{S}^{2}\right) V}
$$

is the the Fourier transform of the Coulomb potential.

The processes of impact ionization and auger recombination can contribute significantly to conduction-electron dynamics and the change of conduction electron density.

Following the diagrams in Figure 6.1, the total scattering-in/scattering-out rates for the final electron state $|\vec{k}\rangle$ due to impact ionization as a second-order two-particle Coulomb scattering process, proportional to $\left|V^{(c)}(q)\right|^{2}$ are [31]: 


$$
\begin{aligned}
& \Gamma_{k}^{(\mathrm{in})(i m p)}=\frac{2 \pi}{\hbar} N_{e f f}\left(1-n_{k}^{e}\right)^{2} \sum_{\vec{q}}\left|V^{(c)}(\mathrm{q})\right|^{2} g_{v}(q)\left(1-n_{|\vec{k}-\vec{q}|}^{h}\right) n_{|\vec{k}+\vec{q}|}^{e} \delta\left(2 \varepsilon_{k}^{e}-\varepsilon_{|\vec{k}+\vec{q}|}^{e}+E_{G}+\varepsilon_{|\vec{k}-\vec{q}|}^{h}\right) \\
& \Gamma_{k}^{(\text {out })(i m p)}=\frac{2 \pi}{\hbar} N_{e f f} n_{k}^{e} \sum_{\vec{q}}\left|V^{(c)}(\mathrm{q})\right|^{2} g_{v}(q)\left(1-n_{|\vec{k}-\vec{q}|}^{e}\right)^{2}\left(1-n_{|\vec{k}-2 \vec{q}|}^{h}\right) \delta\left(2 \varepsilon_{|\vec{k}-\vec{q}|}^{e}-\varepsilon_{k}^{e}+E_{G}-\varepsilon_{|\vec{k}-2 \vec{q}|}^{h}\right)
\end{aligned}
$$
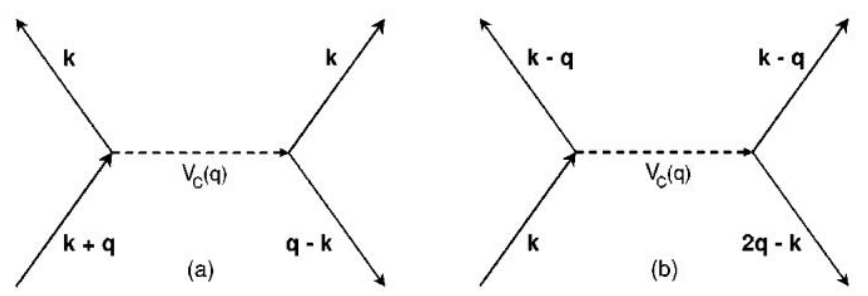

Figure 6.1. Diagrams for impact ionization with electron scattering into state $|\vec{k}\rangle$ in a) and scattering out of state $|\vec{k}\rangle$ in b). Here, the upward and downward solid arrows represent the electron and hole states with the wave vector indicated, respectively. The horizontal dashed arrows stand for the Coulomb interaction $V^{(c)}(q)$ between two electrons. Figure from Ref. [31].

$N_{\text {eff }}$ is the effective number of conduction electrons with energy close to $E_{G}$ and $g_{v}(q)$ is the interband-transition form factor.

The Auger recombination process is also a second-order two particle Coulomb scattering process proportional to $\left|V^{(c)}(q)\right|^{2}$ and it is the inverse process of impact ionization only in the case that the principle of detailed balance can be applied. Following the diagrams in Figure 6.2 (a-b) the total scattering-in/scattering-out rates due to Auger recombination are:

$$
\begin{gathered}
\Gamma_{k}^{(\mathrm{in})(\text { rec })}=\frac{2 \pi}{\hbar}\left(1-n_{k}^{e}\right) \sum_{\vec{q}}\left|V^{(c)}(\mathrm{q})\right|^{2} g_{v}(q) n_{|\vec{k}-2 \vec{q}|}^{h} \delta\left(\varepsilon_{k}^{e}-2 \varepsilon_{|\vec{k}-\vec{q}|}^{e}-E_{G}-\varepsilon_{|\vec{k}-\overrightarrow{2 q}|}^{h}\right)\left(n_{|\vec{k}-\vec{q}|}^{e}\right)^{2} \\
\Gamma_{k}^{(\text {out })(\text { rec })}=\frac{2 \pi}{\hbar}\left(n_{k}^{e}\right)^{2} \sum_{\vec{q}}\left|V^{(c)}(\mathrm{q})\right|^{2} g_{v}(q) \delta\left(\varepsilon_{|\vec{k}+\vec{q}|}^{e}-2 \varepsilon_{k}^{e}-E_{G}-\varepsilon_{|\vec{k}-\vec{q}|}^{h}\right) n_{|\vec{k}-\vec{q}|}^{h}\left(1-n_{|\vec{k}+\vec{q}|}^{e}\right)
\end{gathered}
$$
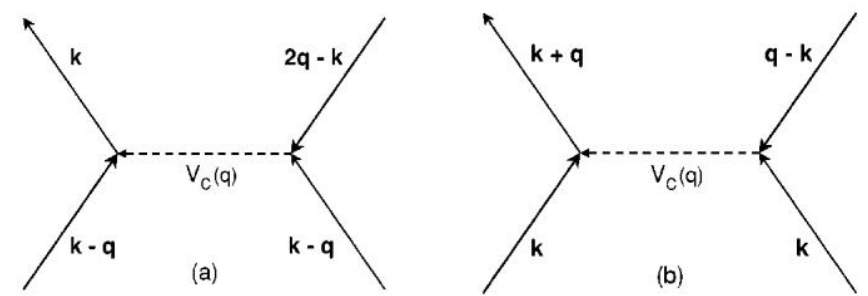

Figure 6.2. Diagrams for Auger recombination with electron scattering into state $|\vec{k}\rangle$ in a) and scattering out of state $|\vec{k}\rangle$ in b). Here, the upward and downward solid arrows represent the electron and hole states with the wave vector indicated, respectively. The horizontal dashed arrows stand for the Coulomb interaction $V^{(c)}(q)$ between two electrons. Figure from Ref. [31]. 


\subsubsection{Electron phonon scattering in the absence of the laser field}

After the conclusion of the laser pulse the electron-phonon scattering is given by equation (6.7) when the laser electric field is 0 . Then electrons experience scattering with phonons transferring energy from the electronic system to the crystal lattice vibrations. Following the diagrams in Figure $6.3(\mathrm{a}-\mathrm{d})$ the total electron-phonon scattering-in/scattering-out rates for the final electron state $|\vec{k}\rangle$ are:

$$
\begin{aligned}
& W_{\vec{k}}^{(\mathrm{in})}=\sum_{\vec{q}}\left(W_{\vec{k}-\vec{q} \rightarrow \vec{k}}^{(a b)}+W_{\vec{k}+\vec{q} \rightarrow \vec{k}}^{(e m)}\right) \\
& =\frac{2 \pi}{\hbar}\left(1-n_{k}^{e}\right) \sum_{q^{\vee} \lambda}\left|C_{q \lambda}\right|^{2} \\
& \times\left[\delta\left(\varepsilon_{k}^{e}-\varepsilon_{|\vec{k}-\vec{q}|}^{e}-\hbar \omega_{q \lambda}\right) N_{q \lambda}^{p h} n_{|\vec{k}-\vec{q}|}^{e}\right. \\
& \left.+\delta\left(\varepsilon_{k}^{e}-\varepsilon_{|\vec{k}+\vec{q}|}^{e}+\hbar \omega_{q \lambda}\right)\left(N_{q \lambda}^{p h}+1\right) n_{|\vec{k}+\vec{q}|}^{e}\right] \\
& W_{\vec{k}}^{\text {(out) }}=\sum_{\vec{q}}\left(W_{\vec{k} \rightarrow \vec{k}-\vec{q}}^{(a b)}+W_{\vec{k} \rightarrow \vec{k}+\vec{q}}^{(e m)}\right) \\
& =\frac{2 \pi}{\hbar} n_{k}^{e} \sum_{\vec{q} \lambda}\left|C_{q \lambda}\right|^{2} \\
& \times\left[\delta\left(\varepsilon_{k}^{e}-\varepsilon_{|\vec{k}+\vec{q}|}^{e}+\hbar \omega_{q \lambda}\right) N_{q \lambda}^{p h}\left(1-n_{|\vec{k}+\vec{q}|}^{e}\right)\right. \\
& \left.-\delta\left(\varepsilon_{k}^{e}-\varepsilon_{|\vec{k}-\vec{q}|}^{e}-\hbar \omega_{q \lambda}\right)\left(N_{q \lambda}^{p h}+1\right)\left(1-n_{|\vec{k}-\vec{q}|}^{e}\right)\right]
\end{aligned}
$$
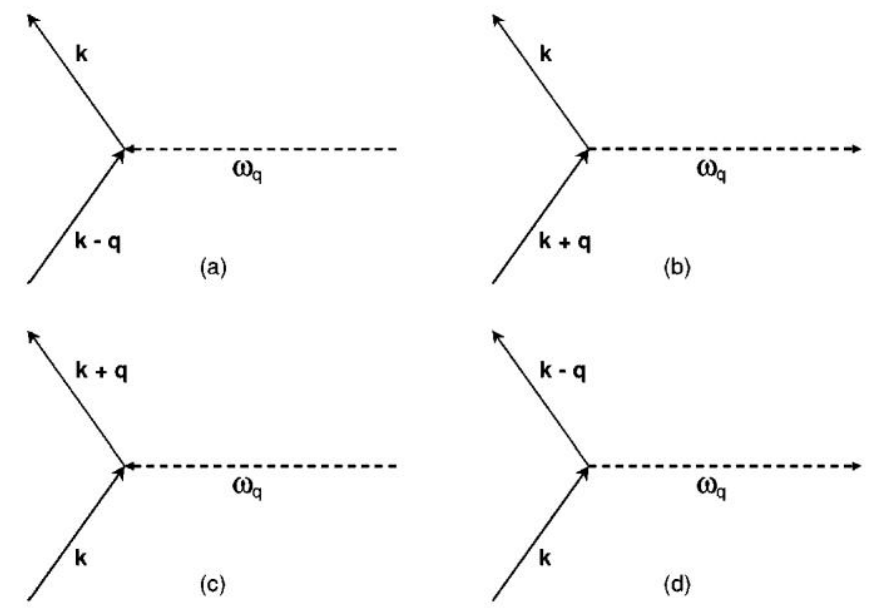

Figure 6.3. Diagrams for electron scattering into state in $|\vec{k}\rangle$ with a) phonon absorption! and b) with phonon emission and for electron scattering out of state $|\vec{k}\rangle$ in c) with phonon emission and d) with phonon absorption. Here, the upward solid arrows represent the electron states with the wave vector indicated. The horizontal dashed arrows indicate the phonon states with the phonon frequency $\omega_{\vec{q}}$. Figure from Ref. [31].

As can be seen from Equation (6.19) and Equation (6.20), the effect of Pauli exclusion is included in the factors of $\left(1-n_{k}^{e}\right)$ and $\left(1-n_{|\vec{k} \pm \vec{q}|}^{e}\right)$ for the final electron states during the transitions. 


\subsubsection{Exciton formation (XF) and non-radiative decay (NRXD)}

The strong Coulomb interaction between an electron and a hole forces the pair to form an exciton. The collision integral for self-trapped exciton (STE)-formation $W_{k}^{(e x t)} n_{k}^{e}$ in Boltzmann equation (6.7) is included semi-empirically [51]. The dimensionless cross section is an overlap integral of the STE-wavefunction, which is a Gaussian with a radius $a_{D}$, and the plane-wave conduction band electron function

$\sigma_{e l}(\varepsilon)=\exp \left[-\frac{1}{2 \pi} \frac{\varepsilon}{\varepsilon_{S T E}}\right]$

where the exciton binding energy is:

$\varepsilon_{S T E}=\frac{\hbar^{2}}{2 m_{e} a_{D}^{2}}$

A rate equation for exciton formation is given by:

$\left(\frac{d N_{X}}{d t}\right)_{X F}=-\frac{d N_{C}}{d t}=\int d E n_{k} W_{k}^{(e x c)}$

where $N_{x}$ represents the exciton density and $N_{c}$ the electron densityin the conduction band calculated by the Boltzmann equation. Radiative exciton decay is not considered due to the characteristic lifetimes. Excited excitons can decay non-radiatively [52] when an energy barrier between the ground and excited electronic states of a localized or self-trapped exciton can be overcome. The non-radiative decay can be accounted for with a term that depends on lattice temperature $\mathrm{T}$.

\subsubsection{Photoionization and scattering rates from first principles}

In chapter 5.3 .3 a first-principles version of the Kadanoff-Baym equations [53,54] employing the collision approximation [55] to reduce the non-equilibrium Green's function dynamics to a single time variable was used to reproduce the carrier dynamics in bulk silicon subjected to an external laser pulse. The equation for the evolution of the non-equilibrium electron (hole) occupation $f_{n \vec{k}}(t)\left(f_{n \vec{k}}^{e}(t)=f_{n \vec{k}}(t), f_{n \vec{k}}^{h}(t)=1-f_{n \vec{k}}(t)\right)$ contains a coherent generation term and ab initio carrier-carrier and carrier-phonon lifetimes $\gamma^{e . h}(t)$ whose formal derivation is performed by using the NEGF theory and that formally correspond to the carrier-carrier and carrier-phonon scattering in the semi classical Boltzmann equation obtained using Fermi Golden Rule. $\gamma^{e . h}(t)$ are time-dependent and non-linear functions of the occupations and are re-calculated at each time step to take into account the presence of the external field. The generation term is derived from Green's functions approach based on DFT Kohn-Sham Hamiltonian and includes Hartree and exchange correlation effects. The expressions for carrier-carrier and carrier phonon scattering include the statically screened Coulomb interaction and the screened ionic potential derivatives, calculated within the density-functional perturbation theory [56].

\subsection{Practical examples}

The pulsed laser field drives the distribution of electrons away from the thermal-equilibrium distribution to a non-thermal one. Figure 6.4 displays a comparison between calculated 
nonequilibrium electron distributions $n_{\vec{k}}$ with and without including in the calculation the effect of pair scattering and the thermal-equilibrium Fermi-Dirac distribution of electrons (dash-dotted curve) at $T=77 \mathrm{~K}$. The value of the peak electric field is $E_{L 0}=100 \mathrm{kV} / \mathrm{cm}$ and $\hbar \omega_{L}=25 \mathrm{meV}$ [46]. The calculation is carried out for doped GaAs with initial number density of electrons $n_{3 D}^{e}=$ $10^{18} \mathrm{~cm}^{-3}$. It can be seen that the impurity- and phonon-assisted photon absorption processes create multiple small peaks (dashed curve) on the high-energy tail (above $54 \mathrm{meV}$ ) of the FermiDirac distribution. The occurrence of these high-energy peaks are attributed to electrons that have predominantly been scattered-out of low-energy states below the Fermi energy (at $54 \mathrm{meV}$ ), which can be seen from terms with $M \neq 0$ in Eqs. (6.9), Eqs. (6.10) and (6.11), (6.12). At the same time, the combination of the electrons that have been scattered out from below the Fermi energy and the electrons that have been scattered out from the band edge leave an oscillation of the electron distribution there. From the conservation of electron number in the conduction band, we expect that the electron distribution below the Fermi energy will drop as the distribution spreads towards high-energy states.

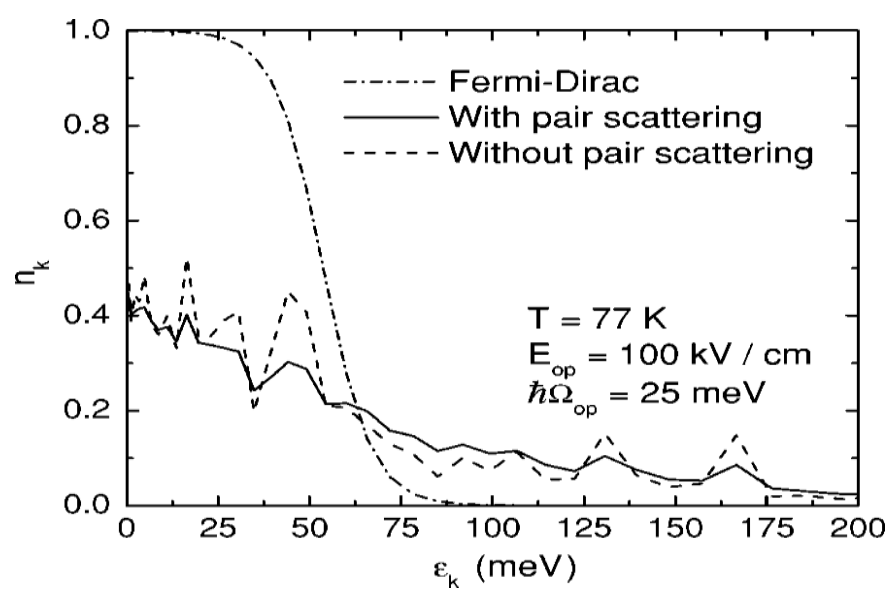

Figure 6.4. Calculated electron distribution $n_{\vec{k}}$ for a bulk GaAs as a function of the kinetic energy $\varepsilon_{k}$ of electrons at T=77. The dash-dotted curve is for the thermal-equilibrium Fermi-Dirac function. The solid curve is for the calculated nonequilibrium electron distribution with the effect of pair scattering, while the dashed curve represents that without the effect of pair scattering. Figure from Ref. [47].

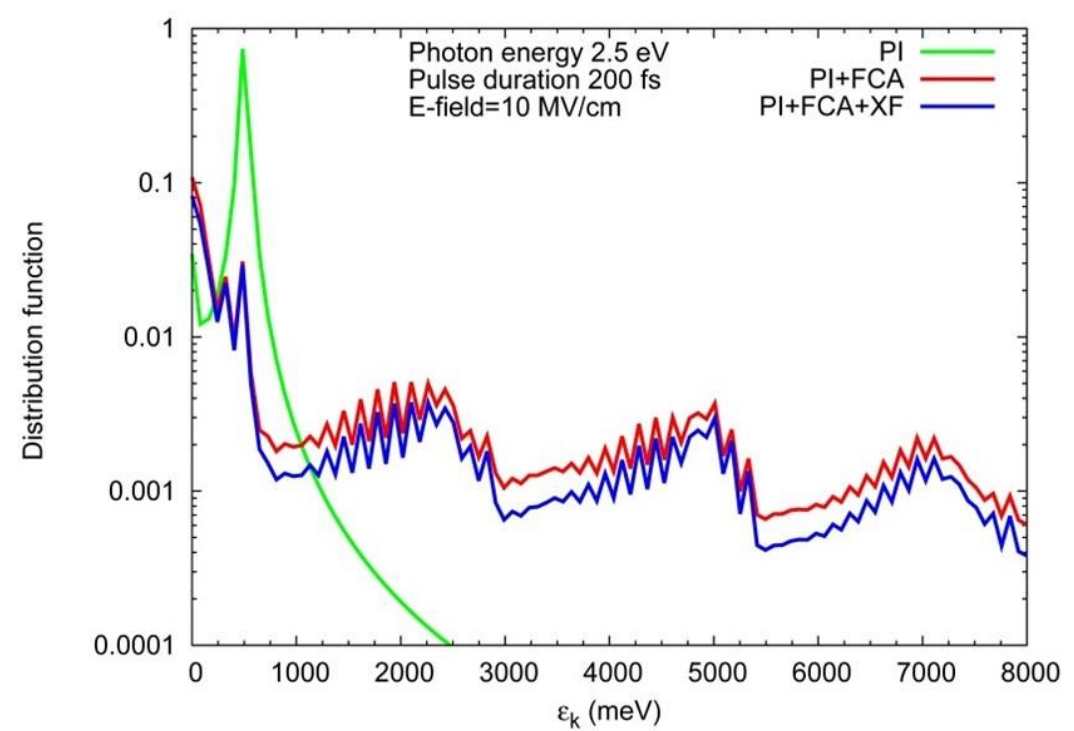

Figure 6.5. Calculated electron distribution $n_{\vec{k}}$ for a bulk $\mathrm{SiO}_{2}$ as a function of the kinetic energy $\varepsilon_{k}$ of electrons for photon energy $5 \mathrm{eV}$ and pulse duration 200 fs. Figure from Ref. [40]. 
In Figure 6.5 we show the electron distribution function of $\mathrm{SiO}_{2}$ irradiated with a laser pulse of duration 200 fs. Three different processes are included in the calculation - photoionization, inverse bremsstrahlung and exciton formation. The electron distribution curve peaks at $500 \mathrm{meV}$, which is the calculated detuning of the photionization term from the bottom of the conduction band. In the other two curves the initial peak diminishes and electron distribution is transferred to higher energy states, where peaks are observed at $2.5 \mathrm{eV}, 5 \mathrm{eV}, 7.5 \mathrm{eV}$, etc., accompanied by small peaks as a result of the inverse bremsstrahlung.

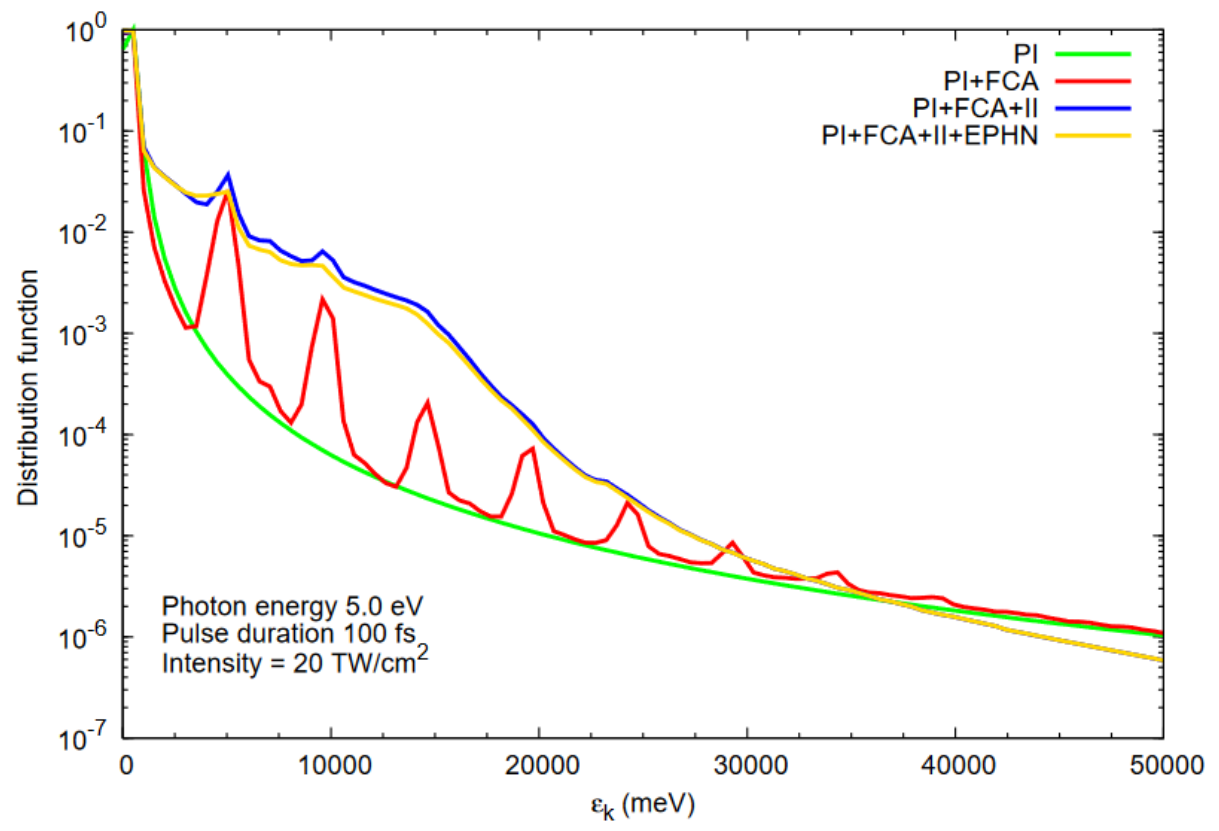

Figure 6.6. Calculated electron distribution $n_{\vec{k}}$ for a bulk $\mathrm{SiO}_{2}$ as a function of the kinetic energy $\varepsilon_{k}$ of electrons for photon energy $5 \mathrm{eV}$ and pulse duration 100 fs. Figure from Ref. [57].

In Figure 6.6 we show the electron distribution function of $\mathrm{SiO}_{2}$ irradiated with a laser pulse of duration $100 \mathrm{fs}$ and photon energy of $5 \mathrm{eV}$. Four different processes are included in the calculation - photoionization, inverse bremsstrahlung and impact ionization and electron-phonon scattering. The curve representing the effect of PI peaks at the same energy of $500 \mathrm{meV}$, which is the calculated detuning of the photionization term from the bottom of the conduction band. In the other three curves the initial peak diminishes and electron distribution is transferred to higher energy states, where peaks are observed at $5 \mathrm{eV}, 10 \mathrm{eV}, 15 \mathrm{eV}$, etc. as a result the free carrier absorption process. It is also evident that the impact ionization leads to an appreciable increase of number of electrons at lower energies in comparison with Figure 6.5, while the electronphonon interaction slightly lowers the magnitude of the distribution function uniformly.

In the calculations we denote $n_{k}^{e}(t)=n^{e}\left(\varepsilon_{k}^{e}, t\right)$ and define $f_{k}^{e}=\rho_{k}^{e} n_{k}^{e}$ with the density of states per unit volume $\rho_{k}^{e}=C_{0} \sqrt{\varepsilon_{k}^{e}}$ in a parabolic dispersion model, where

$C_{0}=\frac{1}{2 \pi^{2}} \frac{\left(2 m^{*}\right)^{3 / 2}}{\hbar^{3}}$

Integrating $f_{k}^{e}$ over all conduction band energy states the conduction band density $n_{3 D}^{e}=\int_{0}^{+\infty} f^{e}\left(\varepsilon_{k}\right) d \varepsilon=\int_{0}^{+\infty} n^{e}\left(\varepsilon_{k}\right) \rho\left(\varepsilon_{k}\right) d \varepsilon=\frac{k_{F}^{3}}{\pi^{2}} \int_{0}^{+\infty} \bar{k}^{2} n^{e}(\bar{k}) d \bar{k} \quad$ is obtained, where the 
dimensionless wave vector is $\bar{k}=k / k_{F}$ where $k_{F}=\left(3 \pi^{2} n_{3 D}^{e}\right)^{1 / 3}$ is the Fermi wave number. The energy density is determined by the

$\varepsilon^{e}=\int_{0}^{+\infty} \varepsilon f^{e}\left(\varepsilon_{k}\right) d \varepsilon=\int_{0}^{+\infty} \varepsilon n^{e}\left(\varepsilon_{k}\right) \rho\left(\varepsilon_{k}\right) d \varepsilon=\frac{\varepsilon_{F} k_{F}^{3}}{\pi^{2}} \int_{0}^{\infty} \bar{k}^{4} n^{e}(\bar{k}) d \bar{k}$ where

$\varepsilon_{F}=\frac{\hbar^{2}\left(3 \pi^{2} n_{3 D}^{e}\right)^{\frac{2}{3}}}{2 m_{e}^{*}}$

is the Fermi energy of conduction electrons at zero temperature.

Figure 6.7 shows the evolution of conduction band electron density for photon energy $5 \mathrm{eV}$ when contributions from the processes of photoionization, free carrier absorption, impact ionization and electron-phonon scattering are included. The density increase due to impact ionization as the pulse progresses is very pronounced for this photon energy and the effect of electron-phonon interaction is also appreciable.

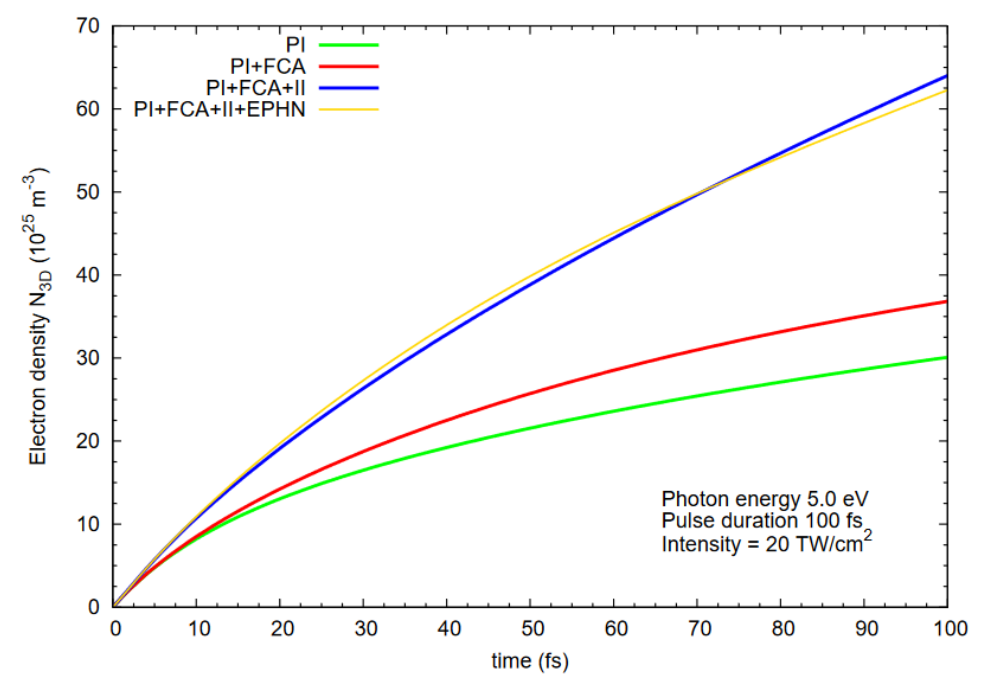

Figure 6.7. Electron density as a function of pulse duration for photon energy $5 \mathrm{eV}$. Figure from Ref. [57].
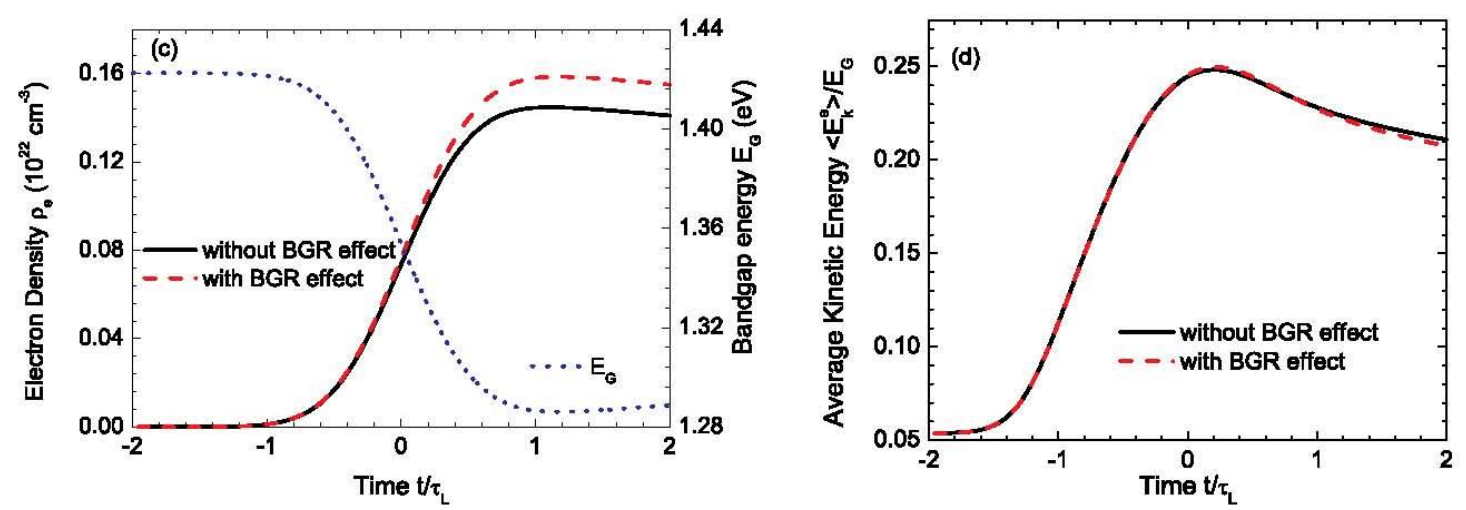

Figure 6.8. Conduction electron density (c), and scaled average electron kinetic energy (d) transients for irradiation of bulk GaAs with pulsed 100fs laser with photon energy $1.55 \mathrm{eV}$ and intensity $0.1 \mathrm{TW} / \mathrm{cm}^{2}$.The Gaussian pulse is centered at 0 . Band gap renormalization (BGR) effect is included (dashed curve) or excluded (solid curve), respectively. Figure from Ref. [58]. 

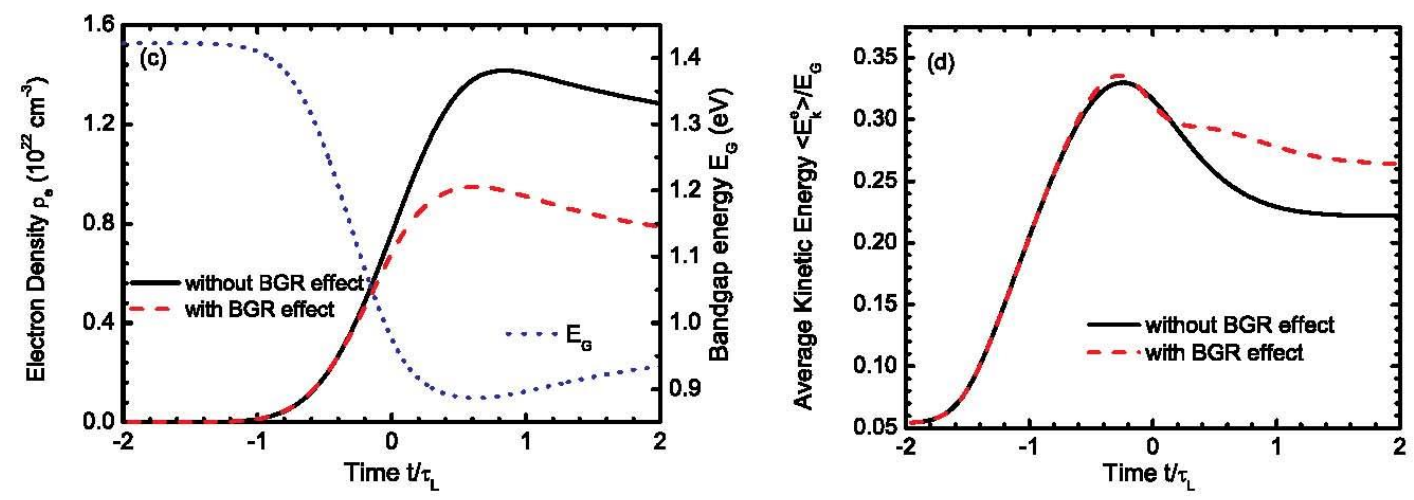

Figure 6.9. The same transients, as in Figure 6.8 for laser intensity of $1 \mathrm{TW} / \mathrm{cm}^{2}$. Figure from Ref. [58].

Finally optical damage criteria can be formulated using the definitions for the conduction band electron density and average energy. If the incident laser field is absorbed by an intrinsic undoped bulk semiconductor, the electrons in the completely filled valence band will cross the band gap to the conduction band. The free-electron density increases with the intensity of the incident laser field. The excited electrons in the conduction band can form an electron plasma when the density is high. and thus the Coulomb interaction is strong. The existence of this plasma increases the reflection of the incident light.

When the condition

$\omega_{L} \geq \frac{e^{2} n_{3 D}^{e}}{\varepsilon_{0} \varepsilon_{r} m_{e}^{*}}$

is satisfied, the incident light will be totally reflected from the surface of the semiconductor. At this point, the semiconductor becomes opaque, and the electron density reaches its maximum value $n_{\text {critical }}^{e}$. In this case, the opaque semiconductor is optically damaged by the laser field for optoelectronic uses since the incident light can no longer be coupled into it. The atoms in semiconductors, such as $\mathrm{Si}$ and GaAs, are chemically connected by covalent bonds with cohesive energy $V_{b} \sim E_{g}$ in a crystal since $E_{g}$ prohibits the creation of free electrons from bound electrons in the valence band. If the intrinsic semiconductors are exposed to an incident laser field, the statistically averaged kinetic energy per electron can be written as

$\left\langle\varepsilon_{k}^{e}\right\rangle=\frac{\int_{0}^{+\infty} \varepsilon_{k}^{e} f_{k}^{e} d \varepsilon_{k}^{e}}{\int_{0}^{+\infty} f_{k}^{e} d \varepsilon_{k}^{e}}$

which is related to the electron temperature $T_{e}$ by the expression

$\Delta \bar{\varepsilon}=\left\langle\varepsilon_{k}^{e}\right\rangle-\frac{3}{5} \varepsilon_{F}=\frac{3}{2} k_{B} T_{e}$.

When the electron distribution function peaks at higher and higher energies, $\Delta \bar{\varepsilon}$ increases although $n_{3 D}^{e}$ could be small at this time. If $\left\langle\varepsilon_{k}^{e}\right\rangle=E_{g}$, there is an instability for chemical bonds in semiconductors. In this case the semiconductor structurally damaged by the laser field for semiconducting-material uses. 


\subsection{Summary}

Starting from a quantum kinetic formalism based on the density matrix a Boltzmann scattering equation was established for modeling intense ultra-short pulse induced high electronic excitation in band gap materials. The processes of photo - ionization and laser energy absorption by including the impurity- and phonon-assisted photon absorption as well as and electron thermalization by Coulomb scattering of electron-electron pairs were considered. Impact ionization and Auger recombination were also considered. The transient conduction electron distribution functions were obtained. We have quantified multiple peaks in the energy distribution functions and analyzed the effect of different microscopic mechanisms. Conduction electron densities and the average electron energies during the fs-laser pulse interaction with semicinductors and dielectrics were evaluated. Some possible types of damage in semiconductors and dielectrics were discussed.

\section{Acknowledgements}

This chapter is based on research performed in collaboration with my colleagues from the US Air Force Research Laboratory (D. Huang, A. Cardimona, P. Alsing) and Instituto de Fusión Nuclear "Guillermo Velarde" (A.Rivera, J.M. Perlado). COST Action CA17126 and support from the Bulgarian National Science Fund (KP-06-KOST) are acknowledged.

\section{References}

[1] Shah, J., Ultrafast Spectroscopy of Semiconductors and Semiconductor Nanostructures (Springer, Berlin), (1999).

[2] Thomas E., Shah J., Rota L., and Lugli P., Phys. Rev. Lett. 66, 1757, (1991)

[3] Kurz H. Semicond. Sci. Technol. 7 B124, (1992)

[4] Kash, J. A., and J. C. Tsang in Light Scattering in Solids IV: Electron Scattering, Spin Effects, SERS and Morphic Effects, edited by M. Cardona and G. Guntherodt, Topics in Applied Physics Vol. 54 (Springer, Berlin), p. 423, (1989)

[5] Kash, J. A., J. C. Tsang, and J. M. Hvam, Phys. Rev. Lett. 54, 2151, 1985

[6] Bradley C.W.W., Taylor R.A., Ryan F., Solid-State Electronics, 32, 1173, (1989)

[7] P. C. Becker, H. L. Fragnito, C. H. Brito Cruz, R. L. Fork, J. E. Cunningham, J. E. Henry, and C. V. Shank, Phys. Rev. Lett. 61, 1647, (1988)

[8] K. Leo, M. Wegener, J. Shah, D. S. Chemla, E. O. Göbel, T. C. Damen, S. Schmitt-Rink, and W. Schäfer, Phys. Rev. Lett. 65, 1340, (1990)

[9] K. Leo, E. O. Göbel, T. C. Damen, J. Shah, S. Schmitt-Rink, W. Schäfer, J. F. Müller, K. Köhler, and P. Ganser, Phys. Rev. B 44, 5726, (1991)

[10] A. Mysyrowicz, D. Hulin, A. Antonetti, A. Migus, W. T. Masselink, and H. Morkoç, Phys. Rev. Lett. 56, 2748, (1986)

[11] W. H. Knox, D. S. Chemla, D. A. B. Miller, J. B. Stark, and S. Schmitt-Rink, Phys. Rev. Lett. 62, 1189, 1989

[12] Pötz W. and Kocevar P. Electronic power transfer in pulsed laser excitation of polar semiconductors Phys. Rev. B 28, 7040, (1983)

[13] Leo K. and Collet J. H. Influence of electron-hole scattering on the plasma thermalization in doped GaAs, Phys. Rev. B 44, 5535, (1991) 
[14] Zheng T.F., Cai W., Hu P., Lax M., Simulation of ultrafast relaxation of photoexcited electrons via analytical distribution functions, Solid-State Electronics, 32, (1989)

[15] Fadel M., Rieger M., Vaissiere J.C., Nougier J.P., Kocevar P., Hot phonon-hot electron coupled Boltzmann equations, Solid-State Electronics, 32, 1229 (1989)

[16] Grinberg Anatoly A. and Luryi Serge, Nonstationary quasiperiodic energy distribution of an electron gas upon ultrafast thermal excitation, Phys. Rev. Lett. 65, 1251 (1990)

[17] Binder R., Scott D., Paul A. E., Lindberg M., Henneberger K., and Koch S. W., Carrier-carrier scattering and optical dephasing in highly excited semiconductors, Phys. Rev. B 45, 1107 (1992)

[18] Lugli P., Bordone P., Reggiani L., Rieger M., Kocevar P., and Goodnick S. M., Monte Carlo studies of nonequilibrium phonon effects in polar semiconductors and quantum wells. I. Laser photoexcitation, Phys. Rev. B 39, 7852, (1989)

[19] Stanton C. J., Bailey D. W., and Hess K., Femtosecond-pump, continuum-probe nonlinear absorption in GaAs, Phys. Rev. Lett. 65, 231; Erratum Phys. Rev. Lett. 66, 242, (1991)

[20] Ferry D. K., Kriman A. M., Hida H., and Yamaguchi S., Collision retardation and its role in femtosecondlaser excitation of semiconductor plasmas, Phys. Rev. Lett. 67, 633, (1991)

[21] Tilmann Kuhn and Fausto Rossi, Monte Carlo simulation of ultrafast processes in photoexcited semiconductors: Coherent and incoherent dynamics, Phys. Rev. B 46, 7496, (1992)

[22] Schmitt-Rink S., Ell C., and Haug H., Many-body effects in the absorption, gain, and luminescence spectra of semiconductor quantum-well structures, Phys. Rev. B 33,1183, (1986)

[23] Schmitt-Rink S., Chemla D. S., and Haug H., Nonequilibrium theory of the optical Stark effect and spectral hole burning in semiconductors, Phys. Rev. B 37, 941, (1988)

[24] Kuznetsov A. V., Interaction of ultrashort light pulses with semiconductors: Effective Bloch equations with relaxation and memory effects, Phys. Rev. B 44, 8721, (1991)

[25] Schmitt-Rink S. and Chemla D. S., Collective Excitations and the Dynamical Stark Effect in a Coherently Driven Exciton System, Phys. Rev. Lett. 57, 2752, (1986)

[26] Zimmerman R., Transverse Relaxation and Polarization Specifics in the Dynamical Stark Effect, Phys status solidi b 159, 317, (1990)

[27] Tilmann Kuhn and Fausto Rossi, Monte Carlo simulation of ultrafast processes in photoexcited semiconductors: Coherent and incoherent dynamics, Phys. Rev. B 46, 7496, (1992)

[28] Stefan Haas, Fausto Rossi, and Tilmann Kuhn, Generalized Monte Carlo approach for the study of the coherent ultrafast carrier dynamics in photoexcited semiconductors, Phys. Rev. B 53, 12855, 1996

[29] Fausto Rossi and Tilmann Kuhn, Theory of ultrafast phenomena in photoexcited semiconductors, Rev. Mod. Phys. 74, 895, 2002

[30] Danhong Huang and D. A. Cardimona, Effects of off-diagonal radiative-decay coupling on electron transitions in resonant double quantum wells, Phys. Rev. A 64, 013822, (2001)

[31] T. Apostolova, D. H. Huang, P. M. Alsing, J. Mclver, and D. A. Cardimona, Effect of laser induced antidiffusion on excited conduction electron dynamics in bulk semiconductors, Phys. Rev. B 66, 075208, 2002

[32] T. Apostolova, Nuclear Instruments and Methods in Physics Research A 653 72, (2011)

[33] E. M. Epshtein, Sov. Phys. Solid State 11, 2213 , (1970)

[34] W. Quade, E. Scholl, F. Rossi, C. Jacoboni, Quantum theory of impact ionization in coherent high-field semiconductor transport, Phys. Rev. B 50 7398, (1994)

[35] Kuhn T. Density matrix theory of coherent ultrafast dynamics. In: Schöll E. (eds) Theory of Transport Properties of Semiconductor Nanostructures. Electronic Materials Series, vol 4. Springer, Boston, MA, (1998)

[36] Alfred Leitenstorfer, Andrea Lohner, Thomas Elsaesser, Stefan Haas, Fausto Rossi, Tilmann Kuhn, W. Klein, G. Boehm, G. Traenkle, and G. Weimann, Ultrafast Coherent Generation of Hot Electrons Studied via Band-to-Acceptor Luminescence in GaAs, Phys. Rev. Lett. 73, 1687, (1994) 
[37] D.H. Huang, Apostolova, T., Alsing, P.M., and Cardimona, D.A., Phys. Rev. B, 69 075214, (2004)

[38] Ziman, J.M. Principles of the Theory of Solids, 1st edn, Cambridge University Press, London, (1964)

[39] Yan, W.-X., Bao, S.-Q., Zhao, X.-G., and Liang, J.-Q. Theory of dynamical conductivity of interacting electrons. Phys. Rev. B, 61, 7269, (2000)

[40] T. Apostolova, J.M. Perlado, A. Rivera, Nuclear Instruments and Methods in Physics Research Section B, 352,167 (2015)

[41] Keldysh L. V. Ionization in the Field of a Strong Electromagnetic Wave, Sov. Phys. J. Exp. Theor. Phys. 20, 1307, (1965).

[42] Gruzdev V.E. Photoionization rate in wide band-gap crystals, Phys. Rev. B 75, 205106, (2007)

[43] Shcheblanov N. S., Derrien T. J. Y., and Itina T. E., Femtosecond laser interactions with semiconductor and dielectric materials, AIP Conf. Proc. 1464, 79 (2012); N. Shcheblanov, Etude numŭrique des interaction d'un laser femtoseconde avec des cibles dielectriques: applications a la determination du seuil d'endommagement des composants optiques, (2013).

[44] Shcheblanov, N. S., M. E. Povarnitsyn, P. N. Terekhin, S. Guizard, and A. Couairon, Nonlinear photoionization of transparent solids: A nonperturbative theory obeying selection rules, Phys. Rev. A 96, 063410, (2017).

[45] T. Apostolova, Danhong Huang, and D. A. Cardimona, , Phys. Rev. B 67, 205323, (2003)

[46] Frohlich, H. and Paranjape, B.V. Dielectric breakdown in solids. Proc. Phys. Soc., Sect. B, 69, 21, (1956)

[47] Huang, D.H., Alsing, P.A., Apostolova, T., and Cardimona, D.A. Effect of photon-assisted absorption on the thermodynamics of hot electrons interacting with an intense optical field in bulk GaAs. Phys. Rev. B, 71, 045204, (2005)

[48] B. K. Ridley, Quantum Processes in Semiconductors (Clarendon, 1999)

[49] Lindberg, M. and Koch, S.W. Effective Bloch equations for semiconductors. Phys. Rev. B, 38, 3342, (1988)

[50] Moloney, J.V., Indik, R.A., Hader, J., and Koch, S.W. Modeling semiconductor amplifiers and lasers: from microscopic physics to device simulation. J. Opt. Soc. Am. B, 11 2023, (1999)

[51] J. Zeller, A.J. Sabbah, M. Mero, P.M. Alsing, J. McIver, W.G. Rudolph, Femtosecond dynamics of highly excited dielectric thin films, in: G.J. Exarhos, A.H. Guenther, N. Kaiser, K.L. Lewis, M.J. Soileau, C.J. Stolz (Eds.), pp. 515-526, 2003

[52] A. Rivera, A. Mundez, G. Garcia, J. Olivares, J.M. Cabrera, F. Agully-Lypez, Ion beam damage and nonradiative exciton decay in LiNbO3, J. Lumin. 128 703, (2008)

[53] Marini A. Competition Between the Electronic and Phonon-Mediated Scattering Channels in the OutOf-Equilibrium Carrier Dynamics of Semiconductors: An Ab-initio Approach. J Phys Conf Ser 427, 012003 (2013)

[54] Sangalli D, and Marini A. Ultra-fast Carriers Relaxation in Bulk Silicon Following Photo-Excitation with a Short and Polarized Laser Pulse. EPL 110, 47004 (2015)

[55] Sangalli D, and Marini A, Complete collisions approximation to the Kadanoff-Baym equation: a firstprinciples implementation J. Phys.: Conf. Ser. 609, 12006, 2015

[56] Baroni S, de Gironcoli S, Dal Corso A, and Giannozzi P. Phonons and Related crystal Properties from Density-Functional Perturbation Theory. Rev Mod Phys 73, 515 (2001)

[57] T. Apostolova, P. Detistov, M.V. Ivanov, Y. Orieult, J.M. Perlado, A. Rivera, "Simulation of Ultra-Intense Laser Irradiation Induced High Electronic Excitation of Bandgap Materials", Nuclear Theory, Vol. 33 (2014), ISSN 1313-2822.

[58] Tzveta T. Apostolova, Andrey A. Ionin, Sergej Ivanovich Kudryashov, Leonid V. Seleznev, Dmitriy V. Sinitsyn, "Self-limited ionization in bandgap renormalized GaAs at high femtosecond laser intensities", Optical Engineering 51 (12), 121808 (2012). 


\title{
7. Semiclassical Monte Carlo methods
}

\author{
Nikita Medvedev* and Vladimir Lipp
}

Here we consider semiclassical Monte Carlo (MC) method applied to model particle transport in matter, focusing on photons, electrons and swift heavy ions. The basics of $\mathrm{MC}$ will be discussed in the context of the material irradiation scenario. The characteristic timescales of the problem, the dominant effects, and methods of their modelling are presented. As the most important parameter in a Monte Carlo model, the cross sections of various processes are discussed. The first Born approximation results in expression of the cross sections in terms of the complex dielectric function of the target. We discuss how such cross sections may be constructed and used in MC simulations. This chapter also contains comments on limits of applicability and approximations in MC models, which are rarely mentioned in Monte Carlo modelling textbooks. Throughout the text, references to useful resources (such as available databases, books and reviews on simulations and codes) for MC modelling are provided. It thus can serve as a brief guide to Monte Carlo methods in radiation transport in matter.

*Contact: nikita.medvedev@fzu.cz 


\subsection{Introduction}

\subsubsection{Monte Carlo methods}

There is no "the" Monte Carlo (MC) method, only "a" Monte Carlo method [1]. There is a huge variety of them. Any model involving random numbers can, in principle, be classified as belonging to the Monte Carlo group. Just to name a few, there is Metropolis MC (tracing of ensemble evolution [2]), biological MC (modelling evolution of organisms and/or chemical reactions in them [3]), comparison of risk analysis (investment banking [4]), direct simulation MC (e.g. for tracing hydrodynamics in gas and fluid flow [5]), dynamic MC (used in chemistry [6]), kinetic MC (describing behaviour of defects in solids [7]), quantum MC (solving ab-initio problems with random sampling [8]), etc. We will consider here only the MC for particle transport, which includes subclasses of event-by-event (or analog) MC and condensed history MC [9].

\subsubsection{Transport processes and their timescales}

Irradiation of matter with fast particles induces a number of observable effects, important in various field of research and applications [10].

We start here by discussing photons in the range from extreme ultraviolet (some $\sim 50-100 \mathrm{eV}$ energy) up to gamma rays $(\sim \mathrm{MeV})$. A photon propagating in a solid target will experience the following processes: photoabsorption, inelastic (Compton) scattering, elastic (Rayleigh, or Thomson) scattering, and $\mathrm{e}^{-} \mathrm{e}^{+}$pair production (for photon energies above $2 \mathrm{~m}_{\mathrm{e}} \mathrm{c}^{2}$ ) [9]. For photon energies below some $100 \mathrm{keV}$, the dominant process is photoabsorption by the deepest possible atomic shell (with ionization potential just below the photon energy, $I_{p}<\hbar \omega$ ). During such a process, an electron from this shell is emitted and a core hole is created.

An impact of an elementary charged particle, such as an electron, induces a sequence of processes. Typically, an electron will scatter inelastically (impact ionization) or elastically, or emit a photon via Bremsstrahlung mechanism. Inelastic scattering event is accompanied by ionization of a new electron from a core shell or valence/conduction band, or - via scattering on collective electron modes of the target - by excitation of plasmons. Elastic scattering means electron scattering on the atomic system without its ionization (sometimes called "quasi-elastic" because some energy is still exchanged with the target). In this event, energy is transferred to an atom increasing its kinetic energy, or to collective atomic modes, phonons.

A swift heavy ion (SHI) penetrating through a target experiences almost the same processes as an incident electron. It loses most of its energy to inelastic scattering exciting bound or conduction band electrons. The difference is that an SHI may transfer so much energy to a target atom that it strips it of many electrons at once, leading to multiple ionization states [11]. For a review of SHI specific processes, we refer an interested reader to Refs. $[12,13]$.

All these irradiation scenarios take place at atto- to femto-second timescales, as depicted in Figure 7.1.

Let us now discuss how the target responds to the introduced excess energy. Typically, noticeable target response occurs after the incident particle (e.g. photon, electron or SHI) is already long gone from a point of interaction: photon may be scattered or absorbed, while charged particles 
continued their motion away from the scattering sight. Response of the target includes the following processes: Auger decays of core-shell holes (emitting an electron after another atomic electron fills the core hole) and radiative decays of core-shell holes (with emission of a photon) occurring at $\sim$ fs timescales [14]; transport of produced valence holes and excited electrons lasting typically from sub-fs to ps timescales, $10^{-15} \mathrm{~s}$ to $10^{-12} \mathrm{~s}$ [15]; atomic motion induced by energy transfer from the excited electrons and valence holes typically at ps timescales [15]; if sufficient energy was transferred, atomic disorder may take place at 1-10 ps timescales [16]; partial or full recovery of the structure requires $\sim 100$ ps -1 ns [16]; macroscopic relaxation of atomic defects and macroscopic strain relaxation may last nano- to micro-seconds; see schematics in Figure 7.1.

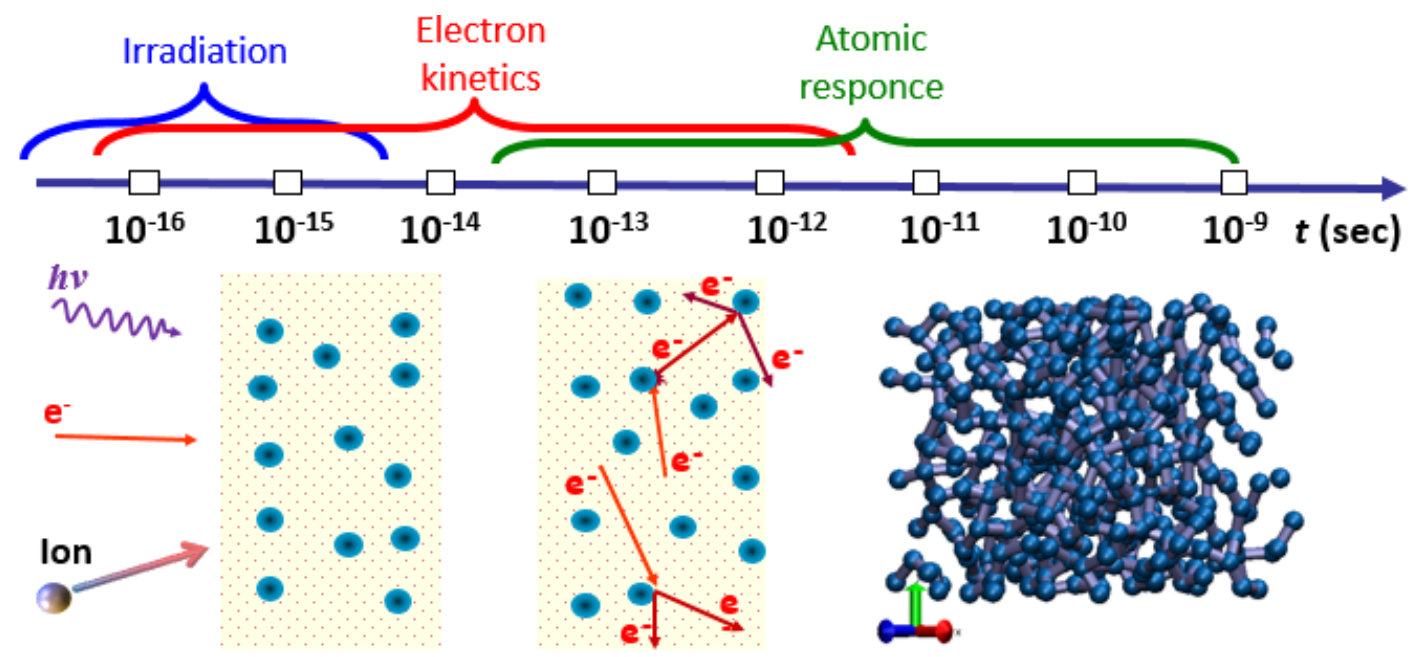

Figure 7.1. Schematics of characteristic timescales in ultrafast irradiation problem.

A sufficiently high-energy incident particle can be treated as a point-like projectile travestying through a solid. This makes it possible to model irradiation-induced processes via particles methods, such as transport Monte Carlo method.

\subsection{Core principles of Monte Carlo}

For a fast particle travelling through a solid, its trajectory may often be approximated as a sequence of straight lines suddenly changing its direction at points of scattering, see schematic Figure 7.2. If the distance between the interaction points is much larger than the characteristic size of a region of scattering, the scattering events may be considered as independent. In other words, the energy exchange in an interaction must be fast in comparison with other characteristic processes (the travel between scattering events, creation of emitted quasiparticles, etc. - note that it is fundamentally limited by the so-called quantum speed limit theorem [17]). In such as case, the particle transport may be described as Markovian process.

In the other case, when scattering process takes so long that it overlaps with the subsequent event, the process is non-Markovian (as illustrated in the bottom panel of Figure 7.2). It is a typical situation for slow particles, or dense, degenerate and strongly interacting systems. In this case, simple Monte Carlo algorithms cannot be applied, and more advanced methods must be used (e.g., time-discretized simulations such as molecular dynamics, MD). 
Markov chain: duration of scattering event $<<$ free flight time
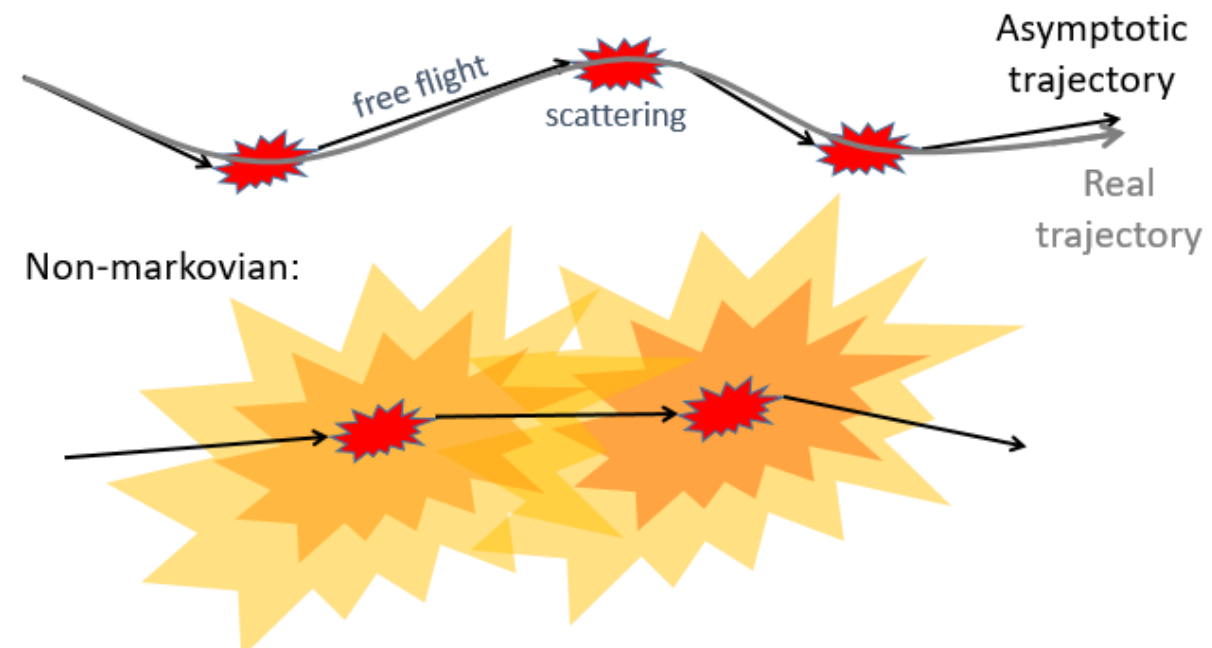

Figure 7.2. Schematics of particle transport in media. Top panel: an example when particle transport can be approximated as Markovian process. Bottom panel: a non-markovian process example.

Within the Markovian approximation, particle transport can be traced as a sequence of independent events, each modelled with its own probability. A classical particle in an absence of external fields travels along a straight line until it meets a scattering centre (a target atom, electron, plasmon, phonon). In external fields, a particle trajectory must be traced including forces acting on them $[9,18]$.

\subsubsection{A Monte Carlo algorithm}

The basic model of particle transport within Monte Carlo framework can then be split into a sequence of repeating events, see Figure 7.3: (a) free flight until a scattering event, (b) scattering, exchanging energy and momentum (which may be accompanied by creation of secondary particles), (c) change of particle's energy and momentum, defining the next free flight (see example of MC algorithm in Figure 7.4) [19]. This is a building block of an MC algorithm. We will consider particular details below.

Looking deeper into it, in practice an MC algorithms vary significantly. One of the most important distinctions is an event-by-event simulation scheme vs. condensed history MC. An event-by-event (or analog) MC models every single collision, while condensed history simulations model distant collisions (with a small deflection angle and energy exchange) using average energy loss along the path. Only close collisions (with large deflection angles) are typically sampled individually in condensed history simulations [18]. A schematic representation of these two approaches is shown in Figure 7.4.

A condensed history MC scheme can significantly speed up the simulation, paying for it with accuracy. It provides incomplete information, which may be insufficient for certain purposes such as modeling response of the target, not only transport of the incident particle. Since it misses a portion of the secondary electrons created, it may not be accurate enough for tracing material damage and similar processes. 


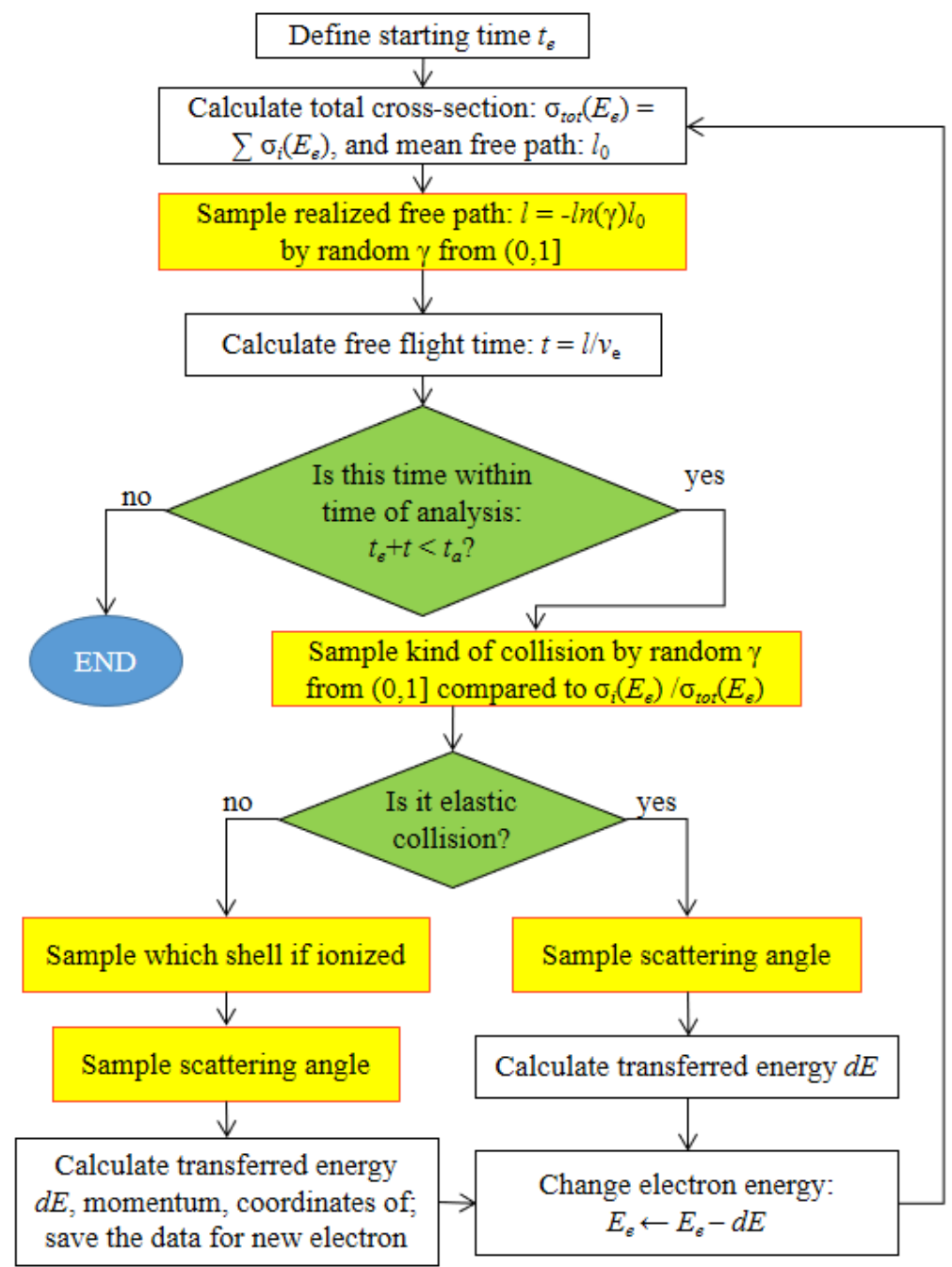

Figure 7.3. Schematics of a typical block in MC algorithm. Yellow blocks involve random numbers sampling. Reproduced from Ref. [20].
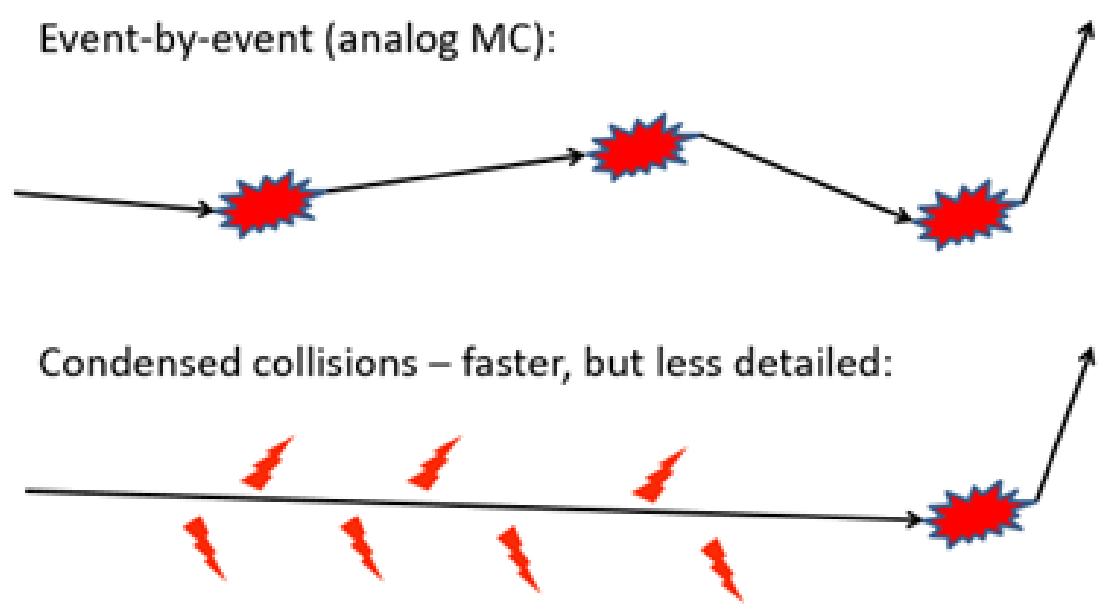

Figure 7.4. Schematics of event-by-event (or analog) MC simulation scheme vs. condensed history MC.

In a transport MC simulation, many processes are described with the help of random numbers. First, the free flight distance - the path until a scattering event - is usually sampled assuming a random meeting of a target scattering center (section 7.2.2). Then, a nature of the scattering event 
(elastic vs. inelastic scattering, Bremsstrahlung emission, etc.) is chosen randomly according to partial probabilities of different events considered. Third, scattering angle and/or transferred energy (section 7.2.2) are chosen randomly according to given probability distributions.

To take into account and average over all possible microscopic trajectories, the entire Monte Carlo simulation has to be repeated many times with different sampled random numbers. The averaging provides statistically reliable data, such as distributions, mean numbers of excited particles, or average energies in different systems. As typical for such integral methods, there are fluctuations in the average values, which decrease as $\sim 1 / \sqrt{N}$, with $N$ being the number of MC iterations.

\subsubsection{Probabilities and cross sections}

Monte Carlo simulations usually assume that the target is composed of random homogeneous arrangement of atoms. By neglecting atomic structure of the material, we gain the capability to simplify its description significantly. In a homogeneous media, the travelled distance of an incident particle between collisions can be defined according to the Poisson distribution [9]. The mean value of the distribution is defined as the mean free path, $l_{0}: P(x)=\exp \left(-x / l_{0}\right)$. In the framework of MC, each probability is replaced with a uniformly sampled random number $\gamma \in$ : $P \rightarrow \gamma$. Then, the corresponding free flight distance can be obtained via the mean free path and a generated random number as:

$$
x=-l_{0} \ln (\gamma)
$$

The mean free path in a homogeneous medium is connected with the cross section of scattering, $\sigma: l_{0}=1 /\left(n_{a} \sigma\right)$, where $n_{a}$ is the atomic density (assuming the cross section is normalized per atom). In evaluation of the total mean free path, the total cross section is a sum of cross sections of all possible scattering events. Note that the total mean free path (as well as total mean free time) can correspondingly be defined via Matthiessen rule considered partial mean free paths of all scattering channels $i: 1 / l_{0}=\sum_{i} 1 / l_{i}$.

Next, during the scattering event, transferred energy or momentum (and scattering angle) is defined via the differential cross section. For example, the probability to transfer energy $\delta E$ is:

$$
P(\delta E)=\frac{\delta \sigma}{\sigma}
$$

Thus, following the same procedure of replacing the probability with a (different) random number, the transferred energy can be defined by solving the equation:

$$
\gamma \sigma=\int_{E_{\min }}^{\delta E} \int_{q_{-}}^{q_{+}} \frac{d^{2} \sigma}{d \varepsilon d q} d \varepsilon d q
$$

written for the double differential scattering cross section over the transferred energy and momentum [21]. The integration limits for momentum and energy are defined by the conservation laws [9,22]. In practice, for finding the transferred momentum and energy, some approximations for the double differential cross sections are used, such that allow for an analytical solution of Eq. (7.3), significantly simplifying and speeding up simulations [9]. However, exact numerical solutions may also be used [15,22]. 
In an inelastic scattering event, the shell $n$ to be ionized is found via a random number and partial scattering cross sections $\sigma_{i}$ for each shell $i$ as follows:

$$
\gamma \sigma=\sum_{i}^{n} \sigma_{i}
$$

Thus, we see that cross sections are the key parameter in transport MC simulations.

In solid targets, scattering on valence or conduction band electrons requires defining energy state, from which an electron will be excited. The choice is made according to the probability distribution defined by the material's density of states [23].

\subsubsection{Specifics of different particles}

Each kind of particle interacts with solids via its own processes, which were mentioned above in Section 7.1.1. Let us discuss a few of them.

Photons entering the target interact mainly with electrons (except for wavelengths corresponding to optical phonons, which can be absorbed by atomic oscillations, typical energies of a fraction of $\mathrm{eV}$ ). Photons can be absorbed by electrons, scatter elastically (Rayleigh or Thomson scattering) or inelastically (Compton scattering), and disappear in a particle-antiparticle pair creation (for energies above twice the rest energy of the corresponding particle).

Photoabsorption produces a photoelectron and leaves a core hole in the shell the electron is emitted from. The cross sections of photoabsorption for each shell of each element for almost entire Periodic Table can be found, e.g., in EPICS-2017 database (which includes EPDL, EEDL, and EADL) [24]: https://www-nds.iaea.org/epics/. Total cross sections of photoabsorption (in term of attenuation length) can also be found in Henke's tables [25]: http://henke.lbl.gov/optical constants/atten2.html. EPICS-2017 database also contains the cross sections for other photon scattering processes. The data can be read and directly used in MC simulations. For a quick look, one can also use the X-ray Data Booklet [26].

However, for photons of low energies (below some 50-100 eV), the collective effects in solids become important. Atomic cross sections can no longer be used, and they have to be replaced with those accounting for the electronic structure of the material. This can be done via the connection between the optical absorption coefficients with the complex refractive index and the complex dielectric function of the material. Optical coefficients can be converted into the complex dielectric function, which in turn is connected with the photon attenuation length [15,22]. Optical coefficients for many materials may be found, e.g., in $[27,28]$.

Electrons in solids can be modelled as free particles for electron energies above some $50 \mathrm{eV}$. They will experience inelastic scattering (impact ionization and scattering on plasmons of the target), elastic scattering (interaction with targets atoms without ionization), and Bremsstrahlung emission of photons. The cross sections of Bremsstrahlung photon emission can be found in EPICS-2017 database (EEDL) [24], or calculated according to PENELOPE model [9].

During inelastic scattering, an incident electron creates another free electron, a secondary electron. Secondary electrons will perform their own scattering and create even more secondary 
particles. This process has multiple names, depending on the field: in MC modelling it is usually called "electron cascades", in laser physics it is referred to as "electron avalanche", while in relativistic particle impacts (by cosmic rays or particle accelerators) it's called "showers". The cross sections of inelastic electron scattering will be discussed in the next section. Ionization potentials of atoms, which are used in cross sections of scattering, can be found in EPICS-2017 database [24], or in experimental [29] and calculated works [30].

Elastic scattering of electrons transfers their kinetic energy to the target atoms without exciting secondary electrons. Depending on the electron energy, it interacts either with collective atomic modes (phonons), or with individual atoms in the target [31]. The energy defining the kind of interaction can be estimated from the characteristic time of electron travelling through the phonon wavelength (atomic correlation length), depending on electron velocity, in comparison with the phonon characteristic time (inverse phonon frequency). It corresponds to electron energies of a few tens of eV: slower electrons interact with the collective atomic modes, whereas for faster electrons the target atoms are too slow and thus essentially immobile. In the latter case, the incident electron interacts with each atom independently [31,32]. At such energies, the scattering cross sections may be approximated with the atomic ones, for instance with Mott's cross sections with modified Molier screening parameter [18].

Core holes left after photo- or impact ionization will decay via multiple channels. They can decay with emitting an electron, i.e., Auger (and Koster-Kronig) decay, or with emitting a photon, i.e., radiative or fluorescent decay [14]. The new created hole(s) in an upper shell will also decay, until all created holes will reach the valence or conduction band of the material. Characteristic times of the Auger as well as radiative decays for each shell of each element can be found in (atomic) EPICS2017 database [24], and in many experimental works [14]. The database also includes probabilities for electrons from different shells to participate in the decay, which allows to sample the shells in an MC procedure.

It is important to point out that apart from intra-atomic decays, in molecules and in solids, interatomic decays are possible. In solids, they are known as Knotek-Feibelman processes [33], while in molecular physics and chemistry the corresponding processes are called ICD, ETI, and a class of related processes [34]. These processes may be of fundamental importance in certain cases. For example, in lithium atoms, $\mathrm{K}$-shell hole can only decay via radiative process, whereas neighboring atoms can provide electrons to participate in (interatomic) Auger decay. These processes are rarely taken into account in MC codes, although they should be [35].

Also, in contrast to atoms (gases and plasma), in solids valence holes are mobile and can travel [19]. They behave essentially as slow electrons in the conduction band, but with its own effective mass [36]. They may scatter elastically and, in case of a narrow band gap material, inelastically. These effects can significantly affect the energy transport and distribution in insulators and semiconductors, and thus influence observable material damage [37].

In case of a swift heavy ion (SHI) penetrating through a solid, the same effects as in electron scattering can take place, with addition of a few specific effects. First, an ion can also capture or lose its own electrons [38]. These processes eventually lead to SHI charge equilibration [38,39]. Thus, in MC simulations, often an assumption of equilibrium charge is used (typically, assuming Barkas formulae [40], or a similar expression [15,41]). In some cases, it may also be important to consider a finite size of the ion, which is often done with the Brandt-Kitagawa model [42]. It seems, 
the most prominent effect of the finite size is a slight increase of the energy losses around the Bragg peak - the maximum of SHI energy loss [43].

Another interesting peculiarity of an SHI is that its mean free path is significantly shorter than the interatomic distance in a target. It is not unphysical: in fact, it reflects the situation that an ion impact ionizes multiple electrons from the same atom [44], and may also ionized not only the closest atom but the next layer of atomic neighbors. There are multi-ionized atoms left in a trail of an SHI [11,44]. These stripped target atoms may not have enough electrons left to undergo a decay on their own, but again, the presence of surrounding atoms of the media will provide electrons to compensate for it and enable decay channels [45].

As we could see in every case discussed, most of the data available are for individual atoms, not for solids. Most of the standard MC codes are relying on the atomic approximation. The atomic approximation works well in many cases, especially when considering highly energetic particles. However, in certain cases it misses some effects qualitatively, which might have to be incorporated into the code manually [45].

\subsubsection{Cross sections within linear response theory}

Cross sections of scattering of fast charged particles in solids can be calculated in the first Born approximation, known also as the linear response theory [31,32]. First order perturbation theory holds for electrons with energies above some $\sim 50 \mathrm{eV}$ (for heavier particles, with energy rescaled correspondingly by the mass ratio, which e.g. makes it $\sim 0.1 \mathrm{MeV}$ for protons). In the first Born approximation, which assumes first order perturbation theory and the plane waves for the wave function of the incident particle, the cross section of scattering on an ensemble of interacting particles (atoms or electrons of the target) can be factorized into the terms related to the scattering on an individual scattering center, $A$, and the dynamic structure factor, $S(\omega, k)[31]$ :

$$
\frac{d^{2} \sigma}{d \Omega d \varepsilon}=A S(\omega, k)
$$

where the double differential cross section over scattering angle $\Omega$ and energy depend on the transferred energy $\varepsilon=\hbar \omega$ and momentum $k=q$.

The presence of the dynamic structure factor essentially means that during the scattering event, while receiving energy from the incident particle, an atom (or an electron) of the target exchanges this energy with other atoms of the target. This changes the way that the atom receives energy and momentum from the projectile.

Utilizing the fluctuation dissipation theorem [46], the dynamic structure factor can be recast in terms of the complex dielectric function (CDF) $[32,47]$. Then, the scattering cross section becomes [48]:

$$
\frac{d^{2} \sigma}{d(\hbar q) d \varepsilon}=\frac{2\left(Z_{e f f} e\right)^{2}}{n_{a} \pi \hbar^{2} v^{2}} \frac{1}{\hbar q}\left[1-\exp \left(\frac{-\hbar \omega}{T}\right)\right]^{-1} \operatorname{Im}\left(\frac{-1}{\epsilon(\omega, q)}\right)
$$

Here $Z_{e f f}$ is the effective charge of the incident particle (for discussion on the effective charge on an incident ion, see e.g. [15]); $e$ is the electron charge; $v$ is the speed of the incoming particle; $T$ is 
the temperature of the target (in the units of energy); and $\epsilon(\omega, q)$ is the complex dielectric function. The term $\operatorname{Im}\left(\frac{-1}{\epsilon(\omega, q)}\right)$ is also called the loss function. Eq. (7.6) is nonrelativistic; for the relativistic version, see e.g. [9,47].

Note that we used the fluctuation dissipation theorem in derivation of the cross section. It implies that the obtained cross sections are valid only in thermal equilibrium. If the system is perturbed out of equilibrium, Eq. (7.6) is no longer valid, but to the best of our knowledge, there is no nonequilibrium alternative known. It is still an open question in the field.

The temperature term in the square brackets in Eq. (7.6) is often neglected due to the fact that solids are usually modelled at normal temperature (however, in some circumstances effects of the target temperature may be important [48]). It is necessary to include it in modeling of plasma.

Eq. (7.6) can describe both, inelastic as well as elastic scattering, via setting the electronic (for inelastic) or atomic (for elastic) CDF [21].

Although $\epsilon(\omega, q)$ can be calculated exactly with ab-initio techniques [49], it is difficult to do that in practice, and hard to apply in MC simulations. The standard practice is to approximate the CDF with some analytical expressions. Some of the most common models are Ritchie-Howie (Drudelike model) [50], Ashley model [51] and Penn algorithm [52] (both providing only total but not differential cross sections), Mermin-based approach [53], Liljequist approximation [54] (expressed in equivalent terms of the so-called generalized oscillator strengths), and recently proposed delta-functional approximation [47].

Most of the models rely on the optical coefficients to extract the complex dielectric function in the optical limit $\epsilon(\omega, q=0)$ [22]. Once the optical limit is known, it can be approximated with some analytical functions, which are then analytically extended into $q>0$ region [22,53]. This provides approximate analytical expressions for $\operatorname{CDF}$ in the entire $(\omega, q)$ plane, thereby allowing for a solution of Eq. (7.6) to obtain the scattering cross section. Coefficients for CDF within RitchieHowie model for many materials can be found e.g. in [15].

Note that in case of very fast particles, or, more precisely, for large transferred energies, the DSF tends to the static structure factor (and CDF, correspondingly, to unity - unscreened potential), meaning the scattering cross section reduces to the scattering on an individual atom of the target. In this case, one can use atomic impact ionization cross sections, and atomic elastic scattering cross sections. For electrons, one may use e.g. so-called binary-encounter Bethe (BEB) cross sections for impact ionization of atoms [55] (or its relativistic version, RBEB [56]). A comparison of the inelastic mean free paths of electrons in solid silicon, calculated with Eq. (7.6) and coefficients from Ref. [15] vs. BEB-calculated cross sections (with the ionization potentials and kinetic energies of atomic energy levels from EPICS-2017 database) are shown in Figure 7.5 obtained with TREKIS code [15]. We can see that above some 50-100 eV, the cross sections are reasonably close. 


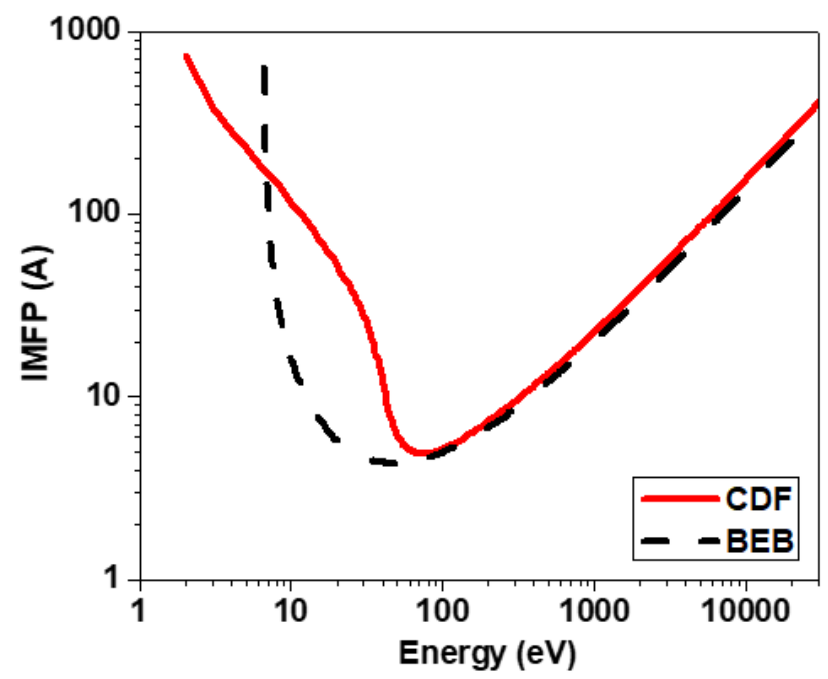

Figure 7.5. Inelastic mean free path of electrons in silicon, calculated within CDF formalism vs. atomic BEB approximation.

In compound targets, the total cross sections of scattering can be calculated using Eq. (7.6) with a good precision, whereas it is a known problem to obtain it in the atomic approximation. The standard way is to combine the total cross sections from the atomic ones using the so-called Bragg additivity rule, which suggests to add the atomic cross sections of electrons with the weights defined by the stoichiometry of the chemical formula of the target [57]. This rule sometimes works very poorly, and can easily introduce an error of some 30\% [57]. This is because in solids electrons from one element may be attracted to another one, especially in ionic materials, thus making the scattering cross section to differ from the atomic ones. Note that Liljequist approach from Ref. [54] and, similarly, delta-functional approximation [47] allow for an analytical solution for the differential and total cross section, and thus provide the speed of calculations in MC codes comparable to the atomic cross sections with noticeably better description of complex materials.

\subsubsection{Assessment of models quality}

Any Monte Carlo code should be thoroughly cross checked before application. It is usually done on several levels. First, the applied cross sections of each process modelled are checked against experimental data wherever available. Then, the integral values are checked. And finally, the experimentally observable output should be checked, which usually includes spectra of emitted electrons (or photons and other modelled particles).

Scattering cross sections are usually compared to the experimental data on particle mean free paths. For example, for the electronic mean free path in many materials, one may use the NIST database [58]. It provides experimental data, as well as those calculated by standard models.

It is a common practice to compare the energy loss, or the stopping power, $S_{e}(E)$, of a particle in the target, which defines the average energy deposition per unit of path travelled. The stopping power can be obtained as the first moment of the differential cross section: 


$$
S_{e}(E)=-\frac{d E}{d x}=n_{a} \int_{\mathrm{E}_{\min }}^{\mathrm{E}_{\max }} \int_{\mathrm{q}_{-}}^{\mathrm{q}_{+}} \varepsilon \frac{d^{2} \sigma}{d \varepsilon d(\hbar q)} d(\hbar q) d \varepsilon
$$

One can find experimental data on ion stopping in many materials e.g. here: https://wwwnds.iaea.org/stopping/. It is also considered a standard in the ion beam community to compare the stopping with the SRIM calculated database [59]: http://www.srim.org/. However, it is important to remember that SRIM data are only cross checked against experiments for $\mathrm{H}$ and $\mathrm{He}$ ions, whereas heavier ions are extrapolated from those via scaling rules. Comparisons with abinitio simulations demonstrated that one should use such extrapolations with caution as they are not always reliable [60]. There are also other ion stopping codes available, such as e.g. CasP [61], PASS [62], etc., see overview in [62].

Calculated stopping power allows one to extract another integral value, the transport range (i.e. the distance between the initial position of the electron/ion and it's final position, when its energy reaches $E_{\text {low }}$ ):

$$
R_{0}(E)=\int_{E_{\text {low }}}^{E} \frac{d \varepsilon}{S_{e}(\varepsilon)}
$$

Where the lower integration threshold is typically chosen (somewhat arbitrarily) as $E_{\text {low }}=10 \mathrm{eV}$. For electrons, data on electron ranges in a few materials may be found e.g. in X-ray Data Booklet [26], and obtained from standard transport codes [63] (this reference also includes data for other particles).

Having assessed the quality and correct implementation of cross sections, the MC model and code itself can be evaluated via direct comparisons with experimentally measurable values (and other standard codes, see Sec. 7.3.3. A typical value to compare with is spectra of emitted electrons. Often researchers calculate the spectra of emitted and backscattered electrons from a thin slab of material $[64,65]$.

\subsection{Practical implementation details}

\subsubsection{Random number generators}

Random number generators are the problem that is discussed a lot in older MC textbooks. It is no longer an issue. There has been developed a theoretical framework describing generation of random numbers based on the Lyapunov exponent estimation of correlation between two successive numbers drawn [66]. Based on this formalism, a few random number generators were developed with theoretically proven randomness. Some examples include:

- ranlux: http://luscher.web.cern.ch/luscher/ranlux/;

- mixmax: https://mixmax.hepforge.org/.

In practice, in the particle transport simulations, even standard random number generators often perform satisfactorily. This is due to the fact that in such a simulation, random numbers are used 
to sample many different processes of many different particles. Thus, artificial correlations introduced cancel out to some degree (in contrast to simulations where the same process is sampled repeatedly, such as e.g. spin-flips in the Ising model).

\subsubsection{Energy cut-off vs. time cut-off}

The standard way to build a Monte Carlo algorithm contains two points that need to be discussed in the context of this book:

(a) Typically, a particle is traced until its energy falls below a certain cut off;

(b) Typically, every MC iteration stops when all traced particles lose their energies below certain cut off. Reliably physical quantities are found by averaging over many iterations.

The point (a) allows to define such physical quantities as deposited dose, which is the spatial distribution of the energy density (per volume or mass of the target), or all the energy in all "stopped" particles as well as energy lost to other channels along its path. It may be a sufficient integral quantity for designing experimental rooms, or even for tracing biological dose in organisms, but it is not sufficient for microscopic modelling of material modifications. For that, one needs the distribution of energy in space at the given time, taking into account that electrons (as well as other particles) do not actually stop at energies below any given cut-off. It has been shown that the total dose evaluated with an energy cut off does not resemble actual distribution at any given time [67].

For microscopic modelling of material damage, the appropriate way is to trace electrons of lower energies too. It may be done either with an alternative model, or by tracing all particles in MC (provided cross sections of scattering at such energies are known). The energy/particle distribution obtained with MC may then be transferred into another model (such as twotemperature model, or another appropriate model) which could trace further dynamics of the target. More details on that will be given in Chapter 15 on combining MC methods with other models.

The point (b) reflects the fact that independent sequential execution of MC iterations is the simplest implementation of the MC algorithm. However, if one needs to combine an MC code with other models with on-the-fly feedback, a different approach to programming is required. All MC iterations need to be propagated in time simultaneously, to allow for a construction of average quantities required in other models on the fly (see an example in [68]). This method consumes significantly more memory, but allows to build combinations of MC models with other models with feedback, tracing evolution of materials properties during the particles transport simulated via MC. More details on that will also be discussed in Chapter 15.

\subsubsection{A few examples of standard codes}

For many practical purposes, especially with respect to applications to experiments design and engineering, standard Monte Carlo codes are sufficient and very well suited, and there is no need to write your own MC code. Some freely available Monte Carlo codes for radiation transport simulations are listed in Table 7.2. The list is not exhaustive; new codes are regularly being developed and appearing for public use. 
Table 7.2. Selected examples of Monte Carlo codes for radiation transport in matter.

\begin{tabular}{ccc}
\hline Type of particles & Code & Reference \\
\hline Ions, hadrons, etc. & GEANT4 & $\underline{\text { https://geant4.web.cern.ch/ }}$ \\
\hline Ions and hadrons & FLUKA & $\underline{\text { http://www.fluka.org/fluka.php }}$ \\
\hline Photons and electrons & PENELOPE & $\underline{\text { http://pypenelope.sourceforge.net }}$ \\
\hline Photons and electrons & MCNP & $\underline{\text { https://mcnp.lanl.gov/ }}$ \\
\hline Neutrons and photons & TART & $\underline{\text { http://redcullen1.net/homepage.new/tart2016.htm }}$ \\
\hline Photons and electrons & XCASCADE & $\underline{\text { https://xm.cfel.de/research/scientific software/xcascade/ }}$ \\
\hline
\end{tabular}

\section{Acknowledgements}

NM gratefully acknowledges financial support from the Czech Ministry of Education, Youth and Sports (Grants No. LTT17015, No. EF16\_013/0001552).

\section{References}

[1] D.P. Kroese, T. Taimre, Z.I. Botev, Handbook of Monte Carlo Methods, Wiley, 2011. https://www.wiley.com/en-au/Handbook+of+Monte+Carlo+Methods-p-9780470177938 (accessed October 4, 2020).

[2] W.K. Hastings, Monte carlo sampling methods using Markov chains and their applications, Biometrika. 57 (1970) 97-109. https://doi.org/10.1093/biomet/57.1.97.

[3] C.J. Mode, Applications of Monte Carlo Methods in Biology, Medicine and Other Fields of Science, InTech, 2012. https://doi.org/10.5772/634.

[4] D.L. McLeish, Monte Carlo Simulation and Finance, 1st ed., Wiley, Hoboken, New Jersey, 2005. https://www.wiley.com/en-be/Monte+Carlo+Simulation+and+Finance-p-9780471677789 (accessed October 4, 2020).

[5] G.A. Bird, Molecular Gas Dynamics and the Direct Simulation of Gas Flows, Clarendon Press, Oxford, 1994. https://books.google.de/books?id=Bya5QgAACAAJ.

[6] B. Meng, W.H. Weinberg, Monte Carlo simulations of temperature programmed desorption spectra, J. Chem. Phys. 100 (1994) 5280-5289. https://doi.org/10.1063/1.467192.

[7] A.F. Voter, Introduction to the kinetic monte carlo method, in: Radiat. Eff. Solids, Springer Netherlands, 2007: pp. 1-23. https://doi.org/10.1007/978-1-4020-5295-8_1.

[8] M.P. Nightingale, C.J. Umrigar, eds., Quantum Monte Carlo Methods in Physics and Chemistry, 1st ed., Springer Netherlands, 1999. https://www.springer.com/gp/book/9780792355519 (accessed October 4, 2020).

[9] F. Salvat, M. Fern, PENELOPE-2014 - A Code System for Monte Carlo Simulation of Electron and Photon Transport, 2015th ed., NUCLEAR ENERGY AGENCY, Organisation for Economic Co-operation and Development, Barcelona, Spain, 2015.

[10] G. Claus, R. Mark, Radioactivity and Radiation - What They Are, What They Do, and How to Harness Them | Claus Grupen | Springer, Springer International Publishing, 2016. https://www.springer.com/gp/book/9783319423296 (accessed October 4, 2020).

[11] J. Rzadkiewicz, A. Gojska, O. Rosmej, M. Polasik, K. Słabkowska, Interpretation of the Si K $\alpha \quad x-r a y$ spectra accompanying the stopping of swift Ca ions in low-density SiO 2 aerogel, Phys. Rev. A. 82 (2010) 012703. https://doi.org/10.1103/PhysRevA.82.012703.

[12] M. Lang, F. Djurabekova, N. Medvedev, M. Toulemonde, C. Trautmann, Fundamental Phenomena and Applications of Swift Heavy Ion Irradiations, in: Ref. Modul. Mater. Sci. Mater. Eng., Elsevier, 2020. https://doi.org/10.1016/B978-0-12-803581-8.11644-3. 
[13] R.A. Rymzhanov, N. Medvedev, J.H. O’Connell, V.A. Skuratov, A. Janse van Vuuren, S.A. Gorbunov, A.E. Volkov, Insights into different stages of formation of swift heavy ion tracks, Nucl. Instruments Methods Phys. Res. Sect. B Beam Interact. with Mater. Atoms. 473 (2020) 27-42. https://doi.org/10.1016/j.nimb.2020.04.005.

[14] O. Keski-Rahkonen, M.O. Krause, Total and partial atomic-level widths, At. Data Nucl. Data Tables. 14 (1974) 139-146. https://doi.org/10.1016/S0092-640X(74)80020-3.

[15] N.A. Medvedev, R.A. Rymzhanov, A.E. Volkov, Time-resolved electron kinetics in swift heavy ion irradiated solids, J. Phys. D. Appl. Phys. 48 (2015) 355303. https://doi.org/10.1088/0022$3727 / 48 / 35 / 355303$.

[16] R.A. Rymzhanov, N. Medvedev, J.H. O’Connell, A. Janse van Vuuren, V.A. Skuratov, A.E. Volkov, Recrystallization as the governing mechanism of ion track formation, Sci. Rep. 9 (2019). https://doi.org/10.1038/s41598-019-40239-9.

[17] S. Deffner, S. Campbell, Quantum speed limits: from Heisenberg's uncertainty principle to optimal quantum control, J. Phys. A Math. Theor. 50 (2017) 453001. https://doi.org/10.1088/17518121/aa86c6.

[18] T.M. Jenkins, W.R. Nelson, A. Rindi, eds., Monte Carlo Transport of Electrons and Photons, Springer US, Boston, MA, 1988. https://doi.org/10.1007/978-1-4613-1059-4.

[19] C. Jacoboni, L. Reggiani, The Monte Carlo method for the solution of charge transport in semiconductors with applications to covalent materials, Rev. Mod. Phys. 55 (1983) 645-705. https://doi.org/10.1103/RevModPhys.55.645.

[20] N.A. Medvedev, Excitation and relaxation of the electronic subsystem in solids after high energy deposition Dissertation, TU Kaiserslautern, Kaiserslautern, 2011. http://dnb.info/1015869106/34.

[21] J.-C. Kuhr, H.-J. Fitting, Monte Carlo simulation of electron emission from solids, J. Electron Spectros. Relat. Phenomena. 105 (1999) 257-273. https://doi.org/10.1016/S0368-2048(99)00082-1.

[22] A. Akkerman, T. Boutboul, A. Breskin, R. Chechik, A. Gibrekhterman, Y. Lifshitz, Inelastic Electron Interactions in the Energy Range $50 \mathrm{eV}$ to $10 \mathrm{keV}$ in Insulators: Alkali Halides and Metal Oxides, Phys. Status Solidi B. 198 (1996) 769-784. https://doi.org/10.1002/pssb.2221980222.

[23] B. Ziaja, R.A. London, J. Hajdu, Unified model of secondary electron cascades in diamond, J. Appl. Phys. 97 (2005) 064905. https://doi.org/10.1063/1.1853494.

[24] D.E. Cullen, A Survey of Atomic Binding Energies for use in EPICS2017, Vienna, 2018. https://wwwnds.iaea.org/publications/iaea-nds/iaea-nds-224_Rev1_2018.pdf (accessed August 24, 2018).

[25] B.L. Henke, E.M. Gullikson, J.C. Davis, X-Ray Interactions: Photoabsorption, Scattering, Transmission, and Reflection at $E=50-30,000 \mathrm{eV}, \mathrm{Z}=1-92$, At. Data Nucl. Data Tables. 54 (1993) 181-342. https://doi.org/10.1006/adnd.1993.1013.

[26] A. Thompson, D. Vaughan, J. Kirz, D. Attwood, E. Gullikson, M. Howells, K.-J. Kim, J. Kortright, I. Lindau, P. Pianetta, A. Robinson, J. Underwood, G. Williams, H. Winick, X-Ray Data Booklet, 2009th ed., Center for X-ray Optics and Advanced Light Source, Lawrence Berkeley National Laboratory, Berkeley, CA, USA, 2009. http://xdb.lbl.gov/ (accessed August 26, 2015).

[27] E.D. Palik, Handbook of optical constants of solids, 1998. https://doi.org/10.1080/716099804a.

[28] S. Adachi, The Handbook on Optical Constants of Semiconductors: In Tables and Figures, World Scientific Publishing Company, New Jersey, London, Singapore, 2012. http://www.amazon.com/The-Handbook-Optical-Constants-Semiconductors/dp/9814405973 (accessed August 1, 2013).

[29] J.A. Bearden, A.F. Burr, Reevaluation of X-Ray Atomic Energy Levels, Rev. Mod. Phys. 39 (1967) 125142. https://doi.org/10.1103/RevModPhys.39.125.

[30] Z. Jurek, S.-K. Son, B. Ziaja, R. Santra, XMDYN and XATOM: versatile simulation tools for quantitative modeling of X-ray free-electron laser induced dynamics of matter, J. Appl. Crystallogr. 49 (2016) 1048-1056. https://doi.org/10.1107/S1600576716006014. 
[31] L. Van Hove, Correlations in Space and Time and Born Approximation Scattering in Systems of Interacting Particles, Phys. Rev. 95 (1954) 249-262. https://doi.org/10.1103/PhysRev.95.249.

[32] U. Fano, Penetration of Protons, Alpha Particles, and Mesons, Annu. Rev. Nucl. Sci. 13 (1963) 1-66. https://doi.org/10.1146/annurev.ns.13.120163.000245.

[33] M.L. Knotek, P.J. Feibelman, Stability of ionically bonded surfaces in ionizing environments, Surf. Sci. 90 (1979) 78-90. http://www.sciencedirect.com/science/article/pii/0039602879900116 (accessed December 13, 2013).

[34] L.S. Cederbaum, J. Zobeley, F. Tarantelli, Giant Intermolecular Decay and Fragmentation of Clusters, Phys. Rev. Lett. 79 (1997) 4778-4781. http://link.aps.org/doi/10.1103/PhysRevLett.79.4778 (accessed December 13, 2013).

[35] N.A. Medvedev, A.E. Volkov, B. Rethfeld, N.S. Shcheblanov, Effect of inter-atomic Auger processes on relaxation of electronic vacancies at deep levels of highly ionized atoms in swift heavy ion tracks, Nucl. Instruments Methods Phys. Res. Sect. B Beam Interact. with Mater. Atoms. 268 (2010) 28702873. https://doi.org/10.1016/j.nimb.2010.03.021.

[36] R.A. Rymzhanov, N.A. Medvedev, A.E. Volkov, Effects of model approximations for electron, hole, and photon transport in swift heavy ion tracks, Nucl. Instruments Methods Phys. Res. Sect. B Beam Interact. with Mater. Atoms. 388 (2016) 41-52. https://doi.org/10.1016/j.nimb.2016.11.002.

[37] P.N. Terekhin, R.A. Rymzhanov, S.A. Gorbunov, N.A. Medvedev, A.E. Volkov, Effect of valence holes on swift heavy ion track formation in Al203, Nucl. Instruments Methods Phys. Res. Sect. B Beam Interact. with Mater. Atoms. 254 (2015) 200-204. https://doi.org/10.1016/j.nimb.2015.01.069.

[38] J.P. Rozet, C. Stéphan, D. Vernhet, ETACHA: a program for calculating charge states at GANIL energies, Nucl. Instruments Methods Phys. Res. Sect. B Beam Interact. with Mater. Atoms. 107 (1996) 67-70. http://www.sciencedirect.com/science/article/pii/0168583X95008004 (accessed December 13, 2013).

[39] O. Osmani, P. Sigmund, Charge evolution of swift-heavy-ion beams explored by matrix method, Nucl. Instruments Methods Phys. Res. Sect. B Beam Interact. with Mater. Atoms. 269 (2011) 813-816. https://doi.org/10.1016/J.NIMB.2010.12.015.

[40] W.H. Barkas, Nuclear research emulsions., Vol. 1, Academic Press, New York, 1963. http://hdl.handle.net/2027/uc1.b3533401 (accessed November 20, 2013).

[41] H.-D. Betz, Charge States and Charge-Changing Cross Sections of Fast Heavy Ions Penetrating Through Gaseous and Solid Media, Rev. Mod. Phys. 44 (1972) 465-539. https://doi.org/10.1103/RevModPhys.44.465.

[42] W. Brandt, M. Kitagawa, Effective stopping-power charges of swift ions in condensed matter, Phys. Rev. B. 25 (1982) 5631-5637. https://doi.org/10.1103/PhysRevB.25.5631.

[43] N.A. Medvedev, R.A. Rymzhanov, A.E. Volkov, Complex dielectric function formalism for description of the electron kinetics in swift heavy ion tracks in LiF and Y203, Nucl. Instruments Methods Phys. Res. Sect. B Beam Interact. with Mater. Atoms. 315 (2013) 85-89. https://doi.org/10.1016/j.nimb.2013.04.090.

[44] J. Rzadkiewicz, O. Rosmej, A. Blazevic, V.P. Efremov, A. Gójska, D.H.H. Hoffmann, S. Korostiy, M. Polasik, K. Słabkowska, A.E. Volkov, Studies of the Ka X-ray spectra of low-density SiO2 aerogel induced by Ca projectiles for different penetration depths, High Energy Density Phys. 3 (2007) 233236. https://doi.org/10.1016/j.hedp.2007.02.026.

[45] N.A. Medvedev, A.E. Volkov, N.S. Shcheblanov, B. Rethfeld, Early stage of the electron kinetics in swift heavy ion tracks in dielectrics, Phys. Rev. B. $82 \quad$ (2010) 125425. https://doi.org/10.1103/PhysRevB.82.125425.

[46] R. Kubo, Statistical-Mechanical Theory of Irreversible Processes. I. General Theory and Simple Applications to Magnetic and Conduction Problems, J. Phys. Soc. Japan. 12 (1957) 570-586. https://doi.org/10.1143/JPSJ.12.570.

[47] N. Medvedev, A.E. Volkov, Analytically solvable model of scattering of relativistic charged particles in solids, J. Phys. D. Appl. Phys. 53 (2020) 235302. https://doi.org/10.1088/1361-6463/AB7C09. 
[48] S.A. Gorbunov, N. Medvedev, R.A. Rymzhanov, A.E. Volkov, Dependence of the kinetics of Al203 excitation in tracks of swift heavy ions on lattice temperature, Nucl. Instruments Methods Phys. Res. Sect. B Beam Interact. with Mater. Atoms. $435 \quad$ (2018) 83-86. https://doi.org/10.1016/j.nimb.2018.01.005.

[49] C. Ambrosch-Draxl, J.O. Sofo, Linear optical properties of solids within the full-potential linearized augmented planewave method, Comput. Phys. Commun. 175 (2006) 1-14. https://doi.org/10.1016/J.CPC.2006.03.005.

[50] R.H. Ritchie, A. Howie, Electron excitation and the optical potential in electron microscopy, Philos. Mag. 36 (1977) 463-481. https://doi.org/10.1080/14786437708244948.

[51] J.C. Ashley, Interaction of low-energy electrons with condensed matter: stopping powers and inelastic mean free paths from optical data, J. Electron Spectros. Relat. Phenomena. 46 (1988) 199214. http://www.sciencedirect.com/science/article/pii/0368204888800197 (accessed October 4, 2015).

[52] S. Tanuma, C.J. Powell, D.R. Penn, Calculations of electron inelastic mean free paths. III. Data for 15 inorganic compounds over the 50-2000 eV range, Surf. Interface Anal. 17 (1991) 927-939. https://doi.org/10.1002/sia.740171304.

[53] I. Abril, R. Garcia-Molina, C.D. Denton, F.J. Pérez-Pérez, N.R. Arista, Dielectric description of wakes and stopping powers in solids, Phys. Rev. A. 58 (1998) 357-366. https://doi.org/10.1103/PhysRevA.58.357.

[54] J.M. Fernández-Varea, F. Salvat, M. Dingfelder, D. Liljequist, A relativistic optical-data model for inelastic scattering of electrons and positrons in condensed matter, Nucl. Instruments Methods Phys. Res. Sect. B Beam Interact. with Mater. Atoms. 229 (2005) 187-218. https://doi.org/10.1016/J.NIMB.2004.12.002.

[55] Y.-K. Kim, M. Rudd, Binary-encounter-dipole model for electron-impact ionization, Phys. Rev. A. 50 (1994) 3954-3967. https://doi.org/10.1103/PhysRevA.50.3954.

[56] Y.-K. Kim, J.P. Santos, F. Parente, Extension of the binary-encounter-dipole model to relativistic incident electrons, Phys. Rev. A. 62 (2000) 052710. https://doi.org/10.1103/PhysRevA.62.052710.

[57] P. Sigmund, A. Schinner, Electronic stopping in oxides beyond Bragg additivity, Nucl. Instruments Methods Phys. Res. Sect. B Beam Interact. with Mater. Atoms. 415 (2018) 110-116. https://doi.org/10.1016/j.nimb.2017.11.023.

[58] C.J. Powell, A. Jablonsky, NIST Electron Inelastic-Mean-Free-Path Database: Version 1.2, (2014) http://www.nist.gov/srd/nist71.cfm. http://www.nist.gov/srd/nist71.cfm.

[59] J.P. Ziegler, U. Biersack, J.F. Littmark, The Stopping and Range of Ions in Solids, Pergamon Press, New York, 1985. http://www.srim.org.

[60] K. Wittmaack, Misconceptions impairing the validity of the stopping power tables in the SRIM library and suggestions for doing better in the future, Nucl. Instruments Methods Phys. Res. Sect. B Beam Interact. with Mater. Atoms. 380 (2016) 57-70. https://doi.org/10.1016/j.nimb.2016.04.057.

[61] P.L. Grande, G. Schiwietz, CasP, Nucl. Instruments Methods Phys. Res. Sect. B Beam Interact. with Mater. Atoms. 267 (2009) 859-863. http://www.casp-program.org/.

[62] A. Schinner, P. Sigmund, Expanded PASS stopping code, Nucl. Instruments Methods Phys. Res. Sect. B Beam Interact. with Mater. Atoms. $460 \quad$ (2019) 19-26. https://doi.org/10.1016/j.nimb.2018.10.047.

[63] M.J. Berger, J.S. Coursey, M.A. Zucker, J. Chang, Stopping-Power and Range Tables for Electrons, Protons, and Helium Ions | NIST, 1998. https://doi.org/https://dx.doi.org/10.18434/T4NC7P.

[64] M. Azzolini, O.Y. Ridzel, P.S. Kaplya, V. Afanas'ev C, N.M. Pugno, S. Taioli, M. Dapor, A Comparison between Monte Carlo Method and the Numerical Solution of the Ambartsumian-Chandrasekhar Equations to Unravel the Dielectric Response of Metals, 2019. https://arxiv.org/pdf/1911.04785.pdf (accessed November 22, 2019). 
[65] D. Emfietzoglou, H. Nikjoo, The effect of model approximations on single-collision distributions of low-energy electrons in liquid water., Radiat. Res. 163 (2005) 98-111. https://doi.org/10.1667/RR3281.

[66] M. Lüscher, A portable high-quality random number generator for lattice field theory simulations, Comput. Phys. Commun. 79 (1994) 100-110. https://doi.org/10.1016/0010-4655(94)90232-1.

[67] M. Murat, A. Akkerman, J. Barak, Electron and Ion Tracks in Silicon: Spatial and Temporal Evolution, $\begin{array}{lllll}\text { IEEE Trans. } & \text { Nucl. } & \text { Sci. } & \text { 3046-3054. }\end{array}$ http://ieeexplore.ieee.org/lpdocs/epic03/wrapper.htm?arnumber=4723756 (accessed January 18, 2016).

[68] N. Medvedev, V. Tkachenko, V. Lipp, Z. Li, B. Ziaja, Various damage mechanisms in carbon and silicon materials under femtosecond X-ray irradiation, 4open. 1 (2018) 3. https://doi.org/10.1051/FOPEN/2018003. 


\title{
8. Brief review of basics of molecular dynamics
}

\author{
Samuel T. Murphy*, K. Nordlund, F. Fjurabekova, and Dorothy M. Duffy
}

In this article, we overview the general algorithms for simulation of equilibrium systems by molecular dynamics. We first briefly present the history of the method and how it can be understood as a general means to solve the N-body equations of motion. We then proceed to describe the basic steps in the molecular dynamics iteration loop, and emphasize that the interatomic potential or force field is the crucial physics input to the method. We finish by providing an overview of the main classes of interatomic potentials and force fields.

*Contact: samuel.murphy@lancaster.ac.uk 


\subsection{Introduction and background}

The purpose of this article is to overview the simulation methodology of the key method for modern materials modelling: molecular dynamics (MD). In this article we focus at some depth on the molecular dynamics method when used in equilibrium modelling. Another article in this collection presents the MD method as applied to radiation effects. The paper presents the basic MD algorithms and a survey of advances in interatomic potential formalism, starting from pair potentials developed in the 1920s up to machine-learning approaches taken into use in the 2010s and up to 2021.

In classical physics, it is well known that the equations of motion of $N$ objects can be solved analytically only when $N=1$ or $N=2$. It is of course of great interest to find methods to be able to predict the motion of a much larger number of objects than 2, whether this is for motion of planets, stars in galaxies, or atoms in molecules. Using computer simulations, one can devise an algorithm to solve iteratively the equations of motion of an arbitrarily large number of objects $N$, in other words, solve the $N$-body problem on a computer. When this is done for atoms as the basic object, the method is conventionally called "molecular dynamics" and usually abbreviated MD.

The MD method was first developed in 1957 by Alder and Wainwright to simulate the motion of two atoms in a dimer molecule [1]. This was shortly followed by the simulation of systems of the order of 100 atoms [2-4], as illustrated in Figure 8.1, which on the computer of that day can be considered an impressive feat.

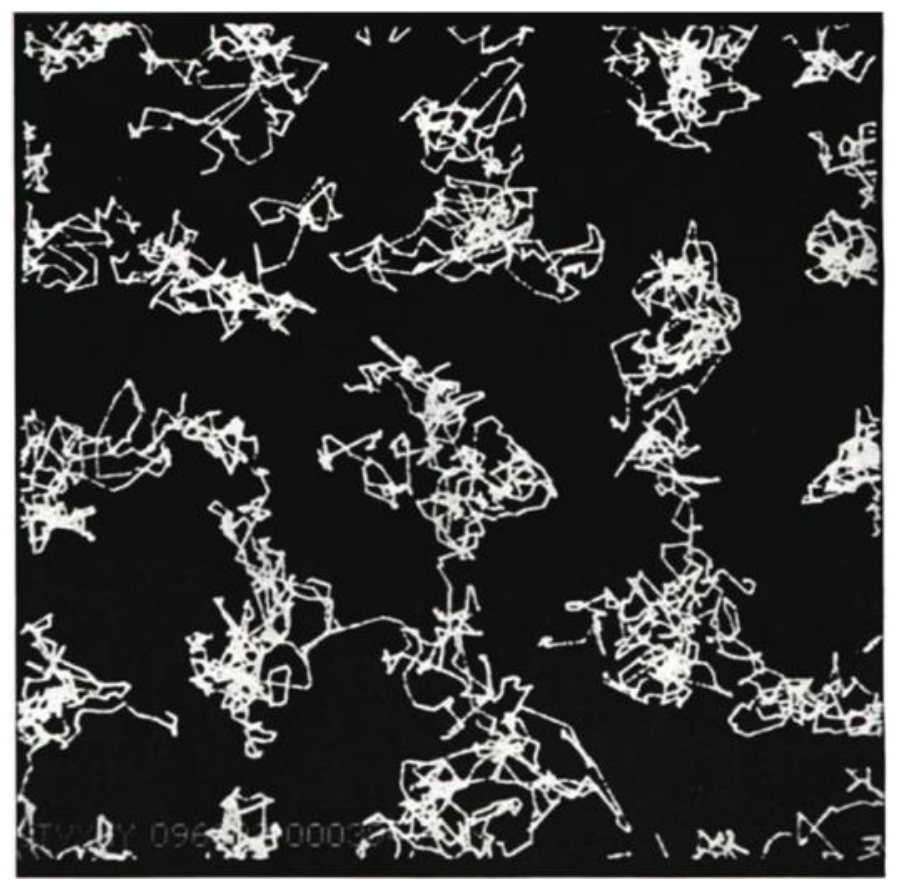

Figure 8.1. Image showing the trajectories from an early molecular dynamics simulation. Image taken from Ref. [2].

These early pioneering simulations have evolved in line with increasing computational resources to enable the study of systems consisting of trillions of atoms [5]. Concomitant with the increase in the number of atoms that can be studied has been the development of complex empirical potentials and force fields [6] to represent the varied interactions present between atoms/ions. Taken together, this means that large, complex systems can be studied with atomic resolution 
using readily available simulation packages. As a consequence, molecular dynamics has been adopted for simulation of a broad range of phenomena including phase transformations [7], mechanical deformation [8], self-assembly [9], radiation damage [10] and protein folding [11] to name a few and is now one of the dominant simulation methods in modern materials physics, biophysics, and chemistry. This statement is supported by a comparative search on Web of Science in Feb 2021 that showed that in 2020, there were 16,000 publications with "molecular dynamics" in the title, abstract or keywords of the paper. The corresponding result for "density functional theory" was 17,600, and for "finite element method" 9600. Any "Monte Carlo" method is less used, since the key word "Monte Carlo" in total gave 14,800 hits, even though there are many completely different Monte Carlo approaches. We note, however, that the searches result in some overlap, since MD can be run, for instance, with forces obtained from density functional theory calculations.

Molecular dynamics simulations are attractive for a number of reasons, including their relative simplicity, flexibility and their connection with statistical mechanics, enabling comparison with experimental observations. There are, however, a number of limitations. These include the relatively short timescales of the simulations and the neglect of electronic information in classical molecular dynamics. Ab initio molecular dynamics maintains some electronic information but such simulations are restricted to very small system sizes and short timescales. There have been a number of approaches to overcome these limitations. Techniques like temperature accelerated dynamics (TAD) [12] and parallel replica dynamics [13] have been developed to extend the timescales that can be reached by classical simulations and some of the electronic information can be reincorporated through approaches like two temperature molecular dynamics.

In this chapter we will explore the underlying principles behind molecular dynamics and describe some of the different potential models that are used for the calculation of forces. For more detailed introductory treatments, the reader is referred to one of the many textbooks on the method, e.g. Refs. 14-18.

\subsection{Basics of molecular dynamics simulations}

\subsubsection{Equations of motion}

Our starting point for the discussion of the key foundational principles of molecular dynamics is the Hamiltonian for an $N$-particle system subject to inter-particle interactions only:

$$
H(\mathrm{p}, \mathrm{r})=H\left(\mathrm{p}_{1}, \ldots \mathrm{p}_{N}, \mathrm{r}_{1}, \ldots \mathrm{r}_{N}\right)=\sum_{i=1}^{N} \frac{\mathrm{p}_{i}^{2}}{2 m_{i}}+U\left(\mathrm{r}_{1}, \ldots \mathrm{r}_{N}\right)
$$

where $\left(\mathrm{r}_{1}, \ldots \mathrm{r}_{N}\right)$ and $\left(\mathrm{p}_{1}, \ldots \mathrm{p}_{N}\right)$ are particles' positions and momenta respectively, $m_{i}$ are the particles' masses and $U\left(\mathrm{r}_{1}, \ldots \mathrm{r}_{N}\right)$ is the potential energy due to inter-particle interactions. Within this framework the forces on each atom can be determined from: 


$$
\mathbf{F}_{i}=-\frac{\partial U}{\partial \mathbf{r}_{i}}
$$

To determine the equation of motion we start from Hamilton's equations:

$$
\dot{\mathbf{r}}_{i}=-\frac{\partial H}{\partial \mathbf{p}_{i}}
$$

and

$$
\dot{\mathbf{p}}_{i}=-\frac{\partial H}{\partial \mathbf{r}_{i}}=-\frac{\partial U}{\partial \mathbf{r}_{i}}=\mathrm{F}_{i}\left(\mathbf{r}_{1}, \ldots \mathbf{r}_{N}\right)
$$

and substitute the time derivative of equation (8.3) into equation (8.4) to give Newton's second law:

$$
m_{i} \ddot{\mathbf{r}}_{l}=\mathbf{F}_{i}
$$

As a consequence, it is only necessary to specify the complete set of particle positions and momenta to determine the entire classical state of the system [19].

\subsubsection{Integration algorithms}

Assuming the classical trajectory is continuous, the positions of the particles at a given time, $t+$ $\delta t$, can be determined from a Taylor expansion, i.e.:

$$
\mathbf{r}_{i}(t+\delta t)=\mathbf{r}_{i}(t)+\delta t \dot{\mathbf{r}}_{l}(t)+\frac{1}{2} \delta t^{2} \ddot{\mathbf{r}}_{l}(t)+\frac{1}{6} \delta t^{3} \dddot{\mathbf{r}}_{l}(t)+\cdots
$$

From there it would appear that we could develop an algorithm to evolve our system of atoms in time. However, equation (8.6) does not take account of the equations of motions and, consequently, will not create realistic trajectories. To enable better approximations to the true positions the use of corrector steps were proposed, where the new positions $r_{i}(t+\delta t)$ were used to determine new forces and hence accelerations that could be used to correct the final position, i.e.:

$$
\mathbf{r}_{i}(t+\delta t)=\mathbf{r}_{i}^{p}(t+\delta t)+c_{0} \Delta \mathbf{a}(t+\delta t)
$$

where the $\mathbf{r}_{i}^{p}(t+\delta t)$ corresponds to the positions predicted using equation (8.6) and $c_{0}$ is a constant. Within this framework, the final positions can be obtained through iterative improvement. However, this iterative correction procedure requires multiple evaluations of the forces, and hence accelerations, for each step, which is computationally intensive.

The task is then to develop an algorithm that can duplicate the classical trajectory while being computationally efficient and also satisfying laws of conservation of energy and momentum, as well as being time reversible. Perhaps the most successful series of methods for integrating the equations of motion are those adopting the approach outlined by Verlet [20]. We start by restating equation (8.7) in a modified form where the derivatives have been replaced, i.e:

$$
\mathbf{r}_{i}(t+\delta t)=\mathbf{r}_{i}(t)+\delta t \mathbf{v}_{i}(t)+\frac{1}{2} \delta t^{2} \mathbf{a}_{i}(t)+\cdots
$$


where, $\mathrm{v}_{i}$ and $\mathrm{a}_{i}$ are the velocity and acceleration of $i$ respectively. As the equations of motion should be time reversible it is equally valid to track backwards in time, such that:

$$
\mathbf{r}_{i}(t-\delta t)=\mathbf{r}_{i}(t)-\delta t \mathbf{v}_{i}(t)+\frac{1}{2} \delta t^{2} \mathbf{a}_{i}(t)-\cdots
$$

Adding equations (8.8) and (8.9) and rearranging allows the elimination of the velocity such that the new positions can be determined from:

$$
\mathbf{r}_{i}(t+\delta t)=2 \mathbf{r}_{i}(t)-\mathbf{r}_{i}(t-\delta t)+\delta t^{2} \mathbf{a}_{i}(t)
$$

The positions predicted in equation (8.10) are correct except for errors of the order $\delta t^{4}$. While it is not necessary to calculate and store the velocities, these are useful for calculating the kinetic energy and can be determined from:

$$
\mathbf{v}_{i}(t)=\frac{\mathbf{r}_{i}(t+\delta t)-\mathbf{r}_{i}(t-\delta t)}{2 \delta t}
$$

It is worth noting that the errors in the velocity are greater than for the positions at $\delta t^{2}$. While the Verlet algorithm is relatively simple and demonstrates excellent conservation of energy and momentum, the handling of the velocities is subject to some numerical imprecision. Modifying the Verlet scheme to address these shortcomings results in the 'leap-frog' algorithm [21], where:

$$
\mathbf{r}_{i}(t+\delta t)=\mathbf{r}_{i}(t)+\delta t \mathbf{v}_{i}\left(t+\frac{1}{2} \delta t\right)
$$

and

$$
\mathbf{v}_{i}\left(t+\frac{1}{2} \delta t\right)=\mathbf{v}_{i}\left(t-\frac{1}{2} \delta t\right)+\delta t \mathbf{a}_{i}(t)
$$

In the leap-frog algorithm it is velocities at the mid-step, i.e. $(t-1 / 2 \delta t)$, that are calculated first according to equation (8.13) and subsequently used to update the positions according to equation (8.12). While the velocities are explicitly calculated as part of the integration procedure, they are not immediately available in the current time step. If required they can be determined from:

$$
\mathbf{v}_{i}(t)=\frac{1}{2}\left(\mathbf{v}_{i}\left(t+\frac{1}{2} \delta t\right)+\mathbf{v}_{i}\left(t-\frac{1}{2} \delta t\right)\right)
$$

The leap-frog algorithm is an improvement of the initial Verlet scheme as it reduces the numerical imprecision associated with taking the difference between two large numbers to give a smaller one. However, the handling of the velocities is still somewhat awkward.

Ultimately, what is desired is an algorithm that stores the current values of $\mathbf{r}_{i}, \mathbf{v}_{i}$ and $\mathbf{a}_{i}$. One such approach is the 'Velocity Verlet' of Swope et al. [22], that takes the form:

$$
\begin{gathered}
\mathbf{r}_{i}(t+\delta t)=\mathbf{r}_{i}(t)+\delta t \mathbf{v}_{i}(t)+\frac{1}{2} \delta t^{2} \mathbf{a}_{i}(t) \\
\mathbf{v}_{i}(t+\delta t)=\mathbf{v}_{i}(t)+\frac{1}{2} \delta t\left[\mathbf{a}_{i}(t)+\mathbf{a}_{i}(t+\delta t)\right]
\end{gathered}
$$

The velocity-Verlet algorithm is performed in stages. The first stage involves the calculation of updated positions using equation (8.15), followed by the velocities at the half time step using:

$$
\mathbf{v}_{i}\left(t+\frac{1}{2} \delta t\right)=\mathbf{v}_{i}(t)+\frac{1}{2} \delta t \mathbf{a}_{i \cdot}(t)
$$


This is followed by a force evaluation and calculation of the acceleration at $(t+\delta t)$ before the the velocity is calculated via:

$$
\mathbf{v}_{i}(t+\delta t)=\mathbf{v}_{i}\left(t+\frac{1}{2} \delta t\right)+\frac{1}{2} \delta t \mathbf{a}_{i}\left(t+\frac{1}{2} \delta t\right) .
$$

As the velocities at the current time are available it is possible to determine the kinetic energy of the system. Further, the potential energy is calculated as part of the force evaluation, which means that all important quantities are available. This, combined with the numerical stability and convenience, means that this algorithm is the most widely adopted in molecular dynamics simulations.

All of the time integration algorithms discussed above require the definition of a time step, $\delta t$. These time steps should be as long as possible to sample phase space rapidly, although not so long that they introduce errors into the trajectory [23]. Time steps that are too large can make particle motion unstable leading to a very big truncation error in the integration process, so the total energy of the system may rapidly increase with time [24]. In general, this limits time steps to $<2$ fs [25], although there are circumstances, where smaller time steps may be required. For example, the high velocities of atoms during a radiation cascade can require very short time steps, therefore, variable time step algorithms have been developed [22,23].

\subsubsection{Ensembles}

If the equations of motion are solved such that a system of $N$ atoms is confined in a fixed volume, $V$ and the forces acting on the atoms are solely related to the potential energy of the system, then the simulation is in the microcanonical (NVE) ensemble. In this case the total energy of the system remains constant despite there being fluctuations in both the kinetic and potential energy as illustrated in Figure 8.2.

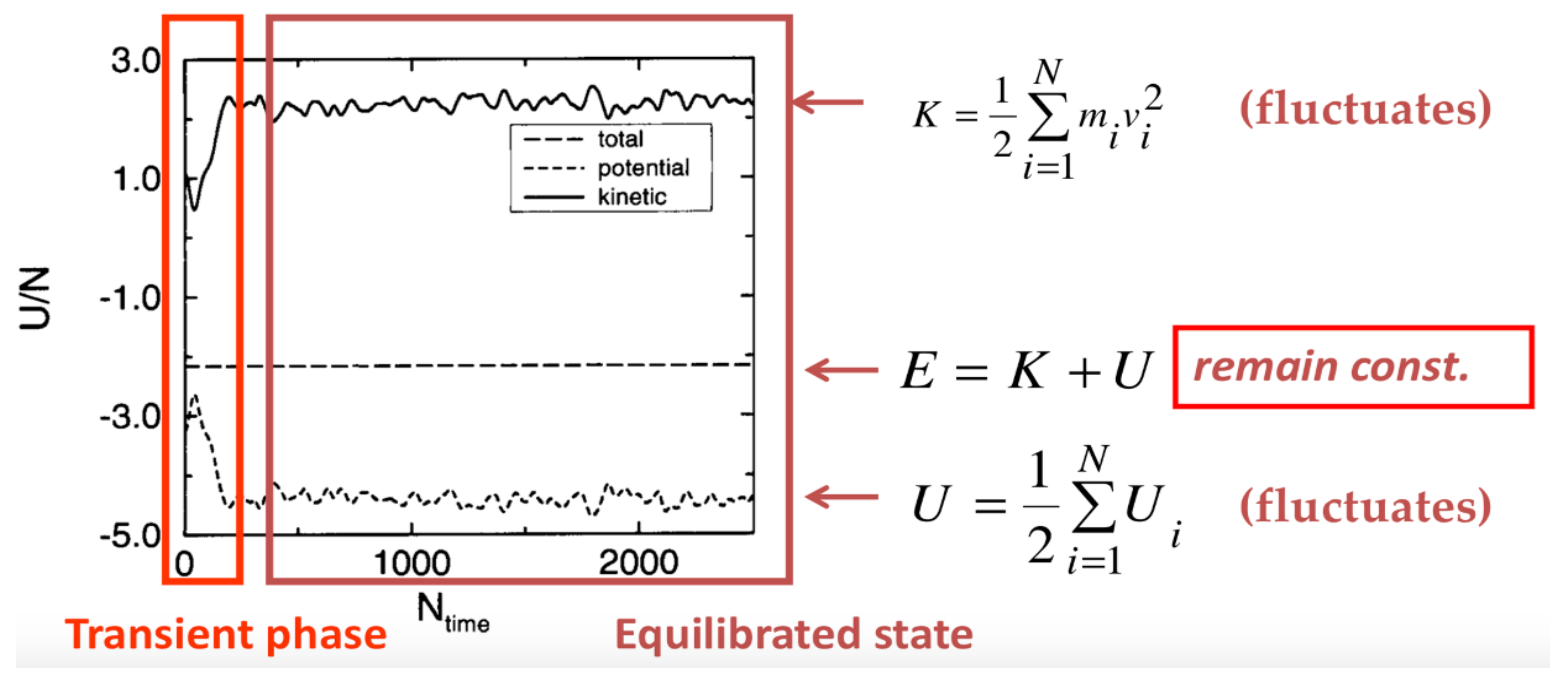

Figure 8.2. Plot showing the kinetic, potential and total energies vary as a function of time during a molecular dynamics simulation within the microcanonical ensemble.

As a consequence of the fluctuations in the kinetic energy the temperature cannot be controlled as: 


$$
k_{B} T(t)=\frac{1}{N} \sum_{i} v_{i}^{2} .
$$

where $k_{B}$ is the Boltzmann constant. However, in many cases it is desirable to control the temperature to reproduce experimental conditions. One method of doing this is to simply rescale the velocities using:

$$
v_{i}^{\prime}=\sqrt{\frac{T}{T(t)}} v_{i} .
$$

While this rescaling approach does maintain the correct temperature it does not reproduce a canonical ensemble and so is rarely used outside of equilibration simulations. An alternative method is to weakly couple the system to a heat bath that adds or removes heat to maintain the desired temperature. The velocities are scaled such that:

$$
\frac{d T}{d t}=\frac{1}{\tau}\left(T_{0}-T\right)
$$

where $T_{0}$ is the target temperature and $\tau$ is a coupling constant corresponding to the time interval between heat exchanges with the heat bath [28]. From here the velocities can be rescaled according to $v_{i}^{\prime}=\lambda v_{i}$, where:

$$
\lambda^{2}=1+\frac{\delta t}{\tau}\left(\frac{T_{0}}{T}-1\right)
$$

In the limit where $\tau>>\delta t$ the coupling is so weak that we have effectively recovered the microcanonical ensemble. At the other extreme, if $\tau=\delta t$ then this reduces to the standard velocity rescaling. It is not clear that this Berendsen thermostat reproduces a canonical distribution.

Another method of controlling the temperature was proposed by Anderson [29]. In this approach the atoms have a small probability of colliding with the heat bath. If an atom does collide with the heat bath, its velocity is drawn from a Maxwell-Boltzmann distribution at the target temperature. While this thermostat does create a canonical (NVT) ensemble, the random changes in velocity disrupt the MD trajectories.

The method proposed by Nose [30] and Hoover [31] introduces a fictitious degree of freedom, $s$, into the energy that represents a heat bath. The potential energy of $s$ is then $(3 N+1) k_{B} T \ln s$ where $3 N+1$ is the number of degrees of freedom. The kinetic energy is given by:

$$
K E(s)=\frac{Q(d s / d t)^{2}}{2}
$$

where $Q$ represents a fictitious mass. If we then consider an extended system where the real system is connected to a heat bath $s$, assuming that the extended system is microcanonical, then the result is that the real system is maintained in a canonical state. The strength of the coupling between the real system and the heat bath is determined by the fictitious mass, $Q$.

The final approach to controlling the temperature we will discuss is the Langevin thermostat [32]. In the Langevin thermostat the equations of motion are modified such that:

$$
m_{i} \ddot{\mathrm{r}}_{l}=\mathrm{F}_{i}-\gamma \dot{r}+\tilde{\mathbf{F}}
$$

where $\gamma$ is a frictional drag force and $\tilde{\mathbf{F}}$ is a stochastic force. In order to satisfy the fluctationdissipation theorem the stochastic forces have a Gaussian distribution, i.e.: 


$$
\begin{gathered}
\langle\tilde{\mathbf{F}}(t)\rangle=0, \\
\left\langle\widetilde{\mathbf{F}}(t) \widetilde{\mathbf{F}^{\prime}}(t)\right\rangle=2 \gamma k_{B} T_{0} \delta\left(t-t^{\prime}\right) .
\end{gathered}
$$

\subsubsection{Constructing a molecular dynamics simulation}

Implicit in all of the time integration procedures is the assumption that the initial positions of all atoms are known and, consequently, these must be provided by the user. As the system sizes available for simulations using classical molecular dynamics are significantly larger than can be studied using electronic structure methods, there is much more emphasis on the development of representative starting structures.

Typically, the initial starting structure is constructed inside a box commonly referred to as a simulation supercell. This supercell can then be subjected to a range of boundary conditions including, free boundary conditions, fixed boundary conditions and periodic boundary conditions. The most common of these is the periodic boundary condition in which the simulation supercell is tessellated infinitely in all directions, as illustrated in Figure 8.3. Further, it is assumed that when an atom crosses the boundary and leaves the box another atom with the same velocity appears on the opposite boundary of the supercell. In many instances, such as when studying surfaces, it is advantageous to mix boundary conditions. For example, the boundary condition in the plane of the surface could be periodic while perpendicular to the surface may have an open boundary.

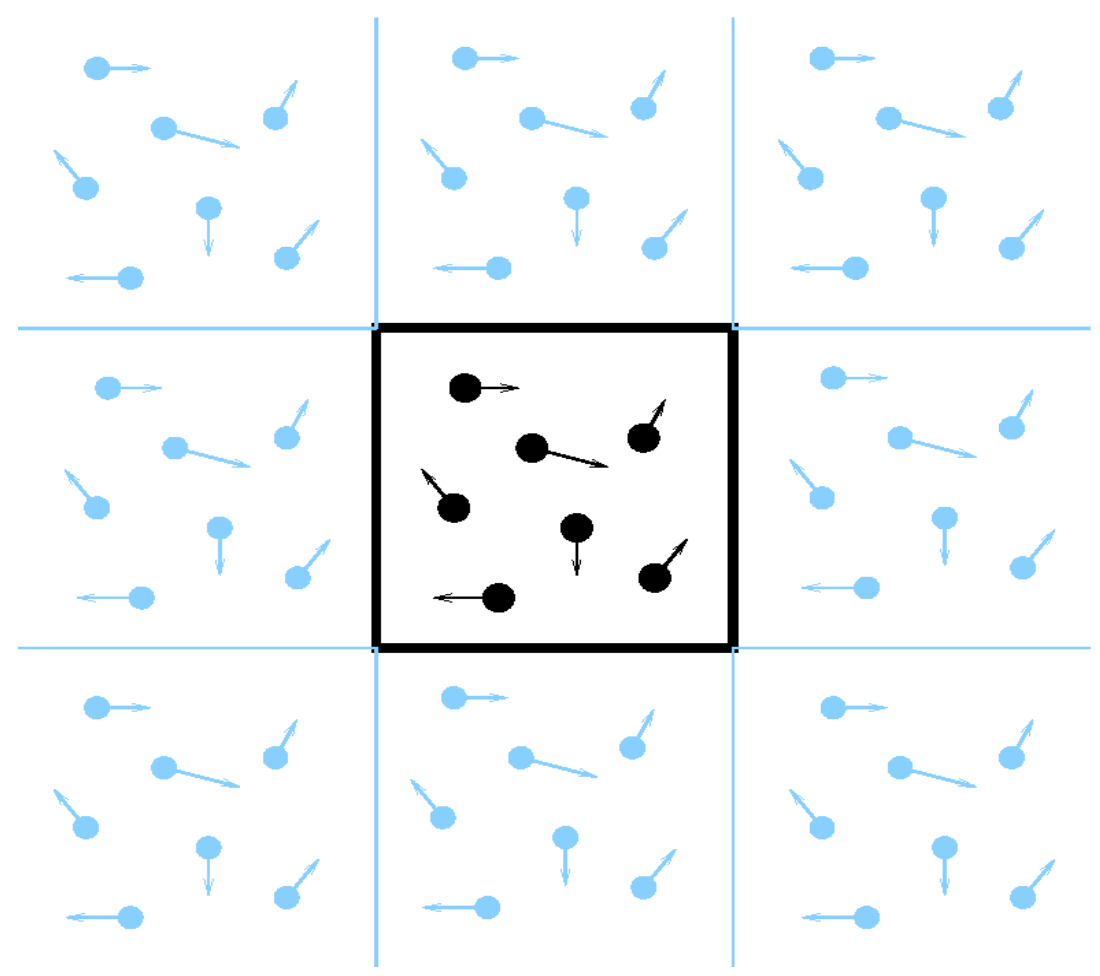

Figure 8.3. Two dimensional representation of the periodic boundary conditions. The cell in black represents the simulation supercell the images in blue represent periodic images. Image taken from Ref. [33].

In addition to defining the positions of the atoms it is also necessary to define their velocities. This must be done such that the velocities represent the temperature of interest, while also ensuring 
that the total linear momentum of the cell is zero. There are two main ways that the velocities can be assigned. The first is to apply a uniform distribution and the second, more popular, approach is to use a Maxwell-Boltzmann distribution, i.e.:

$$
f\left(v_{i}\right)=\sqrt{\frac{m}{2 \pi k_{b} T}} \exp \left[\frac{-m v_{i}^{2}}{2 k_{B} T}\right] .
$$

It should be noted that the system must be equilibrated after the velocities have been generated. With the atom positions and velocities established, the molecular dynamics simulation can proceed if the forces can be determined (methods for calculating forces will be discussed in the next section). Assuming the forces can be calculated, the MD simulation can proceed according to the flowchart illustrated in Figure 8.4.

\section{Simplified schematic of the molecular dynamics algorithm}

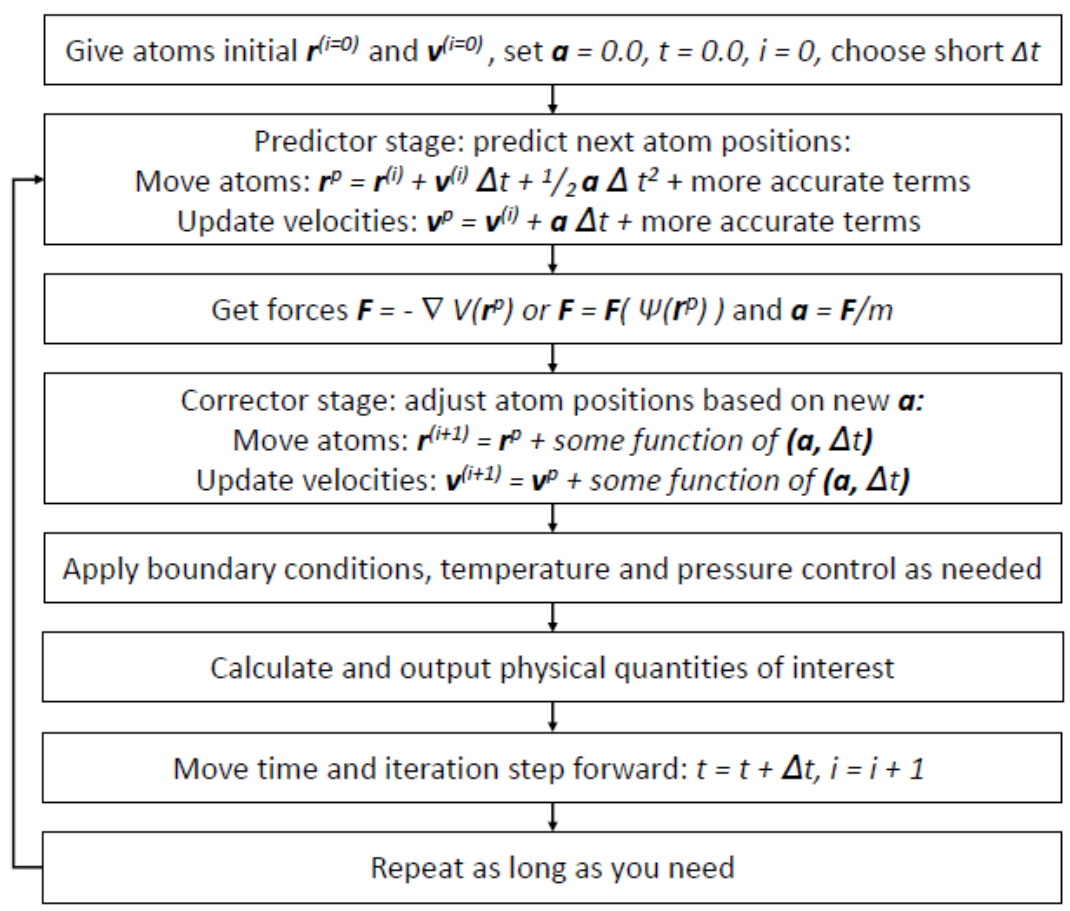

Figure 8.4. Flowchart showing the general outline of a molecular dynamics simulation. Note that in this case the index I stands for the iteration step.

\subsection{Force evaluation}

Evolving the atomic positions according to the equations of motion requires the ability to calculate the forces on the atoms or ions in the supercell. For the analytical potentials, the force $F_{i}$ on atom $i$ is obtained as the gradient of the potential energy with respect to the position of atom $i$ :

$$
\boldsymbol{F}_{i}=\nabla_{\boldsymbol{r}_{i}} E_{\text {total }}
$$

where $E_{\text {total }}$ is the total potential energy of the atomic system. For a pair potential that only depends on the interatomic separation between two atoms $r_{i j}$, the gradient reduces to a simple one-dimensional derivative with respect to the separation. However, for many-body potentials that also depend on the environment or angle between atoms, the gradient becomes considerably 
more complicated. For instance, in a potential that depends on the angles between chemical bonds $\theta_{i j k}$, one has to consider all permutations of the indices $i j k$ when calculating the force on an atom $i$.

The versatility of molecular dynamics is such that these forces may be calculated using empirical potentials or force fields or, alternatively, from quantum mechanics. While ab initio molecular dynamics (AIMD) is now routinely applied on supercells containing several hundreds of atoms, it is not possible to explore the larger systems that are accessible using empirical techniques. The introduction of electronic degrees of freedom in AIMD enables the simulation of a wide range of bonding interactions without the user having to specifically dictate what they are. By contrast, in a classical MD simulation the user must define both the type of interactions present as well as the parameters that define the strength of the interaction. Furthermore, once the potential or force field has been defined, it remains fixed throughout the simulation. Therefore, selection of an appropriate potential and parameterisation is a key part of the classical MD researcher's task.

In order to represent the diverse range of interactions that can occur between atoms/ions in solids, a wide variety of potential forms have been derived. Therefore, we now explore some of the more common potential forms and discuss their applicability.

\subsubsection{Coulombic interactions}

The energy, $E_{\text {Coul }}$, associated with the interaction of two ions, $i$ and $j$, with charges $q_{i}$ and $q_{j}$ is given by Coulomb's law:

$$
E_{\text {Coul }}=\frac{q_{i} q_{j}}{4 \pi \epsilon_{0} r_{i j}}
$$

where $\epsilon_{0}$ is the permittivity of free space. As the magnitude of the Coulombic potential energy decreases as $1 / r_{i j}$, it acts over a relatively long range. The slow convergence implies that the calculation of forces on an atom, $i$, must consider a large number of the surrounding atoms, placing significant demands on computational resources. As a consequence, Ewald devised a method that partitions the sum into a short range, real space component and a long range, reciprocal space component. At long range the reciprocal component is used to mask the charges, thus simplifying the calculation significantly [37]. A number of improvements of this concept have been developed including the particle mesh ewald (PME) [38], the particle-particle particle-mesh (pppm) approach [39] and the Wolf summation [40].

The choice of the charges on the ions in a system is a fundamental part of the construction of a potential model. Many potentials adopt formal charges for the ions; however, the charges can be reduced to represent a degree of covalency (for example the charge on the Ti atom in the Matsui potential for $\mathrm{TiO}_{2}$ is $3.098\|\mathrm{e}\|$ [41]). Similarly, partial charges are widely used in the simulation of glasses [42] and ionic liquids [43]. The exact choice of charge for the ions in ionic crystals, glasses, or ionic liquids can be estimated from quantum mechanical simulations or they can be free parameters for fitting the potential model. 


\subsubsection{Lennard-Jones potential}

The Lennard-Jones potential [44-46] was one of the first potential models employed in molecular dynamics simulations when it was used by Rahman to study atomic motion in liquid Ar [47]. It is currently used extensively to model the interaction between atoms in oganic molecules, such as proteins and polymers, as discussed in section 8.3.4 below.

The Lennard Jones potential can be written in a number of forms, including:

$$
E_{L J}=\sum_{i \neq j}\left(4 \epsilon_{i j}\left[\left(\frac{\sigma_{i j}}{r_{i j}}\right)^{12}-\left(\frac{\sigma_{i j}}{r_{i j}}\right)^{6}\right]\right)
$$

where $\epsilon_{i j}$ and $\sigma_{i j}$ correspond to the minimum in the interaction energy and the separation at which the energy is zero, respectively. As is evident from equation (8.30), the energy depends only on the separation between the atoms $i$ and $j$. An equivalent representation is:

$$
E_{L J}=\sum_{i \neq j}\left(\frac{A}{r_{i j}^{12}}-\frac{C}{r_{i j}^{6}}\right)
$$

where $A=4 \epsilon_{i j} \sigma_{i j}^{12}$ and $B=4 \epsilon_{i j} \sigma_{i j}^{6}$. In both cases, the first term represents a combination of the electrostatic repulsion between the atom's nuclei and the Pauli exclusion principle. As the two atoms approach each other the electron clouds overlap and, as no two Fermions may occupy the same quantum state, this increases the electronic energy. The second term is called the London interaction and it represents the van der Waals interactions [48]. The exponent of 6 in this van der Waals term arises directly from dipole-dipole interactions between induced or permanent dipole moments of the atoms.

\subsubsection{Buckingham potential}

The Buckingham potential is generally used, in conjunction with the Coulomb interaction, to model ionic crystals. Its functional form is similar to the Lennard-Jones potential discussed above, however, the repulsive term is replaced with a Born-Mayer [49] like term to give:

$$
E_{\text {Buck }}=A_{i j} \exp \left(\frac{r_{i j}}{\rho_{i j}}\right)-\frac{C_{i j}}{r_{i j}^{6}}
$$

where $A_{i j}, \rho_{i j}$ and $C_{i j}$ are potential parameters fitted to the specific ion types of $i$ and $j$. The Buckingham potential is frequently combined with the long-range Coulombic interaction to study ionic materials. A plot showing how these terms are combined in the case of the $\mathrm{Mg}^{2+}-\mathrm{O}^{2-}$ interaction is presented in Figure 8.5. 


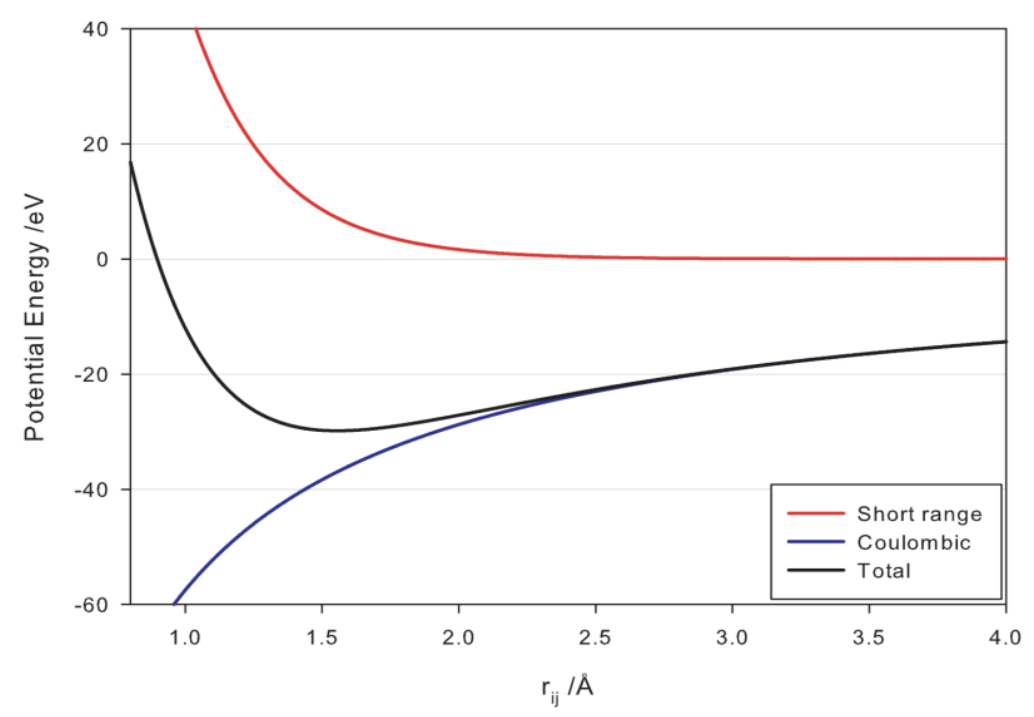

Figure 8.5. Plot showing the potential energy as a function of the separation for the interaction between an $\mathrm{Mg}^{2+}$ and $\mathrm{O}^{2-}$ ion as a function of separation. Figure taken from Ref. [50].

Care must be taken when using the Buckingham potential as at short ranges, (i.e. as $r_{i j} \rightarrow 0$ ) the attractive London term dominates and $E_{B u c k} \rightarrow-\infty$ causing the atoms/ions to fall onto each other. This is sometimes referred to as the Buckingham catastrophe [51].

\subsubsection{Class 1 forcefields}

In general, for inorganic systems such as metals and ionic crystals, empirical potentials are derived for specific materials, however, the flexibility offered by the Class 1 potential energy function means that a small number of force fields can be applied to a diverse range of different systems including proteins, surfactants, polymers and other aqueous or condensed phase systems. These force fields including, Amber, Chemistry at HARvard Macromolecular Mechanics (CHARMM) and the all-atom optimised potential for liquid simulations (OPLS-AA) adopt the functional form:

$$
\begin{array}{r}
E=\sum_{\text {bonds }} K_{b}\left(b-b_{0}\right)^{2}+\sum_{\text {angles }} K_{\Theta}\left(\Theta-\Theta_{0}\right)^{2}+ \\
\sum_{\text {dihedrals }} K_{\phi, n}\left[1+\cos \left(n \phi-\delta_{n}\right)\right]+ \\
\sum_{\text {impropers }} K_{\phi}\left(\phi-\phi_{0}\right)^{2}+E_{\text {Coul }}+E_{L J} .
\end{array}
$$

We start by examining the harmonic terms in equation (8.33). The first term represents interactions between bonded atom pairs, where $b$ is the bond length, $b_{0}$ is the equilibrium bond length and $K_{b}$ is the bond stiffness. Similarly, the second summation is over bonded triplets, where $\theta$ represents the angles between two vectors $i-j$ and $j-k$ and $K_{\theta}$ is the angle stiffness. The fourth term is also harmonic and represents the improper dihedral angles. $\phi$ and $\phi_{0}$ are the dihedral angle and the equilibrium dihedral angles respectively and $K_{\phi}$ is the force constant. The third term is sum over quartets of atoms with bonds between atoms, $i$ and $j, j$ and $k$ and $k$ and $l$, and models the dihedral corresponding to rotation of bonds $i-j$ and $k-l$ around $j-k$. Then, $\phi$, 
$\phi_{0}$ and $K_{\phi, n}$ represent the dihedral angle, equilibrium dihedral and the force constant respectively. The Coulombic interactions, $E_{C o u l}$, are included using the standard form discussed in section 8.3.1. Finally, van der Waals interactions and the Pauli repulsion are included through the Lennard-Jones term, $E_{L J}$.

\subsubsection{Embedded atom model}

First developed by Daws and Baskes [52], the embedded atom method is a many body potential that finds particular utility in the simulation of metals. In contrast to ionic crystals, in which the total cohesive energy can generally be written as the sum of the interaction energies between pairs of atoms, the nature of metallic bonding ensures that this is not the case with metals, as the strength of the interaction between any two atoms is a function of the positions of all the neighbouring atoms. In effect, the ions in a metal are embedded in a "sea" of delocalized electrons and it is the interaction between the ions and the delocalized electrons that is the source of the binding between atoms. In order to capture this effect in the EAM model, ions are assumed to be embedded in the charge density provided by the surrounding ions. The total energy is given as:

$$
E_{\text {tot }}=\frac{1}{2} \sum_{j, j \neq i} \phi_{i j}\left(r_{i j}\right)+\sum_{i}^{N} f_{i}\left(\rho_{h, i}\right)
$$

where $\phi_{i j}\left(r_{i j}\right)$ represents the Coulombic repulsion between the ions and $f_{i}\left(\rho_{h, i}\right)$ is the cohesive energy gained from embedding the ion in charge density represented by $\rho_{h, i}$. The local charge density is approximated as a function, $f$, of the distance between the central ion, $i$, and all the surrounding ions, $j$, within a defined cutoff (equation (8.35):

$$
\rho_{i}=\sum_{j \neq i} f\left(r_{i j}\right)
$$

As a consequence, the calculation of the density is treated like a simple pair-wise interaction. This, coupled with the ability to use cubic splines, that can be readily fitted to first principles data [4], makes the EAM computationally efficient.

An important development of the EAM occurred in 1984 when Finnis and Sinclair, suggested that the embedding energy should be related to the square route of the density, based on the second moment approximation to tight binding theory, i.e.:

$$
f\left(\rho_{i}\right)=\sqrt{\rho_{i}}
$$

The original Finnis-Sinclair model used polynomial functions to define the contribution of an ion to the local density as well as the to describe the pairwise repulsion term. This initial strategy was extended by Dai et al. [53], such that the contribution to the density is now calculated from:

$$
\rho_{i}=\sum_{j \neq i} A^{2} \beta\left(r_{i j}\right)
$$

where:

$$
\beta(r)=\left\{\begin{array}{cc}
\left(r_{i j}-d\right)^{2}+B^{2}(r-d)^{4}, & r \leq d \\
0, & r>d
\end{array}\right.
$$


and $A$ and $B$ are constants and $d$ is a cutoff distance. For the repulsive interaction:

$$
\phi\left(r_{i j}\right)=\left\{\begin{array}{cc}
\left(r_{i j}-c\right)^{2}\left(c_{0}+c_{1} r_{i j}+c_{2} r_{i j}^{2}+c_{3} r_{i j}^{3}+c_{4} r_{i j}^{4}\right), & r \leq c \\
0 \quad & r>c
\end{array}\right.
$$

where $c_{0}, c_{1}, c_{3}$ and $c_{4}$ are fitting constants and $c$ is the cutoff for the potential functional.

The EAM-like potentials have proven to be very well suited to model a wide range of elemental metals and at least binary and ternary metal alloys. An example of results from an MD simulation of a RuAl bilayer system is shown in Figure 8.7.

\section{Melting of crystalline Ru and Al layers due to heat spike ( $t<1 \mathrm{~ns})$}

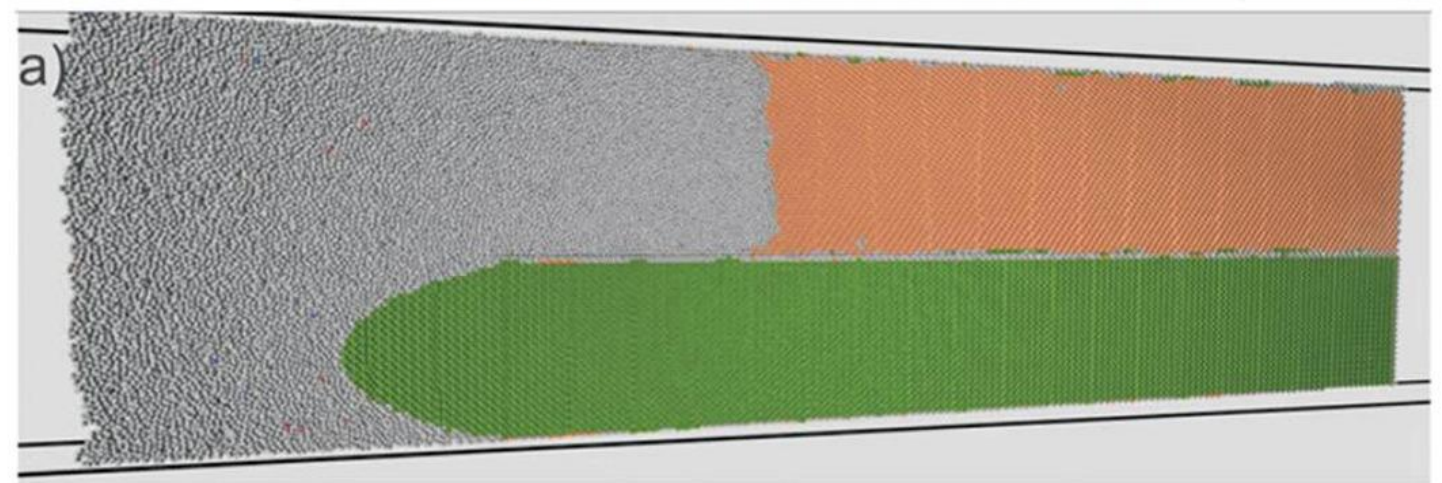

\section{Formation of B2-RuAl nucleus on $\mathrm{Ru} / \mathrm{Al}(\mathrm{Ru})$ interface ( $\mathrm{t} \sim 1 \mathrm{~ns})$}

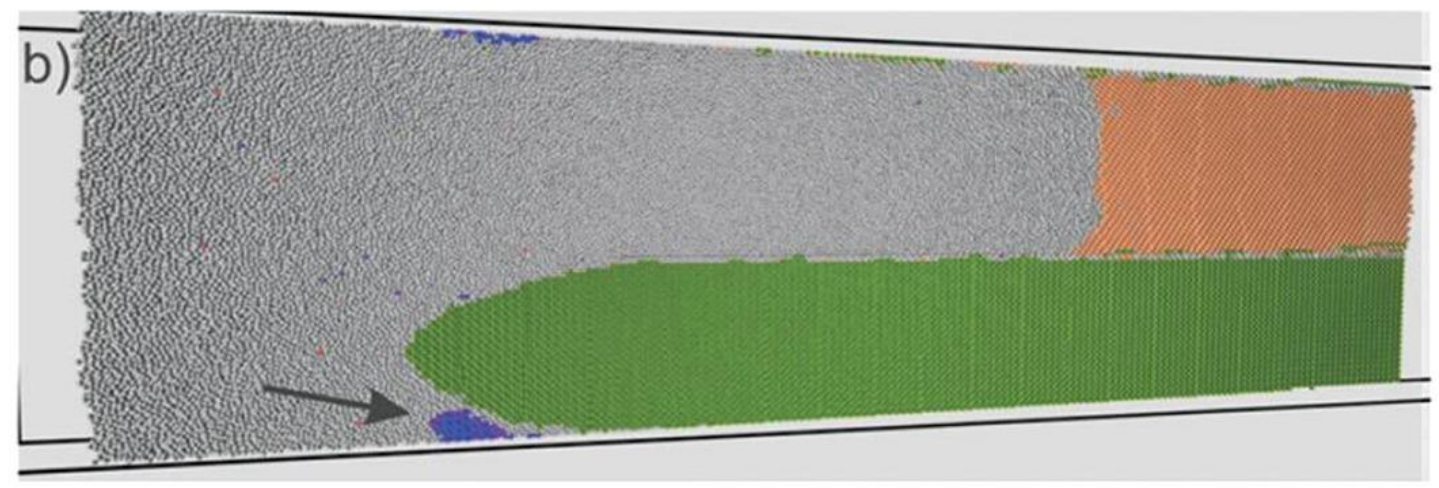

Further growth of B2-RuAl phase $(t>1 n s)$

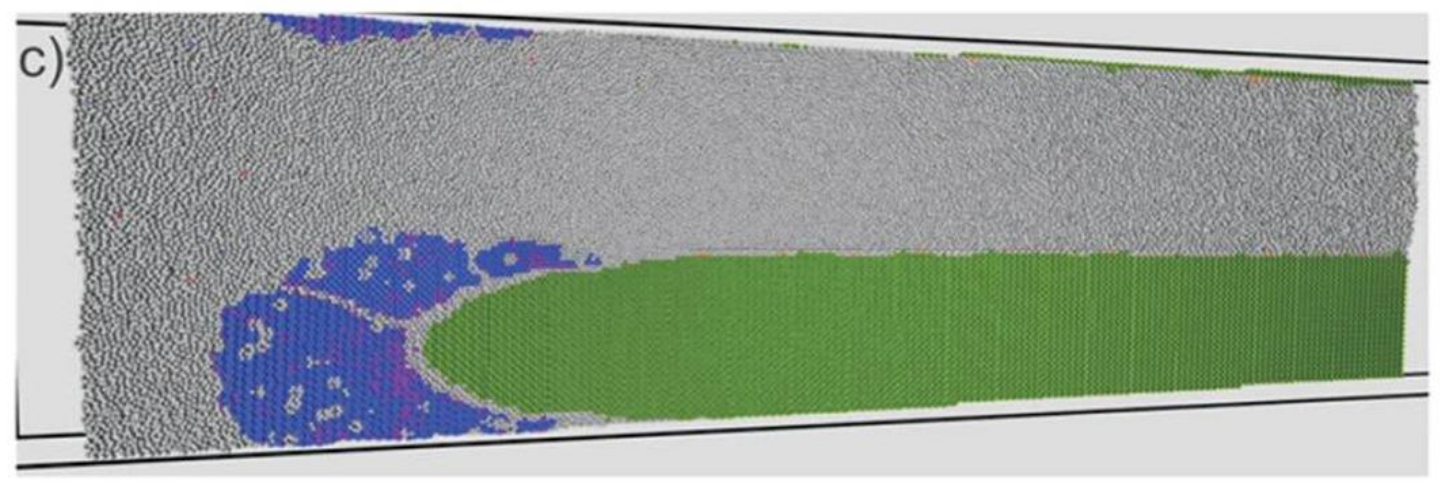

Figure 8.6. Example of a molecular dynamics simulation of spontaneous mixing in a RuAl bilayer metal system. In these simulations (and the corresponding experiments) an initially completely mixed Ru/Al bilayer system was subjected to a sudden heating ("heat spike") in one end. Since the RuAl system has a high negative heat of mixing, this induced energy release and melting of the system (gray atoms). However, when the system cools down, a new binary intermetallic B2 RuAl (blue atoms) starts to form from the melt. For details see Ref. [60], reprinted with open access permissions. 


\subsubsection{Bond order potentials}

The potentials discussed above have been widely used to successfully model ionic crystals, proteins, polymers and metals, however, none of the functional forms discussed so far is able to capture the properties of covalently bonded solids, such as diamond or silicon. As is the case with metals, the interaction between two atoms in a covalent solid is a function of the positions of the neighbouring atoms, however, it is not only a function of the relative distances, but also the angles between the neighbouring bonds. In other words, the strength of a bond is modified by the number and orientation of neighbouring bonds. Bond order potentials, such as the Tersoff potential [61], aim to capture these effects.

Within the framework of the Tersoff potential, the total potential energy for a system of $N$ atoms if given by:

$$
E_{\text {tot }}=\sum_{i=1}^{N} E_{i} .
$$

The site potential for an atom $i$ can then be written:

$$
E_{i}=\frac{1}{2} \sum_{j \neq i}=E_{i j}
$$

and the interaction energy between atoms $i$ and $j$ is then given by:

$$
E_{i j}=f_{C}\left(r_{i j}\right)\left[f_{R}\left(r_{i j}\right)-b_{i j} f_{A}\left(r_{i j}\right)\right] .
$$

where $f_{C}\left(r_{i j}\right)$ is the pairwise cutoff function, otherwise known as a screening function, which goes continuously from 1 to 0 in the neighborhood of the equilibrium bond length and it takes the form:

$$
f_{C}\left(r_{i} j\right)= \begin{cases}1, & r_{i j}<R_{1} \\ \frac{1}{2}\left[1+\cos \left(\pi \frac{r_{i j}-R_{1}}{R_{2}-R_{1}}\right)\right], & R_{1}<r_{i j}<R_{2} \\ 0, & r_{i j} \geq R_{2}\end{cases}
$$

where $R_{1}$ and $R_{2}$ are the inner and out cutoff distances. The screening function ensures that only nearest neighbour atoms are covalently bonded.

The pairwise repulsive function, $f_{R}\left(r_{i j}\right)$ has the functional form:

$$
f_{R}\left(r_{i j}\right)=A e^{-\lambda r_{i j}}
$$

and the pairwise attractive function can be given as:

$$
f_{A}\left(r_{i j}\right)=B e^{-\mu r_{i j}}
$$

where, $A, B, \lambda$ and $\mu$ are all fitting parameters that are greater than zero.

The bond order term, $b_{i j}$, in equation (8.42) is the term responsible for modifying the bond strength as a function of the number, length and orientations of neighbouring bonds. It takes the following form:

$$
b_{i j}=\left(1+\zeta_{i j}^{n}\right)^{1 / 2 n}
$$

where 


$$
\zeta_{i j}=\sum_{k \neq i, j}^{N} f_{C}\left(r_{i k}\right) g\left(\theta_{i j k}\right)
$$

and $n$ is an empirically derived parameter. Increasing the value of $\zeta_{i j}$ leads to a decrease in $b_{i j}$ and hence a weaker bond. By contrast, when $\zeta_{i j}=0, b_{i j}$ reaches a maximum value of 1 . The angular function takes the form:

$$
g\left(\theta_{i j k}\right)=\gamma\left(1+\frac{c^{2}}{d^{2}}-\frac{c^{2}}{d^{2}+\left(\cos \theta_{i j k}-h\right)^{2}}\right)
$$

where $\theta_{i j k}$ is the angle between the bonds between atoms $i$ and $j$ and $i$ and $k$ and $\gamma, c, d$ and $h$ complete the list of potential parameters. The total number of fitting parameters in the Tersoff potential is nine, therefore fitting such bond order potentials is always challenging. Nevertheless, they have been applied very successfully to a number of covalently bonded solids.

\subsubsection{Dipole polarizatibiltiy}

The shell model of Dick and Overhauser [62] envisages an atom or ion as a massive, positively charged core connected to a massless negatively charged shell. The core and the shell are then connected together via a harmonic spring with a force constant, $K_{\text {spring }}$, such that the energy is given as:

$$
E=\frac{1}{2} K_{\text {Spring }} \delta r_{i}
$$

where $\delta r_{i}$ is the separation between the core and the shell. An image showing how the introduction of an external charge polarises and ion based on the shell model is presented in Figure 8.7.
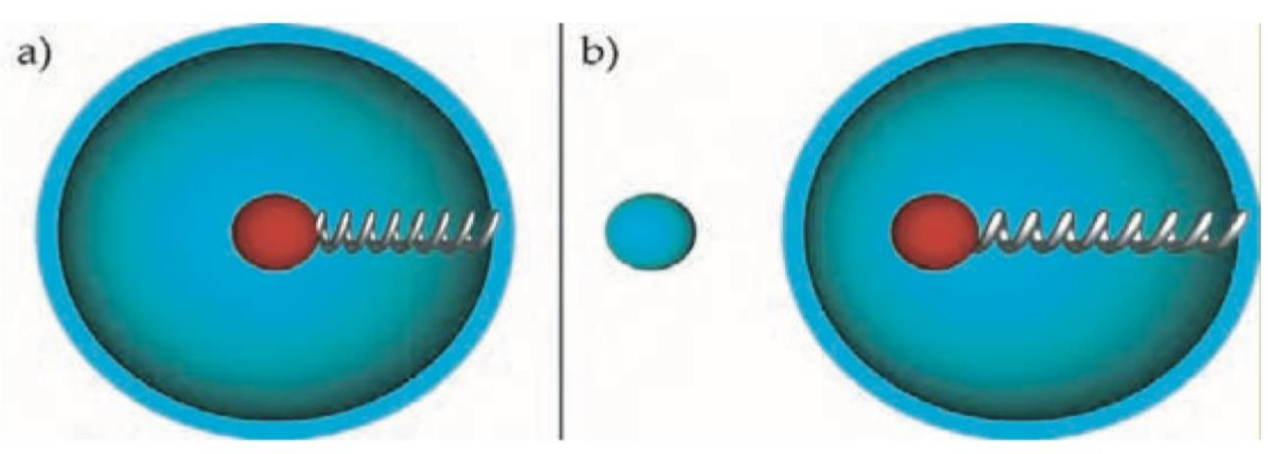

Figure 8.7. Graphical representation of Dick and Overhauser's shell model. (a) shows an unpolarised atom where the core and shell are centered on the same point in space, and (b) shows how the presence of an external electric field can polarise the core-shell arrangement. Image taken from Ref. [50]

The introduction of shells into a molecular dynamics simulation poses significant problems. Whilst it is possible to calculate the force on the shells, as they have no mass, it is not possible to determine an acceleration. As a consequence, it is not possible to evolve their positions in time. There are two solutions to this problem. The first is the adiabatic shell method [63] where the shells are assigned a fraction of the atomic mass, $x$, such that it ensures the natural frequency of vibration of the harmonic spring, $v_{\text {core-shell }}$, i.e.: 


$$
v_{\text {core-shell }}=\frac{1}{2 \pi}\left[\frac{K_{\text {Spring }}}{x(1-x) m}\right]^{1 / 2} .
$$

From a dynamical perspective the core and shell resemble a diatomic molecule, however, there is no exchange of kinetic energy between the core-shell unit and the remainder of the system due to the high vibrational frequency of the bond. The second approach is the relaxed or massless shells model [64]. In this approach the shells have no mass and so their movement cannot be governed by the equations of motion. Therefore, in each timestep the positions of cores at $t+\delta t$ are determined and then the shells are relaxed such that the force on every shell is zero. This neatly mimics the Born-Oppenheimer approximation that states that the electrons can react instantaneously to any change in nuclear positions [65].

\subsubsection{ZBL potential}

The empirical potential and force field models discussed thus far have all assumed that the system is close to equilibrium. However, under extreme conditions, most notably during irradiation where atoms have very high velocities, atoms can become very close to each other requiring a better description of the forces at these short-ranges. Zeigler et al. have shown that the repulsive interaction at short range can be described using a screened Coulomb potential, i.e.:

$$
E_{Z B L}=\frac{1}{4 \pi \epsilon_{0}} \frac{Z_{i} Z_{j} e^{2}}{r_{i j}} \phi\left(r_{i j} / a\right)+S\left(r_{i j}\right)
$$

where $Z_{i}$ and $Z_{j}$ are the charges on the atomic nuclei [56]. The screening length and function are given by equations (8.52) and (8.53), respectively.

$$
\begin{gathered}
a=\frac{0.46850}{Z_{i}^{0.23}+Z_{j}^{0.23}} \\
\phi(x)=0.18175 e^{-3.19980 x}+0.50986 e^{-0.94229 x}+ \\
0.28022 e^{-0.40290 x}+0.02817 e^{-0.20162 x}
\end{gathered}
$$

The ZBL potential is only designed to operate a very close ranges and, therefore, it must be connected the potential model used to describe the system of interest under equilibrium conditions. The final potential is thus described as:

$$
E= \begin{cases}E_{Z B L}, & r_{i j} \leq r_{1} \\ \sum_{n=0}^{7} a_{n} r^{n}, & r_{1}<r_{i j}<r_{2} \\ E_{\text {Original }}, & r_{i j} \geq r_{2} .\end{cases}
$$

where $\sum_{n=0}^{7} a_{n} r^{n}$ is spline function that enables the transition from the ZBL to the original potential [67]. The spline function is fitted to ensure that the overall potential is continuous in both the first and second derivatives. Care should be taken with the selection of the cutoffs between the different functions. As the spline is not governed by any scientific rationale the region of its operation should be as small as possible, however, it is also important to ensure that there are no turning points in the splined region. 


\subsubsection{Reactive potentials}

As described above the lack of explicit electronic detail present within classical molecular dynamics implies that a different potential model is required for each different type of material, i.e. metal, semiconductor, protein etc. This inevitably raises the question of what to do when your target system consists of different types of materials and how should atoms that move between different classes of material be treated. From a fundamental perspective, the issue is that the potentials described so far are unable to model chemical reactivity. Addressing this issue is the motivation for the reactive force field model, typically referred to as ReaxFF [68] and the chargeoptimized many body potential (COMB) potentials [69]. To enable a description of reactivity these potential models adopt a bond order approach which is coupled with a variable ionic charge to produce a flexible interatomic description. As a consequence they are transferable across phases (solid, liquid, gas) and different chemistries, for example an oxygen atom is treated with the same formalism whether it is in $\mathrm{O}_{2}$ gas, liquid $\mathrm{H}_{2} \mathrm{O}$ or solid $\mathrm{SiO}_{2}$.

For the ReaxFF potential the total energy of the system can be determined from:

$$
E_{\text {system }}=E_{\text {bond }}+E_{\text {over }}+E_{\text {angle }}+E_{\text {tors }}+E_{\mathrm{vdWaals}}+E_{\text {Coul }}+E_{\text {Specific }}
$$

where $E_{\text {bond }}$ is the energy associated with forming bonds, $E_{\text {angle }}$ is the bond bending energy, $E_{\text {torsion }}$ is the torsion energy, $E_{\mathrm{vdWaals}}$ is the van der Waals energy and $E_{\text {Coul }}$ is Coulomb energy . In addition to these familiar terms, there is an energy penalty term $E_{\text {over }}$ for deviation from the ideal coordination and a term $E_{\text {spec }}$ to take account of effects not included in the other terms. The bond stretching, bending and torsion terms are all functions of the bond order, $B O$, which is a function of the interatomic separations atoms, $r_{i j}$, in a similar way to the Tersoff potential (described above). This term modifies the strength and angle dependence of the bond, depending on the number and length of neighbouring bonds. For example, the bond order term for carbon is given by the sum of contributions from single, double and triple bonds $\left(B O_{i j}^{\sigma}, B O_{i j}^{\pi}\right.$ and $B O_{i j}^{\pi \pi}$ respectively), as shown in equations (8.56) and (8.57).

$$
\begin{gathered}
B O_{i j}=B O_{i j}^{\sigma}+B O_{i j}^{\pi}+B O_{i j}^{\pi \pi} \\
=\exp \left[p_{b o 1}\left(\frac{r_{i j}}{r_{o}^{\sigma}}\right)^{p_{b 02}}\right]+\exp \left[p_{b o 3}\left(\frac{r_{i j}}{r_{o}^{\pi}}\right)^{p_{b 04}}\right]+\exp \left[p_{b o 5}\left(\frac{r_{i j}}{r_{o}^{\pi \pi}}\right)^{p_{b 06}}\right]
\end{gathered}
$$

The parameters $p_{b o 1}, p_{b o 2}, p_{b o 3}, p_{b o 4}, p_{b o 5}, p_{b o 6}$ and $r_{o}^{\sigma}, r_{o}^{\pi}, r_{o}^{\pi \pi}$ are all fitting parameters. Nonbonded interactions are present between all atom pairs and consequently there is no requirement for explicit switching functions. One caveat is that these terms become unphysically large at short separations requiring shielding functions for the Coulomb and van der Waals terms to compensate.

Similarly, in the COMB approach the total energy is determined from:

$$
E_{\mathrm{CoMB}}=E_{\text {self }}+E_{\text {Coul }}+E_{\text {polar }}+E_{\mathrm{vdWaals}}+E_{\text {bonds }}+E_{\text {other }}
$$


Where $E_{\text {self }}$ is the energy associated with the ionization of an atom, $E_{\text {polar }}$ represents the dipole and higher order elctrostatic interactions and $E_{\text {other }}$ describes specific bond angle configurations [70].

Key to modelling reactivity is the dynamic calculation of atomic charges during the simulation. ReaxFF and COMB both use charge equilbration procedures to distribute charges according to electronegativity differences. While the ability to assign charges dynamically is fundamental for describing reactivity, it is also computationally expensive limiting the system size.

\subsubsection{Machine-learned potentials}

In recent years, machine-learned (ML) interatomic potentials have emerged as an alternative to the analytical potential functionals [71-75]. In these, instead of an analytical functional form or tabulated function, the potential energy and forces are obtained from some machine-learning formalism. The advantage of the approach is that the description of the energy and forces is not limited to the flexibility of the mathematical function. Instead, one can at least in principle fit all the data points in an arbitrarily large database to a desired accuracy.

The downside is that if the ML formalism has no physically motivated approach at all, interatomic configurations outside the fitting database may be arbitrarily badly described. This argument can be illustrated with a simple example. Assume one would make a large database of energies for just 2 isolated atoms as positions of their coordinates in space $r_{i}$ and $r_{j}$, then train a neural network (NN) to reproduce exactly these energies. While with a large enough network all points could be definitely reproduced, this could lead to an effect where the neural network gives different energies to atom positions that have different orientation in space even when the interatomic separation $r_{i j}$ is exactly the same. While the NN would do exactly what it is trained for, the result would be obviously chemically wrong (as a bond energy should not depend on orientation in space).

To avoid such pitfalls, machine-learned approaches for interatomic potentials do utilize some basics physics insights in their implementations. For instance, to avoid the pitfall mentioned above, one can train the ML approach against the interatomic separation $r_{i j}$ rather than coordinates in space $r_{i}, r_{j}$. This idea of "symmetry functions" can also be extended e.g., to bond angles [71,73]. Similarly, to avoid strong local jumps in the energy of very closeby atom positions (which could result from a "blind" ML training even though it is obviously not chemically reasonable) smoothing schemes can be used within the training scheme [73].

After such physical/chemical insight have been included in the ML formalism, the machinelearned potentials have proven to in several materials be able to reach "quantum accuracy", i.e., an accuracy that is comparable to the density-functional theory database against which they are trained. This has been demonstrated to work very well at least in the Gaussian Approximation Potential (GAP) [76,77] approach. An example of the level of agreement that can be obtained is shown in Figure 8.8. Naturally, if the DFT database itself has inaccuracies (which is often the case e.g., for elastic constants), these are carried over to the ML potential.

It is also important to realize that the ML approaches should not be used for extrapolation, as they do not have physically motivated functional forms. For instance, if all interatomic separations in a training database are for normal near-equilibrium chemical separations, interatomic 
configurations with much larger or smaller separations may be arbitrarily poorly described. This was recently shown explicitly for Si and W, for which tests at very small separations were shown to give clearly unreasonable results [78,79]. The issue was resolved by addition of a ZBL-like repulsive potential to the GAP formalism for the short separations [79].

It is worth noting that there is no strict limit between ML and numerically tabulated interatomic potentials, since the ML training schemes can in some formalisms be reduced partly or fully to a final numerically tabulated potential [80]. As in analytical potentials, the implementation of the complexity of the training and final ML force calculation is in the end a trade-off between accuracy and speed: the more complex the formalism, the slower it is. Typical ML potentials are 1-4 orders of magnitude slower than analytical potentials for the same materials, yet at the same time many orders of magnitude faster than DFT.

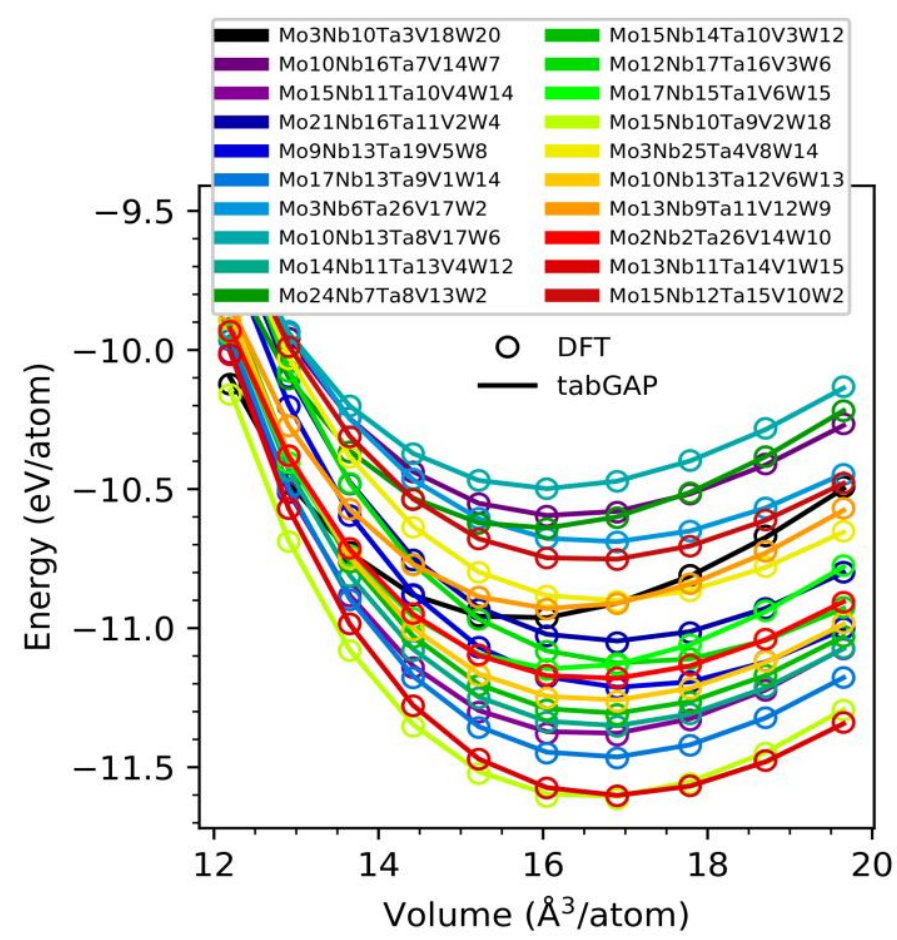

Figure 8.8. Example of the level of agreement that can be obtained between the DFT training data set and a machinelearned interatomic potential. The dots are the DFT data points on the cohesive energy in variour high entropy alloy configurations, and the lines show results from the multielemental tabGAP formalism. From Ref. [80], reprinted with permission from the authors. Copyright the American Physical Scoiety (2021)

\subsubsection{Practical considerations}

The rapid growth in the use of molecular dynamics in scientific research has been accompanied by an increase in the number of potential models developed. For some of the more widely studied materials there have been a large number of potentials developed (see for example the evolution of potentials for $\mathrm{UO}_{2}$ in Figure 8.9). This leads to the inescapable question about which is the correct potential to use. 


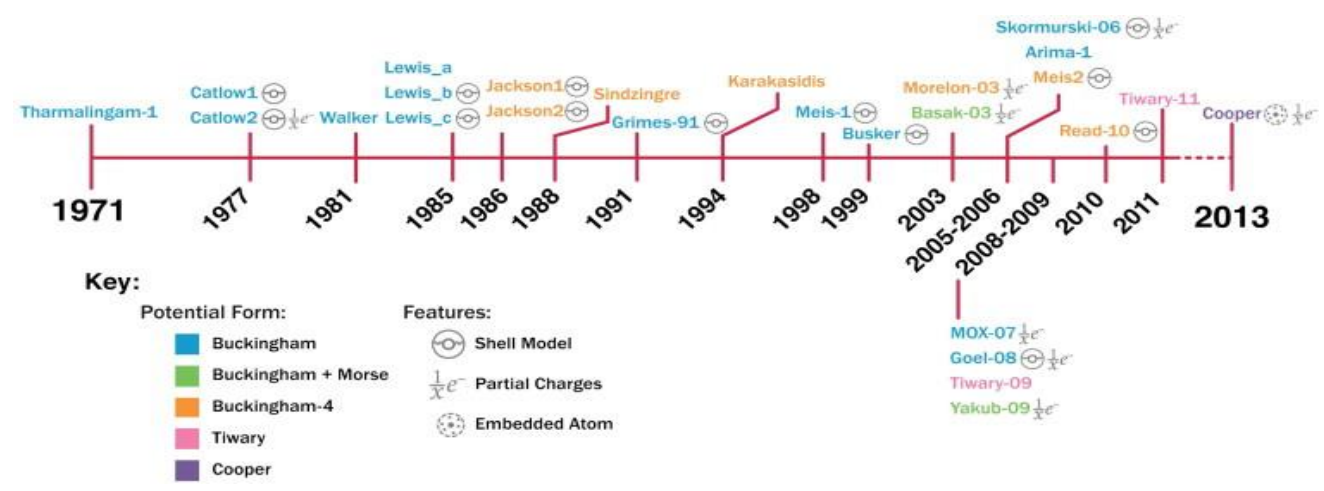

Figure 8.9. Timeline showing the evolution of the potential models for $\mathrm{UO}_{2}$ Image taken from Ref. [81]

There is no simple answer to this question as it largely depends on the properties that the researcher is interested in. It is not the case that the newer potentials are necessarily better than some of the older ones. As discussed by Govers et al. for $\mathrm{UO}_{2}$ the potentials all do a good job of reproducing the properties they were fit to, however, when examining other properties, they perform less well $[82,83]$. Therefore, if a researcher is interested in structure of dislocations in $\mathrm{UO}_{2}$, they might choose to use a potential that reproduces the bulk modulus, even though the same potential does a less good job of the reproducing the thermal expansion. It is, therefore, very important to become familiar with a potential and be aware of its limitations helping to place any results generated in context.

\subsection{Codes available}

From a practical point of view, running molecular dynamics is nowadays rather easy. Historically, many MD codes were developed as pure research codes in one or a few research groups, with well-known shortcomings for both the quality and usability of the codes. During the last decade, a major fraction of MD users has diffused towards using professionally maintained open-source codes: including LAMMPS [91], GROMACS [92] and DL_POLY [93]. The LAMMPS package also supports the GAP ML potentials described in section 8.3.10. These codes have a wide user base, are extendable, and have good documentation. However, it be kept in mind that for reliable scientific research, it is very important that the users do not use the codes as "black boxes" but understand the physics and chemistry of the algorithms.

In addition to the array of simulation packages available to run the MD simulations there are also a whole host of post processing packages to help with the analysis of the atomic trajectories, including OVITO [94,95], Visual Molecular Dynamics (vmd) [96] and pymol [97].

\section{References}

[1] B. J. Alder, T. E. Wainwright, Molecular Dynamics by Electronic Computers, Proc. Intern. Symposium on Transport Processes in Statistical Mechanics, Wiley Interscience, New York, 1957, p. 97.

[2] B. J. Alder, T. E. Wainwright, Studies in Molecular Dynamics. I. General Method, J. of Chem. Phys. 31 (2) (1959) 459.

[3] J. B. Gibson, A. N. Goland, M. Milgram, G. H. Vineyard, Dynamics of Radiation Damage, Phys. Rev 120 (4) (1960) 1229-1253. 
[4] C. Erginsoy, G. H. Vineyard, A. Englert, Dynamics of Radiation Damage in a Body-Centered Cubic Lattice, Phys. Rev. 133 (2) (1964) A595.

[5] N. Tchipev, S. Seckler, M. Heinen, J. Vrabec, F. A. Gratl, M. Horsch, M. Bernreuther, C. Glass, C. Niethammer, N. Hammer, B. Krischok, M. M. Resch, D. Kranzlmueller, H. Hasse, H. Bungartz, P. Neumann, Twetris: Twenty trillion-atom simulation, The International Journal of High Performance Computing Applications 33 (2019) 838-854.

[6] J. A. Harrison, J. D. Schall, S. Maskey, P. T. Mikulski, M. T. Knippenberg, B. H. Morrow, Review of force fields and intermolecular potentials used in atomistic computational materials research, Applied Physics Reviews 5 (3) (2018) 031104. arXiv:https://doi.org/10.1063/1.5020808, doi:10.1063/1.5020808. URL https://doi.org/10.1063/1.5020808

[7] W. C. D. Cheong, L. C. Zhang, Molecular dynamics simulation of phase transformations in silicon monocrystals due to nano-indentation, Nanotechnology 11 (3) (2000) 173-180. doi:10.1088/09574484/11/3/307. URL https://doi.org/10.1088/0957-4484/11/3/307

[8] V. Yamakov, D. Wolf, S. R. Phillpot, A. K. Mukherjee, H. Gleiter, Dislocation processes in the deformation of nanocrystalline aluminium by molecular-dynamics simulation, Nature Materials 1 (1) (2002) $45\{49$. doi:10.1038/nmat700. URL https://doi.org/10.1038/nmat700

[9] M. L. Klein, W. Shinoda, Large-scale molecular dynamics simulations of self-assembling systems,

Science 321 (5890) (2008) 7980-800. arXiv:https://science.sciencemag.org/content/321/

5890/798.full.pdf, doi:10.1126/science.1157834.

URL https://science.sciencemag.org/content/321/5890/798

[10] A. E. Sand, S. L. Dudarev, K. Nordlund, High-energy collision cascades in tungsten: Dislocation loops structure and clustering scaling laws, EPL (Europhysics Letters) 103 (4) (2013) 46003.

doi:10.1209/0295-5075/103/46003. URL https://doi.org/10.1209/0295-5075/103/46003

[11] D. L. Ensign, P. M. Kasson, V. S. Pande, Heterogeneity even at the speed limit of folding: Large-scale molecular dynamics study of a fast-folding variant of the villin headpiece, Journal of Molecular Biology 374 (3) (2007) 806-816. doi:https://doi.org/10.1016/j.jmb.2007.09.069.

URL https://www.sciencedirect.com/science/article/pii/S0022283607012685

[12] R. J. Zamora, B. P. Uberuaga, D. Perez, A. F. Voter, The modern temperature accelerated dynamics approach, Annual Review of Chemical and Biomolecular Engineering 7 (1) (2016) 87-110, pMID: 26979413. arXiv:https://doi.org/10.1146/annurev-chembioeng-080615-033608, doi:10.1146/annurev-chembioeng-080615-033608. URL https://doi.org/10.1146/annurevchembioeng-080615-033608

[13] D. Perez, B. P. Uberuaga, A. F. Voter, The parallel replica dynamics method - coming of age, Computational Materials Science 100 (2015) 90-103, special Issue on Advanced Simulation Methods. doi:https://doi.org/10.1016/j.commatsci.2014.12.011.

URL https://www.sciencedirect.com/science/article/pii/S0927025614008489

[14] M. P. Allen, D. J. Tildesley, Computer Simulation of Liquids, Oxford University Press, Oxford, UK, 1989.

[15] A. R. Leach, Molecular modelling: principles and applications, $2^{\text {nd }}$ Edition, Pearson Education, Harlow, England, 2001.

[16] D. W. Heermann, Computer Simulation Methods in Theoretical Physics, Springer, Berlin, 1986.

[17] R. Lesar, Introduction to Computational Materials Science, Cambridge University Press, 2013. 
[18] D. Frenkel, B. Smit, Understanding molecular simulation: from algorithms to applications, $2^{\text {nd }}$ Edition, Academic Press, San Diego, 2002.

[19] M. E. Tuckerman, G. J. Martyna, Understanding modern molecular dynamics: Techniques and applications, The Journal of Physical Chemistry B 104 (2) (2000) 159-178. doi:10.1021/jp992433y.

URL https://doi.org/10.1021/jp992433y

[20] L. Verlet, Computer "experiments" on classical fluids. I. thermodynamical properties of Lennard-Jones molecules, Phys. Rev. 159 (1967) 98-103. doi:10.1103/PhysRev.159.98. URL https://link.aps.org/doi/10.1103/PhysRev.159.98

[21] R. W. Hockney, Potential calculation and some applications., Methods Comput. Phys. 9 (1970) 135-211. URL https://www.osti.gov/biblio/4079632

[22] W. C. Swope, H. C. Andersen, P. H. Berens, K. R. Wilson, A computer simulation method for the calculation of equilibrium constants for the formation of physical clusters of molecules: Application to small water clusters, The Journal of Chemical Physics 76 (1) (1982) 637-649. URL https://doi.org/10.1063/1.442716

[23] D. Fincham, Choice of timestep in molecular dynamics simulation, Computer Physics Communications 40 (2) (1986) 263-269.

URL https://www.sciencedirect.com/science/article/pii/001046558690113X

[24] S. Kim, Issues on the choice of a proper time step in molecular dynamics, Physics Procedia 53 (2014) 60-62, 26th Annual CSP Workshop on "Recent Developments in Computer Simulation Studies in Condensed Matter Physics", CSP 2013. doi:https://doi.org/10.1016/j.phpro.2014.06.027. URL https://www.sciencedirect.com/science/article/pii/S1875389214000388

[25] T. Schlick, Molecular Modelling and Simulation - An Interdisciplinary Guide, Springer, New York, 2002. ISBN 0-387-95404-X.

[26] N. A. Marks, M. Robinson, Variable timestep algorithm for molecular dynamics simulation of nonequilibrium processes, Nuclear Instruments and Methods in Physics Research Section B: Beam Interactions with Materials and Atoms 352 (2015) 3-8, proceedings of the 12th International Conference on Computer Simulation of Radiation Effects in Solids, Alacant, Spain, 8-13 June, 2014. doi:https://doi.org/10.1016/j.nimb.2014.11.094.

URL https://www.sciencedirect.com/science/article/pii/S0168583X14009951

[27] S. J. Stuart, J. M. Hicks, M. T. Mury, An iterative variable-timestep algorithm for molecular dynamics simulations, Molecular Simulation 29 (3) (2003) 177-186. URL https://doi.org/10.1080/0892702031000089650

[28] H. J. C. Berendsen, J. P. M. Postma, W. F. van Gunsteren, A. DiNola, J. R. Haak, Molecular dynamics with coupling to an external bath, The Journal of Chemical Physics 81 (8) (1984) 3684-3690. URL https://doi.org/10.1063/1.448118

[29] H. C. Andersen, Molecular dynamics simulations at constant pressure and/or temperature, The Journal of Chemical Physics 72 (4) (1980) 2384\{2393. arXiv:https://doi.org/10.1063/1.439486, doi:10.1063/1.439486. URL https://doi.org/10.1063/1.439486

[30] S. Nose, A unified formulation of the constant temperature molecular dynamics methods, The Journal of Chemical Physics 81 (1) (1984) 511-519. URL https://doi.org/10.1063/1.447334

[31] W. G. Hoover, Canonical dynamics: Equilibrium phase-space distributions, Phys. Rev. A 31 (1985) 1695-1697. doi:10.1103/PhysRevA.31.1695. 
URL https://link.aps.org/doi/10.1103/PhysRevA.31.1695

[32] P. Langevin, On the theory of Brownian Motion, C. R. Acad. Sci. (Paris) 146, 530-533 (1908).

[33] E. Hernandez, Molecular dynamics: from basic techniques to applications (a molecular dynamics primer), 2008.

[34] R. Car, M. Parrinello, Unified approach for molecular dynamics and density-functional theory, Phys. Rev. Lett. 55 (1985) 2471-2474. URL https://link.aps.org/doi/10.1103/PhysRevLett.55.2471

[35] G. Kresse, J. Hafner, Ab initio molecular dynamics for liquid metals, Phys. Rev. B 47 (1993) 558-561. doi:10.1103/PhysRevB.47.558. URL https://link.aps.org/doi/10.1103/PhysRevB.47.558

[36] R. Iftimie, P. Minary, M. E. Tuckerman, Ab initio molecular dynamics: Concepts, recent developments, and future trends, Proceedings of the National Academy of Sciences 102 (19) (2005) 6654-6659. URL https://www.pnas.org/content/102/19/6654

[37] P. P. Ewald, Die berechnung optischer und elektrostatischer gitterpotentiale (Jan 1921). doi:10.1002/andp.19213690304.

[38] T. Darden, D. York, L. Pedersen, Particle mesh Ewald: An Nlog(N) method for Ewald sums in large systems, The Journal of Chemical Physics 98 (12) (1993) 10089-10092. URL https://doi.org/10.1063/1.464397

[39] R. Hockney, J. Eastwood, Computer simulation using particles, McGraw-Hill, 1981.

[40] D. Wolf, P. Keblinski, S. R. Phillpot, J. Eggebrecht, Exact method for the simulation of coulombic systems by spherically truncated, pairwise $\mathrm{r}-1$ summation, The Journal of Chemical Physics 110 (17) (1999) 8254-8282. URL https://doi.org/10.1063/1.478738

[41] M. Matsui, M. Akaogi, Molecular dynamics simulation of the structural and physical properties of the four polymorphs of $\mathrm{TiO}_{2}$, Molecular Simulation 6 (4-6) (1991) 239-244. URL https://doi.org/10.1080/08927029108022432

[42] B. Guillot, N. Sator, A computer simulation study of natural silicate melts. part I: Low pressure properties, Geochimica et Cosmochimica Acta 71 (5) (2007) 1249-1265. URL https://www.sciencedirect.com/science/article/pii/S0016703706021971

[43] C. Schroeder, Comparing reduced partial charge models with polarizable simulations of ionic liquids, Phys. Chem. Chem. Phys. 14 (2012) 3089-3102. URL http://dx.doi.org/10.1039/C2CP23329K

[44] J. E. Jones, S. Chapman, On the determination of molecular fields. -- I. From the variation of the viscosity of a gas with temperature, Proceedings of the Royal Society of London. Series A, Containing Papers of a Mathematical and Physical Character $106 \quad$ (738) (1924) 441-462. URL https://royalsocietypublishing.org/doi/abs/10.1098/rspa.1924.0081

[45] J. E. Jones, S. Chapman, On the determination of molecular fields. -II. From the equation of state of a gas, Proceedings of the Royal Society of London. Series A, Containing Papers of a Mathematical and $\begin{array}{llllll}\text { Physical Character } & 106 & \text { (738) } & \text { (1924) } & \text { 463-477. }\end{array}$ https://royalsocietypublishing.org/doi/abs/10.1098/rspa.1924.0082

[46] J. E. Lennard-Jones, Cohesion, Proceedings of the Physical Society 43 (5) (1931) 461-482. doi:10.1088/0959-5309/43/5/301. URL https://doi.org/10.1088/0959-5309/43/5/301

[47] A. Rahman, Correlations in the motion of atoms in liquid argon, Phys. Rev. 136 (1964) A405-A411. doi:10.1103/PhysRev.136 A 405. URL https://link.aps.org/doi/10.1103/PhysRev.136.A405

[48] F. London, The general theory of molecular forces, Trans. Faraday Soc. 33 (1937) 8b-26. URL http://dx.doi.org/10.1039/TF937330008B 
[49] M. Born, J. E. Mayer, Zur gittertheorie der ionenkristalle, Zeitschrift fuer Physik 75 (1) (1932) 1-18. doi:10.1007/BF01340511. URL https://doi.org/10.1007/BF01340511

[50] S. T. Murphy, Atomistic simulation of defects and diffusion in oxides, PhD Thesis, Imperial College London, 2009.

[51] J. Chen, S. D. Niemkiewicz, Multiscale modeling of fracture in barium titanate: fracture toughness estimation and modified g-criterion, ZAMM - Journal of Applied Mathematics and Mechanics / Zeitschrift fuer Angewandte Mathematik und Mechanik 95 (2) (2015) 165-172. URL https://onlinelibrary.wiley.com/doi/abs/10.1002/zamm.201300094

[52] M. S. Daw, M. I. Baskes, Embedded-atom method: Derivation and application to impurities, surfaces, and other defects in metals, Phys. Rev. B 29 (1984) 6443-6453. doi:10.1103/PhysRevB.29.6443. URL https://link.aps.org/doi/10.1103/PhysRevB.29.6443

[53] X. D. Dai, Y. Kong, J. H. Li, B. X. Liu, Extended Finnis-Sinclair potential for bcc and fcc metals and alloys, Journal of Physics: Condensed Matter 18 (19) (2006) 4527-4542. doi:10.1088/09538984/18/19/008. URL https://doi.org/10.1088/0953-8984/18/19/008

[54] S. M. Foiles, M. I. Baskes, M. S. Daw, Embedded-atom-method functions for the fcc metals $\mathrm{Cu}, \mathrm{Ag}, \mathrm{Au}, \mathrm{Ni}$, Pd, Pt, and their alloys, Phys. Rev. B 33 (12) (1986) 7983, Erratum: ibid, Phys. Rev. B 37, 10378 (1988).

[55] M.-C. Marinica, L. Ventelon, M. R. Gilbert, L. Proville, S. L. Dudarev, J. Marian, G. Bencteux, F. Willaime, Interatomic potentials for modelling radiation defects and dislocations in tungsten, J. Phys.: Condens. Matter 25 (2013) 395502.

[56] D. Cereceda, A. Stukowski, M. R. Gilbert, S. Queyreau, L. Ventelon, M.-C. Marinica, J. M. Perlado, J. Marian, Assessment of interatomic potentials for atomistic analysis of static and dynamic properties of screw dislocations in W, J. Phys.: Condens. Matter 25 (2013) 085702.

[57] P. Olsson, J. Wallenius, C. Domain, K. Nordlund, L. Malerba, Two-band modeling of $\alpha$-prime phase formation in Fe-Cr., Phys. Rev. B 72 (2005) 214119, see also Erratum, Phys. Rev. B 74, 229906 (2006).

[58] S. L. Dudarev, P. M. Derlet, A magnetic interatomic potential for molecular dynamics simulations, J. Phys.: Condens. Matter 17 (2005) 1-22.

[59] G. Bonny, N. Castin, D. Terentyev, Interatomic potential for studying ageing under irradiation in stainless steels: the FeNiCr model alloy, Modelling Simul. Mater. Sci. Eng. 21 (2013) 085004.

[60] K. Woll, A. Bergamaschi, K. Avchachov, F. Djurabekova, S. Gier, C. Pauly, P. Leibenguth, C. Wagner, K. Nordlund, F. Muecklich, Ru/Al multilayers integrate maximum energy density and ductility for reactive materials, Sci. Rep. 6 (2016) 19535.

[61] J. Tersoff, New empirical approach for the structure and energy of covalent systems, Phys. Rev. B 37 (1988) 6991-7000. doi:10.1103/PhysRevB.37.6991.

URL https://link.aps.org/doi/10.1103/PhysRevB.37.6991

[62] B. G. Dick, A. W. Overhauser, Theory of the dielectric constants of alkali halide crystals, Phys. Rev. 112 (1958) 90\{103. doi:10.1103/PhysRev.112.90. URL https://link.aps.org/doi/10.1103/PhysRev.112.90

[63] P. J. Mitchell, D. Fincham, Shell model simulations by adiabatic dynamics, Journal of Physics: Condensed Matter 5 (8) (1993) 1031-1038. doi:10.1088/0953-8984/5/8/006.

URL https://doi.org/10.1088/0953-8984/5/8/006

[64] P. J. D. Lindan, M. J. Gillan, Shell-model molecular dynamics simulation of superionic conduction in CaF2, Journal of Physics: Condensed Matter 5 (8) (1993) 1019-1030. URL https://doi.org/10.1088/0953$8984 / 5 / 8 / 005$ 
[65] M. Born, R. Oppenheimer, Zur quantentheorie der molekeln, Annalen der Physik 389 (20) (1927) 457 484. URL https://onlinelibrary.wiley.com/doi/abs/10.1002/andp.19273892002

[66] J. F. Ziegler, J. P. Biersack, U. Littmark, The stopping and range of ions in solids, Pergamon, 1985. ISSN 0891-5490.

[67] J. Byggmaestar, F. Granberg, K. Nordlund, E_ects of the short-range repulsive potential on cascade damage in iron, Journal of Nuclear Materials 508 (2018) 530-539. URL https://www.sciencedirect.com/science/article/pii/S0022311518305166

[68] A. van Duin, S. Dasgupta, F. Lorant, W. Goddard, ReaxFF: A reactive force field for hydrocarbons, Journal of Physical Chemistry A 105 (41) (2001) 9396-9409. doi:-10.1021/jp004368u-.

[69] T. Liang, T.-R. Shan, Y.-T. Cheng, B. D. Devine, M. Noordhoek, Y. Li, Z. Lu, S. R. Phillpot, S. B. Sinnott, Classical atomistic simulations of surfaces and heterogeneous interfaces with the charge-optimized many body (comb) potentials, Materials Science and Engineering: R: Reports 74 (9) (2013) 255-279. URL https://www.sciencedirect.com/science/article/pii/S0927796X13000612

[70] S. R. Phillpot, A. C. Antony, L. Shi, M. L. Fullarton, T. Liang, S. B. Sinnott, Y. Zhang, S. B. Biner, Charge optimized many body (comb) potentials for simulation of nuclear fuel and clad, Computational Materials Science 148 (2018) 231-241. doi:https://doi.org/10.1016/j.commatsci.2018.02.041. URL https://www.sciencedirect.com/science/article/pii/S0927025618301307

[71] J. Behler and M. Parrinello, Generalized Neural-Network Representation of High-Dimensional Potential-Energy Surfaces, Phys. Rev. Lett. 98 (2007) 146401.

[72] A. P. Bartok and R. Kondor and G. Csanyi, Gaussian Approximation Potentials: The Accuracy of Quantum Mechanics, without the Electrons, Phys. Rev. Lett. 104 (2010) 136403.

[73] A. P. Bartok and M. C. Payne and R. Kondor and G. Csanyi, On representing chemical environments, Phys. Rev. B 87 (2013) 184115.

[74] A. P. Thompson and L. P. Swiler and C. R. Trott and S. M. Foiles and G. J. Tucker, Spectral neighbor analysis method for automated generation of quantum-accurate interatomic potentials, J. Comput. Phys. 285 (2014) 316-330.

[75] J. Behler, Perspective: Machine learning potentials for atomistic simulations, J. Chem. Phys. 145 (2016) 170901.

[76] W. J. Szlacht and A. P. Bartok and G. Csanyi, Accuracy and transferability of Gaussian approximation potential models for tungsten, Phys. Rev. B 90 (2014) 104108.

[77] A. P. Bartok and J. Kermode and N. Bernstein and G. Csanyi, Machine learning a general-purpose interatomic potential for silicon, Phys. Rev. X 8 (2018) 041048.

[78] A. Hamedani, J. Byggmaaestar, F. Djurabekova, G. Alahyarizadeh, R. Ghaderi, A. Minuchehr, K. Nordlund, Insights into the primary radiation damage in silicon by machine learning interatomic potential, Mater. Res. Lett. 8 (2020) 364-372.

[79] J. Byggmaestar and A. Hamedani and K. Nordlund and F. Djurabekova, Machine-learning interatomic potential for radiation damage and defects in tungsten, Phys. Rev. B 100 (2019) 144105.

[80] J. Byggmaestar, K. Nordlund, F. Djurabekova, Modeling refractory high-entropy alloys with efficient machine-learned interatomic potentials: Defects and segregation, Phys. Rev. B 104 (10) (2021) 104101.

[81] S. T. Murphy, M. J. Rushton, R. W. Grimes, A comparison of empirical potential models for the simulation of dislocations in uranium dioxide, Progress in Nuclear Energy 72 (2014) 27-32, symposium E @ E- 
MRS 2013 SPRING MEETING Scientific basis of the nuclear fuel cycle. URL https://www.sciencedirect.com/science/article/pii/S0149197013001881

[82] K. Govers, S. Lemehov, M. Hou, M. Verwerft, Comparison of interatomic potentials for $\mathrm{UO}_{2}$. Part I: Static calculations, Journal of Nuclear Materials 366 (1) (2007) 161-177. URL https://www.sciencedirect.com/science/article/pii/S0022311507000098

[83] K. Govers, S. Lemehov, M. Hou, M. Verwerft, Comparison of interatomic potentials for $\mathrm{UO}_{2}$. Part II: Molecular dynamics simulations, Journal of Nuclear Materials 376 (1) (2008) 66-77. URL https://www.sciencedirect.com/science/article/pii/S0022311508000986

[84] D. E. Harrison, P. Kelly, B. J. Garrison, N. Winograd, Low energy ion impact phenomena on single crystal surfaces, Surface Science 76 (2) (1978) 311-322. doi:https://doi.org/10.1016/0039-6028(78)901000. URL http://www.sciencedirect.com/science/article/pii/0039602878901000

[85] M. W. Finnis and A. H. Harker, Moldy6 - A molecular dynamics program for simulation of pure metals, Tech. Rep. AERE R-13182, UK AEA Harwell Laboratory (1988).

[86] T. Diaz de la Rubia and M. W. Guinan, Progress in the development of a molecular dynamics code for high-energy cascade studies, J. Nucl. Mater. 174 (1990) 151-157.

[87] B. J. Garrison, A. Delcorte, K. D. Krantzman, Molecule liftoff from surfaces, Accounts of Chemical Research 33 (2) (2000) 69-77.

[88] J. Phillips, R. Braun, W. Wang, J. Gumbart, E. Tajkhorshid, E. Villa, C. Chipot, R. Skeel, L. Kale, K. Schulten, Scalable molecular dynamics with NAMD, J. Computat. Chem 26 (16) (2005) 1781. doi: 10.1002/jcc.20289

[89] G.J.Ackland, K. D Mellow, S. L. Daraszewicz, D. J. Hepburn, M.Uhrin, K.Stratford, The MOLDY short-range molecular dynamics package, Computer Physics Communications 182 (2011) 2587\{2604.

[90] PARCAS Computer code, available open source at https://gitlab.com/acclab/parcas/.

[91] S. Plimpton, Fast parallel algorithms for short-range molecular dynamics, J. Comput. Phys. 117 (1) (1995) 1-19. URL http://www.sciencedirect.com/science/article/pii/S002199918571039X

[92] D. Van der Spoel, E. Lindahl, B. Hess, G. Groenhof, A. Mark, H. Berendsen, GROMACS: Fast, flexible, and free, J. Comput. Chem. 26 (16) (2005) 1701. doi:\{10.1002/jcc.20291\}.

[93] I. T. Todorov, W. Smith, K. Trachenko, M. T. Dove, DL_POLY 3: new dimensions in molecular dynamics simulations via massive parallelism, J. Mater. Chem. 16 (2006) 1911-1918. doi:10.1039/B517931A. URL http://dx.doi.org/10.1039/B517931A.

[94] A. Stukowski, Visualization and analysis of atomistic simulation data with OVITO - the open visualization tool, Modell. and Simul. in Mat. Sci. and Eng. 18 (1) (2010) 015012.

[95] A. Stukowski, V. V. Bulatov, A. Arsenlis, Automated identification and indexing of dislocations in crystal interfaces, Modell. and Simul. in Mat. Sci. and Eng. 20 (8) (2012) 085007.

[96] W. Humphrey, A. Dalke, K. Schulten, Vmd: Visual molecular dynamics, Journal of Molecular Graphics 14 (1) (1996) 33-38. URL https://www.sciencedirect.com/science/article/pii/0263785596000185

[97] Schrödinger, LLC, The AxPyMOL molecular graphics plugin for Microsoft PowerPoint, version 1.8 (November 2015). 



\title{
9. Molecular dynamics for radiation effects
}

\author{
Kai Nordlund*, Flyura Djurabekova, Samuel T. Murphy, Dorothy M. Duffy
}

In this article, we review the molecular dynamics (MD) methods for use in simulations of radiation effects across a broad energy range. We examine the physical limitations of the classical MD for simulation of radiation effects, and present the modifications required to improve MD's description of high-kinetic energy events. In the high-energy regime, which is known as swift heavy ions, we overview the existing models of extending the classical molecular dynamic methods to include effects of electronic excitations. At the end, we also discuss the complications associated with modelling complex radiation effects and challenges, which remain to be resolved.

* Contact: kai.nordlund@helsinki.fi 


\subsection{Introduction}

Computer simulation of radiation effects has an interesting history, as the very first computer simulation ever was about motion of neutrons in fissile uranium, in a project that for well-known reasons was well funded [1]. Since then, the development of several key computational methods was associated with developments in irradiation physics (for a thorough review, see Ref. [2]).

The purpose of this article is to overview the simulation methodology of the key method for modern modelling of radiation effects: molecular dynamics (MD). While this approach is, of course, by far not the only relevant method [3-6], it has been the most used one for the last couple of decades [2]. Moreover, several recent articles have reviewed the overall framework of multiscale modelling of radiation effects [3,5,7-9]. Hence in this article, we choose to focus at some more depth on the molecular dynamics method.

The basic MD algorithms are described in an early chapter in this book, therefore, in this chapter, we describe the special solutions that are crucial to describe radiation effects properly in molecular dynamics. We describe methods needed both for handling the nuclear collision regime (collision cascades, section 9.2) as well as the electronic collision regime when that produces materials modification (swift heavy ion track regime, section 9.4).

\subsection{Molecular dynamics for collision cascades}

\subsubsection{Special considerations for radiation effects}

The approaches discussed in the previous chapter all related to MD simulation of equilibrium or non-equilibrium thermodynamic systems. Radiation effects are, of course, neither. In fact, when atoms or ions move with $\mathrm{keV}$ or higher energies, the initial stages of the collision cascade are not a thermodynamic system at all. This is because the definition of thermodynamic systems states that the processes occur slow compared to the relaxation time $\tau$ of the system [11].

A simple calculation shows that atoms with kinetic energies in the keV range do not fulfill the criterion for a thermodynamic system. For a hard solid, $\tau$ can be estimated to be of the order of the inverse of the lattice Debye vibration frequency $v$. Since $v \sim 10^{13} \mathrm{~Hz}$ [12], $\tau \sim 100 \mathrm{fs}$. By comparison, for example a $1 \mathrm{keV} \mathrm{Cu}$ atom has the velocity

$$
v=\sqrt{ }\left(2 E / m_{-} C u\right)=55 \mathrm{~km} / \mathrm{s}=0.55 \AA / f s
$$

Hence a $1 \mathrm{keV} \mathrm{Cu}$ atom moves about $55 \AA$ in $100 \mathrm{fs}$, which is multiple times the interatomic distance of about $2.6 \AA$. Since thermodynamic equilibration involves collective lattice vibrations, it is clear there is not sufficient time for equilibration to occur when an atom traverses several interatomic distances during one lattice vibration. 


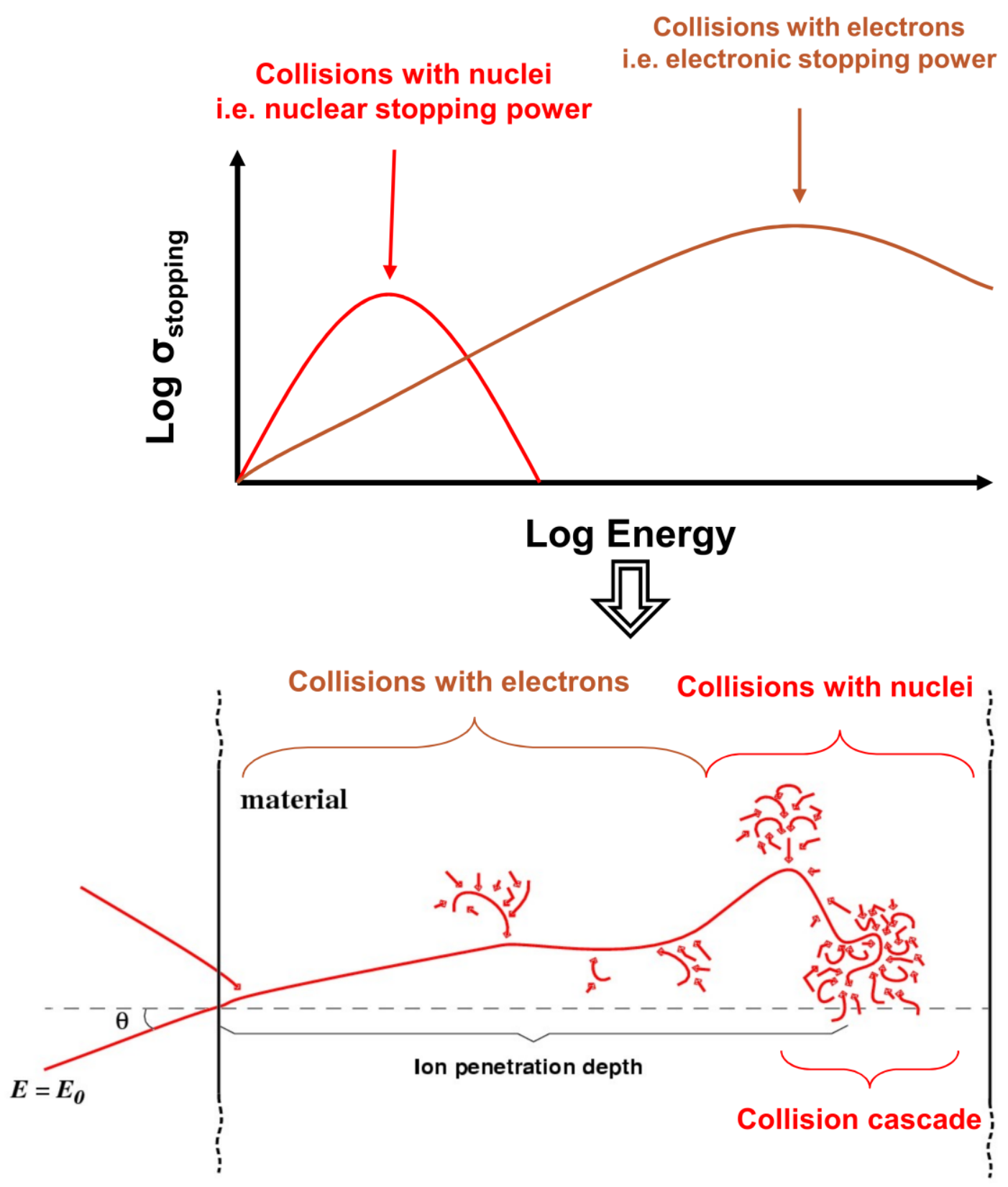

Figure 9.1. Schematic of the energy dependence of stopping power and how this relates to ion movement and collisions in a material. The regime where nuclear collisions dominate is that where collision cascades occur. Swift heavy ion track production may occur at energies around the maximum in electronic stopping power.

Indeed, decades of work with binary collision approximation (BCA) simulations [13-18] have shown that the high-energy part of radiation can be well treated based on a ballistic collision model. This is because at high energies (10's of keV's or more), the ions travel mostly in a straight line, and one can separate the collisions from each other, see Figure 9.1. However, at the end of this so-called ballistic phase, after about $200 \mathrm{fs}$, the maximum energy in the system has decreased to be comparable to the bond energies, of the order of $1-5 \mathrm{eV}$. In this regime, the collisions become dense and local. This is illustrated in a binary collision picture in Figure 9.2 and in a molecular dynamics picture in Figure 9.3). 


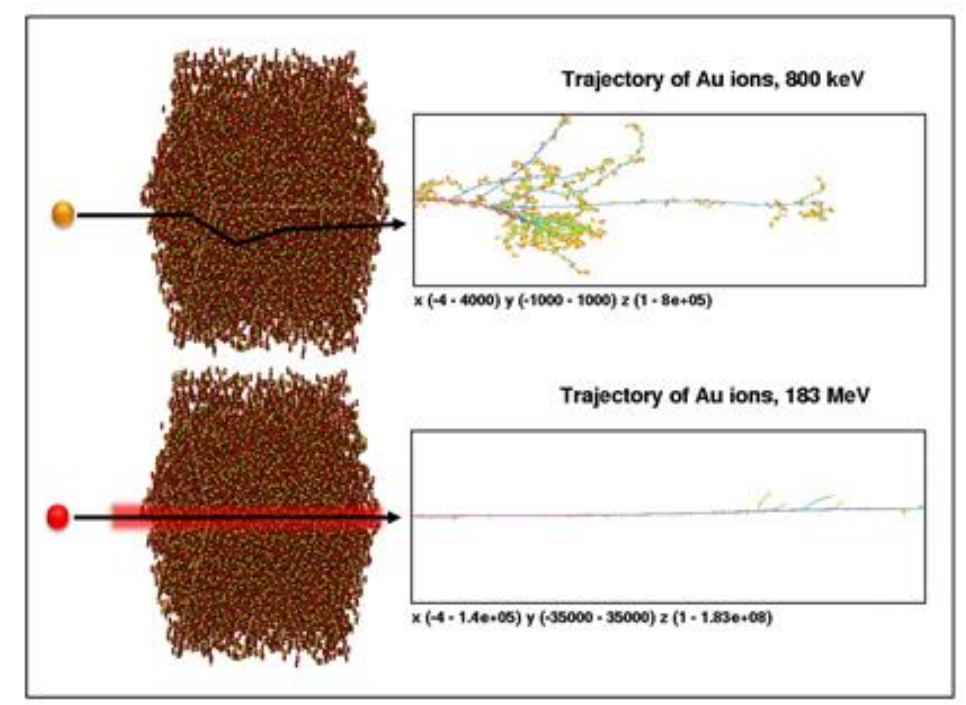

Figure 9.2. Comparison of the ion movement and damage production regions in the nuclear (top) and electronic (bottom) collision regime. The atomic images (left) are from MD simulations of silica, and the trajectory and recoil image from a SRIM simulation [10]. Note also the very different scale in the images, in the top part the plotted width is 400 $\mathrm{nm}$, in the lower $14000 \mathrm{~nm}$.

At this stage, the binary collision approximation breaks down [19] and the atoms start to move initially by complex many-body interactions. After that, in about $1 \mathrm{ps}$, the atoms act as a very hot pressurized liquid $[20,21]$. At this stage, the system also becomes one, where thermodynamics does apply. The many-body interactions may be handled partly by the BCA, however, the transition to the thermodynamic behaviour of the system cannot be addressed using binary collisions only. However, since MD by definition handles all atomic interactions at once, it can at least in principle handle this. However, the regular MD algorithms cannot handle the ballistic phase. 

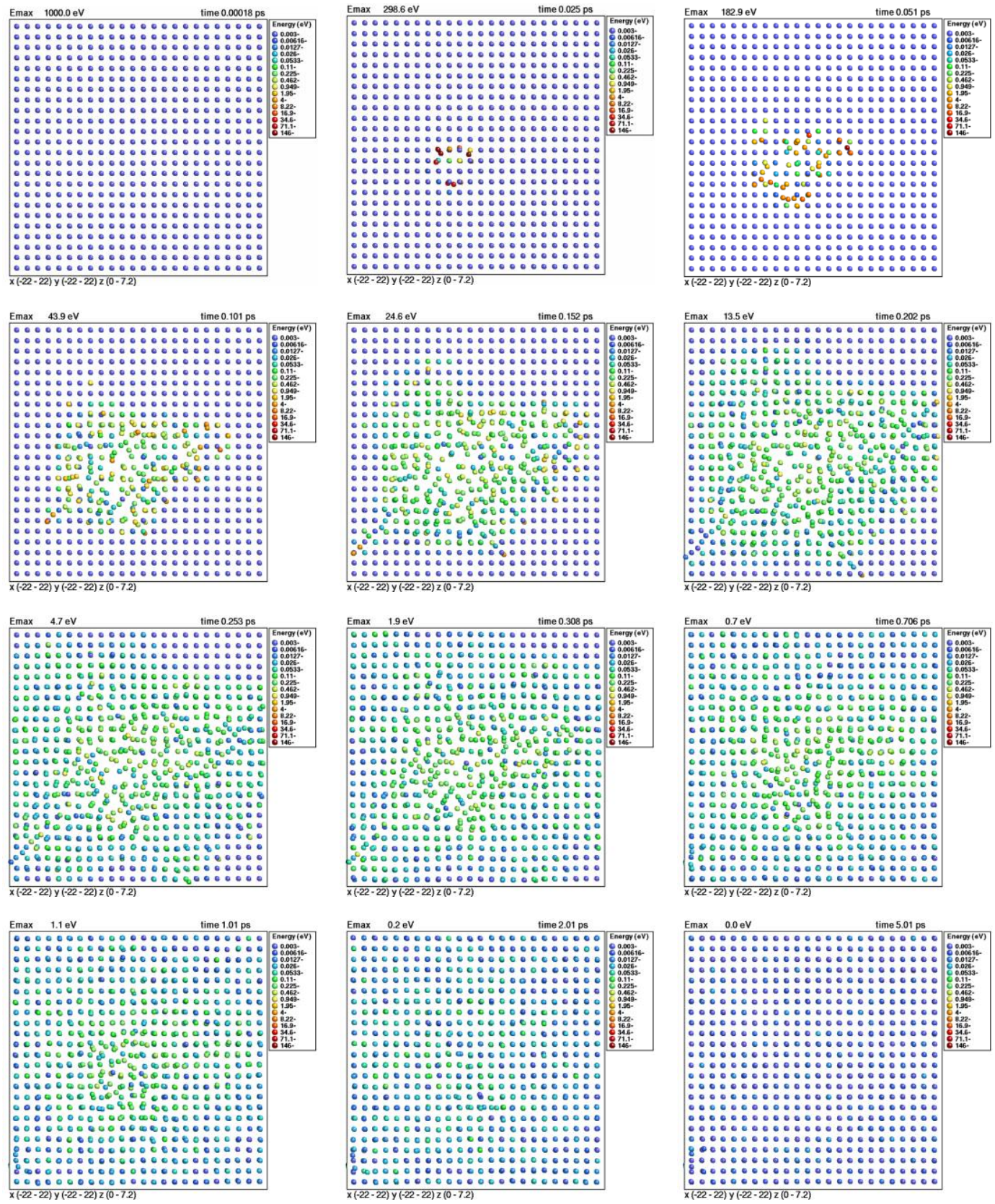

Figure 9.3. Illustration of the thermalization of a low-energy cascade, one induced by a $1 \mathrm{keV} \mathrm{Cu}$ recoil in $\mathrm{Cu}$. The figure shows snapshots of atom positions in a 4-atom layer thick segment of $\mathrm{Cu}$, with the cell size given in the bottom in units of Å. The colors indicate the kinetic energy of each atom. For each snapshot, the maximum kinetic energy of any atom in the system is shown on the top left corner. As the numbers show, at 0.2 ps the maximum kinetic energy (13.5 eV) is still above the cohesive energy of about $4 \mathrm{eV}$. However, already at 0.3 ps the maximum kinetic energy is clearly below the cohesive energy.

In the following subsection, we describe the extensions to the MD method to enable description of both the high-energy effect and the transition to the thermodynamic regime in a single, unified simulation formalism with no discontinuities. The modifications needed in the core algorithm are illustrated in Figure 9.4. 


\section{Simplified schematic of the molecular dynamics algorithm for radiation effects}

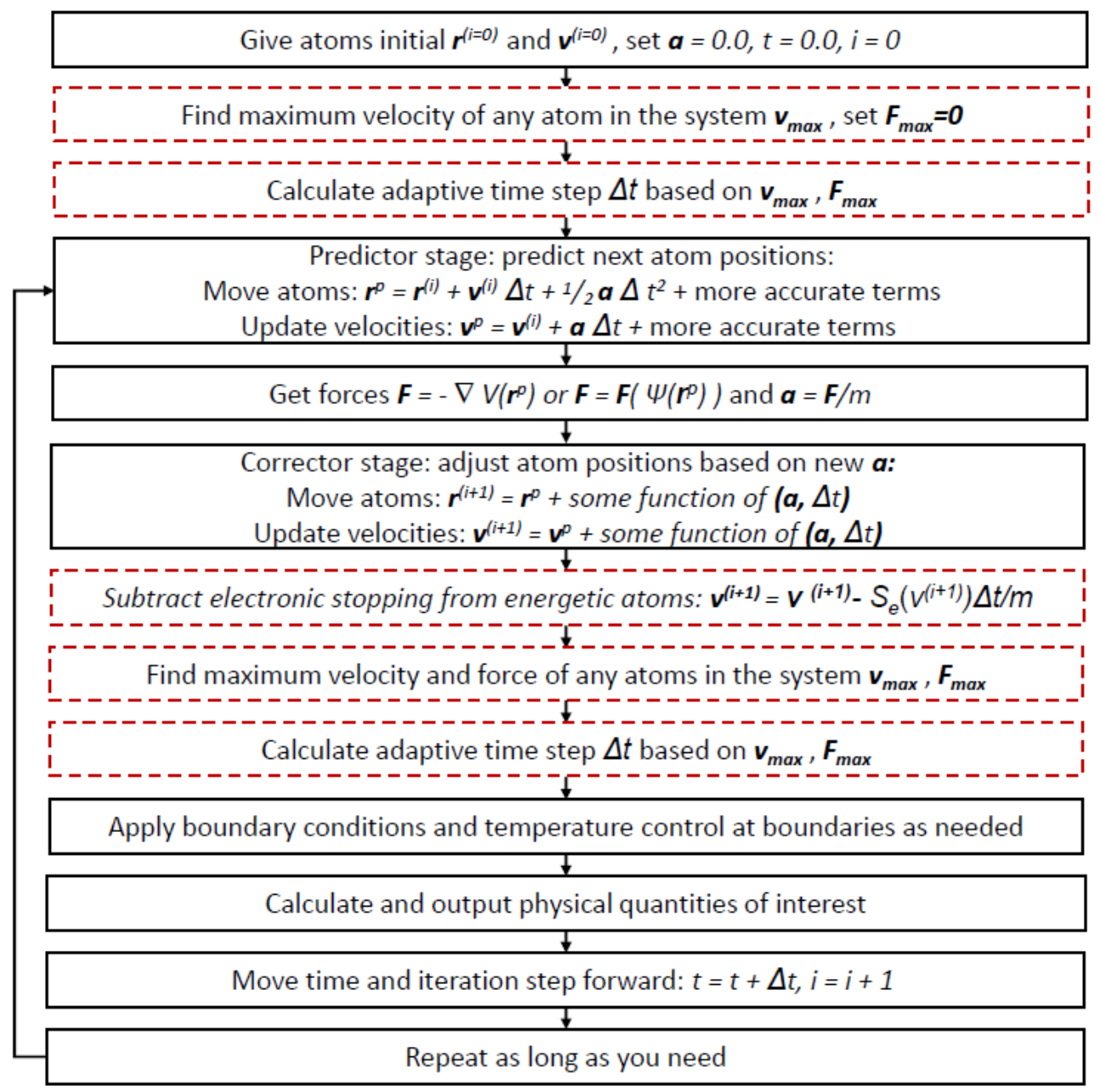

Figure 9.4. The molecular dynamics algorithm for studying radiation effects. The boxes with dashed lines indicate the additional steps needed for radiation effects modelling. It is also very important to have a realistic repulsive potential (see text), however, this is embedded in the interatomic potential $V(r)$ and hence does not affect the main algorithm. $S_{e}$ stands for the electronic stopping power and the index $i$ for the iteration step. In a 2T-MD approach, the solution of the electronic temperature is done separately from this algorithm, and then coupled to the atomic motion e.g. in connection to the temperature control stage.

\subsubsection{Special solutions in MD for nuclear collision effects}

\subsubsection{Necessity of not using thermostats or barostats}

From the discussion above, it is clear that for simulating radiation effects in the collisional phase one should not use any thermostat or barostat. The reason is simply that since the initial ballistic phase is not a thermodynamic system, using any control algorithm that strives to conserve a thermodynamic quantity is by definition not appropriate. Fortunately, the core MD solution of the equations of motion describe atom motion by Newton's laws inherently. Thus, the classical MD algorithm can be used to describe radiation effects as well. In thermodynamic terminology, this corresponds to simulating the $N V E$ ensemble.

However, after the collision phase is over and the extra temperature introduced by the radiation 
has cooled down completely, it may be desirable to use a thermostat or a barostat (to enable modelling e.g. radiation-induced plastic flow). This can be naturally achieved by turning on the thermostat or barostat after the initial recoil event was completed.

\subsubsection{Adaptative time step}

As noted in the chapter describing equilibrium molecular dynamics, the time step used in equilibrium MD is typically a few fs. Even for a relatively short equilibrium time step of 2 fs, this poses a big problem for radiation effects. E.g., in the example of $1 \mathrm{keV} \mathrm{Cu}$ atom, which was given above, this would mean that the atom moves $1.1 \AA$ in a single time step, about half of the interatomic distance. It is clear that this would lead to a huge difference in potential energy and force over a single MD integration step, leading to a major inaccuracy in the solution of the equations of motion. This qualitative deduction can easily be checked by energy conservation tests in an actual MD simulation, and is illustrated in Figure 9.5.

For numerically stable solution of the MD algorithms of motion, it is thus important that the potential and force do not change too much over a single time step. In practical terms, this can be translated to a requirement that the displacement of an atom over a single time step is not too large. Tests like the one shown in Figure 9.5 show that when the maximum displacement of an atom is about $1 / 20$ of the interatomic distance, one can achieve stable solution of the equations of motion. Hence, one could always make MD conserve energy by shortening the time step to match this criterion. However, since in an irradiation event, the atoms initially move very fast, but after the about 200 fs have slowed down to thermal energies, there is no reason to continue using the very short time step over the long simulation times. The very short time step over a long iterative run would make the simulations extremely slow.

An obvious solution to this issue is to use an adaptive time step that is inversely proportional to the maximum velocity of any atom in the system $v_{\text {max }}$ :

$$
\Delta t=\frac{k_{t}}{v_{\max }}
$$

Here $k_{t}$ is a constant that gives the maximum displacement of any atom in the system. This approach alone is, however, sufficient, because in a strong collision between two atoms, the atoms actually slow down when they go up in potential energy. Equation (9.2) alone would then lead to a shorter rather than longer time step in a strong collision, which is contrary to the purpose of stable solution of the equations of motion. This issue can be solved by making the time step also proportional to the maximum potential energy or force in the system, and then picking the shorter of the two alternatives. Moreover, it is also desirable to prevent too large increases of the time step over a single iteration. All of these requirements can be achieved by using a time step

$$
\Delta t=\min \left(\frac{k_{t}}{v_{\max }}, \frac{k_{t}}{F_{\max } v_{\max }}, 1.1 \Delta t_{\text {prev }}, \Delta t_{\text {equilibrium }}\right)
$$

Here the constants $k_{t}$ and $E_{t}$ are then set using energy conservation tests for a given system and energetic atom species. Typically $k_{t} \sim 0.1 \AA ̊$ and $E_{t} \sim 30 \mathrm{eV}$ give stable integration even in very high-energy collisions. 


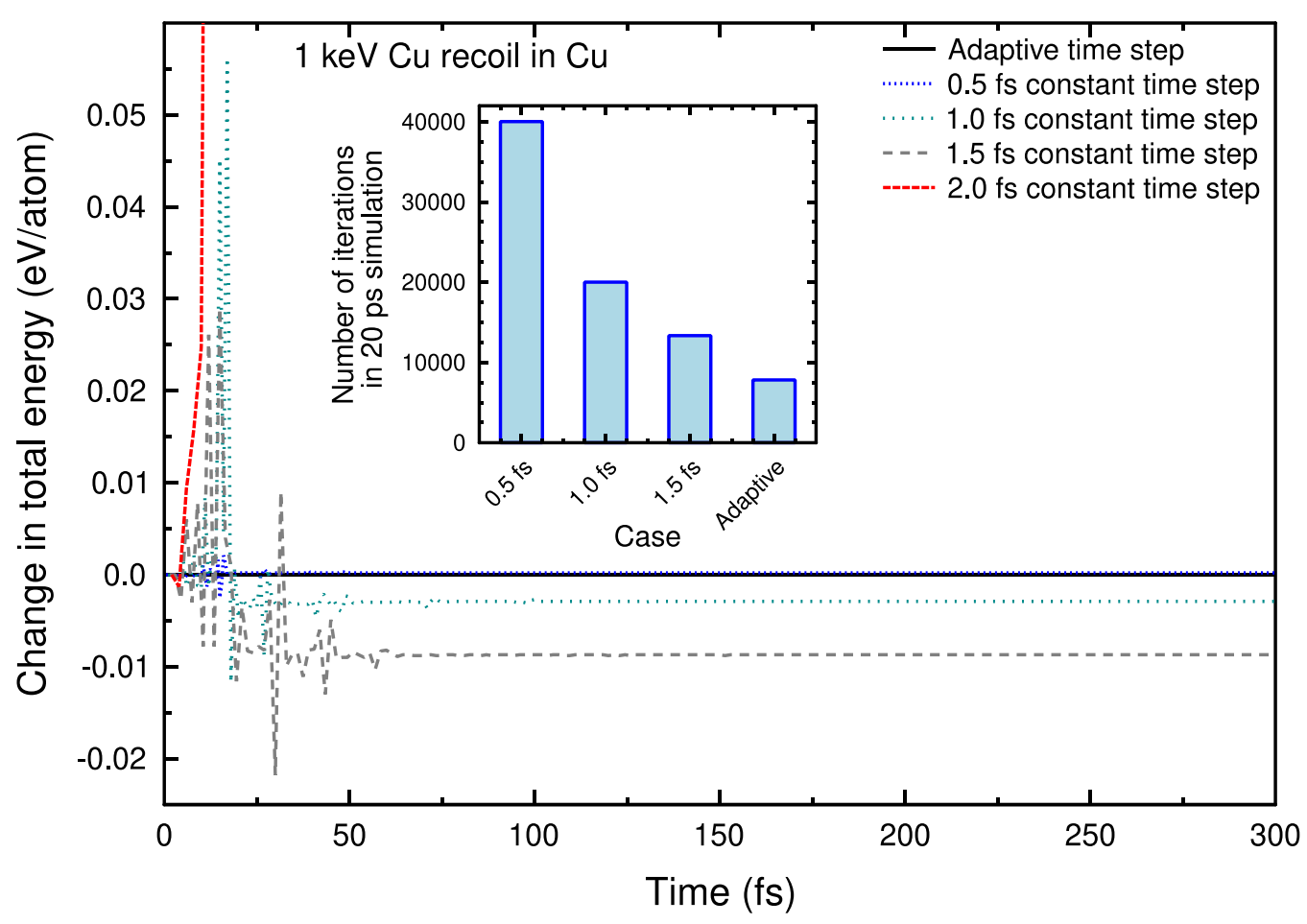

Figure 9.5. Illustration of how the time step selection affects energy conservation in a molecular dynamics simulation of a $1 \mathrm{keV} \mathrm{Cu}$ self-recoil in a 16,384 atom Cu cell with all periodic boundary conditions. The case of "Adaptive time step" is implemented with the equation described in the main text, with the constants $k_{t}=0.1 \AA$ and $E_{t}=30 \mathrm{eV}$ and the equilibrium time step $\Delta t_{\text {equilibrium }}=4.06 \mathrm{fs}$. In the other cases, the simulation was run with a constant time step with the value of the legend. The integrator used was the 5 th order Gear predictor-corrector [22]. The inset shows how many MD iterations (time steps) were needed to simulate the cascade until $20 \mathrm{ps}$. For the constant time step of $2 \mathrm{fs}$, the system was completely unstable and hence it was not even possible to simulate until 20 ps. Note that even if a change in total energy of $-0.01 \mathrm{eV} /$ atom may sound small, in terms of temperature this corresponds to an about $100 \mathrm{~K}$ artificial loss of heat from the system.

\subsubsection{High-energy repulsive potential}

In most conventional equilibrium potentials, the functional form of the short-distance parts, when the interatomic distance $r \rightarrow 0$, is completely unsuitable for simulating radiation effects. For instance, in the Lennard-Jones potential the short-distance part $\propto 1 / r^{12}$ and in the Morse potential $\propto \exp \left(-A / r-r_{0}\right)$. However, consider that all atoms have a positively charged nucleus, the physically reasonable form of the interatomic potential $V$ at small separations must be

$$
V=\frac{1}{4 \pi \varepsilon_{0}} \frac{Z_{1} Z_{2}}{r} \phi(r)
$$

where the term with $Z_{1}$ and $Z_{2}$ is of course the Coulomb potential between the nuclei. For two bare nuclei without any electrons, this term alone would be the exactly correct interatomic potential at all distances except for the nuclei overlap. The $\phi(r)$ is the screening function that describes how the electrons of the atoms in any real solid screen the Coulomb potential of the bare nuclei.

The form of $\phi(r)$ has been the subject of ion beam theory and experiments for decades, for a historical review see Ref. [23]. By far the most commonly used repulsive potential is the universal Ziegler-Biersack-Littmark (ZBL) potential. This potential is essentially an averaging fit to a set of 
Thomas-Fermi electronic structure calculations [24]. It has the major advantage that it is only a function of $Z_{1}$ and $Z_{2}$ and hence can be easily implemented for any atom pair. However, due to its nature as an averaging fit, it can be easily off $5-10 \%$ for a given pair. An alternative, newer universal screening function fit has been recently developed by Zinoviev [25].

Atom pair-specific quantum mechanical calculations of a dimer can be used to obtain more accurate potentials for small interatomic separations [26-28]. Recent comparisons with experiments indicate that these are quite accurate, at least when obtained from all-electron DFT calculations [29].

\subsubsection{Electronic stopping power and electron-phonon coupling}

The electronic stopping power $S_{e}$ (cf. Figure 9.1), i.e., loss of energy of a high-energy ion to electronic excitations, can for kinetic energies $>>$ the cohesive energy of the material be readily implemented in molecular dynamics as a frictional force affecting the motion of all high-energy atoms. Since the electronic stopping power $S_{e}(v)[23,30,31]$ has units of force, the energy loss can be implemented simply by subtracting $\Delta t S_{e}(v) / v$ from the velocity of an energetic atom over every time step of length $\Delta t$. This approach is consistent with the one used in BCA simulations [14], and has been implemented in molecular dynamics by several groups since the 1990's [3238]. It can be validated against experiments by direct comparison of simulated and experimental ion range profiles [35, 38-41].

This simple approach to electronic stopping power thus works well for high energies. However, when the atom energies approach thermal, it is no longer so clear how the electronic stopping power should be treated. The traditional electronic stopping theory predicts that at low energies $S(v) \propto v$ down to $v=0$. However, if this kind of electronic stopping would be implemented directly in MD, it would also "eat away" all thermal kinetic energy, i.e., quench down any system to $0 \mathrm{~K}$, which obviously is not consistent with the everyday experience of us living in an environment with a reasonable stable temperature around 270 - $300 \mathrm{~K}$ (mostly even in Finland).

Hence at the lowest kinetic energies, similar to thermal, the electronic stopping must behave differently. What happens is that when the atoms are in the many-body collisional regime where thermodynamics start to apply, one can assign a local temperature to the hot regions. When this occurs, the atomic system starts to couple with the electronic one via electron-phonon coupling (EPC) [42]. In this context, the name is somewhat misleading since for radiation collision cascades, it is the atomic system that is initially hot, and due to the high kinetic energies, one cannot talk about equilibrium phonons. Hence a more descriptive term would actually be atomic-electronic temperature coupling.

Regardless of the term used, in this regime, the system can be considered as an atomic lattice subsystem with temperature $T_{l}(\vec{r})$ and an electronic one with temperature $T_{e}(\vec{r})$, and these strive towards thermodynamic equilibrium when $T_{l}=T_{e}$. When this condition is met everywhere in the system, the radiation event is over and the concept of electronic stopping power no longer has meaning.

The existence of electron-phonon coupling for radiation effects has been recognized for a long time on a qualitative level of understanding [42-47], only recently has it started to become clearer how the electronic stopping transits into an electron-phonon coupling regime and how the low- 
energy coupling works [48-50]. In section 9.3 a systematic approach for including electronphonon coupling and linking it directly to the local lattice temperature $T_{l}$ is described in detail.

\subsubsection{Boundary conditions and system size}

As emphasized in the beginning, the collisional phase of the cascade can only be simulated within the NVE ensemble. However, modelling of an entire simulation cell only in the NVE ensemble is usually not desirable for at least two reasons. First, since the recoiling atom carries momentum, and momentum is conserved in the MD algorithm, the entire cell starts moving from a recoil if no control is done.

Second, the typical physical situation modelled is irradiation of a small piece of a macroscopic system. In such a system, there is of course in reality a very large thermal bath available, and the extra kinetic energy from a recoil would be dissipated by heat waves and heat conduction into the macroscopic system. Moreover, especially in dense materials the recoil event gives rise to a pressure and heat wave, which can be transmitted over the periodic boundaries back to the collisional zone. To prevent the temperature increase and damp the pressure wave, the conventional solution is to use the NVE ensemble in the center, but then either velocity scaling or a thermostat at the boundary zones of the simulation cell, far from the ballistic cascade $[51,52]$.

A barostat should never be used during the same time as energetic collisions are active, since the collisions give rise to high virials which translate to high pressure spikes that a barostat would try to scale out, even though the transient strong virials are physically correct. Moreover, if a system has a free surface, using a conventional MD barostat can lead to weird artefacts, as it would try to scale away the surface tension, even though a real surface of course should have a surface tension. In systems with no free surfaces, one may be able to use a barostat after the system has cooled down to the ambient temperature.

The minimal system size needed can be estimated from a simple heating consideration, using the connection between kinetic energy and temperature

$$
E=\frac{3}{2} k_{B} T
$$

In a finite simulation cell the energy introduced by a recoil would remain, and would lead to an artificial heating of the system. For instance, if a $E_{r}=5 \mathrm{keV}$ recoil is simulated in a 100,000-atom system, then the temperature would rise by about $T=E_{r} / 2 /\left(2 / 3 N k_{B}\right)=193 \mathrm{~K}$. Here $E$ is divided by two because of the equipartition theorem, and the equation is not exact because a small fraction of the energy is stored as potential energy of the defects introduced [53].

This simple heating calculation can also be used to estimate the minimum number of atoms needed in a cell. Considering that hard solids have melting points around $1000-3000 \mathrm{~K}$ [54], it is rather clear the energy introduced by the recoil must not heated the supercell to close to the melting point. If one sets e.g., $200 \mathrm{~K}$ as the maximal allowed heating, then using the same equation as above, one can calculate a simple rule of thumb for number of atoms $N$ a simulation cell needs to have per $\mathrm{eV}$ of introduced kinetic energy:

$$
\frac{\Delta E}{2}=\frac{3}{2} N k_{B} \Delta T \Rightarrow \frac{N}{E_{r}}=\frac{1 / 2}{\frac{3}{2} k_{B} \Delta T}=19.3 \frac{\text { atoms }}{\mathrm{eV}}
$$


In short, this calculation give rise to the simple rule of thumb that one should have at least 20 atoms per $\mathrm{eV}$ of recoil energy in the simulation cell. This argument considers only the lattice heating. In a high-energy cascade with a fractal distributed structure, one may need a much larger simulation cell size to prevent any recoil atoms from entering the simulation cell border, where temperature control is performed.

Finally, we note that even though the boundary control issue may seem complicated, this is one case where simple size scaling tests can always be used to determine what is a "large enough" size. By simply increasing the system size from small to larger and calculate for each size the physical quantities of interest (remembering the need for averaging over several independent events to get statistically significant results), one can always figure out which size is sufficiently large to give converged results.

\subsection{The two-temperature model}

One notable limitation of classical molecular dynamics for the study of radiation effects is the neglect of explicit electronic effects. As noted in section 9.2.2.4, electronic effects acting on the ion can be implemented as a high-energy electronic stopping power. This can be readily implemented in molecular dynamics as a frictional force. However, this approach does not indicate what the possible materials modification due to electronic stopping is, nor whether a hot atomic system can feed energy back to the electronic system. To address these issues, a two-temperature model description is needed.

\subsubsection{The analytical two temperature model (TTM)}

The approach pioneered by Toulemonde and coworkers for modelling swift heavy ions (SHIs) [55], the inelastic spike model, is based on the two-temperature model (TTM) [56], [57] . The TTM is used extensively for non-equilibrium systems in which the electrons are out of equilibrium with the lattice, such as laser irradiation of solids [58], [59]. An implicit assumption of the model is that the electrons and atoms are in quasi-equilibrium, that is the electrons have a Fermi Dirac energy distribution with an electronic temperature $T_{e}$ and the phonons have a Bose Einstein distribution, with a lattice temperature $T_{l}$. However, the temperatures associated with these two distributions are not necessarily equal, with a temperature difference signalling that the system is out of equilibrium. The temperatures of the electrons and the lattice evolve via two coupled thermal diffusion equations (9.7) and (9.8).

$$
\begin{gathered}
C_{e}\left(T_{e}\right) \frac{\partial T_{e}}{\partial t}=\nabla \kappa_{e} \nabla T_{e}-G\left(T_{e}\right)\left(T_{e}-T_{l}\right)+A(r, t), \\
C_{l} \frac{\partial T_{l}}{\partial t}=\nabla \kappa_{l} \nabla T_{l}+G\left(T_{e}\right)\left(T_{e}-T_{l}\right),
\end{gathered}
$$

Here $A(r, t)$ is a source term representing energy deposition to the electrons as a function of position, $r$, and time, $t$, as discussed in section 9.4.1. Energy exchange between the lattice and electrons occurs via electron-phonon coupling, which is quantified by the electron-phonon coupling constant $G . C_{e}$ and $C_{l}$ are the electronic and lattice heat capacities, respectively. $\kappa_{e}$ and $\kappa_{l}$ are the electronic and lattice thermal conductivities. Each of these parameters may vary as a 
function of the electronic temperature and/or the lattice temperature.

The TTM is used to calculate the evolution of the lattice temperature following a radiation event which deposits energy into the electronic system. Although the model is analytical, in practise solution of the equations is typically done numerically. Typical scenarios are swift heavy ion irradiation, where equations (9.7) and (9.8) are solved with cylindrical coordinates, and laser irradiation of planar surfaces, where 1-dimensional equations are generally appropriate. The TTM is widely used to estimate the electron-phonon coupling constant by fitting to experimental measurements of the time evolution of $T_{e}$ and $T_{l}$ following laser irradiation to the equations (9.7) and (9.8). There is one significant limitation, however. The model only considers the spatial and temporal evolution of $T_{e}$ and $T_{l}$, therefore, there is no information about the details of the atomic dynamics. Effects such as superheating and recrystallisation are, consequently, neglected and the defect or amorphous structure created by a radiation event is not accessible from TTM simulations. This limitation can be overcome by coupling 2TM with MD, as described in the next subsection.

\subsubsection{Two-temperature molecular dynamics}

Two temperature molecular dynamics (2T-MD) is the name given to the modelling method which couples the two-temperature model with molecular dynamics, with the aim of introducing the effects of excited electrons into classical molecular dynamics. As outlined in section 9.3, the twotemperature model (TTM) describes a system using two temperatures, which evolves via two coupled thermal diffusion equations (9.7) and (9.8).

In the 2T-MD model, the lattice thermal diffusion equation (9.8) is replaced by the MD equation of motion for a particle of mass $m$, subjected to a force $\mathbf{F}$ [60], [61].

$$
m \frac{\partial \mathbf{v}}{\partial t}=\mathbf{F}-\gamma \mathbf{v}+\tilde{\mathbf{F}}
$$

The friction term $-\gamma \mathbf{v}$ is introduced to represent energy loss by interaction with electrons, by electronic stopping for example, and the stochastic force $\tilde{\mathbf{F}}$ serves to deposit energy to the lattice from the electrons via electron-phonon coupling. The stochastic force must have the following properties to satisfy the fluctuation dissipation theorem:

$$
\begin{gathered}
\langle\tilde{\mathbf{F}}(t)\rangle=0 \\
\left\langle\tilde{\mathbf{F}}^{\alpha}(t) \tilde{\mathbf{F}}^{\beta}\left(t^{\prime}\right)\right\rangle=2 k_{B}\left(T_{e}\right) \gamma \delta^{\alpha \beta} \delta\left(t-t^{\prime}\right) .
\end{gathered}
$$

Here, $k_{B}$ is Boltzmann's constant and $\alpha$ and $\beta$ are Cartesian components of the force $\mathbf{F}$. The temperature for the Langevin thermostat in equation (9.9) is the local electronic temperature, $T_{e}$, which evolves according to the electronic thermal diffusion equation (9.7). Coupling between the atoms and the electrons is achieved, in practice, by dividing the MD simulation cell into voxels with tens or hundreds of atoms in each voxel. This defines the grid for the numerical solution of the electronic thermal diffusion (9.7). Thus, an electronic temperature, $T_{e}$, is associated with each voxel of the MD simulation cell. The thermal diffusion equation is solved numerically by iterating 
concurrently with the MD simulation, however, to ensure numerical stability, the time step generally needs to be smaller than the MD time step, therefore several electronic time steps may be taken during each MD time step. Energy is transferred between the lattice and electrons at every MD time step and energy conservation is ensured by relating the energy lost by the electrons to energy gained by the lattice and vice versa [60]. Thus, for energy conservation the friction coefficient $\gamma$ is related to the electron-phonon coupling constant $G$ by:

$$
\gamma=\left(\frac{V}{N}\right)\left(\frac{m}{3 k_{B}}\right) G
$$

Here $V$ is the volume of the voxel and $N$ is the number of atoms in the voxel. Note that the lattice is effectively thermostatted to the local, time dependent electronic temperature $T_{e}$ and the strength of the thermostat is related to the electron-phonon coupling constant. Thus a high value of $G$ will result in a high rate of energy transfer between the electrons and the lattice which, in practice, means that energy deposited in the electrons will results in rapid heating of the lattice.

\subsubsection{Parameters for 2T-MD}

It is clear from equations (9.7) and (9.9) that the 2T-MD model requires the input of a number of material parameters for successful implementation. Not all parameters are accurately known and the challenges are enhanced by the fact that several are dependent on the electronic temperature. We discuss each in turn.

\subsubsection{Electronic heat capacity}

One of the most important parameters of the 2T-MD model is the electronic heat capacity as it determines how much energy is stored in the electrons and, therefore, the temperature rise for a given heat input. The electronic heat capacity $\left(C_{e}\right)$ is accurately known for most metals and its temperature dependence, at least for low $T_{e}$, varies linearly with $T_{e}$, as determined by the Sommerfeld model. In the electronic temperature regimes relevant to 2T-MD, however, the Sommerfeld model fails and the $T_{e}$ dependence depends on the details of the density of states near the Fermi level. The $T_{e}$ dependence of heat capacities can be calculated by density functional theory (DFT) and it has been tabulated for a wide range of metals by the Zhiglei group [62] and freely available on their website http://www.faculty.virginia.edu/CompMat/electron-phononcoupling/. The $T_{e}$ variation of $C_{e}$ for band gap materials (semiconductors and insulators) can be calculated by the same method. For such materials $C_{e}$ is effectively zero at low $T_{e}$, as the number of electrons in the conduction band is infinitesimally small, and it becomes finite only at electronic temperatures close to the band gap, when the conduction band becomes populated with free electrons. An accurate evaluation of the $T_{e}$ variation of $C_{e}$ is necessary to capture band gap effects in 2T-MD, as has been demonstrated for swift heavy ion simulations of Si [63] (for additional discussion, see section 9.4).

\subsubsection{Electron-phonon coupling strength}

The strength of the electron phonon coupling determines the rate of energy exchange between the electrons and the atoms and, therefore, the rate of lattice heating. This property is not as well characterized as the heat capacity but it has been measured for a limited number of metals by 
fitting the measured time evolutions of the electronic and lattice temperatures, following laser irradiation, to two temperature models. The electron phonon coupling constant $G$ of metals can also be calculated using DFT and good agreement has been found between the calculated and measured values for W [64]. As with $C_{e}, G$ also varies with $T_{e}$, as it depends on the density of states close to the Fermi level.

$$
G\left(T_{e}\right)=\frac{G_{0}}{g^{2}\left(\epsilon_{f}\right)} \int_{-\infty}^{+\infty} g^{2}(\epsilon)\left(-\frac{\partial f}{\partial \epsilon}\right) d \epsilon
$$

Here $g(\epsilon)$ is the electronic density of states and $f$ is the Fermi-Dirac distribution. $G\left(T_{e}\right)$ has been calculated for a number of metals by Lin et al. and the values are tabulated on the website: http://www.faculty.virginia.edu/CompMat/electron-phonon-coupling/.

\subsubsection{Electronic thermal conductivity}

The thermal conductivity of metals is generally dominated by electronic heat transport and accurate measurements are available for most metals. Simple models exist for the $T_{e}$ variation of $\kappa_{e}$ but the applicability of such models may be limited. One such model is the Drude model:

$$
\kappa_{e}=1 / 3 v_{f}^{2} C_{e} \tau_{e}
$$

where $v_{f}$ is the Fermi velocity and $\tau_{e}$ is the electron scattering time. This has two contributions, one from the electron-electron scattering $\left(\tau_{e e}\right)$ and one from electron-phonon scattering $\left(\tau_{e p}\right)$. The total scattering time is given by:

$$
\frac{1}{\tau_{e}}=\frac{1}{\tau_{e e}}+\frac{1}{\tau_{e p}}
$$

A more detailed discussion of the various approximations and how the results are affected by these approximations are presented in [65] and also chapter 5.2 of [66]. For band gap materials the thermal conductivity is related to the electronic diffusivity, $D_{e}$, by $\kappa_{e}=D_{e} C_{e}$.

\subsubsection{Examples of use of 2T-MD}

The 2T-MD model is applicable to radiation scenarios which drive electrons out of equilibrium with the lattice, however, the practical implementation depends on the details of the energy deposition. Some types of radiation interaction, lasers and very energetic ions for example, interact primarily with electrons and for these scenarios the initial energy deposition goes into the electronic system. For ions the regime of strong electronic interaction is the one around the electronic stopping $S_{e}$ maximum. Low or moderate energy ion interact primarily with the atoms in a solid, therefore, these simulations are initiated by giving one atom in the MD cell a high velocity, as in a cascade simulation. In this subsection, we discuss the implementation of 2T-MD briefly for moderate energy ions and laser irradiation, which represent a wide variation of types of energy deposition. The relevance and use of both the 2T-MD model to examine swift heavy ions is discussed in detail in section 9.3.4.3. 


\subsubsection{Cascades}

Ion irradiation has traditionally been modelled using molecular dynamics in the form of cascade simulations, as described in section 9.2. The effects of electronic stopping are often included in cascades by adding a friction term to the MD equation of motion, cf. section 9.2.2.4. The magnitude of the friction coefficient can be determined e.g., using the SRIM code, as described in [60]. Note, however, that energy is not conserved by this method. The friction term gradually removes energy from the atoms and, as the electronic system is not included in this model, this energy is lost. Indeed, the velocity of the atoms will tend to zero and the simulation will eventually freeze. This undesirable situation is generally avoided by introducing a cut off velocity, below which the electronic stopping is considered to be zero. This removes the problem of freezing dynamics but there is little physical justification for the cut off and results (residual defect distributions) are found to be sensitive to the value chosen for the velocity cut off [66]. The 2T-MD model resolves this issue in cascades in a physically motivated way, because the energy lost in electronic stopping is transferred to the electronic system and subsequently redeposited in the lattice via electronphonon coupling. The lattice is effectively thermostatted to the local electronic temperature which increases above the ambient value and then gradually decreases to ambient as the excess energy diffuses from the impact site.

The practical implementation of 2T-MD for cascades involves superimposing a 3-dimensional grid on the atomistic supercell for the numerical solution of the electronic heat diffusion equation and the grid is extended well beyond the supercell in all 3 directions (Figure 9.6). The function of the extended grid is to transport the energy that has been deposited in the electrons via electronic stopping away from the initial radiation event, via electronic thermal diffusion. The atomistic cell has periodic boundary conditions in all 3 dimensions, which effectively traps the radiation energy in the relatively small cell. In standard cascade simulations a boundary thermostat is generally introduced to dissipate the excess energy by simulating transport to the bulk solid. In this case, one can optimize the time constants of the thermostat by tests of whether it affects the final results. For instance, for the Berendsen thermostat a time constant around $200 \mathrm{fs}$ was found to provide good boundary dissipation for Au cascades [67]. The 2T-MD model presents an alternative approach to energy dissipation. The energy is transferred to the electrons by both electronic stopping and electron-phonon coupling and this electronic energy diffuses away from the central cell via electronic thermal diffusion. This energy must be dissipated at the boundaries of the extended electronic cell to simulate heat diffusion into the bulk, which can be done by implementing Dirichlet (infinite flux) or Robin boundary conditions. Robin boundary condition is a mixture between Dirichlet boundary conditions and Neumann (zero flux) boundary conditions and it is an effective method for the gradual dissipation of the electronic energy. 


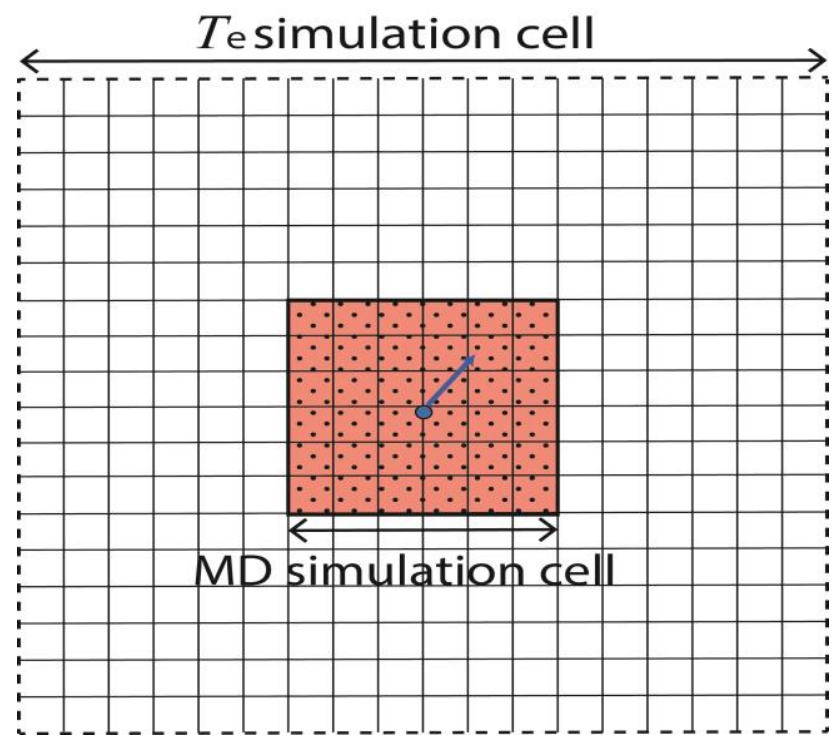

Figure 9.6. Schematic illustration of a 2T-MD simulation of a cascade simulation. The square grid represents the voxels for the numerical solution of the electronic heat diffusion equation. The cascade is initiated by giving a finite velocity to one atom (the PKA) of the simulation.

One potential pitfall to be aware of when implementing the 2T-MD model for cascades relates to the very high velocity of the primary knock-on atom (PKA) during the initial simulation period. The atomistic velocity distribution is far from equilibrium and it is not, therefore, possible to define a realistic lattice temperature. The average velocity in the voxel containing the PKA atom will result in an unphysically high $T_{l}$ and, consequently, too much energy being lost to the electronic system. For this reason, the electron-phonon coupling should not be switched on until the atomic velocity distribution approaches equilibrium. In the initial simulation period only, electronic stopping is active as one atom, or a few atoms, have velocities well in excess of the average. After thermalization of the atomistic velocities the electron phonon coupling is switched on and the lattice and electronic temperatures gradually return to equilibrium. In metals, the thermalization (in the sense that the atoms reach a Maxwell-Boltzmann velocity distribution) occurs after about 3 lattice vibrations [21].

\subsubsection{Laser irradiation}

Modelling laser irradiation by 2T-MD has been pioneered by Zhigilei in a series of seminal papers [62], [68], [69], [70]. The details of the methodology vary slightly from that outlined above but the methods are essentially equivalent. As with SHI, the laser deposits energy to the electrons and this energy is transferred to the atoms via electrn-phonon coupling. A schematic illustration of a 2TMD simulation of an SHI simulation is shown in Figure 9.7. In this case the laser energy impinges on a 2-dimensional solid surface so the atomistic simulation cell is a slab with periodic boundary conditions in the $x$ and $y$ directions. The electronic cell is also periodic in 2 dimensions and there is overlap between the atomistic and the electronic simulation cells in all dimensions (Figure 9.7). 


\section{Laser irradiation}

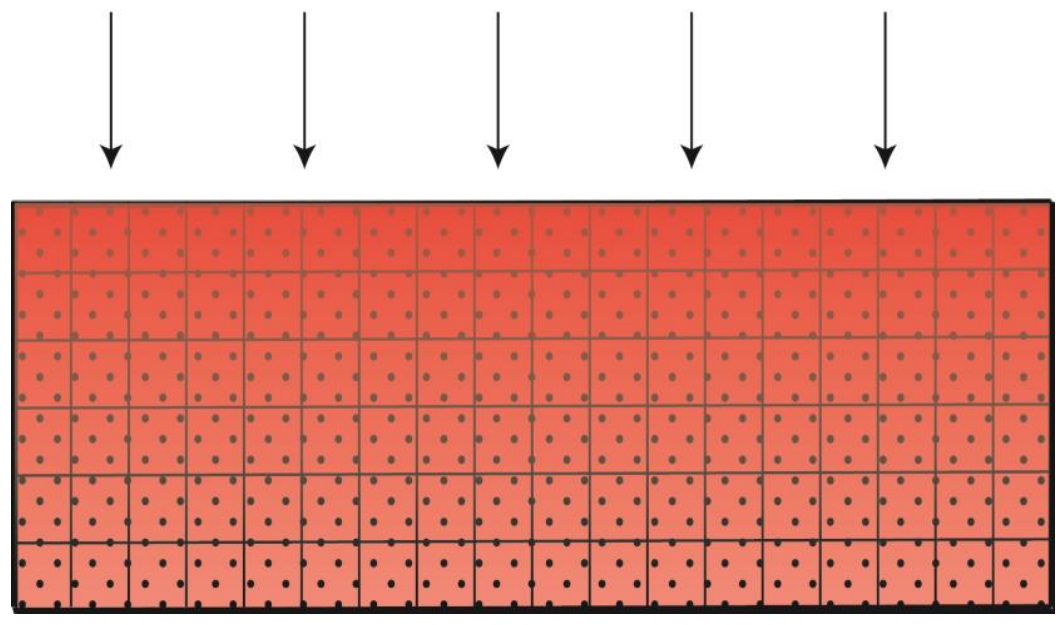

Figure 9.7. Schematic illustration of a 2T-MD simulation of laser irradiation. In this case periodic boundary conditions are imposed in the $\mathrm{x}-\mathrm{y}$ directions of both the MD and the electronic simulation cells.

The justification for this configuration is that the laser irradiates a large surface area, therefore, from an atomistic perspective, it is considered infinite and heat transport in the lateral direction can be neglected. Thin films can be modelled directly by this method but thick films should be coupled to a continuum model in the $z$ direction to model heat transport through both the lattice and the electrons into the bulk material.

The initial energy $A(z, t)$ is deposited homogeneously in the $x-y$ plane with a spatial and temporal distribution given by:

$$
A(z, t)=\frac{2 F}{L \tau_{p}} \sqrt{\frac{\ln 2}{\pi}} \exp -\left[\frac{4 \ln \left(2\left(t-t_{0}\right)^{2}\right.}{\tau_{p}^{2}}\right] \exp -\left[\frac{Z}{L}\right]
$$

Here $F$ is the absorbed fluence, $L$ is the optical penetration depth, $\tau_{p}$ the duration of the pulse taken at the full width half maximum, and $t_{0}$ is the time zero, which is defined as the time when the laser pulse maximum arrives on the sample surface. As with the SHI simulations, the electronic energy diffuses through the system and transfers to the atoms via electron phonon coupling. If the thickness of the sample is similar to the optical penetration depth, then the $\mathrm{z}$ dependence can be neglected as the energy diffuses through the thickness of the film within a few femtoseconds. The electronic temperature distribution is homogeneous for such simulations. The lattice heats up in a timescale that is determined by the strength of the electron phonon coupling and, for sufficiently high fluence, melting and/or ablation occurs. 2T-MD has been highly successful for modelling laser irradiation under a range of scenarios, with excellent agreement between the simulations and ultrafast experimental measurements [71].

\subsubsection{2T-MD Codes}

Two widely used, freely available MD codes, DL_POLY_4.09 and LAMMPS, have 2T-MD functionality. Both codes are very efficient and suitable for multimillion atom simulations on high performance computing platforms. Instructions for registering and down loading DL_POLY can be found on: 
https://www.scd.stfc.ac.uk/Pages/DL_POLY.aspx

A comprehensive user manual and a range of examples are available, including examples of each of the radiation scenarios discussed above. LAMMPS is distributed as an open-source code under GPL: https://lammps.sandia.gov/. The 2T-MD (ttm/mod) functionality is only enabled if LAMMPS was built with the USER-MISC package. See https://lammps.sandia.gov/doc/Build_package.html for more information on building LAMMPS.

\subsection{Molecular dynamics for swift heavy ion effects}

The collision cascades described previously in this article are typically initiated by ions or recoils at energies up to hundreds of $\mathrm{keV}$, as shown in Figure 9.1. The upper part of Figure 9.1 shows that the increase of ion energy leads to a decrease in nuclear stopping power (energy deposited via collision cascades) and gradual increase in electronic stopping power, which starts dominating at energies $\sim 1 \mathrm{MeV} /$ a.m.u. and ion masses about $\gtrsim 40$ a.m.u. At these high energies, the probability of an ion colliding with atoms of the material is small. This means that the ion may pass hundreds of nanometers along a straight line before its trajectory starts diverting due to collisions with target atoms as shown in the lower part of Figure 9.1. Instead, the strong electronic stopping power for ions with energy $\gtrsim 10 \mathrm{keV} / \mathrm{nm}$ may also produce damage in materials. The swift heavy ion damage has been observed experimentally numerous times, and typically takes the shape of elongated roughly cylindrical regions of amorphous material surrounded by pristine crystal, known as "ion tracks" [72-76], although many other forms of swift heavy ion damage also exist [77-80]. The materials with track damage typically do not exhibit signs of cascade-like localized damage. This feature allows to approach the problem of simulating high energy impacts considering only electronic effects in the lattice.

The trick to include electronic stopping power via a simple friction force as described in Sec. 9.2.2.4 or by applying Langevin thermostat as done in [60] only allows representation of the effect of thermal conduction in irradiated material, leaking the energy from the hot region, however, this energy is not returned back into the lattice. To emulate the effect of thermal heating, which can be associated with excitations of electronic subsystem, additional modification of the MD algorithm is required.

The models to include swift heavy ion effects can, however, start from the electronic stopping power $S_{e}=-\left(\frac{d E}{d x}\right)_{e l}[10,23]$. Since this quantity is measured in units of force, $S_{e}=\Delta E / \Delta z$ and hence $\left[S_{e}\right]=\mathrm{keV} / \mathrm{nm}$, one can calculate the maximum electronic energy that may be deposited to the lattice simply from the length of the cell along the track direction $S_{z}: \Delta E=S_{e} S_{z}$, estimating the total amount of energy that was deposited by ions to the electronic subsystem. Deep inside the solid the electronic stopping power must eventually somehow all result in lattice heating (close to the surface, electrons may also escape to the vacuum). However, there is still an uncertainty of the lateral distribution of deposited energy, since the damage created by these ions is known to appear in the shape of elongated but laterally highly localized disordered regions along the ion tracks. 


\subsubsection{Track energy deposition models}

\subsubsection{Instantaneous cyclindrical deposition}

In the simplest energy deposition models, the energy can be simply deposited uniformly in a cylindrical region around the center of the cell [81, 82], assuming some radius for the energy deposition, which is a free parameter of the model.

\subsubsection{Instantaneous deposition from TTM calculations}

The two-temperature model (TTM) described above in section 9.3.1 [72, 75, 83] can be solved numerically in one dimension (the radial one), and has proven to give good agreement with experiments on many relevant quantities such as track radii [75] and electronic sputtering [84]. Hence, using radial energy deposition profiles from the TTM model as an input into MD is likely a better motivated approach than the homogeneous cylindrical models. Using MD is necessary if one wishes to model how swift heavy ions cause structural changes such as amorphization, density change, or defect profiles in the material.

The two-temperature energy deposition can in fact be implemented in MD in two ways. The simplest is instantaneous deposition, where an energy deposition profile to atoms is obtained soon after the ion has passed, e.g., $50 \mathrm{fs}$, and all this energy is deposited to the lattice. Since MD does simulate lattice heat conductivity, this approach should be reasonable at least for insulators. Indeed, this approach has given good agreement with experimental track radii and even density profiles in many cases $[77,85,86]$.

\subsubsection{2T-MD modelling}

An approach logically following from the TTM is to use the 2T-MD model described in detail in section 9.3.2. In this model, the energy deposition for swift heavy ions, can be modelled by considering an electronic simulation cell that is extended beyond the MD cell in perpendicular to the ion's direction of travel. An atomistic simulation cell, with 3-dimensional periodic boundary conditions, is superimposed on the electron cell simulations cell (Figure 9.8). As with the cascade simulations, the electronic simulation cell is divided into a grid for the solution of the electronic thermal diffusion equation. Robin boundary conditions are generally imposed on the electronic cell in the $x$ and $y$ directions Neumann boundary conditions are imposed in the $z$ direction.

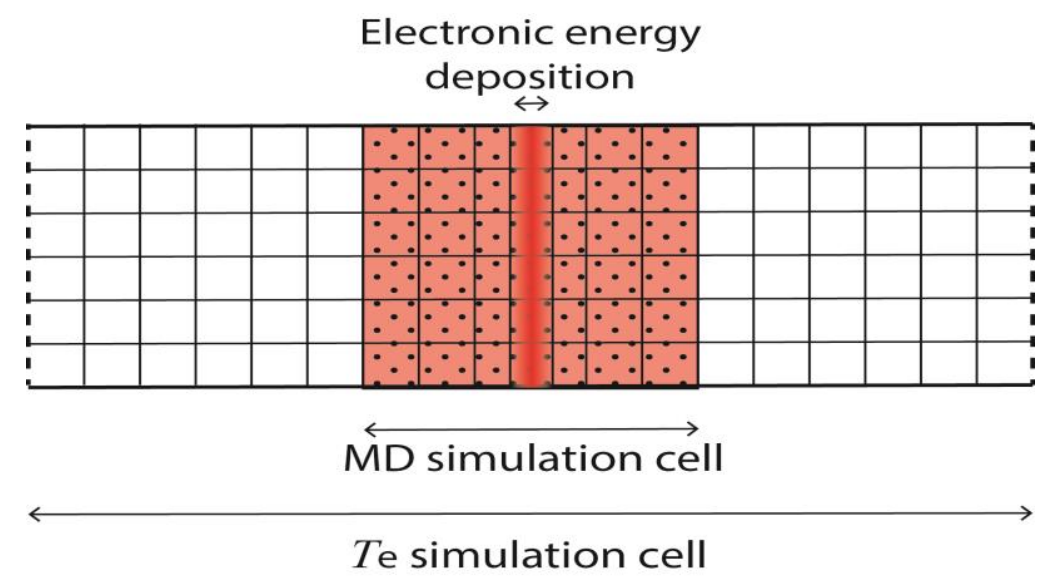

Figure 9.8. Schematic illustration of a 2T-MD simulation of an SHI simulation. The square grid represents the voxels for the numerical solution of the electronic heat diffusion equation. The electronic energy is initially deposited in a cylindrical region through the centre of the simulation cell. 
Energy is deposited in the electronic system during the initial stages of the simulation. The energy deposited by the SHI is quite well represented by a Gaussian spatial distribution $D(r)$ and an exponentially decaying temporal distribution.

$$
A(r, t)=A D(r) \alpha e^{-\alpha t}
$$

Here $r$ is the lateral distance from the ion path and $\alpha=1 / \tau_{d}$, the inverse of characteristic temporal deposition time $\tau_{d}$, which is generally of the order of a few femtoseconds. The Gaussian spatial distribution is given by:

$$
D(r)=\frac{S_{e}}{\sqrt{2 \pi \sigma^{2}}} \exp -\left[\frac{\left(r-r_{0}\right)^{2}}{2 \sigma^{2}}\right]
$$

where $\sigma$ is the standard deviation of the Gaussian spatial distribution and $S_{e}$ is the electronic stopping power. $A$ is a normalization constant that ensures that the total energy deposited equals the stopping power.

$$
S_{e}=\int_{0}^{5 \tau_{D}} d t \int_{0}^{r_{\max }} 2 \pi A(r, t) d r
$$

The electronic energy diffuses radially from the ion path and, simultaneously, transfers energy to the lattice via electron-phonon coupling. The electronic temperature decays with time near the track, however the lattice temperature increases. For sufficiently high energy deposition the lattice temperature exceeds the melting temperature in a finite region around the ion path and incomplete crystallization on solidification can, in some materials, create a disordered or defective region commonly known as an ion track. 2T-MD has been successfully employed to model ion tracks in metals [87], semiconductors [63], insulators [88] and graphene [89].

\subsubsection{Monte Carlo simulations of electrons and holes motion}

A completely different starting point is to do a Monte Carlo simulation of the explicit motion of single electrons and holes in a material [90-93]. While this approach has the advantage of a more explicit description of electron and hole dynamics, the model does not include electron-hole recombination which may affect the energy deposition.

\subsubsection{Coulomb explosion models}

The fourth model used to model swift heavy ions is that based on the idea of Coulomb explosions, i.e., that the passing swift heavy ion excites multiple electrons from lattice atoms, which then due to the high positive charge state undergo a Coulomb explosion [81, 94, 95]. Although simulating charged atoms with a fixed charge is easy in molecular dynamics, this original idea has the obvious weakness from the point of view of MD simulations that the electrons do not leave permanently, but are attracted back to the nucleus. One possibility to handle this is provided by considering the system as a plasma, and use the "Particle-in-cell" (PIC) plasma simulation model to deal with also the attraction [96].

\subsubsection{Comparative discussion on track models}

Taken together, it might seem strange that five different approaches can be used to simulate the same physical phenomenon. All of them are physically motivated, and it is clear that the key physics motivations are at least qualitatively relevant to the phenomenon. This is because it is clear from electronic stopping theory that heavy ions do ionize core electrons [97], so some aspect of (screened) Coulomb explosion must initially be present. It is also clear that some excited 
electrons do move far from the track core as delta electrons [98], so following electrons explicitly is reasonable. Also, at least after the electron collisions have led to a heating of the electron cloud, there must be electron-phonon coupling going on, as this effect is well established to be active from laser irradiation studies [99-102]. However, none of the models include all physical effects relevant, i.e., in other words, none of the models is wrong, and none is completely right. What does remain unclear is the relative contribution of each effect to atomic motion and hence materials damage, and to what extent the models can be combined for a comprehensive description of swift heavy ion effects.

\subsubsection{Practical method considerations for track simulations}

While the energy of the swift heavy ion is very high, and the delta electrons can easily have keV energies, the energy that the atoms receive is comparably low. Although the models described in the previous subsection have considerable variation, they do agree that the kinetic energy the atoms receive is at most of the order of $\sim 10 \mathrm{eV}$. Hence not all out of the special solutions described for modelling collision cascades may be needed to model swift heavy ions.

Most equilibrium interatomic potentials are still reasonable about $10 \mathrm{eV}$ above the ground state (with the likely exception of Lennard-Jones potentials, whose $1 / r^{12}$ repulsion is completely unrealistic at short distances). Hence using a high-energy repulsive potential (section 9.2.2.3) is not necessary. For the same reason, it is not necessary to use an electronic stopping power (section 9.2.2.4). On the other hand, the velocities associated with even $10 \mathrm{eV}$ energy are high enough that it is useful to use an adaptive time step (section 9.2.2.2). Likewise, the reasoning on why one must not use temperature or pressure control for cascades apply equally to track modelling (section 9.2.2.1), i.e., the track core and its vicinity should be modelled in the NVE ensemble. Hence also the boundary condition reasoning from section 9.2.2.5 applies well. In the most common case of wishing to model a segment of a track deep in the material, it is natural to use periodic boundaries in all three dimensions, model the track energy deposition parallel to the $z$ axis, and then apply temperature control at the $x$ and $y$ boundaries. This also has the advantage that the cell size in the $z$ direction may be relatively small. The size in the $x$ and $y$ needs to be large enough to absorb the pressure and heat wave from the track.

To select the system size, one also has to consider the heating of the entire cell due to the swift heavy ion. As a simple estimate, if the electronic stopping power is $10 \mathrm{keV} / \mathrm{nm}$, and one chooses a system thickness of $5 \mathrm{~nm}$, the energy deposition may be as high as $50 \mathrm{keV}$. The system size needed can then be estimated using the same consideration as in section 9.2.2.5, i.e, for $50 \mathrm{keV}$ at least $50000 \times 20=1$ million atoms are needed. For a typical atomic density of 50 atoms $/ \mathrm{nm}^{3}$, this requires a cell volume of $V=20,000 \mathrm{~nm}^{3}$, i.e., a lateral size $S_{x}=S_{y}$ of about $60 \mathrm{~nm}$. However, as noted earlier, one can and should always do a size scaling test to ensure that the system size is sufficient.

\subsubsection{Electronic temperature-dependent interatomic potentials}

We still consider a special molecular dynamics feature that may be relevant in particular to ion tracks and other cases where the electronic excitation level is particularly high. For moderate fluence laser irradiation, the agreement between ultrafast electron diffraction experiments and 
2T-MD models demonstrates the validity of the model, however, at high fluence there are significant discrepancies between modelling and experiment, with experiment detecting melting on a faster timescale than that predicted by simulation [103]. The implication of these results is that the interactions between the atoms are modified under extreme electronic excitation. This result is not unexpected since interatomic interactions are dominated by the electrons, therefore exciting these electrons to higher energy orbitals will affect interatomic interactions. The basic interatomic potentials used in MD rely on the Born-Oppenheimer approximation, i.e., that the electron state has time to finds its equilibrium before the nuclei move appreciably [104]. However, for fs laser pulses, or swift heavy ions which pass an atom on as timescales, and atoms also get into motion at above thermal energies, there may not be sufficient time for the electrons to equilibrate while nuclei move, and a conventional interatomic potential may not be valid. The modified interactions can be investigated theoretically using finite temperature density functional theory (FTDFT). The electronic temperature at which the interatomic interactions are significantly modified is signalled by two effects. The first is a change in the phonon dispersion and the second is a noticeable change in the equilibrium lattice parameter. In the case of $\mathrm{W}$, an investigation of the phonon dispersion signalled the presence of a bcc to fcc phase transformation at high electronic temperature [105]. This transformation was later confirmed using ab initio MD [106]. The variation in lattice parameter can be investigated by relaxing a unit cell of the element or compound at a range of temperatures using FTDFT.

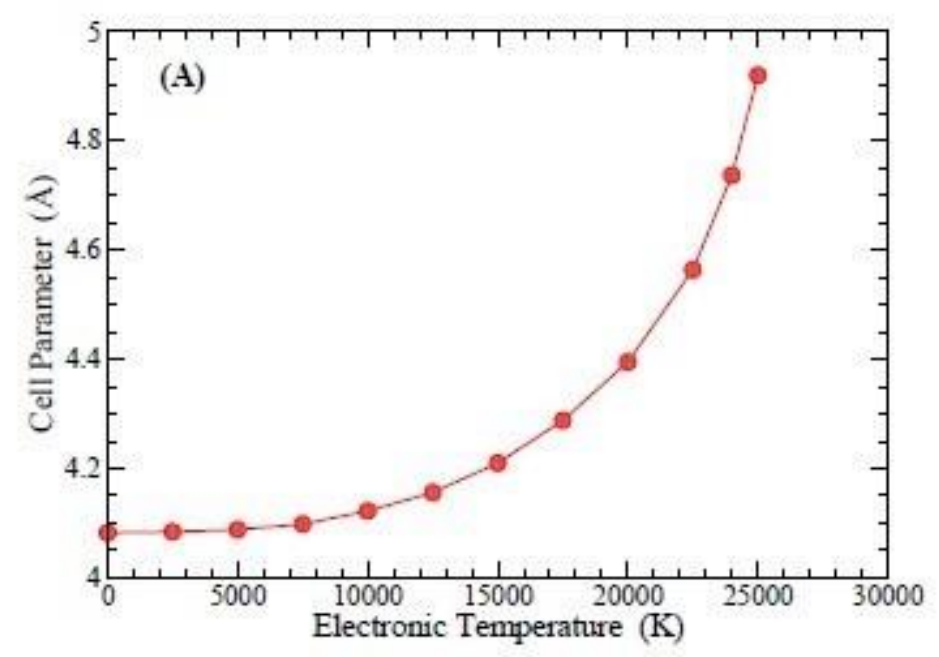

Figure 9.9. Plot of the equilibrium lattice parameter of W against electronic temperature, calculated by FTDFT.

A plot of the equilibrium lattice parameter versus temperature generally shows a significant deviation from zero gradient at a finite temperature. In the example shown for $\mathrm{W}$ in Figure 9.9 this temperature is around $10^{4} \mathrm{~K}$.

The implications of this plot are that if electronic temperatures exceed $10^{4} \mathrm{~K}$, it is necessary to modify the interatomic potentials for accurate simulations and, indeed, these potentials should depend on the local electronic temperature, which is generally time dependent. Such $T_{e}$ dependent potentials can be developed from data obtained from FTDFT calculations, either by force fitting to ab initio molecular dynamics simulations [107] or by fitting to the energy versus lattice parameters curves for a range of $T_{e}$ [106], [108]. There has been some discussion about whether 
free energy of enthalpy should be used for the fitting but a convincing argument for the enthalpy has been presented in [108] and this should be employed for future $T_{e}$ dependent potentials development. Employing $T_{e}$ dependent potentials for radiation scenarios that result in highly excited electrons can capture important effects, such as non-thermal melting and ultrafast phase transformations, that are inaccessible to standard 2T-MD simulations. The rapid change in interatomic interactions causes large forces on the atoms. For example, consider an atom in an equilibrium configuration at ambient temperature. A radiation event may rapidly increase the electronic temperature, which results in a sudden change in the interatomic interactions (Figure 9.10). The atom is no longer close to the minimum of the potential energy curve and, consequently, it is subjected to a finite force. Such non-thermal forces, as they are known, can rapidly destabilize an ordered crystal structure and lead to rapid (non-thermal) melting.

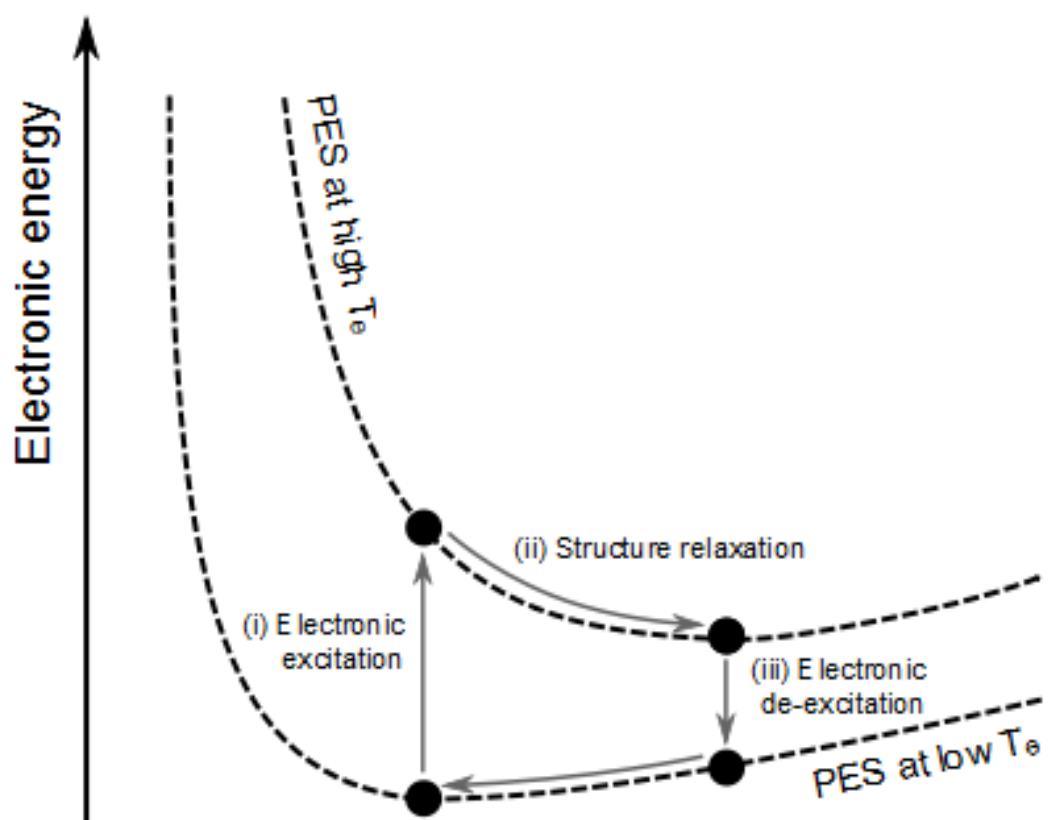

(iv) Structure relaxation

Atomic position

Figure 9.10. Schematic illustration of the effect of high electronic temperature on the potential energy surface.

The implementation of $T_{e}$ dependent potentials to 2T-MD must be performed in such a way that energy is conserved. The $T_{e}$ can change continuously following a radiation event which implies that the potential energy of the atoms also changes continuously, even in the scenario where the atomic configuration is fixed. This is apparent in Figure 9.6. The energy lost, or gained, by this potential energy shift must be compensated for elsewhere in the simulation. In practice this is achieved by rederiving the 2T-MD equations, (9.7) and (9.9), with an extra term in the Hamiltonian that states explicitly that the potentials energy $(U)$ is a function of $T_{e}$. The result is a modified electronic heat capacity that adds an additional term to account for the potential energy variation [108]:

$$
C_{e}=\frac{d E_{e}}{d T_{e}}+\frac{\partial U}{\partial T_{e}}
$$


This modification ensures energy conservation, but there is a potential pitfall that needs to be avoided. Consider the case where $\frac{\partial U}{\partial T_{e}}<\frac{d E_{e}}{d T_{e}}$ then $C_{e}<0$. The implication of this is that adding heat decreases the temperature, which is clearly unphysical. The solution is to design $T_{e}$ dependent potentials or simulations that avoid such scenarios [108].

$T_{e}$ dependent potentials are not currently implemented in the common MD codes that include 2TMD (LAMMPS and DL_POLY), however a locally modified version of LAMMPS is available by contacting the corresponding author of [108].

\subsection{Challenges remaining}

In the previous sections, we described in brief terms the state-of-the-art approaches in radiation effects modelling. As it is evident from the discussion, some aspects of the physical understanding and modelling are very well under control, such as how to solve the equations of motion, select the time step, or include the high-energy electronic stopping. However, several challenges do remain.

In general terms, the major challenge for radiation - and also equilibrium - MD modelling is the reliability of interatomic potentials. Even though for the most commonly studied materials like $\mathrm{Si}$, there are literally dozens of potentials [109], none of them model all aspects of radiation damage reliably. A promising avenue for improvement of this are the Machine-Learning (ML) interatomic potentials that can be systematically trained to be reproduce a very wide range of reference data points from more reliable DFT calculations [110-113]. The downside with machine learning is that it is inherently an interpolation method, and hence attempting to simulate configurations outside the fitting database can lead to significant artefacts. Analytical potentials may actually be better for extrapolation if their functional forms are based on sound physical principles, like e.g., the Tersoff-like [114-117] tight-binding [118, 119] and effective-medium theory [120, 121] potentials. Indeed, for radiation effects the lack of a fit of very short interatomic separations in a machine-learning potential database can lead to major artefacts in the simulations [122]. Very recently, Byggmastar et al. developed a way to train machine-learning potentials in a way that includes a realistic repulsive potential [122-124].

Another possible way of improving the reliability of MD is to obtain the forces directly from DFT calculations. Due to the very high computational cost of DFT, this approach has been limited to small system sizes and low recoil energies [125-127]. This may soon change when exascale computing with new kinds of accelerators may allow running density functional theory simulations on unprecedented scales. However, one always has to keep in mind that also the quantum mechanical density-functional theory approach is approximative, and has known shortcomings [128]. Hence the final test of any simulation model should be against experiments.

Considering challenges related to electronic effects, as became evident from section 9.4, it is not known how to model swift heavy ion effects on materials fully, and related to this, it is also not known how to handle the low-energy limit of electronic stopping power. While the 2TMD approaches have brought considerable advances on the treatment of these, there are still uncertainties related to how the parameters should be chosen. Moreover, as discussed in section 9.4.1.6, there are several physical effects occurring in tracks and currently no model includes them 
all. Both issues clearly need further model development and verification against experiments.

Another class of radiation effects that cannot be modelled very reliably are those involving bond breaking due to electronic excitations. Here the qualitative picture is fairly clear: high-energy electrons, photons or ions can excite electrons in bonds to an antibonding state, which then make the interaction repulsive [129]. It is relatively easy to make an antibonding interatomic potential to model such effects qualitatively [130]. However, due to the complex nature of excited states in solids, describing this quantitatively is very challenging. Time-dependent DFT (TDDFT) methods can in principle model this, and may provide advances on the topic in the future.

In summary, in this article we have reviewed the key aspects of molecular dynamics modelling of radiation effects in materials, both for the nuclear and electronic collision regime. We showed that some parts of the effects are well understood both from a physics and computational methods point of view, but that major unresolved questions still remain for other aspects. Hence there is still room for plenty of interesting and important basic research in the field.

\section{References}

[1] E. Fermi, R. D. Richtmyer, Note on census-taking in Monte Carlo calculations, A declassified report by Enrico Fermi. From the Los Alamos Archive (1948).

[2] K. Nordlund, Historical review of computer simulation of radiation effects in materials, J. Nucl. Mater. 520 (2019) 273, Invited review in Diamond Anniversary issue.

doi:10.1016/j.jnucmat.2019.04.028.

[3] K. Nordlund, F. Djurabekova, Multiscale modelling of irradiation in nanostructures, J. Comput. Electr. 13

(1) (2014) 122, invited review paper for special issue on device modelling.

[4] A. A. Leino, F. Djurabekova, K. Nordlund, Radiation effects in nanoclusters embedded in solids, European Physical Journal B 87 (2014) 242.

[5] J. Marian, C. S. Becquart, C. Domain, S. L. Dudarev, M. R. Gilbert, R. J. Kurtz, D. R. Mason, K. Nordlund, A. E. Sand, L. L. Snead, T. Suzudo, B. D. Wirth, Recent advances in computational materials modeling of tungsten as plasma-facing material for fusion energy applications, Nuclear Fusion 57 (2016) 092008.

[6] K. Nordlund, S. J. Zinkle, A. E. Sand, F. Granberg, R. S. Averback, R. Stoller, T. Suzudo, L. Malerba, F. Banhart, W. J. Weber, F. Willaime, S. Dudarev, D. Simeone, Primary radiation damage: a review of current understanding and models, J. Nucl. Mater. 512 (2018) 450-479.

[7] A. F. Voter, M. R. Sorensen, Accelerating atomistic simulations of defect dynamics: hyperdynamics, parallel replica dynamics, and temperature-accelerated dynamics, in: V. V. Bulatov, T. D. de la Rubia, R. Phillips, E. Kaxiras, N. Ghoniem (Eds.), Multiscale Modelling of Materials, Vol. 538 of MRS Symp. Proc., Materials Research Society, Pittsburgh, 1999, p. 427.

[8] L. A. Marques, L. Pelaz, P. Lopez, M. Aboy, I. Santos, J. Barbolla, Atomistic simulations in Si processing: Bridging the gap between atoms and experiments, Mater. Sci. Engr. B 124-125 (2005) 72.

[9] K. Nordlund, C. Bjoerkas, T. Ahlgren, A. Lasa, A. E. Sand, Multiscale modelling of plasma-wall interactions in fusion reactor conditions, J. Phys. D: Appl. Phys. 47 (2014) 224018, Invited paper for Special Issue on Fundamentals of plasma-surface interactions.

[10] J. F. Ziegler, SRIM-2013 software package, available online at http://www.srim.org.

[11] F. Mandl, Statistical Physics, 2nd Edition, Wiley, Chichester, UK, 1988. 
[12] N. W. Ashcroft, N. D. Mermin, Solid State Physics, Saunders College, Philadelphia, 1976.

[13] O. S. Oen, D. K. Holmes, M. T. Robinson, Ranges of Energetic Atoms in Solids, J. Appl. Phys. 34 (1963) 302.

[14] M. T. Robinson, I. M. Torrens, Computer Simulation of atomic-displacement cascades in solids in the binary-collision approximation, Phys. Rev. B 9 (12) (1974) 5008-5024.

[15] M. T. Robinson, Computer simulation studies of high-energy collision cascades, Nucl. Instr. Meth. Phys. Res. B 67 (1992) 396.

[16] M. Hou, Z. Pan, Collision cascades in Cu, $\mathrm{Au}$ and $\mathrm{Cu} 3 \mathrm{Au}$ : a comparison between molecular dynamics and the binary collision approximation, Nucl. Instr. Meth. Phys. Res. B 90 (1994) 469.

[17] F. Djurabekova and T. S. Pugacheva and F. Umarov and S. V. Yugay, Computer simulation of ion implantation with visual observation of the implantation profiles, IEEE Xplore (2000) 2282000 International conference on ion implantation technology, proceedings.

[18] G. Hobler, D. Kovac, Dynamic binary collision simulation of focused ion beam milling of deep trenches, Nucl. Instr. Meth. Phys. Res. B 269 (14) (2011) 1609.

[19] G. Hobler, G. Betz, On the useful range of application of molecular dynamics simulations in the recoil interaction approximation, Nucl. Instr. Meth. Phys. Res. B 180 (2001) 203-208.

[20] T. Diaz de la Rubia, R. S. Averback, R. Benedek, W. E. King, Role of Thermal Spikes in Energetic Collision Cascades, Phys. Rev. Lett. 59 (1987) 1930-1933, see also erratum: Phys. Rev. Lett. 60 (1988) 76.

[21] H. Zhu, R. S. Averback, M. Nastasi, Molecular dynamics simulations of a $10 \mathrm{keV}$ cascade in $\beta$-NiAl, Phil. Mag. A 71 (4) (1995) 735.

[22] C. W. Gear, Numerical initial value problems in ordinary differential equations, Prentice-Hall, Englewook Cliffs, NJ, USA, 1971.

[23] J. F. Ziegler, J. P. Biersack, U. Littmark, The Stopping and Range of Ions in Matter, Pergamon, New York, 1985.

[24] P. Gombas, Die Statistische Theorie des Atoms und ihre Anwendungen, Springer-Verlag, Vienna, Austria, 1949.

[25] A. N. Zinoviev, Electron screening of the Coulomb potential at small internuclear distances, Nucl. Instr. Meth. Phys. Res. B 354 (2015) 308-312.

[26] J. E. Northrup, Structure of GaAs (001) surfaces: The role of electrostatic interactions, Phys. Rev. B 50 (3) (1994) 2015-2018.

[27] D. Danailov, R. Pfandzelter, T. Igel, H. Winter, K. Gaertner, Deduction of the He-Fe interaction potential in eV-range from experimental data by computer simulation in grazing ion-surface scattering: Row model, Nucl. Instr. Meth. Phys. Res. B 180 (2001) 265-273.

[28] M. A. Karolewski, Repulsive interatomic potentials for noble gas bombardment of $\mathrm{Cu}$ and Ni targets, Nucl. Instr. Meth. Phys. Res. B 243 (1) (2005) 43.

[29] A. N. Zinoviev, K. Nordlund, Comparison of repulsive interatomic potentials calculated with an allelectron DFT approach with experimental data, Nucl. Instr. Meth. Phys. Res. B 406 (2017) 511-7.

[30] J. Lindhard, M. Schar_, H. E. Schiøtt, Range concepts and heavy ion ranges, Kgl. Danske Vid. Selskab, Mat.- Fys. Medd. 33 (14) (1963) 1-42.

[31] J. F. Ziegler, J. P. Biersack, M. D. Ziegler, SRIM - The Stopping and Range of Ions in Matter, SRIM Co., Chester, Maryland, USA, 2008. 
[32] M. H. Shapiro, T. A. Tombrello, Molecular dynamics simulations of inelastic energy loss ffects in sputtering, Nucl. Instr. Meth. Phys. Res. B 90 (1994) 473-476.

[33] K. Nordlund, Molecular dynamics simulation of ion ranges in the 1-100 keV energy range, Comput. Mater. Sci. 3 (1995) 448.

[34] M.-J. Caturla, L. A. M. T. Diaz de la Rubia, G. H. Gilmer, Ion-beam processing of silicon at keV energies: A molecular-dynamics study, Phys. Rev. B 54 (24) (1996) 16683.

[35] D. Cai, N. Grønbech-Jensen, C. M. Snell, K. M. Beardmore, Phenomenological electronic stopping-power model for molecular dynamics and Monte Carlo simulation of ion implantation into silicon, Phys. Rev. B 54 (23) (1996) 17147.

[36] L. Sandoval, H. M. Urbassek, Influence of electronic stopping on sputtering induced by cluster impact on metallic targets, Physical Review B 79 (14) (2009) 144115.

[37] S. T. Nakagawa, H. J. Whitlow, A predictive model for the electronic stopping force for molecular dynamic simulation (I), Nucl. Instr. Meth. Phys. Res. B 268 (19) (2010) 3287-3290.

[38] J. Sillanpää, J. Peltola, K. Nordlund, J. Keinonen, M. J. Puska, Electronic stopping calculated using explicit phase shift factors, Phys. Rev. B 63 (2001) 134113.

[39] P. Haussalo, J. Keinonen, U.-M. Jäske, J. Sievinen, Trapping of hydrogen impurities in helium-implanted niobium and tantalum, J. Appl. Phys. 75 (1994) 7770.

[40] K. M. Beardmore, N. Grønbech-Jensen, An Efficient Molecular Dynamics Scheme for the Calculation of Dopant Pro_les due to Ion Implantation, Phys. Rev. E 57 (1998) 7278.

[41] J. Peltola, K. Nordlund, J. Keinonen, Electronic stopping power calculation method for molecular dynamics simulations using local Firsov and free electron-gas models, Rad. Eff. \& Def. in Sol. 161 (9) (2006) 511-521.

[42] C. P. Flynn, R. S. Averback, Electron-phonon interactions in energetic displacement cascades, Phys. Rev. B 38 (1988) 7118.

[43] A. Caro, M. Victoria, Ion-electron interaction in molecular-dynamics cascades, Phys. Rev. A (General Physics) 40 (5) (1989) 2287-91.

[44] S. Pronnecke, A. Caro, M. Victoria, T. Diaz de la Rubia, M. W. Guinan, The effect of electronic energy loss on the dynamics of thermal spikes in Cu, J. Materials Research 6 (3) (1991) 483-91.

[45] I. Koponen, Energy transfer between electrons and ions in dense displacement cascades, Phys. Rev. B 47 (21) (1993) 14011.

[46] K. Nordlund, M. Ghaly, R. S. Averback, Mechanisms of ion beam mixing in metals and semiconductors, J. Appl. Phys. 83 (3) (1998) 1238-1246.

[47] K. Nordlund, L.Wei, Y. Zhong, R. S. Averback, Role of electron-phonon coupling on collision cascade development in Ni, Pd and Pt, Phys. Rev. B (Rapid Comm.) 57 (1998) 13965-13968.

[48] A. Lim, W. M. C. Foulkes, A. P. Horsfield, D. R. Mason, A. Schleife, E. W. Draeger, A. A. Correa, The electron elevator: excitations across the band gap via a dynamical gap state, Phys. Rev. Lett. 116 (2016) 043201.

[49] A. Tamm and M. Caro and A. Caro and G. Samolyuk and M. Klintenberg and A. A. Correa, Langevin Dynamics with Spatial Correlations as a Model for Electron-Phonon Coupling, Phys. Rev. Lett. 120 (2018) 185501.

[50] A. E. Sand, R. Ullah, A. A. Correa, Heavy ion ranges from first-principles electron dynamics, npj Computational Materials 5 (1) (2019) 43. doi:https://doi.org/10.1038/s41524-019-0180-5. 
[51] T. Diaz de la Rubia and M. W. Guinan, Progress in the development of a molecular dynamics code for high-energy cascade studies, J. Nucl. Mater. 174 (1990) 151-157.

[52] A. V. Krasheninnikov, K. Nordlund, Ion and electron irradiation-induced effects in nanostructured materials, J. Appl. Phys. (Applied Physics Reviews) 107 (2010) 071301.

[53] R. H. Telling, C. P. Ewels, A. A. El-Barbary, M. I. Heggie, Wigner defects bridge the graphite gap, Nature Materials 2 (2003) 333.

[54] C. Kittel, Introduction to Solid State Physics, 3rd Edition, John Wiley \& Sons, New York, 1968.

[55] Z. Wang, C. Dufour, E. Paumier, M. Toulemonde, The S(E) sensitivity of metals under swift-heavy-ion irradiation - a transient thermal-process, Journal of physics-condensed matter 6 (34) (1994) 6733-6750. doi:10.1088/0953-8984/6/34/006.

[56] M. Kaganov, I. Lifshitz, L. Tanatarov, Relaxation between electrons and the crystalline lattice, Soviet physics JETP-USSR 4 (2) (1957) 173-178.

[57] F. Seitz, J. S. Koehler, Displacement of Atoms during Irradiation, in: F. Seitz, D. Turnbull (Eds.), Solid State Physics, Vol. 2, Academic Press, New York, 1956, p. 307.

[58] S. Brorson, J. Fujimoto, E. Ippen, Femtosecond electronic heat-transport dynamics in thin gold-films, Physical review letters 59 (17) (1987) 1962-1965. doi:\{10.1103/PhysRevLett.59.1962\}.

[59] J. Fujimoto, J. Liu, E. Ippen, N. Bloembergen, Femtosecond laser interaction with metallic tungsten and nonequilibrium electron and lattice temperatures, Phys. Rev. Lett. 53 (19) (1984) 1837-1840. doi:\{10.1103/PhysRevLett.53.1837\}.

[60] D. M. Duffy, A. M. Rutherford, Including the effects of electronic stopping and electron-ion interactions in radiation damage simulations, J. Phys. Cond. Matter 19 (2007) 016207.

[61] R. Darkins, D. M. Duffy, Modelling radiation effects in solids with two-temperature molecular dynamics, Comput. Mater. Sci. 147 (2018) 145-153. doi:\{10.1016/j.commatsci.2018.02.006\}.

[62] Z. Lin, R. A. Johnson, L. V. Zhigilei, Computational study of the generation of crystal defects in a bcc metal target irradiated by short laser pulses, Physical review B 77 (21) (JUN 2008). doi:\{10.1103/PhysRevB.77.214108\}.

[63] G. S. Khara, S. T. Murphy, S. L. Daraszewicz, D. M. Duffy, The influence of the electronic specific heat on swift heavy ion irradiation simulations of silicon, Journal of physics-condensed matter 28 (39) (2016).

[64] S. L. Daraszewicz, Y. Giret, H. Tanimura, D. M. Duffy, A. L. Shluger, K. Tanimura, Determination of the electron-phonon coupling constant in tungsten, Applied physics letters 105 (2) (JUL 14 2014). doi:\{10.1063/1.4890413\}.

[65] V. P. Lipp, B. Rethfeld, M. E. Garcia, D. S. Ivanov, Atomistic-continuum modeling of short laser pulse melting of Si targets, Physical Review B 90 (24) (DEC 24 2014). doi: \{10.1103/PhysRevB.90.245306\}.

[66] S. L. Daraszewicz, The modelling of electronic effects in molecular dynamics simulations, PhD Thesis, University College London, 2014.

[67] J. Samela, J. Kotakoski, K. Nordlund, J. Keinonen, A quantitative and comparative study of sputtering yields in Au, Nucl. Instr. Meth. Phys. Res. B 239 (4) (2005) 331-346.

[68] J. Smirnova, L. Zhigilei, B. Garrison, A combined molecular dynamics and finite element method technique applied to laser induced pressure wave propagation, Computer physics communications 118 (1) (1999) 11-16. doi:\{10.1016/S0010-4655(98)00175-1\}.

[69] D. Ivanov, L. Zhigilei, Combined atomistic-continuum modeling of short-pulse laser melting and disintegration of metal films, Physical review B 68 (6) (AUG 2003). doi:\{10.1103/PhysRevB.68.064114\}. 
[70] Z. Lin, L. V. Zhigilei, Time-resolved diffraction profiles and atomic dynamics in short-pulse laserinduced structural transformations: Molecular dynamics study, Physical review B 73 (18) (MAY 2006). doi:\{10.1103/PhysRevB.73.184113\}.

[71] Y. Giret, N. Naruse, S. L. Daraszewicz, Y. Murooka, J. Yang, D. M. Duffy, A. L. Shluger, K. Tanimura, Determination of transient atomic structure of laser-excited materials from time-resolved diffraction data, Applied Physics Letters 103 (25) (DEC 16 2013). doi:\{10.1063/1.4847695\}.

[72] A. Meftah, F. Brisard, J. M. Costantini, E. Dooryhee, M. Hage-Ali, M. Hervieu, J. P. Stoquert, F. Studer, M. Toulemonde, Track formation in SiO2 quartz and the thermal-spike mechanism, Phys. Rev. B 49 (1994) 12457-12463.

[73] C. Trautmann, S. Klaumuenzer, H. Trinkaus, Effect of Stress on Track Formation in Amorphous Iron Boron Alloy: Ion Tracks as Elastic Inclusion, Phys. Rev. Lett. 85 (17) (2000) 3648.

[74] D. Kanjijal, Swift heavy ion-induced modi_cation and track formation in materials, Current Science 80 (12) (2001) 1560.

[75] M. Toulemonde, W. Assmann, C. Dufour, A. Meftah, F. Studer, C. Trautmann, Experimental phenomena and thermal spike model description of ion tracks in amorphisable inorganic insulators, Mat. Fys. Medd. Kong. Dan. Vid. Selsk. 52 (2006) 263.

[76] D. Schauries, M. Lang, O. H. Pakarinen, S. Botis, B. Afra, M. D. Rodriguez, K. Nordlund, D. Severin, M. Bender, C. Trautmann, R. C. Ewing, N. Kirby, P. Kluth, Temperature dependent formation of ion tracks in natural quartz and apatite, J. Appl. Crystallography 46 (6) (2013) 1558\{1563.

[77] P. Kluth, C. S. Schnohr, O. H. Pakarinen, F. Djurabekova, D. J. Sprouster, R. Giulian, M. C. Ridgway, A. P. Byrne, C. Trautmann, D. J. Cookson, K. Nordlund, M. Toulemonde, Fine structure in swift heavy ion tracks in amorphous SiO2, Phys. Rev. Lett. 101 (2008) 175503.

[78] M. Jolivet, A.-E. Lebatard, J.-L. Reyss, D. Bourlès, H. T. Mackaye, F. Lihoreau, P. Vignaud, M. Brunet, Can fossil bones and teeth be dated using_ssion track analysis? Chemical Geology 247 (2008) 81-99.

[79] V. A. Skuratov, J. O'Connell, N. Kirilkin, J. Neethling, On the threshold of damage formation in aluminum oxide via electronic excitations, Nucl. Instr. Meth. Phys. Res. B 326 (2014) 223.

[80] J. Hopster, R. Kozubek, B. Ban-d'Etat, S. Guillous, H. Lebius, M. Schleberger, Damage in graphene due to electronic excitation induced by highly charged ions, arXiv:1310.6233v1 (2013).

[81] E. M. Bringa, R. E. Johnson, R. M. Papaleo, Crater formation by single ions in the electronic stopping regime: Comparison of molecular dynamics simulations and experiments on organic films, Phys. Rev. B 65 (2002) 094113.

[82] A. Rivera, J. Olivares, A. Prada, M. L. Crespillo, M. J. Caturla, E. M. Bringa, J. M. Perlado, O. Pena-Rodriguez, Permanent modifications in silica produced by ion-induced high electronic excitation: experiments and atomistic simulations, Sci. Rep. 7 (2017) 10641.

[83] M. Toulemonde, C. Trautmann, E. Balanzat, K. Hjort, A. Weidinger, Track formation and fabrication of nanostructures with MeV-ion beams, Nucl. Instr. Meth. Phys. Res. B 216 (0) (2004) 1-8.

[84] M. Toulemonde, W. Assman, C. Trautmann, F. Gruner, H. D. Mieskes, H. Kucal, Z. G. Wang, Electronic sputtering of metals and insulators by swift heavy ions, Nucl. Instr. Meth. Phys. Res. B 212 (2003) 346-57.

[85] B. Afra, M. D. Rodriguez, C. Trautmann, O. H. Pakarinen, F. Djurabekova, K. Nordlund, T. Bierschenk, R. Giulian, M. C. Ridgway, G. Rizza, N. Kirby, M. Toulemonde, P. Kluth, SAXS investigations of the morphology of swift heavy ion tracks in $\alpha$-quartz, J. Phys.: Condens. Matter 25 (2013) 045006. 
[86] M. C. Sequeira and J. G. Mattei and H. Vazquez and F. Djurabekova and K. Nordlund and I. Monnet and P. Mota-Santiago and P. Kluth and C. Grygiel and S. Zhang and E. Alves and K. Lorenz, Unravelling the secrets of the resistance of GaN to strongly ionising radiation, Communications physics 4 (1) (2021) 51.

[87] G. S. Khara, S. T. Murphy, D. M. Duffy, Dislocation loop formation by swift heavy ion irradiation of metals, Journal of physics-condensed matter 29 (28) (JUL 19 2017). doi:\{10.1088/1361-648X/aa74f8\}.

[88] A. A. Leino, S. L. Daraszewicz, O. H. Pakarinen, K. Nordlund, F. Djurabekova, Atomistic two-temperature modelling of ion track formation in silicon dioxide, EPL 110 (1) (2015) 16004. URL http://stacks.iop.org/0295-5075/110/i=1/a=16004

[89] H. Vazquez, E. H. Ahlgren, O. Ochedowski, A. A. Leino, R. Mirzayev, R. Kozubek, H. Lebius, M. Karlusic, M. Jaksic, A. V. Krasheninnikov, J. Kotakoski, , M. Schleberger, K. Nordlund, F. Djurabekova, Creating nanoporous graphene with swift heavy ions, Carbon 114 (2017) 511.

[90] N. A. Medvedev, A. E. Volkov, N. S. Shcheblanov, B. Rethfeld, Early stage of the electron kinetics in swift heavy ion tracks in dielectrics, Physical Review B 82 (12) (2010) 125425.

[91] M. Ridgway, T. Bierschenk, R. Giulian, B. Afra, M. D. Rodriguez, L. Araujo, A. P. Byrne, N. Kirby, O. H. Pakarinen, F. Djurabekova, K. Nordlund, M. Schleberger, O. Osmani, N. Medvedev, B. Rethfeld, W. Wesch, P. Kluth, Track and voids in Amorphous Ge Induced by Swift Heavy-Ion Irradiation, Phys. Rev. Lett. 110 (2013) 245502.

[92] S. A. Gorbunov, N. A. Medvedev, R. Rymzhanov, P. N. Terekhind, A. E. Volkov, Excitation and relaxation of olivine after swift heavy ion impact, Nucl. Instr. Meth. Phys. Res. B 326 (2014) 163-168.

[93] N. A. Medvedev, R. A. Rymzhanov, A. E. Volkov, Time-resolved electron kinetics in swift heavy ion irradiated solids, Journal of physics D-applied physics 48 (35) (2015) 355303.

[94] S. Klaumuenzer, M. D. Hou, G. Schumacher, Coulomb explosions in a metallic-glass due to the passage of fast heavy-ions, Phys. Rev. Lett. 57 (7) (1986) 850.

[95] E. M. Bringa, Molecular dynamics simulations of Coulomb explosion, Nucl. Instr. Meth. Phys. Res. B 209 (2003) 1-9.

[96] Y. Cherednikov, S. N. Sun, H. M. Urbassek, Hybrid particle-in-cell/molecular dynamics simulation of swift-ion tracks in lif, Phys. Rev. B 87 (2013) 245424.

doi:10.1103/PhysRevB.87.245424. URL http://link.aps.org/doi/10.1103/PhysRevB.87.245424

[97] G. Schiwietz, P. L. Grande, Introducing electron capture into the unitary-convolution approximation energy-loss theory at low velocities, Phys. Rev. A 84 (2011) 052703. doi:10.1103/PhysRevA.84.052703. URL http://link.aps.org/doi/10.1103/PhysRevA.84.052703

[98] E. Aprile, W. H.-M. Ku, J. Park, Delta electron production and the ultimate energy resolution of liquid argon ionization detectors, IEEE Transactions on Nuclear Science 35 (1) (1988) 37.

[99] A. Miotello, R. Kelly, Laser irradiation effects in Si-implanted SiO2, Nucl. Instr. Meth. Phys. Res. B 65 (1992) 217.

[100] L. Zhigilei, P.B.S.Kodali, B.J.Garrison, A Microscopic View of Laser Ablation, J. Chem. Phys B 102 (1998) 2845.

[101] D. S. Ivanov, L. V. Zhigilei, Combined atomistic-continuum modeling of short pulse laser melting and disintegration of metal_lms, Phys. Rev. B 68 (2003) 064114.

[102] A. K. Upadhyay, N. A. Inogamov, B. Rethfeld, H. M. Urbassek, Ablation by ultrashort laser pulses: Atomistic and thermodynamic analysis of the processes at the ablation threshold, Phys. Rev. B 78 (2008) 045437. 
[103] S. L. Daraszewicz, Y. Giret, N. Naruse, Y. Murooka, J. Yang, D. M. Du_y, A. L. Shluger, K. Tanimura, Structural dynamics of laser-irradiated gold nanofilms, Physical review B 88 (18) (NOV 5 2013). doi:\{10.1103/PhysRevB.88.184101\}.

[104] M. Born and J. R. Oppenheimer, Zur Quantentheorie der Molekeln, Annalen der Physik 389 (20) (1927) 457-484.

[105] Y. Giret, S. L. Daraszewicz, D. M. Duffy, A. L. Shluger, K. Tanimura, Nonthermal solid-to-solid phase transitions in tungsten, Physical review B 90 (9) (SEP 4 2014). doi:\{10.1103/ PhysRevB.90.094103\}.

[106] S. T. Murphy, S. L. Daraszewicz, Y. Giret, M. Watkins, A. L. Shluger, K. Tanimura, D. M. Duffy, Dynamical simulations of an electronically induced solid-solid phase transformation in tungsten, Physical review B 92 (13) (OCT 15 2015). doi:\{10.1103/PhysRevB.92.134110\}.

[107] B. Bauerhenne, M. E. Garcia, Performance of state-of-the-art force fields for atomistic simulations of silicon at high electronic temperatures, European Physical Journal-Special Topics 227 (14) (2019) 16151629, Conference of the Collaborative-Research-Center (SFB) 716 on Particle Methods in Natural Science and Engineering, Heidelberg, GERMANY, SEP 24-26, 2018. doi:\{10.1140/epjst/e2019-800181-3\}.

[108] R. Darkins, P.-W. Ma, S. T. Murphy, D. M. Duffy, Simulating electronically driven structural changes in silicon with two-temperature molecular dynamics, Physical review B 98 (2) (JUL 13 2018). doi:\{10.1103/PhysRevB.98.024304\}.

[109] H. Balamane, T. Halicioglu, W. A. Tiller, Comparative study of silicon empirical interatomic potentials, Phys. Rev. B 46 (4) (1992) 2250.

[110] J. Behler and M. Parrinello, Generalized Neural-Network Representation of High-Dimensional Potential-Energy Surfaces, Phys. Rev. Lett. 98 (2007) 146401.

[111] A. P. Bartok and R. Kondor and G. Csanyi, Gaussian Approximation Potentials: The Accuracy of Quantum Mechanics, without the Electrons, Phys. Rev. Lett. 104 (2010) 136403.

[112] A. P. Bartok and M. C. Payne and R. Kondor and G. Csanyi, On representing chemical environments, Phys. Rev. B 87 (2013) 184115.

[113] J. Behler, Perspective: Machine learning potentials for atomistic simulations, J. Chem. Phys. 145 (2016) 170901.

[114] G. C. Abell, Empirical chemical pseudopotential theory of molecular and metallic bonding, Phys. Rev. B 31 (1985) 6184.

[115] J. Tersoff, New Empirical approach for the structure and energy of covalent systems, Phys. Rev. B 37 (1988) 6991.

[116] D. W. Brenner, Empirical potential for hydrocarbons for use in simulating the chemical vapor deposition of diamond_lms, Phys. Rev. B 42 (15) (1990) 9458; idem, 46, 1948 (1992).

[117] K. Albe, K. Nordlund, R. S. Averback, Modeling metal-semiconductor interaction: Analytical bondorder potential for platinum-carbon, Phys. Rev. B 65 (2002) 195124.

[118] F. Cleri, V. Rosato, Tight-binding potentials for transition metals and alloys, Phys. Rev. B 48 (1) (1993) 22.

[119] G. C. Kallinteris, N. I. Papanicolaou, G. A. Evangelakis, D. A. Papaconstantopoulos, Tight-binding interatomic potentials based on total-energy calculation: Application to noble metals using moleculardynamics simulation., Phys. Rev. B 55 (4) (1997) 2150-2156.

[120] M. J. Puska, R. M. Nieminen, M. Manninen, Atoms embedded in an electron gas: Immersion energies, Phys. Rev. B 24 (6) (1981) 3037. 
[121] M. Manninen, Interatomic interactions in solids: An effective-medium approach, Phys. Rev. B 34 (12) (1986) 8486.

[122] J. Byggmaestar and A. Hamedani and K. Nordlund and F. Djurabekova, Machine-learning interatomic potential for radiation damage and defects in tungsten, Phys. Rev. B 100 (2019) 144105.

[123] A. Hamedani, J. Byggmaaestar, F. Djurabekova, G. Alahyarizadeh, R. Ghaderi, A. Minuchehr, K. Nordlund, Insights into the primary radiation damage in silicon by machine learning interatomic potential, Mater. Res. Lett. 8 (2020) 364-372.

[124] J. Byggmaestar and K. Nordlund and F. Djurabekova, Gaussian approximation potentials for bodycentered cubic transition metals, Phys. Rev. Materials 4 (9) (2020) 093802.

[125] E. Holmstroem, A. Kuronen, K. Nordlund, Threshold defect production in silicon determined by density functional theory molecular dynamics simulations, Phys. Rev. B 78 (4) (2008) 045202.

[126] F. Gao, H. Xiao, W. Weber, Ab initio molecular dynamics simulations of low energy recoil events in ceramics, Nucl. Instr. Meth. Phys. Res. B 269 (14) (2011) 1693-1697. doi:10.1016/j.nimb.2011.01.131.URL http://www.sciencedirect.com/science/article/pii/S0168583X11001649

[127] P. Olsson, C. S. Becquart, C. Domain, Ab initio threshold displacement energies in iron, Mater. Res. Lett. 4 (2016) 216. doi:http://dx.doi.org/10.1080/21663831.2016.1181680.

[128] R. J. Meier, Are DFT level calculations the answer to real-world molecular systems, Comput. Mater. Sci. 27 (2003) 219-223.

[129] F. Spaepen, D. Turnbull, Crystallization processes, Academic Press, New York, 1982, Ch. 2, pp. 15-42.

[130] J. Frantz, J. Tarus, K. Nordlund, J. Keinonen, Mechanism of electron-irradiation induced recrystallisation in Si, Phys. Rev. B 64 (2001) 125313. 


\title{
10. Radiative hydrodynamics
}

\author{
Elisa Vázquez, Manuel Cotelo and Eduardo Oliva*
}

In many naturally occurring systems and laboratory experiments, radiative energy transfer and hydrodynamics are closely intertwined. These systems span from astrophysical structures to laboratory astrophysics experiments, inertial confinement fusion and plasma based soft X-ray lasers, to name some of them. In this chapter we will briefly justify the need of radiative hydrodynamics models to study these systems and explain the physics and numerical methods used to implement these models.

\footnotetext{
*Contact: eduardo.oliva@upm.es
} 


\subsection{Introduction}

Radiative hydrodynamics phenomena are characterized by the fact that radiation and hydrodynamics are intertwined, so the dynamics of the system is governed by both processes. On a day-to-day basis, we do not experience these phenomena, since radiation becomes important in hydrodynamic processes when the system attains temperatures of millions of degrees. However, we can think of many naturally occurring systems and experimental conditions in which matter is driven to such extreme conditions that radiative hydrodynamics plays a fundamental work. Many astrophysical systems, as the interior of stars and supernovae, and their laboratory counterparts require the use of radiative hydrodynamics to model their evolution. Laboratory plasmas created and heated with powerful (terawatt class) lasers attain temperatures of hundreds of electronvolts, attaining the radiative hydrodynamics regime. These plasmas appear in a broad range of experiments and applications. In this section, we will highlight three: laboratory astrophysics, inertial confinement fusion and plasma based soft X-ray lasers.

\subsubsection{Laboratory astrophysics}

Laboratory experiments are a very attractive way to understand the dynamical processes that take place in such astrophysical phenomena as supernovae, the so-called elephant trunks (photoevaporated clouds, like the pillars of creation on the Eagle nebula), planetary interiors, etc... Ideally, an experiment can be repeated, its initial and boundary conditions are controlled and varied, and the number of diagnosed variables is higher than in astronomical observations. However, since there is a difference of 10-20 orders of magnitude between laboratory experiments and their astrophysical counterparts, this task could be thought as impossible. Fortunately, the mathematical structure of the equations of ideal hydrodynamics makes possible to establish the so-called Euler similarity. These equations, also called the Euler equations, take the following form for a polytropic gas:

$$
\begin{gathered}
\rho\left(\frac{\partial v}{\partial t}+v \cdot \nabla v\right)=-\nabla p \\
\frac{\partial \rho}{\partial t}+\nabla \cdot(\rho v)=0 \\
\frac{\partial p}{\partial t}-\gamma \frac{p}{\rho} \frac{\partial \rho}{\partial t}+v \cdot \nabla p-\gamma \frac{p}{\rho} v \cdot \nabla \rho=0
\end{gathered}
$$

In these equations, $\rho$ is the density, $v$ the fluid velocity, $p$ the pressure and $\gamma$ the adiabatic index. The Euler equations for a polytropic gas cover most of the important features of different systems of astrophysical importance like supernovae remnants and photoevaporation clouds. The Euler similarity [1] states that two systems, lets denote them by subindices as (astrophysical) and lab (laboratory) will behave similarly if their initial conditions can be written as

$$
v_{t=0}=\tilde{v} F(r / h), p_{t=0}=\tilde{p} G(r / h), \rho_{t=0}=\tilde{\rho} H(r / h)
$$


where $F, G$ and $H$ are dimensionless functions and $\tilde{v}, \tilde{p}, \tilde{\rho}$, hare scaling factors that differ between the astrophysical and laboratory frames. Thanks to the invariance of Euler equations under some scale transformations, the two systems will evolve similarly if the so-called Euler number is the same in both systems, i.e.

$$
E u \equiv \tilde{v}_{a s} \sqrt{\frac{\tilde{\rho}_{a s}}{\tilde{p}_{a s}}}=\tilde{v}_{l a b} \sqrt{\frac{\tilde{\rho}_{l a b}}{\tilde{p}_{l a b}}}
$$

In addition to this constraint, to ensure the validity of Euler's equations describing the dynamics of the system we must enforce the validity of a fluid treatment (i.e., the system needs to be "collisional"), energy flows due to heat conduction and radiation must be negligible (i.e., the corresponding Peclet number must be large enough) and the viscous dissipation must also be negligible (i.e., the Reynolds number must be large enough). When magnetic fields play a fundamental role, ideal magnetohydrodynamics (MHD) equations control the dynamics of the system. A similar analysis states that two different scaled systems will evolve similarly if, in addition to the previous conditions, the adimensional quantity $B / \sqrt{p}$ is invariant and the ohmic dissipation is negligible (i.e., the magnetic Reynolds number must be large enough). These conditions define the Euler-Alfvén similarity [2]. In some cases, it is possible to weaken some of these assumptions. Indeed, similarity can be extended to systems where radiative mechanisms (radiation cooling/heating, trapping, ionization) are of importance, thus driven by radiationhydrodynamics equations [3,4].

Although the conditions for Euler similarity to hold may seem highly restrictive, many different astrophysical systems can be studied in laboratory experiments [5], like the hydrodynamics of core-collapse supernovae and photoevaporated clouds, dynamics of supernova remnants, accretion disks and columns.

Several examples of astrophysical systems described by these equations are radiative shock waves [6], collision of supernovae remnants [7], hydrodynamics of core-collapse supernovae [8], astrophysical jets [9], photoevaporated molecular clouds (like the pillars of creation) [10] and even systems were magnetic fields play a restricted role, like supersonic accretion columns in binary star systems [11].

\subsubsection{Inertial confinement fusion (ICF)}

The aim of inertial confinement fusion is to produce energy from the nuclear fusion of two light nuclei (usually, deuterium and tritium). In order to generate a net energy gain, the fuel must be heated to extreme (tens of kiloelectronvolts) temperatures and confine it in a volume so enough fusion reactions take place to obtain energy. In ICF, the fuel is imploded so its own inertia compresses and confines it during a short time.

At these conditions, radiative transfer plays an important role in the compression dynamics of the fuel. Moreover, one of the methods of compression, the so-called indirect drive, relies in the production of an X-ray thermal bath inside a hohlraum that will drive the ablation of the external layer of the target and subsequent compression of the fuel. It is clear that a full treatment of radiation and hydrodynamics is required to model these experiments. 


\subsubsection{Plasma-based soft X-ray lasers}

A laser can be briefly described as a light amplifier, via stimulated emission. The simplest theoretical requirement for a laser is to have a system with two energy levels, whose difference in energy equals the photon energy. A population inversion, i.e. there must be more components of the system in the excited (upper level) state than in the lower, must be created by means of a pumping system, i.e. the injection of enough energy in a short time to drive the system out of equilibrium, creating the population inversion. Practically, to reach this population inversion, it is necessary to have a system with at least three energy levels.

In order to amplify soft X-ray lasers, we need to have a system with the required energy difference between levels. One possibility is to use highly charged ions and create a population inversion between two of its optical levels. For example, Ne-like Zinc ( $\mathrm{Zn}^{20+}$ ) amplifies $\lambda=21.2 \mathrm{~nm}$ radiation, while Ni-like Silver ( $\left.\mathrm{Ag}^{19+}\right)$ and Ni-like Molibdenum ( $\left.\mathrm{Mo}^{14+}\right)$ amplify $\lambda=13.7 \mathrm{~nm}$ and $\lambda=18.9 \mathrm{~nm}$ respectively. Thus, in this case, the system in which we will amplify soft X-rays is a plasma, created by an intense infrared pulse that interacts with a target of the required material.

As aforementioned, the pumping system must be powerful enough to drive the level populations out of equilibrium. In the case of plasma-based soft X-ray lasers, terawatt-class lasers can be used. At this power level, the plasma reaches temperatures of the orders of hundreds of electronvolts, and radiative transfer of energy between hot and cold regions of the plasma plays a fundamental role in both the dynamics of the population inversion and the hydrodynamic evolution of the plasma.

For example, in Figure 10.1, temperature maps in false colors of Molybdenum (a), Titanium (b), Silver (c) and Zinc (d) plasmas are shown. There are striking differences in the maximum temperatures attained and its gradients. Radiative cooling of hot regions (where the laser energy is absorbed) is more efficient in high Z elements (Molybdenum and Silver) than in low Z elements (Titanium and Zinc). Thus, the latter show a higher temperature region and strong gradients surrounding it. Further evolution of atomic processes inside the plasma is strongly affected by these differences since collisional rates depend strongly on the temperature. Thus, in order to model these systems, radiative hydrodynamics must be taken into account. 


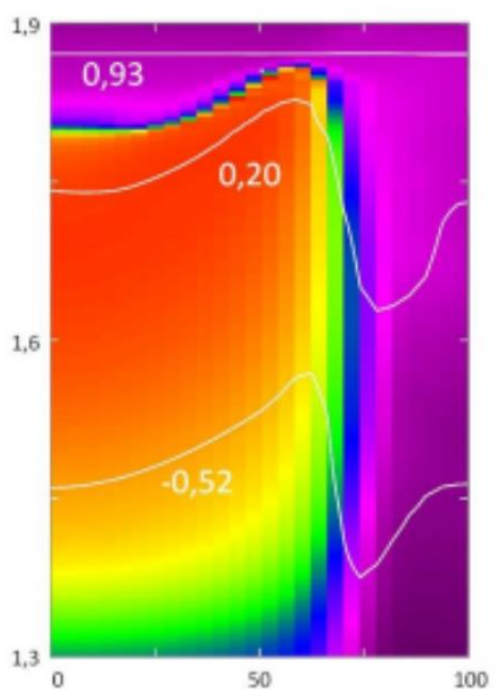

(a) Molibdeno

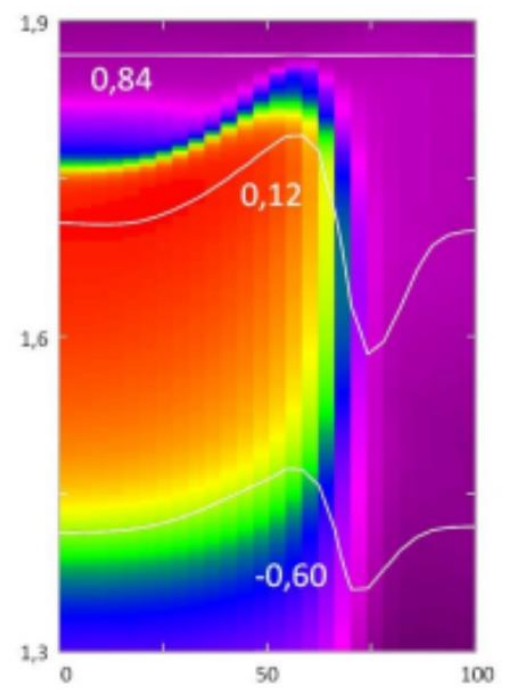

(c) Plata
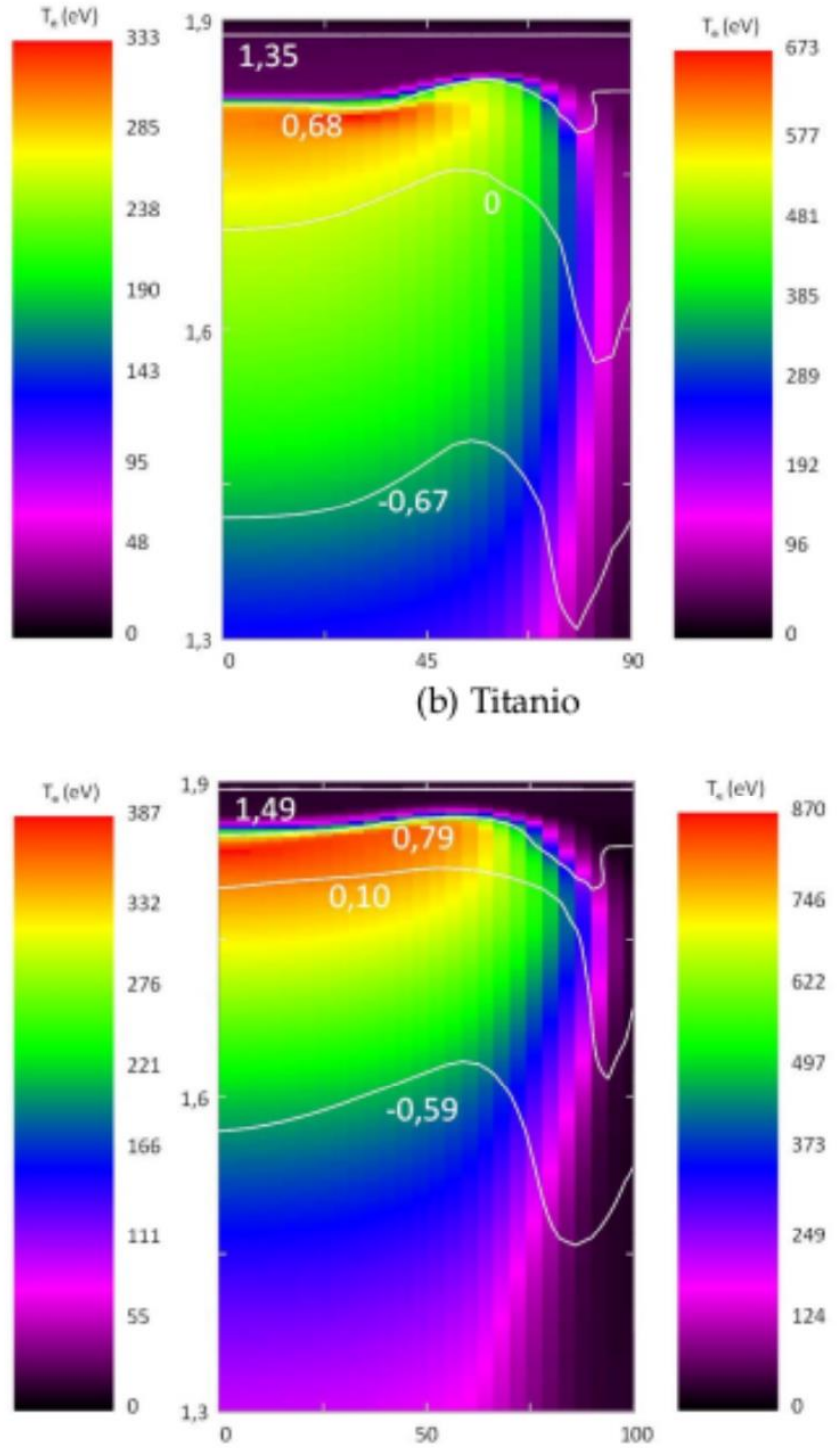

(d) Zinc

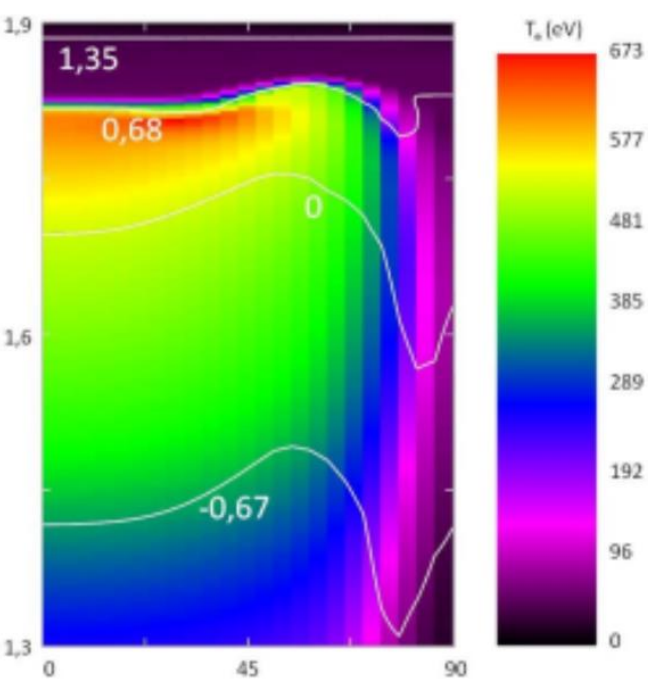

(b) Titanio
Figure 10.1. Temperature maps of Mo, Ti, Ag and Zinc plasmas that will serve as amplifiers of soft X-rays.

\subsection{Radiative hydrodynamics}

\subsubsection{Model}

The number of interactions and processes taking place in a plasma is huge and the modelling of the system must identify the most significant processes and select the correct physics models able to reproduce the phenomena under study. The processes that occur in a plasma range from microscopic scales such as atomic calculations to compute the optical properties of the plasma to macroscopic scales responsible for reproducing the general movement of the plasma based on conservation equations. 


\subsubsection{Two-temperature plasma}

The kind of systems we are going to focus on are based on targets that will be shot by a high intensity and high energy laser that will cause a sudden heating of the matter and will take it to the plasma state. In such conditions, plasma could deviate from equilibrium, and we must adopt a model for the plasma that can include these effects to some extent. In this text we are going to adopt the two-temperature plasma model, where we consider the plasma as a mixture of two species, an electronic fluid and a ionic fluid, each with different thermodynamic properties as temperature, pressure or internal energy. Each specie is in equilibrium within itself but not necessary with the other species. The ionic fluid and the electronic fluid will be related through an exchange of energy due to electron-ion collisions in the plasma that will make the plasma evolve to full equilibrium and eventually reach the same temperature.

This model can be extended to a three-temperature model that includes photons as the third species, but the time scales for the radiation transport enables us to decouple photon transport from the fluidynamics as we will show in next sections.

\subsubsection{Fluid dynamics}

Fluid dynamics has been the paradigm of systems of conservation laws since the pioneer work of Riemann in 1860, rising some of the key ideas in the analysis of quasilineal of hiperbolic partial differential equations. Some of these ideas are [12]:

- The need to consider discontinuous solutions. Even in the case of smooth initial conditions, the solution can develop discontinuities at a finite time.

- The need to impose entropic conditions. Without these conditions, the initial value problem has multiple discontinuous solutons.

- The advantege of the interpretation of the solutions of conservation laws based on patterns of elemental waves.

The dynamic evolution of a fluid is determined by the principles of conservation of mass, momentum, and energy. To obtain a complete mathematical description, the conservation equations must be complemented with constitutive relations of the system that characterizes the properties of the fluid. The properties of the material strongly influence the structure and dynamics of the waves that arise in any continuous mechanical system. Constitutive relationships are studied at the most fundamental level of the fluid and condensed into the equilibrium equation of state (EOS) because the physical principles of thermodynamics impose very restrictive conditions on EOS. It is therefore of great importance to explore the maximum possible range of physical phenomena that occur in fluid dynamics and to recognize their implications for general systems of conservation laws.

The physical model that governs the movement of plasma is based on the conservation of mass, momentum and energy within our system that will form our fluid control volume. These conservation laws take the mathematical form of hyperbolic partial derivative equations of the general form, provided that the properties do not present discontinuities [13,14]. 


$$
\frac{\partial \mathbf{u}}{\partial t}+\sum_{i=1}^{k} \frac{\partial \mathbf{F} \cdot e_{i}}{\partial \mathbf{x}_{i}}=\mathbf{b}(x, t)
$$

where $u$ is the conserved variable, in this case $u \equiv(\rho, \rho \mathbf{v}, \rho e)$. The function $F=F(u(\mathbf{x}, t)$ if the flux function for each conserved variable and represents the rate of exchange of mass, momentum and energy through the border of the control volume. The right-hand side $b=b(\mathbf{x}, t)$ is the volumetric source term for the conserved variables. The integral form of equation (10.6) is more convenient for numerical applications and also accepts more general discontinuous solutions.

$$
\frac{d}{d t} \int_{\Omega(t)} \mathbf{u}(\mathbf{x}, t) d \mathbf{x}+\int_{\partial \Omega(t)} \mathbf{F}(\mathbf{u}(\mathbf{x}, t)) \cdot \mathbf{n} d S=\int_{\Omega(t)} \mathbf{b}(\mathbf{x}, t) d \mathbf{x}
$$

To resume, the conserved variables $u$, flux function $\mathbf{F}$ and source term $b$ are:

$$
\mathbf{u}=\left[\begin{array}{c}
\rho \\
v \rho \\
\left(e+\frac{1}{2} v v\right) \rho
\end{array}\right] \quad \mathbf{F}(\mathbf{u}(\mathbf{x}, t))=\left[\begin{array}{c}
\rho v^{t} \\
v \rho v^{t}+\overline{\bar{I}} p \\
{\left[\rho\left(e+\frac{1}{2} v v\right)+p\right] v^{t}}
\end{array}\right] \quad b=\left[\begin{array}{c}
0 \\
g \rho \\
g v \rho
\end{array}\right]
$$

These are the well-known Euler equations for fluid dynamics where all viscous effect in the plasma are neglected [15]. We have also included the gravity as a mass force that acts on the plasma and affects the conservation of momentum (plasma weight) and the conservation of energy (potential energy). Finally, these contributions will also be neglected as they are several orders of magnitude lower than the main processes in the plasma.

The plasma has other properties in addition to the conserved magnitudes such as the degree of ionization of the material, electronic fluid internal energy or others. These properties are transported with the general motion of the plasma, also called advection.

The most common systems encountered in this field include multiple materials with well-defined interfaces, which we call multimaterial fluids. Each material has differerent EOS and opacities and we need to include variables to fully define the state of the fluid in the control volume. These extra variables added to the conserved magnitudes are the partial density $\rho_{i}$, the partial internal energy $e_{i}$ and the fraction of volume $f_{i}$ occupied by each material. Finally, a control volume containing more than one material will produce interfaces between the different materials present. The variables for each material are related to the conserved variables through these relations:

$$
\begin{aligned}
1 & =\sum_{i=1}^{n} f_{i} \\
\rho & =\sum_{i=1}^{n} f_{i} \rho_{i} \\
\rho e & =\sum_{i=1}^{n} f_{i} \rho_{i} e_{i}
\end{aligned}
$$


In addition to these relations, we must assure the mechanical equilibrium between interfaces of different materials within the control volume $[16,17,18]$. Therefore, the materials that form an interface need to have the same pressure. This equilibrium condition imposes additional restrictions involving the EOS of each material because the pressure is a thermodynamic function that depends on the density and the internal energy of each material.

\subsubsection{Radiation transport}

The particles that compose the plasma (electrons, ions and possibly neutrals) interact with electromagnetic radiation, absorbing and emitting photons through a variety of different processes. Depending on the characteristics of the plasma, the radiation emitted in one region of the plasma might be absorbed in another one, effectively transferring energy from hotter regions to colder regions. Thus, sources and sinks appear in the energy equation of hydrodynamics [19] due to radiation transport. In addition to this, we have to supply our hydrodynamics equations with another one that takes into account the dynamics of these photons.

The transport of neutral particles in a medium is modelled by the so-called Boltzmann equation. In its time-dependent, lineal form, it can be written as:

$$
\frac{1}{v(E)} \frac{\partial}{\partial t} \Psi(x, \Omega, E, t)+\Omega \cdot \nabla \Psi(x, \Omega, E, t)+\Sigma_{t}(x, E, t) \Psi(x, \Omega, E, t)=S(x, \Omega, E, t)
$$

where the angular photon flux $\Psi(x, \Omega, E, t)$ depends in the energy $E$, the position $x \equiv(x, y, z)$, the direction $\Omega \equiv(\phi, \theta)$ and time $t$. A photon propagates through the medium with a speed given by $v(E) . \Sigma_{t}(x, E, t)$ is the collision total cross section for photons and the last term, $S(x, \Omega, E, t)$, takes into account all the processes that act as a source or sink of, in this case, photons with some energy and direction. We can develop further this term:

$$
S(x, \Omega, E, t)=j(x, E, t)+\int_{4 \pi} d \Omega^{\prime} \int_{0}^{\infty} d E^{\prime} \Psi\left(x, \Omega^{\prime}, E^{\prime}, t\right) \Sigma\left(x, \Omega^{\prime} \rightarrow \Omega, E^{\prime} \rightarrow E, t\right)
$$

where $j(x, E, t)$ is the emissivity of the medium. It's temporal dependence is embedded in the evolution of other parameters, as the temperature of the medium.

For many applications in radiation hydrodynamics, it is possible to simplify the Boltzmann equation by only taking into account its stationary solutions. This approximation is valid when the characteristic velocity of the medium is much smaller than that of photons. When this condition holds, we can assume that the photon distribution adjusts instantaneously to the changes of the medium.

\subsubsection{Heat conduction}

In addition to the energy that is carried away by the movement of the fluid and by electromagnetic radiation, free electrons can transfer energy from higher to lower temperature regions. As done with radiation, we can write and solve a transport equation for electrons and add the corresponding sources and sinks to the energy equation [20]. 
The transport of electrons in a plasma can be modelled by a Fokker-Planck equation. The fact that electrons are charged particles makes necessary to introduce long-range electromagnetic interactions with other charged particles. The resulting equation is very complex so in many cases of interest it is worth to solve an approximation to the transport equation: a diffusion equation.

Since the plasma is composed of electrons and ions, we can assume a two-temperature model and write two diffusion equations, one of each species:

$$
\begin{gathered}
\rho c_{v e} \frac{\partial T_{e}}{\partial t}=\nabla\left(\kappa_{e} \nabla T_{e}\right)+S_{e} \\
\rho c_{v i} \frac{\partial T_{i}}{\partial t}=\nabla\left(\kappa_{i} \nabla T_{i}\right)+S_{i}
\end{gathered}
$$

where $c_{v e}, c_{v i}$ are the corresponding specific heat at constant volume, $\kappa_{e}, \kappa_{i}$ the thermal conductivity and $S_{e}, S_{i}$ the corresponding sources and sinks.

As aforementioned, collisions allow the flow of energy among electrons and ions, thermalizing both fluids. This flow of energy can be written as

$$
W_{e i}=w_{e i}\left(T_{e}-T_{i}\right)
$$

where $w_{e i}$ is a coefficient related to the electron-ion collision frequency. This term appears as a source for one fluid and as a sink (i.e. the sign of the term is changed) for the other. Indeed, as written, this term will appear as a sink $S_{e}=-W_{e i}+S_{o t h e r}$ in the electron conduction equation (it will be negative when $T_{e}>T_{i}$ ) and as a source in the ion conduction equation $S_{i}=W_{e i}+$ $S_{\text {other }}$

It is worth mentioning that in several applications, the plasma is heated by laser light. The laser electromagnetic field interacts mainly with electrons, which will absorb its energy. Thus, this energy source can be embedded in the source term of equation (10.12). The next subsection will be devoted to the treatment of the laser energy source.

\subsubsection{Propagation and absorption of laser energy}

As aforementioned, several applications of interest that can be modelled with radiationhydrodynamics imply the propagation of a laser through a plasma. The density gradients will bend the trajectory of the laser, being reflected at the so-called critical density $\rho_{c}=\frac{\epsilon_{0} m_{e}}{e^{2}} w_{l}$ (i.e. where the plasma frequency equals de laser frequency $w_{l}$ ). Part of the energy carried by the laser beam is transferred to the electron fluid. This energy must be taken into account in the corresponding source terms and thus it is required to compute the trajectory of the laser beam and the energy deposited within the plasma.

The propagation of the laser beam can be modelled with the so-called ray-tracing method. The laser beam is divided in "rays" that carry a fraction of the total energy. The trajectory of each ray follows the following equation: 


$$
\frac{d^{2} r}{d s^{2}}=\frac{d r}{d s} \times\left(\frac{\nabla n}{n} \times \frac{d r}{d s}\right)
$$

where $r=(x, y, z)$ is the position, $s$ is the arc length of the curve and $n$ the refractive index. The trajectory mainly depends in the gradient of the refractive index which in a plasma corresponds to the electron density gradient. Solving this equation (or a simplification of it) allows to compute the trajectory of each ray in the plasma and thus that of the laser beam.

Once the trajectory is known, it is possible to compute the energy absorbed by the electron fluid. In the majority of the plasma, the main absorption mechanism is inverse brehmsstrahlung. The energy absorbed is given by $E_{a b s}=E_{0}\left(1-e^{-\kappa_{i b} l}\right)$, where $E_{0}$ is the initial energy, $l$ is the length of the trajectory and $\kappa_{i b}$ is the inverse brehmsstrahlung absorption coefficient, given by:

$$
\kappa_{i b}\left(c m^{-1}\right) \approx 3.4 \frac{Z \ln \Lambda}{\lambda_{\mu m}^{2}\left(k T_{e}\right)_{k e V}^{3 / 2} \sqrt{1-n_{e} / n_{c}}}\left(\frac{n_{e}}{n_{c}}\right)^{2}
$$

where $Z$ is the mean ionization, $\ln \Lambda$ is the Coulomb logarithm, $n_{e}, n_{c}$ are respectively the electron density and critical density, $\lambda_{\mu m}$ is the laser wavelength (in micrometers) and $\left(k T_{e}\right)_{k e V}$ is the electron temperature in energy units (kiloelectronvolt).

\subsubsection{Equation of state}

The physical model described previously requires a relationship between state variables (i.e. $\rho, \mathrm{P}, \mathrm{e})$ in order to be closed. This relationship, which varies from systems and materials, is the socalled equation of state (EOS). The EOS is a thermodynamic relationship between three state variables of the system. To solve the hydrodynamic equations, an EOS in the form $P=P(\rho, e)$ is required. In addition to this, the transport/diffusion equations require the temperature, which can be obtained from the full EOS $e=(\rho, s)$, where $s$ is the specific entropy, via the relationship $T=\left.\frac{\partial e}{\partial s}\right|_{\rho}$ There are several methods to compute the EOS of a material, whether deducing it from first principles (statistical quantum mechanics), time-dependent density functional theory (TDDFT), molecular dynamics (MD) and from interpolation of experimental data, among others.

Usually, the EOS is tabulated so the code that solves the radiation-hydrodynamic equations reads the data in the table and interpolates it to obtain the required value. It is worth mentioning that this interpolation must be done carefully, in order to preserve the properties of the EOS (i.e. monotonicity, convexity...) [21].

\subsection{Interaction of radiation with matter: absorption spectrum}

Our knowledge about plasmas is mostly based on the study of the radiation emitted and absorbed from them. Emitted spectrum tell us about the local temperature and density conditions, giving information about the atomic evolution of the plasma. Absorption spectrum allow us to compute 
opacities and hence radiation transport, needed to study the plasma from an hydrodynamic point of view.

To study plasma properties such as opacity, internal energy, ionization..., etc it is necessary to know the atomic processes (collisionals and radiatives) that take place in it as they determine the atomic population distributions and thereby plasma thermodynamic state. The local thermodynamic equilibrium (LTE) is the simplest scenario, but a non-LTE description is sometimes necessary. LTE can be assumed when collisional processes are dominant over the radiative ones. That happens in plasmas with smaller dimensions than the mean free path, $\lambda_{m f p}$, of the photons emitted from the plasma, i.e., in optically thick plasmas, and the emitted photons are easily reabsorbed, as it will be explained later.

In this section, we will focus on the absorption spectrum, paying attention only to the processes by which plasma particles absorb photons from the radiation field. We consider optically thick plasmas under LTE conditions, close to thermal equilibrium, so that the radiation field can be described by the Planck black-body distribution. For further details see $[22,23,24,25]$.

\subsubsection{Absorption of photons by a material}

Photons emitted from a plasma may be absorbed within the same plasma, what it is called reabsorption, or by other material between the plasma and the detector.

When studying the absorption of photons we mainly find two independent problems. One of them is the absorption of photons by the individual plasma constituents, electrons and ions, what we could call the basic absorption mechanisms. The other is related to the radiation transport of a photon beam inside the plasma. This is a collective effect of the plasma particles, being successively emitted and reabsorbed and it is very important when absorption plays an important role in the energy balance. Here we focus on the basic absorption mechanisms.

Suppose that we want to study the interaction of a beam of photons with a material of thickness $D$. Some of the of the photons (and hence part of the intensity), are absorbed and some are transmitted.

The intensity transmitted in an infinitesimal slice of thickness $d x$ is proportional to the intensity in the beam, to the slice thickness and to the $\kappa(x ; \hbar)$ absorption coefficient of the material:

$$
d I(x, t ; \hbar w)=-\kappa(x ; \hbar) I(x, t ; \hbar w) d x
$$

In an homogeneous material, where the absorption coefficient is independent of the position, the integration of the previous equation yields a decreasing intensity emerging from the material:

$$
I(x=D, \hbar w)=I_{0}(x=0, t ; \hbar w)^{-\kappa(\hbar w) D} .
$$

From this equation it follows that plasmas can be divided into two categories, according to their absorption properties:

If $\kappa(\hbar w) D \ll 1$ (optically thin plasmas), most of the beam intensity is transmitted so only a small part of the photons is absorbed. In this case, the mean free path of the photons emitted from the 
plasma (the distance along the way of the photon, in which, on average, one reaction occurs) is of the order of the plasma dimensions.

If $\kappa(\hbar w) D \geq 1$ (optically thick plasmas), most of the beam intensity is absorbed so a significant part of the photons is absorbed. In this case, the mean free path is shorter than the plasma dimensions, so the probability of reabsorption of photons is reasonably large and LTE can be attained.

The absorption coefficient, $\kappa(\vec{r}, \hbar w)$, describes the attenuation of the intensity of the radiation passing through a material. It is given by the product of the density of absorbing particles, $n(\vec{r})$ and the total absorption cross section, $\sigma(\hbar w)$ :

$$
\kappa(\vec{r}, \hbar w)=n(\vec{r}) \sigma(\hbar w)
$$

where $\sigma(\hbar w)$ is the sum of the cross sections of all the processes that can absorb radiation: photoionization, resonant photoabsorption and inverse bremsstrahlung, and also some scattering processes.

\subsubsection{Absorption mechanisms}

If we are interested in the absorption spectrum we need to know how the different absorption processes contribute to it. But first of all, we need to understand how the plasma constituents interact to give rise to these processes. A plasma consists of three kinds of particles: electrons, ions and photons. Electrons can be divided into bound electrons, when occupying negative-energy states or bound states, and free electrons, which are positive-energy electrons that move freely in the plasma.

There are different ways to classify ionic processes. According to the kind of interacting particles (photon-ion, electron-ion, ion-ion, photon-electron), or to the number of interacting particles (single-particle processes, two-particle processes...), or according to the initial and final state of the absorbing electron (bound-bound transitions, bound free and free-free). Using this latter option, the classification of the absorption processes is:

- Bound-bound (BB) absorption: resonant photoabsorption

- Bound-free (BF) absorption: photoionization

- Free-free (FF) absorption: inverse bremsstrahlung

The absorption spectrum has two parts: the continuous spectrum and the line spectrum.

\subsubsection{Continuous spectrum}

Various processes contribute to the continuous spectrum. The first is the photoionization. In photoionization a photon is absorbed by a bound electron that is ejected into the continuum, ionizing the ion. It is an important process in plasmas if the photon density is high enough to induce large rate of photoionization relative to other processes. This happens in optically thick plasmas while in optically thin plasmas photoionization has a negligibly influence.

This absorption process occurs only above the "edges", that correspond to the binding energies of the bound electrons. The simplest way to define photoionization cross section is: 


$$
\begin{cases}\sigma_{0}\left(\frac{\hbar w_{0}}{\hbar w}\right)^{\alpha} & \hbar \omega \geq \hbar \omega_{0} \\ 0 & \hbar \omega<\hbar \omega_{0}\end{cases}
$$

where $\hbar w_{0}$ is the energy of the edge, $\alpha$ a constant close to three, and $\sigma_{0}$ the cross section at the endpoint of the edge.

The bound-free absorption spectrum has edge structure. The different edges correspond to the binding energy of the bound electrons, which implies that photons with energies lower than this threshold cannot ionize the ion. Looking at this spectrum it is easy to note that just below the absorption edges there is a region where the absorption coefficient is low. That implies that transmission is higher in this spectral region.

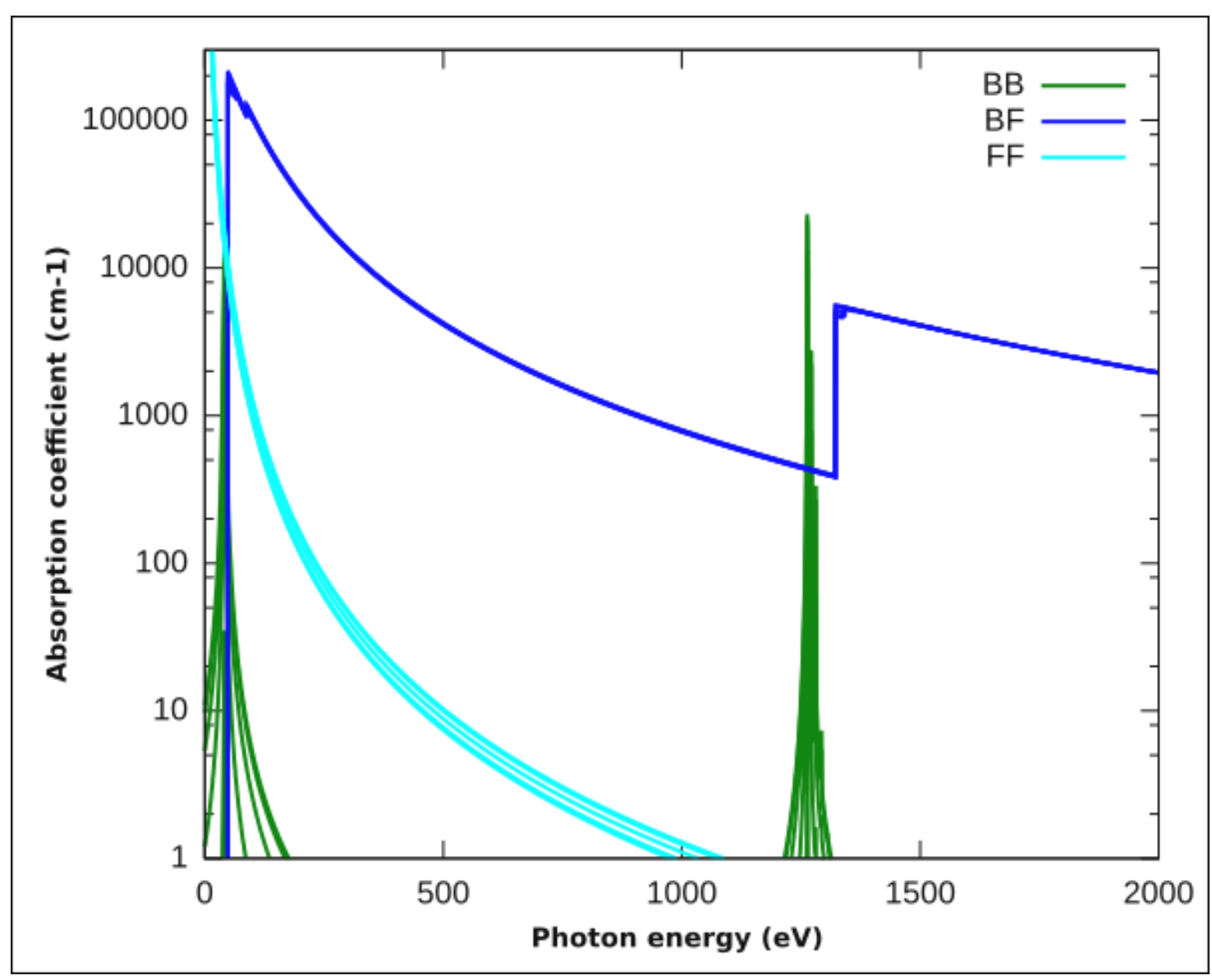

Figure 10.2. Absorption cross sections of an aluminium sample, for various electron temperatures

The second absorption process that contributes to the continuous spectrum is the inverse bremsstrahlung. In this case an electron moving near an ion absorbs a photon from the radiation field. It has influence at very low energies, below the lowest absorption edge. This process does not impinge to the population distribution of bound states but its effect is crucial to determine the energy balance in a dense radiating plasma.

The inverse bremsstrahlung absorption coefficient, $\kappa_{i b}$, decreases as the third power of the photon energy $(\hbar w)$ and can be written as [26]: 


$$
\kappa_{i b}=\frac{32 \pi^{3}}{3 \sqrt{3}} \frac{e^{6}(\hbar c)^{2}}{m c^{2}} \frac{(\hbar w)^{-3}}{\sqrt{2 \pi m c^{2} T_{e}}} \bar{Z}^{2} n_{i} n_{e} g_{i b}(\hbar w),
$$

where $n_{e}$ and $n_{i}$ are de electron and ion densities respectively, $\bar{Z}$ is the average charge state and $g_{i b}$ is the Gaunt factor, that takes into account the quantum mechanical effects and is close to one in most cases.

Since at low energies the wavelength is long, collective absorption by free electrons should be taken into account.

There is a third absorption process that contributes to the continuous spectrum only at very high photon energies, of the order of keV, that is the Compton scattering. Such high energy photons can be found just in femtosecond laser-produced plasmas and in some astrophysical plasmas, and only during highly non-equilibrium state, before thermalizing. Cross section can be calculated as:

$$
\sigma_{s}(w)=\frac{8 \pi}{3} \alpha^{4} a_{0}^{2} \bar{Z}^{2} f(E, T),
$$

where $\alpha$ is the finite-structure constant, $a_{0}$ is the Bohr radius and $\bar{Z}$ is the average charge state (so the average number of free electrons). The distribution function, $f(E, T)$, (where $\mathrm{E}$ is the photon energy and $\mathrm{T}$ the temperature of the plasma, both expressed in $\mathrm{keV}$ ), is an analytical function obtained from an interpolation formula that can be found in [22,27].

\subsubsection{Line spectrum}

Resonant photoabsorption, also called resonant absorption or spectral line absorption, is the most important process in high-Z plasmas, relative to which photoionization and inverse bremsstrahlung provide a continuous background radiation. In the case of high-Z plasmas, most of the radiative energy is concentrated in the line spectrum. In resonant photoabsorption, a photon with an energy equal to the energy difference between two ionic states is absorbed from the radiation field and it induces an upward transition of the ion. This process occurs, mainly, just below the absorption edges, being the main absorption mechanism in that area where the transmission is higher.

In order to account for these transitions between bound states, it is necessary to describe in detail all the possible states of the ions in the plasma and find the probability of realization of such transitions. The cross section for resonant photoabsorption, for the transition from an initial state $i$ to a final state $j$ can be written as:

$$
\sigma_{i j}^{r p}=\frac{\pi e^{2}}{m c} f_{i j} \Psi_{i}(w)
$$

where $f_{i j}$ is the oscillator strength, required to determine the intensity of the transition and for that, we have to compute the wave functions, $\Psi_{i}$, of all the possible ionic states in the plasma. 


\subsubsection{Opacity}

Plasma opacity calculations play an important role in laboratory and astrophysical applications, being necessary in radiation transport codes to better understand the evolution of the produced plasmas.

Dividing the absorption coefficient by the mass density of the plasma, in $\mathrm{g} / \mathrm{cm}^{3}$, one obtains the opacity, $k(\vec{r}, \hbar w)$ in units of $\mathrm{cm}^{2} / g$ :

$$
k(\vec{r}, \hbar w)=\frac{\kappa(\vec{r}, \hbar w)}{\rho(\vec{r})}
$$

Opacity describes the ability of a medium to absorb radiation and so it determines the radiative heat conductivity of matter at high temperatures. Total plasma opacities are due to the absorption of electromagnetic radiation by $\mathrm{BB}, \mathrm{BF}$ and $\mathrm{FF}$ electronic transitions and also electron scattering processes, as Compton effect:

$$
\kappa_{\text {Total }}(w)=\kappa_{\text {abs }}(w)+\kappa_{\text {scat }}(w)=\kappa_{B B}(w)+\kappa_{B F}(w)+\kappa_{F F}(w)+\kappa_{\text {scat }}(w)
$$

Therefore to compute opacity it is necessary detailed information about ion energy levels and wave functions and, for that, we have to consider all the microscopic absorbing processes that take place in the plasma (photoionization, resonant photoabsorption and inverse bremsstrahlung) [28]. Because of the resonant nature of resonant photoabsorption, BB transitions have the largest absorption cross sections. However, for wavelengths far from resonance, they are not important and BF absorption may become dominant.

Because of the large possibility of reabsorption, opacity and radiation transport are significant at high densities or in large plasmas (i.e., in optically thick plasmas) but they can be neglected in optically thin plasmas.

\subsubsection{Planck and Rosseland mean free opacities}

In optically thick plasmas, when radiation is not close to LTE with the plasma, some radiation transport problems arise as it was explained in previous section. But if the plasma is in LTE, it will be possible to make use of important averaged absorption parameters that measure the tendency of a material to absorb radiation of all frequencies:

Rosseland mean opacity, yields the correct value for the integrated energy flux in an optically thick plasma, i.e., when the system approaches to equilibrium. The largest contributions to this opacity comes from the regions with small $\kappa(\hbar w)$, that is, the more transparent regions. It is calculated from $\kappa_{v}^{\text {total }}$ in the following way:

$$
\frac{1}{\bar{\kappa}_{R}}=\frac{\int_{0}^{\infty} \frac{\partial B_{w}(T)}{\partial T} \frac{1}{\kappa_{w}^{T o t a l}(\rho, T)} d w}{\int_{0}^{\infty} \frac{\partial B_{w}(T)}{\partial T} d w}
$$

where $B_{v}(T)$ is the normalised Planck function. 
Planck mean opacity, also called "emision mean" opacity. It yields the correct value of the integrated thermal emission in an optically thin plasma. Unlike Rosseland m.o., it emphasizes the regions of large $\kappa(\hbar w)$, that is, the more opaque spectral regions. It is obtained from the total absorption opacity:

$$
\bar{\kappa}_{P}=\frac{\int_{0}^{\infty} B_{w}(T) \kappa_{w}^{a b s}(\rho, T) d w}{\int_{0}^{\infty} B_{w}(T) d w}
$$

where $\kappa_{v}^{a b s}$ is the spectral absorption coefficient and $B_{v}(T)$ is the normalised Planck function.

\subsection{Conclusions}

Many physical systems of interest in science and engineering (astrophysical systems and laboratory astrophysics, Inertial Confinement Fusion, plasma based soft X-ray lasers, etc) are described by the so-called radiative hydrodynamics equations. The dynamics described by this set of equations involve a fluid description of the plasma, using Euler equations. This description is enhanced with energy sources and sinks and their corresponding transfer equations: electron heat conduction, radiation transport and laser energy propagation and deposition. The set of equations is closed by adding the equation of state (EOS) of matter.

All these processes are solved sequentially, updating the matter properties and transport coefficients so the accuracy is high enough. Indeed, not only an excellent agreement with experiments is usually found but also radiative hydrodynamics models have predictive capabilities.

\section{Acknowledgments}

The authors acknowledge the funding and networking activities carried out within the EU funded COST Action CA17126 (TUMIEE). E.O. acknowledges the Universidad Politécnica de Madrid and Comunidad Autónoma de Madrid, línea de actuación estímulo a la investigación de jóvenes doctores, project CROM and the Spanish Ministerio de Ciencia e Innovación through a Ramón y Cajal RYC2018-026238-I fellowship.

\section{References}

[1] D. Ryutov, R. P. Drake, J. Kane, E. Liang, B. A. Remington and W. M. Wood-Vasey, Similarity criteria for the laboratory simulation of supernova hydrodynamics, Astrophys. J. 518 (1999)

[2] D. D. Ryutov, R. P. Drake and B. A. Remington, Criteria for scaled laboratory simulations of astrophysical MHD phenomena, Astrophys. J., Suppl. Ser. 127 (2000)

[3] J. E. Cross, B. E. Reville and G. Gregori, Scaling of magneto-quantum-radiative hydrodynamic equations: from laser-produced plasmas to astrophysics, Atrophys. J. 795 (2014)

[4] É. Falize, C. Michaut and S. Bouquet, Similarity properties and scaling laws of radiation hydrodynamic flows in laboratory astrophysics, Astrophys. J. 730:96 (2011) 
[5] B. A. Remington, R. P. Drake and D. D. Ryutov, Experimental astrophysics with high power lasers and Z pinches, Rev. Mod. Phys. 78 (2006)

[6] M. Cotelo, P. Velarde, A. G. De la Varga, D. Portillo, C. Stehlé, U. Chaulagain, M. Kozlova, J. Larour, F. SuzukiVidal, Simulation of radiative shock waves in Xe of last PALS experiments, High Energy Density Phys. 17 (2015)

[7] P. Velarde, D. García-Senz, E. Bravo, F. Ogando, A. Relaño, C. García and E. Oliva, Interaction of supernova remnants: From the circumstellar medium to the terrestrial laboratory, Phys. Plasmas 13, 092901 (2006)

[8] D. Arnett, Supernovae and Nucleosynthesis, Princeton University Press (1996)

[9] J. M. Foster, B. H. Wilde, P. A. Rosen, T. S. Perry, M. Fell, M. J. Edwards, B. F. Lasinski, R. E. Turner and M. L. Gittings, Supersonic jet and shock interactions, Phys. Plasmas 9, 2251-2263 (2002)

[10] J. H. Oort and L. Spitzer, Acceleration of interstellar clouds by O-type stars”, Astrophys. J. 121, 6-23 (1955)

[11] J. E. Cross et al, Laboratory analogue of a supersonic accretion column in a binary star system Nature Communications 7:11899 (2016)

[12] Menikoff, R. and Plohr, B. The Riemann problem for fluid flow of real materials. Rev. Mod. Phys. 61, 1, 75-130 (1989)

[13] R. J. LeVecque, Numerical methods for conservation laws, Birkhäuser, 1992

[14] R. J. LeVecque, Finite volume methods for hyperbolic problems, Cambridge University Press, 2002

[15] E. F. Toro, Riemann solvers and numerical methods for fluid dynamics, Springer-Verlag 1999

[16] D. Portillo, Desarrollo de un método numérico para flujos multimaterial en 2D en ARWEN, PhD thesis, Universidad Politécnica de Madrid (2015)

[17] Colella, P., Glaz, H. M. and Ferguson, R. E., Multifluid algorithms for eulerian finite difference methods, (1996)

[18] Miller, G. H. and Puckett, E. G., A high-order Godunov method for multiple condensed phases, J. Comput. Phys. 128, 134 (1996)

[19] Ogando, F. and Velarde, P., Development of a radiation transport fluid dynamic code under AMR scheme, J. Quant. Spectrosc. Rad. Transf. 71 (2001)

[20] Cotelo, M. Mejora y optimización de un código de simulación hidrodinámico con transporte de radiación para el estudio de sistemas en condiciones de alta densidad de energía, PhD thesis, Universidad Politécnica de Madrid (2014)

[21] Cotelo, M., Velarde, P., de la Varga, A. G. and García Fernández, C., Equation of State for laboratory astrophysics applications, Astrophysics and Space Science 336 (2011)

[22] A. Nikiforov, V.Novikov, and V. Uvarov, Quantum-Statistical Models of Hot Dense Matter: Methods for Computation Opacity and Equation of State (Birkhäuser, 2005).

[23] G. J. Tallents, An Introduction to the Atomic and Radiation Physics of Plasmas (Cambridge University Press, 2018).

[24] D. Salzmann, Atomic Physics in Hot Plasmas (Oxford University Press Inc, 1998).

[25] K. Siegbahn (Eds.), Alpha-, Beta- and Gamma-Ray Spectroscopy (Elsevier Science, 1965).

[26] Y. Zeldovich and Y. Raizer, Physics of Shock Waves and High Temperature Hydrodynamic Phenomena (Academic Press, New York, 1966).

[27] P. A. D. Sampson D. H., Electron-impact cross sections for complex ions, 28, 323-334 (1974).

[28] U. Aharony and R. Opher, Radiation opacity in a dense plasma, 79, 27-30 (1979) 



\title{
11. FEM Modeling and Simulations in Laser Matter Interactions and Plasma MHD problems
}

\author{
Vasilis Dimitriou*
}

The dynamic response of matter irradiated by a laser pulse source or loaded by an electric current, depends on its thermomechanical properties, as well as on the characteristics of the loading forces. The numerical modelling and simulations can describe these interactions in detail. A method able to compute time-dependent displacements, stresses, and temperature gradients at all the points of the computational domain is the Finite Element Method (FEM). FEM has no limitations by the geometrical characteristics of the computational domain compared to other analytical or numerical methods [1,2]. Because of these numerical advantages FEM will be here introduced and briefly presented. Furthermore, the FEM modelling strategy discussed in section 11.1 is extended in section 11.2 to the computational study on the plasma generation, on its features and properties.

\footnotetext{
*Contact: dimvasi@hmu.gr
} 


\subsection{The finite element method}

The finite element method is a numerical method that had initially been developed for the branch of solid mechanics. Today, the finite elements are commonly used to solve multiphysics problems. The advantages of the method can be summarized to the handling of complex shape of model geometries and various loading and boundary conditions, the unrestricted type of multiphase materials, the easily refinement and improvement of the analysis accuracy using various element types and approximation schemes and the ability of introducing static or time dependent dynamic effects. In general, the types of problems that scientists aim to solve may be hardly categorized, since a short list would include the: i. structural analysis problems: a cantilever beam, a building, a bridge, etc.; ii. solid mechanics problems: a gear, an air wing, etc.; iii. dynamic problems: vibration of a membrane, of a tower, an earthquake, etc.; iv. thermal analysis problems: heat conduction, radiation of a surface, etc.; v. electrical analysis problems: electrical signal propagation, piezoelectric actuators, etc.; vi. biomechanical problems: human organs, bones, tissues, etc.; vii. fluid mechanical problems; viii. electromagnetic analysis problems... and these are only some of the basic fields that FEM numerical simulations are applied. The multiphysics problems may include some or even all these categories of problems, that may be mathematically described by the help of differential equations.

The computational scheme of the FEM is commonly used to solve field problems. The basic numerical idea of FEM is the division of the computational domain into a finite number of finite elements. In the elements, interpolation functions are used with assumptions on the variation of the unknown dependent variables. This approximated variation is quantified in terms of solution values at the nodes of the finite elements. This process is called discretization or mesh generation and leads to the setup of an algebraic system of equations for certain unknown nodal values that approximate the continuous solution. Figure 11.1 shows a two dimensional (2D) discretized domain. Triangular finite elements are used having three nodes on their vertices.

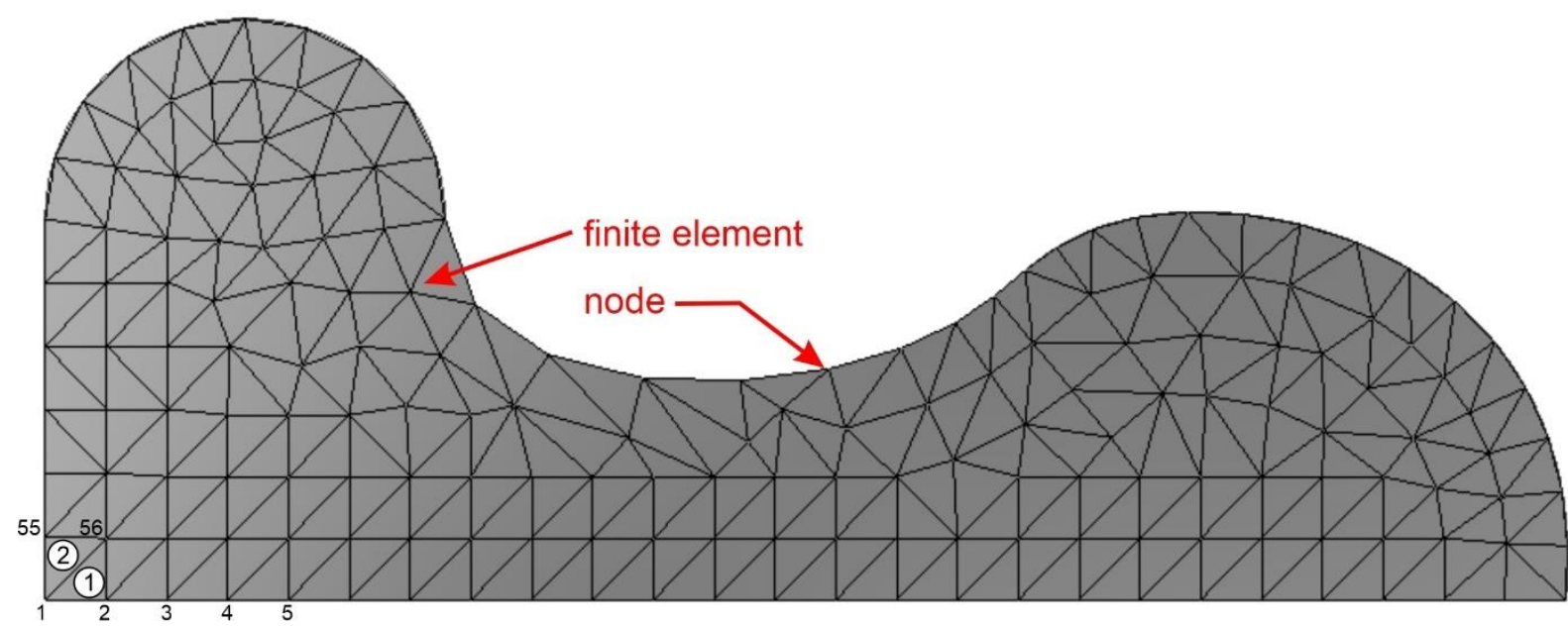

Figure 11.1. A 2D discretized domain by triangular finite elements. The details of the elements and nodes numbering, in circles at the center of the elements and next to the nodes respectively, are highlighted. 


\subsubsection{A classic problem for FEM}

To demonstrate the mathematical modelling philosophy of the finite element method, we assume a solid having known material properties and a well-defined continuous geometry, as presented in Figure 11.2. For this axisymmetric solid, the known data that define it can be summarised in four categories:

i. The boundaries, that are schematically described by a blue line (2D), or the light grey surface enclosed by the blue lines (3D)

ii. The interior of the solid, a dark gray surface (2D) or a dark gray volume (3D)

iii. The boundary conditions, that are prescribed quantities (e.g. prescribed displacements, velocities, stresses, strains etc.)

iv. The loading conditions, that may be a force, pressure, thermal source, electro, magnetic loads etc.
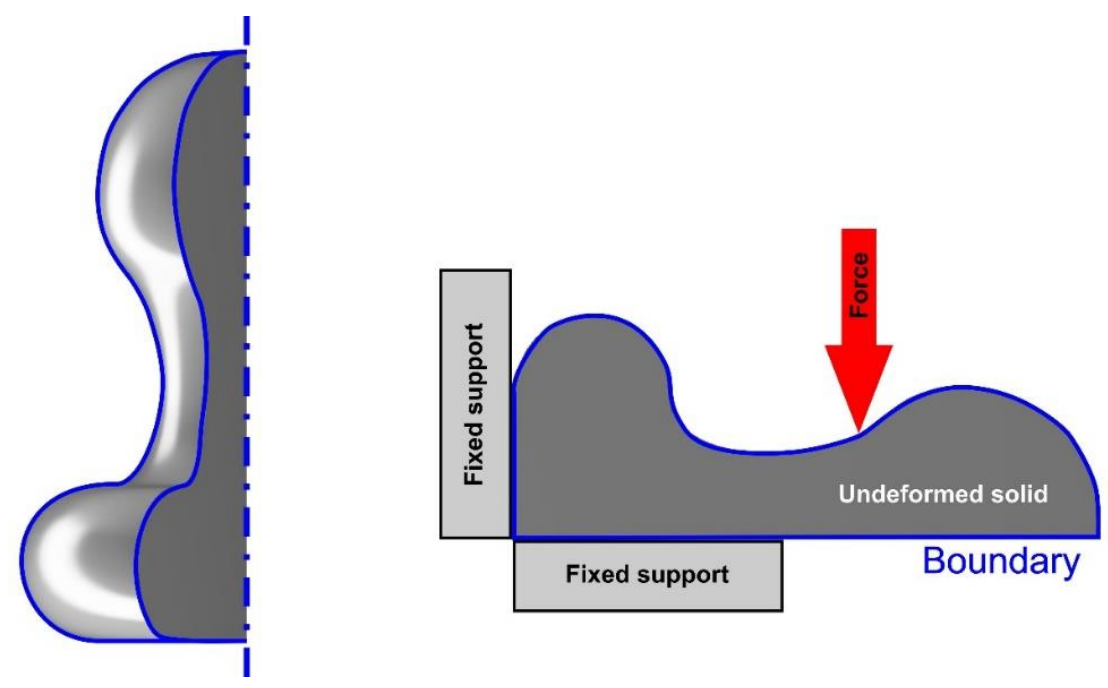

Figure 11.2. A force acting on a solid.

FEM can describe what will happen to the well-defined solid of Figure 11.2 if the known red force is applied and compute the values of the displacements, the stresses, the strains at every point of the solid body [1,2]. The method demands the input data of the categories i-iv given as a mathematical input to the numerical method that may compute the numerical output and schematically represent it for the whole solid body as shown in Figure 11.3.

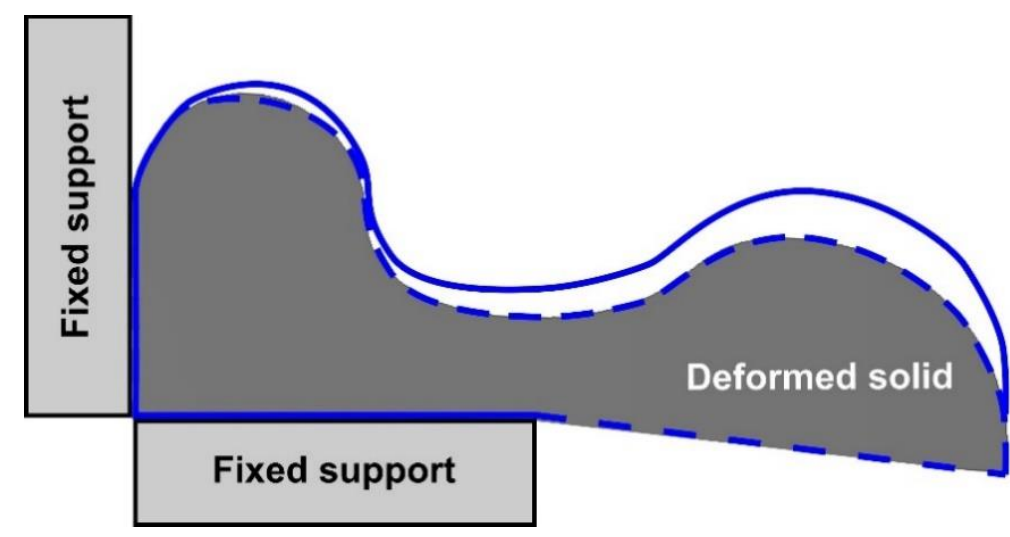

Figure 11.3. The deformed solid. 


\subsubsection{The FEM modeling and simulation process}

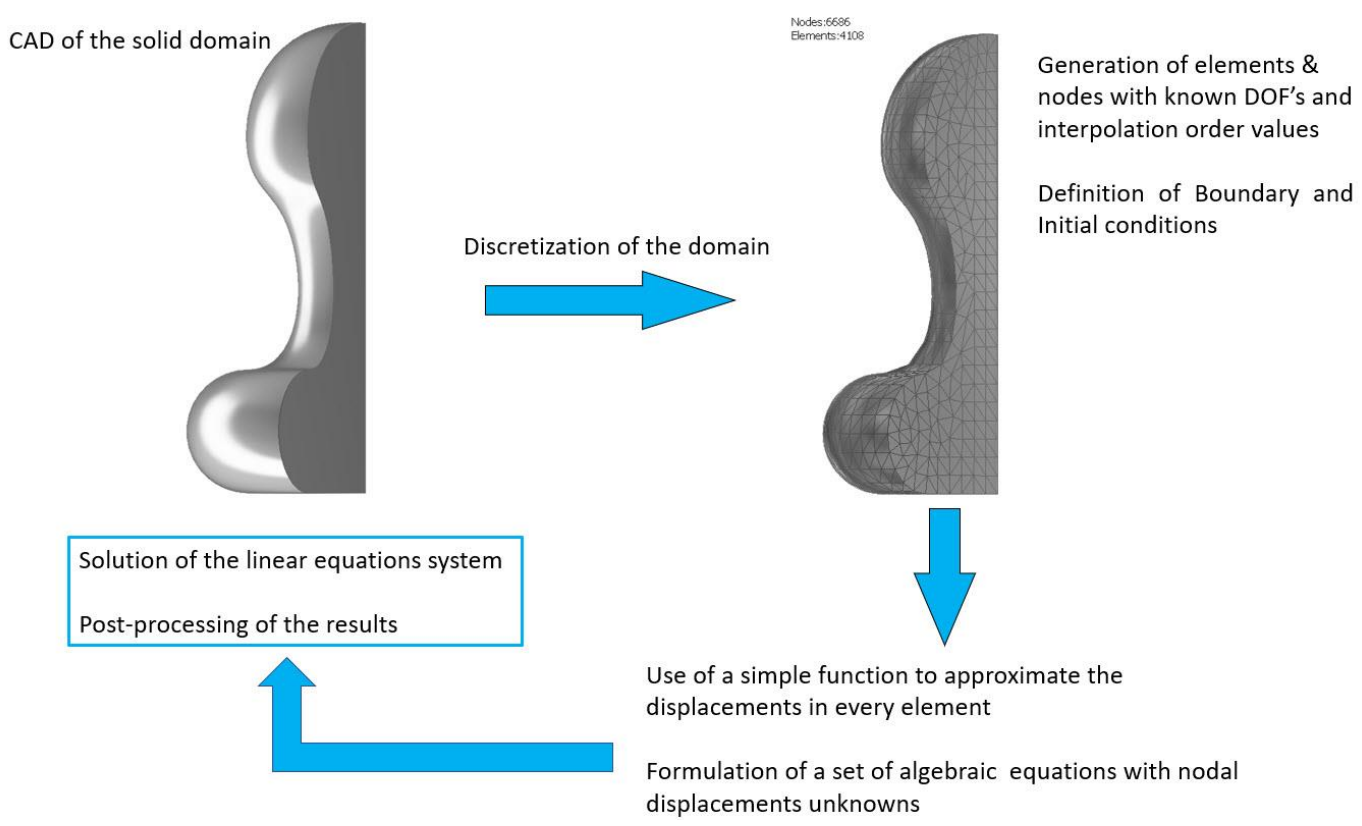

Figure 11.4. The basic modeling steps of FEM.

The basic modeling and simulation steps of FEM are presented in Figure 11.4. The solid domain is geometrically represented, usually by the help of a Computer Aided Design (CAD) system and is further discretized, as shown in Figure 11.4. The finite elements are generated by the help of the nodes that have known Degrees Of Freedom (DOF's) and interpolation order values. A simple function is used to approximate the displacements in every element and a set of linear equations with displacements at each node as unknowns is formulated and finally the algebraic system of equations is solved. The flowchart presented in Figure 11.5 describes these basic steps. It must be noticed that the blue arrows denote that the flow direction of the process is never in one way. After the completion of each step, even if the solution is achieved, cross checks and comparisons to analytical or experimental established results are needed for corrections, updates, refinements etc to take place and lead the FEM simulation to accurate results.

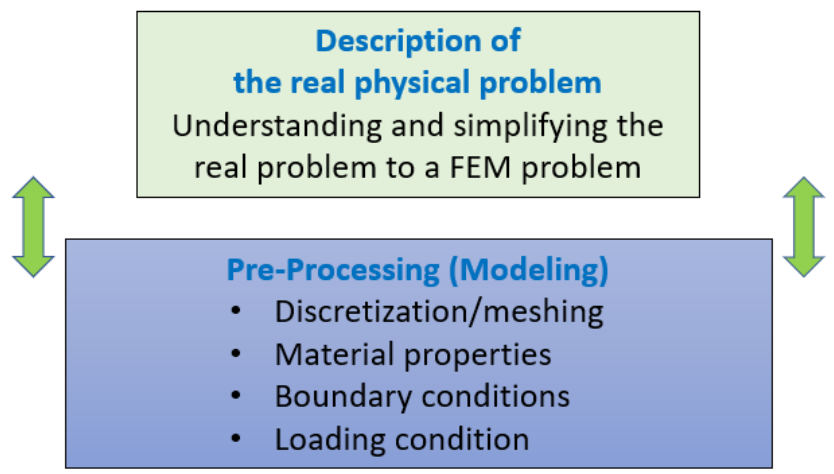

Processing (Solver)

- Approximation functions Formulation of equations

- Solution of the equations system
Post-processing (Results) Obtain, Visualize the Results. Understand/Explain the Results.

Figure 11.5. Flowchart of the FEM modeling and processing (pre- and post- processing). 
To conclude, a FEM analysis demands the type of analysis, the geometry of the domain that is given by mesh nodes, the type of the finite elements, the material properties, the boundary conditions and if needed for transient simulations, the time functions.

\subsubsection{Understanding and describing the physical problem}

The numerical simulations are performed to describe the real physical problems. The physical models represent the real problems to FEM problems. During the development of a physical model the real problem must be understood, and the computational cost of the model simulation must be considered. A very detailed mathematical description in a FEM model may introduce a huge computational cost in the FEM simulation and even make its solution impossible. This computational cost depends on the number of nodes generated, their numbering and on their degrees of freedom that influence the decompositions of the matrices and the number of the algebraic operations that will be done, on the number of interpolation and integration points used in the elements and the type of the analysis. The type of analysis that will be performed can be static steady state, dynamic transient, linear or nonlinear, modal, harmonic, 2D, 3D, implicit, explicit etc. and must be defined during the development of the physical model [1-3].

The basic mathematical concept of the FEM is based on the physical assumption that any continuous solution field such as displacement, stress, temperature, pressure, etc. can be approximated by a discrete model composed of a set of piecewise continuous functions defined over a finite number of subdomains (therefore FEM is a finite method). An example of such an approximation is shown in Figure 11.6, for an 1D thermal problem with a known analytical solution [1-3]. The exact solution $\mathrm{T}(\mathrm{x})$ is plotted in Figure $11.6 \mathrm{left}$, and the approximation piecewise linear solution in Figure 11.6 right.
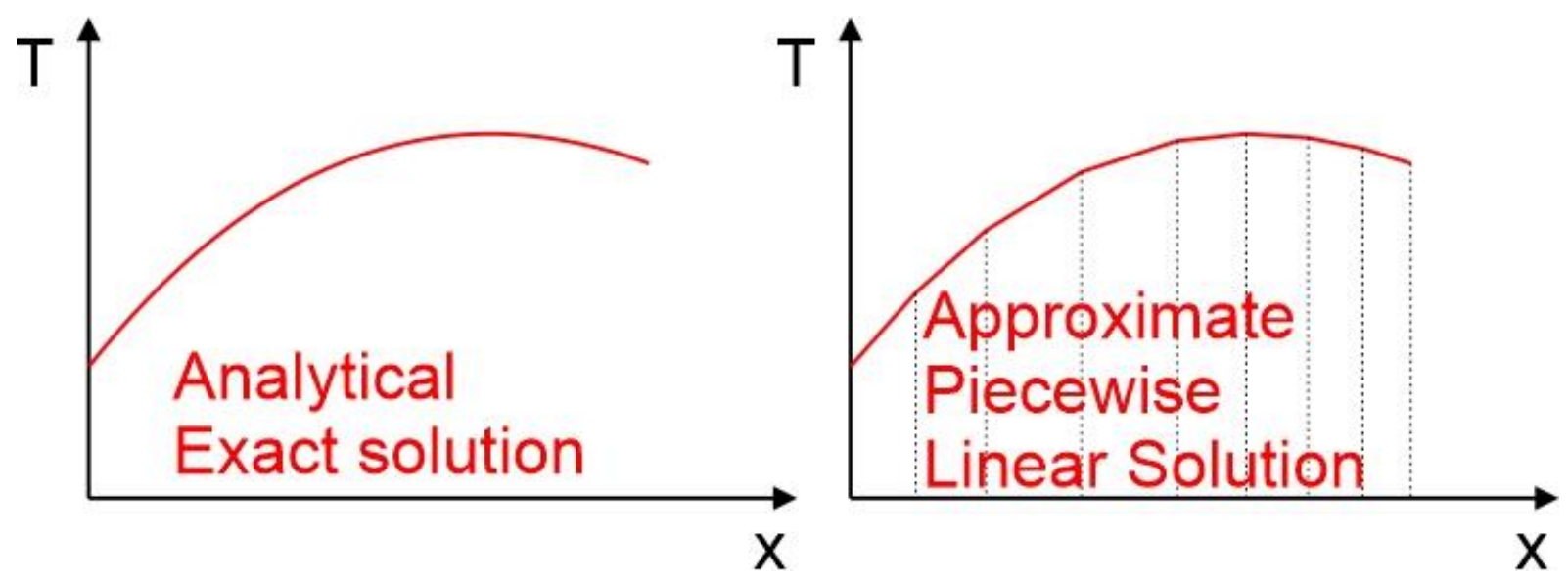

Figure 11.6. Approximation of an exact solution.

In order to model this 1D heat transfer problem using FEM, various approximations can be applied, following the FEM simulation process described in 11.1.2 aiming to numerically describe the physical model. Some representative discretization schemes that can be applied are shown in Figure 11.7. The 1D domain is on the $\mathrm{X}$ axis and is divided in four and two segments in order to be interpolated by four (linear) or two (quadratic) elements. As it can be noticed, the C0 or C1 continuity on the common nodes of the elements, will further affect the continuity of the solution approximation. The chosen order of interpolation in the $\mathrm{k}$ element corresponds to the shape 
function that will be further used to approximate the solution. Every finite element is characterized by the number of nodes that define it and the correspondent degree of interpolation in relation to the shape functions. Typical types of elements for 1D, 2D and 3D computational domains and the linear and quadratic shape functions are presented. When a $\mathrm{p}^{\text {th }}$ order of approximation is used, there are $\mathrm{N}_{\mathrm{p}}+1$ global nodes.

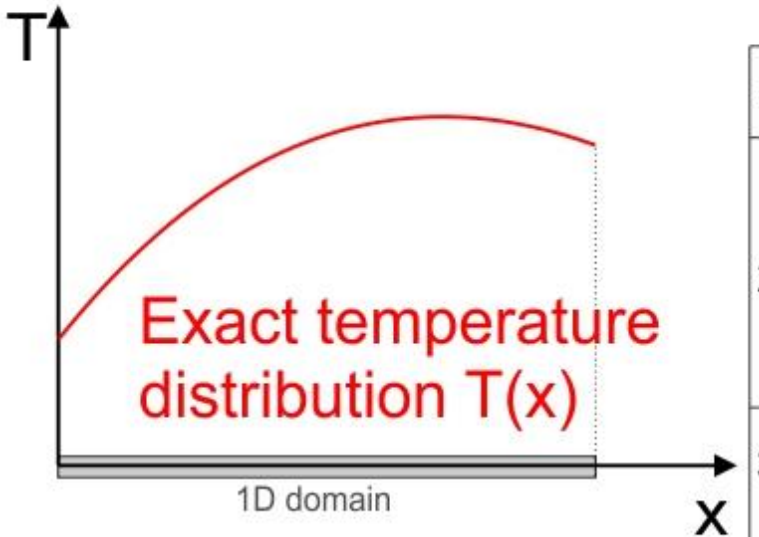

finite elements and shape functions
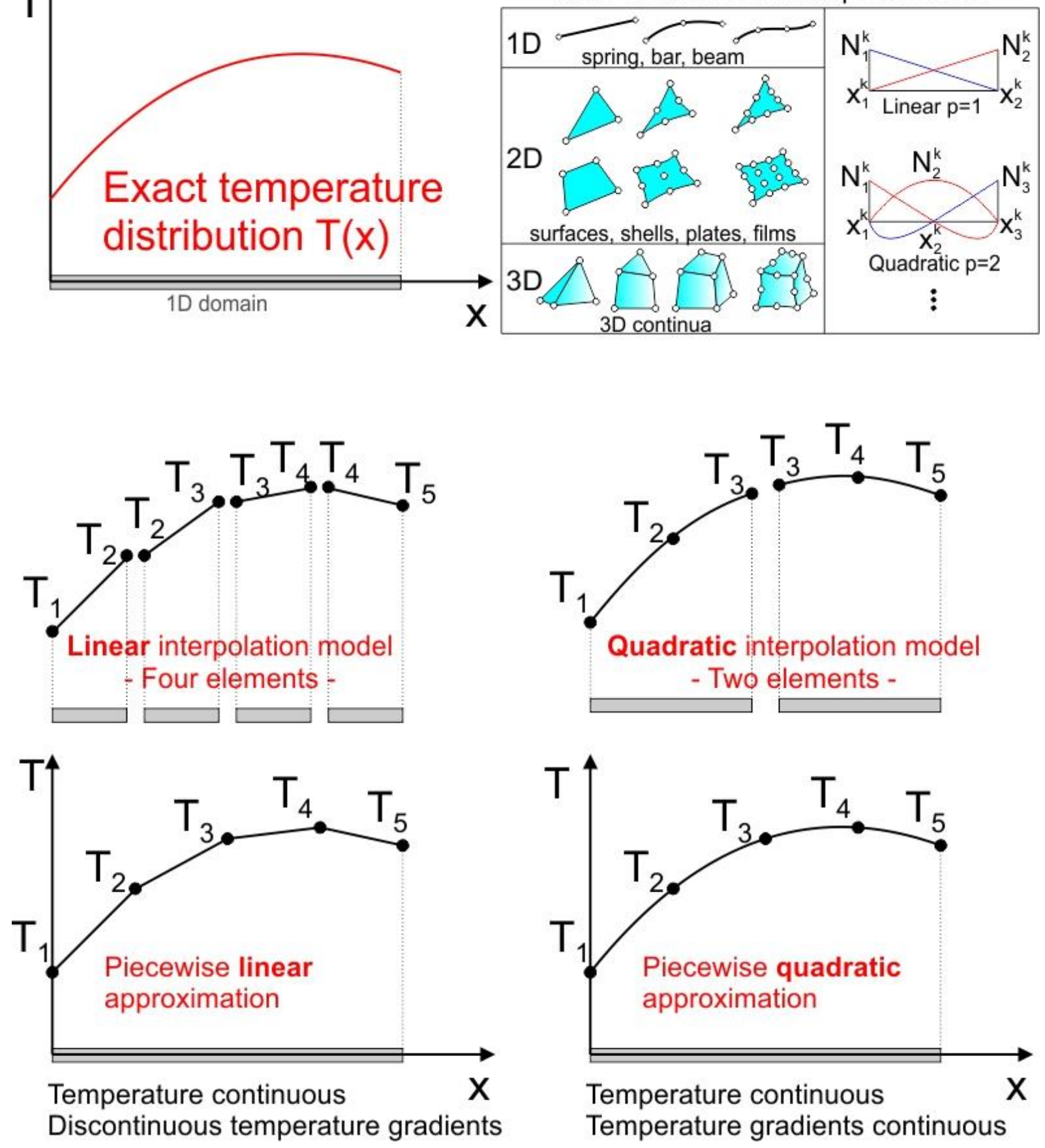

Figure 11.7. Discretization, element types and shape functions. 
Following the modeling steps of the FEM and after the domain discretization and the element type selection, the finite element equations (Variational and Energy methods) are derived. The element equations are then assembled to form a global system of equations, that for time independent problems results in a matrix form to:

$$
[K]\{U\}=\{F\}
$$

Where $[K]$ is the stiffness (properties) matrix, $\{U\}$ is the unknown nodal displacements vector and $\{F\}$ the nodal forces vector. The boundary and the initial conditions are further incorporated to the system of (11.1). Finally, this assembled system of equations is solved for the primary unknows, the nodal displacements and the secondary unknowns of the stress and strain values. The FEM equations to be solved must include all of the appropriate physics of the physical problem and are commonly developed using Direct, Variational-Virtual Work or Weighted Residual Methods. The direct method is based on physical reasoning and enhances the physical understanding of the process. The variational-virtual work method is based on the concept of virtual displacements and leads to relations between internal and external virtual work and to minimization of system potential energy for equilibrium. The weighted residual method starts with the governing differential equation, special mathematical operations develop the "weak form" that is incorporated into a FEM equation. This method is particularly suited for problems that have no variational statement. For stress analysis problems, a Ritz-Galerkin weighted residual method results identically to the variational methods results [1-3].

\subsubsection{Materials excitation modeling and simulations by FEM}

The pulsed laser beams are today a precious tool used for the irradiation of targets of different material properties. Many applications that demand alteration of the surface of the irradiated material are now based on this method [4-14].

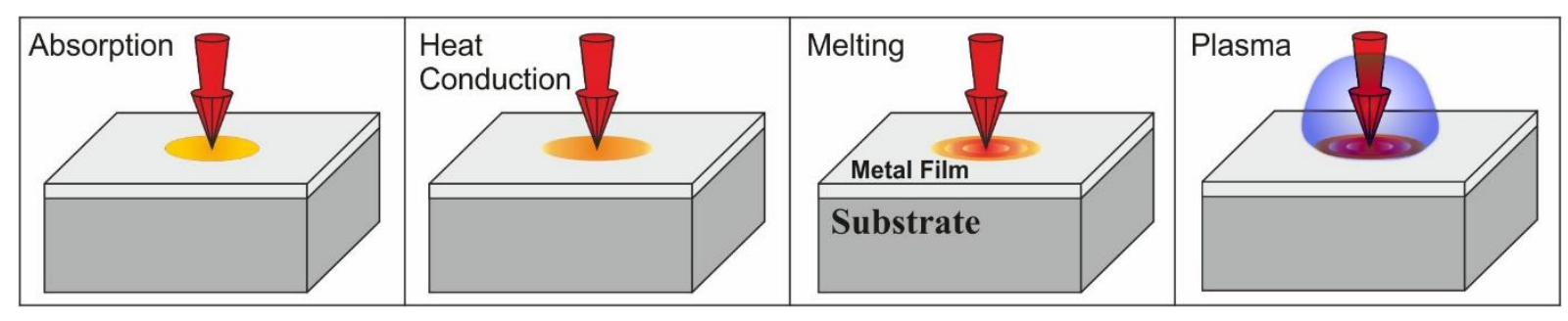

Figure 11.8. The thermoelastic, (absorption and heat conduction), the melting and the plasma phase change regimes of pulsed laser film-substrate interaction.

There are three basic regimes when a laser interacts with a solid and deposits energy to it. These regimes are shown schematically in Figure 11.8 and are namely the thermoelastic, the melting and ablation/plasma regime. At the beginning of the interaction of a nanosecond laser pulse with a solid, the solid is heated and absorbs the laser energy. The material properties of the target and mainly the parameters of absorption and reflection, the thermal conductivity and the specific heat of the metal target determine the way that the solid target will react. The energy absorbed by the solid target expands locally the matter and fields of stress and ultrasonic surface acoustic waves (SAWs) propagate the surface and volume of the solid [15-17]. If the intensity of the laser is 
increased the melting point of the material is reached and the solid changes phase and thus the material properties change. The same holds when the boiling point is reached, if the intensity of the laser is increased more and furthermore, when material removal takes place the laser ablation is achieved and finally, above the ablation threshold, plasma is formed [18-26]. During these procedures, SAWs are generated and propagate the target. The formation of the laser plasma includes electrons, ions and excited neutrals that absorb the laser light.

\subsubsection{FEM mathematical modeling for the simulation of irradiated materials by nanosecond laser pulses}

This FEM model was originally presented in [24-26] and is based on the governing PDE of the problem, the thermal conduction equation:

$$
\rho(T) C_{\rho}(T) \frac{\partial T(x, y, z, t)}{\partial t}-\nabla[k(T) \nabla T(x, y, z, t)]=Q(x, y, z, t)-L_{i}
$$

where, $T$ is the transient temperature, $k$ is the thermal conductivity, $C_{p}$ is the specific heat, $Q$ is the heat source and $L_{i}$ is the latent heat (if $T<T_{m}$ then $L_{i}=0$, if $T \geq T_{m}$ then $L_{i}=L_{m}$, if $T \geq T_{b}$ then $L_{i}=L_{v}$, where $T_{m}$ and $T_{v}$ are the melting and vaporization temperatures). The structural mechanics problem is described by:

$$
\rho \frac{\partial^{2} U(x, y, z, t)}{\partial t^{2}}=\mu \nabla^{2} U(x, y, z, t)+(\lambda+\mu) \nabla[\nabla U(x, y, z, t)]-\mathrm{a}(3 \lambda+2 \mu) \nabla T(x, y, z, t)
$$

where, $\rho$ is the density, $U$ is the unknown displacements vector, $\lambda, \mu$ are the Lamé constants and a is the coefficient of the thermoelastic expansion. A Gaussian type of nanosecond laser heat source is used to introduce the loading that is described by:

$$
Q(x, y, z, t)=I_{s}(t)(1-R) \exp \left(-4 \ln 2\left(t / t_{0}\right)\right) \exp \left(-\left(x^{2}+y^{2}\right) / r_{0}^{2}\right) a \exp (-a z)
$$

$I_{S}$ is the laser pulse energy per unit area per second, $R$ is the optical reflectivity of the sample, $\alpha$ is the optical absorption coefficient and $1 / \alpha$ is the optical penetration depth, $t_{0}$ and $r_{0}$ are the FWHM laser pulse duration and the beam radius on the sample surface, respectively. To include the plasma absorption, the density of the absorbed radiation energy $E_{a}$ is used [27] and in this regime the temporal laser irradiance may be given by:

$$
I_{s}(t)=I_{0} e^{-\Lambda(t)}, \Lambda(t)=b h(t)+d E_{a}(t)
$$

where, $I_{0}$ is the incident laser pulse energy per unit area per second, $\Lambda(t)$ is the optical thickness of the ablation plume, $h$ is the ablation depth, $\mathrm{b}$ and $\mathrm{d}$ are free parameters, time independent coefficients, to be identified.

In the terms of the FEM approach, with the heat capacity matrix $[C]$ and the conductivity matrix $[K]$, the heat source vector $\{Q\}$, the temperature vector $\{T\}$ and the temperature rate vector $\{\partial T / \partial t\}$, the thermal conduction equation can be expressed in terms of vectors by: 


$$
[K]\{T\}+[C]\left\{\frac{\partial T}{\partial t}\right\}=\{Q\}
$$

When temperature exceeds the melting point, $L_{m}$ is subtracted from $\{Q\}$, and the same holds for $L_{v}$. By the same means, if damping is ignored and with the mass matrix [M], the stiffness matrix $[S]$, the displacements vector $\{U\}$, the acceleration vector $\left\{\partial^{2} \mathrm{U} / \partial \mathrm{t}^{2}\right\}$ and $\{F\}$ the force vector, the wave propagation equation is given by:

$$
[M]\left\{\frac{\partial^{2} U}{\partial t^{2}}\right\}+[S]\{U\}=\{F\}
$$

Using the thermal strain vector $\left\{\varepsilon_{0}\right\}$, the transpose of the derivatives of the shape functions $[B]^{T}$ and the material matrix $[D]$, an external force vector for an element can be expressed by:

$$
\{F\}=\int_{V}[B]^{T}[D]\left\{\varepsilon_{0}\right\} d V
$$

The thermal and the mechanical fields are coupled for the solution of this $3 \mathrm{D}$ transient multiphysics problem [28]. The previously mentioned input data are given once in the beginning and thus the computational cost is decreased. A coupled-field 3D solid element type is used with eight nodes, having up to six degrees of freedom. The weak field coupling is performed by the computation of the finite element matrices $[25,28]$.

\subsubsection{FEM results}

Following the steps described at the previous sections, the physical model is simplified to reduce the computational cost. Therefore, the quarter symmetry of the real problem is adopted. The 3D symmetric model presented in Figure 11.9 simulates the laser matter interaction with the solid thin film-substrate, to describe the target's transient thermal-structural response. The target is manufactured by a Golden (Au) thin metal film of $0.6 \mu \mathrm{m}$ thickness deposited on a glass (BK7) substrate of $200 \mu \mathrm{m}$ thickness. This model is capable to simulate the laboratory experiments performed and is validated by the resulting experimental data in every regime and under various loading conditions, as originally published in [25,26,29].

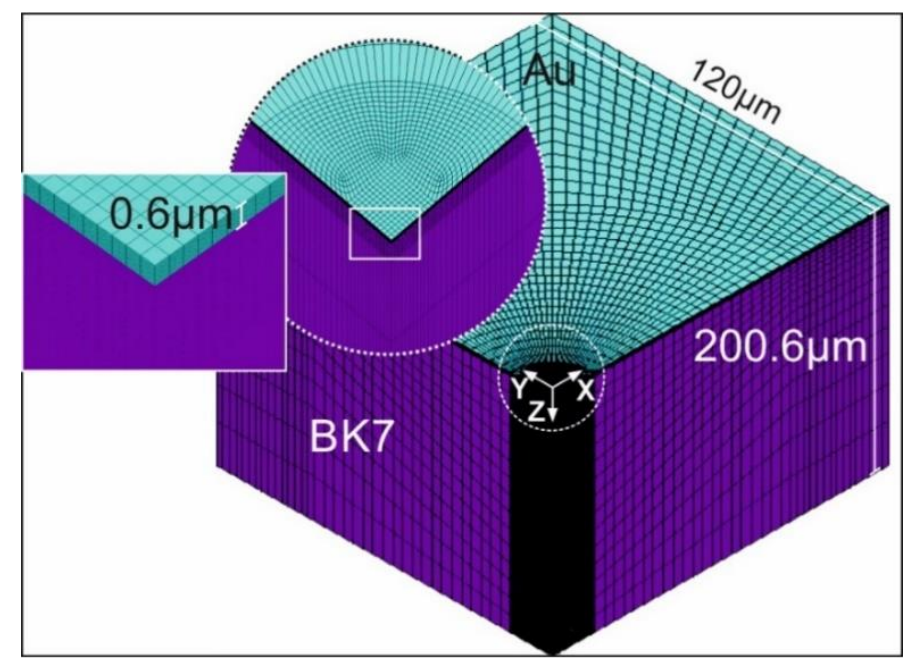

Figure 11.9. The 3D quarter symmetric FEM model and mesh details. 
For this model, a very fine mesh is necessary to resolve the resulting temperature distributions in the thin metallic film and especially in the very small region that is irradiated, in comparison to the large computational 3D domain. The zoomed detail of the irradiated surface in Figure 11.9 presents the mesh of the cyclic area of the laser beam spot. The temperature gradients change rapidly on the boundaries of this area where a locally adaptive fine mesh is used [30]. The positive Z-axis of the model follows the direction of the laser beam and its origin is on the center of the quarter symmetry, where the maximum gradients of thermal effects occur, and the melting and vaporization take place. A heat generation function is used to load the FEM model, as previously explained. The initial temperature is set to be $27{ }^{\circ} \mathrm{C}$ and a total time of $60 \mathrm{~ns}$ after the excitation are considered. The laser energy in the simulations is in the range of 0.8 to $20 \mu \mathrm{J}$, the FWHM laser pulse duration, $\mathrm{t}_{0}$, is $6 \mathrm{~ns}$ and the FWHM beam radius on the sample surface, $\mathrm{r}_{0}$, is $11.5 \mu \mathrm{m}$. The simulation is accomplished when the total time of the transient analysis is reached. Representative results of the simulation are shown in Figure 11.10 and Figure 11.11 [31-33]. Further details for the models and results can be found in the references [24-26,29,31-33].

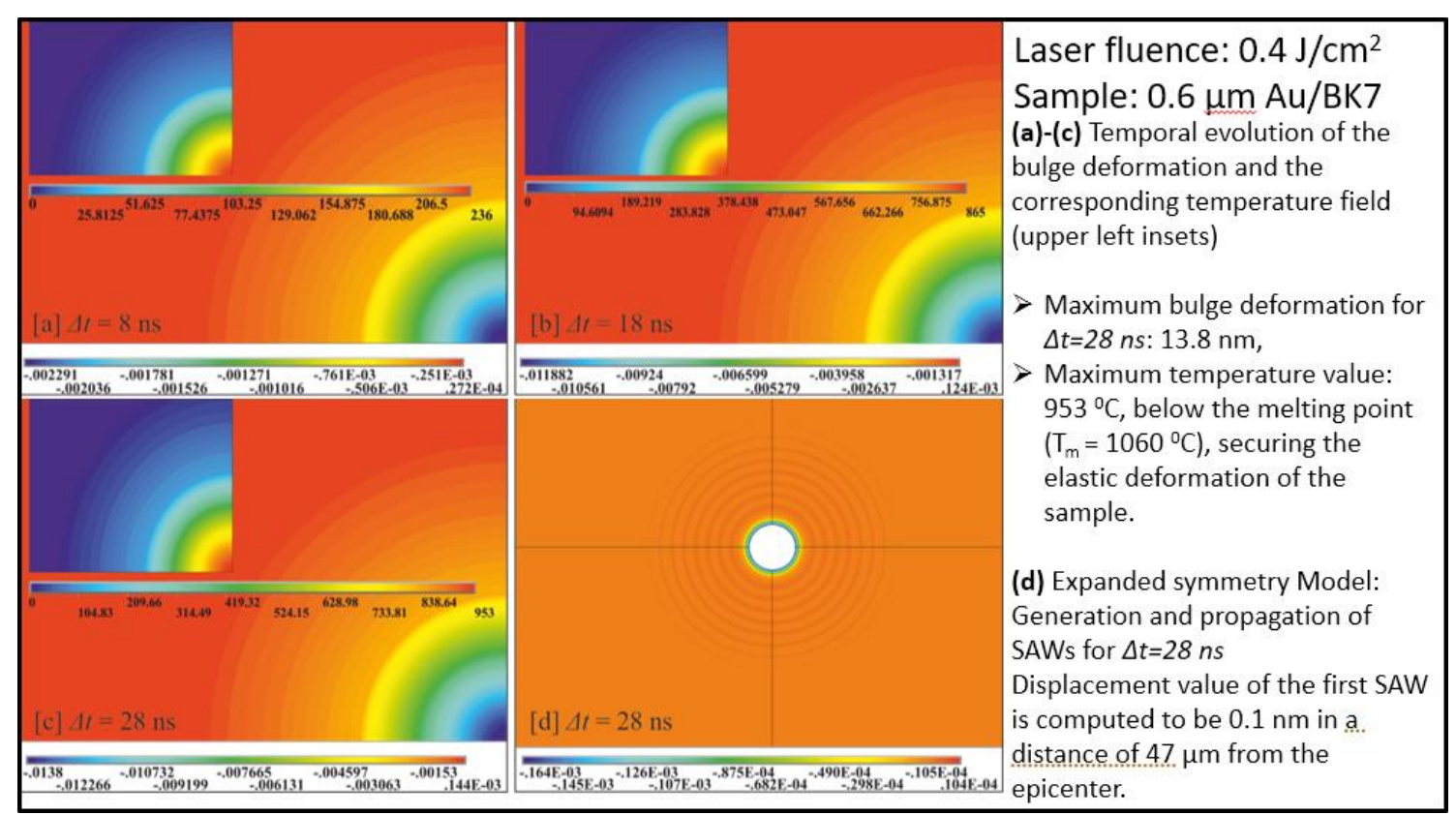

Figure 11.10. FEM results in the thermoelastic regime [32]. 


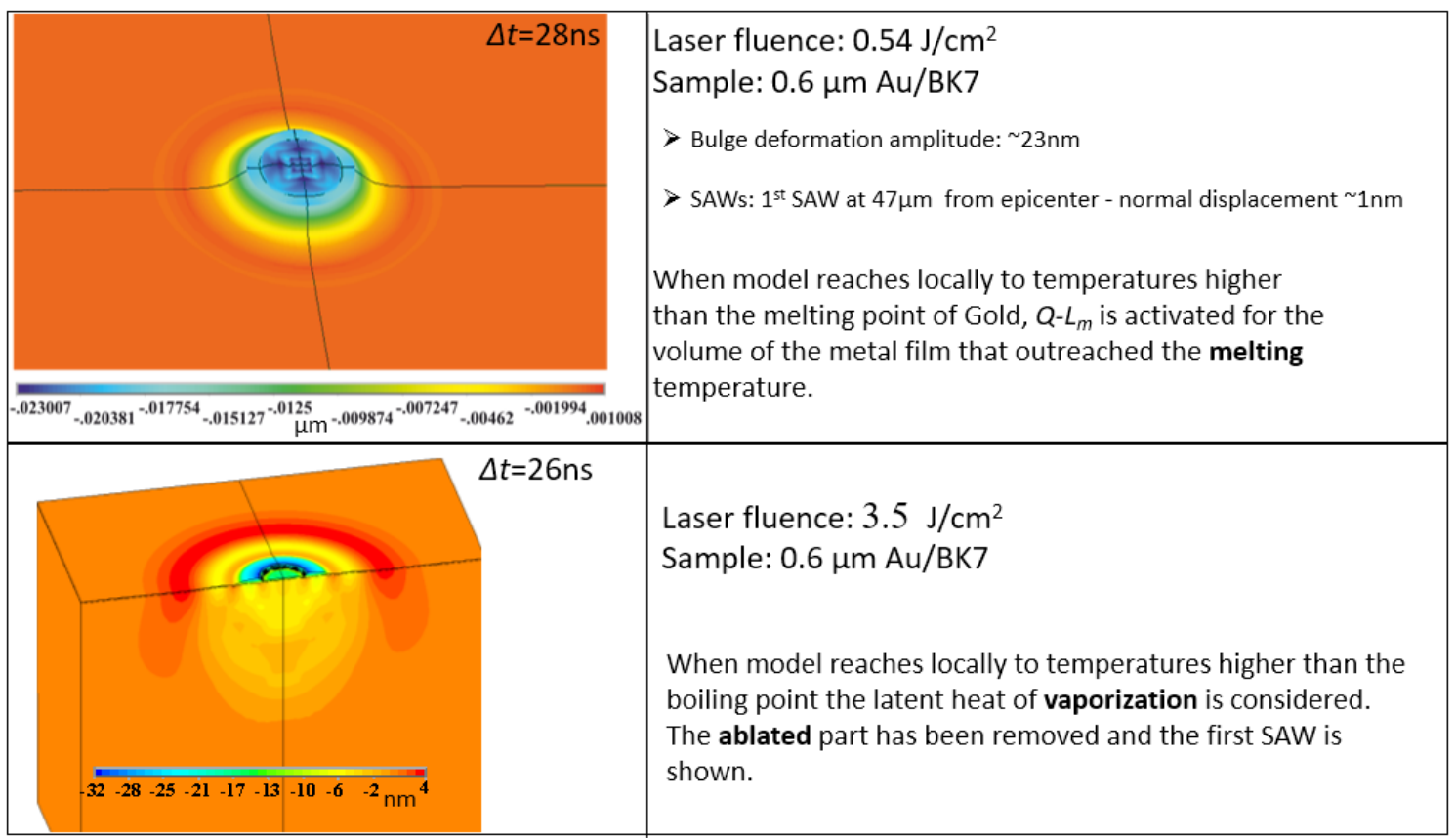

Figure 11.11. FEM results in the melting and ablation/plasma regimes [31,32].

\subsection{Multiphysics FEM simulations for MHD analysis}

The Finite Element Method may provide valuable insights for the phase changes of matter when multiphysics simulations are performed. Taking the advantages of the FEM, that were discussed in the previous paragraphs, the method can be used for the plasma generation and expansion studies by its connection to MHD codes. The complex phenomena that take place during the phase transitions from the solid to the plasma phase are difficult to be simulated by simplified models. Therefore, a numerical approach has to be compared and evaluated by experimental results at every regime [34-36]. Following this philosophy, the boundary conditions and the loads needed for the liquid and gas phases and the plasma studies using MHD analysis may be accurately computed.

The Z-pinch pulsed powered device of the Institute of Plasma Physics and Lasers - IPPL of the Hellenic Mediterranean University - HMU, Research Centre is ideal for such solid to plasma phase transition studies. The Z-pinch experiments are implemented in a mode of producing a peak current of $35 \mathrm{kA}$ with a rise time $(10 \%-90 \%)$ of $60 \mathrm{~ns}$ and a detailed description of the experimental work can be found in [34]. The development of a FEM model able to simulate this type of experiments must include the electric, the magnetic, the thermal and the structural fields of physics. The multiphysics simulations may consider the change of the material properties of the target using a Johnson-Cook strength material model and an equation of state (EOS Gruneisen) $[42,43]$. The numerical results of these transient simulations include the deformations the stresses and the strains, the temperatures, the pressures, the current density, the expansion rate, etc. at every point of the target material studied. The determined values of all these important parameters of the model can set accurately the initial boundary and loading conditions of the MHD problem for the further study of the plasma dynamics of the exploded matter. By using this approach in [37-40] and considering the phase changes of the electrical exploding thick metallic 
copper wires in pulsed-power Z-Pinch experiments, the formation of plasma is studied in detail [34-36].

The development of such a 3D coupled FEM multiphysics simulation also demands the Boundary Element Method (BEM) that models the vacuum region around the metallic wire. The FEM model of the wire considers the skin depth effect and by the help of the eddy-current approximation, the Maxwell equations are solved. The electromagnetic field is computed, and for each node of the discretization the Lorentz force is calculated. These forces are passing to the mechanical solver and the wire's deformation is determined. The temperature fields are processed by the thermal solver using the joule heating power term [41]. As stated before, an analytical Gruneisen EOS [42] and a Johnson-Cook [43] strength material model and additionally the Burgess EOS [44], are considered for the simulation of the MHD response of the copper wire, the elastoplastic behaviour, and the electrical conductivity versus temperature and density, respectively. The ends of the copper wire are fixed at $27{ }^{\circ} \mathrm{C}$. The loading of the model is the alternating current adopted by the experiments performed. The mathematical model of this simulation is defined by the Maxwell equations, the mass conservation, the momentum, and the energy equations, presented in summary in Figure 11.12.

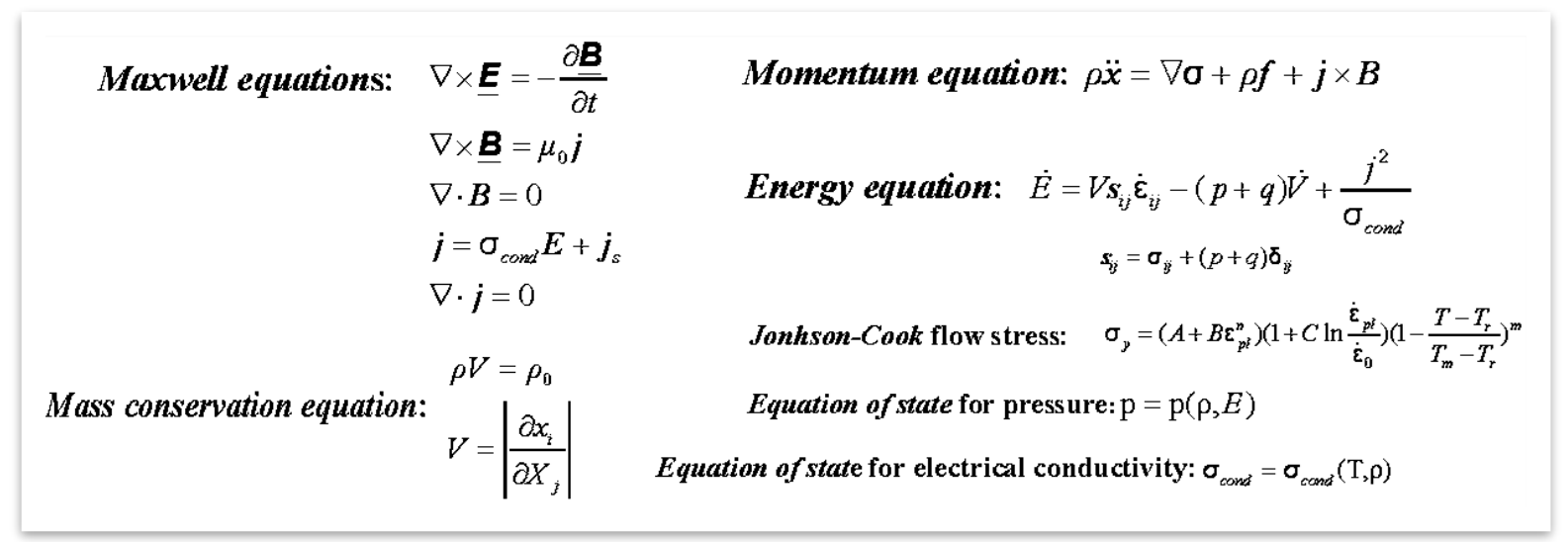

Figure 11.12. The mathematical FEM modeling of the problem [34,35].

In the beginning of the simulation, the metallic wire is thermally expanded affected by the Joule heating and also melting and vaporization take place. The finite element mesh of the model and representative results of the simulation are presented in Figure 11.13 and in Figure 11.14. The comparison of the displacements on the diameter of the wire after $140 \mathrm{~ns}$ from the current start to the initial geometry of the wire results to an expansion of $7 \mu \mathrm{m}$ that is in agreement with the corresponding experimental measurements [35]. This agreement shows that the developed simulation describes accurately the dynamic expansion of the wire for temperatures below the boiling point. Satisfactory agreement of experimental versus the FEM results is also observed concerning the initial times of corona plasma formation. 


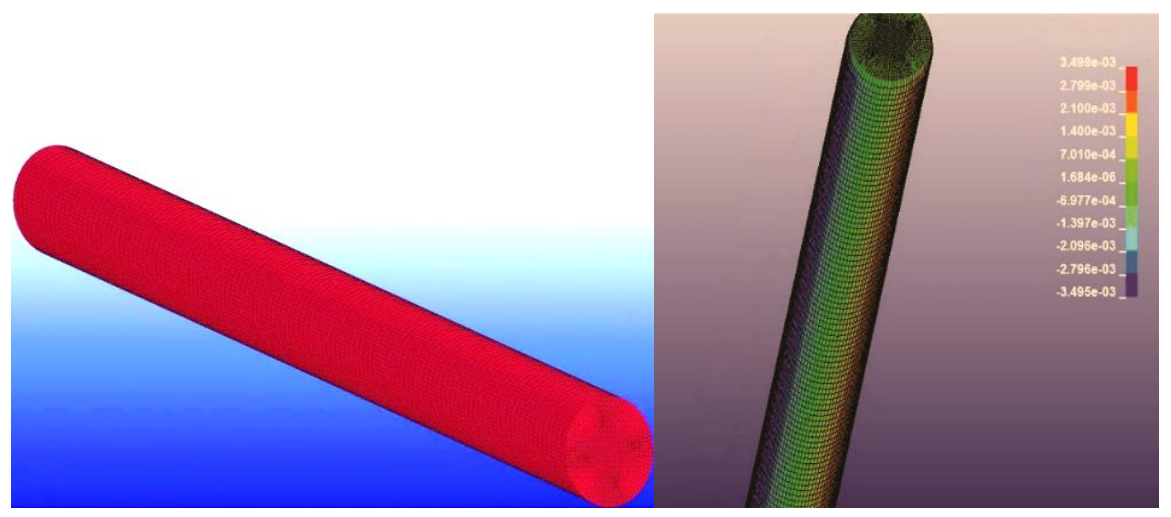

Figure 11.13. The FEM model and the mesh (left) and the resulting displacements (mm) on the X-axis (right) [45].

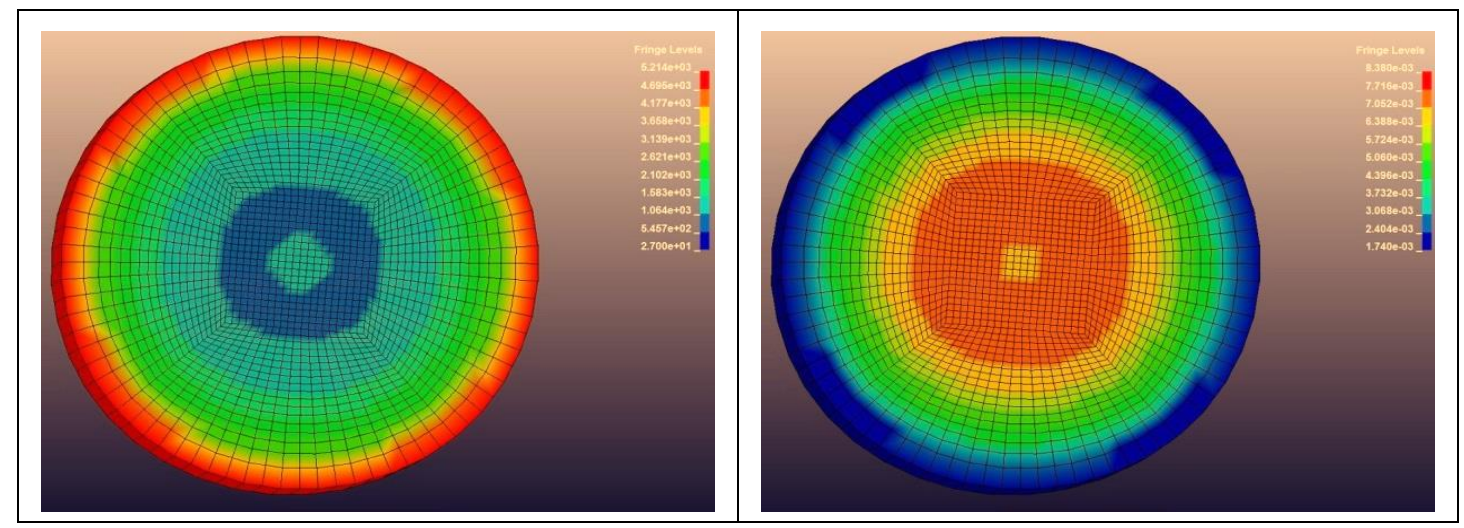

Figure 11.14. The distributions of the temperature $\left(\mathrm{left},{ }^{\circ} \mathrm{C}\right.$ ) and the density (right, $\mathrm{g} / \mathrm{cm}^{3}$ ) on a cross section of the wire at $220 \mathrm{~ns}$ from current start [45].

The FEM simulation results of the geometrical characteristics and the density distributions computed at every last of the solution are connected to the resistive MHD finite difference/volume code PLUTO [34]. For the MHD transient simulation, a fluid and a plasma region surrounded by a vacuum region, are modelled. This choice is based on experimental results suggesting that the plasma of the Z-pinch target consists of a dense fluid that surrounds the solid, which persists for a long time during the current discharge, surrounded by a low-density hot coronal plasma. This interconnection of the FEM and the MHD numerical methods is ideal for studies on the development of plasma instabilities [35]. Further details for the simulations and the experiments may be found in [35] and the supplementary material therein. Figure 11.15 shows representative MHD simulation results at the times of $210 \mathrm{~ns}$ and $240 \mathrm{~ns}$.

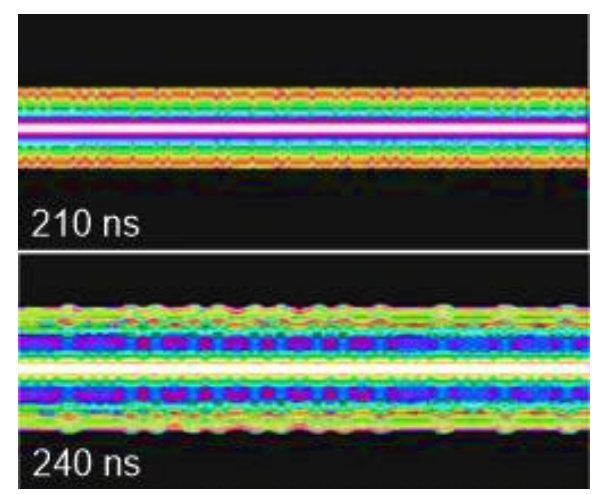

Figure 11.15. MHD simulation results based on the FEM results at $210 \mathrm{~ns}$ and $240 \mathrm{~ns}$ [34]. 


\section{Acknowledgements}

The work has been performed in the Institute of Plasma Physics and Lasers - IPPL of the Hellenic Mediterranean University - HMU, Research Centre. The model development and the simulations were performed during the PhD Thesis of E. Kaselouris that worked with Prof. V. Dimitriou and with the contributions of the Head of the Institute Prof. M. Tatarakis and the Vice Rector of HMU Prof. N. Papadogiannis, that supervised the experimental work and the research. M. Bakarezos, Y. Orphanos, I. Chatzakis, N. Vainos, I Fitilis, A. Skoulakis performed the experiments. G. Koundourakis and A. Skoulakis have contributed to the MHD modeling and simulations. We acknowledge the support by computational time granted from the Greek Research \& Technology Network (GRNET) in the National HPC facility ARIS under projects LaMIPlaS- I, II and III.

\section{References}

[1] O. Zienkevich and R. Taylor, The Finite Element Method, Vol. 1, McGraw-Hill, New York (1991)

[2] Dr. H. "Jerry" Qi, Finite Element Analysis, Lecture Notes, Colorado Edu. (2006).

[3] Prof. M. H. Sadd, Introduction to Finite Element Analysis, Wave Motion \& Vibration in Continuous Media Spring 2005.

[4] M. Hatami, Weighted Residual Methods Principles, Modifications and Applications, Academic Press, Cambridge, Massachusetts, United States (2018).

[5] H. Podlesak, T. Schnick, L. Pawlowski, S. Steinhäuser, B. Wielage, Microscopic study of Al-SiC particulate composites processed by laser shocks, Surf. Coat. Tech. 124 (2000) 32-38.

[6] Al.A. Kolomenskii, H.A. Schuessler, V.G. Mikhalevich, A.A Maznev, Interaction of laser-generated surface acoustic pulses with fine particles: Surface cleaning and adhesion studies, J. Appl. Phys. 84 (1998) 2404-2410.

[7] M. Sakamoto, X. Cai, S.S. Kim, M. Fujitsuka, T. Majima, Intermolecular electron transfer from excited benzophenone ketyl radical, J. Phys. Chem. A 111 (2007) 223-229.

[8] S.C. Chen, D.G. Cahill, C.P. Grigoropoulos, Melting and surface deformation in pulsed laser surface micromodification of Ni-P disks, J Heat Transf 122 (2000) 107-112.

[9] F. Reverdy, B. Audoin, Ultrasonic measurement of elastic constant of anisotropic materials with laser source and laser receiver focused on the same interface, J. Appl. Phys. 90(9) (2001) 4829-4835.

[10] S.J. Davies, C. Edwards, G.S. Taylor, S.B. Palmer, Laser-generated ultrasound: its properties, mechanisms and multifarious applications, J. Phys. D Appl. Phys. 26 (1993) 329.

[11] R.E. Green, Non-contact ultrasonic techniques, Ultrasonics 42 (2004) 9-16.

[12] A. Moura, A.M. Lomonosov, P. Hess, Depth evaluation of surface-breaking cracks using laser-generated transmitted Rayleigh waves, J. Appl. Phys. 103 (2008) 084911-16.

[13] S. Dixon, S.E. Burrows, B. Dutton, Y. Fan, Detection of cracks in metal sheets using pulsed laser generated ultrasound and EMAT detection, Ultrasonics 51 (2011) 7-16.

[14] S. Yashiro, J. Takatsubo, H. Miyauchi, N. Toyama, A novel technique for visualizing ultrasonic waves in general solid media by pulsed laser scan, NDT\&E Int. 41 (2008) 137-144.

[15] D. Schneider, T.A. Schwarz, H.J. Scheibe, M. Panzner, Non-destructive evaluation of diamond and diamond-like carbon films by laser induced surface acoustic waves, Thin Solid films 295 (1997) 107116.

[16] K. Nomura, H. Ohta, K. Ueda, T. Kamiya, M. Hirano, H. Hosono, Thin-film transistor fabricated in singlecrystalline transparent oxide semiconductor, Science 300 (2003) 1269-1272.

[17] A.A Volinsky, N.R. Moody, W.W. Gerberich, Interfacial toughness measurements for thin films on 
substrates, Acta. Mater. 50 (2002) 441-466.

[18] N.A. Vasantgadkar, U.V. Bhandarkar, S.S. Joshi, A finite element model to predict the ablation depth in pulsed laser ablation, Thin Solid films 519 (2010) 1421-1430.

[19] S. Amoruso, R. Bruzzese, N. Spinelli, R. Velotta, Characterization of laser-ablation plasmas, J. Phys. B: At. Mol. Opt. Phys. 32 (1999) R131-R172.

[20] B.Q. Xu, Z.H. Shen, X.W. Ni, J. Lu, Finite element model of laser-generated surface acoustic waves in coating-substrate system, J. Appl. Phys. 95 (2004) 2109-2115.

[21] B.Q. Xu, Z.H. Shen, X.W. Ni, J. Wang, J. Guan, J. Lu, Thermal and mechanical finite element modeling of laser-generated ultrasound in coating-substrate system, Opt. Laser Technol. 38 (2006) 138-145.

[22] V. Oliveira, R. Vilar, Finite element simulation of pulsed laser ablation of titanium carbide, Appl. Surf. Sci. 253 (2007) 7810-7814.

[23] H.S. Lim, J. Yoo, FEM based simulation of the pulsed laser ablation process in nanosecond fields, J. Mech. Sci. Technol. 25 (2011) 1811-1816.

[24] Y. Orphanos, V. Dimitriou, E. Kaselouris, Efthimios Bakarezos, N. Vainos, Michael Tatarakis, N.A. Papadogiannis, An integrated method for material properties characterization based on pulsed laser generated surface acoustic waves, Microelectron. Eng. 112 (2013) 249-254.

[25] V. Dimitriou, E. Kaselouris, Y. Orphanos, E Bakarezos, N. Vainos, I.K. Nikolos, M. Tatarakis, N. A. Papadogiannis, The thermo-mechanical behavior of thin metal films on dielectric substrates under nanosecond laser pulse excitation above the thermoelastic regime, Appl. Phys. A. 118 (2015) 739-748.

[26] V. Dimitriou, E. Kaselouris, Y. Orphanos, M. Bakarezos, N. Vainos, M. Tatarakis, N. A. Papadogiannis, Three dimensional transient behavior of thin films surface under pulsed laser excitation, Appl. Phys. Lett. 103 (2013) 114104.

[27] A.V. Bulgakov, N. M. Bulgakova, Thermal model of pulsed laser ablation under the conditions of formation and heating of a radiation-absorbing plasma, Quantum Electron 29 (1999) 433-437.

[28] ANSYS Inc., Coupled-field analysis guide, Release 12.0, Southpointe Canonsburg, April 2009.

[29] E. Kaselouris, I.K. Nikolos, Y. Orphanos, E Bakarezos, N. A. Papadogiannis, M. Tatarakis, V. Dimitriou, Elastoplastic study of nanosecond-pulsed laser interaction with metallic films using 3D multiphysics fem modeling, International Journal of Damage Mechanics 25(1) 42-55, 2016.

[30] V. Dimitriou, A. Kanarachos, D. Koulocheris, An Approach to Unstructured Finite Element Mesh Generation Using Coons Mapping and Smoothing Techniques, WSEAS Transactions on Circuits and Systems 2 (2003) $473-478$.

[31] V. Dimitriou, E. Kaselouris, Y. Orphanos, E. Bakarezos, N. Vainos, I. K. Nikolos, N. A. Papadogiannis and M. Tatarakis, Matter dynamics under the interaction with laser pulses in the thermoelastic \& plasma regimes, 40th EPS Conference on Plasma Physics, Helsinki, Finland, July 2013.

[32] E. Kaselouris, Y. Orphanos, V. Dimitriou, E. Bakarezos, N. Vainos, M. Tatarakis, N. A. Papadogiannis, 3D finite element modeling of laser-generated surface acoustic waves in film-substrate systems validated by experiments, 10th HSTAM International Congress on Mechanics, 25-27 May 2013, Chania.

[33] E. Kaselouris, V. Dimitriou, I.K. Nikolos, Y. Orphanos, E. Bakarezos, N.A. Papadogiannis, M. Tatarakis, Numerical simulations for the study of matter behavior dynamics governed by the interaction with laser pulses or external strong currents, 10th HSTAM International Congress on Mechanics, 25-27 May 2013, Chania.

[34] E. Kaselouris, V. Dimitriou, I. Fitilis, A. Skoulakis, G. Koundourakis, I. K. Nikolos, E. L. Clark, M. Bakarezos, N. A. Papadogiannis and M. Tatarakis, The influence of the solid to plasma phase transition on the generation of plasma instabilities, Nature Commun. 8 (1) (2017) 1713.

[35] E. Kaselouris, V. Dimitriou, I. Fitilis, A. Skoulakis, G. Koundourakis, I. K. Nikolos, E. L. Clark, J. Chatzakis, M. Bakarezos, N. A. Papadogiannis and M. Tatarakis, Preliminary investigation on the use of low current pulsed power Z-pinch plasma devices for the study of early stage plasma instabilities, Plasma Phys. Control. Fusion 60 (1) (2018) 014031.

[36] E. Kaselouris, V. Dimitriou, A. Skoulakis, I. Fitilis, Y. Orphanos, I.K. Nikolos, E. Bakarezos, N.A. 
Papadogiannis and M. Tatarakis, Experimental \& Numerical study of the initial stages of explosion of thick single wire z-pinch, 41st EPS Conference (2014).

[37] M.G. Haines, A review of the dense Z-pinch, Plasma Phys. Control. Fusion 53 (2011) 093001.

[38] D.A. Hammer and D.B. Sinars, Single-wire explosion experiments relevant to the initial stages of wire array z pinches, Laser Part. Beams 19 (2001) 377-391.

[39] M. Tatarakis et al., Optical probing of fiber z -pinch plasmas, Phys. Plasmas 5 (1998) 682.

[40] V.I. Oreshkin et al., Wire explosion in vacuum: Simulation of a striation appearance, Phys. Plasmas 11 (2004) 4771-76.

[41] G. Le Blanc et al., Ramp wave compression in a copper strip line: comparison between MHD numerical simulations (LS-DYNA) and experimental results (GEPI device), 10th International LSDYNA Conference, 2008.

[42] K. Nagayama, Introduction to Grüneisen Equation of State and Shock Thermodynamics, Kindle Edition.

[43] G.R Johnson and W.H. Cook, Fracture characteristics of three metals subjected to various strains, strain rates, temperatures and pressures, Eng. Fract. Mech 21 (1985) 31-48.

[44] T. Burgess, Electrical resistivity model of metals, 4th International Conference on Megagauss Magneticfield generation and related topics, Santa Fe, NM, 1986.

[45] I. Fitilis, A. Skoulakis, E. Kaselouris, I. K. Nikolos, E. Bakarezos, N. A. Papadogiannis, V.M. Dimitriou, M. Tatarakis, Diagnosing the initial stages from solid to plasma phase for dense plasma explosions, Proceedings of Science,1st EPCD Conference on Plasma Diagnostics, 14-17 April 2015, Frascati, Italy. 
SECTION IV

CASE STUDIES 



\title{
12. Multiscale modeling with MBN Explorer and MBN Studio
}

\author{
Alexey V. Verkhovtsev*, Ilia A. Solov'yov, and Andrey V. Solov'yov
}

This chapter provides an overview of the computational multiscale modeling approach based on utilization of the powerful and universal software packages MBN Explorer and MBN Studio. Three illustrative case studies of multiscale modeling are presented in relation to (i) development of novel sources of monochromatic high-energy radiation based on the crystalline undulators, (ii) controlled fabrication of nanostructures using the focused electron-beam induced deposition, and (iii) ion-beam cancer therapy. These examples illustrate the key algorithms and unique methodologies implemented in the software in different research areas arising in connection with the development of novel and emerging technologies.

*Contact: verkhovtsev@mbnexplorer.com 


\subsection{Introduction}

Computational multiscale modeling encompasses a wide range of end-products and a great number of applications in avionics and automobile industry for designing of nanostructured materials, functionalized surface coating, as well as stronger and lighter materials for aircrafts and cars; in mechanical engineering for virtual design of superhard nanostructured materials; in medical applications for nanostructured implants or novel biomaterials and materials technologies for implantable devices and tissue regeneration; in electronic and chemical industry for development of highly efficient batteries and catalyzers; in pharmaceutical industry for drug design, etc. In most of these applications it is necessary to identify and/or design specific properties of the system determined by its molecular structure on the nanoscale and to ensure transfer of these properties to the macroscopic scale in order to make them functional and usable. Such a transition implies multiscale modeling approaches which rely on a combined use of quantum mechanics methods together with classical molecular dynamics (MD), or linkage of MD and Monte Carlo (MC) simulations, or the application of efficient computational algorithms allowing to perform simulations across the scales.

This chapter provides an overview of the computational multiscale modeling approach [1] based on utilization of the powerful and universal software packages MBN Explorer [2] and MBN Studio [3]. The chapter begins with a brief overview of different theoretical methods for modeling MesoBio-Nano (MBN) systems, and the limitations of these methods. It is followed by a brief description of the MBN Explorer and MBN Studio software packages and the areas of their application. Next, several case studies of multiscale modeling by means of MBN Explorer and MBN Studio are presented, which illustrate the main algorithms and unique methodologies implemented in the software.

There are many concrete examples of novel and emerging technologies benefiting from computational multiscale modeling. As an illustration, a few of them are highlighted in this chapter: (i) development of novel sources of monochromatic high-energy radiation based on the crystalline undulators, (ii) controlled fabrication of nanostructures using the focused electronbeam induced deposition (FEBID), and (iii) ion-beam cancer therapy. These examples illustrate the new possibilities that computational multiscale modeling provides to novel and newly emerging technologies.

\subsection{Computational approaches in Meso-Bio-Nano Science}

MBN Science is an interdisciplinary field of research studying structure formation and dynamics of animate and inanimate matter on the nano- and the mesoscales. This field bundles up a number of traditional topics in theoretical physics and chemistry at the interface with life sciences and materials research under a common theme.

The range of open challenging scientific problems in the field of MBN Science is very broad $[1,4]$. They may include: structure and dynamics of clusters, nanoparticles, biomolecules, and many other nanoscopic and mesoscopic systems; clustering, self-organization, growth and structureformation processes, and their multiscale nature; assemblies of clusters/nanoparticles and biomacromolecules; hybrid bio-nano systems; nanostructured materials; surface phenomena; 
nanoscale phase and morphological transitions; thermal, optical and magnetic properties; collective and many-body phenomena; electron transport and molecular electronics; collision, fusion, fission and fragmentation processes; channeling effects; radiation effects; radiobiological effects, and many more.

MBN Science has been boosted immensely over the past two decades by the fast development of computer powers and related computational techniques that became broadly available. This development resulted in a significant increase of the efficiency of available computer codes for scientific research. Such codes are usually focused on particular systems, their particular sizes and phenomena involved (see Figure 12.1), and thus are limited in their ability to model physical, chemical or biological phenomena that go across the scales. Therefore, much effort of research communities has been devoted in recent years to the computational approaches and modeling techniques allowing to overcome this drawback and open the new horizons in theoretical and computational research.

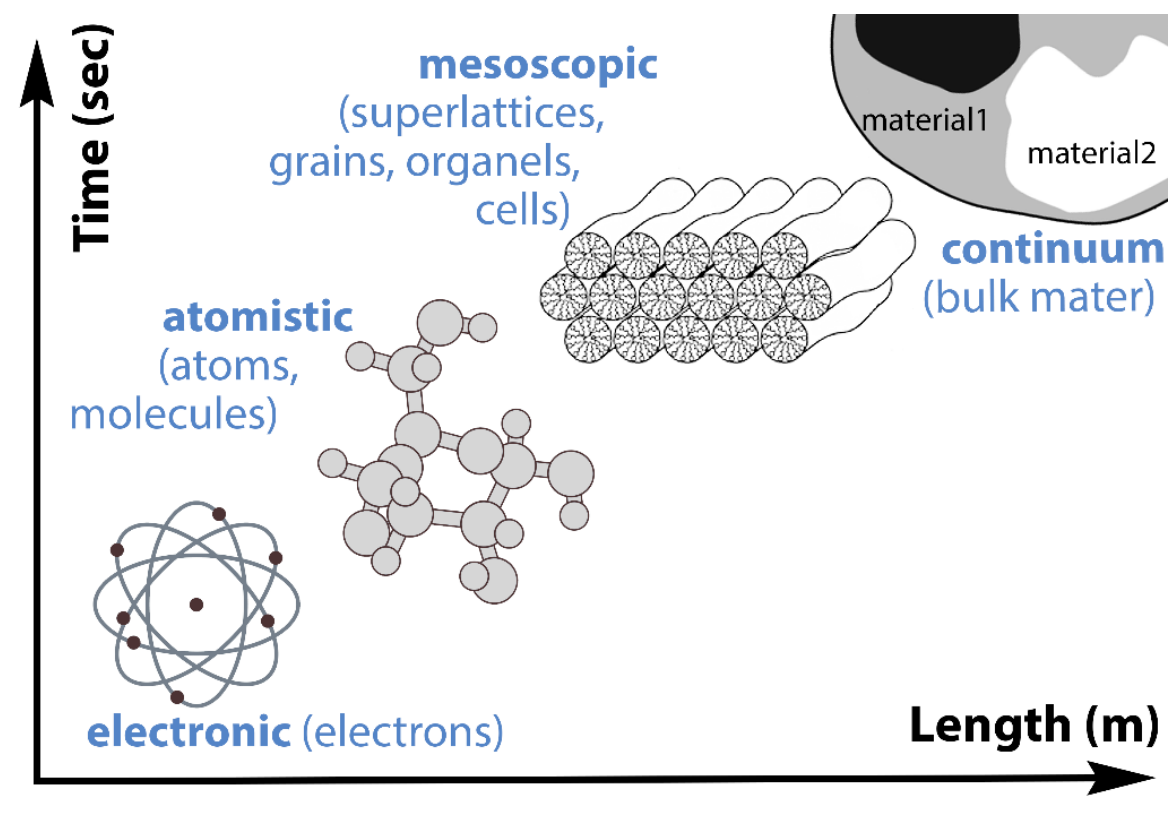

Figure 12.1. An illustration of entities in the different computational techniques. The figure is adapted from Ref. [1]

\subsubsection{Atomic and nano-scales}

The characteristic quantum processes in MBN systems often involve electronic excitation, relaxation, fragmentation or transport dynamics. Often, these effects in molecular and nanosystems occur when they are embedded into larger scale molecular environments. The quantitative description of the mentioned processes requires inclusion of both short-time/(sub-) nanoscale quantum aspects and long-time/nano- and mesoscale environment effects. Dynamical descriptions of molecular systems on the quantum atomic/subnano- and nano-/mesoscales are presently performed with disconnected theoretical and simulation tools. There is no serious hope to explore in near future both ranges within simply extending one tool to the other domain. Bridging this gap implies interfacing quantum atomic/molecular and nano-/mesoscopic approaches. These efforts will result from merging into a common model a time-dependent quantum mechanical (QM) approach and classical molecular dynamics (MD). This will enable the 
exploration of new types of dynamics in a broad range of molecular systems, not accessible for analysis by other theoretical and computational means.

Attempts have been made during the recent years in various realizations of quantum mechanical/molecular mechanical (QM/MM) methods. State-of-the-art achievements of these methods have been reviewed in several publications [5-7], and the field will experience a significant development in the future. The reason for this is that many well-established quantum and classical MD codes, being developed during the long period of time entirely independently, now reach the point when they require coupling in order to go further across disciplines. As this goal could not be achieved in a single universal approach, its realization often requires the solution for each particular pair of quantum and classical codes with their particular areas of application and the communities of researches standing behind.

\subsubsection{Nano- and meso-scales}

Description of structure and dynamics of molecular and nanosystems on the basis of classical MD principles provides significant computational advantages as compared to the quantum descriptions due to the simplicity of the formalism involved. Numerical realizations of this approach nowadays enable to treat the structures consisting of tens millions of atoms and to access dynamical time scales of molecular processes up to tens of microseconds, although for smaller systems, typically on the scale of hundreds of thousands of atoms. A review of achievements made on the construction of software packages built on these principles can be found in [8-10]. The existing codes are based on the utilization of different classical force fields suitable for the description of certain types of molecular systems, e.g. biomolecular systems, carbon or metallic nanosystems, etc. However, many of the MD packages do not have the possibility (at least in sufficiently elaborated form) to combine different force fields for the description of hybrid molecular systems and molecular environments due to the high level of optimization tuned for the specific type of force fields.

In recent years, it has become clear that a detailed understanding of numerous quantum molecular processes happening in larger scale molecular environments becomes possible due to new advances in theoretical and experimental tools developed in atomic and molecular physics and nanotechnology. An impressive example is the discovery of resonant mechanisms at the origin of radiation damage caused by very low-energy electrons [11,12] via the dissociative electron attachment (DEA) mechanism. The identification of such a quantum effect in this context had an enormous impact: it paved the way to new research on possible low-energy radiation damage effects [13]. Another example where the detailed knowledge of atomic and molecular processes in larger scale molecular environments is crucial concerns novel radiosensitizers, e.g. metallic nanoparticles which can enhance radiation effects on biological targets [14]. An important feature of the aforementioned processes is that they occur on relatively short times (tens of femtoseconds) and in relatively small spatial domains (up to few nanometers).

By exploiting the locality of the QM processes, one can isolate the active part of the system and describe it at the pure QM level. In this case, however, possible large-scale effects coming from the other parts of the system might be missing. For instance, this can be the case for a chromophore molecule in solvating shells or impurities in a solid. The size of the QM system can be enlarged but the convergence of such a strategy, in terms of accuracy and/or simulation time, can become slow. 
One can instead explicitly include the environment and treat it classically at MM level. The development of such hierarchical techniques over past decades mainly operates in the field of biological chemistry or surface science, where large sizes and/or environment effects are crucial. These methods allow one to tackle with systems of thousands of atoms (a few tens at the QM level).

All-atom MD simulations on very large systems may require significant computer resources that are not easily affordable. Similarly, simulations of processes on long timescales (beyond $\sim 1 \mathrm{~ms}$ ) are prohibitively expensive, because they require too many integration time steps to be carried out. In these cases, one can sometimes tackle the problem by using reduced representations of the system, where instead of explicitly representing every atom of the system, groups of atoms are represented by "pseudo-atoms". Such representations are sometimes called as the coarse-grained models [15]. The parametrization of these models must be done empirically by matching the behavior of the model to appropriate experimental data or all-atom simulations. Coarse-grained models have been used successfully to examine a wide range of questions in structural biology, liquid crystal organization, and polymer glasses [15].

\subsubsection{Monte Carlo approach and finite element method}

Kinetic Monte Carlo (KMC) method is designed to model time evolution of many-particle systems stepwise in time. Instead of solving dynamical equations of motion the KMC approach assumes that the system undergoes a structural transformation at each step of evolution with a certain probability. The new configuration of the system is then used as the starting point for the next evolution step. The transformation of the system is governed by several kinetic rates which are chosen according to the model considered. Due to its probabilistic nature this methodology permits studying dynamical processes involving complex molecular systems on the time scales significantly exceeding the characteristic time scales of conventional MD simulations [16-18]. The KMC method is ideal in the situations when certain minor details of dynamical processes become inessential, and the major transition of the system to new states can be described by only a few kinetic rates being determined through the corresponding physical parameters.

Quantitative structural analysis of macroscopically large systems as well as the description of a number of processes occurring on the macroscopic scale (such as heat transfer, fluid flow, mass transport, etc.) can be effectively carried out by means of finite element methods (FEM) [19]. For systems involving complicated geometries, loadings, and material properties, it is generally not possible to obtain analytical mathematical solutions. FEM enables to formulate the problem in terms of a set of algebraic equations, rather than requiring the solution of differential equations. These numerical methods yield approximate values of the unknowns at discrete numbers of points in the continuum. Hence this process of modeling a body by dividing it into an equivalent system of smaller bodies or units (finite elements) interconnected at points common to two or more elements (nodal points or nodes) and/or boundary lines and/or surfaces is called discretization. In FEM, instead of solving the problem for the entire body in one operation, one formulates the equations for each finite element and combines them to obtain the solution of the whole body. 


\subsection{MBN Explorer and MBN Studio}

Multiscale modeling of MBN systems is one of the hot topics of modern theoretical and computational research. To fully understand and exploit all the richness and complexity of the MBN-world, especially its dynamics, one needs to consult many disciplines ranging from physics and chemistry to materials and life sciences, exploiting technologies from software engineering and high-performance computing. This general trend brought up the idea and then development of MBN Explorer [2] and MBN Studio [3]. These software packages have been designed as powerful and universal instruments of computational research in the field of MBN Science, which are capable to explore, simulate, record and visualize both structure and dynamics of MBN systems, reproduce its known features and predict the new ones.

MesoBioNano Explorer (MBN Explorer) [2] is a software package for the advanced multiscale simulations of structure and dynamics of various MBN systems. It has many unique features and a wide range of applications in physics, chemistry, biology, and materials science. It is suitable for classical non-relativistic and relativistic molecular dynamics (MD), Euler dynamics, reactive and irradiation-driven molecular dynamics (RMD and IDMD) simulations, as well as for stochastic dynamics or kinetic Monte Carlo (KMC) simulations of various randomly moving MBN systems or processes. These algorithms are applicable to a large range of systems, such as nano- and biological systems, nanostructured materials, composite/hybrid materials, gases, plasmas, liquids, solids, and their interfaces, with the sizes ranging from atomic to mesoscopic.

\subsubsection{Main features of MBN Explorer}

MBN Explorer enables calculations of energies of a large variety of MBN systems and optimization of their structures. The software package supports different types of molecular dynamics for MBN systems. The program operates with a large library of interatomic potentials thus allowing to model many different molecular systems. It is also possible to simulate dynamics of MBN systems in present of external fields - electric, magnetic, gravitational, and electromagnetic waves.

Apart from the standard algorithms, MBN Explorer is equipped with unique algorithmic implementations that enhance significantly the computational modeling capacities in various research and technological areas. The complete list of algorithms implemented in MBN Explorer can be found in $[1,20,21]$.

In particular, MBN Explorer has unique algorithmic implementations for multiscale modeling. By means of stochastic dynamics (KMC, random walk dynamics) algorithms MBN Explorer allows simulating the dynamics of MBN systems on the time scales significantly exceeding the limits for the conventional atomistic MD simulations. Such multiscale dynamics approach is ideal for systems in which details of their atomistic dynamics become excessive and the overall behavior of a system can be reproduced through kinetic rates for the dominating modes of motion and probabilities of the key processes occurring in the system. This important feature of MBN Explorer expands significantly its application areas and goes beyond the limits of other MD codes usually unable to deal with multiscale modeling.

In the case of ultra-relativistic charged particle propagation through different media the implemented algorithms enable simulations of particle dynamics on the macroscopically large 
distances with the atomistic accuracy. These algorithms enable to obtain the necessary atomistic insides into macroscopically large systems and processes occurring therein. For instance, one can simulate the operation of novel intensive sources of high-energy monochromatic $\gamma$-rays based on irradiation of oriented crystals by beams of ultra-relativistic electrons and positrons.

\subsubsection{Main features of MBN Studio}

In order to facilitate the practical work with MBN Explorer a special multi-task software toolkit, called MBN Studio, has been developed [3]. It simplifies modeling of MBN systems, setting up and starting calculations with MBN Explorer, monitoring their progress and examining the calculation results. The software can be utilized for any type of calculations that are supported by MBN Explorer.

MBN Studio enables the Project set-up (standard as well as application-specific). Applicationspecific projects are usually designed for utilization in the specific application areas, e.g. related to novel or emerging technologies. Often application-specific projects also involve special algorithms. A special modeling plug-in allows one to construct and prepare application-specific projects for simulation quickly and efficiently.

MBN Studio has an advanced MBN system modeler, a built-in tool for design of MBN systems. By means of this plug-in one can easily assemble molecular systems of different geometry and composition for their further simulations with MBN Explorer.

MBN Studio supports various standard input/output data formats and links to numerous online databases and libraries with coordinates and geometries for atomic clusters, nanoparticles, biomolecules, crystals and other molecular systems that can be utilized in simulations with MBN Explorer.

MBN Studio is equipped with the output data handling, visualization and analytic tools that allow calculation and analysis of specific characteristics determined by the output of MD simulations. Examples include calculations of diffusion coefficients, heat capacities, melting temperatures for solids, atomic radial distribution functions and many others.

MBN Studio also enables video rendering of the dynamics of MBN systems simulated with MBN Explorer.

To summarize, MBN Explorer and MBN Studio are powerful tools for computational modeling in numerous different areas of challenging research arising in connection with development of the aforementioned technologies. There are several such areas, in which simulations performed by means of MBN Explorer and MBN Studio contributed immensely to their development. For instance, one of the areas concerns the development of novel light sources based on charged particles channeling in crystalline undulators [22, 23]. Another example concerns controllable fabrication of nanostructures with nanometer resolution using tightly-focused electron beams [24-26]. The third example deals with simulations of the nanoscopic molecular processes playing the key role in the ion-beam cancer therapy [13, 27, 28]. MBN Explorer combined with the visualization interface of MBN Studio in many cases can substitute expensive laboratory experiments by computational modeling making the software play a role of a "computational nano- and microscope". The following sections illustrate the capabilities of MBN Explorer and MBN Studio for advanced multiscale modeling in the three aforementioned technological areas. 


\subsection{Computational modeling of novel crystal-based gamma- ray light sources}

MBN Explorer and MBN Studio provide unique possibilities for computational multiscale modeling of the physical processes and phenomena [23, 29-32] that may facilitate the design and practical realization of novel gamma-ray Crystal-based Light Sources (CLS) [22]. Such light sources can be constructed through exposure of oriented crystals (linear, bent, periodically bent) to beams of ultra-relativistic charged particles (e.g. electrons or positrons). Construction of novel CLSs is a challenging task which constitutes a highly interdisciplinary field entangling a broad range of correlated activities. CLSs provide a low-cost alternative to conventional X-ray LSs based on free electrons lasers (FEL) and have enormous number of applications in the basic sciences, technology and medicine [22].

The development of LSs for wavelengths $\lambda$ well below $1 \AA ̊$ (corresponding photon energies $E_{\mathrm{ph}} \gtrsim$ $10 \mathrm{keV}$ ) is a challenging goal of modern physics. Sub-angstrom wavelength powerful spontaneous and, especially, coherent radiation will have many applications in nuclear and solid-state physics, as well as in life sciences. At present, several X-ray Free-Electron-Laser (XFEL) sources are operating (European XFEL, FERMI, LCLS, SACLA, PAL-XFEL) or planned (SwissFEL) for X-rays down to $\lambda \sim 1 \AA[33,34]$. However, no laser system has yet been commissioned for shorter wavelengths due to the limitations of permanent magnet and accelerator technologies. Modern synchrotron facilities $[35,36]$ provide radiation of shorter wavelengths but of much less intensity which falls off very rapidly as $\lambda$ decreases.

Therefore, to create a powerful LS in the range well below $1 \AA$, i.e. in the hard X- and gamma-ray band, one has to consider new approaches and technologies. Novel gamma-ray CLSs can generate radiation in the photon energy range where the technologies based on the charged particles motion in the fields of permanent magnets become inefficient or incapable. The limitations of conventional LSs is overcome by exploiting very strong crystalline fields that can be as high as $\sim 10^{10} \mathrm{~V} / \mathrm{cm}$, which is equivalent to a magnetic field of $3000 \mathrm{~T}$, whilst modern superconducting magnets provide 1-10 $\mathrm{T}$ [37]. The orientation of a crystal along the beam enhances significantly the strength of the particles' interaction with the crystal due to strongly correlated scattering from lattice atoms. This allows for the guided motion of particles through crystals of different geometry and for the enhancement of radiation.

Practical realization of CLSs often relies on the channeling effect. The basic phenomenon of channeling is in a large distance which a projectile particle penetrates moving along a crystallographic plane or axis and experiencing collective action of the electrostatic fields of the lattice atoms [38]. In the planar regime, positrons channel in between two adjacent planes whereas electrons propagate in the vicinity of a plane thus experiencing more frequent collisions. As a result, a typical distance covered by a particle before it leaves the channeling mode due to uncorrelated collisions (the so-called dechanneling length) for positrons is much larger than for electrons. To ensure enhancement of the emitted radiation due to the dechanneling effect, the crystal length must be chosen of the order of the dechanneling length [29-31].

The motion of a projectile and the radiation emission in bent and periodically bent crystals (BCs and PBCs) are similar to those in magnet-based synchrotrons and undulators. The main difference is that in the latter the particles and photons move in vacuum whereas in crystals they propagate 
in a medium, thus leading to a number of limitations for the crystal length, bending curvature, and beam energy. However, the crystalline fields are so strong that they steer ultra-relativistic particles more effectively than the most advanced magnets. Strong fields bring bending radius in $\mathrm{BC}$ down to the $\mathrm{cm}$ range and bending period $\lambda_{\mathrm{u}}$ in PBCs to the hundred or even ten microns range. These values are orders of magnitude smaller than those achievable with magnets [33]. As a result, the radiators can be miniaturized thus lowering dramatically the cost of CLSs as compared to that of conventional LSs. Figure 12.2 matches the magnetic undulator for the European XFEL with the CU manufactured in University of Aarhus and used in recent experiments [39].

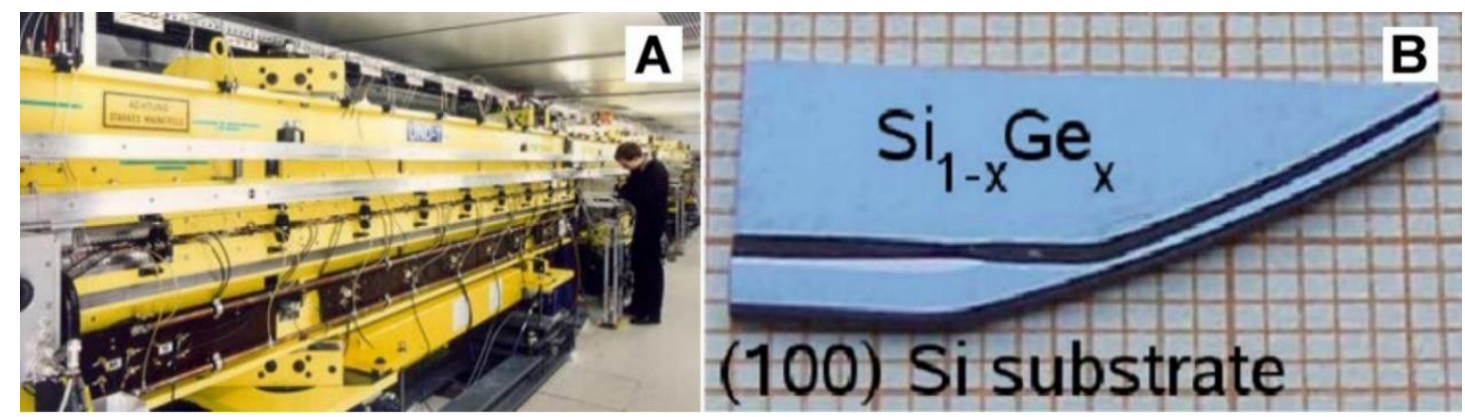

Figure 12.2. (A) Magnetic undulator for the European XFEL and (B) a $\mathrm{Si}_{1-\mathrm{x}} \mathrm{Ge}_{\mathrm{x}}$ superlattice CU build atop the silicon substrate with the face normal to the $<100>$ crystallographic direction [1]. In the superlattice, the (110) planes are bent periodically. The picture of a CU is courtesy of J.L. Hansen, A. Nylandsted and U. Uggerhøj (University of Aarhus).

One of the important practical realizations of the CLSs is based on the concept of a Crystalline Undulator (CU) $[23,29,30]$. A CU device contains a PBC and a beam of ultra-relativistic positrons or electrons undergoing planar channeling, see Figure 12.3. In such a system, in addition to the channeling radiation (ChR) [40], the undulator radiation appears due to the periodic motion of the particles which follow the bending of the planes. A light source based on a CU can generate photons in the energy range from tens of $\mathrm{keV}$ up to the $\mathrm{GeV}$ region [41] (the corresponding wavelengths range starts at $0.1 \AA$ and goes down to $10^{-6} \AA$ ). The intensity and characteristic frequencies of the CU radiation (CUR) can be varied by changing the beam energy, the parameters of bending and the type of crystal. Under certain conditions a CU can become a source of the hard $\mathrm{X}$ - and gamma-ray laser light within the range $\lambda=10^{-2}-10^{-1} \AA$ [23], which cannot be reached in existing and planned FELs based on magnets.

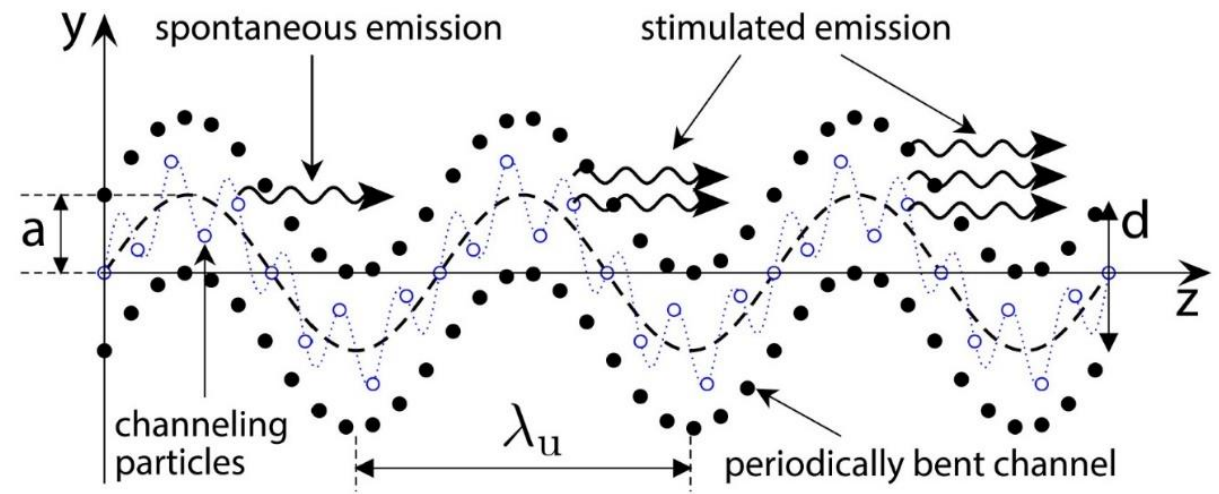

Figure 12.3. Schematic representation of a CU [23, 30,31]. Closed circles mark the atoms of crystallographic planes which are periodically bent with the amplitude $a$ and period $\lambda_{\mathrm{u}}$. Thin dotted line illustrates the trajectory of the particle (open circles) which propagates along the center line (the undulator motion) and simultaneously undergoes so-called channeling oscillations. The periodic mode leads to the emission of the undulator-type radiation, and, under certain conditions, may result in the stimulated radiation. 
The mechanism of the photon emission by means of CU is illustrated by Figure 12.3 which presents a cross section of a single crystal. The $z$ axis is aligned with the mid-plane of two neighboring non-deformed crystallographic planes (not shown) spaced by the distance $d$. The closed circles denote the nuclei of the planes which are periodically bent with the amplitude $a$ and period $\lambda_{\mathrm{u}}$. The harmonic (sinusoidal) shape of periodic bending, $y(z)=a \sin \left(2 \pi z / \lambda_{u}\right)$, is of a particular interest since it results in a specific pattern of the spectral-angular distribution of the radiation emitted by a beam of ultra-relativistic charged particles (the open circles in the figure) propagating in the crystal following the periodic bending.

The operational principle of a CU does not depend on the type of a projectile. Provided certain conditions are met, a particle undergoes channeling in a PBC [23, 29-31]. Its trajectory contains two elements. First, there are channeling oscillations due to the action of the interplanar force. Their frequency $\Omega_{c h}$ depends on the projectile energy $\varepsilon$, on the maximal value of the interplanar force and the interplanar distance $d$. Second, there are undulator oscillations due to periodicity of the bending whose frequency is $\Omega_{u} \approx 2 \pi c / \lambda_{u}$. The spontaneous emission is associated with both of these oscillations. The typical frequency of ChR is $\omega_{c h} \approx 2 \gamma^{2} \Omega_{c h}$, where $\gamma=\left(1-v^{2} / c^{2}\right)^{-1 / 2}$ is the relativistic Lorenz factor with $v$ being the speed of a projectile and $c$ the speed of light. The frequency of CUR is $\omega_{u} \approx 2 \gamma^{2} \Omega_{u}$. If $\Omega_{u} \ll \Omega_{c h}$ then the ChR and CUR frequencies are well separated. In this case the characteristics of CUR are virtually independent on the channeling oscillations $[23,29,30]$, and the operational principle of a CU is the same as of a magnet-based one [42-44] in which the monochromaticity of radiation is due to constructive interference of the photons emitted from similar parts of trajectory.

The feasibility for the construction of a PBC-based light source, a CU, was verified theoretically rather recently [29-31, 45, 46]. In these papers, as well as in the subsequent publications (see the latest review [23]), the essential conditions and limitations which must be met were formulated. These papers gave rise to coordinated theoretical, computational, technological and experimental studies of a number of related phenomena.
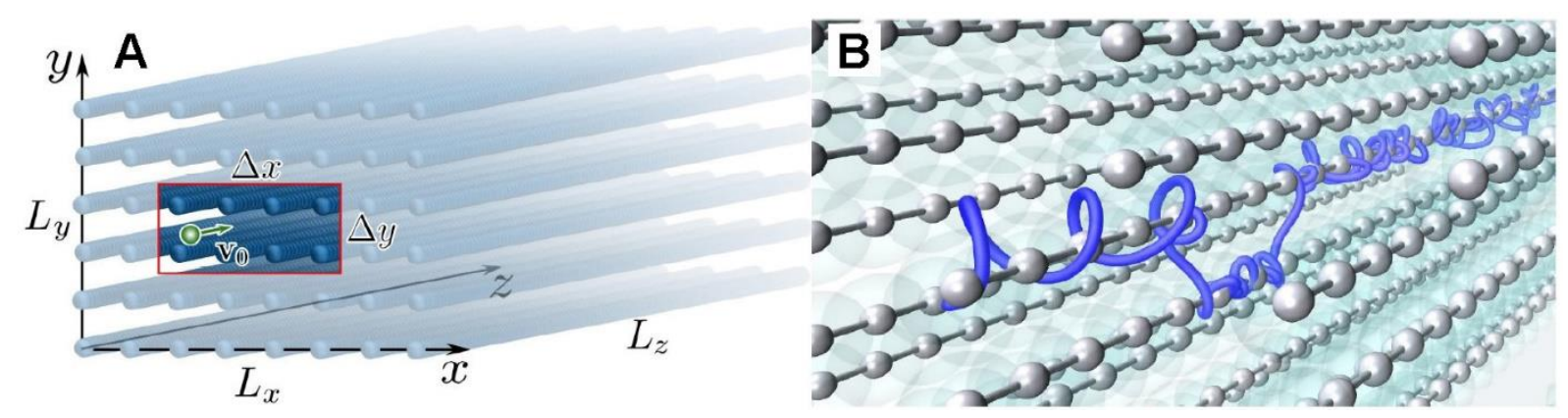

Figure 12.4. A: schematic representation of the channeling process [20]. The $z$-axis is aligned with the incident beam direction ( $v_{0}$ denotes the initial velocity) and is parallel to the crystallographic direction, along which the channeling is simulated. The $y$-axis is perpendicular to the plane. At the entrance, the $x$ and $y$ coordinates of the particle are randomly chosen to lie in the central part of the $(x y)$-plane (the highlighted rectangle). B: illustration of the trajectory of an axially channeling particle.

MBN Explorer enables simulations of propagation of various (positively and negatively charged, light and heavy) particles in various media, such as hetero-crystalline structures (including superlattices), bent and periodically bent crystals, amorphous solids, liquids, nanotubes, fullerites, biological environment, and many more. The applicability of the code to different 
structures can be adjusted either by choosing a proper interaction potential or, if necessary, by including a new potential [32].

By these means the channeling phenomenon $[23,38]$, which takes place when a charged particle enters a crystal at small angles with respect to a crystallographic direction, can be modeled. The particle becomes confined and forced to move through the crystal preferably along the crystallographic direction, experiencing collective action of electrostatic field of the lattice ions (see Figure 12.4). Since the field is repulsive for positively charged particles, they are steered into the interatomic region, while negatively charged projectiles move in close vicinity of ion strings or planes.

\subsubsection{Relativistic equations of motion}

In order to simulate motion of relativistic particles, MBN Explorer considers [32] relativistic equations of motion:

$$
\left\{\begin{array}{c}
\dot{\boldsymbol{v}}=\frac{1}{m \gamma}\left(\boldsymbol{F}-\boldsymbol{v} \frac{\boldsymbol{F} \cdot \boldsymbol{v}}{c^{2}}\right) \\
\dot{\boldsymbol{r}}=\boldsymbol{v}
\end{array}\right.
$$

where

$$
\gamma=\frac{1}{\sqrt{1-v^{2} / c^{2}}}
$$

is the relativistic Lorenz factor with $v$ being the speed of a projectile and $c$ the speed of light.

Applied to the propagation in a medium, equations (12.1) describe the classical motion of a particle in the electrostatic field of the medium atoms. They do not account for random events of inelastic scattering of a projectile from individual atoms leading to the atom's excitation or ionization. The impact of such events on the projectile motion is twofold. First, they result in a gradual decrease in the projectile energy due to the ionization losses. Second, they lead to a chaotic change in the direction of the projectile motion.

Rigorous treatment of the inelastic collision evens can only be achieved by means of quantum mechanics. However, taking into account that such events are random, fast and local they can be incorporated into the classical mechanics framework according to their probabilities [45]; this approach is implemented in MBN Explorer.

\subsubsection{Dynamic simulation box}

To simulate propagation of particles through an infinite medium, MBN Explorer utilizes the dynamic boundary conditions. In this case all particles in the system are separated in two groups: the moving particles and the fixed ones.

For a single moving particle interacting with fixed atoms of the medium, the dynamic boundary algorithm is illustrated in Figure 12.5. Once the particle approaches the border of the simulation box, a new box of the same size is generated with its center placed at the geometric center of the particle. To avoid spurious change in the force acting on the projectile, the positions of the atoms located in the intersection of the two boxes are conserved. The remaining part of the new box is filled with atoms of the medium following the specifications in the input file. 


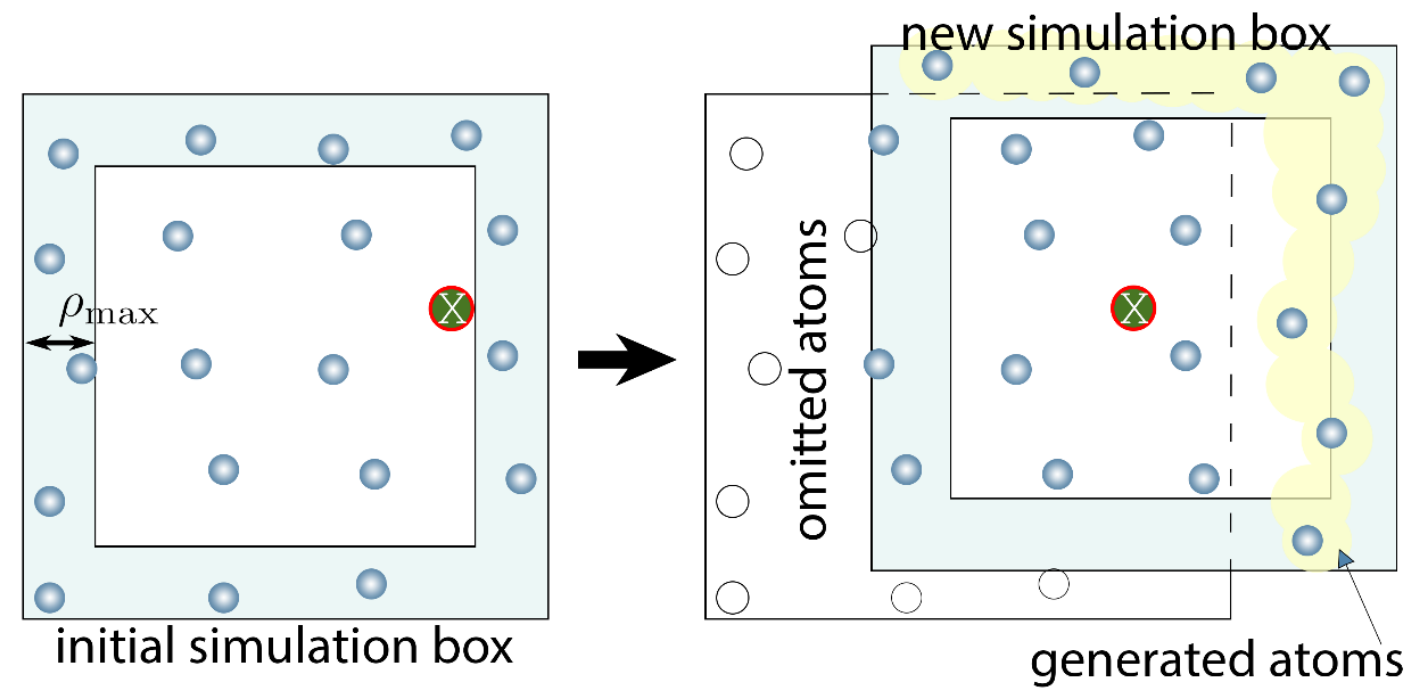

Figure 12.5. Illustration of the dynamic simulation box algorithm [32]. Once an X-marked projectile approaches the face of the simulation box (left panel), a new box of the same size is generated (right panel) with the particle placed approximately in its center. Positions of atoms (small shadowed circles) located in the intersection of the old and new boxes do not change. In the rest part of the new box the atomic positions are generated anew.

MBN Explorer enables to simulate classical trajectories for different types of projectiles and their energies, different crystals (straight, bent and periodically bent) and crystallographic directions. Figure 12.6 shows typical trajectories of $6.7 \mathrm{GeV}$ positrons (panel A) and electrons (panel B) propagating in a $105 \mu \mathrm{m}$ thick silicon crystal. The particles were initially collimated along Si(110) crystallographic planes. Horizontal dashed lines indicate the planes separated by the distance $d=$ $1.92 \AA$, that is the (110) interplanar distance in the silicon crystal at $T=300 \mathrm{~K}$. Figure 12.6 illustrates a difference in channeling motions experienced by electrons and positrons. Positrons (Figure 12.6A) move along the (110) planes bouncing between two neighboring planes, their oscillations exhibit nearly harmonic character. In contrast, electrons (Figure 12.6B) propagate oscillating in the vicinity of a plane. As a result, their trajectories are strongly non-harmonic and they tend to leave the channeling mode of motion earlier as compared to positrons.
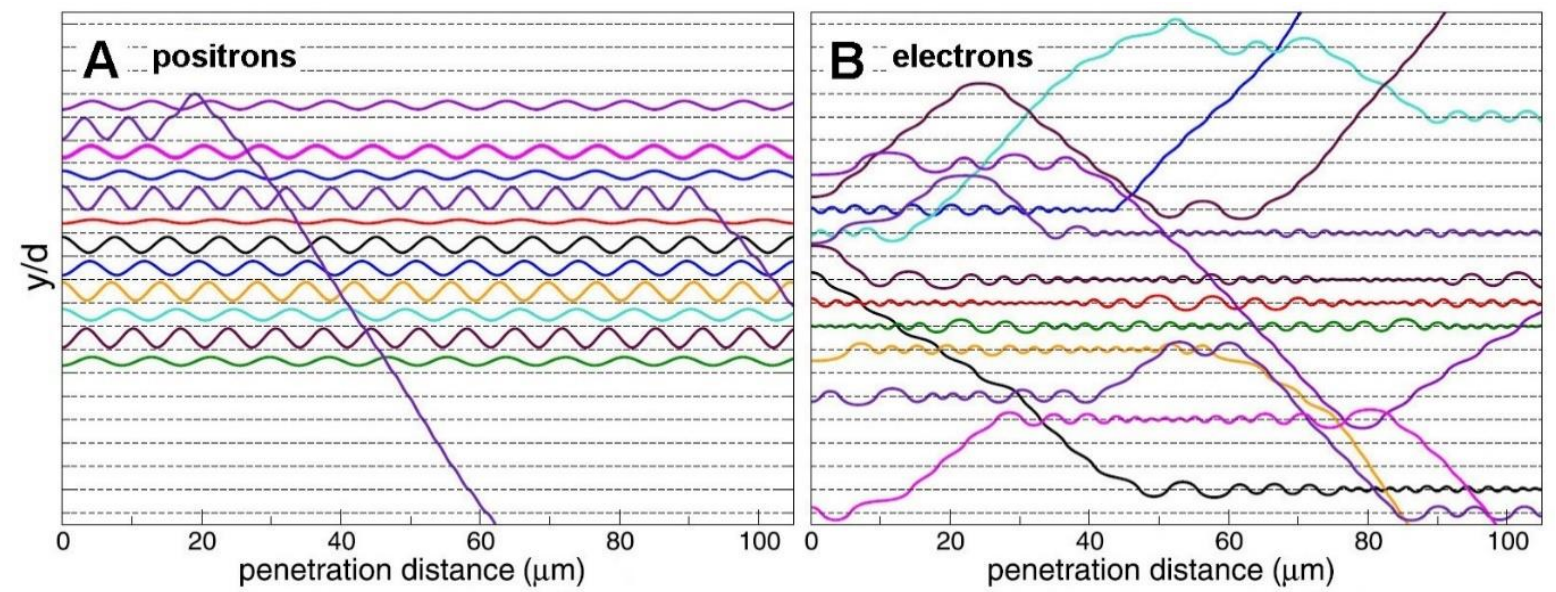

Figure 12.6. Channeling of $6.7 \mathrm{GeV}$ positrons (A) and electrons (B) in a $105 \mu \mathrm{m}$ thick silicon crystal. The plots show typical trajectories of the particles initially collimated along Si (110) crystallographic planes. The trajectories are simulated by means of the dedicated channeling module of MBN Explorer [32]. Horizontal dashed lines indicate the planes separated by the distance $d=1.92 \AA$. 
Simulated trajectories can be used further for statistical characterization of the radiation emitted by a projectile. For each set of simulated trajectories of the total number $N_{\text {traj }}$, the code provides an option to calculate the spectral distribution of the energy $d E$ emitted within the cone $\theta \leq \theta_{0} \ll$ 1 along the direction of the incident particle beam:

$$
\left\langle\frac{d E}{d(\hbar \omega)}\right\rangle=\frac{1}{N_{\text {traj }}} \sum_{n=1}^{N_{\text {traj }}} \frac{d E_{n}}{d(\hbar \omega)^{\prime}}, \quad \frac{d E_{n}}{d(\hbar \omega)}=\int_{0}^{2 \pi} d \varphi \int_{0}^{\theta_{0}} \theta d \theta \frac{d^{2} E_{n}}{d(\hbar \omega) d \Omega}
$$

Here, $d^{2} E_{n} /(\hbar \omega) d \Omega$ stands for the spectral-angular distribution emitted by a projectile moving along the $j^{\text {th }}$ trajectory. In MBN Explorer, this quantity is calculated within the framework of the quasi-classical approximation [47] that combines classical description of the particle's motion with the quantum corrections due to the radiative recoil and using the algorithm described in Refs. $[23,32]$. The sum in Eq. (12.3) is carried out over all simulated trajectories, i.e. it accounts for the contribution of the channeling segments of the trajectories as well as of those corresponding to the non-channeling regime.

Figure 12.7 shows the dependencies $d E_{n} /(\hbar \omega)$ calculated for $6.7 \mathrm{GeV}$ electrons and positrons aligned along $\mathrm{Si}(110)$ crystallographic plane at the crystal entrance [32]. Statistical uncertainties due to the finite number of the analyzed trajectories $(\approx 500$ trajectories in each case) are indicated by the error bars which correspond to the probability $\alpha=0.999$.

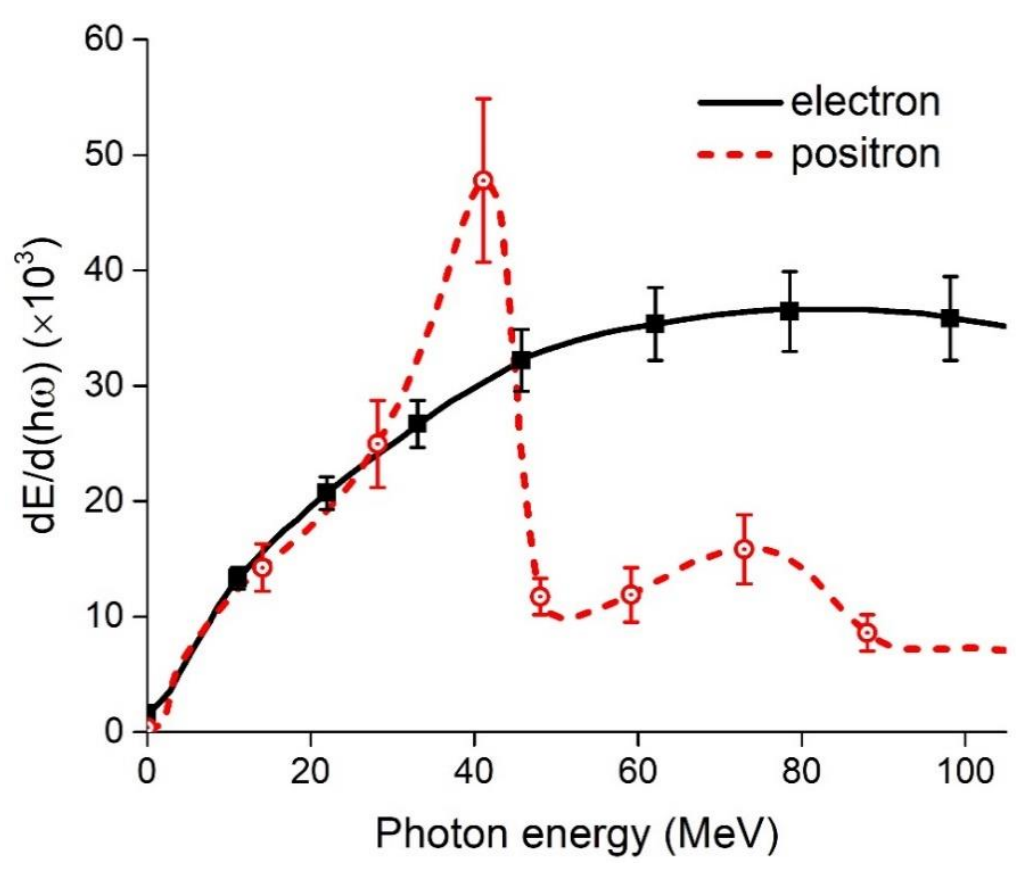

Figure 12.7. Radiation spectra from $6.7 \mathrm{GeV}$ electrons (solid line) and positrons (dashed line) channeling through a 105 $\mu \mathrm{m}$ thick $\mathrm{Si}(110)$ crystal [32]. The lines are drawn over ca. 200 photon energy points in which the spectra were calculated. The symbols mark a small fraction of the points and are drawn to illustrate typical statistical errors (due to a finite number of the simulated trajectories) in different parts of the spectrum.

In connection with the problems of constructing the high-quality undulator material and carrying out a quantitative analysis of the structures manufactured by different methods, MBN Explorer enables all-atom MD simulations to model periodically bent structures under various external conditions. This approach combined with modern numerical algorithms and advanced computational facilities will bring the predictive power of the software up to the accuracy level 
comparable or higher than that achievable experimentally. It can turn computational modeling into a very useful instrumental tool, which in many cases could substitute the actual and expensive laboratory experiments by computational modeling, and thus reduce the experimental and technological costs.

Verification of the channeling module of MBN Explorer against experimental data as well as predictions of other theoretical models has been carried out in a large number of publications [32, 41, 48-52], where channeling of electrons and positions in straight, bent and periodically bent crystals was studied, and the radiation spectra were calculated and successfully compared to experimental data.

\subsection{Computational modeling of the focused electron beam induced deposition process}

The controllable fabrication of nanostructures with nanoscale resolution remains a considerable scientific and technological challenge [53]. To address such a challenge, novel techniques have been developed [25] which exploit irradiation of nanosystems with collimated electron and ion beams. One of such techniques, Focused Electron Beam Induced Deposition (FEBID) [24-26], is based on the irradiation of precursor molecules [54] by high-energy electrons whilst they are being deposited upon a substrate. Electron-induced decomposition releases the metallic component of the precursor which forms a deposit on the surface with size similar to that of the incident electron beam (typically, a few nanometers) [55].

FEBID enables reliable direct-write fabrication of complex, free-standing 3D structures $[55,56]$. Still, as the intended resolution falls below $10 \mathrm{~nm}$, even FEBID struggles to yield the desired size, shape and chemical composition [55,57], which primarily originates from the lack of molecularlevel understanding of the irradiation-driven chemistry (IDC) underlying nanostructure formation and growth $[26,55]$. Computational multiscale modeling provides a new methodology for understanding IDC and, consequently, advancing controllable fabrication of nanostructures.

FEBID operates through successive cycles of organometallic precursor molecules replenishment on a substrate and irradiation by a focused electron beam, which induces the release of metal-free ligands and the growth of metal-enriched nanodeposits. It involves a complex interplay of phenomena that require dedicated computational approaches: (a) deposition, diffusion and desorption of precursor molecules on the substrate; (b) multiple scattering of the primary electrons (PE) through the substrate, with a fraction of them being reflected (backscattered electrons, BSE) and the generation of additional secondary electrons (SE) by ionization; (c) electron-induced dissociation of the deposited molecules; and (d) the follow-up chemistry along with potential thermomechanical effects. While processes (b) and (c) typically happen on the femtosecond-to-picosecond timescale, (a) and (d) may require up to microseconds or even longer.

Until recently, most computer simulations of FEBID and the nanostructure growth have been performed using a Monte Carlo (MC) approach and diffusion-reaction theory [25, 58, 59], which allow simulations of the average characteristics of the process concerning local growth rates and the nanostructure composition. However, these approaches do not provide any molecular-level details regarding structure (crystalline, amorphous, mixed) and the IDC involved. At the 
atomic/molecular level, ab initio methods permit the precise simulation of electronic transitions or chemical bond reorganization $[60,61]$, although their applicability is typically limited to the femtosecond-picosecond timescales and to relatively small molecular sizes. In between these approaches, classical MD [1] and particularly reactive MD [62] have proved to be very useful in the atomistic-scale analysis of molecular fragmentation and chemical reactions up to nanoseconds and microseconds $[62,63]$.

A breakthrough into the atomistic simulation of FEBID was achieved recently by means of Irradiation-Driven Molecular Dynamics (IDMD), a novel and general methodology for computer simulations of irradiation-driven transformations of complex molecular systems [64]. This approach overcomes the limitations of previously used computational methods and describes FEBID-based nanostructures at the atomistic level by accounting for chemical transformation of surface-adsorbed molecular systems under focused electron beam irradiation $[1,64,65]$.

Within the IDMD framework various quantum processes occurring in an irradiated system (e.g. ionization, bond dissociation via electron attachment, or charge transfer) are treated as random, fast and local transformations incorporated into the classical MD framework in a stochastic manner with the probabilities elaborated on the basis of quantum mechanics [64]. Major transformations of irradiated molecular systems are simulated by means of MD with reactive CHARMM (rCHARMM) force field [62, 66] using the MBN Explorer [2] and MBN Studio [3] software packages. Details of the IDMD and rCHARMM are provided in another chapter of this book (see the chapter "Irradiation driven molecular dynamics with reactive CHARMM force field" by A.V. Verkhovtsev, I.A. Solov'yov and A.V. Solov'yov).

In the pioneering study [64] IDMD was successfully applied for the simulation of FEBID of W(CO) 6 precursors on a $\mathrm{SiO}_{2}$ surface and enabled to predict the morphology, molecular composition and growth rate of tungsten-based nanostructures emerging on the surface during the FEBID process. The follow-up study [65] introduced a novel multiscale computational methodology that couples MC simulations for radiation transport with IDMD for simulating the IDC processes with atomistic resolution. The developed multiscale modeling approach enables simulation of radiation transport and effects in complex systems where all the FEBID-related processes (deposition, irradiation, replenishment) are accounted for. The spatial and energy distributions of secondary and backscattered electrons emitted from a $\mathrm{SiO}_{2}$ substrate were used to simulate electroninduced formation and growth of metal nanostructures obtained after deposition of $\mathrm{W}(\mathrm{CO})_{6}$ precursors on $\mathrm{SiO}_{2}$.

Within the IDMD framework, the space-dependent rate for bond cleavage in molecules on the substrate surface is given by:

$$
P(x, y)=\sigma_{\text {frag }}\left(E_{0}\right) J_{P E}\left(x, y, E_{0}\right)+\sum_{i} \sigma_{f r a g}\left(E_{i}\right) J_{S E / B S E}\left(x, y, E_{i}\right)
$$

where $E_{0}$ is the initial energy of the electron beam, $E_{i}<E_{0}$ a discrete set of values for the electron energies lower than $E_{0} ; J_{P E / S E / B S E}\left(x, y, E_{i}\right)$ are space- and energy-dependent fluxes of PE/SE/BSE (electrons per unit area and unit time), and $\sigma_{\text {frag }}\left(E_{i}\right)$ is the energy-dependent molecular fragmentation cross section. The PE beam flux at the irradiated circular spot of radius $R$ is:

$$
J_{0}=\frac{I_{0}}{e S_{0}},
$$


where $I_{0}$ corresponds to the PE beam current, $S_{0}=\pi R^{2}$ to its area and $e$ is the elementary charge. The electron distributions were simulated using the MC radiation transport code SEED (Secondary Electron Energy Deposition) [67, 68]. Molecular fragmentation and further chemical reactions were simulated by means of MBN Explorer [2] while MBN Studio [3] was employed for constructing the molecular system, performing the precursor molecule replenishment phases, as well as for analyzing the IDMD simulation results.
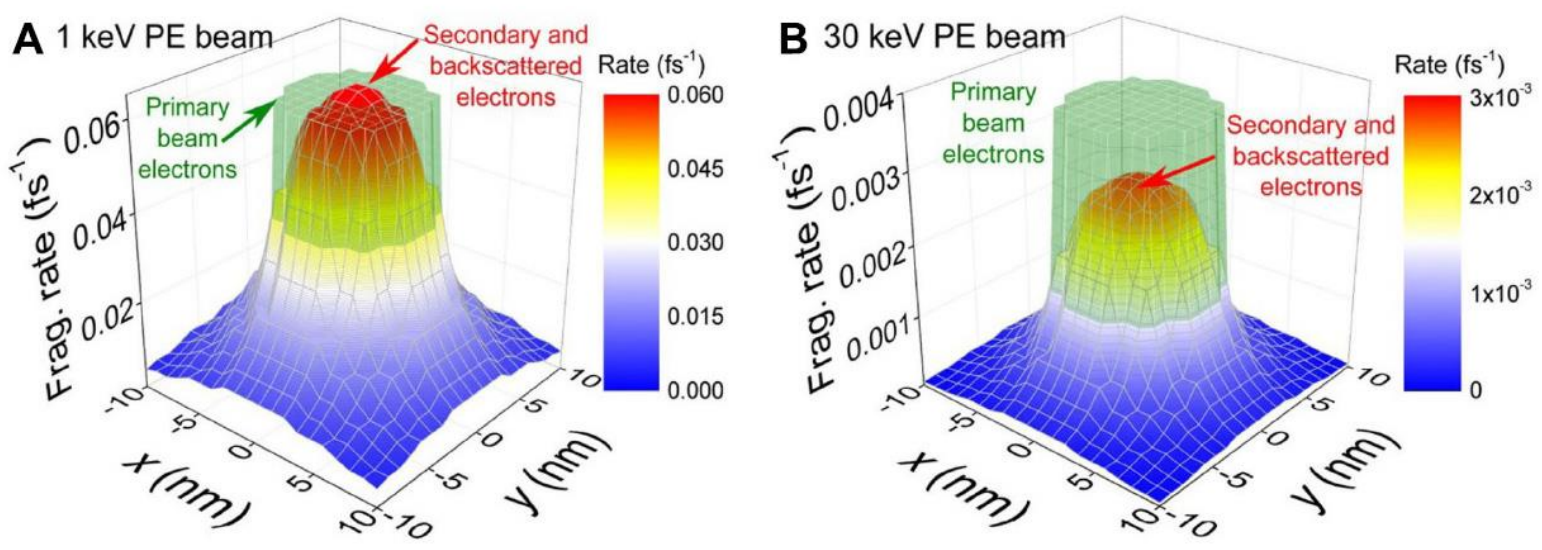

Figure 12.8. Electron-induced fragmentation rates for $\mathrm{W}(\mathrm{CO})_{6}$ precursor molecules irradiated with $\mathrm{PE}$ beams of $E_{0}=1$ $\mathrm{keV}$ (A) and $E_{0}=30 \mathrm{keV}$ (B). The green transparent surface depicts the PE beam area. Adapted from Ref. [65].

Figure 12.8 illustrates the space-dependent fragmentation rates induced by uniform $1 \mathrm{keV}$ (panel A) and $30 \mathrm{keV}$ (panel B) beams of unit PE flux $J_{0}=1 \mathrm{~nm}^{-2} \mathrm{fs}^{-1}$ within a circular area of radius $R=$ $5 \mathrm{~nm}$. Although the number of BSE/SE electrons for $30 \mathrm{keV}$ is small, their large cross section (in relation to $\mathrm{PE}$ ) produces a significant fragmentation probability, but less than that due to $\mathrm{PE}$ at the beam area. However, for $1 \mathrm{keV}$, the fragmentation probability due to BSE/SE ( 80-90\% exclusively due to SE) is very large, and significantly extends beyond the PE beam area. These results clearly demonstrate the very different scenarios to be expected for beams of different energies and which will importantly influence the deposit properties, as well as the prominent role of low-energy SE on molecular fragmentation.

Each irradiation phase lasts for a time known as dwell time, whose typical duration in experiment $(\geq \mu \mathrm{s}$ ) is still computationally demanding for MD. To address this challenge, the irradiation phase was simulated for $10 \mathrm{~ns}$ and simulated PE fluxes $J_{0}$ (and hence PE beam currents $I_{0}$ ) were then scaled to match the same number of PE per unit area and per dwell time as in experiments [64]. As for replenishment, its characteristic times are also typically very long $(\sim \mathrm{ms})$. In simulations, the $\mathrm{CO}$ molecules desorbed to the gas phase are simply removed during the replenishment stages and new $\mathrm{W}(\mathrm{CO})_{6}$ molecules are deposited.

As the irradiation-replenishment cycles proceed, atomic clusters and islands of different sizes and compositions appear on the substrate as the result of IDC, and the process of nucleation of metalenriched islands and its coalescence starts [64]. Figure 12.9 shows the number of atoms (either $\mathrm{W}, \mathrm{C}$ or 0 ) in the largest island is shown for three simulation conditions close to reported in experiments [69]: $30 \mathrm{keV}$ at $I_{0}=0.28 \mathrm{nA}, 10 \mathrm{keV}$ at $I_{0}=2.3 \mathrm{nA}$ and $1 \mathrm{keV}$ at $I_{0}=3.7 \mathrm{nA}$. Smaller clusters tend to merge with time giving rise to larger structures and, eventually, to the largest island displayed in the figure. The jumps in the island size observed with some frequency are due to the merging of independent clusters that grow on the substrate. 


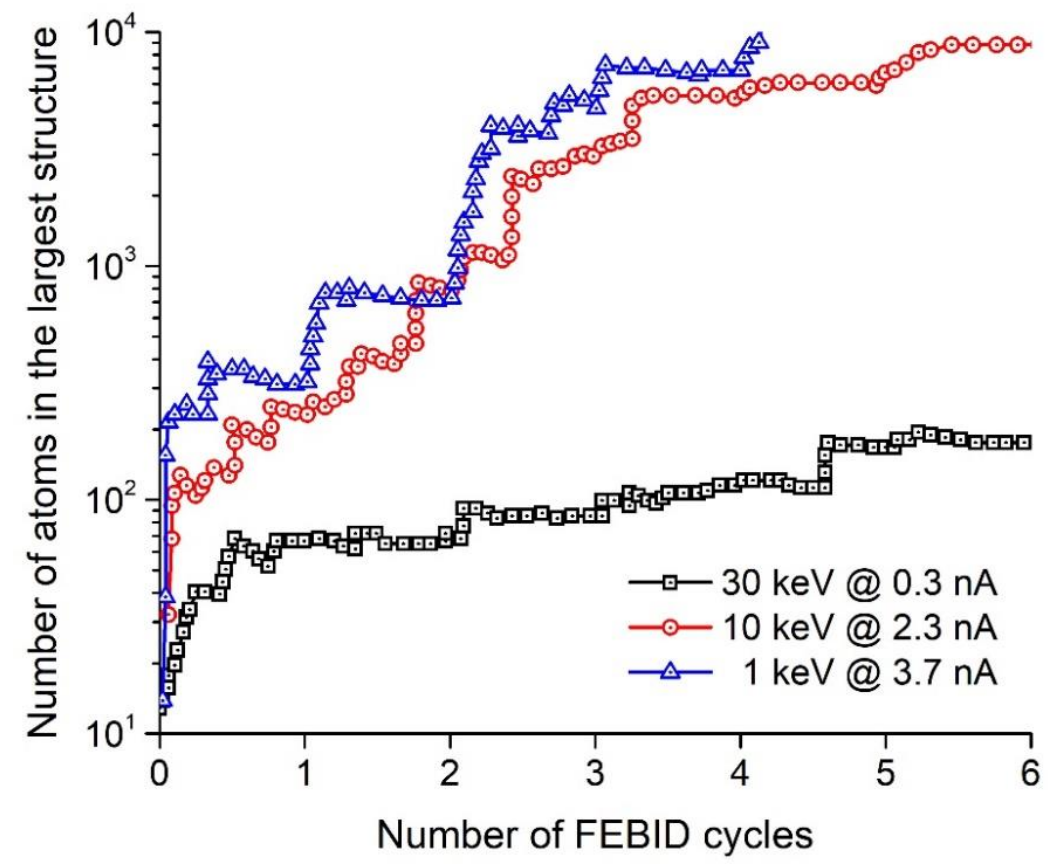

Figure 12.9. Evolution of the number of atoms in the largest simulated islands for PE beams of energies 1,10 and $30 \mathrm{keV}$, for different currents as indicated [65].

Experimental measurements performed to date have been limited to particular values of energy and current due to the characteristics of the electron source [69]. In contrast, the IDMD simulation method permits the exploration of a much broader range of electron beam parameters. Full symbols in Figure 12.10A depict the simulated metal contents of the deposits as a function of experimentally equivalent current $I_{\text {exp }}$. Error bars show the standard deviations obtained from three independent simulations for each case. Experimental results [69] are shown by open symbols. Numbers next to symbols represent the beam energies in keV. It is clearly seen that the results from simulations are within the range of experimental uncertainties, which indicates the predictive capabilities of the simulations.

This analysis provides a detailed "map" of the attainable metal content in the deposits as a function of the beam parameters, which is a valuable outcome for the optimization of FEBID with W(CO) 6 on $\mathrm{SiO}_{2}$. Dashed lines in Figure 12.10A correspond to the limiting values of PE beam energy and current studied. These results clearly show that, within the analyzed energy domain, a decrease in the beam energy and an increase in the current promote the faster growth of the deposit, as well as the augment in its metal content. Simulation results provide the grounds for clearly understanding such trends: an increment in the current means a larger number of PE per unit time, while a reduction in the energy produces an increase in the SE yield. These lead to both the greater size of the deposit and its larger metal content due to the increased probability for bond cleavage (see Figure 12.8).

Figure 12.10B and C show top views of the simulated deposits for 1keV@3.7nA and 10keV@2.3nA, after 5 and 7 irradiation cycles, respectively (the number of atoms in the largest island is similar in these cases, $\sim 12,000$ ). The green circular line marks the area covered by the PE beam (having a radius of $5 \mathrm{~nm}$ ). These figures show that different energy-current regimes lead to distinct deposit microstructures and edge broadenings. While the more energetic $10 \mathrm{keV}$ energy beam produces a deposit almost entirely localized within the PE beam area, the $1 \mathrm{keV}$ beam produces a 
sparser deposit (at least during the early stage of the FEBID process) that significantly extends beyond the PE beam area, producing an undesired edge broadening of the structure.
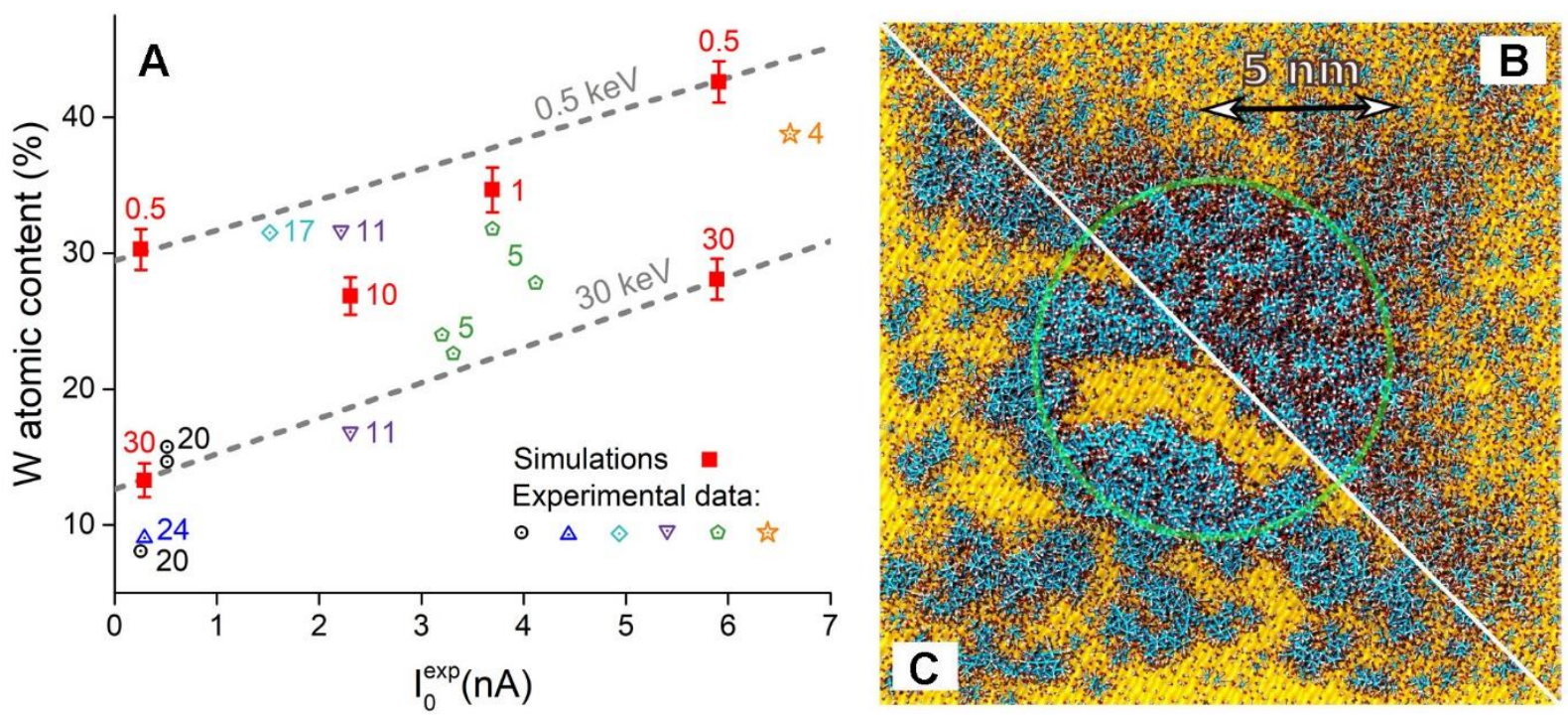

Figure 12.10. Compositions and morphologies of the deposits created by FEBID [65]. (A) Dependence of the deposit metal content on the beam energy $E_{0}$ and current $I_{\text {exp }}$ from experiments (open symbols) [69] and IDMD simulations (full symbols) [65]. Numbers next to symbols represent the beam energy in keV for each case. Panels $\mathbf{B}$ and $\mathbf{C}$ show the top views of the deposits produced by 10keV@2.3nA and 1keV@3.7nA beams, respectively. The green area marks the PE beam spot while blue, white and red spheres represent, respectively, $\mathrm{W}, \mathrm{C}$ and $\mathrm{O}$ atoms; the $\mathrm{SiO}_{2}$ substrate is represented by a yellow surface.

The presented results demonstrate how the novel MC-IDMD approach provides the necessary molecular insights into the key processes behind FEBID, which can be used for its further optimization and development. The simulations (which rely on basic atomic and molecular data such as cross sections for electron scattering and molecular fragmentation) demonstrate [65] a great predictive power, yielding fabricated nanostructure compositions and morphologies in good agreement with available experimental data [69]. In particular, it is shown that the increase in both the growth rate and $\mathrm{W}$-metal content of the deposits with the increase in PE beam current and with the decrease in its energy is related to the increase in the number of ejected low-energy SE. The latter are also responsible for the different microstructures and edge broadenings observed for beams of different energies. This analysis advocates for wide exploitation of the IDMD methodology in FEBID and many other processes in which the irradiation of molecular systems and irradiation driven chemistry play the key role.

\subsection{Computational modeling of ion-induced DNA damage in relation to ion-beam cancer therapy}

MBN Explorer and MBN Studio can be utilized for the evaluation of radiobiological damage created by heavy ions propagating in different media, including biological. Such an analysis is behind the important biomedical technology known as the ion-beam cancer therapy (IBCT) [13, $27,28,70]$. IBCT allows delivery of high doses into tumors, maximizing cancer cell destruction, and simultaneously, minimizing the radiation damage of surrounding healthy tissue. The full potential of such therapy can only be realized if the fundamental mechanisms leading to lethal cell 
damage under ion irradiation are well understood. The key question is whether it is possible to quantitatively predict macroscopic biological effects caused by ion radiation on the basis of physical and chemical effects related to the ion-medium interactions on a nanometer scale.

Recent review papers [13, 71] and the book [28] presented an overview of the main ideas of the MultiScale Approach to the physics of radiation damage with ions (MSA). This approach has the goal of developing knowledge about biodamage at the nanoscale and molecular level and finding the relation between the characteristics of incident particles and the resultant biological damage. The MSA is unique in distinguishing essential phenomena relevant to radiation biodamage at a given time, space and energy scale, and assessing the damage [13, 28, 71]. Temporal and spatial scales are schematically shown in Figure 12.11.

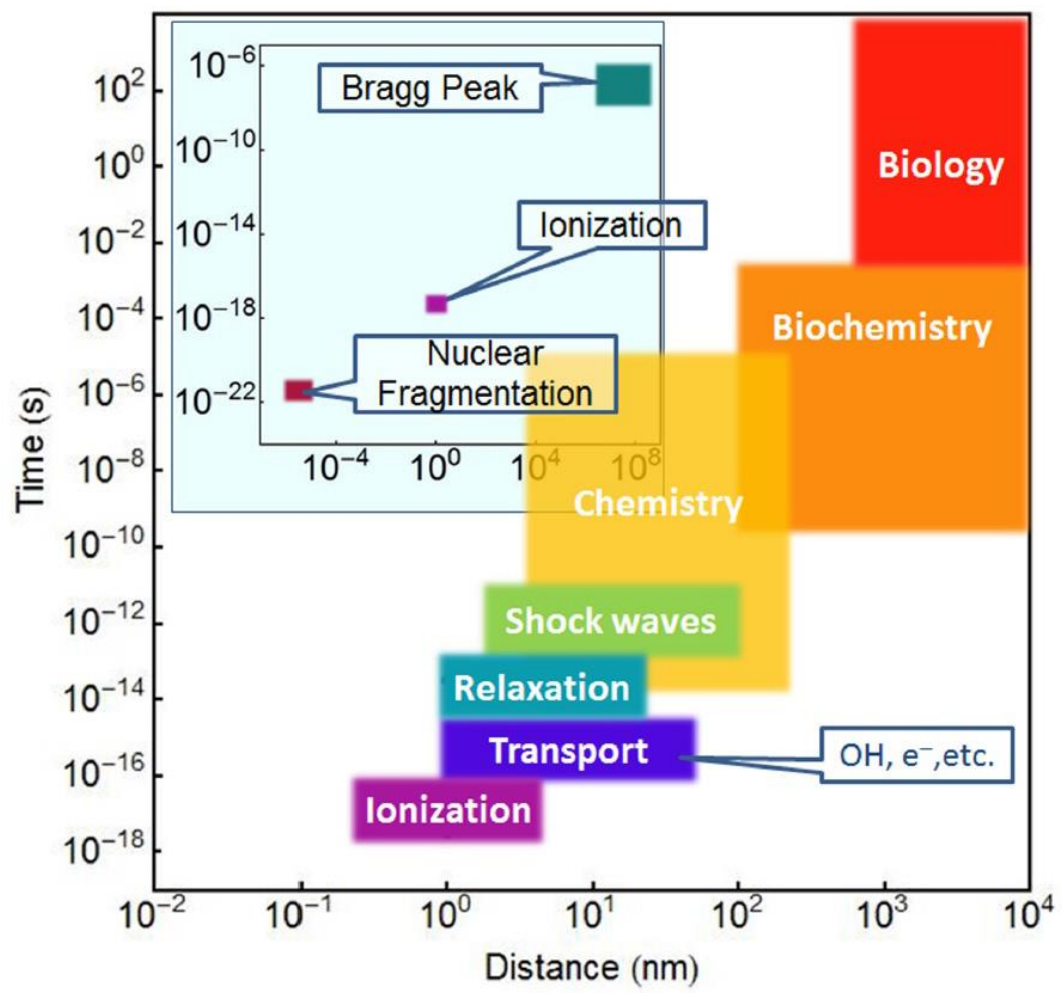

Figure 12.11. Features, processes, and disciplines, associated with radiation therapy, shown in a space-time diagram, which indicates temporal and spatial scales of the phenomena. The history from ionization/excitation to biological effects are shown in the main figure, and features of ion propagation are shown in the inset. Adapted from Ref. [13].

Radiation damage due to ionizing radiation is initiated by the ions incident on tissue. Initially, they have energy ranging from a few to hundreds of MeV per nucleon. In the process of propagation through tissue, the ions lose energy due to ionization, excitation, nuclear fragmentation, etc. Most of the energy loss of the ion is transferred to tissue. Naturally, radiation damage is associated with this transferred energy, and the dose (i.e., deposited energy density) is a common indicator for the assessment of the damage $[13,27,72]$. The profile of the linear energy transfer (LET), that is the energy absorbed by the medium per unit length of the projectile's trajectory, along the ion's path is characterized with a plateau followed by a sharp Bragg peak. The position of this peak depends on the initial energy of the ion and marks the location of the maximum radiation damage. In the process of radiation therapy, a tumor is being "scanned" with the Bragg peak both laterally and longitudinally. 
However, the deposition of large doses in the vicinity of the Bragg peak does not explain how the radiation damage occurs, since projectiles themselves only interact with a few biomolecules along their trajectory and this direct damage is only a small fraction of the overall damage. It is commonly understood that the secondary electrons and free radicals produced in the processes of ionization and excitation of the medium with ions are largely responsible for the vast portion of the biodamage.

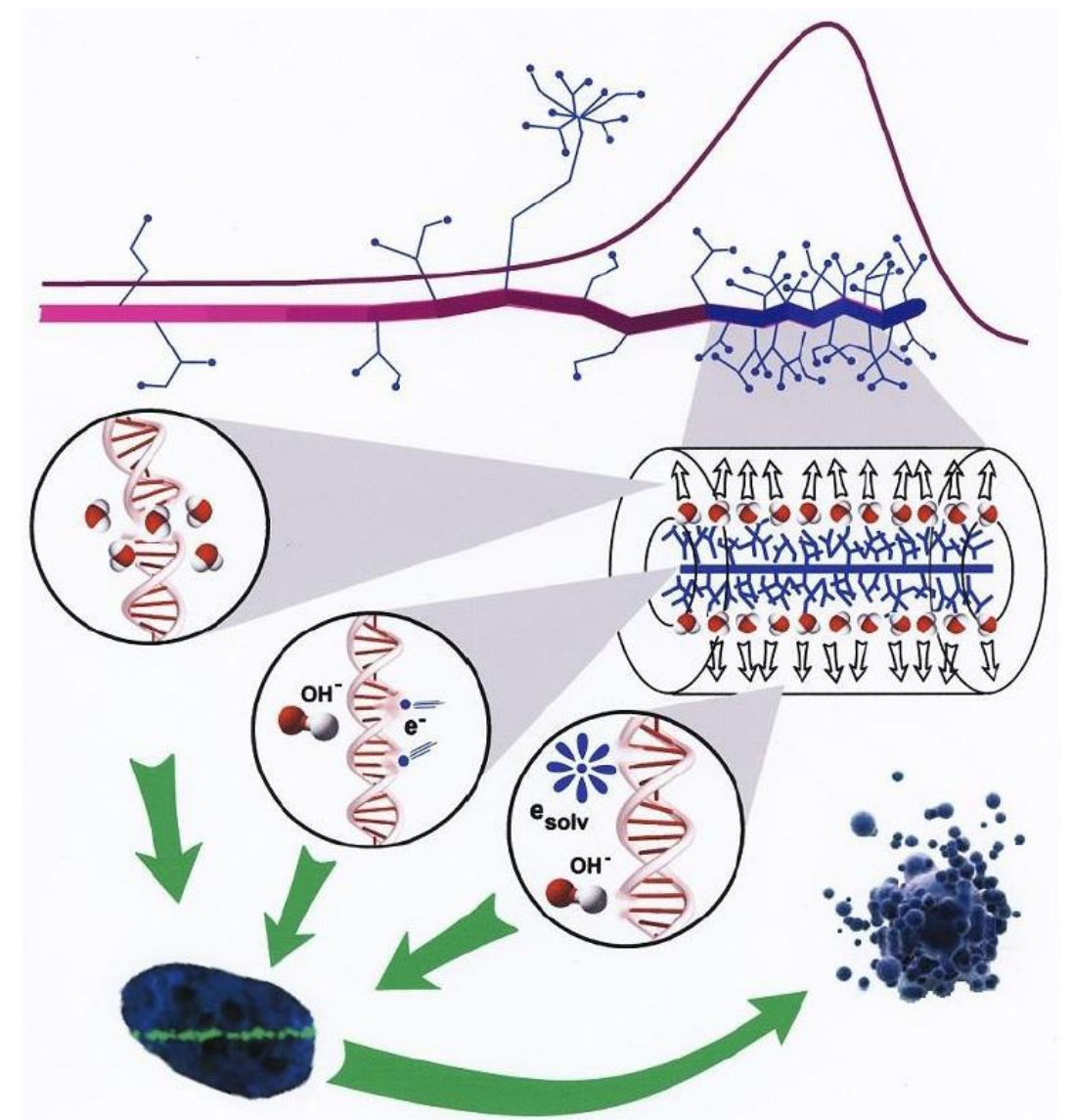

Figure 12.12. The scenario of biological damage with ions. Ion propagation ends with a Bragg peak, shown in the top right corner. A segment of the track at the Bragg peak is shown in more detail. Secondary electrons and radicals propagate away from the ion's path damaging biomolecules (central circle). They transfer the energy to the medium resulting in the rapid temperature and pressure increase inside the cylinder. The shock wave (shown in the expanding cylinder) due to the pressure increase damages biomolecules by stress (left circle), but it also effectively propagates reactive species to larger distances (right circle). A living cell responds to all shown DNA damage by creating foci (visible in the stained cells), in which enzymes attempt to repair the induced lesions. If these efforts are unsuccessful, the cell dies (the lower right corner). Adapted from Ref. [13].

Secondary electrons are produced during a short time of $10^{-18}-10^{-17} \mathrm{~s}$ following the ion's passage. The energy spectrum of secondary electrons has been extensively discussed in the literature (see the review [13]) and the main result is that most secondary electrons have energy below $50 \mathrm{eV}$. This has several important consequences. First, the ranges of propagation of these electrons in tissue are rather small, around $10 \mathrm{~nm}$ [73]. Second, the angular distribution of their velocities, as they are ejected from their original host and as they scatter further, is largely uniform [74]; this allows one to consider their transport using a random walk approach [75-78].

The next time scale $10^{-16}-10^{-15} \mathrm{~s}$ corresponds to the propagation of secondary electrons in tissue. In liquid water, the mean free paths of elastically scattered and ionizing $50-\mathrm{eV}$ electrons are about 
0.43 and $3.5 \mathrm{~nm}$, respectively [74]. This means that they ionize a molecule after about seven elastic collisions, while the probability of second ionization is small [79]. Thus, the secondary electrons are losing most of their energy within first 20 collisions and this happens within 1-2 nm of the ion's path [80]. After that they continue propagating, elastically scattering with the molecules of the medium until they get bound or solvated electrons are formed. It is important to notice that these low energy electrons remain important agents for biodamage since they can attach to biomolecules like DNA causing dissociation [81]. The solvated electrons may play an important role in the damage scenario as well $[82,83]$.
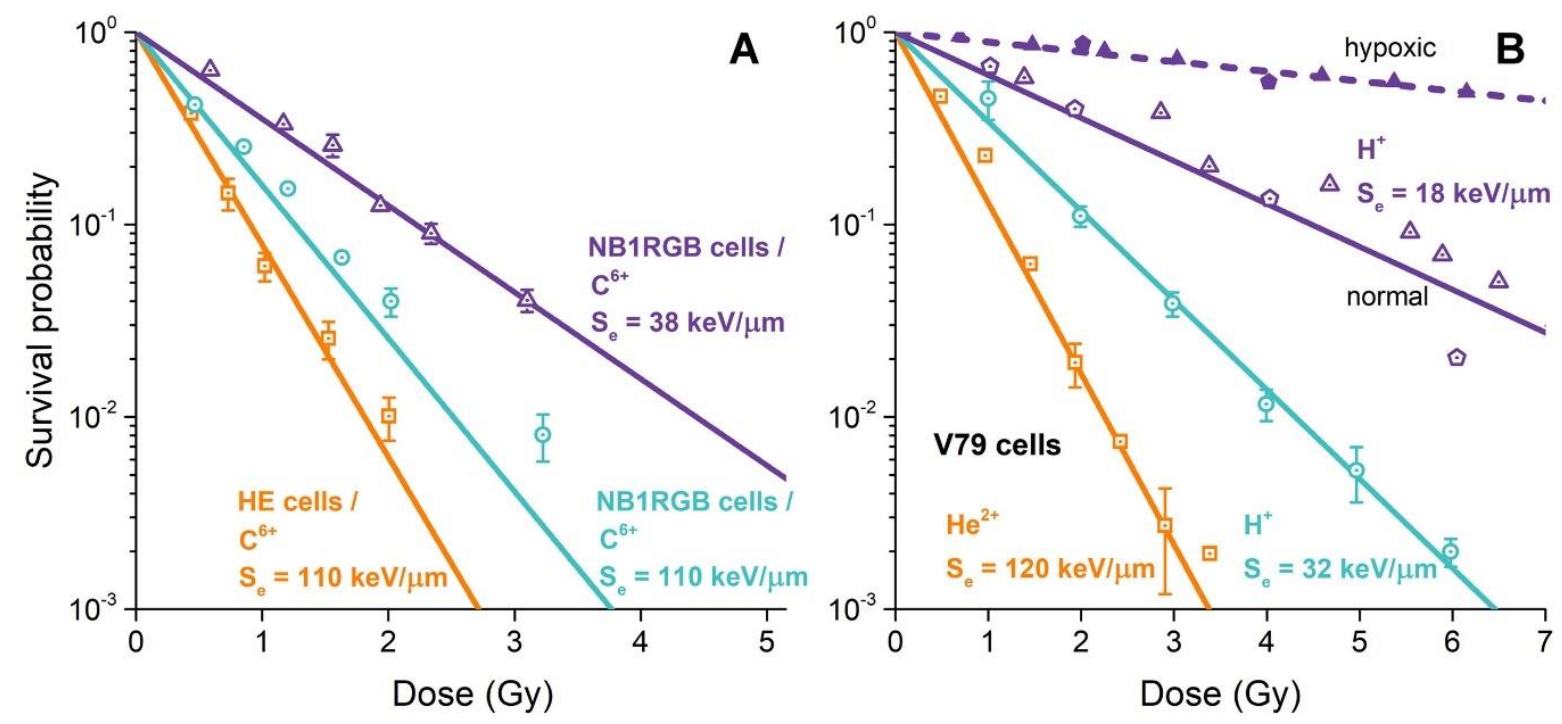

Figure 12.13. Survival probabilities as a function of deposited dose for different human (A) and Chinese hamster ? ? ? cell lines. The survival probabilities calculated within the MSA [13] at the indicated LET values are shown with lines. Experimental data for cells measured at a specific dose, either at normal or hypoxic conditions, are shown by symbols. For further details see $[92,93]$.

The energy lost by secondary electrons in the processes of ionization and excitation of the medium is transferred to its heating (i.e. vibrational excitation of molecules) due to the electron--phonon interaction. As a result, the medium within a 1-2-nm cylinder (for ions not heavier than iron) surrounding the ion's path is heated up rapidly [80, 84]; this cylinder is referred to as the "hot" cylinder. The pressure inside this cylinder increases by several orders of magnitude (e.g. by a factor of $10^{3}$ for a carbon ion at the Bragg peak [84]) compared to the pressure in the medium outside the cylinder. This pressure builds up by about $10^{-14}-10^{-13} \mathrm{~s}$ and it is a source of a cylindrical shock wave [85] which propagates through the medium for about $10^{-13}-10^{-11} \mathrm{~s}$. Its relevance to the biodamage is as follows. If the shock wave is strong enough (the strength depends on the distance from the ion's path and the LET), it may inflict damage directly by breaking covalent bonds in a DNA molecule [66, 80, 86-89]. Besides, the radial collective motion of the medium induced by the shock wave is instrumental in propagating the highly reactive molecular species, such as hydroxyl radicals and solvated electrons, to large radial distances (up to tens of nanometers) thus increasing the area of an ion's impact [90,91].

The assessment of the primary damage to DNA molecules and other parts of cells due to the above effects is done within the MSA. This damage consists of various lesions on DNA and other biomolecules. Some of these lesions may be repaired by the living system, but some may not and the latter may lead to cell inactivation. 
The scenario described above is illustrated in Figure 12.12 [13]. The detailed comparison of its outcomes with experimental observations on the survival probability of irradiated cells was performed in Refs. [92, 93]. Figure 12.13 highlights this comparison for several exemplar case studies.

\subsubsection{Simulation of the thermomechanical damage of the DNA by the ion-induced shock wave}

The direct thermomechanical damage of the DNA molecule as a result of interaction with the ioninduced shock wave has been explored using MBN Explorer [66, 80, 86, 87]. In the earlier investigations [80, 86, 87], the DNA damage by ion-induced shock waves was studied by means of classical MD simulations using non-reactive molecular mechanics force fields. In those simulations the potential energy stored in a particular DNA bond was monitored in time as the bond length varied around its equilibrium distance [80,87]. When the potential energy of the bond exceeded a given threshold value, the bond was considered broken.
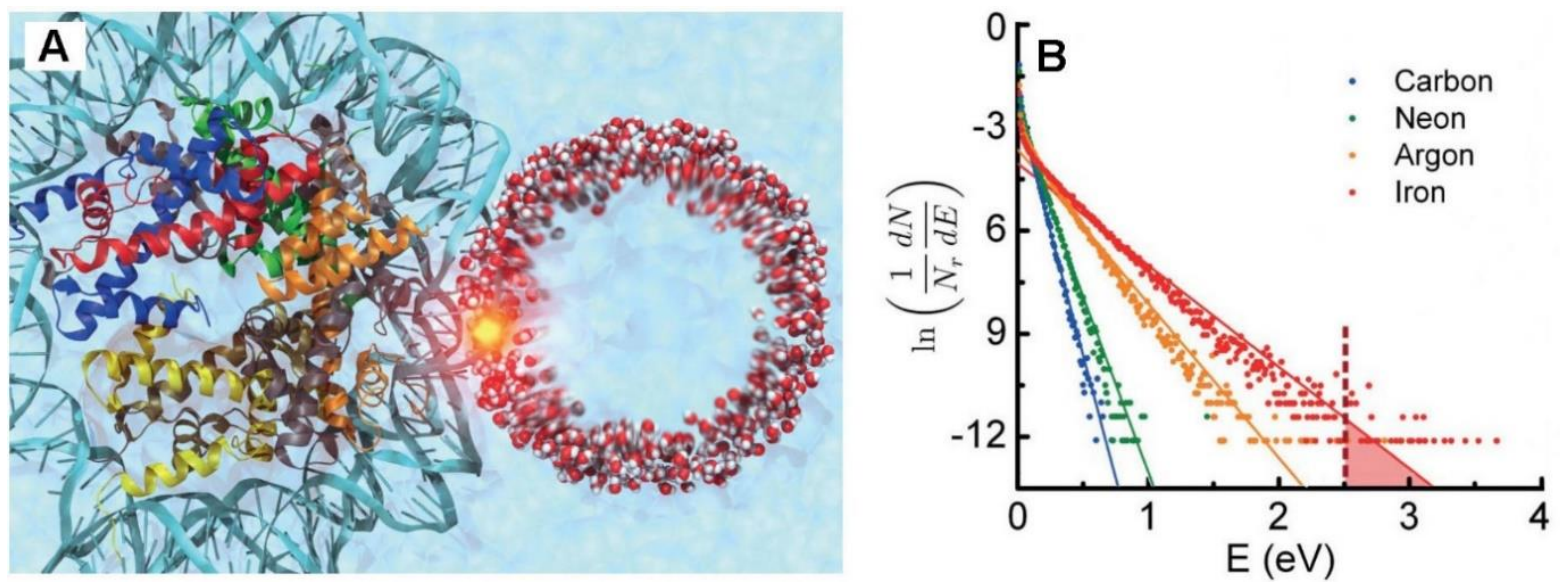

Figure 12.14. A: The cylindrical shock wave front in water (on the right; ion's path is the axis of this cylinder, perpendicular to the figure plane) interacts with a nucleosome (on the left) with a segment of a DNA molecule on the surface. The yellow dot indicates the place where damage occurs. The medium is very dense following the wave front and is rarefied in the wake. B: The dependence of the logarithm of the normalized number of the covalent bond energy records for the selected DNA backbone region per $0.01 \mathrm{eV}$ energy interval on the bond energy for four values of LET: $900,1730,4745$, and $7195 \mathrm{eV} / \mathrm{nm}$, corresponding to the Bragg peak values for carbon, neon, argon, and iron ions, respectively [80]. Straight lines correspond to the fits of these distributions. Adapted from Ref. [80].

In the pioneering study [80] the MD simulations were focused on the interaction of the cylindrical shock wave originating from ion's path with a fragment of a DNA molecule situated on the surface of a nucleosome, see Figure 12.14A. Nucleosomes, histone-protein octamers wrapped about with a DNA double helix, are the primary structural units of chromatin, which is a principal component of the cell nucleus in eukaryotic cells. The simulations [80] were done for four values of LET, namely 900, 1730, 4745 and $7195 \mathrm{eV} / \mathrm{nm}$, corresponding to the Bragg peak values for carbon, neon, argon and iron ions, respectively. Carbon ions are clinically used for cancer treatment, whereas heavier ions up to iron are present in galactic cosmic rays, being potentially damaging for humans during space missions $[94,95]$

The simulations were performed using the standard CHARMM force field [96] which implies the harmonic approximation for describing the interaction potentials for covalent bonds and thus 
does not allow to observe bond breaking events directly. Therefore, in order to study whether the covalent bonds in the DNA backbone can be broken during the shock wave action, the energy temporarily deposited to these bonds was calculated. The analysis of MD simulations performed for four values of LET $(900,1730,4745$ and $7195 \mathrm{eV} / \mathrm{nm})$ gives the distributions of the bond energy records. These records can be represented by a histogram that assigns to every interval of energy $(\varepsilon, \varepsilon+\delta \varepsilon)$; the number of records corresponding to the bond energies from this interval. For each value of LET, the bond energy distribution was constructed. These distributions (normalized to the total number of records $N_{\mathrm{r}}$ for each value of LET) are shown in Figure 12.14B, where $\ln \left(1 / N_{r} d N / d \varepsilon\right)$ is plotted versus the corresponding energy interval. Next, the number of energy records of selected covalent bonds of the DNA backbone exceeding a given threshold was counted. This was done by direct counting of bond energy records, for which $E>E_{0}$, where $E_{0}$ is a variable threshold; e.g., the records counted for $E_{0}=2.5 \mathrm{eV}$ are shown to the right of the dashed vertical line in Figure 12.14B.

A more quantitative description of the ion-induced shock wave phenomenon has become possible by means of reactive MD simulations that permitted explicit simulation of covalent bond rupture and formation [62]. A recent study [66] presented a detailed computational protocol for modeling the shock wave induced DNA damage by means of the rCHARMM force field [62].

The target DNA molecule studied in [66] contained 30 complementary DNA base pairs. The molecule was placed in a water box extending $17 \mathrm{~nm}$ from the DNA in the $x$ - and $y$-directions and $8 \mathrm{~nm}$ in the $z$-direction. The total system size was $1,010,994$ atoms.

It is widely established that one of the key events of radiation-induced DNA damage concerns the formation of single- and double strand breaks (SSBs and DSBs) of the sugar-phosphate backbone. Therefore, the rCHARMM force field was used to describe interatomic interactions in the $\mathrm{C}_{3}$ '-O, $\mathrm{C}_{4}^{\prime}-\mathrm{C}_{5}^{\prime}, \mathrm{C}_{5}$ '-O and $\mathrm{P}-\mathrm{O}$ bonds in the DNA backbone, which connect the sugar ring of one nucleotide and the phosphate group of an adjacent nucleotide. Bond dissociation energies and cutoff distances for bond breakage/formation were determined from density functional theory (DFT) calculations [66]. Covalent interactions in other parts of the DNA molecule were modeled using the standard CHARMM force field.

In the MD simulations, the energy lost by the propagating ion is deposited into the kinetic energy of water molecules located inside a "hot" cylinder of $1 \mathrm{~nm}$ radius around the ion's path. The equilibrium velocities of all atoms inside the "hot" cylinder are increased by a factor $\alpha$ such that the kinetic energy of these atoms reads as $[80,86,97]$ :

$$
\sum_{i}^{N} \frac{1}{2} m_{i}\left(\alpha v_{i}\right)^{2}=N \frac{3}{2} k_{B} T+S_{e} l
$$

Here $S_{\mathrm{e}}$ is the LET of the projectile ion, $l$ is the length of the simulation box in the $z$-direction (parallel to the ion's path), and $N$ is the total number of atoms within the "hot" cylinder. The first term on the right-hand side of Eq. (12.6) is the kinetic energy of the 1-nm radius cylinder at the equilibrium temperature, $T=300 \mathrm{~K}$, whereas the second term describes the energy loss by the ion as it propagates through the medium. 


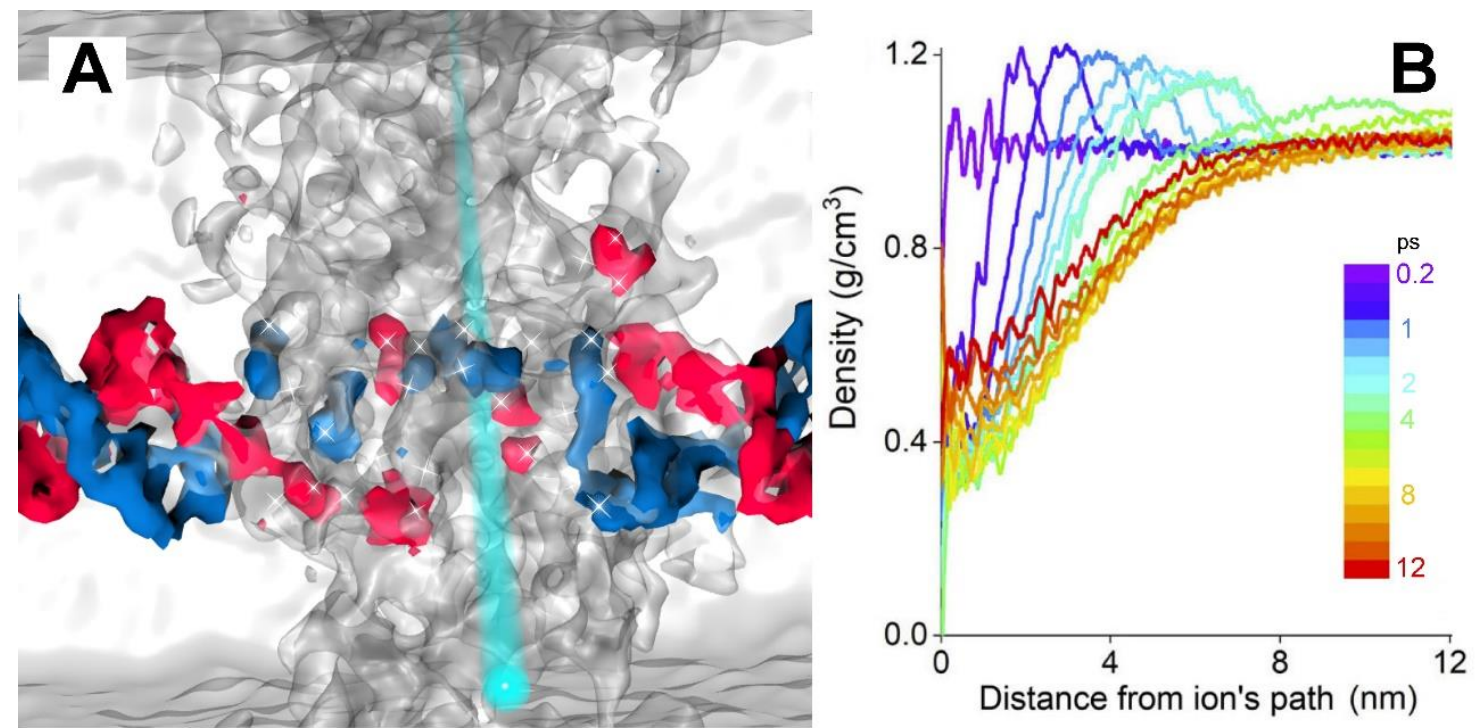

Figure 12.15. A: Illustration of the propagation of the ion-induced shock wave in the vicinity of the DNA segment containing 30 base pairs [66]. Water molecules inside the "hot" cylinder surrounding the ion track (shown by the cyan line) are highlighted. B: The density of water in the radial direction from the ion's path is shown at different instances, ranging from 0 to 12 ps after irradiation.

The shock wave propagates in the molecular system radially away from the ion track, see Figure 12.15A. The range of the shock wave propagation in the aqueous environment can be determined by monitoring the radial density of the water molecules in time. The ion track was in this case placed directly through the geometrical center of the DNA strand. The results of this analysis are shown in Figure 12.15B. The wave front moves toward the edge of the simulation box as time passes, and the wave profile becomes lower and broader, showing that the shock wave relaxes as time passes. This indicates that the impact of the shock wave weakens over time. Figure 12.15B shows that the maximal density of the wave does not change significantly in the range of $2-6 \mathrm{~nm}$ from the ion track, thus a DNA strand placed in this range is expected to receive the strongest impact from the shock wave.

The impact of a shock wave on DNA can be characterized through the probability of strand breaks formation, which can be used to quantify the amount of biodamage induced by the shock wave mechanism [13]. Figure 12.16 shows the number of strand breaks in the DNA segment, caused by a shock wave induced by an argon ion with LET of $2890 \mathrm{eV} / \mathrm{nm}$, a function of simulation time. The number of breaks rises quickly within the first $7 \mathrm{ps}$ of the simulation, where the shock wave front hits both DNA strands of the target molecule. After this moment the DNA damage rate becomes lower lasting until approximately 15 ps, hereafter the number of breaks remains steady. Even if the number of breaks generally rises with time, some fluctuations of this quantity can be seen locally. This effect might be attributed to the broken bonds which can be rejoined if the atoms involved get close to each other after the initial bond breakage. This analysis shows that multiple or even complex stand breaks might be induced by the generated shock waves. 


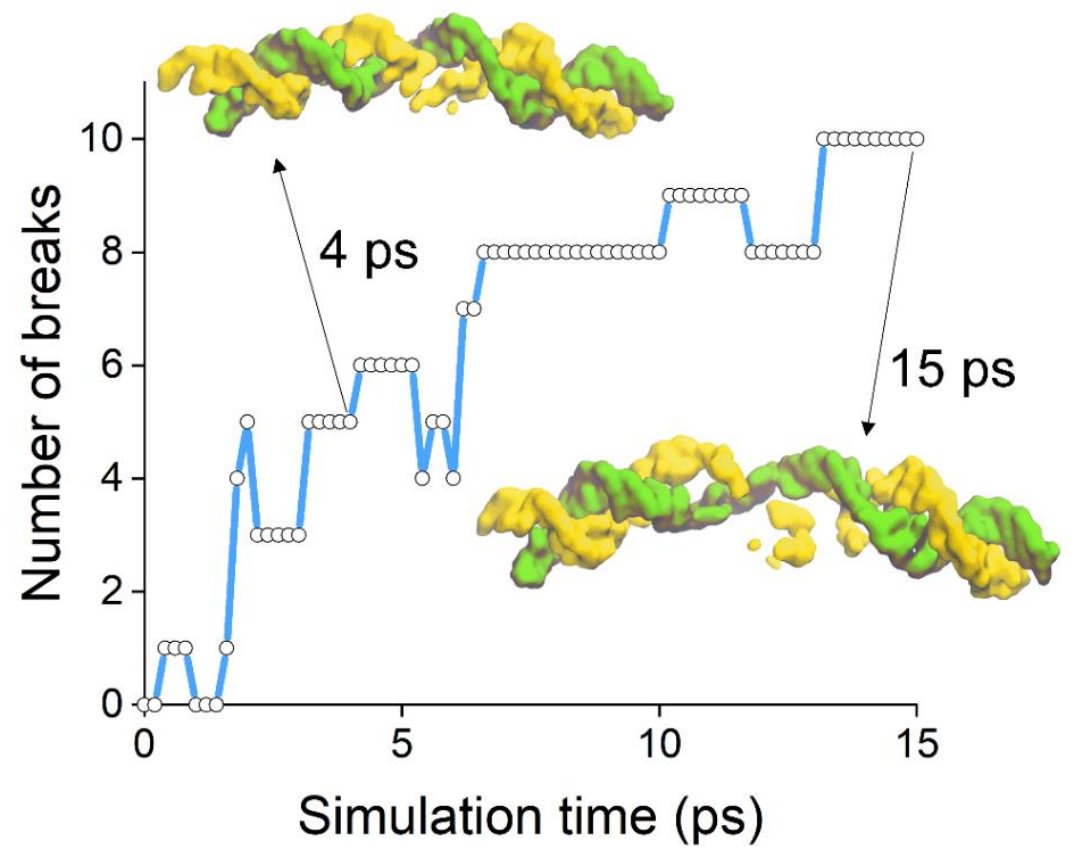

Figure 12.16. The number of strand breaks in the 30 base pairs-long DNA segment induced by the thermomechanical stress by the argon ion with an LET of $2890 \mathrm{eV} / \mathrm{nm}$ as a function of simulation time [66]. Two insets show the breakage of the DNA strands at simulation time instances of 4 ps and 15 ps.

The methodology reviewed here can be applied in further computational studies considering irradiation of DNA with different ions and different orientations between the ion's path and the DNA molecule. Such analysis is important for understanding the radiation damage with ions on a quantitative level, focusing on particular physical, chemical, and biological effects that bring about lethal damage to cells exposed to ion beams $[13,92,93]$.

\subsection{Conclusions}

MBN Explorer and MBN Studio are the powerful tools for computational modeling in different areas of challenging research arising in connection with development of the aforementioned technologies. Illustrative case studies of multiscale modeling have been presented in this chapter in relation to three emerging technologies, namely (i) development of novel sources of monochromatic high-energy radiation based on the crystalline undulators, (ii) controlled fabrication of nanostructures using the focused electron-beam induced deposition, and (iii) ionbeam cancer therapy. These examples illustrate that the unique algorithms and methodologies implemented in MBN Explorer, combined with the visualization interface of MBN Studio, in many cases can substitute expensive laboratory experiments by computational multiscale modeling making the software play a role of a "computational nano- and microscope".

\section{Acknowledgements}

The authors are grateful for financial support from the COST Action CA17126 "Towards understanding and modelling intense electronic excitation" (TUMIEE), Deutsche Forschungsgemeinschaft (Projects no. 415716638, 413220201, SFB1372 and GRK1885), the 
Volkswagen Stiftung (Lichtenberg professorship to IAS), and the European Union's Horizon 2020 research and innovation programme - the Radio-NP project (GA 794733) within the H2020MSCA-IF-2017 call, the RADON project (GA 872494) within the H2020-MSCA-RISE-2019 call and the N-LIGHT project within the H2020-MSCA-RISE2019 call (GA 872196).

\section{References}

[1] I.A. Solov'yov, A.V. Korol, A.V. Solov'yov, Multiscale Modeling of Complex Molecular Structure and Dynamics with MBN Explorer (Springer International Publishing, Cham, Switzerland, 2017)

[2] I.A. Solov'yov, A.V. Yakubovich, P.V. Nikolaev, I. Volkovets, A.V. Solov'yov, J. Comput. Chem. 33 (2012) 2412

[3] G.B. Sushko, I.A. Solov'yov, A.V. Solov'yov, J. Mol. Graph. Model. 88 (2019) 247

[4] J.P. Connerade, A.V. Solov'yov (eds.), Latest Advances in Atomic Clusters Collision: Structure and Dynamics from the Nuclear to the Biological Scale (Imperial College Press, 2008)

[5] H.M. Senn, W. Thiel, Angew. Chem. Int. Ed. 48 (2009) 1198

[6] L.W. Chung, W.M.C. Sameera, R. Ramozzi, A.J. Page, M. Hatanaka, G.P. Petrova, T.V. Harris, X. Li, Z. Ke, F. Liu et al., Chem. Rev. 115 (2015) 5678

[7] E. Brunk, U. Rothlisberger, Chem. Rev. 115 (2015) 6217

[8] K.Y. Sanbonmatsu, C.S. Tung, J. Struct. Biol. 157 (2007) 470

[9] G. Zhao, J.R. Perilla, E.L. Yufenyuy, X. Meng, B. Chen, J. Ning, J. Ahn, A.M. Gronenborn, K. Schulten, C. Aiken et al., Nature 497 (2013) 643

[10] R. Schulz, B. Lindner, L. Petridis, J.C. Smith, J. Chem. Theory Comput. 5 (2009) 2798

[11] B. Boudaiffa, P. Cloutier, D. Hunting, M.A. Huels, L. Sanche, Science 287 (2000) 1658

[12] M.A. Huels, B. Boudaiffa, P. Cloutier, D. Hunting, L. Sanche, J. Am. Chem. Soc. 125 (2003) 4467

[13] E. Surdutovich, A.V. Solov'yov, Eur. Phys. J. D 68 (2014) 353

[14] K. Haume, S. Rosa, S. Grellet, M.A. Smialek, K.T. Butterworth, A.V. Solov'yov, K.M. Prise, J. Golding, N.J. Mason, Cancer Nanotechnol. 7 (2016) 8

[15] S. Kmiecik, D. Gront, M. Kolinski, L. Wieteska, A.E. Dawid, A. Kolinski, Chem. Rev. 116 (2016) 7898

[16] V.V. Dick, I.A. Solov'yov, A.V. Solov'yov, Phys. Rev. B 84 (2011) 115408

[17] M.A. Panshenskov, I.A. Solov'yov, A.V. Solov'yov, J. Comput. Chem. 35 (2014) 1317

[18] P. Moskovkin, M.A. Panshenskov, S. Lucas, A.V. Solov'yov, Phys. Stat. Sol. B 251 (2014) 1456

[19] D.L. Logan, A First Course in the Finite Element Method (6th ed.) (Cengage Learning, Boston, MA, 2016)

[20] I.A. Solov'yov, G.B. Sushko, A.V. Solov'yov, MBN Explorer Users' Guide. Version 3.0 (MesoBioNano Science Publishing, Frankfurt am Main, 2017)

[21] I.A. Solov'yov, G.B. Sushko, A.V. Verkhovtsev, A.V. Korol, A.V. Solov'yov, MBN Explorer and MBN Studio Tutorials: Version 3.0 (MesoBioNano Science Publishing, Frankfurt am Main, 2017)

[22] A.V. Korol, A.V. Solov'yov, Eur. Phys. J. D 74 (2020) 201 
[23] A.V. Korol, A.V. Solov'yov, W. Greiner, Channeling and Radiation in Periodically Bent Crystals (2nd ed.) (Springer Series on Atomic, Optical, and Plasma Physics, vol. 69. Springer-Verlag, Heidelberg, New York, Dordrecht, London, 2014)

[24] W.F. van Dorp, C.W. Hagen, J. Appl. Phys. 104 (2008) 081301

[25] I. Utke, S. Moshkalev, P. Russel (eds.), Nanofabrication Using Focused Ion and Electron Beams (Oxford University Press, 2012)

[26] M. Huth, F. Porrati, C. Schwalb, M. Winhold, R. Sachser, M. Dukic, J. Adams, G. Fantner, Beilstein J. Nanotechnol. 3 (2012) 597

[27] D. Schardt, T. Elsässer, D. Schulz-Ertner, Rev. Mod. Phys. 82 (2010) 383

[28] A.V. Solov'yov (ed.), Nanoscale Insights into Ion-Beam Cancer Therapy (Springer International Publishing, 2017)

[29] A.V. Korol, A.V. Solov'yov, W. Greiner, J. Phys. G: Nucl. Part. Phys. 24 (1998) L45

[30] A.V. Korol, A.V. Solov'yov, W. Greiner, Int. J. Mod. Phys. E 8 (1999) 49

[31] A.V. Korol, A.V. Solov'yov, W. Greiner, Int. J. Mod. Phys. E 13 (2004) 867

[32] G.B. Sushko, V.G. Bezchastnov, I.A. Solov'yov, A.V. Korol, W. Greiner, A.V. Solov'yov, J. Comput. Phys. 252 (2013) 404

[33] P. Emma, R. Akre, J. Arthur, R. Bionta, C. Bostedt, J. Bozek, A. Brachmann, P. Bucksbaum, R. Coffee, F.J. Decker et al., Nature Photonics 4 (2010) 641

[34] B.W.J. McNeil, N.R. Thompson, Nature Photonics 4 (2010) 814

[35] V. Ayvazyan et al., Eur. Phys. J. D 20 (2002) 149

[36] M. Yabashi, H. Tanaka, Nature Photonics 11 (2017) 12

[37] K.A. Olive et al. (Particle Data Group), Chin. Phys. C 38 (2014) 090001

[38] J. Lindhard, K. Dan. Vidensk. Selsk. Mat. Fys. Medd. 34 (1965) 1

[39] L. Bandiera, E. Bagli, G. Germogli, V. Guidi, M. Mazzolari, H. Backe, W. Lauth, A. Berra, D. Lietti, M. Prest et al., Phys. Rev. Lett. 115 (2015) 025504

[40] M.A. Kumakhov, Phys. Lett. 57A (1976) 17

[41] V.G. Bezchastnov, A.V. Korol, A.V. Solov'yov, J. Phys. B: At. Mol. Opt. Phys. 47 (2014) 195401

[42] V.L. Ginzburg, Izv. Akad. Nauk SSSR (in Russian) 11 (1947) 165

[43] H. Motz, J. Appl. Phys. 22 (1951) 527

[44] P. Rullhusen, X. Artru, P. Dhez, Novel Radiation Sources using Relativistic Electrons (World Scientific, Singapore, 1998)

[45] A.V. Korol, A.V. Solov'yov, W. Greiner, J. Phys. G: Nucl. Part. Phys. 27 (2001) 95

[46] A.V. Korol, A.V. Solov'yov, W. Greiner, Int. J. Mod. Phys. E 9 (2000) 77

[47] V.N. Baier, V.M. Katkov, V.M. Strakhovenko, Electromagnetic Processes at High Energies in Oriented Single Crystals (World Scientific, Singapore, 1998)

[48] G.B. Sushko, A.V. Korol, A.V. Solov'yov, Nucl. Instrum. Meth. B 355 (2015) 39

[49] A.V. Korol, V.G. Bezchastnov, G. Sushko, A.V. Solov'yov, Nucl. Instrum. Meth. B 387 (2016) 41 
[50] H. Shen, Q. Zhao, F.S. Zhang, G.B. Sushko, A.V. Korol, A.V. Solov'yov, Nucl. Instrum. Meth. B 424 (2018) 26

[51] A.V. Pavlov, A.V. Korol, V.K. Ivanov, A.V. Solov'yov, J. Phys. B: At. Mol. Opt. Phys. 52 (2019) 11LT01

[52] A.V. Pavlov, A.V. Korol, V.K. Ivanov, A.V. Solov'yov, Eur. Phys. J. D 74 (2020) 21

[53] Z. Cui, Nanofabrication. Principles, Capabilities and Limits (Springer International Publishing, Cham, Switzerland, 2017)

[54] S. Barth, M. Huth, F. Jungwirth, J. Mater. Chem. C 8 (2020) 15884

[55] I. Utke, P. Hoffmann, J. Melngailis, J. Vac. Sci. Technol. B 26 (2008) 1197

[56] M. Huth, F. Porrati, O.V. Dobrovolskiy, Microelectron. Eng. 185-186 (2018) 9

[57] R.M. Thorman, T.P. Ragesh Kumar, D.H. Fairbrother, O. Ingólfsson, Beilstein J. Nanotechnol. 6 (2015) 1904

[58] J.D. Fowlkes, P.D. Rack, ACS Nano 4 (2010) 1619

[59] D. Sanz-Hernández, A. Fernández-Pacheco, Beilstein J. Nanotechnol. 8 (2017) 2151

[60] K. Muthukumar, H.O. Jeschke, R. Valentí, E. Begun, J. Schwenk, F. Porrati, M. Huth, Beilstein J. Nanotechnol. 3 (2012) 546

[61] K. Muthukumar, H.O. Jeschke, R. Valentí, Beilstein J. Nanotechnol. 9 (2018) 711

[62] G.B. Sushko, I.A. Solov'yov, A.V. Verkhovtsev, S.N. Volkov, A.V. Solov'yov, Eur. Phys. J. D 70 (2016) 12

[63] P. de Vera, A. Verkhovtsev, G. Sushko, A.V. Solov'yov, Eur. Phys. J. D 73 (2019) 215

[64] G.B. Sushko, I.A. Solov'yov, A.V. Solov'yov, Eur. Phys. J. D 70 (2016) 217

[65] P. de Vera, M. Azzolini, G. Sushko, I. Abril, R. Garcia-Molina, M. Dapor, I.A. Solov'yov, A.V. Solov'yov, Sci. Rep. 10 (2020) 20827

[66] I. Friis, A. Verkhovtsev, I.A. Solov'yov, A.V. Solov’yov, J. Comput. Chem. 41 (2020) 2429

[67] M. Dapor, Transport of Energetic Electrons in Solids (3rd ed.) (Springer International Publishing, Cham, Switzerland, 2020)

[68] M. Azzolini, M. Angelucci, R. Cimino, R. Larciprete, N.M. Pugno, S. Taioli, M. Dapor, J. Phys.: Condens. Matter 31 (2019) 055901

[69] F. Porrati, R. Sachser, M. Huth, Nanotechnology 20 (2009) 195301

[70] U. Linz (ed.), Ion Beam Therapy: Fundamentals, Technology, Clinical Applications (Springer, 2012)

[71] E. Surdutovich, A.V. Solov'yov, Cancer Nanotechnol. 10 (2019) 6

[72] E.L. Alpen, Radiation Biophysics (Academic Press, San Diego, London, Boston, New York, Sydney, Tokyo, Toronto, 1998)

[73] J. Meesungnoen, J.P. Jay-Gerin, A. Filali-Mouhim, S. Mankhetkorn, Radiat. Res. 158 (2002) 657

[74] H. Nikjoo, S. Uehara, D. Emfietzoglou, F.A. Cucinotta, Radiat. Meas. 41 (2006) 1052

[75] E. Surdutovich, D.C. Gallagher, A.V. Solov'yov, Phys. Rev. E 84 (2011) 051918

[76] E. Surdutovich, A.V. Solov'yov, Eur. Phys. J. D 66 (2012) 206

[77] A.V. Solov'yov, E. Surdutovich, E. Scifoni, I. Mishustin, W. Greiner, Phys. Rev. E 79 (2009) 011909

[78] M. Bug, E. Surdutovich, H. Rabus, A.B. Rosenfeld, A.V. Solov'yov, Eur. Phys. J. D 66 (2012) 291 
[79] E. Surdutovich, O. Obolensky, E. Scifoni, I. Pshenichnov, I. Mishustin, A. Solov'yov, W. Greiner, Eur. Phys. J. D 51 (2009) 63

[80] E. Surdutovich, A.V. Yakubovich, A.V. Solov'yov, Sci. Rep. 3 (2013) 1289

[81] Y. Park, Z. Li, P. Cloutier, L. Sanche, J. Wagner, Radiat. Res. 175 (2011) 240

[82] C. von Sonntag, The Chemical Basis of Radiation Biology (Taylor \& Francis, London, 1987)

[83] M. Smyth, J. Kohanoff, J. Am. Chem. Soc. 134 (2012) 9122

[84] M. Toulemonde, E. Surdutovich, A.V. Solov'yov, Phys. Rev. E 80 (2009) 031913

[85] E. Surdutovich, A.V. Solov'yov, Phys. Rev. E 82 (2010) 051915

[86] A.V. Yakubovich, E. Surdutovich, A.V. Solov'yov, AIP Conf. Proc. 1344 (2011) 230

[87] P. de Vera, N.J. Mason, F.J. Currell, A.V. Solov'yov, Eur. Phys. J. D 70 (2016) 183

[88] A. Fraile, M. Smyth, J. Kohanoff, A.V. Solov'yov, J. Chem. Phys. 150 (2019) 015101

[89] D. Bottländer, C. Mücksch, H.M. Urbassek, Nucl. Instrum. Meth. B 365 (2015) 622

[90] E. Surdutovich, A.V. Solov'yov, Eur. Phys. J. D 69 (2015) 193

[91] P. de Vera, E. Surdutovich, N.J. Mason, F.J. Currell, A.V. Solov'yov, Eur. Phys. J. D 72 (2018) 147

[92] A. Verkhovtsev, E. Surdutovich, A.V. Solov'yov, Sci. Rep. 6 (2016) 27654

[93] A. Verkhovtsev, E. Surdutovich, A.V. Solov'yov, Cancer Nanotechnol. 10 (2019) 4

[94] M. Durante, F.A. Cucinotta, Rev. Mod. Phys. 83 (2011) 1245

[95] A. Kronenberg, F.A. Cucinotta, Health Phys. 103 (2012) 556

[96] A.D. MacKerell, Jr., D. Bashford, M. Bellott, R.L. Dunbrack, Jr., J.D. Evanseck, M.J. Field, S. Fischer, J. Gao et al., J. Phys. Chem. B 102 (1998) 3586

[97] A.V. Yakubovich, E. Surdutovich, A.V. Solov'yov, Nucl. Instrum. Meth. B 279 (2012) 135 



\title{
13. Irradiation driven molecular dynamics with reactive CHARMM force field
}

\author{
Alexey V. Verkhovtsev*, Ilia A. Solov'yov, and Andrey V. Solov'yov
}

This chapter describes Irradiation Driven Molecular Dynamics (IDMD) - a novel computational methodology for atomistic simulations of the irradiation-driven transformations of complex molecular systems, implemented in the MBN Explorer software package. Within the IDMD framework various quantum processes occurring in an irradiated system are treated as random, fast and local transformations incorporated into the classical MD framework in a stochastic manner with the probabilities elaborated on the basis of quantum mechanics. Major transformations of irradiated molecular systems (such as topological changes, redistribution of atomic partial charges, alteration of interatomic interactions) and possible paths of their further reactive transformations can be simulated by means of MD with reactive force fields, in particular with the reactive CHARMM (rCHARMM) force field implemented in MBN Explorer. This chapter reviews the general concept of the IDMD methodology and the rCHARMM force field and provides several exemplary case studies illustrating utilization of these methods.

\footnotetext{
*Contact: verkhovtsev@mbnexplorer.com
} 


\subsection{Introduction}

There are numerous examples where chemical transformations of complex molecular systems are driven by irradiation. Often such modifications carry important outcomes to the functional properties of the irradiated molecular systems. Enough to mention the radiobiological phenomena, in which living cells are inactivated by irradiation due to the induced complex DNA strand breaks [1-3]; the formation and composition of cosmic ices and dusts in the interstellar medium and planetary atmospheres is largely a result of the interplay of the molecular surface adsorption and surface irradiation [4]; the formation of biologically relevant molecules under extreme conditions involving irradiation [5], and many more.

Irradiation driven chemistry (IDC) is nowadays utilized in modern nanotechnology, such as focused electron beam deposition (FEBID) [6-8] and ultraviolet lithography (UVL) $[9,10]$. These technologies belong to the next generation of nanofabrication techniques allowing the controlled creation of complex three-dimensional nanostructures with nanometer resolution which is attractive in both basic and applied research. Fabrication of increasingly smaller structures has been the goal of the electronics industry for more than three decades and still remains one of this industry's biggest challenges. Furthermore, irradiation driven chemistry is a key element in nuclear waste decomposition technologies [11] and medical radiotherapies [1, 3, 12].

IDC studies transformations of molecular systems induced by their irradiation with photon, neutron, or charged particle beams. IDC is also relevant for molecular systems exposed to external fields, mechanical stress or put in plasma environment. A rigorous quantum-mechanical description of the irradiation driven molecular processes e.g. within time-dependent density functional theory (TDDFT) is feasible but only for relatively small molecular systems containing, at most, a few hundred atoms [13-16]. This strong limitation makes TDDFT of limited use for the description of the IDC of complex molecular systems.

Classical molecular dynamics (MD) could be considered as an alternative theoretical framework for modeling complex molecular systems. For instance, by employing the classical molecular mechanics approach it is feasible to study structure and dynamics of molecular systems that are constituted of millions of atoms $[17,18]$ and evolve on time scales up to hundreds of nanoseconds [19-21]. In the molecular mechanics approach, the molecular system is treated classically, i.e., the atoms of the system are interacting with each other through a parametric phenomenological potential that relies on the network of chemical bonds in the system. This network defines the socalled molecular topology, i.e. a set of rules that impose constraints on the system, and permit maintaining its natural shape as well as its mechanical and thermodynamical properties. The molecular mechanics method has been widely used throughout the last decades and has been implemented, for instance, in the well-established computational packages CHARMM [22], AMBER [23], GROMACS [24] and NAMD [25].

In spite of the manyfold advantages, standard classical MD is unable to simulate irradiation driven processes as it typically does not account for coupling of the system to incident radiation, nor does it describe quantum transformations in the molecular system induced by the irradiation. These deficiencies have been overcome recently by introducing Irradiation Driven Molecular Dynamics (IDMD) [26], a new methodology allowing atomistic simulation of IDC in complex molecular systems. 
The IDMD approach has been implemented in the MBN Explorer software package [27] capable to operate with a large library of classical potentials, many-body force fields and their combinations. MBN Explorer is an advanced software package for simulations of complex biomolecular, nano- and mesoscopic systems [27-30]. It is suitable for classical non-relativistic and relativistic molecular dynamics (MD), Euler dynamics, reactive and irradiation-driven molecular dynamics (RMD and IDMD) simulations, as well as for stochastic dynamics or Monte Carlo (MC) simulations of various randomly moving Meso-Bio-Nano (MBN) systems or processes.

The reactive CHARMM (rCHARMM) force field [31, 32] implemented in MBN Explorer enables the description of bond rupture events and the formation of new bonds by chemically active atoms in the system, monitoring all the changes of the system's topology that occur during its transformations. Chemically active atoms carry information about their partial charges, interactions with other atoms in the system, valences and multiplicities of the bonds that can be formed with other reactive atoms in the system. Being an extension of the commonly used standard CHARMM force field [33-35], rCHARMM is directly applicable to organic and biomolecular systems. Its combination with other force fields [36] enables simulations of even broader variety of molecular systems experiencing chemical transformations whilst monitoring their molecular composition and topology changes $[26,32,36,40]$.

This chapter provides an overview of the IDMD methodology exploiting rCHARMM force field and complements it with several illustrative examples.

\subsection{Irradiation driven molecular dynamics}

IDMD methodology has been designed for the atomistic simulations of the irradiation-driven chemistry processes, and it is applicable to any molecular system exposed to radiation $[26,28,29$, 40]. Within the framework of IDMD various quantum collision processes (e.g. ionization, electronic excitation, bond dissociation via electron attachment, or charge transfer) are treated as random, fast and local transformations incorporated into the classical MD framework in a stochastic manner with the probabilities elaborated on the basis quantum mechanics. This can be achieved because the aforementioned quantum processes happen on the sub- to femtosecond time scales (i.e. during the periods comparable or smaller than a typical single time step of MD simulations) and involve typically a relatively small number of atoms.

The probability of each quantum process is equal to the product of the process cross section and the flux density of incident particles [41]. The cross sections of collision processes can be obtained from (i) ab initio calculations performed by means of various dedicated codes, or (ii) analytical estimates and models, (iii) experiments, as well as (iv) atomic and molecular databases. The flux densities of incident particles are usually specific for the concrete problem and the system considered. The properties of atoms or molecules (energy, momentum, charge, valence, interaction potentials with other atoms in the system, etc.) involved in such quantum transformations are changed according to their final quantum states in the corresponding quantum processes.

In the course of a quantum process the energy and momentum transferred to the system through irradiation is absorbed by the involved electronic and ionic degrees of freedom, and chemically reactive sites (atoms, molecules, molecular sites) in the irradiated system are created. The follow- 
up dynamics of the reactive sites may be described by the classical MD and the thermodynamic state of the system until the system undergoes further irradiation-driven quantum transformations. The chemically reactive sites may also be involved in the chemical reactions leading to the change of their molecular and reactive properties, and participate in the formation of stable and chemically neutral atoms and molecules.

The IDMD methodology accounts for the major dissociative transformations of irradiated molecular systems and possible paths of their further reactive transformations [26] which can be simulated by means of MD with reactive force fields [31]. The necessary input parameters for such simulations can be elaborated on the basis of the quantum chemistry methods. IDMD simulations are sensitive to the statistical mechanics factors, like the number density of the reactive species, their mobility, diffusion, temperature and pressure of the medium, etc. All these factors may be accounted for through either Newtonian or Langevin framework of MD.

IDMD simulations allow to account for the dynamics of secondary electrons and the mechanisms of energy and momentum transfer from the excited electronic subsystem to the system's vibrational degrees of freedom, i.e. to its heat. For small molecular systems being in the gas phase the ejected electrons can often be uncoupled from the system and excluded from the analysis of the system's post-irradiation dynamics. For the extended molecular and condensed phase systems the interaction of secondary electrons with the system can be treated within various electron transport theories, such as diffusion [2, 42] or Monte Carlo (MC) approach [43], and be considered as additional irradiation field imposed on the molecular system [40]. Such an analysis provides the spatial distribution of the energy transferred to the medium through irradiation. Finally, immobilized electrons and electronic excitations transfer the deposited energy to the system's heat via the electron-phonon coupling, which lasts typically up to the picosecond time scale [44]. The IDMD approach accounts for the key outcomes of this relaxation process determining its duration, the temporal and spatial dependence of the amount of energy transferred into the system's heat. As such, IDMD allows the computational analysis of physicochemical processes occurring in the systems coupled to radiation on time and spatial scales far beyond the limits of quantum mechanics based computational schemes (e.g. DFT and TDDFT, nonadiabatic MD, Ehrenfest dynamics, etc.). Such an analysis is still based on the atomistic approach as any other form of traditional MD.

IDMD relies on several input parameters such as the bond dissociation energies, molecular fragmentation cross sections, amount of energy transferred to the system upon irradiation, energy relaxation rate, spatial region wherein the energy is relaxed. These characteristics originating from smaller spatial and temporal scales can be obtained by accurate quantummechanical calculations by means of the aforementioned computational schemes. If such calculations become too expensive, the required parameters can still be obtained from experimental data or by means of analytical models/methods.

Due to the limited number of parameters that enter IDMD and the reactive molecular force fields, and a much larger number of various output characteristics accessible for simulations and analysis, the IDMD approach allows the computational analysis of the system properties in the spatial and temporal domains inaccessible by other computational means. As such, it opens unique possibilities for modeling of irradiation driven modifications and chemistry of complex molecular systems beyond the capabilities of either pure quantum or pure classical MD. IDMD 
approach enables to link outputs of numerous MC codes (e.g. Geant4 $[45,46]$ ) simulating radiation and particle transport in different media with the inputs of IDMD and thus to achieve the multiscale description of irradiation driven molecular dynamics, chemistry and structure formation in many different MBN systems. These important capabilities of MBN Explorer have been demonstrated in the recent work on the FEBID case study [40]. The similar methodology can be used for simulations of numerous molecular systems placed into radiation fields of different modality, geometry and temporal profile.

The developed IDMD framework provides a broad range of possibilities for multiscale modeling of the IDC processes that underpin emerging technologies ranging from controllable fabrication of nanostructures with nanometer resolution (see e.g. Refs. [8, 47] for FEBID and Refs. [9, 10] for UVL) to radiotherapy cancer treatment (see, e.g. [1-3]), both discussed further in this Chapter.

The IDMD algorithm was validated through a number of case studies of collision and radiation processes including atomistic simulations of the FEBID process and related IDC $[26,40]$, collisioninduced multifragmentation of fullerenes [36], electron impact induced fragmentation of $\mathrm{W}(\mathrm{CO})_{6}$ [39], thermal splitting of water [31], radiation chemistry of water in the vicinity of ion tracks [37], DNA damage of various complexity induced by ions [32], and other [28]. Several case studies from the aforementioned list are discussed in greater details below in Sections 4-6.

\subsection{Reactive CHARMM force field}

MBN Explorer allows simulations of dissociation and formation of covalent bonds through the reactive CHARMM (rCHARMM) force field [31], which is an extension of the standard CHARMM force field [33-35]. This extension requires specification of two additional parameters that define the dissociation energy of a covalent bond and the cutoff radius for bond breaking or formation.

By specifying the additional parameters for the bonded interactions, MBN Explorer considers all molecular mechanics interactions, i.e., bonded, angular, dihedral, using an alternative parametrization. If the distance between a given pair of atoms becomes greater than the specified cutoff radius, this particular bonded interaction is removed from the system's topology and not considered in future calculations.

The standard CHARMM force field [33] employs harmonic approximation for describing the interatomic interactions, thereby limiting its applicability to small deformations of the molecular system. In case of larger perturbations, the potential should decrease to zero as the valence bonds rupture. In order to permit rupture of covalent bonds in molecular mechanics force field, MBN Explorer uses a modified interaction potential describing interaction of atoms connecting by chemical bonds. The standard CHARMM force field describing covalent bonds is defined as

$$
U^{(\text {bond })}\left(r_{i j}\right)=k_{i j}^{b}\left(r_{i j}-r_{0}\right)^{2}
$$

where $k_{i j}^{b}$ is the force constant of the bond stretching, $r_{i j}$ is the distance between atoms $i$ and $j$, and the parameter $r_{0}$ is the covalent bond length. This parametrization describes well the bond stretching regime in the case of small deviations from $r_{0}$ but gives an erroneous result for the larger distortions. 
For a satisfactory description of the covalent bond rupture, it is reasonable to use the Morse potential. This potential requires one additional parameter if compared to the aforementioned harmonic potential. This parameter accounts for energy of the bond dissociation. For a pair of atoms the Morse potential reads as:

$$
U^{(\text {bond })}\left(r_{i j}\right)=D_{i j}\left[e^{-2 \beta_{i j}\left(r_{i j}-r_{0}\right)}-2 e^{-\beta_{i j}\left(r_{i j}-r_{0}\right)}\right]
$$

where $D_{i j}$ is the dissociation energy of the covalent bond, and $\beta_{i j}=\left(k_{i j}^{b} / D_{i j}\right)^{1 / 2}$ determines the steepness of the potential. Figure 13.1 illustrates the Morse potential for the CN7-CN8B bond that is one of the covalent bonds in the DNA backbone; for small deviations from $r_{0}$ the Morse potential and the harmonic approximation are close to each other.

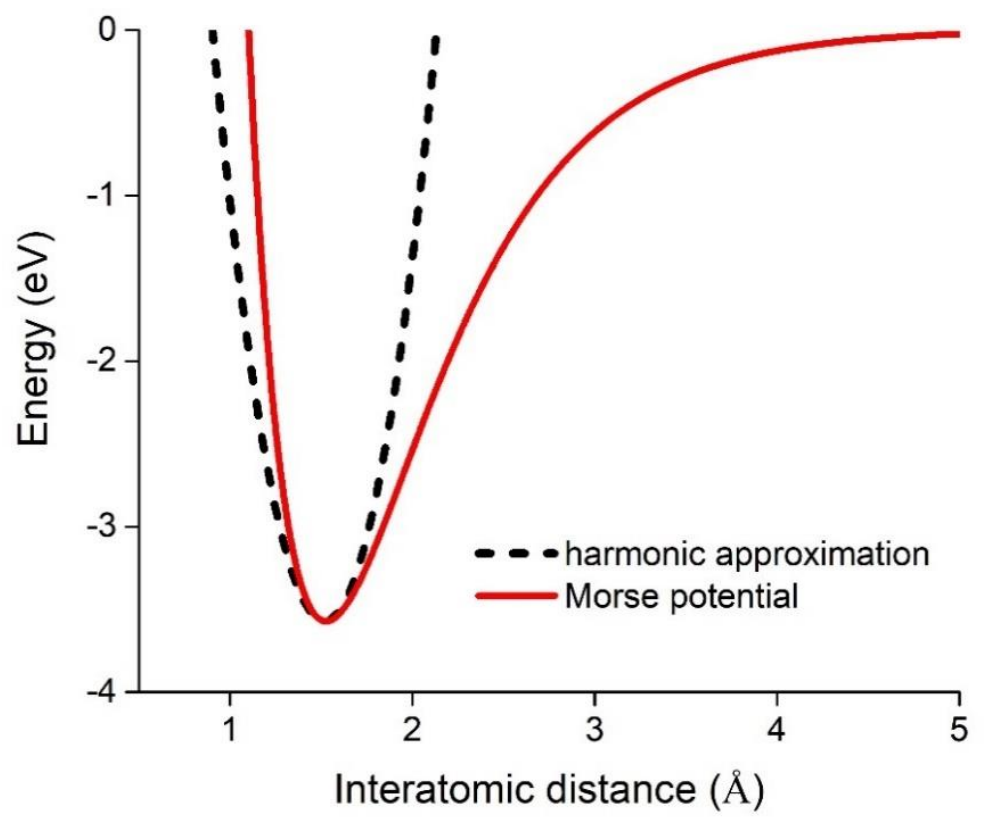

Figure 13.1. The pairwise carbon-carbon (type CN7-CN8B) interaction potential in harmonic approximation (dashed line) and modeled with the Morse potential (solid line).

The rupture of covalent bonds in the course of simulation automatically employs an improved potential for the valence angles. In the CHARMM force field, the potential associated with the change of a valence angle between bonds with indices $i j$ and $j k$ reads as:

$$
U^{(\text {angle })}\left(\theta_{i j k}\right)=k_{i j k}^{\theta}\left(\theta_{i j k}-\theta_{0}\right)^{2}
$$

where $k_{i j k}^{\theta}$ and $\theta_{0}$ are parameters of the potential, and $\theta_{i j k}$ is the actual value of the angle formed by the three atoms. This potential grows rapidly with increasing the angle, and it may lead to nonphysical results when modeling the covalent bond rupture. In order to avoid such cases, the harmonic potential (13.3) is substituted in the modified force field with an alternative parametrization, which reads as:

$$
U^{(\text {angle })}\left(\theta_{i j k}\right)=2 k_{i j k}^{\theta}\left[1-\cos \left(\theta_{i j k}-\theta_{0}\right)\right] \text {. }
$$

At small variations of the valence angle, this parametrization is identical to the harmonic approximation (13.3) used in the standard CHARMM force field. For larger values of the angle, the new parametrization (13.4) defines an energy threshold which becomes important for an accurate modeling of bond breakage. 
The rupture of a covalent bond is accompanied by ruptures of the angular interactions associated with this bond. The effect of bond breakage on the angular potential can be described through a smoothed step function $\sigma\left(r_{i j}\right)$, defined as

$$
\sigma\left(r_{i j}\right)=\frac{1}{2}\left[1-\tanh \left(\beta_{i j}\left(r_{i j}-r_{i j}^{*}\right)\right)\right]
$$

with $r_{i j}^{*}=\left(R_{i j}^{v d W}+r_{0}\right) / 2$. This function introduces a correction to the angular interaction potential, assuming that the distance between two atoms involved in an angular interaction increases from the equilibrium value $r_{0}$ up to the van der Waals contact value $R_{i j}^{v d W}$. Since an angular interaction depends on two bonds connecting the atoms with indices $i j$ and $j k$, the potential energy describing the valence angular interaction that is subject to rupture is parameterized as

$$
\widetilde{U}^{(\text {angle })}\left(\theta_{i j k}\right)=\sigma\left(r_{i j}\right) \sigma\left(r_{j k}\right) U^{(\text {angle })}\left(\theta_{i j k}\right) .
$$

As seen from Eq. (13.6), the angular potential decreases with the increase of the bond length between any of the two pairs of atoms $i j$ or $j k$. As an illustration, Figure 13.2A shows the CN8BON2-P angular potential which arises, for instance, when modeling DNA nucleotides. The presented angular potential is calculated using Eq. (13.6) assuming the breakage of the bond between the oxygen and the phosphorous atoms. For the sake of illustration, the CN8B-ON2 bond length in this case is taken equal to its equilibrium value $r_{0}$.
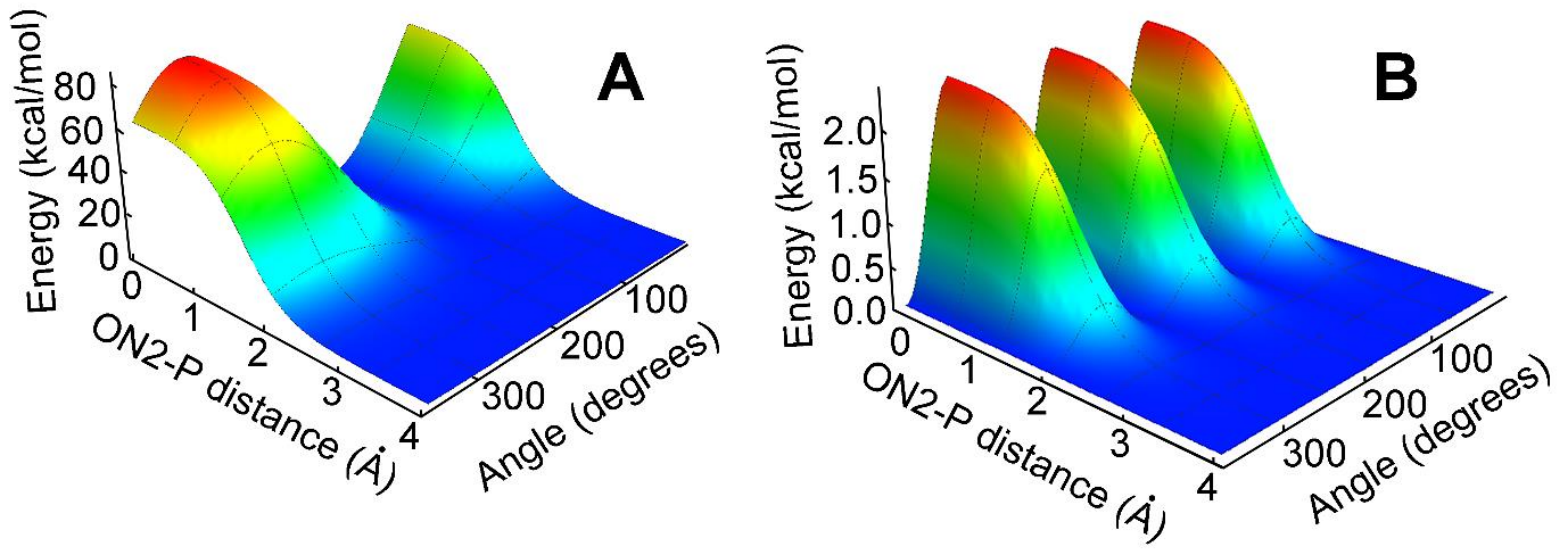

Figure 13.2. A: The CN8B-ON2-P angular potential calculated using Eq. (13.6) with account for the ON2-P bond rupture. B: The CN4-P-ON2-CN7 dihedral potential calculated using Eq. (13.8) with account for the ON2-P bond rupture.

Dihedral interactions arise in the conventional molecular mechanics potential due to the change of the dihedral angles between every four topologically defined atoms. Let us consider a quadruple of atoms with indices $i, j, k$ and $l$, bound through an interaction which is governed by a change of the dihedral angle. In this case, the dihedral angle stands for the angle between the plane formed by the atoms $i, j$ and $k$, and the plane formed by the atoms $j, k$ and $l$. In the harmonic approximation, the dihedral energy contribution reads as:

$$
U_{i j k l}^{(\text {dihedral })}=k_{i j k l}^{d}\left[1+\cos \left(n_{i j k l} \chi_{i j k l}-\delta_{i j k l}\right)\right]
$$

where $k_{i j k l}^{d}, n_{i j k l}$ and $\delta_{i j k l}$ are parameters of the potential, and $\chi_{i j k l}$ is the angle between the planes formed by atoms $i, j, k$ and $j, k, l$. 
The dihedral interactions also become disturbed upon covalent bond rupture; therefore, Eq. (13.7) should be modified to properly account for this effect. The rupture of a dihedral interaction between a quadruple of atoms $i, j, k$ and $l$ should account for three bonds that contribute to this interaction. Thus, the potential energy describing the dihedral interaction with account for the bond rupture reads as:

$$
\widetilde{U}_{i j k l}^{(\text {dihedral })}=\sigma\left(r_{i j}\right) \sigma\left(r_{j k}\right) \sigma\left(r_{k l}\right) U_{i j k l}^{(\text {dihedral })}
$$

where $U_{i j k l}^{(\text {dihedral })}$ is the potential in Eq. (13.7) describing the dihedral interaction within the framework of the standard CHARMM force field. The functions $\sigma\left(r_{i j}\right), \sigma\left(r_{j k}\right)$ and $\sigma\left(r_{k l}\right)$ are defined by Eq. (13.5); they are used to limit the dihedral interaction upon increasing the corresponding bond length. Figure 13.2B shows a profile of a CN4-P-ON2-CN7 dihedral interaction potential with accounting for the rupture of the central ON2-P bond. This dihedral interaction is also important for modeling bond breakages in DNA nucleotides.

\subsection{Multi-fragmentation of carbon fullerenes upon collision}

Irradiation- and collision-induced processes involving carbon fullerenes have been widely studied over the past several decades both experimentally and theoretically [48-51]. In particular, collisions involving neutral and charged $\mathrm{C}_{60}$ and $\mathrm{C}_{70}$ fullerenes have been widely studied $[48,52-$ 56], and a lot of experimental information has been obtained on the probability of fullerene fusion and the production of smaller clusters due to subsequent fragmentation.

Collision-induced fusion and fragmentation of $\mathrm{C}_{60}$ fullerenes was studied by means of classical MD simulations with the rCHARMM force field, performed with MBN Explorer [36]. In particular, the many-body Brenner (reactive empirical bond-order, REBO) potential [57] was used to model interactions between carbon atoms and combined together with rCHARMM to enable monitoring changes of the molecular topology and the yields of different atomic and molecular fragments produced.

2000 simulations of $\mathrm{C}_{60}+\mathrm{C}_{60}$ collisions were performed to reflect the statistical nature of the fusion and fragmentation processes; the duration of the simulation was set to 10 ps. In each simulation the fullerenes were randomly oriented with respect to each other. The input geometries were prepared by means of MBN Studio [58] - a multi-task software toolkit for MBN Explorer. The quantitative information on time evolution of the fragments produced (i.e., the number of fragments of each type) was obtained directly from the output of the simulations. Ensemble-averaged fragment size distribution was calculated for each collision energy by summing up the data from each individual trajectory and normalizing the resulting value to the total number of fragments.

Figure 13.3 shows the average size of the molecular system recorded at the end of the simulations as a function of the center-of-mass collision energy. The average system size was defined as the total number of atoms divided by the total number of molecular species corresponding to a given collision energy. Data extracted from the different trajectories at a given collision energy were summed up and normalized to the total number of collision products, including different molecular fragments as well as non-fragmented $\mathrm{C}_{60}$ molecules and fused $\mathrm{C}_{120}$ compounds. 


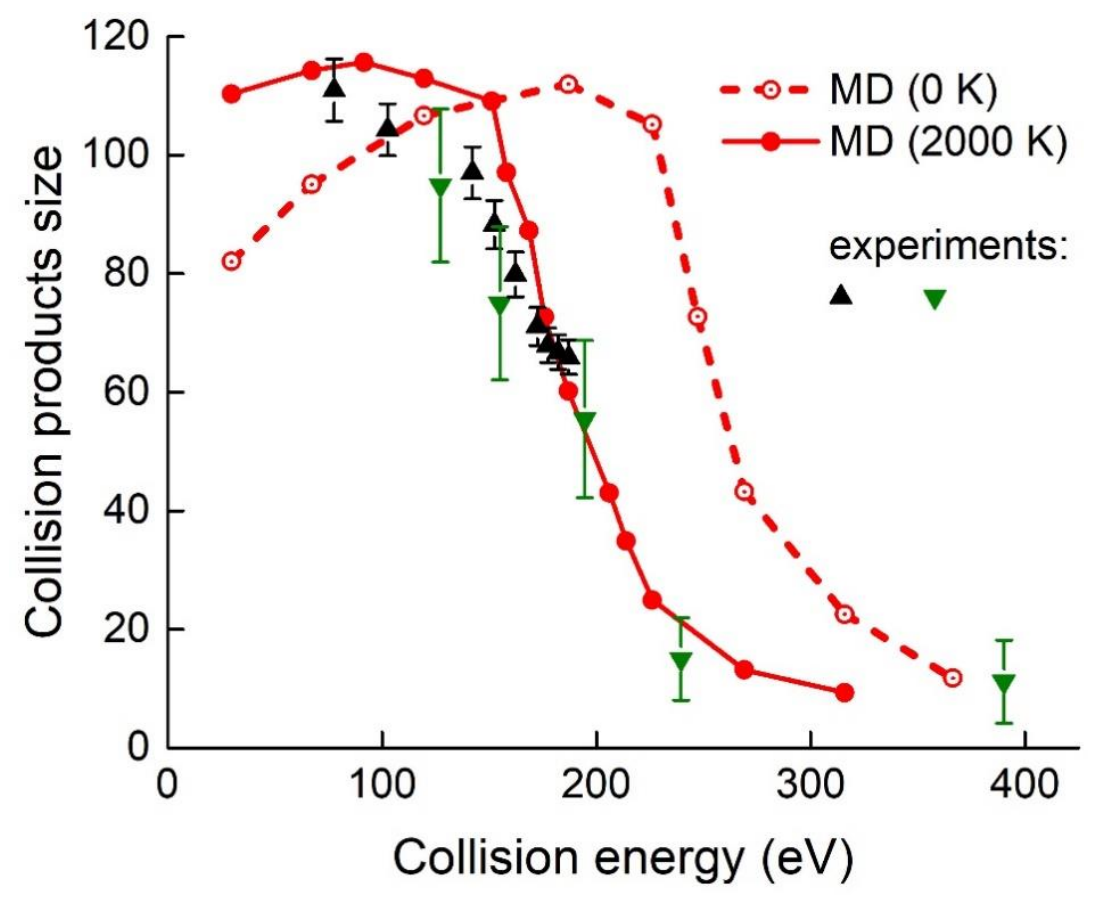

Figure 13.3. The average size of molecular products produced in $\mathrm{C}_{60}-\mathrm{C}_{60}$ collisions as a function of the collision energy [36]. The collision products, including different molecular fragments as well as non-fragmented $\mathrm{C}_{60}$ molecules and fused $\mathrm{C}_{120}$ compounds, were recorded after 10 ps of the simulations. Open and filled circles describe the simulations performed at the fullerene initial temperature of $0 \mathrm{~K}$ and $2000 \mathrm{~K}$, respectively. Other symbols represent experimental data [53,59]. In the experiments, an average temperature of the colliding fullerenes was estimated around $2000 \mathrm{~K}$.

Open circles in Figure 13.3 represent the results obtained at the zero temperature of fullerenes. Figure 13.3 shows that the maximal average size of molecular products and hence the maximal fusion probability is obtained at collision energies of about $200 \mathrm{eV}$, which is significantly higher than experimental results obtained for $\mathrm{C}_{60}{ }^{+}+\mathrm{C}_{60}$ collisions $[53,59]$.

In the experiments, the average temperature of the colliding fullerenes was estimated around $2000 \mathrm{~K}$ [53]. For a better match with the experimental conditions, we performed simulations where the fullerenes were given an initial temperature of $2000 \mathrm{~K}$. As a result, each thermally excited molecule had an initial internal kinetic energy of about $30 \mathrm{eV}$. Different initial structures and velocities used for the collision simulations were obtained from a 10 ns-long constanttemperature simulation of a single $\mathrm{C}_{60}$ molecule being at $T=2000 \mathrm{~K}$. In this simulation, temperature control was achieved by means of the Langevin thermostat with a damping time of 0.1 ps. Note that similar simulations performed at different fullerene temperatures suggest that $\mathrm{C}_{60}$ resembles its intact cage-like structure up to $T \approx 2300 \mathrm{~K}$. At higher temperatures, a transition, which is usually considered as the fullerene melting takes place. It corresponds to an opening of the fullerene cage and the formation of a highly-distorted but still non-fragmented structure [60, 61].

The results of the simulations at $T=2000 \mathrm{~K}$ are shown in Figure 13.3 by filled circles. The results reveal that simulations with thermally excited fullerenes agree much better with the experimental results: taking into account that statistical uncertainty of the calculated average size of collision products to be about $10 \%$, the calculated numbers agree well with the experimental data. We found that the largest average product size and hence the highest probability of fusion is for collisions with energies of about $90-120 \mathrm{eV}$, which is significantly lower than the value of about 
$200 \mathrm{eV}$ simulated at zero initial temperature. The fusion barrier decreases due to the thermal energy stored in the fullerenes.
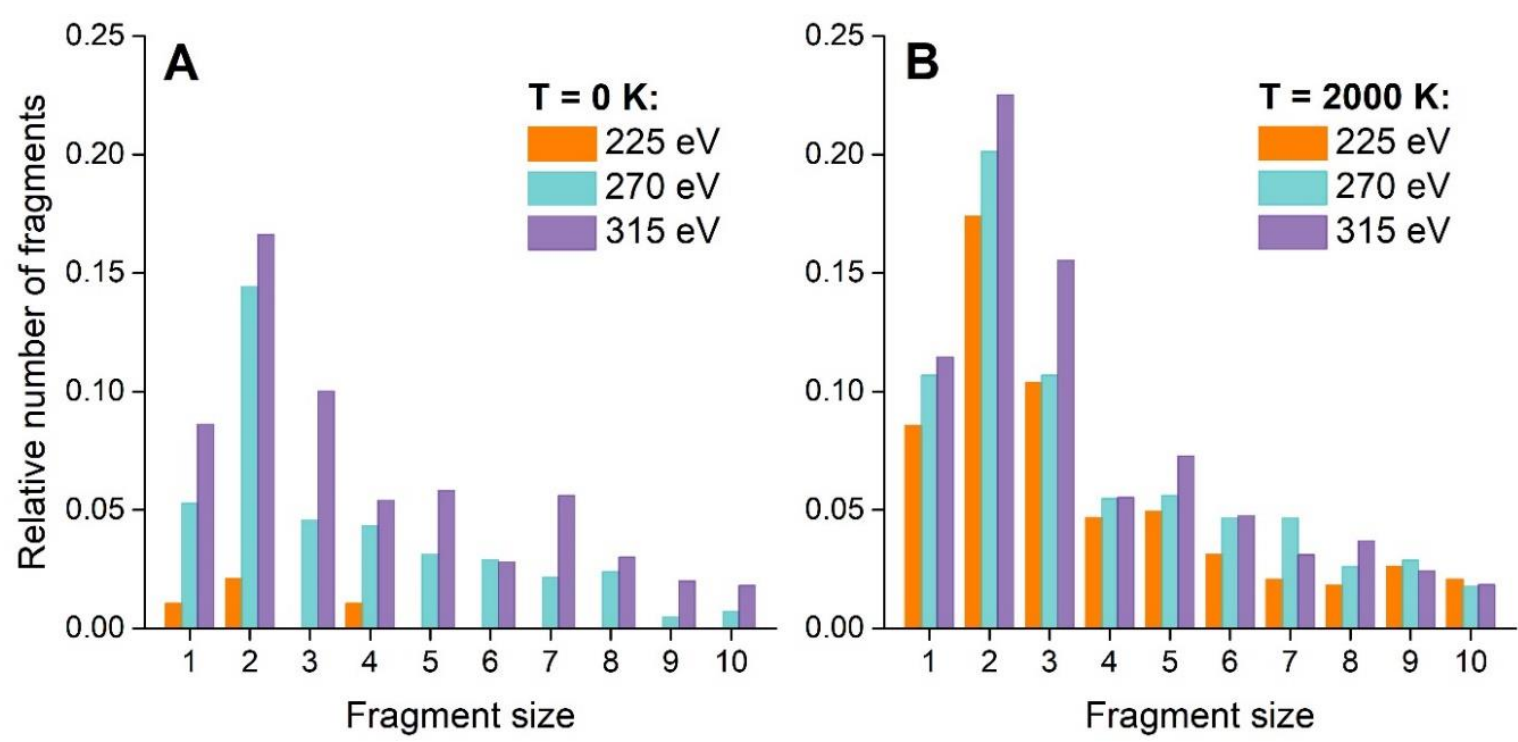

Figure 13.4. Number of $\mathrm{C}_{n}(n \leq 10)$ fragments, normalized to the total number of fragments produced after $10 \mathrm{ps}$, for the center-of-mass collision energies of 225, 270 and $315 \mathrm{eV}$ [36]. Panels A and B show the results obtained at the $0 \mathrm{~K}$ and $2000 \mathrm{~K}$ temperature of colliding fullerenes, respectively.

To analyze the impact of the fullerene initial temperature on the fragmentation dynamics, the size distribution of fragments containing up to 10 carbon atoms formed after 10 ps-long simulations was analyzed. The results of this analysis are shown in Figure 13.4. The simulations where fullerenes prior collisions had zero temperature (Figure 13.4A) show that the collision energy of $225 \mathrm{eV}$, only a few fragmentation events have been observed, while at the energy of $270 \mathrm{eV}$ a phase transition has taken place leading to multi-fragmentation of the fullerenes and the formation of multiple small-size fragments. The results of simulations for fullerenes at initial temperature of $2000 \mathrm{~K}$ (Figure 13.4B) demonstrate that the phase transition takes place at lower collision energy. In this case the multi-fragmentation regime starts at the collision energy of about $185 \mathrm{eV}$. The most prominent effect of the fullerene finite temperature is an increase in the number of $\mathrm{C}_{2}$ and $\mathrm{C}_{3}$ fragments. The data shown in Figure 13.4 demonstrate that at $315 \mathrm{eV}$ collision energy the relative number of larger fragments is about 3-6\% of the total number of fragments produced, and these values are almost independent on the initial energy stored in the system.

It is known that the size distribution of small fragments $C_{n}$ produced in collisions involving fullerenes follows a $n^{-\lambda}$ power law $[62,63]$. Observing that the simulated distributions of fragments are peaked at $n=2$, the results for $n \geq 2$ were fitted with a power function. The fitting procedure gives the value of $\lambda=1.47 \pm 0.04$, which is close to the value of 1.54 , obtained in earlier MD statistical trajectory simulations at $500 \mathrm{eV}$ center-of-mass collision energy [64].

\subsection{Transport of chemically reactive species around energetic ion tracks}

MBN Explorer can be utilized for the evaluation of radiobiological damage created by heavy ions propagating in different media, including biological. An energetic charged particle propagating 
through the medium loses its energy in inelastic collisions with the medium constituents. The transport of produced secondary particles as well as the radiation damage induced by them are the objects of experimental, numerical and computational studies [3, 65]. In particular, the physics and chemistry of radiation damage caused by irradiation with protons and heavier ions has recently become a subject of intense interest because of the use of ion beams in cancer therapy $[1-3,12]$.

Radiation damage due to ionizing radiation is initiated by the ions incident on tissue. The initial kinetic energy of the ions ranges from a few to hundreds of $\mathrm{MeV}$ per nucleon. In the process of propagation through tissue, they lose energy due to ionization, excitation, nuclear fragmentation, etc. Most of the energy loss of the ion is transferred to tissue. For an energetic ion propagating in a medium the dependence of the energy deposited into the medium on the penetration distance is characterized by a sharp maximum, the Bragg peak, in the region close to the end of ion's trajectory. It is commonly understood that the secondary electrons and free radicals produced in the processes of ionization and excitation of the medium with ions are largely responsible for the vast portion of the biodamage. In the Bragg peak region, the secondary electrons lose most of their energy within 1-2 nm of the ion's path [66]. After that the electrons continue propagating, elastically scattering with the molecules of the medium until they get bound or solvated electrons are formed [2, 3]. Such low-energy electrons remain important agents for biodamage since they can attach to biomolecules like DNA causing dissociation [67].

The energy lost by secondary electrons in the processes of ionization and excitation of the medium is transferred to its heating (i.e. vibrational excitation of molecules) due to the electron-phonon interaction. As a result, the medium within the cylinder of the $\sim 1-2 \mathrm{~nm}$ radius surrounding the ion's path is heated up rapidly and the pressure inside this cylinder increases by several orders of magnitude (e.g. by a factor of $10^{3}$ for a carbon ion at the Bragg peak [68]) compared to the pressure in the medium outside the cylinder. This pressure builds up by $10^{-14}-10^{-13} \mathrm{~s}$ and it is a source of a cylindrical shock wave [69] which propagates through the medium for about $10^{-13}-10^{-11} \mathrm{~s}$.

If the shock wave is strong enough, it can inflict damage by the thermomechanical stress and induce breakage of covalent bonds in the DNA molecule [32, 66, 70-73]. Besides, the radial collective motion of the medium induced by the shock wave is instrumental in propagating the highly reactive molecular species, such as hydroxyl radicals and solvated electrons, to large radial distances (up to tens of nanometers) and preventing their recombination [37, 42].

The hydroxyl radical, $\mathrm{OH}^{\bullet}$, is one of the main chemically reactive species involved in the indirect damage of DNA molecules. It can form upon fragmentation of ionized $\left(\mathrm{H}_{2} \mathrm{O}^{+}\right)$or electronically excited water molecules $\left(\mathrm{H}_{2} \mathrm{O}^{*}\right)$ :

$$
\begin{aligned}
& \mathrm{H}_{2} \mathrm{O}^{+} \rightarrow \mathrm{OH}^{\bullet}+\mathrm{H}^{+} \\
& \mathrm{H}_{2} \mathrm{O}^{*} \rightarrow \mathrm{OH}^{\cdot}+\mathrm{H}^{\bullet} .
\end{aligned}
$$

The hydroxyl radicals can damage DNA, but they can also annihilate, among other reactions [74], via a recombination forming hydrogen peroxide:

$$
\mathrm{OH}^{\bullet}+\mathrm{OH}^{\bullet} \rightarrow \mathrm{H}_{2} \mathrm{O}_{2} \text {. }
$$

This reaction was studied earlier [37] by means of the rCHARMM force field implemented in MBN Explorer. For the sake of simplicity, this reaction was considered as a representative example of 
the induced radiation chemistry, so all other chemical reactions were disregarded. Pre-solvated electrons and hydrogen radicals were not included in these simulations.

Figure 13.5 illustrates the effects of a carbon ion-induced shock wave on the $\mathrm{OH} \cdot$ chemistry in the Bragg peak region. Figure 13.5A shows the $\mathrm{OH} \cdot$ mean squared displacement (MSD) in a simulation where the shock wave is allowed to develop (solid line) and in another one where the shock wave is artificially "switched off" (dashed line). The shock waves develop naturally in the MD simulations as a result of the energy deposited along the ion's track. However, the computer simulations allow one to artificially "switch off" this process in order to distinguish its influence in the evolution of the radiation chemistry scenario. The earlier study [37] assessed how the shock wave modifies the radiation chemistry as compared to the traditional picture of biophysical models, where shock waves have not yet been considered.
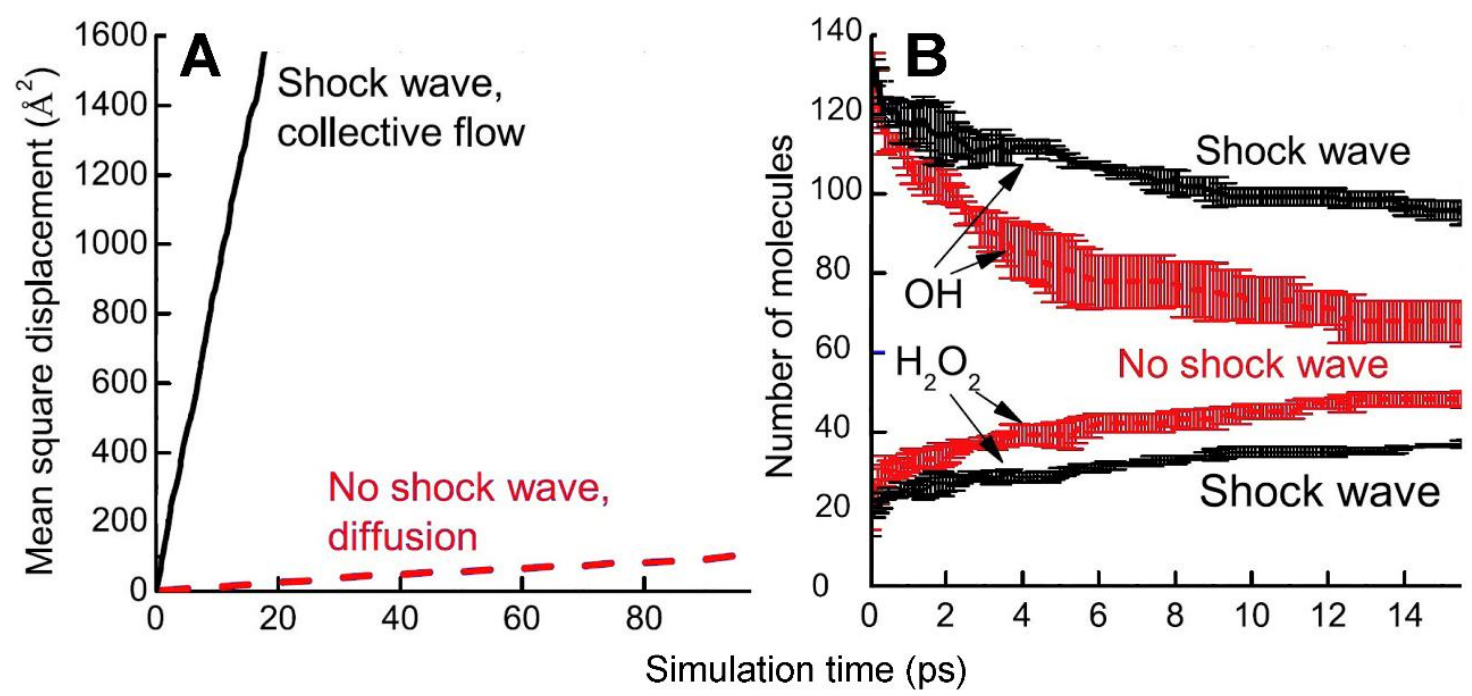

Figure 13.5. A: Mean squared displacement of the $\mathrm{OH} \bullet$ radicals produced around a $200 \mathrm{keV} / \mathrm{u}$ carbon ion path. B: Time evolution of the number of $\mathrm{OH} \bullet$ radicals and produced $\mathrm{H}_{2} \mathrm{O}_{2}$ molecules [37, 75]. Results of simulations where the shock wave is allowed to develop (transport by the collective flow, solid lines) and where it is artificially "switched off" (transport by diffusion, dashed lines) are shown, in order to demonstrate the shock wave effects on the radiation chemistry.

Figure 13.5A compares the transport of $\mathrm{OH}^{\cdot}$ radicals in simulations where pure diffusion of hydroxyl radicals in the static medium is considered (by artificially switching off the shock wave, as explained above) and their transport by the collective flow induced by the shock wave. For the latter case, the radicals are transported almost 80 times faster than diffusion, clearly demonstrating the capacity of the collective flows initiated by shock waves to effectively transport reactive species in the medium.

The reactivity of the $\mathrm{OH} \cdot$ radicals in the presence of the shock wave is illustrated in Figure 13.5B. It depicts the average number of $\mathrm{OH} \cdot$ and $\mathrm{H}_{2} \mathrm{O}_{2}$ molecules in the two cases where the shock wave is artificially switched off and naturally allowed to develop, respectively. The error bars represent standard deviations corresponding to three independent simulation runs. As clearly seen, the evolution of the number of molecules is different when the collective flow is either present or absent. The transport of radicals by the collective flow does not only propagate the radicals much faster than diffusion, but also prevents their recombination, both by their spreading and by 
creating harsh conditions in which the formation of the 0-0 bond is suppressed [37]. These results are in line with the observations made for the only reported data for water radiolysis with very high-LET ions [76], where unusually large amounts of radicals escaped ion tracks.

\subsection{Atomistic simulation of the FEBID process}

One of the technological applications of irradiation driven chemistry is focused electron beam induced deposition (FEBID) - a novel and actively developing nanofabrication technique that allows controllable creation of metal nanostructures with nanometer resolution [6-8]. FEBID is based on the irradiation of precursor molecules (mainly, organometallic) [77] by keV electron beams whilst they are being deposited on a substrate. Electron-induced decomposition of the molecules releases its metallic component which forms a deposit on the surface with size similar to that of the incident electron beam (typically a few nanometers) [47].

To date, a popular class of precursors for FEBID is metal carbonyls $\operatorname{Me}_{m}(\mathrm{CO})_{n}[8,78]$ which are composed of one or several metal atoms (Me) bound to a number of carbon monoxide ligands. Metal carbonyls have been widely studied experimentally and a substantial amount of data on thermal decomposition and electron-induced fragmentation have been accumulated over the past decades [79-85]. Strong interest in studying properties of these compounds has been attributed to their peculiar structure containing strong $\mathrm{C}-\mathrm{O}$ bonds and relatively weak $\mathrm{Me}-\mathrm{C}$ bonds. While the former are difficult to cleave, the latter dissociate easily, what usually happens through a sequential loss of $\mathrm{CO}$ groups when sufficient internal energy is available.

FEBID operates through successive cycles of precursor molecules replenishment on a substrate and irradiation by a tightly-focused electron beam, which induces the release of metal-free ligands and the growth of metal-enriched nanodeposits. This process involves a complex interplay of phenomena, taking place on different temporal and spatial scales and thus requiring a dedicated theoretical and computational approach [28]: (i) deposition, diffusion, aggregation and desorption of precursor molecules on the substrate; (ii) transport of the primary, secondary and backscattered electrons; (iii) electron-induced dissociation of the deposited molecules; (iv) the follow-up chemistry; and (v) relaxation of energy deposited into electronic and vibrational degrees of freedom, and resulting thermomechanical effects.

Until recently, simulations of the FEBID process and the nanostructures growing have been performed only by means of the Monte Carlo (MC) approach and the diffusion theory. These methods allow simulations of the average characteristics of the process but do not provide molecular-level details of the adsorbed material. The IDMD approach [26] goes beyond this limit and provides a description of the nanostructures created by the FEBID process on the atomistic level accounting for the quantum and chemical transformations within the absorbed molecular system.

In the earlier study [26] atomistic IDMD simulations of the FEBID process of tungsten hexacarbonyl, $\mathrm{W}(\mathrm{CO})_{6}$ on a $\mathrm{SiO}_{2}$ surface were performed using MBN Explorer. The results of the simulations were validated through the comparison with experimental data [86].

The rCHARMM force field used to model the structure and dynamics of irradiated $\mathrm{W}(\mathrm{CO})_{6}$ molecules atop the hydroxylated $\mathrm{SiO}_{2}$ surface, requires specification of several parameters (the 
equilibrium bond lengths, bonds stiffness and dissociation energies). Additionally, one need to define the dissociative chemistry of precursors including the definition of the molecular fragments and atomic valences. In the model considered only the dissociation and formation of the W-C and $\mathrm{W}$-W bonds was permitted, while the $\mathrm{C}-\mathrm{O}$ bonds were treated within the harmonic approximation, Eq. (13.1), preventing those bonds from breakage.

A snapshot of the MD simulation of the first irradiation phase is shown in Figure 13.6. The irradiation by an electron beam of the cylindrical shape is considered. Only those precursors molecules that move inside the cylinder are exposed to radiation that may induce their dissociation. The dissociation rate of the precursor molecules was evaluated from the experimental data [86].

The FEBID process was modeled [26] with the rescaled computationally accessible parameters (the irradiation time and the beam current). These parameters may differ from experimental values, but they have to be chosen so that they correspond to a given (in experiment) number of electrons $N_{\mathrm{e}}$ targeting the system (the electron fluence) and thus producing the irradiation induced effects on the same scale as in the experiment. Following this idea, the irradiation time in IDMD simulations is typically decreased as compared to the corresponding experimental values.

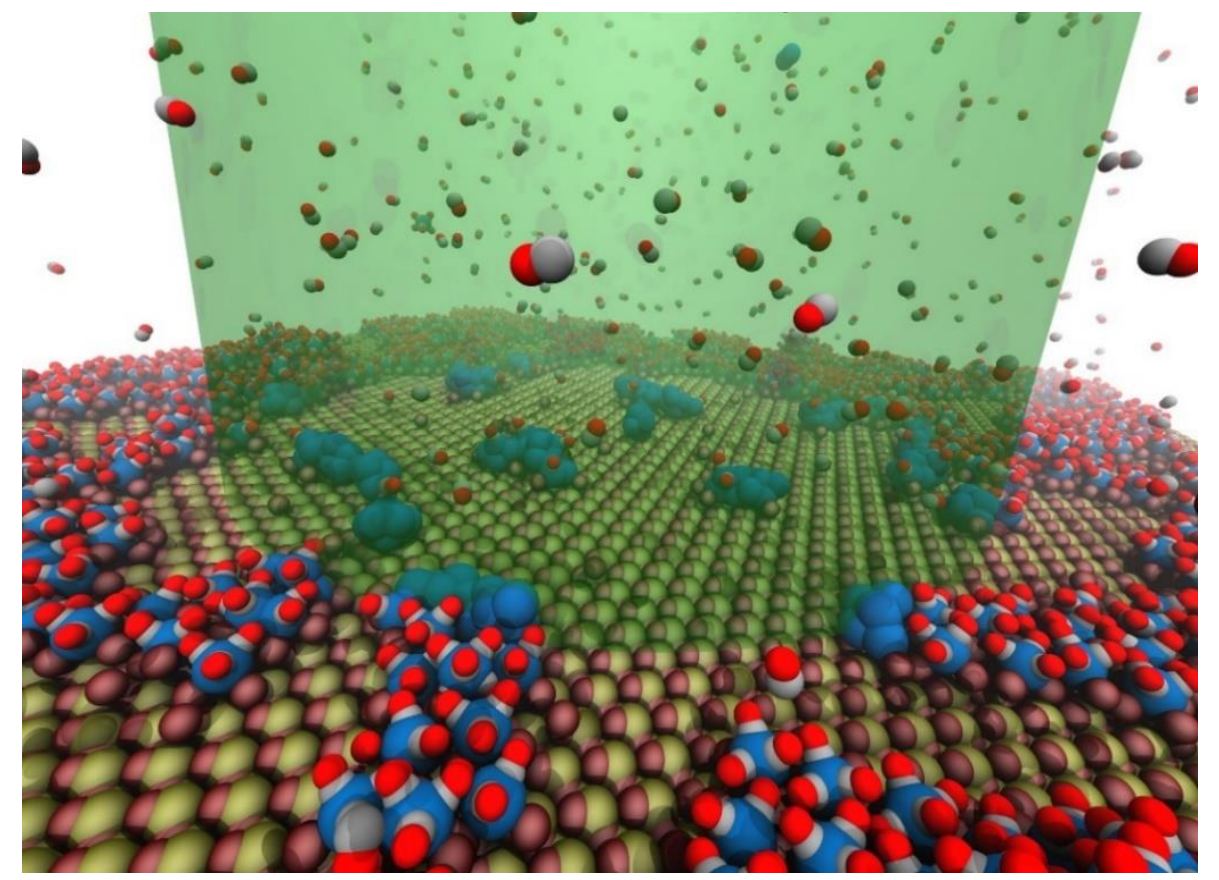

Figure 13.6. A: Snapshot of the MD simulation [26] of adsorption of $\mathrm{W}(\mathrm{CO})_{6}$ precursor molecules atop the $\mathrm{SiO}_{2}$ surface at the early stage of irradiation by an electron beam (a transparent green cylinder). The interaction of deposited precursor molecules with the beam leads to the fragmentation of precursors and to the formation of tungsten clusters, shown in blue.

The probability of $\mathrm{W}-\mathrm{C}$ bonds dissociation in $\mathrm{W}(\mathrm{CO})_{6}$ caused by collisions with electrons per unit time can be evaluated as

$$
P=\sigma \times J_{0}
$$

where $\sigma$ is the bond dissociation cross section and

$$
J_{0}=\frac{I_{0}}{e \times S_{0}}
$$


is the electron flux density targeting the precursor. Here $I_{0}$ is the electron beam current, $e$ is the electron charge and $S_{0}=\pi R^{2}$ is the beam cross section equal with $R$ being the beam radius. Substituting Eq. (13.12) into Eq. (13.11) one derives

$$
P_{0}=\frac{I_{0}}{e} \frac{\sigma}{S_{0}}
$$

Estimating $I_{0}=4 \mu \mathrm{A}$ and $\sigma=1.2 \times 10^{-2} \mathrm{~nm}^{2}$ [86], one derives $P_{0}=3.8 \times 10^{-6} \mathrm{fs}^{-1}$. This analysis can be refined on the basis of the accurate calculations of the corresponding fragmentation cross section of the precursor, as well as the yield and the spatial distribution of the secondary electrons, which all depend on the energy of the primary beam [40]. That study also demonstrated the possibility to link the input characteristics for the IDMD simulations with outputs of MC codes simulating radiation and particle transport in different media and thus to achieve the multiscale description of IDMD of the FEBID process. This methodology is general and can be used for simulations of numerous molecular systems placed into radiation fields of different modality, geometry and temporal profile.

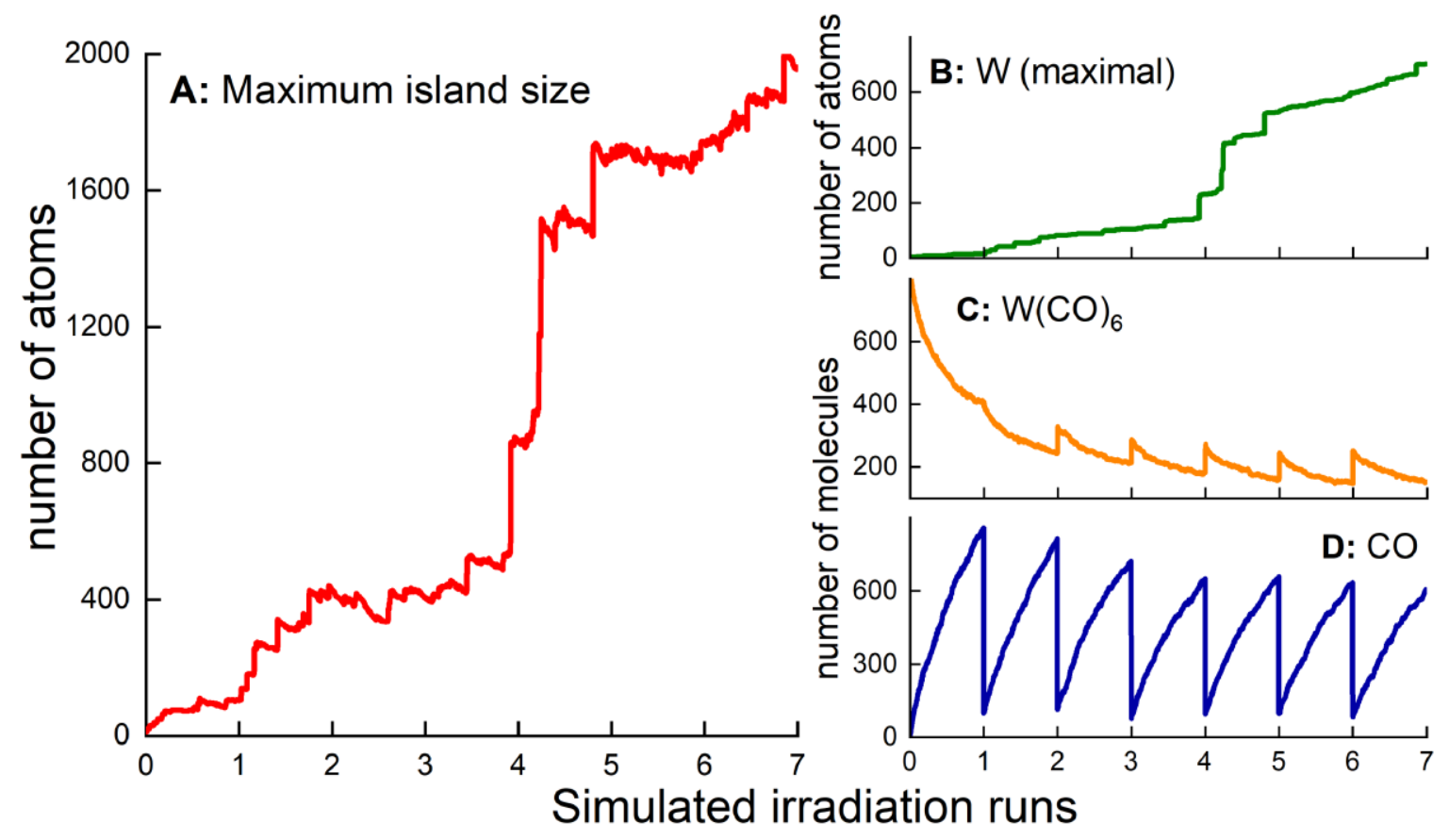

Figure 13.7. Time evolution of the size of the largest W-enriched island (A), the number of W atoms (B), the number of $\mathrm{W}(\mathrm{CO})_{6}$ (C) and CO (D) molecules in the system during the irradiation periods [26]. The irradiation periods are marked by the successive numbers. The duration of each period is $10 \mathrm{~ns}$. In simulations the electron beam radius $R$ is equal to $5 \mathrm{~nm}$ and the beam current $I_{0}=4 \mu \mathrm{A}$.

Figure 13.7A shows the time dependence of the size of the largest $\mathrm{W}$-enriched island emerged in the simulations. Panels B, C and D show this evolution for the numbers of $\mathrm{W}$ atoms, $\mathrm{W}(\mathrm{CO})_{6}$ and $\mathrm{CO}$ molecules in the system during the irradiation periods, respectively. The irradiation periods are marked in the plots by the successive numbers. The duration of each period is $10 \mathrm{~ns}$. The replenishment periods are excluded from the plots. However, the drops in the number of $\mathrm{CO}$ molecules and the number of $\mathrm{W}(\mathrm{CO})_{6}$, corresponding to the changes of the system that occur during the replenishment periods, are well seen. Both the size and the number of $\mathrm{W}$ atoms in the islands grow due to the attachment of new atoms to the islands in the course of the FEBID process. The panels A and B indicate the coalescence of smaller islands into a larger single nanostructure 
(ripening process) during the initial stage of the FEBID process. The irregular spikes on the curves arise at the instants when separate islands merge together.

The $\mathrm{W}$-(CO) bonds in precursors dissociate during the irradiation periods leading to appearance of the $\mathrm{CO}$ molecules. Most of these are created in the vicinity of the surface and later are evaporated into the vacuum chamber. The evaporation process continues during the replenishment periods. Figure 13.7D shows that during each irradiation period the number of CO molecules grows nonlinearly. To account for the evaporation process of CO during the replenishment periods, the $\mathrm{CO}$ molecules are removed from the simulation box after each irradiation phase. After that the new $\mathrm{W}(\mathrm{CO})_{6}$ molecules are deposited on the surface according to the chosen deposition rate and the duration of the deposition process. This results in the abrupt decrease of the $\mathrm{CO}$ molecules and increase of $\mathrm{W}(\mathrm{CO})_{6}$ numbers before the start of each new cycle irradiation.

The results reviewed here demonstrate that the IDMD approach [26] provides a powerful computational tool to model the growth process of W-granular metal structures emerging in the FEBID process at the atomistic level of detail. The morphology of the simulated structures, their composition and growth characteristics are consistent with the available experimental data. The performed analysis indicates also the need of further wide exploitation of the IDMD methodology in FEBID and many other processes in which the irradiation of molecular systems and irradiation driven chemistry play the key role.

\subsection{Conclusions}

Irradiation Driven Molecular Dynamics is a unique computational methodology implemented in the MBN Explorer software package which enables atomistic-level modeling of irradiation-driven transformations involving complex molecular system. The concept of IDMD is general and applicable to any molecular system treated with any classical force field implemented in the software. IDMD is capable to describe systems modeled through pairwise potentials, many-body force fields, molecular mechanics force fields (including the reactive CHARMM force field) and their combinations. The limited number of parameters that determine molecular force fields and their irradiation-driven perturbations results in a countable number of modifications that could occur in a molecular system upon irradiation and makes the method efficient and accurate. This implementation opens a broad range of possibilities for modeling of irradiation-driven modifications and chemistry of complex molecular systems.

\section{Acknowledgements}

The authors are grateful for financial support from the COST Action CA17126 "Towards understanding and modelling intense electronic excitation" (TUMIEE), Deutsche Forschungsgemeinschaft (Projects no. 415716638, SFB1372 and GRK1885), the Volkswagen Stiftung (Lichtenberg professorship to IAS), and the European Union's Horizon 2020 research and innovation programme - the Radio-NP project (GA 794733) within the H2020-MSCA-IF-2017 call and the RADON project (GA 872494) within the H2020-MSCA-RISE-2019 call. 


\section{References}

[1] D. Schardt, T. Elsässer, D. Schulz-Ertner, Rev. Mod. Phys. 82 (2010) 383

[2] E. Surdutovich, A.V. Solov'yov, Eur. Phys. J. D 68 (2014) 353

[3] A.V. Solov'yov (ed.), Nanoscale Insights into Ion-Beam Cancer Therapy (Springer International Publishing, 2017)

[4] A.G.G.M. Tielens, Rev. Mod. Phys. 85 (2013) 1021

[5] G. Horneck, C. Baumstark-Khan (eds.), Astrobiology: The Quest for the Conditions of Life (Springer-Verlag Berlin Heidelberg, 2002)

[6] W.F. van Dorp, C.W. Hagen, J. Appl. Phys. 104 (2008) 081301

[7] I. Utke, S. Moshkalev, P. Russel (eds.), Nanofabrication Using Focused Ion and Electron Beams (Oxford University Press, 2012)

[8] M. Huth, F. Porrati, C. Schwalb, M. Winhold, R. Sachser, M. Dukic, J. Adams, G. Fantner, Beilstein J. Nanotechnol. 3 (2012) 597

[9] B. Wu, A. Kumar, J. Vac. Sci. Technol. B 25 (2007) 1743

[10] A.M. Hawryluk, L.G. Seppala, J. Vac. Sci. Technol. B 6 (1988) 2162

[11] F. Heidet, N.R. Brown, M. Haj Tahar, Rev. Acc. Sci. Technol. 8 (2015) 99

[12] U. Linz (ed.), Ion Beam Therapy: Fundamentals, Technology, Clinical Applications (Springer, 2012)

[13] P.M. Dinh, P.G. Reinhard, E. Suraud, Phys. Rep. 485 (2010) 43

[14] J.A. Maruhn, P.G. Reinhard, E. Suraud, Simple Models of Many-Fermion Systems (Springer-Verlag Berlin Heidelberg, 2010)

[15] D. Jacquemin, V. Wathelet, E.A. Perpéte, C. Adamo, J. Chem. Theory Comput. 5 (2009) 2420

[16] A.D. Bochevarov, E. Harder, T.F. Hughes, J.R. Greenwood, D.A. Braden, D.M. Philipp, D. Rinaldo, M.D. Halls, J. Zhang, R.A. Friesner, Int. J. Quant. Chem. 113 (2013) 2110

[17] K.Y. Sanbonmatsu, C.S. Tung, J. Struct. Biol. 157 (2007) 470

[18] G. Zhao, J.R. Perilla, E.L. Yufenyuy, X. Meng, B. Chen, J. Ning, J. Ahn, A.M. Gronenborn, K. Schulten, C. Aiken et al., Nature 497 (2013) 643

[19] P. Husen, I.A. Solov'yov, J. Am. Chem. Soc. 138 (2016) 12150

[20] R. Salomon-Ferrer, A.W. Götz, D. Poole, S. Le Grand, R.C. Walker, J. Chem. Theory Comput. 9 (2013) 3878

[21] A.C. Pan, T.M. Weinreich, S. Piana, D.E. Shaw, J. Chem. Theory Comput. 12 (2016) 1360

[22] B. Brooks, R. Bruccoleri, B. Olafson, D. States, S. Swaminathan, M. Karplus, J. Comput. Chem. 4 (1983) 187

[23] D.A. Case, T.E. Cheatham III, T. Darden, H. Gohlke, R. Luo, K.M. Merz Jr., A. Onufriev, C. Simmerling, B. Wang, R.J. Woods, J. Comput. Chem. 26 (2005) 1668

[24] D.V.D. Spoel, E. Lindahl, B. Hess, G. Groenhof, A. Mark, H. Berendsen, J. Comput. Chem. 26 (2005) 1701

[25] J.C. Phillips, R. Braun, W. Wang, J. Gumbart, E. Tajkhorshid, E. Villa, C. Chipot, R.D. Skeel, L. Kalé, K. Schulten, J. Comput. Chem. 26 (2005) 1781

[26] G.B. Sushko, I.A. Solov'yov, A.V. Solov'yov, Eur. Phys. J. D 70 (2016) 217 
[27] I.A. Solov'yov, A.V. Yakubovich, P.V. Nikolaev, I. Volkovets, A.V. Solov'yov, J. Comput. Chem. 33 (2012) 2412

[28] I.A. Solov'yov, A.V. Korol, A.V. Solov'yov, Multiscale Modeling of Complex Molecular Structure and Dynamics with MBN Explorer (Springer International Publishing, Cham, Switzerland, 2017)

[29] I.A. Solov'yov, G. Sushko, A.V. Solov'yov, MBN Explorer Users' Guide. Version 3.0 (MesoBioNano Science Publishing, Frankfurt am Main, 2017)

[30] I.A. Solov'yov, G. Sushko, A. Verkhovtsev, A.V. Korol, A.V. Solov'yov, MBN Explorer and MBN Studio Tutorials: Version 3.0 (MesoBioNano Science Publishing, Frankfurt am Main, 2017)

[31] G.B. Sushko, I.A. Solov'yov, A.V. Verkhovtsev, S.N. Volkov, A.V. Solov'yov, Eur. Phys. J. D 70 (2016) 12

[32] I. Friis, A. Verkhovtsev, I.A. Solov'yov, A.V. Solov'yov, J. Comput. Chem. 41 (2020) 2429

[33] A.D. MacKerell Jr., D. Bashford, M. Bellott, R.L. Dunbrack, Jr., J.D. Evanseck, M.J. Field, S. Fischer, J. Gao, H. Guo et al., J. Phys. Chem. B 102 (1998) 3586

[34] A.D. MacKerell Jr, N. Banavali, N. Foloppe, Biopolymers 56 (2001) 257

[35] R.B. Best, X. Zhu, J. Shim, P.E.M. Lopes, J. Mittal, M. Feig, A.D. MacKerell Jr., J. Chem. Theory Comput. 8 (2012) 3257

[36] A. Verkhovtsev, A.V. Korol, A.V. Solov'yov, Eur. Phys. J. D 71 (2017) 212

[37] P. de Vera, E. Surdutovich, N.J. Mason, F.J. Currell, A.V. Solov'yov, Eur. Phys. J. D 72 (2018) 147

[38] E. Surdutovich, A.V. Solov'yov, Eur. Phys. J. D 73 (2019) 241

[39] P. de Vera, A. Verkhovtsev, G. Sushko, A.V. Solov'yov, Eur. Phys. J. D 73 (2019) 215

[40] P. de Vera, M. Azzolini, G. Sushko, I. Abril, R. Garcia-Molina, M. Dapor, I.A. Solov'yov, A.V. Solov'yov, Sci. Rep. 10 (2020) 20827

[41] L.D. Landau, E.M. Lifshitz, Quantum Mechanics (Non-relativistic Theory) (Elsevier ButterworthHeinemann, 1977)

[42] E. Surdutovich, A.V. Solov'yov, Eur. Phys. J. D 69 (2015) 193

[43] M. Dapor, Transport of Energetic Electrons in Solids (3rd ed.) (Springer International Publishing, Cham, Switzerland, 2020)

[44] L.G. Gerchikov, A. Ipatov, A.V. Solov'yov, W. Greiner, J. Phys. B: At. Mol. Opt. Phys. 33 (2000) 4905

[45] S. Agostinelli, J. Allison, K. Amako, J. Apostolakis, H. Araujo, P. Arce, M. Asai, D. Axen, S. Banerjee, G. Barrand et al., Nucl. Instrum. Meth. A 506 (2003) 250

[46] J. Allison et al., Nucl. Instrum. Meth. A 835 (2016) 186

[47] I. Utke, P. Hoffmann, J. Melngailis, J. Vac. Sci. Technol. B 26 (2008) 1197

[48] E.E. Campbell, Fullerene Collision Reactions (Springer Science \& Business Media, 2003)

[49] J.P. Connerade, A.V. Solov'yov (eds.), Latest Advances in Atomic Clusters Collision: Structure and Dynamics from the Nuclear to the Biological Scale (Imperial College Press, 2008)

[50] E.E.B. Campbell, F. Rohmund, Rep. Prog. Phys. 63 (2000) 1061

[51] M. Gatchell, H. Zettergren, J. Phys. B: At. Mol. Opt. Phys. 49 (2016) 162001

[52] E.E.B. Campbell, V. Schyja, R. Ehlich, I.V. Hertel, Phys. Rev. Lett. 70 (1993) 263

[53] F. Rohmund, A.V. Glotov, K. Hansen, E.E.B. Campbell, J. Phys. B: At. Mol. Opt. Phys. 29 (1996) 5143 
[54] H. Bräuning, R. Trassl, A. Diehl, A. Theiß, E. Salzborn, A.A. Narits, L. Presnyakov, Phys. Rev. Lett. 91 (2003) 168301

[55] J. Jakowski, S. Irle, B.G. Sumpter, K. Morokuma, J. Phys. Chem. Lett. 3 (2012) 1536

[56] J. Handt, R. Schmidt, Europhys. Lett. 109 (2015) 63001

[57] D.W. Brenner, Phys. Rev. B 42 (1990) 9458

[58] G. Sushko, I. Solov'yov, A. Solov'yov, J. Mol. Graph. Model. 88 (2019) 247

[59] A.V. Glotov, E.E.B. Campbell, Phys. Rev. A 62 (2000) 033202

[60] S.C. Kim, D. Tománek, Phys. Rev. Lett. 72 (1994) 2418

[61] A. Hussien, A.V. Yakubovich, A.V. Solov'yov, W. Greiner, Eur. Phys. J. D 57 (2010) 207

[62] A. Rentenier, P. Moretto-Capelle, D. Bordenave-Montesquieu, A. Bordenave-Montesquieu, J. Phys. B: At. Mol. Opt. Phys. 38 (2005) 789

[63] L. Horváth, T.A. Beu, Phys. Rev. B 77 (2008) 075102

[64] J. Schulte, Int. J. Mass. Spectrom. Ion Proc. 145 (1995) 203

[65] G. García Gómez-Tejedor, M.C. Fuss (eds.), Radiation Damage in Biomolecular Systems (Springer Science + Business Media B.V., 2012)

[66] E. Surdutovich, A.V. Yakubovich, A.V. Solov'yov, Sci. Rep. 3 (2013) 1289

[67] Y. Park, Z. Li, P. Cloutier, L. Sanche, J. Wagner, Radiat. Res. 175 (2011) 240

[68] M. Toulemonde, E. Surdutovich, A.V. Solov'yov, Phys. Rev. E 80 (2009) 031913

[69] E. Surdutovich, A.V. Solov'yov, Phys. Rev. E 82 (2010) 051915

[70] A.V. Yakubovich, E. Surdutovich, A.V. Solov'yov, AIP Conf. Proc. 1344 (2011) 230

[71] P. de Vera, N.J. Mason, F.J. Currell, A.V. Solov'yov, Eur. Phys. J. D 70 (2016) 183

[72] A. Fraile, M. Smyth, J. Kohanoff, A.V. Solov'yov, J. Chem. Phys. 150 (2019) 015101

[73] D. Bottländer, C. Mücksch, H.M. Urbassek, Nucl. Instrum. Meth. B 365 (2015) 622

[74] C. von Sonntag, The Chemical Basis of Radiation Biology (Taylor \& Francis, London, 1987)

[75] P. de Vera, E. Surdutovich, A.V. Solov'yov, Cancer Nanotechnol. 10 (2019) 5

[76] J.A. LaVerne, R.H. Schuler, J. Phys. Chem. 100 (1996) 16034

[77] S. Barth, M. Huth, F. Jungwirth, J. Mater. Chem. C 8 (2020) 15884

[78] T.P. Ragesh Kumar, P. Weirich, L. Hrachowina, M. Hanefeld, R. Bjornsson, H.R. Hrodmarsson, S. Barth, D.H. Fairbrother, M. Huth, O. Ingólfsson, Beilstein J. Nanotechnol. 9 (2018) 555

[79] V.H. Wysocki, H.I. Kenttämaa, R.G. Cooks, Int. J. Mass Spectrom. Ion Proc. 75 (1987) 181

[80] S. Beranová, C. Wesdemiotis, J. Am. Soc. Mass Spectrom. 5 (1994) 1093

[81] R.G. Cooks, T. Ast, B. Kralj, V. Kramer, D. Žigon, J. Am. Soc. Mass Spectrom. 1 (1990) 16

[82] K. Wnorowski, M. Stano, W. Barszczewska, A. Jówko, Š. Matejčík, Int. J. Mass Spectrom. 314 (2012) 42

[83] K. Wnorowski, M. Stano, C. Matias, S. Denifl, W. Barszczewska, Š. Matejč́́k, Rapid Commun. Mass Spectrom. 26 (2012) 2093

[84] M. Neustetter, E. Jabbour Al Maalouf, P. Limão Vieira, S. Denifl, J. Chem. Phys. 145 (2016) 054301 
[85] M. Lacko, P. Papp, K. Wnorowski, Š. Matejčík, Eur. Phys. J. D 69 (2015) 84

[86] J.D. Fowlkes, P.D. Rack, ACS Nano 4 (2010) 1619 


\title{
14. Fundamentals of Monte Carlo particle transport and synergies with quantum dynamics for applications in ion-irradiated materials in Space and radiobiology
}

\author{
Fabiana Da Pieve*, Natalia Koval, Bin Gu, Daniel Muñoz-Santiburcio, \\ Johannes Teunissen, Emilio Artacho, Jorge Kohanoff, and Fabrizio Cleri
}

First-stage radiation effects induced in detectors in accelerators, electronic devices and solar arrays in space, shielding materials for spacecraft, materials in nuclear power plants, and in water samples as proxies for biological matter are traditionally studied via Monte Carlo particle transport approaches. In this chapter, we introduce the basic notions of Monte Carlo particle transport and some of the practicalities of the related codes, also mentioning the low energy extension specific to the case of radiation effects in biological matter. Then, we will discuss some applications in the studies of radiation effects in space and planetary missions and in radiobiology, where synergistic studies between the Monte Carlo community and the condensed matter/chemical-physics community are possible.

\footnotetext{
*Contact: fabiana.dapieve@gmail.com
} 


\subsection{Introduction}

The study of radiation-induced effects on materials and biological systems is of importance for several fields of science, including the study of the damage of detectors in high energy particle physics experiments, the performance of materials in reactors, the damage of spacecraft components, the biological risks in missions to Space and ion-beam cancer therapy. In many applications, the primary radiation to be investigated are hadrons (such as protons), neutrons or heavy ions; such primary particles produce secondaries, which include other hadrons, electrons and photons. For all these particles (including high-energy photons, treated as quantummechanical particles) the associated interactions must be taken into account, with different degrees of detail according to the required accuracy of the simulation.

Given the high number of generated particles through the medium and the high number of scattering integrals (related to different interactions the primary and secondary particles can have, in different energy ranges), the early-stage radiation effects for the above mentioned applications are traditionally studied via Monte Carlo (MC) radiation transport methods, in which a stochastic approach, based on an approximation to the Boltzmann transport equation, is used to simulate the statistically averaged trajectories of model particles as they travel across the matter.

In the following, after a brief summary of the fundamentals of charged particles interactions with matter in a large energy range, we illustrate the fundamental notions of MC particle transport $[1,2,3,4]$, in both the so-called condensed history approach suitable for high energy applications and in the track structure approach for the study of radiation effects in biological targets at the micro- and nanoscale. We then present some technicalities of the MC calculations and in the end we make a brief excursion in the applications related to radiation-induced effects in spacecraft components, in water/icy targets of interest for planetary exploration, and in radiobiology. In particular, we will focus on possible synergies between the first-principles chemicalphysics/condensed matter approaches and the MC particle transport approaches, for problems related to radiation-induced effects by those interactions (among the many considered in MC approaches used in the applications mentioned above) occurring in the low-energy regime. While in MC approaches there is actually no real dynamics of the systems, coupled electron-nuclear dynamics in TDDFT [5] and ab-initio Molecular Dynamics (MD) [6] allow to study the dynamical response of the system and can provide insights into the quantities provided by MC codes, ameliorate some input datasets used by the latter in the low energy regime and clarify the accuracy of internationally recommended datasets commonly used to benchmark MC results.

\subsubsection{Brief summary of interactions}

In many applications, the transport of protons and other charged particles is of relevance. The number of interactions these can have with matter is daunting, and cross sections are based, depending on the energy range, either on experimental data, or on sole (not parameter-free) models or on parameterized models which extend reduced set of experimental data to larger energy windows. Differently from Chapter 7, we will mention here not only the interactions that the incoming particle can have with the electrons in the target material, but also the other interactions that a particle in high energy applications can induce in the system. The main classes of interactions for protons and charged hadrons are: 
- electronic interactions with electrons, such as ionization/excitations, described through the electronic stopping power;

- multiple Coulomb scattering at small angles (elastic deflection without energy loss, or very small inelastic loss);

- inelastic nuclear reactions ( $>30-50 \mathrm{MeV}$ ): these are high-energy nuclear reactions in which a target nucleus struck by an incident particle $(p+, n$, $\pi$, etc.) of energy typically above a few tens $\mathrm{MeV}$, can lead to fragmentation into secondary lighter nuclei and/or lighter particles. Such nuclear reactions are considered to take place in two steps:

1) a fast direct stage intranuclear cascade $\left(\sim 10^{-22} \mathrm{sec}\right)$, where the incident proton/ion knocks out several nucleons; At low projectile energies $(<100 \mathrm{MeV})$, all interactions occur just between nucleons and the process is called nucleon cascade [7]. With increasing energy, firstly pions come out (at energies of about hundreds of $\mathrm{MeV}$ ), then at higher energies $(\sim 2-10 \mathrm{GeV})$ heavier hadrons are produced. These heavier particles can also participate in the intra-nuclear cascade and interact between each other, in what is called a hadron cascade. Particles that gain high enough energy to escape from the nucleus are emitted mainly in the direction of the incident particle; the rest of the energy is distributed in the nucleus, which is left in a highly excited state. In the intranuclear cascade collisions are treated as a series of two-body collisions $[8,9,10]$ or via Quantum Molecular Dynamics models [11], where the reaction is treated in a semi-classical many-body framework, dealing with all correlations among the ejects;

2) an "evaporation" phase [12] in which the excited nucleus decays by the evaporation of charged particles and neutrons, forming a continuous distribution of products [Figure 14.1]. The evaporated fragments have energies up to $\sim 40 \mathrm{MeV}$. In this phase, residual particles are emitted in isotropic manner; the reaction yields show different regions according to the different reaction pathways (spallation, fission, fragmentation);

- elastic nuclear interactions $(<10-20 \mathrm{MeV})$ : unlike the small angle scattering events at the previous point, the scattering taking place in an elastic nuclear collision is not a glancing one. After penetrating the electron cloud of the target atom, the proton interacts with an unscreened nucleus. Elastic scattering is generally modelled as a hard-sphere collision. In a crystal lattice, atoms are displaced from their initial positions, creating vacancies and interstitials. If the recoil energy of the primary knock-on nucleus is higher than the threshold for atomic displacement, it can produce displacement of a further nucleus, initiating a collisional displacement cascade. Although qualitatively similar, the displacement cross sections for protons are generally larger than those of neutrons, because of the electromagnetic interaction, and pions, because of the larger mass, for a given material (see e.g. [13]); all cross sections rapidly decrease above a few tens of $\mathrm{MeV}$ in any material.

Neutrons, being neutral particles, cannot ionize materials. Apart from the inelastic, two-step collisions described above, reactions that may occur in a target under neutron impact are elastic scattering and inelastic scattering with neutron-capture. Elastic scattering is a collision between the neutron and the nucleus, where after reaction, the neutron is deviated from its trajectory with a transfer of kinetic energy and the nucleus is left in the ground state. For inelastic interaction, a neutron is captured by the nucleus and then re-emitted with different energy and momentum (a $\left(n, n^{\prime}\right)$ reaction). The resulting nucleus, in an excited state, can de-excite by emission of a $\gamma$-ray, 
charged particles, or other neutrons. For neutrons with energy below $0.5 \mathrm{MeV}$, the probability of neutron capture increases [2]: the neutron is absorbed by the nucleus, usually accompanied by a $\gamma$-ray (the $(n, \gamma)$ radiative capture).

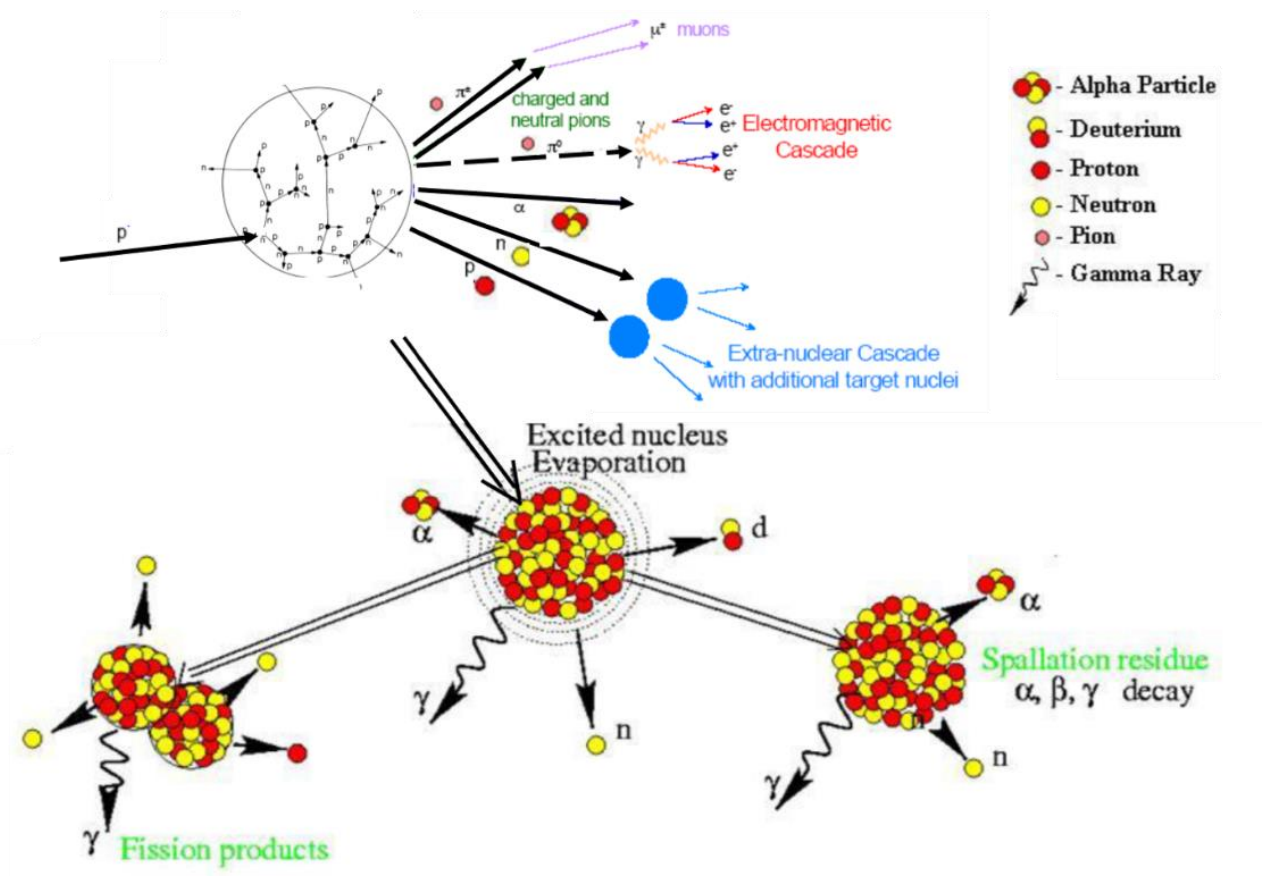

Figure 14.1. Schematic example of a high-energy proton interaction with a heavy target nucleus, and some typical outcomes of the subsequent particle cascade. Adapted from [14] and [15].

Electrons constitute the predominant secondary particle species generated as charged particles traverse matter. Elastic interactions are those in which either the electron passes straight through the sample, or is deflected from its path by Coulomb interaction with the positive potential inside the electron atomic cloud, with no or negligible energy loss. The closer the electron comes to the nucleus, the larger is the scattering angle. The backscattering probability is peaked at energies of 1-10 KeV and rapidly decreases, and gets larger the larger the atomic number $Z$ of the target.

In inelastic electron interactions, the energy transferred to the target can cause emission of secondary electrons. These can be Auger electrons, or other electrons emitted with low energies from the valence states, and with smaller probability higher energy electrons, also called $\delta$-rays, with energies of several hundred keV. Collective excitations such as plasmons, phonons, ... can also be induced. Also, an electron passing an atom within its electron cloud is decelerated by the Coulomb force of the nucleus; this inelastic interaction generates a continuous spectrum of highenergy X-rays, with maximum energy equal to that of the incident beam, the Bremsstrahlung emission.

At electromagnetic field energies $E=h c / \lambda$ for which the wavelength $\lambda$ becomes comparable with the atomic size, that is a few $\mathrm{keV}$, the photons can be practically considered as particles with zero mass and momentum $p=E / c$. They can interact with matter through: $\mathrm{a}$ ) the photoelectric effect; $\mathrm{b}$ ) Compton scattering, where the photon transfers part of its energy to an atomic electron that is emitted with well defined energy and momentum; c) coherent (Rayleigh) scattering from the atomic electron cloud, with a relatively small contribution for $\mathrm{keV}$ energies where the photoelectric dominates, and negligible for $\mathrm{MeV}$ energies; d) positron-electron pair production 
when the energy of the photon reaches twice the rest energy of an electron (1.022 MeV), and rapidly increasing with increasing photon energy. The positron-electron pair share the excess photon energy (above $1.022 \mathrm{MeV}$ ) as kinetic energy. After coming to rest, the positron annihilates with an atomic electron, and a pair of annihilation photons with an energy of $0.511 \mathrm{MeV}$ is produced.

\subsection{The Boltzmann equation}

When the incoming particle beam is very intense, it becomes meaningless to follow each primary particle individually, and to track all the huge shower of its secondary particles. Instead, a statistical approach is sought, centred on the fundamental notions of phase space and of particle distributions (in position, energy, angle, momentum, time) in the phase space. Each particle $i$ is represented by a point with 6 coordinates in phase space, each phase space coordinate corresponding to one of the degrees of freedom of the particle: three correspond to the position $\{x, y, z\}$ and the other three to the vector components of the particle kinetic momentum $\left\{p_{x} p_{y}, p_{z}\right\}$ (also expressed as the triplet of energy and two direction angles, $\{E, \vartheta, \phi\})$; if required, spin, particle type, etc. may be considered by adding additional degrees of freedom.

The aim is thus to determine the expected number of particles in a phase space element $\left(d^{3} r d^{3} p\right)$ at time $t$, that is the probability density $\mathrm{F}=\mathrm{F}\left(\vec{r}_{i}, \vec{p}_{i}, t\right), i \in N$, which requires to follow the 6 phasespace coordinates of a macroscopic number of particles $N$, of the order of Avogadro's number. The most accurate, though approximate, equation that describes particle transport in terms of the phase space density is the Boltzmann transport equation, that is obtained from the full equation for the density distribution (the Liouville equation) by rewriting the equation for one representative particle, called the 1-particle density distribution $f$, and averaging over the ensemble of the other particles. By following the time evolution of the 1-particle density $f=$ $f(\vec{r}, \vec{p}, t)=f(\vec{r}, E, \vec{\Omega}, t)$ (where $\vec{\Omega}$ is the unitary vector defined by $\vartheta, \phi)$ ), a complete "history" of the radiation tracks can be obtained (that is, an average space-time distribution of the expected energy-momentum behaviour of the particle beam, transported and scattered across the target).

The Boltzmann equation is an integro-differential non-homogeneous equation, based on the principle of detailed balance in phase space: at any phase space point, the time derivative of $f$ is the sum of a contribution from all the particles that arrive at the point $\vec{r}$ with momentum $\vec{p}$ in absence of collisions ("streaming" or free-flight term), plus a contribution from all the particles that arrive at $\vec{r}$ by changing their momentum $\vec{p}^{\prime} \rightarrow \vec{p}$ as a result of an interaction ("collision" term). In practice, the Boltzmann equation is often written in terms of the particle flux

$$
\psi(\vec{r}, E, \vec{\Omega}, t)=|\vec{v}| \cdot f(\vec{r}, E, \vec{\Omega}, t)
$$

with units of [particles]/([surface][time]) [16]:

$$
\underbrace{\frac{1}{v} \frac{\partial \psi(x)}{\partial t}}_{\text {time-dependent }}+\underbrace{\vec{\Omega} \nabla \psi(x)}_{\text {translation }}+\underbrace{\mu_{\mathrm{t}} \psi(x)}_{\text {absorption }}-\underbrace{S(x)}_{\text {source }}=\underbrace{\int_{\Omega} \int_{E} \psi(x) \mu_{\mathrm{s}}\left(x^{\prime} \rightarrow x\right) d x^{\prime}}_{\text {scattering }}
$$


where $x$ represents all phase-space coordinates $\vec{r}, \vec{\Omega}, E, t\}$ and $\psi(x)$ is thus the flux of particles at $\vec{r}$ moving along the direction $\vec{\Omega}$ with kinetic energy $E$ at time $t$. The different terms in the equation have the following physical meaning:

$a$. the first term, $1 / v \partial \psi(x) / \partial t$ is the time-dependent flux change, due to particles escaping from the system boundaries, or disappearing by an absorption reaction, or radioactive decay.

b. $\vec{\Omega} \nabla \psi(x)$ and $\mu_{t} \psi(x)$ are respectively the flux change due to translation without change of energy and direction (free flight) and the absorption term, with $\mu_{t}(\vec{r}, E, t)$ being the total macroscopic absorption cross section integrated over angles (or attenuation coefficient, inverse of the mean free path $=1 / \lambda_{t}=\sigma_{t} N_{A} \rho / A$, where $\lambda_{\mathrm{t}}$ is the mean free path and $\sigma_{t}$ the interaction probability per atom $/ \mathrm{cm}^{2}$ ).

c. $S(x)$ represents the particle source, external (e.g., a particle beam irradiating the target volume) or internal (e.g. neutrons from fission reactions in the volume).

$d$. the term on the r.h.s. represents the effect of collisions, or scattering (change in particle flux from changes in direction and energy, without a change of particle position); $\mu_{s}(\vec{r}$ : $\left.\overrightarrow{r^{\prime}}, \vec{\Omega}: \overrightarrow{\Omega^{\prime}}, E: E^{\prime}, t: t^{\prime}\right)$ is the angle-dependent macroscopic scattering cross section, $\sigma_{s} N_{A} \rho / A$. Note that in the writing of Eq. (14.2), the collision term has been linearized, by assuming that the density of particles in the source beam is low enough to neglect particle-particle collisions in the source, and only collisions with the target atoms and nuclei are considered.

The analytical solution of the Boltzmann equation has been attempted for simple geometries, assuming a monoenergetic source and a homogenous isotropic scattering medium (see e.g., $[17,18])$. In any practical case, recourse to numerical methods is necessary to obtain solutions for complex geometries, multi-material targets, and sources with complex energy and momentum spectra. Particle transport is an inherently stochastic problem where all quantities and processes are naturally described by probability distributions. Considering the daunting number of possible interactions that a particle can undergo in the target, and the different type of secondaries that can be generated, the MC family of numerical techniques represent a very convenient solution method. Instead of integrating the probability distributions under given initial and boundary conditions, in the MC method the same distributions are randomly sampled, producing a collection of representative particle "histories" (usually in the figure of many millions), from which physical quantities of interest can be derived by statistical averaging from these distributions. It can be said that MC methods essentially create a statistical sample of a complex problem for which a direct solution is unattainable.

To construct a MC simulation, it is more convenient to put the Boltzmann equation (14.2) in integral form, by performing the integration over the time variable via a conveniently chosen integrating factor. According to the Ergodic Theorem of Birkhoff and Von Neumann [19], the average of a function along the time trajectory is equal to the ensemble average over all phase space, in the limits of infinite time and sample size, respectively. On this basis, one can replace the time variable with a pathlength variable $s$ along the direction $\vec{\Omega}$, by defining: $\vec{r}^{\prime}=\vec{r}-s \vec{\Omega}$, which allows to write the translation term as [4, p. 403]:

$$
\frac{1}{v} \frac{\partial \psi}{\partial t}+\frac{\partial \psi}{\partial s}+\mu_{\mathrm{t}} \psi(x)=S+q
$$


where $q$ indicates the scattering (double) integral in the r.h.s. of equation (14.2). A detailed treatment of this equation can be found in the many reviews about particle transport [1-4]. By introducing the integrating factor $\beta=\int_{0}^{s} \mu_{\mathrm{t}} s d s$, also called optical thickness, the Boltzmann equation turns into:

$$
\psi(\vec{r}, E, \vec{\Omega})=\int_{0}^{\infty} d s e^{-\beta}\left[S(\vec{r}, E, \vec{\Omega})+\int_{0}^{E m a x} d E^{\prime} \int_{4 \pi} d \Omega^{\prime} \mu_{s}\left(\vec{r}, E^{\prime}, \vec{\Omega}^{\prime}\right) \psi\left(\vec{r}, E^{\prime}, \vec{\Omega}^{\prime}\right)\right]
$$

This form of the equation can be approximated as a series of events, alternating collisions and free flights (Von Neumann series): (i) a particle is started by sampling the source $S(\vec{r}, E, \vec{\Omega})$; (ii) it is transported to a position $\vec{r}$, sampled from the path-length distribution $\beta$; (iii) at this site, it makes a collision, and changes its direction and (possibly) its energy to values sampled from the collision kernel, $\mu\left(\vec{r}, E^{\prime}, \vec{\Omega}^{\prime}\right) \psi\left(\vec{r}, E^{\prime}, \vec{\Omega}^{\prime}\right)$; then it moves to a successive position $\vec{r}^{\prime}$ as in (ii), where it will make a new collision (iii), and so on, until it is absorbed, or escapes from the system boundaries. This succession of free-flights and collisions (the particle "history") is the essence of the MC algorithm, which can be also explicited in an integral-kernel form, for the sake of mathematical formalism; a detailed treatment of the MC approximation to the Boltzmann equation can be found in the many reviews about particle transport [1-4].

\subsection{The Monte Carlo method}

As hinted in the previous section, in the MC method particles are transported step-by-step accounting for the stochastic nature of their microscopic interactions. The interactions of individual primary ions and their secondaries are sampled to build a history of charged particle passage and energy deposition in 3-D geometries $[4,16]$. Depending on the type of incoming radiation and its energy, different interaction processes have to be considered (see Figure 14.1), whose details (energy- and angle-dependent cross sections) are provided by experimental data and/or theoretical models of the elementary interactions.

Each particle individual path is modelled statistically while undergoing the different interaction processes. The final result is calculated by considering a large enough statistical sample of the path (i.e., time-) trajectories to obtain a good approximation of the phase space density, or the particle flux.

At each step of the Von Neumann series, the path length of free flight and the collisional interactions (and their outcomes) follow from a random selection of the corresponding variable from the appropriate probability distributions. Indeed, a key aspect of the MC technique is the ability to generate a sample of phase space points $x$ distributed according to a Probability Density Function (PDF) $f(x)$ of the $x$ variable ( $x$ can be multidimensional). The samples are (pseudo)random values of a variable distributed according to a PDF that embodies the physics of the interaction, which implies a random sampling of the outcome of physical events from probability distributions.

The "golden rule" of the Monte Carlo method is based on the use of a homogeneous pseudorandom number generator in the interval $[0,1]$, to sample any arbitrary probability density. Starting from the density $f(x)$, a positive quantity defined on some interval $[a, b]$ with integral 
normalized to 1 , the cumulative density function $F(x)=\int_{a}^{b} f(x) d x$ is constructed (CDF). By definition, $F(x)$ is monotonically increasing from 0 to 1 in $[a, b]$; then, a random number $\xi \in[0,1]$ is used to invert the CDF, as $y=[F(\xi)]^{-1}$. By construction, $y$ is an approximant of the functional derivative of the CDF, that is $y \rightarrow f(x)$, the original PDF. With this general method, any PDF can be sampled, be it given in analytical, numerical, or tabular form, for any variable in the MC simulation: the pathlength $s$, the scattering angles $\Omega=(\theta, \phi)$, the cross sections for any atomic/nuclear events (elastic scattering, photoelectric, Compton, capture, fission etc.), and so on.

The use of Pseudo Random Numbers (PRN) generator algorithm is needed as computers can not generate true random number sequences. A PRN is a deterministic algorithm that, given the previous state in the sequence, can generate the next number with (almost) no correlation with the preceding one. Such algorithms need a seed to start a sequence and, being deterministic they will always produce the same sequence when initialized with that state. This allows to reproduce the same results when the same code is run on different computers (and it also obviously helps with the debugging of MC codes). For the practical applications of MC codes, very efficient PRN algorithms exists, that can generate very long sequences of uncorrelated numbers (up to $2^{241}$ or $>10^{70}$ for the most recent PRNs.

\subsubsection{Continuous slowing down approximation, condensed history technique and thresholds}

The Monte Carlo method can be applied independently on the type and properties of the transported particles. However, there are some important differences when dealing with charged particles, compared to the case of neutral particles. The latter (neutrons, photons) experience occasional interactions with individual target particles (electrons, nuclei) separated by a relatively large free pathlength, giving rise to the characteristic exponential attenuation profile. Instead, when a charged particle passes through a material it actually experiences a huge density of interactions per unit pathlength, both with the nuclear electric field, the mean electron field, and with individual outer shell electrons of the single atoms, because of the long range of the Coulomb field [4]. Therefore, unless the particle velocity falls below a cut-off value roughly corresponding to its de Broglie wavelength, $\lambda=h / m v$, being much larger than the interatomic distances (that is, about $10^{-3} \mathrm{c}$ for an electron, or $10^{-6} \mathrm{C}$ for a proton), it is normal practice to model the energy loss of charged particles as continuous, with a rate equal to the electronic stopping power, given by the Bethe Bloch equation [20]:

$$
-\left(\frac{d E}{d x}\right)=\frac{4 \pi \rho Z r_{e}^{2} m_{e} c^{2}}{A} \frac{z^{2}}{\beta^{2}}\left(\ln \frac{2 m_{e} c^{2} \beta^{2} \gamma^{2}}{I^{2}}-\beta^{2}+C-\delta\right)
$$

with $\beta=v / c$ the particle velocity, $\gamma=\left(1-\beta^{2}\right)^{-1}$ the Lorentz relativistic factor, $Z$ and $z$ the electric charges of the target atom and of the moving particle, $\rho$ the medium density $\left(\mathrm{g} / \mathrm{cm}^{3}\right), I$ the mean excitation potential of the target atom, and $C$ and $\delta$ are to corrections respectively due to excitation close to shell boundaries (relevant for small energies) and a density correction due to polarization, relevant for high energies. The so called Barkas correction [20] is sometimes added, representing a correction due to the electron capture of the positively charged protons at low energies in the domain of the Bragg peak and behind, leading to a slightly increased range of the moving 
particle, whereas the negatively charged anti-protons cannot capture electrons from the environmental electrons.

The attenuation coefficient along a pathlength segment $d s$ can then be written as the sum of two terms

$$
\mu_{s}\left(\vec{r}, \widehat{\Omega}: \hat{\Omega}^{\prime}, E: E^{\prime}\right)=\frac{d \psi}{d E} \frac{d E}{d s}+\mu\left(\widehat{\Omega}: \hat{\Omega}^{\prime}\right) \cdot \delta\left(E^{\prime}-E\right)
$$

The first term represents the continuous energy loss involving no scattering, while the second term describes the scattering without the energy loss. In this scheme, called continuous-slowingdown approximation (CSDA), the particle attenuation is no longer exponential, but has a one-toone correspondence with the energy loss. The travelled distance is obtained by integrating the reciprocal of the total stopping power over each discrete energy loss $\Delta E$, and the maximum penetration depth (or CSDA range) corresponds to the integral from the initial particle energy $E_{0}$ to zero. The CSDA range marks the limit at which all charged particles of a given type with the same energy are absorbed in a given medium. Due to the particular form of the stopping power (14.6), the energy loss increases rapidly with decreasing energy, concentrating the maximum of energy deposition in the target towards the end of the trajectory (the so-called Bragg peak of energy deposition).

Notably, assuming the CSDA implies that all particles with the same energy are assumed to travel the same average distance [21]: this is why the CSDA range is a close approximation to the average path length travelled by a charged particle as it slows down to rest. However, in reality particles lose energy in random individual encounters, and their energy after a collision is a continuous distribution, rather than a fixed value: this problem, called energy straggling, is more important for lighter than for heavy penetrating particles (typically electrons, which obviously have the same mass of atomic electrons, and therefore can share the kinetic energy in all possible proportions from 0 to $E_{0}$ ). A correction scheme due to L. Landau [22] allows to take into account this energy spreading, in the form of a universal function applied to the distribution of $\Delta E$ values, for a given traveled pathlength $\Delta s$ (see below).

An approximation similar to the CSDA for the energy loss is adopted also for the angular deflection, mostly originating from the elastic scattering, by calculating an average angular distribution (Goudsmit-Saunderson, or Molière approximations [4]) that results from the cumulative effect of a large number of small deflections, along a pathlength segment $\Delta s$. The above ensemble of approximations for particle kinetics, common to most MC particle transport codes, makes up the so-called condensed-history Monte Carlo method for charged particle transport simulations [16], in which many discrete events are replaced by a straight continuous step, and the corresponding energy losses and changes of direction are condensed into a sum of losses (stopping power) and an overall scattering angle. In a condensed history, a large numbers of interactions along a small segment $\Delta s$ of the radiation track is accounted via a "cumulative" effect and the typical spatial resolution is of the order of $\mathrm{mm}$. 


\subsubsection{Computational efficiency and variance reduction techniques}

MC calculations results are obtained as the most probable value of a quantity $x$ of interest (e.g., flux, number of created isotopes, energy deposition in a volume, etc.), calculated on the ensemble of particle "histories", indeed a statistical sample of the actual transport problem. Given the finite size of the statistical sample, such "results" are extracted from a suitable average of the quantity of interest, over the set of samples. By their very nature, MC methods suffer from statistical uncertainty, or variance, due to finite sampling. According to the central limit theorem, the uncertainty is inversely proportional to the square root of the number of independent samples (this is strictly true for Gaussian statistics, and in practice this is the case for an unbiased sample). Thus, for each particle, for each desired quantity, averages can be computed as well as their standard deviation $\sigma$ :

$$
\bar{x}=\frac{1}{N} \sum_{n=0}^{N} x_{\mathrm{n}} \quad, \quad \sigma(x)=\sqrt{\frac{1}{N-1} \sum_{n=0}^{N}\left(x_{\mathrm{n}}-x_{0}\right)^{2}}
$$

with $x_{n}$ being the contribution of the $n_{t h}$ element in the sample (one particle "history").

Several techniques of biased sampling must be used in practical applications, in order to reduce the variance (for a given amount of computing time) and accelerate convergence (to reduce the overall computing time). The general approach is to modify the PDF corresponding to some interaction event, in order to artificially increase or decrease the corresponding probability in a controlled way, while taking into account the effect of the biasing by a numerical correction factor. Such methods go under the general name of "variance reduction techniques", in the following we give some details of the most important ones.

Modified collision density. Since the number of events necessary to achieve a given statistical precision may be very high, it is sometimes necessary to artificially increase the number of collision events in specific zones. Such approach is often necessary for neutral particles, which may experience rare collisions in a small volume, or in a low-density medium. Collisions can be "forced" by locally increasing the value of a cross section; to compensate for the artificial increase, the score (energy deposition, particle absorption, etc.) is reduced by a factor ("weight") inversely proportional to the biased cross section. This goes also under the name of pathlength stretching, since the distance to the next collision is given by the exponential of the cross section. The notion of particle weight is generally useful in a MC method, as a multiplicative factor that relates to the importance of an event. Particles can carry a weight larger or smaller than 1 (the unbiased value) and their contribution to any event is weighted by this factor.

Range cut-off. If secondary charged particles have a residual range (that is, the integral of $(d E / d x)^{-}$ 1 from the current value of energy to zero) insufficient to reach the volume of interest, they are suppressed by releasing their residual energy on the spot. Alternatively, the pathlength step time can be adjusted, by adopting the best value in each zone.

Adjusted energy thresholds. Transport and production thresholds are helpful for reducing computer time, but also are needed because of limitations in the validity of the physics model used to describe the cross sections of the different processes. Production energy thresholds are needed for explicit production of Bremsstrahlung radiation (below such a threshold, the electron radiative energy loss is included in the stopping power, $d E / d x$ ), or for the explicit production of 
secondaries by photons and electrons. A $\delta$-ray threshold sets the limit between discrete and continuous ionization energy losses. Transport thresholds are such that the transport of a particle is stopped when its energy is lower than the preset threshold; in this case, its remaining energy is deposited at that point, or better, in the case of a charged particle, is distributed along its residual range [16].

\subsubsection{Some (other) approximations}

To conclude this brief introduction to the technical aspects of MC methods in particle transport, it may be relevant to discuss some of the underlying assumptions and approximations that are common to most MC transport codes.

Firstly, the transport of particles is described as a Markovian process: after each time interval during which the distribution of the phase-space points migrates from one cell to another one, all the correlations are destroyed. This means there are no memory effects, i.e. the fate of a particle depends only on its actual present properties, and not on previous events or histories. This is a valid assumption if the characteristic times of the fluctuations (the scattering processes) in each phase-space cell are very little with respect to the characteristic times of the distribution evolution (the transport processes) [4]. While this assumption does not seem to present particular problems, the additional assumption that particles do not interact with each other is not valid in extremely intense radiation fields.

Target materials are assumed to be amorphous, that is the macroscopic interaction cross sections are obtained by a simple stoichiometric averaging of the elemental cross sections. This is a reasonable approximation for macroscopic conditions, but effects like channelling of ions within crystallographic planes is starting to be considered in new implementations of MC codes for high energy [23]. In almost all applications, materials are considered as continuous, homogeneous and isotropic. For the important problem of transport of cosmic rays in the atmosphere of planets, which would require a description of the variable density and temperature of the atmosphere, MC codes usually approximate the continuous density variation by many discrete layers of uniform density [24]. Furthermore, materials are static: there is no dynamics induced in them by the impact of primaries and the generation and passage of secondaries.

The accuracy of MC calculations does not depend only on the number of particle histories, with statistical convergence possibly accelerated by biasing, but also on the models or data on which the probability distribution functions are based. It has actually been shown that deficiency in the accuracy or in the knowledge of the fundamental light fragment and neutron production cross sections is critical, in particular for the study of galactic cosmic rays transmission through matter (shielding, spacecraft components, and human tissue) [25].

The CSDA previously mentioned continuous uses as input the electron stopping power of the projectile in the material being considered. The CSDA implies a one-to-one correspondence between the particle energy and the travelled distance at each step. However, energy loss has a statistical nature, which means that actually the probability of losing an energy in a certain range $\Delta \mathrm{E}$ around $\mathrm{E}$, when travelling for a certain path-length, should be considered. The fluctuations around a mean value of the energy loss (straggling) are described by a distribution that is sampled within the transport operator as an energy-dependent step function used to boost the energy loss 
by quantities $d \Delta E$ with probability $p(\Delta E, s)$, with respect to the continuously decreasing energy loss from the transport operator [4]. As a matter of fact, without an appropriate correction, the CSDA overestimates the effective projected ranges, because of the range straggling of charged particles, especially at low energies where nuclear collisions and multiple scattering become dominant. For high energy ions, on the contrary, the effective stopping power is the same as the CSDA stopping power, because the loss of energy is mainly by collisions with the atomic electrons and by nuclear interactions.

\subsubsection{Geant4 and the suite of applications for space studies}

An overview of several MC particle transport codes currently in use in the scientific community is provided in Table 14.1. Among these, the Geant4 code [26] is especially well-designed to simulate high-energy processes with nearly any kind of particle as source. The software suite, with the source code written in $\mathrm{C}++$, is freely available. Geant4 is not a unique code, but a set of libraries which can be used to build specific applications. The user can modify and combine any parts of the code according to the requirements of each particular system to be simulated (https://geant4.web.cern.ch/support/user documentation). Other widely used codes, such as MCNP6 [27], FLUKA [28], HETC-HEDS [29] and SHIELD [30] do not seem to be as flexible as GEANT4. MCNP6 also requires a license and is a trademark of Los Alamos National Security (LLC, Los Alamos National Laboratory); FLUKA (a code mostly devoted to high-energy physics) requires development to be done in FORTRAN 77, which excludes many features available using more modern programming languages (although only partly true, as routines can be added in F95 and later cross-linked). While PHITS [31], MCNP [27] and GEANT4 [26] support multithreading and Message Passing Interface (MPI), thus being adapted to massively-parallel computing environment, the other codes require splitting large simulations into smaller runs with different random number seeds, and merging the results at the end.

Table 14.1. Comparison of MC codes, particularly used for applications related to space missions

\begin{tabular}{|c|c|c|c|}
\hline Code/ Developers & Particles transported & $\begin{array}{c}\text { Maximum } \\
\text { energy }\end{array}$ & Language/Threading \\
\hline $\begin{array}{c}\text { MNCP } \\
\text { Los Alamos National Lab, USA }\end{array}$ & $p, n, \mu, \gamma, \pi$ and HZE ions & $100 \mathrm{GeV}$ & $\begin{array}{l}\text { Fortran/MPI and } \\
\text { OpenMP }\end{array}$ \\
\hline $\begin{array}{c}\text { Geant4 } \\
\text { CERN and Geant4 } \\
\text { collaboration }\end{array}$ & $\begin{array}{l}p, n, \mu, \gamma, \pi \text { and HZE ions and } \\
\text { other subatomic particles }\end{array}$ & $\begin{array}{l}\text { Over } 1 \\
\text { PeV }\end{array}$ & $\begin{array}{l}\mathrm{C}++, \text { POSIX Threading, } \\
\text { MPI and TOPC }\end{array}$ \\
\hline $\begin{array}{c}\text { FLUKA } \\
\text { INFN and CERN, Italy }\end{array}$ & 61 particles and $\gamma$ & $1 \mathrm{GeV}$ & Fortran/Sequential \\
\hline $\begin{array}{c}\text { PHITS } \\
\text { JAEA, Japan }\end{array}$ & $p, n, \eta, n u c l e i, e-$ and $\gamma$ & $200 \mathrm{GeV}$ & Fortran/OpenMP \\
\hline $\begin{array}{c}\text { SHIELD } \\
\text { JINR and NIR, Russia }\end{array}$ & $\begin{array}{l}\text { any }(\mathrm{A}, \mathrm{Z}) \text { nuclei, } p, n, \pi, K, \\
\text { antinucleons and } \mu\end{array}$ & $1 \mathrm{TeV}$ & Fortran/Sequential \\
\hline $\begin{array}{c}\text { HETSC-HEDS } \\
\text { Oak Ridge National Lab and } \\
\text { NASA, USA }\end{array}$ & $p, n, \mu, \gamma, \pi$ and HZE ions & & Fortran/Sequential \\
\hline
\end{tabular}


A large set of physics "lists" (collection of models/available data for interactions over different energy ranges) is included in Geant4. Such lists have to be combined in order to cover a wide energy range, and even if not the case, to describe the interactions of different charged particles and neutral particles. There exist a very large developer and user communities for this code, which strongly contribute to the further improvement of the physics lists.

In studies of radiation effects induced on both biological and spacecraft components during a space mission, Geant4 is rarely used as standalone tool. A suite of programs and frameworks are used that operate on top of the various Geant4 modules. Examples are the MUlti-LAyered Shielding SImulation Software (MULASSIS), a Monte Carlo-based space radiation analysis tool for use with simple 1-D geometries [32]; the Geant4 Radiation Shielding tool (GRAS) a fully 3-D, MCbased radiation analysis tool tailored for use in space radiation [33]; and more-general frameworks on top of several Geant4 modules, also allowing to obtain the radiation spectra during a space mission, like the SPENVIS online system [34], developed by ESA. Once the energy spectrum of source particles is determined, it can then be used to calculate the effect of interest for the user. Some of these quantities are:

- the degradation of the output current in spacecraft solar cells linked to atomic displacements;

- the absorbed dose in water (see Paragraph 14.2.2), used as proxy for biological tissue reactions in humans, which appropriately scaled can allow to predict the increase of the likelihood for an astronaut to develop cancer [11] and induced short-term effects (cataracts, acute radiation sickness, ...) [35];

- the Total Ionising Dose, an important parameter for electronic components and material degradation over the duration of a space mission;

- the so-called Single-Event Effects in microelectronics, causing failure of circuits and sometimes loss of instruments [36].

\subsubsection{MC track structure codes}

The study of biological effects of ionizing radiation at the cellular level is very important both in the field of ion-beam cancer therapy and in the science that aims at developing more reliable risk assessment models for astronauts and future explorers on the Moon and Mars. The effects at cellular level are strongly linked to the details of the spatial pattern of the energy deposition at the nanoscale induced (mostly) by ionizations ("ionization clusters") within subcellular structures (e.g., DNA, proteins, cell membrane). At such spatial scale, the transport of low-energy radiation becomes a critical component of any quantitative analysis of radiation effects [37], as the penetration of such radiation in matter becomes comparable to the target dimensions (less than $1 \mu \mathrm{m})$. Evidence has accumulated through the years that the most important damage to biological molecules is due to secondary electrons with energy around $100 \mathrm{eV}$ (the ionization peak of liquid water and DNA nucleotides) and much below, even $<20 \mathrm{eV}[38,39,40]$.

In standard, condensed history MC dosimetric calculations, where the elementary steps $\Delta s$ represent track segments that are sufficiently long compared to the electron mean free path, and thus group a considerable number of similar collisions, electrons below $1 \mathrm{keV}$ are actually considered to be absorbed on the spot and thus are not tracked anymore below such energy. While 
such an approach makes the MC simulation of charged particle transport computationally feasible up to very high energies ( $\mathrm{MeV}-\mathrm{GeV})$, it is not appropriate to describe the interactions of low energy radiation, as for the latter a resolution down to the $\mathrm{nm}$ scale is necessary.

Microdosimetry and nanodosimetry allow, respectively, for a quantitative description of the stochastic aspects of energy deposition in irradiated media at the $\mu \mathrm{m}$ scale and for a quantitative description of key quantities at the nm scale that show connection to the probability of double strand breaks in DNA, such as the distribution of the size of the ionization clusters produced both along the core of the track of the primary particle and in the region affected by secondary electrons (the penumbra) [42]. For very-low-energy electrons, the MC simulation of individual interactions down to $\sim 10 \mathrm{eV}$ energies (event-by-event tracking) can be performed by the explicit calculation of the track structure: in practice, this is a step-by-step MC method where electrons are followed in their succession of individual collisions and free flights, with the input of the appropriate electron-electron cross sections (shell ionization, electronic excitation, elastic scattering, etc). The description in the condensed-history approach is clearly not appropriate for resolving the events at very low energies and dimensions, if one is interested in the distribution of clusters of ionization events at the scale of DNA or proteins (a few nanometers) (see Figure 7.4 for the difference between the condensed history and the track structure approach).

A whole family of MC track structure codes has appeared in the past years, like PARTRAC [43], KURBUC [44], RETRACKS (RITRACKS) [45], Geant4-DNA [46] and NOREC [47], today considered the state-of-the-art for nanoscale electron transport; among these, Geant4-DNA is the only opensource code. Most models consider that electrons stop propagating below $\sim 10 \mathrm{eV}$ energy. Notably, the high-degree of detail in track structure models makes the development of the algorithms highly complex, therefore such models are commonly tailored to the single target water, as representative of biological tissues (also considering that a large part of the biological damage to DNA and proteins comes indirectly, from free radicals produced by water radiolysis). The cross sections are derived from semi-empirical linear response theory-based models which make use of the first Born approximation and extrapolate the experimentally available data of the energy loss function at $\overrightarrow{\boldsymbol{q}}=0$ to $\overrightarrow{\boldsymbol{q}} \neq 0$, where $\overrightarrow{\boldsymbol{q}}$ is the momentum of the perturbation [37].

Recent studies have reported a potentially relevant effect of the different models used for the dielectric function of water on ionization clustering [48] and DNA damage induction [49]. Also, there is a high degree of uncertainty as the low energy range cross sections become sensitive to the details of the electronic structure of the target [50]. Recent works have started to implement more realistic targets in Geant4-DNA [51].

\subsection{Examples of applications of Monte Carlo particle transport and synergies with quantum dynamics in the electromagnetic sector}

In MC codes, the wide coverage of physics comes from a mixture of experimentally available data or modelled cross sections from parametrized models. Each cross-section table or physics model has its own applicable energy range. Combining more than one tables/models, one physics process can have enough coverage of energy range for a wide variety of simulation applications. 
Here below we will present some applications which deal with particles of an initially high energy and which allow us to highlight a possible synergy with the approaches in the condensed matter/chemical physics community when particles get slowed down in passing through the target and quantities can be sensitive to the many body physics of the target. Indeed, for low energy interactions, accurate modelling of electronic stopping for ions from real time TDDFT, of defects creation by ab-initio MD and of collisional cascades induced by scattering among nuclei by injecting electronic stopping from real time TDDFT into MD cascades simulations, can well provide better input to MC codes or refine the output from the latter. We will focus on applications on materials of use in Space missions, water and ice targets in astrobiology and radiobiology.

\subsubsection{Electronic excitations and atomic displacements in solar cells for space missions}

As seen in previous paragraphs, atomic displacements are created by both elastic Coulomb interactions (described by the concept of nuclear stopping) and nuclear (inelastic and elastic) collisions. The resulting structural defects evolve in time, some eventually healing while others leaving defective structures in the system. The solar cells of spacecraft are affected by such displacements, mostly induced by the accumulated impact of the radiation trapped in the Van Allen belts in Earth's vicinity during repeated orbits, and eventually by Solar Energetic Particles (SEPs) events either in Earth vicinity, or in deep Space or on airless bodies like the Moon (not protected by any atmosphere which could eventually attenuate the incoming radiation). The induced cumulative structural defects cause trapping of the charge carriers, degrading the output current. The so-called Non-Ionizing Energy Loss (NIEL) model, which calculates the energy imparted to atomic displacements, is generally used by the radiation-effects community in the field of Space mission design for the prediction and study of the degradation of the performance of the solar cells. The NIEL formula is given by

$$
-\left(\frac{d E}{d \chi}\right)_{\text {NIEL }}=\frac{N}{A} \int_{T d}^{E_{R}^{\max }} E_{R} L\left(E_{R}\right) \frac{d \sigma\left(E, E_{R}\right)}{d E_{R}} d E_{R}
$$

where $\chi=x \rho_{A}$, with $\rho_{A}$ being the absorber density in $\mathrm{g} / \mathrm{cm}^{3}, x$ the penetration depth of the particle in the material, and $N$ the Avogadro's number, $A$ is the atomic mass of an atom in the material, and $E$ is the kinetic energy of the incident particle. The integration is done over all possible recoil energies $E_{R}$ between the threshold displacement threshold $T_{d}$, the minimum recoil kinetic energy to create a stable defect averaged over all crystallographic directions, and the maximum energy transferred to the recoil nucleus $E_{R}{ }^{\max } . L\left(E_{R}\right)$ is the Lindhard partition function [52] which gives the fraction of the stopping power that goes to NIEL and $d \sigma\left(E ; E_{R}\right) / d E_{R}$ is the elastic Coulomb scattering differential cross section for protons (or other incoming particles) on nuclei. The NIEL generally correlates well with the degradation of semiconductors induced by displacement damage [53]. In several cases there is a linear relationship between the NIEL and the number of displacements when using the modified Kinchin Pease model (Norgett, Robinson and Torrens NRT - model [54]), based on the BCA (successive independent two-body collisions) for calculations, which partially takes into account a modified efficiency for displacements due to the electronic excitations compared to the hard sphere-model. Such a linear relationship means that indeed the efficiency of producing electrically active defects is a function of the NIEL, and that the damage only depends on the number of defects introduced and not on their variety. 
Most of the damage to solar cells in Space comes from protons $<10 \mathrm{MeV}$ [55]. For this low energy regime (from few keV to some MeV), Geant4 [26], SR-NIEL [56] or other Geant4-based tools used for space radiation-induced effects use similar assumptions for the Coulomb contribution to the NIEL. In particular, for the energy loss to electronic degrees of freedom, two models are used in all Geant4 electromagnetic physics lists: for protons, for energies below $2 \mathrm{MeV}$ NIST PSTAR/SRIM (TRIM) [57,58] stopping power based on the Lindhard dielectric response theory and above 2 $\mathrm{MeV}$ the Bethe-Bloch formula with shell, Barkas, and Bloch and density effect corrections. While the SRIM database is usually assumed to be the accurate reference for stopping power, it is important to note that SRIM electronic stopping values are produced by bringing together a limited number of available experimental results in the form of ratios with respect to He stopping, $r\left(Z_{1}, H e, v\right)=S_{e}\left(Z_{1}, Z_{2}, v\right) / S_{e}\left(H e, Z_{2}, v\right)$, where $Z_{1}$ and $Z_{2}$ denote the atomic numbers of projectiles (with velocity $v$ ) and target atoms, respectively [59]. For the nuclear stopping, the Ziegler, Biersack, Littmark screened Coulomb potential, fitted among a large set of data $[60,61]$ and the BCA are used. Both the electronic and nuclear stopping power are calculated according to the Bragg's rule, i.e. the (electronic, nuclear) stopping for a compound is obtained as a weighted sum in which each material contributes proportionally to the fraction of its atomic weight. The $T_{d}$ is a fixed number for each element, independently on the compound in which the element is found. Moreover, the system is considered amorphous and thus channelling effects, where the ions travel in between crystallographic planes, and any dependence of the $T_{d}$ (the minimum energy to displace an atom and create a stable defect) on the crystalline direction are neglected. In addition, collisional cascades are always considered as adiabatic, e.g. there is no synergy between the electronic and nuclear degrees of freedom, which also means that there is no dependence of the $T_{d}$ on accompanying electronic excitations produced by the impacting particle or by the recoil atoms.

Deviations from the NIEL scaling hypothesis have been observed, at both high and low energy [6267]. Microscopic, device-dependent modelling of displacement damage, combining both more sophisticated energy deposition computations and theoretical modelling of the electrical properties of defects and disordered regions is necessary [65,68]. At present, the many approximations done in SRIM are being intensively examined by the condensed matter community [69-73]. Accurate MD calculations [67 and refs. therein] have demonstrated that, in reality, the number of defects can be different from the one described by the NRT model often used in NIEL calculations. Multiscale approaches for radiation damage starting from Geant4 calculations of the produced recoil ions and considering also parameterized approaches for electron and phonon excitations have also been presented [74]. Recent studies show the importance of including electronic stopping effects in cascade formation, in terms of the number of formed defects and cascade morphology [75-83]. The energy transfer from the projectile to the target recoils is a nonionizing event, but the impacting particle and the recoils may deposit locally some energy to the electronic excitations which may alter the formation of defects.

In Figure 14.2 (left panel) we show the results of a MC particle transport calculation performed with the Geant4-based tool MULASSIS for the impact of protons trapped in the Van Allen belts onto a triple-junction solar cells $\left(\mathrm{Ga}_{0.5} \mathrm{In}_{0.5} \mathrm{P} / \mathrm{GaAs} / \mathrm{Ge}\right)$ and their passage through the layers of the cell [72], for a 3-year mission in low Earth orbit, with same inclination and altitude of the International Space Station. Taking into account the relative motion between the satellite and the bombarding particles, we can consider that proton and electron irradiation inside the inner Van 
Allen belt have an isotropic incidence. The MC calculations show that the range of energies passing through the different layers is essentially as large as the one of the impacting particles, though in such "passing protons" through the layers also protons generated via nuclear reactions are here considered. Nevertheless, many irradiation ground testing experiments are done with unidirectional and monoenergetic $1 \mathrm{MeV}$ proton beams on unshielded solar cells (without the typical protective $\mathrm{SiO}_{2}$ coverglass), on the basis of previous studies that showed an equivalence between the damage induced in a realistic space environment on shielded solar cells and irradiation of unidirectional monoenergetic protons in ground experiments on unshielded solar cells [62, 85-87]. SRIM results for such unidirectional proton beam (right panel of Figure 14.2) show that $1 \mathrm{MeV}$ protons stop in the Ge bottom layer. As said above, in the calculations of the number of defects via SRIM, which are usually done via the NRT model (in the quick cascade mode, see [73]), the $T_{d}$ used as input for SRIM is non-direction dependent, is a fixed number for each chemical element, and is not affected by any possible local energy deposition to the electronic degrees of freedom which may influence the displacements.

In Table 14.2 we show the results from ab-initio MD calculations (classical equations of motion for atoms + DFT for electrons) for the $T_{d}$ for different channeling directions in the Ge layer of the solar cells and, for the PKA in the [110] direction, the different values of the $T_{d}$ obtained with an ad-hoc consideration of different electronic temperatures (inserted via Fermi-Dirac smearing), reflecting the influence of electronic excitations. The results show that the $T_{d}$ is indeed different for different crystalline directions, likely influencing the defects that can be created starting from impacting particles or recoils moving in different trajectories, and that the $T_{d}$ is a dynamical quantity. In Figure 14.3 we show the consequent change in the NIEL curve as calculated from SRNIEL [56] when considering different values of the $T_{d}$ (here we have considered the different values corresponding to the different conditions, as these give a higher change in the $T_{d}$; curves for the $T_{d}$ values corresponding to different electronic temperatures would be in the middle between those shown), Clearly, a correct estimation of the $T_{d}$ has a remarkable impact for proton energies between $0.2 \mathrm{keV}$ and $\sim 8 \mathrm{keV}$, energies which are surely covered by the protons stopping in the Ge layer under ground testing conditions, and for electron energies between $0.3 \mathrm{MeV}$ and $20 \mathrm{MeV}$, which surely will pass through the layer. The implementation of the dynamical nature of $T_{d}$ and of its direction-dependence may be considered respectively in codes such as the recently developed Iradina [88] and the MARLOWE code which allows for crystalline order [89].
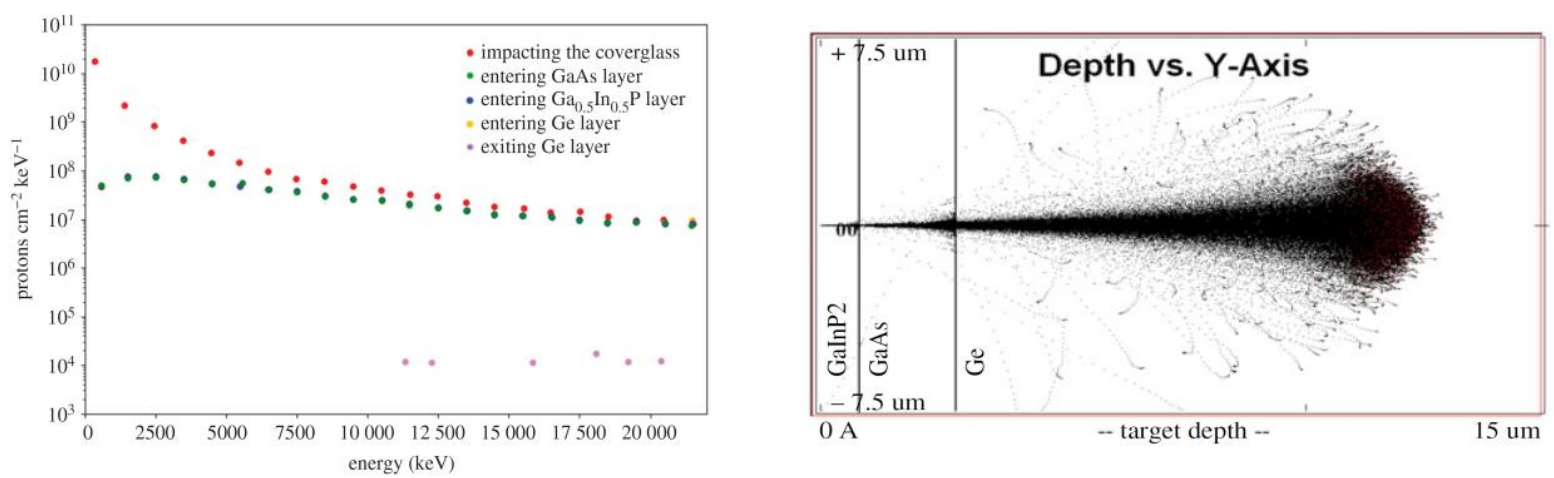

Figure 14.2. Left panel: Fluence of the omnidirectional primary proton radiation across the layers of the solar cell (coverglass included), accumulated during a 3-year International Space Station-like mission, calculated via MC particle transport based on Geant4 (MULASSIS code) [72, 84]. Right panel: ion track trajectory output from TRIM for unidirectional protons of $1 \mathrm{MeV}$. 
Table 14.2. Comparison of threshold diplacement energy value $\left(T_{d}\right)$ obtained from this work and other ab-initio MD results in the literature [90,91], for different channeling directions. In this work, also different values of the electronic temperature $\theta_{\mathrm{e}}=1100 \mathrm{~K}(\sim 0.1 \mathrm{eV}), \Theta_{\mathrm{e}}=3300 \mathrm{~K}(\sim 0.3 \mathrm{eV})$ and $\Theta_{\mathrm{e}}=4400 \mathrm{~K}(\sim 0.4 \mathrm{eV})$ for the PKA along the [110] direction are considered.

\begin{tabular}{cccc}
\hline Work & $\operatorname{Td}[111](\mathrm{eV})$ & $\operatorname{Td}[110](\mathrm{eV})$ & $\operatorname{Td}[001](\mathrm{eV})$ \\
\hline [this work] & 10 & 29 & 18 \\
& & $27\left(\Theta_{\mathrm{e}}=1100 \mathrm{~K}\right)$ & \\
& & $24\left(\theta_{\mathrm{e}}=3300 \mathrm{~K}\right)$ & \\
{$[90]$} & 9.5 & $23\left(\theta_{\mathrm{e}}=4400 \mathrm{~K}\right)$ & 18 \\
{$[91]$} & $10.5 \pm 0.5$ & 28.5 & 18 \\
\hline
\end{tabular}
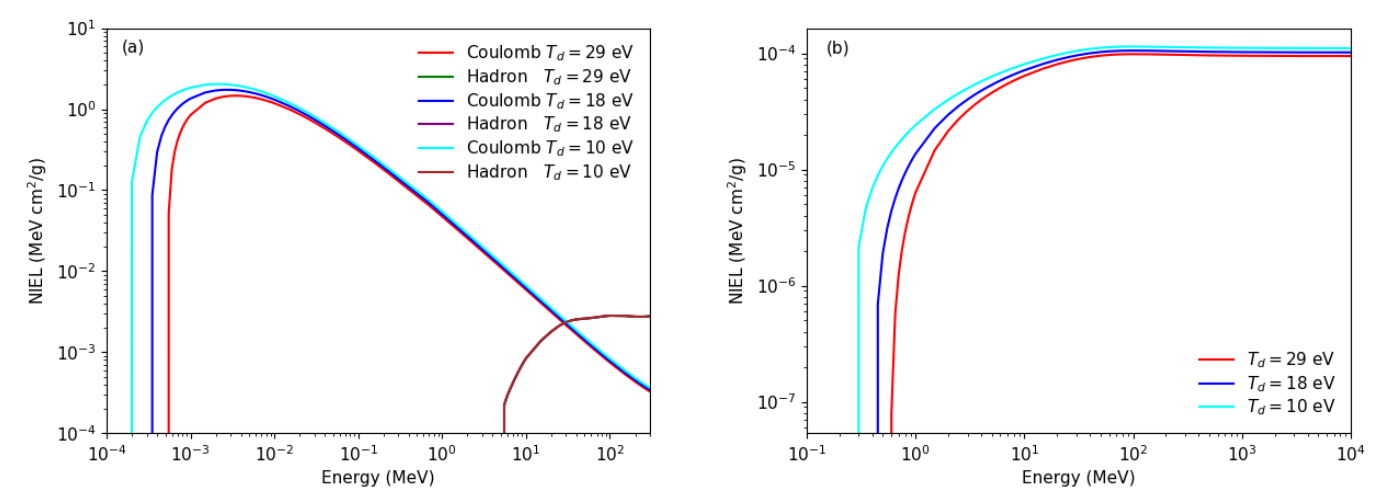

Figure 14.3. a) contributions to the NIEL from both Coulomb interactions and hadron interactions (for protons), and b) from Coulomb interactions (for electrons) for the different $T_{d}$ in Table 14.2.

\subsubsection{Radiation protection in space and astrobiology}

There are at least three important possible synergies between the MC particle transport community and the chemical-physics/condensed matter community using ab-initio methods for ion-irradiation studies in the field of radiation protection in space and astrobiology.

MC particle transport codes (in the condensed history approach) are used extensively in studies about the radiation environment and radiation-induced doses around Earth [92], at a spacecraft during possible interplanetary travel missions [93] and around and at the surface of other bodies such as Mars [24,94] and the Moon [95]. The amount of energy deposited by ionizing radiation in the target material (often a water "phantom", considered as a proxy to biological matter) per unit mass is termed the absorbed dose (or simply "dose"), measured in J/kg, or Grays (Gy), is defined as:

$$
D(G y)=\frac{\Delta E}{m}=\frac{1}{\rho} \int_{E 0}^{\infty} \frac{d \varepsilon}{d x} f(E) d E
$$

where $m$ is the mass of the volume of interest in the target, $d \varepsilon / d x$ is the (unrestricted) collisional stopping power (corresponding to the electronic stopping power, e.g., the energy lost by the particle per unit path length), $f(E)$ is the particle fluence (the flux of particles integrated over the time period of interest), with $E$ being the energy of impacting particles, and $\rho$ is the density in the 
volume of interest. The aim of such studies is to estimate the absorbed dose which is then transformed into effective dose, via radiation quality factors which take into account the fact that the same amount of energy deposited in material by two different types of radiation can bring different effects, and thus different levels of risk. The results can then be compared with limits for the effective dose for stochastic effects induced by low doses prolonged in time, such as exposure from the ubiquitous, constant, low flux of Galactic Cosmic rays - GCRs (it must be noted that short term effects induced by high doses, such as those by transient intense events on the Sun, are also of concern).

On the Martian surface, the radiation environment is constituted, under quiet solar conditions, by primary GCRs particles reaching the top of the thin atmosphere and going down without any interaction (apart from ionization), secondaries that are generated via spallation/fragmentation and ionization processes in the planetary atmosphere, and upward secondaries generated by the interactions of the radiation reaching the ground and going below the surface with the regolith of the planet. In particular, Geant4-based calculations (see Figure 14.5) show that the radiation environment as induced by the impact of the GCRs protons and $\alpha$-particles is mostly constituted by protons $\geq 100 \mathrm{MeV}, e$ and $\gamma$-particles in the range $1 \mathrm{keV}$ up to $100 \mathrm{MeV}$, and neutrons spanning a wide energy range down to thermal energies [96].

For most of the energies of the particles reaching the Martian surface, the range of such particles in water (the main constituent of biological matter) is larger than the few nanometers typical of subcellular structures. Thus, dosimetry estimations, at the basis of risk assessment studies for human Mars surface exploration [96] (and at the basis of studies in the astrobiology field, such as those on potential survival of microorganisms, half-lives of eventual biomolecules [97,98], or those on astrophysical ices such as comets in search for organics [99]) are indeed commonly performed on the basis of dose estimations using the MC condensed history approach and the CSDA. For operational radiation protection (measurements and assessment of doses in the body), quality factors, Qs defined as a continuous function of the LET of the radiation (ICRU 2007 [100]) are commonly used.

However, it is now widely accepted by the scientific community that the radiation quality factors are not only related to the (restricted) Linear Energy Transfer (LET, the energy deposited in the system "locally", equal to the electronic stopping power of the particle minus the energy brought away from the volume by high energy secondary electrons), but also to the pattern of energy deposition on the microscopic and nano-scale, the track structure [101,102,103]. It is recognized that particles (of different charge and different speed) with the same LET may show differences in the final biological effects. This is however neglected in specifying $Q$ as a simple function of LET [104]. Microdosimetry, the study of the pattern of the energy deposition at micrometer length scale [105], provides useful insights on a combination of several stochastic processes, including the restricted LET, track length distribution and the energy-loss straggling of the primary particles [106]. Nevertheless, it is now the field of nanodosimetry which is at the basis for the development of new approaches for radiation quality factors. Nanodosimetry provides information on the details of the spatial pattern of energy deposition at the nanometer scale, particularly relevant for the low energy electrons as mentioned in paragraph 14.1.8. A new model has recently been developed at NASA [107] that allows for incorporation of these track structure features in the formulation of new quality factors, which do not only dependent on the LET but also on the charge and velocity of the particle. This is an important conceptual difference between the quality factors 
used by NASA for projection of risk from space exposures and the quality factors recommended by the ICRP which only depends on the LET, which are more appropriate for operational radiation protection on Earth. Real time TDDFT modelling could provide information on the spatial pattern of ionization events as induced by particles of different charge and speed, for the low speed regime, the energy range where non perturbative effects appear.

A second important possible synergy comes from the need of MC track structure codes to have as input the cross sections, for both elastic and inelastic processes, for the incoming and secondary particles. The quality of this cross sections obviously influences the accuracy of the final results, and currently this is a main issue especially for the hazardous low energy electrons, but improvements for low energy protons are also needed. The cross sections at low energy and datasets internationally recommended to serve as benchmark for the MC track structure results still have a high level of uncertainty. This is valid in first instance for the hazardous low energy electrons, but improvements for low energy protons are also needed. In this context, an opportunity for synergistic studies with the ab-initio molecular/chemical-physics community is offered by the fact that ab-initio approaches can in principle provide high-accuracy cross sections, the main input needed by MC track structure codes. Such calculations are nowadays done in different flavors, where actually ab-initio techniques are mixed with some semi-empirical modelling to different extents [108-110], although recently an approach has been put forward to extract cross sections from TDDFT calculations in a fully ab-initio manner [111].

Nanodosimetry calculations have also been recently performed for the study of irradiation of astrophysical ices [95]. Recent results from the MC track structure code Geant4-DNA [112] have shown that the radius of a cylindrical volume comprising the trajectories of the primary ion and all secondary electrons, which will collectively determine the effects on an icy target, has a certain dependence on the electronic stopping power. The study of radiation impact on icy targets is not only relevant for astrophysical ices, such as comets or icy grains, but can also be relevant for underground ice on Mars. Protons reaching the subsurface of Mars are slowed down by the interaction with the regolith, and can reach even the $\mathrm{keV}$ range, as shown by Geant 4 calculations $[113,114]$. This is relevant as it is likely in the subsurface ice permafrost that we have a chance, if any, to find traces of life, as it has been demonstrated that some microorganisms are able to thrive on environments subject to regular radiation [115] and to survive in a dormant state in icy environments. Thus, improved modelling of the energy loss processes of protons in subsurface ice permafrost is desirable, to answer the question of how much energy can be deposited in such ice layer, what are the effects of the radiation on it at the atomistic scale, and whether we can predict the radiolysis products of the Martian subsurface as these would be potential nutrients of those putative microorganisms. Real time TDDFT calculations can determine very accurately quantities such as the electronic stopping power of protons on an icy sample, shown as a function of the trajectory of the projectile in Figure 14.4. The electronic stopping for $100 \mathrm{keV}$ protons impacting onto ice shows a strong dependence on the impact parameter [116]. This observation is of general importance, for both possibly organic samples trapped in ice and in radiobiology: indeed, since the size of the target volumes considered in nanodosimetry is always smaller than the lateral extension of the penumbra of the primary particle track, the distribution of the clustered ionization events depends critically on the geometrical position of the nanodosimetric target volume with respect to the particle track. The dependence on the impact parameter does indeed affects the hole and excitation populations of the different orbitals [117] (also seen in localized 
representations such as the Wannier function picture), which makes it possible to make predictions regarding the radiolysis mechanism of the different chemical species, considered by some authors as a mechanism providing potential availability of nutrients to possible microorganisms [115].
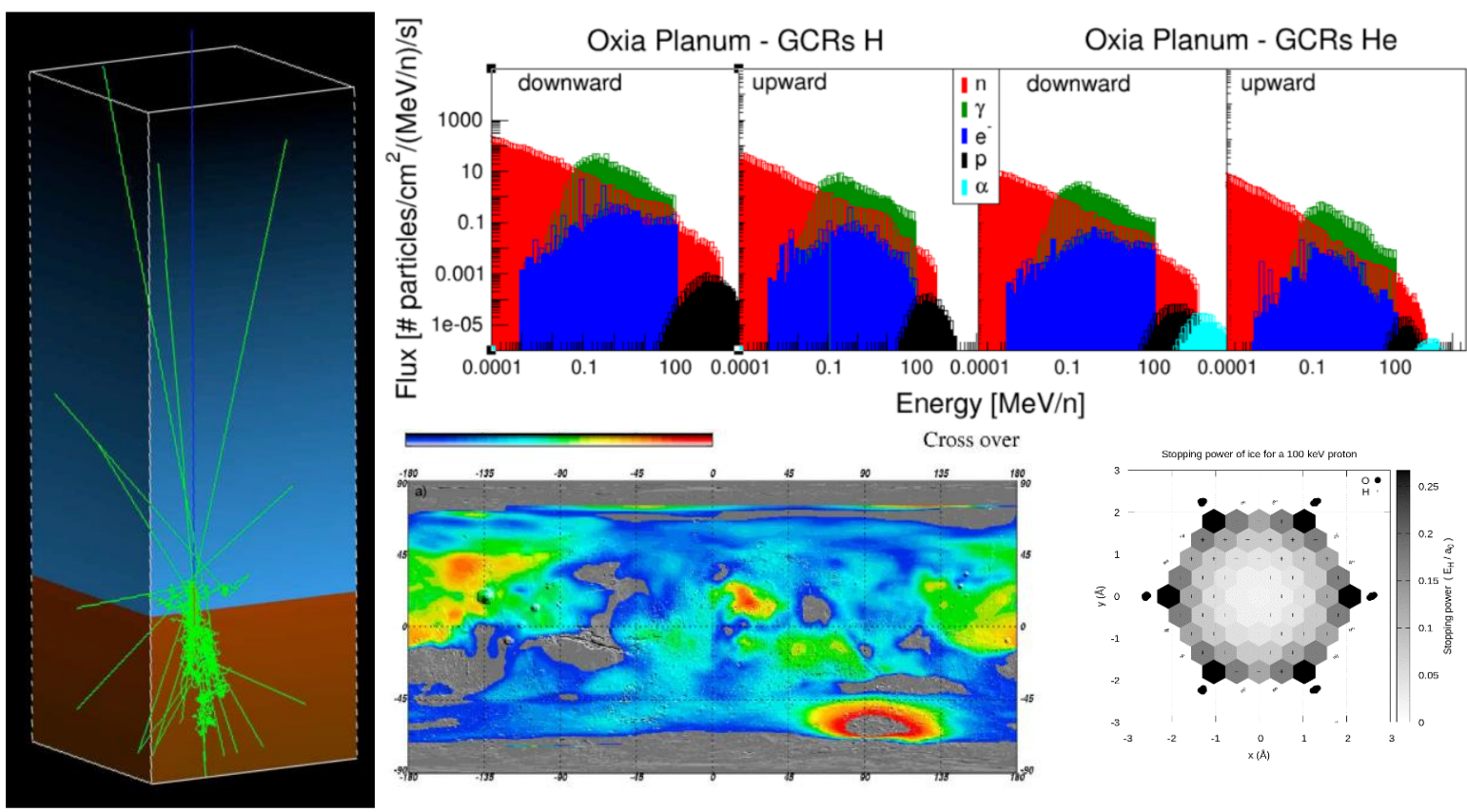

Figure 14.4. Illustration of the geometry used in GEANT4 calculations as a section of 150X150X250 km3. The air shower was initiated by Galactic Cosmic Rays protons and $\alpha$-particles [96]. Tracks from a low statistic simulation are for a pure illustrative scope [97]. A Map of subsurface ice on Mars [119]. Electronic stopping power for a $100 \mathrm{keV}$ proton impacting on ice, as a function of the impact parameter, from TDDFT calculations combined with Ehrenfest dynamics.

\subsubsection{Ion-beam cancer therapy: down to complete stopping}

A last example where ab-initio methods based on TDDFT can be of use is the field of ion-beam therapy, where irradiation with light ions is getting increasingly popular compared to traditional photon-based radiotherapy. Such increased use of light ions beams is due to the better confinement of the achieved dose distributions in the region of the tumour and the increased relative biological effectiveness (RBE), the ratio of biological effectiveness of such ionizing radiation relative to $\mathrm{X}$-rays, given the same amount of absorbed energy.

The optimal use of ion radiotherapy heavily relies on modelling. The treatment planning has to account primarily for the distribution of the deposited dose, for a significantly enhanced biological effectiveness of the impacting protons relative to photons at a sub-mm section at the track ends, and also for an accurate estimation of the penetration depth. For real time TDDFT methods, for disordered systems like water, the main component of biological matter, it is still a challenge to obtain a meaningful electronic stopping with a single or few trajectories. Sampling trajectories on a uniform grid, or stochastically generated, require a relatively large number of trajectories for convergence [120]. However, recently, an ion trajectory pre-sampling method has been developed which greatly improve the computational efficiency [121]. This method selects trajectories on a geometric basis, by comparing the probability distribution function (PDF) of the distance between the projectile and the nearest atoms for the supercell to be simulated via RT-TDDFT, with the converged distribution obtained for a large sample. The trajectories are scored according to the 
overlap between all sampled trajectories accumulatively and converged distributions for all chemical species present in the target, e.g. $\mathrm{H}$ and $\mathrm{O}$ for the case of water.

Figure 14.5 left panel depicts the electronic stopping curves as a function of velocity for protons in liquid water, obtained by real time TDDFT calculations, based on Gaussian basis sets, with only 8 pre-sampled short trajectories at the length of $20 \AA$, and the comparison with available experiments. It can be seen that, with the geometry pre-sampling of ion trajectories, TDDFT calculations can reproduce an accurate electronic stopping $\left(\mathrm{S}_{\mathrm{e}}\right)$ curve around the Bragg peak for protons in liquid water, with a relatively small demand of computational resources.

An important quantity in radiation therapy is the penetration depth. Protons beams (and in general radiation beams) have a finite penetration depth in a given material. They exhibit a relatively low ionisation density at the surface; their ionisation density increases near the end of the beam range, where there is a narrow region of high ionisation density, i.e. the Bragg peak. The radiation dose from a proton beam falls off sharply both laterally and distally [122], which leads to a limited damage induced in healthy tissues surrounding the tumor. For clinical applications, several beams can be combined to achieve the appropriate dose in the volume of interest, via the superposition of Bragg peaks of different intensities and energies ("spread-out Bragg peak", SOBP). Particles undergo a rapid, non-linear change in their energy deposition characteristics for the end of the range and the increase of LET combined with dose falloff can cause range uncertainties of 1-2 mm [123,124]. Therefore, improved calculations for describing the energy dependence of the RBE down to the stopping limit [125], and removal of the uncertainties regarding the range are critically needed.

In Figure 14.5 right panel we report how the electronic stopping curves around the Bragg peak derived from TDDFT and SRIM affect the penetration range (R). The red line shows the differences of $R(\Delta R)$ for protons in liquid water based on the $S_{e}$ curves of TDDFT and SRIM (red dashed and gray solid lines). The contribution from the nuclear stopping $\left(\mathrm{S}_{\mathrm{n}}\right)$ of the SRIM tables is also shown as green line. It can be seen that, on the left side of the Bragg peak and below $0.1 \mathrm{MeV}, \Delta \mathrm{R}$ induced by the difference in Se between TDDFT and SRIM tables is up to $80 \mathrm{~nm}$, as a relative overestimation in Se by TDDFT. For higher energy, the value of $\Delta R\left(\mathrm{~S}_{\mathrm{e}}\right)$ decreases to 0 and then goes up in the opposite direction to $-11 \mathrm{um}$ at about $2.5 \mathrm{MeV}$. The negative sign means that the penetration depth given by SRIM table is bigger than that of TDDFT. For proton beams used in therapy, the typical energy is between $50 \mathrm{MeV}$ and $250 \mathrm{MeV}$ with a range over $10 \mathrm{~mm}$ [126]. The $11 \mu \mathrm{m}$ difference due to the difference in $\mathrm{S}_{\mathrm{e}}$ between TDDFT and SRIM around the Bragg peak is negligible. In comparison, the contribution from nuclear stopping $\left(\Delta R\left(S_{n}\right)\right)$ is very tiny around the Bragg peak. It will be increased continuously to about $0.16 \mathrm{~mm}$ at $250 \mathrm{MeV}$ (not shown in the figure). 

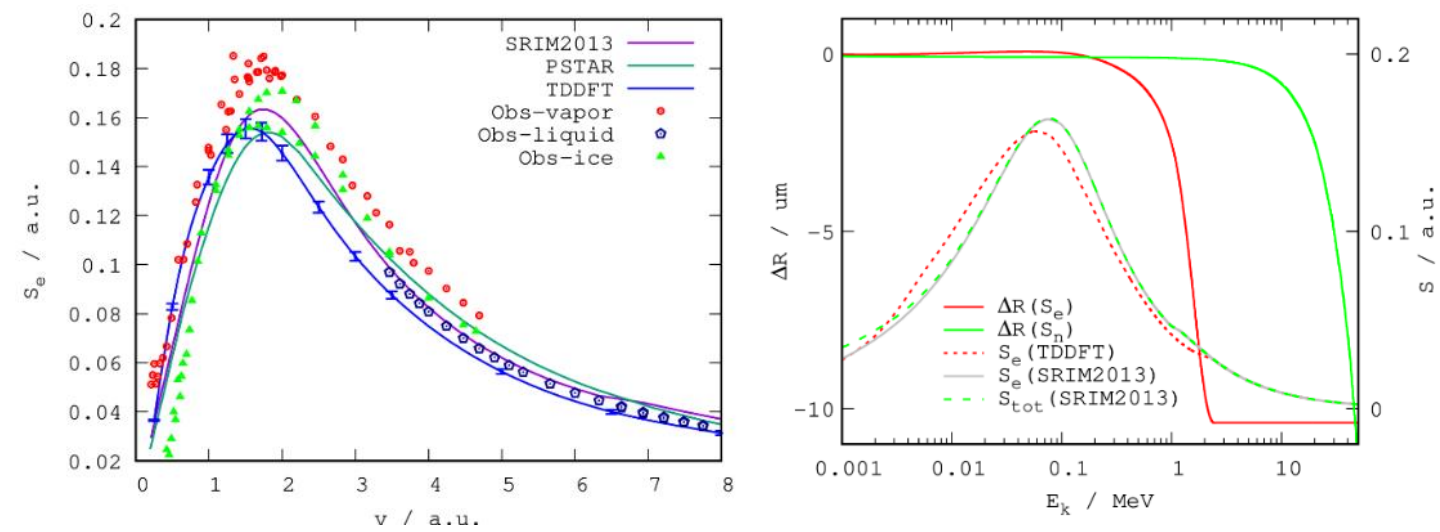

Figure 14.5. Right panel: The purple and dark-green lines show the empirical tables of SRIM2013 and PSTAR, individually. TDDFT calculations,based on Gaussian basis sets, and with 8 pre-sampled short trajectories at the length of $20 \AA$, are given as blue line with error bars [121]. Available observations of the electronic stopping power for proton in water and ice are also reported, [https://www-nds.iaea.org/stopping/stopping_hydr.html, and references therein]; Left panel: penetration range difference $\Delta \mathrm{R}$ for proton in liquid water based on the electronic stopping curves of TDDFT and SRIM.

\section{Acknowledgments}

This work has received funding from the Research Executive Agency under the EU's Horizon2020 Research and Innovation program ESC2RAD (grant ID 776410). This work benefited from networking activities carried out within the EU funded COST Action CA17126 (TUMIEE) and represents a contribution to it.

\section{References}

[1] M. H. Kalos and P. A. Whitlock. Monte Carlo Methods, volume I, Basics. John Wiley \& Sons, New York, (1986)

[2] I. Lux and L. Koblinger, Monte Carlo Particle Transport Methods: Neutron and Photon Calculations (CRC Press, 1990)

[3] A. Bielajew, Fundamentals of the Monte Carlo method for neutral and charged particle transport, http://www-personal.engin.umich.edu/ bielajew/MCBook/book.pdf

[4] F. Cleri, Monte-Carlo methods for the study of the diffusion of charged particles through matter, in Proceedings of the IX international School on Nuclear Physics, Neutron Physics And Nuclear Energy, edited by W. Andrejtscheff, D. Elenkov (1990) 401-439

[5] F. Agostini B. F. E. Curchod, R. Vuilleumier, I. Tavernelli and E. K. U. Gross, "TDDFT and QuantumClassical Dynamics: A Universal Tool Describing the Dynamics of Matter", in Handbook of Materials Modeling : Methods: Theory and Modeling, pp 1-47 (2018) Springer

[6] R. Iftimie, P. Minary and M. E. Tuckerman, Ab initio molecular dynamics: Concepts, recent developments, and future trends, Proc. Nat. Acad. Sci. 102 (19) (2005), 6654-6659

[7] J. Cugnon, Cascade Models and Particle Production: A Comparison. In: Gutbrod H.H., Rafelski J. (eds) Particle Production in Highly Excited Matter. NATO ASI Series (Series B: Physics), vol 303. Springer, Boston, MA (1993) https://doi.org/10.1007/978-1-4615-2940-8_12

[8] D.H. Wright and M.H. Kelsey, The Geant4 Bertini Cascade, Nucl. Instrum. Methods Phys. Res., Sect. A 804. Supplement C (2015) 175 -188 
[9] G. Folger, V. Ivanchenko and J.P. Wellisch, The Binary Cascade, The European Physical Journal A Hadrons and Nuclei 21.3 (2004) 407-417

[10] P. Henrotte. Extension du modèle de cascade intranucléaire pour les réactions de spallation". PhD thesis (2005) Université de Liège

[11] M. Durante and F.A. Cucinotta, Physical basis of radiation protection in space travel, Rev. Mod. Phys. 83 (2011) 1245-1281

[12] J. Benlliure. Spallation Reactions in Applied and Fundamental Research. In: The Euroschool Lectures on Physics with Exotic Beams, Vol. II. Ed. by Jim Al-Khalili and Ernst Roeckl. Berlin, Heidelberg: Springer Berlin Heidelberg (2006) pp 191-238

[13] M. Guthoff, W. de Boer and S. Müller, Simulation of beam induced lattice defects of diamond detectors using FLUKA, Nucl Instr Meth A 735 (2013) 223

[14] J. Miller. Shielding Strategies for Human Space Exploration, chapter Database development and laboratory validation. NASA CP-3360, Langley Research Center, Maryland, USA (1997)

[15] G. Santin. NSREC Short Course notes (2006)

[16] A. Fassò, A. Ferrari and P.R. Sala, Radiation transport calculations and simulations, Radiat Prot Dosimetry 137 (2009) 118-33

[17] V. Boffi and V. Molinari, Solution to the monoenergetic neutron Boltzmann equation for a finite parallelepiped, Transp. Theory Stat. Phys. 1 (1971) 313

[18] V. Kumar and D.C. Sahni, Integral transform method for solving multi-dimensional neutron transport problems with linearly anisotropic scattering, Ann. Nucl. Energy 8 (1981) 173

[19] M.G. Nadkarni, "Ergodic Theorems of Birkhoff and von Neumann" in Basic Ergodic Theory, Texts and Readings in Mathematics 6 (2013). Hindustan Book Agency, Gurgaon. https://doi.org/10.1007/978-9386279-53-8_2

[20] P. Sigmund, Particle Penetration and Radiation Effects. Springer Series in Solid State Sciences, 151 (2006) Berlin Heidelberg: Springer-Verlag. ISBN 3-540-31713-9

[21] R.E. Johnson, Energetic Charged-Particle Interactions with Atmospheres and Surfaces, Physics and Chemistry in Space No. 19 (1990) Berlin: Springer-Verlag

[22] A, Pedro, and A. Brahme. "Restricted Energy-Loss Straggling and Multiple Scattering of Electrons in Mixed Monte Carlo Procedures." Radiation Research 100 (1) (1984) 16-29. JSTOR, www.jstor.org/stable/3576517

[23] E. Bagli, M. Asai, .D Brandt, A. Dotti, V. Guidi and D.H. Wright, A model for the interaction of high-energy particles in straight and bent crystals implemented in Geant4, The European Physical Journal C 74 (8) (2014) 1-11

[24] J Guo, TC Slaba, C Zeitlin, RF Wimmer-Schweingruber, FF Badavi, Dependence of the Martian radiation environment on atmospheric depth: Modeling and measurement, Journal of Geophysical Research: Planets 122 (2017), 329-341

[25] D. Matthiä, B. Ehresmann, H. Lohf, J. Köhler, C. Zeitlin, et al, The Martian surface radiation environment - a comparison of models and MSL/RAD measurements. J. Sp. Weather Sp. Clim. 6 (2016) A13

[26] J. Allison, K. Amako; J. Apostolakis; H. Araujo; P. Arce Dubois et al., Geant4 Developments and Applications, IEEE Trans. Nucl. Sci. 53 (2006) 270-278

[27] J. E. Sweezy, T. Booth, F. Brown et al., Mcnp-a general Monte Carlo n-particle transport code, version 5, MCNP user manual, Report LA-UR-03-1987, Los Alamos, CA: Los Alamos National Laboratory, (2003) 
[28] G. Battistoni, The FLUKA code, galactic cosmic ray and solar energetic particle events: From fundamental physics to space radiation and commercial aircraft doses, IEEE Nucl. Sci. Symp. Conf. Rec., IEEE (2008) 1609-1615

[29] L. W. Townsend, T. M. Miller and T. A. Gabriel, Hetc radiation transport code development for cosmic ray shielding applications in space, Radiation Protection Dosimetry 116 (2005) 135-139

[30] A. V. Dementyev and N. M. Sobolevsky, Shield - universal Monte Carlo hadron transport code: scope and applications, Radiation Measurements 30 (1999) 553-557

[31] T. Sato, K. Niita, N. Matsuda, S. Hashimoto, Y. Iwamoto et al., Particle and heavy ion transport code system, PHITS, version 2.52, J. Nucl. Sci. Technol. 50 (2013) 913-923

[32] F. Lei, P.R. Truscott, C.S. Dyer, B. Quaghebeur, D. Heynderickx, P. Nieminen, H. Evans, and E. Daly, MULASSIS: A Geant4-Based Multilayered Shielding Simulation Tool. IEEE Trans. Nucl. Sci., 49 (2002) 2788-2793

[33] G. Santin, V. Ivanchenko, H. Evans, P. Nieminen and E. Daly, GRAS: a general-purpose 3-D Modular Simulation tool for space environment effects analysis. IEEE Trans. Nucl. Sci. 52 (2005) 2294 - 2299

[34] D. Heynderickx, B. Quaghebeur, E. Speelman and E. Daly, ESA's Space Environment Information System (SPENVIS) - A WWW interface to models of the space environment and its effects. In 38th Aerospace Sciences Meeting and Exhibit. AIAA (2000)

[35] F.A. Cucinotta,, M. Alp, B. Rowedder and M.-H. Y. Kim, Safe days in space with acceptable uncertainty from space radiation exposure, Life Sciences and Space Research 5 (2015) 31-38

[36] P. Jiggens, M. -A. Chavy-Macdonald, G. Santin, A. Menicucci, H. Evans and A. Hilgers, The magnitude and effects of extreme solar particle events, J. Space Weather Space Clim. 4 (2014) A20

[37] H. Nikjoo, D. Emfietzoglou, T. Liamsuwan, R. Taleei, D. Liljequist and S. Uehara, Radiation track, DNA damage and response: a review, Reports on Progress in Physics 79 (2016) 1166601

[38] E. Alizadeh, T. M. Orlando and L. Sanche, Biomolecular damage induced by ionizing radiation: The direct and indirect effects of low-energy electrons on DNA, Annual Review of Physical Chemistry 66, (2015) 379-398

[39] S. Denifl, T. D. Märk and P. Scheier, "The role of secondary electrons in radiationdamage," in Radiation Damage in Biomolecular Systems, edited by G. García Gómez-Tejedor and M. C. Fuss (2012) pp 45-58, Springer Netherlands, Dordrecht

[40] H. Nikjoo and L. Lindborg, RBE of low energy electrons and photons, Phys. Med. Biol. 55 (2010) R65

[41] I. El Naqa, P. Pater and J. Seuntjens, Monte Carlo role in radiobiological modelling of radiotherapy outcomes, Phys. Med. Biol. 57 (2012) R75-R97

[42] H. Rabus, Nanodosimetry - on the "tracks" of biological radiation effectiveness, Z. Med. Phys. 30 (2020) 91-94

[43] M. Dingfelder, R.H. Ritchie, J.E. Turner, W. Friedland, H.G. Paretzke et al., Comparisons of calculations with PARTRAC and NOREC: transport of electrons in liquid water, Radiation research 169, (2008) 584594

[44] T. Liamsuwan, D. Emfietzoglou, S. Uehara and H. Nikjoo, Microdosimetry of low-energy electrons, Int. J. Rad. Biol. 88 (2012) 899-907

[45] I. Plante and F. A. Cucinotta, Cross sections for the interactions of $1 \mathrm{eV}--100 \mathrm{MeV}$ electrons in liquid water and application to Monte-Carlo simulation of HZE radiation tracks, New Journal of Physics 11, (2009) 063047 
[46] S. Incerti, I. Kyriakou, M.A. Bernal, M.C. Bordage, Z. Francis, et al., Geant4-DNA example applications for track structure simulations in liquid water: A report from the Geant4-DNA Project, Medical physics 45 (2018) e722--e739

[47] V.A. Semenenko, J.E. Turner and T.B. Borak, TB, NOREC, a Monte Carlo code for simulating electron tracks in liquid water, Radiation and environmental biophysics 42 (2003) 213-2017

[48] C. Villagrasa, M. -C. Bordage, M. Bueno, M. Bug, S. Chiriotti, E. Gargioni, B. Heide, H. Nettelbeck, A. Parisi and H. Rabus, Assessing the contribution of cross-sections to the uncertainty of Monte Carlo calculations in micro- and nanodosimetry, Radiation Protection Dosimetry 183 (2018) 11-16

[49] N. Lampe, M. Karamitros, V. Breton, J. M. C. Brown, I. Kyriakou, D. Sakata, D. Sarramia and S. Incerti, Mechanistic DNA damage simulations in Geant4-DNA part 1: A parameter study in a simplified geometry, Phys. Med. 48 (2018) 135-45

[50] R. Garcia-Molina, I. Abril, I. Kyriakou and D. Emfietzoglou, Inelastic scattering and energy loss of swift electron beams in biologically relevant materials, Surf. Interf. Anal. 49, (2017) 11-17

[51] S. A. Zein, M.-C. Bordage, Z. Francis, G. Macetti, A. Genoni, C.Dal Cappello, dW.-G. Shina and S. Incerti, Electron transport in DNA bases: An extension of the Geant4-DNA Monte Carlo toolkit, Nucl. Instrum. Meth. B 488 (2021) 70-82

[52] M. Scharff, P. V. Thomsen J. Lindhard and V. Nielsen, Integral equations governing radiation effects, Mat. Fys. Medd. Dan. Vid. Selsk., 33 (10) (1963)

[53] C. Inguimbert, P. Arnolda, T. Nuns and G. Rolland, "Effective NIEL" in Silicon: Calculation Using Molecular Dynamics Simulation Results, IEEE Trans. Nucl. Sci. 57 (4) (2010) 1915 - 1923

[54] M.J. Norgett, M.T. Robinson and I.M. Torrens. A proposed method of calculating displacement dose rates, Nuclear Engineering and Design, 33(1) (1975) 50-54

[55] C. Poivey, ESA-CERN-SCC Workshop CERN, May 9-10, 2017 https://indico.cern.ch/event/635099/contributions/2570676/attachments/1456363/2249942/4_Ra diation_Effects_and_RHA_ESA_Internal_Course_4_10_May_2017_DD_CP.pdf

[56] http://www.sr-niel.org/

[57] NIST: Introduction of ESTAR, PSTAR, and ASTAR. See http://physics.nist.gov/PhysRefData/Star/Text/intro.html

[58] J.F. Ziegler, M.D. Ziegler and J.P. Biersack, SRIM - The stopping and range of ions in matter, Nucl. Instrum. Meth. B 268 (2010) 1818-1823

[59] K. Wittmaack, Misconceptions impairing the validity of the stopping power tables in the SRIM library and suggestions for doing better in the future. Nucl. Instrum. Meth. B 380 (2016) 57-70

[60] J. F. Ziegler, J. P. Biersack and U. Littmark, The Stopping and Range of Ions in Solids, Volume 1 of The Stopping and Ranges of ions in Matter, edited by J.F. Ziegler, Pergamon Press, 1985;

[61] S.R. Messenger, E. A. Burke, M. A. Xapsos, G. P. Summers, R. J. Walters, I. Jun and T. Jordan, NIEL for Heavy Ions: An Analytical Approach, IEEE Trans. Nucl. Sci. 50 (2003) 1919 - 1923

[62] S.R. Messenger, G. P. Summers, E. A. Burke, R. J. Walters, and M. A. Xapsos, Modeling solar cell degradation in space: A comparison of the NRL displacement damage dose and the JPL equivalent fluence approaches, Prog. Photovolt.: Res. Appl. 9 (2001) 103

[63] S.R. Messenger, E.A. Burke, G.P. Summers, M.A. Xapsos, R.J. Walters, E.M. Jackson and B.D. Weaver, Nonionizing energy loss (NIEL) for heavy ions, IEEE Trans. Nucl. Sci. 46 (1999) 1595-1602 
[64] S.R. Messenger, E.A. Burke, G.P. Summers and R.J. Walters, Application of displacement damage dose analysis to low-energy protons on silicon devices, IEEE Trans. Nucl. Sci. 49 (2002) 2690-2694

[65] R.A. Weller, M.H. Mendenhall and D.M. Fleetwood, A screened Coulomb scattering module for displacement damage computations in Geant4, IEEE Trans. Nucl. Sci. 51 (2004) 3669 - 3678

[66] M. Huhtinen, Simulation of non-ionising energy loss and defect formation in silicon, Nucl. Instrum. Meth. A 491 (2003) 194

[67] F. Gao, E. Hernandez-Rivera, D. Huang and P. D. LeVan, Displacement damage and predicted nonionizing energy loss in GaAs , J. Appl. Phys. 121 (2017) 095104

[68] G. Herrero-Saboya, L. Martin-Samos, A. Jay, A. Hémeryck and N Richard, A comprehensive theoretical picture of E centers in silicon: From optical properties to vacancy-mediated dopant diffusion, J. Appl. Phys. 127 (2020) 085703

[69] P. Sigmund and A. Schinner, Electronic stopping in oxides beyond Bragg additivity, Nucl. Instrum. Meth. B 415 (2018) 110-116

[70] M. Caro, A. A. Correa, E. Artacho and A. Caro, Stopping power beyond the adiabatic approximation, Sci. Rep. 7 (2017) 2618

[71] R. Ullah, E. Artacho and A.A. Correa, Core electrons in the electronic stopping of heavy ions, Phys. Rev. Lett. 121 (2018) 116401

[72] N. Koval, F. Da Pieve and E. Artacho, Ab initio electronic stopping power for protons in Ga0.5In0.5P/GaAs/Ge triple-junction solar cells for space applications, Royal Open Science Society 7 (2020) 200925

[73] W.J. Weber and Y. Zhang, Predicting damage production in monoatomic and multi-elemental targets using stopping and range of ions in matter code: Challenges and recommendations, Curr. Opin. Solid State Mater. Sci. 23 (4) (2019) 100757

[74] M. Raine, A. Jay, N. Richard, V. Goiffon, S. Girard, M. Gaillardin and P. Paillet, Simulation of single particle displacement damage in silicon-Part I: Global approach and primary interaction simulation, IEEE Trans. Nucl. Sci. 64 (2017) 133-40

[75] M. Caro, A. Tamm, A.A. Correa and A. Caro, Role of electrons in collision cascades in solids. I. Dissipative model, Phys. Rev. B 99 (2019) 174301

[76] A. Tamm, M. Caro, A. Caro, and A.A. Correa, Role of electrons in collision cascades in solids. II. Molecular dynamics, Phys. Rev. B 99 (2019) 174302

[77] C.W. Lee, J.A. Stewart, S.M. Foiles, R. Dingreville and A. Schleife, Multi-scale simulations of electron and ion dynamics in self irradiated silicon, Phys. Rev. B 102 (2020) 024107

[78] T. Jarrin, A. Jay, A. Hémeryck and N. Richard, Parametric study of the Two-Temperature Model for Molecular Dynamics simulations of collisions cascades in Si and Ge, Nucl. Instrum. Meth. B 485 (2020) $1-9$

[79] M. Toulemonde, W.J. Weber, G. Li, V. Shutthanandan, P. Kluth, T. Yang, Y. Wang and Y. Zhang, Synergy of nuclear and electronic energy losses in ion-irradiation processes: the case of vitreous silicon dioxide, Phys. Rev. B 83 (2011) 054106

[80] W. J. Weber, D.M. Duffy, L. Thomé and Y. Zhang, The role of electronic energy loss in ion beam modification of materials, Curr. Opin. Solid State Mater. Sci. 19 (2015) 1-11 
[81] E. Zarkadoula, D.M. Duffy, K. Nordlund, M.A. Seaton, I.T. Todorov, W.J. Weber and K. Trachenko, Electronic effects in high-energy radiation damage in tungsten, J. Phys. Condens. Matter 27 (2015) 135401

[82] M. Backman, F. Djurabekova, O.H. Pakarinen, K. Nordlund, Y. Zhang, M. Toulemonde and W.J. Weber, Cooperative effect of electronic and nuclear stopping on ion irradiation damage in silica, J. Phys. D: Appl. Phys. 45 (2012) 50525

[83] T. Jarrin, J. Teunissen, F. Da Pieve, N. Richard, A. Hémeryck, unpublished

[84] F. Lei, R.R. Truscott, C.S. Dyer, B. Quaghebeur, D. Heynderickx, P. Nieminen, H. Evans and E. Daly, MULASSIS: a Geant4-based multilayered shielding simulation tool, IEEE Trans. Nucl. Sci. 49 (2002) 2788-2793.

[85] G.P. Summers, S.R. Messenger, E.A. Burke, M.A. Xapsos and R.J. Walters, Low energy proton-induced displacement damage in shielded GaAs solar cells in space. Appl. Phys. Lett. 71 (1997) 832-834

[86] G.P. Summers, S.R. Messenger, E.A. Burke, M.A. Xapsos and R.J. Walters, Contribution of low-energy protons to the degradation of shielded GaAs solar cells in space, Prog. Photovolt. Res. Appl. 5 (6) (1997) $407-413$

[87] G. Summers, E. Burke, M. Xapsos, C. Dale, P. Marshall and E. Petersen, Displacement damage in GaAs structures, IEEE Trans. Nucl. Sci. 35 (1998) 1221-1226

[88] C. Borschel and C. Ronning, Ion beam irradiation of nano-structures-A 3D Monte Carlo simulation code, Nucl. Instrum. Meth. B 269 (2011) 2133-2138

[89] C.J. Ortiz, A. Souidi, C.S. Becquart, C. Domain and M. Hou, Recent radiation damage studies and developments of the Marlowe code, Radiation Effects and Defects in Solids, 169 (2014) 592-602

[90] M. Jiang, H. Xiao, S. Peng, G. Yang, Z. Liu, L. Qiao and X. Zu, A Theoretical Simulation of the Radiation Responses of Si, Ge, and Si/Ge Superlattice to Low-Energy Irradiation, Nanoscale Res. Lett. 13 (2018) 133

[91] E. Holmström, K. Nordlund and A. Kuronen, Threshold defect production in germanium determined by density functional theory molecular dynamics simulations, Phys. Scr. 81 (2010) 035601

[92] T. Ersmark, P. Carlson, E. Daly, C. Fuglesang, I. Gudowska, B. Lund-Jensen, P. Nieminen, M. Pearce and G. Santin, Geant4 Monte Carlo Simulations of the Galactic Cosmic Ray Radiation Environment On-Board the International Space Station/Columbus, IEEE Trans. Nucl. Sci. 54 (2007) 1854-1862

[93] C.J. Mertens and T.C. Slaba, Characterization of solar energetic particle radiation dose to astronaut crew on deep-space exploration missions, Space Weather 14 (2019) 1650-1658

[94] D. Matthiä, D.M. Hassler, W. de Wet, B. Ehresmann, A. Firan, et al., The radiation environment on the surface of Mars-Summary of model calculations and comparison to RAD data, Life Sciences in Space Research 14 (2017) 18-28

[95] G. Reitz, T. Berger and D. Matthiä, Radiation exposure in the Moon environment, Planetary and Space Science 74 (2012) 78-83

[96] F. Da Pieve, G. Gronoff, J. Guo, C.J. Mertens, L Neary, B. Gu, N.E. Koval, J. Kohanoff, A.C. Vandaele and F. Cleri, Radiation environment and doses on Mars at Oxia Planum and Mawrth Vallis: support for exploration at sites with high biosignature preservation potential, J. Geophys Res.: Planets 126 (2021) e2020JE006488

[97] L. R. Dartnell, L. Desorgher, J.M. Ward, and A. J. Coates, Martian sub-surface ionising radiation: biosignatures and geology, Biogeosciences, 4 (2007) 545-558 
[98] A. A. Pavlov, G. Vasilyev, V. M. Ostryakov, A. K. Pavlov and P. Mahaffy, Degradation of the organic molecules in the shallow subsurface of Mars due to irradiation by cosmic rays, Geophys. Res. Lett. 39, (2012) L13202

[99] G. Gronoff, R. Maggiolo, G. Cessateur, W. B. Moore, V. Airapetian, J. De Keyser, F. Dhooghe, A. Gibbons, H. Gunell and C. J. Mertens, The Effect of Cosmic Rays on Cometary Nuclei. I. Dose Deposition, The Astrophysical Journal 890 (2020)

[100] ICRP (103) (2007). The 2007 Recommendations of the International Commission on Radiological Protection. Annals of the ICRP, Publication 103. The International Commission on Radiological Protection

[101] H. Nikjoo, S. Uehara, W. E. Wilson, M. Hosh and D. T. Goodhead, Track structure in radiation biology: theory and applications, Int. J. Rad. Biol., 73 (1998) 355-364

[102] H Rabus. Nanodosimetry - on the "tracks" of biological radiation effectiveness. Z Med Phys., 30 (2020) 91-94

[103] T. Friedrich, K. Ilicic, C. Greubel, S. Girst, J. Reindl, M. Sammer, B. Schwarz, C. Siebenwirth, D. W. M. Walsh, T. E. Schmid, M. Scholz and G. Dollinger, DNA damage interactions on both nanometer and micrometer scale determine overall cellular damage, Sci. Rep. 8 (2018) 16063

[104] D.T. Goodhead. Track structure and the quality factor for space radiation cancer risk (reid). Technical report (2018) https://three.jsc.nasa.gov/articles/Track_QF_Goodhead.pdf

[105] H.H. Rossi and M. Zaider. "Microdosimetry and its applications" London, UK (1996) Springer

[106] H.G. Menzel, The complexity of quantities in radiation dosimetry: the issue of radiation quality, Radiat. Prot. Dosimetry 183 (2019) 3-10

[107] F.A. Cucinotta, Review of NASA approach to space radiation risk assessments for mars exploration, Health Physics, 108 (2015) 131-42

[108] M. U. Bug, W. Yong Baek, H. Rabus, C. Villagrasa, S. Meylan and A. B. Rosenfeld, An electron-impact cross section data set $(10 \mathrm{eV}-1 \mathrm{k} \mathrm{eV})$ of DNA constituents based on consistent experimental data: A requisite for Monte Carlo simulations, Rad. Phys. Chem. 130 (2017) 459-479

[109] J.R. Madsen and G. Akabani, Low-energy cross-section calculations of single molecules by electron impact: a classical Monte Carlo transport approach with quantum mechanical description, Phys. Med. Biol. 59 (2014) 2285-305

[110] S. Taioli, P. E. Trevisanutto, P. de Vera, S. Simonucci, I. Abril, R. Garcia-Molina and M. Dapor, Relative Role of Physical Mechanisms on Complex Biodamage Induced by Carbon Irradiation, Phys. Chem. Lett. 12 (2021) 487-493

[111] N. Koval, P. Koval, F. Da Pieve, J. Kohanoff, E. Artacho, D. Emfietzoglou, unpublished

[112] C. N. Shingledecker, S. Incerti, A. Ivlev, D. Emfietzoglou, I. Kyriakou, A. Vasyunin, and P. Caselli, CosmicRay Tracks in Astrophysical Ices: Modeling with the Geant4-DNA Monte Carlo Toolkit, The Astrophysical Journal $904(2020) 189$

[113] L. Röstel, J. Guo, S. Banjac, R.F. Wimmer-Schweingruber and B. Heber, Subsurface Radiation Environment of Mars and Its Implication for Shielding Protection of Future Habitats, J. Geophys Res.: Planets 125 (2020), e2019JE006246

[114] P. M. Silva de Magalhães, Radiation environment and its effects on the Martian surface and underground, Master Thesis (2016) University of Lisbon Técnico Lisboa 
[115] D. Atri, On the possibility of galactic cosmic ray-induced radiolysis-powered life in subsurface environments in the Universe, Journal Royal Society Interface 13 (2016) 20160459

[116] D. Muñoz-Santiburcio and E. Artacho, unpublished

[117] K. G. Reeves and Y. Kanai, Electronic Excitation Dynamics in Liquid Water under Proton Irradiation, Sci Rep. 7: 40379 (2017)

[119] W. C. Feldman, A. Pathare, S. Maurice, T. H. Prettyman, D. J. Lawrence, R. E. Milliken and B. J. Travis, Mars Odyssey neutron data: 2. Search for buried excess water ice deposits at nonpolar latitudes on Mars, J. Geophys Res.: Planets 116 (2011) E11009

[120] A. A. Correa, Calculating electronic stopping power in materials from first principles. Computational Materials Science 150 (2018) 291

[121] B. Gu, B. Cunningham D. Muñoz-Santiburcio, F. Da Pieve, E. Artacho and J. Kohanoff, Efficient calculation of electronic stopping in disordered systems: application to liquid water, J. Chem. Phys. 153 (2020) 034113

[122] J. Chen, Microdosimetric characteristics of proton beams from $50 \mathrm{keV}$ to $200 \mathrm{MeV}$, Radiation Protection Dosimetry 143 (2010) 436-439

[123] L. Paganetti, M. Gerweck and H. Goitein, The general relation between tissue response to $\mathrm{x}$-radiation $(\alpha / \beta$ values) and the relative biological effectiveness (RBE) of protons: prediction by the Katz trackstructure model, Int. J. Rad. Biol. 76 (2000) 985-998

[124] J.A. Lomax, Myths and realities of range uncertainty, British J Radiol. 93 (2011) 582

[125] W. Friedland, E. Schmitt, P. Kundrát, M. Dingfelder, G. Baiocco, S. Barbieri and A. Ottolenghi, Comprehensive track-structure based evaluation of DNA damage by light ions from radiotherapyrelevant energies down to stopping, Sci. Rep. 7 (2017) 45161

[126] W. D. Newhauser and R. Zhang, The physics of proton therapy, Phys. Med. Biol., 60 (2015) R155 


\title{
15. Combining Monte Carlo methods with other techniques
}

\author{
Nikita Medvedev* and Vladimir Lipp
}

Here we describe a methodology of combining Monte Carlo method of particles transport in matter with other simulation techniques. The general ideology of so-called hybrid models is presented, placing the multiscale models in the proper context. Then, peculiarities of the Monte Carlo models are discussed with respect to the task of combining them with other methods. We address the models without and with feedback, emphasising their limits of applicability, and practical implementation. A few examples of such approaches are then given, demonstrating the power of combined approaches.

*Contact: nikita.medvedev@fzu.cz 


\subsection{General outlook}

The main idea of a so-called hybrid model rises from the traditional theoretical methodology of identifying parameters, with respect to which certain approximations can be made. Traditionally, identifying those parameters allows to simplify a theory; for example, a semiclassical free-electron approximation can be used when kinetic energy of an electron is much greater than its potential energy of interaction with an external system, whereas a tight binding (TB) approximation can be used in the opposite case.

The problem is, in practice we are usually dealing with dynamical processes of excitation of an electronic system in a solid, for which a part of the system a part of the time can be approximated as free electrons, whereas another part is rather in the regime of TB. A rigorous way to deal with such a problem would be deriving a nonperturbative theory applicable across multiple regimes of excitation. Unfortunately, such theories, where exist, are often unsolvable with present day computers. Alternative approaches are thus required.

\subsubsection{Ideology behind hybrid methods}

Hybrid models tackle this problem differently. Within the hybrid philosophy, let us notice that we can divide the whole system into (artificial) subsystems, each of which can be described with its own efficient model: in the above-mentioned example, a part of electrons can be described as free, whereas another part as tightly bound. There is, of course, some intermediate region where electrons do not strictly belong to either fraction, but if this region is not highly populated, or if it is passed through very quickly, it allows to neglect influence of these few transiently living electrons transitioning from the "free" to the "tightly bound" regime. Thus, a proper hybrid model would reliably describe the two subsystems with their own appropriate methods, and add some interconnection between them.

This example demonstrates the general idea of a hybrid model: divide and conquer. Firstly, one would identify the parameters space, and find which parameter allows for a division of the entire system into subsystems that can be described efficiently with already existing (or easily developed) models. Then, a proper and efficient interconnection between the models should be identified. Below we will discuss a few standard parameters, along which the division can be made while setting a hybrid model.

If we have a situation in which different regions of the parameter space are weakly coupled to each other, they can be described independently with individual appropriate methods (for illustration, see Figure 15.1). In this case, the work mainly reduces to a proper description of the coupling between the different methods.

For example, when a problem may be separated in time (see Figure $15.1 \mathrm{a}$ ), one may describe ultrafast effects (where equilibrium is not reached) with nonequilibrium methods, whereas long timescales can be modelled within the thermodynamic theory, as was done e.g. in $[1,2]$. Time is a very convenient parameter to use for dividing the system or models, because the computer codes are also executed sequentially in time. Thus, the models can often be split into independent executions and only exchange information between the two models by means of output-input files (a model without feedback, as will be described below) [1,2]. 
Similar to division in time, often systems can be divided in space due to peculiarities of the problem. For example, a Gaussian spatial shape of a laser beam means that the centre of the pulse has much higher intensities than its tails. While there might be damage occurring in the centre, the tails will be only slightly heated preserving the atomic structure, which implies that two regions in space can be described with different approaches. For example, the centre requires description with an atomistic model to trace structural evolution, whereas the tails are perfectly well described with a thermodynamic approach, tracing only the evolution of the material temperature (MD with TTM) [3].

Models that use different methods for processes separable in space and/or time are known as 'Multiscale models' [4]. It is a subclass of hybrid approaches.

(a)

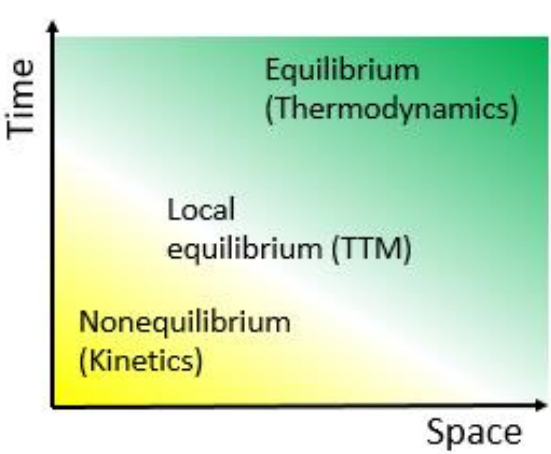

(c)

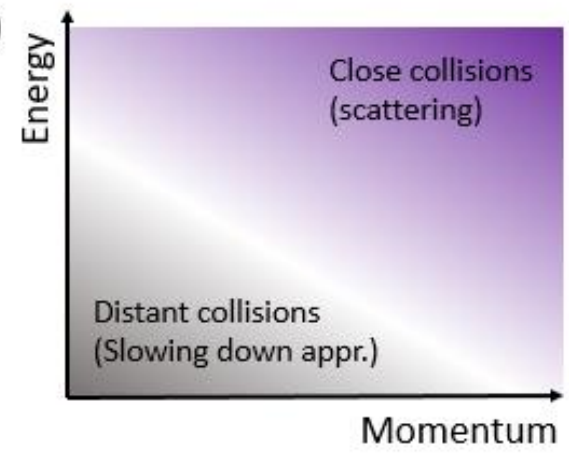

(b)

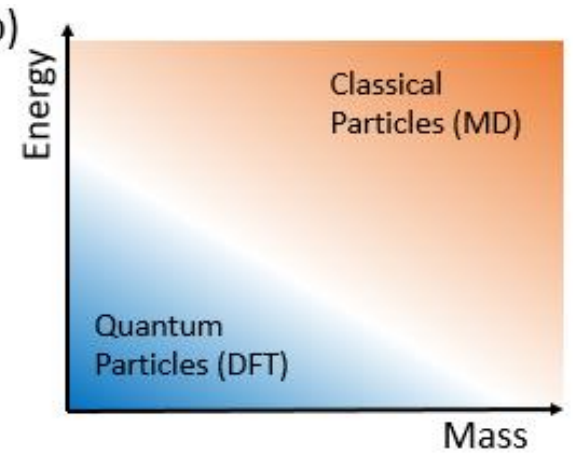

(d)

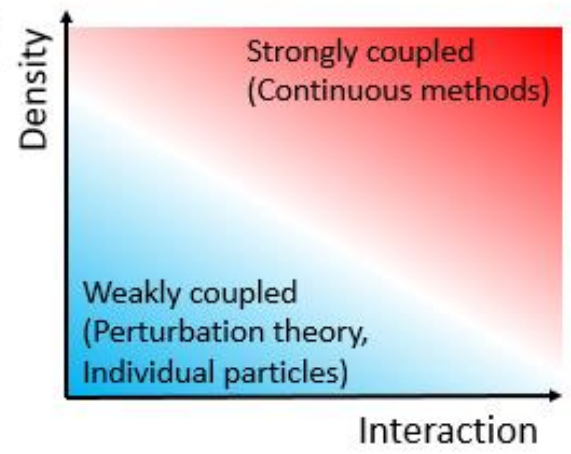

Figure 15.1. Schematics of cross sections of parameter space along various parameters. (a) In real space and time. (b) In energy and mass of particles. (c) In energy and momentum space. (d) In density of particles and strength of their interaction. A few examples of methods used to describe particles in various region of parameter space are presented.

Particles with very different masses have noticeably different kinetics (Figure $15.1 \mathrm{~b}$ ). This fact was used in classical approaches such as Born-Oppenheimer (BO) approximation, which assumes that electrons are infinitely faster than atoms (or atoms are infinitely heavier than electrons). Electrons thus can be treated with quantum mechanical methods (such as density functional theory, DFT), whereas ions may often be traced as classical particles (within molecular dynamics simulations, MD). This led to creation of a hybrid approach, DFT-MD [5]. It also resulted into the two-temperature model (TTM), within which electrons thermalize among themselves much faster than with atoms, thereby transiently establishing two different equilibrium distributions: for electrons and for ions [6-8]. 
The idea to divide the processes in the momentum space (Figure $15.1 \mathrm{c}$ ) was used since the classical Boltzmann equation, in which the left-hand side described long-range fields which change a particle momentum continuously (small change of momentum at short time), whereas the right-hand side describes collisions (instantaneous large changes of momentum) [9]. This idea also found another application within the MC modelling, identifying the so-called close and far collisions [10]. Since a majority of scattering events are distant collisions (with only small energy and momentum exchange), they can be averaged and treated as continuous energy loss of a particle. In close (or head-on) collisions, the energy and momentum exchange is significant, and thus they are treated as individual scattering events. Simulation schemes that use such a separation in energy-momentum space are called condensed history (or condensed collisions) MC, whereas methods that treat all collisions individually, without average energy loss, are referred to as event-by-event (or analog) MC (see Chapter 7) [10].

In case if different kinds of particles under consideration have different densities, they may also be described with different approximations. Dense and strongly interacting or delocalized ensembles may be approximated with continuous methods (such as kinetic equation, hydrodynamics, or two temperature model), while low-density ensembles of particles can be traced individually (with Monte Carlo simulations) [11], see Figure $15.1 \mathrm{~d}$. Another example of a combination of atomistic and continuum methods is a two-temperature molecular dynamics, TTM-MD [12] (discussed in Chapter 8).

Of course, Figure 15.1 shows but a few examples of cutting the parameter space used so far in the literature. Depending on the peculiarities of the problem, one may find different parameters along which the parameter space can be divided. According to these conditions, various models may be chosen to describe different processes. This can serve as the basis for constructing a hybrid model.

The beautiful thing is, the hybrid modelling approach does not restrict one in using only one division along a chosen parameter, but allows for chopping up the parameters space along multiple lines. For example, one can divide the space in energy and time domains simultaneously, at the same time dividing the system into subsystems of particles with different masses [13]. If useful, one can keep dividing the models along other parameters, thereby ending up with a large number of simple models, but complexly interconnected. An example of such a code, XTANT [14], will be given below in Sec. 15.3. And even if the hybrid modelling is implemented by dividing along a single parameter (e.g., time), one can use more than two models: for instance, different models for short, middle, and long timescales. An example of such a model can be found in [15] (where the short timescales are modelled with Monte Carlo).

\subsubsection{Specifics of Monte Carlo methods}

MC models require a large number of iterations repeating the same processes over and over again with different random numbers sampled to cover all possible events and trajectories and to average over them. The standard way an MC code is constructed is to run a single iteration from start to end (defined by the energy cut off or simulation time), then start the next iteration, and so on. An illustration of this scheme is shown in Figure 15.2a.

The problem with this method is that it only allows to obtain the final results after all iterations are computed. When the MC model needs to be combined with other methods, it only allows for 
combination of models without feedback. It means, the other model cannot influence the MC simulation on the fly, it can only receive data from it. For instance, data on electron transport and the spatial distribution of the deposited dose obtained in an MC simulation may be passed to another code, such as TTM [1]. But the transient TTM data (such as target temperature) are not passed back into MC, and electrons are still modelled as if they propagate in an unperturbed cold target.

To construct a model with feedback allowing the data to be passed between the models both ways on equal footing, a Monte Carlo code needs to have a time discretization synchronized with the other model. In such a case, all the MC iterations need to be divided in time so that at each time step information could be collected from all of them, and passed to each of them. This way, models could communicate and respond to a change of parameters simulated by its counterpart. Schematics is shown in Figure 15.2b. This way, for example, a change of the atomic density modelled with MD enters into MC on the fly, affecting the mean free paths, and energy transferred from electrons to atoms is transferred from MC to MD on each time step [16]. Running all MC iterations simultaneously - or, in practise, sequentially, but discretized in the same time steps and propagated together - is memory consuming, but modern computers and clusters can handle it.

(a)

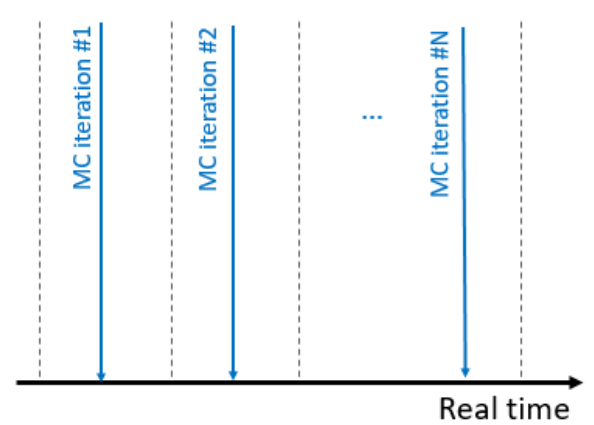

(b)

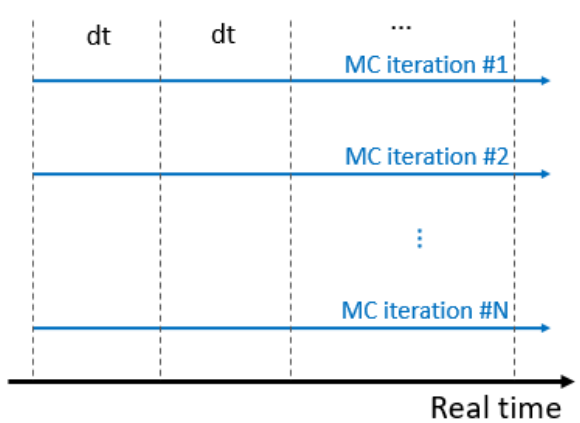

Figure 15.2. Schematics of execution of Monte Carlo algorithms. (a) Standard scheme, in which MC iterations are performed independently. (b) Alternative way, in which all iterations are propagated simultaneously step by step with intermediate synchronizations.

\subsection{Hybrid models without feedback}

If the regions of application of different models do not overlap, or overlap only weakly, the models can be executed independently and only connected by means of one-directional information exchange. For example, below we will discuss a hybrid scheme consisting of MC and TTM and MD models, in which the MC pre-calculates a distribution of energy in space and time due to photoabsorption and secondary electron cascades [1,2]. The distribution of energy is then passed as a source term into the TTM (or MD), after which the TTM (MD) proceeds in calculating the heat transport and energy exchange with the lattice, resulting into local melting or phase transitions. Such combinations are possible under condition that electronic kinetics is short-lived, during which the target does not change its properties significantly - then a one-way passing of information between the models is justified. In this case, it suffices to use standard MC algorithm of independent iterations Figure 15.2a, and gathering the averaged information only after a completion of the entire MC simulation. 


\subsubsection{Example: XCASCADE(3D)}

The Monte Carlo code XCASCADE (which stands for X-ray-induced electron cascades) describes effects induced in electronic system of irradiated target upon X-ray photon impact $[17,18]$. It relies on atomic approximation for the target as described in Chapter 7, and uses EPICS-2017 database for photoabsorption cross sections [19]. Photo-electrons are then modelled assuming BEB impact ionization cross sections [20], which implies that during electron cascades, the target remains practically unaffected. This limits XCASCADE applicability to "low-dose" regime, where "low dose" in practice may be enough to eventually cause ablation of the target.

Applied to X-ray free electron lasers, the XCASCADE code predicted that electron cascades are typically over within a few to a few tens of femtoseconds, for typical irradiation parameters (photon energies from a $\sim 30 \mathrm{eV}$ up to $\sim 25 \mathrm{keV}$ ) [17]. This fact allows to perform the Monte Carlo simulation at the early stages, before further models are applied, and use its output as input for those models. It is justified by the fact that during the timescales of a few tens of femtoseconds target's atoms are practically frozen. Thus, cascades of high-energy electrons takes place within almost unaffected target. Examples of the situations when it is not the case will be considered in Section 15.3.

Thanks to the assumption of the low dose, i.e., low number of excited electrons, we can assume that excited electrons almost never interact with each other and electron cascades evolve independently for each absorbed photon. It can significantly simplify simulation scheme: it is thus sufficient to model only a single photon absorption (in each MC iteration), and then place the evolved electron cascade at the actual position where the photon was absorbed (repeat for all photons and their absorbed places according to the laser spot shape and their in-depth distribution). This is equivalent to applying spatial convolution with the laser shape, which in practice greatly simplifies numerical implementation of the Monte Carlo algorithm [2,21].

Within XCASCADE, the electron cascades may be considered over when electron energy drops below a certain cut-off. Low-electron electrons are more strongly affected by ongoing modification of the electronic system of the target. XCASCADE(3D) was recently combined (without a feedback) with the two-temperature model that traces low-energy electrons. Such combination allowed to estimate the damage threshold of ruthenium metal under intense FEL irradiation [2]. Later, the TTM part was replaced with two-temperature hydro-dynamics, which allowed to trace evolution of the pressure waves in the target and describe its ablation [22]. To trace atomic dynamics with a good precision, a Molecular Dynamics simulation was added to the mix at later simulation times, thereby creating a combination of three models, Monte Carlo, hydrodynamics and molecular dynamics [15].

Such a combination of three models helped to gain insights into microscopic processes of ablation of ruthernium under XUV and X-ray irradiation, from the initial photon absorption, to electron kinetics and transport, to atomic dynamics of material damage. In particular, it delivered such interesting results as proving similarity in damage for different photon energies from optical to hard X-rays [22], and showed an effect of two-level ablation in ruthenium [15] in a good agreement with experiments, see example in Figure 15.3 

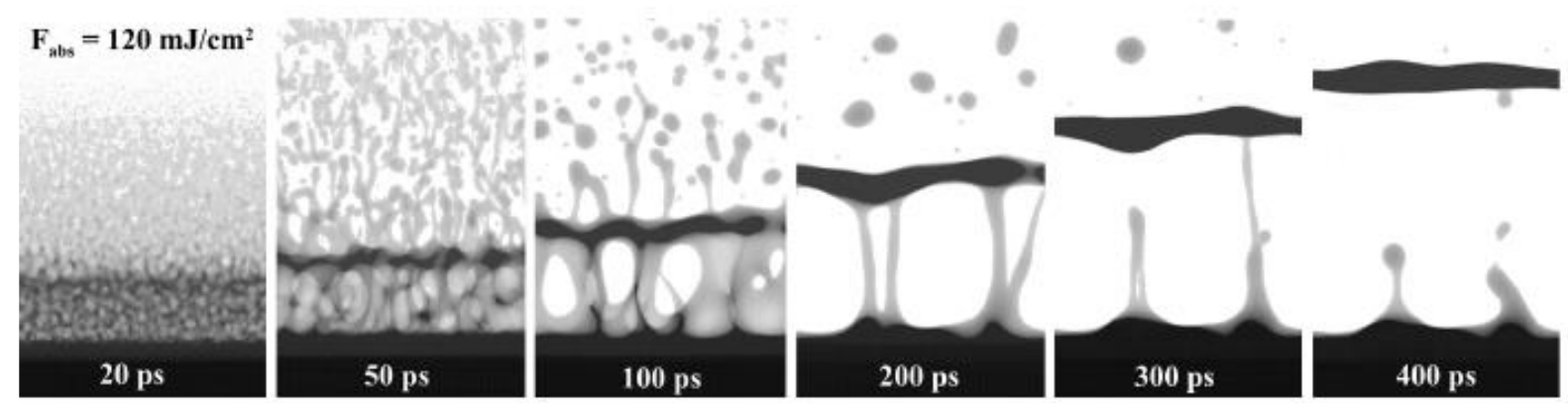

Figure 15.3. Mass distribution of ruthenium irradiation with $100 \mathrm{fs} X U V$ pulse with fluence of $120 \mathrm{~mJ} / \mathrm{cm}^{2}$ obtained with a hybrid approach, at different time instants after irradiation. Figure is reproduced from Ref. [15].

\subsubsection{Example: TREKIS}

Let us discuss another example of a combination of Monte Carlo method with molecular dynamics simulation, this time applied to modelling of swift heavy ion track creation. The MC code TREKIS (the acronym stands for Time-Resolved Electron Kinetics in SHI-Irradiated Solids $[23,24]$ ) was combined with MD simulation, performed with the simulation package LAMMPS [25].

The Monte-Carlo code TREKIS is an event-by-event individual particle simulation [23,24]. The used scattering cross sections are based on the complex dielectric function formalism as described in Ref.[23]. Based on these cross sections, TREKIS models the following processes: (a) traversing of an ion, producing ionization of a target and creating primary electrons (so-called delta electrons) and electronic holes (core and valence band); (b) scattering of delta electrons on atoms and target electrons, and the kinetics of all secondary generations of electrons; (c) Auger decays of core holes, also producing secondary electrons; (d) radiative decays of core holes, ensuing photon transport and photoabsorption exciting new electrons and holes; (e) valence holes transport and their scattering on the target atoms [23,24].

The MC algorithm uses sequential iterations (Figure 15.4a). As a result, it delivers the radial distributions of the density and energy of electrons, holes in the valence band and atomic shells, and the energy transferred to the atomic system. For a typical SHI energy produced on linear accelerators, electron cascades nearly finish within $\sim 100 \mathrm{fs}$ [26].

The following simple but effective approximation was proposed to couple TREKIS with MD simulation in Refs. [27-30]: at the instant of $100 \mathrm{fs}$, the energy transferred to the atoms via elastic scattering of electrons and valence holes, as well as remaining potential energy of valence holes and electrons, is passed from MC to MD simulation. This energy is delivered as a momentary increase of the kinetic energies of lattice atoms in the simulation cell via assigning random additional momenta to atoms. The velocities of atoms in cylindrical layers counted from the SHI trajectory are set with the Gaussian-like dispersion of the kinetic energy, and a uniform distribution of momenta of atoms within each layer. This distribution of the velocities is then used to simulate the lattice response in the following $\sim 150 \mathrm{ps}$ applying the classical MD code LAMMPS [31]. 


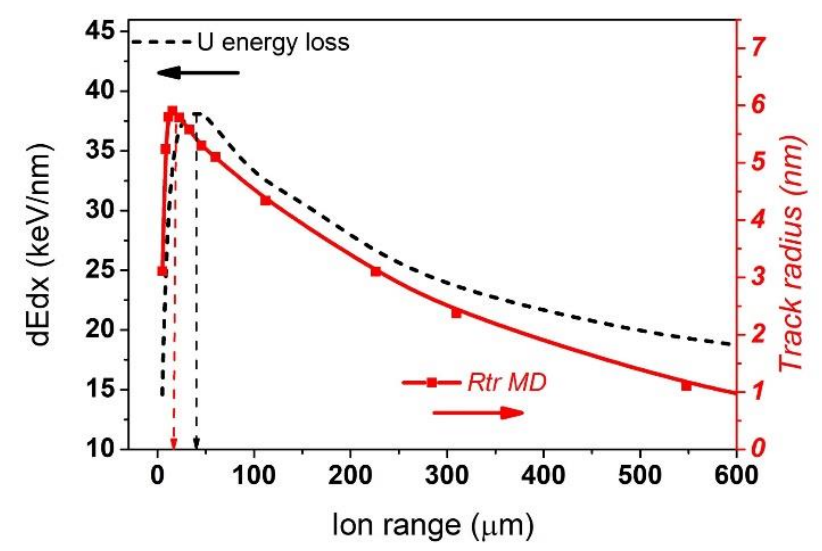

Figure 15.4. Results of $\mathrm{MC}+\mathrm{MD}$ simulation of $\mathrm{U}$ ion impact on $\mathrm{Al}_{2} \mathrm{O}_{3}$ : an overlay of the stopping power (left axis) as a function of the ion penetration range, and the created track radius (right axis). Figure is reproduced from Ref. [29].

Such an approximation enables, e.g., to describe the radial sizes and atomic structure of SHI tracks in a reasonable agreement with experiments [32,33]. It revealed a curious effect that the created damage in insulators along an SHI path does not coincide with the deposited dose profile [29]. An example of this mismatch is shown in Figure 15.4, where one can see that the maximal damage is located deeper along an SHI path than the peak of the energy deposition (the Bragg peak). This demonstrates that estimation of the deposited dose alone (as typically done with standard MC codes) is insufficient to evaluate the formed material damage, and later stages of material response are necessary to consider (e.g. with an MD simulation).

Calculations with this multiscale approach also showed an importance of valence holes kinetics, since valence holes transfer to atoms a significant amount of energy [27]. Standard MC codes usually do not include these effects.

At later stages, it is found to be crucial to consider relaxation kinetics in detail: recrystallization around an SHI trajectory is the dominant factor defining the final material modifications [30]. Meaning, in different materials with different recrystallization efficiency, identical energy deposition and identical initial transient damage results in different final stable tracks [30]. So, it is also insufficient to estimate only the region where the melting temperature is reached, to evaluate where the final damage will be observed (as usually done with the so-called thermal spike model). Only a hybrid model, describing properly ultrafast nonequilibrium electron kinetics as well as long-term atomic dynamics, could reveal such new effects.

\subsection{Hybrid models with feedback}

A feedback between the models is needed when processes are not so clearly separable in the parameter space (time, real space, reciprocal space, etc., see Section 15.1.1). This occurs when parameters modeled with one model are noticeably affect some parameters of the other model on the fly. If various regions in the parameter space affect each other, the models describing them must be able to influence each other during a simulation run. First models with feedback included such methods as DFT-MD [5], and TTM-MD [12] combinations, which became standard tools in modeling laser irradiation of solids. 
When subsystems in different parameter subspaces interact strongly, a self-consistency must be ensured. That means, a feedback loop between the models must be included, so that each model would react to the information passed from the other one. In such a case, the models cannot be executed sequentially; a parallel execution must be performed, exchanging information at each time step of the simulation. In this case, the hybrid code needs to have a unifying framework that would allow the models to be executed in parallel and exchange information on the fly. That being said, within each (sufficiently small) time step the models can still be executed sequentially, but before the next time step, they must exchange information and react to it.

In practise, many models may in fact have different time steps, and some of them may not use time steps at all, such as the typical Monte Carlo simulations. For constructing a hybrid code, it is important to synchronize time steps in the models, and introduce them when needed (see description of alternative MC algorithms in section 15.1.2). For different time discretizations in different models, a larger time steps must be a multiple of the smaller time steps.

Combining an MC approach with other models implies that average quantities are passed from the MC module into other models. Such quantities must be averaged over the statistics (over all iterations) at each time step (see Figure 15.2b, Section 15.1.2). Monte Carlo codes utilizing this algorithm were tested and proved their capabilities to be combined with other models, as will be shown in the next section.

\subsubsection{Example: XTANT}

Here, we will discuss an example of a recently developed hybrid code XTANT (the acronym stands for X-ray-induced Thermal And Nonthermal Transitions [14]). The idea behind this hybrid approach comes from the specifics of its application: to model a material response to irradiation with X-ray femtosecond lasers (free-electron lasers, FEL [34]). An FEL pulse creates a transient nonequilibrium electron distribution and electron cascades. It has been demonstrated that in materials under FEL irradiation the transient electron distribution function is in nonequilibrium state which consists of two distinct parts in the energy space: a low energy fraction of electrons that are in near-thermal equilibrium (which can be described with thermodynamic approach), whereas high-energy electrons are highly nonequilibrium (can be described with nonequilibrium methods such as MC) [11]. Such a distribution was demonstrated with different theoretical methods in various materials, and thus can be assumed a universal transient distribution under FEL irradiation [11,35-37]. This shape of the distribution was named a 'bump-on-hot-tail' [38]. 


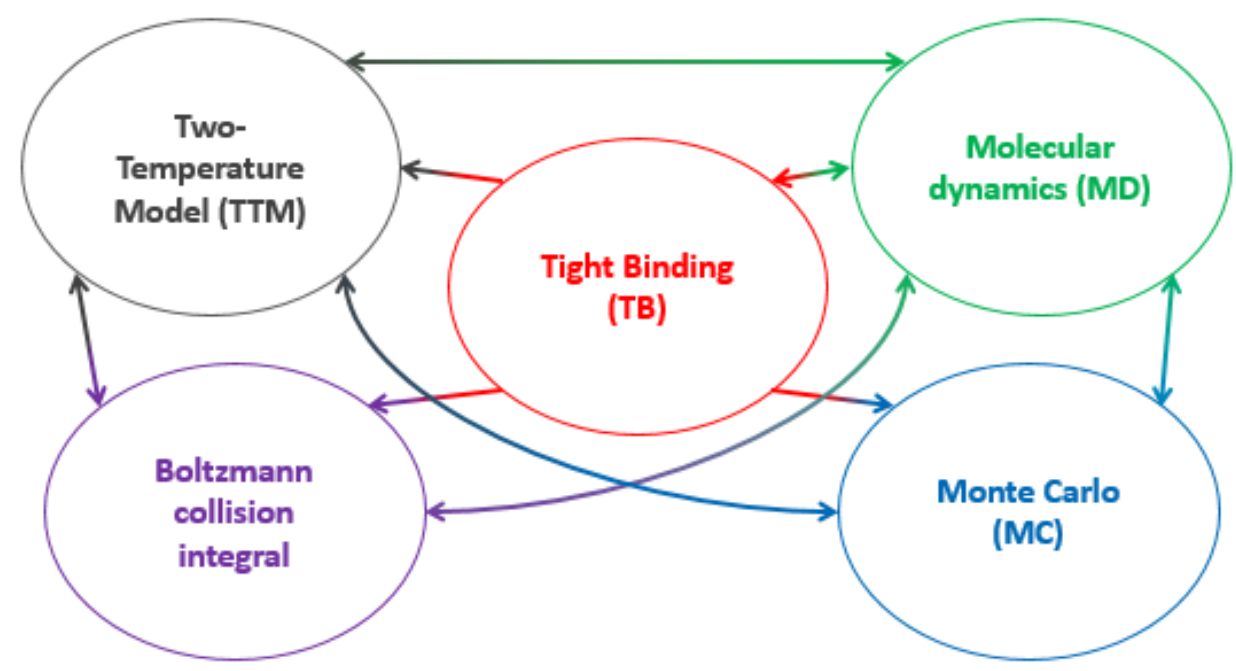

Figure 15.5. Schematics of XTANT. Different modules incorporated in the codes are depicted. Arrays show the data flow in connections between them. Figure adapted from Ref. [14].

Having two distinct region in the energy space - the majority of low-energy electrons in near thermal equilibrium, and a minority of high-energy out-of-equilibrium electrons - immediately hints at a construction of a hybrid approach. Additionally, the electrons and atoms can be treated with independent methods, with proper connections between them. All models must be executed in parallel, as they are not separated in time, and an explicit feedback between the models must be taken into account [16]. Thus, XTANT code was constructed from the following modules (as schematically depicted in Figure 15.5):

(a) A Monte Carlo module that follows nonequilibrium high-energy electron and core-hole kinetics. The cross sections of inelastic scattering used are based on the complex-dielectric function formalism as developed by Ritchie and Howie [39]. On each time step, the data from all MC iterations are collected to pass into other models. The following parameters are passed: the number of electrons entering or leaving the low-energy domain (with energies below cut-off of $10 \mathrm{eV}$ counted from the bottom of the conduction band), the energy of these electrons, and the energy transferred to atoms via elastic scattering of high-energy electrons. As a feedback, MC receives the details of the band structure and adjusts the probabilities of electron ionization and corresponding mean free paths every time step.

(b) The module tracing a temperature evolution of valence and low-energy conduction-band electrons relies on the Fermi distribution (a thermodynamic approximation). This distribution populates a transient and evolving band structure of the material modelled with the tight binding method mentioned below. The evolution of the electronic distribution is defined by the sources and sinks of particles and energies from the MC module and the energy exchange with the atomic system.

(c) The energy exchange (coupling) of low-energy electrons with atoms is calculated via the Boltzmann collision integral beyond simple Fermi's Golden Rule [40]. The Boltzmann collision integral is calculated with the probabilities of electron transitions calculated via overlap of the electronic wave functions obtained within the tight binding method. It thus depends on the transient positions of all the atoms within the simulation box [41]. 
(d) Molecular dynamics traces the atomic trajectories of all atoms in the super-cell with periodic boundary conditions [42,43]. The energy transferred from both MC (a) and TTM (b) electron fractions is fed to atoms on each time step via velocity scaling [40]. The transient potential energy surface defining the forces acting on atoms in MD simulation is calculated with the tight binding method.

(e) The transferable tight binding method is used for calculations of the transient Hamiltonian, electronic band structure, collective potential energy surface for atoms, and matrix elements used in the electron-ion (electron-phonon) coupling entering Boltzmann collision integral $[16,40]$. This method allows to include influence of electron distribution on the interatomic potential, thereby allowing to model nonthermal phase transitions $[44,45]$.

This hybrid code was cross checked against experiments with free-electron lasers irradiation of diamond [46-48]. It demonstrated an excellent agreement with the femtosecond time-resolved experiments, which measured optical properties of the irradiated diamond [47]. Under intense femtosecond irradiation, diamond experiences ultrafast phase transition into graphite, completed within $\sim 200 \mathrm{fs}$, the fastest solid-solid phase transition observed so far [47]. XTANT predicted that this phase transition takes place as a sequence of the following processes [16]: first, photoabsorption induces nonequilibrium electron cascades, see Figure 15.6a. The cascades excite secondary electrons across the band gap of diamond into the conduction band. During these processes, the high-energy electrons lose their energy and disappear from the MC-energy domain leading to decrease of their density.

Once the density of the low-energy electrons in the conduction band (shown in Figure 15.6b) overcomes the threshold of $\sim 1.5 \%$ of the valence electrons, it induces a band gap collapse (Figure 15.6c) - the material turns from an insulating into a semimetallic. The shrinkage and collapse of the band gap promotes even more electrons into the conduction band, and having overcome the threshold of $\sim 3-3.5 \%$ it induces diffusionless atomic transition into the graphite phase, see snapshots in Figure 15.6e $[14,16]$. All of these phases influence the optical properties of the material, and thus could be tracked in experiment, validating the model [47].

(a)

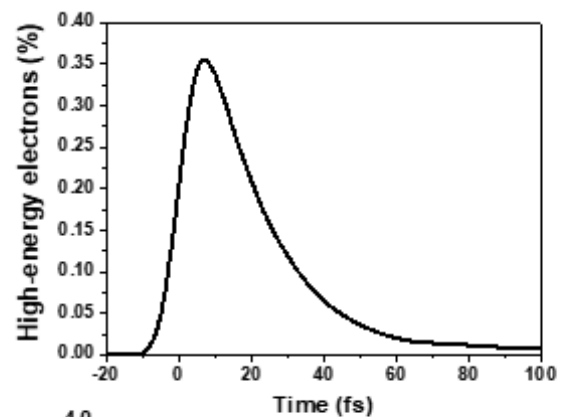

(b)

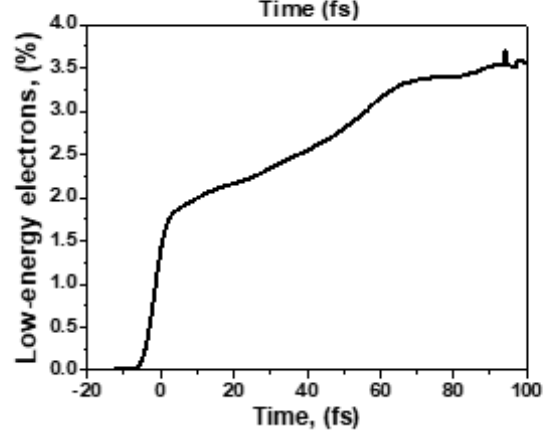

(c)

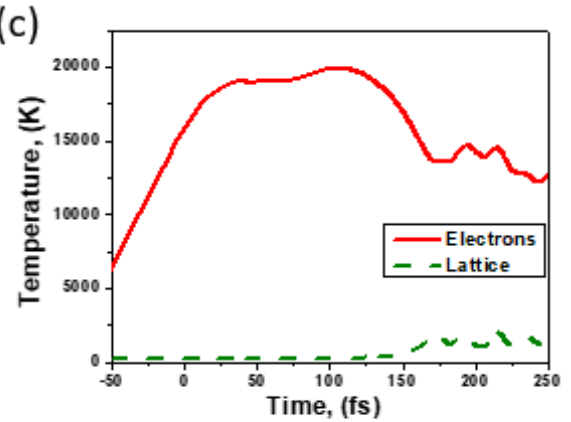

(d)

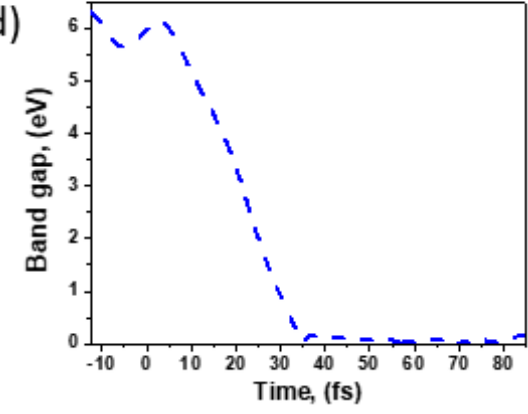



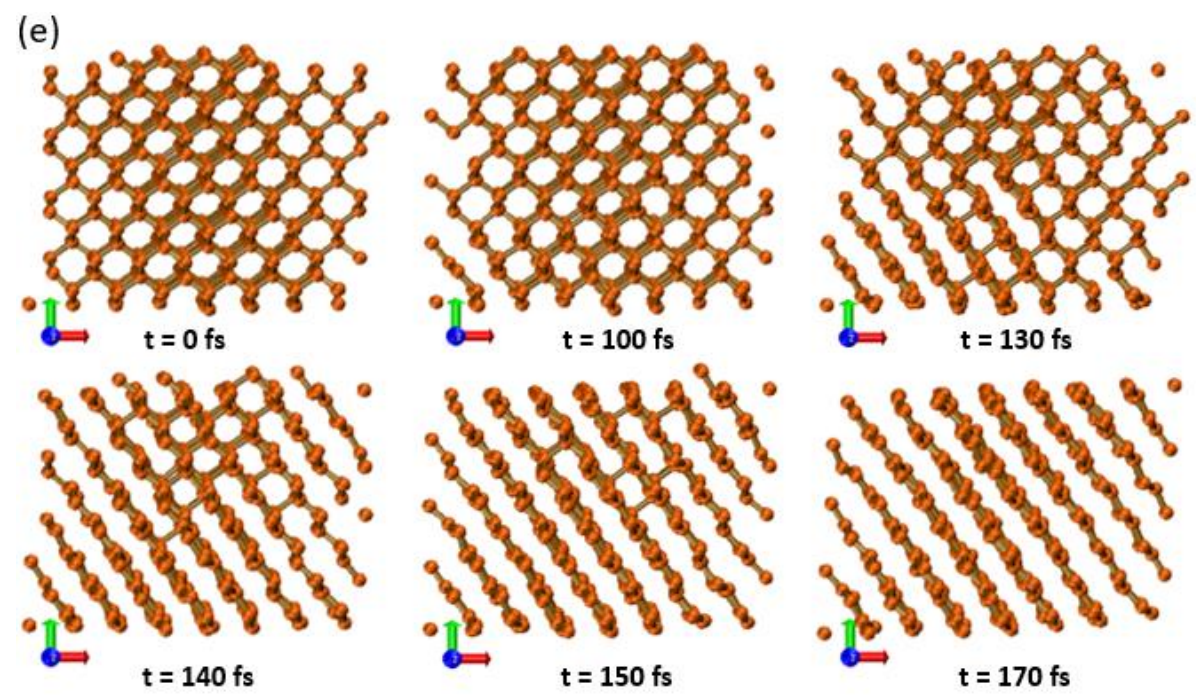

Figure 15.6. Example of results obtained with XTANT for diamond irradiated with $92 \mathrm{eV}$ photon pulse, 10 fs FWHM duration, $0.85 \mathrm{eV} /$ atom deposited dose. (a) Density of high-energy (Monte Carlo) electrons. (b) Density of low-density (TTM) electrons in the conduction band. (c) Electronic and atomic temperatures. (d) Band gap of the material. (e) Atomic snapshots at different time instants after irradiation. Figures are compiled from Refs. [16,46,49].

Such a sequence of processes leading to the phase transition is of a clearly nonthermal nature [16]: the phase transition is induced by the modification of the interatomic potential due to electron excitation, and not by the heating of the atomic system via electron-phonon coupling. At such timescales, electron-phonon coupling does not transfer any significant energy to atoms, so the atomic temperature remains low (Figure 15.6d) [47]. The atomic temperature increases during the phase transition, but as a result of changes of atomic potential energy surface which accelerates the atoms and not as the cause of the phase transition. We emphasize that this increase at 150-200 fs is not the result of the electron-phonon coupling, but a response to nonthermal phase transition $[14,16]$.

XTANT code also allowed to calculate the interplay of the thermal and nonthermal effects, which is significant in silicon [40,50] and group III-V semiconductors [51]. The nature of damage in this materials depends on the fluences: at low laser fluences, it is typically slow thermal melting (limited by the timescales of the electron-phonon coupling, a few picoseconds), whereas at high fluences, nonthermal melting takes place at sub-picosecond timescales [50].

Simulations with the hybrid code XTANT predicted existence of nonthermal phase transitions in oxides, including creation of such exotic matter as superionic state in highly electronically excited $\mathrm{Al}_{2} \mathrm{O}_{3}[52,53]$. The superionic state is a phase that is liquid and solid simultaneously [54] - in case of $\mathrm{Al}_{2} \mathrm{O}_{3}$ irradiation, oxygen subsystem melts into liquid, whereas aluminium subsystem stays crystalline [52,53].

XTANT also predicted nonthermal phase transitions in finite size metals, in contrast to bulk metals where nonthermal phonon hardening takes place under irradiation [55]. It calculated electronion (electron-phonon) coupling in metals across the Periodic Table [41].

Overall, the hybrid approach combining Monte Carlo and other methods with feedback proved it capabilities, thereby showing the power of such methods. 


\section{Acknowledgements}

NM gratefully acknowledges financial support from the Czech Ministry of Education, Youth and Sports (Grants No. LTT17015, No. EF16\013/0001552).

\section{References}

[1] O. Osmani, N. Medvedev, M. Schleberger, B. Rethfeld, Energy dissipation in dielectrics after swift heavy-ion impact: A hybrid model, Phys. Rev. B. 84 (2011) 214105. https://doi.org/10.1103/PhysRevB.84.214105.

[2] I. Milov, I.A. Makhotkin, R. Sobierajski, N. Medvedev, V. Lipp, J. Chalupský, J.M. Sturm, K. Tiedtke, G. de Vries, M. Störmer, F. Siewert, R. van de Kruijs, E. Louis, I. Jacyna, M. Jurek, L. Juha, V. Hájková, V. Vozda, T. Burian, K. Saksl, B. Faatz, B. Keitel, E. Plönjes, S. Schreiber, S. Toleikis, R. Loch, M. Hermann, S. Strobel, H.-K. Nienhuys, G. Gwalt, T. Mey, H. Enkisch, F. Bijkerk, Mechanism of single-shot damage of Ru thin films irradiated by femtosecond extreme UV free-electron laser, Opt. Express. 26 (2018) 19665. https://doi.org/10.1364/OE.26.019665.

[3] D.S. Ivanov, A.I. Kuznetsov, V.P. Lipp, B. Rethfeld, B.N. Chichkov, M.E. Garcia, W. Schulz, Short laser pulse nanostructuring of metals: Direct comparison of molecular dynamics modeling and experiment, Appl. Phys. A Mater. Sci. Process. 111 (2013) 675-687. https://doi.org/10.1007/s00339-013-7656-9.

[4] K. Nordlund, C. Björkas, T. Ahlgren, A. Lasa, A.E. Sand, Multiscale modelling of plasma-wall interactions in fusion reactor conditions, J. Phys. D. Appl. Phys. 47 (2014) 224018. https://doi.org/10.1088/0022-3727/47/22/224018.

[5] R. Car, M. Parrinello, Unified approach for molecular dynamics and density-functional theory, Phys. Rev. Lett. 55 (1985) 2471-2474. https://doi.org/10.1103/PhysRevLett.55.2471.

[6] V.L. Shabansky, V.P. Ginzburg, Kinetic Electron Temperature in Metals and Anomalous Electron Emission, Reports Acad. Sci. USSR. 100 (1955) 445.

[7] M.I. Kaganov, I.M. Lifshitz, L.V. Tanatarov, Relaxation between electrons and crystalline lattice, Sov. Phys. JETP. 4 (1957) 173.

[8] S.I. Anisimov, B.L. Kapeliovich, T.L. Perel-man, Electron emission from metal surfaces exposed to ultrashort laser pulses, J. Exp. Theor. Phys. 39 (1974) 375.

[9] V. Vedenyapin, A. Sinitsyn, E. Dulov, Kinetic Boltzmann, Vlasov and related equations, Elsevier Science Ltd, 2011. http://www.sciencedirect.com/science/book/9780123877796 (accessed March 7, 2017).

[10] T.M. Jenkins, W.R. Nelson, A. Rindi, eds., Monte Carlo Transport of Electrons and Photons, Springer US, Boston, MA, 1988. https://doi.org/10.1007/978-1-4613-1059-4.

[11] N. Medvedev, U. Zastrau, E. Förster, D.O. Gericke, B. Rethfeld, Short-Time Electron Dynamics in Aluminum Excited by Femtosecond Extreme Ultraviolet Radiation, Phys. Rev. Lett. 107 (2011) 165003. https://doi.org/10.1103/PhysRevLett.107.165003.

[12] D. Ivanov, L. Zhigilei, Combined atomistic-continuum modeling of short-pulse laser melting and disintegration of metal films, Phys. Rev. B. 68 (2003) 064114. https://doi.org/10.1103/PhysRevB.68.064114.

[13] S.A. Gorbunov, P.N. Terekhin, N.A. Medvedev, A.E. Volkov, Combined model of the material excitation and relaxation in swift heavy ion tracks, Nucl. Instruments Methods Phys. Res. Sect. B Beam Interact. with Mater. Atoms. 315 (2013) 173-178. https://doi.org/10.1016/j.nimb.2013.04.082.

[14] N. Medvedev, V. Tkachenko, V. Lipp, Z. Li, B. Ziaja, Various damage mechanisms in carbon and silicon materials under femtosecond X-ray irradiation, 4open. 1 (2018) 3. https://doi.org/10.1051/FOPEN/2018003.

[15] I. Milov, V. Zhakhovsky, D. Ilnitsky, K. Migdal, V. Khokhlov, Y. Petrov, N. Inogamov, V. Lipp, N. Medvedev, B. Ziaja, V. Medvedev, I.A. Makhotkin, E. Louis, F. Bijkerk, Two-level ablation and damage 
morphology of Ru films under femtosecond extreme UV irradiation, Appl. Surf. Sci. (2020) 146952. https://doi.org/10.1016/j.apsusc.2020.146952.

[16] N. Medvedev, H.O. Jeschke, B. Ziaja, Nonthermal phase transitions in semiconductors induced by a femtosecond extreme ultraviolet laser pulse, New J. Phys. 15 (2013) 015016. https://doi.org/10.1088/1367-2630/15/1/015016.

[17] N. Medvedev, Femtosecond X-ray induced electron kinetics in dielectrics: application for FEL-pulseduration monitor, Appl. Phys. B. 118 (2015) 417-429. https://doi.org/10.1007/s00340-015-60054 .

[18] V. Lipp, N. Medvedev, B. Ziaja, Classical Monte-Carlo simulations of x-ray induced electron cascades in various materials, in: Proc. SPIE 10236, Damage to VUV, EUV, X-Ray Opt. VI, 102360H, International Society for Optics and Photonics, 2017: pp. 102360H-102360H-9. https://doi.org/10.1117/12.2267939.

[19] D.E. Cullen, A Survey of Atomic Binding Energies for use in EPICS2017, Vienna, 2018. https://wwwnds.iaea.org/publications/iaea-nds/iaea-nds-224_Rev1_2018.pdf (accessed August 24, 2018).

[20] Y.-K. Kim, M. Rudd, Binary-encounter-dipole model for electron-impact ionization, Phys. Rev. A. 50 (1994) 3954-3967. https://doi.org/10.1103/PhysRevA.50.3954.

[21] I. Milov, V. Lipp, N. Medvedev, I.A. Makhotkin, E. Louis, F. Bijkerk, Modeling of XUV-induced damage in $\mathrm{Ru}$ films: the role of model parameters, JOSA B. 35 (2018) B43-B53. https://doi.org/10.1364/JOSAB.35.000B43.

[22] I. Milov, V. Lipp, D. Ilnitsky, N. Medvedev, K. Migdal, V. Zhakhovsky, V. Khokhlov, Y. Petrov, N. Inogamov, S. Semin, A. Kimel, B. Ziaja, I.A. Makhotkin, E. Louis, F. Bijkerk, Similarity in ruthenium damage induced by photons with different energies: From visible light to hard X-rays, Appl. Surf. Sci. 501 (2020) 143973. https://doi.org/10.1016/J.APSUSC.2019.143973.

[23] N.A. Medvedev, R.A. Rymzhanov, A.E. Volkov, Time-resolved electron kinetics in swift heavy ion irradiated solids, J. Phys. D. Appl. Phys. 48 (2015) 355303. https://doi.org/10.1088/0022$3727 / 48 / 35 / 355303$.

[24] R.A. Rymzhanov, N.A. Medvedev, A.E. Volkov, Effects of model approximations for electron, hole, and photon transport in swift heavy ion tracks, Nucl. Instruments Methods Phys. Res. Sect. B Beam Interact. with Mater. Atoms. 388 (2016) 41-52. https://doi.org/10.1016/j.nimb.2016.11.002.

[25] S. Plimpton, LAMMPS WebPage, (2016) http://lammps.sandia.gov.

[26] N. Medvedev, A.E. Volkov, B. Ziaja, Electronic and atomic kinetics in solids irradiated with freeelectron lasers or swift-heavy ions, Nucl. Instruments Methods Phys. Res. Sect. B Beam Interact. with Mater. Atoms. 365 (2015) 437-446. https://doi.org/10.1016/j.nimb.2015.08.063.

[27] R. Rymzhanov, N.A. Medvedev, A.E. Volkov, Damage threshold and structure of swift heavy ion tracks in Al203, J. Phys. D. Appl. Phys. 50 (2017) 475301. https://doi.org/10.1088/1361-6463/aa8ff5.

[28] R.A. Rymzhanov, N. Medvedev, A.E. Volkov, J.H. O'Connell, V.A. Skuratov, Overlap of swift heavy ion tracks in Al203, Nucl. Instruments Methods Phys. Res. Sect. B Beam Interact. with Mater. Atoms. 435 (2018) 121-125. https://doi.org/10.1016/j.nimb.2017.11.014.

[29] R.A. Rymzhanov, S.A. Gorbunov, N. Medvedev, A.E. Volkov, Damage along swift heavy ion trajectory, Nucl. Instruments Methods Phys. Res. Sect. B Beam Interact. with Mater. Atoms. 440 (2019) 25-35. https://doi.org/10.1016/j.nimb.2018.11.034.

[30] R.A. Rymzhanov, N. Medvedev, J.H. O'Connell, A. Janse van Vuuren, V.A. Skuratov, A.E. Volkov, Recrystallization as the governing mechanism of ion track formation, Sci. Rep. 9 (2019). https://doi.org/10.1038/s41598-019-40239-9.

[31] S. Plimpton, Fast Parallel Algorithms for Short-Range Molecular Dynamics, J. Comput. Phys. 117 (1995) 1-19. https://doi.org/10.1006/jcph.1995.1039.

[32] R.A. Rymzhanov, N.A. Medvedev, A.E. Volkov, Effect of valence holes kinetics on material excitation in tracks of swift heavy ions, Nucl. Instruments Methods Phys. Res. Sect. B Beam Interact. with Mater. Atoms. 365 (2015) 462-467. https://doi.org/10.1016/j.nimb.2015.08.043. 
[33] R.A. Rymzhanov, N. Medvedev, J.H. O’Connell, V.A. Skuratov, A. Janse van Vuuren, S.A. Gorbunov, A.E. Volkov, Insights into different stages of formation of swift heavy ion tracks, Nucl. Instruments Methods Phys. Res. Sect. B Beam Interact. with Mater. Atoms. 473 (2020) 27-42. https://doi.org/10.1016/j.nimb.2020.04.005.

[34] J. Rossbach, J.R. Schneider, W. Wurth, 10 years of pioneering X-ray science at the Free-Electron Laser FLASH at DESY, Phys. Rep. (2019). https://doi.org/10.1016/J.PHYSREP.2019.02.002.

[35] R.R. Fäustlin, T. Bornath, T. Döppner, S. Düsterer, E. Förster, C. Fortmann, S.H. Glenzer, S. Göde, G. Gregori, R. Irsig, T. Laarmann, H.J. Lee, B. Li, K.-H. Meiwes-Broer, J. Mithen, B. Nagler, A. Przystawik, H. Redlin, R. Redmer, H. Reinholz, G. Röpke, F. Tavella, R. Thiele, J. Tiggesbäumker, S. Toleikis, I. Uschmann, S.M. Vinko, T. Whitcher, U. Zastrau, B. Ziaja, T. Tschentscher, Observation of Ultrafast Nonequilibrium Collective Dynamics in Warm Dense Hydrogen, Phys. Rev. Lett. 104 (2010) 125002. https://doi.org/10.1103/PhysRevLett.104.125002.

[36] N. Medvedev, B. Rethfeld, Transient dynamics of the electronic subsystem of semiconductors irradiated with an ultrashort vacuum ultraviolet laser pulse, New J. Phys. 12 (2010) 073037. https://doi.org/10.1088/1367-2630/12/7/073037.

[37] S.P. Hau-Riege, Nonequilibrium electron dynamics in materials driven by high-intensity $x$-ray pulses, Phys. Rev. E. 87 (2013) 053102. https://doi.org/10.1103/PhysRevE.87.053102.

[38] D.A. Chapman, D.O. Gericke, Analysis of Thomson Scattering from Nonequilibrium Plasmas, Phys. Rev. Lett. 107 (2011) 165004. https://doi.org/10.1103/PhysRevLett.107.165004.

[39] R.H. Ritchie, A. Howie, Electron excitation and the optical potential in electron microscopy, Philos. Mag. 36 (1977) 463-481. https://doi.org/10.1080/14786437708244948.

[40] N. Medvedev, Z. Li, V. Tkachenko, B. Ziaja, Electron-ion coupling in semiconductors beyond Fermi's golden rule, Phys. Rev. B. 95 (2017) 014309. https://doi.org/10.1103/PhysRevB.95.014309.

[41] N. Medvedev, I. Milov, Electron-phonon coupling in metals at high electronic temperatures, Phys. Rev. B. 102 (2020) 064302. https://doi.org/10.1103/PhysRevB.102.064302.

[42] L. Verlet, Computer "Experiments" on Classical Fluids. I. Thermodynamical Properties of LennardJones Molecules, Phys. Rev. 159 (1967) 98-103. https://doi.org/10.1103/PhysRev.159.98.

[43] D.C. Rapaport, The Art of Molecular Dynamics Simulation, Cambridge University Press, 2004. http://books.google.com/books?id=iqDJ2hjqBMEC\&pgis=1 (accessed November 20, 2014).

[44] C.W. Siders, Detection of Nonthermal Melting by Ultrafast X-ray Diffraction, Science (80-. ). 286 (1999) 1340-1342. http://www.sciencemag.org/content/286/5443/1340.abstract (accessed December 14, 2015).

[45] S.K. Sundaram, E. Mazur, Inducing and probing non-thermal transitions in semiconductors using femtosecond laser pulses., Nat. Mater. 1 (2002) 217-24. https://doi.org/10.1038/nmat767.

[46] J. Gaudin, N. Medvedev, J. Chalupský, T. Burian, S. Dastjani-Farahani, V. Hájková, M. Harmand, H.O. Jeschke, L. Juha, M. Jurek, D. Klinger, J. Krzywinski, R.A. Loch, S. Moeller, M. Nagasono, C. Ozkan, K. Saksl, H. Sinn, R. Sobierajski, P. Sovák, S. Toleikis, K. Tiedtke, M. Toufarová, T. Tschentscher, V. Vorlíček, L. Vyšín, H. Wabnitz, B. Ziaja, E. Al., Photon energy dependence of graphitization threshold for diamond irradiated with an intense XUV FEL pulse, Phys. Rev. B. 88 (2013) 060101(R). https://doi.org/10.1103/PhysRevB.88.060101.

[47] F. Tavella, H. Höppner, V. Tkachenko, N. Medvedev, F. Capotondi, T. Golz, Y. Kai, M. Manfredda, E. Pedersoli, M.J. Prandolini, N. Stojanovic, T. Tanikawa, U. Teubner, S. Toleikis, B. Ziaja, Soft x-ray induced femtosecond solid-to-solid phase transition, High Energy Density Phys. 24 (2017) 22-27. https://doi.org/10.1016/j.hedp.2017.06.001.

[48] N. Medvedev, B. Ziaja, Multistep transition of diamond to warm dense matter state revealed by femtosecond X-ray diffraction, Sci. Rep. 8 (2018) 5284. https://doi.org/10.1038/s41598-01823632-8.

[49] N. Medvedev, V. Tkachenko, B. Ziaja, Modeling of Nonthermal Solid-to-Solid Phase Transition in Diamond Irradiated with Femtosecond x-ray FEL Pulse, Contrib. to Plasma Phys. 55 (2015) 12-34. https://doi.org/10.1002/ctpp.201400026. 
[50] N. Medvedev, Z. Li, B. Ziaja, Thermal and nonthermal melting of silicon under femtosecond x-ray irradiation, Phys. Rev. B. 91 (2015) 054113. https://doi.org/10.1103/PhysRevB.91.054113.

[51] N. Medvedev, Z. Fang, C. Xia, Z. Li, Thermal and nonthermal melting of III-V compound semiconductors, Phys. Rev. B. 99 (2019) 144101. https://doi.org/10.1103/PhysRevB.99.144101.

[52] R.A. Voronkov, N. Medvedev, A.E. Volkov, Superionic State in Alumina Produced by Nonthermal Melting, Phys. Status Solidi - Rapid Res. Lett. $14 \quad$ (2020) 1900641. https://doi.org/10.1002/pssr.201900641.

[53] N. Medvedev, Nonthermal phase transitions in irradiated oxides, J. Phys. Condens. Matter. 32 (2020) 435401. https://doi.org/10.1088/1361-648X/aba389 (accessed July 30, 2020).

[54] M. Millot, F. Coppari, J.R. Rygg, A. Correa Barrios, S. Hamel, D.C. Swift, J.H. Eggert, Nanosecond X-ray diffraction of shock-compressed superionic water ice, Nature. 569 (2019) 251-255. https://doi.org/10.1038/s41586-019-1114-6.

[55] N. Medvedev, I. Milov, Nonthermal phase transitions in metals, Sci. Rep. 10 (2020) 12775. https://doi.org/10.1038/s41598-020-69604-9. 


\title{
16. Molecular dynamics simulations of collision cascades in semiconducting materials
}

\author{
Thomas Jarrin*
}

In radiative environments like space or nuclear facilities, the interaction of energetic particles with semiconducting materials may trigger the creation of electron-hole pairs or displace atoms from their equilibrium position, thus degrading the microelectronic devices performances. Although the effects of the displacement of atoms, known as displacement damage (DD), are well identified from a technological point of view, with the appearance of phenomena like Dark Current (DC) or Random Telegraph Signal (RTS) arising into image sensors for example, a complete fundamental picture capable of explaining those technologically observed effects is still missing [1]. In this purpose, we have been developing a multiscale simulation approach, capable of treating physical processes ranging from particle-matter interactions to electronic properties of the defects, thus involving simulation techniques of very different size and time scales. Among those simulation techniques, Molecular Dynamics (MD) is employed for simulating the propagation of the collision cascades in the materials. The nature of collision cascades, stochastic highly out of equilibrium phenomena encompassing nuclear and electronic stopping power physics, makes it a challenge to be accurately simulated with MD. This chapter clearly presents the issues related to collision cascades simulations and offers solutions.

*Contact: thomas.jarrin@cea.fr 


\subsection{Introduction}

DC and RTS both correspond to a measured current in an image sensor in the absence of light. When the measured current is constant it is called DC, whereas when it oscillates between discrete values of current, it is called RTS. Both mechanisms can be explained by the promotion of electrons in the conduction band, and holes in the valence band, via mechanisms which do not involve photons. Such events are highly improbable in bulk materials without energy levels in the band gap created by the presence of defects in the material which reduces the energy necessary for the promotion of electrons. Figure 16.1 illustrates the processes made possible by the creation of defect levels in the band gap, named Shockley-Read-Hall (SRH) mechanisms [2].

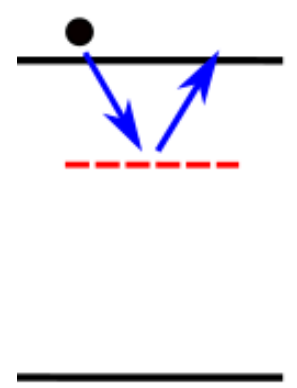

(i)

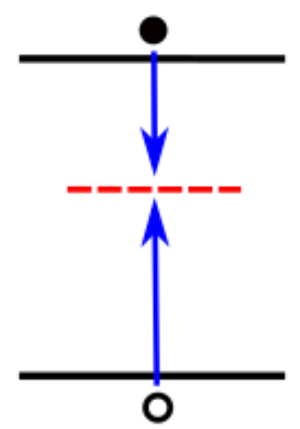

(ii)

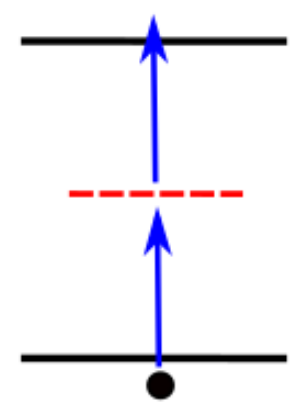

(iii)
Conduction

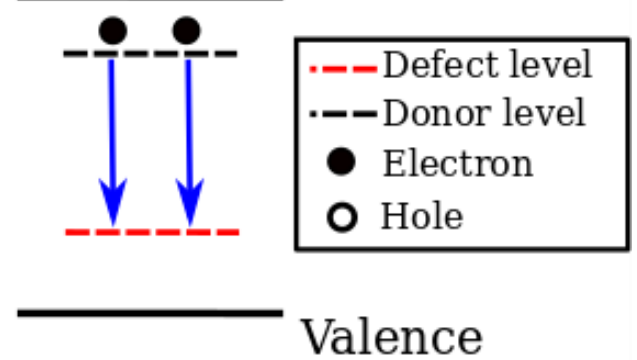

(iv)

Figure 16.1. Mechanisms of devices degradation due to defects level: (i) trapping; (ii) recombination: (iii) thermal generation; (iv) majority carriers (here electrons) concentration decrease.

Among those four processes, only thermal generation (iii) results in an increase in the number of carriers (electrons in conduction, holes in valence) and can thus be considered responsible for DC and RTS in image sensors. However, linking specific defects structures to DC-RTS signal is not an easy task. Indeed, the carrier generation rate of defects levels in the band gap are different for every defect, as each defect type has its own properties (energy level, electronic activity ...) and will therefore contribute differently to DC-RTS. Moreover, experimentally linking a particular defect with DC-RTS measurements is very difficult, as the intrinsic electronic properties of defects are hardly accessible experimentally. Thus, getting to understand the technologically observed phenomena of DC-RTS from a fundamental point of view requires resorting to simulations.

We have developed a global simulation approach, already entirely applied on $\mathrm{Si}$ [3-5] and partially on $\mathrm{Ge}$ and $\mathrm{Si}-\mathrm{Ge}$ alloys [6], whose purpose is to simulate the complete process of DD in semiconductors, in order to link simulation-based atomistic observations with experimentally observed electronic phenomena like DC and RTS. Figure 16.2 represents the scheme of the global simulation approach.

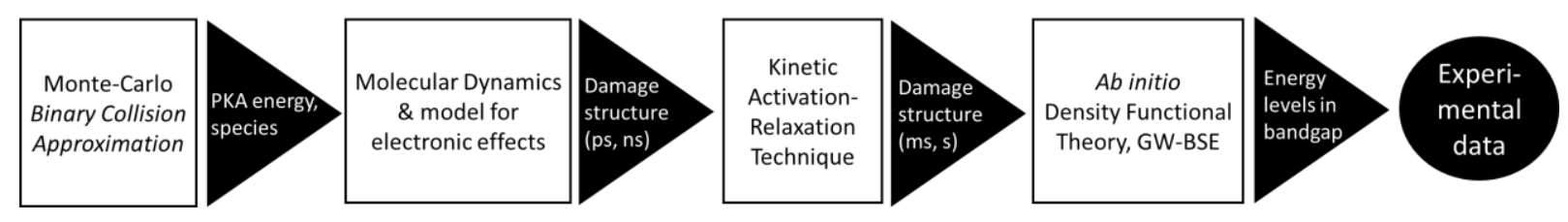

Figure 16.2. Scheme of the global simulation approach.

The first step in the process of DD and also the first step of our simulation approach is the interaction between the incident energetic particle and the matter. It is performed with a Monte 
Carlo (MC) code in the Binary Collision Approximation (BCA) [7], Geant4 [8-10]. Matter being described as continuous and not discrete (atomistic description) within Geant4, the main information which can be obtained from this step is the energy of the created Primary Knock-On atoms (PKAs) following the interaction with the incident energetic particle. The typical energies retrieved from the MC step will be employed to obtain an atomistic description of the propagation of the cascades of collisions in the materials thanks to the MD code LAMMPS [11]. The MD step is initiated by giving one atom (the PKA) a direction and an energy representative of the energies obtained in the previous step. It allows to investigate collisions between atoms and thus the creation of defects up to a simulation time of few ns at most, after which there is not enough energy in the system to cross the energy barriers and further relax the system. This is the reason why, in order to reach longer timescales, we employ the kinetic Activation Relaxation Technique (kART) implemented in the code of the same name $[12,13]$, specifically designed to observe defects diffusion and structure relaxation on long timescales (up to the second and more). At the end of the $k A R T$ run, the damage structure is relaxed on time scales comparable to experimental timescales. The defects remaining in the structure at the end of this step can be classified and the most prominent ones in terms of frequency should be characterized with $a b$ initio techniques aiming at accessing their structural, vibrational and electronic properties, finally making the link with experiments [14]. This consists in the fourth and last step of our simulation approach.

Each step of this simulation approach requires expertise in the simulation technique employed and the physical processes simulated. In this case study, we will focus on the second step, i.e., MD. Collision cascades are highly out of equilibrium phenomena, due to the high energies involved during the collision phase. The interatomic potential employed should be adequate for calculating forces between atoms separated by very short distances (less than $1 \AA$ ). However, commonly employed classical interatomic potentials are not accurate at short interatomic distances. Moreover, collision cascades are stochastic. Depending on the initial direction of the PKA (the direction taken by the PKA after the interaction with the neutron), the cascades properties, i.e., mainly size, shape, number of defects and clusters, can be drastically different. MD simulations of collision cascades should explicitly cope with the stochasticity of the cascades. Finally, electronic stopping power, although it contributes less to total stopping than nuclear stopping power at the considered energies (tens of $\mathrm{keV}$ ), is not negligible and should be included. This means incorporating the effects caused by inelastic collisions between ions and electrons in a simulation technique where the electrons are not explicitly taken into account. The first part of the case study explains how to derive an adequate interatomic potential, the second part presents in detail the issue related to stochasticity and propose solutions and the last part focuses on the inclusion of electronic effects into MD simulations.

\subsection{Interatomic potential}

Most of the applications for which MD is used for concerns materials in equilibrium or near equilibrium. Interatomic potentials are thus designed to correctly model the materials when the interatomic distances are not too far from the equilibrium ones [15]. For collision cascades, the physics of the phenomena at stake makes their use inappropriate. At first glance, a collision cascade can be divided into two phases. A first phase in which some atoms having a very high kinetic energy (the PKA and the atoms it shocked, Secondary Knock-On Atoms (SKAs)) collide 
with other atoms in the matter and thus get very close one from each other (the higher the energy, the shorter the interatomic distance). A second phase, during which there is no collision anymore because the atoms have lost most of their kinetic energy through shocks, and during which the defective structure will relax and defects eventually heal. The second phase can be described with general purpose interatomic potential, but the first collisional phase requires an accurate description of the physics at short interatomic distances, which goes beyond the capabilities of general-purpose commonly employed potentials. It is therefore mandatory to employ a potential accurate in a very broad range of interatomic distances.

Developing an interatomic potential from scratch is a cumbersome work that may repel many. In order to benefit from the huge amount of work carried out on the development of interatomic potentials during the last decades, we present a method, already employed in early studies of DD with MD [16] which eases the development of an interatomic potential suitable for collision cascades simulations. The idea is to combine an existing general-purpose potential to an existing repulsive two-body potential accurate to describe collisions. In the case of $\mathrm{Si}$ and Ge, we have successfully combined the two-body part of the well-known Stillinger-Weber (SW) [17] potential to the Ziegler-Biersack-Littmark (ZBL) [18] repulsive potential with a Fermi function $F(r)$ as follows:

$$
V_{t o t}(r)=(1-F(r)) V_{Z B L}(r)+F(r) V_{S W}(r)
$$

With:

$$
F(r)=\frac{1}{1+\exp \left(b_{f}\left(r-r_{f}\right)\right)}
$$

Where $V_{\text {tot }}$ is the final total potential, $b_{f}$ and $r_{f}$ parameters of the Fermi function that needs to be chosen so that the transition is smooth for the potential but also for the first and if possible second derivatives of this potential. Equation (16.1) ensures accurate forces calculations for every possible interatomic distance in a simple and efficient way.

\subsection{Stochasticity}

At the very early stage of the collision cascade, right after the collision with the neutron, the PKA can be ejected from its equilibrium site in an infinity of direction. Each possible direction will lead to a different sequence of collisions and therefore to different cascades sizes and number of defects created. To base observations on steady, trustworthy ground, it is thus necessary to work with statistical quantities obtained by running large number of simulations in various directions. As an example, and to prove the necessity of treating collision cascades results in a statistically rigorous way, we have performed a convergence study of cascades properties in Si initiated with PKAs of $1 \mathrm{keV}$, with respect to the number of simulations performed. In this study, we look at three properties: the number of defects, the number of clusters and the PKA penetration depth. We are interested in the mean values and the whiskers plot distributions for each of these properties with respect to the simulation performed. Moreover, two methods to choose the initial direction of the PKA are compared. In the first one the initial directions of the PKA are randomly chosen, whereas in the second one the initial directions of the PKA are chosen based on the symmetry of the material under investigation. 
For sake of conciseness, only the main conclusions of this study are presented here (see more details in [19]). We have found that even for a simple material like $\mathrm{Si}$, and energies as low as $1 \mathrm{keV}$, the cascades properties cannot be considered converged, i.e., reproducible and precise, with less than 50 simulations performed: important variations in mean values and distributions are observed. We would recommend performing at least 70 simulations. Moreover, the methods based on crystal symmetry to choose the initial directions of the PKA gave better results in terms of reproducibility. This study performed on $1 \mathrm{keV}$ collision cascades in Si reveals the convergence of cascades results with respect to the number of simulations performed is not trivial at all. It should always be checked, even for low energy cascades. We know a complete study like the one we did cannot be performed prior to every study, but a lighter computationally non-prohibitive study should be performed each time. Also, statistical quantities should always be clearly presented with quantitative incertitude, error bars and graphical representation of distributions.

\subsection{Electronic effects}

In MD simulations of Swift Heavy Ion (SHI) irradiation, the inclusion of electronic effects is a mandatory established step as the defects track produced by the passage of the ion is directly linked to electronic excitations [20-22]. Although the main energy loss mechanism for the PKAs and SKAs in collision cascades is nuclear stopping power, the effects of electronic stopping are far from being negligible. According to SRIM, a $10 \mathrm{keV} \mathrm{Si}^{+}$ion loses up to $18 \%$ of its energy to electrons in Si.

The most widely employed model to include electronic effects into MD simulations is the derivation of the Two-Temperature Model (TTM) [23] specifically designed to be used with MD, by Duffy et al. [24]. Electronic stopping and electron-ion coupling effects are incorporated into the simulations via the formalism of a Langevin thermostat $[25,26]$ : ions are immersed into a bath of electrons. Electronic stopping power is described by a friction force and the coupling between electrons and ions both by a friction force and a random stochastic force term. The classical equations of motions of MD thus have to be modified accordingly:

$$
\begin{gathered}
m_{I} \frac{d \vec{v}_{I}}{d t}=-\Delta U_{I}-\gamma_{I} \vec{v}_{I}+\vec{F}_{I}^{\text {stoch }} \\
\gamma_{I}=\left\{\begin{array}{cc}
\gamma_{p}, & \left\|\overrightarrow{v_{I}}\right\|<v_{0} \\
\gamma_{p}+\gamma_{s}, & \left\|\overrightarrow{v_{I}}\right\| \geq v_{0}
\end{array}\right.
\end{gathered}
$$

Where $m_{I}$ is the mass of ion $I, v_{I}$ its velocity, $-\Delta U_{I}$ the gradient of adiabatic forces deriving from the interatomic potential, $\gamma_{I}$ the friction coefficient being composed of $\gamma_{S}$ the electronic stopping power and $\gamma_{p}$ the electron-ion coupling, $v_{0}$ is the cutoff velocity for the electronic stopping power and $\vec{F}_{I}^{\text {stoch }}$ the stochastic force term.

Within the TTM, the simulation box is divided into a regular grid of cubic cells. In each cell is defined an electronic temperature $T_{e}$ (the temperature of the bath) and an ionic temperature $T_{a}$. Heat diffusion in the electronic subsystem is governed by the following heat diffusion equation: 


$$
C_{e} \frac{\partial \mathrm{T}_{\mathrm{e}}}{\partial \mathrm{t}}=\nabla\left(\kappa_{e} \nabla T_{e}\right)-g_{p}\left(T_{e}-T_{a}\right)+g_{s} T_{a}^{\prime}
$$

Where $C_{e}$ is the electronic specific heat, $\kappa_{e}$ the electronic heat conductivity $g_{p}$ a parameter deriving from $\gamma_{p}, g_{s}$ a parameter deriving from $\gamma_{s}$ and $T_{a}$ ' a source term homogeneous to a temperature.

The TTM is implemented in many open access MD codes (LAMMPS, DL_POLY [27]), has proven to allow the study within MD of complex phenomena involving electrons [28] and is not computationally demanding. However, it suffers from some limitations.

Firstly, many parameters have to be specified by hand by the user. The influence of those parameters on collision cascades outputs is not very well known and understood. Moreover, choosing the values for the parameters of the TTM is tricky as in some cases trustworthy experimental or theoretical values are missing. For example in $\mathrm{Si}$, two values different by a factor of three exist in the literature for $\gamma_{p}[29,30]$ and $C_{e}$ is known only in a narrow temperature range compared to the temperature range spanned during the simulations [31]. In order to assess the influence of the TTM parameters on simulation results in Si and Ge we have realized a parametric study [32] covering the main parameters of the TTM. Again, for sake of conciseness, only main conclusions are presented here. Readers are referred to the original article for more details. This study pointed out the significant influence $\gamma_{s}$ and $\gamma_{p}$ have on the number of defects created. Increasing both parameters results in a drastic decrease of the number of defects.

Table 16.1 shows the mean (over 100 simulations) number of defects obtained in Si and Ge depending on the values chosen for $\gamma_{s}$ and $\gamma_{p}$ for self PKAs of $10 \mathrm{keV}$. The parameter chosen for the first line of Table 16.1 corresponds for $\gamma_{s}$ to the value calculated with SRIM at $10 \mathrm{keV}$ and for $\gamma_{p}$ to the value given by [29]. As no value is available for $\gamma_{p}$ in Ge, we choose to use the same as for $\mathrm{Si}$. The significant influence of those parameters on the number of defects highlights the fact that they have to be chosen with care. As far as $\gamma_{s}$ is concerned, the value can be expected to be accurate as it can be calculated with SRIM. However, $\gamma_{p}$ values are badly defined and the incertitude on those values is huge, sometimes pushing people to treat it as a parameter and to perform simulations with a range of values [24].

Table 16.1. Mean number of defects in Si and Ge at the end of collision cascades initiated with self PKAs of $10 \mathrm{keV}$ with respect to the $\gamma$ parameters chosen. Mean values are calculated over 100 simulations. Between brackets is indicated the Standard Error of the Mean. The number of defects is calculated based on the Lindemann [33] methods described in [6]. Units given are the one used within LAMMPS for "metal units".

\begin{tabular}{cc|cc}
\hline \multicolumn{1}{c|}{ Si } & & \multicolumn{2}{c}{ Ge } \\
\hline Parameters & Defects & Parameters & Defects \\
\hline$\gamma_{\boldsymbol{s}}=39.4 \mathrm{~g} / \mathrm{mol} / \mathrm{ps}$ & \multirow{2}{*}{$622(13)$} & $\gamma_{s}=39.4 \mathrm{~g} / \mathrm{mol} / \mathrm{ps}$ & $2361(58)$ \\
$\gamma_{\boldsymbol{p}}=24.4 \mathrm{~g} / \mathrm{mol} / \mathrm{ps}$ & & $\gamma_{p}=24.4 \mathrm{~g} / \mathrm{mol} / \mathrm{ps}$ & \\
$\gamma_{\boldsymbol{s}}=100.0 \mathrm{~g} / \mathrm{mol} / \mathrm{ps}$ & \multirow{2}{*}{$416(9)$} & $\gamma_{s}=100.0 \mathrm{~g} / \mathrm{mol} / \mathrm{ps}$ & $1569(44)$ \\
$\gamma_{\boldsymbol{p}}=24.4 \mathrm{~g} / \mathrm{mol} / \mathrm{ps}$ & & $\gamma_{p}=24.4 \mathrm{~g} / \mathrm{mol} / \mathrm{ps}$ & \\
$\gamma_{\boldsymbol{s}}=24.4 \mathrm{~g} / \mathrm{mol} / \mathrm{ps}$ & \multirow{2}{*}{$410(8)$} & $\gamma_{s}=24.4 \mathrm{~g} / \mathrm{mol} / \mathrm{ps}$ & $1338(33)$ \\
$\gamma_{\boldsymbol{p}}=100.0 \mathrm{~g} / \mathrm{mol} / \mathrm{ps}$ & & $\gamma_{p}=100.0 \mathrm{~g} / \mathrm{mol} / \mathrm{ps}$ & \\
\hline
\end{tabular}

The second significant limitation of the TTM is the treatment of electronic density. In the TTM, electronic density is considered constant in the entire simulation box. As there is no concept of 
locality and position dependence of the density, the electronic stopping power does not vary with the density. As a matter of fact, a projectile in a channelling direction will lose as much energy to the electrons as a projectile passing very close to other ions whereas it is known both by experiments [34] and Real-Time Time Dependent Density Functional Theory (RT-TDDFT) calculations [35] that the electronic stopping power is crystal direction (i.e. electronic density) dependent.

The two limitations exposed in this section constitute strong motivations to try to refine the TTM. A novel model by Caro et al. [36] has been developed in this sense. In this model, the concept of locality arises in the electronic density and the electronic stopping power is not scalar anymore but tensorial. It is therefore dependent on electronic density (and thus position of other atoms) and velocity of nearby atoms. Moreover, electron-ion coupling and electronic stopping power are treated as originating from the same physical basis, making unnecessary the specification by the user of parameter values both for electronic stopping and electron-ion coupling. In practice, the relation between electronic stopping power and the electronic density seen by the projectile has to be derived from RT-TDDFT calculations and given as input to the code under the form of tabulated values. The same tabulated values are used by the code to treat the electron-ion coupling part of the model. For details about the model and data used as input see [37] and [38]. This model overtakes the main practical and theoretical limitations of the TTM.

\subsection{Conclusions}

A global simulation approach aiming at simulating DD in semiconducting materials has been presented. The MD step of this simulation approach has been covered. Extensive care should be given to the interatomic potential to accurately describe collisions. Coping with the stochasticity of the cascades requires running large sets of simulations and working with statistical quantities. Concerning electronic effects, we have seen that their inclusion has significant outcomes on cascades results [32]. The most widely used model so far, also the model we have been using in our research group, the TTM, suffers from some intrinsic limitations which should be overtaken to more accurately assess the influence of electronic effects into collision cascades. A very recently developed model specifically targets the limitations of the TTM by benefiting from the recent developments in TDDFT calculations of electronic stopping power. We believe this model will become in the next few years the standard procedure for incorporating electronic effects into MD simulations.

\section{References}

[1] J.R. Srour, J.W. Palko, Displacement Damage Effects in Irradiated Semiconductor Devices, IEEE Trans Nucl Sc. 60 (2013) 1740-1766. https://doi.org/10.1109/TNS.2013.2261316.

[2] Physics of Semiconductor Devices, 3rd Edition / Wiley, Wiley.Com. (n.d.). https://www.wiley.com/enus/Physics+of+Semiconductor+Devices\%2C+3rd+Edition-p-9780471143239 (accessed December 10, 2020).

[3] M. Raine, A. Jay, N. Richard, V. Goiffon, S. Girard, M. and Gaillardin, P. Paillet, Simulation of Single Particle Displacement Damage in Silicon - Part I: Global Approach and Primary Interaction Simulation, IEEE Trans Nucl Sc. 64 (2017) 133-140. https://doi.org/10.1109/TNS.2016.2615133.

[4] A. Jay, M. Raine, N. Richard, N. Mousseau, V. Goiffon, A. Hémeryck, P. Magnan, Simulation of Single 
Particle Displacement Damage in Silicon-Part II: Generation and Long-Time Relaxation of Damage Structure, IEEE Trans Nucl Sc. 64 (2017) 141-148. https://doi.org/10.1109/TNS.2016.2628089.

[5] A. Jay, A. Hémeryck, N. Richard, L. Martin-Samos, M. Raine, A. Le Roch, N. Mousseau, V. Goiffon, P. Paillet, M. Gaillardin, P. Magnan, Simulation of Single Particle Displacement Damage in Silicon-Part III: First Principle Characterization of Defect Properties, IEEE Trans Nucl Sc. 65 (2018) 724-731. https://doi.org/10.1109/TNS.2018.2790843.

[6] T. Jarrin, A. Jay, M. Raine, N. Mousseau, A. Hémeryck, N. Richard, Simulation of Single Particle Displacement Damage in Si1-xGex alloys - Interaction of Primary Particles with the Material and Generation of the Damage Structure, IEEE Trans Nucl Sc. 67 (2020) 1273-1283. https://doi.org/10.1109/TNS.2020.2970488.

[7] M.T. Robinson, The binary collision approximation: Background and introduction, Rad Eff Def Sol. null (1994) 3-20. https://doi.org/10.1080/10420159408219767.

[8] S.A. <i>et al.</i>, GEANT4, a simulation toolkit, Nulc Instr Meth Phys R A. 506 (2003) 250-303. https://doi.org/10.1016/S0168-9002(03)01368-8.

[9] J.A. <i>et al.</i>, Geant4 developments and applications, IEEE Trans Nucl Sc. 53 (2006) 270-278. https://doi.org/10.1109/TNS.2006.869826.

[10] J.A. <i>et al.</i>, Recent develompents in Geant4, Nulc Instr Meth Phys R A. 835 (2016) 186-225. https://doi.org/10.1016/j.nima.2016.06.125.

[11] S. Plimton, Fast Parallel Algorithms for Short-Range Molecular Dynamics, J Comput Phys. 117 (1995) 1-19. https://doi.org/10.1006/jcph.1995.1039.

[12] F. El-Mellouhi, N. Mousseau, L.J. Lewis, Kinetic activation-relaxation technique: An off-lattice selflearning kinetic Monte Carlo algorithm, Phys Rev B. 78 (2008) 153202. https://doi.org/10.1103/PhysRevB.78.153202.

[13] N. Mousseau, L.K. Béland, P. Brommer, J.F. Joly, F. El-Mellouhi, E. Machado-Charry, M.C. Marinica, P. Pochet, The Activation-Relaxation Technique: ART Nouveau and Kinetic ART, J Mol Opt Phys. 2012 (2012) 925278. https://doi.org/10.1155/2012/925278.

[14] A comprehensive theoretical picture of E centers in silicon: From optical properties to vacancymediated dopant diffusion: Journal of Applied Physics: Vol 127, No 8, (n.d.). https://aip.scitation.org/doi/abs/10.1063/1.5140724 (accessed December 10, 2020).

[15] K. Nordlund, J. Keinonen, A. Kuronen, Effect of the interatomic Si-Si potential on vacancy production during ion implantation of Si, Phys Scr. T54 (1994) 34-37. https://doi.org/10.1088/00318949/1994/T54/007.

[16] K. Nordlund, M. Ghaly, R.S. Averback, M. Caturla, T. Diaz de la Rubia, J. Tarus, Defect production in collision cascades in elemental semiconductors and fcc metals, Phys Rev B. 57 (1998) 7556-7570. https://doi.org/10.1103/PhysRevB.57.7556.

[17] F.H. Stillinger, T.A. Weber, Computer simulation of local order in condensed phases of silion, Phys Rev B. 31 (1985) 5262-5271. https://doi.org/10.1103/PhysRevB.31.5262.

[18] J.F. Ziegler, J.P. Biersack, U. Littmark, The Stopping Range of Ions in Solids, 1st ed., Pergamon Press, N.Y, 1983.

[19] T. Jarrin, A. Jay, N. Richard, A. Hémeryck, Coping with the stochasticity of collision cascades in Molecular Dynamics simulations, Nucl. Instrum. Methods Phys. Res. Sect. B Beam Interact. Mater. At. 500-501 (2021) 1-9.

[20] D.A. Young, Etching of Radiation Damage in Lithium Fluoride, Nature. 182 (1958) 375-377. https://doi.org/10.1038/182375a0.

[21] E.C.H. Silk, R.S. Barnes, Examination of fission fragment tracks with an electron microscope, Philos. Mag. J. Theor. Exp. Appl. Phys. 4 (1959) 970-972. https://doi.org/10.1080/14786435908238273.

[22] N. Itoh, D.M. Duffy, S. Khakshouri, A.M. Stoneham, Making tracks: electronic excitation roles in forming swift heavy ion tracks, J. Phys. Condens. Matter. 21 (2009) 474205. https://doi.org/10.1088/09538984/21/47/474205. 
[23] A. Caro, M. Victoria, Ion-electron interaction in molecular dynamics cascade, Phys Rev A. 40 (1989) 2287-2291. https://doi.org/10.1103/PhysRevA.40.2287.

[24] D.M. Duffy, A.M. Rutherford, Including the effects of electronic stopping and electron-ion interactions in radiation damage simulations, J Phys Cond Matter. 19 (2007) 1-11. https://doi.org/10.1088/09538984/19/1/016207.

[25] Sur la théorie du mouvement brownien - Wikisource, (n.d.). https://fr.wikisource.org/wiki/Sur_la_th\%C3\%A9orie_du_mouvement_brownien (accessed December 10, 2020).

[26] G.E. Uhlenbeck, L.S. Ornstein, On the Theory of the Brownian Motion, Phys. Rev. 36 (1930) 823-841. https://doi.org/10.1103/PhysRev.36.823.

[27] I.T. Todorov, W. Smith, K. Trachenko, M.T. Dove, DL_POLY_3: new dimensions in molecular dynamics simulations via massive parallelism, J. Mater. Chem. 16 (2006) 1911-1918. https://doi.org/10.1039/B517931A.

[28] R. Darkins, D.M. Duffy, Modelling radiation effects in solids with two-temperature molecular $\begin{array}{llllll}\text { dynamics, } & \text { Comput. } & \text { Mater. } & \text { Sci. } & 147 & \text { (2018) }\end{array}$ https://doi.org/10.1016/j.commatsci.2018.02.006.

[29] O. Osmani, I. Alzaher, T. Peters, B.Ban d'Etat, A. Cassimi, H. Lebius, I. Monnet, N. Medvedev, B. Rethfled, M. Scleberger, Damage in crystalline silicon by swift heavy ion irradiation, Nulc Instr Meth Phys R B. 282 (2012) 43-47. https://doi.org/10.1016/j.nimb.2011.08.036.

[30] A. Chettah, H. Kucal, Z.G. Wang, M. Kac, A. Meftah, M. Toulemonde, Behavior of crystalline silicon under huge electronic excitations: A transient thermal spike description, Nulc Instr Meth Phys R B. 267 (2009) 2719-2724. https://doi.org/10.1016/j.nimb.2009.05.063.

[31] C.J. Glassbrenner, G.A. Slack, Thermal Conductivity of Silicon and Germanium from 3K to Melting Point, Phys Rev A. 134 (1966) 1058-1069. https://doi.org/10.1103/PhysRev.134.A1058.

[32] T. Jarrin, A. Jay, A. Hémeryck, N. Richard, Parametric study of the Two-Temperature Model for Molecular Dynamics simulations of collisions cascades in Si and Ge, Nucl. Instrum. Methods Phys. Res. Sect. B Beam Interact. Mater. At. 485 (2020) 1-9. https://doi.org/10.1016/j.nimb.2020.09.025.

[33] H. Hensel, H.M. Urbassek, Implantation and damage under low-energy Si self-bombardment, Phys Rev B. 57 (1998) 4756. https://doi.org/10.1103/PhysRevB.57.4756.

[34] S. Lohmann, D. Primetzhofer, Disparate Energy Scaling of Trajectory-Dependent Electronic Excitations for Slow Protons and He Ions, Phys. Rev. Lett. 124 (2020) 096601. https://doi.org/10.1103/PhysRevLett.124.096601.

[35] C.-W. Lee, J.A. Stewart, R. Dingreville, S.M. Foiles, A. Schleife, Multiscale simulations of electron and ion dynamics in self-irradiated silicon, Phys. Rev. B. 102 (2020) 024107. https://doi.org/10.1103/PhysRevB.102.024107.

[36] A. Tamm, M. Caro, A. Caro, G. Samolyuk, M. Klintenberg, A.A. Correa, Langevin Dynamics with Spatial Correlations as a Model for Electron-Phonon Coupling, Phys. Rev. Lett. 120 (2018) 185501. https://doi.org/10.1103/PhysRevLett.120.185501.

[37] M. Caro, A. Tamm, A.A. Correa, A. Caro, Role of electrons in collisions cascades in solids. 1. Dissipative model, Phys Rev B. 99 (2019) 174301. https://doi.org/10.1103/PhysRevB.99.174301.

[38] A. Tamm, M. Caro, A. Caro, A.A. Correa, Role of electrons in collision cascades in solids. 2. Molecular dynamics, Phys Rev B. 99 (2019) 174302. https://doi.org/10.1103/PhysRevB.99.174302. 



\title{
17. Modelling ion implantation ranges with $a b$ initio electronic stopping power
}

\author{
Andrea E. Sand*
}

We present the results of combining high-fidelity quantum mechanical simulations of ion-electron interactions, using real-time time-dependent density functional theory (rt-TDDFT), with larger scale atomistic simulations. The larger scale simulations employ a molecular dynamics-based method for efficiently simulating ion ranges. The combination of these methods offers a way to directly compare rt-TDDFT predictions to experimentally measureable quantities in the form of ion ranges and implantation profiles.

*Contact: andrea.sand@aalto.fi 


\subsection{Introduction}

Fast charged particles interact with both the electrons and the nuclei in a material, leading to a loss of kinetic energy of the traveling particle. The average rate of energy losses, $\mathrm{dE} / \mathrm{dx}$, is known as the stopping power. Accurate values of the stopping power for projectiles in different materials are needed in order to correctly predict ion implantation ranges, energy deposition, and damage production under different irradiation conditions. Different mechanisms dominate the stopping power, depending on the nature and kinetic energy of the projectile.

Tungsten $(\mathrm{W})$ is a prime candidate material for future fusion reactors, where radiation damage predictions are of paramount importance for safety considerations. Under neutron irradiation, energetic atomic recoils become charged projectiles in the target material, and the resulting radiation damage depends sensitively on the energy dissipation mechanisms from such recoils. Therefore we investigate here the energy losses of $\mathrm{W}$ projectiles in $\mathrm{W}$. Tungsten recoils from the $14 \mathrm{MeV} \mathrm{D}-\mathrm{T}$ fusion neutrons have velocities in the range $\mathrm{v}<\mathrm{v} 0$, where $\mathrm{v} 0$ is the Bohr velocity.

For stopping power considerations, this energy range is generally referred to as the low-energy range. The stopping power of low-energy ions can be divided into (i) the contribution from energy losses to electrons through excitation and ionization of atoms in the target materials and of the projectile itself, called the electronic stopping power Se, and (ii) the nuclear stopping power Sn, which describes the average slowing down of an energetic projectile due to elastic collisions with nuclei in the target material.

Several theories have been developed to describe Se for low-energy ions. The LSS theory, developed by Lindhard, Scharff and Schiott [15], is based on the assumption of a homogeneous electron density, as is the theory by Fermi and Teller [9]. The electronic stopping for a homogeneous electron density has also be derived quantum mechanically by Echenique, Nieminen and Ritchie [6]. The Firsov model, on the other hand, describes the electronic stopping as a series of atomic collisions, where the stopping is atom-specific. These theories all have in common a linear dependence on the projectile velocity $\mathrm{v}$.

Typical for the theories of electronic stopping in this energy range is that they contain parameters that are difficult to determine, such as the charge state of the projectile, or the impact parameter of the atomic collisions. Semi- empirical models address such shortcomings by extensive fitting to experimental data. A commonly used software package providing this type of semi-empirical prediction of the stopping power is the Stopping and Range of Ions in Matter (SRIM) [27]. SRIM gives very good predictions of the stopping power for cases where experimental data is available, but can deviate up to $10-20 \%$ in cases where data is unavailable [25].

The stopping power can be determined experimentally based on the range profile of projectiles implanted in a target $[7,8]$, or by measuring the energy change of projectiles passing through a thin foil $[19,16]$. Even an experimentally rigorous method and accurate measurement requires the separation of the different components of the stopping power, which relies heavily on theoretical considerations. For projectiles with increasing energy, the electronic stopping increasingly dominates over the nuclear stopping, at some point allowing the latter to be neglected. For lower energy projectiles, nuclear stopping can be strongly reduced by employing channeling trajectories. However, even for such trajectories, the nuclear stopping cannot be completely disregarded, and it in- creases as the projectile energy decreases. This is demonstrated 
in the work by Eriksson et al. [7], who base their analysis on the expected velocity proportionality of the electronic stopping. A linear dependence on velocity is clear in the experimental data down to the velocities where also nuclear stopping is assumed to affect results, but a definitive separation of $\mathrm{Se}$ and $\mathrm{Sn}$ is not possible in the lower velocity limit.

For low-energy projectiles, the electronic band structure of the material, and the orbital structure of the projectile, play an important role in the interactions between the projectile and the electrons. The first principles method of real-time time-dependent density functional theory (rtTDDFT) allows us to explicitly calculate energy losses accounting for both band structure, local atomic orbitals, as well as the specific trajectories or local environments of the projectile. One of the original TDDFT calculations of electronic stopping demonstrated the effect of the band gap in insulators, providing an explanation for the experimental observation of a non-linearity in the electronic stopping in LiF [20].

Here, we show how the electronic stopping power of $\mathrm{W}$ ions in $\mathrm{W}$ calculated from first principles can be used in a multi-scale approach, allowing us to benchmark the TDDFT predictions against experimental ion implantation ranges.

\subsection{Electronic stopping power calculations}

A review of the method for calculating electronic stopping using rt-TDDFT can be found in [4]. We give a brief description of the main elements of the procedure here.

The total energy of the system to be simulated consists of several components, which roughly can be considered as a) the kinetic energy of the projectile, b) the kinetic energy of the target atoms, c) the interaction, or potential energy, of all the particles in the system, and d) the excitation energy of the electronic system. The goal is to determine the change in (d) as a function of the projectile displacement. We can approach the problem through a number of simplifications.

(1) We fix the velocity of the projectile to a constant value. This makes the analysis more straightforward, and is motivated by the dependence of the electronic stopping power on the velocity of the projectile, and our desire to determine the value for a given velocity. Furthermore, the simulation covers a very short period of time, on the order of a femtosecond, and the projectile velocity would not change considerably during this time along a channeling trajectory.

(2) We fix the positions of the target atoms. This approach is motivated by the fact that the projectile travels through the material significantly faster than the target atoms respond to the interaction with the projectile, which is particularly true for a channeling trajectory.

(3) We identify the potential energy of the interactions as the ground state energy in the BornOppenheimer (BO) approximation of the system with the given atomic configuration, including the projectile. With the above computational simplifications and approximations, we can identify the energy increase of the system, minus the BO energy, as the excitation energy of the electrons [2]. This is the energy gained by the electronic system, and hence lost by the projectile. When we average this quantity over the (periodic) trajectory, we obtain a value for the electronic stopping power experienced by the projectile in the given geometry. To obtain an average value for trajectories in an amorphous target, or equivalently for random trajectories in a crystalline target, 
one would need to average over many different trajectories [12]. However, in the current study we are interested specifically in the channeling geometry, hence only one trajectory is needed.

The rt-TDDFT calculations [23] were carried out with the Qb@ll code [13, 5, 24], where KohnSham wave functions expanded in a plane-wave basis were used to represent the electrons. A cutoff energy value of 140 Ry provided converged results for this geometry. The adiabatic local density approximation (ALDA)

[3] was used to represent the exchange and correlation (XC) functional. The ions were represented by normconserving nonlocal pseudopotentials, factorized in the Kleinman-Bylander form [14].

The initial state of the system can be obtained either with or without the projectile. Obtaining the ground state without the projectile can be motivated in the view that the material through which the projectile travels exists without the projectile prior to its approach. However, since our projectile W has a fairly high Z-number, the sudden introduction of this into the lattice would likely cause severe disruptions. The initial ground state was therefore obtained with the projectile already present in the channel.

As the projectile begins to move, there is an initial transient period as the system adjusts to the movement, but a steady state is reached quite quickly. The steady state captures the response of the target material to the traveling projectile, in other words the quantity identified with the electronic stopping power. Figure 17.1 shows the electron density perturbation caused by the traveling $\mathrm{W}$ projectile in the $\mathrm{W}$ lattice. The perturbation of the electronic system extends past the nearest atomic rows. The image also illustrates how the perturbations are more pronounced in the wake of the projectile. When a simulation cell with periodic boundaries is used, care must also be taken to avoid traversing the same region of the material multiple times [4]. For a periodic trajectory and oscillating energy loss, an average rate of energy transfer can be obtained straightforwardly [21]. Figure 17.2 shows the Born-Oppenheimer energy for the trajectory in the $\left\langle\begin{array}{lll}1 & 0 & 0\end{array}\right\rangle$ channel in $\mathrm{W}$, the excitation energy of the electronic system obtained with TDDFT for a range of projectile velocities along this trajectory, and the energy difference, which in the center of the $\left\langle\begin{array}{lll}1 & 0 & 0\end{array}\right\rangle$ channel shows only minimal oscillations.

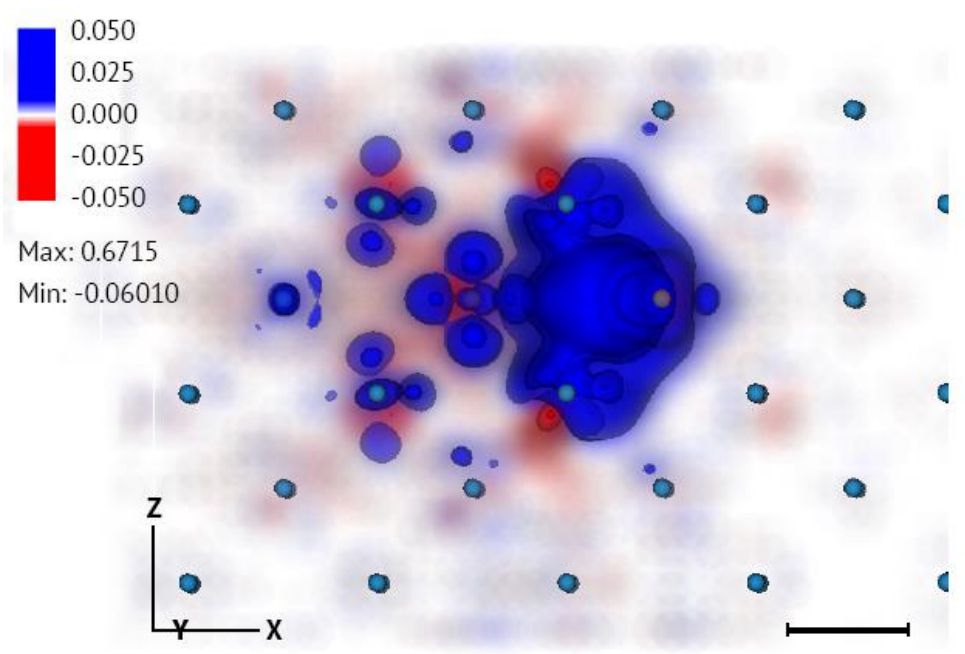

Figure 17.1. Perturbation of the electron density around a W projectile in the $\left\langle\begin{array}{lll}1 & 0 & 0\end{array}\right\rangle$ channel in W. 
Obtaining a value for the electronic stopping power with rt-TDDFT nevertheless involves a number of approximations, as well as computational restrictions due in particular to the many core electrons participating in the energy dissipation. For increasing projectile energies, and decreasing impact parameters, increasing numbers of core electron states must be calculated explicitly. While it has been shown that the projectile core electrons play a more important part than the target atom core electrons [26], computational limits are rapidly approached with the high-Z species W. For these calculations, a pseudopotential with 12 explicit electrons was used, and a number of runs were tested with a 20- electron pseudopotential. No difference was found in the predicted electronic stopping value. A further uncertainty arises from the fact that trajectories with small impact parameters may be inaccurately captured with the restricted dynamics employed in the computational technique that allows extracting the electronic energy losses from the total energy of the system. Although the channeling geometry minimizes these uncertainties, validating the predictions of rt-TDDFT by comparison to experiments is nevertheless desirable.

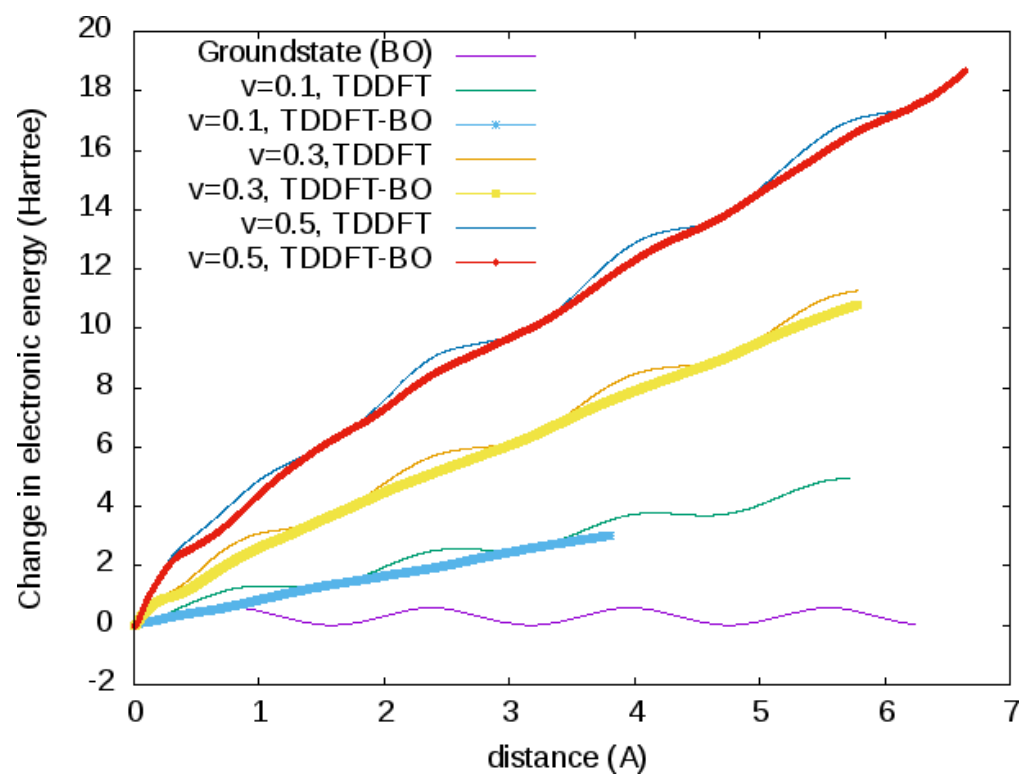

Figure 17.2. The BO and TDDFT electronic energies for $\mathrm{W}$ ions travelling through the $\langle 100\rangle$ channel in $\mathrm{W}$.

\subsection{Validation of rt-TDDFT predictions}

Fitting to the steady state region for different velocities gives the results shown in Figure 17.3(a) [23]. The predicted stopping power is proportional to the projectile velocity, in agreement with theoretical predictions. Figure 17.3(a) also shows the SRIM prediction of the stopping power for $\mathrm{W}$ in (amorphous) W. The stopping power predicted by rt-TDDFT in the $\left\langle\begin{array}{lll}1 & 0 & 0\end{array}\right\rangle$ channel is lower than that given by SRIM, by a factor of three. However, a lower value from these calculations can be expected based on electron density considerations for the channeling trajectory. Furthermore, the rt-TDDFT value is in the expected range compared to the experimentally measured values for other projectile species through the $\left\langle\begin{array}{lll}1 & 0 & 0\end{array}\right\rangle$ channel in tungsten (Figure 17.3(b)).

An experimental value for the electronic stopping power for low velocity $\mathrm{W}$ projectiles in the $\mathrm{W}$ $\left\langle\begin{array}{lll}1 & 0 & 0\end{array}\right\rangle$ channel is not available. However, a value for the maximum ion range Rmax is given in [7]. 
We therefore employ a multi-scale approach to compare the rt-TDDFT predictions directly to the experimentally measured range of the channeled ions. This involves explicitly including also the nuclear interactions. Here, the universal Ziegler-Biersack-Littmark (ZBL) potential [28] is used to describe the $\mathrm{W}$-W interaction, but increased accuracy could be obtained by using DFT-based repulsive potentials fitted for the specific projectile-target combination under study [29].
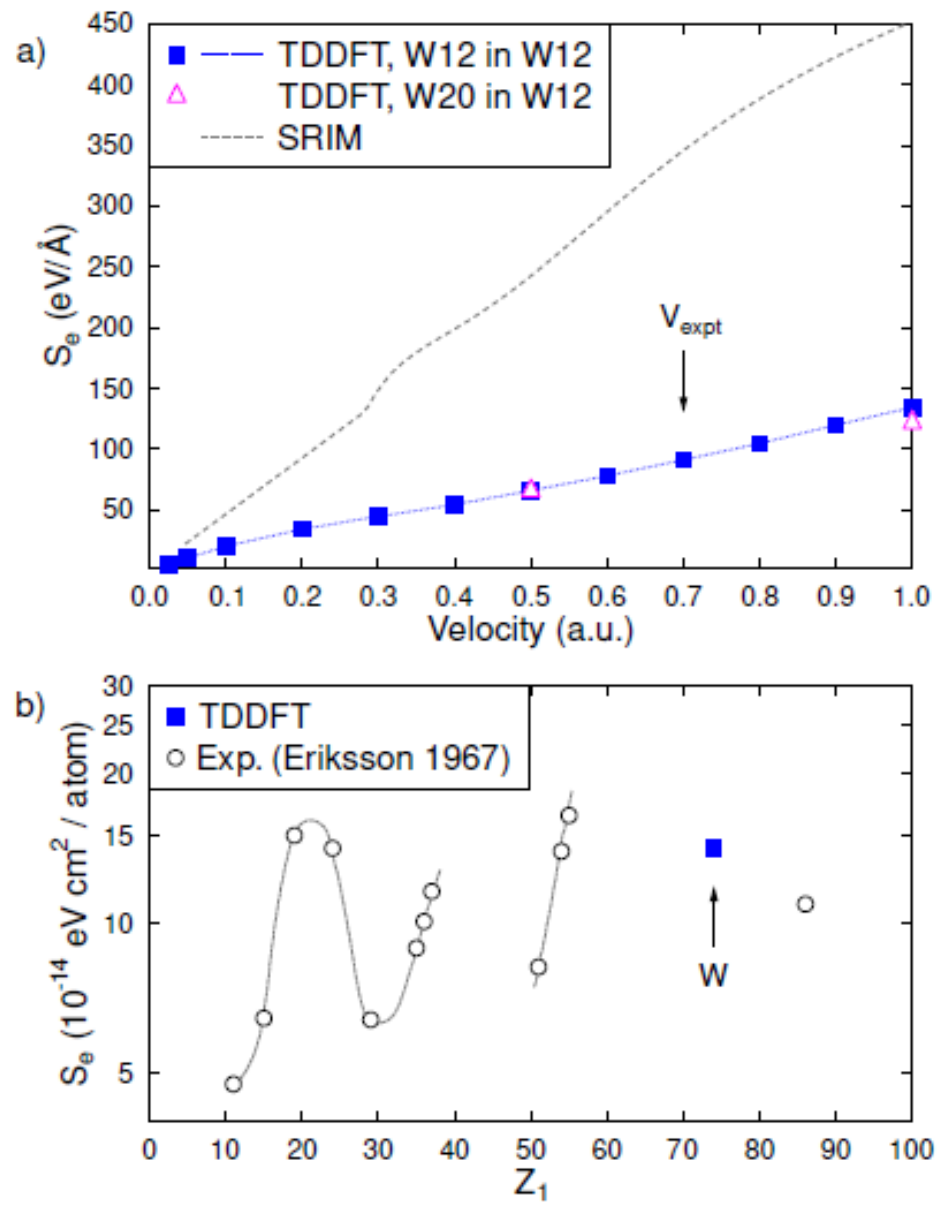

Figure 17.3. Electronic stopping prediction by rt-TDDFT, compared to SRIM and experiment.

It is illustrative to investigate the effect of the nuclear stopping along an ideal channeling trajectory. Although nuclear stopping is much reduced in the channeling geometry, it is not completely negligible. This is demonstrated by a calculation of the ideal center-channel path with and without the effect of the nuclear repulsive potential. Figure 17.4 illustrates the impact of the nuclear stopping on the range, and the stopping power deduced from the range calculation. The experimental ion energy of $50 \mathrm{keV}$ is indicated in the graphs, and it can be seen that the value of the total stopping power calculated from the range where nuclear interactions were included would not be significantly different from the electronic stopping power. The effect on the range is nevertheless non-negligible. If one integrates the equations of motion only accounting for electronic stopping, with no nuclear interactions, the total distance travelled would be overestimated by $57 \%$ [23]. Hence, it is indeed necessary to take into account also the nuclear interactions when calculating the range of the projectile. 


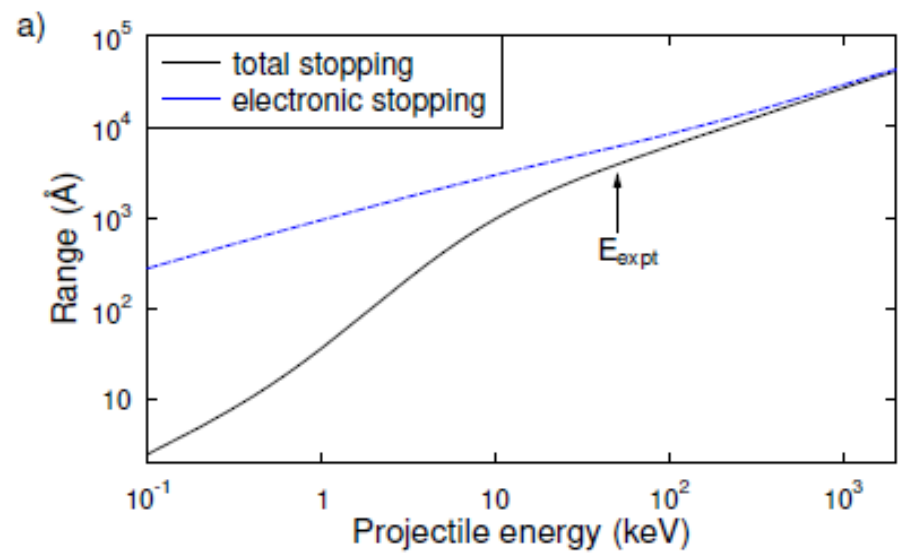

b)

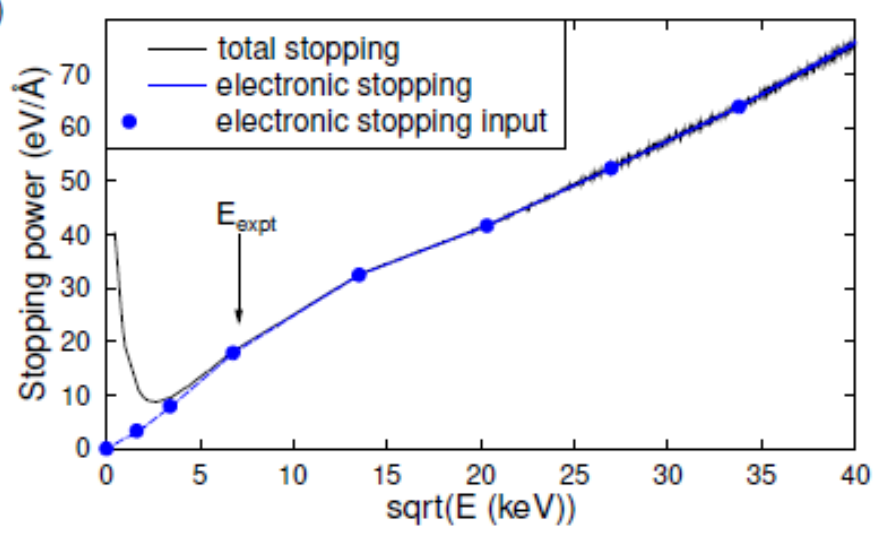

Figure 17.4. Ranges and stopping power calculated along an ideal channeling trajectory with and without nuclear interactions.

\subsection{Reaching experimental scales}

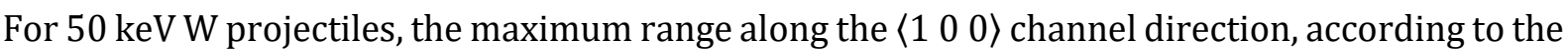
experimental work [7], is almost $350 \mathrm{~nm}$. In order to simulate the full trajectory of such particles, a simulation cell with tens of thousands of atoms would be needed. In comparison, rt-TDDFT calculations can manage around 200 atom systems, depending on the number of explicit electrons modeled per atom.

Here, we are interested in the depth the projectile reaches in the material, and not in the effect on the material itself, such as damage formation. The binary collision approximation (BCA), used e.g. in the codes SRIM [27], or MARLOW [22], would offer a computationally efficient method for simulating ion ranges. The latter code also takes crystal structure into account, which in principle allows for simulating the channeling geometry. However, BCA is not able to treat well simultaneous collisions $[22,10]$, in other words the interactions that take place when an atom passes centrally between two or more neighbors within interaction distance. This is a source of uncertainty in particular for channeling trajectories [10], which are the focus of this study. The channeling phenomenon is also essentially a many-body interaction, with the projectile experiencing the collective "string" potential along the atomic rows adjacent to a channel [11]. One can therefore question the validity of the asymptotic trajectory obtained as a solution to the binary scattering integral during the multiple soft collisions experienced by the channeling ion, in 
particular at the end of the trajectory when the time spent in the collision grows. These considerations motivate the use of a molecular dynamics (MD) based method [1].

While full MD simulations can be performed for multi-million atom systems using modern supercomputers, such simulations nevertheless require large amounts of computing resources. This would not be a problem for a single ion trajectory, but the maximum range Rmax measured in ion implantation experiments refers to the depth reached by $0.1 \%$ of the incident ions. This is because many ions de-channel along the way, and when the goal is to measure the stop- ping power along the channel, the quantity of interest is the depth reached by the furthest traveling projectiles, which can be assumed to stay in the channel for the entire trajectory. The individual trajectories are strongly stochastic, so to obtain enough statistics for a reliable comparison requires simulating on the order of a million trajectories. This requires a computationally more efficient method than full MD simulations.

Here, we use a "stripped down" molecular dynamics method called RIA (re- coil interaction approximation), developed explicitly for simulating ion ranges with high accuracy and in different crystalline structures, and implemented in the MDRANGE code [17]. In RIA, the interactions of the projectile with the atoms in the target material are computed using the molecular dynamics method of integrating the equations of motion taking interactions with all neighbors into account. However, in RIA only interactions with the projectile are considered, while interactions between target atoms are neglected. To take into account the effects of target temperature within this framework, target atoms are given initial thermal displacements which are determined based on the Debye model [18]. Since energetic projectiles interact strongly and rapidly with target atoms, compared to the interactions and relaxation time scale of the solid, neglecting the target-target interactions provides a reasonable approximation. This offers a significant increase in efficiency, while retaining the full many-body treatment, capable of handling simultaneous interactions, which is inherent in the MD formalism. With this method, it is also not necessary to model the whole system at all times. Instead, only the region around the projectile is modelled, and the target material is continuously replicated in front of the projectile as it moves through the material.

The RIA calculations thus include explicitly the effect of nuclear stopping through the nuclear repulsive potential. The nuclear stopping power is small for the strongly channeled ions, but dominates over the electronic stopping power immediately if the trajectory exits the channel. After ejection from the channel, the trajectory is effectively random. For such trajectories, the total path outside the channel is on the order of 10 Ångstroms at most, hence the value of the electronic stopping for those trajectories is of no consequence, and the constant value obtained with rt-TDDFT for the center channel can therefore be used everywhere in the RIA simulations. The ions that reach the Rmax depth, i.e. the depth past which exactly $0.1 \%$ of the projectiles travel, all channel for the full extent of their trajectory. The RIA simulations are terminated at the point when the projectile reaches thermal velocity, and that position is taken as the final site of deposition of the projectile. 


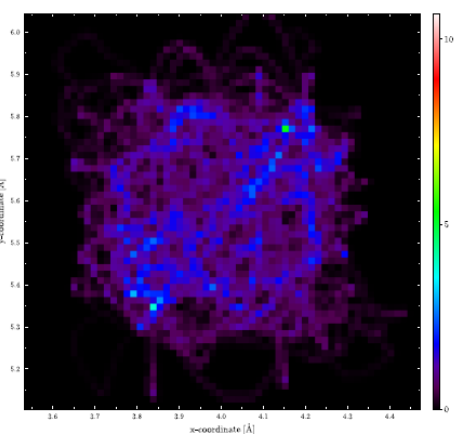

(a)

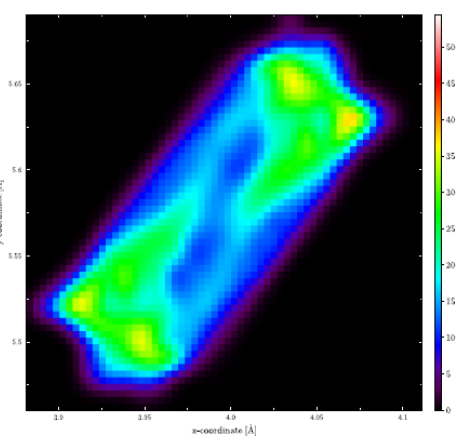

(b)

Figure 17.5. Trajectories of strongly channeled ions.

It is known theoretically that channeling projectiles oscillate around the midpoint of the channel, and this behaviour is also observed in the RIA simulations. The electronic stopping power predicted by rt-TDDFT for off-center trajectories shows an increase compared to the center channel trajectory, which is also expected based on electron density considerations. Hence, the applicability of the constant electronic stopping value obtained for the ideal, centered straight trajectory was investigated for realistic trajectories computed with the RIA method, by determining the mean deviation of the projectiles' position from the center of the channel, measured over the full trajectory.

Investigation of all trajectories that reached to the depth Rmax, or beyond, showed that the channeling paths can display a range of different configurations in the perpendicular plane, from fairly random to very focussed. Figure 17.5 illustrates two typical trajectories. In all of the channeling trajectories, however, the projectile stays within $0.6 \AA$ of the center-channel position. This observation holds for both low temperature (with negligible thermal displacement of lattice atoms in the RIA simulations), and for initial displacements corresponding to room temperature. The electron density within this region of the channel is very flat [23], hence the electronic stopping is expected to vary only negligibly for strongly channeling ions. The RIA simulations therefore a posteriori validate the use of a constant electronic stopping power in this particular geometry.

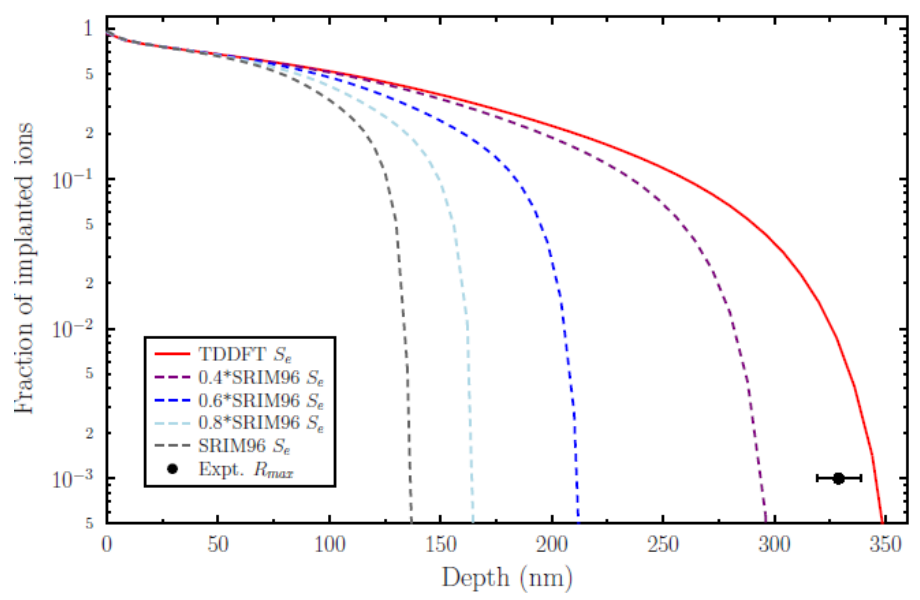

Figure 17.6. Integral range distributions predicted by simulations of implanted $50 \mathrm{keV} \mathrm{W}$ in W along the $\langle 100\rangle$ lattice direction. 
One million RIA simulations were performed for each case, with initial ion positions randomly distributed above the tungsten bcc lattice surface. Ions were given a velocity corresponding to 50 keV kinetic energy perpendicular to the tar- get surface, with the incident direction oriented along the $\left\langle\begin{array}{lll}1 & 0 & 0\end{array}\right\rangle$ crystal axis. Many of the ions scatter from the target surface atoms, and subsequently follow random trajectories through the material. These experience strong nuclear stop- ping, and travel only a short distance. The same is observed in experiment, seen as a shallow peak in the differential range distribution [8]. A large portion of the ions finds a channel, however, and travel further. Channeling ions nevertheless experience a non-zero probability of ejection from the channel, giving rise to a somewhat uniform depth distribution beyond the initial peak. Those ions that stay in the channel for the whole trajectory travel the furthest, experiencing the weakest total stopping. These all reach more or less the same depth, giving rise to a second, smaller peak in the differential range distribution. This depth is marked by a sharp drop in the integral range distribution, and is identified as the maximum range Rmax, which is also clearly present in experimental data. Figure 17.6 shows the integral range distribution obtained by the RIA simulations using the rt-TDDFT predicted electronic stopping, compared to the distribution obtained from simulations where the electronic stopping power predicted by SRIM'96 was used, along with a range of scaled stopping power values. One can see how decreasing the electronic stopping power yields identical distributions at shallow depth, where the atoms have been ejected from the channel and travel along random trajectories where the nuclear stopping dominates. The progressively deeper tails observed for decreasing electronic stopping correspond to the longer trajectories travelled within the channel. The experimentally measured Rmax value is also plotted, together with the $3 \%$ uncertainty reported for the measurement [7].

\subsection{Discussion}

As was noted above, the nuclear stopping is not completely negligible when measuring ion ranges for channeling trajectories. Furthermore, the channeling ion is sensitive to any perturbations along the channel, which occur as a result of thermal motion of the target atoms. The magnitude of this thermal effect was also studied with the RIA method, and it was found that even a small local heating above room temperature within the irradiated material would result in a decrease in Rmax.

Other possible effects in real materials include the likely presence of a thin oxide layer on the surface of the sample, and impurities and defects within the sample. These were not accounted for in the RIA simulations, but would potentially result in a slightly smaller Rmax than that obtained for the ideal crystal. Considering these effects, the agreement between simulation and experiment is very good, and shows the applicability of the rt-TDDFT method for calculating electronic stopping even for the heavy transition metal W.

\section{Acknowledgements}

This work has been carried out with support from the Academy of Finland through project No. 311472. 


\section{References}

[1] K. M. Beardmore and N. Grønbech-Jensen. Efficient molecular dynamics scheme for the calculation of dopant profiles due to ion implantation. Phys. Rev. E, 57(6):7278-7287, 1998.

[2] M. Caro, A. Tamm, A. A. Correa, and A. Caro. Role of electrons in collision cascades in solids. i. dissipative model. Phys. Rev. B, 99(174301), 2019.

[3] D. M. Ceperley and B. J. Alder. Ground state of the electron gas by a stochastic method. Phys. Rev. Lett., 45:566-569, Aug 1980.

[4] A. Correa. Calculating electronic stopping power in materials from first principles. Computational Materials Science, 150:291 - 303, 2018.

[5] Erik W. Draeger, Xavier Andrade, John A. Gunnels, Abhinav Bhatele, André Schleife, and Alfredo A. Correa. Massively parallel first-principles simulation of electron dynamics in materials. Journal of Parallel and Dis- tributed Computing, 106:205-214, aug 2017.

[6] P.M. Echenique, R.M. Nieminen, and R.H. Ritchie. Density functional calculation of stopping power of an electron gas for slow ions. Solid State Communications, 37(10):779-781, 1981.

[7] L. Eriksson, J. A. Davies, and P. Jespersgaard. Range measurements in oriented tungsten single crystals (0.1-1.0 mev). i. electronic and nuclear stopping powers. Phys. Rev., 161:219-234, Sep 1967.

[8] L. Eriksson, J. A. Davies, and P. Jespersgaard. Range measurements in oriented tungsten single crystals (0.1-1.0 mev). i. electronic and nuclear stopping powers. Phys. Rev., 161:219-234, Sep 1967.

[9] E. Fermi and E. Teller. The capture of negative mesotrons in matter. Phys. Rev., 72:399-408, Sep 1947.

[10] K. Gärtner, M. Nitschke, and W. Eckstein. Computer simulation studies of low energy b implantation into amorphous and crystalline silicon. Nucl. Intsr. Meth. Phys. Res. B, 83:87-94, 1993.

[11] Donald S. Gemmell. Channeling and related effects in the motion of charged particles through crystals. Rev. Mod. Phys., 46:129-227, Jan 1974.

[12] Bin Gu, Brian Cunningham, Daniel Muñoz Santiburcio, Fabiana Da Pieve, Emilio Artacho, and Jorge Kohanoff. Efficient ab initio calculation of elec- tronic stopping in disordered systems via geometry pre-sampling: Appli- cation to liquid water. The Journal of Chemical Physics, 153(3):034113, 2020.

[13] F. Gygi. Architecture of qbox: A scalable first-principles molecular dynam- ics code. IBM Journal of Research and Development, 52(1.2):137-144, Jan 2008.

[14] Leonard Kleinman and D. M. Bylander. Efficacious Form for Model Pseu- dopotentials. Physical Review Letters, 48(20):1425-1428, may 1982.

[15] J. Lindhard, V. Nielsen, M. Scharff, and P. V. Thomsen. Integral equations governing radiation effects (notes on atomic collisions, iii). Mat. Fys. Medd. Dan. Vid. Selsk., 33(10):1-42, 1963.

[16] S. Lohmann, R. Holenak, and D. Primetzhofer. Trajectory-dependent elec- tronic excitations by light and heavy ions around and below the bohr ve- locity. Phys. Rev. A, 102(062803), 2020.

[17] K. Nordlund. Molecular dynamics simulation of ion ranges in the 1-100kev energy range. Computational Materials Science, 3(4):448 - 456, 1995.

[18] K. Nordlund and G. Hobler. Dependence of ion channeling on relative atomic number in compounds. Nuclear Instruments and Methods in Physics Research Section B: Beam Interactions with Materials and Atoms, 2017.

[19] J. H. Ormrod, J. R. Macdonald, and H. E. Duckworth. Some low-energy atomic stopping cross sections. Can. J. Phys., 43:275-284, 1965.

[20] J. M. Pruneda, D. Sánchez-Portal, A. Arnau, J. I. Juaristi, and Emilio Artacho. Electronic 
stopping power in LiF from first principles. Phys. Rev. Lett., 99:235501, Dec 2007.

[21] Edwin E. Quashie, Bidhan C. Saha, and Alfredo A. Correa. Electronic band structure effects in the stopping of protons in copper. Phys. Rev. B,94:155403, Oct 2016.

[22] Mark T. Robinson and Ian M. Torrens. Computer simulation of atomic- displacement cascade in solids in the binary-collision approximation. Phys. Rev. B, 9(12), 1974.

[23] E. Sand, R. Ullah, and A. A. Correa. Heavy ion ranges from first- principles electron dynamics. npj Comput Mater, 5:43, 2019.

[24] Andr'e Schleife, Erik W. Draeger, Yosuke Kanai, and Alfredo A. Correa. Plane-wave pseudopotential implementation of explicit integrators for time- dependent Kohn-Sham equations in large-scale simulations. The Journal of Chemical Physics, 137(22):22A546, 2012.

[25] W.H. Trzaska, G.N. Knyazheva, J. Perkowski, J. Andrzejewski, S.V. Khleb- nikov, E.M. Kozulin, T. Malkiewicz, M. Mutterer, and E.O. Savelieva. New experimental stopping power data of 4he, 16o, 40ar, 48ca and $84 \mathrm{kr}$ pro- jectiles in different solid materials. Nuclear Instruments and Methods in Physics Research Section B: Beam Interactions with Materials and Atoms, 418:1-12, 2018.

[26] Rafi Ullah, Emilio Artacho, and Alfredo A. Correa. Core electrons in the electronic stopping of heavy ions. Phys. Rev. Lett., 121:116401, Sep 2018.

[27] J. F. Ziegler. SRIM-2008.04 software package, available online at http://www.srim.org., 2008.

[28] J. F. Ziegler, U. Littmark, and J. P. Biersack. The stopping and range of ions in solids. Pergamon New York, 1985.

[29] A.N. Zinoviev and K. Nordlund. Comparison of repulsive interatomic po-tentials calculated with an all-electron dft approach with experimentaldata. Nuclear Instruments and Methods in Physics Research Section B: Beam Interactions with Materials and Atoms, 406:511-517, 2017. 


\section{Case studies on FEM and MHD}

Evaggelos Kaselouris, George Koundourakis, and Vasilis Dimitriou*

Case studies of finite element method (FEM) and magnetohydrodynamics (MHD) simulations will be presented.

*Contact: dimvasi@hmu.gr 


\subsection{Laser matter interaction FEM simulations- Introduction using LS-DYNA/LS-PREPOST}

The aim of the Finite Element Method - FEM, study is to provide to the readers the ability of understanding and implementing simulations of laser matter interaction thermal-structural problems using LS-DYNA FEM software. In this first introductory case, the users learn how to use the LS-PrePost tool that acts as a pre-processor and post-processor to the LS-DYNA FEM solver and a characteristic simple transient structural problem is demonstrated.

\subsubsection{LS-DYNA}

LS-DYNA from Livermore Software Technology Corporation (LSTC), is a highly advanced general purpose nonlinear finite element program that is capable of simulating complex real-world problems. The distributed and shared memory solver provides very short turnaround times on desktop computers and clusters operated using Linux, Windows and UNIX.

LS-DYNA is suitable to investigate phenomena involving large deformations, sophisticated material models and complex contact conditions for structural dynamic problems. The software allows switching between explicit and different implicit time stepping schemes. Disparate disciplines, such as coupled thermal analyses, Computational Fluid Dynamics (CFD), fluidstructure interaction, Smooth Particle Hydrodynamics (SPH), Element Free Galerkin (EFG), Corpuscular Method (CPM), Discrete Element Method (DEM) and the Boundary Element Method (BEM) can be combined with structural dynamics. For pre- and post-processing, LS-DYNA comes with the LS-PrePost tool, which can be utilized to generate inputs and visualize numerical results.

\subsubsection{Keyword format input files}

An LS-DYNA input file is a text-file in so called Keyword format usually with a *.k, *.key or *.dyn suffix, e.g. laser.k. A finite element model in LS-DYNA is built up by different keywords, which is defined for all definitions and parameters in a model (e.g. *PART, *NODE). A short overview of the basic structure of such an input file for a basic 1 element finite element model is provided.

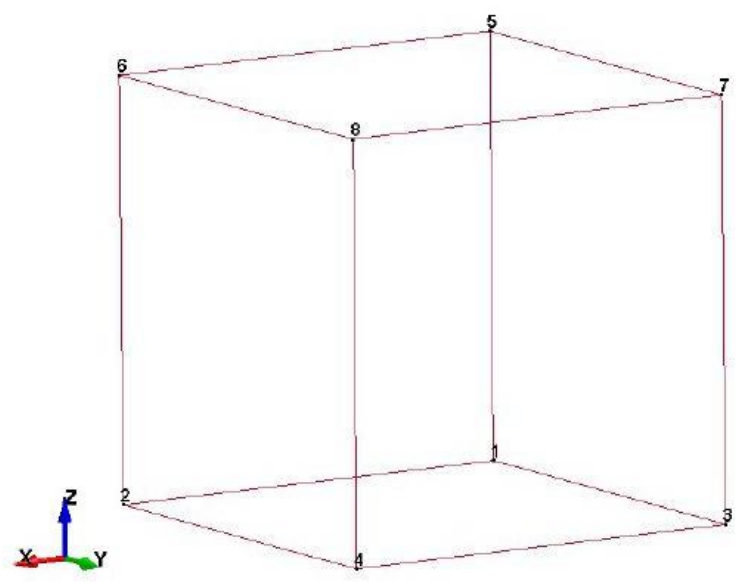

Figure 18.1 Cube consisting of one element with eight node points 
The *PART keyword is used to begin the definition of the finite element model. The keyword *PART contains data that points to other attributes of this part, e.g. material properties. Keywords for these other attributes, in turn, point elsewhere to additional attribute definitions.

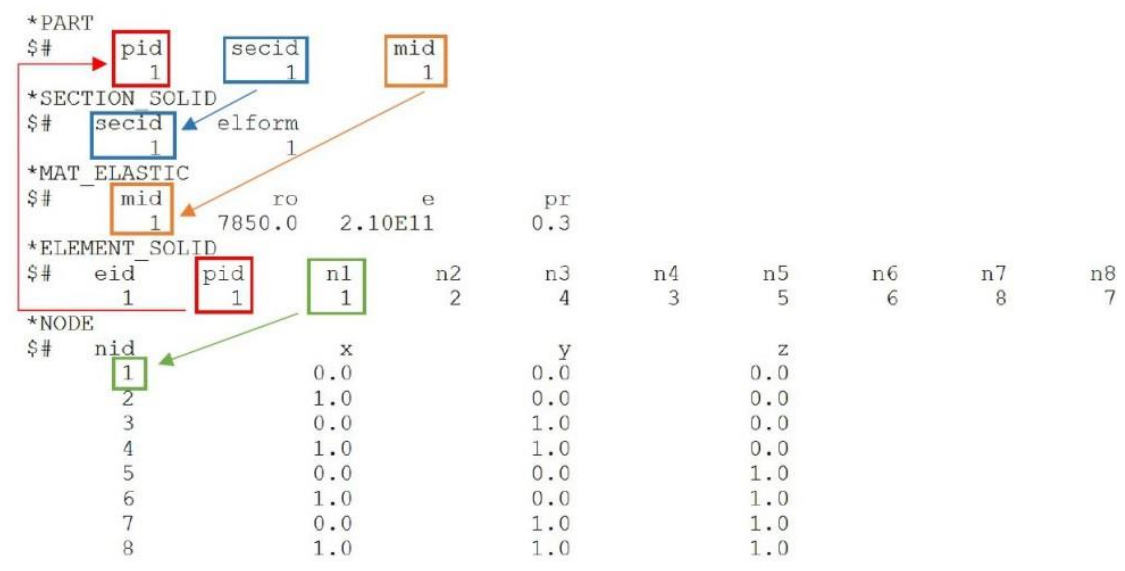

Figure 18.2 Organization of the keyword input for the cube

A brief description follows:

*PART: We have one part with identification pid=1. This part has attributes identified by section identification secid=1 and material identification $\operatorname{mid}=1$.

*SECTION_SOLID: Parts definitions that reference secid=1 are defined as constant stress 8 node brick elements (elform $=1$ ).

*MAT_ELASTIC: Parts definitions that reference mid=1 are defined as an elastic material with density, Young's modulus and Poisson's ratio.

*ELEMENT SOLID: The element with identification eid=1 are defined by nid=1 to nid=8 and belongs to pid=1.

*NODE: The node identified by nid has coordinates $\mathrm{x}, \mathrm{y}, \mathrm{z}$.

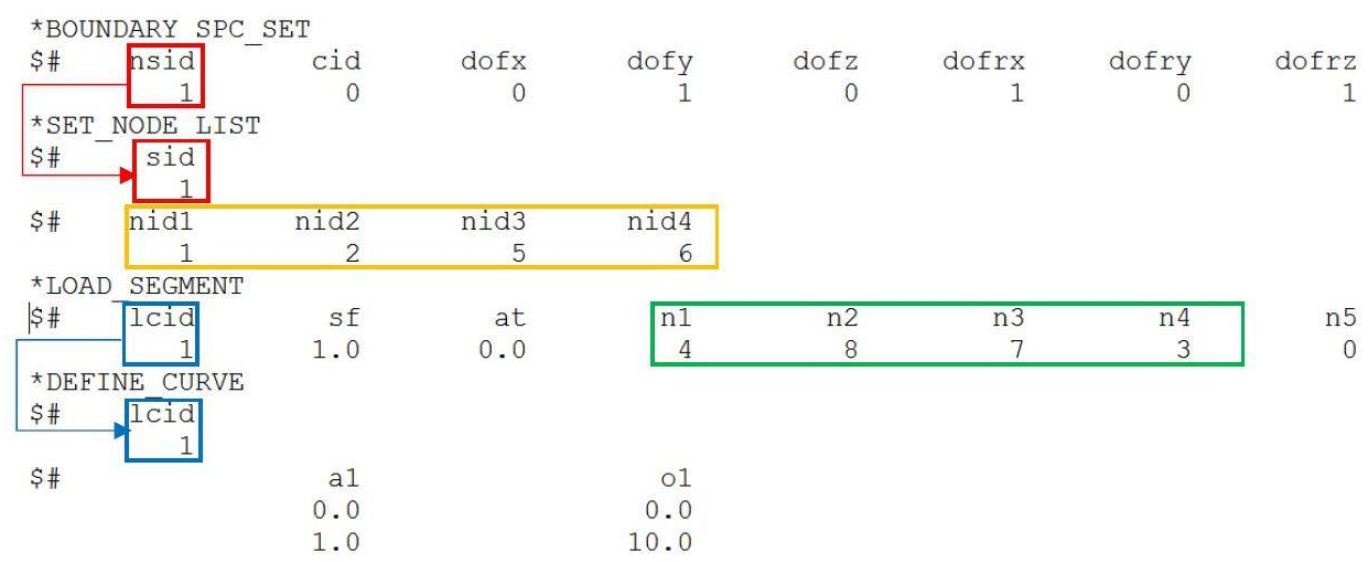

Figure 18.3 Definition of boundary conditions and loads on the cube

Boundary conditions and time dependent loads are also set by keywords and are usually applied on nodes, elements, segments or parts. Set definitions are often used to define groups of these 
entities. Since all loads are time dependent, curves need to be defined that states time vs load unit (force, pressure etc.).

About the keywords above:

*BOUNDARY_SPC_SET: The node set with identification nsid=1 are constrained in y-translation and $\mathrm{x}$ - and z-rotations.

*SET_NODE_LIST: This keyword defines that node 1, 2, 5 and 6 belongs to node set sid=1.

*LOAD_SEGMENT: A pressure load is applied on a segment that are defined by node 4, 8, 7 and 3. *DEFINE_CURVE: The curve consists of two points that defines the time vs pressure. This curve with identification lcid=1 is used for the load.

\subsubsection{LS-PrePost}

LS-PrePost is an advanced pre- and post-processor designed specifically for LS-DYNA. It is developed for Windows, Linux and Apple and it is free to download from the web link http://ftp.lstc.com/anonymous/outgoing/lsprepost/4.5/.

LS-Prepost main functions contain:

- $\quad$ Full support of LS-DYNA keyword files

- $\quad$ Full support of LS-DYNA result files

- $\quad$ Robust handling of geometry data (new CAD engine)

- $\quad$ Pre-processing (meshing, model clean-up, entity creation)

- $\quad$ Post-processing (animation, fringe plotting, curve plotting)

\subsubsection{Graphical user interface}

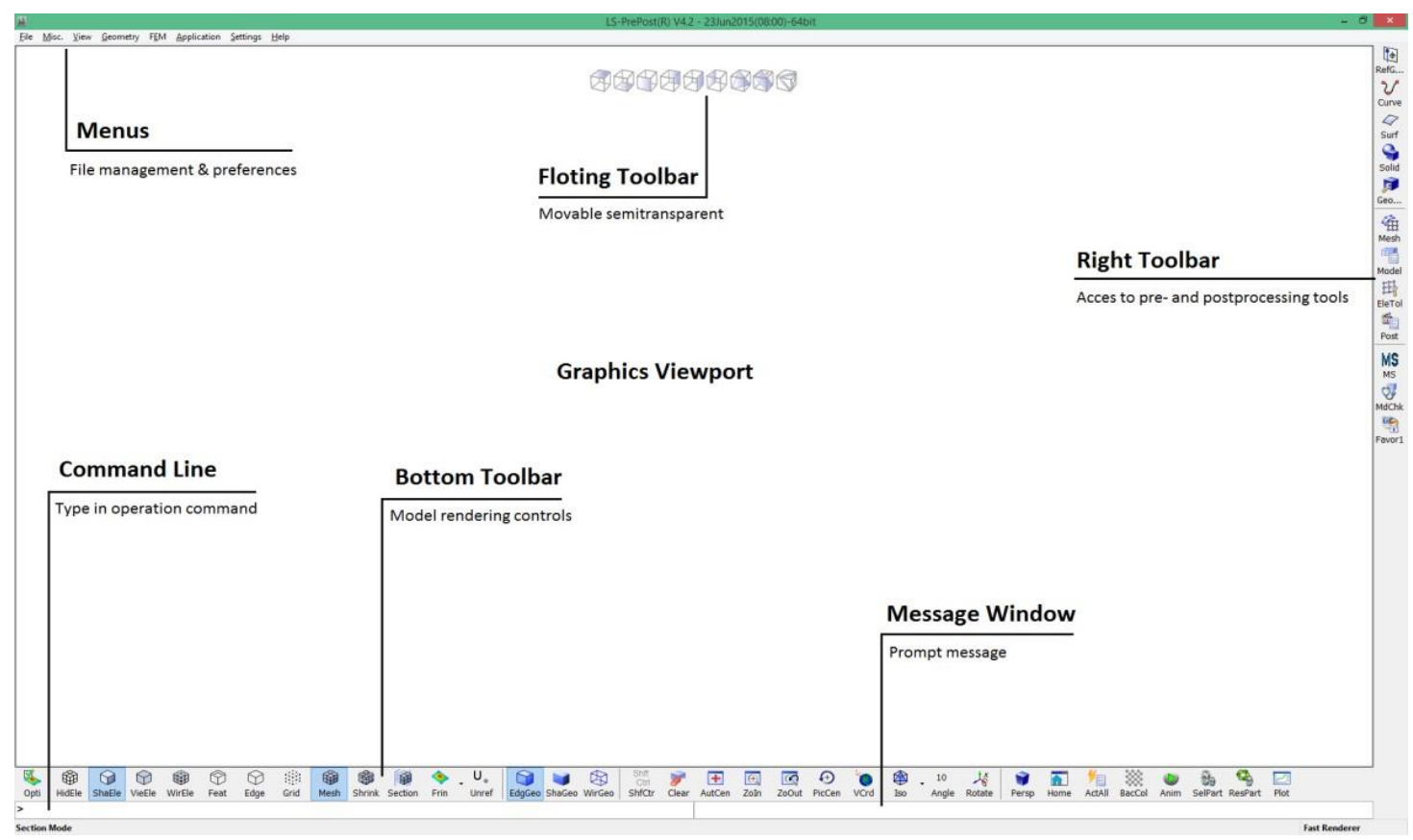

Figure 18.4 Graphical interface of the LS-PREPOST 
On the right-hand side of figure 18.4, you can see the main toolbar. When clicking on one of these, a sub-toolbar just to the left will be shown. That is the location where you'll find most of the tools needed to create/modify/delete entities in your model.

In the bottom toolbar, are found the tools which are the most used to determine how LS-PrePost should render mesh/surfaces, orient the model, etc. There are a couple of the drop-down menus on the top left corner that you will use frequently: File/View/Application/Settings. The Floating Toolbar is used to toggle between different views.

\subsubsection{Menus}

Here are presented the useful menus for the user, the File Menu, the Geometry Menu and the FEM menu.

\section{File Menu}

\begin{tabular}{l|lr}
\hline File & Misc. View & \\
\hline New & \\
Open & \\
Import & \\
Recent & \\
\hline Save & \\
Save As & \\
\hline Update & Ctrl+U \\
Run LS-DYNA & \\
\hline Print... & Ctrl+P \\
Movie... & Ctrl+M \\
\hline Exit & Ctrl+X \\
\hline Save and Exit & \\
\hline
\end{tabular}

New - Launch a new session of LS-PrePost, all model/data will be closed

Open - Open file (new model created for each file opened)

Import - Import file (adds keyword data to current model)

Recent - Open recent files (stored in /user/.Ispp_recent)

Save - Over-write current Keyword or Project file

Save As - Save any of the following file formats using advanced options: Keyword, Active Keyword (visible data), Project, Post.db (condensed d3plot data), Geometry, Keyword and Project (using the same file name).

Update - Load new d3plots for run in progress

Run LS-DYNA - Pop up LS-DYNA job submission dialog, currently only limited to the same local machine LS-PrePost is running

Print... - Launch printing interface (send to printer or image file)

Movie... - Launch movie generation interface

Exit - Exit LS-PrePost

Save and Exit - Save data to current file and exit LS-PrePost

Figure 18.5 File menu 


\section{Geometry Menu}

Geometry FEM Application S
[f] Reference Geometry ,
if Surve
Surface
Solid

Reference Geometry - Access tools for creating and editing reference geometry (Axis, Plane, Coordinate System, Point, Reference Geometry Edit)

Curve - Access tools for creating and editing curves (Point, Line, Circle, Circular Arc, Ellipse, Elliptical Arc, BSpline Curve, Helix, Composite Curve, Break Curve, Merge Curve, Bridge Edge, Smooth Curve, Middle Curve, Morphing Curve, Fillet Curve, Parabola, Hyperbola, Function, Polygon, Convert, Sketch)

Surface - Access tools for creating and editing surfaces (Plane, Cylinder, Cone, Sphere, Torus, Ellipsoid, Fill Plane, Extrude, Revolve, Sweep, Loft, N-Side Surface, Patch Surface, Bridge Two Faces, Combine Faces, Fit From Points/Mesh, Middle Surface, Surface Morphing, Fit Primary Surface, Break Surface)

Solid - Access tools for creating and editing solids (Box, Cylinder, Cone, Sphere, Torus, Extrude, Revolve, Sweep, Loft, Fillet, Chamfer, Draft, Thicken, Wedge, Boolean, Prism)

Geometry Tools - Access other geometry tools (Delete Face, Blank Entity, Extend Curve, Extend Face, Intersection, Offset, Project, Replace Face, Stitch Faces, Trim, Transform, Reverse Direction, Copy Entity, Management, Heal, Topology Simplify, Measure, Text Object, Array flow)

Figure 18.6 Geometry menu

FEM Menu

\begin{tabular}{|c|c|c|}
\hline FEM & Application & 51 \\
\hline 苜 & Element and Mesh & ' \\
\hline 畕䡒 & Model and Part & • \\
\hline ad & Element Tools & • \\
\hline 뜰 & Post & • \\
\hline
\end{tabular}

Element and Mesh - Access mesh creation tools (Shape Mesher, Auto Mesher, Solid Mesher, Block Mesher, N-Line Mesher, 2D-Mesher, Tetrahedral Mesher, Blank Mesher, BulkF Mesher, Element Generation, Node Editing, Element Editing, Nurbs, Mass Trimming, Spot Welding, SPH Generation, Disc Sphere Generation, Multiple Solver Mesh, Result Mapping)

Model and Part - Access model and part tools (Assembly and Select Part, Keyword Manager, Create Entity, Part Data, Display Entity, Reference Check, Renumber, Section Plane, Model Selection, Subsystem Manager, Group, View, Part Color, Appearance, Annotation, Split Window, Explode, Lighting Setup, Reflect Model, Trace Light)

Element Tools - Access element tools (Identify, Find, Blank, Move or Copy, Offset, Transform, Normals, Detach, Duplicate Nodes, Node Editing, Element Editing, Measure, Morph, Smooth, Part Trim, Part Travel, Edge Face, Regionalize)

Post - Access post-processing tools (Fringe Component, Fringe Range, History, XY Plot, ASCII, Binary Output, Follow, Trace, State, Particle, Circle Grid, Chain Model, FLD, Output, Setting, Vector)

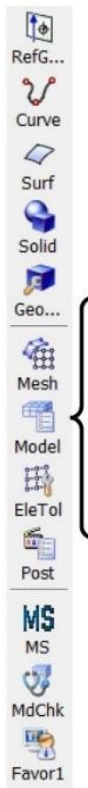

Figure 18.7 FEM menu

\subsubsection{Transient structural problem}

Consider the deformation of a cube on the ground with an applied pressure on the top surface. The task is to compute the vertical displacement of the cube due to this pressure. 


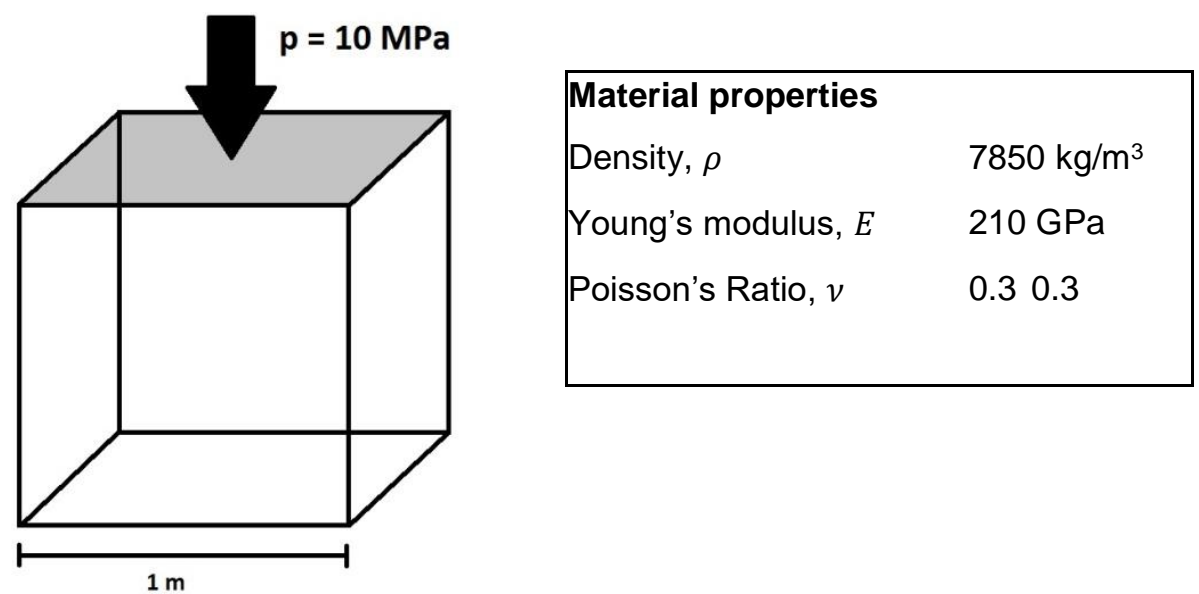

Figure 18.8 Applied pressure on the top surface and problem material properties

The S2 unit system is used in this tutorial.

\begin{tabular}{|c|c|c|}
\cline { 3 - 3 } \multicolumn{1}{c}{ S1 } & S2 \\
\hline \hline length & meter & millimeter \\
\hline time & second & second \\
\hline mass & kilogram & tonne \\
\hline force & Newton & Newton \\
\hline Young's modulus of stecl & $210.0 \mathrm{E}+09$ & $210.0 \mathrm{E}+03$ \\
\hline density of stecl & $7.85 \mathrm{E}+03$ & $7.85 \mathrm{E}-09$ \\
\hline gravitation & 9.81 & $9.81 \mathrm{E}+03$ \\
\hline
\end{tabular}

Figure 18.9 Unit system

\subsubsection{Preparation LS-PrePost}

The most common way to work with/open LS-PrePost is to have a short-cut on the desktop directly. This gives you control over which version of LS-PrePost you would use and you can easily update LS-PrePost separately.

In LS-PrePost, go to View > Toolbar and activate Text and Icon (Right) and Text and Icon (Bottom). This is done to easier navigate through the different toolbars.

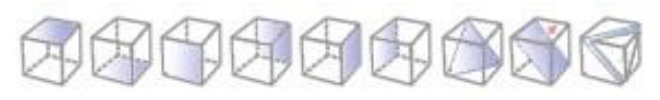

Check so you can see your Floating Toolbar in the LS-PrePost window.

If not, activate it by clicking on Opti > ISO View in the bottom toolbar.

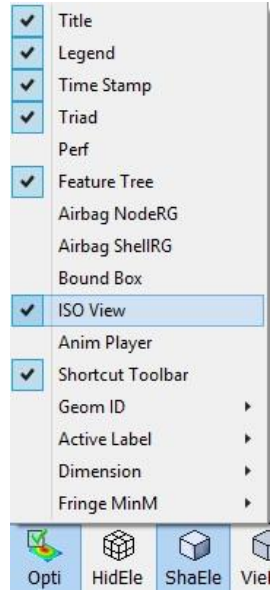




\subsubsection{Create model}

18.1.3.2.1 Geometry and mesh

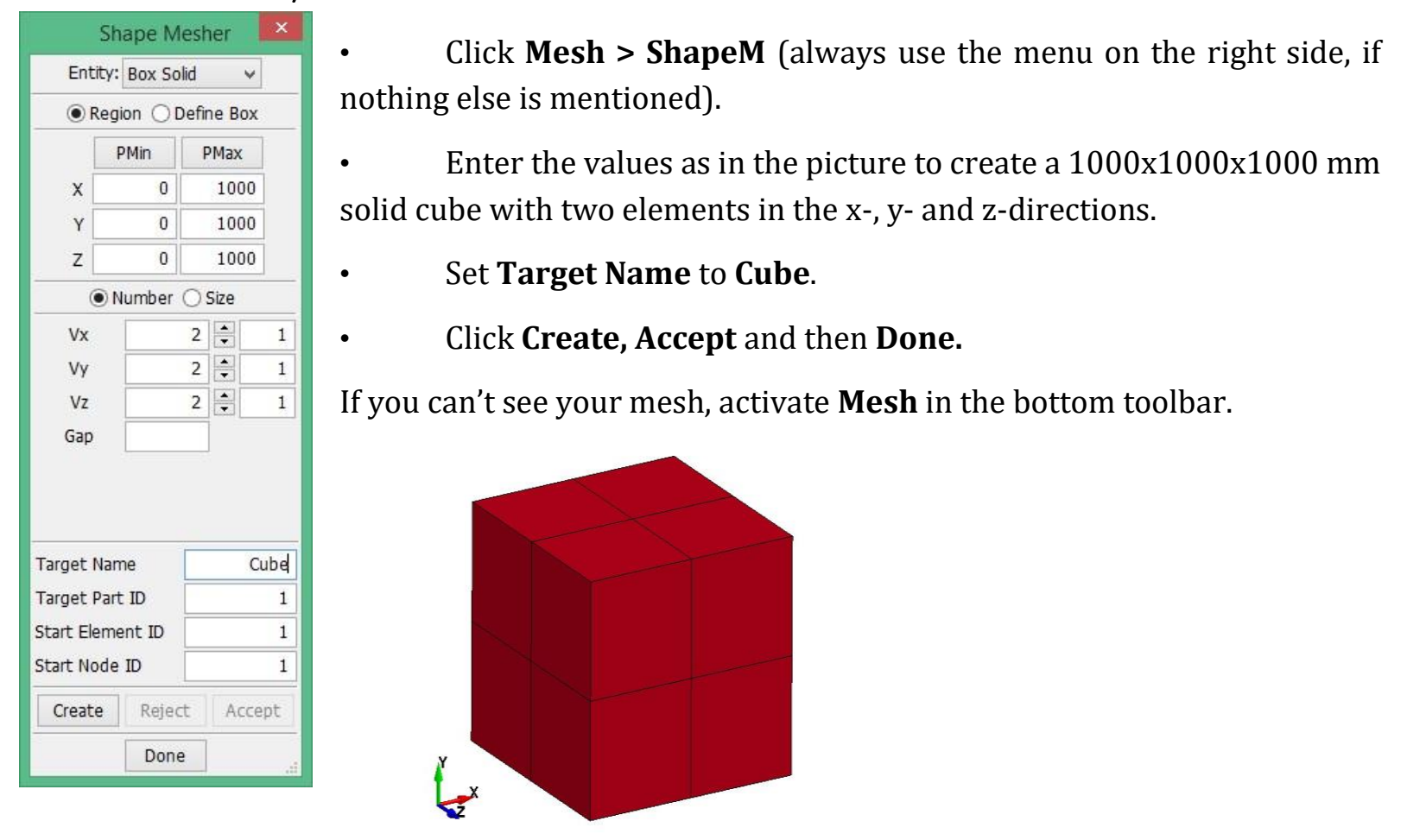

\subsection{Boundary conditions}

Apply boundary conditions to fix one side of the cube:

- $\quad$ Click Model > CreEnt

- In the Entity Creation box, double-click on Boundary and click Spc in the dropdown that appears.

- Select Cre

- Set shall be activated.

- Select XOZ as Sym Plane, Y, RX and RZ will then be activated. The boundary conditions will then have a translational constraint in global y -direction and rotational constraints about $\mathrm{x}$ - and $\mathrm{z}$-axis.

- From the ISO-views on the top of the screen, click at the one called Top.

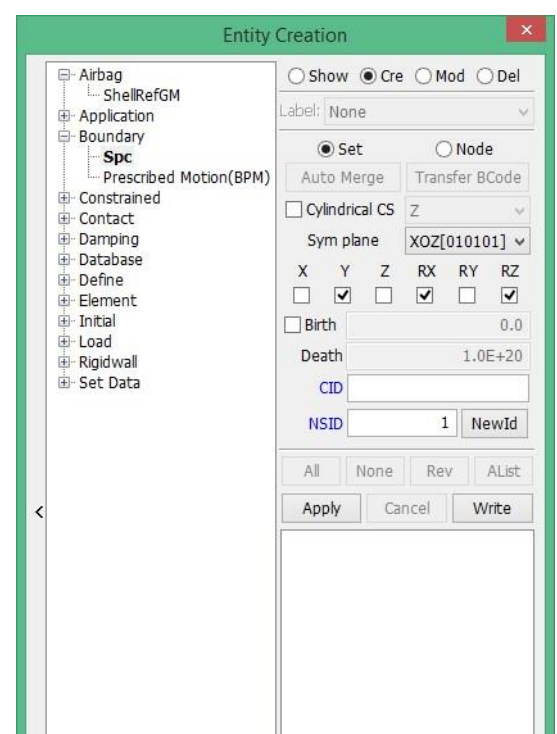



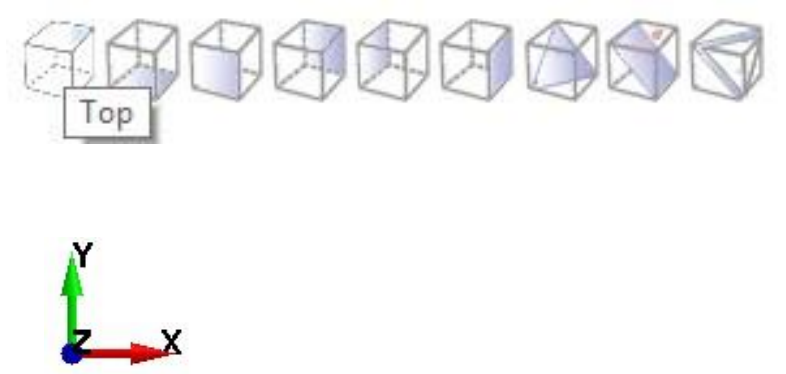

Check that your coordinate system looks like the one above. To show the coordinate system, click Opti and activate Triad.

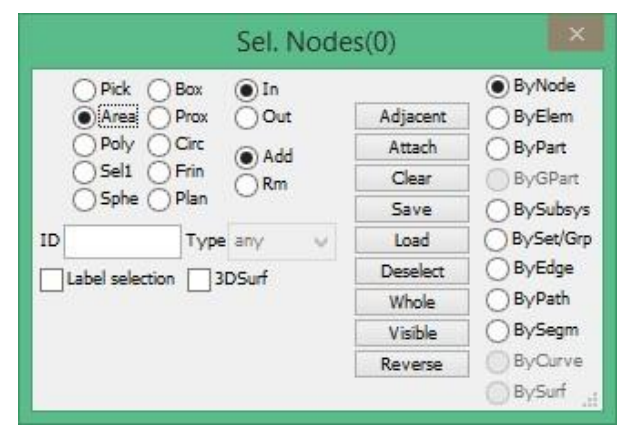

This box shows alternatives to select the nodes. Select Area.

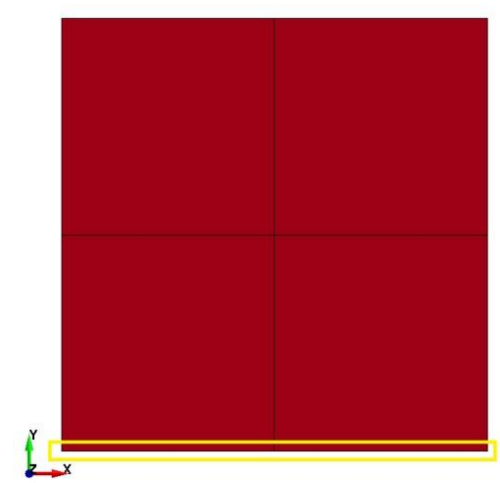

- Select the nodes in the yellow square by making a box with the mouse.

- A node set will be created from the nodes that were chosen, NSID = 1 in Entity Creation indicates that it will get an Id $=1$.

- Click Apply, then Done in the Entity Creation box.

The nodes are now constrained.

\subsection{Apply the load}

For loads, a curve must be defined that states the variation of the load over time. Click Model > Keywrd. In the dialog window that opens, there one can change between Model and All. Model shows the keywords that already have been created. All shows all possible keywords that are available in LS-DYNA.

Select All at the top of Keyword Manager window. Double-click DEFINE > CURVE. Name the curve Curve - Pressure for example. All titles are optional, but it is good practice to make use of them to make the model clear and structured. The points for the curve will be written in $\mathbf{A 1}$ and $\mathbf{0 1}$ : 


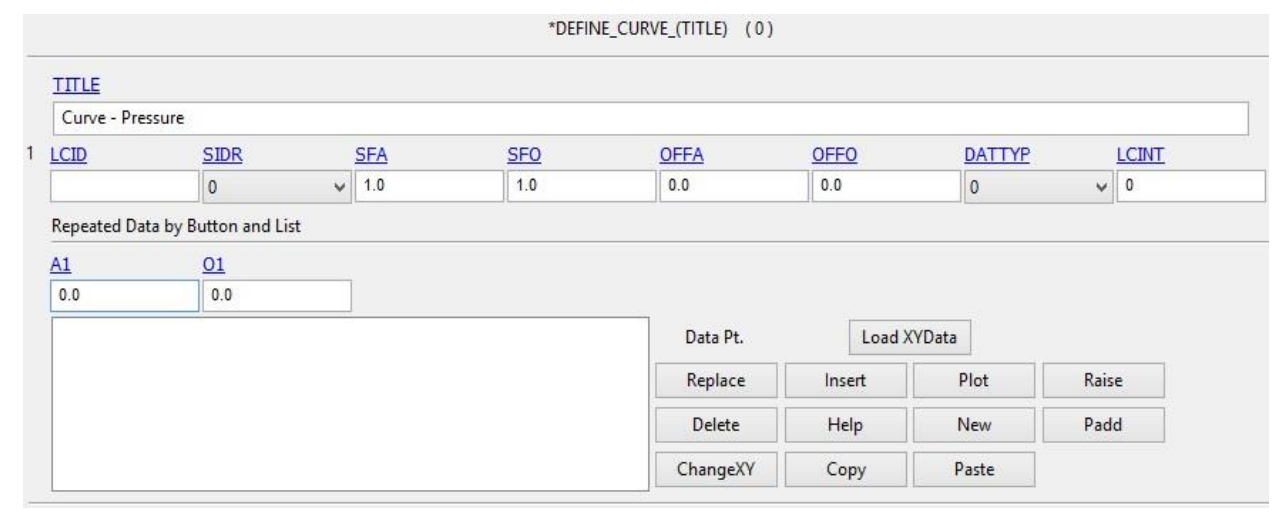

- Write $\mathbf{0}$ and $\mathbf{0}$, Click Insert.

- $\quad$ Then $\mathbf{1}$ and $\mathbf{1}$, Insert.

- Finally, 1.1 and 1, Insert.

- Click Accept

It is important that the curve extends beyond the end time of the simulation. The simulation will have the termination time $1 \mathrm{~s}$ (will be set later). Therefore, the last point of 1.1 was added.

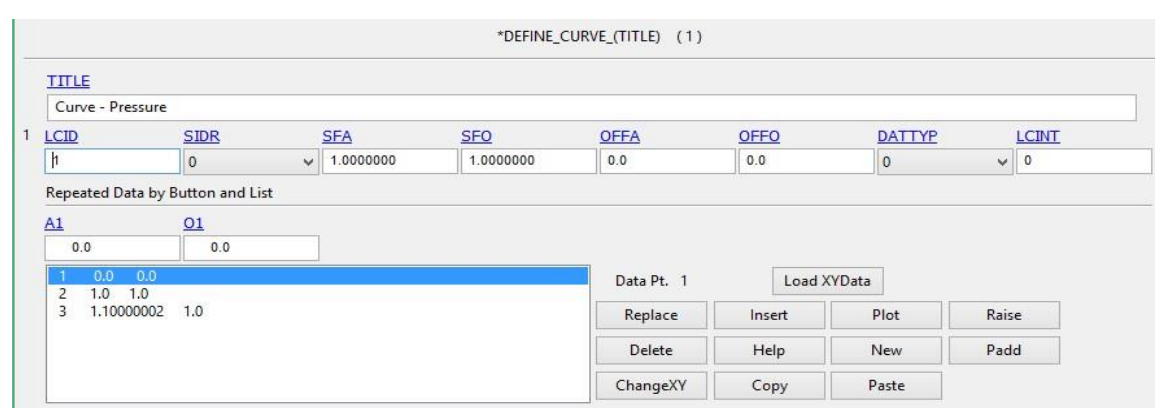

To view the curve, Click Plot. Close the PlotWindow (X or Quit) and the *DEFINE_CURVE (Done) window.

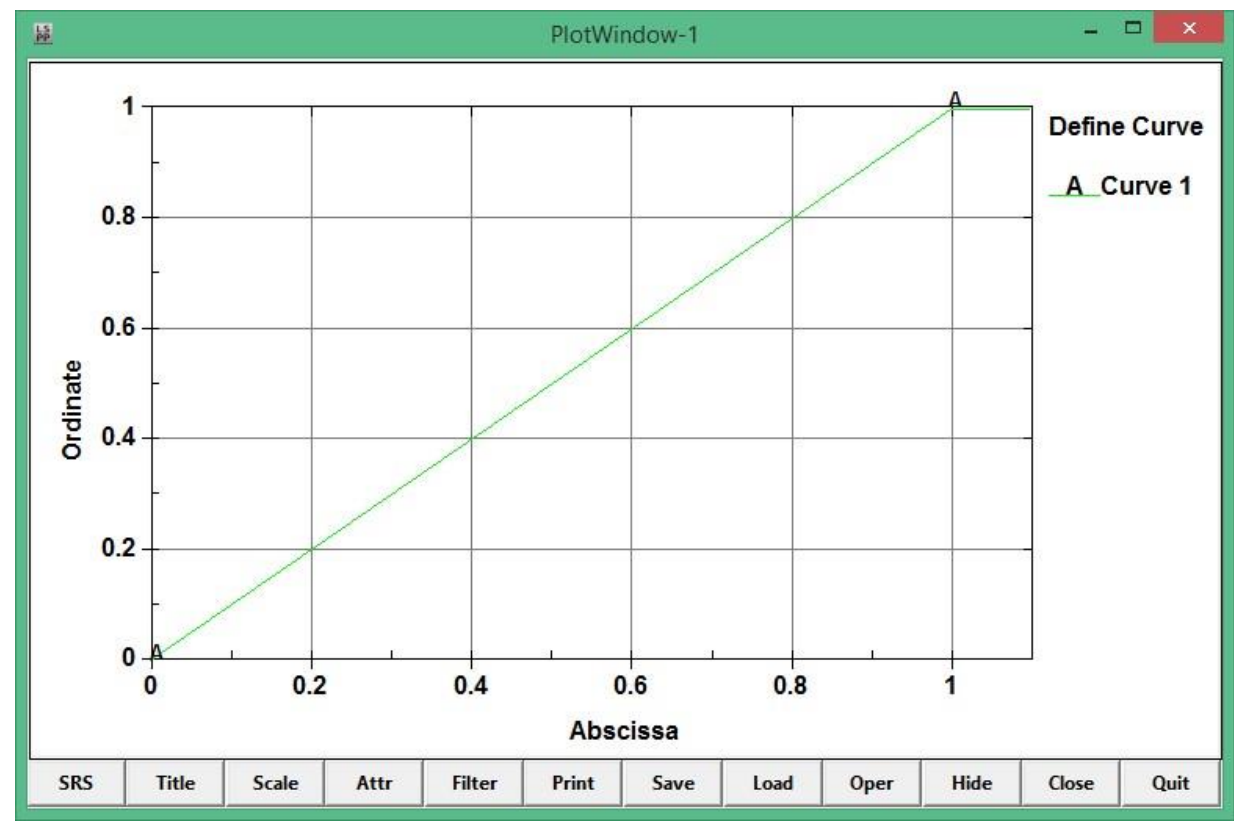


Now click Model > CreEnt:

- In the Entity Creation box, double-click on Load and click on Segment in the dropdown that appears.

- Click Cre

- Change Type: to LOAD_SEGMENT_SET.

- Give the load the title Pressure.

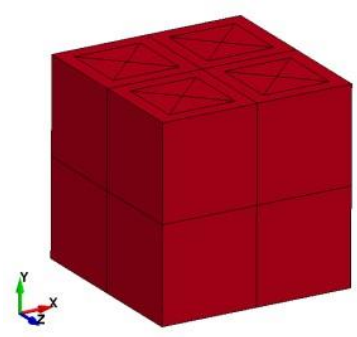

- Click on LCID and select 1 Curve - Pressure, press Done.

- To obtain a pressure of $10 \mathrm{MPa}$, the scale factor $\mathbf{S F}$ will be used.

- Set SF to $\mathbf{1 0}$ (the pressure unit is MPa for the selected unit system).

- From the selection box, Pick can be activated.

- Click on the four segments on the top of the cube, as in the figure. If necessary, deactivate entities with right mouse button.

- Click Apply, then Done

\subsection{Termination}

The end time for the simulation needs to be set. This keyword is almost always mandatory for any simulation using LS-DYNA:

- $\quad$ Click Model > Keywrd.

- Double-click CONTROL > TERMINATION

- Set ENDTIM to 1. The simulation will then last for 1 time unit, which is second in this case.

- Accept, then Done.

\begin{tabular}{l|l|l|l|l|} 
& & & & *CONTROL_TERMINATION \\
& & & & \\
& & & \\
& & & \\
\hline ENDTIM & ENDCYC & DTMIN & ENDENG & ENDMAS \\
\hline 1.0 & 0 & 0.0 & 0.0 & 100000000.0 \\
\hline
\end{tabular}

\subsection{Output}

The user must request all the data needed to post-process an analysis using LS-DYNA, before starting the simulation. We will create a d3plot file, which gives complete output states of the simulation:

- $\quad$ Click Model > Keywrd.

- Double-click DATABASE > BINARY_D3PLOT

- Set DT to 0.1. This implies that results will be printed every 0.1 time unit.

- Accept, then Done 


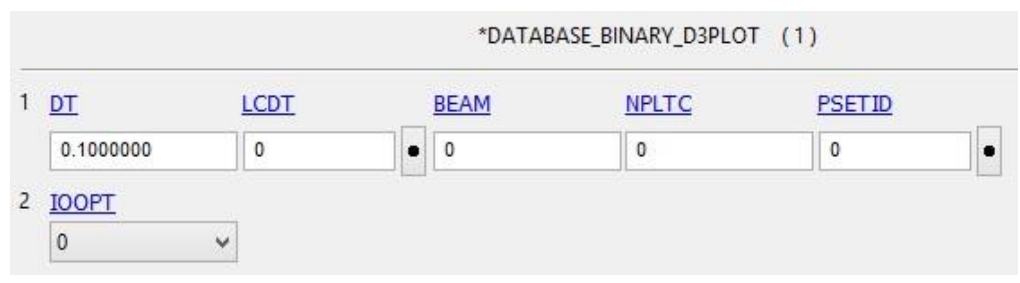

\subsection{Material properties}

- To create a material card to define the material properties:

- $\quad$ Click Model > Keywrd

- Double-click MAT > 001-ELASTIC. This is an isotropic elastic material

- Name the material to Steel.

- Set the material properties RO, E and PR as in the figure below (also stated in section 1.3).

- Click Accept, then Done.

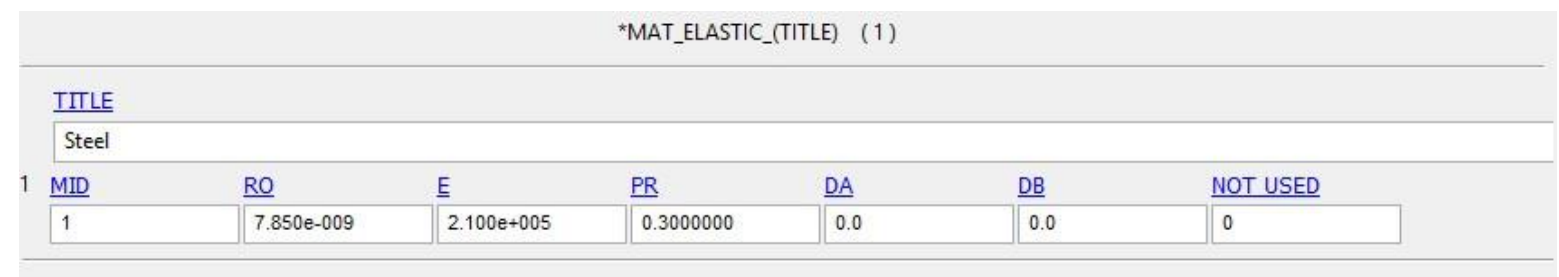

\subsection{Element properties}

The element type to be used:

- From Keyword Manager, double-click SECTION > SOLID.

- Name the section to Cube

- Use ELFORM = 1, which is the default element formulation.

- $\quad$ Click Accept, then Done.

\begin{tabular}{|c|c|c|c|c|c|}
\hline & & & & & *SECTION_SOLID_(TITLE) (1) \\
\hline & IITLE & & & & \\
\hline & Cube & & & & \\
\hline 1 & $\underline{\text { SECID }}$ & ELFORM & & AET & \\
\hline & 1 & 1 & $\checkmark$ & 0 & \\
\hline
\end{tabular}

Now apply the material and element properties to the part. Since the part already is created, one can activate Model, instead of All, in the Keyword Manager. This makes it easier to navigate through the list of keywords.

- Double-click PART > PART

- Click on the black dot next to SECID, defined entities will then be shown. Select your newly created section (1 Cube) and Accept and then Done

- Do the same thing for MID. Click Accept, then Done. 
The result should be as shown below.

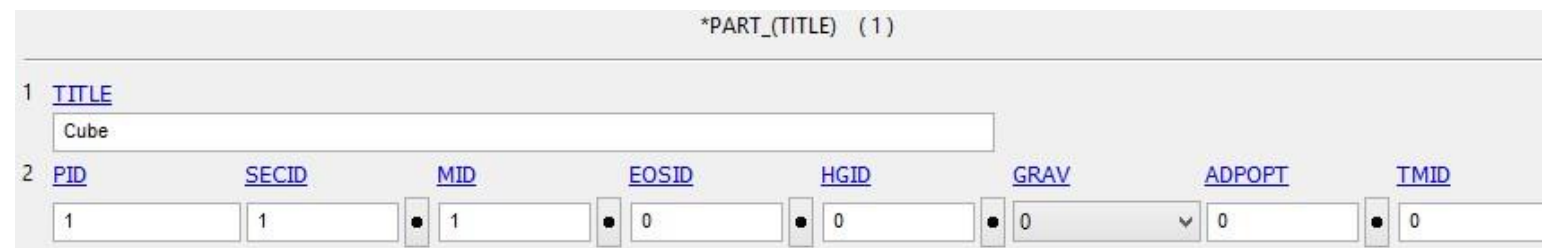

\subsection{Check the model before running}

Now check for errors using the Model Check:

- From the top menu, click Application > Model Checking > General Checking.

- Switch to Keyword Check. The warnings and error should not exist.

- Click Done.

Note that even if no errors or warnings occurs, the model can still be incomplete or wrong. There is no way for any pre-processor to know your intended use of the model. Hence, the loadings and boundary conditions can only be checked if they make any sense, not if they are correct with respect to your load case.

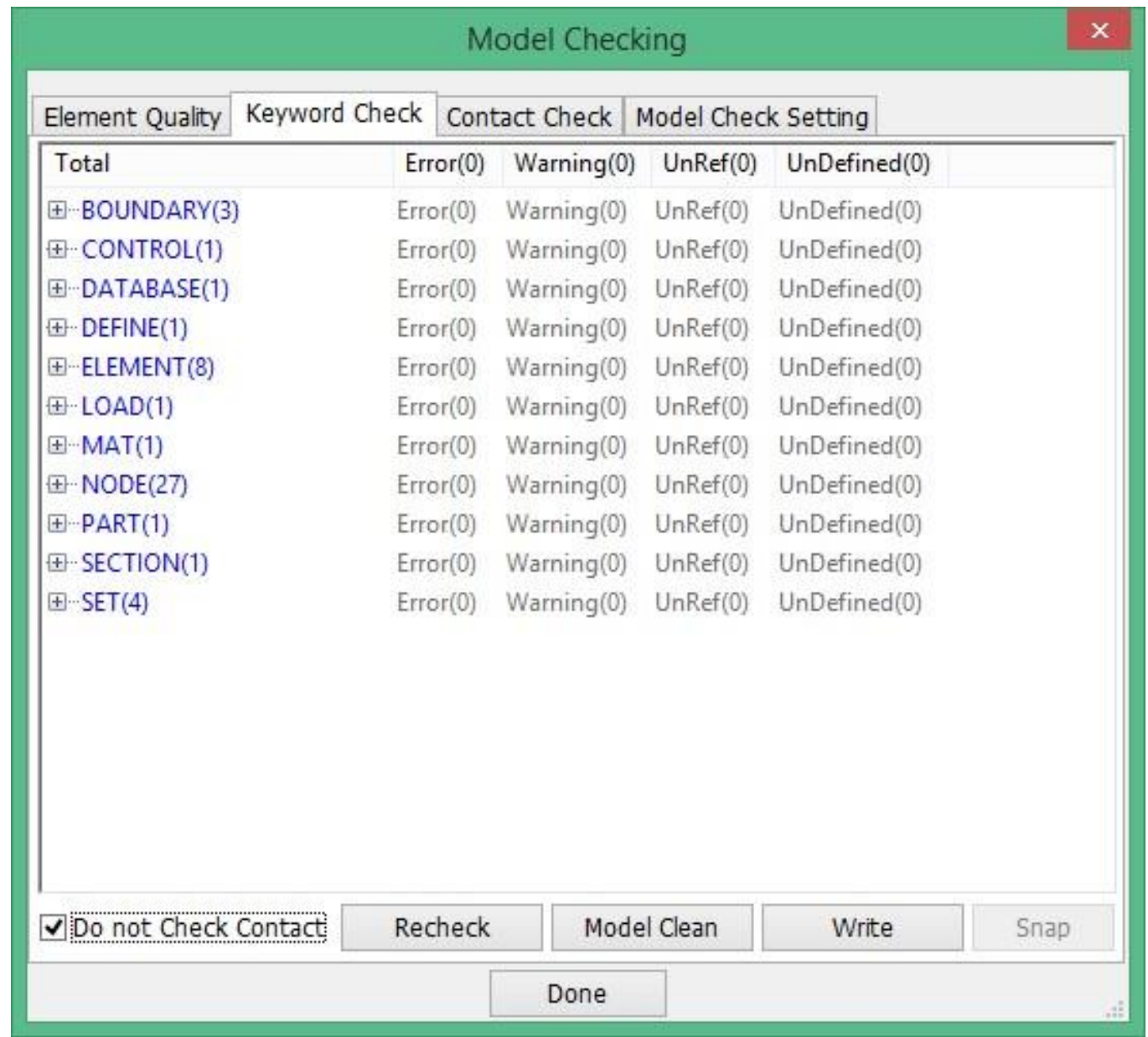




\subsubsection{Save and run the simulation}

It is preferable to run each simulation in a separate folder, thus create one before saving if you have not done so, e.g. CUBE. First save the finished keyword model from LS-PrePost in the new "CUBE" folder that you have created on your computer using File $>$ Save As $>$ Save Keyword As. Use the file name cube.k, note the. $\mathbf{k}$ suffix.

To run the model first open LS-Run $\stackrel{=5}{>} \mid$ from the start menu|in Windows.

Select the cube.k file on the INPUT file using the file menu button to the right of the INPUT field or by drag-and-dropping the file cube.k onto the INPUT field. Select solver "SMP Single" and set NCPU to 2. This should yield the result below. Note: To learn more about LS-Run, see the Help menu.

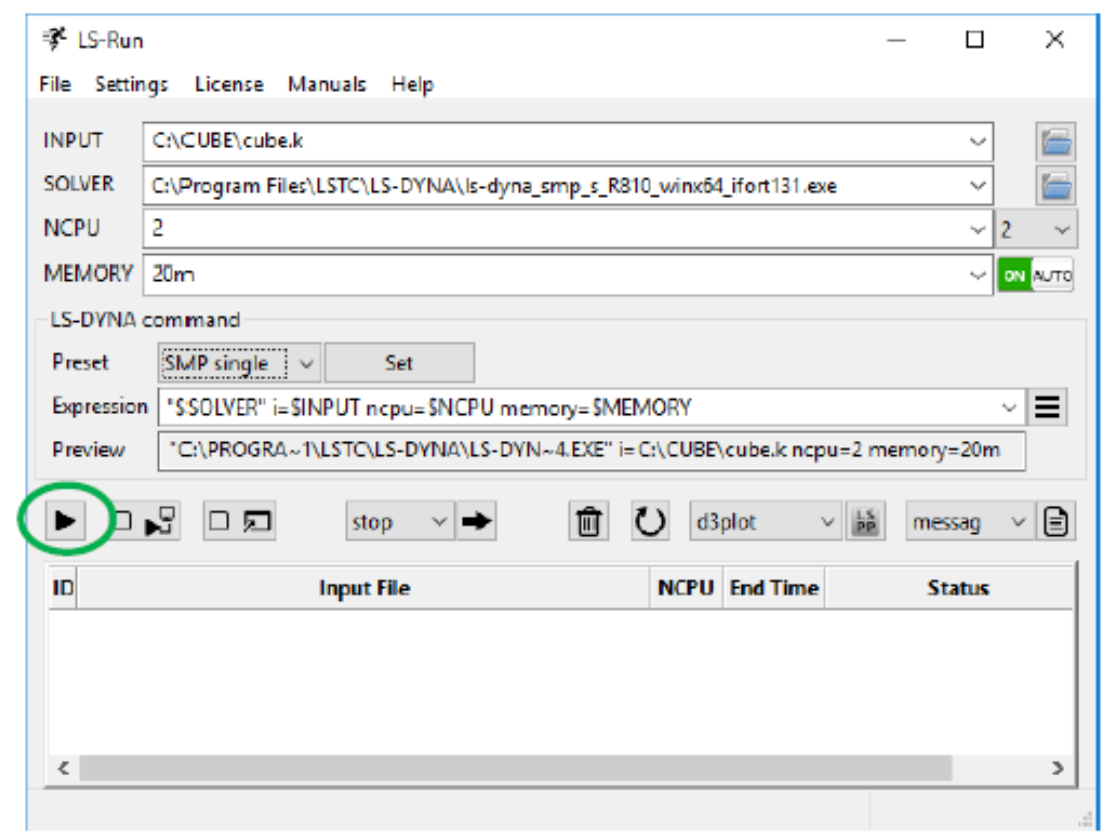

To start the simulation, press the play button circled in green in the image above. In just a few second the simulation should be done yielding "Finished (Normal Termination)".

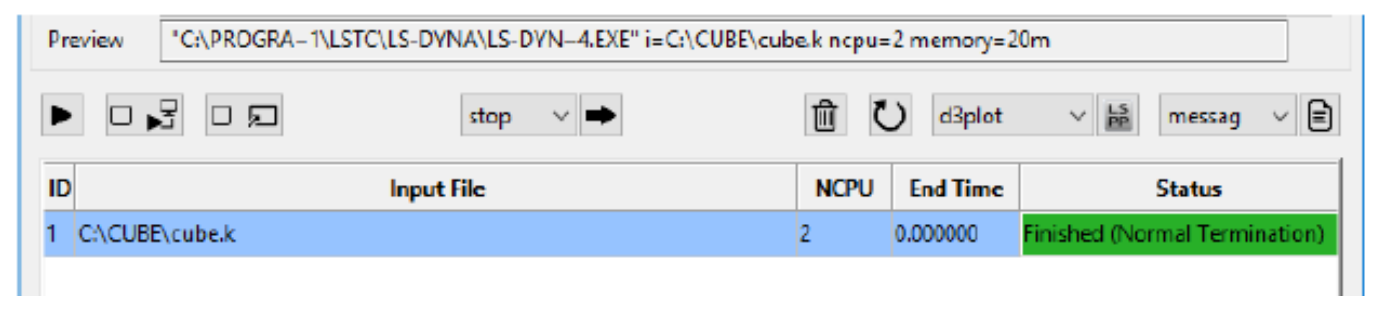

\subsubsection{Post processing}

To visualize the results, you need to open the d3plot result file. This is done by selecting File $>0$ pen $>$ LS DYNA Binary Plot. Once Binary Plot is opened, the animate toolbar is the tool that enables you to step through the different states of the simulation. Hold the mouse over the different buttons, a text box will pop up and show information about the different possibilities using the animator toolbar. Play around with the buttons to see what happens. Note that the deformations are very small, therefore you will probably not notice that anything happens with the cube. 


\begin{tabular}{|c|c|c|c|c|c|}
\hline \multicolumn{5}{|c|}{ Animate } & $x$ \\
\hline$\square$ Eigen First: & 1 Last: & 12 Inc: & 1 Time: & 0 State: & 1 \\
\hline$\square$ Animate & & & & $\checkmark$ & \\
\hline
\end{tabular}

To easier see what happens, you can scale up the deformations:

\begin{tabular}{|c|c|c|}
\hline \multicolumn{3}{|c|}{ Post Settings } \\
\hline \multicolumn{3}{|c|}{$\begin{array}{l}\text { ODisplacement Scale Factor } \\
\text { OReflections } \\
\text { OHIC/CSI Constants } \\
\text { OConcrete Crack Width } \\
\text { OCoordinate Scale Factor } \\
\square \text { FLD E'Strain } \\
\square \text { Local B'Strain } \\
\square \text { Extrapolate }\end{array}$} \\
\hline OWhole & OP & \\
\hline \multicolumn{3}{|c|}{ Displacement Scale Factor: } \\
\hline & \begin{tabular}{l|l}
5000 & 1.0 \\
\end{tabular} & $\checkmark$ \\
\hline$\nabla x$ & ఐY & $\square 2$ \\
\hline \multirow[t]{2}{*}{ Apply } & Clear & Reset \\
\hline & Done & \\
\hline
\end{tabular}

- Click Settings > Post Settings in the top menu.

- Select Displacement Scale Factor, write $\mathbf{5 0 0 0}$ as the factor. X, Y and Z shall be activated, which implies that the displacements will be scaled in all directions.

- Click Apply, then Done.

Play around with the animate toolbar again and see how the cube deforms.

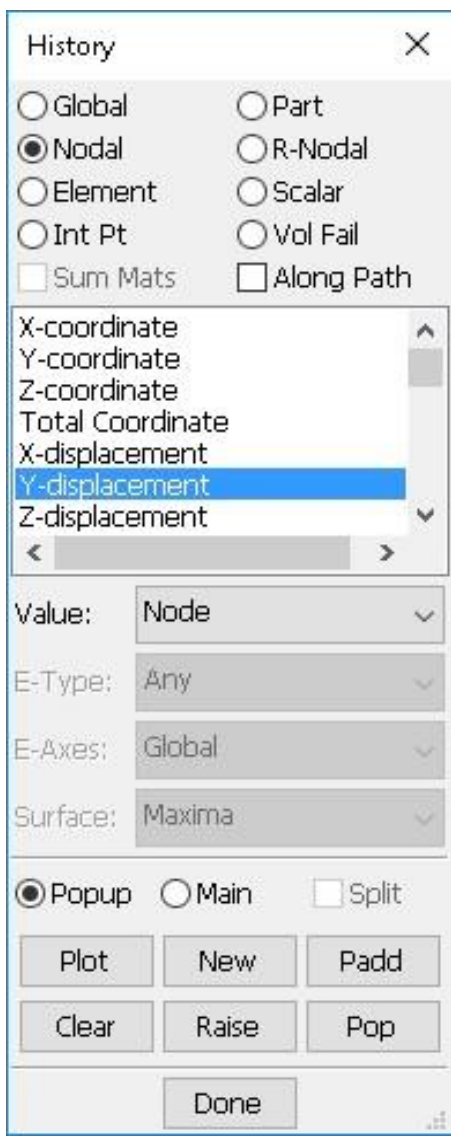

Then plot the deformation history as a curve:

- Click Post > History.

- In the History Box, select Nodal > Y-displacement.

- Select a node on the top of the box.

- Click Plot.

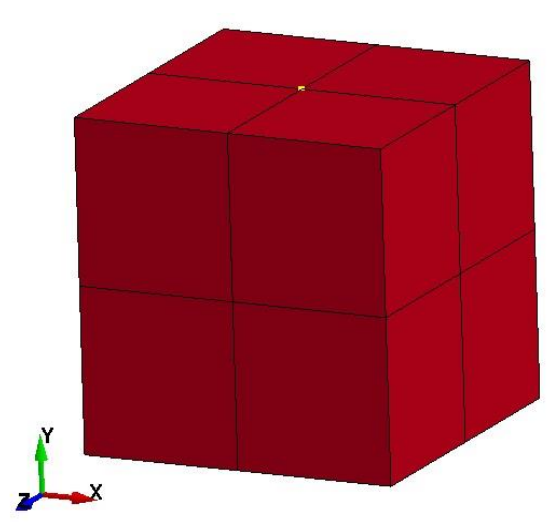


In the Plot Window, Y-displacement vs Time are stated. Zoom by pressing Ctrl and make a box with the mouse. A right-click will reset the window to original. Zoom in on the curve around Time=1, click on the final state. Note that the Y-displacement is $\mathbf{- 0 . 0 4 7 6} \mathbf{~ m m}$.

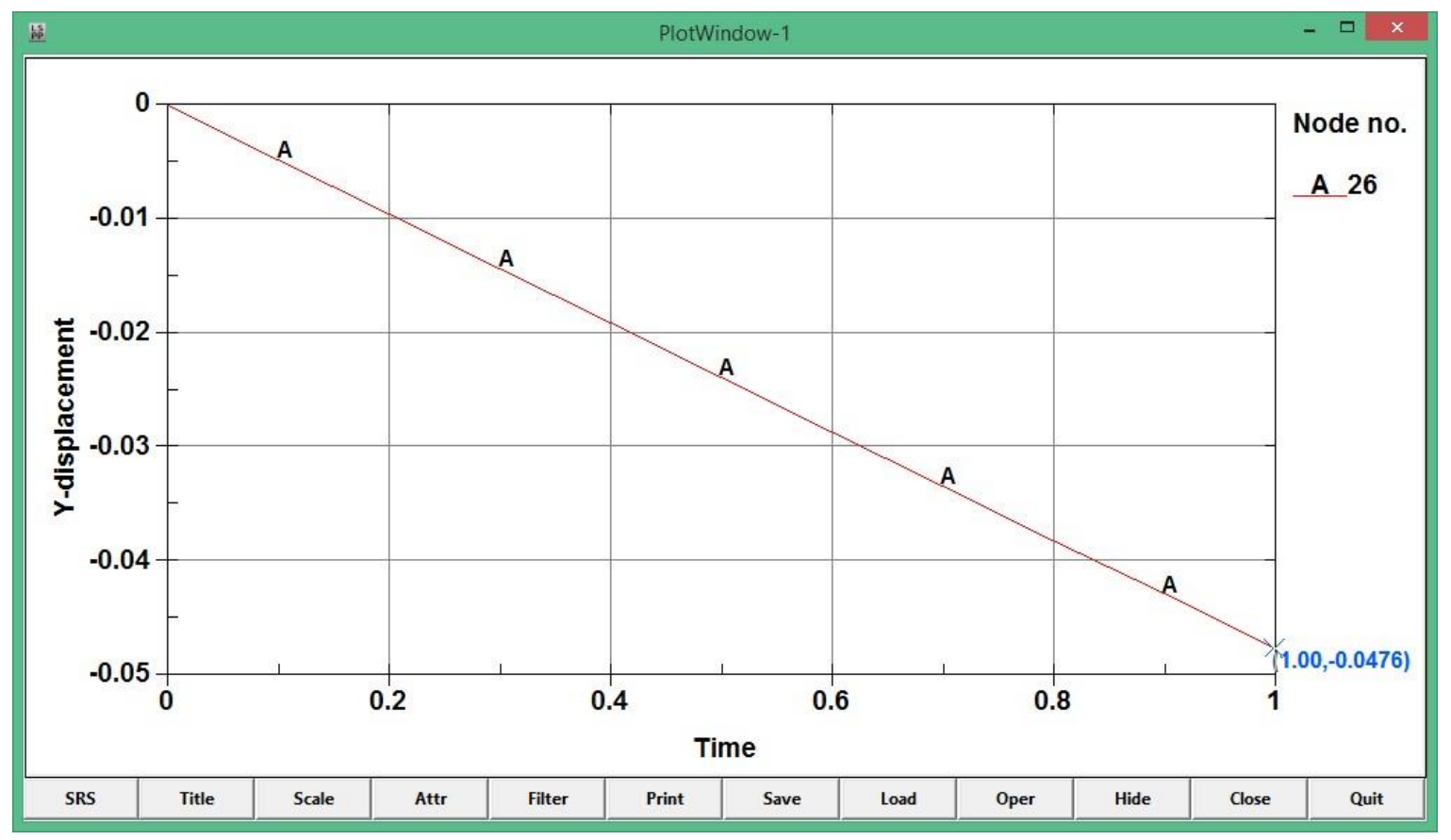

When you click on the curve, information about the picked point will be stated in the Message box, which is located at the bottom of the LS-PrePost window. If you double-click in this box, a bigger box will pop up.

Close the Message box and Plot Window.

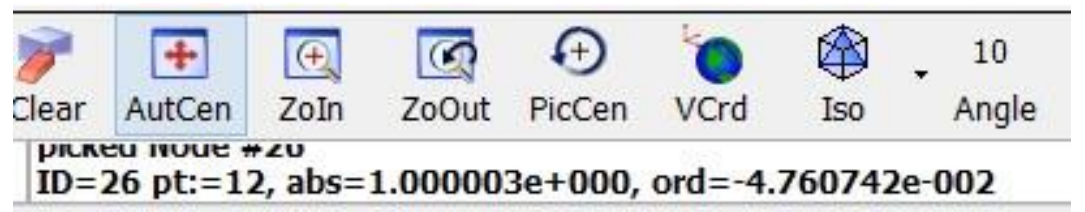

Now, click Post $>$ FriComp $>$ Ndv > y-displacement. Use the Animate toolbar and go to the last state. The values in the Fringe Level shows that the maximum y displacement is $-4.761 \mathrm{e}-\mathbf{0 2} \mathbf{~ m m}$.

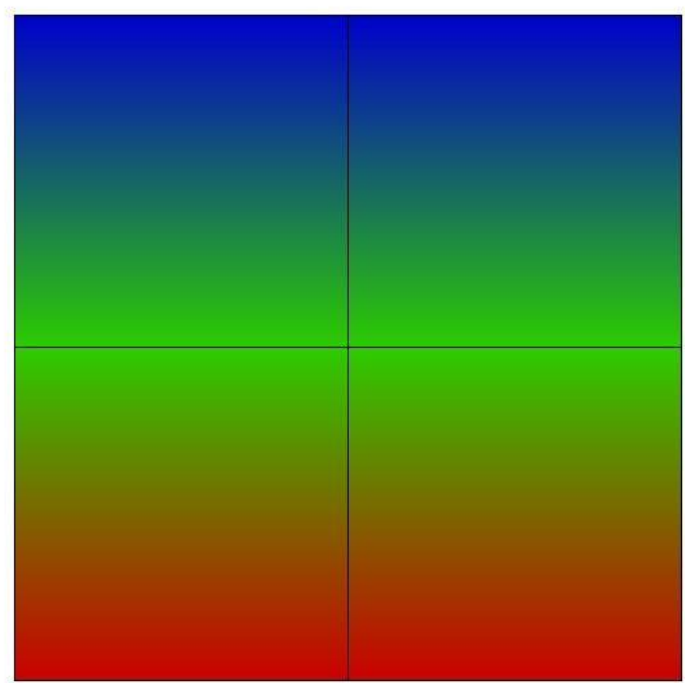




\subsubsection{Analytical solution}

The analytical solution of the vertical displacement due to a $10 \mathrm{MPa}$ pressure load is derived from Hooke's law.

$$
\text { Displacement }=\frac{p L}{E}=\frac{10 e^{6} \cdot 1}{210 e^{9}}=4.76 e^{-5} \mathrm{~m}=0.0476 \mathrm{~mm}
$$

The simulation result should be nearly identical to the analytical solution.

\subsubsection{Exercises}

There are several possibilities to try out different features in LS-DYNA with this simple model. What happens if you change:

material?

- boundary conditions?

- $\quad$ element formulations?

- $\quad$ load level?

\subsubsection{References}

Tutorials taken from:

https://www.dynasupport.com/tutorial/introduction-ls-dyna-ls-prepost-for-explicit-andimplicit-analysis

https://www.dynasupport.com/manuals

\subsection{Laser matter interaction FEM simulations - Tensile stress $\&$ thermal stress problem}

The aim is to understand a structural problem with elastoplastic properties taken into account and a thermal stress problem, since thermal stresses are developed during laser irradiation. Initially, a tensile test on a flat specimen is performed. The end of the specimen is constrained while a prescribed motion is applied on the other end.

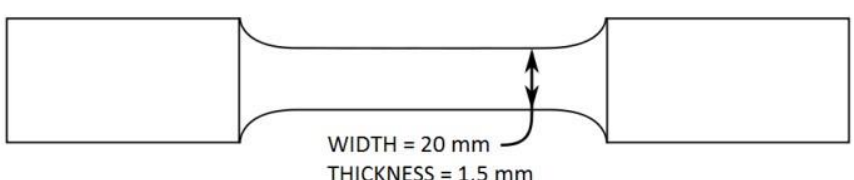

Figure 18.10 Tensile test and problem material properties

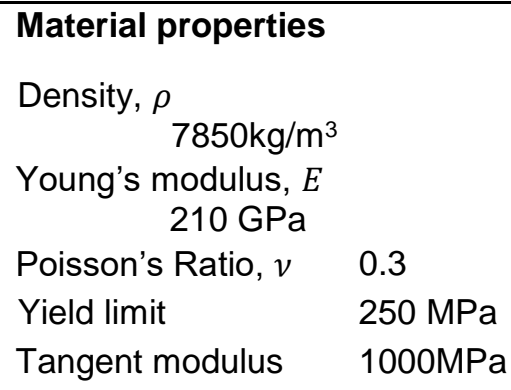




\subsubsection{Explicit elastoplastic structural analysis}

Open tensile_test.k in LS-PrePost, which contains the geometry of the test specimen.

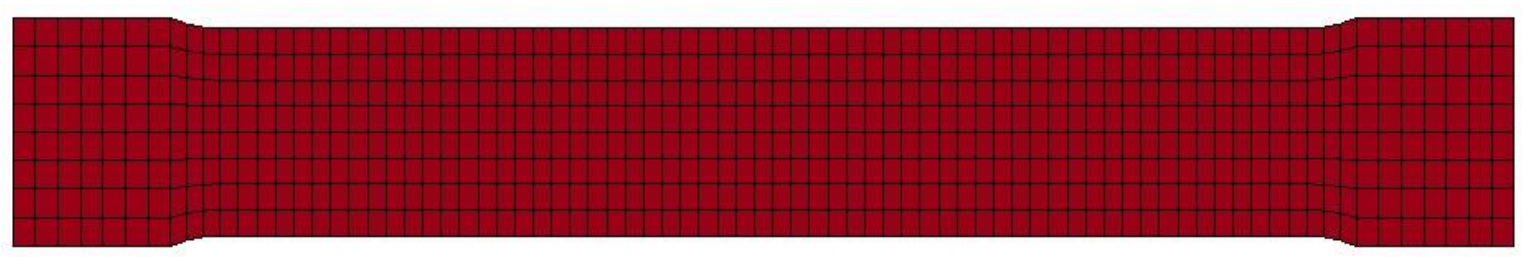

Figure 18.11 Geometry and mesh of the specimen

\subsubsection{Material properties}

LS-DYNA accepts, for most materials, input in terms of true stress vs. true strain. Normally an experimental uniaxial tension test is performed and engineering stress and strain data are obtained. Before these data are used as an input in a material model in LS-DYNA, they are converted to true stress and strain. The curves behave as in the figure.

Engineering stress is the applied load divided by the original cross-sectional area of a material, while true stress is the applied load divided by the actual cross-sectional area (changing area with respect to time) of the specimen at that load.

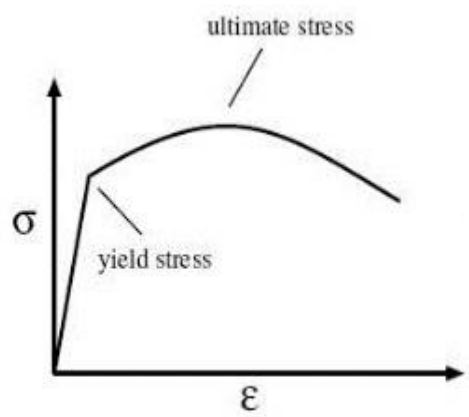

engineering stress-strain

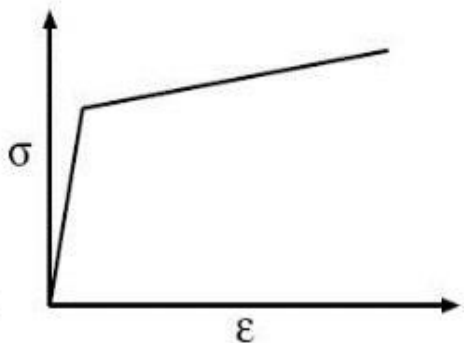

true stress-strain

Figure 18.12 Engineering and true stress-strain curves

To create the material card:

Click Model > Keywrd. Activate All in the Keyword Manager. Double-click MAT > 024PIECEWISE_LINEAR_PLASTICITY, which is an elasto-plastic material.

Define Yield stress SIGY and Tangent Modulus ETAN, which gives linear hardening.

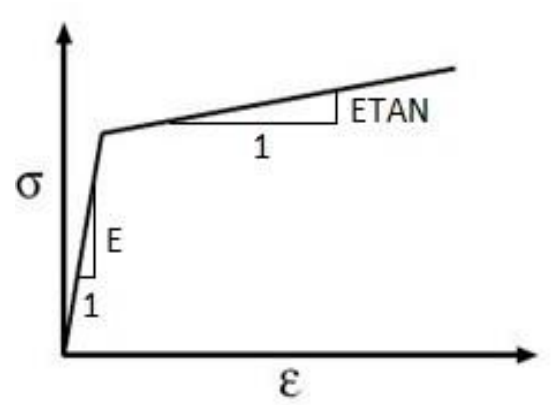




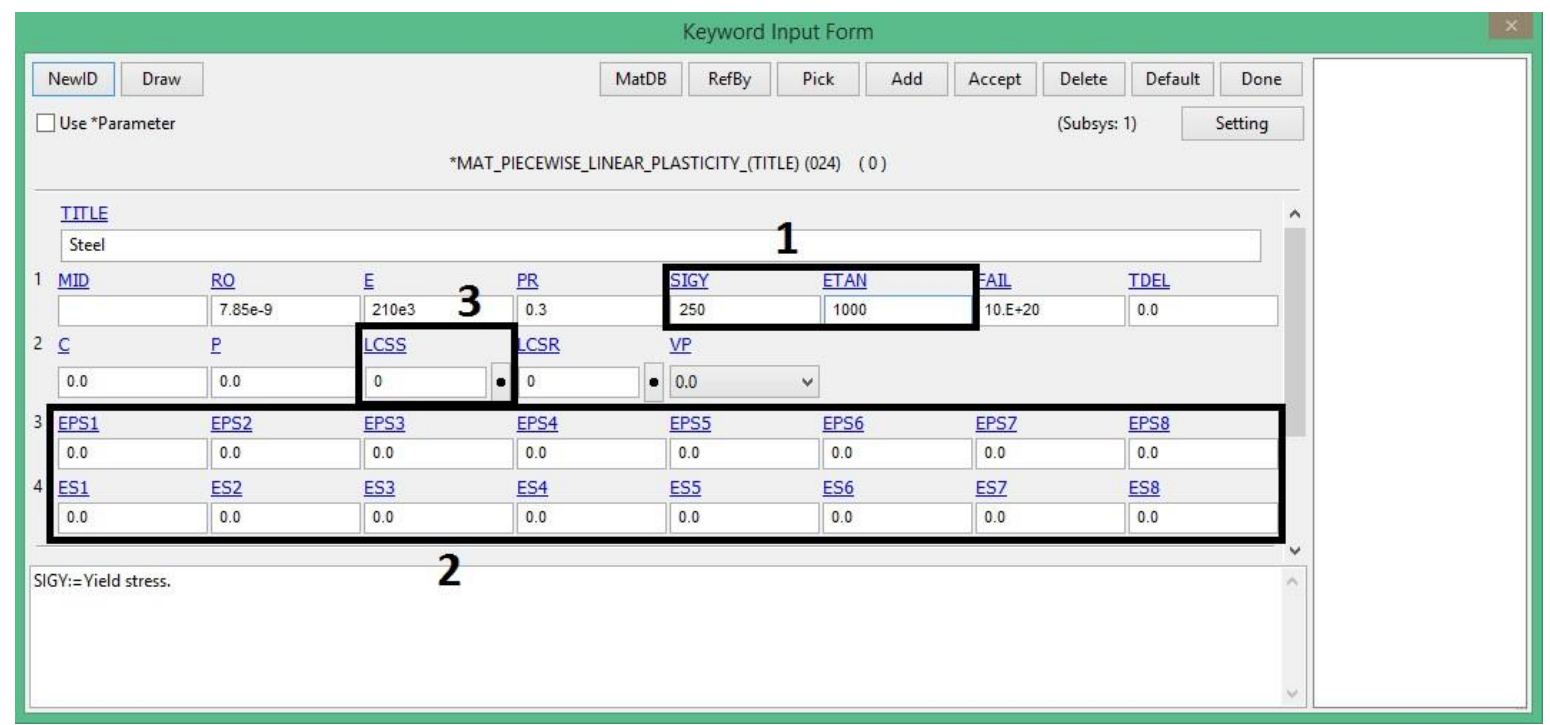

Enter the title and the values as in the figure above and click Accept, then Done.

\subsubsection{Element properties}

To set the element formulation and properties do as follows:

- Double click SECTION > SHELL in the Keyword Manager.

- Enter Specimen_shell as TITLE.

- Set T1 to 1.5 and press Enter, T2-T4 will then be changed to $\mathbf{1 . 5}$ as well and this defines the thickness of the nodes in every element.

- Click Accept, then Done.

Now go to Part in the Keyword Manager and assign the newly created material and section. There one can also change the name of the part, to Tensile specimen for example. Click Accept, then Done.

\subsubsection{Boundary conditions}

Apply the fixed boundary conditions as follows:

- Click on the Bottom view in the Floating Toolbar.

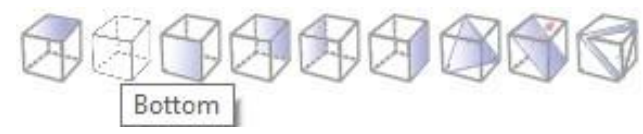




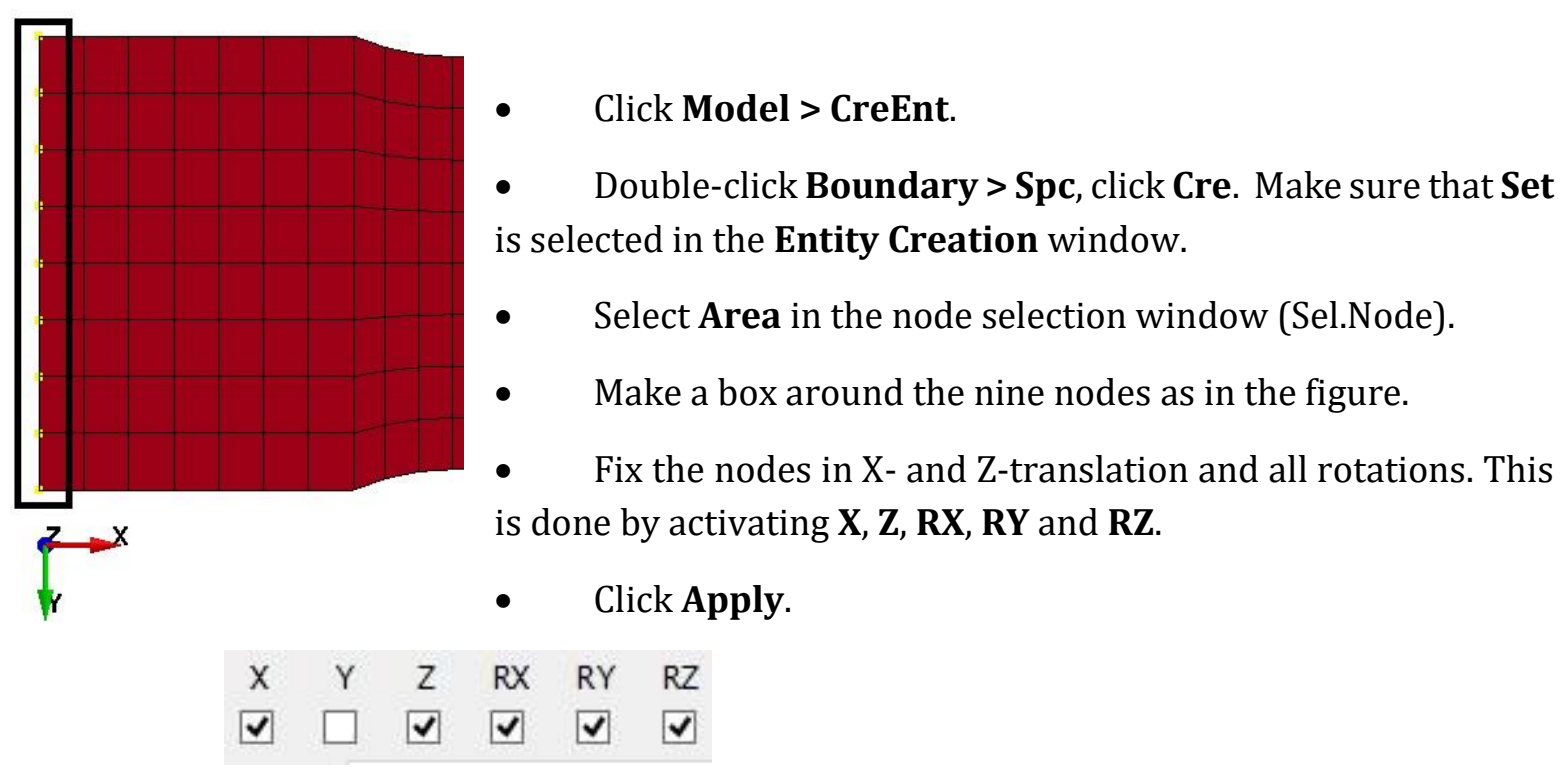

Now create another boundary condition:

- In the node selection window, write $\mathbf{8 0}$ in the ID box and press Enter. Write $\mathbf{8 7}$ and press Enter. Two nodes will now be selected at the outer edges of the test specimen. Fix the nodes in $\mathrm{Y}$ translation by only activating $\mathbf{Y}$.

- Click Apply.

- Close Entity Creation window.

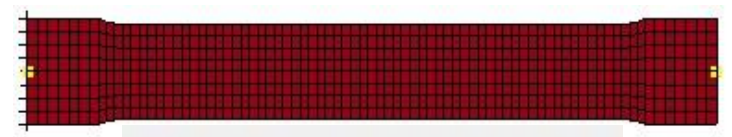

$\begin{array}{llllll}X & Y & Z & R X & R Y & R Z\end{array}$

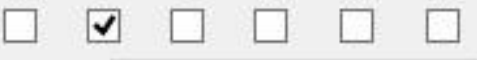

\subsubsection{Prescribed motion/displacement}

For motions and loads, a curve must be defined that states the variation of the load/displacement/velocity etc. over time.

First create the curve defining the motion:

- In the top menu, click Application > Tools > CurveGen

- Change Method to X-Y.

- Deactivate Smooth.

- Write $\mathbf{X = 0}$ and $\mathbf{Y = 0}$ (ignore the value of smooth)

- Click Insert. Then 0.01 and 1, Insert.

- Finally, 0.011 and 1, Insert (the termination time will be $0.01 \mathrm{~s}$ for this tutorial).

- Change Curve Name to e.g. Motion.

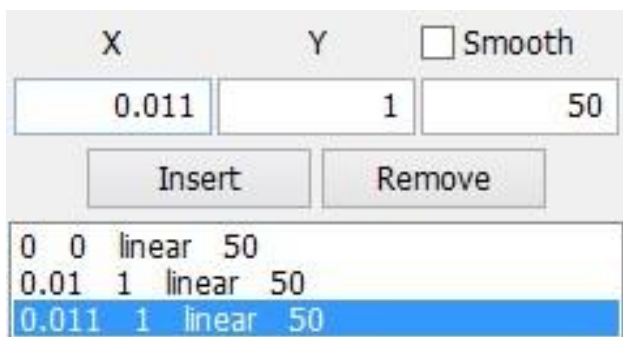

- Click Create, then Done. 
Note that the curve could alternatively have been created using DEFINE > CURVE in the Keyword Manager. A curve created in CurveGen will contain a larger amount of points, compared to a curve created by DEFINE > CURVE.

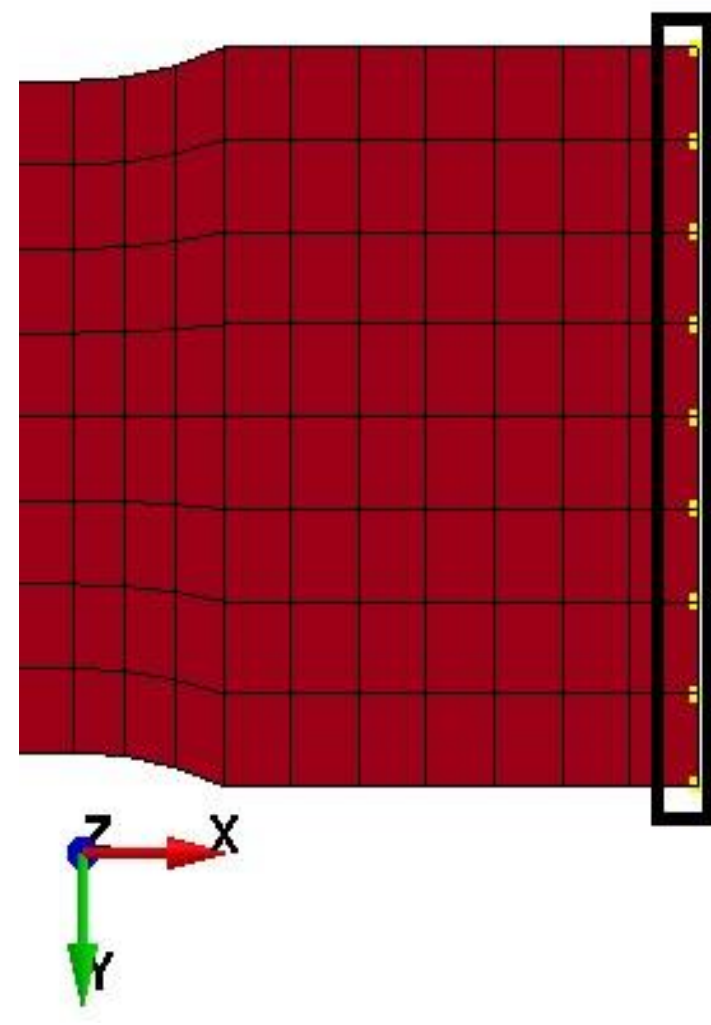

Now apply the motion to the end of the tensile specimen:

- Click Model > CreEnt.

- In the Entity Creation window, double-click Boundary > Prescribed Motion.

- Select Cre. Change Type to SET and activate Pick.

- Make a box around the nine nodes as in the figure.

- Set DOF = 1 (X-translational as degree of freedom) and $\mathbf{V A D}=\mathbf{2}$ (displacement as the prescribed nodal quantity, there are possibilities to prescribe the nodal velocity or acceleration as well).

- Click on LCID and select the previously created curve.

- Set $\mathbf{S F}=\mathbf{2 0}$, which implies that the node set will be moved $20 \mathrm{~mm}$.

- Click Apply, then Done.

\subsubsection{Set the termination time}

To set the termination time:

- $\quad$ Click Model > Keywrd.

- Double-click CONTROL > TERMINATION.

- Set ENDTIM to 0.01. The simulation will then last for 0.01 seconds.

- Click Accept and then Done.

\subsubsection{Output}

Here the output to be saved by LS-DYNA during the simulation is defined, first specify d3plot output:

- Create d3plots.

- $\quad$ Click Model > Keywrd.

- Double-click DATABASE > BINARY_D3PLOT.

- $\quad$ Set $\mathbf{D T}=\mathbf{5 e - 4}$.

- Click Accept and then Done.

Since the task was to compare the stress vs. strain curve for an element with the material curve, we want to obtain this data frequently. The time-history graphs created by History, use 
information found in the d3plot files. To obtain data more frequently we can specify which type of data are of interest and how often it will be printed for selected elements. Therefore:

- $\quad$ Click Model > Keywrd.

- Double-click DATABASE > ASCII_option. We are interested in the stress and strains in the elements, therefore activate ELOUT. On the same row, set DT = 5e-6.

- Click Accept, then Done.

The element/elements that we want to gather data from must be defined:

- Double-click DATABASE > HISTORY_SHELL in the Keyword Manager.

- Write, for example, shell element number $\mathbf{3 1 4}$ under ID1, which is an element close to the center of the specimen.

- Click Insert, Accept, then Done.

- Check the model to see that everything looks okay.

\subsubsection{Save and run the simulation}

The model is now ready to be saved. File $>$ Save As $>$ Save Keyword As. Choose a folder path and name your file tensile_test_model.k for example. Note that the folder path cannot contain any spaces. Close LS-PrePost and run the simulation, as shown in 4.1.3.3.

\subsubsection{Post processing}

Open the d3plot in LS-PrePost from LS-Run or from the File menu of LS-PrePost. Start to press the Forward button in Animate toolbar to see what happens with the specimen. Now select the elout file, first plot and save stress vs. time:

- $\quad$ Click Post > ASCII.

- Select elout* and click Load.

- Select Sh-314, Ip-1 or Ip-2 (doesn't matter which integration point in this case) and 9Effective Stress (v-m).

- Click Plot.

- In the PlotWindow, click Save.

- Let the Output Type be Curve file.

- Enter Stress as the Filename (also set correct folder path).

- Click Save (located at the bottom toolbar, see red square).

Quit 


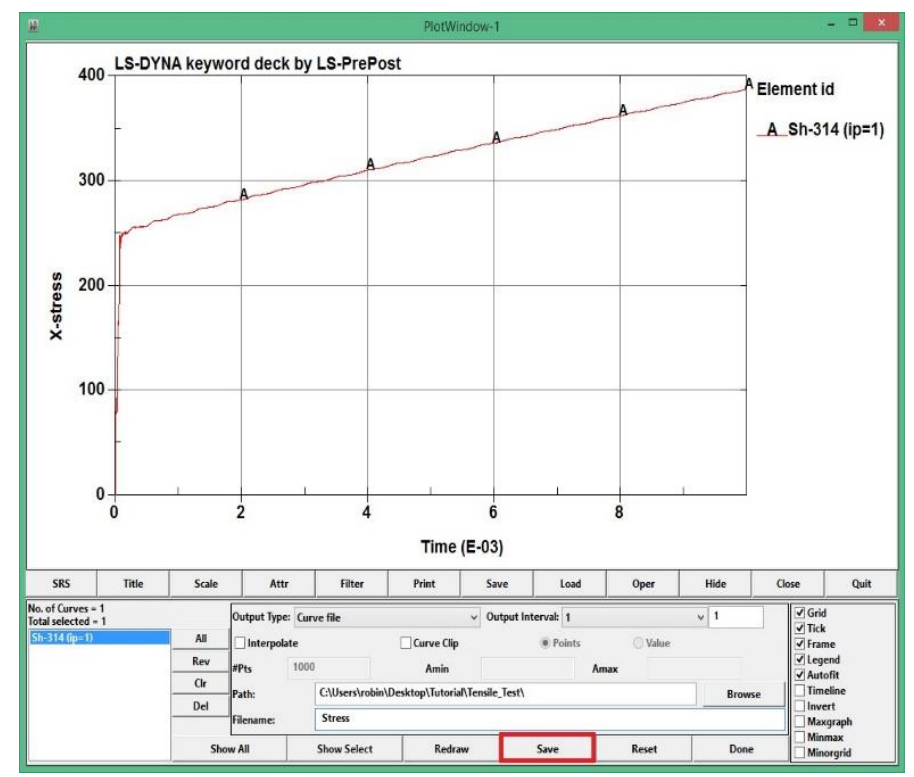

\subsubsection{D Thermal stress problem}

This problem addresses the unconstrained expansion of a block due to heating. The model consists of one 8 node brick element at an initial temperature of $10^{\circ} \mathrm{C}$. The brick material is given a volumetric thermal generation rate. It holds:

$$
q V t=m c \Delta T
$$

Where:

volumetric heat generation rate volume

$$
\begin{aligned}
& \mathrm{q}=10 \mathrm{~W} / \mathrm{m}^{3} \\
& \mathrm{~V}=1 \mathrm{~m}^{3} \\
& \mathrm{~m}=\rho \mathrm{V}=(1 .)(1 .)=1 . \mathrm{kg} \\
& \mathrm{c}=1 \mathrm{~J} / \mathrm{kg} \mathrm{C} \\
& \mathrm{t}=3 \mathrm{sec}
\end{aligned}
$$$$
\text { mass }
$$$$
\text { heat capacity }
$$$$
\text { time for heat generation }
$$

The block increases in temperature by $30 \mathrm{C}$. The final block temperature is $10 \mathrm{C}+30 \mathrm{C}=40 \mathrm{C}$.

A tangent coefficient of thermal expansion is defined by

$$
\lambda=2.0 e-07 * T
$$

The thermal expansion can be calculated by

$$
\Delta l=\int_{10}^{40} 2.0 e-07 * T d T=\left[1.0 e-07 * T^{2}\right]_{10}^{40}=1.5 e-04
$$

The problem should be specified as a coupled thermal structural analysis in the $\left({ }^{*}\right.$ CONTROL_SOLUTION) section. 
LS-DYNA uses a tangent coefficient of thermal expansion, which is defined as the slope of the thermal strain versus temperature curve for the material.

The mechanical mass scaled time step is set to 0.01 seconds (*CONTROL_TIMESTEP) and the thermal time step is set to 0.1 seconds (*CONTROL_THERMAL_TIMESTEP). Explicit time integration is used for the structural calculations and implicit time integration is used for the thermal calculations. Implicit time integration is unconditionally stable and, therefore, a larger thermal time step can be taken.

Task: You are given the LS-DYNA input file and the results. Your task is to understand the building of the model and the usage of the keywords.

\subsubsection{Code piece}

The following keywords are needed for the simulation:

\section{*CONTROL_SOLUTION}

\$\# soln nlq isnan lcint

$\begin{array}{llll}2 & 0 & 0 & 100\end{array}$

The Keyword *CONTROL_SOLUTION is used to specify the analysis solution procedure when thermal, coupled thermal or structural analysis is performed.

Variables:

Soln: Analysis solution procedure:

0 : Structural analysis only,

1: Thermal analysis only,

2: Coupled structural thermal analysis.

\section{*CONTROL_TERMINATION}

\$\# endtim endcyc dtmin endeng endmas nosol
$\begin{array}{lllll}1.2 & 0 & 0.0 & 0.01 .000000 \mathrm{E} 8 & 0\end{array}$

The Keyword *CONTROL_TERMINATION is used to set the termination conditions.

Variables:

endtim: It defines the termination time of the analysis. It is a mandatory variable for the LSDYNA software.

endcyc: It defines the maximum number of steps that will be analyzed, according to the step time (dtstart) that we will set for out problem's analysis. Always variable endtim is more important and analysis will be terminated if we reach endtim value. 
dtmin: It defines the minimum time between steps that is allowed. When the time step drops to dtmin, LS-DYNA terminates. By default, is inactive.

endeng: This variable sets the maximum total energy change ratio that is allowable. If succeeded analysis is terminated. By default, is inactive.

endmas: This variable sets the maximum total mass change ratio that is allowable. If succeeded analysis is terminated. By default, is inactive.

\section{*CONTROL_THERMAL_SOLVER}

\$\# atype ptype solver cgtol gpt eqheat fwork sbc

$\begin{array}{lllllll}1 & 2 & 31.00000 \mathrm{E}-6 & 1 & 1.0 & 1.0 & 0.0\end{array}$

\$\# msglvl maxitr abstol reltol omega unused unused tsf

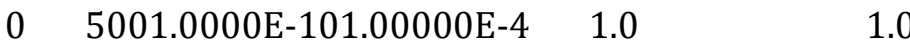

The keyword *CONTROL_THERMAL_SOLVER sets options for the thermal solution in a thermal only or coupled structural-thermal analysis. To use it is required the usage of the proper solver (variable soln) in *CONTROL_SOLUTION keyword.

\section{*CONTROL_THERMAL_TIMESTEP}

$\$$ ts tip its tmin tmax dtemp tscp lcts

$$
\begin{array}{lllllll}
0 & 0.5 & 0.0011 .00000 \mathrm{E}-7 & 0.0139 & 100.0 & 0.5 & 0
\end{array}
$$

The keyword *CONTROL_THERMAL_TIMESTEP sets timestep for a thermal only or coupled structural-thermal analysis. To use it is required the usage of the proper solver (variable soln) in *CONTROL_SOLUTION keyword and the *CONTROL_THERMAL_SOLVER keyword.

\section{*CONTROL_TIMESTEP}

\$\# dtinit tssfac isdo tslimt dt2ms lctm erode ms1st

$$
\begin{array}{llllllll}
0.0 & 0.6 & 0 & 0.0 & 0.0 & 0 & 0 & 0
\end{array}
$$

\$\# dt2msf dt2mslc imscl unused unused rmscl
$0.0 \quad 0 \quad 0$
0.0

The keyword ${ }^{*}$ CONTROL_TIMESTEP sets the timestep for structural problem analysis.

dtini: This variable defines the initial timestep size. By default, is blank or 0 and LS-DYNA determines initial step size. 


\section{*DATABASE_BINARY_D3PLOT}

\$\# dt lcdt beam npltc psetid

$\begin{array}{lllll}0.0 & 0 & 0 & 100 & 0\end{array}$

\$ ioopt

0

The keyword *DATABASE_BINARY_D3PLOT defines parameters for the output from the entire model. From these outputs by post processing them we will conclude to an final presentable output of our solution analysis.

dt: In this variable we set the time interval between output states.

nr: In this variable we set Number of RUNning ReStart Files, RUNRSF, written in a cyclical fashion. The default is 1, i.e., only one runrsf file is created and the data there in is overwritten each time data is output.

npltc: In this variable we calculate DT = ENDTIME/NPLTC applies to D3PLOT and D3PART only. This overrides the DT specified in the first field.

\section{*DATABASE_GLSTAT}

\$\# dt binary lcur ioopt

$\begin{array}{llll}0.003 & 0 & 0 & 1\end{array}$

The keyword *DATABASE has many options to define to obtain output files containing results information. One of them is the *DATABASE_GLSTAT, which provide us with the global data of the solution analysis of our model.

Dt : In this variable we se the time step between outputs. If is set to 0 then no output is printed. binary : In this variable we set the type for binary output.

1 : Data written to an ASCII file default for SMP LS-DYNA.

2 : Data written to binary database "binout", default for MPP LS-DYNA.

3 : Both ASCII and binary outputs.

\section{*PART}

$\$ \#$ title

\$\# pid secid mid eosid hgid grav adpopt tmid

$\begin{array}{llllllll}2 & 1 & 1 & 1 & 0 & 0 & 0 & 1\end{array}$


The keyword *PART is essential for the model identification and characterization in the solution analysis. Within it variables we combine various properties and meshes to form the parts we want to analyze. To form a part in LS-DYNA we combine the identity of a formed mesh with the identity of the material, with the identity of the used Equation Of State for that material, with the thermal properties identity, etc.

pid: In this variable we put the part id number

secid: In this variable we put the section id number, defined in a *SECTION keyword.

mid: In this variable we put the material id number, defined in a *MAT keyword.

eosid: In this variable we put the Equation Of State id number, defined in a *EOS keyword.

tmid: In this variable we put the thermal properties id number, defined in a *MAT_THERMAL keyword.

\section{*SECTION_SOLID}

\$\# secid elform aet

$1 \quad 1 \quad 0$

In this keyword, we define section properties for solid continuum and fluid elements.

secid: In this variable we put the section id number, defined in a *SECTION keyword.

elform: Element formulation options:

-2: Fully integrated S/R solid intended for elements with poor aspect ratio, accurate formulation

-1: Fully integrated S/R solid intended for elements with poor aspect ratio, efficient formulation

0: 1 point corotational for *MAT_MODIFIED_HONEYCOMB

1: Constant stress solid element: default element type.

2: Fully integrated S/R solid

3: Fully integrated quadratic 8 node element with nodal rotations

4: S/R quadratic tetrahedron element with nodal rotations

5: 1 point ALE

6: 1 point Eulerian

7: 1 point Eulerian ambient

8: Acoustic

9: 1 point corotational for *MAT_MODIFIED_HONEYCOMB

10: 1 point tetrahedron

11: 1 point ALE multi-material element

and various others. 


\section{*MAT_ELASTIC_PLASTIC_THERMAL}

Task: Determine the usage of this material keyword.

\section{*MAT_THERMAL_ISOTROPIC}

\$\# tmid tro tgrlc tgmult tlat hlat

$\begin{array}{llllll}1 & 0.0 & 0.0 & 0.0 & 0.0 & 0.0\end{array}$

$\$ \#$ hc tc

560.03.70000E-5

This keyword *MAT_THERMAL_ISOTROPIC defines thermal isotropic properties to the material that selects it.

tmid: In this variable we set the unique ID number of the thermal material identification.

tro: In this variable we set the thermal density of the material. If set to 0.0 then it is equal to the structural density.

hc: In this variable we set the specific heat of the material

tc: In this variable we se the thermal conductivity of the material.

\section{*ELEMENT_SOLID}

$\begin{array}{cccrrrrrrrrr}\text { \$\# } & \text { eid } & \text { pid } & \mathrm{n} 1 & \mathrm{n} 2 & \mathrm{n} 3 & \mathrm{n} 4 & \mathrm{n} 5 & \mathrm{n} 6 & \mathrm{n} 7 & \mathrm{n} 8 & \\ 1 & 1 & 4313 & 4309 & 5283 & 5313 & 4308 & 4308 & 5138 & 5138 \\ 2 & 1 & 5313 & 5283 & 5284 & 5314 & 5138 & 5138 & 5139 & 5139 \\ 3 & 1 & 5314 & 5284 & 5285 & 5315 & 5139 & 5139 & 5140 & 5140\end{array}$

In this keyword *ELEMENT_SOLID we define each element of the created mesh of our model that we are going to perform solution analysis. Elements are defined in three-dimensional solid elements including 4 noded tetrahedrons and 8-noded hexahedrons. Most common are the 8noded hexahedrons.

eid: In this variable we set the unique ID number of the element.

pid: In this variable we set part ID number from with this element comes from.

n1 - n8: In these variables we set the nodal points ID that consists the element. If we use tetrahedron shape for our elements then we set only $n 1-n 4$ nodal point variables.

$\begin{array}{crccccc}\text { *NODE } & & & & & & \\ \text { \$\# nid } & \mathrm{x} & \mathrm{y} & \mathrm{z} & \text { tc } & \mathrm{rc} & \\ 1 & -0.027596 & 0.019934 & 0.2715 & 0 & 0\end{array}$




$\begin{array}{rrrrrr}2 & 0.0576701 & 1.0 & 0.2715 & 0 & 0 \\ 3 & -0.027596 & 0.019934 & 0.1285 & 0 & 0\end{array}$

In the keyword *NODE we define the nodes of our model. We also can define boundary conditions for the nodes. Nodes are defined by setting their coordinates at X-Y-Z axis.

nid: In this variable we set the unique node ID number

$\mathrm{x}$ : In this variable we set the $\mathrm{X}$ coordinate of the node

y: In this variable we set the Y coordinate of the node

z: In this variable we set the $\mathrm{Z}$ coordinate of the node

tc: In this variable we set the translational constraint:

0: no constraints,

1: constrained $\mathrm{x}$ displacement,

2: constrained y displacement,

3: constrained z displacement,

4: constrained $\mathrm{x}$ and $\mathrm{y}$ displacements,

5: constrained y and $\mathrm{z}$ displacements,

6: constrained $\mathrm{z}$ and $\mathrm{x}$ displacements,

7: constrained $\mathrm{x}, \mathrm{y}$, and $\mathrm{z}$ displacements.

rc: In this variable we set the rotational constraint:

0: no constraints,

1: constrained $\mathrm{x}$ rotation,

2: constrained y rotation,

3: constrained $\mathrm{z}$ rotation,

4: constrained $\mathrm{x}$ and $\mathrm{y}$ rotations,

5: constrained $\mathrm{y}$ and $\mathrm{z}$ rotations,

6: constrained $\mathrm{z}$ and $\mathrm{x}$ rotations,

7: constrained $\mathrm{x}, \mathrm{y}$, and $\mathrm{z}$ rotations.

*INITIAL_TEMPERATURE_NODE

It defines the initial temperature for the selected nodes. 


\subsubsection{References}

Tutorials taken from:

https://www.dynasupport.com/tutorial/introduction-ls-dyna-ls-prepost-for-explicit-andimplicit-analysis

https://www.dynaexamples.com/thermal/thermal-stress

\subsection{Laser matter interaction FEM simulations- Laser heating of a metal}

The heating of an AISI H13 steel workpiece is examined during irradiation by a CW continuous laser. 3D transient coupled thermal-structural numerical simulations $[1,2]$ take place using LSDYNA. Similar procedure may be followed to study a problem of matter's electronic excitation.

The workpiece is defined as a deformable body. The laser beam is modeled as a Gaussian moving heat source. For the dynamic elastoplastic behavior of the workpiece: a Johnson-Cook constitutive strength material model will be used [3-5].

\subsubsection{Governing equations}

18.3.1.1 3D transient heat conduction equation

$$
\rho c\left(\frac{\partial T}{\partial t}+V_{x} \frac{\partial T}{\partial x}\right)=\frac{\partial}{\partial i}\left(\kappa(T) \frac{\partial T}{\partial i}\right)+\dot{Q}-a_{T} T_{0}(3 \lambda+2 \mu) \dot{\varepsilon}_{i i}
$$

$i=x, y, z$ coordinates, $t$ time

$\rho$ the density, $c$ the specific heat, $V_{x}$ the laser scanning speed along the x-direction

$k$ thermal conductivity and $\dot{Q}$ the power generation per unit volume

$T$ temperature, $T_{0}$ ambient temperature, $\alpha_{T}$ thermal expansion coefficient,

$\lambda, \mu$ lame coefficients, $\dot{\varepsilon}_{i i}$ strain rate

\subsubsection{3D transient mechanical equation}

$$
\rho \frac{\partial^{2} U_{i}}{\partial^{2} t}=\mu \frac{\partial^{2} U_{i}}{\partial^{2} k}+(\lambda+\mu) \frac{\partial}{\partial i}\left(\frac{\partial U_{k}}{\partial k}\right)-(3 \lambda+2 \mu) \alpha_{T} \frac{\partial T}{\partial i}
$$

$U$ the displacement, $\rho$ the density, $\lambda$ and $\mu$ lame coefficients, $\alpha_{T}$ thermal expansion coefficient 
The strain tensor

$$
\varepsilon_{i j}=\frac{1}{2}\left(\frac{\partial U_{i}}{\partial U_{j}}+\frac{\partial U_{j}}{\partial U_{i}}\right)
$$

The stress tensor

$$
\sigma_{i j}=2 \mu \varepsilon_{i j}+\lambda \varepsilon_{k k} \delta_{i j}-(3 \lambda+2 \mu) a_{T}\left(T-T_{0}\right) \delta_{i j}
$$

\subsubsection{Modeling of laser heat source}

The Gaussian distribution of the $q(r)$ absorbed laser heat flux or laser power intensity is given by:

$$
q(x, z)=\frac{2 P_{t o t}}{\pi r_{b}^{2}} e^{-\left(\frac{2\left(\left(x-t V_{x}\right)^{2}+z^{2}\right)}{r_{b}^{2}}\right)}
$$

where $P_{\text {tot }}$ is the total absorbed power and $r_{b}$ is the laser beam radius.

It also holds that:

$$
P_{\text {tot }}=\eta P_{\text {inc }}
$$

where $P_{i n c}$ is the incident laser power, $\eta$ is the average absorptivity of the workpiece material and $t$ is the time.

The laser heat flux is applied to the top surface of the workpiece. The boundary condition on the top laser irradiated surface takes into account the heat flux, convection and radiation and it holds:

$$
-k \frac{\partial T}{\partial y}=q(x, z)-h\left(T-T_{0}\right)-\sigma \varepsilon\left(T^{4}-T_{0}^{4}\right)
$$

where $h$ is the convective heat transfer coefficient, $T_{0}$ is the ambient temperature, $\sigma$ is the StefanBoltzmann constant $\left(5.6710^{8} \mathrm{~W} / \mathrm{m}^{2} \mathrm{~K}^{4}\right)$ and $\varepsilon$ is emmisivity. Heat flux is considered to be normal to the laser irradiated surface, while the motion of the laser beam is considered along the $\mathrm{X}$ direction.

Moreover, the laser beam diameter is considered to be $\mathbf{2 5 0} \boldsymbol{\mu m}$, the laser power is $\mathbf{9 8 . 2} \mathbf{W}$ and the heat flux is $\mathbf{2} \mathbf{k W} / \mathbf{m m}^{2}$. 


\subsubsection{Finite Element Modeling}

The workpiece dimensions are $3.0 \times 0.4 \times 0.5 \mathrm{~mm}$. The workpiece is modeled with approximately 115,000 solid elements. The mesh of the workpiece is shown below.

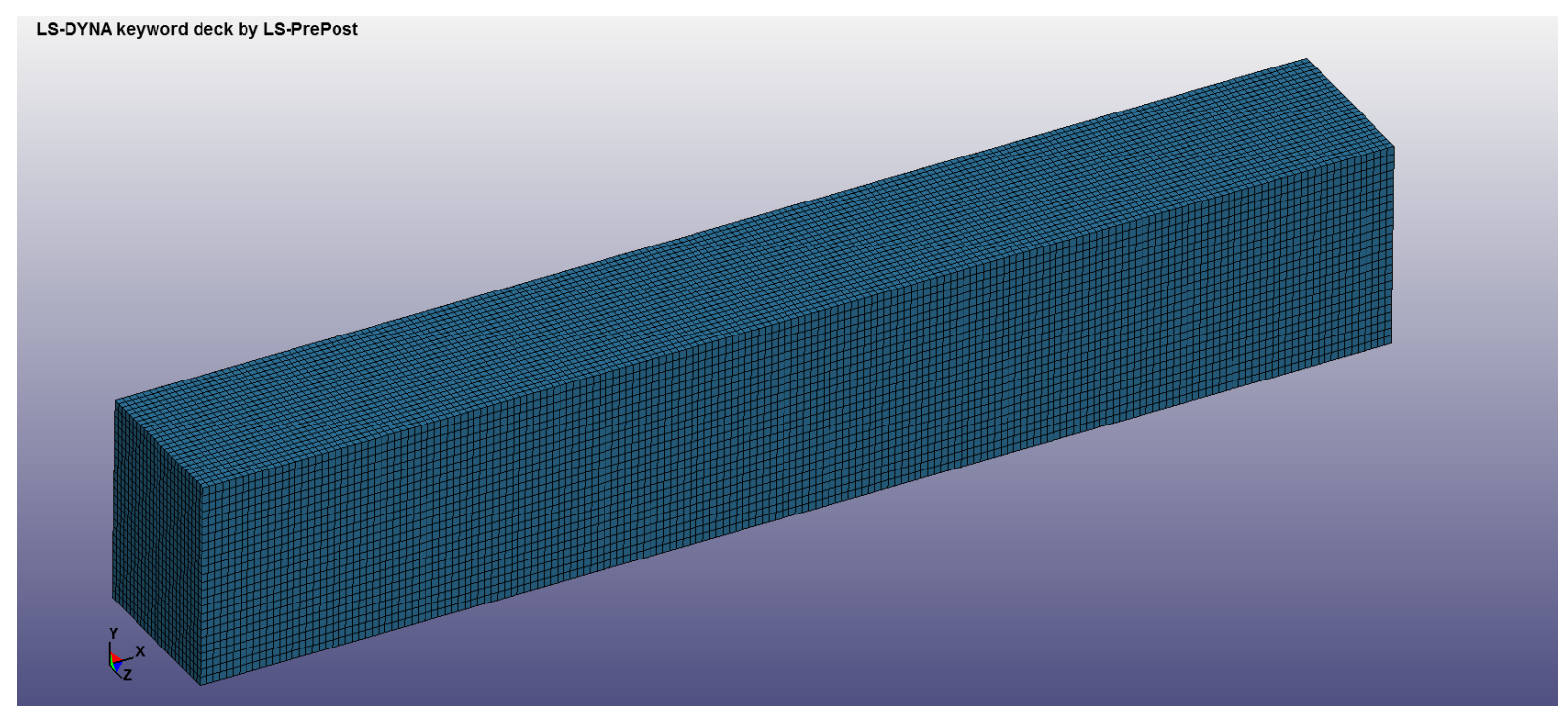

Figure 18.13 Mesh and geometry of the workpiece

Regarding the initial and boundary conditions, all translations and rotations of the bottom and the right side of the workpiece are fully constrained and $\mathrm{Z}$ translations at its front and back side are also constrained. The ambient temperature is assumed to be $20^{\circ} \mathrm{C}$.

\subsubsection{Material model}

The adoption of a suitable material-constitutive model for the workpiece is critical. The selected material model is the Johnson-Cook (J-C), a purely empirical one that takes into account the effect of plastic strain, strain rate and temperature. The flow stress is expressed as:

$$
\sigma_{y}=\left(A+B \varepsilon^{n}\right)\left(1+C \ln \frac{\dot{\varepsilon}}{\dot{\varepsilon}_{0}}\right)\left(1-\frac{T-T_{r}}{T_{m}-T_{r}}\right)^{m}
$$

where $\varepsilon$ is the equivalent plastic strain, $\dot{\varepsilon} / \dot{\varepsilon}_{0}$ is the dimensionless plastic strain rate, $\dot{\varepsilon}_{0}=1 \mathrm{~s}^{-1}$ is a reference strain rate used to normalize the strain rate, $A$ is the yield stress, $B$ is the hardening constant, $C$ is the strain rate sensitivity, $n$ is the hardening exponent, $m$ is the thermal softening exponent, $T_{m}$ is the melting temperature of the workpiece and $T_{r}$ the room temperature. The material constants $A, B, C, n, m$ are determined from experimental results.

\subsubsection{Material properties}

The physical properties of the AISI H-13 steel workpiece and the J-C material model and failure parameters of the workpiece are listed. 
Table 18.1. Mechanical and physical properties of AISI H-13 in room temperature.

\begin{tabular}{|l|l|}
\hline Property & Workpiece \\
\hline Density $\left[\mathrm{kg} / \mathrm{m}^{3}\right]$ & 7800 \\
\hline Elastic modulus [GPa] & 211 \\
\hline Poisson's ratio $[-]$ & 0.28 \\
\hline Specific heat $[\mathrm{J} / \mathrm{kg} \mathrm{K}]$ & 560 \\
\hline Thermal conductivity $[\mathrm{W} / \mathrm{m} \mathrm{K}]$ & 37 \\
\hline Thermal expansion $\left[10^{-6} \mathrm{C}\right]$ & 10.4 \\
\hline Melting Point $[\mathrm{K}]$ & 1700 \\
\hline
\end{tabular}

Table 18.2 Johnson-Cook material model and failure parameters of AISI H-13

\begin{tabular}{|l|l|l|l|l|l|}
\hline $\begin{array}{l}\text { Material model } \\
\text { Parameters }\end{array}$ & A [MPa] & B [MPa] & n [-] & C [-] & m [-] \\
\hline Values & 674.8 & 239.2 & 0.28 & 0.027 & 1.3 \\
\hline
\end{tabular}

Furthermore, a mean value of absorptivity $\eta=\mathbf{0 . 5}$ and a mean value of emmisivity $\varepsilon=\mathbf{0 . 4}$ are considered based on the work of Singh et al. For the convectional heat transfer to the surrounding air, a heat transfer coefficient of $h=5 \mathrm{~W} / \mathrm{m}^{2} \mathrm{~K}$ is also considered. The laser scanning speed is considered to be $150 \mathrm{~m} / \mathrm{min}$.

\subsubsection{Work to be done}

1) Read the keywords of the code so you can understand what each of them does.

2) From the code that you will be given identify first which consistent units you use.

3) Create a mesh that will have dimensions $3 \times 0.4 \times 0.5 \mathrm{~mm}$ along the $\mathrm{x}, \mathrm{y}, \mathrm{z}$ directions and the discretization will be $225 \times 30 \times 30$ respectively resulting in a total number of 202500 elements using the Shapemesher command. 


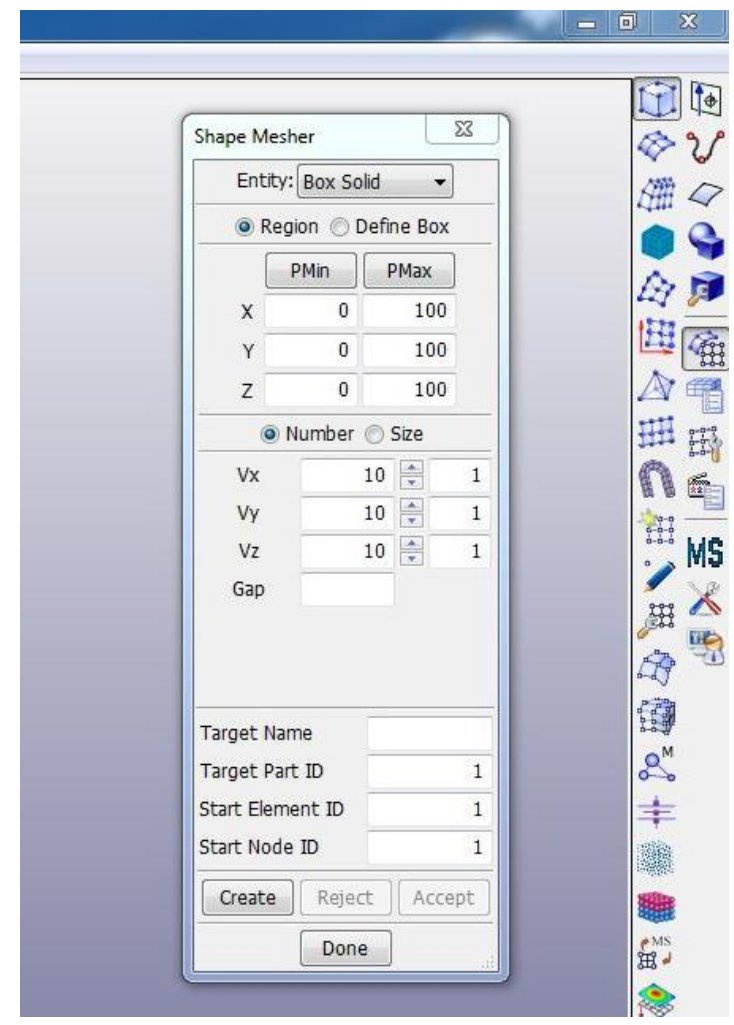

Figure 18.14 Shapemesher

4) Constrain all the translations and rotations at the bottom and the right side of the workpiece. Moreover constrain the $\mathrm{Z}$ translations at its front and back side. For this purpose you will need to use the keywords *BOUNDARY_SPC_SET, *SET_NODE_LIST.

5) Define the surface where the laser heat flux is applied. This will be on the top of the workpiece. Use the *SET_SEGMENT command.

6) Then your work will be examined by the instructor and he will further help you to complete the simulation.

\subsubsection{Code pieces}

The following keywords needed for the simulation are explained:

\section{*CONTROL_SOLUTION}

\$\# soln nlq isnan lcint

$2 \quad 0 \quad 0 \quad 100$

The Keyword *CONTROL_SOLUTION is used to specify the analysis solution procedure when there is thermal, coupled thermal or structural analysis is performed.

Variables:

Soln: Analysis solution procedure:

0 : Structural analysis only, 
1: Thermal analysis only,

2: Coupled structural thermal analysis.

\section{*CONTROL_TERMINATION}

$\$ \#$ endtim endcyc dtmin endeng endmas nosol

$$
\begin{array}{lllll}
1.2 & 0 & 0.0 & 0.01 .000000 \mathrm{E} 8 & 0
\end{array}
$$

The Keyword *CONTROL_TERMINATION is used to set the termination conditions.

Variables:

endtim: It defines the termination time of the analysis. It is a mandatory variable for the LSDYNA software.

endcyc: It defines the maximum number of steps that will be analyzed, according to the step time (dtstart) that we will set for out problem's analysis. Always variable endtim is more important and analysis will be terminated if we reach endtim value.

dtmin: It defines the minimum time between steps that is allowed. When the time step drops to dtmin, LS-DYNA terminates. By default, is inactive.

endeng: This variable sets the maximum total energy change ratio that is allowable. If succeeded analysis is terminated. By default, is inactive.

endmas: This variable sets the maximum total mass change ratio that is allowable. If succeeded analysis is terminated. By default, is inactive.

\section{*CONTROL_THERMAL_SOLVER}

\$\# atype ptype solver cgtol gpt eqheat fwork sbc

$$
\begin{array}{lllllll}
1 & 2 & 31.00000 \mathrm{E}-6 & 1 & 1.0 & 1.0 & 0.0
\end{array}
$$

\$\# msglvl maxitr abstol reltol omega unused unused tsf

$0 \quad 5001.0000 \mathrm{E}-101.00000 \mathrm{E}-4 \quad 1.0$

The keyword *CONTROL_THERMAL_SOLVER sets options for the thermal solution in a thermal only or coupled structural-thermal analysis. To use it is required the usage of the proper solver (variable soln) in *CONTROL_SOLUTION keyword.

\section{*CONTROL_THERMAL_TIMESTEP}

\$\# ts tip its tmin tmax dtemp tscp lcts

$$
\begin{array}{lllllll}
0 & 0.5 & 0.0011 .00000 \mathrm{E}-7 & 0.0139 & 100.0 & 0.5 & 0
\end{array}
$$

The keyword ${ }^{*}$ CONTROL_THERMAL_TIMESTEP sets timestep for a thermal only or coupled structural-thermal analysis. To use it is required the usage of the proper solver (variable soln) in *CONTROL_SOLUTION keyword and the *CONTROL_THERMAL_SOLVER keyword. 


\section{*CONTROL_TIMESTEP}

\$\# dtinit tssfac isdo tslimt dt2ms lctm erode ms1st

$\begin{array}{llllllll}0.0 & 0.6 & 0 & 0.0 & 0.0 & 0 & 0 & 0\end{array}$

\$\# dt2msf dt2mslc imscl unused unused rmscl
$\begin{array}{lll}0.0 & 0 & 0\end{array}$
0.0

The keyword *CONTROL_TIMESTEP sets the timestep for structural problem analysis.

dtini: This variable defines the initial timestep size. By default, is blank or 0 and LS-DYNA determines initial step size.

\section{*CONTROL_THERMAL_NONLINEAR}

\$\# refmax tol dcp lumpbc thlstl nlthpr phchpn

$\begin{array}{lllllll}100 & 0.0 & 1.0 & 0 & 0.0 & 0 & 0.0\end{array}$

The keyword *CONTROL_THERMAL_NONLINEAR set parameters for a nonlinear thermal or coupled structural-thermal analysis. The control card, *CONTROL_SOLUTION, (variable soln) is also required.

\section{*DATABASE_BINARY_D3PLOT}

$\begin{array}{cccccc}\text { \$\# } & \text { dt } & \text { lcdt } & \text { beam } & \text { npltc } & \text { psetid } \\ & 0.0 & 0 & 0 & 100 & 0 \\ & \text { \$\# } & & & & \end{array}$

0

The keyword *DATABASE_BINARY_D3PLOT defines parameters for the output from entire of the model. From these outputs by post processing them we will conclude to an final presentable output of our solution analysis.

dt: In this variable we set the time interval between output states.

nr: In this variable we set Number of RUNning ReStart Files, RUNRSF, written in a cyclical fashion. The default is 1, i.e., only one runrsf file is created and the data there in is overwritten each time data is output.

npltc: In this variable we calculate DT = ENDTIME/NPLTC applies to D3PLOT and D3PART only. This overrides the DT specified in the first field. 


\section{*DATABASE_GLSTAT}

\$\# dt binary lcur ioopt

$\begin{array}{llll}0.003 & 0 & 0 & 1\end{array}$

The keyword *DATABASE has many options to define to obtain output files containing results information. One of them is the *DATABASE_GLSTAT, which provide us with the global data of the solution analysis of our model.

Dt: In this variable we se the time step between outputs. If is set to 0 then no output is printed. binary: In this variable we set the type for binary output.

1: Data written to an ASCII file default for SMP LS-DYNA.

2: Data written to binary database "binout", default for MPP LS-DYNA.

3: Both ASCII and binary outputs.

\section{*MAT_ADD_THERMAL_EXPANSION}

\$\# pid lcid mult lcid multy lcid multz

$\begin{array}{lllllll}2 & 69 & 1.0 & 0 & 1.0 & 0 & 1.0\end{array}$

With this keyword *MAT_ADD_THERMAL_EXPANSION we add thermal expansion properties to all nonlinear solid, shell, thick shell and beam elements and all material models.

pid: In this variable we put the PART ID of the part that we add thermal expansion

lcid: For isotropic material models, LCIDY, MULTY, LCIDZ, and MULTZ are ignored, and LCID is the load curve ID defining the thermal expansion coefficient as a function of temperature. If zero, the thermal expansion coefficient is constant and equal to MULT.

mult: Scale factor scaling load curve given by LCID.

\section{*PART}

$\$ \#$

title

\$\# pid secid mid eosid hgid grav adpopt tmid

$\begin{array}{llllllll}2 & 1 & 1 & 1 & 0 & 0 & 0 & 1\end{array}$

The keyword *PART is essential for the model identification and characterization in the solution analysis. Within it variables we combine various properties and meshes to form the parts we want 
to analyze. To form a part in LS-DYNA we combine the identity of a formed mess with the identity of the material, with the identity of the used Equation Of State for that material, with the thermal properties identity, etc.

pid: In this variable we put the part id number

secid: In this variable we put the section id number, defined in a *SECTION keyword.

mid: In this variable we put the material id number, defined in a *MAT keyword.

eosid: In this variable we put the Equation Of State id number, defined in a *EOS keyword.

tmid: In this variable we put the thermal properties id number, defined in a *MAT_THERMAL keyword.

\section{*SECTION_SOLID}

\$\# secid elform aet

$1 \quad 1 \quad 0$

In this keyword, we define section properties for solid continuum and fluid elements.

secid: In this variable we put the section id number, defined in a *SECTION keyword.

elform: Element formulation options:

-2: Fully integrated S/R solid intended for elements with poor aspect ratio, accurate formulation

-1: Fully integrated S/R solid intended for elements with poor aspect ratio, efficient formulation

0: 1 point corotational for *MAT_MODIFIED_HONEYCOMB

1: Constant stress solid element: default element type.

2: Fully integrated S/R solid

3: Fully integrated quadratic 8 node element with nodal rotations

4: S/R quadratic tetrahedron element with nodal rotations

5: 1 point ALE

6: 1 point Eulerian

7: 1 point Eulerian ambient

8: Acoustic

9: 1 point corotational for *MAT_MODIFIED_HONEYCOMB

10: 1 point tetrahedron

11: 1 point ALE multi-material element

and various others 


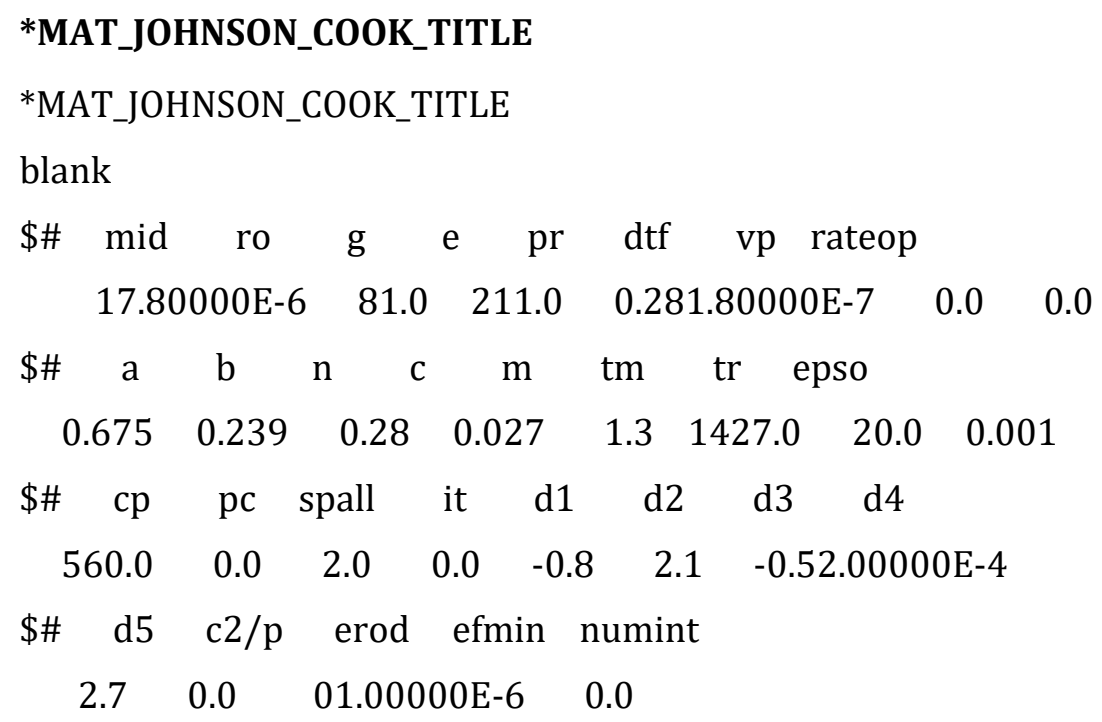

Johnson-Cook is an empirical model for the flow stress and strain rate. It is the most common used Viscoplasticity and yield stress model.

So in this keyword (*MAT_JOHNSON_COOK_TITLE) we set the variables of the Johnson-Cook model for a material.

mid: In this variable we put the material identification number.

ro: In this variable we set the mass density of the material

g: In this variable we set the shear modulus of the material.

e: In this variable we set the Young's modulus of the material

pr: In this variable we set the Poisson's ratio of the material

dtf: In this variable we set the Minimum time step size for automatic element deletion (shell elements).

vp: In this variable we set the formulation for rate effects:

0.0 for scale yield stress

1.0 for Viscoplastic formulation

a,b,n,c,m: These variables are the input constants of the Johnson-Cook formula

tm: In this variable we set the melt temperature of the material

tr: In this variable we set the room temperature

cp: In this variable we set the specific heat

pc: In this variable we set the tensile failure stress or tensile pressure cutoff $(\mathrm{PC}<0.0)$ 


\section{*EOS_LINEAR_POLYNOMIAL}

*EOS_LINEAR_POLYNOMIAL

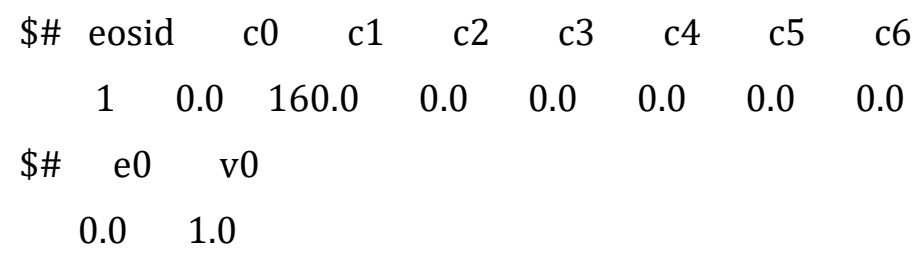

This is the keyword *EOS_LINEAR_POLYNOMIAL where we define coefficients for a linear polynomial Equation Of State and initialize the thermodynamic state of the material.

$$
P=C_{0}+C_{1} \mu+C_{2} \mu^{2}+C_{3} \mu^{3}+\left(C_{4}+C_{5} \mu+C_{6} \mu^{2}\right) E
$$

eosid: In this variable we set the unique number ID of the EOS

c0: In this variable we set the 0th polynomial equation coefficient.

c1: In this variable we set the 1st polynomial equation coefficient (when used by itself, this is the elastic bulk modulus)

\section{*MAT_THERMAL_ISOTROPIC}

\$\# tmid tro tgrlc tgmult tlat hlat

$$
\begin{array}{llllll}
1 & 0.0 & 0.0 & 0.0 & 0.0 & 0.0
\end{array}
$$

\$\# hc tc

560.03.70000E-5

This keyword *MAT_THERMAL_ISOTROPIC defines thermal isotropic properties to the material that selects it.

tmid: In this variable we set the unique ID number of the thermal material identification.

tro: In this variable we set the thermal density of the material. If set to 0.0 then it is equal to the structural density.

hc: In this variable we set the specific heat of the material

tc: In this variable we se the thermal conductivity of the material.

\section{*ELEMENT_SOLID}

$\begin{array}{cccrrrrrrrr}\text { \$\# } & \text { eid } & \text { pid } & n 1 & n 2 & n 3 & n 4 & \text { n5 } & \text { n6 } & \text { n7 } & \text { n8 } \\ 1 & 1 & 4313 & 4309 & 5283 & 5313 & 4308 & 4308 & 5138 & 5138 \\ 2 & 1 & 5313 & 5283 & 5284 & 5314 & 5138 & 5138 & 5139 & 5139 \\ 3 & 1 & 5314 & 5284 & 5285 & 5315 & 5139 & 5139 & 5140 & 5140\end{array}$


In this keyword *ELEMENT_SOLID we define each element of the created mesh of our model that we are going to perform solution analysis. Elements are defined in three-dimensional solid elements including 4 noded tetrahedrons and 8-noded hexahedrons. Most common are the 8noded hexahedrons.

eid: In this variable we set the unique ID number of the element.

pid: In this variable we set part ID number from which this element comes from.

n1 - n8: In these variables we set the nodal points ID that consists the element. If we use tetrahedron shape for our elements then we set only $\mathrm{n} 1-\mathrm{n} 4$ nodal point variables.

\section{*NODE}

\$\# nid $\quad \mathrm{x} \quad \mathrm{y} \quad \mathrm{z} \quad$ tc $\quad$ rc

$\begin{array}{llllll}1 & -0.027596 & 0.019934 & 0.2715 & 0 & 0\end{array}$

$\begin{array}{llllll}2 & 0.0576701 & 1.0 & 0.2715 & 0 & 0\end{array}$

$\begin{array}{llllll}3 & -0.027596 & 0.019934 & 0.1285 & 0 & 0\end{array}$

In the keyword *NODE we define the nodes of our model. We also can define boundary conditions for that nodes. Nodes are defined by setting their coordinates at X-Y-Z axis.

nid: In this variable we set the unique node ID number

$\mathrm{x}$ : In this variable we set the $\mathrm{X}$ coordinate of the node

y: In this variable we set the Y coordinate of the node

$\mathrm{z}$ : In this variable we set the $\mathrm{Z}$ coordinate of the node

tc: In this variable we set the translational constraint:

0: no constraints,

1: constrained $\mathrm{x}$ displacement,

2: constrained y displacement,

3: constrained z displacement,

4: constrained $\mathrm{x}$ and $\mathrm{y}$ displacements,

5: constrained y and z displacements,

6: constrained $\mathrm{z}$ and $\mathrm{x}$ displacements,

7: constrained $\mathrm{x}, \mathrm{y}$, and $\mathrm{z}$ displacements.

rc: In this variable we set the rotational constraint:

0: no constraints, 
1: constrained $\mathrm{x}$ rotation,

2: constrained y rotation,

3: constrained z rotation,

4: constrained $\mathrm{x}$ and $\mathrm{y}$ rotations,

5: constrained $\mathrm{y}$ and $\mathrm{z}$ rotations,

6: constrained $\mathrm{z}$ and $\mathrm{x}$ rotations,

7: constrained $\mathrm{x}, \mathrm{y}$, and $\mathrm{z}$ rotations.

\section{*DEFINE_FUNCTION}

\$\# fid heading

3

\$\#

function

$\mathrm{f}(\mathrm{x}, \mathrm{z}$, time,temp $)=-(2) /(\exp (128 *((\mathrm{x}-2.5 *$ time $) *(\mathrm{x}-2.5 *$ time $)+(\mathrm{z}-0.2) *(\mathrm{z}-0.2))))+\left(5^{*} 10^{* *}(-\right.$

8) $) *\left(\right.$ temp-20) $+\left(5.7 * 10^{* *}(-17)\right) * 0.5 *(($ temp $* * 4)-160000)$

In this keyword *DEFINE_FUNCTION we define a function that can be used by other keywords.

fid: In this variable we set the function ID number

heading: In this variable we set an optional descriptive heading.

function: In this variable we set the arithmetic expression that represents our function.

\section{*BOUNDARY_FLUX_SET}

\$ ssid

1

\$\# lcid mlc1 mlc2 mlc3 mlc4 loc nhisv fid

$\begin{array}{llllllll}3 & 1.0 & 1.0 & 1.0 & 1.0 & 0 & 0 & 3\end{array}$

The keyword *BOUNDARY_FLUX is to apply a flux boundary condition.

ssid: In this variable we set the set ID number

lcid : In this variable we set the ID of a reference load curve

mlc1-mlc4: In these variables we set the curve multipliers.

fid: in this variable we set the ID number of the function we use 


\section{*SET_SEGMENT}

$\begin{array}{cccccccccccc}\text { \$\# } & \text { sid } & \text { da1 } & \text { da2 } & \text { da3 } & \text { da4 } & \text { solver } & & & \\ & 1 & 0.0 & 0.0 & 0.0 & 0.0 \text { MECH } & & & & \\ \text { \$\# } & \text { n1 } & \text { n2 } & \text { n3 } & \text { n4 } & \text { a1 } & \text { a2 } & \text { a3 } & \text { a4 } & & \\ 129285 & 129645 & 129647 & 129287 & 0.0 & 0.0 & 0.0 & 0.0 \\ 123165 & 123525 & 123527 & 123167 & 0.0 & 0.0 & 0.0 & 0.0 \\ 117045 & 117405 & 117407 & 117047 & 0.0 & 0.0 & 0.0 & 0.0\end{array}$

In this keyword *SET_SEGMENT we define set of segments with optional identical or unique attributes.

sid: In this variable we set the unique set ID number.

solver: In this variable we set the name of the solver of the set.

n1-n4: In this variable we set the nodal points

\subsubsection{References}

[1] V. Dimitriou, E. Kaselouris, Y. Orphanos, E Bakarezos, Nikolaos Vainos, M. Tatarakis, N. A. Papadogiannis, Three dimensional transient behavior of thin films surface under pulsed laser excitation, Appl. Phys. Lett. 103 (2013) 114104.

[2] E. Kaselouris, I.K. Nikolos, Y. Orphanos, E Bakarezos, N. A. Papadogiannis, M. Tatarakis, V. Dimitriou, Elastoplastic study of nanosecond-pulsed laser interaction with metallic films using 3D multiphysics fem modeling, Int. J. Damage Mech. 25 (2016) 42-55.

[3] E. Kaselouris, A. Skoulakis, Y. Orphanos, K. Kosma, T. Papadoulis, I. Fitilis, E. Clark, A.P. Markopoulos, M. Bakarezos, N.A. Papadogiannis, M. Tatarakis, and V. Dimitriou, Analysis of the heat affected zone and surface roughness during laser micromachining of metals, Key Engineering Materials 827 (2020) 122-127.

[4] E. Kaselouris, A. Baroutsos, T. Papadoulis, N.A. Papadogiannis, M. Tatarakis, and V. Dimitriou, A study on the influence of laser parameters on laser-assisted machining of AISI H-13 steel, Key Engineering Materials 827 (2020) 92-97.

[5] R. Singh, M.J. Alberts and S.N. Melkote, Characterization and prediction of the heat-affected zone in a laser-assisted mechanical micromachining process, Int. J. Mach. Tools Manuf. Nucl. 48 (2008) 9941004.

\subsection{Plasma-pinch MHD simulations}

MHD simulations are performed to describe matter/plasma dynamics when heated by high pulsed currents.

The goal is to give the User a first insight of the PLUTO code $[1,2]$ and test it in a simple example as a Z-pinch one wire configuration. 


\subsubsection{What is PLUTO?}

PLUTO (version 4.2) is an astrophysical code that has been modified to simulate laboratory plasmas. It is a finite volume/finite difference, shock-capturing code designed to integrate a system of conservation laws of the form:

$$
\frac{\partial U}{\partial t}+\nabla T(U)=S(U)
$$

$U$ represents a set of conservative quantities, $T(U)$ is the flux tensor and $S(U)$ defines the source terms. An equivalent set of primitive variables $V$ is more conveniently used for assigning initial and boundary conditions.

The sets of conservative and primitive variables $\mathrm{U}$ and $\mathrm{V}$ are correlated by:

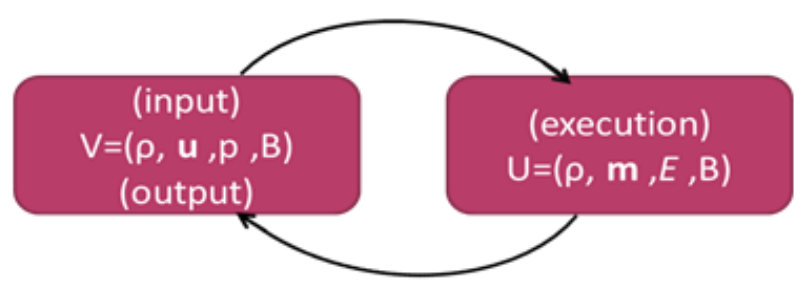

Figure 18.15 Conservative and primitive variables [6]

where $\rho$ is the material density, $m$ is the momentum density with $m=\rho u, E$ is the total energy density, $\mathrm{B}$ is the magnetic field, $\mathrm{p}$ is the thermal pressure and $\mathrm{u}$ the bulk velocity. The open $\mathrm{C}$ and $\mathrm{C}++$ program environment of PLUTO is flexible for the introduction of:

- user geometries,

- functions of topologies e.g. of the magnetic field,

- functions of the densities e.g. inhomogeneous plasmas

- boundary conditions at the computational domains to simulate the real physical problem.

The PLUTO manual $[3,4]$ can be downloaded from:

http://plutocode.ph.unito.it/files/userguide.pdf

\subsubsection{PLUTO Configuration}

Five different files are important:

- definitions.h

- pluto.ini

- init.c 
- res_eta.c

- cooling.c

The first three are necessary for all physical problems while the last two are needed for our case. With these five files and Pluto code implemented in a Unix/Linux platform the User can run the code modifying its file via the physical problem. The analysis of each one follows.

\subsubsection{1 definitions.h}

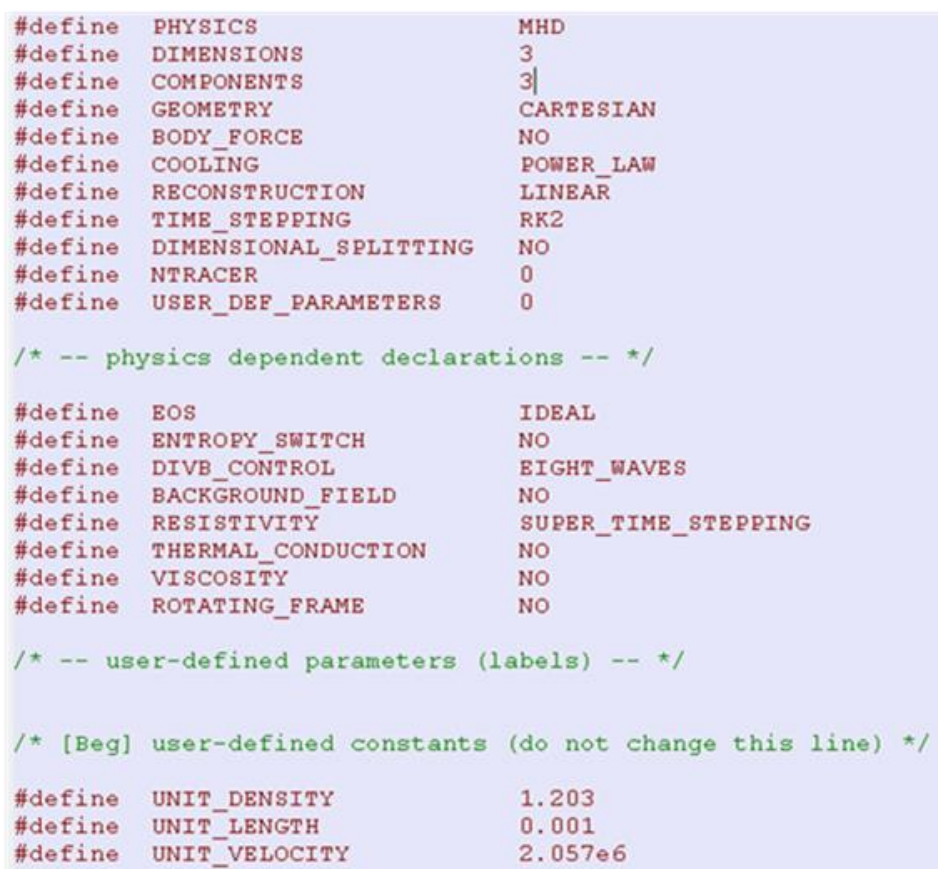

In this header file the user must specify: physics equations (e.g. MHD), dimensions, geometry, optically thin cooling losses and so on. For our case crucial is the dimensionalization process through the definition of three physical constants:

- $\mathrm{L}_{0}\left(\mathrm{UNIT} \_L E N G T H\right)$ in $\mathrm{cm}$ (e.g. $0.001 \equiv 0.001 \mathrm{~cm}=10 \mu \mathrm{m}$ is the picture's unit length)

- $\quad \rho_{\mathrm{o}}\left(\right.$ UNIT_DENSITY) in $\mathrm{gr} / \mathrm{cm}^{3}$

- $\quad \mathrm{u}_{\mathrm{o}}\left(\mathrm{UNIT}_{-}\right.$VELOCITY) in $\mathrm{cm} / \mathrm{s}$

All other units are derived from the combination of these three:

- $\mathrm{t}_{0}=\mathrm{L}_{\mathrm{o}} / \mathrm{u}_{\mathrm{o}}($ time unit $)$

- $\mathrm{p}_{\mathrm{o}}=\rho_{\mathrm{o}} \mathrm{u}_{\mathrm{o}}^{2}$ (thermal pressure unit)

- $\quad B_{0}=u_{o}\left(4 \pi \rho_{0}\right)^{1 / 2}$ (magnetic field unit).

For resistivity implementation $n=\left(c^{2} / 4 \pi\right) n_{r}$, where $n$ is the diffusivity tensor $n \equiv \operatorname{diag}\left(n_{x 1}, n_{x 2}, n_{x 3}\right)$, $c$ the speed of light and $\mathrm{n}_{\mathrm{r}}$ the resistivity tensor. So $\mathrm{n}_{\mathrm{o}}$ is in $\mathrm{cm}^{2} / \mathrm{s}$ :

- $\mathrm{n}_{0}=\mathrm{L}_{0}^{2} / \mathrm{t}_{0}=\mathrm{u}_{0} \mathrm{~L}_{0}$ (diffusivity unit) 
The User sets the dimensionless units in PLUTO files by dividing the physical variables with the above units. Therefore:

$\mathrm{L}=\mathrm{L}_{\mathrm{cgs}} / \mathrm{L}_{0}, \quad \rho=\rho_{\mathrm{cgs}} / \rho_{0}, \quad \mathrm{u}=\mathrm{u}_{\mathrm{cgs}} / \mathrm{u}_{0}, \quad \mathrm{t}=\mathrm{t}_{\mathrm{cgs}} / \mathrm{t}_{0}, \mathrm{p}=\mathrm{p}_{\mathrm{cgs}} / \mathrm{p}_{0}, \quad \mathrm{~B}=\mathrm{B}_{\mathrm{cgs}} / \mathrm{B}_{0}, \mathrm{n}=\mathrm{n}_{\mathrm{cgs}} / \mathrm{n}_{\mathrm{o}}$

The modification of the other switches is in the manual. This file is modified every time the user runs the python script.

\subsubsection{2 pluto.ini}

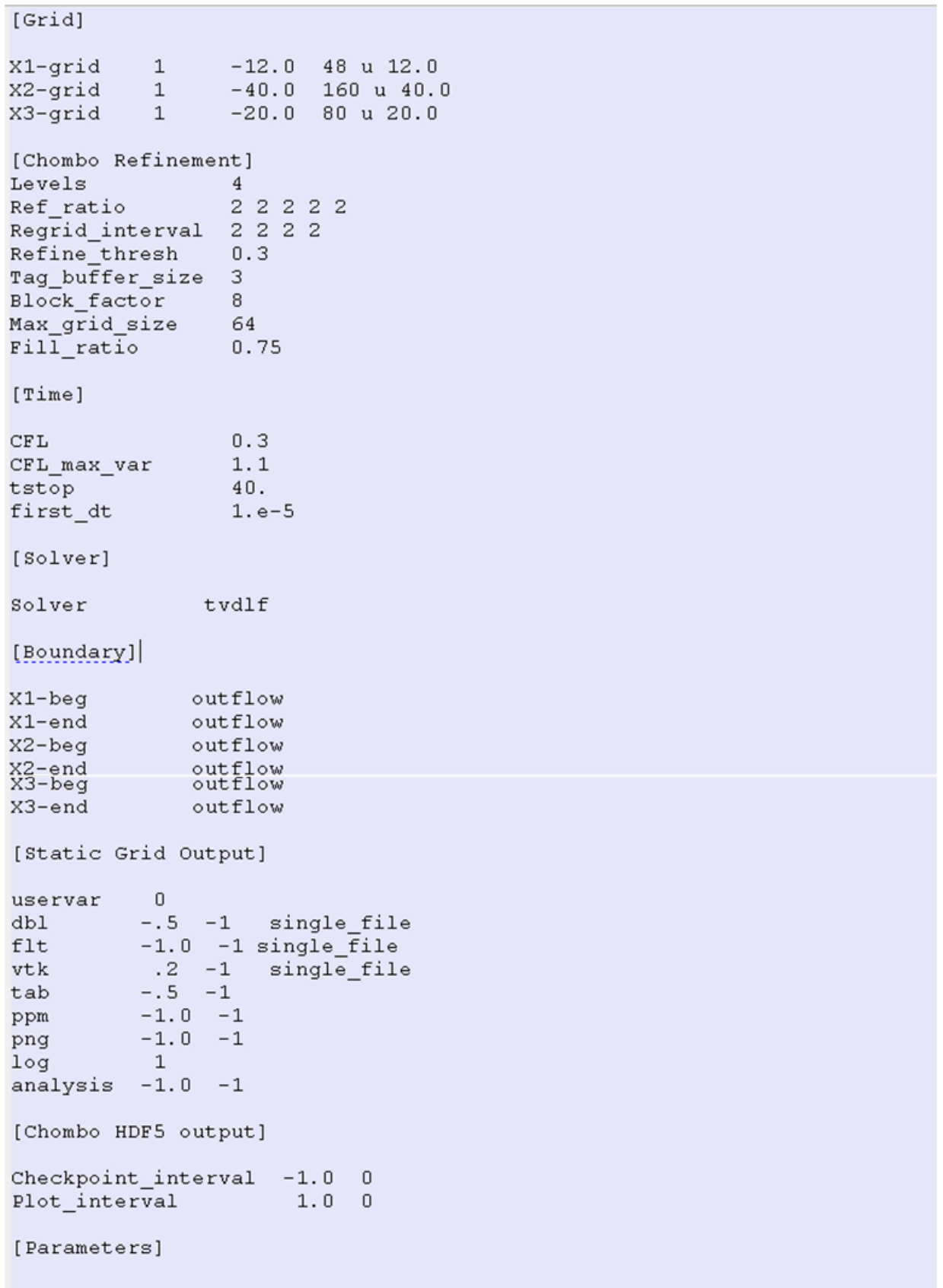

This file defines the physical domain. The first three lines give the size of the computational domain for each dimension. For the above picture $\mathrm{X} 1$ is the $\mathrm{X}$ axes (Cartesian grid) and has $24 \mathrm{~L}_{\mathrm{o}}$ (UNIT_LENGTH) length. The same counts for the other axes. The number of computational zones (points) is the middle number. So in our example the $\mathrm{X}$ axes is from $-12<\mathrm{X}<12 \mathrm{~L}_{\mathrm{o}}$ units and is separated in 48 zones. The same holds for the other axes. The whole mesh is $48 \times 160 \times 80=614400$ 
unit cubes. If the unit length $\mathrm{L}_{0}$ is $0.001 \mathrm{~cm}=10 \mu \mathrm{m}$ the $\mathrm{X}$ axes is $240 \mu \mathrm{m}$ long and the computational unit cube has a spatial volume of $5 \times 5 \times 5=125 \mu \mathrm{m}^{3}$.

The tstop stands for the computational time execution.

For our example tstop $=40$ PLUTO units. For the real time we have tstop(real) $=40 t_{0}=40 \mathrm{~L}_{0} / \mathrm{u}_{0}=$ $=40\left(0.001 \mathrm{~cm} / 2.057 \cdot 10^{6} \mathrm{~cm} / \mathrm{s}\right)=19.44 \mathrm{~ns}$.

The static grid output comes with a variety of files. Here we chose vtk file format (the only positive value of 0.2 ) at every 0.2 unit time to be saved. So for the whole execution time we will have $40 / 0.2=200$ vtk files. Each file has the information of the primitive physic variables. So for this example 200 time frames can be visualized for each variable from $t=0$ to $t=19.44 \mathrm{~ns}$, every $19.44 / 200=0.0972 \mathrm{~ns}$.

For every other switch the User can see the manual.

\subsubsection{3 init.c}

This is the most important file for the physical problem. The User sets the initial condition physical variables functions for the physics model.

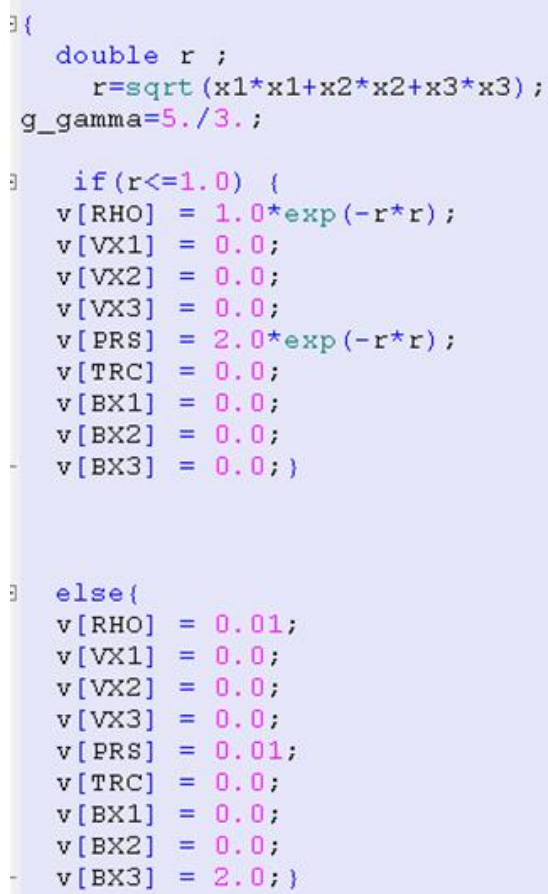

The programming language is $\mathrm{C}$. In the above code is defined a physics problem of a plasma sphere $(\mathrm{r}<1.0)$, where $\mathrm{r}$ is defined in a Cartesian grid $\mathrm{x} 1, \mathrm{x} 2, \mathrm{x} 3$ corresponding to the $\mathrm{X}, \mathrm{Y}, \mathrm{Z}$ axes coordinates respectively. RHO is the mass density $(\rho), \mathrm{VX} 1, \mathrm{VX} 2, \mathrm{VX} 3$ are the three components of bulk velocity, PRS is the thermal pressure (p) and BX1,BX2,BX3 are the three components of the magnetic field. Inside the sphere we have Gaussian shaped mass density and thermal pressure while the magnetic field is zero. Outside the sphere the plasma is thinner and has a constant density (0.01) and pressure (0.01) while the magnetic field is constant at the $\mathrm{Z}$ axis. 
If we have the dimensions of definitions.h file of the previous picture the interpretation of the dimensionless units of the init.c file is:

$\mathrm{r}<=1.0 \rightarrow \mathrm{r} / \mathrm{L}_{0}<=1.0 \rightarrow \mathrm{r}<=\mathrm{L}_{0}=10 \mu \mathrm{m}$

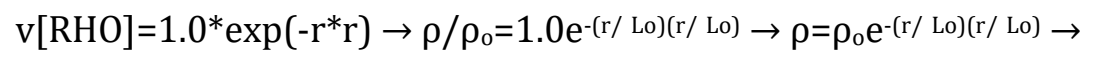

$\rho=1.203 \mathrm{e}^{-(\mathrm{r} / 0.001 \mathrm{~cm})(\mathrm{r} / 0.001 \mathrm{~cm})}$ where $\mathrm{r}$ in $\mathrm{cm}$ and $\rho$ in $\mathrm{gr} / \mathrm{cm}^{3}$ etc.

For all the other variables and implementations the User can see the documentation.

\subsubsection{4 res_eta.c}

The resistive module is enabled by setting RESISTIVITY to SUPER TIME STEPING in definitions.h file or running the code and setting it at the Python script.

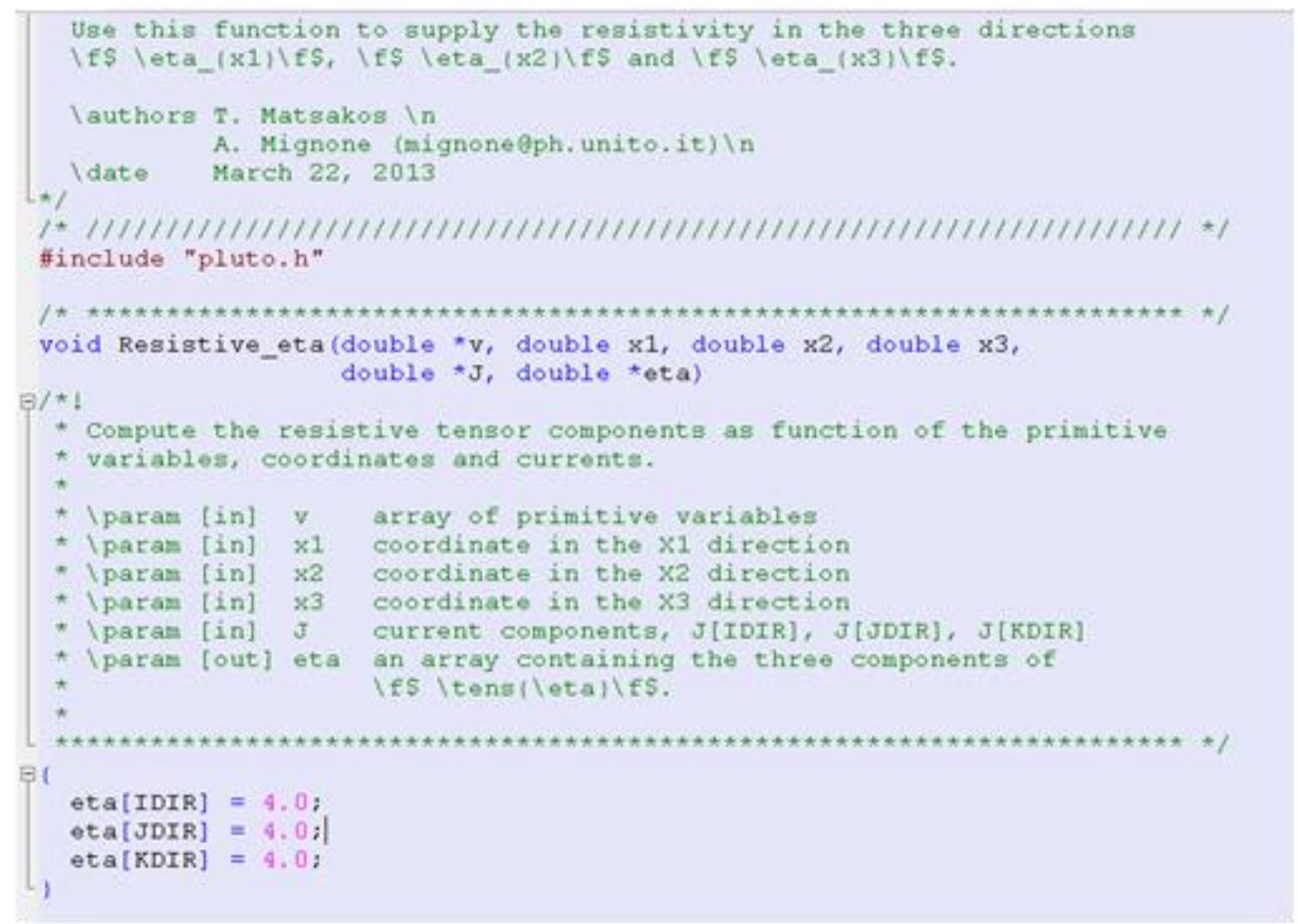

Here we can see the three components of the resistivity tensor as eta[IDIR], eta[JDIR], eta[KDIR]. The dimensions are in $\mathrm{n}_{0}$ units. So in this example the three components are constant and $\mathrm{nx}=\mathrm{ny}=\mathrm{nz}=4.0 \mathrm{n}_{0}=4.0 \mathrm{~L}_{0} \mathrm{u}_{0} \rightarrow \mathrm{nx}=\mathrm{ny}=\mathrm{nz}=4 \cdot 0 \cdot 0.001 \mathrm{~cm} \cdot 2 \cdot 057 \cdot 10^{6} \mathrm{~cm} / \mathrm{s}=8 \cdot 228 \cdot 10^{3} \mathrm{~cm}^{2} / \mathrm{s}$.

\subsubsection{5 cooling.c}

The cooling module is implemented in definitions file or through the running procedure by setting it at the Python script. The cooling module we will use is the Power Law Cooling that accounts for bremsstrahlung cooling with the appropriate correction for the mean molecular weight of the plasma Tungsten ions and electrons.

\subsubsection{Running the code}

PLUTO file is in a separate working directory in the hard drive of the computer. In the same directory is the specific file of the test case e.g. Z_pinch_test. The procedure is the following. 
If the User is using a bash shell the first line command is:

>export PLUTO_DIR=/home/User/PLUTO

Changing directory to the test problem:

$>c d Z_{-}$pinch_test

Next step is invoking the Python script with:

>python PLUTO_DIR/setup.py

The next screen will appear:

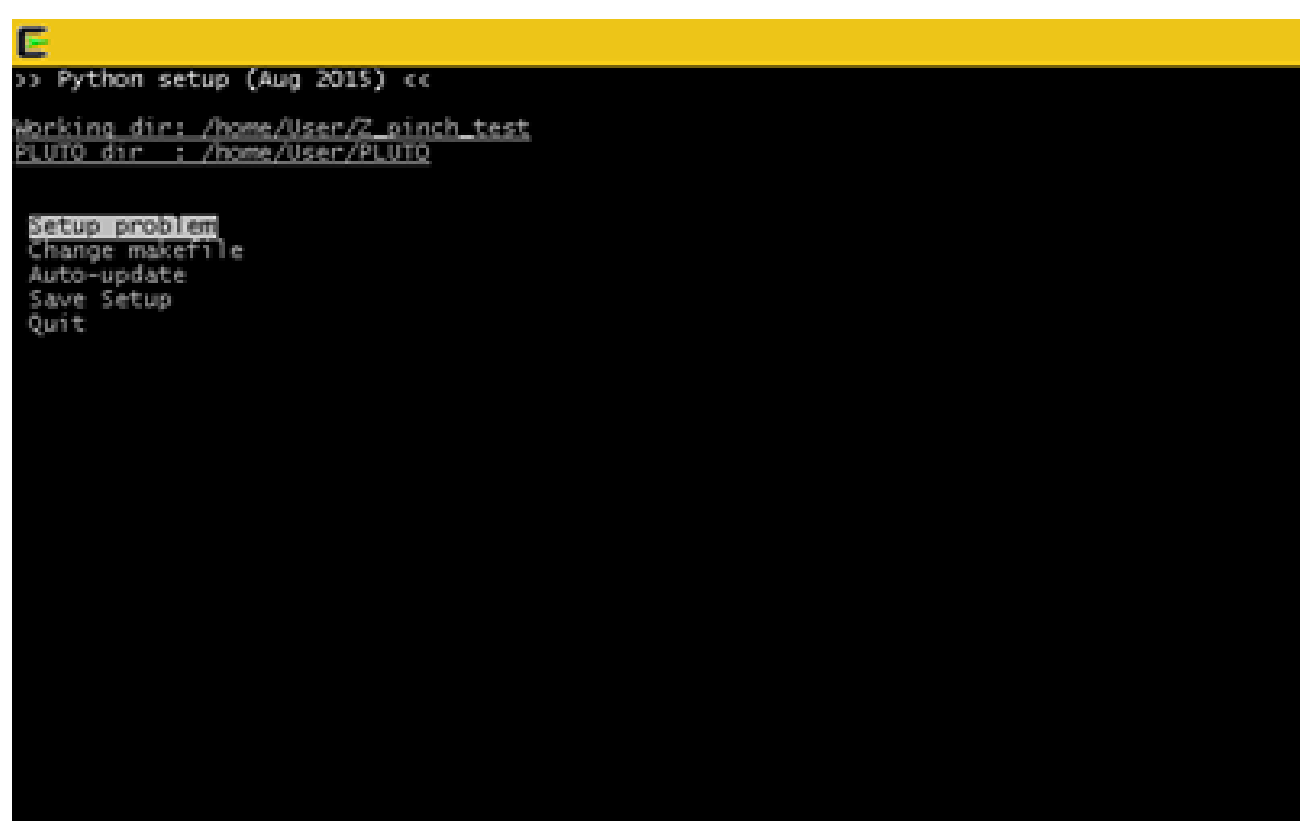

Selecting the Setup problem The User begins to modify the definitions.h file. For the specific case an implementation example is: 


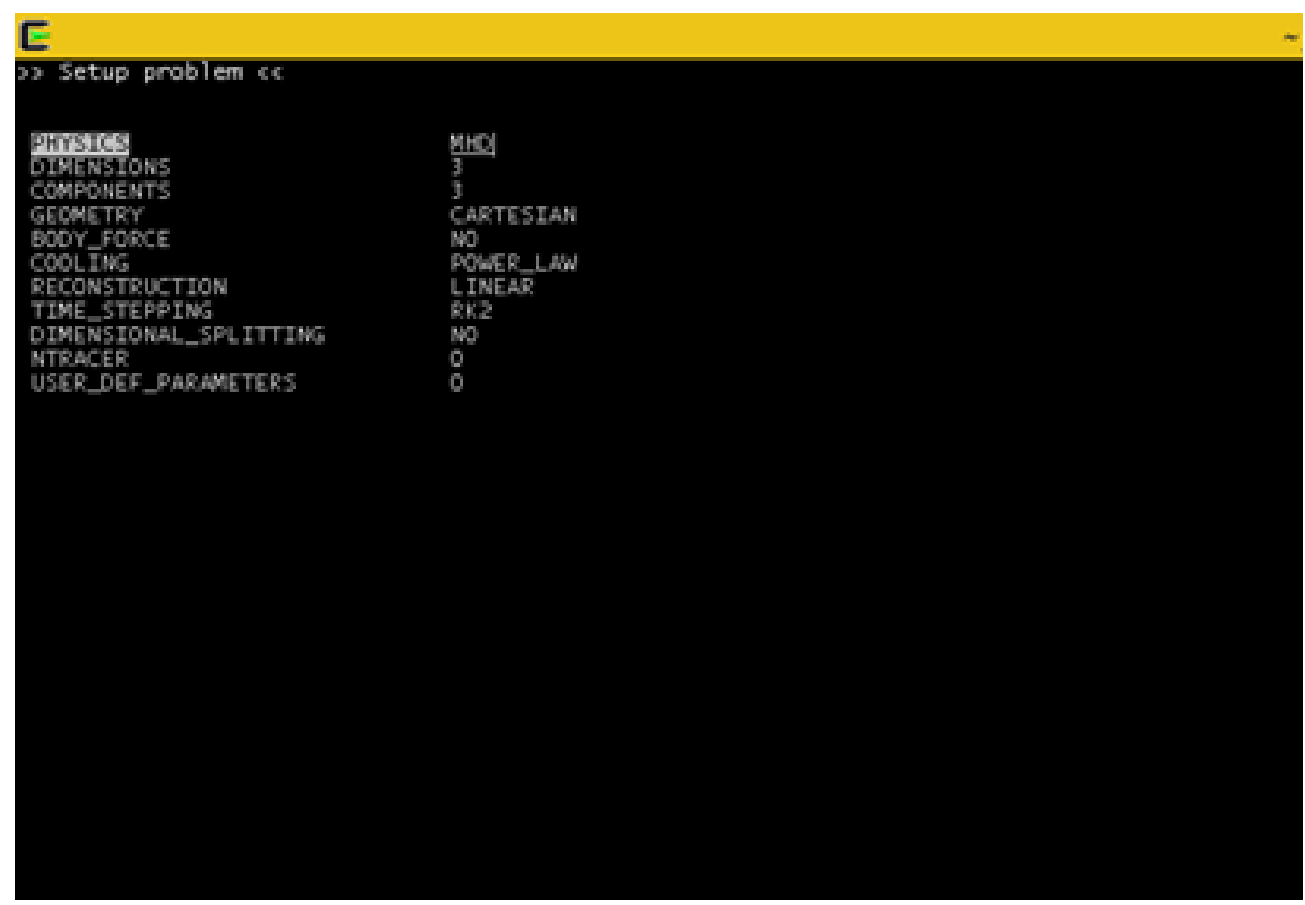

the next screen is:

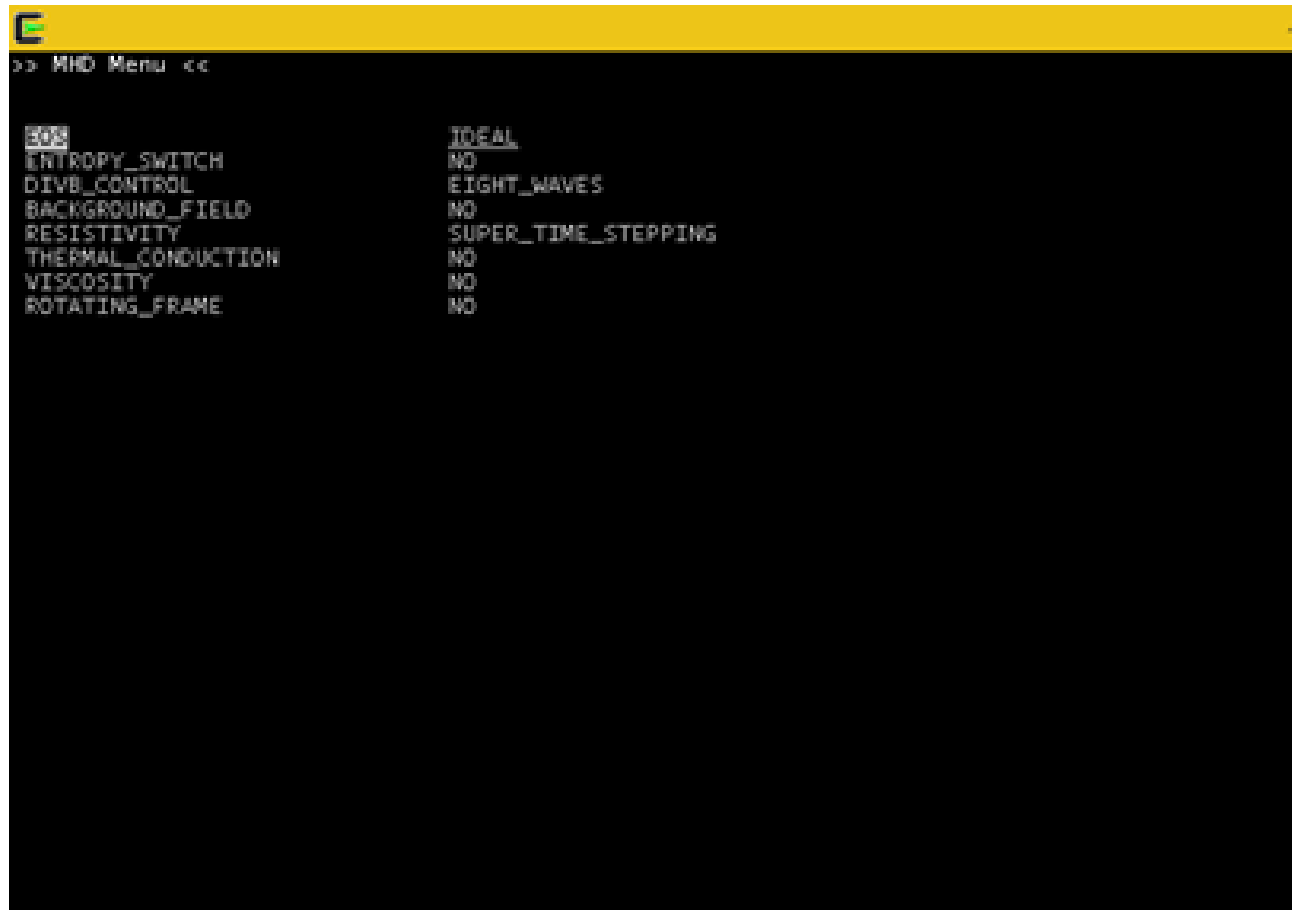

For the first run the User chooses the makefile file to compile PLUTO and link C source code files. For every next run if the User selects Change makefile from the Python setup, a new makefile will be created, otherwise the existing one will be automatically updated. 


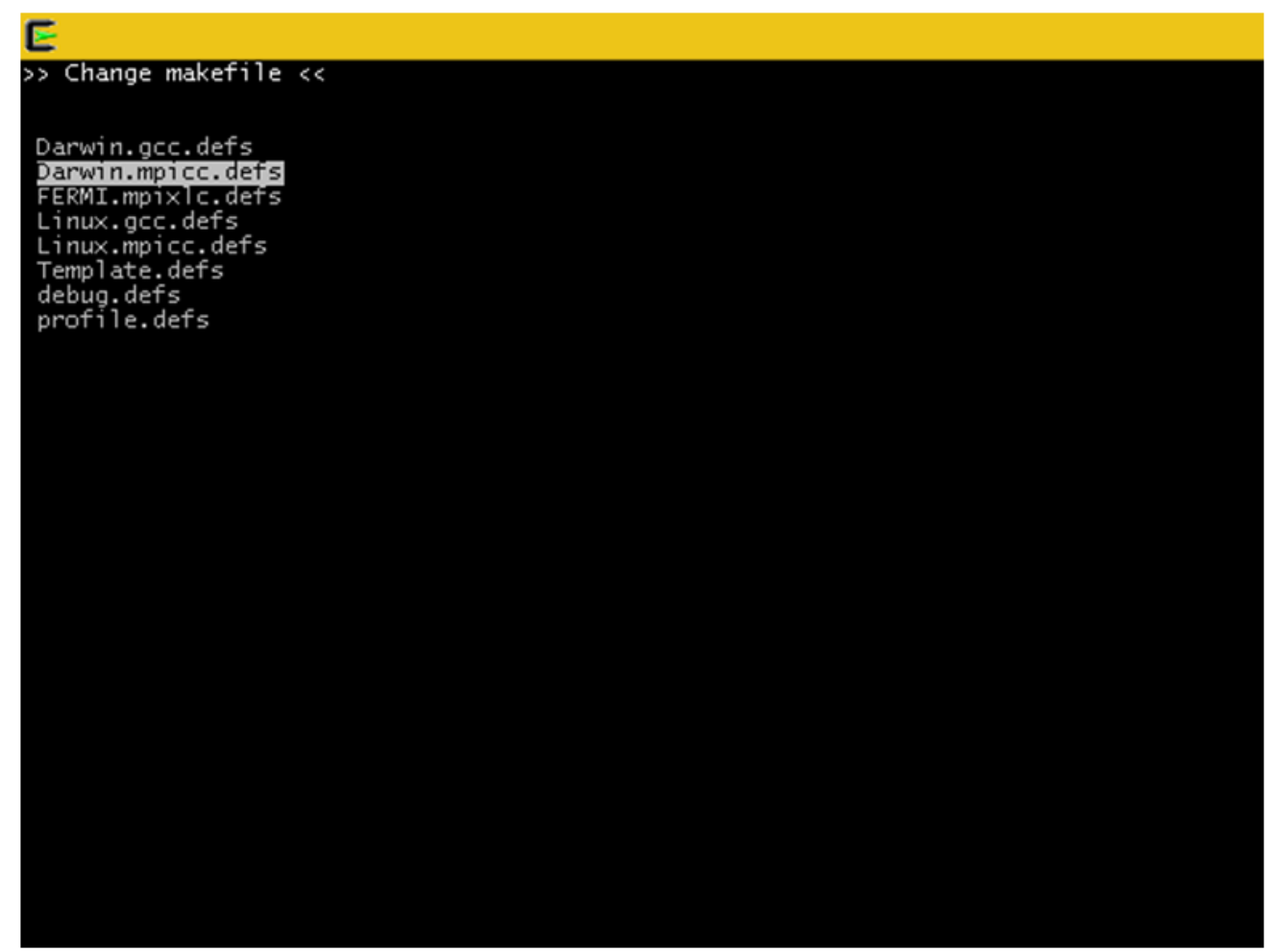

After the creation of the makefile the Python script returns to the first screen and the User selects Quit. If the compilation is successful the User can compile the code with the command:

$>$ make

If the compilation is successful, the User can type:

$>$./pluto for a single run, or

> mpirun ./pluto for a parallel run.

After, the execution of the code begins.

\subsubsection{Setting up the test case of a Z-pinch single wire with the initial form of the code}

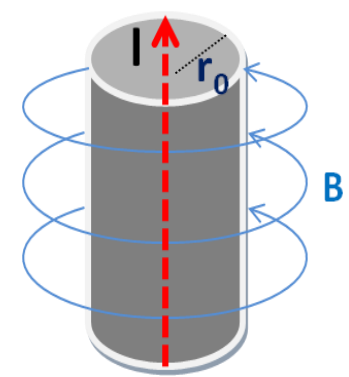

Figure 18.16 Plasma Z-pinch column, the plasma current at Z-axis and its magnetic field lines

$>$ Cylindrical Plasma Column + An Axisymmetric magnetic field

Interplay between thermal pressure (outwards) and magnetic pressure (inwards) 
For $\mathbf{j}_{\mathbf{z}}=$ const., we have in cylindrical coordinates, inside the cylinder, from the Ampere's Law $\left(\mathrm{r}_{0}\right.$ is the plasma radius):

$\mathbf{B}_{\Theta}=\mathbf{B}_{0} \cdot \mathbf{r}$ (Magnitude of the Magnetic field) and $\mathbf{p}=\mathbf{f}(\mathbf{r}$ ) (Plasma pressure) [if there is an equilibrium state $\left.\mathbf{p}=\mathbf{p}_{\mathbf{0}} \cdot\left(\mathbf{1}-\mathbf{r}^{2} / \mathbf{r}_{\mathbf{0}}{ }^{2}\right)\right]$.

And outside:

$\mathbf{B}_{\Theta}=\mathbf{B}_{0} \cdot \mathbf{r}_{\mathbf{0}} / \mathbf{r}$ and $\mathbf{p}=\mathbf{0}$. The maximum magnetic field $\mathrm{B}_{0}$ is calculated as a simple application of the Ampere's Law. The function of the thermal pressure is depending whether it is considered plasma equilibrium or not. The distribution of the mass density can be any function that preserves the conservation of the initial mass distribution. Usually a good choice of both $\rho$ (mass density) and $p$ (thermal pressure) are Gaussian functions due to their smooth transition from the dense plasma regions to the thinner ones.

\subsubsection{Visualization with VisIt}

VisIt is an Open Source, interactive, scalable, visualization, animation and analysis tool [5]. From Unix, Windows or Mac workstations, users can interactively visualize and analyze data ranging in scale from small ( $<10$ core) desktop-sized projects to large $\left(>10^{5}\right.$ core) leadership-class computing facility simulation campaigns. Users can quickly generate visualizations, animate them through time, manipulate them with a variety of operators and mathematical expressions, and save the resulting images and animations for presentations. VisIt contains a rich set of visualization features to enable users to view a wide variety of data including scalar and vector fields defined on two- and three-dimensional (2D and 3D) structured, adaptive and unstructured meshes. Our VisIt version is 2.10.2. It can be downloaded from https://visit.llnl.gov/.

The first page is the following with the command window at the left and the visualization at the right. The first button to press is the Open button and the User can find the files that he wants to visualize from a specific directory. The next step is to use the $A d d$ button to choose the kind of the visualization plot and the physical variable that wants to be visualized. We usually use the Contour and Pseudocolor plot. The User has a lot of choices such as 2D or 3D plots of all the physical variables and he/she can create any variable expression through the Controls $\rightarrow$ Expressions button. 


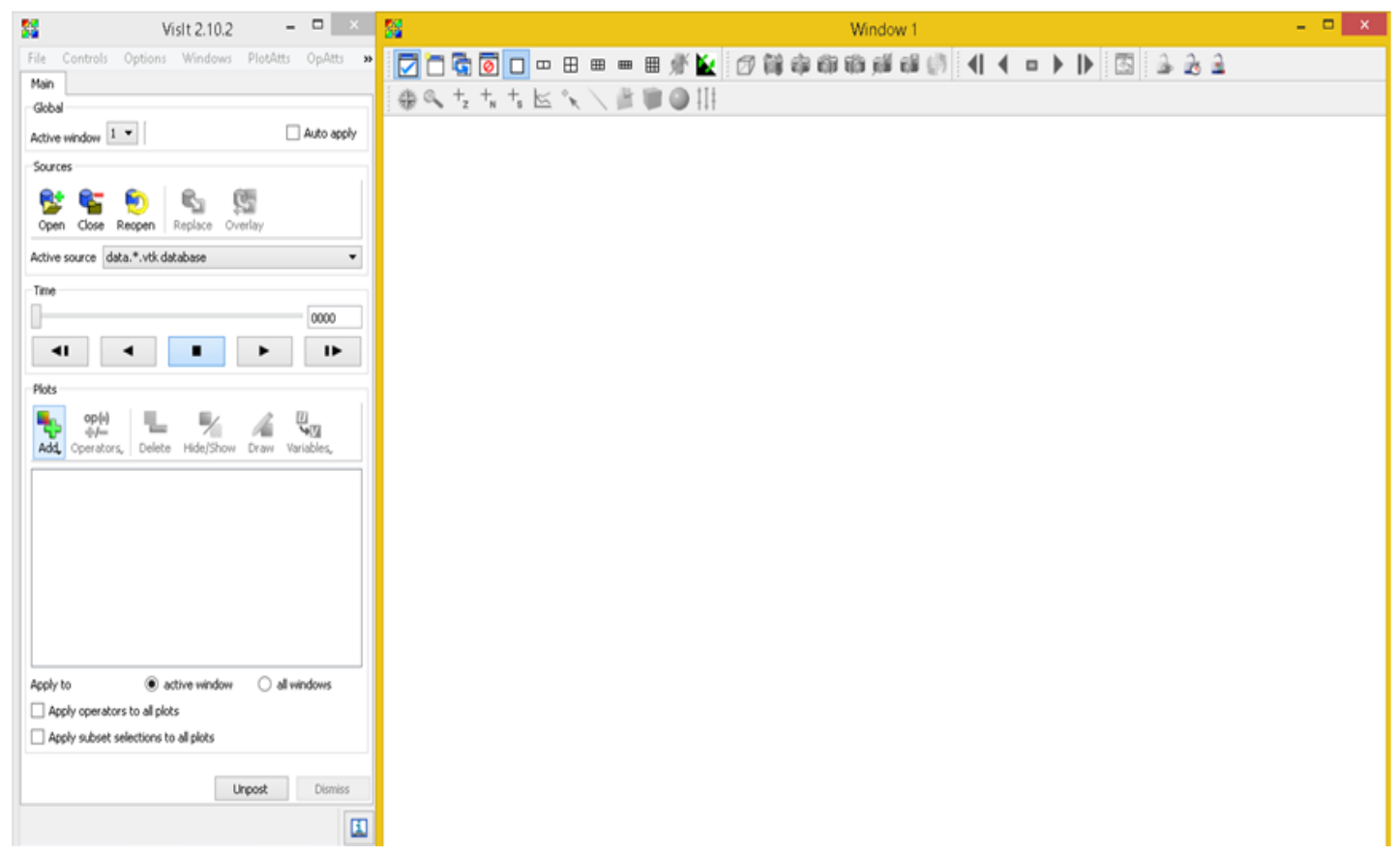

Figure 18.17 VisIt main window

An example of the visualization of the mass density, of the 75 vtk file at the XZ plane of a Z-pinch model is next shown

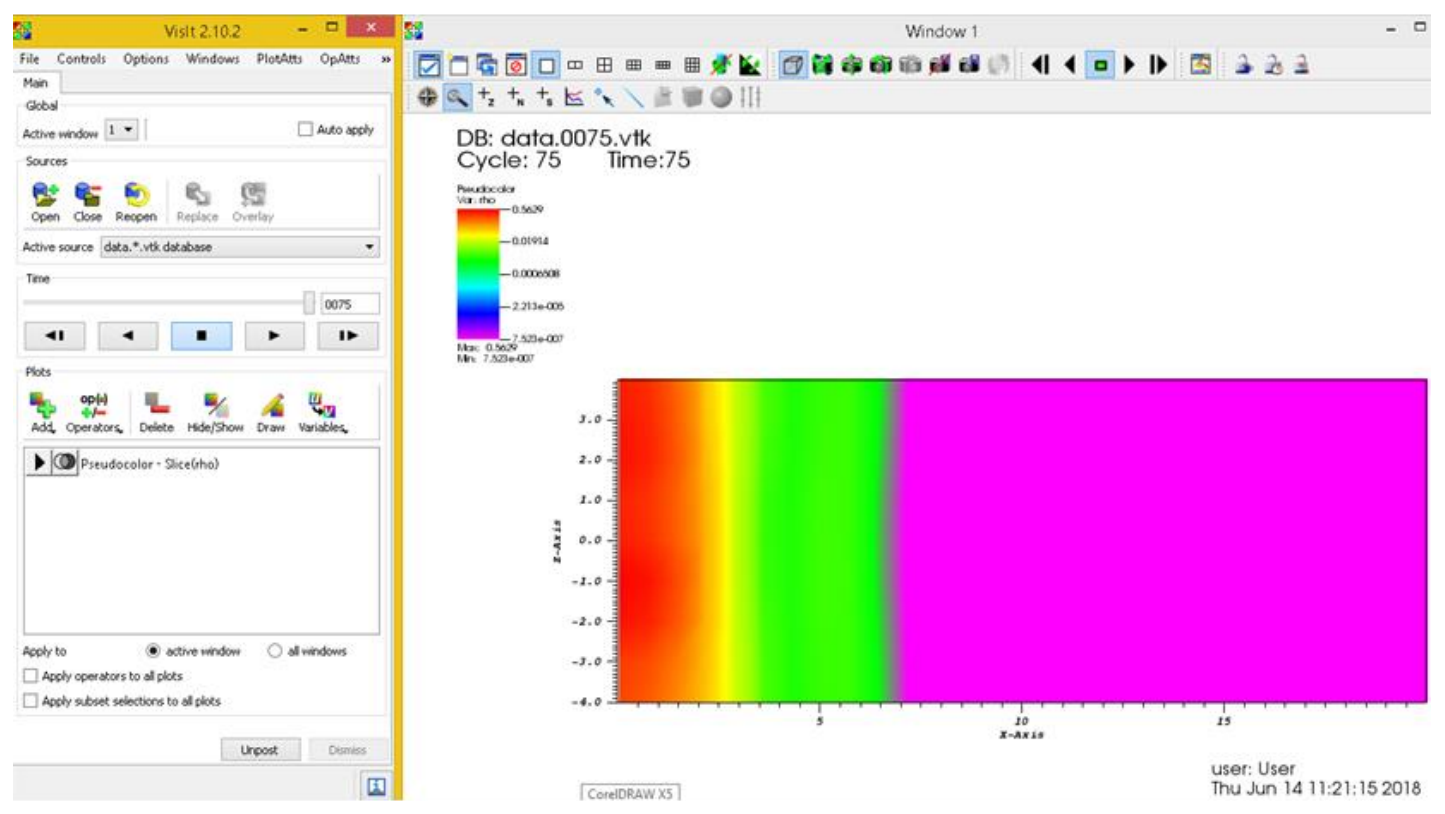

Figure 18.18 Visualization of the mass density

For more information about the plots and the use of VisIt the User can see the manual from VisIt window.

\subsubsection{Running a test case-Exercise 1}

It is time to run a test case. The model of our choice is a Z-pinch single wire. No modifications of the original code exist at this test case. 
- Make a Z_pinch_test file at the working directory. Inside this file are the five important files we mentioned earlier (definitions.h, pluto.ini, init.c, res_eta.c, cooling.c)

- Inside definitions.h the three user defined constants have the same values as in the definitions.h picture. Define the $t_{0}$ (unit time), $p_{0}$ (unit pressure), $B_{0}$ (magnetic field unit) and $\mathrm{n}_{\mathrm{o}}$ (diffusivity unit) in cgs units.

- Inside pluto.ini make a spatial window of $500 \times 500 \times 500 \mu \mathrm{m}$ with 50 points(zones) at each axis with the beginning of the axes at the center of the spatial window. Select tstop=5.0 and evaluate it in nanoseconds. The vtk file format will be selected and we want 10 vtk files at the end of the run.

- The init.c file must be in Cartesian coordinates. Two different regions exist one cylindrical $(\mathrm{r}<=2.0)$ plasma region and the vacuum one $(r>2.0)$. We want Gaussian shapes of the prs (thermal pressure) and rho (mass density) of the form:

$\mathrm{v}[\mathrm{RHO}]=1.0 * \exp \left(-\mathrm{r}^{*} \mathrm{r}\right), \mathrm{v}[\mathrm{PRS}]=1.0 * \exp \left(-\mathrm{r}^{*} \mathrm{r}\right.$ ) for $\mathrm{r}<=2.0$ (material plasma) and $\mathrm{v}[\mathrm{RHO}]=0.01$, $\mathrm{v}[\mathrm{PRS}]=0.01$ for $\mathrm{r}>2.0$ (vacuum plasma).

- Define 2.0 for the plasma radius and 1.0 for the density and pressure, in cgs units. The magnetic field geometry is the one described at the Z-pinch single plasma distribution. The two components BX1, BX2 will have the azimuthal form of the previous description in Cartesian coordinates while the BX3 is zero. The Bo maximum value is 1.0 and must be defined in cgs units also.

- The res_eta.c file must have three components eta[IDIR]=2.0, eta[JDIR]=2.0, eta[KDIR]=2.0 spatial constant. Estimate 2.0 in cgs units.

- Run the code following the steps described previously.

- After the end of the run open VisIt and make 3D Contour plot of the mass density and see the cylindrical plasma evolution along time. Then make 2D Pseudocolor plots at the XY and XZ plane of the mass density and the magnetic field magnitude. The Expressions button must be opened and Bmag=sqrt $(\mathrm{bx} 1 * \mathrm{~b} \times 1+\mathrm{b} \times 2 * \mathrm{bx} 2+\mathrm{bx} 3 * \mathrm{~b} \times 3)$ must be created and applied. See the evolution of the magnetic field. At the end make a lineout plot of the magnetic field at the XY plane at $\mathrm{t}=0$ and confirm the linear rising until the plasma surface and the inverse radius decrease in the vacuum region.

\subsubsection{References}

[1] A. Mignone et al., PLUTO: A Numerical Code for Computational Astrophysics, Astrophys. J. Supplement Series, 170 (2007) 228-242.

[2] G Koundourakis, A Skoulakis, E Kaselouris, I Fitilis, EL Clark, J Chatzakis, M Bakarezos, N Vlahakis, NA Papadogiannis, V Dimitriou, M Tatarakis, A numerical study on laboratory plasma dynamics validated by low current x-pinch experiments, Plasma Phys. Control Fusion 62 (2020) 125012.

[3] http://plutocode.ph.unito.it/

[4] http://plutocode.ph.unito.it/files/userguide.pdf

[5] https://visit.llnl.gov/ 


\section{Acknowledgements}

Acknowledgements: The work has been performed in the Institute of Plasma Physics and Lasers - IPPL of the Hellenic Mediterranean University - HMU, Research Centre. A. Skoulakis has contributed to the MHD modeling and simulations. We acknowledge the support by computational time granted from the Greek Research \& Technology Network (GRNET) in the National HPC facility ARIS under projects LaMIPlaS- I, II and III. 




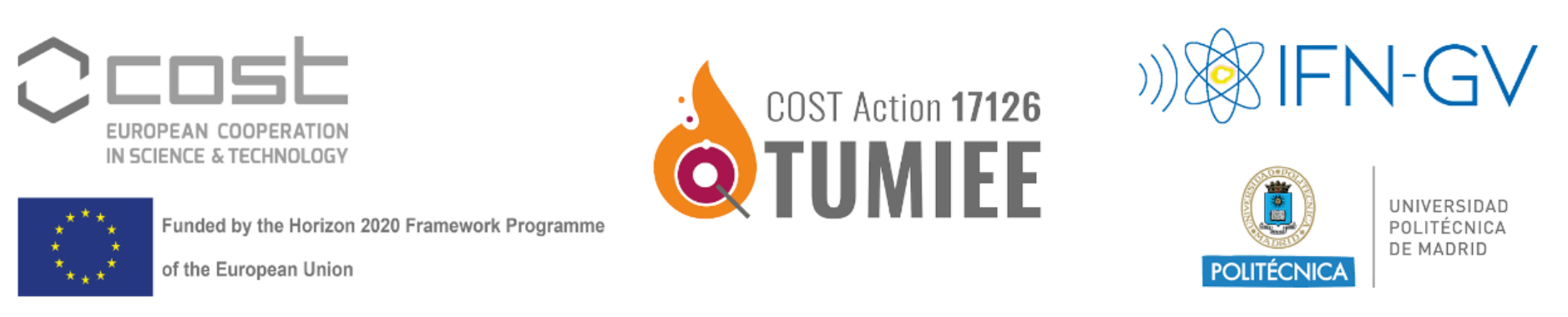

